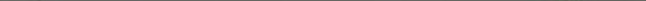




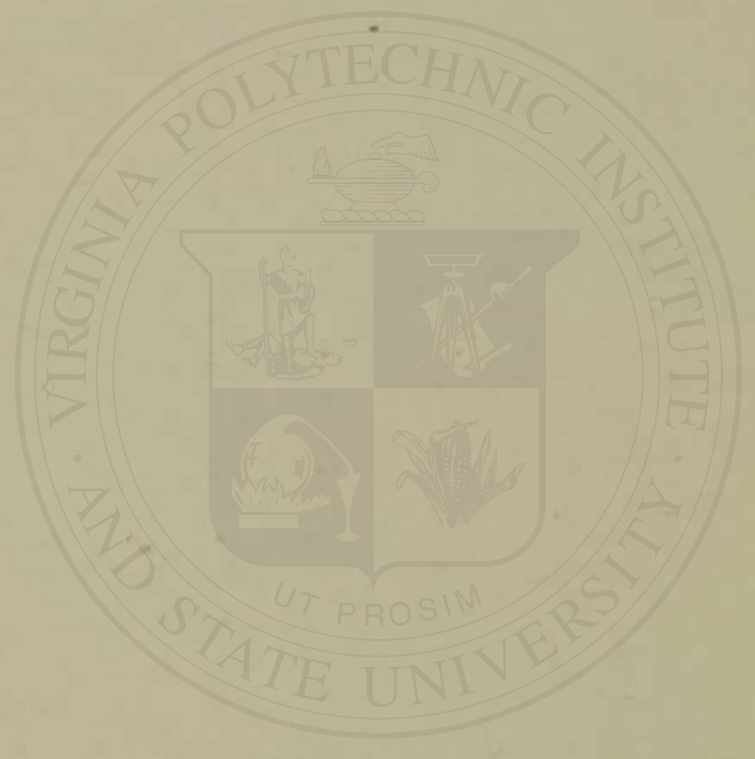


Sarteara Seeder

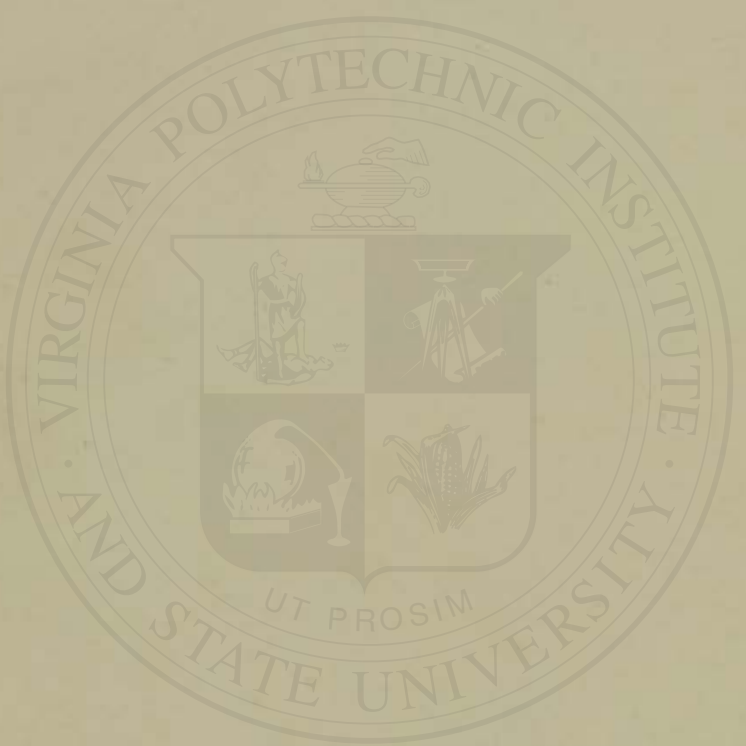

SCANNED AT VIRGINIA POLYTECHNIC INSTITUTE AND STATE UNIVERSITY 


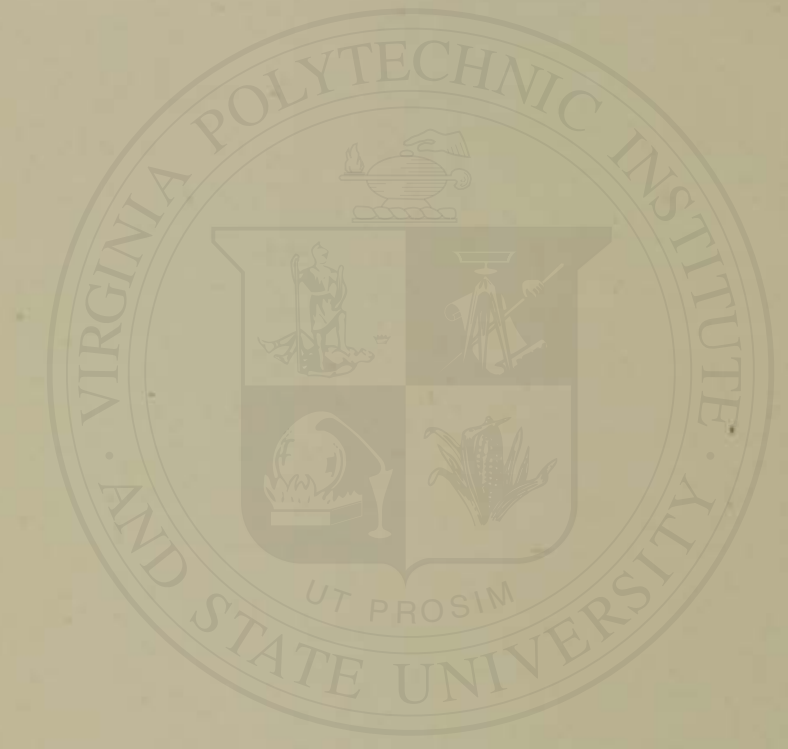




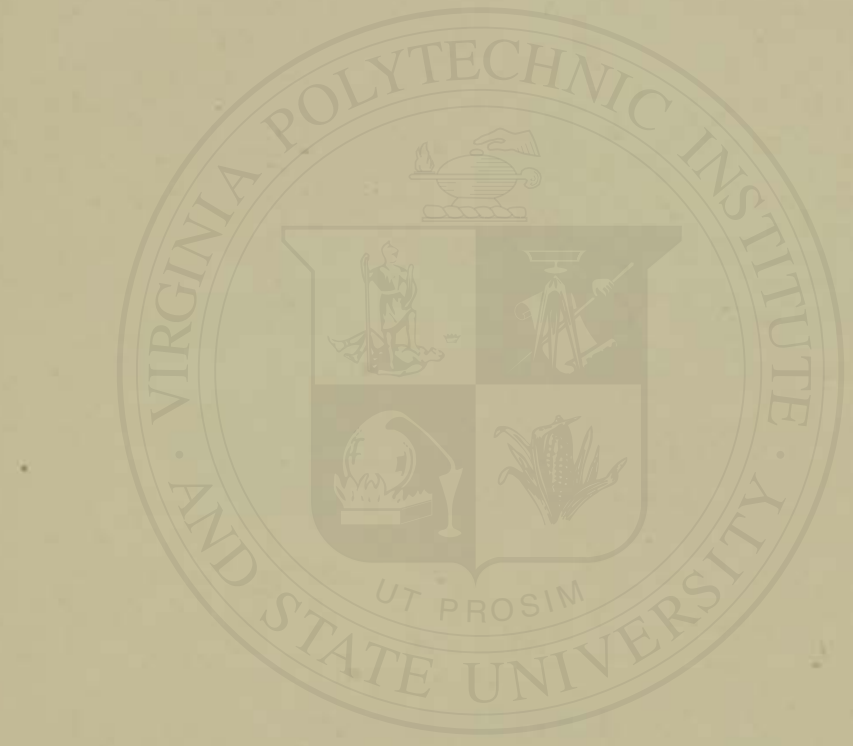




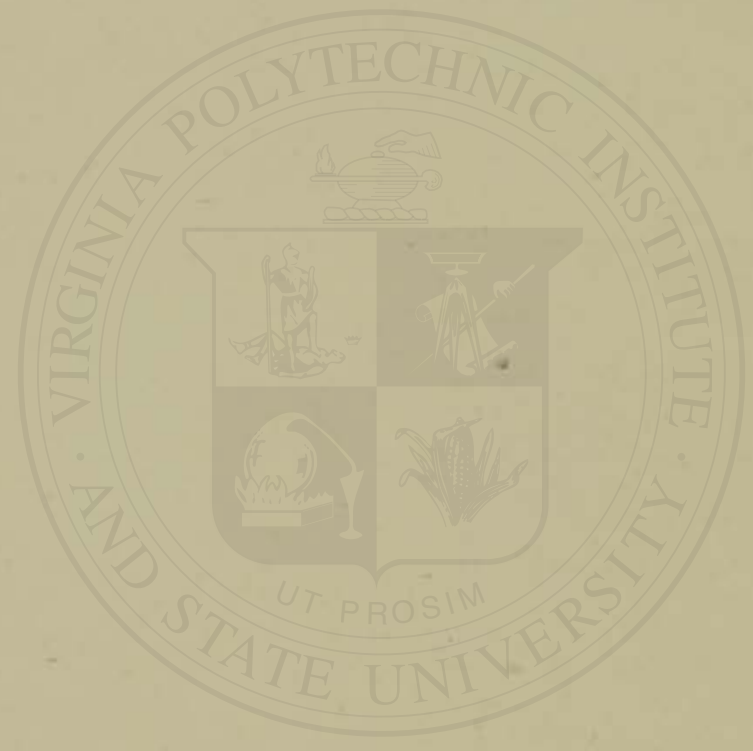




\section{THE NEW DIETETICS}

\section{A Guide to Scientific Feeding in Health and Disease}

\section{BY JOHN HARVEY KELLOCG \\ M. D.. LI. D., F, A. C. S.}

Fellow of the Royal Society of Medicine of Great Britain, of the American Medical Association, Member of the American Economic Association, and of the National Geographical Society, Member of the Societé d'Hysiene of France, Late Member of the Michizan State Board of Health, Editor of "Good Health," Superintendent of the Battle Creek Sanitarium, President of Battle Creek College.

\section{REVISED EDITION}

THE MODERN MEDICINE PUBLISHING CO.

Battle Creek, Michigan, U. S. A. 


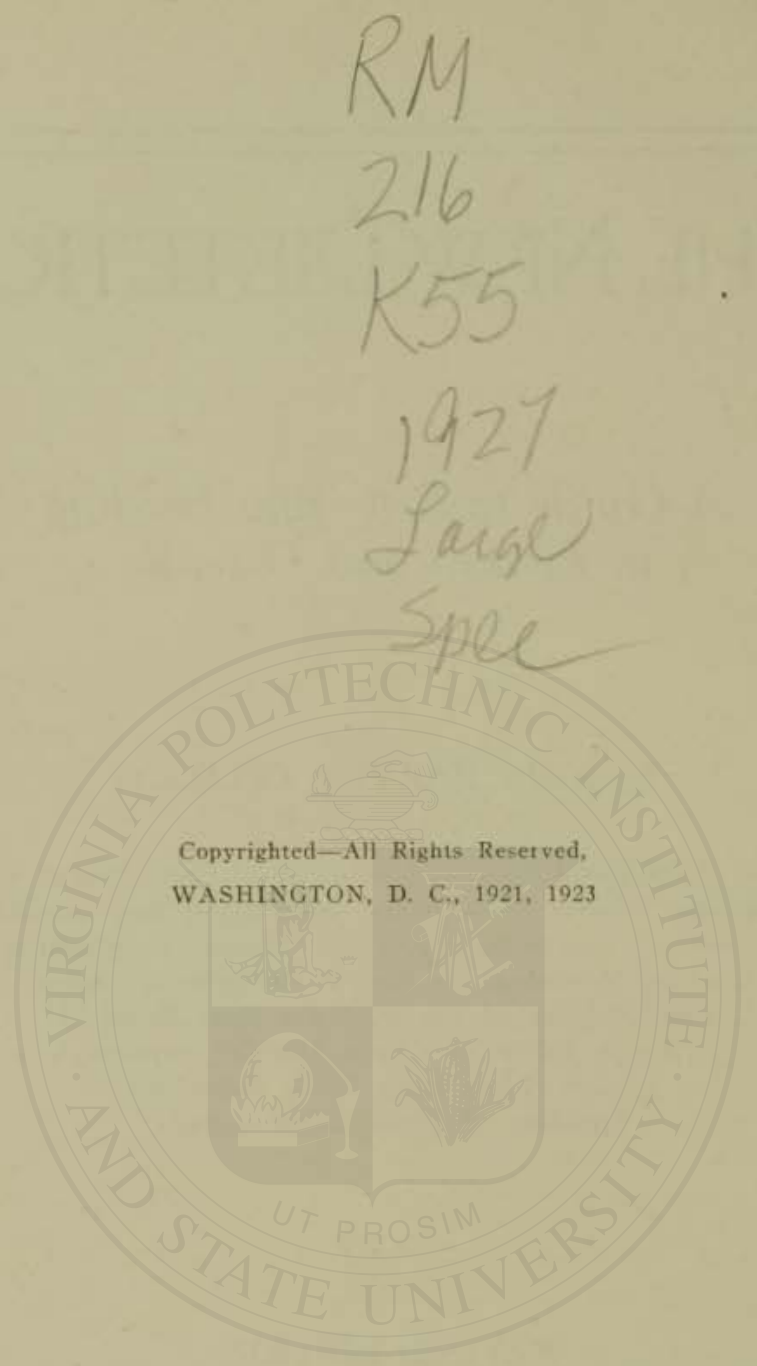




\section{The New Dietetics}




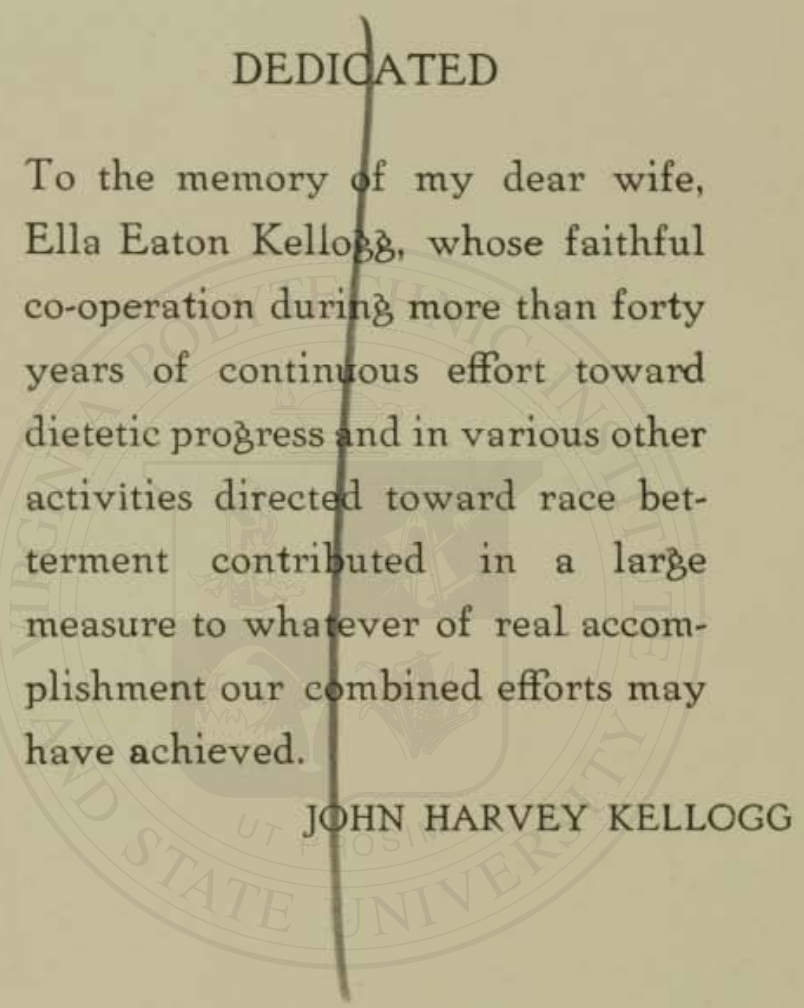




\section{PREFACE}

Within twenty years a new science of dietetics has been developed. As the result of the patient labors of many men, numerous mysteries have been cleared up, many hoary-headed theories have been exploded, and a great flood of light has been thrown upon one of the most important questions related to human existence.

It is now possible to say with a considerable degree of certainty how much food is needed daily by a man, woman or child at rest or at work, and to indicate what particular foodstuffs are best suited to meet any dietetic need which may be indicated in any condition of health or disease. It is also possible to state with reference to every common foodstuff what is its value as a source of energy and what are its special dietary properties and characteristics.

In other words, there now exists a real science of dietetics based on scientific facts worked out in laboratory researches, verified by animal feeding experingents and confirmed by clinical experience. Dietetics is no longer the jumbled mass of empirical notions of which it largely consisted even so late as two decades ago.

Thanks to the painstaking researches of Pavlov, Rubner, Atwater, Benedict, Chittenden, Mendel, Osborne, McCollum and other investigators perhaps equally worthy of mention, we now possess knowledge instead of assumptions, facts instead of fancies, scientific standards in place of empirical though time-honored notions and sanctions.

There is perhaps no place in the world where the successive steps of scientific progress in the knowledge of nutrition and dietetics have been watched with greater care and interest than at the Battle Creek Sanitarium. For more than forty years this institution has been a great clinical laboratory in which an intensive study of foodstuffs and of their effects upon the human body has been continuously carried on by the writer and his associates. As new discoveries have been made in the nutrition laboratories of this country and Europe, the results have been carefully scrutinized and tested in actual clinical practice. It is not claimed that complete knowledge has yet been attained. There is still much to be learned about human nutrition, and the rapid progress which is being made in these days affords good foundation for the hope 
that the advances within the next few years will be fully equal to those made in the past.

In the preparation of this work the author has made use of all available, well attested facts to be found in the authoritative works on human nutrition, and has added thereto the results of nearly a half century's careful study of the subject, theoretically, clinically and experimentally. As editor of "Good Health" since 1873 and Medical Director of the Battle Creek Sanitarium since 1876 , the writer has been in constant contact with men and women enquiring the way to health, and has devoted his life in a large measure to finding the correct answers to the questions, What is the biologic way in eating? How may each particular foodstuff be best utilized as a means of promoting the cure of disease? and similar queries.

So far as the writer knows, this is the first work in which full recognition has been accorded the important discoveries of Bouchard, Metchnikoff, Tissier, and others in relation to the influence of diet upon the intestinal flora through the effect upon the food residues which form the nutrient media for the various organisms, benign and pernicious, which by various means find their way into the human alimentary canal.

In this work a conscientious attempt has been made to set forth only such recommendations as have a solid basis of physiologic fact verified by actual practical experience.

The constant aim of the author has been to present the known facts relating to human nutrition in such a way as to be of greatest service to the physician, the trained nurse, the intelligent housewife, and to every student of nutrition, as well as to the professional dietitian. To this end numerous tables have been prepared by the use of which the dietary may be balanced not only for protein, fats and carbohydrates, but also for cellulose, lime and iron, and even acids and bases. These tables are based upon the data supplied by the tables of Atwater, Bunge, Gautier, Kônig, and Sherman. They have been in constant use at the Battle Creek Sanitarium and elsewhere for several years.

The writer especially desired to acknowledge his indebtedness to a large number of colleagues and assistants who during the last forty-five years have co-operated in his efforts to solve dietetic problems of various sorts, the solution of some of which have resulted in the production of some of the most popular of the present day breakfast foods. First of all I am indebted to my dear wife, 
Mrs. E. E. Kellogg, recently deceased, for most faithful co-operation during many years in the experimental work which resulted in the establishment of the dietary system of the Battle Creek Sanitarium.

To Miss Lenna Cooper, Dean of the Battle Creek Sanitarium School of Home Economics, I am indebted for great assistance in perfecting and systematizing the dietary methods and improving the cuisine upon which the practical suggestions of this book are based.

To Professor Irving Fisher is due credit for first proposing the plan of "food portions" consisting of quantities of food representing 100 calories of energy or a simple fraction of this amount.

To my sister, Mrs. Clara K. Butler, and to Miss Gertrude Estill, I am very greatly indebted for most efficient and indispensable assistance extending over many months in compiling a great mass of notes and menoranda accumulated during many years, and painstaking supervision of copy and proof sheets while going through the press.

I desire, also, to express my appreciation of the courtesy of Robert Smith, Ph. D, of the Sheffield Scientific School of Yale University, in giving the proof sheets of the work a careful reading.

Finally, in presenting this work to the public as the result of a life time study of dietetics from the standpoint of a physician and a believer in Nature as the wisest of all teachers, the writer desires to ask the reader's consideration of the fact that however widely the ideas and methods herein presented may differ from those current in popular and professional usage, they cannot be justly looked upon as simply theoretical or experimental, since they are in daily and successful practical use in a large institution, in the development of which during the last forty-five years they are believed to have been the most important factor. However novel the methods presented may seem to some readers, they are by no means new; they are based upon biologic principles which are as old as the human race and only need a fair trial to demonstrate their value. That the candid reader will accord the views presented the test of actual practical experience with open mindedness and in the spirit of scientific inquiry, is the earnest hope of the writer.

January, 1922.

John Harvey Kellogg. 


\section{PREFACE \\ THE SECOND EDITION}

That a second edition of this work is called for within 15 months of the publication of a good sized first edition is gratifying evidence to the author that the york is appreciated and that it is meeting a recognized need.

It is encouraging to note that the prominence given to the antitoxic diet and changing the intestinal flora in the first edition has been so fully justified by the developments of the last two years that the stress laid upon these most important of all dietetic measures need be in no degree lessened. The importance of changing the intestinal flora is now almost universally recognized by leading clinicians, and the methods pointed out in this book for changing the flora and maintaining a normal intestinal flora have become so thoroughly established by scientific research and extended clinical observation that their value is not questioned by any reputable authority.

The writer desires to expless his appreciation of the many friendly criticisms and valuable suggestions which have been offered by various friends, and especially for suggestions by Helen S. Mitchell, Ph. D. (Yale), Director of the Nutrition Laboratory of the Battle Creek Sanitarium and Professor of Nutrition in Battle Creek College. The author desires also to make amends for an oversight in the first edition by acknowledging his obligations to Dr. E. S. Risley for conducting several laborious chemical inquiries which furnished the data for tables VIII and IX, to Dr. Paul Roth for original data pertaining to metabolism, and to Dr. Welcome Lewis for original data used in tables showing the lime and iron content of several foodstuffs:

September, 1923.

\section{THE THIRD EDITION}

J. H. K.

In preparing this edition for the press several hundred additions have been made to the text and many minor changes and corrections have been made, all of which, it is hoped, will add to the value of the work as a manual and textbook of diet and nutrition based upon the recognized facs and principles of human physiology and the latest findings of the nutrition laboratory research.

April, 1927.

J.H.K. 


\section{Table of Contents}

FOODS

Food as Fuel-The Chemical Composition of the BodyDifferences in the Composition of the Body in Infants and Adults-The Source of Food.

\section{Food Principles}

Proteins-Complete Proteins-A Poisonous Nucleus in Proteins-Anaphylaxis-Carbohydrates-Fats-Food Salts-Vitamins-Cellulose.

\section{THE DIGESTIVE PROCESSES}

Mouth Digestion-The Saliva - Conditions Which Hinder Salivary Activity-Protective Properties of the SalivaThe Saliva in Disease-Regulating Influence of the Sense of Taste-Gastric Digestion-The Fasting Juice-The Gastric Juice-Appetite Juice-The Chemical Juice-The Gastric Acid-Regulation of Acidity and Control of the PylorusPepsin-Lipase-Functions of the Hydrochloric Acid of the Gastric Juice-Bitters Do Not Increase the Secretion of Gastric juice-Recent Studies of the Influence of Food Upon the Gastric Secretions-Influence of Paraftin upon Gastric Secretion-The Influence upon Gastric Secretion of the Introduction into the Rectum of Different Kinds of Oils-Digestion in the Small Intestine-The Pancreatic Juice-The Intestinal Juice-The Bile-Intestinal Absorption and Excretion-Transpertation of the Food-Swallowing-Movements of the Stomach-Passage of Foods from the StomachThe Effects of Heat and Cold-The Movements of the Small Intestine - The Movements of the Colon-The Nerves of the Alimentary Canal-The Normal Intestinal Rhythm.

The Liver

The Liver Aids Digestion-The Liver Stores Food Supplies-The Liver Stores Metallic Poisons-The Liver Probably Detoxicates Organic Poisons-The Liver Makes Ferments and Hormones-The Liver Makes Bile.

Mastication

Spallanzani's Discoveries About Mastication - Horace Fletcher's Discoveries - Special Indications for Thorough Mastication-When the Food Should Be Chewed LessHorace Fletcher's Fatal Mistake.

\section{Gastric Sensations}

Appetite-Satiety-Repletion-Nausea-Hunger in InfantsThe Hunger of Starvation-Hunger During Sleep-Mental and Nervous Influence on Hunger-Absence of HungerAbsence of Hunger in Disease-Increase of Hunger in Disease-Modifications of Hunger-Bitters-Thirst-Air Hunger. 
METABOLISM $\ldots \ldots \ldots \ldots \ldots \ldots \ldots \ldots$

The Energy of Food-The Calorie . . . . . . . . 99-120

The Energy Output of the Body and the Work Equivalent of Food-Vital Work-Metabolism-Basal Metabolism or Metabolism During Rest-The Source of Animal HeatMetabolism and Mental Work-Metabolism and Muscular Work-Energy Expenditure in Work of Various Sorts-Energy Output and Food Requirement of Persons Engaged in Different Occupations-The Energy Expended in Housework-The Relation of Work to Total Food Requirement - The Rate of Growth-Food Requirements by Men, Women and Children-Changes Which Take Place in the Body in Old Age-The Quantity of Food Required in Old Age-Physique and Diet-Diet and Metabolism-Diet and Development-The High Protein Ration.

THE PHYSIOLOGY OF EATING

Protein . . . . . . . . . . . . . . . . . . 124-140

The Chittenden Protein Standard-Luxus Consumption of Protein-Effects of Protein Intake Upon the Urine-A High Protein Diet Increases Intestinal Putrefaction-Complete Proteins-The Normal Protein Requirement-Advantages of a Low Protein Ration.

Fats

Vegetable Fats-Kernel Oils.

Carbohydrates-Starches and Sugars

The Digestibility of Raw Starch-Sugars-Dates vs. Cane Sugar-Substitutes for Sugar-The Nation's Candy BillHoney-Some Odd Sugars-The Consumption of Sugar Should be Reduced.

Food Salts

The Iron Ration-The Daily Requirement of Iron-Where to Find the Necessary Food Iron-The Iron Content of Foodstuffs-To Determine the Iron Content of a Ration or a Dietary by the Use of the Table-Foods Rich in IronThe Lime Ration-The Daily Loss of Lime-Food Lime Is Necessary for Plants as well as Animals-The Influence of Lime on Egg Production-The Lime Content of FoodsFoods Deficient in Lime-Acids and Bases of Foods-The Chemical Balance.

Vitamins

What the Lack of Vitamins Does-The Antiscorbutic Vitamin-The Antineuritic or Anti-beri-beri Vitamin-Yeast Extract Very Rich in Vitamins-The Fat-soluble Vitamin -Vitamin D-Vitamin E-Chronic Avitaminosis-How Vitamins Are Destroyed. 
Cellulose

Bran-Bran Does Not Irritate-Agar-Agar-ManyallaThe Laxative Effects of Clay.

\section{The Acids of Foods}

Tartaric Acid-Malic Acid-Citric Acid-Phytic AcidUnwholesome Acids-Acetic Acid-Oxalic Acid-Tannic Acid-Benzoic Acid-Butyric Acid-Lactic Acid-Uric Acid - The Toxic Effects of Uric Acid-Normal Food Acids do not Acidify the Blood and Tissues.

\section{WHOLESOME AND UNWHOLESOME FOODS}

The Calorie on the Bill of Fare-Method of Converting Percentages in Food Composition Into Calories Per Ounce - Changes in Foods Produced by Cookery-Uncooked Foods.

\section{Cereals}

The Protein of Cereals-The Iron Content of Cereals and Breads-The Lime Content of Cereals-Breads are Deficient in Lime-Wheat-Analysis of Wheat and the Products of Roller Milling-Bread Making-Baking Powders-Wheat Bran-How to Use Bran-The Anti-Graham CampaignCorn-Cornmeal-Cornmeal Mush and Milk-Corn OilWhite and Yellow Corn-Grits-Corn Starch-Corn Syrup -Pop Corn-Green Sweet Corn-Toasted Corn FlakesGofio-Oats-Rice-Polished Rice-Barley-Pearl BarleyRye - Millet - Quinoa - Kaoliang - Buckwheat-Breakfast Cereals-Ready-to-Eat Breakfast Foods-Toasted Cereal Flakes-Sesame Seed Meal.

\section{Vegetables}

Uncooked Vegetables-Roots and Tubers-Root Starches-

The Iron Content of Vegetables-The Lime Content of Vegetables-The White Potato-Potato Poisoning-Potato Cookery-The Sweet Potato-The Dasheen-The TaroSago-The Cassava-Tapioca-The Yautia-The OnionThe Garlic-The Leek-Lily Bulbs-The Water Chestnut.

\section{Green Vegetables}

The Cabbage-Sauerkraut-Brussels Sprouts-CauliflowerBroccoli-Seakale-The Pai ts'ai or Chinese Cabbage-Palm Cabbage - Celery - Lettuce - Asparagus - Endive - White Chicory-Okra-The Artichoke-Iceland Moss-Irish Moss -Agar-Agar-Mushrooms-Yeast and Yeast ExtractGreens-Greens as Roughage-The Iron Control of Greens - The Lime Content of Greens-Spinach-Salads.

\section{Root Vegetables}

The Beet-The Parsnip-The Carrot-The Turnip-Salsify - Vegetable Oyster-The Radish-The Horse RadishKohl-rabi (Turnip Cabbage) - The Jerusalem ArtichokeCeleriac. 


\section{Vegetable or Garden Fruits}

The Tomato-Vitamins in Tomato Juice-The CucumberThe Eggplant-The Watermelon-The Muskmelon-The Squash and Vegetable Marrow-The Pumpkin-The Chayote.

\section{Legumes}

Digestibility of Legumes-Alkalies to be Avoided-The Iron Content of Legumes-The Lime Content of LegumesBeans-The Mung Bean-The Adsuki Bean-String Beans - Shelled Beans-Succotash-The Soy Bean-Composition of the Soy Bean-Soy Bean Milk-To Fu-Soy SauceSoy Bean Sprouts-The Pea-Green Peas-Lentils-Dried Vegetables.

Fruit Acids-Disinfection of Fruits-Fruit Seeds-The Fruit Diet-The Germicidal Properties of Fruits-The Iron Content of Fruits-The Lime Content of Fruits-The Ripening Process in Fruits-Arsenic in Fruits-The Apple -Apple Juice-The Pear-The Quince-The Peach- Prunes and Plums-The Cherry-The Persimmon-The Olive-The Grape-Grape Juice-The Composition of Grape Juice-Berries-The Strawberry-The Currant and the Gooseberry - The Cranberry - The Huckleberry or Blueberry-The Blackberry and Loganberry-The RaspberryThe Mulberry-Wild Berries-Citrus Fruits-The Orange - The Lemon-The Lime-Tropical Fruits-The BananaBanana Flour-Banana Figs-The Fig-The Date-The Guava-The Mango-The Pawpaw-The Papaw-The Pineapple-The Pomegranate-The Avocado, or Alligator Pear-The Karob-Dried Fruits-Canned Fruits-Fruit Juices.

Nuts

The Lime Content of Nuts-The Iron Content of NutsThe Vitamins of Nuts-The Almond-Almond Milk-The Hickory Nut-The Pecan-The Walnut-The English Walnut-The Butternut-The Pine Nut-The Chestnut-The Chinquapin-Filberts, Cob Nuts, and Hazelnuts-The Beechnut-The Acorn-Composition of the Acorn-The Ground Nut-The Brazil Nut-The Paradise Nut-The CoconutCoconut Butter-The Cashew Nut-The Peanut-Peanut Butter-Flour from the Peanut-Peanut Milk-Malted Nuts-Nut Meats-Protose-The Litchi or Chinese Hazelnut-Nut Meals-Nut Creams.

Animal Foods

Special Characteristics of Animal Foods-Meats-The Iron of Meats-Lime Deficient in Flesh-The Acids of Meat Foods-Beef-Mutton-Goat-Venison- Pork - Fowls Fish-Sea Foods-Oysters-Meat Extractives-The Poisons of Meat-Viscera Highly Objectionable-High Meats-Putrefaction Products in Meat-The Bacteria of Meat-Acid- 
osis from a Flesh Diet - Effect of Flesh Eating on Animals - Pernicious Effects of a Meat Diet Experimentally Proven - Parasites of Meat-Canned Meats Usually Not SterileFlesh Meat Not Essential-Raw Beef not an Antiscorbutic - Special Contraindications of Flesh Foods-Eggs-Egg Yolk-Egg White-The Cooking of Eggs-Influence of Food on Eggs-Eggs and Biliousness-Eggs and AcidosisThe Food Iron of Eggs-The Lime Content of Eggs-Egg Substitutes-Preserved Eggs-Bad Eggs-Candling of Eggs -Egg Poisoning-When Eggs Should Not Be Eaten-Milk -Why Milk Sours while Meat Putrefies-The Special Value of Milk Proteins-Milk Rich in Vitamins-Influence of Food of the Cow Upon the Vitamins of Milk-Milk Rich in Salts-The Lime Content of Milk and Cheese-The Iron Content of Milk-Dried Milk-Cream-Butter-Butter Color-Cheese-Cheese Bacteria-Skimmed-Milk-Canned Milk-Buttermilk-Whey-Modified Milk-Milk Must be Clean-Sour Milk-Bitter Milk-Slimy and Colored MilkBacteria on Milk Bottles-Infections Due to Unclean MilkInfected Milk a Cause of Tuberculosis-Injurious Effects of Sterilizing or Pasteurizing of Milk-Certified Milk-A Person May Be Sensitized to Milk-Medical Uses of Miik-How to Eat Milk-Milk a Cheap Food-Relation of Diet to Growth -Goat's Milk.

\section{Condiments}

The Use of Condiments as Preservatives-The Taste for Condiments Unnatural-Chloride of Sodium-Common Salt - Salt Hunger-Poisonous Effects from Salt-Harmful Effects from the Excessive Use of Salt-The Salt-Free Diet - Borax versus Salt-Alcohol-Alcohol Lessens Vital Resistance-Alcohol Lessens Nerve Sensibility-Alcohol Hinders Digestion-Alcohol is a Discredited Drug.

\section{Tea and Coffee}

Tea and Coffee are Poisonous Drugs - Chronic Caffen Poisoning-Coffee Raises Blood Pressure-Tea, Coffee, and Uric Acid-Coffee Cripples the Liver-Other Coffee Poisons-Some Poisonous Effects of Tea and Coffee-Tea and Coffee Neurasthenia-Coffee Insomnia-Tea, Coffee and Indigestion-Decaffeinated Coffee (Kaffee Hag)-MatéCocoa Drinks - Caffein the Cause of Goiter-Soda Fountain Drinks Containing Cafiein-Coffee Substitutes.

Tobacco

The Effects of Tobacco-Tobacco a Heart Poison.

Poisoned Foods

Food Toxins-Hamburger Steak-Moldy Meat-Poisons in the Flesh or Milk of Sick or Overworked Animals-Milk from Tuberculous Cows Poisonous-Poison in Clams-Typhoid from Oysters-Poisonous Ice Cream-Cheese Bacteria - Cheese Poisoning-Poisons in Plant Foods-The Poisons of Molds-Lathyrism-Ergotism-Actinomycosis-Infected Vegetables-The Germs of Fruit Skins-Infection from Flies-Botulism-Parasitic Infection of Foods-Adulterated Foods-Benzoate of Soda-Toxicity of Certain FoodstuffsKeeping the Soil Clean. 
Water Drinking $\ldots \ldots \ldots \ldots \ldots \ldots \ldots \ldots \ldots \ldots .524-530$

Tests for the Purity of Water-Reagents Necessary for Qualitative Examination of Water-Mineral Waters-Distilled Water-Purification of Water with Chlorin.

\section{MEDICAL DIETETICS}

Common Dietetic Errors-Overeating-Underfeeding-Deficiency Diet-Irregular Meals-Hasty Eating-Drinking at Meals-Bad Food Combinations-Highly Seasoned FoodsThe Use of Condiments-Insipid Foods-A Monotonous Diet-Indigestible Foods-Cold Foods-Hot Foods-Eating When Exhausted-Misinformation and Pseudo-Scientific Teaching.

Intestinal Toxemia-Autointoxication

Poisons Produced by Putrefactions in the Colon-Intestinal Antiseptics Harmful-Gastric Toxicity.

Scientific Tests of the Nutritive Functions

Examination of the Mouth-Significance of the Coated Tongue-The Teeth-The Saliva-Ewald Test BreakfastThe Fractional Test Gruel Meal-Advantages of the Fractional Method-Acidity-Bile Regurgitation and Secretion of Mucus-Rate of Emptying-The Water Meal-Test for the Chemical Secretion-Test for the Psychic SecretionTest for the Condition of the Gastric Glands-The Barium Meal-The Motility Test-Examination of the Gall Bladder -Examination of the Stools-Bacteriological Examination of the Stools-The Blood-Chemical Examination of the Blood-Waste Constituents of the Blood-The Urine-Tests of the Urine-Collection of the Specimen-Metabolism Tests-Tests for Acidosis-The Body Weight-Strength Tests-Tests of Heart and Circulation.

Regimens and Dietaries

All Dietaries Must Supply Lime. Iron and Vitamins-The Fruit Regimen-How Fruits Differ-The Daily RoutineThe Green Regimen-When the Tongue Remains CoatedDisorders in Which the Fruit Regimen is Indicated-The Milk Regimen-Technic of the Milk Regimen-Amount of Milk to be Taken-How the "Milk Regimen" Differs from the "Milk Diet"-Practical Suggestions-Results of the Milk Regimen-Supplementary Feeding-Method of Changing the Intestinal Flora-Why the Intestinal Flora Needs to be Changed-The Low Protein Diet-Increased Intestinal Activity-The Displacement of Harmful, Putrefactive Bacteria by Harmless, Acid-forming Organisms-Change of the Intestinal Flora Without the Use of Cultures-To Keep the Flora Changed-When Is the Intestinal Flora Changed? -Diseases in Which the Intestinal Flora Must Be Changed - The Antitoxic Diet-The Low Protein Diet-Protein- 
Free Diet-Fever Diet-The Laxative Diet-Bran-When the Laxative Diet Is Indicated-Tissue-Building or Fattening Regimen-The Fattening Diet-The Fasting RegimenThe Fasting Body Feeds upon Itself-Loss of Weight During Fasting - Objections to Fasting - Safe and Scientific Fasting -The Bland or Non-Laxative Diet-Carbohydrate-Free Diet-Fat-Free Diet-Dry Diet-The Potato RegimenFresh or Raw Diet-Rectal Feeding-Intravenous FeedingWater Drinking-Hot Water Drinking-Lavage of the Blood and Tissues-Gavage.

\section{Diet in Digestive Disorders}

Gastric Symptoms-Significance of Symptoms a Possible Cause of Gastric Symptoms-Reverse Peristalsis-Vomiting -Nausea-A Sense of Fullness-Lack of Appetite-Belching-Heart Burn - Waterbrash - Regurgitation-GlobusBiliousness-Coated Tongue and Foul Breath-Diet in $\mathrm{Hy}$ perchlorhydria or Hyperacidity-Gastric and Duodenal Ulcer - Peptic Ulcer-Dietetic Treatment in Cases of Gastric and Duodenal Ulcer-The Gluten Feeding Method-Lenhartz's Method--The Alkaline Treatment-Gastric Acidity Due to Organic Acids-Gastric Pain-Abnormal Peristalsis-Gastric Motility-Post-Operative Feeding-Gastric Atony-Diet for Hypochlorhydria and Achylia-Pepsin of Little ValuePitters Do Not Stimulate the Stomach-Special Dangers in Achylia-Acute Dilation of the Stomach-MerycismAerophagia-Cyclic Vomiting-Hiccough or SingultusAcute Gastro-Enteric Infection-Stomatitis-Urticaria-A Classification for Dyspepsia Based upon Causes-Dyspepsia Due to Erroneous Habits - Gastric Catarrh in ChildrenDiet in Acute Gastritis-Diet in Chronic Gastritis-Amylaceous Dyspepsia-Diet for Decay of the Teeth-Diet in Constipation-Use of the Enema-Rules for the Care of the Colon to Combat Constipation-The Elimination of Disease Germs from the Alimentary Tract-Diet in Chronic Colitis - Acute Colitis-Dysentery-Diet in Intestinal Obstruction - Diet for Ulceration of the Colon-Diet in Hirschsprung's Disease-Diet for Hemorrhoids-Diet in Appendicitis-Diet in Disorders of the Liver-Tests for the Liver FunctionDiet for Biliousness-Diet for Gallstones and Gall Bladder Disease-Cirrhosis and other Degenerations of the Liver-Diet in Disease of the Pancreas-Diet in DiarrheaDiet in Acute Diarrhea-Dysentery-Diet in Tropical Dysentery-Diet in Parasitic Disease of the Intestine-Diet in Incompetency of the Ileocecal Valve-Atonic ConstipationTapeworm-Diet in Esophageal Stricture.

\section{Diet in Disorders of Nutrition}

Diet in Obesity - Intermittent Fasting-Reduction of Weight by Exercise-Obesity Due to Endocrine Disturbance-The Water Intake-Karell's Method-Oertel's Method-Von Noorden's Method-Banting's Method-The Salisbury Method-Diet in Emaciation-Diabetes-The Dietetic Treatment of Diabetes-The Fat Allowance in DiabetesThe Low Protein Ration Established-The Best Food for Diabetics-Foods to be Wholly Avoided by Diabetics-To Remove Starch from Vegetables-List of Foods Showing 
the Quantities of Each in Which Are Found Five Grams of Carbohydrates-Foods Grouped According to Carbohydrate Content-Insulin-The Pancreatic Hormone-The Use of Insulin (Iletin) - A Simple Method of Approximating the Proper Ration for a Diabetic Patient-Diabetes and Renal Disease-Diahetes in the Aged-Pregnancy with DiabetesDiet in Diabetic Coma-Examination of the Urine-Saccharine-Recipe for Giuten Bread-Gluten Products-SugarFree Milk-Diet in Simple Goiter-Iodine per Ton in Common Foodstuffis-Exophthalmic Goiter-HypothyroidismDyspituitarism-Diet in Addison's Disease-Uremia-The Anemias-Pernicious Anemia-Diet for Acidosis-Infantile Acidosis-Post-Operative Acidosis - Sprue - Pellagra Hodgkin's Disease-Hemophilia-Diet in Scurvy-Purpura -Septicemia - Osteomalacia-Anorexia-Pica.

\section{Diet in Joint Diseases.}

Chronic Rheumatism-Osteoarthritis-Rheumatoid Arthritis - Acute Polyarthritis-Diet in Acute Rheumatism-Diet in So-Called Lric Acid Disorders-Gout.

Diet in Disease of the Heart and Blood-Vessels. . . . 846-855

High Blood Pressure-Apoplexy-Angina Pectoris-Diet in Myocarditis-Senile Heart.

Diet in Nervous Disorders

Headache-Mental Disease-Fpilepsy-Hysteria-Neurasthenia-Sitophobia - Neuralgia - Neuritis - Vagatonia - Diet in Seasickness-Carsickness-Myasthenia-Herpes ZosterTic-Douloureux - Nervousness.

\section{Diet in Disorders of the Genito-Urinary Organs}

Diet in Diseases of the Kidneys Bichlorid Nephritis- Water Drinking-Diet in Cases in Which One Kidney Has Been Removed-Albuminuria-Oxaluria-Diet in Abscess of the Kidney-Gravel and Stone-Pyelitis-Inflammation of the Prostate Gland - Diet in Urethritis - Gonorrhea Cystitis.

\section{Diet in Diseases of Women}

Diet During Pregnancy-Vomiting of Pregnancy-Diet of the Lying-in Woman-Diet in Eclampsia.

\section{Dietetic Management of Fevers.}

Foods Which May Be Used in Fevers-Foods to be Avoided in Fevers-Acute Infectious Fevers-Whooping CoughPoliomyelitis-Cholera-Typhoid Fever-Typhus FeverErysipelas - Influenza-Mumps-Pyemia - Tetanus - Pneumonia and Pleurisy-Pleurisy with Effusion-Diet in Asthma. 
Diet in Chronic Infectious Diseases . . . . . . . . 898-918

Pulmonary Tuberculosis-The Diet in Intestinal Tuberculosis-The Diet in Syphilis-Chronic Parasitic InfectionsDiet in Diseases of the Skin-Diet in Cancer-Cancer of the Colon.

Diet in Surgical Cases . . . . . . . . . . . 19 1931

Post-Operative Feeding-Feeding After Gastro-Enterostomy, Pylorectomy and the Polya Operations - Diet in PostOperative Peritonitis-Jejunal Feeding-Diet After Operation on the Gall Bladder-Diet in Accidents and Emergency Cases-Diet in Coma-A Meatless Hospital Dietary.

Diet in Diseases of the Eye, Ear, Nose and Throat. . 932-934

Infant Feeding $\ldots \ldots \ldots \ldots \ldots \ldots \ldots \ldots \ldots \ldots .935$.956

Feeding Schedule-A Simple Plan for Artificial Feeding of Infants - Infaut Feeding Table-Purées-Vegetable BrothsMalt Sugar-Vitamins-Fats Often a Disturbance-Dangerous Foods-Anemic Infants-Vegetable Soups--The Infant's Stools-Nursing-Weaning-Mixed Feeding-Vomiting of Infants-Diarrhea in Infants and Cholera Infantum-Pyloric Obstruction-Feeding During Fever-Hypersensitiveness of Infants to Certain Foodstuffs-Anaphylaxis-Infant Foods-Vegetable Milk-Humanized Food.

Diet of Young Children

THE WORLD'S FOODS

Edible Foods Growing Wild in the United States-Wild Foods-Stems or Leaves-Roots and Tubers-Seeds-Nuts -Fruits-Plants Cultivated by the Natives of the New World before 1492-Origin of Food Plants.

Making the Bill of Fare

Bill of Fare for Low Protein Diet-Bill of Fare for Antitoxic Diet-Bill of Fare for Fever-Bill of Fare for Protein-Free Diet-Bill of Fare for Fattening Diet-Bill of Fare for Laxative Diet-Bill of Fare for Fruit RegimenBill of Fare for Restorative or Blood-Making Diet-Bill of Fare for Reducing Diet-Bill of Fare for Basic Diet-Bill of Fare for Fresh or Raw Diet-Bill of Fare for Hyperchlorhydria-Bill of Fare for Ulcer Diet-Bill of Fare for Modified Ulcer Diet-Bill of Fare for HypochlorhydriaBill of Fare for Achylia Gastrica-Reducing Diet List-Bill of Fare for Dry Diet-Table of Food Values. 
Index 1012-1031

\section{TABLES}

Table I.

Table II.

Table III.

Table IV.

Table V.

Table VI.

Table VII.

Table VIII

Table IX.

Table X.

Table XI.

Table XII.

Table XIII.

Table XIV.

Table XV.

Table XVI.

Table XVII.

Table XVIII.

Table XIX.

Table XX.

Table XX-A

Table XXI.

Table XXII.

Table XXIII.

Table XXIV.

Table XXV.

Table XXVI.

Table XXVII.

Table XXVIII.

Table XXIX.

Table XXX.

Table XXXI.

Table XXXII.
Chemical Composition of the Body 27

Table Showing Basal Food Requirement ......... 103

Showing Annual Increase in Weight of Boys and

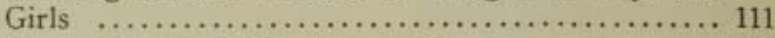

Normal Rate of Development in Boys .......... 112

Showing Normal Rate of Development in Girls.... 113

Showing Normal and Deficient Weight for Different Heights in Boys and Girls ................ 114 Food Requirement of Infants and Young Children.. 118

Food Requirement of Children and Youth ......... 119

Food Requirement of Adults ................. 119

Table of Heights and Weights of Men .......... 120

Table of Heights and Weights of Wornen........ 120

Showing the Effects of High Protein and Low Pro-

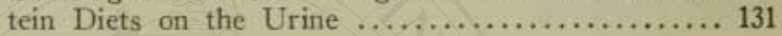

Amount of Intestinal Putrefaction in the Feces with Various Diets ....................... 134

Showing the Amount of Indol Produced in Equal

Quantities of Various Foodstuffs...$\ldots \ldots \ldots \ldots .135$

Comparison of Protein of Nuts with that of Milk.. 138

Showing the Iron Content of Foodstuffs ....... 171

Foods Rich in Iron ...................... 175

The Lime Content of Fcodstuffs .............. 184

Foods Rich in Lime ...................... 188

Foods Rich in Both Lime and Iron ............ 188

Foods Poor in Lime and Iron................... 190

Acid and Basic Properties of Foods ............ 192

Vitamin A. ............................ 208

Vitamin B. . . . . . . . . . . . . . . . . . . 208

Vitamin C. ............................... 209

Vitamin Content of Common Foodstuffs ....... 210

Showing the Relative Value of Various Foods...... 214

Showing the Cellulose in Cereals .............. 220

Showing the Cellulose in Vegetables ............ 220

Showing the Cellulose in Fruits ............... 221

Oxalic Acid in Various Foodstuffs . ........... 237

Uric Acid in Foods (Hall) ................. 240

Percentage of Water in Various Foods before and after Cooking..$\ldots \ldots \ldots \ldots \ldots \ldots \ldots \ldots \ldots \ldots .248$

Gain of Water by Vegetables in Cooking.......... 249

Composition of Corn Products ................. 268

Iron and Lime Content of Greens ............ 308

Amount of Sugar in Common Fruits ........... 331

Showing the Proportions of Acid, Sugar, and Pec-

tin in Some Common Fruits ................... 331

Composition of Fruits .................... 339 
Table XXXIII. Percentage Composition of Dried Fruits ........ 362

Table XXXIV. Composition of Canned Fruits ................ 364

Table XXXV. Composition of Fruit Juices ................. 366

Table XXXVI. Composition of Lime Juices ............... 367

Table XXXVII. Composition and Edible Parts of Nuts .......... 369

Table XXXVIII. Showing the Caloric Value of Nuts Compared to Milk ................................. 374

Table XXXIX. Composition of Milk and Milk Products ........ 436

Table XL. Average Composition of Condensed Milks ....... 449

Table XLI. Composition of the Milk of Animals ........... 469

Table XLII. Sodium Chlorid in Foods .................. 482

Table XLIII. Digestion Table ....................... 997

Table XLIV. Percentage Composition of Foodstuffs and Energy

Value (Calories) per Ounce .................. 1002 


\section{LIST OF ILLUSTRATIONS}

Dr. William Beaumont $\ldots \ldots \ldots \ldots \ldots \ldots \ldots \ldots \ldots \ldots \ldots \ldots \ldots$. 44

Alexis St. Martin ....................... 45

Prof. J. P. Pavlov-Pavlov's Dogs ............... 46

Experiment to Demonstrate Appetite Juice-Pavlov's Pouch 47

The Alimentary Procession (colored) $\ldots \ldots \ldots \ldots \ldots \ldots, 72$

The Alimentary Procession (colored) $\ldots \ldots \ldots \ldots \ldots \ldots, 73$

Starch Granules ...................... 146

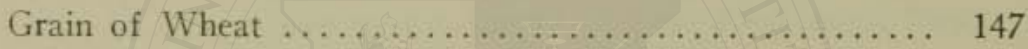

Vitamins-Experiments with Pigeons ............ 196

Vitamins-Experiments with Rats .............. 197

Changes Due to Rickets-Deficiency of Fat Soluble A ... 202

Parasites of Meat-Tapeworm ................ 410

Parasites of Meat-Trichinæ $\ldots . \ldots \ldots \ldots \ldots \ldots \ldots .411$

Appearance of Eggs when Candled ............... 434

The Battle Creek Sanitarium Dairy Barn ........... 435

Veillon Tube Cultures ...................... 568

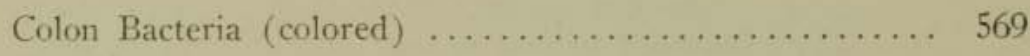

Duodenal Feeding - The Stomach after Gastroenterostomy and Polya's Operation ................... 692 


\section{The New Dietetics}

\section{FOODS}

Any substance to be properly regarded as human food must be capable of supplying the body with the material needed for the repair of its tissues and for maintaining its various functions, while at the same time working no injury to the body and in no way interfering with its activities. There are many substances which possess some of the properties of foods and might be useful nutrients except for the fact that they contain special substances which are inimical to the interests of the body, and hence are injurious. There are, in fact, numerous organic compounds, products of the vegetable kingdom, which are not only incapable of sustaining the life of human beings but prove rapidly destructive. Such substances are not foods but poisons. Alcohol, for example, though oxidized or burned in the body as is starch and sugar, is not to be regarded as a food, for the reason that it possesses toxic properties which render its use unsafe.

A pronounced difference between a food and a poison is the fact that food is harmful only when taken in excess of the body needs, whereas a poison is injurious in all doses, small as well as large. Quantities of poison so small that apparently no effect at all is produced by single doses, when given daily for long periods, produce cumulative effects which generally appear as degenerations of heart, blood vessels, nerves, liver, kidneys or other glands.

\section{Food as Fuel.}

Life has been likened to a consuming fire-a flame. One of the chief functions of food is to serve as fuel by the burning of which the animal heat is maintained, and the energy required for every sort of bodily activity is supplied.

The wastes dismissed from the body through the bowels and the kidneys represent the smoke and the ashes which result from 
vital combustion, which is not simply a burning in the body, but actual destruction or burning of the body.

The vital combustion which constitutes life differs from the fire which burns in a stove or a furnace, not only in the fact that it burns without a luminous flame and in the presence of water, which is fatal to an ordinary fire, but in the fact that while in the case of the stove or furnace the container itself does not burn, having such properties as enable it to resist the action of fire, in the case of the combustion within the bodies of human beings and animals, the body itself, the container, as well as the fuel, is slowly consumed, as would be the case with a stove or a furnace constructed of wood. Indeed, it is even possible that the iron walls of a stove itself may be in time destroyed. Iron will rust if long exposed to air and moisture, and this rusting is a sort of burning akin to the combustions which occur within the body through the agency of the oxygen of the air.

Burning is oxidation. When the oxidation takes place rapidly, the burning is accompanied by a flame and intense heat. If the process is sufficiently slowed down, there is no flame and the heat is moderate in intensity, although the total amount of heat produced by the burning or oxidation of any substance is exactly the same whether the burning is rapid and accompanied by a flame or so slow that no rise of temperature is perceptible to the senses.

That the body is not destroyed by vital combustion is due to the fact that the tissues are continually reproduced or recreated from new material furnished in the form of food.

Food thus supplies material for repair of the living machinery of the body, as well as fuel to keep the body warm and energy for muscular work and other forms of vital activity. Food is also a stimulant to the body cells. Many so-called stimulant drugs, such as alcohol, are, in fact, not stimulants but narcotics. They paralyze the living cells and lower their working efficiency. They waste and do not replenish the energy of the body. Wholesome food is a normal and useful cell stimulant. Drug stimulants are unnatural and harmful.

\section{The Chemical Composition of the Body.}

By an examination of the smoke and ashes resulting from the burning of a house or an animal, it may be possible to determine the nature of the elements which entered into its structure. That 
is, an examination of the ashes of a burned house will show how much iron, lime, and other minerals existed in the house before it burned. Investigations made in relation to the chemical composition of the body have shown it to be as follows:

\section{TABLE I.}

Showing the Chemical Composition of the Adult Human Body. In man weighing

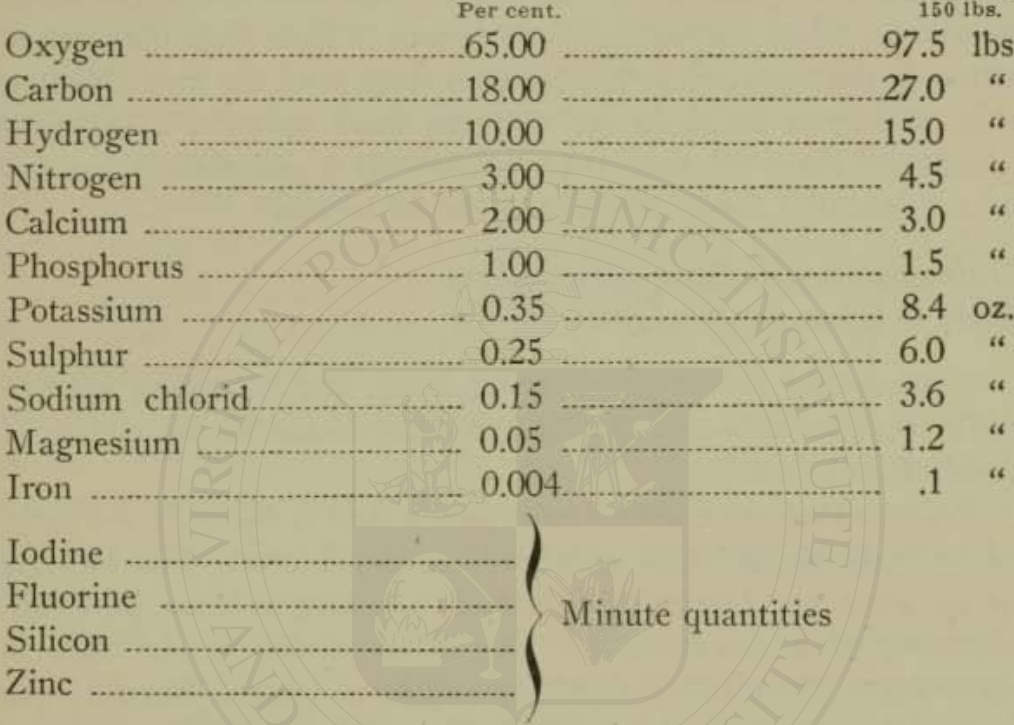
food.

Oxygen is obtained chiefly from the air, but also from the

Hydrogen is one of the constituents of water and is found in nearly all foods.

Nitrogen is obtainable only from the proteins of the food. The air is four-fifths nitrogen, but atmospheric nitrogen can be used by the body only after it has first been assimilated by plants under the influence of the sunlight.

Carbon is obtained chiefly from starch and sugar, but it is also obtained from fats, in which it is found in combination with much hydrogen and a little oxygen, and in small amount from protein.

Iron and lime and other salts are chiefly found associated with protein. They are most abundant in the bran or outer coats of seeds and in the green parts of plants, - the leaves and tender shoots-and in milk and the yolk of eggs. 
The amount of iodine found in the body is very small, yet this minute quantity appears to be essential for the function of the thyroid gland, in the secretion of which it is found.

Recent researches have shown that the metal zinc is a constant constituent of the tissues and of foodstuffs and in about the same proportion as iron. The total amount of zinc in the body of a man weighing 150 pounds is about 30 grains.

The most important mineral constituents of the body from a dietetic standpoint are potash, soda, lime and iron. The magnesia and other mineral substances which enter into the composition of the body are so closely associated with the lime and iron that if care is taken to see that the food contains a sufficient amount of these elements there is scarcely a probability that any of the essential food salts will be found lacking.

\section{Differences in the Composition of the Body in Infants and Adults.}

According to the Chemiker-Zeitung, the following marked differences have been found in the composition of the bodies of children and adults :

The water-content of a new-born infant is about 75 per cent. of its body weight; that of an adult, 58 per cent. The muscles of an adult are 77 per cent. water. Growing infants need considerable quantities of water. An infant needs to take daily in some form, about one-seventh its weight of water. Most of this is absorbed from its food. An adult requires daily about onethirtieth his body weight of water in food and drink.

The amount of water is determined somewhat by the kind of food eaten. When considerable protein is taken, much water is required to carry off through the kidneys the urea and other waste products resulting from its metabolism. Carbohydrates, that is, starch and sugar, require the retention in the body of three times their weight of water, while retained salt requires more than one hundred times its own weight of water.

The cartilages of an infant contain 2.24 per cent. of lime; the bones of a person nineteen years of age contain more than three times as much.

The blood of an infant contains a much larger number of white blood cells than does that of an adult.

Between infancy and the attainment of adult size there is a notable change in the relative size of various organs. The brain 
of an adult is four times as large as the infant brain. The adult liver is eleven times as large, the heart twelve to thirteen, and the lungs twenty times as great as that of the infant.

\section{The Source of Food.}

For all the thousands of various substances which are capable of sustaining animal life we are indebted to the sun. The energy of the sunlight, captured by the mysterious alchemy of the chlorophyl grains found in green leaves, is the source of all human energy as well as of the energy displayed in the manifold activities of animal life in its varied forms. Indeed, almost every form of energy with which we are acquainted is derived from the sun. It is sunlight in modified form which turns all the windmills and water wheels and the machinery which they drive. It is the energy derived from coal and petroleum (fossil sunlight) which propels our steam and gas engines, our locomotives and automobiles. The chlorophyl grains of the green leaf capture the rays of light as they flit by at the rate of 186,000 miles a second, and with this magic force bind together the atoms which form the so-called organic molecules and endow with the mysterious properties of living matter the lifeless particles of hydrogen, oxygen, iron, lime and other chemical elements which enter into the composition of animal and vegetable structures.

Food is simply sunlight in cold storage. The function of the plant is to store the energy borrowed from the sun, preparing it for the service of man and animals that, like the steam engine, the furnace or the lamp, must be regarded as mechanisms for using or expending energy. The heat which glows in the fire on the hearth, the light which shines out from the incandescent lamp or the brilliant electric arc are nothing more than resuscitated sunlight; so likewise the heat of our bodies and the energy of mind and muscle which we are able to display are transmuted sunshine. 


\section{Food Principles}

The many thousands of different substances which may serve as food for human beings and for animals, notwithstanding the enormous differences in appearance, flavor and other qualities, are made up essentially of six simple kinds of materials. These are:

1. Proteins.

2. Carbohydrates.

3. Fats.

4. Salts or food minerals.

5. Vitamins.

6. Cellulose.

It is necessary to understand the chief facts relating to these several food princples in order to have any proper understanding of scientific dietetics.

\section{Proteins.}

The proteins, or albuminous substances, contain, in addition to the carbon, hydrogen and oxygen of the carbohydrates, nitrogen and sometimes also sulphur or phosphorus and iron. The protein molecule is very large, having a molecular weight from five hundred to a thousand times that of water.

As known to the chemist, proteins are the dead remains of the protoplasm or living substance of plants and animals. Proteins are produced by plants. They cannot be produced by the chemist, and the tissues of animals are able only to slightly modify the proteins which plants produce.

Protein supplies the body with the nitrogen, sulphur and phosphorus which, with hydrogen, oxygen and carbon, are the chief chemical elements which enter into the composition of the living structures of the body, the nerves, muscles, glands and other parts which constitute the vital machine. Associated with the protein in all animal structures are to be found iron, lime and other mineral elements, some of which are known to form part of the protein molecule. The white of egg, the lean of meat, the curd of milk and the gluten of wheat are examples of protein. 
Proteins generally contain sulphur, but some do not contain phosphorus. The offensive odors arising from the burning of protein is due to the poisonous compounds which are formed from nitrogen and sulphur. Compounds of these substances formed either in the chemical laboratory or in the mysterious laboratory of the plant are among the most poisonous known to man. Nitrogen is an essential constituent of the high explosives used in warfare. It enters into the venoms of snakes and the virulent poisons produced by bacteria.

Proteins are not so simple in their composition as are other food principles. The protein molecule is, indeed, very complex. It is made up of a considerable number of substances loosely held together by the energy derived from the sun. These simpler bodies or "building stones" which, in a pure state, appear in a crystalline form, are known to the chemist as amuno acids. There are known more than forty of these amino acids. Of these, only eighteen enter into the composition of the human body.

Vegetable proteins differ very greatly in their composition. There are found in the vegetable proteins a considerable number of amino acids which do not enter into the composition of the human body and hence cannot be utilized for tissue building. The number of these "building stones" which enter into the various vegetable proteins differs widely, as well as the proportions in which the different ones are found.

\section{Complete Proteins.}

Every plant produces its own variety of protein, and hence there are almost as many kinds of proteins as there are different sorts of plants. Some proteins are lacking in certain elements (tryptophan, lysin) which are needed for building animal tissues. These are known as "incomplete" proteins. The proteins of meat, eggs and milk are of course "complete."

Most plant proteins are lacking in some one or more of the "building stones" which are absolutely essential to support growth or repair of the living tissues. It is interesting to note that those foodstuffs which have become staple articles of diet throughout the world are the ones which contain the highest quality of protein. Rice, millet, and the potato owe their popularity, in large part at least, to the fact that they furnish an excellent quality of protein. Among the few vegetable products which furnish proteins which are complete may be mentioned 
nuts of various sorts. The soy bean is also notable in this respect. It is particularly interesting to note that while the soy bean furnishes a complete protein, that is, a protein 100 per cent. of which may be utilized in tissue building, the proteins of ordinary peas and beans are worth for tissue building much less than their face value.

The researches of Osborne, Mendel and others indicate that proteins to be able to support growth must contain lysin, an amino-acid which the body is unable to produce. This essential element was found in different proteins in the following proportions :

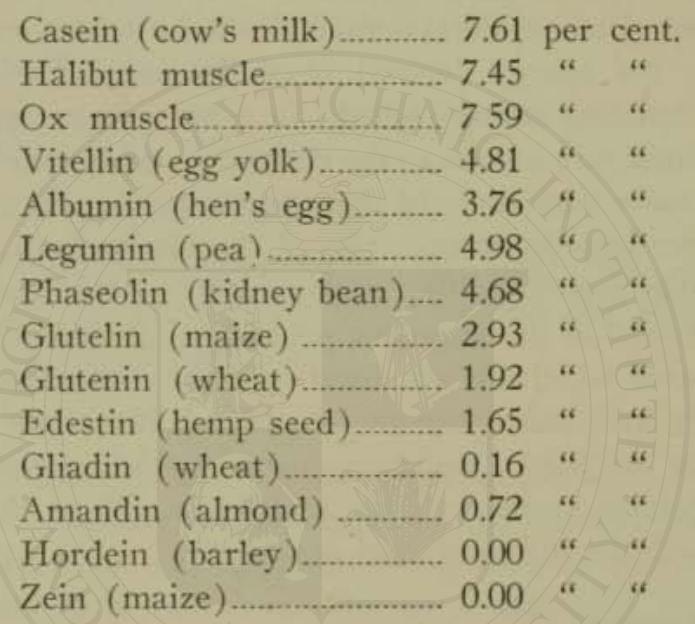

It is interesting to note that those proteins which are biologically intended to promote the growth of very young animals, such as milk and egg proteins, are rich in lysin, which is essential for the formation of animal proteins.

A person who undertakes to live upon a low protein or nonflesh diet must take care to make such a selection of foodstuffs as will furnish a full supply of complete protein; otherwise the body will languish. Lack of energy, and low resistance to disease are the natural result of a deficient supply of complete protein. Ignorance of this fact or neglect to consider it in arranging bills of fare has been the cause of numberless failures in attempts to dispense with the use of flesh foods. Lean meat furnishes complete proteins, which fact is probably one of the chief reasons for its use as a foodstuff. It is important to remember, however, that the proteins of milk and eggs are equally as complete as meat proteins. 


\section{A Poisonous Nucleus in Proteins-Anaphylaxis.}

It is now known that every protein molecule contains a group of atoms which under some circumstances may act as a poison. When protein is digested in the normal way and broken up into its "building stones," this poison nucleus is destroyed; but when the proteins are introduced directly into the blood, the breaking up of the molecule is incomplete and the poison nucleus is set free, and highly toxic effects are produced. These effects are known as anaphylaxis. They are seen in persons who have been sensitized to certain forms of protein, as in strawberry poisoning, buckwheat poisoning, poisoning from the use of oysters, eggs, shellfish, and various other foodstuffs. Hay fever, or pollinosis, is due to the absorption of pollen protein through the mucous membrane of the nose. More will be said further on with reference to sensitization to various foodstuffs.

\section{Carbohydrates.}

Carbohydrates are food principles composed wholly of carbon, hydrogen and oxygen. The hydrogen and oxygen are present in the proportions to form water. There are many different carbohydrates. Those which the body can make use of as food are starch, dextrin, sugar and fruit acids. The utilizable sugars are cane sugar, malt sugar, milk sugar, dextrose and levulose.

Starch is the most abundant of all the carbohydrates, being found in all cereals, in unripe fruits and a few ripened fruits, in certain nuts and in most vegetables.

Dextrin is derived from starch and is practically identical with it in chemical composition. Sugars are also derived from starch.

There are many different kinds of starches. Each plant produces its own variety, the form of which is so characteristic that it may be used as a means of identification.

Of the various vegetable acids, the only ones which are utilized by the body are citric acid, malic acid and tartaric acid. The last named is less fully utilized than the others. Oxalic acid, benzoic acid and some other acids of vegetable origin are not to any extent oxidized or broken up in the body. As found in vegetable foods organic acids are in combination with soda or potash in the form of acid salts. When the acids are burned or utilized in the body, the alkaline soda or potash is left behind; so that the ultimate effect of the utilizable organic acids when eaten is to increase the alkalinity of the vital fluids and not to 
acidify the body fluids, as was formerly supposed to be the case. Carbohydrates are almost exclusively derived from vegetable sources.

Glycogen is an animal starch produced by the liver of animals from sugar brought to the liver in the portal vein after absorption from the stomach and intestines. The body of a fullsized man contains about two pounds of glycogen, which is stored in the liver and the muscles. It is converted into sugar as needed for use as fuel, which is its sole function in the body.

Milk sugar is another carbohydrate peculiar to animals, being exceedingly rare in the vegetable kingdom.

The carbohydrates are used in the body to maintain animal heat and to supply energy for work. Every muscular contraction, every heart beat, every bodily movement of any sort involves the burning or destruction of carbohydrate.

If more carbohydrate is eaten than is required for immediate use, it may be stored in the form of fat, a residual tissue which is intended to be drawn upon when the food supply is cut off or reduced.

Every protein molecule contains a carbohydrate group; and when protein is broken up in the body about half its weight becomes carbohydrate and is thus utilized by the body like sugar or starch, the remainder being for the most part excreted as urea.

The human constitution seems to be adapted to a dietary very rich in carbohydrates. A well-balanced bill of fare will contain more than half, at least three-fifths, of its energy value in the form of carbohydrates. Energy in this form is apparently more readily and easily utilized than in any other form. Sugar, in other words, is the natural fuel of the body.

The natives of the Arctic regions, who are compelled to live on a diet consisting almost exclusively of flesh foods, have an intense craving for sweets. In the short summer season these unfortunate people gather as large a store as possible of berries, leaves, and every edible thing of a vegetable character which the soil of that region produces. Shackleton mentions the intense craving experienced by his men for puddings and other dishes prepared from flour during their first long winter night in the Antarctic.

These facts agree with the observations of modern physiologists that carbohydrates, to the amount of two or three ounces daily, are absolutely essential to the maintenance of good health, 
for the reason that the utilization of fats requires the presence of carbohydrates. As Naunyn says, "Fats burn in a fire of carbo hydrates." A deficiency of carbohydrate soon causes acidosis.

\section{Fats.}

Fats are derived from both animal and vegetable sources They consist chiefly of carbon and hydrogen, with a small amount of oxygen. Every animal produces a fat peculiar to itself. Such fat is formed by the conversion of starch and other carbohydrates into fat by the action of the body cells.

When a surplus of food is eaten, fat is deposited in the tissues in the same form as that in which it is swallowed; that is, if lard is eaten by man it may not be transformed into the same sort of fat which the body forms from starch and sugar, but may remain lard, and be deposited in the tissues as lard. The same is true of beef or mutton tallow and of other fats. A dog fed upon mutton fat becomes round and hard like a sheep and is known in the laboratory as "a mutton dog." It is an interesting fact that vegetable fats are more nearly like the natural fat which the body produces from starch and sugar than are the fats found in the ox, the sheep and the hog.

It is generally helieved that a certain amount of fat in the diet is essential for the maintenance of health; but Hindhede, the eminent Danish physiologist, has shown in recent years that a man may live in perfect health and sustain his strength for many months on a diet from which fat is excluded.

Hindhede's research seems to show conclusively that fat is not so essential as an article of food as it has heretofore been supposed to be. This is the natural result of the fact that the body is able to make its own fat from starch and other carbohydrates. Another fact of great interest has an important bearing upon this point. A subtle element, the growth-promoting vitamin, essential for health as well as development, is often, though not always, associated with fats. When the fat is lacking or deficient in quantity, this element is likely also to be lacking; and because of this association, which has only recently come to be understood, injuries resulting from the withholding of fats have very naturally been attributed to lack of the fat itself rather than to the absence or deficiency of the growth-promoting vitamin with which the older physiologists were not acquainted. This element is abundant in butter but is deficient in lard, in some vegetable oils and 
many other fats. It is also plentiful in greens, which Hindhede used as an essential part of his bread and potato diet.

Fats, if not absolutely essential, are a wholesome and useful constituent of the dietary. More than any other food, they produce the sense of satiety or satisfaction after eating. They are the most concentrated of all forms of foodstuffs, having an energy value more than double that of either carbohydrates or proteins. If eaten in excess, fats usually produce a rapid gain in flesh, encourage intestinal putrefaction, with rancid stools, and are apt to give rise to a troublesome and even dangerous condition known as acidosis. It is strictly physiologic that fats be eaten with cereals or breadstuffs, for the starch which is found abundantly in cereals, as has already been shown, is necessary for the proper utilization of fats by the body cells.

Hindhede of Copenhagen informed the writer that one of his subjects, an athlete, lived for twenty-two months on a diet from which fats of all sorts were rigidly excluded. The food consisted of bread prepared from the whole wheat, with potatoes and greens. It was remarked that the consumption of large quantities of greens were found necessary to keep the patient in good condition.

When on a visit to Hindhede's laboratory in Copenhagen recently, the writer had the pleasure of meeting the subject of this experiment, Mr. Madsen, and found him to be an exceedingly robust and athletic man, apparently enjoying perfect health, and in no way endangered by his long abstention from fats and the numerous other dietary experiments of which he has been the subject during the last twenty years.

\section{Food Salts.}

Eight mineral elements, viz., calcium, sodium, potassium, magnesium, iron, phosphorus, sulphur and chlorin, constitute about 4 per cent. of the weight of the body, or about five or six pounds in a person of ordinary weight. These so-called minerals or mineral salts are not for the most part found to any extent either in the body or in foodstuffs in a strictly inorganic-state. It is true that chlorin and sodium in the form of sodium chlorid or common salt and phosphorus in the form of phosphate of lime are found in small amounts in foods in an inorganic state; but iron, sulphur, phosphorus and calcium, in general, exist in the body only in organic form. The presence of iron in its ordinary metallic state may be easily recognized by the application of sev- 
eral chemical tests. But organic iron, that is, food iron and the iron of the tissues, cannot be detected by any of these tests, showing that in the chemistry of the plant some subtle change is effected which gives to the so-called mineral elements of the food properties which in their inorganic state they do not possess-in other words, prepares them for use by animals as food.

The body loses daily in the excretions from two-thirds of an ounce to an ounce of salts-chlorids, sulphates, phosphates and other salts of calcium, magnesium, sodium and potassium. To make good this loss, the daily food must contain at least an equal amount of organic or food salts.

\section{Vitamins. See fu. 194}

Vitamins are subtle substances other than carbohydrates, fats, proteins or salts which are essential for growth, development and good nutrition. Their purpose seems to be to activate or stimulate the various functional activities of the body. The term vitamin was given by Funk to a substance which he isolated from foodstuffs and which in minute quantities was found to be capable of curing and preventing beri-beri, a fatal disease very common among rice eating people. This substance is found abundantly in the bran and germ of seeds, grains and yeast and in greenstuffs, fruits and to some extent in most foods. It is deficient or absent in polished rice, superfine flour, new process or degerminated corn meal, sugars, many other denatured foods and in fats. This vitamin was given the name water-soluble B by McCollum. When the word vitamin is used alone, it is generally understood that reference is made to vitamin B.

Several other vitamins have since been discovered, the most important of which are fat-soluble A, found in butter, cod liver oil and other fats and in green leaves and young sprouts, and water-soluble $\mathrm{C}$, the so-called antiscorbutic vitamin, the absence of which gives rise to scurvy. This vitamin is found most abundant in green sprouts, fresh vegetables and the juice of the orange and the tomato.

Vitamin D is a fat soluble substance the presence of which prevents rickets. Recent studies have shown that rickets may result from a deficiency of lime or phosphorus, or the absence of vitamin D. The discovery that animals or human beings could be cured by sunlight, or by exposure to the ultra-violet rays, led to investigations which have shown that vitamin D is produced by the action of the sunlight upon cholesterol. 
In the absence of vitamin D, reproduction fails. There is also diminished vital resistance and an increased susceptibility to infectious diseases. This is especially true with reference to diseases of the lungs and air passages.

Vitamin D is generally found associated with vitamin A, from which it has only recently been distinguished. It is most abundant in butter, yolks of eggs, green vegetables, whole milk and cod liver oil.

Exposure of the skin to the sunlight will cause the disappearance of rickets without a change of diet, doubtless for the reason that vitamin $\mathrm{D}$ is produced by the action of the sun upon cholesterol, which is always found in the skin.

The importance of vitamin $\mathrm{D}$ will be recognized when it is recollected that in some sections of the country, such as the Great Lake region, there is wide prevalence of rickets. Surveys have shown that in certain sections nearly nine-tenths of the population gave evidence of injury from rickets.

Vitamin E is a fat-soluble substance which is essential to reproduction. When this factor is lacking, gestation occurs but is not completed, the developing embryo being absorbed.

This vitamin is found in milk and butter and is abundant in seeds and green leaves. Wheat embryo is very rich in it, and it is found to be present in corn oil and cottonseed oil. It is not present in any quantity in liver or cod liver oil.

No evidence has been offered that a deficiency of vitamin E is responsible for sterility in human beings, and it is so abundant in most natural foodstuffs that it is not of great importance from a practical standpoint.

Vitamins are produced only by plants. They are found to a limited extent in some animal tissues, particularly in the liver.

\section{Cellulose.}

Cellulose is an insoluble substance which forms the framework of plants and plant products. It is not acted upon by the fluids of the human digestive apparatus, although it is digested to some degree by herbivorous animals and even constitutes the principal food of certain low forms of animal life. Cellulose is a highly important constituent of the diet of human beings serving to give to the food mass the necessary bulk to enable it to move along the alimentary canal at the proper rate. 


\section{THE DIGESTIVE PROCESSES}

A complete account of the chemical changes which take place in foodstuffs in passing through the alimentary canal would require the space of a large volume. The essentials of the whole subject may, however, be condensed into a brief space. Here is the subject in a nutshell:

There are practically four digestible foodstuffs-starch, albumin or protein, fats and sugar.

There are five food laboratories - the mouth, the stomach, the small intestine, the colon and the liver.

There are five digestive fluids - the saliva, the gastric juice, the bile, the pancreatic juice and the intestinal iuice.

The saliva digests starch.

The gastric juice digests protein.

The bile aids in the digestion of fats.

The pancreatic juice digests starch, protein and fats.

The intestinal juice digests all foodstuffs.

Starch is digested by the saliva, the pancreatic juice and the intestinal juice.

Protein is digested by the gastric juice, the pancreatic juice and the intestinal juice.

Fats are digested by the bile, the pancreatic juice and the intestinal juice.

Sugars are digested by the intestinal juice.

The "salts" are dissolved by the several digestive fluids.

\section{Nouth-Digestion.}

The mouth is a mill. It grihds the food by a gradual reduction process, while it is at the same time being macerated or softened and mixed with the saliva But the reduction of the food to the consistency of a soft paste is but a small part of what happens in the mouth laboratory. The chewing of the food sets in operation the whole series of food laboratories. When the movements of the jaw begin, the salivary glands start to pour out the saliva, the gastric glands secrete gastric juice, and all the other laboratories prepare for the work that they are expected to do: 
and beside this, the movement $\$$ which carry the food into the stomach, while depositing the foodstuffs in this chamber for further attention, send on beydnd the stomach peristaltic waves which reach the colon, and with such vigor that its contents move forward four times as fas during the eating of a meal as in the intervals between meals.

\section{The Saliva.}

The three pairs of salivary glands together produce daily three or four pints of saliva, about the same amount of secretion produced by the skin and also by the kidneys. The saliva differs from the sweat and the urine in the fact that it is reabsorbed.

The salivary secretion is absent in fishes. The saliva of dogs contains no ferment or digestive principle, probably because meat contains no carbohydrate for the saliva to act upon. The same is true of the saliva of infants.

Human saliva, like that of the big apes and other frugivorous animals, is very rich in ferments. In man, the character of the saliva is influenced by the nature of the substances in the mouth during mastication.

Nearly thirty years ago, the writer showed, experimentally, that the quality of dryness in the food is one of the most powerful stimulants of salivary activity. In the mastication of an ounce of dry toast without other flavoring than a small amount of salt twice its weight of saliva was secreted in five minutes. An ounce of water held in the mouth for the same length of time was scarcely at all increased in weight. The movements of mastication increase the activity of the salivary glands, perhaps by increasing their blood supply. Acid and alkaline substances powerfully stimulate the flow of saliva, as do, also, savory substances and foods which appeal to the appetite. Pepper, as well as other irritating and distasteful substances, causes an abundant flow of saliva, as also do substances which give rise to nausea.

The chewing of hard, insoluble and hence tasteless substances does not increase the flow of saliva to any considerable extent. The saliva produced by the chewing of food is much more active than that produced between meals. The saliva produced by the chewing of paraffin will in four hours digest twice its volume of a one per cent. solution of boiled starch.

The saliva contains two digestive ferments. One of these simply liquifies the starch. Another, amylase, converts the starch first into dextrin and then into malt sugar or maltose. 
The saliva acts very slowly upon raw starch, and on the whole is much less active than is the pancreatic juice in the digestion of starch.

When first secreted, the amylase of the saliva is inactive. It is made active by very minute quantities of acids and alkalies, but is rendered inactive by strong acids and alkalies, also by bacteria.

The action of the saliva is not completed in the mouth, but continues for an hour or two after the food passes into the stomach, or until the gastric contents become sufficiently acid to arrest the activity of the salivary ferments. Recent experiments indicate that the activity of the saliva is resumed in the small intestine after it comes in contact with the alkaline intestinal secretions.

\section{Conditions Which Hinder Salivary Activity.}

Careful determination of the activity of the saliva, made in the laboratories of the Battle Creek Sanitarium under the writer's supervision, show enormous differences in the digestive activity of different persons. In some cases the activity was found to be sufficiently great to digest all the starch ordinarily eaten, while in others the degree of activity was scarcely onetenth or even one-twentieth as great.

The average of a thousand cases taken at random from a series of more than forty thousand in which the activity of the saliva was carefully determined by the method devised by Sir William Roberts showed the complete digestion of about half of all the starch liquified in the mouth and stomach.

Bacterial infections of the mouth and stomach interfere with the function of the saliva by rendering its ferment inactive. The saliva is also deteriorated by the excessive secretion produced by gum chewing and the chewing of tobacco. The saliva in such persons has very little ferment. The prolonged stimulation exhausts the secreting cells so that the saliva becomes inactive.

Taylor very aptly says of gum chewing and tobacco chewing:

"Vicious habits, like the vulgar chewing of tobacco or gum, keep the salivary glands constantly at work, and the results are as bad physiologically as the practices are reprehensible to good taste."

Recently reported observations indicate that imperfect insalivation of the food and incomplete salivary digestion inter- 
fere w'th gastric digestion not only by introducing the food into the stomach in an imperfectly comminuted form, but through the absorption of the pepsin by the undigested starch. It is thus evident that thorough chewing of the food is necessary both to insure the complete digestion of starch and also to promote gastric digestion. It is probable that in the majority of persons not more than a fourth of the starch eaten is digested by the saliva, and in many cases the proportion must be very much smaller than this.

Sir William Roberts, many years ago, showed that acetic and oxalic acids interfere with digestion by preventing the action of the saliva upon starch. The amount of acetic acid found in a teaspoonful or two of vinegar was shown to be sufficient entirely to stop the action of the saliva. Oxalic acid diluted to one part in 10,000 was found to arrest the action of the saliva completely. The injurious action of the fruit acids, citric, malic and tartaric, is comparatively slight.

Citric acid powerfully stimulates the flow of saliva, and the saliva produced is very active in digesting starch.

Tannic acid interferes with the action of the saliva. Hence tea and coffee interfere with the digestion of starch, and for this reason alone should be discarded, since they cause indigestion as well as other disorders of various sorts.

\section{Protective Properties of the Saliva.}

It is known that wounds in the mouth generally heal quite readily. Clairmont, of Vienna, has shown that the saliva possesses valuable properties as a cleansing agent. Although it has no direct bactericidal action upon such pathogenic organisms as the bacilli of typhoid fever and tetanus, the colon bacillus or pusproducing organisms, some bacteria are destroyed by contact with human saliva. The saliva of goats and other ruminants, especially parotid saliva, was found, moreover, to have quite marked bactericidal properties. Clairmont's observations led him to believe that the saliva maintains in the mouth conditions unfavorable for the growth of micro-organisms which might otherwise fix themselves upon the teeth and gums and set up processes of decay or ulceration.

Pickerill and Gies urge the use of acids and sweets to stimulate the secretions of the mouth as the most efficient means of 
preserving the teeth. The teeth of the Maoris are immune to caries (Pickerill).

\section{The Saliva in Disease.}

Fleckseder (Zentralblatt für Innere Medicin) calls attention to the fact that the saliva is acid in diabetes and also in cancer of the stomach, in leukemia, in pernicious anemia, jaundice, and sometimes in chlorosis. The chlorids may be increased or diminished according to the disease. When the chlorids of the urine are diminished, there is usually also a diminution of chlorids in the saliva. Sugar is sometimes present in severe cases of diabetes.

Diminished saliva (oligosialia) is present in many severe maladies, especially in cases of profuse sweating, insistent vomiting, dropsy, diabetes, anemia, cachexia accompanying carcinoma of the stomach, pernicious anemia, fever, uremia and cirrhosis of the liver. When scanty, the saliva is cloudy and acid, and sometimes has a peculiar sweetish odor. In ptyalism the saliva is thin, alkaline and clear. A profuse flow of saliva occurs in pregnancy, in stomatitis, in most of the painful gastric affections, in nausea and ulcer of the stomach. An intermittent flow is frequently observed in cases of facial neuralgia, in the gastric crises of locomotor ataxia and in neurasthenia.

\section{Regulating Influences of the Sense of Taste.}

While food is in the mouth it is not only chewed but tasted. The recognition of the gustatory properties of the food by the nerves of taste, through reflex centers in the brain, prepares the stomach, pancreas, liver and other digestive organs for the work which they are to do. Tasting the food also in some mysterious way regulates the process of nutrition by cutting off the appetite for one food principle after another as the body has received a sufficiency of each particular item.

At the back of the mouth is found a remarkable structure, the soft palate, which is peculiarly sensitive to contact with solid objects. If the food is imperfectly chewed, the contact of coarse particles with the uvula and other portions of the soft palate give rise to a reverse movement by which the food is carried back between the teeth to be more thoroughly masticated. This action of the soft palate is a sort of inspection intended to secure not only thorough mastication of the food but rejection of substances which are not food and which might do injury if swallowed. 
The longer the food is retained in the mouth and the more thoroughly it is chewed, the larger the amount of gastric juice which will be found in the stomach ready to act upon the food after it reaches the gastric laboratory. It is part of the function of the mouth laboratory, then, not only to digest the food and prepare it for the action of the other digestive organs but to regulate the nourishing of the body. In order that this regulating process shall be successful, it is necessary that the food should be so thoroughly chewed that its sapient properties may be fully developed and thoroughly appreciated. When this instinctive regulator of nutrition has been trained to normal action, it is a very dependable guide in determining not only the quality of the various foodstuffs which are needed to meet the requirements of the body, but also the amount of food which should be taken.

The importance of thorough mastication of the food will be discussed elsewhere. It may be mentioned here, however, that it is essential to good digestion, not only because by thorough chewing the food is comminuted, but for still another reason. Recent observations have shown that when large quantities of starch enter the stomach without having been changed by the action of the saliva, the pepsin may be absorbed by the undigested starch and thus gastric digestion may be interfered with and the acid of the gastric juice left free (Maxwell). The excess of free acid gives rise to gastric spasm, pain and various other inconveniences.

\section{GASTRIC DIGESTION}

The chief function of the stomach is to prepare the food for digestion in the intestine and to serve as a reservoir to hold the food while it is being doled out to the intestine in small quantities by the action of the pylorus. While the food is in the stomach about half the starch is liquefied, one-fourth the starch is converted into sugar, and three-fourths of the protein is liquefied, but none of it is completely digested.

In the mucous membrane of the stomach are glands, some of which produce pepsin, others the gastric acid which is known to the chemist as hydrochloric acid. These two remarkable products co-operate in the digestion of the proteins of the food. Rennin, a milk-curdling ferment, is also produced in the stomach. The digestive work done in the stomach is not complete. It is rather of a preliminary character intended to complete the breaking-up of the food so as to render each particle easily accessible to the other more powerful digestive juices which are brought in con- 


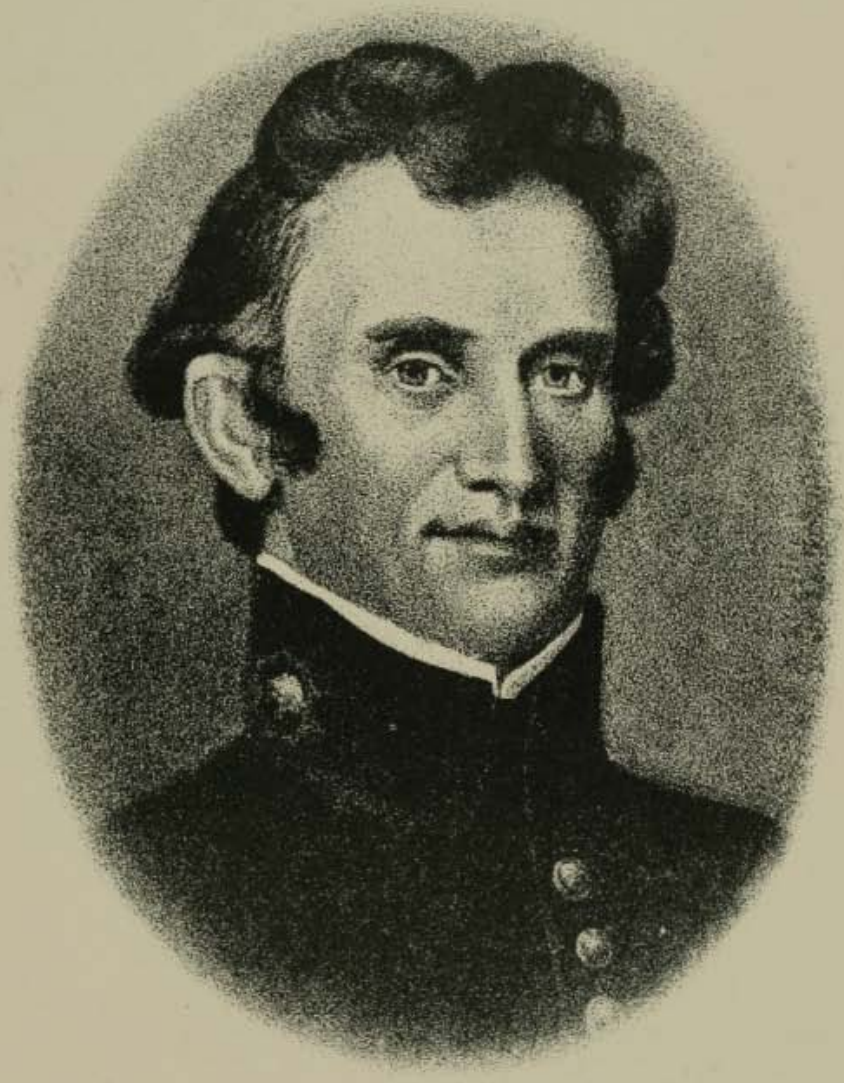

DR. WILLIAM BEAUMONT

(From "Life and Letters of Dr. William Beaumont," C. L. Mosby Co., St. Louis, Mo.) 


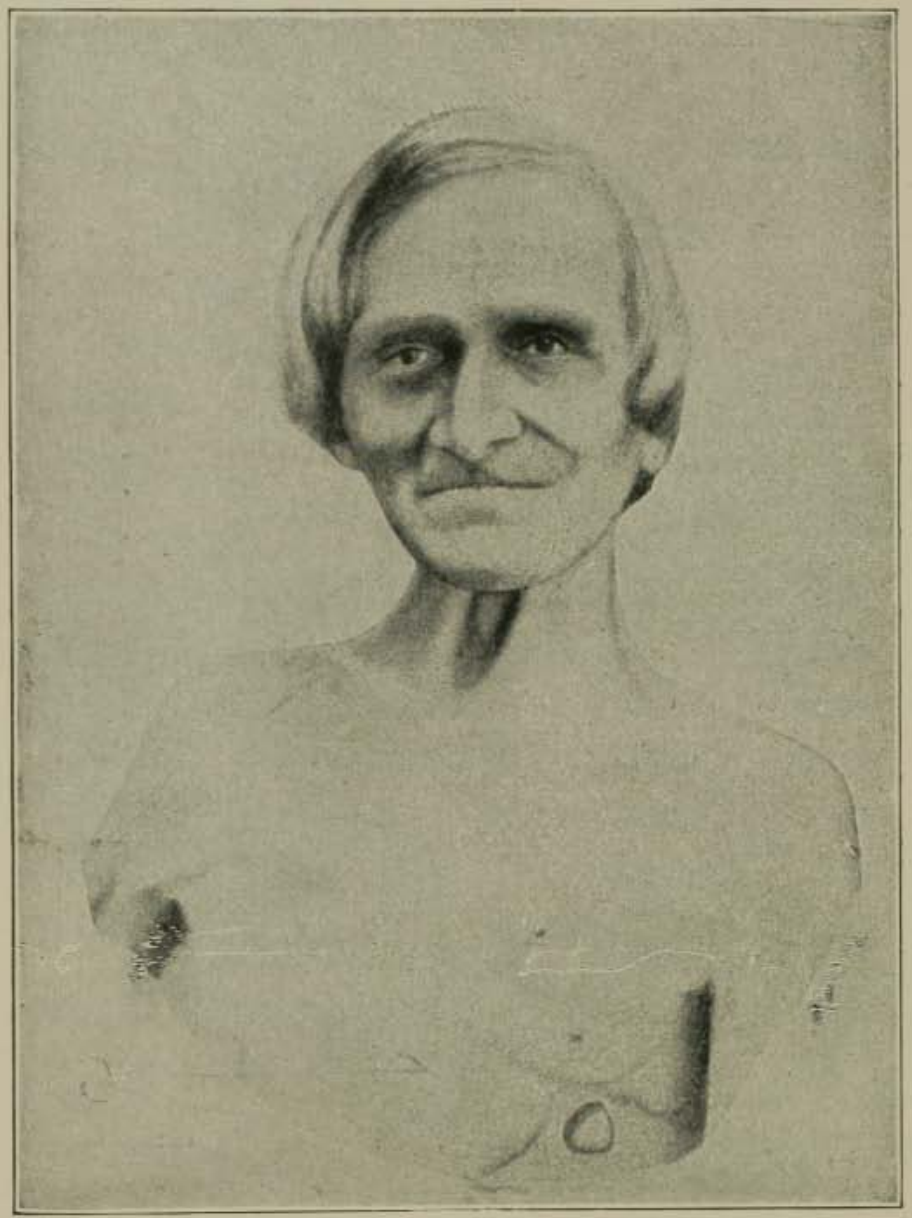

ALFXIS ST, MARTIN

Showing Opening in Left Side

(From "Life and Letters of Dr. William Beaumont," C. L. Mosby Co,, St. Louis, Mo.) 
tact with the food after it leaves the stomach. It very naturally follows that very little absorption takes place in the stomach. The stomach, indeed, may be considered as an antechamber to the real food laboratory, the small intestine, where the chief work of digestion and absorption are done. According to the comparative anatomists, the stomach of man does only about one-fifth of the digestive work, while in the goat and the ox the stomach does seventy per cent. of the work of transforming the food.

\section{The Gastric Juice.}

The quantity of gastric juice produced by the stomach in 24 hours is about three pints. The amount required for the digestion of a hearty meal, such as a dinner, the principal meal of the day, has been estimated as a little less than one and one-half pints.

The gastric juice is the product of six different sets of glands. Three of these secrete ferments, pepsin, rennin and lipase, a fat-digesting ferment. Other glands secrete mucus, still others acid, and by another set of glands there is secreted a serous fluid termed diluting juice, which serves to regulate the acidity and digestive activity of the gastric juice.

It was formerly supposed that gastric juice is produced only during digestion. This belief was largely based upon the observations of Beaumont. But it may be noted that this shrewd observer remarked that he usually found the stomach empty, or at least that it contained only a small quantity of gastric juice, which he states sometimes amounted to one or two ounces. This quite agrees with the later observations of Carlson on a man having a gastric fistula similar to that of St. Martin, upon whom Beaumont's experiments were made. Carlson holds that the secretion is continuous, usually not exceeding one to two ounces per hour, but sometimes three to five ounces per hour. This continuous secretion possesses the properties of ordinary gastric juice, though usually less acid than the juice produced during digestion. The continuous secretion is absent in fevers and also when the stomach is the seat of inflammation or gastritis.

The rate of secretion and doubtless the volume also varies greatly in different persons. It is known that several pints of gastric juice are required for the digestion of each meal. Recent experiments show that the rate of secretion of the gastric juice varies from one-third of an ounce to two and one-third ounces in ten minutes, or a maximum of nearly one pint an hour. The 
activity of the stomach is greatly influenced by the character and volume of the food.

After the appearance in 1834 of Beaumont's interesting account of his experiments upon St. Martin, the knowledge of digestion was advanced very little until the publication in 1897 of Pavlov's remarkable treatise. "The Work of the Digestive Glands." This work provided, for the first time, a basis for rational medical dietetics. Previously so little was actually known respecting the physiology of gastric and intestinal digestion that medical dietetics, at least in relation to gastric and intestinal digestion, was almost wholly empirical or based upon vague and unproven theories.

The facts set forth by Pavlov were so extraordinary and so revolutionary that the writer thought it worth while, in 1907, to visit Petrograd for the purpose of getting a first-hand acquaintance with the work and views of this distinguished investigator, whom he found the most approachable, courteous, and communicative of men. The experiments which the professor and his able assistants performed for the writer's benefit not only confirmed the facts made known in his published works but made us acquainted with additional facts which at that time had not yet been published to the world.

Some of the most important facts in relation to gastric digestion were discovered by Pavlov through experiments made possible by an ingenious operation devised by him whereby the stomach of a dog was divided into two compartments, one of which was made use of by the dog for the digestion of his food. while the experimenter employed the other for his scientific observations.

\section{Appetite Juice.}

Pavlov found that within a few moments after a dng began chewing the food offered it, a stream of freshly secreted gastric juice started to pour from the little stomach into the receptacle provided to receive it. In fact, the secretion of gastric juice by the little stomach often began even before food had been taken into the mouth of the dog, provided it was allowed to see and smell appetizing food.

By another experiment Pavlov proved that if food was simply chewed and never allowed to enter the stomach, the stomach produced an abundant secretion of highly active gastric juice, which continued as long as the chewing continued. In one 


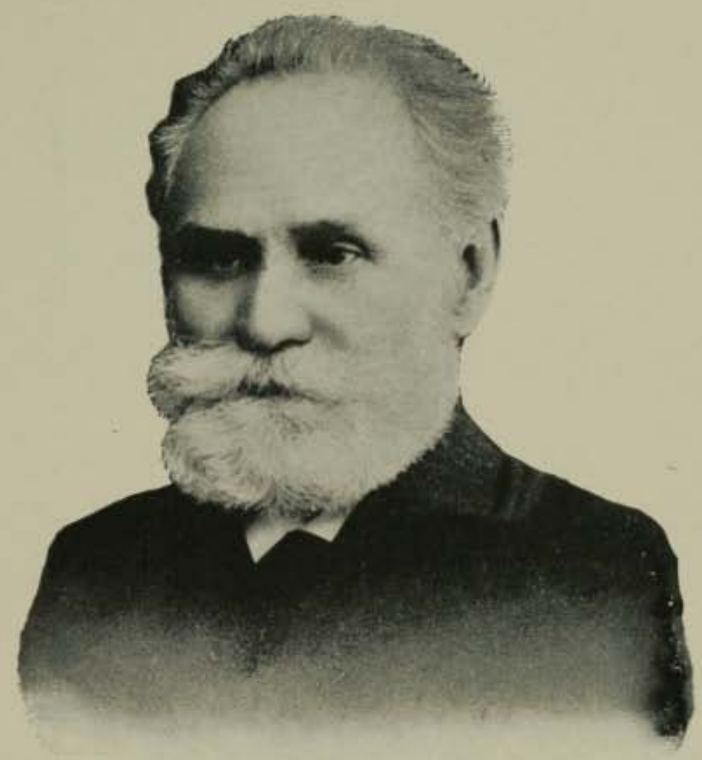

PROF. J. P. PAILOV OF PETROGRAD

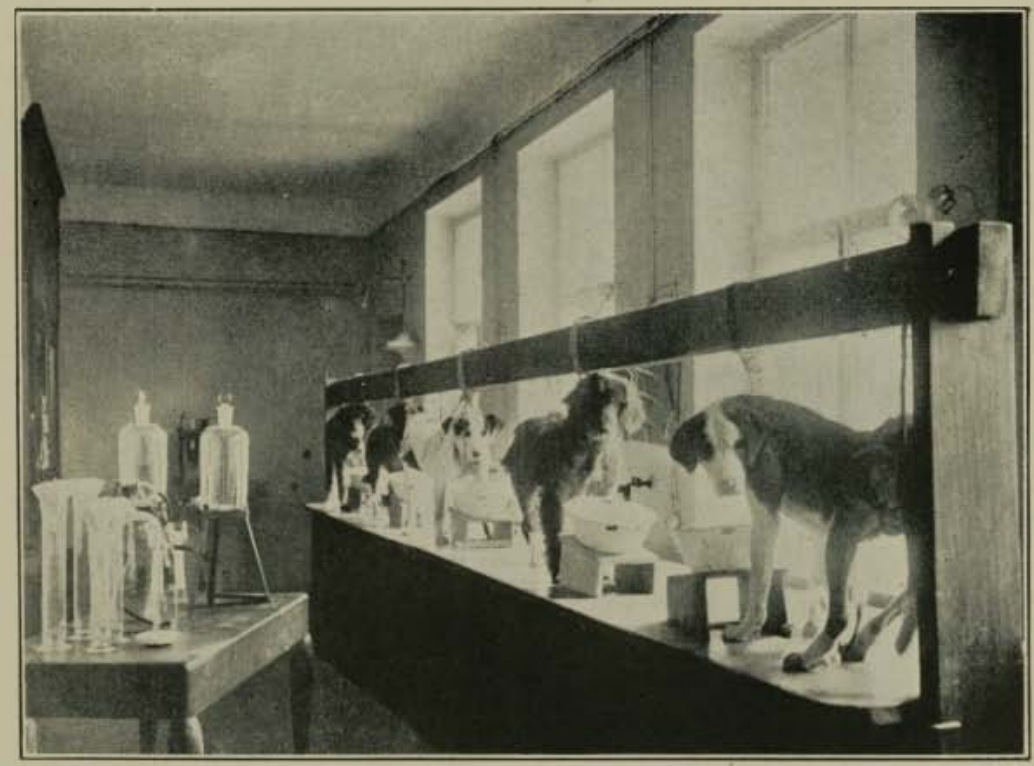

PAVLOV'S DOGS PRODUCING GASTRIC JUICE 


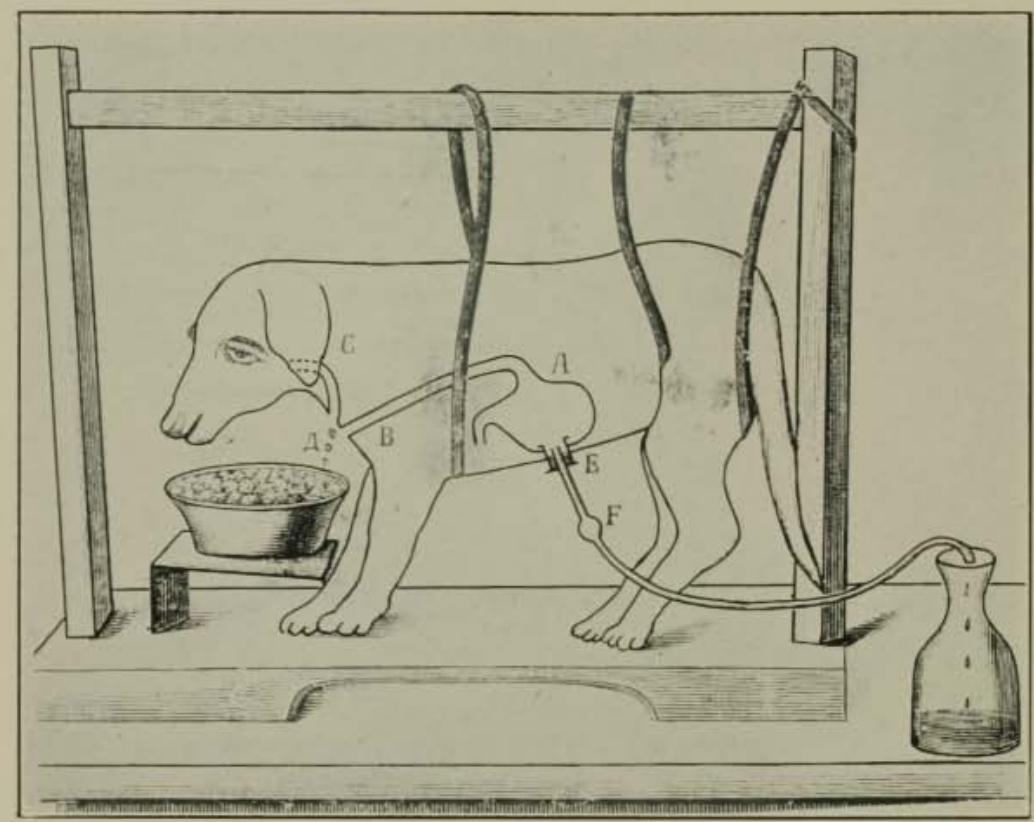

Fig. 1.-Experiment to Demonstrate Appetite Juice.

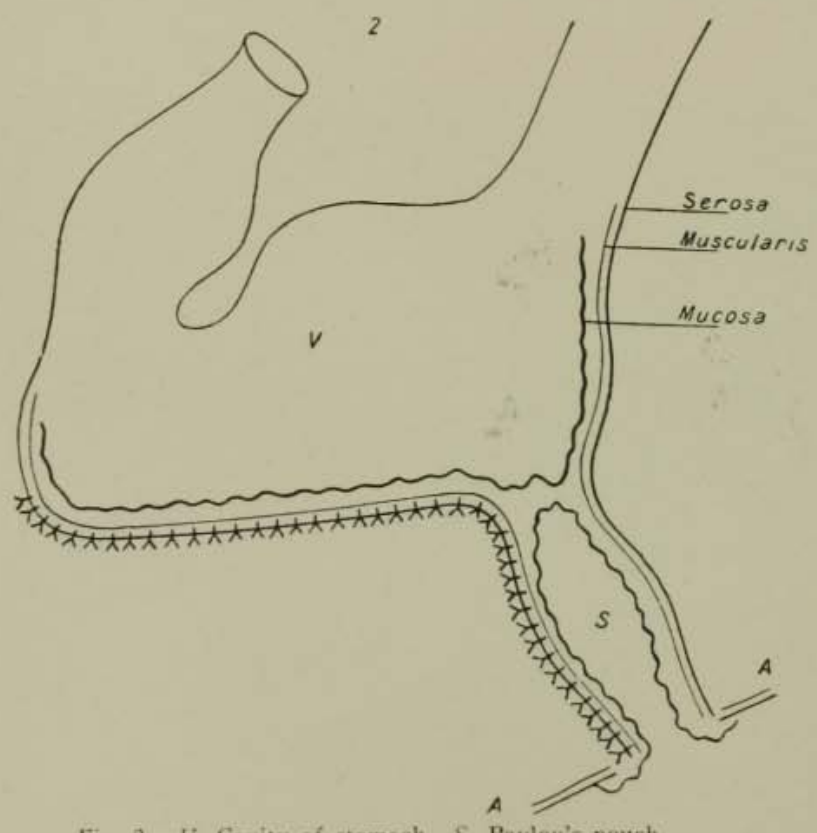

Fig. 2.- V. Cavity of stomach. S. Pavlov's pouch.

A..4. Abdominal wall. 
of the accompanying cuts is shown a group of dogs as seen by the writer at ten o'clock in the morning in Pavlov's laboratory, after they had been actively chewing food since six o'clock in the morning. Each dog had produced nearly a quart of gastric juice, although not a particle of food had entered the stomach, it being prevented from so doing by an operation upon his esophagus, whereby the food, when swallowed, was made to drop back into the pan from which he ate, instead of going into his stomach. The gastric juice produced under these conditions, by the simple chewing of food in the mouth, Pavlov designated by the term "appetite juice."

Miller and others have shown that the sight of food is a more powerful stimulus to gastric secretion than odor. Unpalatable food produces little or no appetite juice, though it may be ultimately well digested. Worry and mental strain cause delay in the development of appetite juice and hinder digestion. This effect is not produced by entertaining mental activity, such as reading a newspaper during meals.

The gastric juice secretion thus evoked by the tasting and chewing of food is strongly acid and possessed of a very high degree of digestive activity. At the time of the writer's visit the laboratory was doing quite an extensive business in the collection of gastric juice from the stomachs of dogs and shipping it to specialists in various parts of Europe for use by patients. Very remarkable results were attributed to its use, far greater than have ever been found to result from the use of the pepsin obtained from the stomach of the hog.

Appetite juice is produced at the average rate of about oneeighth of an ounce per minute, though it may be generated at a rate four times as great as this. Its secretion ceases as soon as the taste of food disappears from the mouth.

Appetite juice, like the continuous gastric secretion, is either greatly diminished or entirely absent in gastritis or any inflammatory disorder of the gastric mucous membrane, as well as in fevers. It is also stopped by pain and by strong emotions, as by anger and fear. Pavlov believed the function of the appetite juice to be to start the digestive process. He regarded it, in fact, as the most important part of the gastric secretion. The correctness of this view has been called in question in view of the fact that normal infants have no appetite juice, and that the digestion is apparently carried on in a normal way in dogs in which the nerves of the stomach have been divided so as to prevent the 
development of appetite juice, and also in persons having a gastric fistula through which food may be passed into the stomach without having been placed in the mouth. The fact is also recalled that in many cases of achylia, a condition in which no gastric juice is formed, a digestion is apparently carried on by the small intestine in a perfectly normal way. The same is also true in cases in which the stomach has been removed. However, it cannot be denied that appetite juice is a highly useful factor in digestion, and it seems probable that its absence must sooner or later give rise to impairment of digestion and nutrition.

When the tongue is coated, the food flavors cannot be appreciated by the nerves of taste, and as a result the gustatory reflexes are destroyed, appetite juice is not formed, and digestion may be suspended. (Roger).

Fats mixed with the food delay the development of appetite juice and diminish its quantity. Meat introduced into the stomach of a dog without passing through the mouth digests after a very long time, but when taken through the mouth, five times as much will be digested in the same time, through the aid of the appetite juice which is produced. This experiment affords a most forcible demonstration of the importance of appetite and of mastication as aids to digestion. Bread placed in the stomach produced no secretion whatever, but when chewed in the mouth and swallowed, appetite juice was produced and the bread was readily digested. When a portion of the digesting bread was introduced directly into the stomach of another dog, gastric juice was promptly secreted.

Fats not only hinder the formation of appetite juice, but their presence in the stomach leads to a diminution in the production of chemical juice as well.

Pavlov showed that fats remain longer in the stomach than any other food principle, but after leaving the stomach they pass rapidly through the intestine.

\section{The Chemical Juice.}

The gastric secretion which appears in the stomach as the result of contact of the food with the gastric mucous membrane is called by Pavlov "chemical juice." The chemical juice appears to be called forth by various stimulating substances found in foodstuffs, particularly by the extractives of meat. Recent experiments, however, have shown that the most powerful stimulant of the gastric glands is gastrin, a hormone which is formed when 
protein food is brought in contact with normal gastric juice in the stomach.

The chemical secretion, as well as the appetite juice and the zontinuous secretion, is arrested by fever, especially when the body temperature rises considerably. In health, active secretion of gastric juice is produced when gastrin is injected under the skin or introduced into a vein. When fever is present, this does not occur.

\section{Fasting Juice.}

Gastric juice is usually found in the stomach in the intervals between meals, as in the morning before breakfast. The amount varies from a fraction of an ounce to several ounces. The acidity varies according to the amount of bile or mucus present. Bile is found in nearly half the cases.

\section{The Gastric Acid.}

One of the most remarkable physical properties of the gasiric juice is its acidity. The contents of the healthy stomach during digestion are always found to be highly acid. This is rarely ever due to fermentation but rather to the presence of hydrochloric acid. According to Debove, an eminent French physiologist, the hydrochloric acid formed by the gastric glands differs from the ordinary hydrochloric acid of the laboratory in being much more active. As secreted by the gastric glands, the acid has the same degree of acidity as that possessed by one-half of one per cent. solution of hydrochloric acid, about 1.25 per cent. of the ordinary C. P. hydrochloric acid of the laboratory.

A solution containing hydrochloric acid in this proportion, if applied to the skin, would produce powerful corrosive effects. As soon as secreted, however, the strong acid solution is diluted by the gastric mucus so that the normal acidity of the gastric contents corresponds to a solution containing about two parts of hydrochloric acid in a thousand parts of water, or two-tenths of one per cent.

It is evident, then, that when matters vomited or regurgitated from the stomach have a strong acid taste, it is no certain indication that the food has fermented in the stomach, but may be proof that the stomach is making at least a normal amount of gastric acid. When there is fermentation of the gastric contents, a rather rare occurrence, vomited matters have not only a sour taste but a sour smell as well, due to the presence of acetic acid, 
which is volatile at the temperature of the body, whereas hydrochloric acid is not volatile.

The normal stomach produces each day from one dram to two-thirds of an ounce of pure hydrochloric acid. Just how the stomach is able to produce this strong acid is not known, nor how the gastric mucous membrane alone, of all other body tissues, is protected from the destructive action of this highly corrosive liquid.

The amount of acid formed by the stomach in the digestion of a meal depends primarily upon the character of the food. Rehfuss and Hawk confirm the observation of Pavlov that animal foodstuffs produce a higher degree of acidity than do vegetable foods, the average acidity of beef being 120, eggs 80 , and vegetables 70 . Milk produces greater acidity than do eggs. Bread and cereals produce the lowest degree of acidity. The degree of acidity produced by animal foodstuffs is equal to that which is universally regarded as pathological and indicative of disease. From this fact the inference may be clearly drawn that the habitual use of meats must tend to produce a diseased condition of the stomach.

The acidity of the gastric contents during digestion is not usually found so high as one-half of one per cent., being normally about one-fifth of one per cent. This is also the acidity of the continuous secretion found in the empty stomach when mixed with saliva which has been swallowed and liquids which have entered the stomach from the intestine.

\section{Regulation of Acidity and Control of the Pylorus.}

Boldyreff demonstrated that the acidity of the gastric contents is chiefly regulated by reflux or regurgitation of the alkaline contents of the duodenum, and that hyperacidity is most frequently the result of the failure of this regulating function. This important observation has apparently been generally overlooked by clinicians, but the mechanism discovered by Boldyreff is now recognized as the efficient means by which hyperacidity is normally prevented.

As shown by Hunter, hyperacidity is nearly always present in cases of obstruction of the common duct by gallstones, the hyperacidity apparently being due to absence of bile and pancreatic juice. The alkaline secretion of the pyloric portion of the stomach is also an important factor in neutralization.

Hyperacidity is due (Ryle) to (I) excessive secretion; 
hypertonus of the pylorus, preventing duodenal reflux; (3) excessive intragastric tension (Apperly); (4) failure of the duodenum, which normally acts as a positive factor in preventing hyperacidity by forcing its contents back into the stomach (Boldyreff).

The pylorus is probably the main factor in controlling duodenal reflux (Goodhart and Campbell). The intragastric pressure nuust be below that of the duodenum for reflux to occur even when the pylorus is open.

In the "steerhorn" or hypertonic stomach, the prepyloric portion of the stomach is higher than the duodenum, so reflux does not easily occur. In the hypotonic or " $\mathrm{J}$ "-shaped stomach, the prepyloric portion of the stomach is below the pylorus, which facilitates reflux.

Hyperchlorhydria is probably due to a hypertonic condition of the whole pyloric portion of the stomach rather than of the pylorus alone, while a hypotonic condition of the pyloric portion of the stomach predisposes to hypochlorhydria, because it facilitates reflux and neutralization of the gastric acid.

The "steerhorn" or hypertonic stomach is most common in cases of duodenal ulcer, a condition in which hyperchlorhydria occurs more often than in any other condition.

That some other factor besides the pyloric sphincter influences hyperchlorhydria, however, is shown by the fact that hyperchlorhydria may persist after gastro-jejunostomy in cases of duodenal ulcer.

The acid control of the stomach is probably due to a tida: movement of fluids between the duodenum and the stomach, regulated by reciprocal changes in tension between the stomach and duodenum. The pyloric sphincter resists solid particles but probably allows more or less interchange of liquids most of the time.

The relief of pain afforded by the use of alkalies is probably due to the production of gas, which causes a reflex relaxation of tension the same as does the taking of food. The taking of thirty minims of dilute hydrochloric acid three times a day promptly relieves the gastrogenous diarrhea sometimes observed in achylia, probably in increasing the tonus of the pyloric portion of the stomach.

\section{Pepsin.}

This ferment is the most important of the several ferments found in the gastric juice. As secreted, it is not active, but only 
becomes an active digestive agent when activated by combining with hydrochloric acid.

The amount of pepsin contained in the pint and a half of gastric juice produced in twenty-four hours is about seven and one-half grains. The twenty or more ounces of gastric juice required for the digestion of a hearty dinner contain four grains of pepsin, sufficient to digest one and two-thirds pounds of egg white or three and one-third ounces of dried albumin, nearly double the amount required for a full day's ration. It is thus apparent that the daily production of pepsin is sufficient to digest four times the amount of protein which the body requires.

Except when the gastric glands are destroyed by degeneration, pepsin is usually secreted in abundance. It is for this reason that the feeding of pepsin as a remedy is useless as an aid to gastric digestion. Some years ago an experiment upon a dog was made by a French physiologist which still more clearly demonstrated this fact. By means of tubes passed into the dog's stomach, a stream of water was allowed to flow into the stomach through one tube and out of the other, until more than 2,000 quarts of water had been passed through the stomach. The digestive activity of this water was tested by the addition of hydrochloric acid and was found to be such that the amount of pepsin present was sufficient to digest an amount of flesh more than equal to the weight of the dog.

When the gastric acidity is excessive, the digestive power of the gastric juice may be greatly lessened by the destructive action of the acid upon the pepsin.

The presence of bile in the stomach prevents the action of pepsin, even though the gastric contents may still remain acid. The bile precipitates the pepsin. Pepsin is readily digested by trypsin, a ferment produced by the pancreas, and hence its action does not continue after the food leaves the stomach.

\section{Lipase.}

Roger, of Paris, and others have in recent years called attention to the interesting fact that the stomach secretes a lipase, or fat-digesting ferment, which is very active in the digestion of emulsified fats such as cream and egg yolk.

In this connection it is interesting to note the fact that while the principal digestive work of the stomach is to digest proteins, provision is made also for the digestion of starch and fat. Thus, by the co-operation of these several digestive agents in the stom- 
ach, the food may be completely broken down and so prepared for the more vigorous digestive action of the pancreatic juice and the finishing work of the intestinal juice to which the food is subjected in the intestine. When large quantities of fat are eaten, the intestinal fluid, consisting chiefly of a mixture of bile and pancreatic juice, is often regurgitated, the evident purpose being to aid in the digestion of the fat when the quantity is greater than the gastric lipase is able to deal with. This regurgitation of bile from the intestine is most likely to occur in cases of high acidity, a condition which interferes with the action of the lipase of the stomach and may even destroy it. The alkaline intestinal fluids, by neutralizing the acid gastric juice, aid the digestion of the fat and so hasten its departure from the stomach.

\section{Functions of the Hydrochloric Acid of the Gastric Juice.}

The hydrochloric acid of the gastric juice plays a remarkably versatile rôle, as will be seen by the following enumeration of its uses:

1. Activates pepsin. As secreted, pepsin has no digestive activity, but in the presence of hydrochloric acid it acquires an astonishing digestive potency by which it is able to transform the various proteins of the food into peptone, although it does not actually complete the digestive process. Pepsin-hydrochloric acid is the only agent by which raw connective tissue can be digested in the body. It is evident, then, that in achylia, when pepsin and hydrochloric acid are no longer produced in the stomach, flesh foods should be entirely discarded.

It may be mentioned in this connection that in the absence of hydrochloric acid pepsin may be to some degree activated by food acids-citric, malic and tartaric acids. It should also be noted that when both hydrochloric acid and pepsin are absent from the gastric juice the ferments sometimes may be made to reappear by the introduction of hydrochloric acid or acid protein into the stomach (Taylor). If after the use of hydrochloric acid in this way the ferments do not appear, it is evident that the gastric glands have undergone degeneration. It is desirable that this test be made in all cases of achylia.

2. Activates chymosin and lipase. The chymosin, or rennet, of the gastric juice coagulates milk and the lipase digests fat.

3. Regulates reflux of alkaline liquids from the duodenum. 
It is known that this is the normal means of regulating gastric acidity. It is known, however, that in achylia, in which there is no acid in the stomach, the pylorus often remains open and the food passes out more quickly than normal.

4. Closes the pylorus. When the acid contents of the stomach enter the duodenum, the pylorus is closed by a reflex action. Failure of the pylorus to close is probably the cause of the rapid emptying of the stomach in achylia.

5. Stimulates the secretion of pancreatic juice.

6. Causes contraction and emptying of the gall bladder.

7. Disinfects the food, destroying bacteria and other organisms. The gastric juice does not destroy all bacteria, but it has been demonstrated that most pathogenic or disease-producing bacteria do not survive prolonged contact with normal gastric juice. glands.

8. Stimulates the production of pepsin by the gastric

9. Stimulates the movements of the stomach. Cannon proved that the tonus of the stomach walls, as shown by the pressure within the stomach, was four times as great with acid contents as with neutral contests.

10. Closes the cardiac orifice after the swallowing of food.

11. The hydrochloric acid of the gastric juice protects the body against the poisons eliminated through the bile by precipitating them and thus preventing their reabsorption. It is possible that injury may be done by the use of soda in large doses through the prevention of the precipitation of the bile salts. These salts are irritant poisons. When they are absorbed, they are again excreted. The bile thus becomes more and more concentrated.

The acidity of the gastric juice is increased by the following:

Hunger

Water

Gastric juice

Lean meats

Meat extracts, bouillon and animal broths

Yeast extract

Milk, especially buttermilk and skimmed milk

Cane sugar

Undigested cooked starch

Dextrin

Sodium chlorid (common salt) 
Carbonated water

Agreeable foods

Strongly flavored foods

Condiments

Cold baths

Sleeping after meals

Imperfect mastication of food (chemical juice)

Prolonged chewing of food (appetite juice)

Acids (especially butyric, oxalic and tannic)

Tea and coffee, alcohol and tobacco

Worry

Disease of the gall bladder

Gastric or duodenal ulcer

Putrefaction in the intestine (Ivy).

According to Taylor, "There are experiments with animals indicating that increased bacterial action in the intestine, aiding the formation of a large amount of fatty acid (also other toxic substances, such as bacterial peptone, etc.), provokes in some way an increased secretion of hydrochloric acid in the stomach." The writer has noted a very constant association between intestinal toxemia and gastric hyperacidity or hyperhydrochloria.

Hypersecretion, or hyperacidity, may be intermittent or continuous. It is a common symptom in connection with hysteria, chlorosis and neurasthenia, so-called nervous dyspepsia, a condition which is probably due to chronic intestinal toxemia.

The acidity of gastric juice is lessened:

1. By fats, cream and oils, especially olive oil when perfectly fresh and free from rancidity.

2. Sugar.

3. By the use of soft foods and purées, when eaten with very little mastication.

4. By bland foods.

5. By a saltless diet.

6. By sweating baths or by profuse perspiration induced by exercise, which causes the elimination of salt.

7. By fatigue.

8. By disease-fever, cancer, degeneration of gastric glands (achylia), cachexia, pernicious anemia. Hydrochloric acid is often absent or diminished in Bright's disease, chlorosis and pronounced secondary anemia.

9. About one-fourth of the hydrochloric acid secreted by the stomach is absorbed or neutralized by mucus. 
10. The gastric juice may be neutralized by a reflux of fluids, bile and pancreatic juice, from the duodenum.

According to Pavlov, egg albumin has no effect upon gastric secretion. He found the same true of undigested starch, solid fats and hydrochloric acid.

\section{Bitters Do Not Increase the Secretion of Gastric Jurice.}

The observations of Carlson and others have clearly demonstrated that, contrary to popular belief, bitters do not increase the production of gastric juice. Reichmann and Schœffer have shown, in fact, that the presence of bitters in the stomach has the effect of lessening the secretion of gastric juice. From these experiments, it is evident that the age-long faith in bitters as a means of promoting digestion has no scientific basis. Bitters hinder instead of aiding gastric digestion.

It has long been known that alcohol increases the activity of the gastric glands; but Chittenden, in his classical research made many years ago for the Committee of Fifty, showed that while alcohol increases the secretion, it at the same time destroys the activity of the gastric juice by precipitating the pepsin. Wines, as well as strong liquors were found to be decidedly detrimental to digestion.

In general, it may be regarder as clearly established by scientific reasearch that the only safe and proper stimulants for the stomach are those normal gastric activators, the flavors, hormones and vitamins which are found in natural foodstuffs, and which are often deficient or lacking altogether in artificial and denatured foods. The best stomach tonics or peptic stimulants are to be found in the unsophisticated products of the orchard, garden and field.

\section{RECENT STUDIES OF THE INFLUENCE OF FOODS UPON THE GASTRIC SECRETION}

The study by Pavlov of the gastric juice by the aid of the isolated stomach, begun in 1894, furnished the foundation for a clear conception of the relation between food and the several digestive processes, especially the influence of food upon gastric secretion. But the work of Pavlov was practically confined to animal foodstuffs. It has long seemed to the writer most desirable to possess similar data respecting the influence of the various vegetable products among our common foodstuffs, especially as these so greatly exceed foodstuffs of animal origin both in num- 
ber and in diversity of composition. By request of the writer, is study of this question has recently been made by Professor Boldyreff, for many years associated with Professor Pavlov of Leningrad, now director of the Pavlov Institute of the Battle Creek Sanitarium. Professor Boldyreff's familiarity with the Pavlov technic, from his long service with the great physiologist as his chief assistant, gives to his observations special value as a contribution to the growing body of exact knowledge respecting the reaction of the stomach to various foodstuffs.

The main facts developed by Professor Boldyreff's research are the following:

The research was conducted with dogs, with isolated stomachs prepared by the method of Heidenhain, in which the vagus nerves of the stomach are cut, so that the small or experimental stomach is influenced only by the sympathetic nerves. Under these conditions, only chemical juice is produced; that is, the gastric secretion which is produced by the sight, taste and smell of food, does not appear. The secretion produced is known as chemical juice. Its production is the results of the stimulating influence of the food upon the gastric glands through the absorption of the soluble elements of the food.

The following table shows the degree of digestive activity evoked by different common foods:

\begin{tabular}{|c|c|c|c|c|c|}
\hline Sunstance (Extract) & $\begin{array}{l}\text { Duration } \\
\text { Hours }\end{array}$ & $\begin{array}{l}\text { Quantity } \\
\text { C.C. }\end{array}$ & $\begin{array}{l}\text { Acidity } \\
\% \mathrm{HCl}\end{array}$ & $\begin{array}{c}\text { Pepsin } \\
\text { Mett's Test }\end{array}$ & $\begin{array}{l}\text { Digestive } \\
\text { Activity } \\
Q \times P\end{array}$ \\
\hline Apples ................ & 2.50 & 20.1 & .29 & 5.1 & 102 \\
\hline Mushrooms .......... & 2.75 & 18.5 & 40 & 5.1 & 94 \\
\hline Raisins .... & 3.00 & 27.8 & .44 & 3.3 & 92 \\
\hline Cabbage........... & 2.00 & 16.2 & .40 & 4.6 & 76 \\
\hline Savita (yeast extract)... & 2.00 & 17.6 & .40 & 4.1 & 72 \\
\hline Carrots ............... & 2.25 & 19.2 & .41 & 3.6 & 69 \\
\hline Beef bouillon $\ldots \ldots \ldots$ & 2.00 & 15.9 & .39 & 3.0 & 48 \\
\hline Apricots $\ldots \ldots \ldots \ldots$. & 2.75 & 16.0 & .41 & 3.0 & 48 \\
\hline Beets $\ldots \ldots \ldots \ldots \ldots \ldots$ & 2.25 & 16.0 & .42 & 3.0 & 48 \\
\hline Rutabagas ... & 2.00 & 14.8 & 38 & 3.0 & 44 \\
\hline Prunes ....... & 3.00 & 20.0 & .42 & 2.0 & 40 \\
\hline Lentils ........ & 1.50 & 9.6 & .29 & 3.3 & 32 \\
\hline Potato $\ldots \ldots \ldots \ldots . .$. & 2.00 & 6.2 & .38 & 4.1 & 25 \\
\hline Grape juice .......... & 2.00 & 7.1 & .34 & 2.8 & 20 \\
\hline Tomato juice ......... & 1.50 & 4.8 & .35 & 3.0 & 14 \\
\hline Onions $\ldots \ldots \ldots \ldots \ldots$ & 1.75 & 4.7 & .27 & 2.5 & 12 \\
\hline Oats $\ldots \ldots \ldots \ldots \ldots . . . . .$. & 2.25 & 9.8 & .41 & 1.0 & 10 \\
\hline Rice $\ldots . . . \ldots \ldots \ldots . . .$. & 1.50 & 1.3 & .00 & 0.0 & 0 \\
\hline Cane Sugar ( $10 \%$ Sol.) & 0.00 & 0.0 & .00 & 0.0 & 0 \\
\hline Dextrose...$\ldots \ldots \ldots$ & + & + & trace & 0 & 0 \\
\hline Levulose ............. & + & + & “ & 0 & 0 \\
\hline
\end{tabular}


In reviewing the above table, it will be noted that several vegetable extracts or bouillons are more active in awakening gastric activity than is meat extract or beef bouillon. Savita, a yeast extract, resembling meat extract in appearance and flavor, evoked a degree of digestive activity more than fifty per cent greater than beef bouillon. It is quite possible that the superiority of Savita may be due to the fact that it is very rich in vitamin $B$, which is a vital stimulant and has been shown by McCarrison, Boldyreff and others to have a powerful and energizing influence upon the digestive organs.

\section{The Influence of Paraffin Oil upon Gastric Secretion.}

Pavlov, Sokoloff and others have shown experimentally that oils inhibit gastric secretion. These investigations also showed that the inhibition originated from the small intestine. It was further demonstrated that the actual cause of the lessened secretion is the presence in the oils of fatty acids. These acids not only lessen the activity of the gastric glands but also lessen the acidity of the gastric juice and the amount of pepsin present. Oils which are perfectly neutral are less active than those which have become slightly rancid by the development of fatty acids; although neutral oils also lessen gastric secretion because of the rapid development of fatty acids after they enter the stomach or the intestine.

These facts, experimentally proven by observations upon laboratory dogs, have been abundantly verified in clinical experience.

Some medical writers have strongly denounced the use of mineral oil as a remedy for constipation, claiming that, like other oils, it lessens the activity of the gastric glands, and thus interferes with digestion.

The fact that mineral oil is not acted upon by the digestive juices, and that it is not decomposed by bacteria, that, in fact, it undergoes no chemical change in its passage through the alimentary canal; and the further fact that it is not taken up by the absorbents which drink up with so great avidity the products of digestion and many other substances from the intestinal canal, have furnished an apparently sound basis for the belief in the innocuousness of this valuable remedy for delayed bowel action. 
The question has been definitely and finally settled by research recently undertaken by Professor Boldyreff.

The experiment consisted of administering a dose of paraffin oil to a dog with a Pavlov pouch, thirty minutes before feeding seven ounces of raw meat, the rate of gastric juice production after a meal of this sort having been previously determined. The immediate effect of the administration of mineral oil was found to be a slight increase (about seven per cent) of the amount of gastric juice produced, with no change in the acidity or the pepsin content of the juice. Later, a slight diminution of the gastric secretion was observed, but this was soon followed by a gradual return to normal. It thus appears that the use of paraffin or mineral oil has no detrimental effect upon gastric secretion.

\section{The Influence upon Gastric Secretion of the Introduction into the Rectum of Different Kinds of Oil.}

The writer observed many years ago (1905), that the introduction of linseed oil into the rectum as a means of encouraging bowel action, a method then much in vogue, apparently interfered with gastric digestion, lessening the appetite the following day, causing coating of the tongue and other symptoms of gastric disturbance.

Since Pavlov had shown that animal and vegetable oils have the effect to lessen gastric secretion, the conclusion was drawn that the introduction of oil into the rectum probably produced a similar effect. This led the writer to discontinue the use of the oil enema. Recently, at the writer's request, Professor Boldyreff conducted a series of experiments the results of which fully confirmed the conclusions reached by the writer from clinical observations.

The following is a summary of the conclusions drawn from the results of this research:*

"1. The mineral oils (liquid paraffin oil) do not diminish the appetite, and do not have any harmful effect upon gastric digestion.

"2. Of the animal oils, butter has no inhibiting action on digestion because it is not liquid at body temperature, and therefore cannot move into the small intestine. Cod liver oil inhibits digestion but in a relatively small degree.

“3. The vegetable oils (olive oil and linseed oil) inhibit very strongly the psychical phase of digestion.

- "The Influence of Different Kinds of Oil Introduced into the Rectum upon Gastric Secretion," by W. N. Boldyreff, M.D., and J. H. Kellogg. M.D., Bulletin of the Battle Creek Sanitarium and Hospital Clinic, Vol. XX, No. 2, 1925. 
"4. The fatty acids are the active substance in the oils.

"5. Commercial oils have a stronger inhibitory action, as they contain free fatty acids, but neutral oil also possesses this action, though in a less degree. Moreover, the action of neutral oil is slower.

"6. The inhibiting action of oils introduced into the rectum consists only in decreasing the quantity of gastric juice; its acidity and pepsin content remain nearly constant."

It is clearly evident from the above that animal and vegetable oils when introduced into the rectum have a very pronounced injurious effect upon gastric digestion by decreasing the production of appetite juice. The effect of these oils is doubtless the result of the entrance of a portion of the oil into the small intestine through reversed peristalsis. Even in cases in which the ileocecal valve is intact, it is quite possible that some portion of the oil should enter the small intestine, and it is, of course, quite possible that the inhibitory effect may be the result of the absorption of fatty acids produced in the cecum, where absorption is known to be rapid.

The worst effects would naturally occur in cases of pronounced incompetency of the ileocecal valve, a condition which exists in a very large proportion of cases of chronic constipation. It is, in fact, the rule rather than the exception in such cases.

In view of these findings, it is clear that the practice of introducing vegetable oils into the rectum for the relief of constipation, should be discarded. If it is desired to use oil for this purpose, mineral oil should be employed, since this does not disturb the appetite nor produce any other harmful effect on gastric digestion.

The effect of cod liver oil was found less marked than other oils in experiments with the dogs, for the reason that dogs are very fond of fish, and the odor probably stimulates the flow of juice.

\section{DIGESTION IN THE SMALL INTESTINE}

The small intestine performs the greater part of the digestive work and is the only part of the alimentary canal in which the work of digestion is carried to completion. It is also the chief organ of absorption, practically no absorption of digested foods taking place from either the stomach or the colon. Both the stomach and the colon may be removed without serious interference with digestion or nutrition; but removal of any considerable portion of the small intestine is dangerous to life.

Using a domestic analogy, the stomach may be considered the kitchen of the body and the small intestine the dining-room. 
In this part of the alimentary canal, which is about twenty-two feet in length, the food is disposed of so rapidly that at the end of eight hours from the time the food enters the stomach all of its usable elements have been digested and absorbed.

The small intestine absorbs something more than six quarts every twenty-four hours. This amount includes not only the liquids taken in the form of food and drink, but the pancreatic juice, the gastric juice and other digestive fluids which are poured into it. The rapid absorption which takes place in the small intestine is made possible by the five million villi which hang out into the interior of the gut and which in some respects resemble the minute rootlets of a plant. Indeed, the villi may be regarded as the roots of the body, while the foodstuffs which pass over them along the intestinal canal correspond accurately to the soil which surrounds the rootlets of a plant. Each of the five million villi of the small intestine absorbs about one ounce of liquid in a lifetime of sixty years. So minute are the villi and so delicate is this part of the digestive machinery that a single villus working steadily for eight weeks is able to absorb barely one drop of liquid.

In the small intestine the digestive process is fully completed, the useful products of digestion are absorbed, and the unusable residues, together with the bile, mucus and various body wastes which are eliminated by the intestine, are pushed on through the ileocecal valve into the colon, their return to the small intestine being prevented by the check-valve action of this remarkable anatomical structure.

\section{The Pancreatic Juice.}

In the intestine the food comes in contact with three digestive juices: the pancreatic juice, the bile and the intestinal juice. The pancreatic juice contains four ferments, besides activating substances. These are as follows: trypsin, which digests protein; lipase, which digests fat by converting it into soap; amylase, which converts starch and dextrin into malt sugar, chymosin, or rennet, which coagulates milk.

Acids stimulate the secretion of pancreatic juice. This is true of food acids as well as of the hydrochloric acid of the gastric juice. Hence, when the normal gastric juice is absent, acid fruits should be freely used to encourage pancreatic secretion 
and intestinal digestion. Water drinking also increases pancreatic secretion, as do fats and alkalies; but alkalies diminish the activity of the pancreatic juice while increasing its volume. An excessive quantity of hydrochloric acid lessens the activity of the pancreatic juice, although increasing the amount.

There is an appetite pancreatic juice as well as an appetite gastric juice. The secretion of appetite pancreatic juice begins, in fact, before the appetite gastric juice secretion begins. Pancreatic secretion is greatly increased by gastric hyperacidity.

The trypsin of the pancreatic juice, like pepsin of the gastric juice, is not active when first formed by the glands. It only becomes a potent agent for digestion when it has been activated by the bile or by bacterial products. Sometimes bile as well as bacteria reach the pancreas through the pancreatic duct and activate the pancreatic ferments, giving rise to autoligestion of the pancreas and pancreatitis. This occurs as the :esult of disease of the gall bladder and bile ducts, and is probably a common cause of diabetes.

The amylase of the pancreatic juice differs from that of the saliva in the fact that it is much more active and is able to digest raw starch. It is for this reason that the starch of the food is usually all digested, little or no starch being found in the feces. This is, unfortunately, a disadvantage, since starch or sugar is needed in the colon to promote the growth of lactic acid-forming germs which are necessary to maintain the activity of the colon and to hinder the growth of putrefactive organisms.

Amylase, or the starch-digesting ferment of the pancreatic iuice, is not often found in the colon. It is likely to be found, however, when starch is present, as starch seems in some way to protect the ferment. On this account it is desirable to take regularly with the food a sufficient amount of raw starch to insure the presence of a certain amount of starch in the feces. This may be accomplished by eating freely of uncooked vegetables containing starch, or by avoiding overcooking of oatmeal and other cereals. The Scotch eat their oatmeal scalded rather than cooked. This practice has been in vogue in Scotland for centuries and doubtless with great advantage to the Scotch people. For some years the writer has followed a similar plan, requiring oatmeal to be cooked only 8 or 10 minutes instead of 30 minutes or more, the customary time. The use of cereals prepared in this way renders great service in regimens for changing the intestinal flora. 
Hyperacidity impairs the activity both of the amylase and of the trypsin of the pancreatic juice and hence may interfere with the digestion of starch and protein in the intestine as well as in the stomach. In cases of diabetes the pancreatic secretion may be so much depreciated that the protein of the food is not properly digested. This matter should be investigated in such ases. It is possible to obtain a sample of the pancreatic juice for examination by means of a duodenal tube.

\section{The Intestinal Juice.}

The intestinal juice is in some respects the most remarkable of all the digestive fluids. It contains several ferments as follows: Erepsin, which completes the digestion of protein and is in this respect the most efficient of all the protein digesting ferments; chymosin, which coagulates milk; maltase, which converts maltose into dextrose ; lactose, which digests milk sugar, converting it into glucose and galactose; sucrose, which digests cane sugar, changing it into dextrose and levulose; and entero-kinase, which activates trypsin.

\section{The Bile.}

The bile, produced by the liver, is secreted continuously, even during starvation, although it is increased after meals. The amount of bile produced depends somewhat upon the diet. It varies from a pint to a quart daily, averaging about twenty ounces.

The importance of the bile is shown by the fact that the liver of the horse secretes thirteen pints of bile in twenty-four hours. The liver of an ox secretes nearly one-half as much; that of a sheep three-quarters of a pint.

A portion of the bile is absorbed and again secreted, so that it becomes in this way concentrated. There is in this fact some justification for the idea that the bile becomes vitiated so that one is benefited by getting rid of a portion of it. Lauder Brunton noted many years ago that the bile from a biliary fistula lacks the bitter taste of bile, doubtless for the reason that it has not been concentrated by reabsorption.

Brugsch and Rother have recently (1922) found that the liver eliminates urine acid in the bile. 
The amount of bile produced is greatly increased by a hearty meat diet. The free use of fats also increases the secretion of bile. Bile contains no digestive agent, but aids in the digestion of fats and also encourages the absorption of digested fats. It also contains substances which activate the ferments of the pancreas. The presence of bile hinders the growth of bacteria, and it is also believed that it neutralizes to some extent the poisonous products produced by bacteria.

It is evident that in the absence of bile the amount of protein and fat should be reduced, so as to lessen the liability to injury from putrefactive processes in the colon. The digestion of fats is interfered with by the absence of bile; hence the intake of fat should be reduced when the entrance of bile into the intestine is interfered with, as in jaundice and during drainage of the gall bladder.

The loss of bile through drainage of the gall bladder does not apparently produce any serious impairment of health, as the body seems to be able to readily compensate the loss of the bile. It is necessary, however, that great care be taken to regulate the diet so as to lessen to the greatest degree possible the tendency to intestinal putrefactions.

\section{Intestinal Absorption and Excretion.}

The great activity of the small intestine as compared with the colon is seen in the fact that the latter organ, capacious as it is, ordinarily absorbs only about ten ounces of liquid in twentyfour hours, or one-twentieth as much as the small intestine. This great activity of the small intestine as an absorbing organ is an indication of the great importance of the function of the ileocecal valve in preventing the reflux of refuse, perhaps in a putrefying condition, from the colon into the small intestine. Comparatively little harm results so long as these refuse matters remain in the colon, even though there is some putrefaction, although normally no putrefaction occurs in any part of the alimentary canal. But when putrefying materials back up into the small intestine, they are rapidly absorbed, and serious mischief results, as shown bv attacks of so-called biliousness, headache, sleeplessness, skin disorders, and the highly varied symptoms of intestinal toxemia.

The large intestine is the channel through which the waste 
iron, lime and other metals are eliminated. Practically all of the iron, amounting to about one-sixth of a grain daily, finds its way out of the body through this channel.

\section{TRANSPORTATION OF THE FOOD}

The statements made in this section are based upon the careful observations of Cannon, Hertz, Carlson, Case and others who have made extensive observations upon the movements of the stomach and intestines by the aid of the X-rays.

The alimentary canal is a transportation system as well as a series of laboratories, the tube being divided into sections separated by gates through which the food is moved from one part to the next by contraction of its muscular walls. This movement is termed peristalsis.

There are ten gates. Of these, one is placed at each end of the canal-the mouth to guard the entrance and the anus to protect the exit.

Next to the mouth we find the soft palate, by which the food is inspected as to its consistency and fitness for swallowing.

The third gate is located at the upper end of the esophagus, or gullet, and is kept constantly closed except when food or water is being swallowed.

The entrance of the stomach is guarded by a circular muscle, the cardiac orifice, which keeps the food in the stomach while it is being subjected to churning and kneading processes during gastric digestion.

The outlet of the stomach, the pylorus, constitutes the fifth gate. Its function is to hold back the food in the stomach until it is prepared to enter the small intestine, then to pass it out from the stomach into the intestine in spoonful doses as the intestine is prepared to receive it.

At the lower opening of the small intestine is found a sixth gate, the ileocecal sphincter, which might be properly regarded as a second pylorus. It holds back the food in the small intestine until digestion and absorption have been practically completed, then relaxes and permits the unused residues to pass on through the ileocecal valve into the colon. This structure, constituting the seventh gate, acts as a check valve to prevent the reflux of fecal matters from the colon into the small intestine. 
In the center of the transverse colon is found what may be called an eighth gate, where contraction waves are started in opposite directions, forward and backward, simultaneously. The backward traveling waves, so-called antiperistaltic waves, have for their purpose the retaining in the cecum of the dilute material received from the small intestine until two-thirds of its water content have been absorbed. By this means the consistency of the colon contents is increased to such a degree as to secure periodical rather than constant evacuations.

A free loop of the colon, known as the pelvic colon, which joins the rectum, constitutes the ninth gate. After an evacuation of the bowels the pelvic colon collapses and lies low in the left side of the abdomen. As the loop of intestine is gradually filled with fecal matters, it rises again, and when fully distended, contracts and discharges its contents; hence this might be properly called the discharging gate. In many cases of chronic constipation, the pelvic colon, on account of being the seat of colitis, has become adherent while fallen down and collapsed, and being unable to rise as filled, a serious obstacle to bowel movement develops. This condition may fortunately be relieved by an operation.

The anus is the tenth or exit gate.

\section{Swallowing.}

After the food has been chewed it is gathered upon the surtace of the tongue, the tip and edges of which are pressed against the teeth and the roof of the mouth, the soft palate is drawn up so as to close the posterior opening into the nasal cavity, and by a backward movement the tongue is made to act like a piston, forcing the food against the soft palate and the back of the pharynx. At the same time the larynx rises and is pulled forward, opening the upper end of the esophagus, and the epiglottis moves downward with a quick jerk which tosses the food into the open gullet. As the act of swallowing terminates, the solid substances are grasped by the circular muscles at the upper end of the gullet and by peristaltic movements are slowly moved toward the stomach, into which they enter at intervals as the cardiac sphincter relaxes. Liquid foods move down more rapidly than solids, being squirted some distance down the esophagus. A partial vacuum exists in the lower part of the esophagus which by suction greatly facili- 
tates the movement of food in the right direction, a device which is certainly marvelously well adapted to the purpose for which it is designed.

In the mouth the food is acted upon by voluntary muscies. When the work of these muscles is done, the food is tossed or squirted down the throat to come within grasp of the involuntary muscles which from this point automatically control the movement of the food along the digestive canal. The act of swallowing is reflex. One cannot swallow except when some object is brought in contact with certain sensitive spots located at the base of the tongue, the upper surface of the soft palate and sspecially the back wall of the throat. These sensitive spots fiffer from other reflex areas in the body in that they do not become fatigued but are always ready to respond when stimulated. This makes it possible to swallow many times in succession without difficulty. When a morsel of food starts on its journey lown the esophagus, the cardiac orifice is notified and opens at che right time to receive the morsel when it arrives. If cocaine is applied to the throat, the sensitive areas are benumbed and ihe swallowing reflex is lost. The opening of the cardiac orifice to permit the entrance of the food into the stomach is in harmony with a general law governing the entire digestive tube, by which a wave of relaxation always precedes a wave of contraction.

Certain drugs, as adrenalin, cause relaxation of the cardiac orifice. The cardiac orifice contracts more strongly after cold drinks and carbonated water than with warm water. It is for this reason that warm water is taken to encourage vomiting. The cardiac orifice contracts with sufficient vigor to resist a pressure equivalent to ten inches of water or about six ounces to the square inch.

The gastric walls contract when the stomach is empty, gradually relaxing to accommodate themselves to the accumulating contents during the taking of food or liquids. This adjustment of the stomach to the volume of its contents is shown in the disappearance of fullness, sometimes felt during a meal, after ceasing to eat for a few minutes. The contraction of the cardiac sphincter is due to stimulation by the acid of the gastric juice as the pylorus is closed by a reflex produced by contact of the gastric juice with the intestinal mucous membrane. When the gastric acid is deficient, both the pylorus and the cardiac orifice may be relaxed, 
a condition which encourages intestinal infection and autointoxication.

When gas escapes from the stomach it is due to the fact that gases accumulated in the upper part of the stomach prevent contact of the acid gastric juice with the mucous membrane near the cardiac orifice. In consequence its closure is prevented. As the gas escapes, the fluid rises and comes in contact with the mucous membrane about the cardiac sphincter, which is then closed.

\section{Movements of the Stomach.}

The movements of the stomach have been very carefully studied by many observers. It was formerly supposed that, when empty, the stomach remained quiet. The observations of Carlson have demonstrated that this is an error and that the empty stomach is always active. A high degree of activity gives rise to so-called hunger pains. Hunger is always associated with contractions of the stomach. When food is received the movements become slower and one is no longer conscious of them.

During digestion contraction waves pass over the stomach at the rate of three per minute. When the contents of the stomach are highly acid the rate may be increased to five or even six waves per minute. There are also other influences which control the rate of gastric peristalsis. When considerable fat is present in the food the waves are less frequent and less vigorous. Proteins produce more frequent waves than fats. The most frequent waves are caused by the presence in the stomach of starch or sugar. These gastric contractions continue until the stomach is emptied, which sometimes requires several thousand waves, although if the pylorus opened at each wave, the entire contents of the stomach would be passed into the intestine in two minutes.

When the gastric juice is diluted by the swallowing of liquids, the waves are slowed, increasing again when the gastric contents become strongly acid. In vomiting, the cardiac end of the stomach is wholly relaxed, although strong waves may occur in the pyloric end of the stomach.

When vomiting is induced the cardiac end of the stomach is relaxed. The diaphragm and the abdominal muscles contract in such a way as to press the stomach between them, squeezing out its contents. The act of vomiting is almost wholly passive. When contracted, the stomach is reduced in circumference to 
about one-fifth that in its relaxed condition. In adjustment to accommodate the gastric contents when the stomach is distended, the muscular fibres of its walls slip by, and the same thing happens with the abdominal muscles in relaxing to adjust themselves to distending contents. Consequently the pressure within the abdominal cavity may not be increased although the volume of the abdominal contents, as in the case of a dog after a large meal, may be doubled.

In the taking of food the successive morsels are arranged in strata in the cardiac end of the stomach, the last food swallowed being found in the center.

The two parts of the stomach really have two distinct functions, the left or cardiac end is a reservoir in which the food is dissolved and disinfected. This end of the stomach also secretes hydrochloric acid which disinfects the food. The right half of the stomach kneads, churns, and manipulates the food, reducing it to the consistency of soup and finally spurting it into the duodenum.

The pylorus has a curious selective action which was noted by the ancients; hence its name, which literally means gate keeper.

\section{Passage of Foods from the Stomach.}

In passing from the stomach into the duodenum, the gastric contents are spurted some distance along the intestinal canal.

Carbohydrates pass out of the stomach most rapidly. Next in order are the proteins and lastly, the fats. Bread begins to enter the small intestine within ten minutes after eating. Potato leaves the stomach rapidly, perhaps because it is acted upon by the gastric juice only to a very small extent. At the end of half an hour eight times as much carbohydrates have left the stomach as of protein. Fats retard the passage of both carbohydrates and protein from the stomach when present in considerable amount. When the gastric contents of the stomach remain alkaline their passage from the stomach is delayed.

It has been shown by Vogeler and others that hypersecretion and acidity are accompanied by low motility and the reverse. Where there is an excess of acid in the gastric fluids, the entrance of this strongly acid material into the duodenum causes a prolonged closure of the pylorus. 
Acids delay the passage of food from the stomach by increasing the secretion of gastric acid.

Acid protein, that is, protein to which acid has been added, passes through the pylorus much more rapidly than does ordinary protein.

Protein is discharged more slowly than starch because it zombines with the free hydrochloric acid and so delays the opening of the pylorus.

Fat remains long in the stomach for the reason that when fat enters the duodenum it closes the pylorus just as does the gastric acid. The purpose of this is to prevent the entrance of fats into the intestine except at such a rate as will permit them to be absorbed.

Another reason for the slow passage of fats from the stomach is that they lessen the activity of the acid-forming glands.

Fat stimulates the flow of pancreatic juice. This is necessary because in the presence of fats the secretion of hydrochloric acid, which is also a stimulant for the pancreatic secretion, is lessened.

When fats are taken in large quantities the pancreatic juice and bile flow back into the stomach.

Water begins to pass into the intestine almost as soon as it is swallowed. A pint of water may pass out of the stomach in thirty minutes.

Water excites the acid-forming glands. This is an important provision whereby the normal stomach is kept supplied with gastric juice even when containing no food, which is necessary to prevent the growth of molds and bacteria in the stomach.

When fats and carbohydrates are fed together the carbohydrates are discharged slowly. Fats and protein fed together are discharged still more slowly. Fats hinder the secretion of gastric acid and the protein absorbs a considerable portion of what little acid is secreted, thus causing great delay in the opening of the pylorus.

Hyperacidity causes delay in the emptying of the stomach by keeping the pylorus closed. A solution of hydrochloric acid of a strength of seven-tenths to eight-tenths of one per cent. causes continued spasm of the pylorus.

Since it has been shown that the gastric acid is never secreted at greater concentration than one-half of one per cent., it seems clear that pyloric spasm is really the result of a hypersensitive 
state of the gastric mucous membrane or a reflex disturbance from a diseased gall bladder or some other source.

Gas in the stomach hinders peristalsis by preventing contact of the food with the gastric walls. This is especially true when the position is horizontal.

\section{The Effects of Heat and Cold.}

There are clinical facts which indicate that extreme heat and cold applied to the surface of the body influence the action of the intestinal muscles. For example, it is well known that the cold bath and exposure to cold air have a decided influence in producing hunger and appetite. The prolonged cold sitz bath increases constipation by producing a spastic or contracted condition of the colon.

For many years the writer has regularly prescribed as a means of encouraging appetite an ice bag placed over the region of the stomach for half an hour before meals. Hot applications over the stomach lessen appetite. Hot applications over the abdomen and the hot enema cause relaxation of the spastic colon. Levin has shown that warm water leaves the stomach much faster than cold water.

\section{The IMovements of the Small Intestine.}

Peristalsis is the process by which the food is moved along the alimentary canal from one section to another. There are three forms of peristalsis:

First, a slowly advancing contraction which moves through a short distance in an intestinal loop. This is the ordinary method by which the food is moved along the digestive tube. The rate of advancement is half an inch to an inch a minute.

A second form of peristalsis is a wave movement that sweeps rapidly over a large part of the intestine, sometimes from one end to the other, without pause. This form of peristalsis is known as peristaltic rush.

Another movement which occurs in the small intestine is the so-called segmentation, which consists of a churning movement and simple manipulation of the food with little or no advancement. Peristaltic movements are in general in one direction, from above downward, but under some circumstances the direction of the movement may be reversed. The peristaltic rush occurs after the administration of a purge and is sometimes ob- 
served after a stimulating enema, as of soap suds, or diluted molasses or lemon juice.

The time required for food to pass from the stomach to the colon differs with different foods, being four hours for carbohydrates, five hours for fats, and six hours for proteins. Cannon observed that bran not only stimulates gastric peristalsis but also moves through the small intestine with unusual rapidity. Vegetable foods in general pass through the intestine more rapidly than animal foods because of the presence of cellulose and the larger amount of protein and fat in flesh foods.

According to Cannon, when a horse eats oats, although its intestine is more than twice as long as the human, the material passes through the small intestine in a shorter time than in man.

Irritation of the colon slows the passage of food from the stomach and also its movement through the small intestine. (Carlson.)

\section{The Movements of the Colon.}

The contents of the small intestine, when discharged into the cecum, are nine-tenths water. In the cecum two-thirds of this water is absorbed. According to Roith, the contents of the transverse colon have as great a consistency as those of the rectum, which shows that little absorption occurs in the lower half of the colon.

When the colon becomes filled with fecal matters, peristalsis ceases or is greatly slowed.

The peristaltic waves of the colon begin in the middle of the transverse colon. The duration of the wave movements is four to five minutes. Antiperistaltic waves, starting at this point, pass backward to the cecum at intervals of ten to twenty minutes. These waves occur in groups at the same rate as the peristaltic waves of the stomach, about five per minute.

Enemas excite antiperistalsis in the colon and stimulate the movements of the small intestine. The antiperistaltic waves set up by the enema are often very vigorous and may often continue for an hour or more. This is doubtless one cause of the frequent retention of the enema, the water injected being held in the cecum by antiperistalsis.

Activity of the colon is stimulated by the entrance of food from the small intestine through the ileocecal valve. Both peristaltic and antiperistaltic waves occur. Although strong antiperis- 

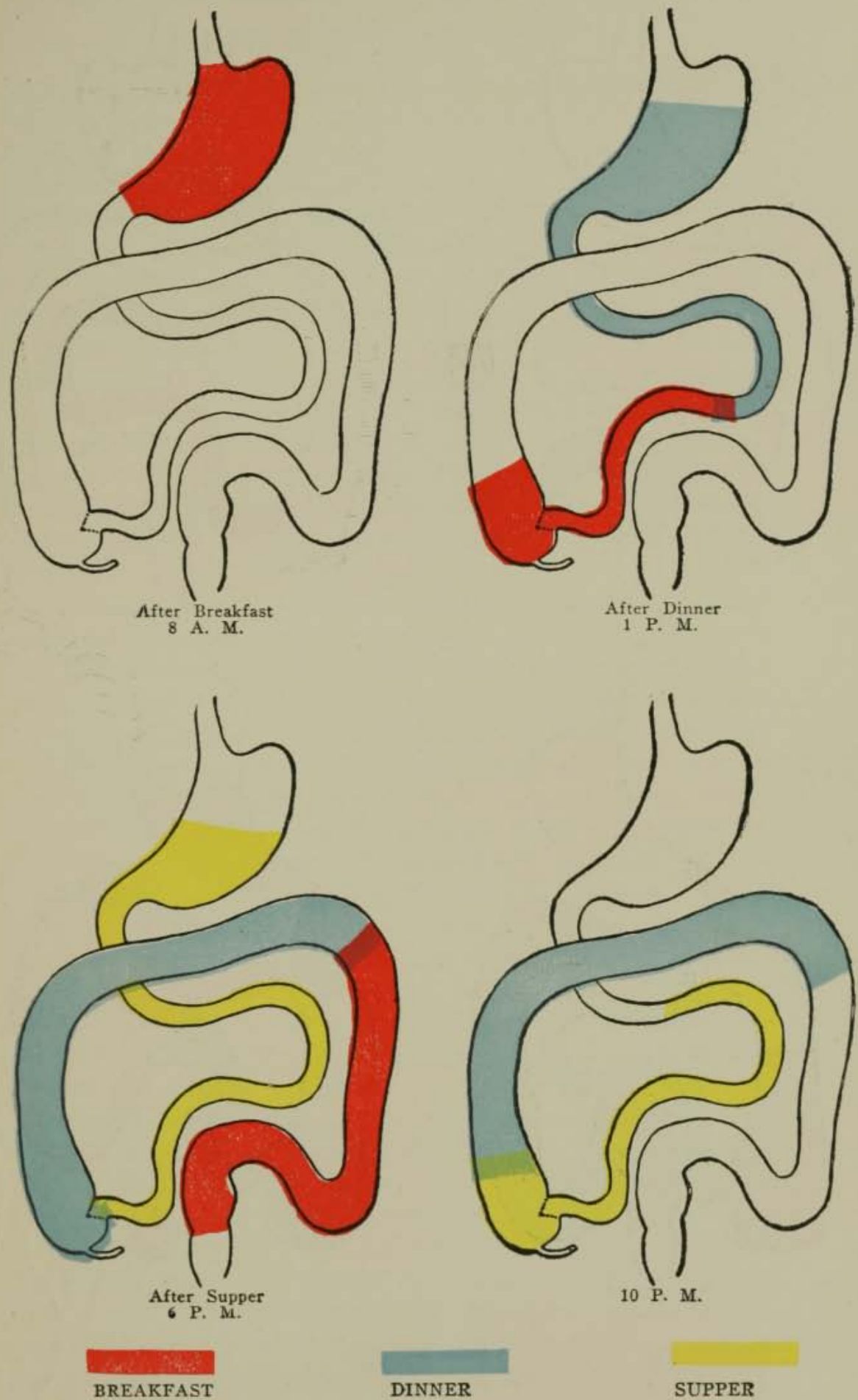

DINNER

\section{SUPPER}

THE ALIMENTARY PROCESSION AS SHOWN BY THE X-RAY 

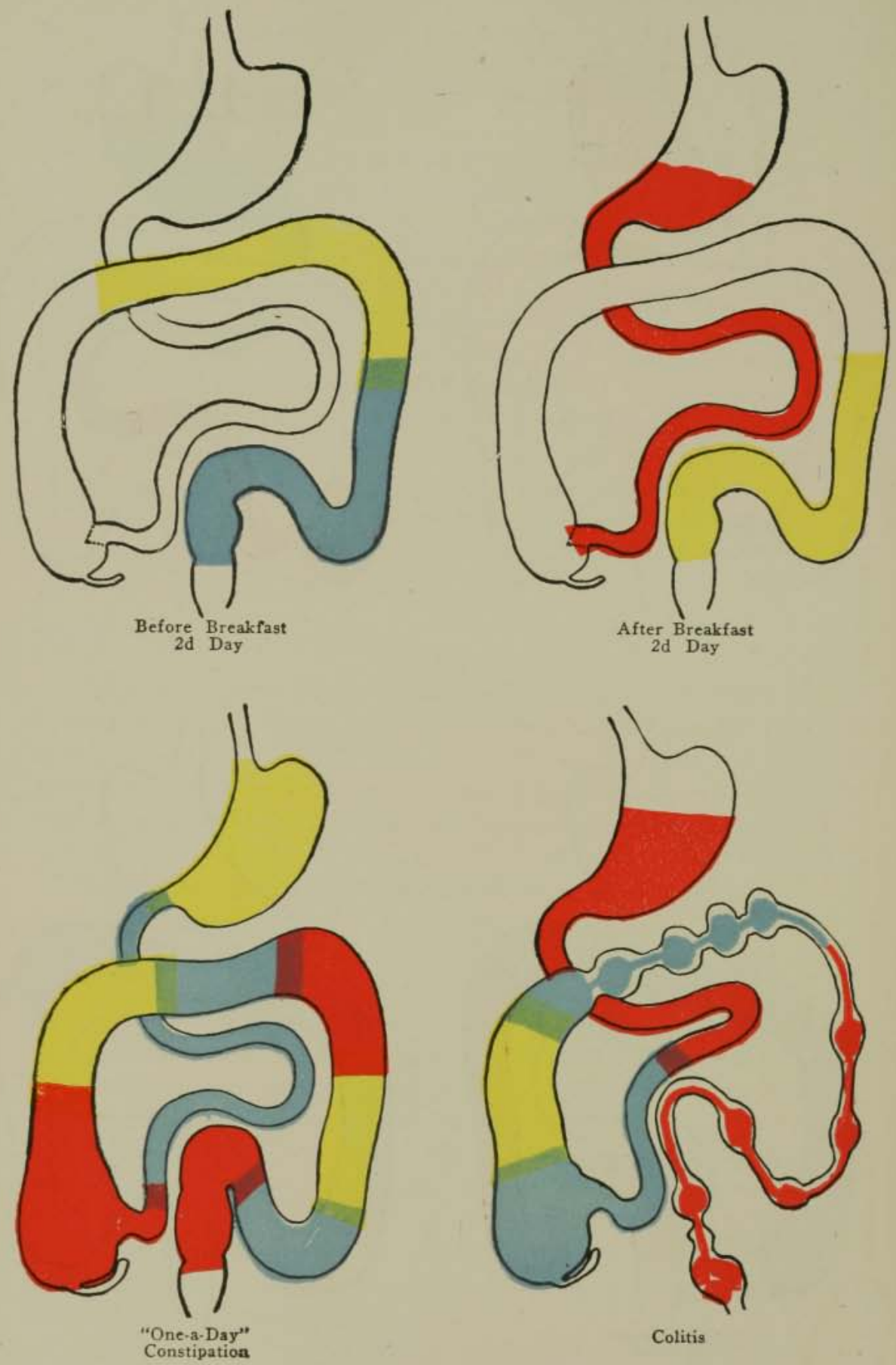

Colitis

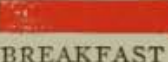

DINNER

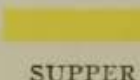

THE ALIMENTARY PROCESSION AS SHOWN BY THE X-RAY 
taltic waves force the contents of the cecum back against the ileocecal valve, this structure is so efficient a check valve it prevents the passage of material from the colon into the small intestine. At least this is true when the valve is intact. Cannon reports that although he has watched this movement hundreds of times, he has never but twice seen material pass from the colon into the small intestine. In one of these cases the colon of the animal had been greatly distended by the introduction of water.

The ileocecal sphincter, which is located just behind the ileocecal valve, is provided with a special nervous control distinct from that of any other part of the intestinal canal, showing that this is an important and essential structure. Its evident purpose is to hold back in the small intestine the digesting foodstuffs until the processes of digestion and absorption are complete and the wastes ready to be passed on into the colon for dismissal from the body.

The function of the ileocecal valve is to maintain the normal forward movement of the food residues and wastes pushed into the colon from the small intestine. Without the ileocecal valve antiperistalsis would continually drive back into the small intestine the contents of the colon and thus prevent its forward movement.

Says Cannon: "Antiperistalsis in the colon gives new meaning and value to the location of a sphincter or valve at the opening of the ileum. For, inasmuch as the valve is normally competent, the constrictions repeatedly coursing toward it force the food before them into a blind sac. The effect on the contents must be the same as the effect seen in the stomach when the pylorus remains closed before the advancing waves. The confined material is pressed upon by the approach of each constriction; but since it cannot go onward in the blind sac, and is, moreover, subjected to increasing pressure as the constriction comes nearer, it is forced into the only way of escape-i. e., away from the cecum through the advancing constricted ring. About twenty-five waves in the cat affect thus every particle of food in the colon during each normal period of antiperistalsis. The result must be a thorough mixing of the contents, and a bringing of these contents into close contact with the absorbing wall-a process which has already been variously repeated many times in the stomach and in the small intestine. The last remnants of value in the food, with some of the water, are here removed; and the waste is passed onward into the distal colon to be ejected from the body." 
Case established by extensive studies conducted in the X-ray laboratories of the Battle Creek Sanitarium the same antiperistaltic function for the human colon that Cannon first observed in animals.

According to Hurst, the time required for the food to pass through each of the three parts of the colon is two hours.

The movements of the colon are about 50 per cent. less active during sleep than during the waking hours.

The taking of food greatly accelerates the movements of the colon. Hurst observed more progress of the residues in the colon during a meal hour than during four previous hours. It appears, in fact, that most of the progress of the food residues in the colon occurs during the taking of food and shortly after. Hence the importance of taking ample time to masticate.

Under normal conditions, when the colon is kept free from accumulating residue, the new materials brought into it "are moved onward into an empty distal section by a sudden push lasting a few seconds." (Holzknecht.) Three or four such pushes of three seconds each are sufficient to transmit the food residues from one end of the colon to the other.

According to Hurst, the entire colon is emptied by a single contraction in normal defecation. In constipation, and in those whose bowels move but once a day, only the lower part of the colon is emptied.

The highly detrimental effect of constipation is clearly shown by the fact observed by Cannon that "When the colon is full it is usually quiet."

The intra-abdominal pressure in the rectum during defecation is four to eight times the ordinary pressure. It may amount to two to four pounds to the square inch. When the abdominal muscles are weak, as the result of bad posture, improper dress or neglect of exercise the intra-abdominal pressure may be insufficient to secure thorough emptying of the colon. This is undoubtedly one of the most common causes of constipation.

As regards the time required for the complete transit of the alimentary tract. Cannon says: "If approximately nine hours are required for material to reach the descending colon in man, the waste from food taken at eight o'clock in the morning might be discharged at five o'clock in the afternoon."

Evidently the normal rhythm would secure three bowel movements daily, or a bowel movement after each meal. Unfor- 
tunately the normal condition is rarely found among civilized people, who almost universally suffer from chronic constipation through neglect. Cannon says: "If the signal is not soon obeyed, it ceases to be given; the feces may then remain long in the rectum without calling forth sensations, and the defecation reflex be to that extent impaired."

The effects of certain drugs upon peristalsis is very pronounced. Nicotine at once abolishes peristalsis in the small intestine. Adrenalin stops peristalsis of the stomach immediately and causes intra-gastric pressure to fall to zero.

\section{The Nerves of the Alimentary Canal.}

The activities of the alimentary canal are controlled by three sets of nerves. First, the motor nerves, consisting of the two pneumogastric nerves which control the food tube from the esophagus to the lower end of the ileum; and the sacral or pelvic nerves which control the colon and the ileocecal valve. Second, the sympathetic nerves, the action of which is to inhibit or control the action of the motor nerves. Third, nerve ganglia located in the walls of the gut.

The tonus or tonic contraction of the stomach, when empty, is due to the local nerves in the walls of the stomach. The peristaltic waves may continue even after the stomach has been removed from the body of an animal provided it is kept in a warm salt solution. Peristaltic and antiperistaltic waves of the colon and the segmenting or churning movements of the small intestine are likewise due to local nerves.

The pneumogastric nerves control the pylorus and cardiac orifice. When these nerves are stimulated, the pylorus contracts and the cardiac orifice relaxes, an admirable arrangement which makes vomiting possible as a means of emptying the stomach. This arrangement also provides for the entrance and rejection of food in the stomach.

When food is swallowed, the branches of the pneumogastric nerves in the throat are stimulated; as a result the pylorus contracts, the cardiac orifice opens in eight or ten seconds from the time food starts from the mouth, the pressure in the stomach falls to zero, and the stomach increases in size to enable it to accommodate the incoming food. The closure of the pylorus during swallowing is necessary to prevent the reflux of bile and other matters from the small intestine. 
The appetite juice, or psychic secretion, is produced through the action of the pneumogastric nerves, which are reflexly stimulated through the nerves of taste. There is likewise a psychic gastric tonus which causes the stomach to contract when food is taken into the mouth.

The stomach, intestines, and other viscera do not possess the ordinary sensation of pain or touch. Hurst and others have shown that gastric pain and most other gastric symptoms are due to exaggeration of gastric tonus which may arise from various causes.

Pain referred to the abdomen is believed to be really due to the extreme sensitiveness of the peritoneum or lining membrane of the abdominal wall, which is very highly endowed with the prin sense, and when in the state of inflammation is exquisitely sensitive,

The muscular sense is highly developed in the walls of the stomach and intestine. Violent contractions of the muscular walls of the intestine, or cramp, give rise to pain much the same as does muscular cramp in any other part of the body.

Because of the close connection of the duodenum and the colon with the abdominal wall, inflammation and violent contractions of these parts are more likely to give rise to pain than when they occur in other parts.

Stretching is the most efficient means of stimulating the intestinal wall to contraction. This is the cause of the pain of colic.

The introduction of fluid into the colon gives rise to both antiperistaltic and peristaltic waves by distending the colon. If the quantity of liquid introduced is so large as to overstretch the gut, severe colic pains may be induced.

When the sympathetic nerves are stimulated the result is complete relaxation of the entire alimentary tract except the pylorus, ileocecal sphincter and the anal sphincters. Nicotine irritates the sympathetic nerve and thus has the effect of paralyzing the pneumogastric nerves, abolishing their action upon the stomach and intestine.

The pain of gastric ulcer is due to inflammation of the lymphatic vessels and glands in the vicinity of the ulcer. In this condition a very weak acid solution may give rise to pain, whereas in a healthy stomach no sensation whatever is produced by a strong solution of hydrochloric acid (one-half of one per cent.).

Great weakness or depression may cause complete suspension 
of the peristaltic waves of the stomach and intestine so that the food remains stationary. Anger, anxiety, mental or physical distress may cause the movements of the stomach to cease at once. Cannon observed this in the cat, rabbit, dog and guinea pig.

The antiperistaltic waves of the colon are abolished by excitement, which may be a cause for the occasional occurrence of sudden diarrhea under this condition.

The excitation of the sympathetic nervous system by emotional excitement may cause cessation of both secretion and motion in the entire alimentary canal.

The tonus of the stomach, which starts digestion, depends upon appetite. Appetite gives rise to hunger, and Cannon has shown that hunger is due to contraction (tonus) of the gastric muscles.

\section{The Normal Intestinal Rhythm.}

When at the end of eight hours the unusable residue is pushed through into the colon it is ready to be dismissed from the body and should be dismissed without any considerable delay. The colon possesses no digestive function. It is a waste disposal organ, and when properly trained hastens along the refuse received from the small intestine, together with the various body wastes which are excreted into it, and pushes them out of the body before they have had time to undergo putrefactive changes.

Recent X-ray studies of the colon show that it possesses a controlling mechanism somewhat similar to that which controls the heart. When undamaged by mistreatment it handles the food residues in a wonderfully intelligent and efficient manner. A check valve placed between the small intestine and the colon prevents the reflux of the waste matters from the colon, which is highly important. The rapid transit of the foodstuffs through the small intestine affords no opportunity for putrefactive changes. The small intestine never rests until its undigested residues have all been pushed on into the colon, a task which is practically always completed at the end of eight hours from the beginning of a meal. Fortunately, the movements of the small intestine are wholly independent of the will, and its expeditious action is rarely interfered with by any cause whatever. With the colon, however, the case is different. Whether or not the colon is emptied of its contents depends more or less upon individual whim and convenience. 
If evacuation of the bowels at the proper time is neglected, the waste products which are continually being pushed forward from the small intestine accumulate and the colon becomes filled from end to end and even distended by its undue accumulation. On the other hand, if the colon is permitted to function in a perfectly normal manner, the waste products which reach the center of the colon eight hours after the taking of a meal are by the impetus received by the next meal pushed forward nearly to the point of exit and by the impetus received from the following meal are pushed out of the body.

A moment's thought will show that when this order of things exists, the food residues and body wastes are never retained long enough to permit putrefactive changes to occur. For example, if breakfast is taken at 8 o'clock the residue may be dismissed by 10 o'clock P. M. or at the end of 14 hours; the dinner residue will be dismissed soon after rising the next morning or at the end of 17 to 19 hours; and the supper residue will be dismissed after breakfast or, at the latest, after dinner the next day or at the end of 15 to 20 hours. So long as this happy order is maintained, there is no putrefaction in the colon, no absorption of putrefaction products, and in consequence freedom from biliousness, headaches, lack of appetite, and the long list of miseries which are the natural result of the absorption from the colon of the poisons of various sorts which are always present when constipation exists; that is, when the bowels move but once a day or at less frequent intervals.

The universal use of pills, mineral waters and other means of stimulating the activity of the bowels is the natural result of the abuses to which the colon is subjected by wrong diet, neglect to attend to the call of nature and various other disturbing influences which are almost universally active among civilized people.

Our dogs, cats and other household pets are "house-broken" and in consequence become constipated like their masters who are also house-broken. The poor colon has suffered to an extraordinary degree from the conditions imposed by house dwelling and civilized life. An Arab, when offered a good situation in a neighboring city, declined for the reason that in the city it was often inconvenient to evacuate the bowels promptly when nature demanded. 


\section{THE LIVER}

The liver is one of the most remarkable and versatile organs in the body. The weight of the liver, the largest gland in the body, is three and one-half pounds. It contains more than twenty million bile-making cells which together produce every twentyfour hours twenty ounces of bile. It has been estimated that each cell makes in a lifetime of sixty years, ten drops of bile!

These facts place before the mind at once a vivid picture of the wonderfully delicate structure of this marvelous organ. The liver performs more different kinds of work than any other organ of the body. Here is a brief summary of the chief kinds of work performed by the liver:

1. The Liver Aids Digestion. When food leaves the intestine and enters the portal vein the digestive work is not yet quite completed. It requires certain delicate touches which only the liver can give; hence the portal vein carries to the liver the products of gastric and intestinal digestion and there the digestive work is completed. Levulose or fruit sugar is by the liver converted into glucose or dextrose, the only form in which the body cells can make use of sugar. Galactose, derived from milk sugar, is also converted into dextrose by the liver. The liver converts wastes and surplus protein into ammonia and further converts ammonia into urea. Finally, the liver aids digestion by secreting and pouring into the intestine 20 ounces of bile every 24 hours.

2. The Liver Stores Food Supplies. The sugars of various sorts absorbed from the intestine are by the liver converted into glycogen or animal starch and stored up in its cells, a source of energy to be drawn upon until the supply is replenished by another meal. From moment to moment, as needed, the liver changes the insoluble glycogen back to sugar and pours it into the blood to be sent to the heart and other muscles for use.

The liver also stores up iron. This is particularly true in infancy. The liver of the new born infant contains nearly all the iron that will be required to produce the hemoglobin of the blood until the infant has attained the age of nine months. This is necessary for the reason that the mother's milk contains but a very small percentage of iron. The livers of guinea pigs store no iron. No storage is required because the guinea pig begins the very day it is born to eat grass which is rich in iron. But milk contains little iron and as the human infant receives little 
iron-containing food until after it is weaned, its liver is stocked with iron before its birth to furnish the material needed for blood making during the nursing period.

3. The Liver Stores Metallic Poisons. Metals, such as lead, which may be found in water which has passed through lead pipes, mercury used as medicine or absorbed by other means, and various other metallic substances found in hard waters and mineral waters are captured, in large part, by the liver. The storing of these foreign substances in the liver more or less cripples it and impairs its functions; hence the liver should be protected against damage from this source.

4. The Liver Detoxicates Organic Poisons. It is the liver which stands between the smoker and death. The liver destroys or detoxicates nicotine and prepares it for elimination by the kidneys. This is true of the numerous poisons absorbed from the colon and the various poisons produced by the body as well as of poisons received through food and drink, such as alcohol, tea, coffee, etc. When the liver has more of this work than it can well perform the blood becomes saturated with poisons and a state of autointoxication is produced. In this condition a person suffers from the symptoms which are commonly known as "biliousness." The indication is not that the liver is inactive but that more work is imposed upon it than it is possible for it to perform.

Detoxication of the normal body wastes and preparation of these substances for elimination by the kidneys is the normal work of the liver. To require of this heavily loaded organ to do unnecessary work in dealing with such poisons as alcohol, nicotine, caffein, and the poisons of pepper, mustard and similar condiments, is unnatural and must result in the premature wearing out and failure of the liver. Boix showed that pepper is six times as active in causing gin liver or cirrhosis as is gin, and acetic acid, the acid of vinegar, twice as active.

5. The Liver Probably Makes Ferments and Hormones. These subtle substances are essential for the working of the wonderfully intricate mechanism of the body. Nearly all of the vital changes which take place in the body are the result of the action of activating substances, that is, substances which set in motion vital or chemical changes by their mere presence. Many of these hormones and ferments are known, but it is believed that the unknown activating agents greatly outnumber those which are as yet known. 
6. The Liver Makes Bile. In the bile are found highly poisonous alkaline wastes. Bouchard showed that the bile is six times as poisonous as the urine. The alkaline substances which the bile contains serve a useful purpose in neutralizing the acidity of the gastric contents, which must be neutralized quickly after they enter the small intestine to prevent injury to the delicate mucous membrane of these parts, which is not able to withstand the action of acids as is the mucous membrane of the stomach. Bile activates the pancreatic juice which, when it leaves the pancreas, possesses no digestive activity. Bile also emulsifies fats, aids the absorption of fats and other digestive foodstuffs and inhibits or hinders the growth of germs and the production of germ poisons, possibly also serving in some degree to neutralize the poisons produced by certain bacteria in the intestine.

Recent observations indicate that the bile contains uric acid which the liver excretes with other poisons.

Bouchard showed that the bile is six times as poisonous as is the urine. It should be promptly eliminated by the discharge of the bile with other wastes by evacuation of the bowels. When constipation exists, the bile is reabsorbed and again excreted by the liver, thus becoming concentrated. This concentration of the bile is probably a common cause of what is generally known as "biliousness."

7. Attention has recently been called to the fact that the liver destroys foreign proteins which find their way into the blood stream without having undergone complete digestive transformation. This function is a protection against anaphylaxis. When inefficiency of the liver exists, this function fails and the condition may be detected by Widal's test (See Index). 


\section{MASTICATION}

Grinding, mixing, spreading, transporting and discharging are the several mechanical factors involved in the process of digestion. In chewing, the cheeks and the tongue co-operate in keeping the food between the teeth, which by a gradual reduction process grind hard substances to the proper fineness for swallowing. The consistency of the food is tested by the cheeks, the gums, the tongue and the soft palate. When found imperfectly chewed the food is automatically returned to the grinding surfaces for further chewing. The soft palate, the membranous veil which hangs in the back of the throat, is endowed with a delicate tactile sense which especially equips it for the inspection of the foodstuffs which come in contact with it in the act of swallowing. When properly chewed, much of the food becomes liquid, being dissolved by the saliva and to some extent liquefied by the digestive action of the salivary ferment. The undissolved portion is reduced by thorough mastication to particles not more than onetwelfth of an inch in diameter. It is not uncommon, however, to find in vomited matters masses half an inch or more in diameter, because of insufficient mastication and the consequent failure of the stomach to reduce the food to the liquid state necessary for intestinal digestion.

The amount of pressure exerted in chewing, when tested with the dynamometer, is found to be surprisingly great, amounting in a person with well developed jaws to as much as 270 pounds when the teeth are brought together with as much force as possible. In the chewing of delicate or friable substances a pressure of not more than 1 or 2 pounds is sufficient. Tough meat requires a pressure of 40 pounds to divide its fibers. In the chewing of bread crust the pressure rises to 60 pounds, though when well softened with water or saliva it is easily crushed with a pressure of 3 pounds.

The act of chewing does more than merely to mechanically divide the food. It promotes the flow of saliva and prepares the stomach for the reception of the food. Chewing of palatable food causes the stomach to contract and causes the gastric glands to pour out the appetite juice which is necessary to start the process of gastric digestion after the food is received into the stomach. If the food is tasteless, or indifferent, mastication has no effect upon the stomach. When food is hurried through the 
mouth instead of being properly chewed, overeating is likely to occur as the gustatory reflexes through which the body instinctively makes a selection of foodstuffs suited to its needs have no opportunity to operate.

\section{Spallanzani's Discoveries About Mastication.}

Probably many people imagine that Horace Fletcher was the first apostle of thorough mastication; but the fine art of mastication really had a much more ancient origin. To Spallanzani, a man of science who flourished in the eighteenth century (1729-1799) belongs the credit of having been the first to demonstrate the importance of thorough chewing of the food. In his great work on "Digestion," Spallanzani gives an account of experiments which demonstrate the importance of thoroughly chewing the food. Spallanzani was the first to study experimentally the properties of the gastric juice. He made small hollow spheres with perforated sides. Into these he introduced foodstuffs of various sorts, then swallowed the spheres and after they had passed through the body, examined the contents, noting the changes which had been wrought by digestion. By this ingenious method, he also studied the effects of mastication.

We quote his own account of his experiments and his conclusions therefrom from an old work, "The Art of Invigorating Life," by Wm. Kitchiner:

I took two pieces of Mutton, each weighing 45 grains, and having chewed one as much as I used to chew my food-enclosed them in two separate spheres-and swallowed them at the same time-these tubes were voided at the same time- of the masticated meat there remained only 4 grains - of the other there were 18 left.

The necessity of Mastication is sufficiently known-there is perhaps no person who has not, some time or other, suffered from Indigestion, for want of having chewed his food properly. The reason is obvious. Not to mention the saliva which moistens the food, and predisposes it to be dissolved, it cannot be doubted, that when it is reduced to pieces by the action of the Teeth, the gastric fluid penetrates, and attacking it at more points, dissolves it more speedily than when it was whole. This is true of menstrua in general, which always dissolve bodies sooner when they have been previously broken to pieces. This is also the reason why, in other experiments, masticated bread and dressed flesh were more readily dissolved than unchewed bread and raw flesh. The boiling had made it tenderer, and consequently disposed it to allow ingress to the gastric fluid. 
Three-quarters of a century later, the famous Dr. Arbuthnot tells us that "Mastication is a very necessary Preparation of solid Aliment, without which there can be no good Digestion"; and that

To Chew long, and leisurely, is the only way to extract the essence of our food-to enjoy the taste of it, and to render it easily convertible into laudable Chyle, by the facility it gives to the gastric juices to dissolve it without trouble.

The pleasure of the Palate, and the health of the Stomach, are equally promoted by this salutary habit, which all should be taught to acquire in their infancy.

From 30 to 40 (according to the tenderness of the meat) has been calculated as the mean number of Munches, that solid meat requires, to prepare it for its journey down the Red Lane; less will be sufficient for tender, delicate, and easily digestible white meats.

Finke, in his "Medical Geography," published more than a hundred years ago, remarks,-

Slave dealers are well acquainted with the characteristic signs of perfect Health-any defect of which much diminishes the value of a Slave. The want of a Tooth makes a Slave worth two Dollars less.

Mastication is the source of all good Digestion;-with its assistance, almost any thing may be put into any stomach with impunity:without it, Digestion is always difficult, and often impossible: and be it always remembered, it is not merely what we eat, but what we digest well, that nourishes us.

Brillat-Savarin, a French advocate and judge who at the time of the French revolution sought refuge in this country, in an interesting work entitled "Physiologie du Gout," calls attention to the importance of mastication, telling us that the foodstuffs "must be comminuted by the teeth, impregnated with the saliva and other gustatory juices, and pressed against the palate by the tongue till the juice so yielded makes a favorable impression upon the gustatory papillæ, and the triturated body receives from them the passport necessary to enter the stomach.

In performing this function, the tongue unites the food in a pulpy mass in the middle of the mouth; and then, supporting itself against the lower jaw, it lifts up its central portion, so that it forms at its root a declivity through which the food slips into the back part of the mouth, where it is received by the pharynx, which, contracting in its turn, propels it into the esophagus, of which the peristaltic motion conducts it into the stomach.

Beaumont a few years later in the epoch making work in which he recorded the results of his "experiments and observa- 
tions on the gastric juice and the physiology of digestion" made on St. Martin, tells us that "Mastication is absolutely necessary to healthy digestion." He adds, "If food is swallowed rapidly, more will generally be taken into the stomach before the sensation of hunger is allayed than can be digested with ease."

The noted Count Rumford, and Sinclair, the famous author of "The Code of Health," likewise advocated thoroughgoing mastication.

The late Dujardin-Beaumetz, of Paris, called special attention to the importance of mastication many years before the advent of Fletcherism. Said this eminent medical teacher:

It is not sufficient simply to prescribe a vegetarian regimen, abstinence from alcoholic drinks, and the use of alkaline waters; it is necessary to require of the patient that he give to his meals sufficient time to secure complete mastication of his food. Miahle maintained many years ago that all dyspepsia is the result of insufficient mastication. Without going so far as this, one may say, however, that the presence of badly masticated foods is one of the most frequent causes of gastro-duodenitis. Such foods really act as foreign bodies, which irritate the pyloric region of the stomach and the mucous membrance of the duodenum. The same is true of an excessive quantity of foods. Great eaters are very subject to gastritis. It is necessary, then, to eat rationally and to masticate slowly.

Sir Andrew Clark, physician to Queen Victoria, used to say that since the mouth contains thirty-two teeth every mouthful of food should receive thirty-two bites.

It is thus very evident that the importance of thorough mastication of the food is by no means a new or a modern discovery, but is simply the recovery of a much neglected truth, the value of which was fully appreciated by our remote ancestors as well as other members of the animal kingdom.

Since 1875 the writer has frequently demonstrated experimentally in public lectures the necessity for thorough chewing of the food to secure the digestion of starch. During the entire period it has been his practice to recommend to his patients the thorough mastication of dry food for the purpose of promoting the health of the teeth and good digestion. This practice, in fact, was begun by the writer himself more than fifty years ago as a result of an elementary study of human physiology. Mr. Fletcher certainly did not discover the necessity for thorough mastication. His Fletcherizing movement was a renaissance. 


\section{Special Indications for Thorough Mastication.}

Special pains should be taken to masticate the food to the extent of reduction to a soft pulp in the following cases:

1. Achylia, a condition in which the stomach has ceased to make hydrochloric acid so that the action of the gastric juice is suspended. It is true that in these cases salivary digestion continues so that the starch may be more completely digested than in the normal stomach. Nevertheless, the food is no longer subjected to the solvent action of the gastric juice, and hence if the food enters the stomach in large lumps, these masses will be pushed on into the small intestine and disturbances of intestinal digestion will be likely to result. It must be remembered, also, that in cases of achylia the duration of gastric digestion is greatly reduced, the food leaving the stomach often in two or three hours instead of being retained for four or four and a half hours. It is evident, then, that very thorough mastication is required in cases in which the normal gastric acid is not present.

2. In cases in which the operation of gastro-enterostomy or the polya or any other reconstructive operation has been performed upon the stomach, thorough mastication of the food is highly important, for such a stomach is more or less crippled at the best, and mouth digestion should be advanced as far as possible so as to lighten the work of the stomach.

3. In cases of obesity, thorough mastication is important as a means of limiting the amount of food eaten. In diabetes, in which the food is necessarily limited, thorough mastication should be enjoined upon the patient as a means of securing satigfaction of the appetite without a large intake of food. In bulimia (excessive appetite), epilepsy and in persons subject to hiccough and aerophagia, thoroughness of mastication is necessary.

4. Prolonged mastication is also a useful aid to peristalsis and should be practiced by persons subject to constipation. Hurst, Case and others have shown that the food residues move along in the colon four times as rapidly during the taking of a meal as during the intervals between meals. In other words, mastication of food seems to set the whole alimentary canal in motion. In a recent paper Hurst asserts that the movement of residues in the colon occurs almost exclusively during the taking of food. This fact emphasizes the necessity for regular and deliberate eating and suggests the taking of fruit between meals and especially at night as an aid to efficient colon activity. 
5. Efficient and prolonged mastication of the food is also highly important in cases in which the gall bladder has been removed. The reason for this is that by prolonged mastication the food will enter the stomach more slowly and hence will pass out into the small intestine more slowly. This is important for the reason that when the gall bladder is removed the liver is not able to store up any considerable quantity of alkaline bile with which to neutralize a large quantity of highly acid material coming from the stomach within a short time.

6. Very thorough mastication of the food is required whenever it is necessary to prescribe a diet of dry or solid food.

Professor Strümpell made an experiment for the purpose of determining the influence of imperfect mastication upon the digestion and absorption of protein. He swallowed, half chewed, a plate of boiled lentils. He found that only 40 per cent. of the aitrogen was absorbed, or less than half the amount that should have been taken up.

According to Gautier, the same is found to be true of other regetables and also with bread when eaten in too soft a state, and when care is not taken to secure a thorough and abundant mixture of saliva.

\section{When the Food Should Be Chewed Less.}

While the thorough mastication of food is usually essential to good digestion and good health, there are conditions in which much mastication is to be avoided, the food being taken in liquid or purée form and so needing no mastication to prepare it for swallowing.

1. It is well known that the chewing of food stimulates the formation of gastric acid. It is evident that in cases in which an excess of acid is formed much chewing will tend to aggravate the difficulty and hence hard and dry foods which need thorough chewing to prepare them for swallowing should be avoided.

2. In cases of gastric and duodenal ulcer, also in cases of gall bladder disease accompanied by pyloric spasm or "hunger pains," chewing should be avoided.

3. Chewing is also to be restricted in cases of gastric hypersensitiveness in which pain follows eating of solid foods although the gastric juice contains no excess of acid as shown by tests after a test meal. Soft foods should be eaten. 


\section{GASTRIC SENSATIONS}

In health there are but four distinct sensations associated with the stomach; namely, hunger, appetite, satisfaction or satiety, and repletion.

The nausea which sometimes occurs in persons who are apparently in good health, though in such cases transient, may be properly regarded as a morbid symptom indicating some derangement of the normal gastric functions.

Hunger appears to be a very universal sensation. Minute vegetable organisms, such as the swarm cells of spore-producing plants, according to Lister, may behave very much like hungry animals, swallowing microbes and digesting them with avidity while resting, then swimming off to find more food.

People who eat several times a day often never experience the sensation of real hunger. Hibernating animals have no hunger. Insects are not hungry during the winter. The myriads of salmon which ascend the Columbia River are said never to eat, notwithstanding the great exertion in swimming hundreds of miles up stream. On the other hand, birds appear to be hungry all the time; at least they eat continually, though food is always found in their crops. The caterpillar does nothing but eat and defecate; but in certain species, after the caterpillar becomes a butterfly it never eats at all. In some insects, according to Faber, there is no provision for hunger, the digestive organs being lacking in the fullest developed insect.

Hunger may become so intense as to cause great lassitude, weakness, nervous irritability and even headache and nausea. In extreme cases fainting may occur.

The seat and cause of hunger have been the cause of much research and discussion by physiologists for centuries. Haller thought the sensation of hunger to be caused by the grinding or rubbing together of the folds of the mucous lining of the stomach, due to contraction of its walls, observing that in a hungry man "the stomach is always contracted" (pinched). In this opinion the sagacious scientist came very near the truth as we shall see later. Darwin, on the other hand, thought hunger to be due to the absence of contractions in the stomach. Beaumont regarded hunger as the result of "a distension of the gastric vessels" or glands with gastric juice. Ewald considers hunger as purely a psychic sensation due to stimulation of the hunger center by an impoverished state of the blood. Boldyreff discovered, in 1905, 
by placing inflated balloons in the stomachs of starving dogs, that strong contractions of the stomach occurred at quite regular intervals. In 1912, by similar experiments upon human beings, Cannon proved that these contractions are the cause of hunger. Each contraction of the stomach gives rise to a hunger pang lasting about half a minute. The contractions may be repeated rhythmically during 15 minutes to an hour or even longer in extreme hunger, and in very intense hunger the contraction may become continuous.

There are two elements in hunger, one relating to the stomach itself, the other relating to the body as a whole.

A very strong sensation of hunger is accompanied by a feeling of emptiness which may affect the whole abdomen. This may be relieved or temporarily abolished by pressure upon the abdominal wall. The starving Indian tightens his belt to relieve his discomfort.

\section{Appetite.}

There is a definite distinction between hunger and appetite. One may have hunger without appetite, a not uncommon experience, but appetite without hunger is the daily experience of those who eat desserts at the end of a full meal. Hunger is referred to the stomach, whereas appetite is nearly always referred to the mouth and throat. One's mouth may water in the presence of savory viands although the sensation of hunger is not felt. Hunger, as Cannon has shown, is due to actual contractions of the gastric walls and is not felt in the absence of these contractions; but appetite is a product of the memory of pleasant sensations of taste and smell associated with eating.

The researches of Carlson have extended the discoveries of Cannon and have thrown much additional light upon the nature of hunger and appetite and the relations of one to the other.

Ryle thus summarizes the modern views of the characteristics which distinguish the sensations of appetite and hunger ("Gastric Function of Health and Disease"):

The first is a pleasant sensation; the second unpleasant. The first may occur in an inadequately fed subject, may be present even when the stomach contains food in plenty, is stimulated rather than diminished by pleasant foods and beverages, and is only abolished by repletion. The second results only from undue abstention from food or excessively rapid 
utilization of food, is absent when the stomach is filled, and is relieved by eating. The first is a daily experience in health; the second is only an occasional experience under the usual conditions of civilized life. The first has a sustained quality; the second is felt as a recurring or intermittent sensation.

Other senses besides taste may stimulate appetite, and in man sights, smells, sounds, and the general sensory comforts of pleasant environment may all react in its favor. To some extent it is undoubtedly a "conditioned reflex." The localization of the appetite sensation is not so accurate as that of the hunger sensation, but this is partly because appetite is a less clamant spokesman. Some refer it to the pharynx or esophagus, some to the epigastrium or to all three. Personally $\mathrm{I}$ experience the sensation mainly in the gastric zone. In any case this ability to localize it suggests that, as with hunger, there may be a local visceral process concerned, and there are various clinical, radiographic, and pathological observations which lend support to the view that appetite, while differing from hunger in many respects, has also a local origin in the upper alimentary tract. These observations point to the tonic activity of the gastric and, perhaps, the esophageal plain muscle as the probable factor, while for the appreciation of hunger peristaltic activity is-as Carlson's experiments suggest-a necessary addition. Peristaltic activity (in the same manner as the activity of skeletal muscles) requires for its initiation a certain degree of passive tension or postural tonus; appetite may occur without hunger, but hunger seldom, if ever, occurs without appetite. It may be urged, too, that just as general well-being and preparedness for physical effort are associated in idea and fact with a favorable state of tonus and adaptability of the skeletal muscles, so, too, digestive well-being and preparedness for digestive effort are associated with a favorable state of what may be termed "anticipatory" tonus in the plain muscle of the stomach or upper alimentary tract generally. Certainly there is a close inter-relationship between the two states, for general well-being and good appetite are associated phenomena, and it will be agreed that appetite is stronger in the vigorous athletic type of individual, and that it varies directly with constitution and habit. Gastric tonus from the radiographic point of view in healthy individuals shows a similar variation with physical type.

In the light of existing knowledge it becomes fair to conclude that we have in the "symptoms of health"-hunger, appetite, satisfaction, and repletion, and the readiness with which they appear and disappear-a measure not only of the muscular sensibility, but also of the muscular and motor efficiency of the stomach. These symptoms, however, afford no index of its secretory behavior.

\section{Satiety.}

Satiety is the state of satisfaction resulting from taking sufficient palatable food to satisfy hunger and slightly distend the stomach. An Irish laborer, accustomed to a diet of potatoes and buttermilk, is not satisfied with a meal consisting of con- 
centrated food less in bulk, even though the actual amount of nourishment taken may be much greater. Fats more than any other foodstuff contribute to the sense of satiety. A very small amount of fat often satisfies the appetite. Many persons find it difficult to eat fat in any but very small quantity on this account.

Repletion.

When the stomach is over-filled and more or less uncomfortable, or even a painful sense of distention is experienced. This sense of repletion may also be experienced as the result of a too rapid filling of the stomach with either solids or liquids.

The sensation of repletion is due to the fact that the stomach is not sufficiently relaxed to accommodate its contents, so the intragastric tension is excessive. Such a condition may exist even when the quantity of salts or liquids swallowed may not be abnormally great. Failure of the stomach to relax sufficiently to receive a proper amount of food is a condition very frequently encountered in case of chronic disease, and is one of the obstacles to be overcome in cases in which the habitual intake of food is insufficient.

\section{Nausea.}

Nausea is in some respects closely akin to hunger; in fact, hunger and nausea are sometimes felt simultaneously. In nausea, however, there is absence of true hunger pangs. Violent contractions occur in the pyloric region, accompanied by a dilatation of the cardiac orifice-a pressure pain sensation and sinking feeling, headache, perspiration, usually giddiness and often great prostration.

\section{Hunger in Infants.}

In adults, hunger usually occurs four to six hours after a full meal. In an infant, hunger occurs in two or three hours. The hunger contractions of the stomach are much stronger in young persons than in those advanced in years.

\section{The Hunger of Starvation.}

Hunger contractions appear to be increased in vigor by starvation. Carlson states that in himself this change was very marked and lasted for some time after a five days' fast. He also 
suggests that this effect may be analogous to the general rejuvenating effect of starvation in certain animals, and even intimates that an occasional period of starvation may exercise a beneficial influence in the prolongation of life and in renewing vigor and capacity for work.

\section{Hunger During Sleep.}

It is a curious fact that while the "tone" of the general muscular system, the blood vessels, the bladder, and other muscular parts decreases during sleep, this is not true of the hunger contractions of the stomach, which not only continue in full vigor but even may become so strong as to cause awakening from sleep in adults as well as in children and dogs.

\section{Mental and Nervous Influence on Hunger.}

Ordinary mental processes do not influence hunger, but the sensation is temporarily abolished by fear, anger, joy and perhaps other emotions.

When a starving person sees or smells appetizing food the nunger pangs are more intensely felt, although the hunger contractions are not increased.

Hunger contractions have been proven to be due to the local nervous mechanism rather than to the pneumogastric or sympathetic nerves. Even when completely isolated from the central nervous system, the stomach makes hunger contractions.

Chewing or tasting palatable foods diminishes hunger contractions. Movement of the jaws, when the mouth is empty, has no influence upon the hunger contractions; but chewing gum, paraffin, even straw or wood, may lessen hunger. Hunger is temporarily relieved by very small quantities of food.

\section{Absence of Hunger.}

Hunger contractions differ from the movements of the stomach during digestion in the fact that the movements of the stomach during digestion are almost wholly confined to the pyloric region, while hunger contractions involve the left portion of the stomach.

Hunger, when present, may be diminished by taking water into the stomach, particularly hot water. Small pieces of ice, when swallowed, have a decided effect in lessening hunger. 
Hunger may also be temporarily abolished by passing a tube into the stomach. Rectal feeding decreases hunger.

Strong acid solutions have the effect of diminishing the hunger contractions, as do also strong alkaline solutions.

The introduction of air into the stomach produces no effect.

Smoking lessens hunger by stimulating the nerves of the mouth. Any stimulus applied to the mouth has a similar effect.

In nausea the cardiac orifice and the fundus of the stomach are completely relaxed; hence hunger is necessarily absent.

Pressure over the stomach by means of a belt lessens the hunger sensation but does not abolish it. A smaller amount of food is required to appease hunger when a belt is applied before the meal. This fact suggests a method of aiding control of the appetite in cases in which it is necessary to restrict the amount of food necessary to appease hunger by preventing the expansion of the abdominal wall which takes place simultaneously with the receptive relaxation of the stomach.

\section{Absence of Hunger in Disease.}

The partial or complete absence of hunger and appetite is common in cases of neurasthenia and hysteria. Absence of appetite is also a frequent result of constipation, especially when the tongue is coated. In such cases there might be hunger without appetite. The absence of hunger in fever has been shown to be associated with the absence of hunger contractions.

Appetite is less in advanced age than in youth, for the reason that the hunger contractions are less vigorous.

\section{Increase of Hunger in Disease.}

In bulimia, hunger is not appeased by eating, or reappears very shortly after a full meal; if food is not taken, headache, weakness, and prostration develop just as in a normal person suffering from extreme hunger. This symptom, which frequently occurs in Addison's disease, may also appear in connection with various nervous disorders. The hunger of bulimia usually appears very suddenly and it may be satisfied or even changed to nausea by a few mouthfuls of food. In these cases there is doubtless an abnormally irritable condition of the stomach. The application of the moist abdominal bandage, accompanied by a hot pack over the stomach, is a valuable means of relief in these cases. 
"Hunger pains" are simply the severe gastric pains which appear when the stomach is empty or within three to six hours after a meal in persons suffering from ulcer of the stomach or duodenum or disease of the gall bladder. These pains are due to contractions identical with normal hunger pangs, but painful because of the inflammation or other diseased condition present or because of their intensity. In marked hypersecretion, or hypersensitiveness, the pain may be almost continuous. The pain usually disappears promptly on the taking of food.

The pain of gall bladder disease may be due to hunger contractions or to contractions of the gall bladder, which may give rise to pain closely resembling the hunger pains of ulcer. Contractions of the gall bladder are greatly increased during normal digestion and naturally must be exaggerated in cases of hyperacidity of the gastric juice, as are the contractions of the stomach.

Hunger contractions have been shown to be absent in gastritis, tonsilitis, influenza and colds. Food taken under such conditions sometimes gives rise to nausea. Hunger contractions are shown to be absent in dogs suffering from infection and pneumonia.

\section{Modifications of Hunger.}

Exposure of the skin to cold, as in cold bathing, produces shortly afterwards an increase of hunger contractions. This accounts for the beneficial effects of the cold morning bath in producing a readiness for breakfast. This effect is analogous to the well-known influence of cold water applied to the skin in producing contractions of the bladder. A cold bath, followed by a brisk walk, is the best appetite stimulant with which the writer is acquainted. The injection of the blood of a starving dog into the veins of a normal dog increases hunger contractions in the latter. A similar effect was produced by injecting into healthy dogs blood from an animal suffering from experimental diabetes. Profuse hemorrhage may give rise to hunger contractions. Certain drugs, as pilocarpin and pituitrin, increase hunger contractions, while morphin and adrenalin produce the opposite effect.

Hunger is increased by external cold and decreased by external heat, a characteristic in which it resembles pain sensations. 
Physiologic experiments show that cold increases nerve sensibility, while heat diminishes the same.

\section{Bitters.}

Bitters taken into the mouth diminish the hunger contractions, as do other stimulants applied to the oral membrane. It is thus evident that bitters do not increase the demand for food. It is equally well known that they do not increase but actually diminish the secretion of gastric juice. The only means known which can be relied upon for increasing hunger, appetite and digestive power are a moderate diet, out-of-door life, exposure to sunlight and cold air, cold bath and muscular exercise.

\section{THIRST}

The sensation of thirst is referred to the mouth and throat. Like hunger, thirst is primarily due to changes in the blood. The blood normally contains 78 per cent. of water. This percentage is decreased by the addition of solids, such as chlorid of sodium, sugar or the products of digestion. Water is demanded to restore the blood to its normal state. This nice balance between water solvents and solids in solution is maintained by an automatic mechanism, and thirst is the signal of the necessity for adjustment.

When water is absorbed into the blood, the excess is at once removed by the kidneys or skin. The volume of the blood, as well as its composition, is nicely regulated by an automatic mechanism. An intake of water prompted by the sensation of thirst is almost immediately followed by an output of water through the kidneys or skin.

It is well known that thirst is induced by profuse perspiration resulting from exercise or exposure to heat, by the use of food containing considerable quantities of salt and by the use of alcohol and various other drugs. Fever also creates a demand for water, which is now recognized to be essential as a means of aiding Nature in her efforts to bring about recovery, although less than a century ago the use of water was forbidden to fever patients. At the present time, water externally and internally is recognized as, next to rest, the most important of all means of combating fever.

Cannon has shown that thirst is due to a relaive dryness of the mucous membrane of the mouth and pharynx due to a sup- 
pression or a diminution of the secretion of saliva. This may be the result of excessive perspiration or urination, or of diarrhea, as well as of deprivation of water or the excessive use of salt. Pack has shown that salivation produced by giving pilocarpine destroys thirst in rabbits that have been deprived of water for seven days.

Persons who suffer from autointoxication and whose tongues are usually thickly coated, are particularly likely to neglect water-drinking, greatly to their detriment. Such persons often do not experience the sensation of thirst except when a great loss of water occurs through vigorous perspiration.

The lack of appetite for food, very common in such cases, is very frequently accompanied by lack of thirst, even when the need of water is as great or even greater than the need of food. The absence of the thirst sensation may be due to a sort of paralysis of the thirst center, just as the lack of hunger may possibly result from a like failure of the hunger center. In such cases, the patient must be required to eat, even though he feels no desire for food; and in like manner he should be required to drink freely, even though the sensation of thirst is not experienced. Since it is sometimes very difficult to induce the patient to drink any considerable amount of water at any one time, he should be asked to drink small quantities, from an ounce to three or four ounces, very frequently. An ounce every twelve or fifteen minutes, or four or five ounces every hour when not asleep, will not exceed the needs of the body.

\section{Air Hunger.}

While oxygen is not usually included among foodstuffs, it is really as much a food as is water, and without its active agency, all vital activity would cease. Oxygen supports the vital combustions of the body just as it supports the combustion of wood, oil, and other fuel in our stoves and furnaces. The ordinary air contains a little more than one-fifth (21 per cent.) its volume of oxygen. This amount is more than is needed in ordinary respiration, which requires only about 14 per cent. of oxygen; but during violent exercise a considerably increased consumption of oxygen occurs, and at elevated altitudes where the air is much rarefied the amount of oxygen is diminished. The extra 6 per cent. of oxygen which the air contains is evidently a factor of safety to provide for these emergencies. 
The body may suffer air hunger through receiving too little oxygen in several ways:

1. By an insufficient amount of oxygen in the air, as when the air contains a large amount of carbon dioxid, displacing a part of the oxygen, which occasionally happens in connection with certain industries, as in the burning of lime.

2. A deficient intake of air, as when the chest is confined or the air passages obstructed.

3. Slowing of the circulation of the blood so that the carbon dioxid of the blood is not thrown off and the oxygen taken in with sufficient rapidity.

4. A deficient amount of hemoglobin in the blood, as when the red cells are diminished in number, as in anemia.

5. When the hemoglobin of the blood, which carries oxygen, is damaged, as in carbonous oxid poisoning, so that it does not give up its oxygen readily.

While oxygen is carried by the red cells, it is afforded to the tissues by the plasma or watery portion of the blood. The plasma absorbs the oxygen from the red cells and passes it on to the tissues. The amount of oxygen contained in the plasma is usually small, only about one-fortieth that found in the red cells, but by breathing pure oxygen it may be increased to as much as seven times the normal amount. In this way the plasma may in emergency be made to take the place of the blood cells. For example, a frog from which all the blood is removed, being substituted by a saline solution, lives and breathes in a perfectly normal way in an atmosphere of pure oxygen.

When the supply of oxygen is greatly diminished, giving rise to cyanosis, relief should be obtained as quickly as possible by supplying oxygen. A delay may result in so great an injury to the nervous system that later efforts may fail when more prompt action would have been successful.

It should be remembered that rapid breathing does not necessarily mean an increased supply of air to the lungs. Very rapid breathing is likely to be shallow breathing. The deeper parts of the lungs are not emptied. If the patient is not able to breathe deeply he should be assisted by artificial respiration or by rhythmic compression of the lower part of the chest or pressing up against the diaphragm from below. A peculiar form of respiration, known as Cheyne-Stokes' breathing, indicates failure of the 
respiratory center and the necessity for giving inhalations of oxygen.

The inhalation of oxygen is always indicated when there is syanosis or rapid breathing. This condition may exist as the result of extreme anemia. Rapid respiration is sometimes noted in cases of diabetes. In all such cases oxygen may be inhaled by means of a stream of oxygen passed into one nostril through a soft rubber tube by means of a mask placed over the nose and the mouth of the patient, or better, by means of a respiratory chamber.

In the British Medical Journal of March 6, 1920, Meakins shows that in normal individuals the blood contains about nineteen-twentieths as much oxygen as it is capable of holding; in other words, lacks 5 per cent. of being completely saturated. In pneumonia and conditions of cyanosis the under-saturation may amount to as much as 18 per cent. By causing the patient to breathe pure oxygen, the under-saturation, even in pneumonia, may be reduced to 3 per cent.; in other words, the blood of such a patient may be made to hold and carry even more oxygen than it usually carries in health. This fact shows the importance of oxygen inhalation in pneumonia. This measure should not be left for use as a last resort when the patient is cyanotic and nearly ready to die, but should be used as the best known means of preventing the development of dangerous conditions. 


\section{METABOLISM}

\section{The Energy of Food - The Calorie}

Life involves constant change of substance and expenditure or loss of energy. The function of food is to supply energy and to keep the resources of the body from becoming exhausted. All energy is convertible into heat. For this reason, the amount of heat into which any form of energy may be converted is taken as a measure of energy. There are two kinds of heat units in use: 'The British thermal unit (B. T. U.) is the amount of heat required to raise one pound of water one degree Fahrenheit in temperature. The more modern unit, devised by the French, is the amount of heat required to raise one kilogram (2.2 pounds) of water one degree Centigrade $\left(1.8^{\circ} \mathrm{F}\right.$.) in temperature. This unit is known as the calorie.

One calorie is equal to four B. T. U.'s $(2.2 \times 1.8=3.96)$ : that is, the amount of heat required to raise the temperature of four pounds of water $1^{\circ} \mathrm{F}$.

The equivalent of one calorie in mechanical work is approximately one and one-half foot tons (3,088 foot pounds). Eleven calories are approximately equivalent to one horse power minute.

\section{The Energy Output of the Body and the Work Equivalent of Food.}

The body, like a locomotive, is a machine. It is a mechanism which is capable of transforming the potential energy of food into the dynamic energy of work. Considered from an economic standpoint, the animal body is superior to all other mechanisms, since it transforms fuel into work with less loss than any machine which human ingenuity has ever constructed. For example, in the case of the steam engine not so much as onefifth of the energy of the fuel can be utilized under the best 
conditions, and ordinarily the proportion of energy utilized is much less than this. In the case of the human machine the energy value of food-fuel in relation to work done is three to one or even as low as two and one-half to one.

It is to be remembered, however, that a considerable amount of energy is required to carry on the internal work of the body For example, in a resting man the heart utilizes about 10 per cent. of the total energy intake in forcing the blood through the circulation; the chest muscles in maintaining the movement of air through the lungs consume 20 per cent. of the total energy; 10 per cent. is utilized in the work of digestion and in gland action; 30 to 50 per cent. of the total energy intake is required to maintain the muscle tonus or tension, a rapid rhythmical con traction ( 16 to 20 per second) whereby bodily heat is maintained; 20 to 30 per cent. is consumed in other vital work.

It is highly important to note that even when a person is in a state of complete rest, as much as one-half of the total energy expenditure of the body may be consumed by tension under the influence of worry, fear, nervousness or excitement of any sort. This form of energy expenditure may be increased to many times the normal amount, which explains why a person becomes tired, even to exhaustion, while simply waiting, and why a worried person or a harried animal cannot be made to gain in flesh, and emphasizes the importance of complete rest as a condition favor. able for increasing the body weight.

The amount of energy expended in a state of complete rest is termed the base ration. This for the average person amounts to about 25 calories per kilogram of body weight, or a little less than 12 calories per pound of body weight. This estimate sup. poses that the body weight is approximately the normal weight for a person of a given height, which may be determined by reference to Table II.

\section{Vital Work-Metabolism.}

The various forms of vital activity carried on in the body, especially the intimate cell activities on which all bodily functions depend, are collectively known as metabolism. The vital work, or metabolism, which has for its purpose the building up of the body or storing up energy, is known as constructive metabolism, while the vital work which involves the breaking down of tissues and the consumption of energy is known as destructive metabolism. 
In childhood and youth, constructive metabolism is highly active, resulting in growth or increase in both height and weight.

In adult life, after development is completed, constructive netabolism has for its purpose the keeping of the body cells and tissues in repair, and the maintenance of the body weight by the making good of the losses which result from work.

Plants work as well as animals. Bonnier has shown that peas in germinating produce about five calories of heat a day for each gram of weight.

Experiments made by Langworthy and Milner show that luring the ripening process bananas produce heat at the rate of about one-half calorie per hour per pound. The heat is produced by the destruction of carbohydrates.

Destructive metabolism is continually going on in the body, but is most active during muscular work and least during sleep.

All animals, even plants, require periods of rest, during which destructive metabolism is diminished to such a degree that :onstructive metabolism is in the ascendency so that losses of energy may be made good. When a person is deprived of sleep, even for a few days, the rapid loss of strength and weight are slear indications of the loss and injury which the body sustains from continuous destructive metabolism and of the beneficent functions of sleep. Plants grow most rapidly during the night.

\section{Basal Metabolism or Metabolism During Rest.}

During complete rest, even sound sleep, the body cells are at work. The heart continues to beat, the lungs empty and fill, the bodily heat is maintained; digestion, liver action, bile formation, the secretions of the kidneys, skin and other excretory glands continue-all the general life functions are carried on as during the waking hours, though less actively. The heart uses in twenty-four hours about one-tenth of the total energy output of the body, consuming one calorie of energy for each 400 beats, equal to the lifting of 720,000 foot pounds in twenty-four hours. The heart is able to do this enormous amount of work because each period of intense work is followed by a longer period of rest.

Metabolism during sleep is greater after severe effort because of increased muscular tension. The sleep metabolism must also be greater in a person sleeping amidst noises, even though the sleeper is unconscious of the noise. 
The basal metabolism is modified by various factors, such as age, sex, climate, habits, race, diet and mental state. It is highest in infants, nearly double that of adults, and lowest in advanced age. The rate of energy output is five per cent lower in women than in men, lower in Orientals, (Japanese and Chinese), and higher in athletes than in sedentary men. A man increased his basal metabolism nearly fifty per cent by systematic exercise within a year. Americans living in Brazil showed a metabolism rate one-fourth lower than the average in this country.

Basal metabolism rises during the day, being one-seventh more than during sleep in the morning and nearly a quarter more than during sleep in the late afternoon.

Metabolism must be increased during restless sleep and exciting dreams, accompanied by perspiration and followed by exhaustion.

\section{The Source of Animal Heat.}

One of the most remarkable phenomenon connected with the life of a warm-blooded animal is the maintenance of its temperature at a definite point, with little variation irrespective of external temperatures, which may be either considerably above or considerably below that of the body. The normal temperature of the interior of the human body is constantly maintained at about $100^{\circ} \mathrm{F}$., though the temperature if taken in the mouth is found to be a little less than $98.4^{\circ} \mathrm{F}$.

The body is constantly losing heat through contact with the air, through the breath and the skin. The body loses heat even when the temperature of the surrounding air is above that of the body, through the evaporation of perspiration from the surface. The amount of heat lost by the body depends upon the amount of skin surface, and is regulated by the blood supply to the skin and the excretion of sweat. The amount of heat lost by the body in twenty-four hours is about 2500 calories, which is equivalent to the amount of heat which would be produced by the burning of three-quarters of a pound of coal or a cube of hard wood four and a half inches square or ten ounces of oil.

In the air at a temperature of $86^{\circ} \mathrm{F}$., the loss of heat from the body occurs at practically the same rate as that by which it is produced, so that no clothing is required for warmth. It is for this reason that the natives of certain tropical climates wear 
THE ENERGY OF FOOD - THE CALORIE

항

के

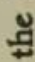

兘

营

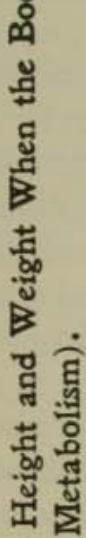

ली

ले

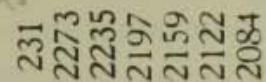

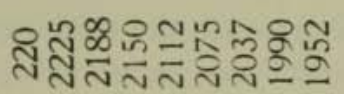

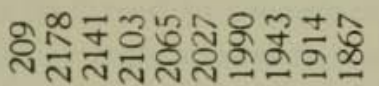

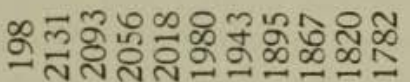

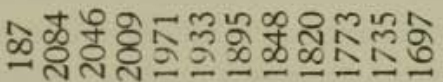

: : : :

서음ำ

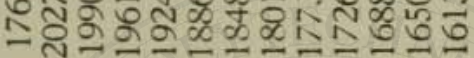

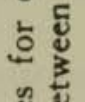

.

ชึ

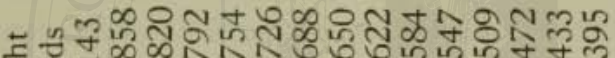

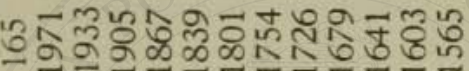

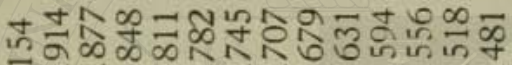

人

造

ลั

호은

뜰

ल)

ฮู

.

ฉ气

오으

$\stackrel{2}{\square}$

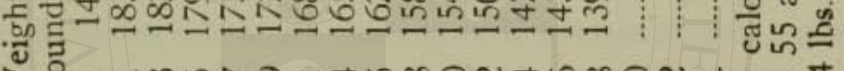

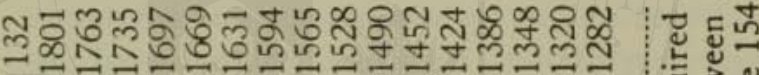

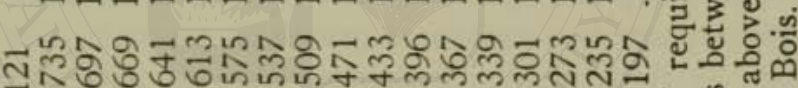

ำ

은

- 38 in

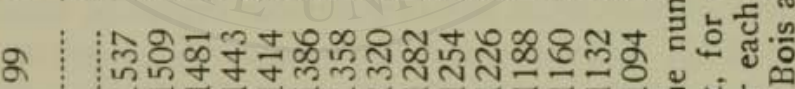

o :

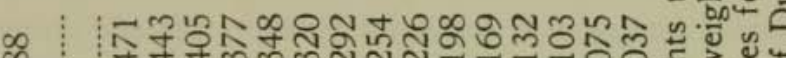

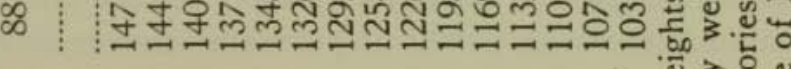

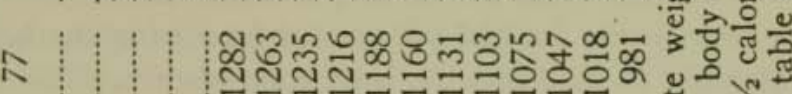

可桴

‡

8 :

뭉

스ニ=ㅇㅇㅇㅇㅇㅈㅇ

范

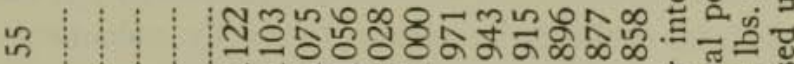

일

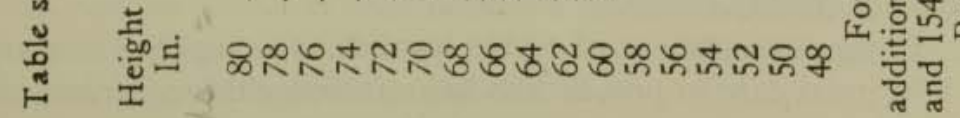

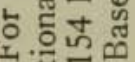


little or no clothing; or, if clothing is worn, its purpose is to protect the body from the heat of the sun rather than for warmth. This neutral air temperature of $86^{\circ} \mathrm{F}$. is the usual temperature of the air surrounding the body underneath the clothing.

The amount of food required by an individual daily, when at rest, depends largely upon the amount of heat lost, and since this depends upon the body surface, it is important to know the body surface for persons of different heights and weights. Various formulae have been developed by which this may be determined in individual cases, but the most convenient method is to refer to a table which has been prepared by Dubois. The accompanying table II, based upon that of Dubois (Lusk's "Science of Nutrition") shows the number of calories required by persons of different heights, from four feet to six feet eight inches, and from a weight of fifty-five pounds to two hundred and thirty-one pounds.

The heat of the body may be regarded as a waste product of metabolism, or cell work. It is for the most part a by-product of muscular work. When a person is at rest, the temperature is maintained by the muscle tension. When the temperature lowers, muscle tension is increased. It is also naturally increased by work as well as by various nervous influences. Fear, worry, anxiety, expectancy, so-called "nervousness," are conditions in which muscular tension is greatly increased. Tension may, in fact, by these means be doubled or even more than trebled. It also explains the fatigue and even exhaustion which is felt after an anxious hour, or a period of great mental and nervous excitement, even without any considerable degree of muscular activity.

Cold increases muscular tension to a very marked degree. This accounts for the phenomenon of shivering, which is simply greatly exaggerated muscular tension. Nature increases muscular tension even to the extent of producing tremor or visible muscular movements for the purpose of increasing the heat production necessary to maintain the body temperature. The strong shivering, with chattering of the teeth, which occurs when the body is exposed to great cold, is a remedial effort by which the lowering of the temperature of the blood is prevented. If the muscles are made to contract by electricity or by voluntary exercise, the shivering quickly ceases.

In the management of feeble invalids, it is highly necessary to take great care to prevent the loss of energy through unneces- 
sary nerve tension. The "rest cure" is often necessary in such cases to prevent the waste of energy through tension and thus afford an opportunity for a gain in weight.

Persons between twenty and fifty years of age require, on the average, about three and two-thirds calories per hour per square foot of body surface, or eighty-eight calories per square foot of surface for each twenty-four hours.

\section{Metabolism and Mental Work.}

There can be no doubt that mental work requires the expenditure of a certain amount of energy. This is clearly indicated by the amount of blood supplied to the brain, nearly one-fifth the total blood supply of the body; but careful experiments by Benedict and Carpenter and other investigators have shown that the amount of energy expended during severe brain work is so small that it cannot be accurately measured. This is not surprising, however, when it is considered that the weight of the brain and nerve substance is only about one-fiftieth of the total weight of the body. It seems probable that the chief reason for the large blood supply of the brain is to carry away the fatigue poisons which are produced by brain activity. It seems to the writer that the effect of mental work upon energy expenditure must depend to some degree at least upon the effect of the work upon "tension." If the work is of such a character as to greatly increase muscle tension, energy expenditure must certainly be increased as it is in worry or mental "strain."

The thyroid gland has a marked influence upon metabolic activity. When the gland is excessively active, as in exopthalmic goiter or Graves' disease, the rate of metabolism may be doubled. This fact explains the emaciation and exhaustion which accompany this disorder. On the other hand, when the thyroid gland is under-developed or inactive, as in myxedema or creatinism, the rate of metabolism may be very greatly diminished.

Metabolism is increased in various conditions of disease, particularly in fever, in lymphatic leukemia, in severe diabetes, in certain cases of heart disease, and sometimes in cancer. In fever and in pernicious anemia there may be disturbances of metabolism, also in disease of the pituitary and other ductless glands. Some observers have noticed a marked increase of metabolism in acidosis, which may result from either disease or a deficiency of carbohydrates in the diet. 
Metabolism is decreased in advanced age, by prolonged fasting and by sleep. It is greatly increased by a diet rich in protein, as after a hearty meal of meat.

\section{Metabolism and Muscular Work.}

Muscular exercise is by all odds the most powerful means of increasing metabolism. In an average man whose metabolism is at the rate of about 80 calories per hour when in a state of complete rest, the expenditure will become 84 calories per hour when sitting up. Active work will increase the expenditure to 300 calories per hour, and a higher rate of expenditure may be developed by very vigorous work. That is, by muscular effort, the rate of metabolism may be increased to four or even eight times what it is in a state of complete rest.

Exercise also increases the expenditure through muscular tension during sleep as well as during the waking hours. After vigorous work, for example, the expenditure of energy during sleep after severe work may be increased from 10 to 25 per cent. above the rate of sleep after rest.

It is evident, then, that in determining the amount of food required in an individual case, it is necessary to take careful note of the number of hours spent in sedentary work and in active exercise.

\section{Energy Expenditure in Work of Various Sorts.}

When lying at rest, the energy expenditure is at a minimum, or approximately 12 calories per pound of body weight. For a person weighing 154 pounds ( 70 kilos), the expenditure would be 1,848 calories for the twenty-four hours. On rising to a sitting position, the output of energy is increased 5 per cent.; standing with the muscles relaxed, produced an increase of 10 per cent. in the energy expenditure; and standing with the muscles rigid, as in the position of "attention," increases the energy output 14 per cent.

In walking an hour at the rate of 3 miles an hour, the maximal velocity for economy, the body expends 1.1 calories for each pound transported; that is, for each pound of body weight plus any weight which may be carried. Walking at the rate of 5.3 miles per hour raises the energy output to 3.6 calories per pound of body weight per hour.

To the energy represented by the work done in walking 
must be added, of course, the basal metabolism or the energy output of rest increased by 10 per cent. for the standing position.

A somewhat different method of calculating the work done in walking, which gives closely similar results, is based upon the observations made by Zuntz, who found that walking 13 feet on a level at the rate of 3 miles an hour requires an energy expenditure about equivalent to that required to lift the body vertically one foot. Consequently, the amount of work done in walking a mile at the rate of 3 miles an hour is found by multiplying the weight transported by $400(5,280 \div 13=406)$.

The work done by the muscles in transporting the body is about three times that represented in the mechanical work done. The energy value of the work done in walking at a moderate gait may be obtained by multiplying the distance traveled in miles at the rate of 3 miles an hour first by 400 , then by 3 , then dividing the product by 3,000 . To determine the actual energy expenditure in the work done, it is necessary to multiply the amount of mechanical work expressed in foot pounds by the factor 3 , since only one-third of the energy of the food is convertible into useful work.

These figures hold good only when a person is walking on a level road. If a hill is climbed, the work done in lifting the body through the vertical height ascended must be added. This is readily obtained in foot pounds by multiplying the weight transported by the number of feet through which it is lifted.

In walking down an incline of 5 per cent., the energy output in walking has been shown by Zuntz to be about one-half that in walking on a level. Descending an incline of 10 per cent., the energy expenditure is the same as that in walking over a level surface. In going down an incline of 25 per cent, the energy expenditure is doubled. The energy expenditure in bicycle riding is about one-half that required in walking the same distance.

Example. A man weighing 160 pounds with a resting metabolism of 1,800 calories walks for one hour at the rate of 3 miles an hour, carrying a weight of 50 pounds and climbing a hill 1,500 feet in height. The weight of the body, plus the weight carried, equals 210 pounds. This multiplied by 1.1 equals 231 , the number of calories required for transporting the weight on a level. The base metabolism during the hour spent in climbing would be 75 calories. Lifting a weight of 210 pounds to a height of 1500 feet will require an expenditure of 315 calories (210 multiplied 
by 1,500 divided by 1,000 equals 315 ). The total expenditure will be 621 calories ( 231 plus 75 plus 315 equals 621 calories for the hour); certainly a large output, but not more than frequently sccurs in such violent exertion as mountain climbing.

Carrying the example further, let us suppose that the individual who has spent an hour at the task above described rests for the remainder of the 24 hours, 8 hours in bed and 15 hours siting. The basal metabolism during the 8 hours in bed would be 75 calories per hour or a total of 600 calories. In sitting, the energy output would be approximately 80 calories per hour. In 15 hours this would amount to 1,230 calories, making a total of 2,428 calories, which would represent the energy output and the food intake required to supply the energy expended for the 24 hours.

\section{Energy Output and Food Requirements of Persons Engaged in Different Occupations.}

The energy expenditure required by different occupations varies very greatly. Careful observations made by various investigators have shown the actual energy requirement of different occupations for a person weighing 154 pounds to be about as follows:

\begin{tabular}{l|l}
$\begin{array}{c}\text { Occupation } \\
\text { Bookbinder }\end{array}$ & $\begin{array}{c}\text { Calories of } \\
\text { metabolism per day }\end{array}$ \\
Shoemaker & 2,440 \\
Carpenter & 2,510 \\
Metal worker & 3,100 \\
Painter & \\
Stonemason & 2,900 \\
Man sawing wood & 2,950 \\
\hline
\end{tabular}

\section{The Energy Expended in Housework.}

Benedict and Johns have made (1919) a valuable series of observations for the purpose of determining the energy expenditure required by ordinary light household activities. The observation was made by a study of the energy output of fifteen or twenty young women before and after entering a large airtight chamber in which they were engaged in performing some household operation, all doing exactly the same thing in the same way.

The conclusions reached may be briefly stated as follows:

1. When sitting quietly in the experimental chamber, two 
lours after a meal, the energy expenditure was one-half calory per pound of body weight per hour.

2. Reading aloud increased the energy expenditure 3 per cent.

3. The energy expenditure when singing was increased 22 per cent.

4. The exercise of plain sewing occasioned a smaller expenditure of energy than singing, being less than two-thirds as much, or 13 per cent.

5. The energy expenditure in standing was 9 per cent. greater than in sitting.

6. Sweeping caused an increase of 150 per cent.

7. Moderate walking increased the energy loss 250 per cent.

8. Dusting increased the loss by 134 per cent.

9. The energy loss occasioned by the act of rising up and sitting down once a minute during three minutes, showed an increased energy loss of 60 per cent. of the energy lost in the sitting posture.

\section{The Relation of Work to Total Food Requirement.}

The amount of external mechanical work done by the body represents but a very small part of the total energy expenditure. It is to be remembered that in the case of the body, as in that of the steam engine, much more energy is lost in heat as a byproduct than is utilized in mechanical work, besides the large amount of energy required for carrying on the various vital processes of the body. In general, it is held by physiologists that the energy of the food intake should be five or six times that represented by the external work done.

In increasing the intake of food to support an increase of work beyond the rest ration requirement it is not necessary to increase the protein, and the fats need be increased but very little. The chief increase should be in carbohydrates, for carbohydrates furnish the sugar which constitutes the fuel of the muscles.

\section{The Rate of Growth.}

The food intake is closely related to age and rate of growth. The younger the animal the more rapidly it grows. The body of an infant increases in weight seven times as fast between the 
ages of one month to three months as between the sixth and ninth month, the average increase being, for the first 3 months, about 3.6 pounds; for the second 3 months, 3.6 pounds; from 6 to 9 months, 3.4 pounds; and from 9 to 12 months, 3 pounds; an average of one and one-tenth pounds per month, or about twothirds of an ounce a day. During the first month the rate of increase is more than an ounce daily.

The crying of a baby may double its metabolism rate. On this account babies that cry much do not gain in weight. However, a certain amount of crying is good exercise for an infant and promotes good digestion and healthy growth.

The growth of the body is notably more rapid during the first five years of life than later. The weight of a healthy infant is three times as great at the end of the first year as at its birth. The weight doubles during the second year.

During the second period of five years, that is, 5 to 10 years, boys grow more rapidly than girls, and at 10 years of age average two or three pounds more in weight. Between the ages of 10 and 15 years, girls grow more rapidly than boys. For the two years, $121 / 2$ to $141 / 2$, girls are taller than boys of the same age, and from $12 \frac{1}{2}$ to $151 / 2$ years exceed boys of the same age in weight by three or four pounds. After 15, boys grow more rapidly than girls, increasing in weight at the rate of nearly seven pounds a year, while girls increase only two and three-quarters pounds yearly. Girls reach their full height and weight at 20 years, while boys continue to grow until 23 and increase considerably in breadth and weight after this age. Girls remain nearly stationary between the ages of 20 and 30 years.

After the age of 35 years, adults increase in weight at the rate of about three-quarters of a pound annually. It is a curious fact that while below the height of 60 inches men are usualiy heavier than women of the same age, above this height women are heavier than men of the same height and age.

The accompanying tables, copied from an admirable paper by Emerson and Manny of the Boston Nutrition Clinic embody the results obtained by Bowditch, Peckham, Boas, Burk, Holt, Greenwood and others in the measurement of more than 400,000 children and afford the best information available respecting the normal rate of growth and development.

A point of much importance which is usually overlooked in the routine use of tables of this sort is the fact that a very con- 
siderable variation from the average may occur within normal limits and must be taken into account. This variation often amounts to as much as 10 to 20 per cent in either direction and sometimes more than this. The natural "build" of the subject, and especially his race must be taken into consideration. Italian and Jewish children are notably shorter than native Americans. It is not wise to assume that a weight 7 per cent below the average is an indication of under-nutrition without other confirmatory evidence. The table is of value, however, in calling attention to such cases and thus suggesting an inquiry which may show the need of better feeding or other aid to better nutrition.

\section{TABLE III}

Showing Annual Increase in Weight of Boys and Girls.

\begin{tabular}{|c|c|c|c|c|c|c|c|}
\hline \multirow{2}{*}{\multicolumn{2}{|c|}{ Age }} & \multicolumn{2}{|c|}{ Year -52 Weeks } & \multirow{2}{*}{$\begin{array}{l}\text { Quarter-13 } \\
\text { Pounds }\end{array}$} & \multirow{2}{*}{$\begin{array}{l}\text { Weeks } \\
\text { Ounces }\end{array}$} & \multicolumn{2}{|c|}{ Week } \\
\hline & & Pounds & Ounces & & & Pounds & Ounces \\
\hline Birth to & 1 year & 13.45 & 215.2 & 3.3625 & 53.8 & .259 & 4.14 \\
\hline 1 to & 2 years & 6.3 & 100.8 & 1.575 & 25.2 & .121 & 1.94 \\
\hline 2 to & 3 years & 5.2 & 83.2 & 1.3 & 20.8 & 100 & 1.60 \\
\hline 3 to & 4 years & 4.3 & 68.8 & 1.075 & 17.2 & .053 & 1.32 \\
\hline 4 to & 5 years & 4.0 & 64.0 & 1.0 & 16.0 & 077 & 1. 23 \\
\hline 5 to & 6 years & 4.0 & 64.0 & 1.0 & 16.0 & 077 & 1.23 \\
\hline 6 to & 7 years & 4.3 & 68.8 & 1.075 & 17.2 & 083 & 1. 32 \\
\hline 7 to & 8 years & 5.0 & 80.0 & 1.25 & 20,0 & 096 & 1.54 \\
\hline 8 to & 9 years & 5.1 & 81.6 & 1.275 & 20.4 & .098 & 1.57 \\
\hline 9 to & 10 years & 5.8 & 92.8 & 1.45 & 23.2 & .112 & 1.79 \\
\hline 10 to & 11 years & 5,3 & 84.8 & 1.325 & 21,2 & .102 & 1.63 \\
\hline 11 to & 12 years & 6.2 & 99.2 & 1.55 & 24.8 & .119 & 1.91 \\
\hline 12 to & 13 years & 7.9 & 126.4 & 1.975 & 31.6 & -152 & 2.43 \\
\hline 13 to & 14 years & 10.4 & 166.4 & 2.6 & 41.6 & .200 & 3.20 \\
\hline 14 to & 15 years & 12.2 & 195.2 & 3.05 & 48,8 & .235 & $3.7 i$ \\
\hline 15 to & 16 vanta & 13.6 & 217.6 & 3.40 & 54,4 & .262 & 4. 18 \\
\hline
\end{tabular}

\begin{tabular}{|c|c|c|c|c|c|c|}
\hline \multirow[b]{2}{*}{ Age } & \multicolumn{2}{|c|}{ Year -52 Weckx } & \multirow{2}{*}{$\begin{array}{l}\text { Quarter- } \\
\text { Pounds }\end{array}$} & \multirow{2}{*}{$\begin{array}{l}\text { Weeks } \\
\text { Ounces }\end{array}$} & \multicolumn{2}{|c|}{ Week } \\
\hline & Pounde & Ounces & & & Pounds & Ounces \\
\hline Bitth to 1 year & 13.34 & 213.44 & 3.335 & 53.36 & .257 & 4.11 \\
\hline 1 to 2 years & 6.0 & 96.0 & 1.50 & 24.0 & .115 & $1.8 \pi$ \\
\hline 2 to 3 yesrs & 5.0 & 80.0 & 1. 25 & 20.0 & 096 & 1.54 \\
\hline 3 to 4 years & 3. 8 & 60.8 & .95 & 15.2 & .073 & 1. 17 \\
\hline 4 to 5 years & 3.6 & 57,6 & .9 & 14.4 & .060 & 1.11 \\
\hline 5 to 6 years & 3.6 & 57.6 & 9 & 14.4 & .069 & 1.11 \\
\hline 6 ta 7 years & 4.3 & 68.8 & 1.075 & 17.2 & .083 & 1.32 \\
\hline 7 to 8 years & $4: 8$ & 76.8 & 1.2 & 19.2 & .092 & 1.47 \\
\hline 8 to 9 yeara & 4.9 & 78,4 & 1.225 & 19.6 & .094 & 1.51 \\
\hline 9 to 10 years & 5.5 & 88,0 & 1.375 & 22.0 & .106 & 1. 69 \\
\hline 10 to 11 years & 6.6 & 105.6 & 1.65 & 26.4 & .127 & 2.03 \\
\hline 11 to 12 years & 9.2 & 147.2 & 2.3 & 36.8 & 177 & 2.83 \\
\hline 12 to 13 yesrs & 10.0 & 160.0 & 2.5 & 40.0 & 192 & 3.06 \\
\hline 13 to 14 years & 9.6 & 153.6 & 2.4 & 38.4 & 185 & 2.9 .5 \\
\hline 14 to 15 years & 8.4 & 134.4 & 2.1 & 33.6 & 175 & $2.5 !$ \\
\hline 15 to 16 years & 5.6 & 89.6 & 1.4 & 22.4 & .108 & 1.72 \\
\hline
\end{tabular}

The effects of the earlier occurrence of puberty in girls than in boys is clearly shown in the above table. Between the ages of 10 to 13 years inclusive, girls increase in weight an average of 25.8 pounds, while boys increase only 19.4 pounds in weight; but in the years 13 to 16 , boys increase in weight by 36.2 pounds, while girls make a gain during these years of only 23.6 pounds. 
TABLE IV

Showing Normal Rate of Development in Boys.

\begin{tabular}{|c|c|c|c|c|c|c|c|}
\hline \multicolumn{8}{|c|}{ BOYS } \\
\hline Years & $\begin{array}{l}\text { Age } \\
\text { Months }\end{array}$ & $\begin{array}{c}\text { Height in } \\
\text { Inches }\end{array}$ & $\begin{array}{c}\text { Weight in } \\
\text { Pounds }\end{array}$ & Years & Montis & $\begin{array}{c}\text { Height in } \\
\text { Inches }\end{array}$ & $\begin{array}{c}\text { Weight in } \\
\text { Pounds }\end{array}$ \\
\hline Birth & 0 & +20.6 & $* 7.55$ & 9 & 0 & 50.0 & 59.6 \\
\hline & 2 & $\$ 22.5$ & +10.4 & 9 & 2 & 50,3 & 60.6 \\
\hline & 4 & $* 24.5$ & $* 13.2$ & 9 & 4 & 50.6 & 61.5 \\
\hline & 6 & $\$ 26.5$ & $* 16.0$ & 9 & 6 & 51.0 & 62.5 \\
\hline & 8 & $* 27.5$ & $* 17.7$ & 9 & 8 & 51,3 & 63.5 \\
\hline & 10 & $\$ 28,5$ & -19.3 & 9 & 10 & 51,6 & 64.4 \\
\hline 1 & 0 & $\$ 29.5$ & $\$ 21.0$ & 10 & 0 & 51,9 & 65.4 \\
\hline 1 & 2 & $* 30.3$ & +22.1 & 10 & 2 & 52.2 & 66.3 \\
\hline 1 & 4 & $* 31.1$ & $* 23.3$ & 10 & 4 & 52.5 & 67.2 \\
\hline 1 & 6 & $\$ 32.0$ & +24.5 & 10 & 6 & 52,7 & 68.0 \\
\hline 1 & 8 & $\div 32.7$ & +25.5 & 10 & 8 & 53.0 & 68,9 \\
\hline 1 & 10 & +33.4 & $\neq 26.4$ & 10 & 10 & 53.3 & 69.8 \\
\hline 2 & 0 & $\$ 34.0$ & $* 27 . \overline{3}$ & 11 & 0 & 53.6 & 70.7 \\
\hline 2 & 2 & +34.7 & $\div 28.2$ & 11 & 2 & 53.9 & 71.7 \\
\hline 2 & 4 & $\$ 35.4$ & $4: 29,1$ & 11 & 4 & 54.2 & 72.7 \\
\hline 2 & 6 & $* 36.0$ & $* 30.0$ & 11 & 6 & 54.5 & 73.8 \\
\hline 2 & 8 & 36.5 & $* 30.8$ & 11 & 8 & 54.8 & 74.8 \\
\hline 2 & 10 & $\$ 37,0$ & $\$ 31.6$ & 11 & 10 & 55.1 & 75.9 \\
\hline 3 & 0 & $\$ 37.5$ & +32.5 & 12 & 0 & 55.4 & 76.9 \\
\hline 3 & 2 & 38.0 & +33.2 & 12 & 2 & 55.8 & 78.2 \\
\hline 3 & 4 & $* 38,5$ & $\$ 34.0$ & 12 & 4 & 56,1 & 79.5 \\
\hline 3 & (5) & +39.0 & +34.7 & 12 & 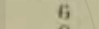 & 56.5 & 80.8 \\
\hline 3 & 8 & $\$ 39,5$ & 435.4 & 12 & 8 & $56 . \mathrm{s}$ & 82,1 \\
\hline 3 & 10 & $* 40.0$ & 36.1 & 12 & 10 & 57.2 & 83,5 \\
\hline 4 & 0 & $* 40.5$ & +36.8 & 13 & 0 & 57.5 & 84.8 \\
\hline 4 & 0 & 39.5 & 37.2 & 13 & 2 & 57.9 & 86.5 \\
\hline 4 & 2 & 39.9 & 37.9 & 13 & 4 & 55.3 & S8. 3 \\
\hline 4 & 4 & 40,2 & 38.5 & 13 & 6 & 58,7 & 90,0 \\
\hline 4 & 6 & 40.6 & 39.2 & 13 & 8 & 59.2 & 91.8 \\
\hline 4 & 8 & 41,0 & 39.8 & 13 & 10 & 59.6 & 93.5 \\
\hline 4 & 10 & 41,4 & 40,5 & 14 & 0 & 60.0 & 95.2 \\
\hline 5 & 0 & 41.7 & 41.2 & 14 & 2 & 60.5 & 97.2 \\
\hline 5 & 2 & 42.1 & 41.8 & 14 & 4 & 61.0 & 99,3 \\
\hline 5 & 4 & 42,4 & 42.4 & 14 & 6 & 61,5 & 101.3 \\
\hline 5 & 6 & 42.8 & 43.1 & 14 & 8 & 61.9 & 103.3 \\
\hline 5 & 8 & 43.2 & 43,8 & 14 & 10 & 62.4 & 105.3 \\
\hline$\because$ & 10 & 43.5 & 44.5 & 15 & $\theta$ & 62, 9 & 107.4 \\
\hline 6 & 3 & 43.9 & 45.2 & 15 & 2 & 63,2 & 109.7 \\
\hline 6 & 2 & 44.3 & 45.9 & 15 & 4 & 63.6 & 111.9 \\
\hline 6 & 4 & 44.7 & 46.6 & 15 & 6 & 63.9 & 114.2 \\
\hline 6 & 6 & 45.1 & 47.3 & 15 & 8 & 64.2 & 116.5 \\
\hline 6 & 8 & 45.4 & 48.1 & 15 & 10 & 64.6 & 118.8 \\
\hline 6 & 10 & 45,7 & 48.8 & 16 & 0 & 64.9 & 121,0 \\
\hline 7 & 0 & 46.0 & 49.5 & 16 & 2 & 6.j. 1 & 122.5 \\
\hline 7 & 2 & 46.5 & 50.3 & 16 & 4 & 65.7 & 124.0 \\
\hline 7 & 4 & 46.9 & 51.2 & 16 & 6 & 65.7 & 125.5 \\
\hline 7 & 6 & 47.4 & 52.0 & 16 & 8 & $6 ; .9$ & 127.0 \\
\hline 7 & 8 & 47.9 & 52.8 & 16 & 10 & 66.1 & 128.5 \\
\hline 7 & 10 & 48.3 & 53.6 & 17 & 0 & 66.5 & 130.0 \\
\hline 8 & 0 & 48.8 & 54.5 & 17 & 2 & 66.7 & 130.9 \\
\hline 8 & 2 & 49.0 & 55.4 & 17 & 4 & 66.8 & 131,7 \\
\hline 8 & 4 & 49.2 & 56.2 & 17 & 6 & 67.0 & 132,6 \\
\hline 8 & 6 & 49.4 & 57.1 & 17 & 8 & 67.2 & 133,4 \\
\hline 8 & 8 & 49.6 & 57.9 & 17 & 1i) & 67.3 & 134,3 \\
\hline 3 & 10 & 49.8 & 58.8 & 18 & 0 & 67.4 & -13.5 .1 \\
\hline
\end{tabular}


THE ENERGY OF FOOD-THE CALORIE

TABLE V

Showing Normal Rate of Development in Girls

\begin{tabular}{|c|c|c|c|c|c|c|c|}
\hline \multicolumn{8}{|c|}{ GIRLS } \\
\hline \multicolumn{2}{|c|}{ Age } & \multirow{2}{*}{$\mid \begin{array}{c}\text { Height in } \\
\text { Inches }\end{array}$} & \multirow{2}{*}{$\begin{array}{c}\text { Weight in } \\
\text { Pounds }\end{array}$} & \multicolumn{2}{|c|}{ Age } & \multirow{2}{*}{$\begin{array}{c}\text { Height in } \\
\text { Inches }\end{array}$} & \multirow{2}{*}{$\begin{array}{c}\text { Weight in } \\
\text { Pounda }\end{array}$} \\
\hline cars & Months & & & Years & Months & & \\
\hline sirth & $\begin{array}{l}0 \\
2\end{array}$ & $* 20.5$ & $* 7.16$ & 9 & 0 & 49.7 & 57.4 \\
\hline & 2 & +22.3 & 49.9 & 9 & 2 & 50.0 & 58.3 \\
\hline & 4 & $* 24.2$ & $* 12.7$ & 9 & 4 & 50.4 & 59.2 \\
\hline & 6 & $\neq 26.0$ & $* 15.5$ & 9 & 6 & 50.7 & 60.2 \\
\hline & 8 & $* 27.0$ & $* 17.2$ & 9 & 8 & 51.0 & 61.1 \\
\hline & 10 & $* 28.0$ & $* 18.8$ & 9 & 10 & 51.4 & 62.0 \\
\hline 1 & 0 & $* 29.0$ & $\neq 20.5$ & 10 & 0 & 51.7 & 62.9 \\
\hline$i$ & 2 & +29.8 & +21.7 & 10 & 2 & 52.1 & 64.0 \\
\hline 1 & 4 & +30.6 & +22.8 & 10 & 4 & 52,4 & 65.1 \\
\hline i & $\hat{6}$ & $* 31.4$ & $\$ 24.0$ & 10 & 6 & 52.8 & 66.2 \\
\hline$i$ & 8 & +32.0 & $\$ 24.8$ & 10 & 8 & 53.2 & 67.3 \\
\hline$i$ & 10 & $\$ 32.7$ & +25.6 & 10 & 10 & 53.5 & 68.4 \\
\hline 2 & 0 & $=33.4$ & +26.5 & 11 & 0 & 53.8 & 69.5 \\
\hline 2 & 2 & $* 34.0$ & $* 27.3$ & 11 & 2 & 54.1 & 71.0 \\
\hline 2 & 4 & $\div 34.6$ & +28.1 & 11 & 4 & 54.5 & 72.6 \\
\hline 2 & 6 & +35.3 & +29.0 & 11 & 6 & 54.9 & 74.1 \\
\hline 2 & 8 & +35.9 & +29.8 & 11 & 8 & 55.3 & +75.7 \\
\hline 2 & 10 & $* 36.5$ & $* 30.6$ & 11 & 10 & 53.7 & 77.2 \\
\hline 3 & 0 & +37.0 & $\neq 31.5$ & 12 & 0 & 56.1 & 78.7 \\
\hline 3 & 2 & +37.5 & $* 32.1$ & 12 & 2 & 56.5 & 80.4 \\
\hline 3 & 4 & +38.0 & +32.7 & 12 & 4 & 56.9 & 82.0 \\
\hline 3 & 6 & $* 38,5$ & +33.3 & 12 & 6 & 57.3 & 83.7 \\
\hline 3 & 8 & $\$ 39.0$ & +34.0 & 12 & 8 & 57.7 & 85.4 \\
\hline 3 & 10 & +39.5 & $* 34.6$ & 12 & 10 & 58.1 & 87.0 \\
\hline 4 & 0 & $* 40.0$ & $\$ 35.3$ & 13 & o & 58.5 & 88.7 \\
\hline 4 & 0 & 39.7 & 36.2 & 13 & 2 & 58.9 & 90.3 \\
\hline 4 & 2 & 39.7 & 36.8 & 13 & 4 & 59.2 & 91.9 \\
\hline 4 & 4 & 40.0 & 37.4 & 13 & 6 & 59.5 & 93.5 \\
\hline 4 & 6 & 40.4 & 38.0 & 13 & 8 & 59.8 & 95.1 \\
\hline 4 & 8 & 40.7 & 38.6 & 13 & 10 & 60.1 & 96.7 \\
\hline 4 & 10 & 41.0 & 39.2 & 14 & 0 & $60^{\circ} .4$ & 98.3 \\
\hline 5 & 0 & 41.3 & 39.8 & 14 & 2 & 60.6 & 99.7 \\
\hline 5 & 2 & 41.6 & 40,4 & 14 & 4 & 60,8 & 101.1 \\
\hline 5 & 4 & 41.9 & 41.0 & 14 & 6 & 61.0 & 102.5 \\
\hline 5 & 6 & 42.3 & 41.6 & 14 & 8 & 61.2 & 103.9 \\
\hline 5 & 8 & 42.6 & 42.2 & 14 & 10 & 61.4 & 105.3 \\
\hline 5 & 10 & 42.9 & 42.8 & 1.5 & 0 & 61.6 & 106.7 \\
\hline 6 & 0 & 43.3 & 43.4 & 15 & 2 & 61.7 & 107.6 \\
\hline 6 & 2 & 43.7 & 44.1 & 15 & 4 & 61.8 & 108.6 \\
\hline 6 & 4 & 44,1 & 44.8 & 15 & 6 & 61.9 & 109.5 \\
\hline 6 & 6 & 44.5 & 45.5 & 15 & 8 & 62.0 & 110.4 \\
\hline 6 & 8 & 44.9 & 46.2 & 15 & 10 & 62.1 & 111.3 \\
\hline 6 & 10 & 45.3 & 46.9 & 16 & 0 & 62.2 & 112.3 \\
\hline 7 & 0 & 45.7 & 47.7 & 16 & 2 & 62.3 & 112.8 \\
\hline 7 & 2 & 46.0 & 48.5 & 16 & 4 & 62.4 & 113.3 \\
\hline 7 & 4 & 46.4 & 49.3 & 16 & 6 & 62.5 & 113.8 \\
\hline 7 & 6 & 46.7 & 50.1 & 16 & 8 & 62.5 & 114.4 \\
\hline 7 & 8 & 47.0 & 50.9 & 16 & 10 & 62.6 & 114.9 \\
\hline 7 & 10 & 47.4 & 51.7 & 17 & 0 & 62.7 & 115.4 \\
\hline 8 & 0 & 47.7 & 52.5 & & & & \\
\hline 8 & 2 & 45.0 & 53.3 & & & & \\
\hline $\mathrm{x}$ & 4 & 48.4 & 54.1 & & & & \\
\hline 8 & 6 & 48.7 & 5,0 & & & & \\
\hline 8 & 8 & 49.0 & $\therefore 8$ & & & & \\
\hline 8 & 10 & 49.4 & 56.6 & & & & \\
\hline
\end{tabular}

Without clothing. 


\section{TABLE VI}

\section{Showing Normal and Deficient Weight for Different Heights in Boys and Girls.}

\begin{tabular}{|c|c|c|c|c|c|c|c|}
\hline \multicolumn{4}{|c|}{ BOYS } & \multicolumn{4}{|c|}{ GIRIS } \\
\hline $\begin{array}{l}\text { Height } \\
\text { Inches }\end{array}$ & $\begin{array}{c}\text { Average } \\
\text { Weight } \\
\text { for Height } \\
\text { Pounds }\end{array}$ & $\begin{array}{l}7 \% \\
\text { Under- } \\
\text { weight } \\
\text { Pounds }\end{array}$ & $\begin{array}{l}10 \% \\
\text { Under- } \\
\text { weight } \\
\text { Pounds }\end{array}$ & $\begin{array}{c}\text { Areroge } \\
\text { Weight } \\
\text { for Height. } \\
\text { Pounds } \\
\end{array}$ & $\begin{array}{c}7 \% \\
\text { Under- } \\
\text { weight } \\
\text { Pounds } \\
\end{array}$ & $\begin{array}{l}10 \% \\
\text { Under- } \\
\text { weight } \\
\text { Pounds }\end{array}$ & $\begin{array}{l}\text { ITeight } \\
\text { Inchex }\end{array}$ \\
\hline+21 & 8.2 & 7.6 & 7.4 & 7.9 & 7.3 & 7.1 & $21 *$ \\
\hline$\neq 22$ & 9.7 & 9.0 & 8.7 & 9.4 & 8.7 & 8.5 & $22 *$ \\
\hline 23 & 11.1 & 10.3 & 10.0 & 11.0 & 10.2 & 9.9 & $23^{*}$ \\
\hline$\div 4$ & 12.5 & 11.6 & 11.3 & 12.5 & 11.6 & 11.3 & $21^{*}$ \\
\hline 25 & 13.9 & 12.9 & 12.5 & 14,0 & 13.0 & 12,6 & $25 *$ \\
\hline 26 & 15.3 & 14.2 & 13.8 & 15.5 & 14.4 & 14.0 & $26 *$ \\
\hline 27 & 16.9 & 15.7 & 15.2 & 17.2 & 16.0 & 15.5 & $27 *$ \\
\hline 28 & 18.5 & 16.2 & 16.7 & 18.8 & 17.5 & 16.9 & $28 *$ \\
\hline 29 & 20.2 & 18.8 & 18.2 & 20.5 & 19.1 & 18.5 & $29 *$ \\
\hline 30 & 21,7 & 20.2 & 19.6 & 22.0 & 20.5 & 19.8 & $30^{*}$ \\
\hline 431 & 23,2 & 21.6 & 20.9 & 23.4 & 21.8 & 21,1 & $31 *$ \\
\hline 32 & 24.5 & 22.8 & 22.1 & 24.8 & 23.1 & 22.3 & $32 *$ \\
\hline 933 & 25.9 & 24.1 & 23.3 & 26.0 & 24.2 & 23.4 & $33 *$ \\
\hline 934 & 27.3 & 25.4 & 24.6 & 27.3 & 25.4 & 24,6 & $34 *$ \\
\hline 35 & 28.7 & 26.7 & 25.8 & 28.6 & 26.6 & 25.7 & $35 *$ \\
\hline 36 & 30.0 & 27.9 & 27.0 & 30.0 & 27.9 & 27.0 & $36 *$ \\
\hline 4837 & 31.6 & 29.4 & 28.4 & 31.5 & 29,3 & 28.4 & $37 *$ \\
\hline 435 & 33.2 & 30.9 & 29.9 & 32.7 & 30.4 & 29.4 & $38 *$ \\
\hline 39 & 36.3 & 33.8 & 32.7 & 35.7 & 33.2 & 32.1 & 39 \\
\hline 40 & 38,1 & 35.4 & 34.3 & 37.4 & 34.8 & 33.7 & 40 \\
\hline 41 & 39.8 & 37.0 & 35.8 & 39.2 & 36.5 & 35.3 & 41 \\
\hline 12 & 41.7 & 38.8 & 37.5 & 41.2 & 38.3 & 37.1 & 42 \\
\hline 43 & 43.5 & 40.5 & 39.2 & 43.1 & 40.1 & 38.8 & 43 \\
\hline 44 & 45.4 & 42.2 & 40.9 & 44.8 & 41.7 & 40.3 & 44 \\
\hline 45 & $47 \cdot 1$ & 43.8 & 42.4 & 46.3 & 43.1 & 41.7 & 45 \\
\hline 46 & 49.5 & 46.0 & 44.6 & 48.5 & 45.1 & 43.7 & 46 \\
\hline 47 & 51.4 & 47.8 & 46.3 & 50.9 & 47.3 & 45.8 & 47 \\
\hline 48 & 53.0 & 49.3 & 47.7 & 53.3 & 49.6 & 48,0 & 48 \\
\hline 49 & 55.4 & 51.5 & 49.9 & 55.8 & 51.9 & 50.2 & 49 \\
\hline 50 & 59.6 & 55,4 & 53.6 & 58.3 & 54.2 & 52.5 & 50 \\
\hline 51 & 62.5 & 58.1 & 56.3 & 61.1 & 56,8 & 55.0 & il \\
\hline 52 & 65.8 & 61.1 & 59.2 & 63.8 & 59.3 & 57,4 & 52 \\
\hline 03 & 68.9 & 64.1 & 62.0 & 66.8 & 62,1 & 60.1 & 53 \\
\hline i4 & 72.0 & 67.0 & 64.8 & 70.3 & 65,4 & 63.3 & $5 t$ \\
\hline 55 & 75,4 & 70.1 & 67.9 & 74.5 & 69.3 & 67.1 & 5.5 \\
\hline 56 & 79.2 & 73.7 & 71.3 & 78.4 & 73.9 & 70.6 & 56 \\
\hline 57 & 82.8 & 77.0 & 74.5 & 82.5 & 76.7 & 74.3 & 57 \\
\hline 59 & 87.0 & 80.9 & 78.3 & 86.6 & 80.5 & 77.9 & 53 \\
\hline 59 & 91.1 & 84.7 & 82.0 & 91.1 & 84.7 & 82.0 & 59 \\
\hline 60 & 95.2 & 88.5 & 85.7 & 96.7 & 89.9 & 87,0 & 60 \\
\hline 61 & 99.3 & 92.3 & 89.4 & 102.5 & 95,3 & 92.2 & 61 \\
\hline 62 & 103.8 & 96.5 & 93.4 & 110.4 & 102.7 & 99.4 & 53 \\
\hline 63 & 108.0 & 100,4 & 97.2 & 118.0 & 109.7 & 106,2 & 63 \\
\hline 64 & 114.7 & 106.7 & 103.2 & 123.0 & 114.4 & 110.7 & 64 \\
\hline 65 & 121.8 & 113,3 & 109.6 & 130.0 & 120.9 & 117.0 & 65 \\
\hline 66 & 127.8 & 118.9 & 115.0 & 137.0 & 127.4 & 123.3 & 66 \\
\hline 67 & 132.6 & 123.3 & 119.3 & 143.0 & 133.0 & 129.7 & 67 \\
\hline 68 & 138.9 & 129.2 & 125.0 & 146.9 & 136.6 & 132.2 & 6s \\
\hline
\end{tabular}

-without Clothing. 


\section{Food Requirements by Men, Women and Children.}

The amount of food needed differs according to the age, the size, and the amount of muscular activity.

Small animals require more food in proportion to their size than do large. Some insects eat all the time. Small birds consume each day an extraordinary amount of food. Infants and children need more food than adults in proportion to their size.

For persons of average weight, the food requirement may be determined by multiplying the body weight in pounds by the number of calories which careful laboratory research has shown to be requisite, as follows:

\begin{tabular}{|c|c|c|c|c|c|c|}
\hline \multirow{3}{*}{ Birth to } & \multicolumn{2}{|c|}{ Age } & $\begin{array}{l}\text { Calories } \\
\text { per Pound }\end{array}$ & \multicolumn{2}{|r|}{ Age } & $\begin{array}{l}\text { Calories } \\
\text { per Pound }\end{array}$ \\
\hline & to 1 t & month & (1) 30 & 6 & years & $34-35$ \\
\hline & & months & 40 & 7 & “ & $32-34$ \\
\hline & 3 & " & 41 & 8- 9 & " & $30-35$ \\
\hline & $4-6$ & " & 43 & $10-12$ & " & $28-32$ \\
\hline & $7-9$ & " & 40 & $12-13$ & " & $25-30$ \\
\hline & $1-2$ & years & 40 & $14-17$ & " & $20-25$ \\
\hline & $3-4$ & " & $37-40$ & Adults & & $16-20$ \\
\hline & 5 & " & $35-37$ & & & \\
\hline
\end{tabular}

The following table, based upon the work of Atwater and Benedict, shows the amount of food required by persons of different sex and age, taking the ration of an adult man as the unit:

\begin{tabular}{l|r} 
Man _.a. & 1.0 \\
Woman . & 0.8 \\
Boys (14-17) & 0.8 \\
Girls (14-17) & 0.7 \\
Children (10-13) & 0.6 \\
Children (6-9) & 0.5 \\
Children (2-4) & 0.4 \\
Children (under 2) & \\
\hline
\end{tabular}

\section{Changes Which Take Place in the Body in Old Age.}

The general tendency throughout the body in advancing age is for the development of cells of a comparatively low type, such as the connective tissue cells. These cells, tending to increase in the liver, kidneys and various other organs, crowd out and destroy the higher cells and hence produce impairment of function in 
important excretory and other organs. The same changes take place in the brain and nerves and are especially active in the arteries, causing arteriosclerosis. Certain of the white blood corpuscles also take part in this degenerative process, as shown in the whitening of the hair, which is due to the robbing of the hair of pigments by white blood cells (macrophags), and destruction of the supporting structures of the bones, causing porosity and weakening of these structures.

\section{The Quantity of Food Required in Old Age.}

The food should be steadily reduced in quantity with advancing age. No doubt many elderly persons eat more than is wholesome for them. As weight and bodily activity diminish, the food intake should be proportionately diminished. According to Maurel and Quatelet, the food requirement of persons in advanced years is as shown in the following table.

\begin{tabular}{|c|c|c|c|c|}
\hline \multirow{2}{*}{$\begin{array}{l}\text { Age } \\
\text { Years }\end{array}$} & \multicolumn{2}{|c|}{ MEN } & \multicolumn{2}{|c|}{ WOMEN } \\
\hline & Welght, Lbs. & Calories & Weight, Lbs. & Calories \\
\hline 60. & 144 & 1963 & 125 & 1700 \\
\hline 70. & .... 139 & 1890 & 118 & 1600 \\
\hline 80 & .... 135 & 1530 & 113 & 1300 \\
\hline 90 & ..... 117 & 1450 & 108 & 1230 \\
\hline & & & & \\
\hline
\end{tabular}

Zimmer has shown that vigorous muscles, even when at rest, destroy more sugar than do feeble ones, a fact which is easily understood when we remember that the muscles are the furnace of the body, and are the chief seat of the vital combustion by which glycogen, or sugar, is consumed. Large and vascular muscles will naturally consume more sugar than feeble and anemic muscles, just as a large furnace with a good draft will consume more fuel than a small furnace with a poor draft. Under the influence of either massage or exercise, the blood is made to go through the muscles; while in a state of rest it goes around rather than through them.

It thus appears that a robust, well developed person with a good muscle tone needs more food than a weak person whose muscles are soft and relaxed. This fact is usually indicated by a keen appetite in the case of the robust person and its absence in the case of the weak one. 


\section{Diet and Metabolism.}

The taking of food increases metabolism. Fasting reduces metabolism about 10 per cent. Food stimulates the activity of the body cells. In the case of fats and carbohydrates, the stimulating effect is small -6 per cent. for carbohydrates and 14.5 per cent. for fats; but in the case of protein the effect is great, amounting to 40 per cent. (Rubner). This is a fact of great importance and should never be lost sight of in the arrangement of bills of fare. Meat has long been regarded as a stimulating or "heating" food, and on that account has been condemned in fevers and as a hot weather diet by most authorities; but it is important to remember that there are numerous conditions of disease in which marked increase of metabolism is present and in which it is most desirable to suppress influences likely to produce a further increase of metabolic activity.

It is worth while to note, also, that in conditions of disease, when the normal balance of metabolic activities is already upset, further disturbance in the same direction may be easily produced, so that a protein ration which might be tolerated in health, may become highly injurious.

In toxic goiter (Graves' disease), lymphatic leukemia, severe diabetes, certain cases of cancer, fever, and advanced cases of heart disease, there may be a marked increase of metabolism. It is now known, in fact, that hyperthyroidism, with consequent exaggerated metabolism, occurs much more frequently than was formerly supposed. In all these conditions, which include a very large number of clinical cases, comprising all febrile cases, it is very evident that a very low protein diet is indicated. This naturally requires the exclusion of meat and meat extracts and broths of all sorts from the diet, in such cases. Every experienced physician can recall cases in which the temperature of a fever patient has risen sharply after the taking of meat. For many years, the writer has excluded meats and meat products of all sorts from the surgical wards under his supervision, and with marked benefit in the avoidance of postoperative fever as well as acidosis and other complications.

\section{Diet and Development.}

The remarkable influence of food upon bodily growth and development is not only a matter of common observation but is most forcibly illustrated by the results of laboratory experiments 
and certain remarkable natural history facts. Gudernatsch, in experiments upon tadpoles, found that if fed on thymus giand they grew to be very large but never changed to frogs; whereas, if they were fed on thyroid gland they quickly changed to frogs but remained extremely small, scarcely larger than flies. The larvae of bees fed on "bee bread" become ordinary workers or infertile females; but if fed "royal jelly," they become queens or fertile females. Canary birds fed on sweet red peppers become red in color.

\section{The High Protein Ration.}

Voit was the first to establish a standard ration. He required for a man weighing 154 pounds approximately 4 ounces of protein, 2 ounces of fat, and 18 ounces of carbohydrates; more exactly, in calories, protein, 484; fat, 521 ; carbohydrates, 2050; total, 3055. The research suggested by Horace Fletcher and conducted by Chittenden and Mendel demonstrated that the protein might be reduced to one-third of Voit's standard with an actual gain in health and vigor. Drs, Murlin and Miller of the United States Army, after a nutritional survey of the United States Army camps, pointed out in their report that "it is the general consensus of opinion among experts in nutrition that an excess of protein is undesirable in the dietary of a hard working man, since muscular work does not involve destruction of muscular tissue beyond the amount sustained in muscular rest."

\section{Food Requirement of Infants and Young Children.}

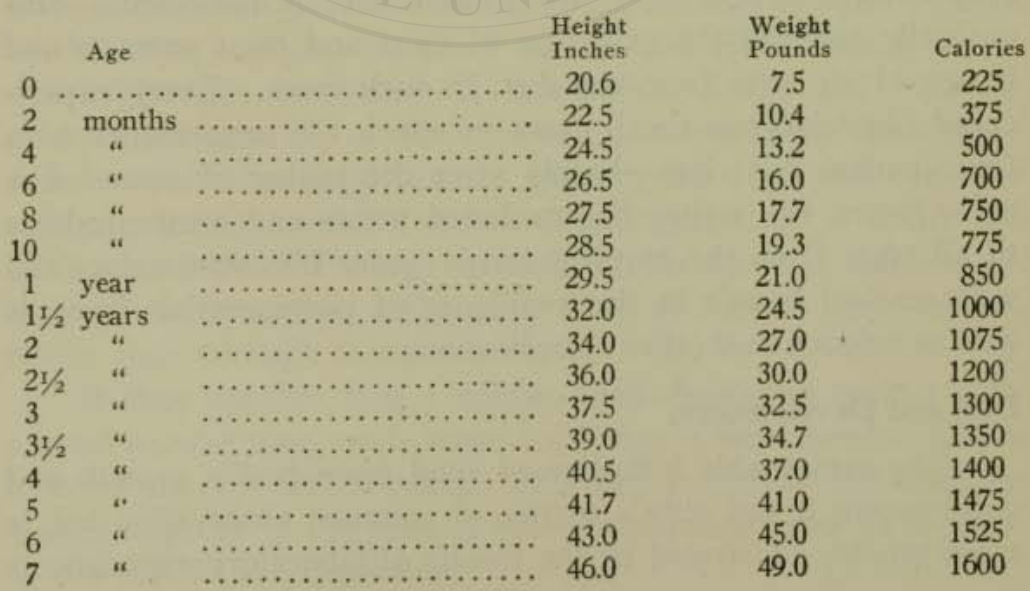




\section{Focd Requirement of Children and Youth.}

\begin{tabular}{cccc|cccc}
\hline $\begin{array}{c}\text { Age } \\
\text { Yrs. Mos. }\end{array}$ & $\begin{array}{c}\text { Height } \\
\text { Inches }\end{array}$ & $\begin{array}{c}\text { Weight } \\
\text { Pounds }\end{array}$ & Calories & $\begin{array}{c}\text { Age } \\
\text { Yrs. Mos. }\end{array}$ & $\begin{array}{c}\text { Height } \\
\text { Inches }\end{array}$ & $\begin{array}{c}\text { Weight } \\
\text { Pounds }\end{array}$ & Calories \\
\multicolumn{6}{c}{ BOYS } & & \multicolumn{5}{c}{ GIRLS } \\
$8-0$ & 48.8 & 54.5 & 1700 & $8-0$ & 47.7 & 52.5 & 1575 \\
$8-6$ & 49.4 & 57.1 & 1750 & $8-6$ & 48.7 & 55.0 & 1600 \\
$9-0$ & 50.0 & 59.6 & 1800 & $9-0$ & 49.7 & 57.4 & 1650 \\
$9-6$ & 51.0 & 62.5 & 1850 & $9-6$ & 50.7 & 60.2 & 1700 \\
$10-0$ & 51.9 & 65.4 & 1900 & $10-0$ & 51.7 & 62.9 & 1750 \\
$10-6$ & 52.7 & 68.0 & 1950 & $10-6$ & 52.8 & 66.2 & 1800 \\
$11-0$ & 53.6 & 70.7 & 2000 & $11-0$ & 53.8 & 69.5 & 1850 \\
$11-6$ & 54.5 & 73.8 & 2050 & $11-6$ & 54.9 & 74.1 & 1900 \\
$12-0$ & 55.4 & 76.9 & 2100 & $12-0$ & 56.1 & 78.7 & 1950 \\
$12-6$ & 56.5 & 80.8 & 2150 & $12-6$ & 57.3 & 83.7 & 2000 \\
$13-0$ & 57.5 & 84.8 & 2200 & $13-0$ & 58.5 & 88.7 & 2050 \\
$13-6$ & 58.7 & 90.0 & 2250 & $13-6$ & 59.5 & 93.5 & 2100 \\
$14-0$ & 60.0 & 95.2 & 2300 & $14-0$ & 60.4 & 98.3 & 2150 \\
$14-6$ & 61.5 & 101.3 & 2350 & $14-6$ & 61.0 & 102.5 & 2200 \\
$15-0$ & 62.9 & 107.4 & 2400 & $15-0$ & 61.6 & 106.7 & 2250 \\
$15-6$ & 63.9 & 114.2 & 2450 & $15-6$ & 61.9 & 109.5 & 2275 \\
$16-0$ & 64.9 & 121.0 & 2500 & $16-0$ & 62.2 & 112.3 & 2300 \\
$16-6$ & 65.7 & 125.5 & 2550 & $16-6$ & 62.5 & 113.8 & 2325 \\
$17-0$ & 66.5 & 130.0 & 2600 & $17-0$ & 62.7 & 115.4 & 2350 \\
$17-6$ & 67.0 & 132.6 & 2650 & & & & \\
\hline
\end{tabular}

Food Requirement of Adults.

\begin{tabular}{lrr|ccc}
\hline \hline $\begin{array}{c}\text { Height } \\
\text { Inches }\end{array}$ & $\begin{array}{c}\text { Weight } \\
\text { Pounds }\end{array}$ & Calories & $\begin{array}{c}\text { Height } \\
\text { Inches }\end{array}$ & $\begin{array}{l}\text { Weight } \\
\text { Pounds }\end{array}$ & Calories \\
& MEN & & & WOMEN & \\
60 & $120-130$ & $1900-2000$ & 59 & $111-126$ & $1775-2000$ \\
61 & $122-132$ & $1950-2100$ & 60 & $113-129$ & $1800-2050$ \\
62 & $124-135$ & $2000-2150$ & 61 & $115-132$ & $1850-2100$ \\
63 & $127-140$ & $2050-2200$ & 62 & $117-136$ & $1875-2175$ \\
64 & $131-143$ & $2100-2250$ & 63 & $120-140$ & $1900-2250$ \\
65 & $134-147$ & $2150-2350$ & 64 & $123-144$ & $1950-2300$ \\
66 & $138-151$ & $2200-2400$ & 65 & $125-148$ & $2000-2350$ \\
67 & $142-156$ & $2250-2500$ & 66 & $128-152$ & $2050-2400$ \\
68 & $146-162$ & $2300-2550$ & 67 & $132-155$ & $2100-2450$ \\
69 & $150-168$ & $2400-2650$ & 68 & $136-160$ & $2150-2500$ \\
70 & $154-174$ & $2450-2750$ & 69 & $140-165$ & $2200-2600$ \\
71 & $159-180$ & $2500-2850$ & 70 & $144-169$ & $2300-2700$ \\
72 & $165-185$ & $2600-2900$ & & & \\
73 & $170-189$ & $2700-3000$ & & & \\
74 & $176-192$ & $2800-3050$ & & & \\
75 & $181-196$ & $2900-3100$ & & & \\
\hline
\end{tabular}


Table of Heights and Weights of Men as Prepared by the Association of Life Insurance Medical Directors*

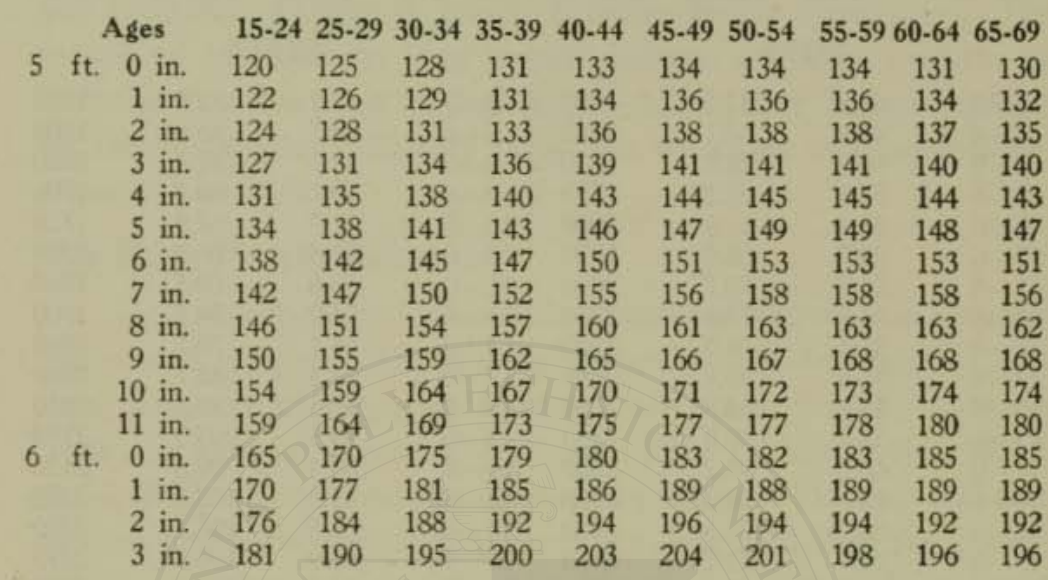

Table of Heights and Weights of Women as Prepared by the Mutual Life Insurance Company of New York*

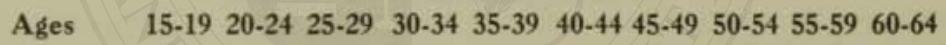

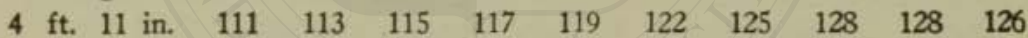

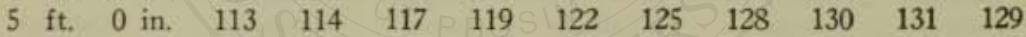
$\begin{array}{lllllllllll}1 \text { in. } & 115 & 116 & 118 & 121 & 124 & 128 & 131 & 133 & 134 & 132\end{array}$ $\begin{array}{llllllllllll}2 \text { in. } & 117 & 118 & 120 & 123 & 127 & 132 & 134 & 137 & 137 & 136\end{array}$

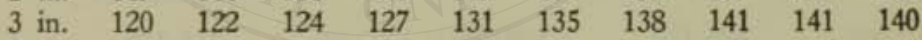

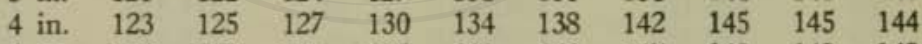
$\begin{array}{lllllllllll}5 \text { in. } & 125 & 128 & 131 & 135 & 139 & 143 & 147 & 149 & 149 & 148\end{array}$

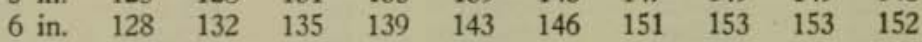
$\begin{array}{lllllllllll}7 \text { in. } & 132 & 135 & 139 & 143 & 147 & 150 & 154 & 157 & 156 & 155\end{array}$ $\begin{array}{lllllllllll}8 \text { in. } & 136 & 140 & 143 & 147 & 151 & 155 & 158 & 161 & 161 & 160\end{array}$ $\begin{array}{lllllllllll}9 \text { in. } & 140 & 144 & 147 & 151 & 155 & 159 & 163 & 166 & 166 & 165\end{array}$

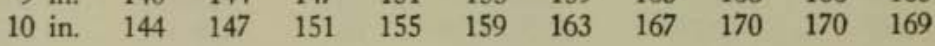

* The figures given include the ordinary house clothing. For net weight subtract 4 per cent. 


\section{THE PHYSIOLOGY OF EA TING}

Physiologic or biologic feeding requires careful adjustment of the food supply in quality and quantity to the nutritive needs of the body. A hundred years ago, even twenty years ago, it was difficult to do this. Many of the most important questions pertaining to nutrition were then only vaguely answerable. The clearly defined straight road in human feeding had not yet been scientifically marked out, but today this is no longer true. Careful laboratory experiment and scientific observation and research have solved so many of the problems and settled so many mooted questions it is now possible to feed human beings in as thoroughly scientific a manner as horses, cows, pigs and chickens are fed, and the results in human beings are equally as striking as those in domestic animals.

Physiologic feeding is as necessary for a man as for a race horse or for a competitor for a prize in a poultry show. The average man knows how to feed his horse so as to keep him in the best possible condition for service, but in feeding himself gives no thought to physiologic requirements but swallows whatever his fancy craves or his wife or hired cook sets before him. The farmer's wife knows how to feed cows or chickens so as to secure the largest returns in milk or eggs, but in feeding herself and her family gives little thought to physiologic needs.

The application of physiologic and biologic principles to the feeding of domestic animals, combined with scientific breeding, has accomplished such wonderful results that we now have cows which give more milk, hens that lay more eggs, fatter pigs and stronger, better and more enduring horses than the world ever knew before. From the results of the thousands of feeding experiments conducted in the Agricultural Experiment Stations and in nutrition laboratories, practical information of the greatest importance may be drawn in relation to human feeding. In recent years, much has been done in this direction, thanks to the labors of Hopkins, Mendel, McCollum and numerous experts 
who have given their attention to this line of research. However, the average man is not yet, to any considerable degree, acquainted with the facts which modern research have developed and placed at our disposal and the application of which ought to accomplish as much for human beings as has been accomplished for domestic animals through the regulation of their nutrition by proper feeding.

Unphysiologic eating is the active or predisposing cause of many chronic maladies. Biologic habits of eating should be formed in childhood and carefully adhered to during adult life. The so-called pleasures of the table are usually illicit enjoyments resulting from the indulgence of artificial tastes and depraved appetites from which a brief temporary felicity is derived at the expense of subsequent long drawn-out misery and disease.

It is of the highest importance to begin the training of children in biologic habits of eating at an early age. Among the things of fundamental importance which should enter into the training of infants and young children are the following:

1. Simplicity of taste. Sweets and the strongly flavored foods are extremely injurious to children. Through lessening or destroying their appetite for simple, mildly flavored natural foodstuffs, sweets and other highly flavored foods pervert the sense of taste, over-stimulate the appetite and lessen or destroy the appetite of the child for the mildly flavored, natural foods to which their digestive organs are adapted.

2. Thorough mastication of the food. Young children may be easily taught to chew their food in a proper manner. In fact, thorough mastication is as natural for a child as for a monkey. The habit of hasty chewing is acquired. A monkey will seize one by one, and place in his mouth a whole handful of cherries, as rapidly as they may be offered to him, but later it will be discovered that he has not swallowed a single one of the luscious fruit, but carefully stored them away in his capacious cheek pouches. Later, at his leisure, he masticates each cherry in the most thorough-going manner, carefully rejecting the seed and the skin. If a child is thoroughly trained in the proper mastication of food when it first begins to chew, the habit will become fixed.

To encourage the habit of thorough mastication, the diet of children should include, from a very early age, or as soon as weaned, a considerable proportion of foods requiring the use of the teeth. Graham or whole-wheat bread toasted until dry and 
crisp, should be made a part of every meal. This will give the muscles of mastication the exercise necessary to develop them, and will at the same time insure an adequate supply of blood to feed the growing teeth with the lime necessary to give them a firm, enduring structure.

Children should be taught to chew even soft food, and to sip milk and other liquid foods slowly so as to insure a proper admixture of saliva. Eating physiologically is a fine art which must be acquired and perfected by careful training by mothers, nurses and teachers. Every domestic board where young children gather should be a training table. Each child should be taught the proper method of eating, the special properties and relative values of foods, and how to select a suitable bill of fare. Early childhood is the time for correcting abnormal appetites, or rather, nipping them in the bud. The liking for hot condiments, or highly spiced ragouts, and rich sauces, are acquired tastes and may easily be diverted in normal channels by checking the earliest tendencies toward these perversions. The writer is fully persuaded that by such careful direction of the appetites in the early years, much may be done toward the prevention of the development of the alcohol and tobacco habits and other physical and normal obliquities in later life.

3. Prompt and regular evacuation of the bowels at least three times a day is another biologic habit the training in which is of the highest importance. It is of far more consequence for a teacher to know whether a child's colon is evacuated regularly and frequently, than to know that he is acquiring proficiency in mathematics, geography or any other of his ordinary school studies. As Herbert Spencer has well said, "The first essential for success is to be a good animal."

Eating requires that the selection and use of food should be in harmony with the facts which modern research has brought forth in relation to human digestion and nutrition. These have been set forth in preceding chapters, the perusal of which should enable the intelligent reader to regulate his feeding in a satisfactory manner. In this chapter the constituents of foodstuffs will be discussed in fuller detail. 


\section{Protein}

Protein is that element of the food which is used for the construction and repair of the highly vitalized or living tissues of the body; that is, the essential vital machinery, the muscles, glands, nerves and other parts concerned in the various activities or functions of the body, consist of protein. Between the periods of infancy and maturity, protein is required both for growth and repair. Recent experiments made in animal feeding indicate that in growing animals more than one-half ( 55 per cent.) of the protein intake is utilized in the formation of new tissue. After maturity, the sole use of protein is for repair. The practical question, then, in the adult individual is, how much protein is required to make good the daily wear and tear of the body, or the necessary loss from the breaking down of the living structures as the result of work.

This question is one of the very greatest practical interest, for Folin and other physiologists have shown that all surplus protein above what is required for repair is not only useless, not being necessary to satisfy any bodily need, but is a positive damage to the body, because it imposes upon the eliminative organs, especially the liver and kidneys, a heavy burden of unnecessary work, the inevitable effect of which must be to wear out these organs prematurely and to interfere with their normal functions of destroying and eliminating the natural body wastes and thus keeping the blood and tissue fluids free from obstructing poisons.

Probably the majority of persons who have given some thought to the question of the protein ration are laboring under the supposition that this question is a very new one, and that it is still a matter of experiment whether or not human life and vigor may be well maintained on a dietary which furnishes not more than one-third or one-half the amount of protein generally consumed. This is, however, quite an error. More than twenty years ago, when the question of the deficiency of protein in the Japanese dietary was raised by Mori, a special investigation was undertaken by a Japanese physiologist, Kumagawa, for the purpose of settling this question. He found that with his ordinary 
diet of Japanese foods only 54 grams of protein were eaten, less than half the amount required by Voit, and still smaller than Atwater's standard. He also found that this diet furnished the body more protein than it actually required and reached the conclusion that a larger amount of protein than this is quite unnecessary, and that only a very small amount of protein is really needed, the important point being that the body shall be furnished with a sufficient amount of fat and carbohydrates to maintain the supply of energy required by the tissues. He found also that an increase in the protein intake did not increase the amount of protein in the body but only increased the amount of urea found in the excretions.

Numerous physiologists have contributed experimental observations toward the settlement of the question, What should be the daily intake of protein?

Klemperer, experimenting upon two young men who weighed respectively 141 and 144 pounds, found the protein consumption to be in one 27.4 grams, a trifle less than one ounce, and in the other 22.4 grams or three-fourths of an ounce.

Sivén, in experiments upon himself, found 28 grams, or one ounce of protein, sufficient to meet his bodily needs.

Many years ago (1887) Hirschfeld had demonstrated the sufficiency of 37.5 grams (one and one-fourth ounces) of protein to supply the needs of the body; and this notwithstanding the fact that his ordinary diet previous to the experiment had contained 130 grams, or more than three times the necessary amount.

Voit, the eminent German physiologist, had previously established a dietetic standard of 118 grams of protein (more than four ounces) as the necessary requirement, and Atwater had considerably raised this standard as the result of observations upon some 15,000 people in whom he found the consumption of protein to vary from 100 to 175 grams (three and one-half to six ounces.) The great difference in the conclusions reached by these different investigators rendered necessary a critical examination of the question. A thoroughgoing and decisive experimental research was finally undertaken, the conclusions of which have not only remained to the present time unshaken but have been so abundantly reinforced by the findings of other investigators that they may now be said to be established for all time. The genesis of this investigation, known as the Chittenden research, is of such unusual interest that it will be worth while to 
devote a little space to its presentation, especially as the facts have never before been made public.

\section{The Chittenden Protein Standard.}

According to Chittenden, one-third gram per pound of body weight of protein, or one and one-third calories per pound of body weight, is quite sufficient to fully meet the requirements of the body. Sherman has recently placed the protein requirements of the body at the still lower level of one calory per pound of body weight. From Folin's experiments this would seem to be ample, since this most reliable investigator has shown that the greater part of the protein taken in the food is excreted by the kidneys in the form of urea without ever having been assimilated or utilized by the tissues. Folin holds that utilized protein is represented in the urine by creatinin, which agrees with the observation of Liebig that in hunted foxes the amount of creatinin found in the muscles is ten times the normal quantity. Tibbles holds that the physiologic minimum of protein is one-half gram per kilogram of body weight, or a little less than one calory per pound of body weight, an observation which agrees with the recent very extensive research of Sherman. The experiments conducted by Sivén led to the same conclusion.

Professor Chittenden says, "With these facts before us, it is difficult to accept the assumption that dietetic customs afford any indication of the food requirements of the body. To the physiologist such a view does not appeal, since there is a lack of any scientific evidence that carries conviction."

For some years after Professor Chittenden announced the results of his notable research, Dr. Benedict and others of the Atwater school vigorously opposed the low protein standard advocated by Chittenden and his associates, pronouncing it unsafe and questioning its scientific basis.

In defending his position, Professor Chittenden quotes Professor Benedict as saying :

Food should be ingested in just the proper amount to repair the waste of the body; to furnish it with the energy it needs for work and warmth; to maintain it in vigor; and, in the case of immature animals, to provide the proper excess for normal growth, in order to be of the most advantage to the body.

Professor Chittenden also remarks that "Voit, among others, has clearly emphasized the general principle that the smallest 
amount of proteid, with non-nitrogenous food added, that will suffice to keep the body in a state of continual vigor is the ideal diet."

No physiologist will question the accuracy of the statement that the ideal diet is the smallest amount of food which is "sufficient to keep up that strength of body and mind that is essential to good health, to maintain the highest degree of physical and mental activity with the smallest amount of friction and the least expenditure of energy, and to preserve and heighten, if possible, the ordinary resistance of the body to disease germs."

All surplus of food is a wasteful burden to the body and a handicap to its energies. This is true even of fats and carbohydrates, an excess of which is readily disposed of by being deposited beneath the skin in the form of fat, a reserve source of energy which may be drawn upon in time of need; but it is especially true of protein, a substance which cannot be stored in the body, which can be utilized only in the small amount required to replace the losses due to natural wear and tear. This amount the above considerations clearly show to be approximately one ounce to an ounce and a quarter for the average male adult, or, expressed in units of energy, 110 to 125 calories, the equivalent of about four ounces of lean meat or six and a half ounces of roast beef.

Another simple, and at the same time most convincing method of determining the necessary protein intake has recently been made known. A few years ago, Rubner made a study of the protein intake of the nursing infant and found it to be one-half gram of protein to the kilogram of body weight, or nine-tenths of a calory per pound of body weight. Careful investigation has shown that an infant uses 50 per cent. of its protein intake for growth, that is, for building up its muscles and glands, blood and other structures. This leaves not more than one-half of one calory per pound of body weight as the amount necessary for tissue repair. It could hardly be claimed that the amount of protein needed for tissue repair per pound of body weight could be greater in an adult than in an infant; hence it is evident that if the intake of protein is fixed at one calory per pound of body weight, much more than Nature provides to the infant to meet its requirement for growth as well as repair, the provision for meeting the demands of the body for repair of its vital machinery will be ample. It is interesting to know that the conclusion 
reached by this short and direct method is practically identical with the results at which Professor Chittenden arrived through his laborious research. Professor Chittenden weighs 125 pounds. His protein intake is 31 grams or 125 calories, or one calory per pound of body weight.

The subject of one of Professor Rubner's experiments pe1formed in one day work equal to nearly one million foot pounds, of which enormous output of energy it was found that protein supplied only one six-hundredth part. This shows again the fallacy of the popular notion that meat and other highly nitrogenous foods are necessary for the maintenance of strength, especially in persons who are engaged in hard labor.

Other observations by Rubner show that the body may be maintained in health and strength on one-half an ounce of dried protein daily. These facts are of the greatest importance and serve still further to fortify the position of Professor Chittenden in regard to the low protein ration, as well as the practice of non-flesh eaters.

The Japanese have for generations "lived and thrived on a daily ration noticeably low in its content of protein," and "generations of low protein feeding, with the temperance and simplicity in dietary methods thereby implied, have certainly not stood in the way of phenomenal development" (Chittenden).

The experience of the war so overwhelmingly proved the sufficiency of the low protein ration, the opposers of the Chittenden standard were compelled to recognize their error. The report brought back by Professor A. E. Taylor of the general adoption in Germany of the low protein ration and the account given by him of the physical accomplishments of the thousands of workers in the great ammunition factories in Germany, presented facts which could not be ignored, and which led Dr. Benedict of the Carnegie Nutrition Laboratory, of Boston, to plan the "Springfield experiment," which demonstrated in the most conclusive manner the sufficiency of the low protein standard established by the classic research of Chittenden.

Sherman, of Columbia University, published recently the results of a new research in the protein requirement which fully confirm the observations made by Chittenden and indicate that the proteins found in corn, wheat and oats, the three principal cereal grains have a higher value than other observers have given them. 
Sherman proved that nine-tenths of the protein of the diet may be derived from the cereals mentioned, provided that the other one-tenth consists of milk or apple proteins. He also notes that the total protein intake need not exceed one-half gram per kilogram, which is equivalent to a little less than one calorie per pound of body weight; that is, a person weighing 150 pounds may maintain his total protein requirement upon 13 ounces of corn meal, 9 ounces of graham flour, or 7 ounces of oatmeal, provided that in each case the cereal protein is supplemented by the protein found in 4 ounces of milk. The equivalent of the milk protein will be found in an ounce of cottage cheese.

Professor Sherman's observations agree with the results of large human experience. Cornmeal mush and milk was a staple food in the pioneer families of the Middle West half a century ago, and produced the sturdy men and women who have made this great section the very heart of American civic and industrial life. Oatmeal with milk is the staple food of the sturdy Scotch; and buttermilk with oatmeal and potatoes has for generations been the chief nutrient of the hardy Irish peasant.

The protein of the ordinary ration appears to be disposed of in two ways:

1. A portion, after conversion into amino acids or "building" stones, is carried by the blood to the tissues, where it is utilized for repairing the waste due to wear and tear of the vital machinery.

2. The remainder, probably the greater part of all the protein eaten, never reaches the tissues at all but is converted into trea in the liver and excreted by the kidneys.

Van Slyke has shown that the major part of the food protein is eliminated within 24 hours in the form of urea. This conversion of the excess of protein takes place chiefly in the liver and begins within a few minutes after meat is eaten.

On a high protein diet the amount of urea excreted by the kidneys is 33 to 37 grams, or an ounce and a quarter. On a free meat diet the amount may rise to 100 grams, or three and onethird ounces. On a protein-free diet the protein output falls to 9 grams, less than one-third of an ounce, and on a diet based upon the Chittenden standard, the amount excreted is from half an ounce to two-thirds of an ounce. From these facts it is evident that the kidneys of the hearty meat eater are compelled to do from two to five times as much work in the elimination of 
urea as is required of the kidneys of a low protein feeder. Since Folin has shown that much of the urea excreted by the kidneys represents surplus protein, that is, food protein which has never been assimilated and hence has not been utilized for tissue building, it will readily appear that a high protein diet is one of the most efficient methods by which the kidneys may be prematurely worn out and life thus abbreviated.

The protein of the food contains a certain proportion of sulphur. This is separated in the process of digestion and com bines with hydrogen, forming hydrogen sulphide, a highly poison. ous gas which causes cyanosis when present in sufficient quantity. This fact is of itself sufficient reason for avoidance of an excess of protein. A pronounced odor of sulphureted hydrogen, usually accompanied by a black or dark brown coloration of the feces, is evidence that protein is being eaten in excess.

\section{Luxus Consumption of Protein.}

Tibbles, the eminent English authority on dietetics, in dis cussing the question of the protein ration, remarks without comment; "Kellogg subscribes to the doctrine that no protein is stored in the system, and considers that all protein above 60 or 70 grams daily is broken down in the alimentary canal by bacteria into toxins such as indol, skatol, phenol, leucin, tyrosin, etc., and cause autointoxication."

The professor is wholly correct in asserting that the writer considers that 60 or 70 grams of protein is amply sufficient to meet the needs of the average man. This amount is, in fact, more than can be utilized to advantage. But the doctor is wrong in thinking that we have at any time entertained the notion that all surplus protein undergoes putrefaction in the intestine. Folin as well as Van Slyke has shown that the surplus protein is elim inated by the kidneys as urea without ever entering into any useful process in the body. Nenchi, MacFayden and others have shown that about one-seventh of the protein undergoes putrefaction in the colon.

The doctrine of "luxus" protein feeding is justly repudiated by Taylor and other modern physiologists. There is not a particle of evidence that protein is needed in the body for any other purpose than the repair of tissues. Any surplus is treated as waste material and is a burden to the organs of elimination, especially the liver and the kidneys.

It cannot be doubted, of course, that reduction of the protein below the actual needs of the body causes a depreciation of 
vital resistance, through disturbance of the nutritive balance. The same is true with reference to any other food principle; but the question under discussion is not the effect of an actual deficiency of protein, but the effect of an excess.

\section{Effects of Ptotein Intake Upon the Urine.}

A few years ago (1908) the writer had made in the laboratory of the Battle Creek Sanitarium a careful study of the urine in 24 men and 22 women, all young healthy subjects living upon a moderately low protein diet (Chittenden standard). The accompanying table shows the marked contrast between the urine of persons living on such a diet and on the ordinary mixed diet. An examination of this table shows that a mixed diet requires the kidneys to do an enormous amount of excess and unnecessary work in the elimination of waste products derived from the food directly and not resulting from necessary or productive vital work.

\begin{tabular}{|c|c|c|c|c|}
\hline & BLE & VII & & \\
\hline & & $\begin{array}{c}\text { Ordinary or } \\
\text { high protein } \\
\text { diet (Folin) } \\
\text { gms. }\end{array}$ & $\begin{array}{l}\text { Low protein } \\
\text { diet with- } \\
\text { out meat } \\
\text { gms. }\end{array}$ & $\begin{array}{r}\text { Excess } \\
\text { per } \\
\text { cent. }\end{array}$ \\
\hline Quantity ........... & c. c. & 1.430 & 1.000 & 43 \\
\hline Total nitrogen... & “ & 16.000 & 6.440 & 148 \\
\hline Urea ....................... & “ & 29.800 & 11.650 & 155 \\
\hline Uric acid ......................... & " & 0.370 & 0.323 & 14 \\
\hline Ammonia, in terms of NH3. & " & 0.850 & 0.247 & 244 \\
\hline Chlorid ……n....... & “ & 10.050 & 6.455 & 56 \\
\hline Creatinin ......... & “ & 1.550 & 0.815 & 90 \\
\hline 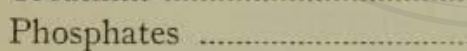 & “ & 3.870 & 1.455 & 166 \\
\hline Total sulphates ............... & “ & 3.140 & 1.071 & 192 \\
\hline Indican .................... & “ & 77.000 & 5.600 & 1275 \\
\hline
\end{tabular}

A study of the table will show at once the enormous amount of extra work imposed upon the kidneys by a high protein diet. It is to be noticed, for example, that the total quantity of urine eliminated by persons taking an ordinary mixed or high protein diet is 43 per cent. greater than the amount required by the low protein diet. The acidity is more than double. The total nitrogen, representing the total amount of nitrogenous wastes eliminated, is increased nearly 150 per cent. The urea is increased an equal amount. The ammonia, which is the result of putre- 
faction in the colon, is increased to nearly three and a half times the normal. Creatinin, which is in large part derived from the meat eaten, is practically doubled. All the products resulting from putrefaction are increased to two or three times that of the low protein urine, and the indican, a direct product of the putrefaction of protein in the colon, is increased more than 1300 per cent.

These facts show beyond any room for doubt that a high protein diet, or even the ordinary mixed diet which, by including meat, comprises an excess of protein, compels the kidneys to do an enormous amount of extra and harmful work in order to remove from the blood the excessive amount of poisonous wastes thrown into it. The total work done is considerably more than double the total work normally required of the kidneys. Is it not a fair conclusion that kidneys required to do double duty cannot be expected to retain their integrity as long as those required to do only the normal and necessary amount of work? The great prevalence of degeneration of the kidneys among flesheating people and flesh-eating animals, bears eloquent testimony upon this point.

The liver and the kidneys co-operate in protecting the body against the destructive influence of the waste and poisonous substances derived respectively from the activities of the body cells and the unutilized protein of the food. It has been clearly shown by extended clinical observation that over-loading these essential vital organs with work by eating an excess of protein is one of the most potent causes of disease. The experiments of Chittenden, by which this was clearly demonstrated, have been fully and repeatedly confirmed by more recent investigations.

It is evident, then, that a diet which is sufficient to maintain the body in a state of health and to fully support its activities while at the same time taxing the liver and the kidneys to a minimum extent, must be in the highest degree suited to promotion of endurance and longevity.

A research conducted by Campbell at Singapore and reported in the Biochemical Journal (1919) clearly demonstrates the advantage of a low protein diet. The work included the study of the urine in a Brahmin, a Chinese, a Tamil, a Malay, a Hindoo, a Eurasian, a Bengali, a Sikh and a European. The Brahmin and the Hindoo were vegetarians; the others lived on a mixed diet. It was found that the Brahmin and the Hindoo eliminated much 
smaller quantities of urea and other waste substances than any of the other subjects and very much less (scarcely half) than the average European ( 0.11 grams $\mathrm{N}$ per kilogram for the Brahmin and 0.127 for the Hindoo).

Modern researches confirm the conception of Liebig that protein is essentially a tissue-building substance, and not primarily intended for body fuel. A dog fed upon lean meat free from fat requires a ration having a much higher caloric value than when fed upon a diet consisting largely of fats and carbohydrates, thus showing that, as a source of energy, protein is much inferior to fats and carbohydrates. The relation of the two classes of food principles appears to be essentially the same as that of metal repairs and coal to the locomotive.

\section{A High Protein Diet Increases Intestinal Putrefaction.}

The careful study of the bacterial flora of the intestine which has been made within recent years by Escherich, Tissier, Metchnikoff, Herter, Levin, Roger, Adami, Cohendy, Gilbert, Dominici, Strassberger and others has shown the immense influence exerted upon the functions and tissues of the body by bacterial toxins formed within the alimentary canal. Absorbed into the portal blood, these poisons are circulated through the liver, where they are in part oxidized and rendered innocuous, and find their way out through the lungs, skin, and especially the kidneys. These investigators and others have shown that there is a close relation between the protein ration and intestinal putrefactions.

Pasteur supposed that bacteria are a necessary aid to intestinal digestion and essential to both animal and plant life. His pupil, Roux, showed that beans may grow in a sterile soil. Nuttal and Thierfelder kept guinea-pigs alive in a sterile medium. Levin examined 480 animals at Spitzbergen-bears, seals, reindeer and other animals -53 species, and established the fact that in the Arctic regions the intestines of mammals are usually sterile.

It seems to be finally settled that bacteria are not necessary for the maintenance of animal life, and certainly that putrefactive bacteria are not only unnecessary, but harmful. Nevertheless, these bacteria are present in the intestines in enormous quantities. Gilbert calculates the number of bacteria daily discharged from the body at twelve trillions. Strassberger makes the number one hundred and twenty trillions. Roger enumerates 240 species of bacteria which have been found in the human intestine. Many of 
these produce substances which are in the highest degree toxic. On a high-protein diet, the poison-forming bacteria rapidly increase, giving rise to intestinal autointoxication. German medical authorities have been rather slow to recognize this condition, but Senator, Backmann, Mester, Brieger, Hoppe-Seyler, Krauss, Ewald, Jaffe, Albu, Hirschler, Munch, Rovighi, Grawitz and others have recognized the validity of the teaching of Bouchard, and have expressed views in harmony therewith.

The writer has for some years pursued studies in relation to this question, and lately by the aid of the improved methods of Herter, Folin and other recent investigators, has made several observations which seem to have a practical bearing. Five healthy young men were fed upon various diets, as indicated in the tables presented herewith, and a careful study was made of the stools and the urine. In making these studies the stools were examined both chemically and bacteriologically.

The amount of the daily ration was from 1,800 to 2,400 calories. The principal carbohydrates were bread and potatoes. Fat in the proportion of about 30 per cent. of the total ration was taken in the form of butter. The protein ration was varied from 125 to 450 calories. Each special dietary was closely followed for five to eight days, and was employed with two or more persons, and in several instances repeated a number of times. The figures given in the accompanying table are the averages obtained from all the observations made, the number of which is indicated in each case.

\section{TABLE VIII}

Showing the Amount of Intestinal Putrefaction as Indicated by the Amount of Indol Found in the

Feces and of Indican in the Urine With the Several Diets Indicated.

\begin{tabular}{|c|c|c|c|c|c|}
\hline & $\begin{array}{l}\text { Daily } \\
\text { ration in } \\
\text { calories }\end{array}$ & $\begin{array}{l}\text { Gms, of } \\
\text { protein } \\
\text { per kilo of } \\
\text { body weight }\end{array}$ & $\begin{array}{l}\text { No. of } \\
\text { obser- } \\
\text { vations }\end{array}$ & $\begin{array}{c}\text { Avg. amt. } \\
\text { indol } \\
\text { in feces } \\
\text { mgms. }\end{array}$ & $\begin{array}{l}\text { Avg. } \\
\text { indican } \\
\text { in urine } \\
\text { (Folin } \\
\text { scale) }\end{array}$ \\
\hline $\begin{array}{l}\text { Group I-Moderately high } \\
\text { protein diet, } 1.00 \text { gram or } \\
\text { more per kilo. of body }\end{array}$ & & & & & \\
\hline $\begin{array}{l}\text { weight } \\
\text { Group II-Low-protein diet }\end{array}$ & 2,187 & $1.00-1.80$ & 39 & 1.2270 & 6.00 \\
\hline $\begin{array}{l}\text { Group II-Low-protein diet } \\
\text { (vegetable) } \\
\text { Group III-Low-protein alec }\end{array}$ & 2,064 & $0.49-0.96$ & 74 & 0.3611 & 0.00 \\
\hline $\begin{array}{l}\text { Group III-Low-protein diec } \\
\text { below Chittenden stand- }\end{array}$ & & & & & \\
\hline $\begin{array}{l}\text { ard (vegetable) } \\
\text { Group IV-Fruit and cereal }\end{array}$ & 2,040 & $0.49-0.80$ & 51 & 0.1227 & 0.00 \\
\hline Glet G-High-protein alet & 2,075 & $0.49-0.70$ & 38 & 0.0567 & 0.00 \\
\hline $\begin{array}{l}\text { Group V-High-protein diet } \\
\text {-meat and vegetables,..... }\end{array}$ & 2,200 & $1.50-1.80$ & 10 & 1.3508 & 9.50 \\
\hline
\end{tabular}


It is most clearly shown by the above tables, giving the results of 212 observations, that the amount of intestinal putrefaction is directly proportional to the amount of protein in the dietary.

Beckmann showed the same to be true of white of eggs, but much less so than with meat.

Mester compared fresh meats and "prime" meat with the following result:

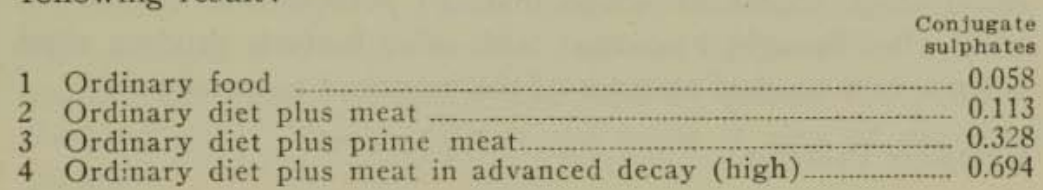

In a second series of observations, to each of various food mixtures of equal weight ( 25 grams) was added a definite quantity (10 grams) of feces which had been previously examined for indol and bacteria. The whole was then placed in an incubator at body temperature for three days. The quantity of indol produced, was determined by distillation and testing by Herter's method.

\section{TABLE IX}

Showing the Amount of Indol Produced in Equal Quantities of Various Foodstuffs (25 Grams) Mixed With 10 Grams of Human Feces and Incubated for Three Days.

Beef (fresh)
$\begin{aligned} & \text { Pork (fresh) } \\ & \text { Mutton (lean) }\end{aligned}$ MEATS
Average

MILK PRODUCTS

Unboiled milk 0.626

Boiled milk

Yogurt cheese 0.126

Average. .426

\section{VEGETABLE FOODS}

Cereals (average of 20) .055

Raw vegetables

Cooked vegetables

Cooked fruits

.147

Bananas

.078

.222 .404

Average .181 
The table shows that the flesh foods on an average produced nearly eight times as much indol as the average produced by vegetable foods. Milk products produced only 3 per cent. as much indol as meats, while yogurt cheese, made with the Bacillus bulgaricus, made less than 1 per cent. as much indol as meat.

These results are entirely in harmony with the results obtained in feeding experiments, and taken with them show very clearly that foodstuffs which undergo putrefaction outside the body when brought in contact with colon bacteria produce when eaten a very marked increase of the intestinal putrefactions.

\section{Complete Proteins.}

Until recently it had been taken for granted that all proteins are essentially alike and capable of replacing one another. It has been learned, however, in recent times, that the protein molecule is very complex, and so variable in composition that the number of proteins possessed of characteristic differences is almost infinite. Every plant produces proteins peculiar to itself, and there are marked differences among animal proteins.

These differences are due to the fact that the protein molecule is made up of simpler bodies known as amino acids which have been called "building stones" because by different combinations of these foundation elements, the various living tissues of the body are built up. Many different amino acids have been discovered, some forty or fifty. Less than half of these enter into the proteins of the human body. A protein which furnishes all the amino acids necessary for building human protein, is known as a complete protein. Such animal proteins as lean meat, eggs, and milk, all contain complete proteins, being of animal origin, and milk and eggs, the yolk at least, being especially prepared for food for young animals. But the proteins of plants differ very widely. Some are complete, but most plant proteins are incomplete. Those foods which the experience of the race has chosen to serve as staples for the feeding of large populations are found by chemical examination to possess the best proteins, that is, those which contain in the best proportions the "building stones" needed for the repair or construction of body protein.

It is interesting to note in this connection that of the several classes of plant foods nuts contain the best protein. Researches by Osborne, Mendel, Clapp, Harris, Nollan, Johns, Jansen, Daniels, Loughlin and Cajori and others have demonstrated that the 
proteins of nuts are complete proteins. This has been shown to be true of all nuts thus far studied, which include the almond, coconut, Brazil nut, black walnut, English walnut, butternut, filbert and hickory.

The great cereal staples, wheat, rice, corn, and oats, contain proteins of fair quality. The same is true of rye, and millet possesses a protein of exceptional value. The protein of the potato, another staple, has been shown to be of a superior order. The proteins of ordinary beans, peas, and lentils are of inferior quality; but those of the soy bean and the peanut are equal to those of milk. The protein of the apple is also of excellent quality.

But the incomplete proteins of most vegetable foods are by no means useless. When eaten with foods containing complete proteins, such as milk, eggs, nuts, soy beans or peanuts, their incomplete proteins are supplemented in such a way as to make them satisfactory nutrients. This is one of the most important facts in food physiology, and supplies an important foundation stone for the rational combination of foodstuffs in the construction of bills of fare. It is manifestly essential that care should be taken to insure the necessary intake of complete proteins, and this is especially important when the protein intake is reduced to a minimum.

Sherman has shown that with a cereal diet the addition of milk sufficient to supply ten per cent. of the protein is sufficient for normal growth and maintenance. This would require a daily intake of about four ounces of milk or an equivalent amount of cheese, say half an ounce to an ounce. Certainly two or three glassfuls of milk daily will supply an ample amount of complete proteins to fully supplement any otherwise sufficient bill of fare.

In the absence of milk, nuts, soy beans or peanuts may be used as complementary foods.

A study of the relative protein content of nuts, milk, and meat shows that, pound for pound, the almond, beechnut, and walnut contain on an average as much protein as does meat and five times as much as is found in milk, and protein which animal feeding experiments recently made prove to be of equal value. The chestnut, the chinquapin, the filbert, the hickory, pecan and pine nut contain on an average as much protein as is found in fish: while the butternut, the peanut and pignolia contain twice 
as much, and 50 per cent. more than is found in the best cuts of meat.

The following table shows the number of ounces of nuts required to furnish the same amount of protein as 4 ounces of milk, the amount required to balance for complete proteins a day's ration of cereals and no milk, eggs or meat:

\section{TABLE X}

\begin{tabular}{|c|c|c|}
\hline \multirow[b]{2}{*}{ Almond } & \multicolumn{2}{|c|}{$\begin{array}{c}\text { Amount equal to protein of } \\
4 \text { ozs. of mllk ( } 336 \text { cals). } \\
\text { Ounces Calories }\end{array}$} \\
\hline & .7 & 134 \\
\hline Beechnut ................. & 6 & 116 \\
\hline Brazil nut.............. & .8 & 165 \\
\hline Butternut .. & .5 & 100 \\
\hline Chestnut . & 1.3 & 120 \\
\hline Filbert & 9 & 187 \\
\hline Hickory nut... & .9 & 190 \\
\hline Malted Nuts ... & ... 6 & 91 \\
\hline Pecan & 1.4 & 305 \\
\hline Peanut & .7 & 85 \\
\hline Peanut meal... & 3 & 33 \\
\hline Pine nut................. & 6 & 122 \\
\hline Walnut (black) ... & .5 & 94 \\
\hline Walnut (English) & .8 & 165 \\
\hline
\end{tabular}

Eggs may also serve as supplementary foods, one egg furnishing about the same amount of supplementary protein as a half pint of milk.

It is evident, then, that meat can no longer be regarded as a necessary source of human food, other foodstuffs capable of supplying any real nutritive need which meat can serve being both diversified and abundant.

\section{The Normal Protein Requirement.}

As already shown, the amount of protein needed daily is small, probably about one calorie per pound of body weight, that is, normal body weight. A fat person needs no more protein than a person of normal weight for the same height. For the average person this will require 120 to 150 calories of protein.

An examination of the table will show that less than an ounce of peanuts, black walnuts or butternuts will supply as much com- 
plete protein as 4 ounces of milk, the full amount actually needed to supplement a bill of fare otherwise sufficient; while an ounce cf almonds, beech nuts, filberts, hickory nuts, pine nuts or English walnuts, and less than an ounce and a half of chestnuts or pecans may take the place of milk as a supplementary food. One-third of an ounce of nut meal or twice as much Malted Nuts serve the same purpose.

As a matter of fact, if the daily bill of fare includes with a sufficiency of other foods one or two ounces of nuts or soy beans, an egg, or half a pint of milk, he need give no thought to the amount of protein he eats provided he does not eat meat, since he will be sure to get enough and not likely to get too much.

In a magazine article, Osborne presented the argument that prutein is needed not only for food, but for stimulation, it having been shown by Rubner and Lusk that protein differs from other food principles in the fact that it excites the cells to do an amount of work in excess of the energy which it supplies to them. The fact is also mentioned that caffein does the same thing, only in the case of caffein the effect is of course wholly that of excitation, this drug having no food value whatever. Since this effect is purely that of stimulation, no equivalent energy being supplied to support the expenditure induced, it must be regarded as harmful rather than desirable. Rubner has shown that the extra energy thus produced cannot be utilized in useful work, but is a total loss.

Many of the greatest of the ancient philosophers were flesh abstainers, of whom special mention may be made of Herodotus, the father of history; of Pythagoras, the father of Greek philosophy, and of his famous pupil, Socrates, whose scarcely less famous pupil, Plato, was likewise a flesh abstainer, as was also Plutarih, the author of the "Lives." Seneca, one of the most noted of the Roman philosophers, was likewise an exponent of the fleshless diet. Guatama Buddha was a strict vegetarian and requirer? his disciples to discard flesh of all sorts as millions of them at the present time do in India and other Eastern countries.

\section{Advantages of a Low Protein Ration.}

The advantages of a lowered protein ration were very clearly shown by experiments conducted with a section of the French army in 1906. Major P. Joly reports the result of an experi- 
ment with two companies numbering respectively 149 and 142 men. It was found that when the four ounces of fresh meat furnished the men was replaced by the same quantity of carbohydrates (cane sugar), there was a decided improvement in their physical condition. They were better able to endure the fatigue of marching, the number of heart beats was diminished, as well as the respiratory movements, and there was less sickness. It was especially noticeable that the men showed less fatigue and were less subject to the depressing effects of heat. During the last three days of manœuvres, the entire ration of five of the men was replaced by eleven ounces of carbohydrates (sugar) without any ill consequences, the men doing the same work as the others without showing any evidence of fatigue.

The advantage of a low protein diet in maintaining high resistance to disease is shown by the observations of Dr. Reid Hunt, who found that animals fed on a low protein diet were able to resist "two or three times the ordinary fatal dose of acetonitrile" (Chittenden).

Numerous further evidences of the advantages of a low protein ration will be presented in other sections of this work, wherein it is clearly shown that a low but sufficient protein ration is conducive to longevity, to physical and mental endurance, to resistance to disease, and freedom from certain maladies, and that the rigorous restriction of the protein intake to the actual needs of the body is one of the most useful means of combating many obstinate bodily disorders and prolonging the life in cases of incurable organic disease.

The limitation of the protein intake, especially the elimination of flesh proteins from the dietary, is of particular service in the correction of morbid conditions of the intestinal flora. The putrefactive changes which occur in the undigested residues of flesh foods when retained in the colon are now recognized as one of the most active causes of chronic disease. The greatest evils due to errors in diet are those which result from the putrefaction of the unused residues of meats while lying in the colon. 


\section{Fats}

Fats, like the starches and the sugars, are fuel foods. Their chief function in the body is to furnish fuel to maintain heat and to serve as a source for the energy expended in muscular work, heart and lung work, gland work, and other forms of vital activity. In recent years it has been found, however, that fats are an essential constituent of protoplasm and hence enter into the composition of the living cells of the body. Of this function of fat little is known.

As fuels, fats differ from starches and sugars in that while the latter substances may be termed quick-burning fuels, fats instead of being burned at once are stored for future use in the form of adipose tissue, sometimes known as residual or reserve tissue.

In chemical composition fats differ from starches and sugars in the much smaller proportion of oxygen which they contain. For this reason they require a much larger amount of oxygen for their consumption and produce a proportionately larger amount of energy when burned. For example, an ounce of starch furnishes 114 calories of energy, whereas an ounce of pure fat supplies 255 calories, more than twice as much.

There are many different kinds of fats, both animal and vegetable. In the process of ripening of certain fruits and nuts the starch of the green fruit is converted into fat. There is abundant evidence that a similar transformation of starch into fat occurs in the human body.

When starch is taken in larger quantity than is required to furnish the amount of sugar needed to produce animal heat and support the muscles in their work, the surplus is converted into fat which is deposited in the cellular tissue beneath the skin and in other parts of the body.

In the process of digestion, starches of all sorts are converted into a common product, dextrose or grape sugar, which is identically the same in character no matter what the source of starch from which it is formed. In the digestion of fats, however, the case is different, since the products of digestion of fats differ 
as widely as do the fats themselves. The changes which take place in fats during digestion are essentially the same as those which occur in soap making. The fats are first emulsified, then broken up into fatty acids and glycerine. The fats combine with the potash and soda furnished by the bile and the pancreatic juice to form soaps. After absorption, the glycerine recombines with the fatty acids. The nature of the fat of an animal may be modified by the fat fed to it.

The fats of herbivorous animals are much harder than those of carnivorous animals. The fats of fishes and cold-blooded animals naturally have a still lower melting point. The true human fat, that is, that made by the body, is formed by the tissues from starch, sugar and other carbohydrates.

Of the several animal fats, beef tallow, mutton tallow, lard, butter and cream, the latter is the most easily digestible. Next in order is butter. Those fats which have the highest melting point are most difficult of digestion.

The vegetable oils are all digested easily and possess about the same value. At least this is true of olive oil, corn, peanut, coconut and cottonseed oil. Crisco and oleomargarine are chiefly or wholly of vegetable origin. Nut margarine is prepared from vegetable oils.

While not differing greatly in their digestibility, fats differ much in food value because of their varying vitamin content. The fat soluble vitamin, necessary for growth and health main tenance, also to prevent sore eyes and rickets in infants and night blindness in adults, is deficient in most oils, in lard, and in oleo margarine and nut margarine. On this account butter and cream should be preferred as sources of fat when available. It is to be said, however, that a deficiency of the fat soluble vitamin may be made up by the free use of greens which are rich in this food essential.

Fat is not altogether an encumbrance, even when present in slight excess. It is a residual tissue, which may be drawn upon in case of food scarcity. The camel's hump serves as a store house from which supplies are drawn when other food is not available in its long desert journeys.

Great excess of fat is highly detrimental to health and preju dicial to longevity. Life insurance examiners penalize applicants for insurance when 10 per cent. or more overweight, because the life expectancy of such persons is known to be below the average 
Ghee, a fat much used in India, Turkey and other Oriental countries, is prepared from butter made from the milk of cows, sheep or goats. The butter is boiled and granulated, and though unsalted, will keep for many years, even a century or two, according to an authentic record of a find in an old castle in Turkey.

\section{Vegetable Fats.}

The principal sources of edible vegetable fats are the following:

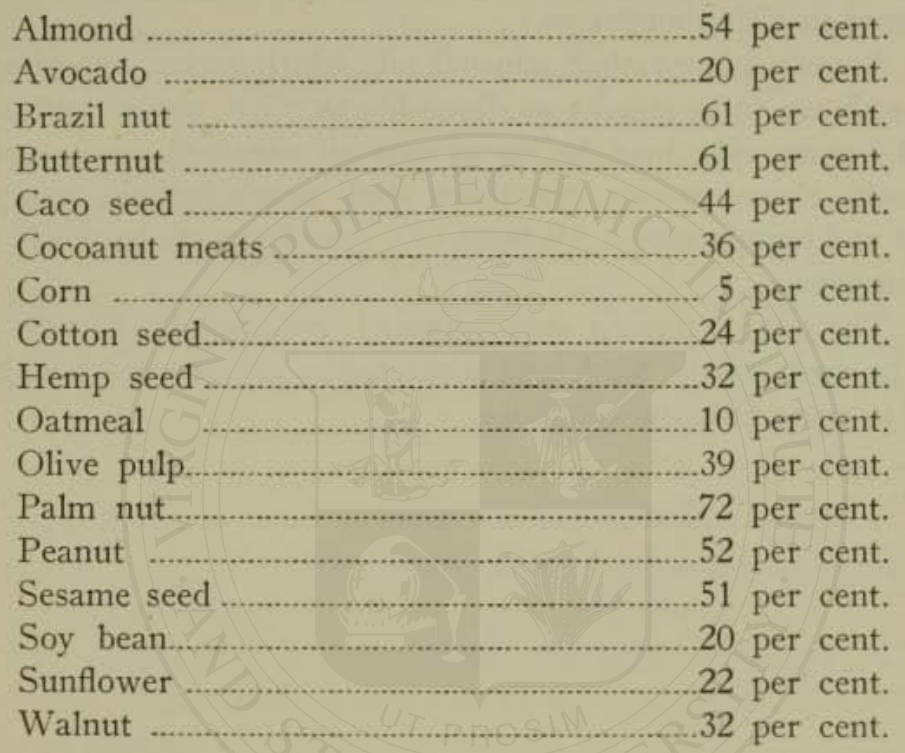

\section{Kernel Oils.}

A most excellent vegetable fat is prepared from the nuts of an African palm, the melting point of which is near that of butter, for which it is much used as a substitute. Palm kernel oil is also prepared from the nuts of the cohune palm. It is grown in Central America, where immense forests of this tree are found. In Spanish Honduras and Guatamala the cohune palm forms 20 to 30 per cent. of the vegetation. The nuts grow in enormous clusters nearly as large as a bunch of bananas. The nut is two or three inches in length and an inch and a half in diameter. The meat, which is about the size of a large nutmeg, contains 40 to 60 per cent. of oil. A tree yields about 1,000 nuts annually, from which may be obtained 10 quarts of oil. It is estimated that the trees growing wild in British Honduras produce annually not 
less than 275,000 tons of oil. Other Central American countries are capable of producing a much larger amount. Cohune oil closely resembles the kernel oil of the African palm, though it has a slightly lower melting point.

While these oils are as digestible as butter fat, it is not as yet certainly known whether they contain the essential vitamins, although it is most probable that they are quite deficient in this respect, and hence when used as butter substitutes must be supplemented by liberal quantities of greens which supply the lacking element in rich abundance.

Among other rather unusual oils which recent experiments have shown to be equally as digestible as olive, peanut and other well known oils, may be mentioned oil obtained from apricot kernels, cherry stones, cantaloup seed, peach kernels, pumpkin seed and tomato seed. All these oils were found to be edible and wholesome.

The oils of tomato seed, mustard seed, apricot kernels and of the seeds of the black and white poppy are also used in Italy and in some Oriental countries. 


\section{Carbohydrates - Starches and Sugars}

The carbohydrate element of foodstuffs consists chiefly of starches and sugars. Many fruits and vegetables contain fruit acids, which are also potential carbohydrates. Lactans and pentosans are found in certain fruits and legumes. These carbohydrates are not utilizable by human beings, although they are apparently readily assimilable by herbivorous animals. Hence, we derive practically all of our carbohydrate nourishment from starches and sugars.

The normal human diet contains about 60 per cent. of carbohydrates, the most of which is starch. The starches are for the most part derived from cereals, but a few roots and vegetables are also rich in starch, particularly the Irish potato, the sweet potato, the manioc root and arrowroot. The taro of the Sandwich Islands and the dasheen, a near relative of the dahlia of our flower gardens, are other valuable sources of edible starch.

Starch appears in plants in the form of granules which differ in size and appearance in different species of plants.

The accompanying illustrations will give something of an idea of the characteristic appearance of some of the more common forms of starch.

The chemical composition of starch (saccharid) is $\mathrm{C}_{6} \mathrm{H}_{10} \mathrm{O}$. Cane sugar, malt sugar and milk sugar have a similar composition, a single molecule of water being added to two molecules of starch- $\mathrm{C}_{12} \mathrm{H}_{22} \mathrm{O}_{11}$. Glucose and levulose have the composition $\mathrm{C}_{6} \mathrm{H}_{12} \mathrm{O}$ e.

Certain vegetables contain peculiar carbohydrates which have the composition $\mathrm{C}_{5} \mathrm{H}_{10} \mathrm{O}$ s, called pentosans. By digestion, these bodies are converted into pentoses, a kind of sugar which the human tissues are unable to utilize. Pentoses are found in the urine after the eating of prunes, cherries and grapes. The soy bean contains a considerable amount of pentosans and pentoses, but only a trace of starch.

Starch and dextrine seem to be the carbohydrates best 
adapted to the use of human beings. As supplied by the plant, starch is insoluble in water and non-absorbable, and must be transformed by the processes of digestion before it can be utilized. Very liberal provision is made for the utilization of starch. Its digestion begins in the mouth, before that of any other food substances. The work of transforming the starch into sugar is continued in the stomach. In the intestine, the starch digestion is carried on by the pancreatic and intestinal juices.

By this arrangement, the sugar formed from the starch is produced and absorbed slowly, so that the blood at no time becomes overcharged with sugar. The normal sugar content of the blood is about one part in one thousand. When this is much exceeded, the kidneys remove the sugar to prevent injury to the blood-vessels and other structures, which would result if the sugar were allowed to accumulate. This explains the appearance of sugar in the urine when large quantities of sugar are taken. If, for example, a half pound of candy or sugar in some other form is eaten at once, within a short time sugar will appear in the urine; but the same amount of sugar taken in the form of starch will never cause the appearance of sugar in the urine of a healthy person. This is the difference between a person in health and a person suffering from diabetes. A diabetic may show sugar in the urine, even though no sugar but only starch has been eaten. But this never occurs in a normal person, no matter how large a quantity of starch may be taken at one time.

As an additional precaution against a too rapid intake of sugar and its undue accumulation in the blood, the liver is endowed with the special function of capturing the sugar and retaining it within itself by changing it into a form of carbohydrate known as glycogen, a sort of animal starch.

The muscles, also, have the ability to convert sugar into glycogen, and thus a certain amount of carbohydrate is stored up to be utilized in maintaining the sugar content of the blood at its normal percentage during the intervals between meals or longer periods of fasting.

During a complete fast, the store of glycogen is practically used up after two or three days.

Starch has the further advantage over sugar as a carbohydrate food in the fact that it has a neutral flavor, and is bland and absolutely unirritating.

Another advantage possessed by farinaceous foodstuffs over 


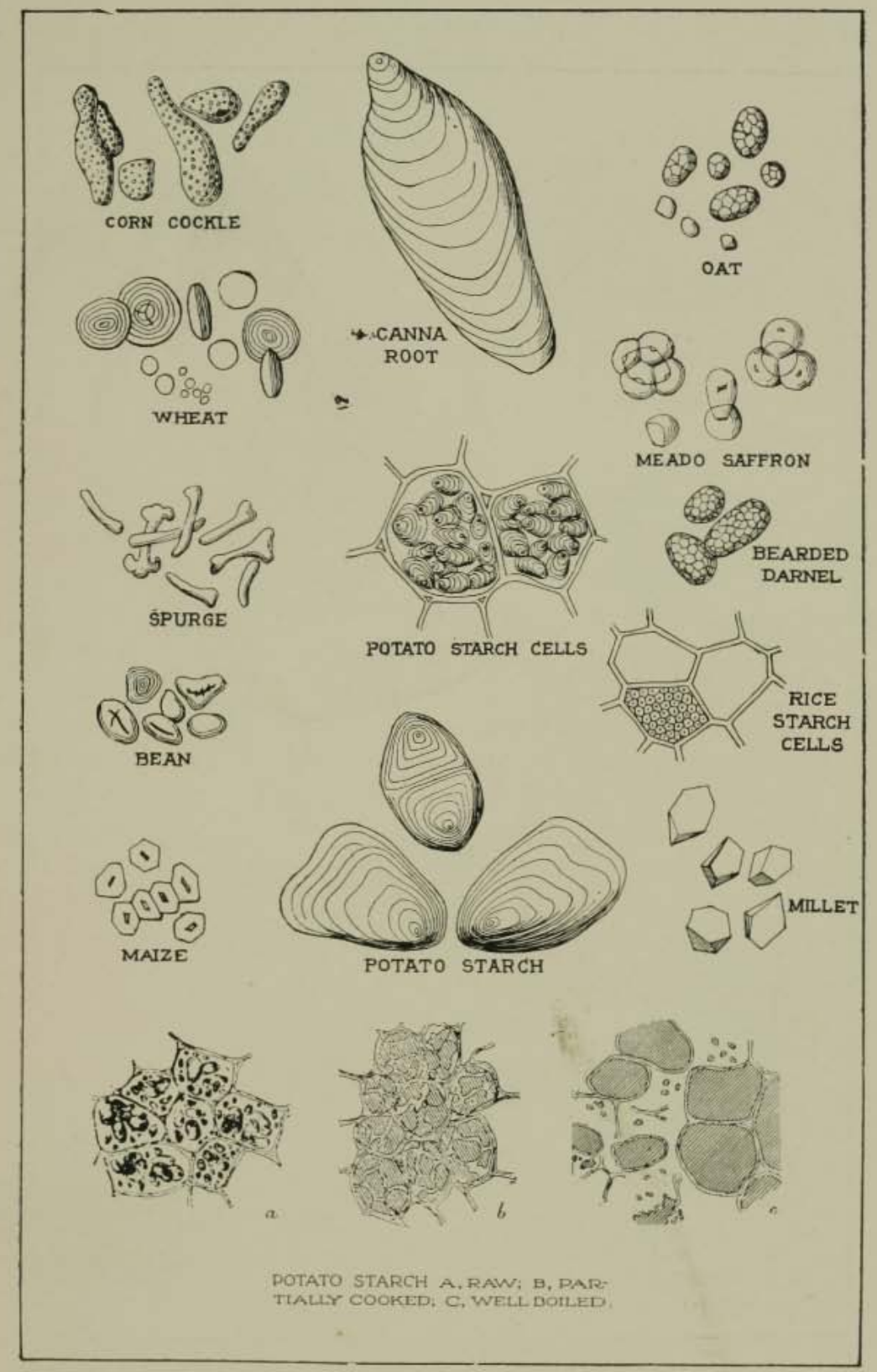

STARCH GRANULES 


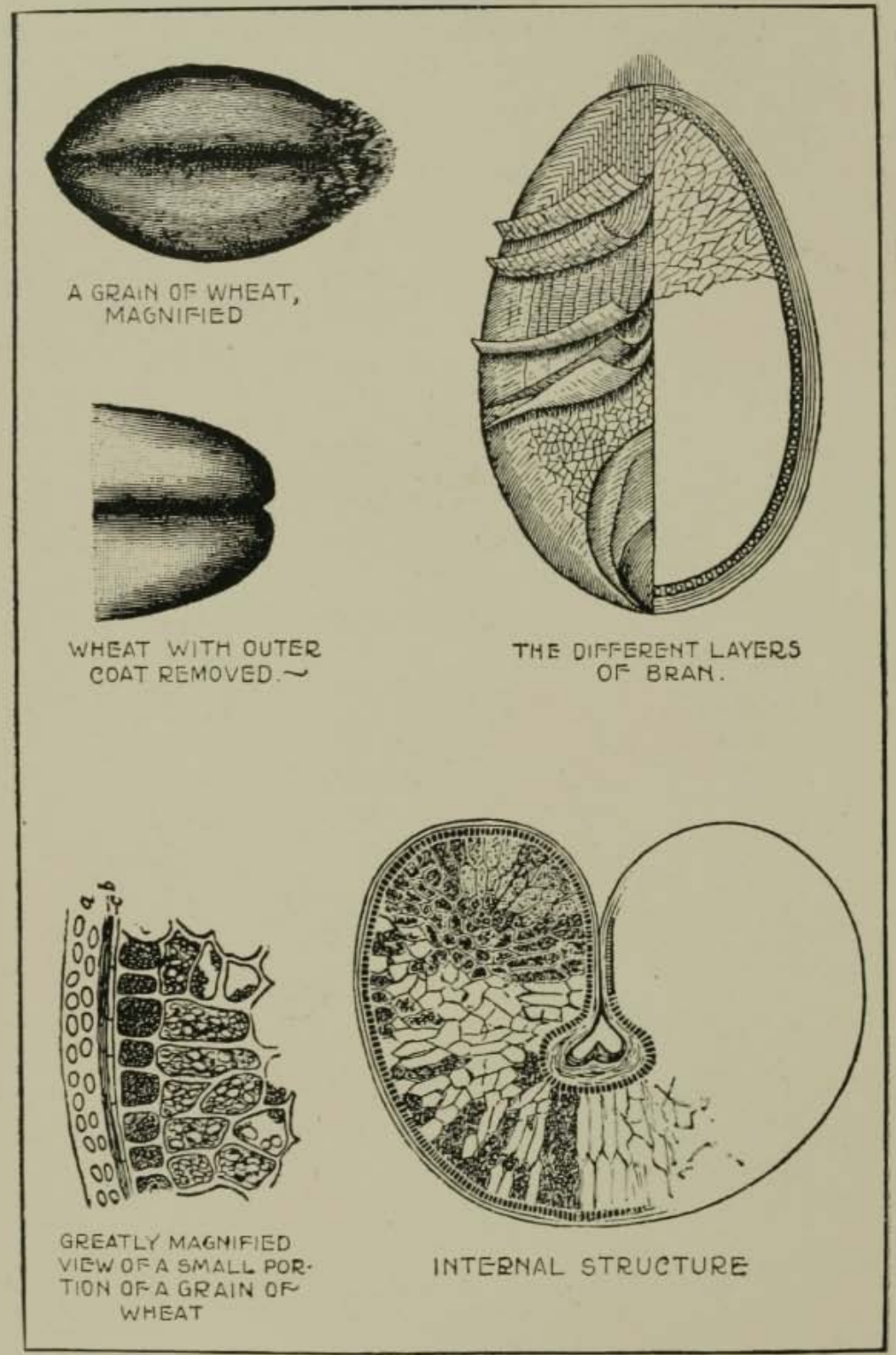


cane sugar consists in the fact that in this form the carbohydrates are not isolated, but are associated with the lime, iron and other salts as well as the precious vitamins, all of which form an essential part of the day's ration.

Some years ago, Grierson made the interesting discovery that there is a marked difference in the digestibility of starch from different sources. Grierson found, for example, that the starch of wheat, corn and rice requires two full hours for digestion, whereas the same quantity of oatmeal starch digests in eighty minutes, and the time required for the digestion of a like quantity of arrowroot starch is but thirty minutes and of potato starch only ten minutes. From which it appears that the root starches are much more easily digestible than are the cereal starches, the starch of wheat, corn and rice requiring twelve times as long for digestion as does the starch of potatoes, and the starch of oatmeal eight times as long.

The prejudice against farinaceous foods which still seems to exist in the minds of many persons, even some physicians, is wholly without sound foundation. Of all the elements of our food, starch is the least likely to give rise to inconvenience or to produce harmful effects, even when taken in excess; and the notion that the free use of starch gives rise to rheumatism, to intestinal indigestion, to gastric or intestinal fermentation, or "sour stomach," is wholly baseless. It is of course possible that the dietary may contain a disproportionate amount of starch, so that an individual may suffer for the lack of other necessary elements; but in such cases the bad effects observed are not to be attributed to the starch itself, but rather to lack of other essential elements.

For example, in the feeding of babies, it has long been observed that infants do not thrive on farinaceous infant foods, a long list of which have been offered as safe substitutes for mother's milk and cow's milk; and this has been found to be true even when these farinaceous food products were combined with a considerable proportion of cow's milk, sufficient to supply the protein and fat required. It is now known that the chief injury sustained by the child under these circumstances is the result of the lack of vitamins, which are supplied by mother's milk. The child gets all the protein, fat and carbohydrate it needs, but lacks the vitamins which are essential to promote growth. This defect may be remedied by the use of orange or tomato juice. 
Daniels and others have recently shown that the growth of babies may be increased by adding to the milk a soup prepared from wheat germs. The notable results obtained are doubtless due to the vitamins contained in the germ of wheat.

The dietaries of adults, when largely made up of farinaceous foodstuffs in their ordinary commercial forms, are equally liable to be lacking in vitamins. For example, fine flour bread, polished rice, the new process corn meal, corn flakes, rice flakes, macaroni, and, in fact, most of the cereals found upon the average breakfast table, are deficient in vitamins.

The hardy Chinese laborer, the gigantic Japanese wrestlers, the sturdy Smyrna porters, the long distance runners of India, the hardy Alpine peasant, all thrive on diets in which natural farinaceous foodstuffs are the staple, but which are supplemented by liberal quantities of greens and other foodstuffs which are rich in vitamins.

Hindhede has clearly demonstrated the safety of a highly farinaceous diet by long continued researches which have brought him into great prominence as an author in human nutrition in his own country, Denmark. although they have received little attention in this country. In a letter received by the writer from Professor Hindhede of Copenhagen, a couple of years ago (1919) he said that he then had under observation a man who had subsisted for twenty-three months on a diet consisting exclusively of bread, potatoes and greens. No fat of any sort was added to the foods named, and not a particle of other food had been eaten, yet the subject was in perfect health, vigorous and athletic, and able to engage in the hardest work. Professor Hindhede remarked that he had noted that large quantities of greens were very essential. McCollum, Mendel and others have shown that both cereals and potatoes are deficient in the fat-soluble, growth-promoting vitamin which is supplied abundantly by greens of all sorts.

When much gas is formed in the intestine as the result of eating freely of starchy foods, the cause is not "dyspepsia" but a "bad flora," that is, the presence in the colon of that most mischievous of micro-organisms, Welch's bacillus or an excessive number of colon bacilli. Without lessening the intake of carbohydrate, even increasing it, the gas, though at first increased, disappears as soon as the flora is "changed," This the writer has witnessed in hundreds of cases. 


\section{The Digestibility of Raw Starch.}

Observations by Langworthy and Deuel (Jour. Biol. Chem., May, 1920) seem to demonstrate that the uncooked starch of corn and of wheat is quite easily digestible, and when eaten by human beings in quantities of ten to twelve ounces are completely digest $d$ and assimilated, no trace being found in the feces. In the case of raw potato starch, 20 per cent. remained undigested.

It was noted, also, that the raw starch did not interfere with rhe digestion of other food principles taken with it, although, in the case of the potato, unpleasant symptoms, particularly a considerable formation of gas and "frequent intestinal cramps," were noted.

An interesting observation was that with the potato diet the feces, when heated, gave off 1.0 fecal odor but rather an odor of scorched bread, which we take to be evidence of the value of raw starch as a means of suppressing intestinal putrefaction, a matter to which the writer has frequently called attention and on which is based the recommendation to cook oatmeal six to ten minutes rather than longer.

Langworthy's results are in accord with laboratory observations made many years ago (1905) by the writer in experiments with raw starch in which it was observed that raw starch was digestible in quantities of four to six ounces. It was noted, however, that different persons differed in this regard. In the case of one person, most of the starch was discharged undigested, and there was diarrhea with much griping and malaise.

\section{SUGARS}

The world's production of cane sugar in 1914 was 42,000,000,000 pounds. Americans use more than any other people.

At the close of the Revolutionary War, the per capita annual consumption of sugar in this country was 7.5 pounds. It is now about 103 pounds. The consumption of sugar has increased in this country since 1900 at the rate of 1 per cent. a year. Diabetes has doubled its death rate in the same period.

Cane sugar was made from bamboo before the sugar cane was used for this purpose. Sorghum has been grown for sugar making in China for centuries. The Indians made sugar from the maple, hickory, birch and watermelon, and the natives of Mexico prepared sugar from the juice of the century plant in pre-Columbian times. 
Of the many sorts of sugar known to the chemist only five enter to any considerable extent into our ordinary dietary. These are sucrose or cane sugar, maltose or malt sugar, lactose or milk sugar, dextrose or grape sugar, and levulose or fruit sugar.

Of these several sugars we are most familiar with cane sugar, which is derived commercially from the sugar cane, sorghum, beet root and maple tree. Cane sugar is the sugar of trees, roots and grasses. It is also found in a few fruits, especially certain varieties of the date.

In the ripening of fruits, cane sugar is converted into glucose and levulose. These sugars are formed from cane sugar by a digestive change identical with that which takes place in the human body. When cane sugar is eaten, it is slowly converted into levulose and dextrose, for cane sugar in its native form cannot be utilized by the body. It must first undergo a digestive process, as does starch. But unlike starch, the digestion of cane sugar does not begin in the mouth, but is delayed until the sugar reaches the intestine.

The enormous consumption of cane sugar in this country, four times that of many other civilized countries, and the growing prevalence of the candy habit as the result of the diligent fostering of the practice by energetic manufacturers, justifies the inquiry whether or not the present extravagant use of the sugar of commerce is wholesome or safe.

Atchison Robertson, in carrying out a series of experiments, injected a half pint of a 20 per cent. solution of sugar into the stomach of a patient who was suffering from chronic gastric catarrh. "Shortly afterwards the patient became sick, and vomited a very acid fluid which put his teeth on edge. He also complained of heartburn and flatulence, and of a severe pain in the region of the stomach." Another experiment was the injection of a solution of fruit sugar (levulose) of the same strength, which produced no discomfort. The experiment was repeated with similar results in other cases of dyspepsia, and in every case the invert sugar produced no unpleasant symptoms. Schule found that from two to six lumps of cane sugar, when taken in an ordinary test breakfast, produced no appreciable effect on the digestion, but when the quantity was increased from ten to twenty lumps, considerable delay of digestion ensued, by reason of "the great outpouring of mucus in the stomach."

Brandi, an eminent German chemist, observed, in experi- 
ments upon a dog, that a solution of cane sugar having a strength of less than 6 per cent. caused irritation, with reddening of the mucous membrane. A 10 per cent. solution produced a dark red color with great irritation; and a 20 per cent. solution gave rise to still greater irritation, and produced such distress that the experiment was terminated. The author has met many cases of grave stomach disorder in which evidently the chief cause was the free use of sugar either in the form of candy or in connection with the use of coffee, oatmeal mush, or other "breakfast foods."

Ogata, in experimenting upon dogs for the purpose of determining the effects of cane sugar upon digestion, observed that the addition of one-third of an ounce of cane sugar to a meal of meat reduced digestion one-fourth.

The Germans made years ago extensive experiments with sugar as a means of supporting muscular work, extending their experiments to horses and other animals as well as soldiers. They found that while the sugar showed remarkable sustaining qualities, it was impossible to use more than a comparatively small amount on account of the irritating effects upon the stomach.

Sugar is freely used by rowing clubs in Holland, and it is reported that it seems "to counteract the bad effects of a meat diet so that the dreaded symptoms of overtraining did not appear." This fact is very interesting as an indication that the effect of overtraining is due to the large quantity of meat consumed. Sugar is antitoxic. When freely used it not only lessens the amount of meat consumed, because of the limitation of the calorie intake, but also through its antitoxic influence combats intestinal putrefactions encouraged by a heavy meat diet.

Molasses is much used in sugar-producing regions as food for horses and dairy cattle. The so-called black strap molasses is commonly fed in quantities of eight to twelve pounds per head. As a food a good quality of molasses is preferable to sugar for the reason that it is rich in iron and lime.

Dextrin is a product intermediate between starch and dextrose. The chemical composition of the three substances is essentially identical, except that in the change from starch to sugar, water is added to the molecule. Dextrin is found in plant juices and fruits. It is present in commercial glucose and in malt sugars to the extent of about 30 per cent. Dextrin may be produced not only by the digestion of starch but by the action of mineral acids and by heating at proper temperatures. The crust of bread and 
the browned portion of toasted bread contain dextrin. Dextrin has been recently shown to have special value as a means of changing the intestinal flora. When it is taken in doses of three ounces three times a day for several days in succession ( 7 to 10 days), the putrefactive flora disappears and acid-forming bacteria become dominant. The stools cease to have a putrid odor.

Dextrose or grape sugar is the sugar of grapes. It is found in most fruits with levulose in about equal quantities. Dextrose is formed from starch and from other sugars by the process of digestion and may be produced from various carbohydrates by chemical processes. All the sugars of foods are converted into dextrose before they are utilized by the body. Dextrose is the chief source of bodily energy. It is the body fuel par excellence.

Glucose is a saccharine product prepared by boiling cornstarch with a dilute mineral acid until the starch is partially converted into dextrose. It contains both dextrose and dextrin. Socalled brewers' sugar is a form of glucose containing about 70 per cent. of dextrose. What is known as " 80 sugar," or "acme sugar," contains 80 per cent. of dextrose. Practically all the cheaper grades of candy, and probably some of the higher grades, consist very largely of glucose. This is a cheap form of sugar about half as sweet as cane sugar.

Malt sugar. There is found in grains ferments or diastases similar to the ferments of the saliva, which are capable of first liquefying starch and then converting it into sugar. These ferments become active in the process of germination, and by the conversion of the starch in the seed furnish food for the young plant. Sprouted or malted barley has long been used in civilized countries for the conversion of starch into sugar in the manufacture of beer. The same process of sugar making has been employed in Japan for at least two thousand years. The Japanese make from rice, corn, millet, and sweet potatoes a preparation which they call ame, a sweet substance which appears in the form of a thick amber colored liquid and also in hard, white masses resembling candy. Ame is much used in Japan, and was in common use there hundreds of years before cane sugar was known in Europe.

It has been experimentally determined (Hammond), in the feeding of infants, that an infant is able to oxidize half a dram of lactose per kilogram of body weight, or practically one-fourth dram per pound. If the quantity is increased 50 per cent. beyond 
this, sugar appears in the urine. The same is true when cane sugar is fed; but in the case of malt sugar, more than three times as much sugar may be given before sugar appears in the urine. In other words, malt sugar is utilized by the infant more than three times as well as is milk sugar or cane sugar. From this it clearly appears that malt sugar is greatly preferable to any other form of sugar as a foodstuff. Cane sugar, the sugar of grasses, is adapted to the digestive organs of herbivorous animals. One of the four stomachs of ruminants supplies a ferment which quickly converts cane sugar into dextrose and levulose, the sugars found in fruits.

Malt sugar has many advantages over cane sugar. It is nonirritating, being native to the body, since it is constantly formed in the digestion of starch. Malt sugar also contains lime, iron and vitamins, all of which are lacking in cane sugar.

The manufacture of malt sugar for food was initiated by the writer more than thirty years ago. The difficulty was to eliminate the bitter flavor which made the sirupy extracts of malt too unpalatable to employ as foods. Malt sugar is now made by several concerns and is sold both in syrup and powder form.

Reid has shown that maltose enjoys an additional advantage over ordinary sugar in that it is absorbed much more rapidly. This is especially true when the mucous membrane is diseased, as in intestinal catarrh. In such conditions malt sugar is much better utilized than any other forms of sugar. This fact explains the observation often made by physicians that patients who are easily able to eat farinaceous foods freely without difficulty and can even take malt preparations in very considerable quantity without experiencing any inconvenience, may be unable to eat the smallest amount of cane sugar without suffering seriously in consequence from formation of gas in the intestines.

Malt sugar is especially valuable as a food for invalids and children. It may be given to young infants advantageously and is far preferable to cane sugar. It is most useful in cases of constipation in infants because of its mild laxative properties. It may well replace cane sugar for table use. Its characteristic flavor combines well with the flavors of fruits and grains.

Persons suffering from gastric hyperacidity, or peptic ulcer, are usually able to eat malt sugar in the form of Meltose, or Malt Honey, without suffering any inconvenience. 
Levulose is the sugar of fruits, and is often called fruit sugar, although dextrose is as truly a fruit sugar as is levulose. In most fruits these two sugars are found in about equal amount. Levulose is formed from cane sugar by the process of digestion. It is converted in the body into dextrose before it can be utilized. Honey consists of about equal parts of levulose and dextrose.

Levulose has been shown to be better tolerated by some diabetics than is dextrose and other sugars and on that account is sometimes very useful in the treatment of cases of diabetes.

Manna, a sweet substance which from the most ancient times has been gathered from certain shrubs growing in the Sinai desert, owes its sweetness to mannite, a sweet substance but not a true sugar. Manna is still gathered from the same locality where the ancient Hebrews found it in their wanderings and may be obtained from any drug store. It is slightly laxative and on this account is used medicinally. Quite recently a sweet substance said to be manna has been occasionally found in considerable quantity on the foliage of the Douglas fir tree in British Columbia. It forms on the leaves in drops the size of peas, and sometimes in larger masses.

Mannite differs from cane sugar in that it is not fermented by yeast. It is somewhat laxative and may be used without injury by diabetics, since it does not increase the sugar of the urine. It is found in the beet root and in the sweet juice of the ash tree.

A sugar known as inosite or American manna is obtained from the sugar pine. It exudes from the heart of the tree and collects on wounds which have been produced by fire, the axe or otherwise, forming irregular masses of a brittle, white substance having much the appearance of candy. It is sweet to the taste and is as well relished by many people as is maple sugar, but one's appetite for it is quickly satisfied. Chemical examination of this material, however, shows it to be quite different from the sugar of the cane and the maple tree. It does not ferment with yeast as does sugar, and though sweet in flavor is not really a sugar. Neither is it the true mannite which is found on the Douglas fir and on the shrubs of the Sinai desert. It is used as a medicine by the California Indians.

Lactose or milk sugar is a form of sugar peculiar to animals. It is in this respect unique, being found only very rarely in the vegetable kingdom. It is of all sugars the most difficult of digestion and assimilation. It has the least tendency to ferment of 
any of the sugars, although it is readily fermentable by the milksouring bacteria which are always found present in the intestines of a healthy infant within a few hours after birth.

Experiments have shown (von Noorden) that lactose is utilized only one-fourth as readily as is maltose, the sugar formed by the digestion of starch.

The value of lactose as a means of encouraging the growth of protective organisms in the intestine is due to the slowness with which it is utilized, permitting it to find its way into the large intestine where its presence is necessary to promote the growth of acid-forming bacteria.

The lactose of milk clings to the casein of the milk, from which it can be separated only by repeated washings. This fact contributes greatly to the success of the milk regimen-which floods the intestine with curds which carry milk sugar into the intestine and there feed the friendly bacteria. Advantage is taken of this fact in reference to changing the intestinal flora. It is found that if a person takes nine or ten ounces of milk sugar in three portions, during the day, for a few days in succession, the stools quickly lose their putrid character and the protective organism known as the bacillus acidophilus makes its appearance, while putrefactive organisms disappear. Similar results follow the use of three pints of acidophilus buttermilk daily.

\section{Dates vs. Cane Sugar.}

There is no doubt that the American people are suffering greatly from the excessive use of cane sugar. Cane sugar differs from the natural sugar found in dates in several important particulars. In the date the sugar is associated with other essential food constituents, especially protein, which is necessary for tissue building, iron, which is essential for the blood, lime, which is essential for the bones, and vitamins, another highly important food constituent necessary for the maintenance of nutrition and to stimulate growth and development in the young; all these food essentials are found in the date but none are present in cane sugar. Consequently, when a person uses cane sugar freely he is certain to suffer from a deficiency of lime, iron and vitamins, as the result of which he is likely to be anemic and to suffer from muscular weakness and lack of tone, as well as from an unhealthy state of the bones and decay of the teeth.

Another disadvantage of cane sugar arises from the fact 
that it is not that form of sugar to which the tissues of the human body are best adapted. Sugar (dextrose) is the fuel of the body, but not cane sugar, which is sucrose. The body uses invert or digested sugar. It cannot make use of cane sugar until after it has been digested. The process of digestion is always slow and often difficult in the human body, although in herbivorous animals the digestion of cane sugar is liberally provided for by a stomach especially adapted to the purpose. The sugar of dates, with the exception of a few varieties, is invert sugar; that is, sugar which is already digested and ready for immediate absorption and utilization. The cane sugar of the green date is during the process of ripening changed to invert sugar. The more mature the fruit, the more complete is this transformation. A similar transformation takes place in other sweet fruits.

Cane sugar has the great disadvantage that it is irritating as well as difficult of digestion. If taken otherwise than in very dilute form it is likely to produce severe irritation and may give rise to ulceration. The use of cane sugar is a common cause of gastric catarrh and hyperacidity. It is believed by many authorities that the free use of cane sugar is a cause of diabetes. It is most desirable that the consumption of cane sugar, which is carried to great excess in this country, should be greatly lessened. The substitution of dates, especially of the invert sugar varieties, should be encouraged. The date is more than a wholesome substitute for sugar, it is a whole food. It may be used as freely as may be desired and without injury. Observations made by Metchnikoff show that by the free use of dates the intestinal flora may be changed; that is, the poison-forming putrefactive bacteria may be suppressed and the harmless protective acidforming bacteria made to grow in their place. An ounce of dates contains more food iron than an ounce of beefsteak.

\section{Substitutes for Sugar.}

The only substitutes for sugar thus far found have been certain coal tar products, chief of which is saccharine, which is 280 times as sweet as cane sugar. This substance has been much used until recently as a substitute for sugar by certain manufacturers and by persons suffering from diabetes. It has been constantly under suspicion, however, and recently (1920) Professor Heitler, a well known authority on heart diseases, has published the results of a research which shows that, while sugar 
stimulates the activity of the heart, saccharine depresses it. The heart is gradually weakened by its use.

Saccharine belongs to the great family of coal tar products, many of which are active heart poisons, hence it is not surprising that careful observation has shown it to be a highly injurious drug. An experiment made some years ago in this country showed that it might be used in minute quantities for some time without producing disturbance of digestion, but apparently the effects of the drug upon the heart were not studied. Certain manufacturers relying upon the report of the government committee, have conducted a widespread campaign intended to promote the use of saccharine, and these efforts have been greatly aided by the recent extraordinarily high price of sugar. Now that the facts are known, it is highly important that the information should be spread abroad as rapidly as possible.

When saccharine is used in larger quantity than one or two grains three times a day it causes disturbance of the stomach.

Cane sugar, when freely used, is often productive of great injury, but to substitute for cane sugar a coal tar product such as saccharine, is to jump from the frying pan into the fire. The proper thing to do is to restrain the appetite for sugar. Sugar, as such, was unknown to our remote ancestors, and was doubtless disliked by many of them as by many persons at the present time. The sugar-eating habit may be acquired like the tobacco or the alcohol habit or the habit of chewing gum. It is a habit which should be combated.

A fortunate discovery by the United States Bureau of Plant Industry may lead to a solution of the sugar substitute problem. When in Washington, some months ago, the writer was showii a specimen of dried leaves from a plant which grows wild in Paraguay, and contains a substance 180 times as sweet as sugar. This curious plant is known as kaa hee by the natives of Paraguay. Another strange plant obtained from Nigeria bears a fruit which, although but slightly sweet itself, possesses the remarkable property of imparting to such sour substances as limes, lemons, or even vinegar, an intense sweet flavor which is retained for several hours.

The best solution of the problem is to be found in the education of the appetite away from sweets. The American sugar tooth has acquired enormously overgrown proportions and should be rigorously restricted in its indulgence in sweets. 


\section{The Nation's Candy Bill.}

The United States consumed in 1919 an average of 13.1 pounds of candy per capita (Financial World) at a cost (retail) of $\$ 1,219,000,000.00$, an increase of more than 1,000 per cent. in forty years.

Most candies contain a large percentage of glucose. Some years ago Roger reported the results of a series of experiments conducted by him for the purpose of determining the influence of various substances upon the protective power of the liver against infection. He found that "large doses of glucose, given by mouth, weaken the protective power of the liver." This fact unquestionably explains the observation made by many persons that the free use of candy makes them "bilious."

The use of candy and of cane sugar in other forms should be forbidden in all deficiency diseases such as scurvy, beri-beri and pellagra, as well as in cases in which lime and iron are deficient.

Bergeim, Rehfuss and their associates found that both cane sugar and glucose when freely used, diminished the secretion of gastric juice and delayed the emptying of the stomach. It is evident that cane sugar must be avoided in all forms of gastric disease and especially in gastric catarrh, gastric and duodenal ulcer, hyperacidity and gall bladder disease. The excessive use of cane sugar is believed by many able clinicians to be the cause of diabetes. There are many cases of persons who are on the border line of diabetes, potential diabetics. Examination of the urine in such cases shows no sugar, but Bergell's reaction is positive. A chemical examination of the blood shows a high sugar content. Such cases should wholly avoid cane sugar and should greatly lessen the intake of carbohydrates in any form.

\section{Honey.}

It is quite possible that good results would follow the exchange of at least a considerable part of the cane sugar we consume for honey. This natural sweet is prepared from the nectar of flowers. It is said that the nectar from 62,000 clover blossoms is required to produce one pound of honey, and that $3,700,000$ visits of bees are required to gather it into the hive.

The sugars of honey are levulose and glucose in about equal parts. Honey possesses the advantage over cane sugar that it requires no digestion. It is prepared for immediate absorption 
and use by the tissues. Formerly, much of the honey sold was adulterated. But since the passage of the pure food laws, this is no longer the case. Comb honey is necessarily pure.

\section{Some Odd Sugars.}

There are numerous sorts of sugar3, some of which would hardly be recognized as real sugars. The people of various countries produce sugars in great variety. The Greeks prepare sugar from currants; the Arabs make sugar from the palm, the date, the karob bean and the raisin. In Mexico and South America brown sugar is put up in loaves of various sizes, large and small, and is often of better flavor than the brown sugar of this country.

\section{The Consumption of Sugar Should Be Reduced.}

The excessive fondness for sweets so common in this country is without doubt a cultivated taste, and should be restrained. A no-sugar movement would doubtless do us good, and the present disposition to boycott the sugar trust on account of the high price of sugar will render better service than mere economic benefit. The per capita consumption of sugar should certainly be reduced one-half at least. Children should be encouraged to eat dates and raisins instead of candies. In general, sweet fruits should be substituted for sugar. When one person in every hundred in the United States is a diabetic, it is certainly time that this grave dietetic error should receive serious consideration. That sugar may be wholly dispensed with is well shown by the observations of Stefansson among the Eskimos. He says in "The Friendly Arctic,"

"Sugar we found in Victoria Island to be peculiarly distasteful to the natives, and even children of no more than four or five objected violently to the taste of candy, sugar, sweet preserves, canned fruit and the like." 


\section{Food Salts}

In addition to starch or sugar (carbohydrates), fats and protein, complete foods contain certain organic salts which are essential to life and health. Kellner fed animals food from which the salts had been extracted and they all died, and sooner than animals that had no food at all.

The principal salts, which should be always abundant in the daily bill of fare, are potash, soda, lime, magnesia and iron. Phosphorus and chlorin are found in combination with the alkaline elements. Recent researches indicate that iodine is also a necessary constituent of the food and that zinc, which is present in foodstuffs in nearly the same proportion as is iron, may possibly be a valuable factor in nutrition. The iodine content of foods is a matter of importance especially in diseases of the thyroid gland.

An eminent French chemist, M. Guerithault, recently (July $19,1920)$ communicated to the Academy of Sciences a report of an investigation of the copper constituent in plants. He finds copper present in varying amounts in "the leaves of lettuce, spinach, leeks, and celery; the roots of the carrot, turnips, radish, salsify, leeks, beets, and cress and the stalks of the latter; bulbs of onions and the tubercles of potatoes; in green beans; in the fruits of the pumpkin, cucumber, tomato, apple, pear, cherry, grape, orange, olive, banana, date and chestnut; and in the seeds of peas, beans, soy beans, lentils, wheat, barley, rye, oats, maize, cress, radish; as also in decorticated rice and in various nuts, including sweet and bitter almonds, walnuts and hazelnuts."

The lime and iron of the food, with other salts, are closely dssociated with protein in vegetable tissues. In animal tissues the lime is found almost exclusively in the bones and the iron in the blood. The practically universal neglect in this country to provide a constant and abundant supply of food salts in the dietary renders this subject sufficiently important to justify a somewhat detailed discussion of it in the interest of food reform and race betterment. For many years the importance of food salts has been recognized in the writer's work at the Battle Creek Sani- 
tarium; and a few years ago he worked out a plan by which the lime and iron constituents of the diet, and incidentally other food salts, may be as easily balanced as are the carbohydrates, proteins and fats, the balancing of which for each, patient has long been a part of the Battle Creek Sanitarium diet system.

In general it is sufficient to make sure that the food contains a sufficiency of lime and iron. When these elements are present in sufficient amounts the other essential salts are practically certain to be present also.

\section{THE IRON RATION}

By the term "iron ration" is meant the necessary daily intake of food iron, or so-called organic iron found in ordinary foodstuffs in varying proportions.

Iron is one of the essential constituents of the body. It is found in all living organisms, both animal and vegetable. Its importance to the growing plant has long been recognized by scientists, and is evidenced by the fact that iron abounds in the germ of cereals and other seeds. It is for the same reason, doubtless, that iron is found in relatively large amount in the embryos of animals, as in eggs, particularly the yolk of the egg. Iron is essential to life from the very outset.

In the animal body, the chief function of iron is to aid in the formation of the hemoglobin of the blood, which is an organic iron compound peculiar to animals, and possesses a special chemical property by means of which it is able to absorb and condense oxygen ( 1.3 times its volume).

This iron-containing hemoglobin is the chief constituent of the red blood corpuscles. Without iron, the body cannot make hemoglobin and hence cannot make blood cells, one of the most highly essential of all the bodily tissues.

The essential character of the blood seems to have been known from the most ancient times, even when the arteries were supposed to be carriers of air. Said the old prophet, "The blood is the life," a dictum which John Hunter's famous experiment proved to be the expression of a profound scientific truth.

The $25,000,000,000,000$ red cells floating in the blood stream of a man of average size have a combined area of about 22,000 square feet, or one-half an acre.

The life of a red blood cell is only about six weeks; hence, of the $25,000,000,000,000$ oxygen carriers, $7,000,000$ die every 
second of our lives, and to keep the number intact 7,000,000 new red corpuscles must be created by the living forces of the body every second, one of the most striking illustrations of creative power at work with which science has made us acquainted.

It has been observed that plants often suffer from anemia, their leaves are pale green and the flowers lack brilliance of color. This condition may in many cases be remedied by the administration of iron. Water containing iron rust poured about the roots of the plant, or even iron filings mixed with the soil about the plant, will often produce a rapid change for the better; the foliage becomes deep green in color and the tints of the flowers become more pronounced. In some instances pink hydrangeas have been made to produce blue blossoms.

When the iron supply is deficient and the blood is depreciated, the face, lips, gums and finger nails become pale because the red blood cells are reduced in number. In normal blood ,a minute cube, half a line in diameter, contains not less than $5,000,000$ red corpuscles with half a square inch of absorbing surface. This number is greatly reduced in anemia, the blood count becoming $3,000,000$ or $1,000,000$ or in very pronounced cases falling as low as 500,000 or even lower. This means that the oxygen-absorbing and carrying element of the blood, that is, the hemoglobin, has been reduced in proportion. Instead of 22,000 square feet of oxygen-carrying surface, there are only 15,000 or 4,000 or even 2,000 square feet, a reduction to one-tenth the normal amount.

This explains why an anemic person is short of breath, even when there is no trouble with the heart or lungs. He pants when he goes up stairs. He cannot run or even walk fast for a short distance without getting out of breath. There is nothing wrong with his breathing apparatus. The air is taken into the lungs properly and it comes in contact with the blood, but the blood does not take up enough oxygen because it is deficient in hemoglobin and so cannot absorb the oxygen brought to it, the effect being just the same as though the air supply were in part shut off by disease or by injury to the lungs.

The anemic person lacks appetite because food is fuel and the oxygen which the iron-containing hemoglobin brings is necessary to burn up the incoming supplies of fuel and to prevent the accumulation of unused and clogging material in the tissues.

The anemic person becomes sallow and the skin is often pig- 
mented as well as pale, because the oxygen supply is insufficient to burn up the wastes of the body and they accumulate in the tissues. It is also probable that in some cases, at least, the pigmentation is due to the production of poisonous pigments produced in such quantity that the adrenals, liver and kidneys are not able to destroy or eliminate them.

A person whose blood count is low feels languid and weak, because oxygen is needed to release the energy supplied by the food and stored up in the muscles and other organs.

In anemia all the life processes are depreciated, every vital function is crippled and the whole body is depleted. Long continued anemia of a severe type may give rise to paralysis as the result of damage to the spinal cord.

In view of the great importance of iron in the life activities of the body, it would naturally be expected that it would figure large among the constituents of the body. But the very opposite is true. It is the very smallest in amount of the twelve principal elements which enter into the composition of the tissues.

As will be seen by reference to Table I, the entire body contains only one-tenth of an ounce or one part of iron in 24,000 parts of body weight. This proportion is about the same as that of a teaspoonful of flour compared with a barrelful. There are hidden away in the bones five hundred times as much lime as there is iron in the whole body; and yet, this minute amount of iron, 44 grains, is just as essential as the oxygen which forms two-thirds the total weight of the body, or as the carbon and other elements which make up the balance.

Iron is found in the body almost exclusively in the form of hemoglobin, the red coloring matter of the blood. The blood constitutes about 7 per cent. of the body weight and contains about 95 per cent, of the iron. The red color of a piece of raw beefsteak is due to the blood in it. By thorough washing in salt water the blood may be removed and the muscle tissue left will be found nearly white in color.

Iron is found, practically, only in the blood because it is through the circulation of the blood that the iron is made to do its peculiar and wonderful work in entrapping the life-giving oxygen and circulating it throughout the body to every minute cell and tissue, bringing it in immediate contact with the smallest waste particles, and carrying back to the lungs the used-up materials, the products of combustion in the form of carbon dioxid 
$\left(\mathrm{CO}_{2}\right)$, by far the most bulky and the most important of all our excretions, amounting to more than half a pound daily, or more than forty times the weight of the urea eliminated and four hundred times the weight of uric acid excreted.

And the elimination of this enormous mass of poisonous debris all depends upon a few grains of iron in the blood. When a person is submerged in water he drowns in three or four minutes because of inability to get the carbon dioxid out of his blood. The blood and tissues become saturated with the poison and the body cells are asphyxiated. A man who has an insufficient amount of iron in his blood is like a half drowned man. The $\mathrm{CO}_{2}$ accumulates in his blood and tissues and the living cells become crippled and incompetent.

\section{The Daily Requirement of Iron.}

The solid as well as the liquid materials which enter into the composition of the body are in a state of perpetual change. An eminent scientist defines an animal as, "a stream of matter flowing through a certain form." The fluids of the body change very rapidly; the soft parts quickly, though less rapidly, and the solid parts more slowly. For example, the daily loss of lime is only 10 or 12 grains, at which rate nine years would be required to exhaust the body's store of 4.6 pounds of lime $(\mathrm{CaO})$. The blood, as already noted, loses all its red cells and replaces them by new ones about once in six weeks according to the estimate of physiologists. This would involve a loss and renewal of practically the entire iron store of the body every six weeks. But nature is less extravagant in her expenditure of iron than in the disposal of her store of lime. She hoards the precious metal with the greatest care. The worn out bodies of the $7,000,000$ red cells which perish every second are carefully worked over and the iron contained in their hemoglobin is recovered and stored in the liver and spleen and used in the making of new cells to take the place of those which are lost. Of course there is some loss of iron, but instead of losing a grain a day the actual loss of iron from the body is only about one-seventh of one grain.

We need food iron to make good this loss. Small though it is, it must be made good or the direst consequences will follow. Animals fed on a diet which contains no food iron die in a month or less.

To make certain that the supply of iron is adequate, the 
physiologists tell us that we require not less than 0.231 grains, practically one-quarter of a grain, per diem. The iron ration for the maintenance of health may be said, then, to be onequarter of a grain of food iron daily.

Women require a larger intake of iron in proportion to their weight than do men, and infants and children need much more than adults. The functions associated with childbearing and nursing make a special demand which requires an iron ration for the average woman fully equal to that needed by the average man of greater weight.

The expectant mother must supply iron for the blood of her infant, as well as for storage in its liver, from her own blood. In other words, the mother must furnish from her own store of blood iron, enough iron for blood-making purposes to meet the needs of her infant not only before birth but for a whole year afterwards, at the end of which time its blood volume and its weight are three times as great as at birth.

The monthly function of women involves a loss of iron which must be made good through a diet especially rich in organic iron. Even before puberty, girls require food richer in iron in proportion to their size than do boys, for two reasons :

(1) Because they grow faster than boys, becoming taller and heavier between the ages of twelve and fifteen years than boys of the same age.

(2) Because they usually eat less than do boys of equal age.

It is perhaps for this reason that women, guided by instinct. are notably more fond of greenstuffs and salads and fresh vegetables than are men. This natural tendency should be encouraged. Women and girls should eat more freely of greens of varions sorts and of all foods rich in iron than do men and boys. Lentils, dates and malt sugar are rich in iron. See Table XII.

The popular notion that cow's milk contains everything need. ful for nutrition is an unfortunate and dangerous error, though one into which a person very naturally falls. Milk is a natural food intended for animal sustenance and is primarily meant to be used as an exclusive diet, but it must be remembered that it is only sufficient as a complete nutrient when fed to very young animals which have been provided by nature with an extra supply of iron stored up in their livers before birth.

A guinea pig becomes anemic and starves on an exclusive diet of cow's milk. The same thing happens to a human adult if 
the diet is too long contınued. Neither the guinea pig nor the human adult has a reserve of iron suitable for blood-making sufficient to last more than a few weeks.

This question becomes one of very great importance in the artificial feeding of infants. According to the best authorities on the chemistry of foods, human milk contains twice as much iron as does cow's milk. This fact insures the nursing infant an adequate supply of iron during the nursing period. A very striking illustration of the marvelously wise adaptations of means to ends in the economy of Nature is afforded by the difference in the composition of human milk and cow's milk. Cow's milk contains nearly four times as much lime to the ounce as does human milk, but only half as much iron. The reason for this striking peculiarity is found in the fact that the calf needs four times as much lime for the formation of bone because it reaches maturity in less than one-fourth the time of the human infant, while, on the other hand, it needs less iron in its maternal food supply because it begins, like its mother, to nibble grass, a food very rich in iron, within a short time after its birth; whereas, the human infant normally is nourished exclusively by its mother's milk until six months of age or older.

When infants are fed other food than mother's milk the management of their diet becomes a matter of exceeding delicacy. It is more than probable that few if any artificially fed infants escape injury more or less serious through an inadequate supply of iron or lime or vitamins or of all these elements, or from lack of balance in the bill of fare in some other particular.

In the feeding of infants experience has shown the necessity for the dilution of cow's milk and the best results are secured by diluting whole milk with water, sweetened with milk sugar or malt sugar. In feeding young infants, equal parts of milk and water are generally employed. An infant fed on such a mixture receives only one-fourth the amount of iron normally found in human milk. On such a diet, the child becomes anemic. Resistance is lowered and the infant becomes subject to a great variety of disorders, the effects of which may remain through life. The free use of orange juice, the use of malt sugar instead of milk sugar and the use of barley meal, malt soup, lentil soup, and soups prepared from spinach and other green vegetables and foods rich in iron, in connection with milk feeding, are measures the value of which has been little appreciated until recent years. It is 
probable that the deficiency of iron in some infant dietaries is to a certain extent compensated by an excess of lime; at least some recent observations would indicate that this may be the case.

\section{Where to Find the Necessary Food Iron.}

Experiments made upon animals (Hall) show that when the food contains no iron at all, 40 per cent. of the entire iron content of the animal's body may be lost in three weeks.

How may the daily requirement of iron be best secured? This would seem to be an extremely simple problem, for no one of all the metals is so abundantly scattered over the face of the earth and in the earth and all about us, facing us at every turn, as is iron; and formerly it was supposed to be a matter of entire indifference in what form the iron was received so long as it was gotten into the body.

More than a third of a century ago Bunge of Bâle, Switzerland, one of the most eminent of those of the world's chemists who devote their lives to the study of the chemical problems relating to the human body, undertook, with the aid of his students, an extended series of experiments for the purpose of settling the question whether the ordinary iron with which we are familiar, the so-called inorganic iron, or any of its compounds could be utilized by the body in keeping good its stock of iron for use in blood making.

First of all, Bunge showed that natural foodstuffs contain iron in a peculiar state so different from that in which it is found in various chemical compounds and minerals that it cannot be recognized by the usual chemical tests for iron. This is known as organic iron. Bunge found in the yolks of eggs considerable quantities of this organic iron (hematogen) which was so similar to hemoglobin as to be easily converted by the body into the coloring matter of the blood, one of the most remarkable of all organic substances. Similar compounds were found in milk, legumes and cereals.

Bandisch has recently shown that ultra-violet light, acting upon the green parts of plants, produces compounds of iron in which the "metallic character of the iron is masked by the organic character of the compound." This organic iron is so different fiom ordinary metallic iron that it cannot be detected by the usual laboratory tests for iron. According to Dr. Bandisch these compounds are produced by a substance formed by the action of 
ultra-violet light to which he gives the name of formaldoxine.

In experiments upon a large number of rats, rabbits and dogs conducted in Bunge's laboratory it was found that the animals supplied with foods rich in organic iron made twice as much hemoglobin as those which were supplied with inorganic iron. It was also noticed that foodstuffs contain iron only in the organic form, "built up by the life processes of plants." This organic iron, according to Bunge, is the source of the iron used by the body in the formation of hemoglobin. The observations of Bunge have since been confirmed by many other investigators.

One observer has shown that rabbits made anemic by bleeding recovered when given chlorophyl much more rapidly than when given inorganic iron.

It is doubtless true, as suggested by Von Noorden and others, that inorganic iron may temporarily stimulate the blood-making process, but apparently it cannot be used, at least to any extent, in the construction of red blood cells. This stimulating effect of chemical iron preparations may be of some possible advantage in certain cases of disease, but the stimulation produced is of very short duration, and on this account such iron preparations should be employed only for very brief periods, if at all. As a matter of fact, they are rarely useful and very seldom needed. The long continued use of inorganic iron in the form of medicine or mineral waters is detrimental, causing constipation and other disorders. To prevent injury to the tissues, the liver, exercising one of its most important protective functions, captures the inorganic iron and holds it in its own tissues until it can be disposed of. This unnecessary work demanded of the liver may seriously interfere with the performance of some of its highly important functions. Thus the long continued use of inorganic iron in mineral waters or otherwise is to be condemned.

The iron ration must be drawn from organic sources; that is, it must be obtained from natural foodstuffs. Chemical, or inorganic iron, even though possibly of some limited service in certain cases of disease, is no proper substitute for food iron. As Sherman well says, in summing up the world's most eminent authorities, "Inorganic iron when absorbed is not utilized *** to any appreciable extent, but remains unused in the tissues. Evidently, then, we should look to food rather than to medicines or mineral waters for the supply of iron needed in normal nutrition."

Egers has shown that the regeneration of the blood after a 
severe hemorrhage goes on much faster with a diet rich in food iron than when dependence is placed upon inorganic iron.

\section{The Iron Content of Foodstuffs.}

Most foods in common use are so poor in ordinary iron that the ordinary bill of fare contains scarcely half the amount of iron needed to supply the daily requirement. As a result, the average American is anemic. Grave forms of anemia are becoming more frequent. School children are almost universally more or less anemic. This fact affords an explanation of the great prevalence of diseased tonsils, teeth and other disorders due to low resistance.

Among the foods richest in iron are found some which we had not even suspected of being so rich in this essential metal food principle until this digest of technical data was made. Here are a few of the most important, the relative richness in iron being in the order as named:

Wheat bran, egg yolk, lentils, beans, peas, wheat, mustard greens, hazelnuts, barley, rye, beef, almonds, graham bread, spin ach, turnip tops, and olives.

Each of the above named foodstuffs contains in one ounce more than 6 per cent. of the iron ration for one day, so that one pound of any of these foods, if eaten, would supply the necessary iron requirement.

In the case of several foods, the percentage of iron is so high that 6 to 10 ounces are sufficient for a full day's ration. It is interesting to note that one vegetable product, the lentil, contains exactly the same percentage of iron as the yolk of egg, and beans are not far behind, 6 ounces of lentils or 7.5 ounces of beans supplying iron for one day. Peas, mustard greens and whole wheat supply iron for a day with less than three-quarters of a pound of their substance.

Of the foods which are poor in iron, 33, or one-fourth of the total number, contain less than 1 per cent. of a day's ration. A large number (36 per cent.) contain between 2 and 3 per cent of iron per ounce, making a total of 69 foodstuffs (51 per cent.) which may be classed as very low in iron. Such foods as cane sugar, confectionery, olive oil, lard and suet contain no iron at all.

It is evident that when these foods with low iron content are made use of they should be supplemented by suitable foods rich in iron, which are named in Table XII.

A study of the average iron content of the several classes of foodstuffs affords useful information. 
When considered with reference to the amount of iron or the percentage of the day's iron ration contained in one ounce of material, we find the legumes, on the average, far ahead of all others (11.35). Greens come next (6.14), then eggs (5.67), meats (4.29), cereals (3.9), nuts (3.49), fruits (1.74), vegetables (1.46), milk products (1.05), and milk (0.47) with less than half of 1 per cent. of the daily requirement.

When examined with reference to the percentage of the iron ration contained in 100 calories, the order of precedence is changed. First in the list is greens (46.4), then meats (15.6), legumes (13.7), vegetables (10.4), fruits (5.6), cereals (4.3), nuts (2.67), and lastly eggs (1.36).

Table XI shows all the essential facts in relation to the iron content of more than 100 common foods. The several columns, numbered 1 to 7 , present the following particulars:

1. The amount of food iron calculated as grains of metallic iron, in one ounce of foodstuff.

2. The percentage of one day's iron ration ( 0.231 grains $)$ in one ounce.

3. The amount (ounces) of foodstuff required for one day's iron ration.

4. The percentage of the iron ration found in 100 calories.

5. The weight in ounces of 100 calories.

6. The weight in ounces of an ordinary serving.

7. The number of calories in an ordinary serving.

By the use of this table it is possible very easily to determine the iron content of the food intake for a meal, a day, or any definite length of time. 
TABLE XI.

\section{The Iron Content of Foodstuffs.*}

The following table shows in column (1) the amount of food iron calculated as grains of metallic iron in one ounce of foodstuff. (2) The percentage of one day's iron ration $(0.231$ grains) in one ounce. (3) The amount (ounces) of foodstuff required for one day's iron ration. (4) The percentage of the iron ration found in 100 calories. (5) The weight in ounces of 100 calories. (6) The weight in ounces of an ordinary serving. (7) The number of calories in an ordinary serving. (8) Amount of iron in an ordinary serving.
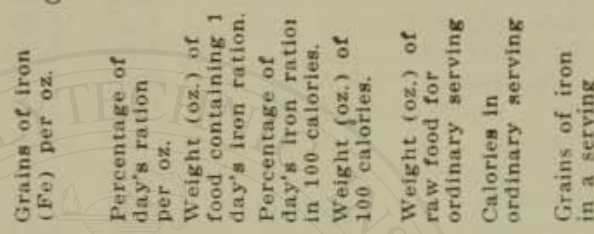

(1)

(2)

(3)

(4) (5)

(6) (7)

Almonds

.0171

$\begin{array}{llll}7.35 & 13.6 & 4.0 & 0.5\end{array}$

Apples, A. P

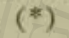

.0013

Apple juice

.0065

Apricots, A. P.

.0009

$\begin{array}{lllll}0.56 & 177.7 & 3.2 & 5.6\end{array}$

$\begin{array}{lllll}2.80 & 35.5 & 3.2 & 1.2\end{array}$

$\begin{array}{lllll}0.39 & 256.6 & 3.0 & 6.0\end{array}$

Apricots, dried

.0013

Asparagus

$\begin{array}{llll}0.56 & 177.7 & 3.5 & 6.1\end{array}$

$0.5 \quad 92$

$\begin{array}{lll}5.9 & 67 & .008\end{array}$

Bananas

.0044

Barley, entire

.0026

Barley, pearled

.0179

Beans, dry

.0088

Beans, lima, dry

.0306

Beans, lima, fresh

.0306

Beans, soy

.0088

Beans, string, fresh

.0248

Beef, all lean

.0048

Beefsteak, med. fat

.0170

Beets

Blackberries

Blood

$\begin{array}{lllll}2.64 & 38.0 & 3.5 & 1.1\end{array}$

$\begin{array}{lllll}1.90 & 52.5 & 30.0 & 15.7\end{array}$

$\begin{array}{llll}1.12 & 90.0 & 4.0 & 3.5\end{array}$

$\begin{array}{llll}7.75 & 13.0 & 7.6 & 1.7\end{array}$

$\begin{array}{llll}3.80 & 26.2 & 3.8 & 1.0\end{array}$

$\begin{array}{lllll}13.25 & 7.5 & 13.5 & 1.0\end{array}$

$\begin{array}{lllll}13.25 & 7.5 & 13.3 & 1.0\end{array}$

$\begin{array}{lllll}3.80 & 26.0 & 10.7 & 2.9\end{array}$

$\begin{array}{llll}10.73 & 9.3 & 9.2 & 0.9\end{array}$

$\begin{array}{lllll}2.08 & 48.0 & 17.7 & 17.0\end{array}$

$\begin{array}{lllll}7.36 & 13.6 & 30.0 & 4.0\end{array}$

$\begin{array}{lll}1.2 & 100 & .009\end{array}$

$\begin{array}{lll}6.0 & 100 & .005\end{array}$

$\begin{array}{lll}3.0 & 46 & .004\end{array}$

$\begin{array}{llll}2.0 & 157 & .012\end{array}$

$\begin{array}{lll}3.0 & 19 & .013\end{array}$

$\begin{array}{rrr}3.0 & 19 & .013 \\ 3.5 & 100 & .009\end{array}$

$\begin{array}{lll}1.0 & 90 & .018\end{array}$

$\begin{array}{llll}1.0 & 100 & .009\end{array}$

$\begin{array}{lll}1.0 & 97 & .031\end{array}$

$\begin{array}{lll}1.0 & 99 & .031\end{array}$

$\begin{array}{lll}4.0 & 62 & .035\end{array}$

$\begin{array}{lll}1.0 & 112 & .025\end{array}$

$\begin{array}{lll}4.0 & 44 & .005\end{array}$

$\begin{array}{llll}4.0 & 100 & .068\end{array}$

$\begin{array}{llll}4.20 & 24.0 & 10.5 & 2.5\end{array}$

$\begin{array}{llll}4.0 & 160 & .038\end{array}$

$\begin{array}{llllllll}.0026 & 1.12 & 90.0 & 9.0 & 7.6 & 2.2 & 29 & .006\end{array}$

.2301

Blueberries ……………………..... 0039

Bran

$\begin{array}{llll}1.12 & 90.0 & 7.0 & 5.9\end{array}$

$\begin{array}{lll}3.0 & 50 & .008\end{array}$

Bread, Boston brown ............... .0131

Bread, entire wheat

$\begin{array}{rrrrr}1.00 & 1.0 & \ldots . & 1.7\end{array}$

$\begin{array}{lll}2.5 & 53 \quad 010\end{array}$

$\begin{array}{lllllll}14.76 & 6.7 & 22.1 & 1.5 & 0.5 & 35 & .0170\end{array}$

Bread, graham …….................... .0109

$\begin{array}{llll}5.60 & 17.5 & 9.0 & 1.2\end{array}$

$\begin{array}{llll}3.03 & 33.0 & 4.3 & 1.4\end{array}$

$\begin{array}{lll}1.2 & 77 & .016\end{array}$

$\begin{array}{llll}4.70 & 21.0 & 6.4 & 1.3\end{array}$

$\begin{array}{lll}2.0 & 139 & .014\end{array}$

Bread, rye …………………......... .0070

$\begin{array}{llll}3.03 & 33.0 & 2.6 & 1.4\end{array}$

$\begin{array}{lll}2.0 & 158 & .012\end{array}$

Bread, entire rye (pumpernickel)

.0088

Bread, white

$\begin{array}{llll}3.81 & 26.2 & 6.5 & 1.7\end{array}$

$\begin{array}{llll}2.0 & 144 & .014\end{array}$

Brose

Brussels Sprouts

.0039

.0200

$\begin{array}{llll}1.70 & 58.0 & 2.3 & 1.3\end{array}$

$\begin{array}{lllll}8.60 & 11.5 & 8.6 & 1.0\end{array}$

$\begin{array}{lll}2.0 & 111 & .018\end{array}$

$\begin{array}{lll}2.0 & 159 & .008\end{array}$

.0048

$\begin{array}{llll}1.0 & 100 & .020\end{array}$

"Based upon the tables of Sherman in "Chemlstry of Food and Nutrition," Macmillan Company.

(The letters A. P. stand for "as purchased.") 


\begin{tabular}{|c|c|c|c|c|c|c|c|c|}
\hline & & (2) & (3) & (4) & (5) & (6) & (7) & (8) \\
\hline & .0009 & 0.40 & 256.6 & 0.2 & 0.5 & 0.5 & 109 & .001 \\
\hline$k$ & .0011 & 0.50 & 210.0 & 4.7 & 10.0 & 6.0 & 60 & .007 \\
\hline ge & .0048 & & 48.0 & 23.4 & & 4.0 & 36 & .019 \\
\hline ge greens & .0079 & 3.40 & 29.0 & 30.0 & & 4.0 & 25 & .032 \\
\hline oupe .......... & .0013 & 0.56 & 178.0 & 4.7 & 9.0 & & 78 & 009 \\
\hline & & 1.12 & 90.0 & 9.0 & 7.3 & 7 & 51 & .010 \\
\hline fower & & & 90.0 & 13.0 & 11.0 & & 25 & 008 \\
\hline & & & 105.0 & 18.0 & 18.0 & & 5 & 002 \\
\hline & & & 21.0 & 44.0 & & & 35 & 030 \\
\hline e & & & 40.5 & 2.0 & & & 62 & \\
\hline 10 & & & 55.0 & 5.8 & & & 62 & 08 \\
\hline & & & 128.0 & 3.4 & 4.7 & & 94 & \\
\hline juice & & & 177.7 & 2.5 & 4.6 & 0 & 100 & \\
\hline & & 1.30 & 77.0 & 2.0 & 1.4 & 0 & 14 & \\
\hline & See & & & & & & & \\
\hline 1 & .00 & 2.21 & 45.5 & 2.0 & 0.9 & .0 & 167 & .00 \\
\hline & & & 18.0 & 5.0 & 1. & & 109 & .013 \\
\hline & & 1.70 & 58.0 & 2.0 & & & 100 & .00 \\
\hline frech & & & 66.0 & 4.8 & & & 86 & \\
\hline , dried & & 5.50 & 18.0 & 5.5 & 1. & 0 & 109 & .013 \\
\hline & & & 35.0 & 2.4 & & & 110 & \\
\hline & & & 90.0 & 9.0 & & & 40 & 000 \\
\hline & & & 231.0 & 0.7 & & & 137 & 12 \\
\hline & & & & 8.0 & & & 10 & \\
\hline & & & 110.0 & 6.0 & & & 48 & \\
\hline Zante & & 4.7 & 21.0 & 6.0 & & & 135 & \\
\hline & & & 19.4 & 30.0 & & & 50 & \\
\hline ed, A. P. & & & 17.5 & 6.0 & & & 100 & .0 \\
\hline w leaved & & 13.40 & 7.5 & & & 0 & & \\
\hline (x) & & & 110.0 & 12.3 & 12. & & 12 & \\
\hline & & 5.67 & 17.5 & 13.6 & 2. & & 70 & \\
\hline$(1.8 \mathrm{oz})$ & & 10.54 & & & & 1.8 & 70 & .024 \\
\hline & & & 580.0 & 1.3 & 4.7 & & 144 & \\
\hline$(1.2 \mathrm{oz})$ & & & & & & 1.2 & 17 & .001 \\
\hline one yol & & & & & & & & \\
\hline $\mathrm{e}(.6 \mathrm{oz})$ & & 9.80 & & & & 2 & 103 & 4. \\
\hline & & 4.60 & 20.0 & 50.5 & 11.0 & 0 & $\begin{array}{l}60 \\
14\end{array}$ & 032 \\
\hline & & & 65.6 & 1.5 & & & 102 & $.00>\mathrm{C}$ \\
\hline & & & & 6.3 & & & 168 & \\
\hline & & & 52 & 6.3 & & .0 & 43 & 0 \\
\hline & & & & 6.3 & & 6.0 & 122 & .017 \\
\hline & & & 45.6 & 2.3 & & ........ & .... & \\
\hline & & & 16.2 & 5.6 & & & ....... & \\
\hline & & & & 9.7 & & ..... & .... & \\
\hline & & & & 1.5 & & & $\ldots . .$. & \\
\hline & & & & 2.5 & & ........ & $\ldots$. & \\
\hline & & & 16.0 & 6.3 & & & & \\
\hline & & 64. & 1.5 & 64.3 & & 0.5 & 62 & .074 \\
\hline & & & & & & & 100 & .060 \\
\hline & & 13 & & 13.0 & & 0 & 100 & .030 \\
\hline & & & 105.0 & 6.0 & & & 40 & .0 \\
\hline & & & & 4.0 & & & 50 & \\
\hline 1. P. & & & 177.0 & 2.0 & 3. & 3.5 & 72 & .005 \\
\hline & & & & & & & & .005 \\
\hline & & & 13.0 & 4.0 & & 0.5 & & .009 \\
\hline & & & 58.0 & 1.7 & & 1.5 & 150 & .006 \\
\hline & & & 77.0 & 2.0 & & 1.6 & 139 & .005 \\
\hline & & & 58.0 & 8.0 & & 5 & 52 & .010 \\
\hline & & & 90.0 & 9.0 & & 4.0 & 35 & \\
\hline
\end{tabular}




\begin{tabular}{|c|c|c|c|c|c|c|c|c|}
\hline & (1) & (2) & (3) & (4) & (5) & (6) & (7) & (8) \\
\hline Lemon juice .. & .0026 & 1.12 & 90.0 & 9.0 & 8.0 & 1.5 & & .004 \\
\hline Lentils, dried & .0377 & 16.32 & 6.1 & 18.0 & 1.1 & 1.0 & 99 & .038 \\
\hline Lettuce (leaf) & .0031 & 1.34 & 74.5 & 52.3 & 18.4 & 1.0 & 6 & .003 \\
\hline Maple syrup .... & .0121 & 0.57 & 17.5 & 7.0 & 1.3 & 1.3 & 108 & .016 \\
\hline Malt sugar & .0214 & 9.30 & 10.8 & 9.3 & 1.0 & 2.0 & 200 & .043 \\
\hline Malted nuts & .0192 & 8.30 & 12.0 & 5.5 & 0.7 & 1.3 & 200 & .025 \\
\hline Macaroni ..... & .0053 & 0.51 & 43.6 & 2.2 & 1.0 & 1.0 & 102 & .005 \\
\hline Milk, whole ....... & .0011 & 0.47 & 210.0 & 2.3 & 5.1 & 6.0 & 115 & .007 \\
\hline Gilk, buttermilk & .0011 & 0.50 & 210.0 & 4.7 & 10.0 & 6.0 & 60 & .007 \\
\hline Milk, skimmed _... & .0011 & 0.47 & 210.0 & 4.5 & 9.6 & 6.0 & 60 & .007 \\
\hline Milk, condensed, sweetened & .0026 & 1.12 & 88.8 & 1.3 & 1.1 & 2.0 & 185 & .005 \\
\hline filk, human .................................... &, 0030 & & & & & & & \\
\hline Molasses ........... & .0320 & 1.38 & 7.3 & 17.0 & 1.2 & 1.2 & 98 & .038 \\
\hline fuskmelon & .0013 & 0.56 & 175.0 & 5.3 & 8.8 & 7.0 & 78 & .009 \\
\hline Mustard greens & .0213 & 9.22 & 10.0 & & & 3.0 & & .065 \\
\hline Nuttolene & .0171 & 7.40 & 13.5 & 14.8 & 2.0 & 2.0 & 100 & .034 \\
\hline Oatmeal & .0166 & 7.19 & 14.0 & 6.4 & 0.9 & 1.0 & 113 & .017 \\
\hline Olives, ripe, A. P. ... & .0127 & 5.49 & 18.0 & 6.5 & 1.2 & 2.0 & 118 & .025 \\
\hline Onions … …… & .0026 & 1.12 & 90.0 & 7.0 & 7.2 & 2.5 & 42 & .007 \\
\hline Oranges & .0009 & 0.42 & 236.6 & 2.6 & 6.8 & 5.0 & 73 & .005 \\
\hline Orange juice & .0009 & 0.42 & 236.6 & 3.0 & 8.0 & 6.0 & 91 & .005 \\
\hline Parsnips …......... & .0026 & 1.12 & 90.0 & 6.0 & 5.4 & 3.0 & 55 & .008 \\
\hline Peaches, A. P. & .0013 & 0.56 & 180.0 & 5.0 & 8.0 & 4.0 & 38 & .005 \\
\hline Peaches, dried & .0052 & 2.29 & 45.0 & 5.0 & 4.0 & 2.0 & 160 & .010 \\
\hline Pears, A. P. & .0013 & 0.56 & 180.0 & 3.1 & 5.5 & 4.0 & 64 & .005 \\
\hline Peas, dry ............ & .0249 & 10.78 & 9.2 & 11.0 & 1.0 & 1.0 & 100 & .025 \\
\hline Peas, green & .0074 & 3.16 & 31.0 & 11.3 & 3.5 & 3.5 & 70 & .026 \\
\hline Peanuts ......... & .0088 & 3.81 & 26.2 & 2.4 & 0.6 & 0.5 & 78 & .004 \\
\hline Pecan nuts & .0114 & 4.93 & 20.3 & 2.3 & 0.5 & 0.5 & 104 & .006 \\
\hline Pineapple ……………........... & .0022 & 0.95 & 105.0 & 8.0 & 8.1 & 4.0 & 50 & .009 \\
\hline Plums ......... & .0022 & 0.95 & 105.0 & 4.0 & 4.1 & 4.0 & 91 & .009 \\
\hline Potatoes ……........ & .0057 & 2.47 & 40.5 & 10.4 & 4.2 & 3.0 & 71 & .017 \\
\hline Potatoes, sweet & .0022 & 0.95 & 105.0 & 2.6 & 2.8 & 3.0 & 105 & .007 \\
\hline Protose & .0249 & 10.80 & 9.2 & 21.5 & 2.0 & 2.0 & 100 & .050 \\
\hline dried, A. P. & .0131 & 5.67 & 17.5 & 7.0 & 1.2 & 2.0 & 145 & .026 \\
\hline Purslane. & .0080 & 3.90 & 25.6 & & & 4.0 & & .032 \\
\hline Pumpkin & .0035 & 1.51 & 66.0 & 8.7 & 13.6 & 4.5 & 25 & .016 \\
\hline Radishe & .0026 & 1.12 & 90.0 & 13.7 & 11,9 & 1.0 & 8 & .003 \\
\hline Raisins (seedless) & .0092 & 4.00 & 25.0 & 9.2 & 1.0 & 1.0 & 98 & .009 \\
\hline Raspberries & .0026 & 1.12 & 90.0 & 6.0 & 5.3 & 4.0 & 75 & .010 \\
\hline & .0280 & 12.90 & 7.8 & & & 4.0 & & .112 \\
\hline Rice, polished & .0039 & 1.68 & 58.0 & 1.7 & 1.0 & 1.5 & 160 & .006 \\
\hline & .0088 & 3.81 & 26.0 & 4.0 & 1.0 & 1.0 & 100 & .009 \\
\hline Romaine & .0525 & 22.75 & 4.4 & & & 2.0 & & .105 \\
\hline Rye flour ... & .0057 & 2.47 & 40.5 & 2.5 & 1.0 & & 100 & \\
\hline entire & .0171 & 7.40 & 13.4 & 9.6 & 1.3 & 1.3 & 100 & .022 \\
\hline Sav & 1.186 & 513.43 & 0.2 & & & 0.2 & 6.4 & .237 \\
\hline 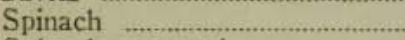 & .0158 & 6.84 & 14.51 & 100.4 & 15.0 & 4.0 & 20 & .063 \\
\hline Spinach, mountain & .0080 & 3.90 & 25.6 & ........ & ....... & 4.0 & ........ & .032 \\
\hline Spinach, New Zealand & & 4.10 & & & & 4.0 & & .036 \\
\hline & .0026 & 1.12 & 30.0 & 0.9 & 7.6 & 4.0 & 46 & .010 \\
\hline Strawberries . & .0035 & 1.51 & 66.0 & 13.7 & 9.4 & 4.0 & 44 & .014 \\
\hline & & 3.03 & 33.0 & 3.0 & 1.0 & 1.0 & 100 & .007 \\
\hline Tomatoes & .0018 & 0.78 & 128.0 & 11.7 & 13.3 & 4.0 & 25 & .007 \\
\hline & & 0.95 & 105.0 & 8.5 & 9.0 & 4.5 & 50 & .010 \\
\hline $\mathrm{Tu}$ & .0152 & 6.58 & 14.0 & & & 4.0 & & .061 \\
\hline & & 4.00 & 25.0 & 2.0 & 0.5 & 0.5 & 94 & .005 \\
\hline & .0083 & 3.60 & 28.0 & & & 1.5 & 10 & .012 \\
\hline 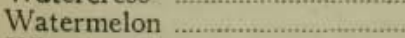 & .0013 & 0.56 & 177.7 & 6.6 & 11.6 & 8.0 & 69 & .015 \\
\hline Wheat, e & .0219 & 9.50 & 10.6 & 9.0 & 1.0 & & 150 & .033 \\
\hline Wheat br & .0341 & 14.76 & & 22.1 & 1.5 & 0.5 & 35 & .017 \\
\hline
\end{tabular}




\section{To Determine the Iron Content of a Ration or a Dietary by the Use of the Table.}

1. See that the weight of the servings of the several articles which compose the bills of fare for the day are the same as indicated in Table XI.

2. Add together the figures for the several items found in Column (7). The total for the three meals together should equal not less than .231. Anemic persons require twice this amount or about .500 or one-half grain of iron daily.

It is important to note that in the case of foodstuffs of which a very large quantity must be taken to supply a day's iron ration the nature of the food is usually such that a large quantity may be taken without inconvenience, so that, while the amount of iron per ounce may be small, it may be possible to take without inconvenience a sufficiently large quantity to more than balance the deficiency. This is particularly true in the case of fruit juices and greens.

To facilitate the work of balancing the bill of fare for the iron content the writer has prepared the accompanying tables (Tables XI and XII).

\section{Foods Rich in Iron.}

The accompanying table of foods rich in iron (Table XII) comprises less than one-third of the foods included in Table XI. Each foodstuff in this list supplies in one ounce at least 4 per cent. of one day's iron ration.

Some of these foods, particularly those which are found in the first half of the list, should enter liberally into each day's bill of fare. Without the free use of such foods, it is impossible to maintain the iron content of the body intact; the blood will deteriorate and all the bodily forces will languish. This is a matter to which every housewife, every individual, should give earnest attention. At a recent (1920) race betterment exhibit in connection with a great health and sanitation exhibition in Chicago, the writer had blood examinations for hemoglobin made in the cases of several thousand persons. The average was found to be 80 , or 20 per cent. below the normal. There can be no doubt that practically the whole American population is anemic because of the lack of iron-containing foodstuffs in the national bill of fare. The average American bill of fare offers almost no ironrich food except meat. The consumption of meat is steadily de- 
creasing because of high prices and the spreading of information respecting the advantages of non-flesh dietary. The effect of discarding meats must be to increase the national anemia unless other more natural sources of iron are added to the bill of fare. Fortunately, the place of meat as a source of food iron may be easily filled, as reference to Table XII will show. Instead of standing at the head of the list of iron-rich foods, steak is fortyninth in the list. More than a dozen vegetable foods contain double the amount of food iron supplied by meat; half a dozen, three times as much; four, more than four times as much; and two, more than ten times as much. Even such common garden weeds as red root and dock contain twice as much iron as does an equal weight of meat and better iron; while bran, molasses, lentils and egg yolk contain about three times as much.

Evidently the old notion that one must eat red meat to enrich the blood is an error; it is in the green things that the richest stores of food iron are to be found.

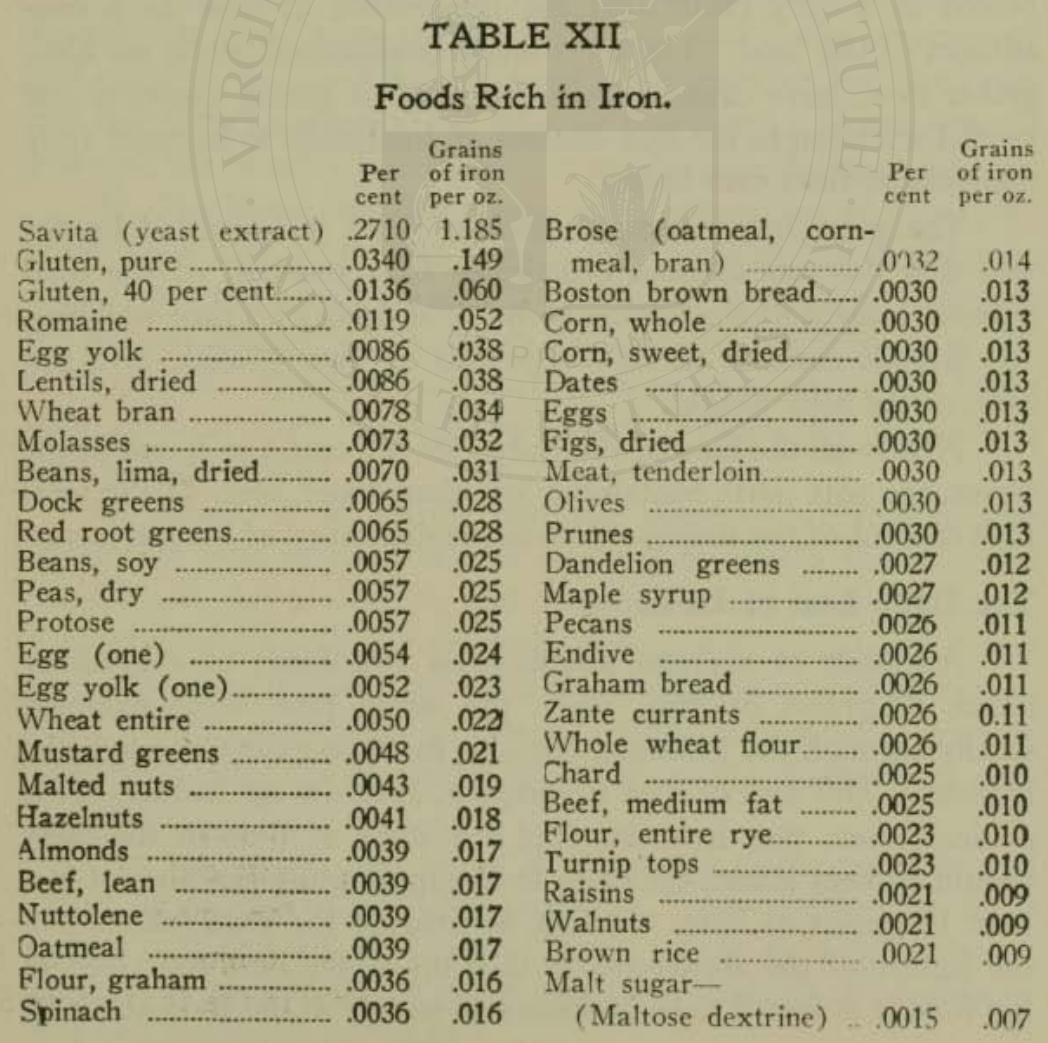




\section{THE LIME RATION}

Taken by itself, in its mineral form, lime possesses no food value, or at least only under exceptional circumstances, as when the body is deprived wholly of lime from other sources. Even then, mineral lime can be utilized only in very small quantities. It was, indeed, long a mooted question among physiologists whether or not inorganic lime ever could be utilized by the animal organism. In recent years carefully conducted experiments by Mendel and Osborne, as well as other investigators, have shown that when an animal is fed with food from which lime has been excluded the deficiency may be made up by feeding inorganic lime; but it is evident that this use of mineral lime is exceptional and the biologic law that vegetables feed upon mineral substances and animals upon organic matters built up by vegetable organisms working under the influence of the sun's rays still holds good.

Recent experimental studies on the feeding of animals have placed in a very clear light the importance of lime as a constituent of the food. The facts lately emphasized, while not altogether new, have caused the importance of giving constant and special attention to the lime content of foodstuffs to be more fully appreciated than ever before.

The great importance of calcium $(\mathrm{Ca})$ is suggested by the fact that this one element constitutes fully one-half of the total mineral content of the human body. The body of a man weighing 154 pounds contains about 4.5 pounds of lime $(\mathrm{CaO})$, or 3 per cent. of the body weight.

Nearly all the lime of the body ( 99 per cent.) is found in the bones, the soft parts containing less than 1 per cent. of the total lime content of the tissues. Hence the deficiency of lime in meats.

\section{The Daily Loss of Lime.}

An adult of average weight loses two-thirds of a gram or about 10 grains of lime, daily, the waste lime being excreted chiefly through the intestine, but also to some extent through the kidneys. This loss of lime is constant, even during fasting. It is evident, then, that in order that the store of lime in the body should be kept intact, the daily food supply must contain not less than 10 grains of lime. In fact, to provide for emergencies and the failure of the absorbents to take up all the lime contained in the food it is found to be necessary to supply in the food about 50 
per cent. more than the actual requirements, or 15 grains of lime daily. Some authorities demand more than this.

Unfortunately, the food commonly eaten by no means always contains this amount. Indeed, Dr. Sherman, of Columbia University, calculates that at least half the people of the United States are suffering from lime starvation. Probably lime is more often deficient in the dietary of the average American than any other element with the exception of iron and cellulose.

The fact that lime is found in every bodily tissue, bones, soft tissues, blood and other fluids, indicates its universal importance in the body.

1. To the bones it gives solidity. The bones of the adult are more than half lime. The amount of lime in the bones is so great that a burned bone retains its form even though the animal part has been wholly destroyed by fire. A deficiency of food lime causes in young children bowlegs and is no doubt one of the causes of decay of the teeth.

2. The ability of the muscles to contract and to do work depends not alone upon their development and to the presence of fuel sugar in the blood and glycogen in the muscles, but also upon just the right proportion of lime in the blood. When lime is deficient, the muscles lose their tone and become flabby and weak.

3. The blood contains a very small proportion of lime, only about 0.8 grains to the pint (cow's milk contains fifteen times as much), but this small amount is absolutely essential to maintain the normal beating of the heart through its influence upon the heart muscle. The mammal heart has been kept beating for hours after removal from the body by passing through it a solution of lime and other salts.

4. Again, it is known that the presence of this minute amount of lime in the blood is essential to enable the blood to coagulate or clot to stop the flow of blood from a wounded vessel. A careful surgeon always examines the patient's blood and determines the coagulation time before undertaking a serious operation. When coagulation is delayed, a transfusion of a pint or more of healthy blood may be given before the operation is performed.

The relation of lime to the development of the bones is well shown by an experiment upon puppies. Those fed on lean meat and fat only had soft and flexible bones. Puppies to which bones were given gnawed at the bones and thus obtained the necessary lime and developed normally. 
Voit, an eminent physiologist who devoted his life to the study of food problems, fed pigeons for a year on foodstuffs which were greatly deficient in lime. There seemed to be no change in the birds, which appeared to be in perfect health, but after they were killed careful examination showed that, while the leg and wing bones contained the normal amount of lime, the bones of the head were almost wholly deprived of lime, being greatly thinned and at some points actually perforated. Evidently the body had sought to maintain the normal blood content of lime by robbing those parts of the skeleton from which it could best be spared.

Sherman, of Columbia, repeated Voit's experiment, employing monkeys instead of pigeons. The results were the same. The leg and arm bones did not suffer much, but the skull bones lost their lime almost altogether, becoming so thin that they could be crushed between the thumb and fingers as easily as an eggshell.

Some years ago the authorities in charge of the London zoo observed that the lion cubs were deformed, bowlegged, clubfooted, dwarfed and always died young. Treves, an eminent London surgeon, was consulted. He asked about the food. He was informed that they were given the very best of meats. He suggested the feeding of bones and bone meal. His advice being followed, the deformities soon disappeared and the cubs ceased to die prematurely.

The cave dwellers, who in prehistoric times were compelled by the rigors of the ice age to subsist on a flesh diet, rounded out their diet by splitting bones and eating portions of them, especially the marrow.

According to Cobez de Baca, one of the Spanish explorers of the Texas coast in the sixteenth century, the natives of that region carefully saved the bones of fishes and other small animals and ate them after reducing them to powder in stone mortars.

The lack of lime in the food is no doubt one cause of the increasing prevalence of bone disease in civilized countries, and may be responsible for the early decay of the teeth which is becoming so nearly universal that the development of a toothless race is threatened. Evidently the teeth suffer loss of lime along with the other bony structures of the head.

Hart and Steenback showed that milch cows require food containing a large amount of lime. If such food is not supplied their bones are robbed of lime. Nature takes care to see that 
milk, the sole food of the very young animal, contains an adequate amount of lime, even at the expense of the mother.

This interesting observation explains the negative results of a very plausible theory advanced many years ago as the basis of a plan for securing painless childbirth. Expectant mothers were told to avoid milk, grains, and lime-containing vegetables, and to make their diet consist chiefly of fruits and nuts which are very poor in lime. The idea was that in so doing the bones of the infant would contain less lime and the infant head, the chief cause of suffering, would thus be less rigid and would easily mold itself to the outlet of the pelvis. Hundreds tried the food formula for "painless" childbirth, but the results were most disappointing. Experiments upon animals showed that no matter how much the lime in the mother's food was reduced, the bones of the offspring always contained the normal amount. The mother's bones were robbed to make good the deficiency of lime in the food.

Here also is found, perhaps, an explanation of the remarkable tendency to decay of the teeth which often appears in the last months of pregnancy, a fact long known but not explained. Recent studies conducted by members of the faculty of the dental department of the University of Michigan show that the saliva becomes very deficient in lime during the last months of gestation.

Infants and young children require two or three times as much lime in proportion to their weight as do adults, since they need lime for building up and solidifying the skeleton as well as for making good the daily waste. A young nursing infant gets daily in its mother's milk more than three grains of lime, or one-fifth as much as the daily requirement for an adult weighing ten to fifteen times as much.

The wonderful care which Nature takes to supply the young animal with an abundance of lime for building its skeleton is well illustrated in the interesting case of the egg. According to the Annals of the Pasteur Institute, before incubating the contents of an egg include one-half grain of lime. During incubation, the amount steadily increases until when hatched the body of the chick contains three grains, or six times as much lime as the embryo contained. The growing chick has in some mysterious way, not yet determined, managed to steal from the shell the needed lime for stiffening its slender leg bones so as to enable them to support its body as soon as it emerges from its calcareous cell. It thus appears that the egg shell is not simply a container but also 
serves as a storehouse of lime to be drawn upon by the developing chick.

Herbst has shown that between the age of six to fourteen boys require three or four times as much lime in proportion to their weight as do adults, since they must store up in their growing bones, 3 to 6 grains of lime daily, or about one-third of the adult maintenance lime ration.

\section{Food Lime is Necessary for Plants as Well as Animals.}

Professor True of the University of Pennsylvania, in a recent address in Toronto before a scientific body, called attention to important biological experiments which demonstrate the high importance of calcium or lime in plant growth. It seems that this remarkable element acts as a regulator of the intricate processes of cell growth. Under its magic influence, the cell walls are able to take up from the plant juices the particular elements which they require. Without lime, the nutrition of the cell fails; its growth stops. Experiment has shown that this regulating influence of calcium prevails throughout the entire plant, from its most minute rootlets to its unfolding buds. A soil which is deficient in lime, though abounding in all the other elements requisite for the maintenance of plant life, remains sterile, as though all plant foods were absent. Without calcium, the growing plant cannot absorb the various other elements which it needs.

These facts account for the universal and abundant presence of calcium in the growing parts of plants; that is, in green leaves, buds, twigs and tender shoots. What is true of plants, is equally true of human beings. Calcium is a regulator of the nutritive processes. It is essential for heart action and muscle action and constitutes an essential element of the blood and tissue fluids, as well as of the brain, nerve, glands and other living structures.

\section{The Influence of Lime on Egg Production.}

Buckner and Martin (Journal Biologic Chemistry) have shown that when fowls are abundantly supplied with food containing lime the egg production may be increased as much as 70 per cent. This experiment clearly shows the influence of diet upon nutrition. Children whose diet does not comprise a sufficient amount of food lime grow slowly because of the lack of lime for bone building and the bones are likely to be poorly developed. On this account housewives should take great care to see 
that the family diet contains an abundance of greens, such as spinach, dandelion, dock, lamb's quarter, chard and other green vegetables.

\section{The Lime Content of Foods.}

The proportion of lime found in different foodstuffs varies greatly. This fact is of much importance in view of the recent observations of Osborne and Mendel as well as of other investigators who have undertaken exhaustive experiments in animal feeding, and have shown that rats do not grow, develop and reproduce normally unless supplied with a full quota of lime.

If the assertion of Professor Sherman that "half the American people are suffering from lime starvation" is true, and there is no reason to doubt it, the study of foods with reference to their lime content is highly essential to our understanding of rational dietetics. This study has heretofore been greatly neglected.

First of all it is interesting to note that in general roots and the green parts of plants (greens) are richest in lime. The legumes (beans, peas and lentils) and certain nuts (almonds, hazelnuts and walnuts) are also rich in lime. Of the grains, oatmeal is the only one which presents a fair proportion of lime. Wheat and corn are both very deficient in lime, and rice contains almost no lime at all.

Of all natural food products, the richest in lime are the hazelnut, the almond, and linseed meal. The last named product, though not commonly used as a food, may be resorted to in emergency and is doubtless entirely wholesome.

Milk is so rich in lime that a pint and a quarter contains sufficient of this necessary element for a full day's supply. But an equal amount of lime would be furnished by half the weight of almonds, and eight or nine ounces of hazelnuts will supply the same quantity, or six ounces of linseed meal.

The foods which contain the largest proportion of lime and in the order named are:-cheese, cottage cheese, hazelnuts, almonds, molasses, beans, chard and other greens, egg yolk, wheat bran, skimmed milk, whole milk, olives. In milk the lime is closely associated with the casein which forms the curd. Milk contains more lime than is found in lime water.

In cheese the lime is of course concentrated, the proportion being about six times as great as in milk.

Since skimmed milk contains practically as much lime as 
does whole milk, it is a highly useful article of food. Not a drop should be wasted. By combining with other foodstuffs as in bread and other cereal preparations, with the addition of fat, it may be very advantageously made a regular part of the daily bill of fare.

The feeding of skimmed milk to pigs is a foolish waste. Our growing boys and girls need every drop of milk which the country produces.

We are losing our teeth and shrinking in stature because we need more lime and milk is one of our most important sources of food lime.

The amount of lime required by the average adult is threefourths of a grain for each 100 calories of food or about 15 grains a day for a person taking a ration of 2,000 calories. This amounts to four-fifths of a pound per annum for each man, woman and child in the United States, aggregating a total lime requirement for the country of 40,000 tons per annum.

That the nation fails to get its full lime ration is due in part to the wasting of milk in our dairy industry and in part to the fact that we unwisely deprive ourselves of an essential food element by feeding the bran of our wheat and other foodstuffs rich in lime to hogs and cattle instead of making use of them ourselves. As a natural result, our domestic animals are steadily improving in physical development while as a nation we are declining in vigor and showing distinct indications of physical degeneracy.

It is interesting to note that our wide-awake agricultural department at Washington is advising the people to buy milk instead of meat, pointing out that its nutritive value is far greater. Even whey contains a notable amount of lime, more than a third as much as whole milk, but less than a twentieth as much as cheese. However, the amount is so large that it should be utilized when possible. When fresh, or only slightly acid, whey is a wholesome food.

Wheat bran contains as much lime as does milk, but of course it cannot be taken in so great quantities as milk, hence is less available as a source of lime.

It is interesting to note that molasses contains a good proportion of lime, more than three times as much as is found in oatmeal, and nearly twice as much as in milk. In this respect molasses is superior to sugar, which contains no lime at all. In 
molasses, the lime found in the natural juices of the cane is concentrated; it is probable that the lime used in the manufacture of sugar may be the source of part of the lime in molasses. Maple syrup contains half as much lime as does molasses.

A careful study of Tables XIII and XIV will develop a considerable fund of most useful information. The method of using Table XIII in balancing the bill of fare for lime is the same as that used in balancing for iron, as already explained.

\section{Foods Deficient in Lime.}

It is very instructive to note the great number of common foods which in the form in which they appear upon our tables are almost wholly lacking in lime. The table shows, in fact, more than fifty common foods which are so poor in lime that one would need to eat not less than six pounds to obtain an adequate amount of lime for a single day. In some instances the lime content is so small that the amount of foodstuff necessary to furnish a day's ration rises to ten, twenty, and even forty pounds.

Among the foods most deficient in lime may be mentioned the following: cane sugar, which contains no lime; honey, which supplies in one pound of wax-free material, only one-fortieth of the lime needed for one day. Apples, bananas, beef and other meats, butter, also lard and oils of all sorts, most of the cereals and nearly all fruits are deficient in lime.

An examination of the average bill of fare will show it to be woefully deficient in food lime as well as in iron, and, naturally, in other salts as well. To assist in remedying this deficiency, we have prepared the accompanying list of foods which are rich in food lime. Foods selected from this list, especially the first third of it, should be made a part of every day's bill of fare, and in liberal quantities. The number of foods rich in lime is so great, it is easy to provide for abundant variety while insuring an abundance of food lime. Three tumblerfuls of milk (20 ounces), four ounces of cottage cheese, five of mustard or red root greens, or half a pound of hazelnuts or filberts will supply a full day's lime ration. Half a pound of soy beans, lamb's quarters or turnip greens will do the same. It is better, however, to secure the needed lime with a greater variety of foods, which may easily be chosen from Table XIV. 


\section{TABLE XIII \\ The Lime Content of Foodstuffs}

The following table shows in column (1) the amount of lime (grains) contained in one ounce. Column (2) shows the percentage of one day's ration in one ounce. Column (3) shows the amount of foodstuff required to supply 15.4 grains of lime $(\mathrm{CaO})$, the amount needed for one day. Column (4) shows the percentage of one day's requirement of lime found in 100 calories. Column (5) shows the weight of 100 calories. Column (6) shows the weight of an ordinary serving. Column (7) gives the number of calories in an ordinary serving. Column (8) gives the amount of lime in an ordinary serving.
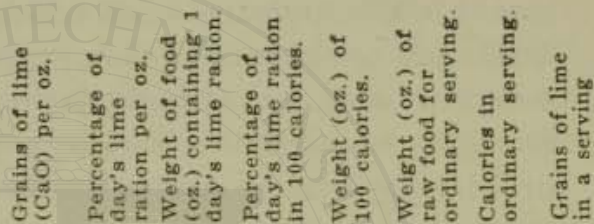

(1)

(2)

(3)

(4)

(5)

(6) (7)

Almonds

1.463

$\begin{array}{lll}9.5 & 10.5\end{array}$

5.2

Apples, A. P.

0.3358 .0

Apples, dried

$\begin{array}{lll}1.3 & 78.5\end{array}$

1.6

Apple juice

$0.5 \quad 314.3$

1.6

0.5

0.592

(8)

Apricots, A. P.

.049

$0.6 \quad 180.0$

1.6

Apricots, dried

$\begin{array}{lrrr}2.6 & 38.0 & 13.2\end{array}$

Asparagus .404

Bananas

$\begin{array}{llll}1.0 & 100.6 & 17.1\end{array}$

$\begin{array}{lll}0.4 & 280.0 & 1.2\end{array}$

Barley, entire

055

Barley, pearled

$\begin{array}{lll}1.7 & 58.5 & 3.0\end{array}$

$\begin{array}{llll}0.8 & 126.2 & 1.1\end{array}$

Beans, dried

122

$\begin{array}{lll}6.4 & 15.7 & 6.5\end{array}$

Beans, lima, fresh ....................171

$\begin{array}{lll}2.8 & 35.4 & 2.8\end{array}$

$\begin{array}{lll}1.1 & 90.0 & 1.1\end{array}$

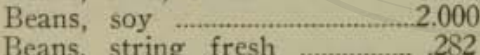

Beans, string fresh ……...........
Beef, tenderloin ……..............

$\begin{array}{lll}13.0 & 7.7 & 11.2\end{array}$

$\begin{array}{lll}1.8 & 54.6 & 15.4\end{array}$

$\begin{array}{lll}0.4 & 270.0 & 0.5\end{array}$

Beets

.178

Blackberries ………....................... 104

Blueberries ………………........... .122

Bran, wheat ………………….... .525

Bread, Boston brown ……...... .790

Bread, entire wheat …………... . 133

Bread, graham ……........................ 176

Bread, rye

1.286 .5

0.7150 .0

0.8126 .2

$\begin{array}{lll}3.5 & 52.5 & 10.2\end{array}$

$\begin{array}{lll}5.1 & 19.5 & 7.9\end{array}$

0.9116 .0

$\begin{array}{lll}5.6 & 5.0 & 67\end{array}$

.73

$\begin{array}{llll}1.0 & 1.2 & 100 & .23\end{array}$

$\begin{array}{llll}1.0 & 1.2 & 100 & .23 \\ 6.0 & 6.0 & 100 & .29\end{array}$

$\begin{array}{llll}6.1 & 3.0 & 46 & .26\end{array}$

$\begin{array}{llll}1.1 & 2.0 & 157 & .81\end{array}$

$\begin{array}{llll}16.1 & 3.0 & 19 & .46\end{array}$

$\begin{array}{llll}3.5 & 3.5 & 100 & .19\end{array}$

$\begin{array}{llll}1.1 & 1.0 & 90 & .26\end{array}$

$\begin{array}{llll}1.0 & 1.0 & 100 & .12\end{array}$

$\begin{array}{llll}1.0 & 1.0 & 97 & .98\end{array}$

$\begin{array}{llll}1.0 & 1.0 & 99 & .43\end{array}$

$\begin{array}{llll}3.0 & 4.0 & 92 & .68\end{array}$

$\begin{array}{llll}0.9 & 0.9 & 112 & 1.80\end{array}$

$\begin{array}{llll}8.4 & 4.0 & 44 & 1.13\end{array}$

Bread, entire rye (pumper-

nickel)

.147

$\begin{array}{lll}1.2 & 90.0 & 2.8\end{array}$

$\begin{array}{lll}1.3 & 2.5 & 200\end{array}$

1.13
.14

$\begin{array}{llll}9.1 & 2.2 & 29 & .39\end{array}$

Bread, white …………………..... 091

Bread, fruit ㄷ………...... .514

1.0105 .0

$\begin{array}{llll}8.0 & 3.0 & 50 & .31\end{array}$

$\begin{array}{llll}4.8 & 2.5 & 52 & .30\end{array}$

$\begin{array}{llll}1.5 & 0.5 & 35 & .37\end{array}$

$\begin{array}{llll}1.2 & 1.2 & 100 & .95 \\ 1.4 & 2.0 & 139 & .27\end{array}$

$\begin{array}{llllll}.0 & 1.3 & 1.4 & 2.0 & 144 & .29\end{array}$

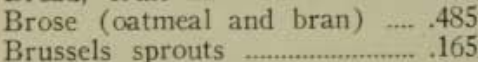

$\begin{array}{lll}1.7 & 60.0 & 2\end{array}$

$0.6 \quad 170.0$

$\begin{array}{ll}3.3 & 30.0\end{array}$

$\begin{array}{ll}3.1 & 32.0\end{array}$

1.193 .3

.239

1.664 .4

(The letters A. P. stand for "as purchased.") 


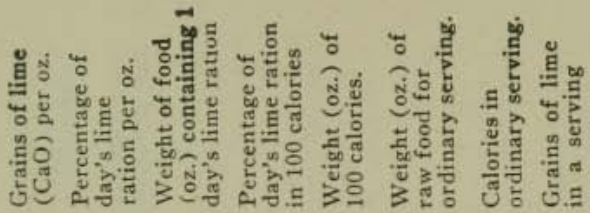

Butter

(1)

(2)

(3)

(4)

(5)

(6)

(7)

(8)

Buttermilk

.092

$\begin{array}{llll}0.6 & 167.4 & 0.3 & 0.5\end{array}$

0.5

$109 \quad .05$

Buttermilk, yogurt …………...........

Cabbage

$\begin{array}{llll}4.2 & 24.0 & 41.1 & 10.0\end{array}$

$\begin{array}{lll}6.0 & 60 & 3.85\end{array}$

Cabbage greens .276

Cantaloupe ………………........ 104

Carrots

$\begin{array}{rrrr}4.8 & 20.6 & 46.3 & 9.6 \\ 1.8 & 56.0 & 20.0 & 11 .\end{array}$

$\begin{array}{lll}6.5 & 75 & 4.85\end{array}$

$\begin{array}{lll}4.2 & 23.7\end{array}$

$47.3 \quad 5.4$

4.0

$\begin{array}{llll}0.7 & 150.0 & 6.1 & 9.0\end{array}$

$4.0 \quad 25 \quad 2.00$

Cauliflower $\quad .753$

Celery ㄴ.._-

Chard

$\begin{array}{lll}2.2 & 44.9 & 17.3\end{array}$

7.8

7.0

$\begin{array}{ll}4.9 & 20.4\end{array}$

56.411 .6

$\begin{array}{llll}3.1 & 32.2 & 58.9 & 19.1\end{array}$

3.5

$\begin{array}{ll}6.0 & 16.7\end{array}$

Cheese

.919

Cheese, cottage …………............. 4.180

Cheese, yogurt ……….............285

Cherries, A. P. 116

Cherry juice

.104

Chestnuts

\begin{tabular}{ll}
37.0 & 2.7 \\
\hline
\end{tabular}

$\begin{array}{ll}55.0 & 9.2\end{array}$

3.0

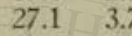

$27.8 \quad 3.6$

$29.7 \quad 0.8$

3.0

0.8133 .0

85.2

3.2

0.5

$0.7 \quad 150.0$

26.0

0.9
4.7

2.0

$1.3 \quad 75.0$

$3.1 \quad 18.1$

0.5
2.2

Chicken, broiler

.066

Coconut, dry _........................................

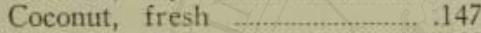

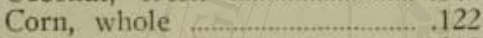

Cornmeal …...................................

Corn, sweet, fresh …………... .037

Corn, sweet, dry _......................... 129

Cow peas

.612

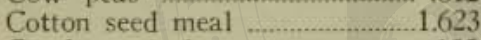

Crackers, soda

.135

Cranberries ………_...................... 110

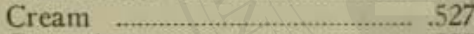

Cucumbers …_.......................

Currants, fresh …………............ 159

Currants, dry, Zante …............502

Dandelion greens …..................643

Dates, dried, A. P. ..._.......... .398

Dock, narrow leaved …..........1.00

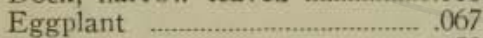

Eggs

Egg, one $(1.8$ oz.) …............... .810

Egg white …………………..............

Egg white, one $(1.2 \mathrm{oz}.) \quad \ldots \ldots . . .116$

Egg yolk ...............................................

Egg yolk, one $(.6 \mathrm{oz}.) \quad \ldots \ldots \ldots \ldots . . .503$

Endive …………........................ 637

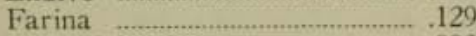

Figs, dried ………………....... 992

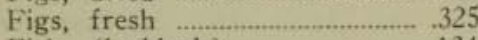

Fish, (haddock) ………………...... 134

Flour, buckwheat ….................... .061

Flour, entire wheat ……............ 190

Flour, graham …….................... 239

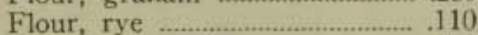

Flour, rye, entire ………….... 293

Flour, white ……………….........122

0.4235 .0

$\begin{array}{lll}1.9 & 1.4\end{array}$

4.5

$2.3 \quad 42.6$

$1.4 \quad 3.3$

$1.0 \quad 105.0$

2.2

0.8126 .2

0.7140 .0

$0.9 \quad 0.9$

$\begin{array}{llll}0.9 & 1.0 & 167 & .15\end{array}$

0.2416 .0

0.8

$0.8 \quad 114.4$

$\begin{array}{ll}4.0 & 25.1\end{array}$

$10.5 \quad 9.5$

0.9114 .0

$0.7 \quad 140.0$

$\begin{array}{lll}3.4 & 29.2\end{array}$

0.7

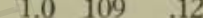

0.6157 .0

0.8

$\begin{array}{llll}1.0 & 1.0 & 100 & .11\end{array}$

$1.0 \quad 96.8$

4.1

$\begin{array}{lll}3.5 & 3.0 & 86\end{array}$

$\begin{array}{llll}1.0 & 1.0 & 109 & .13\end{array}$

9.2

1.0

$0.8 \quad 0.9$

$7.2 \quad 1.8$

$\begin{array}{lll}1.0 & 110 & .14\end{array}$

$\begin{array}{lll}3.3 & 30.7\end{array}$

$\begin{array}{rrrrr}12.6 & 20.0 & 2.0 & 10 & .20\end{array}$

$\begin{array}{lll}3.0 & 40 & .33\end{array}$

$\begin{array}{llllll}3.6 & 1.0 & 1.5 & 135 & .48\end{array}$

$\begin{array}{lllllll}24.0 & 24.1 & 5.8 & 3.0 & 50 & 1.93\end{array}$

$\begin{array}{lllllll}2.6 & 38.6 & 2.6 & 1.0 & 2.0 & 177 & .79\end{array}$

$\begin{array}{ll}6.7 & 15.0\end{array}$

0.4230 .0

$\begin{array}{ll}2.9 & 34.2\end{array}$

$5.2 \quad 19.0$

$0.6 \quad 167.4$

0.7140 .0

$\begin{array}{ll}5.4 & 18.3\end{array}$

$3.3 \quad 30.6$

$\begin{array}{ll}4.1 & 24.0\end{array}$

0.8119 .4

6.415 .6

$\begin{array}{ll}2.1 & 47.4\end{array}$

8.7114 .6

0.5252 .5

1.281 .0

$\begin{array}{ll}1.6 & 64.4\end{array}$

$0.7 \quad 140.0$

$1.9 \quad 52.5$

0.8136 .2

Gluten, pure

.1 .025

$\begin{array}{rrrrr}5.7 & 12.5 & 4.0 & \cdots \ldots . & 4.00 \\ 6.3 & 2.4 & 1.5 & 12 & .10\end{array}$

$\begin{array}{lllll}6.3 & 2.4 & 1.8 & 70 & .81\end{array}$

$\begin{array}{lllll}\ldots . . & \cdots . . & 1.8 & 70 & .81\end{array}$

$\begin{array}{lllll}2.8 & 4.7 & 1.2 & 14 & .11\end{array}$

$\begin{array}{lllll}5.0 & 0.9 & 1.2 & 17 & .11\end{array}$

$\begin{array}{lllll}5.0 & 0.9 & 1.9 & 109 & 1.59\end{array}$

$\begin{array}{llllll}45.5 & 20.9 & 0.6 & 60 & .50\end{array}$

$\begin{array}{rrrrr}0.8 & 1.9 & 3.0 & 14 & 1.91\end{array}$

$\begin{array}{lllll}21.6 & 1.1 & 2.0 & 168 & 1.98\end{array}$

$\begin{array}{lllll}7.2 & 4.3 & 2.0 & 43 & .65\end{array}$

$\begin{array}{lllll}2.6 & 3.0 & 6.0 & 122 & .80\end{array}$

$\begin{array}{lllllll}6.6 & 15.0 & 6.6 & 1.0 & 0.5 & 62 & .51\end{array}$ 


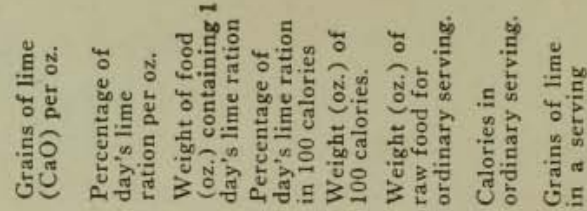

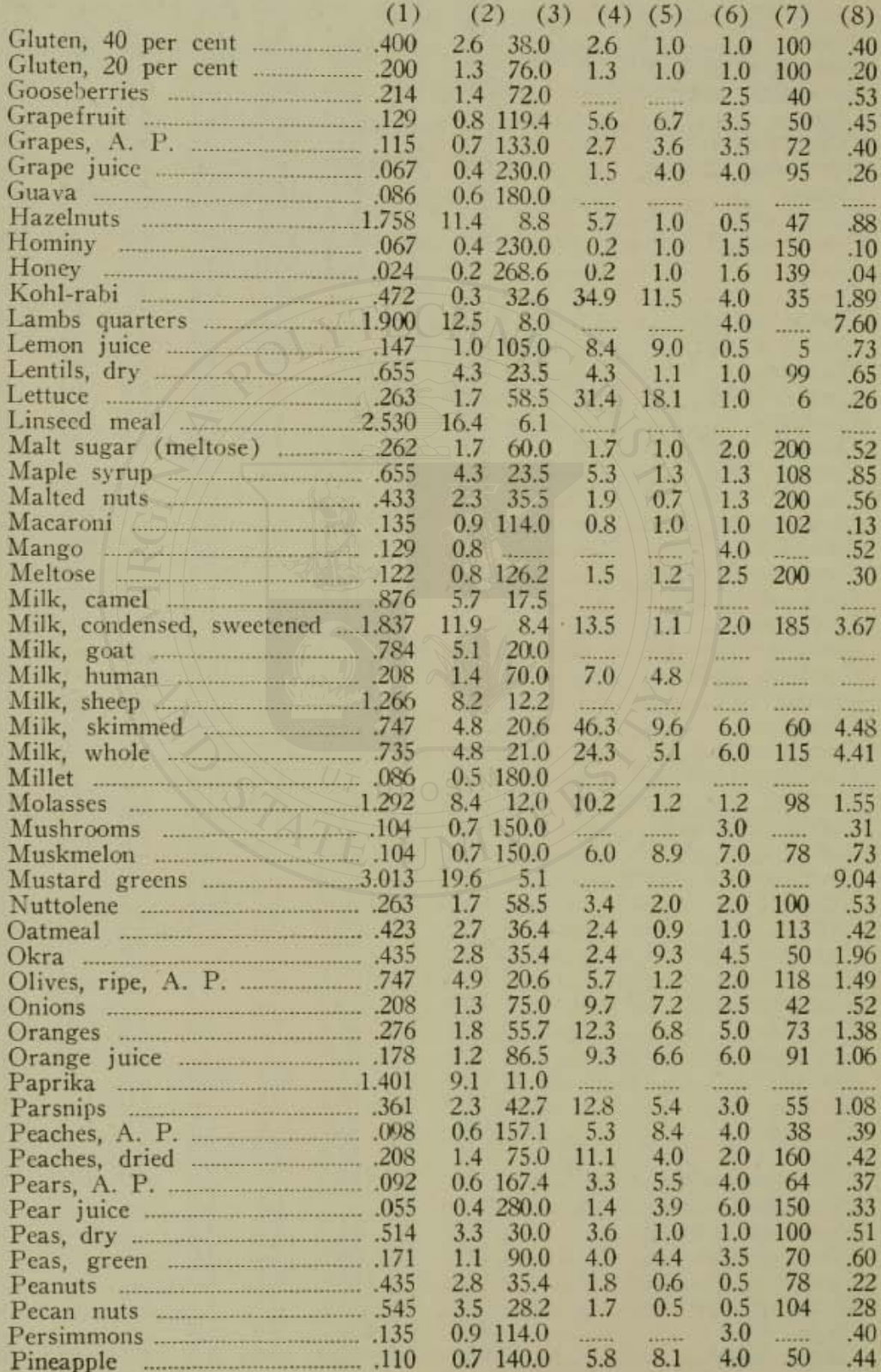




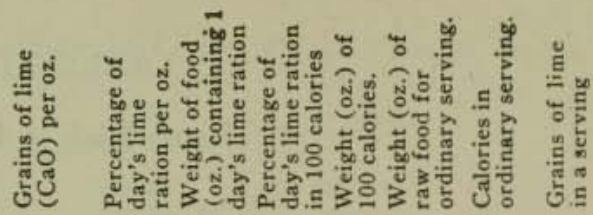

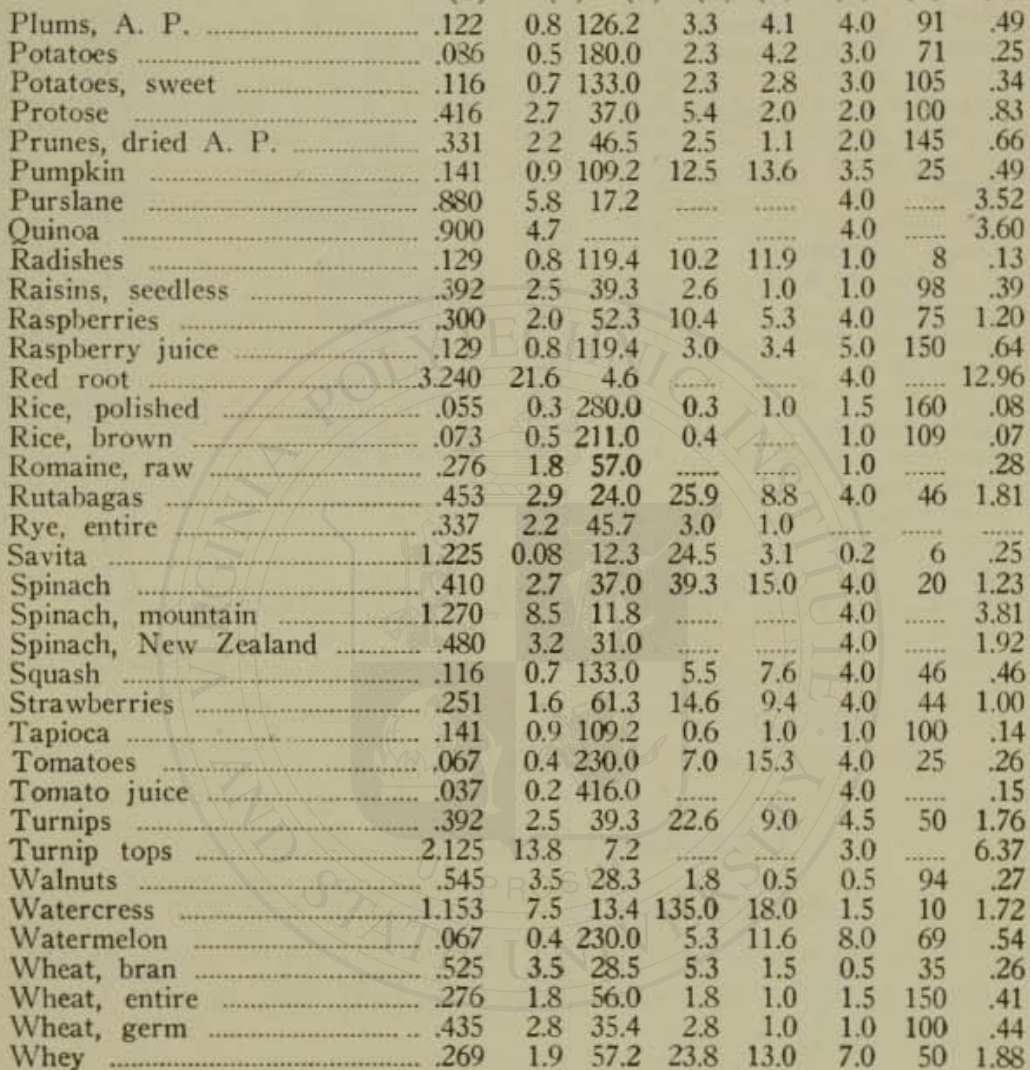




\section{TABLE XIV}

\section{Foods Rich in Lime.}

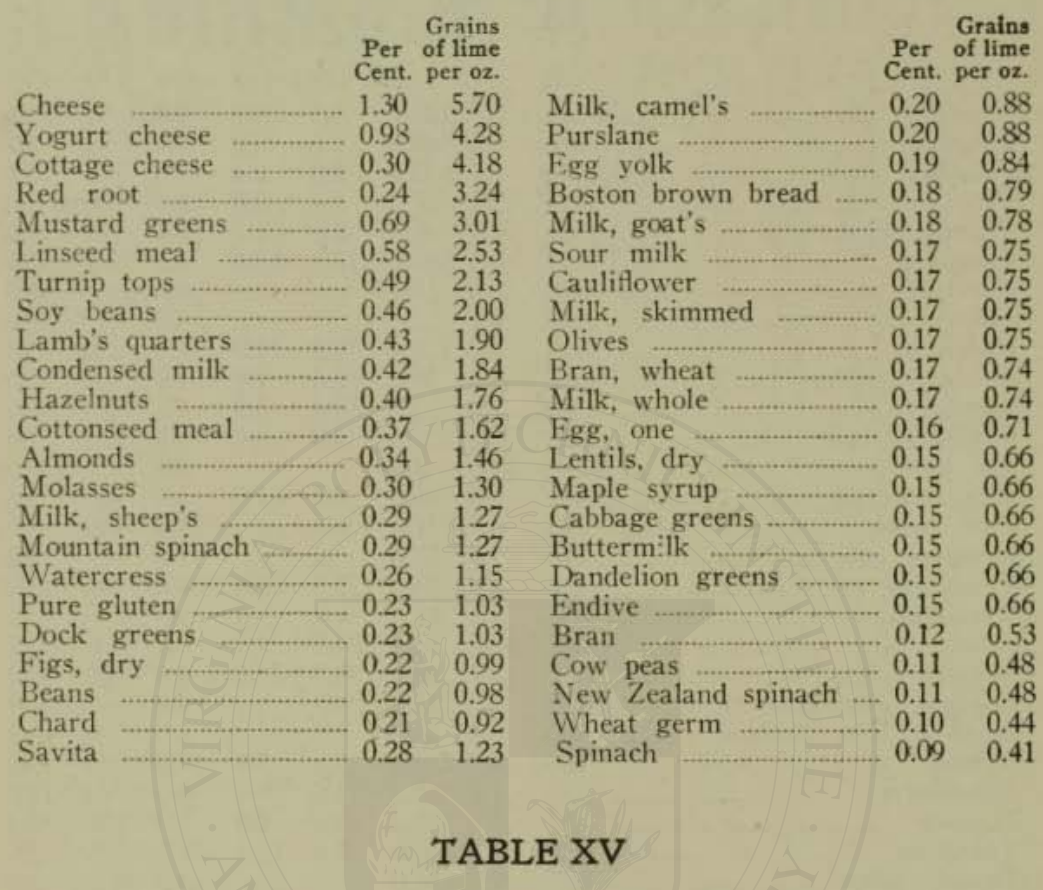

\section{Foods Rich in Both Lime and Iron.}

The following foods rich in both lime and iron should be made conspicuous features of the daily bills of fare; by their liberal use, any serious deficiency in food lime or iron may be very certainly avoided:

$\begin{array}{ll}\text { Almonds } & \text { Endive } \\ \text { Boston brown bread } & \text { Figs, dry } \\ \text { Bran } & \text { Gluten, pure } \\ \text { Chard } & \text { Hazelnuts } \\ \text { Dandelion greens } & \text { Lentils } \\ \text { Dock greens } & \text { Maple syrup } \\ \text { Egg yolk } & \end{array}$

Molasses
Mustard greens
Olives
Red root
Savita
Spinach
Turnip tops




\section{Foods Poor in Iron Foods Poor in Lime}

Bread, white

Candy

Carrots

Cheese

Corn flakes

Corn meal

Corn syrup

Corn starch

Crackers, white

Cream

Egg white

Fats and oils

Fish

Hominy

Macaroni

Milk

Potatoes

Rice

Rice flakes

Sugar

Tomatoes

Turnips

\section{Foods Poor in Both} Lime and Iron

Beef

Bread, white

Candy

Chicken

Corn flakes

Corn meal

Crackers, white

Fats and oils

Fish

Fruit

Hominy

Macaroni

Mutton

Rice

Rice flakes

Sugar, white
Bread, white

Candy

Corn flakes

Corn meal

Corn syrup

Fats and oils

Fish

Hominy

Rice

Rice flakes

Sugar
Crackers, white

\section{Acids and Bases of Foods-The Acid Alkaline Balance}

As pointed out by Bunge many years ago, and as further shown by an extensive research conducted by Sherman, of Columbia University, foodstuffs differ in relation to their acid and basic contents. In certain foods, bases or alkalies predominate, while in others the mineral acids predominate.

The waste products of the body are predominantly acid; consequently it is desirable that the foodstuffs should be predominantly basic. If acids predominate in the ration, and this condition continues for a considerable period of time, the effect will be to disturb the nice balance between acids and bases which is normally maintained in the body fluids and which is essential for life. A deficiency of alkalies constitutes acidosis.

\section{The Chemical Balance}

It is necessary that the alkalinity of the blood should be constantly maintained as the only means by which the acid wastes of the body which are constantly accumulating as the result of all sorts of vital work may be promptly removed. The actual degree of alkalinity of the blood is very slight, so slight in fact that a diminution amounting to so great a change as the difference 
between ordinary hydrant water and distilled water would result in instant death.

The influence of foodstuffs upon the alkalinity of the blood depends entirely upon the residues which they leave behind after having undergone oxidation, or so-called wet combustion, in the body. Certain foods leave alkaline, or basic, residues and help to maintain the alkalinity of the blood, while others leave acid residues which tend to lower the blood alkalinity. All flesh foods leave highly acid residues, and lessen the alkali reserve of the blood and tissue fluids to a marked degree. Eggs tend in the same direction, though less strongly than do meats. Meat extracts, broths, bouillon, meat stocks and gravies, all belong in the group of acidifying foods.

Cereals of all sorts, including breads of, every description, contain a preponderance of acids, though much less than that of meats.

A few fruits, particularly cranberries, plums and prunes, must also be placed in this class because of the presence of benzoic acid, which is not oxidized in the body.

All other fruits, green vegetables and root vegetables of all sorts, are strongly basic and tend to alkalinize the tissue fluids.

Milk is slightly alkaline.

The accompanying table shows the acid and basic properties of most common foodstuffs. In using the table to regulate the chemical balance it is only necessary to multiply the figures found under "ounce" by the number of ounces in a serving of each of the foods eaten. The sums of these quantities for acids and bases will show the number of units of each sort in the bill of fare. If the two are equal, the meal is exactly balanced for acids and bases. If one predominates, the difference represents the excess of acid or base as the case may be. Acids should never be in excess. In general it is decidedly better to select foods which afford a large basic margin so as to add to the alkali reserve of the body, which always tends toward diminution, especially in old age.

A strongly basic diet is especially indicated in cases of arteriosclerosis and disease of the kidneys, in fevers, in pregnant women, in all forms of acidosis, and in old age.

It is evidently necessary that persons who have a tendency to acidosis should avoid meats and eggs and make large use of those foodstuffs which furnish strongly basic residues. The accompanying tables show the pronounced difference in the character of the residues left in the body by different foodstuffs. 
A glance at the tables will show at once the great value of fruits and vegetables as alkalinizers of the blood and tissue fluids and the great harm which must necessarily result from a meat and cereal diet. Indeed, it is apparent that a too exclusively cereal diet, even, may prove harmful. It is to be understood, of course, especially in the case of cereals, that an excess of acid in certain foods, may be offset by taking at the same meal a sufficient amount of foods rich in alkali.

In the marked tendency of meats to acidify the blood and tissue fluids is found a strong biologic objection to the free use of flesh foods in the dietary. A full appreciation of the influence of flesh foods upon nutrition and the risks attending their use in general will lead to their elimination from the diet altogether. The strenuous efforts being made recently by the great packing companies to increase the consumption of meat by an "Eat-MoreMeat Campaign," although professing an altruistic interest in the physical welfare of the American people, is purely commercial in its aims and must be regarded as an unscrupulous attempt to exploit the ignorance of the public in matters pertaining to human nutrition. Fortunately, the effort thus far has shown no marked evidence of success and it is gratifying to note that it has not received support and encouragement from any physiologist of established standing.

At the present time all physiologists are agreed that the highest degree of physical vigor and efficiency may be maintained on a diet which excludes flesh foods and physiologists the world over are unanimous in advising a curtailment rather than an increase in meat consumption. Sherman quotes with approval the statement of Tigerstedt that the extractive substances found in meat may produce disorders of one kind or another in the body. "The metabolism might also take an abnormal or unfavorable form if the fluids of the body were flooded with too much protein," and Professor Sherman adds, "This moderate and conservative warning against too free a use of meat as food was written, it is important to remember, in a Scandinavian textbook, and was therefore addressed to those whose average rate of meat consumption was less than half as much as ours."

The experience of the war taught the American people that meat can be very largely or even wholly dispensed with not only without injury but with profit and this important lesson learned is not likely to be forgotten, notwithstanding the efforts being made by certain commercial interests to deceive the public by the publication of misleading and deceptive statements. 


\section{TABLE XVI}

Excess of Acid or Alkali in one Ounce and in 100 Calorie Portions of Common Foodstuffs.

\begin{tabular}{|c|c|c|c|c|}
\hline & Acids & & & \\
\hline Almonds & 100 Calories & Ounce & 100 Calories & Ounce \\
\hline Apples, fresh & & & 1.8 & 3.3 \\
\hline Apricots, fresh & & & $\begin{array}{r}0.0 \\
11.0\end{array}$ & $\begin{array}{l}1.0 \\
1.8\end{array}$ \\
\hline Asparagus & & & 3.6 & 0.2 \\
\hline 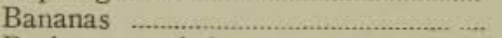 & & & 5.6 & 1.5 \\
\hline 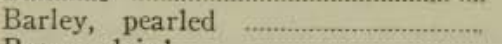 & 2.9 & 2.9 & & \\
\hline 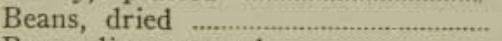 & & & 5.0 & 5.0 \\
\hline Beans, lima, canned & & & 12.0 & 2.7 \\
\hline Beans, lima, dried & & & 12.0 & 12.0 \\
\hline Beans, lima, fresh & & & 11.6 & 4.0 \\
\hline 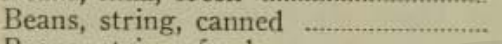 & & & 13.0 & \\
\hline Beans, string, fresh & & & 12.9 & 1.5 \\
\hline Beefsteak, porterhouse & 4.0 & 3.0 & & \\
\hline Beefsteak, round, lean & 6.8 & 3.0 & & \\
\hline 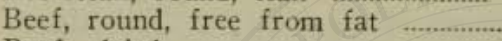 & 10.0 & 3.3 & & \\
\hline 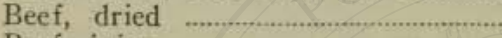 & 8.3 & 4.1 & & \\
\hline 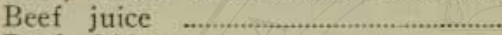 & 9.8 & 0.7 & & \\
\hline Beef, kidney & 7.4 & 2.4 & & \\
\hline Beef, liver & 7.9 & 2.9 & & \\
\hline Beets …......... & & & 23.6 & 3.0 \\
\hline Bread, white & 2.7 & 2.0 & & \\
\hline Bread, whole wheat ....... & 3.0 & 2.1 & & \\
\hline Buckwheat flour .................. & 2.0 & 2.0 & & \\
\hline Buttermilk & & & 6.1 & \\
\hline Cabbage ..... & & & 18.0 & 1.6 \\
\hline Carrots & & & 23.9 & 3.0 \\
\hline Cauliflower & & & 17.5 & 1. \\
\hline Celery & & & 42.1 & 2. \\
\hline - & & & 41.1 & \\
\hline Cheese, cheddar & 1.2 & 1.5 & & \\
\hline Cherries, fresh & & & 7.8 & \\
\hline Chestnuts, dried & & & 3.2 & 3. \\
\hline Chestnuts, fresh & & & 3.1 & 2 \\
\hline Chicken & 10.0 & 3.0 & & \\
\hline $\begin{array}{l}\text { Coconuts } \\
\text { Codfish, salted }\end{array}$ & 12.0 & & 1.2 & \\
\hline $\begin{array}{l}\text { Codfish, salted } \\
\text { Corn, green }\end{array}$ & 1.8 & 0.5 & & \\
\hline 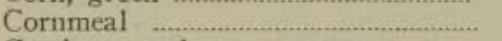 & 1.5 & 1.5 & & \\
\hline Crackers, soda & 2.0 & 2.5 & & \\
\hline eerries $(*)$ & & & 3.7 & \\
\hline & & & 0.3 & 0. \\
\hline - & & & 45.5 & 2.2 \\
\hline zante & & & 1.8 & 1. \\
\hline - & & & 3.2 & 3. \\
\hline Eggs, whole & 7.5 & 3.1 & & \\
\hline - & 9.5 & 1.4 & & \\
\hline Egg yolk & 7.0 & 7.0 & & \\
\hline Figs, dried & & & 32.3 & 29.4 \\
\hline Flour, entire wheat & 3.3 & 3.3 & & \\
\hline Flour, wheat, patent ….................... & $\begin{array}{r}2.7 \\
10.8\end{array}$ & $\begin{array}{l}2.7 \\
2.7\end{array}$ & & \\
\hline - & & & 8.0 & 8.1 \\
\hline & & & 2.8 & \\
\hline - & & & 4.0 & \\
\hline & 12.0 & 2. & & \\
\hline & & $27.7-3=0$ & & \\
\hline
\end{tabular}


Ham, lean

100 Calories

Lamb

Lemons

Lemon juice

Lentils

Lettuce

Milk, condensed, sweetened

Milk, condensed, unsweetened

Milk, skimmed

Milk, whole

Molasses

Mushrooms

Muskme:ons

Oatmeal

Olives

Onions

Oranges

Orange juice

Oysters

Parsnips

Peaches, canned

Peaches, fresh

Peanuts

Pears, fresh

Peas, canned

Peas, dried

Peas, green

Pineapple

Plums (*)

Pork

Pork, sausage

Pork, tenderloin

Potatoes

Potato chips

Potatoes, sweet

Prunes, dried (*)

Pumpkins

Radishes

Raisins

Raspberry juice

Rhubarb (*)

Rice

Rutabagas

Salmon

Sausage, bologna ……………………...

Sardines

Shredded wheat

Spinach

Squash

Soy bean

Tomatoes, fresh

Trout

Turkey

Turnips

Veal

Walnuts, California

Watermelons

Wheat, cracked (Branola)

White fish
5.5

6.3

3.3

2.7

Oz. $\begin{gathered}\text { Base (Alkali) } \\ 100 \text { Calories Oz. }\end{gathered}$

3.7

5.2

2.7

12.3

10.7

$1.5 \quad 1.5$

$38.6 \quad 2.1$

$1.4 \quad 1.2$

$2.7 \quad 2.3$

$\begin{array}{ll}5.0 & 0.5\end{array}$

$2.6 \quad 0.5$

$20.8 \quad 1.7$

$9.0 \quad 1.1$

$18.8 \quad 2.1$

3.0

3.3

$18.9 \quad 16.0$

$3.1 \quad 0.4$

$10.9 \quad 1.6$

$\begin{array}{ll}14.3 & 1.7\end{array}$

$30.0 \bigcirc 4.3$

$18.1 \quad 3.3$

$10.0 \quad 1.3$

$12.2 \quad 1.4$

$0.7 \quad 0.1$

$5.6 \quad 1.0$

$\begin{array}{ll}1.5 & 0.2\end{array}$

$1.4 \quad 1.4$

$1.3 \quad 0.4$

$\begin{array}{ll}15.7 & 1.9\end{array}$

$\begin{array}{ll}7.3 & 1.8\end{array}$

2.8

$13.0 \quad 1.4$

$37.4 \quad 2.5$

$29.8 \quad 3.5$

$5.4 \quad 3.2$

$4.0 \quad 2.6$

$4.2 \quad 3.2$

$3.3 \quad 3.3$

$113.0 \quad 7.6$

$\begin{array}{ll}6.1 & 0.8\end{array}$

$12.0 \quad 12.0$

$\begin{array}{ll}5.4 & 2.6\end{array}$

$3.6 \quad 3.0$

$6.7 \quad 3.0$

$1.1 \quad 2.2$

$3.3 \quad 3.3$

$\begin{array}{ll}7.7 & 3.3\end{array}$ 


\section{Vitamins}

These subtle elements, which have been in recent years proven to be absolutely essential to life and health, are present in food in such small quantities that notwithstanding the prodigious amount of work which has been bestowed upon them their chemical composition is not yet known. The proof of the existence of this class of food principles and their importance to human life rests upon numerous observations which have shown that in their absence various disorders known as deficiency diseases make their appearance.

Vitamin B appears to be an essential constituent of nerve tissue. Funk found vitamin $B$ in ox brain and other investigators have found it in the spinal cord.

When vitamin B is not present in the food in sufficient amount, the vitamin constituent of the nerves is gradually lost and degeneration of the nerve tissue with paralysis follows as the result. Several weeks and even months may be required for the consumption of the store of vitamins in the nerve tissues of the body, but a study of animals subjected to a dietary deficient in vitamins has shown that degenerative changes in the nervous system begin early, as may be demonstrated by miscroscopic examination.

Vitamins are necessary to promote growth. In their absence young animals do not develop, reproduction does not take place, mothers are not able to nurse their young, appetite and nutrition and various diseases develop, among the best known of which are scurvy, beri-beri, rickets, perhaps pellagra, and certain diseases of the eye.

Even malignant growths are influenced by the lack of vitamins. Short asserts that cancer of rats has been shown to grow only one-fourth as rapidly when vitamins are absent.

Ehrlich in experiments upon rats demonstrated that by means of an exclusive diet of rice cancer could be made to cease growth and sometimes nearly to disappear. He remarked to the writer, who visited his laboratory when the experiments were in progress, that it was "necessary to starve the rats nearly to death." Evidently they were suffering from lack of vitamins. 
Vitamins are produced only by vegetables. In order to insure a full supply of all the different vitamins it is important that the dietary should be varied. Infants and invalids are the most likely to suffer from lack of vitamins because they are often fed for long periods upon food in which there is very little variety.

Five distinct vitamins are now recognized and have been carefully studied. They are designated by the letters of the alphabet, $A, B, C, D$, and $E$. The newly discovered vitam ns $D$ and $E$ have not yet been sufficiently studied to fully determine their distribution in foodstuffs.

Dr. Robert McCarrison, an eminent English military surgeon, who has made an extensive study of deficiency diseases, has thus summarized our present knowledge of vitamins $A, B$ and $C$ :

"(1) Vitamins are constant constituents of living tissues. Although present in very small amounts, maintenance of health is dependent on their action.

"(2) Vitamins do not themselves contribute to the energy : upply of the body, but facilitate utilization by it of proteins, fats, carbohydrates and salts of food.

“(3) Proteins, fats, carbohydrates and salts cannot support life without vitamins, nor vitamins without these proximate principles; they are complementary to each other. Without vitamins, the body starves.

"(4) A distinct relationship exists between the amount of vitamin required and the balarce of food in protein, fat, carbohydrate and salt, the efficacy of the vitamin depending on the composition of the food mixture.

“(5) A distinct relation exists between the amount of vitatnin required and the rate of metabolic process.

“(6) Each vitamin plays a specific part in nutrition.

"(7) It appears that vitamin A is associated with the metabolism of liquids and calcium, as well as with chemical reactions requicite for growth and maintenance.

“(8) Vitamin B appears to be associated with the metabolism of carbohydrates and with the chemical reactions and functional perfection of all cells, particularly nerve cells.

"(9) Vitamin C appears to be associated with the metabolism of calcium and with the chemical reactions of growing tissues.

"(10) All vitamins are concerned in the maintenance of orderly balance between destructive and constructive cellular processes. 
"(11) One vitamin cannot replace another, although its function may be interfered with by the absence of another.

"(12) The final result of their efficiency is the same, whatever be the degree of deprivation. The greater the deprivation the more rapid is the onset of symptoms due to it; the lesser the deprivation the slower is the onset of the symptoms due to it.

"(13) Each vitamin exercises a specific influence on the adrenal glands; the effect of their deprivation on these organs is one of the most outstanding features of deficiency diseases.

"(14) Vitamins influence markedly the production of hormones and all external secretions.

"(15) There is reason to believe that the capacity of any given cell for work is impaired in proportion to the degree of vitamin starvation.

"(16) Vitamins aid the tissues in resisting infection.

“(17) Vitamins, especially vitamin B, induce in the human and animal body a desire for food.

"(18) Vitamins are one link in the chain of essential substances requisite for harmonious regulation of chemical processes of healthy cellular action. If the link be broken, harmony ceases or becomes discord as it may cease or become discord if any other link be broken.

"(19) The place of vitamins in human economy must be considered in connection with metabolism as a whole, in connection with their relation to other essential food requisites, with their relation to organs of digestion and assimilation, and with their relation to endocrine regulators of metabolic processes.

"Vitamins are the spark which ignites the fuel mixture of a petrol-driven engine, liberating its energy; a spark is of no use without fuel, nor fuel without spark-nay, more, the efficacy of the spark is dependent in a great measure on the composition of the fuel mixture.

"What happens (when the body goes sick in consequence of deficient foods, usually ill-balanced) is this-in the absence of vitamins or in an inadequate supply, protein, fat, carbohydrate and salts are not properly utilized; some are largely wasted, others yield products harmful to the organism. In these circumstances, life may be sustained for a longer or shorter period, during which the body utilizes its reserve stores of vitamins and sacrifices its less important tissues to this end. But there is a limit beyond which such stores cannot be drawn upon, and once reached, the 


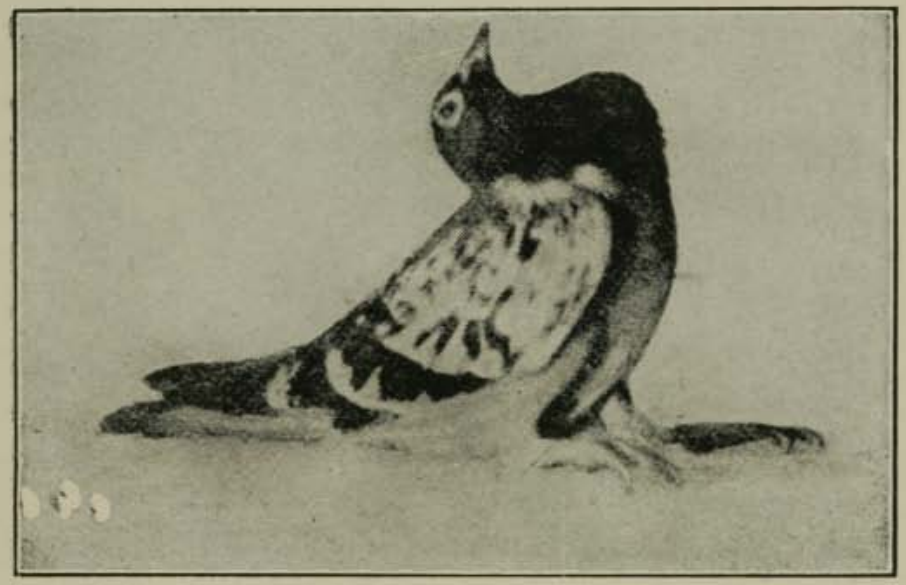

A Bird Nearly Dead from Lack of Vitamins

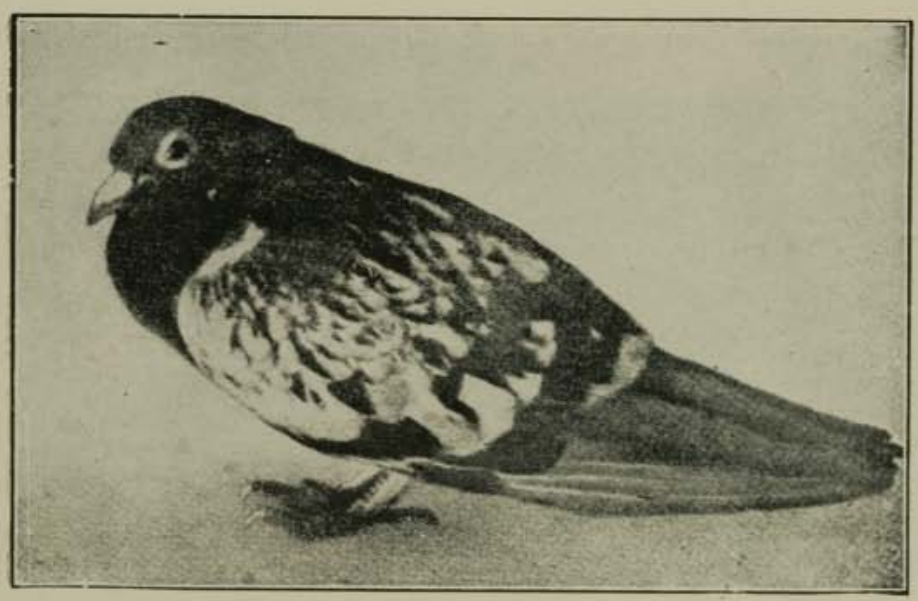

The Same Bird a Few Hours after the Injection of the Needed Vitamins (Funk) 


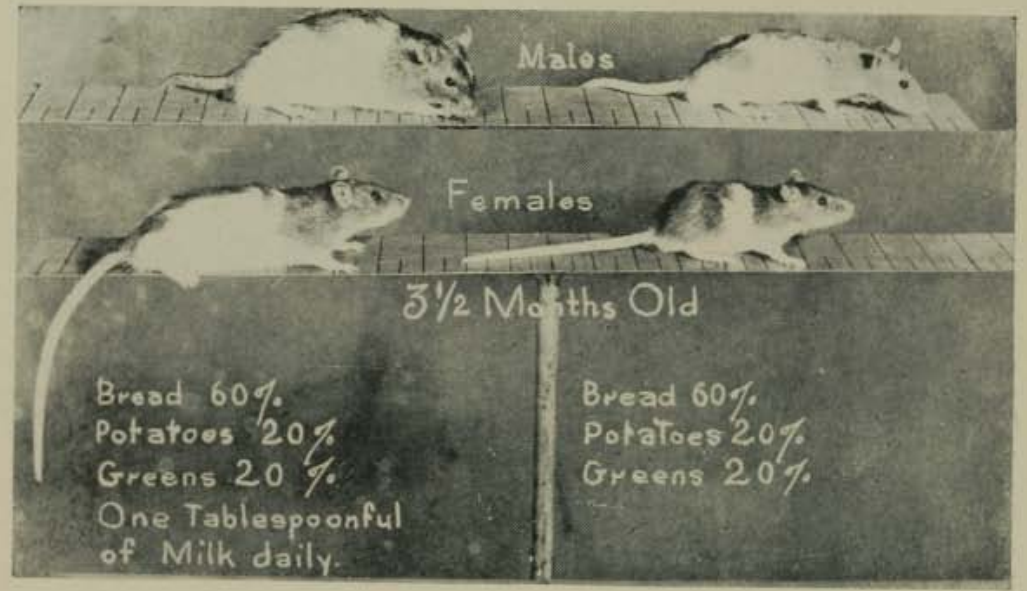

Experiments Showing Deficiency of Lime and Vitamins (Nutrition Laboratory of the Batte Creek Sanitarium.)

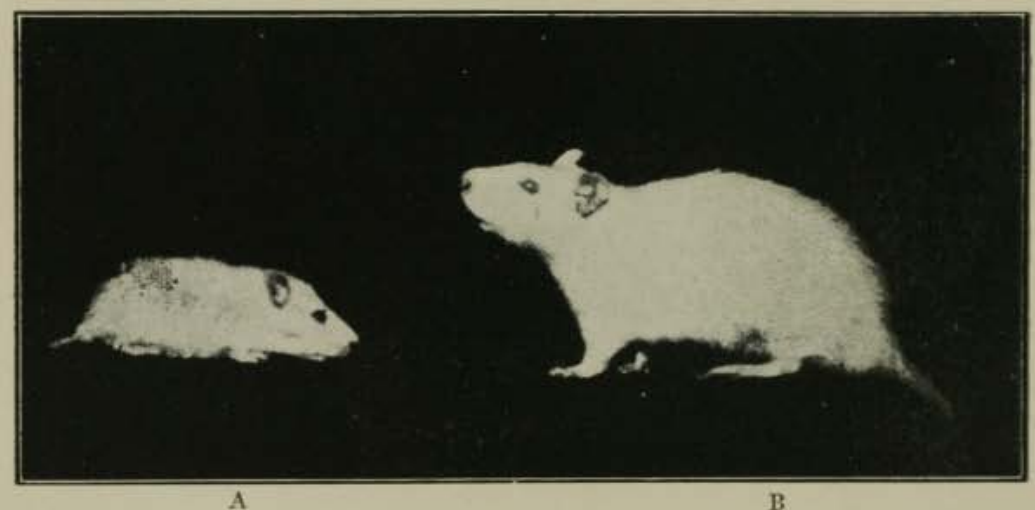

Rats of the same age (12 weeks). The smaller (A) was fed bread and apple, all it would eat, (B) was given bread and milk and weighed five times as much as A. (Sherman.) 
cells of higher function-secretory, endocrine and nerve cellsbegin to lack vigor and depreciate in functional capacity, although the tissues may still hold considerable stores of vitamin. The disintegration process is delayed or hastened, lessened in severity in one direction or increased in another, according as the food constituents are well or ill balanced and according to the character of lack of balance.

\section{What Lack of Vitamin Does.}

"The lack of vitamin disturbs the calcium metabolism; puts an end to regenerative processes; involves with respect to the cells of higher function, the functional depression of many, death and failure of a few. The cardinal effect is depreciation of cellular function, and this depreciation is the foundation upon which disease is built. Extreme deprivation means rapid dissolution and death; partial deprivation means slow dissolution and disease.

"This conception of the function of vitamins holds out wide promise in the cure of disease, due to or favored by their deficiency in food; for though they cannot restore to life cells already dead, they can restore to normal depressed functional capacity the general mass of the body's cells. The conception that vitamins provide the cells of the body with the power-one might almost say will-to work, has this great merit, that it furnishes a working hypothesis on which to frame treatment."

\section{The Antiscorbutic Vitamin-Water Soluble C.}

The absence or deficiency of vitamin $C$ is now known to be the cause of scurvy, a disease which has been recognized from ancient times, but the cause of which was unknown until the discovery of vitamins by modern scientific research. Experience has shown the necessity for fresh, uncooked foods to prevent outbreaks of scurvy among sailors and soldiers and other large bodies of men fed on rations, as well as bottle-fed infants, but the existence of a subtle antiscorbutic substance was not known.

The antiscorbutic vitamin is found in the juices of fresh fruit and vegetables, and particularly in the orange, the lemon and the grapefruit. Recent observations have shown that the antiscorbutic vitamin is also found in abundance in the tomato, the potato and the turnip, and to some extent in the tamarind. It is probable that all green vegetables are more or less antiscorbutic.

The antiscorbutic vitamin is readily destroyed by boiling and 
also by drying. It is absent in salted meats. The process of sterilizing milk destroys the antiscorbutic vitamin and pasteurizing injures it. On this account raw or uncooked vegetables or fruits should make a part of each day's bill of fare.

The orange and the tomato are the richest sources of vitamin C. Hoobler states that one and one-half cubic centimeters (onethird teaspoonful) of orange juice contains as much vitamin $C$ as a pint of milk. Orange juice should be given to all infants after six months and to bottle-fed infants after two months.

It has been found that orange juice may be dried at a low temperature without losing its antiscorbutic properties, especially if air is excluded. This will probably be found to be true of other fruits or fruit juices and green vegetables.

Recent experiments on guinea pigs reported by Zilva and Wells indicate that one of the first effects of scurvy, an effect which seems to have been long overlooked because not easily recognizable, is to be found in the teeth. There was found, even in very slight cases, complete fibrosis of the pulp. It is probable that decay of the teeth is really due in many cases to scurvy, the presence of which was overlooked.

Dr. Howe, of the Forsythe Dental Clinic of Boston, maintains that deficiency of vitamins is the probable cause of pyorrhea as well as of other dental disorders. Hess has shown that general feebleness and a puny or weazened condition in infants are the early effects of scurvy, which later shows itself in a fully developed form in what is known as Barlow's disease, in which there is a swollen condition of the joints and tenderness of the joints and skin.

The Chinese have long made use of sprouted seeds in the form of salad and combined with vegetables in various ways. Sprouted soy beans is one of the constituents of the famous chop suey. Recently Chick and Delf, of London, have made a study of the comparative value of dry and sprouted peas and lentils in preventing scurvy. They found that these seeds when soaked twenty-four hours in water and then sprouted forty-eight hours at room temperature were five or six times as active as dry seeds in preventing scurvy, and in this respect compared well with many fresh vegetables. So small a quantity as one and one-fourth grams (one-third dram) of the sprouted seeds fed daily was found sufficient to prevent scurvy in guinea pigs. This amount is somewhat greater than the amount of raw turnips or cabbage, 
fresh orange juice or lemon juice required, but is less than the amount of raw carrot or beet root needed.

An outbreak of scurvy among Serbian soldiers during the World War was successfully treated by the use of sprouted beans.

The antiscorbutic power of germinated seeds is considerably lessened by boiling.

Holst tells us that Cartier, on his last voyage to Newfoundland in 1535, cured his crew of 103 men, who with the exception of three were sick with scurvy, by administering to them a freshly prepared decoction of pine needles.

The writer is acquainted with a case in which a prospector saved himself from scurvy by eating grass when all other members of his party suffered seriously.

A very good salad may be prepared from sprouted soy bean seeds which have been allowed to grow to the length of about an inch. Sprouted soy bean seeds also add an excellent quality to vegetable soups. The property which these seeds have of producing highly valuable vitamins makes very desirable the encouragement, in this country, of the use of sprouted seeds.

Barley, wheat, lentils, peas and beans are best suited for this purpose. Puncture a dozen holes in the bottom of a tin pan. Fill the pan half full with the beans (soy, or better, mung beans). Fill the pan with water, cover closely and set in a warm place where the water may drain away. Refill the pan with water three times a day so as to keep the beans moist. Allow sprouts to grow until an inch or two in length. The beans should be thoroughly washed before putting to soak, to prevent molding.

Sprouted beans are one of the chief ingredients of the famous chop suey of the Chinese restaurants, and may account for the popularity of this dish.

Fresh fruits are rich in the scurvy-preventing vitamin; at least this is true of citric fruits: but recent observations by Osborne and Mendel have shown that fruits in general do not contain a large amount of vitamin $\mathrm{A}$ which is found in abundance in the branny coat of cereals, in root vegetables and greens.

The effect of cooking upon vitamin $C$ is highly injurious and should be given due consideration. During the war, scurvy broke out in a number of camps, affecting as many as 40 per cent. of the men. It was found that the vegetables were cooked for three hours. When the cooking time was reduced to forty minutes, scurvy rapidly disappeared. 
Sherman has recently called attention to the fact that the long cooking or twice cooking of vegetables destroys the vitamin $C$, even in fresh vegetables in which this vitamin is not destroyed by ordinary cooking. It is important to remember this in feeding diabetics with thrice cooked vegetables. They should be sure tc eat an abundance of lettuce, celery and tomatoes to supply vitamin C. It is important, also, in these cases to see that a good supply of vitamin $B$ is furnished by the use of yeast extract as well as greenstuffs.

It has recently been shown by Delf and Eddy that the cook ing of cabbage destroys 93 to 95 per cent, of the antiscorbutic vitamin. Sherman has shown that the boiling of tomatoes o1 tomato juice for one hour destroys one-half of its vitamin C Four hours' cooking destroys more than two-thirds of this essen tial vitamin.

Hoobler finds that a teaspoonful of orange juice contains as much vitamin $C$ as three pints of milk.

It has also been noticed that in camps located near apple orchards, from which apples were obtained and freely eaten, no scurvy occurred. Lime juice is found to have little value in pre. venting scurvy, but lemon and orange juice are highly valuable, and tomato juice, even the juice of canned tomatoes, is nearly as good. The juice of turnips and a drink prepared from tamarinds has also proved to be valuable, as well as soup made from potatoes.

It has been noticed that scurvy frequently occurs among the inmates of institutions when potatoes are scarce and high in price. Raw cabbage is better than orange juice in preventing scurvy, but cooked cabbage is much less valuable.

Recent studies (1921) at the University of Wisconsin by Steenbock and others seem to show that changes which take place in sauerkraut and silage, destroy the antiscorbutic vitamin.

\section{Vitamin B.-The Antineuritic or Antiberi-beri Vitamin.}

The first vitamin discovered (Funk) is now known as vitamin $B$. In a report, the "Present Status of Our Knowledge of Vitamins and Its Application to the Dietary," by Sherman, Winslow, Fisk, Greenwald and Jones, read before the American Public Health Association at its 51st Annual Meeting at Cleveland, Ohio, October 16,1922 , we are told concerning vitamin $B$ :- 
To this substance the name of vitamin was given by Funk (1911, 1912a. $1912 \mathrm{~b})$, and it is this substance which is ordinarily referred to when the -vord vitamin is used without qualification. Beriberi is due to the use of diets deficient in this vitamin. Most frequently such deficiency has been attributable to an excessive dependence upon white rice; but that the rice itself is not a necessary factor is shown by the occurrence of cases of beriberi in Newfoundland where the dietary deficiency was brought about by too exclusive a use of fine white flour.

The antineuritic vitamin, the absence of which produces beriberi, is found in most vegetables and vegetable juices. In cereals it is almost wholly confined to the outer covering or bran and the germ. Fine wheat flour, polished rice and new process cornmeal contain this vitamin only in infinitesimal amounts, and are equally lacking in other vitamins.

The continuous exclusive use of these foodstuffs causes beriberi, a most distressing malady which may easily prove fatal and which, until recent years, caused the death of many thousands of persons annually in the Philippine Islands from the use of polished rice.

Experiments reported by the United States Public Health Service showed that in fowls fed on fine flour bread, neuritis appeared at the end of twenty to thirty days.

Fowls fed on whole wheat remained in good health.

Fowls fed on corn grits or new process cornmeal showed neuritis in three to seven weeks.

Fowls fed on old-fashioned water-ground cornmeal or the whole corn remained well.

Emmett and Luros, from studies of the effect of heat on brown rice and vitamin $\mathrm{B}$ preparations from yeast and milk, conclude there are two vitamins which have heretofore been classed together as water-soluble B.

Beri-beri is a disease by no means confined to the Philippines, China and Japan, where it has long been known. During the war, beri-beri developed rapidly in the British army, in the Dardanelles and in Mesopotamia, when the soldiers were fed for a time almost exclusively on a diet consisting of wheat, canned meats and jam. Canned foods with the exception of tomatoes contain little or no vitamins. White bread is almost wholly devoid of vitamins, and jam contains none.

Thousands of persons, men, women and children, in civilized life are exposed to all the dangers of a deficient diet. These dangers may be eliminated by taking care to make free use of fresh, uncooked foods daily. Graham bread should be used in- 
stead of fine flour bread and so-called whole wheat bread. Oatmeal is a better breakfast food than rice, cornmeal, corn flakes and other cereals from which the bran has been removed. In experiments upon pigeons, it has been shown that bread made from graham flour supplies all the elements necessary for the prevention of beri-beri, whereas beri-beri quickly develops on a diet of fine flour bread.

McCarrison, of England, who has given great attention to the study of vitamins, and has written a most valuable work upon the subject of deficiency diseases, finds in the lack of vitamin $B$ some explanation of the genesis of that great mass of ill-defined gastro-intestinal disorders and vague ill-health which forms so large a proportion of human ailments at the present day. Osborne and Mendel and Kerr in Mendel's laboratory, have repeatedly demonstrated the marked effect of vitamin upon the appetite.

While beriberi or polyneuritis is the characteristic result of the long-continued lack of vitamin $B$, this grave and even fatal disease must be regarded only as the end result. It is highly important to bear in mind the following warning of the distinguished authors above referred to, who in their report tell us that, "Before the nerve symptoms become apparent the body may be seriously weakened in several other ways and rendered much more susceptible to the inroads of infectious disease. Hence, the importance of such selection of food as shall result in an intake of vitamin $B$ much more liberal than the minimum which suffices for the support of growth and the prevention of beriberi."

Spinach, even dried spinach, is an excellent source of vitamins. Dried spinach contains twice as much of the vitamins as does whole wheat, soy beans, dried eggs or milk powder. It is far superior to cabbage, lettuce and celery.

In general, the juices of raw fruits and vegetables are much richer in growth-promoting vitamins than is milk. Osborne and Mendel have noted that the tomato contains a rich supply of all the vitamins.

Cooper has shown that the amount of antineuritic vitamin required depends to some extent upon the amount of carbohydrate eaten. In the feeding of pigeons, if the amount of starch in the food is increased, the amount of vitamins must also be increased. It is important to bear this fact in mind in the management of bottle-fed infants.

McCarrison has shown that when the water-soluble B vita- 

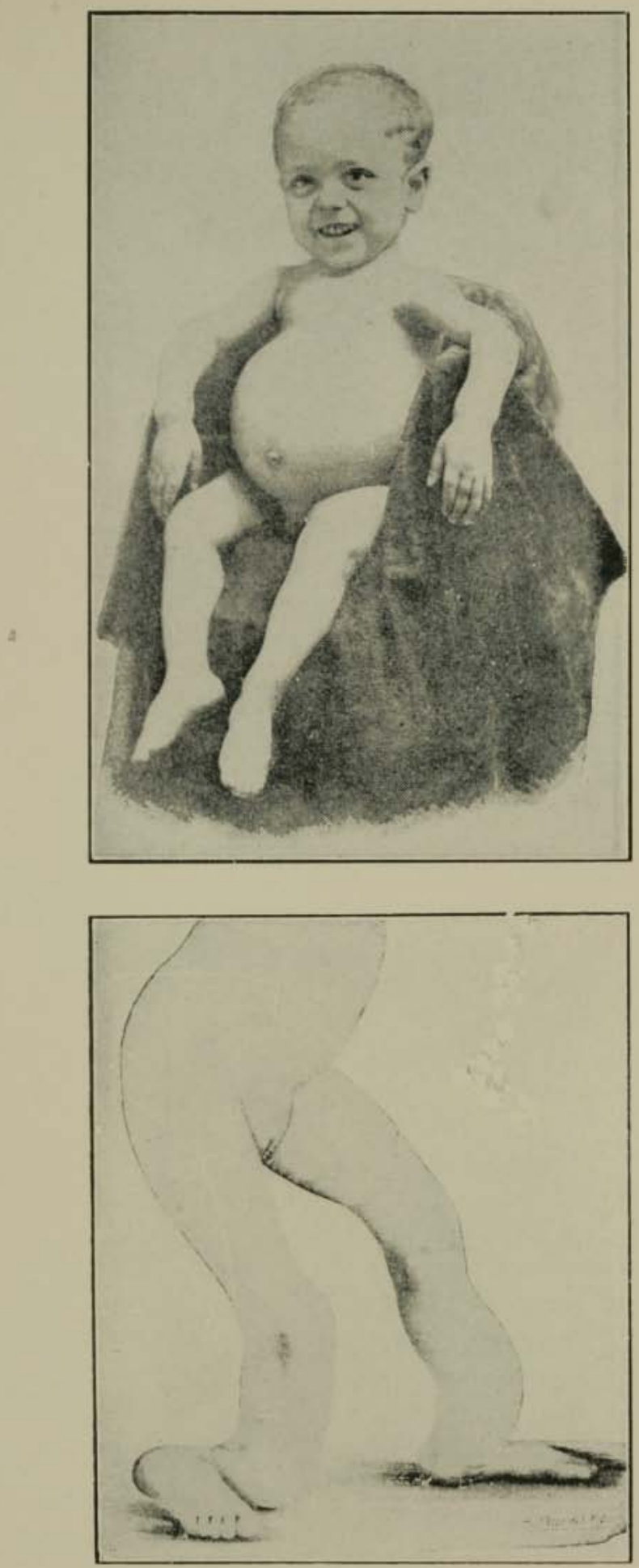

Changes Due to Rickets-Deficiency of Fat-Soluble A. (Holt) 


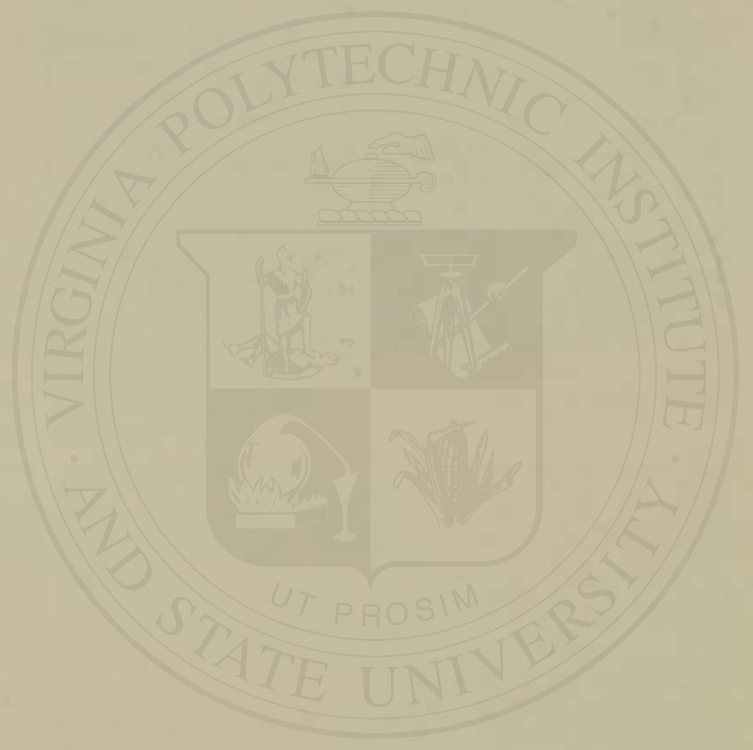

k. 
min, which is supplied by the bran of cereals, greens and root vegetables, is lacking, the resistance of the body to the invasion of the tissues by bacteria is greatly lowered.

McCarrison has also noted that vitamin $B$ aids peristalsis by energizing the intestine.

Dr. Ishiguro, the eminent Japanese military surgeon, who had great opportunities for observation during the Russo-Japanese war, denied that the lack of meat was the cause of beri-beri in the Japanese army, and called attention to the fact that the increased consumption of meat in Japan has been accompanied by an increase in the prevalence of beri-beri.

\section{Yeast Extract Very Rich in Vitamins.}

Yeast is extraordinarily rich in water-soluble vitamin $B$. The wide advertising of yeast preparations has induced many persons to make use of yeast more or less regularly in spite of the unpleasant flavor of crude yeast and the large amount required to produce any perceptible effect. Such persons will be glad to know that all the benefits of yeast may be obtained from its extract which contains all its virtues in such concentrated form that the same results may be obtained with less than onetenth the quantity. An additional advantage of the extract is its delicious flavor. When properly prepared, yeast extract is rich in the osmazomes which give to the mushroom and soy sauce, as well as meats and meat extracts, their attractive flavors. Broths, sauces, soups and bouillons prepared from such extracts can not be distinguished from meat preparations of similar sorts except by a greater attractiveness and uniformity of flavor.

\section{The Fat-soluble Vitamin-A.}

The chief sources of the highly important fat-soluble vitamir are milk, butter and other dairy products, and the green leaf Greens of many sorts are largely used by the Chinese and othes Orientals who make little use of milk. Greens should be much more largely used in this country not only for their precious vitamins but also for the food lime and iron which they supply.

The fat-soluble vitamin is not found in lean meat, but is present in the liver, kidneys and other glands of animals in which certain quantities are stored as a measure of safety.

The Eskimo finds it necessary to eat the raw, frozen liver of 
the seal to prevent the disastrous consequences which follow the deficiency of vitamins in his ordinary diet.

Another delicacy which is greatly enjoyed by dwellers in the Arctic regions is the intestine of the ptarmigan, a bird which lives upon the green leaves of the willow or heather, which are very rich in vitamins. When a bird is killed, the Eskimo opens its abdomen and scoops out its long, squirming intestine, and swal lows it warm and quivering, as an Englishman or an Americar gourmet swallows a raw oyster, thus making available for his own use the vitamins found in the half-digested greenstuffs with which the intestine is filled.

When a young Lapp goes out to hunt reindeer, his best girl charges him to bring her a stomach. These northern people eat with great avidity the half-digested reindeer moss which they find in the reindeer's stomach.

The absence of the fat-soluble vitamin gives rise to sore eyes in rats, the eyes being finally destroyed with ulceration of the cornea if the lacking element is not supplied. Night blindness has also been attributed to this cause.

The observations of Mellanby show that the fat-soluble A vitamin is found in olive oil, cottonseed oil and coconut oil, although absent in linseed oil. It is less abundant in these vegetable oils, however, than in butter.

The fat-soluble vitamin is of especial importance to growing animals. The water-soluble appears from experiments by Mendel and others to be more important for adults.

In a report presented at the last meeting of the American Public Health Association, October 16, 1922, Sherman and his colleagues stated that,

"Recent experiments emphasize strongly the fact that the fatsoluble vitamin is needed by adults as well as during growth. That adults appear less dependent upon the fat-soluble vitamin content of their food is attributable in part to the fact that they have passed the stage of development at which the effect of a deficiency becomes apparent quickly, and in part to the fact that when the adult has grown up on a diet rich in this vitamin he will have acquired a sufficient store to carry him over subsequent periods of inadequate intake, provided these are not too prolonged."

It is also known that the lack of vitamin $A$ not only gives rise to disease of the eyes but to other maladies. Sherman tells us that, "It has been repeatedly found that a diet poor in the fat- 
soluble vitamin (A) leads to weakness in other respects as well as to increased susceptibility to eye disease. Osborne and Mendel (1921) report diarrhea and diminished appetite as frequently resulting from food poor in fat-soluble vitamin; and they have definitely correlated this deficiency with the occurrence of phosphate renal calculi (bladder stones) among their experimental animals. McCollum and Davis $(1913,1914)$ and also Drummond (1919a, 1919b) report increased susceptibility to infections including lung disease among individuals whose diet is poor in this vitamin. Steenbock, Sell and Buell (1921) confirm this and emphasize also the susceptibility to bronchial troubles and abnormalities of the skin. Evans and Bishop (1922) find that diets containing enough fat-soluble vitamin for growth and protection from eye disease may still require enrichment with this vitamir in order to enable the animal to meet the added strain of reproduction.

"With so much direct experimental evidence of widespread weakening of the body when the intake of fat-soluble vitamin is low, it is not surprising that many authorities find reason to correlate low intake of this vitamin with increased susceptibility to such diverse diseases as rickets, pellagra and tuberculosis."

Both the water-soluble and the fat-soluble vitamins are closely associated with growth. When these vitamins are deficient the growth of young animals is impeded and when they are wholly absent growth ceases.

The fat-soluble vitamin is fifteen times as soluble in fat as in water. It is found in young shoots, in the yolk of eggs, and in yellow corn and carrots as well as in milk, cheese and butter. It is apparently associated with the yellow color known as carotin.

Winter milk contains only two-thirds as much vitamin A as does the milk of cows produced in summer.

Skimmed milk contains only one-eighth as much vitamin A as does full milk (Morgan). The most important sources of this highly important element are dairy butter and greens. 
The observations of Osborne and Mendel have demonstrated that dried alfalfa, clover, grass, spinach and carrot are equal to butter fat as sources of the fat-soluble vitamin. The same was found to be true of the dried tomato.

\section{Vitamin D}

Recent studies have revealed the presence in certain foodstuffs and in cod liver oil of another fat-soluble vitamin which is designated by the letter D. When this vitamin is absent, rickets develops. The disease quickly disappears when food containing the vitamin is given. A most surprising discovery was the fact rickets may be cured by exposing the affected animals to the sunlight or to ultra-violet rays with no change in the diet. The inference was that the light rays in some way produces the vitamin. Further experiment showed that animals suffering from rickets as the result of a diet containing no vitamin $D$ recover quickly if their food is exposed to light rays. It appears to be proven that vitamin $\mathrm{D}$ is produced by the action of ultra-violet rays upon cholesterol, a substance found in the oily secretion of the sebaceous glands of the skin.

Rickets is common in regions in which sunshine is deficient, especially in animals and infants in whose diet lime and phosphorus are not present in proper quantities and in proper or balanced proportions. Careful surveys have shown that in cloudy sections a large proportion of the population suffer from rickets, the effects of which are seen in misshapen legs (knock-knees, bow-legs), heads and chests, narrow pelves in women, and low resistance to disease, especially susceptibility to infections of the respiratory organs, influenza, tuberculosis, and pneumonia.

Park observed that exposure of the body to sunlight caused "a greater consumption of food, stimulated activity, improved the appearance, and increased the reproductive capacity."

Vitamin D is present in abundance in greens, whole milk and egg yolk. It is also present in cod liver oil. In cloudy regions, foods ordinarily rich in vitamin $\mathrm{D}$ may be deficient in this element. Spinach raised in a greenhouse in the winter season has been shown to be much less efficient in curing or preventing rickets than spinach raised in the field in summer.

In cloudy countries, the tendency to rickets should be counteracted by means of sun baths. Sea bathing and swimming 
are beneficial, but the sunshine gymnasium is better. Open-air gymnasia in which exercise may be taken with exposure of nearly the whole body surface to contact with fresh air and the sun's rays should be provided in connection with every public school and should be employed by adults as well as by children. The exposure should be sufficiently thorough to give the skin a brown or tanned color, and should be often repeated.

The general use of the sun bath and the addition to the daily diet of greens, bran, oatmeal or other whole grain preparations, and whole milk in place of the white bread, meat, coffee and cane sugar which constitute the staples of the present popular bill of fare, would soon make rickets a rare disease.

The presence of vitamin $D$ in abundance in the daily food and exposure of the body to sunlight, are as necessary to maintain a high state of vigor in adults as to prevent rickets in infants.

\section{Vitamin E}

Still another fat-soluble vitamin, designated as $\mathrm{E}$, has been shown by Evans to be needed for reproduction. This vitamin is found in abundance in seeds and green leaves, lettuce and other greens, and in some vegetable oils. Wheat germ is rich in vitamin $\mathrm{E}$.

\section{Chronic Avitaminosis.}

Savages as well as wild animals who live upon natural foods, do not suffer from avitaminosis, lack of vitamins, but among highly civilized people, because of the general use of sophisticated and denatured foodstuffs, there is widespread injury from this cause. It is probable, indeed, that a majority of all civilized people suffer more or less from vitamin deficiencies. The lack of these subtle food principles is coming to be recognized and the probable cause of a vast number of vague and obscure illnesses, many of which are usually attributed to other causes. Says Plimmer, an eminent English investigator,-

"With slight shortages of the various vitamins characteristic symptoms take a long time in appearing. In most cases the first sign of illness is loss of appetite, followed by digestive disturbance. Animals may have stoppage of the gut, gastric or duodenal ulcer, or die of appendicitis or other troubles before the typical symptoms of the deficiency disease are shown. During the war the slow 
healing of wounds was found to be associated with shortage of vitamin C. Heart and digestive troubles are seen with shortage of vitamin B. Under all variations of vitamin shortage experimental animals are more susceptible to infections of all kinds. Animals fed on vitamin-poor diet have succumbed to epidemics of infectious disease which have not attacked other animals kept side by side with them, but fed on food containing enough of all the vitamins. Details of these experiments cannot be entered into here but they all prove how intimately health depends upon a supply of food containing plenty of all the vitamins. Lack of cleanliness, bad housing, and confinement do not produce disease in properly fed animals."

The thorough study that has now been made of most common foods has put us in possession of such precise information that no one need suffer from a shortage of the necessary vitamins provided only that proper care is exercised in the selection of foodstuffs, since these essential elements are found in abundance in the simplest and cheapest of food materials.

The information condensed in the accompanying tablesXVII to XX-A, will enable the reader to balance his diet for vitamins in such a manner as to insure against any deficiency.

The following tables show the relative amounts of the several vitamins found in various common foodstuffs:

\section{TABLE XVII}

\section{Foods Rich in Vitamin A}

Alfalfa
Avocado
Beans (string)
Beet greens
Butter
Carrots

Chard
Cream
Cheese (cream)
Dandelion greens
Egg yolk
Milk (whole)

\section{Millet}

Peas (green)

Pineapple

Prunes

Tomatoes

Wheat germ

\section{Results of Deficiency in Vitamin A.}

Anemia (probably).

Retarded or dwarfed development.

Malnutrition.

Diminished resistance to infection of eye, ear, nasal sinuses, glands and lungs. 
TABLE XVIII

\section{Foods Rich in Vitamin B}

$\begin{array}{lll}\text { Alfalfa } & \text { Graham bread } & \text { Peas (green) } \\ \text { Asparagus } & \text { Grape fruit } & \text { Pineapple } \\ \text { Beans } & \text { Lemon } & \text { Potatoes } \\ \text { Beet greens } & \text { Lentils } & \text { Rice (brown) } \\ \text { Bran } & \text { Lettuce } & \text { Rutabaga } \\ \text { Branola } & \text { Milk } & \text { Shredded wheat } \\ \text { Cabbage } & \text { Nuts } & \text { Spinach } \\ \text { Carrots } & \text { Oatmeal } & \text { Wheat flakes } \\ \text { Cauliflower } & \text { Okra } & \text { Wheat germ } \\ \text { Celery } & \text { Onions } & \text { Whole wheat } \\ \text { Dandelion greens } & \text { Oranges } & \text { Yeast extract }\end{array}$

Results of Deficiency in Vitamin B.

Malnutrition.

Stunted growth.

Nervous exhaustion

Loss of appetite.

Indigestion, especially constipation.

Polyneuritis.

Beriberi

\section{TABLE XIX}

\section{Foods Rich in Vitamin C}

$\begin{array}{lll}\text { Beans (sprouted) } & \text { Grains (sprouted) } & \text { Raspberries } \\ \text { Cabbage (raw) } & \text { Lemon juice } & \text { Rutabaga } \\ \text { Carrots (raw) } & \text { Lettuce } & \text { Swede } \\ \text { Cloudberries (fresh or } & \text { Onions } & \text { Tomato (fresh or } \\ \quad \text { canned) } & \text { Orange juice } & \text { canned) } \\ \text { Fruit (fresh, raw) } & \text { Pineapple } & \text { Turnip } \\ \text { Fruit juice (fresh, raw) } & \text { Potatoes } & \end{array}$

\section{Results of Deficiency in Vitamin C.}

Stunted growth.

Malnutrition.

Scurvy (sore moutls, tender joints).

Defective teeth, 


\title{
TABLE XX
}

\section{THE VITAMINS OF FOODS *}

\author{
$(\mathrm{A}-\mathrm{B}-\mathrm{C})$
}

[The sign + indicates that the vitamin is present. ++ indicates that the vitamin is present in adequate quantities. +++ indicates that the food is very rich in vitamin. - indicates little or no vitamin present. ? or * indicates doubt or uncertainty.]

\begin{tabular}{|c|c|c|c|}
\hline Food & Vitamin A & Vitan & Vitamin C \\
\hline Alfalfa & & & * \\
\hline Almonds & + & & * \\
\hline Artichoke & - & + & - \\
\hline Apples, raw ........ & + & + & ++ \\
\hline Asparagus ............. & * $\sqrt{1}$ & & $*$ \\
\hline 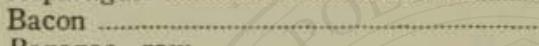 & .. - to $t$ & $t$ & $?$ \\
\hline 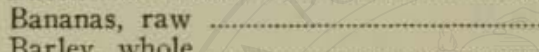 & + & & ++ \\
\hline $\begin{array}{l}\text { Barley, whole } \\
\text { Beans, kidney }\end{array}$ & $\stackrel{+}{+}$ & & $\bar{*}$ \\
\hline $\begin{array}{l}\text { Beans, kidney } \\
\text { Beans, navy }\end{array}$ & + & & * \\
\hline Beans, soy & + & & $*$ \\
\hline Beans, sprouted & + & & \\
\hline Beans, string, fresh, raw & $+t$ & & \\
\hline Beans, string, cooked & & & * \\
\hline $\begin{array}{l}\text { Beechnut } \\
\text { Beef }\end{array}$ & & & - tot \\
\hline Beef juice & & + & -tot \\
\hline Beef fat ........ & + & - & - \\
\hline Beets (roots) & & & * \\
\hline 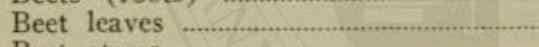 & & 7 & * \\
\hline 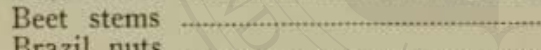 & & & * \\
\hline & & & * \\
\hline $\begin{array}{l}\text { Bread, white, water } \\
\text { Bread, white, milk }\end{array}$ & + & + & $\bar{?}$ \\
\hline Bread, whole wheat, water & H & & ? \\
\hline Bread, whole wheat, milk & & & $?$ \\
\hline $\begin{array}{l}\text { Butter } \\
\text { Buttermilk }\end{array}$ & & & - \\
\hline $\begin{array}{l}\text { Buttermilk .................... } \\
\text { Cabbage, green, raw }\end{array}$ & $+t$ & & $\begin{array}{l}+ \text { variab } \\
+++\end{array}$ \\
\hline $\begin{array}{l}\text { Cabbage, green, raw } \\
\text { Cabbage, head, raw }\end{array}$ & + & & \\
\hline Cabbage, head, cooked & + & & + \\
\hline 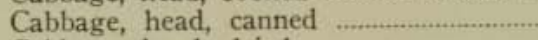 & + & & $?$ \\
\hline Cabbage, head, dried .............................. & & & $+?$ \\
\hline Carrots, fresh, raw & & & ++ \\
\hline Carrots, cooked & & & + \\
\hline Cauliflower & & & ${ }_{*}^{+}$ \\
\hline Celery, bleached stems & $\bar{t}^{\text {to }}$ & & $*$ \\
\hline $\begin{array}{l}\text { Celery, bleached leaves } \\
\text { Celery, green leaves }\end{array}$ & & & * \\
\hline Chard & & & * \\
\hline Cheese, full milk & & & * \\
\hline $\begin{array}{l}\text { Cheese, cottage (skim) } \\
\text { Clover, young }\end{array}$ & & ++ & $\stackrel{*}{*}$ \\
\hline 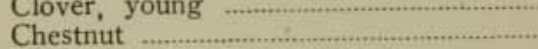 & & & * \\
\hline
\end{tabular}

- Copied with slight modification from Sherman's "Food Products," Macmillan Company, New York 


\begin{tabular}{|c|c|c|c|}
\hline FOOD & Vitamin A Vit & In B & Vitamin C \\
\hline Cloudberries & * & * & $++t$ \\
\hline 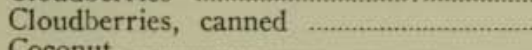 & $*$ & * & \\
\hline Coconut & + & & $*$ \\
\hline & - & 一 & 二 \\
\hline $\begin{array}{l}\text { Codliver oil } \\
\text { Corn (maize), white }\end{array}$ & $\begin{array}{c}t+t \\
t+t\end{array}$ & $\bar{t}$ & $\overline{-}$ \\
\hline & $t^{\text {tot }}$ & & $\overline{-}$ \\
\hline Corn oil & $?$ & 1 & - \\
\hline Cottonseed (flour or meal) ..................... & + & & $*$ \\
\hline 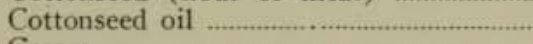 & ? & & - \\
\hline $\begin{array}{l}\text { Cream } \\
\text { Cress }\end{array}$ & $+t+$ & & +variable \\
\hline $\begin{array}{l}\text { Cress } \\
\text { Cucumber }\end{array}$ & $*$ & + & $\stackrel{+}{+}+?$ \\
\hline Dandelion greens & ++ & & + \\
\hline Dasheens ................. & $-?$ & & + \\
\hline Eggs ……. & ++ & $t$ & + ? \\
\hline $\begin{array}{l}\text { Egg white } \\
\text { Egg yolk }\end{array}$ & $\overline{1}$ & & $*$ \\
\hline $\begin{array}{l}\text { Egg yolk } \\
\text { Eggplant }\end{array}$ & & $t$ & * \\
\hline $\begin{array}{l}\text { Eggplant } \\
\text { Endive }\end{array}$ & + & & + \\
\hline Filberts ......................... & * & & $*^{+}$ \\
\hline Fish, fat & + & & * \\
\hline 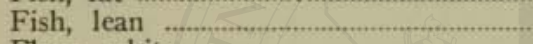 & - to + & & * \\
\hline 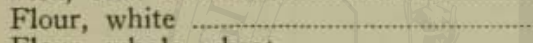 & - & + & - \\
\hline Flour, whole wheat & + & & - \\
\hline whole, dry & $\bar{t}$ & & 二 \\
\hline $\begin{array}{l}\text { Grans, whoole, dity } \\
\text { Grains, sprouted }\end{array}$ & + & & $\overline{t+}$ \\
\hline Grapes & $\neq$ & & t+ \\
\hline Grape juice & * & & + \\
\hline $\begin{array}{l}\text { Grapefruit } \\
\text { Ham }\end{array}$ & $*$ & & $++?$ \\
\hline $\begin{array}{l}\text { Ham } \\
\text { Heart }\end{array}$ & - to + & + & $\bar{I}$ \\
\hline $\begin{array}{l}\text { Heart } \\
\text { Hickory nuts }\end{array}$ & $\stackrel{+}{*}$ & & $+?$ \\
\hline Honey & - & & - \\
\hline $\begin{array}{l}\text { Ice cream (genuine) } \\
\text { Lard }\end{array}$ & +- & & + ? \\
\hline $\begin{array}{l}\text { Lard ............................... } \\
\text { Legumes, sprouted ... }\end{array}$ & -tot & & $\div$ \\
\hline $\begin{array}{l}\text { Legumes, sprouted } \\
\text { Lemon juice }\end{array}$ & & & $\begin{array}{ll}+t \\
+t\end{array}$ \\
\hline Lemon juice, dried & ? & & $\begin{array}{l}t+t \\
+t+\end{array}$ \\
\hline 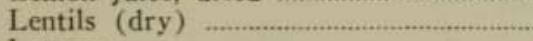 & * & + & 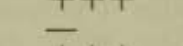 \\
\hline 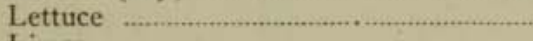 & $+t$ & & ++ \\
\hline Limes …............. & & & + \\
\hline 둔. & $\overline{1}, \quad-$ & & $\div$ \\
\hline 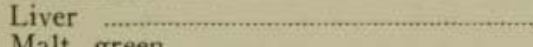 & t+var'ble - & +1 & + \\
\hline $\begin{array}{l}\text { Malt, green } \\
\text { Mango }\end{array}$ & $\begin{array}{l}+ \\
*\end{array}$ & & $t^{t}+$ \\
\hline 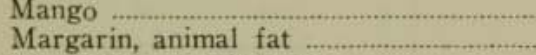 & - tot & - & \pm \\
\hline rin, vegetable fat & - & - & - \\
\hline 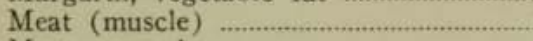 & - to + & & - to + \\
\hline nned & -tot & + & - \\
\hline Meat extract ……... & - & & - . \\
\hline Milk (whole) ... & & ++ & + variable \\
\hline Milk, "scalded" & $\begin{array}{l}t^{2}+t+ \\
+t\end{array}$ & +1 & + variable \\
\hline $\begin{array}{l}\text { Milk, condensed } \\
\text { Milk, evaporated }\end{array}$ & $\begin{array}{l}++ \\
+++\end{array}$ & $\begin{array}{l}++ \\
++\end{array}$ & $\begin{array}{l}\text { +variable } \\
\text {-? }\end{array}$ \\
\hline $\begin{array}{l}\text { Milk, evaporated } \\
\text { Milk, dried, whole }\end{array}$ & $+t+$ & ++ & + variabl \\
\hline
\end{tabular}




\begin{tabular}{|c|c|c|c|}
\hline FOOD & Vitami & Vitar & Vitamin C \\
\hline Milk, dried, skim & . + & & ariab \\
\hline $\begin{array}{l}\text { filk, fresh, skim } \\
\text { fillet }\end{array}$ & $+t$ & & \\
\hline Mulberries ............ & * & * & + \\
\hline Mutton & - to + & + & $?$ \\
\hline Mutton fat & + & - & - \\
\hline $\begin{array}{l}\text { Oatmeal } \\
\text { Oleo oil }\end{array}$ & & & 一 \\
\hline $\begin{array}{l}\text { Oleo oil } \\
\text { Olive oil }\end{array}$ & \pm & - & $\overline{-}$ \\
\hline 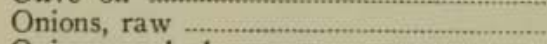 & - & & \\
\hline Onions, cooked & & & + \\
\hline (1). & $+\mathrm{t}$ & & \\
\hline e peel & + & + & \\
\hline $\begin{array}{l}\text { Palm oil } \\
\text { Parsley }\end{array}$ & + & $T$ & $\bar{*}$ \\
\hline Parsnips ……………............... & & & * \\
\hline Peaches, raw ..... & & * & \\
\hline 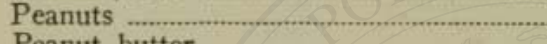 & + & + & * \\
\hline $\begin{array}{l}\text { Peanut butter } \\
\text { Peanut oil }\end{array}$ & + & & $*$ \\
\hline $\begin{array}{l}\text { Peanut oil } \\
\text { Pears }\end{array}$ & * & - & $\bar{*}$ \\
\hline Peas, young, green & & & + \\
\hline Peas, dry & & & \\
\hline Peas, sprouted & + & & \\
\hline 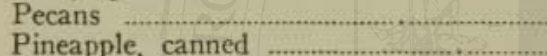 & & & \\
\hline & & & + \\
\hline Pine nuts & & & \\
\hline Pork …_............ & & + & $\bar{*}$ \\
\hline $\begin{array}{l}\text { Potatoes, sweet } \\
\text { Potatoes, white, }\end{array}$ & & t & * \\
\hline $\begin{array}{l}\text { Potatoes, white, raw } \\
\text { Potatoes, white, boiled } 15 \text { minutes } \ldots . . .\end{array}$ & & & \\
\hline $\begin{array}{l}\text { Potatoes, white, boiled } 15 \text { minutes } \\
\text { Potatoes, white, boiled } 1 \text { hour }\end{array}$ & + & & \\
\hline Potatoes, white, baked & + & & + \\
\hline $\begin{array}{l}\text { Prunes } \\
\text { Radish }\end{array}$ & t & & \\
\hline 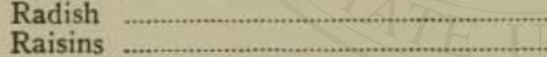 & & & * \\
\hline $\begin{array}{l}\text { Raisins } \\
\text { Raspberries, fre }\end{array}$ & * & * & $\bar{t}$ \\
\hline $\begin{array}{l}\text { Raspberries, tresh } \\
\text { Raspberries, canned }\end{array}$ & $*$ & * & \\
\hline 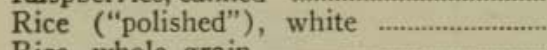 & - & - & - \\
\hline $\begin{array}{l}\text { Rice, whole grain } \\
\text { Rutabagas }\end{array}$ & + & + & - \\
\hline $\begin{array}{l}\text { bagas } \\
\text { whole }\end{array}$ & 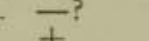 & & $++t ?$ \\
\hline $\begin{array}{l}\text { Rye, whole } \\
\text { Sauerkraut }\end{array}$ & $\begin{array}{l}+ \\
+\end{array}$ & & $\bar{Z}$ \\
\hline Spinach, canned & & * & $*$ \\
\hline Spinach, cooked & & & $*$ \\
\hline $\begin{array}{l}\text { Spinach, dried } \\
\text { Spinach, raw }\end{array}$ & & & \\
\hline $\begin{array}{l}\text { Spinach, raw ......... } \\
\text { Squesh, Hubbard .... }\end{array}$ & & & +++ \\
\hline (1) & - & - & - \\
\hline (1) & - & - & - \\
\hline Swede ……............. & & & ++ \\
\hline Sweetbreads & + & & \\
\hline Tomato, raw & . $+t$ & +++ & +++ \\
\hline 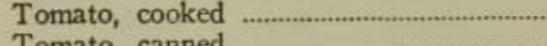 & & & $\begin{array}{l}t+t \\
+t+\end{array}$ \\
\hline $\begin{array}{l}\text { Tomato, canned } \\
\text { Tomato, dried }\end{array}$ & ++ & ++ & ++ \\
\hline & & & \\
\hline
\end{tabular}




\begin{tabular}{|c|c|c|c|}
\hline FOOD & Vitamin A & Vitamin B & Vitam \\
\hline Turnip & - to + & & \\
\hline Veal ....... & & & * \\
\hline Walnuts ....... & * & & * \\
\hline Wheat bran & + & & - \\
\hline Wheat embryo & ++ & & - \\
\hline 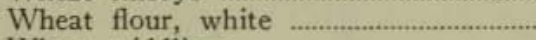 & - & + & - \\
\hline Wheat middlings & * & ++ & - \\
\hline Wheat, whole & + & & - \\
\hline Yeast & 一 & $++t$ & 一 \\
\hline Yeast extract & - & +++ & - \\
\hline
\end{tabular}

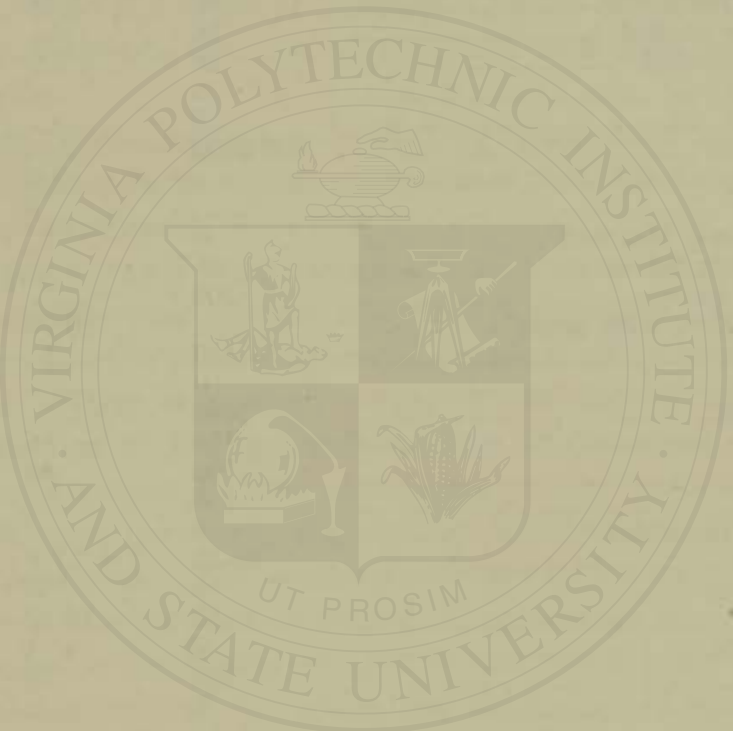


TABLE XXa

SHOWING THE RELATIVE VITAMIN VALUES OF VARIOUS FOODS *

(Compiled from Sherman's "Chemistry of Food and Nutrition")

\begin{tabular}{|c|c|c|c|}
\hline & A & B & $\mathrm{C}^{* *}$ \\
\hline $\begin{array}{l}\text { Apples, raw } \\
\text { Bacon }\end{array}$ & ${ }_{8}^{16}(?)$ & $6-10$ & $10-20$ \\
\hline 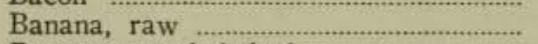 & 100 & $6-10$ & $20-40$ \\
\hline 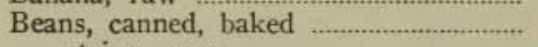 & 16 & & \\
\hline string, raw & 100 & $\begin{array}{r}10 \\
20-24\end{array}$ & $6-13$ \\
\hline $\begin{array}{l}\text { navy, canned, baked ........................... } \\
\text { Beef, lean }\end{array}$ & 16 & $\begin{array}{r}20-24 \\
10\end{array}$ & 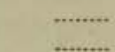 \\
\hline $\begin{array}{l}\text { Beef, lean } \\
\text { fat }\end{array}$ & 150 & & …..... \\
\hline Beets & 5 & ........ & .......... \\
\hline Butter & $500-1400$ & & \\
\hline Cabbage, fresh, raw & 18 & $9-18$ & 100 \\
\hline 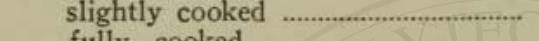 & 18 & ......... & 30 \\
\hline $\begin{array}{l}\text { fully cooked } \\
\text { old }\end{array}$ & $\begin{array}{r}18 \\
6\end{array}$ & ......... & $5-10$ \\
\hline Cauliflower & $\begin{array}{r}6 \\
18\end{array}$ & ........... & ........... \\
\hline Carrots, raw ................................ & $600-2000$ & & ........... \\
\hline 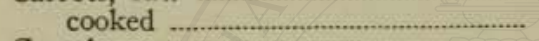 & 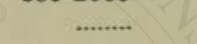 & $7-9$ & .......... \\
\hline Cantaloupe & & 8 & ........ \\
\hline Celery, stems, bleached ............................. & $\begin{array}{r}0 \\
65\end{array}$ & & …..... \\
\hline $\begin{array}{l}\text { bleached, leaves } \\
\text { green leaves }\end{array}$ & 200 & & ............ \\
\hline Cereals, whole, with germ ................ & & $50-75$ & ................ \\
\hline 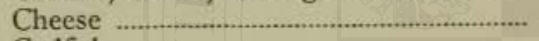 & 600 & ......... & ......... \\
\hline$\ldots+\ldots$ & 0 & ........ & .......... \\
\hline 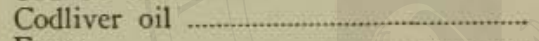 & 5000 & & ........ \\
\hline 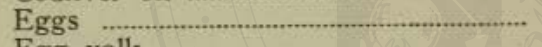 & 550 & 16 & $+\ldots . . .$. \\
\hline $\begin{array}{l}\text { Egg yolk } \\
\text { Egg white }\end{array}$ & $\begin{array}{r}1700 \\
0\end{array}$ & 50 & (........... \\
\hline $\begin{array}{l}\text { Egg white } \\
\text { Flour, white, wheat }\end{array}$ & 0 & (.......... & …....... \\
\hline $\begin{array}{l}\text { Flour, white, wheat } \\
\text { Grapes }\end{array}$ & $16-22$ & $7-9$ & $4-5$ \\
\hline Lemon Juice …………………… & 3 & 10 & $9-18$ \\
\hline Lettuce & 65 & $10-12$ & \\
\hline Milk, whole & $\begin{array}{r}65 \\
500\end{array}$ & $70^{9}$ & $.3-1.5$ \\
\hline $\begin{array}{l}\text { dry } \\
\text { Oatmeal }\end{array}$ & 0 & $\ldots+\ldots . .$. & ........... \\
\hline Olive oil & 13 & & .......... \\
\hline 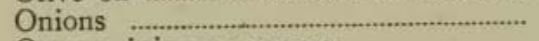 & & 6 & \\
\hline 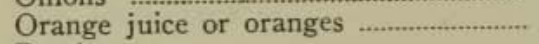 & 20 & & 9-19 \\
\hline Peaches, raw & & $6-10$ & ......... \\
\hline 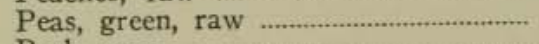 & $150-200$ & 60 & $\ldots+. .$. \\
\hline Pork, raw & & …........ & ........ \\
\hline $\begin{array}{l}\text { Potatoes, raw } \\
\text { cooked }\end{array}$ & 8 & 8 & $1.5-4.5$ \\
\hline sweet, yellow & 200 & & \\
\hline Prunes & 1500 & $\begin{array}{r}38 \\
20-24\end{array}$ & ........ \\
\hline & 170 & $8-16$ & $9-19$ \\
\hline $\begin{array}{l}\text { Tomatoes, raw or canned } \\
\text { Turnips }\end{array}$ & $1.5-10$ & 8 & \\
\hline Wheat ….......................... & 0 & $50-75$ & 0 \\
\hline & ......... & $200-300$ & \\
\hline $\begin{array}{l}\text { sprouted } \\
\text { Yeast (dry) }\end{array}$ & …...... & $800-1200$ & \\
\hline extract & ........ 80 & $000-12000$ & \\
\hline
\end{tabular}

- A blank space indicates "not determined," not "absence."

*. Compared with orange juice as 100 . 
Canned foods should never constitute a large part of the dietary. Fresh foods, rich in vitamins, are needed daily to maintain the nerve tissues in health and to prevent scurvy and other deficiency diseases.

The fat-soluble vitamin is apparently not affected by the temperatures ordinarily employed in cooking. Mendel found the fat-soluble vitamin of butter fat to be uninjured by exposure to a temperature of $230^{\circ} \mathrm{F}$. for sixteen hours.

Vitamins are destroyed by alkalies. For this reason, the use of carbonate of soda in cooking is to be strongly condemned. When bicarbonate of soda is used in the making of bread, it is by the process of baking converted into carbonate of soda, which is strongly alkaline and destroys vitamins. The old-fashioned method of making biscuit by the use of soda and sour milk is likewise objectionable.

Baking powder biscuit, as ordinarily made, have an alkaline reaction which affords sufficient evidence that the vitamins are damaged or destroyed.

Pellagra prevails most extensively in certain districts in the South where the new process cornmeal is generally used. This preparation of corn is almost wholly deprived of vitamins by the process of milling, and when prepared with baking soda what small proportion of vitamins remains may be destroyed.

In mountainous regions contiguous to the localities where pellagra is very prevalent, the disease is either absent or only rarely encountered. These mountaineers are so far away from the cities where new process cornmeal is obtainable that they are obliged to have their corn ground in the small mills scattered through the mountains, in which the old-fashioned stone burrs are still in use. The cornmeal made in this way represents the entire grain and is comparatively rich in vitamins.

Holt and others have called attention to the fact that an increase of carbohydrates in the diet of a child necessitates a liberal increase in the vitamin content of the dietary. If this is neglected, nutrition and development fail. For this reason, the amount of orange juice or tomato juice must always be increased when a child is placed upon a cereal diet or when there is an increase in the malt sugar added to the milk. No doubt this principle applies with equal force in the feeding of adults, so that the free use of cane sugar at meals, or in the form of confectionery at any time, must involve vitamin deficiency since cane 
sugar contains no vitamins while at the same time producing a special demand for an increase of this essential food constituent. No doubt the free use of cane sugar is in this way responsible for many thousands of cases of nutritive disorders of various sorts occurring especially in this country where the use of cane sugar is carried to a very great excess.

This is a matter to which every person who has charge of dietaries should give attention. A person may easily starve to death in the midst of plenty, and while eating abundantly, for lack of the precious vitamins found in spinach and other fresh foods. Every meal should include a liberal supply of spinach or other greens. An abundant supply of greens should be provided for the table during the winter as well as the summer months.

According to Mendel and Osborne, the tomato is unique as a source of vitamins, being rich in all three of the known vitamins, fat-soluble A and water-soluble B and C. This is true not only of the fresh tomato but of both canned and dried tomatoes. This fact places the tomato at the head of our common foods as a means of insuring to the body an adequate supply of all the vitamins. It should be used very freely.

Hess tells us that the juice or purée of canned tomatoes may be regarded as a palatable solution of the three vitamins and a serviceable antiscorbutic for artificially fed infants. It may be used in doses of one ounce daily.

\section{How Vitamins Are Destroyed.}

Vitamins are destroyed by the action of heat or alkalies and are removed by the so-called "high milling" of cereals.

The antiscorbutic vitamins are destroyed by boiling and are damaged by a temperature of 160 degrees $\mathrm{F}$., the usual temperature employed in pasteurizing milk.

A boiling temperature for several hours impairs, but does not destroy the fat-soluble and antineuritic vitamins.

Canned meats and other foods are often heated to a temperature of $240^{\circ} \mathrm{F}$. for two or three hours. This is necessary to destroy the spores of putrefactive germs which are not killed by ordinary cooking temperatures. It is evident that canned foods must be deficient in vitamins. Canned tomatoes are an exception to this general rule. Cooked or canned tomatoes are nearly as good a source of vitamins as the fresh fruit.

Funk found that a dog fed exclusively on canned beef died in two weeks. 


\section{Cellulose}

Cellulose is found exclusively in vegetable products. It forms the framework and fibers of plants, the walls of vegetable cells and the coverings of seeds. Wood consists of cellulose with the addition of layers of cork.

The chemical composition of cellulose closely resembles that of sugar. There are many different celluloses, just as there are many different sugars. Cellulose is formed from sugar and dextrine circulating in the sap or juices of plants and may by chemical processes be re-converted into sugar.

In its ordinary form, cellulose is insoluble in boiling water, and is not acted upon by the digestive fluids of the alimentary canal, although it is digested by the ferments of certain bacteria which sometimes flourish in great numbers in the colon.

Special forms of cellulose are soluble in boiling water. On cooling this dissolved cellulose forms a gelatinous mass. Agar is a cellulose of this sort. It is found in seaweeds which grow along the coast of Japan. In commerce, it is sometimes known as Japanese isinglass, or Ceylon moss.

The cellulose in fresh fruits and vegetables and in tender shoots is softened and broken up in cooking, and to a large extent disappears through the action of bacteria in the colon.

Professor Bunge, in his "Physiologic and Pathological Chemistry" says of cellulose, "It is of great importance in acting as a mechanical stimulus to promote the peristalsis of the intestine. For this reason cellulose is absolutely essential to animals with a long intestinal tract. If rabbits are fed on a diet containing no cellulose, the onward movement of the intestinal contents ceases, inflammation in the intestine ensues, and the animals rapidly die. But if horn-parings be added to the same food, nutrition is normal.

The following are the details of a post-mortem examination of a rabbit which had died for lack of cellulose: "The stomach only contained mucus, and showed signs of incipient inflammation in the region of the pylorus; the small intestine, full of mucus, was much inflamed throughout its whole length, as was also the 
cecum. The latter was largely filled with excrement of the consistency of putty, which adhered firmly to the walls and folds of the cecum. The difference between these contents and those of the cecum of a normally fed rabbit is very noticeable, for here the mass in the cecum is pretty loose, falling almost completely away if the intestine be bent backwards, and this loose consistency is caused only by the tough fibers, by means of which the communication between the anus and the stomach is kept open. This could hardly have been the case in the animal which died."

"The short intestine of carnivora does not require a mechanical stimulus to produce peristaltic action. The intestine of human beings is well known to be of medium length; a man's life therefore is not endangered by deprivation of cellulose, although the normal movement of the intestine might be thereby impeded. The muscular wall of the intestine becomes atrophied like every other muscle, if it has no work to do. We must therefore see that the diet of human beings does not lack woody fibers. The excessive fear of indigestible food which prevails among the wealthier classes may lead to universal debility of the intestinal walls. Habitual constipation would perhaps not be such a common trouble if we were accustomed from our childhood to a dietary containing a sufficient supply of woody fiber."

\section{Bran.}

The outer coverings of seeds consist of cellulose combined with layers of cork cells, a woody substance much more resistant than the cellulose found in green vegetables. Bran is for this reason an excellent form of roughage. It is needed on every table and at every meal as an accessory food to make good the deficiencies of the ordinary bill of fare in cellulose as well as iron, lime, and vitamins.

The cellulose of bran, of seeds, of berries, the coarser fibers of vegetables and agar, escapes the action of bacteria in the colon and hence is of great value in giving to the intestinal contents the bulk necessary to stimulate peristaltic activity.

The composition of bran varies greatly according to the amount of fine middlings included with it. The best bran consists of large flakes of nearly uniform size and free from middlings.

When well softened with water, bran is not irritating but is an emollient. The thin films of cellulose become as soft and 
pliable as wet paper, and excite the bowel, not by scratching or irritating it, but by a gentle titillation, so to speak, and by giving to the food sufficient mass to distend the intestine and stimulate it to vigorous acivity. This was observed by Cannon in his X-ray studies of digestion in cats.

In its ordinary commercial form, bran is seldom fit for use, on account of the large amount of dirt which it contains, including insects and bacteria. For use as a food laxative, it should be carefully prepared by thorough cleaning and washing of the wheat before grinding and sterilization of the bran. Sterilized bran, first introduced by the writer many years ago, is now prepared by various manufacturers and is put up in convenient packages. One or two rounded tablespoonfuls should be taken at each meal, the amount depending upon the character of the other foods taken. The writer has never seen any ill effects from the use of sterilized bran, which he has prescribed for many years, although there are many cases in which it fails to produce the desired effect and has to be supplemented by the use of paraffin oil as a lubricant.

This is particularly true in cases in which the cecum is greatly dilated or crippled by adhesions from chronic appendicitis and in cases in which there is obstruction of other parts of the colon, especially the pelvic colon as the result of adhesions.

The combination of paraffin oil with bran or agar-agar in some form is also useful in cases of spastic contraction due to colitis.

Experience shows that from half an ounce to an ounce of cellulose must be taken with the meals daily to insure sufficient bulk to stimulate the intestine to action. In cases in which the colon is very redundant or is crippled by adhesions, even double this amount may sometimes be needed, at least until the bowel has been trained to normal action. This amount of cellulose is provided by two rounded tablespoonfuls of sterilized bran in addition to other laxative foods.

The amount of food required to furnish two-thirds of an ounce ( 300 grains) of cellulose may be ascertained by reference to the accompanying tables (Tables XXI-XXIII).

In the tables of cellulose, the term cellulose is made to include the cork and other indigestible vegetable matters associated with it. It will readily be seen that bran or agar is almost absolutely 
essential as an addition to ordinary foodstuffs to provide the bulk required for efficient bowel action.

It will be noted that foodstuffs differ greatly in their cellulose content, seedy fruits, greens and legumes, supplying this element in relatively larger amount than any other food.

\section{TABLE XXI}

Showing the Cellulose in Cereals.

\begin{tabular}{|c|c|c|}
\hline & $\begin{array}{l}\text { Number of } \\
\text { cellulose } \\
\text { grains in } \\
\text { one ounce }\end{array}$ & $\begin{array}{l}\text { Grains of } \\
\text { cellulose } \\
\text { in } 100 \\
\text { calories }\end{array}$ \\
\hline Barley (cooked). & 20.0 & 64.0 \\
\hline Beans (dried) .... & 40.0 & 40.0 \\
\hline Corn flakes....... & 10.0 & 9.7 \\
\hline Cornmeal................. & 10.0 & 9.7 \\
\hline Wheat (cooked) & 10.0 & 38.0 \\
\hline Wheat grits (cooked) & 5.0 & 27.0 \\
\hline Rolled wheat (cracked) & 9.0 & 36.0 \\
\hline Graham flour & 10.0 & 9.6 \\
\hline Fine flour & 1.5 & 1.4 \\
\hline Oatmeal (cooked).. & 44.0 & 37.0 \\
\hline Polished rice ........... & 2.0 & 2.0 \\
\hline Unpolished rice ....... & 3.8 & 3.7 \\
\hline Rye (small) ......... & 15.0 & 14.4 \\
\hline Peas (dried) .......... & 20.5 & 28.5 \\
\hline Lentils ......................... & 20.0 & 19.6 \\
\hline Granola (cooked)...... & 10.0 & 9.8 \\
\hline Sterilized bran .................... & 200.0 & $\ldots \ldots$. \\
\hline 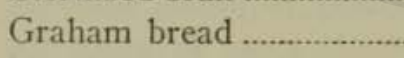 & 6.0 & 23.0 \\
\hline Whole wheat bread..... & 5.0 & 7.0 \\
\hline
\end{tabular}

TABLE XXII

Showing the Cellulose in Vegetables.

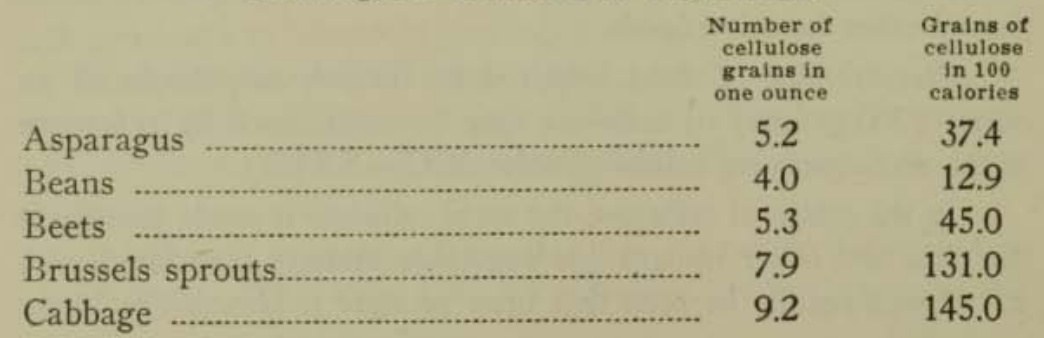


Carrot (raw)

36.0

Cauliflower (steamed) _............................... 4.6

44.6

Celery (raw)

7.0

127.0

Cucumber (raw)

$3.9 \quad 78.0$

Green peas

$9.4 \quad 27.0$

Kohl-rabi (raw)

$7.8 \quad 86.0$
3.7

Lettuce

$3.7 \quad 65.0$

Onion

Parsnips

8.7

50.0

Peas (dried)

28.5

27.0

Potato (baked)

16.6

Pumpkin

5.5

65.6

Spinach

6.1

50.0

Tomatoes

4.7

63.6

Turnip

\section{TABLE XXIII.}

Showing the Cellulose in Fruits.

Prunes (cooked).

Number of

cellulose

grains in

one ounce

Apples

10.0

5.0

Pears

15.0

Peaches

5.0

7.5

Plums

10.0

Cherries

37.0

25.0

Blackberries

61.0

Huckleberries

10.0

Strawberries

23.0

Currants

7.5

Raisins

7.5

Raisins (stewed)

7.4

Oranges

10.0

Bananas

1.5

Grains of

cellulose

in 100

calories

36.0

5.0

81.0

40.0

30.0

44.0

200.0

150.0

300.0

87.0

138.0

36.0

38.4

38.0

67.0

5.2 
Figs

Apricots

Gooseberries (stewed)

It should further be mentioned that in the use of cellulose in concentrated form, as in sterilized bran, the whole amount used at a meal should not be taken at once, as at the beginning or end of a meal, but should be well mixed with the food by taking small portions at frequent intervals during the meal.

It should also be mentioned that for greatest efficiency as a laxative bran should be used in liberal quantities at every meal.

There has been much discussion about the digestibility of bran and the advisability of its use. Naturally its digestibility is less than that of fine flour. Its value depends upon its indigestibility. But it does not interfere with the digestibility of other foodstuffs to any appreciable extent. Mendel and Osborne have shown by feeding experiments, using white rats, that bran may be made to take the place of meat as a source of protein; and Hindhede, food controller of Denmark during the World War, claims to have demonstrated the same. During the war the Danish people lived almost exclusively on barley porridge, vegetables, greens, milk and bread. In making their bread they added wheat bran to their coarse rye meal, and thrived upon it, reducing the annual death rate 34 per cent. No one starved, and no one was made ill by the coarse fare.

It is well known that the digestive organs of herbivorous animals are able to digest cellulose, a function which seems to have been lost by civilized human beings, for it has been shown (Tibbles) that the capacious cecum of the negro of the Nile and the Bushman secretes a ferment which dissolves the cellulose covering of vegetable cells. It is claimed, also, that the vermiform appendix of the negro produces a ferment which digests cellulose.

But the digestibility of cellulose is of much less consequence than its indigestibility since its chief value in human food is to serve as roughage. The importance of cellulose as a food constituent is well known to farmers and to all feeders of animals. Rabbits, when fed on a diet containing no cellulose, rapidly die. When cellulose is not present in their food the movements of the intestine cease, inflammation follows and death soon after.

Experiments by Hart, as well as by Osborne and Mendel, 
have shown the cause of leg weakness in chickens to be lack of roughage. The difficulty disappeared when paper was given as roughage, but no improvement was noted by other changes in the diet which would have corrected any defect which might have arisen from lack of vitamins or salts in the food. These experiments clearly demonstrate the value of bran and other forms of roughage and should be of service to housewives and others who are responsible for the feeding of children, as well as to poultry raisers.

A study of the nutritive value of wood by Haberland showed that wood contains, especially in winter, considerable quantities of sugar, starch and oil, with small quantities of protein. These nutrient materials are found only in the living wood; that is, in the twigs, small branches and sap wood, not in the heart wood of the tree. Hard wood contains a considerable amount of starch, from one-fifth to one-fourth of its weight, while the pine, birch and linden contain considerable oil but little starch. The pine and spruce contain so much resin that they are quite unfit for food, and the oak and the willow are objectionable on account of the considerable amount of tannin which they contain. The best food woods are elm, linden, maple, poplar and birch. When finely ground, these woods may be mixed with cereals and other foods for use by animals, and in emergency by man, and may be made to yield a considerable amount of nourishment.

\section{Bran Does Not Irritate.}

That bran is an excellent laxative is not a new discovery. The fact has been known for years and has been utilized by the farmer in the treatment of his horses, cattle and pigs, but, unfortunately, he has neglected to make use of this most excellent remedy for his own benefit. The general failure to resort to bran for the relief of constipation has probably been due to the wide-spread but erroneous notion that bran is irritating.

We are glad to find this view which we have so long defended endorsed by that most eminent of medical authorities, The Journal of the American Medical Association (Dec. 6, 1919, p. 1768). We quote as follows:

Bran is therefore chiefly to be looked on as a form of almost indigestible carbohydrate, which is endowed with considerable laxative value, not only because it adds by its bulk to the distention of the intestine, but also because of the spicate shape of its particles. Exces- 
sive irritation does not result from these; for when properly moistened and heated, bran becomes as soft and pliable as wet paper, and hence produces merely a gentle titillation and is usable even in patients with a tendency to colic.

Hippocrates was a believer in bran. In one of his famous medical works, he says:

This I know, moreover, that to the human body it makes a great difference whether the bread be fine or coarse; of wheat with or without the hull. . . Melancholic diseases are most particularly exacerbated by beef, for it is of an unmanageable nature, and requires no ordinary powers of stomach to digest it.

The writer quite agrees with Professor Tibbles, who asserts that "the excessive fear of indigestible foods which prevails in the minds of many people is largely responsible for atonic constipation and many of the cases of chronic intestinal catarrh, mucous colitis, and membranous colitis. It is therefore important in the treatment of these diseases that the food does not lack cellulose and especially woody fibre."

Von Noorden, one of the most eminent of living medical authorities, has for many years pointed out the necessity for roughage in the treatment of colitis, a disease for which bland foods are commonly prescribed. Von Noorden insists that in this disease the diet should include coarse bran bread, legumes, including the skins, coarse oatmeal, all sorts of coarse vegetables, fruits, currants, gooseberries and grapes, including the skins and seeds. Von Noorden reports complete and permanent cures of colitis by this regimen in 50 per cent. of all cases, and cure or improvement in more than 75 per cent. of all cases treated. More bran and less beef would be a good general prescription for the American people.

Bran contains a considerable amount of protein and when added in liberal quantities to other foodstuffs, excluding meat, might easily enrich the diet sufficiently to insure all the protein needed. It should be noted, also, that in addition to its richness in protein, bran contains a rich store of lime, iron and vitamins, important food constituents which are deficient in most other foodstuffs.

Roger Crab, who died on 11th September 1680, lived for 3s. $3 d$. a year on broth and bran, with chopped turnip leaves, and drank only water. He had fought and was wounded in the Cromwellian army (Sinclair.) 
The United States Department of Agriculture has published the results of experiments made at the New York Experiment Station which indicate that wheat bran is laxative not only because of its indigestibility which makes it a mechanical stimulant of the intestine, but because of the presence in bran of a peculiar laxative substance, phytin, which is found in very considerable amount in the outer layers of the envelope. This important discovery furnishes a scientific basis for the regular use of bran as a natural laxative which secures increased bowel activity not by irritating the bowel as do medicinal laxatives but by physiologic stimulation so that it may be used an indefinite length of time without losing its effects and without injury.

Carnivorous animals eat bones and other indigestible substances, doubtless to give increased bulk to their ingesta. Dogs often eat grass, and sea lions swallow quantities of pebbles (Norton).

\section{Agar-Agar.}

Agar occurs in commerce in long, flexible strings, which are imported to this country in huge bales. These strings do not consist of the dried seaweed, as many suppose, but is the cellulose which has been dissolved from the seaweed by boiling.

Some years ago, the writer obtained through $\mathrm{Mr}$. George U. West, American consul at Kobe, Japan, the following account of the method of preparing agar:

The process of manufacture may be described as follows: The seaweed is first crushed, each kind separately, to remove shells and other adhering matter, and then washed with clean water. The washed seaweed is placed on a mat and dried until its color becomes white by the action of the sun, frost and dew. This operation takes place during September and October, and when bleached the weight of the seaweed is decreased nearly one-half. After bleaching, the six kinds of seaweed-in the proportion of Iza, 4; Egokusa, 4; Misaki, 3; Hirakusa, 3; Nanbu, 4; and Onikusa, 2-are all put together in a boiler and cooked for about 14 hours, until they become soluble. The liquid is then strained through a sack and a box with a bamboo sieve on one side, from which it runs into a container. From the container the liquid is ladled into trays about $31 / 2$ feet long and 3 inches deep.

After remaining in the trays about 12 hours, these are placed on a low stand, and the isinglass is cut into strips 3 inches wide and 14 inches long, with a knife and a ruler. These strips are then put into a long closed wooden box (the ends of which are 3 inches square, one end being open and one filled in with a wire sieve) and pushed through 
the sieve end in the form of long fine strips. The isinglass is then placed on a low stand, which is covered with a clean mat, and dried in the sun during the day and during the night for two or three weeks during January and February, being watered at midnight. The quality of the isinglass depends upon the weather during this time, the best being made when it is clear and cold, the poorest when it is warm and rainy. When the isinglass is bleached sufficiently, it is compressed and packed in Japanese matting, tied with a straw rope.

It is evident that this crude method of preparation exposes the agar to contamination through the contact of insects, dust, etc. On this account commercial agar requires thorough disinfection and cleansing before use.

The use of agar is to be most highly recommended as a means of giving the necessary bulk to stimulate the intestine to prompt action. When properly prepared it is wholly free from unpleasant flavor, and it manifests such astonishing avidity for water that when it is present in the feces they cannot possibly become dry and hard.

In cases in which constipation is due to "greedy colon" agar or bran is indispensable. In such cases the colon has acquired the power to eat up enormous quantities of the cellulose of the food, so that it is very difficult to increase the bulk of the feces by the use of green vegetables. This is the reason for the disappointment experienced by many who hope to find in the free use of lettuce and like green foods a panacea for their intestinal ills. Agar has been shown by the experiments of Mendel and others to be indigestible by any of the digestive fluids with which it comes in contact in the human body. It must be taken in sufficient quantity to accomplish the object sought. Two-thirds of an ounce to an ounce daily is the quantity usually required for adults. For young children a quarter to a half of this quantity is sufficient.

Agar is easily taken in soup, cereal coffee, fruit juice or stewed fruit. It should be allowed to soften a little and should then be swallowed without chewing.

This remedy should be taken at meals in order that it may be intermingled with the food, and so prevent the formation of hardened residues in the intestine.

Agar may be used with advantage as a substitute for a meal, when food cannot be taken, and when there is no appetite for food, and when so used it maintains the intestinal rhythm which 
would otherwise be lost, resulting in constipation. It should in such cases be taken with fruit juice or fresh or stewed fruit. When one finds at night that the usual amount of food has not been taken, an extra dose of agar with a little fruit may be taken before going to bed. No digestive work is required by either the fruit or the agar except to move it along the digestive canal. It is important to take fruit juice with the agar to excite the necessary peristalsis. Cooked bran may be used in the same way.

Some years ago, the writer received from a trained nurse working among the Indians at Hoonah, Alaska, an interesting specimen of dried seaweed which is collected by the Indians in the summer season for use as a laxative during the long winter months when berries and vegetable foods are not accessible. "After being gathered, the seaweed is spread out on the rocks and dried a little in the sun; then they fold it up and cut it in squares and put it in a coal oil can; a branch of spruce tree between each cake and a weight put on to press it. It is taken out and put in the sun day after day until well dried. Tire seaweed is used as a laxative, for which purpose they eat it raw"

The seaweed thus prepared occurs in blocks about eight inches square and an inch thick. It is jet black, and has a salty, though not unpleasant taste. It differs from Japanese seaweec in being very brittle so that it may be easily eaten dry.

Lewis and Clarke in their famous journey to the Pacific Coast more than a century ago found the Indians making use of boiled moss as a foodstuff in the winter season, doubtless for its laxative effects.

Only among highly civilized people is there general neglect to include in the diet the necessary roughage to maintain a healthy action of the intestines. Savages take care to secure this food essential.

\section{Manyalla.}

Mr. Kennan, in his fascinating volume, "Tent Life in Siberia," gives the following account of the use of partially digested reindeer moss by the Koraks:

"Hold on!" exclaimed Dodd, with a deprecating gesture, "that's enough, I'll eat it;" and taking out a half-spoonful of the dark viscid mass, he put it to his lips.

"Well," said we expectantly, after a moment's pause, "what does it taste like?" 
"Like the mud pies of infancy!" he replied sententiously. "A little salt, pepper, and butter, and a good deal of meat and flour, with a few well-selected vegetables, would probably improve it; but it isn't particularly bad as it is."

Upon the strength of this rather, quivocal recommendation I tasted it. Aside from a peculiar earthy flavor, it had nothing about it which was either pleasant or disagreeable. Its qualities were all negative except its grassiness.

This mixture, known among the Koraks as "manyalla," is eaten by all the Siberian tribes as a substitute for bread, and is the nearest approximation which native ingenuity can make to the staff of life. It is valued, we are told, more for its medicinal virtues than for any intrinsic excellence of taste, and our limited experience fully prepared us to believe this statement. Its original elements are clotted blood, tallow, and half-digested moss taken from the stomach of the reindeer, where it is supposed to have undergone some essential change which fits it for second-hand consumption. These curious and heterogeneous ingredients are boiled up together with a few handfuls of dried grass to give the mixture consistency, and the dark mass is then molded into small loaves and frozen for future use.

This half digested moss is much used by natives of the Arctic regions to combat constipation. It is also no doubt useful as a source of vitamins in which meat is very deficient.

Up-to-date butchers feed cows on bran for some days before slaughtering. They say the flesh keeps better and has a better flavor. Chickens are fed on skimmed milk or buttermilk and bran for some days before killing, for the same purpose. The effect observed is doubtless due to the suppression of colonic putrefactions.

\section{The Laxative Effects of Clay.}

Dr. Case, of the Battle Creek Sanitarium, observed some years ago a laxative effect from the use of fuller's earth in X-ray examinations of the stomach and intestine. Jordan of London has recently confirmed this observation and recommends a tablespoonful of washed kaolin before each meal as an excellent laxative.

More than thirty years ago, the writer was informed by a lady that she had cured her husband of constipation by giving him spoonful doses of sand.

It is quite probable that the eating of clay by various primitive tribes may be based upon its laxative properties. 
Alder, a traveler among the islands of the South Pacific, says of the practice of clay-eating, "The clay is washed down with copious drafts of cocoanut water taken a pint at a-well, 'irrigation' is the only word that seems to suit the process. In response to our stumbling inquiries as to why they eat dirt, they indicate that it is bagoose, or good for them. We came to the conclusion that it must be some mineral substance otherwise lacking in their diet."

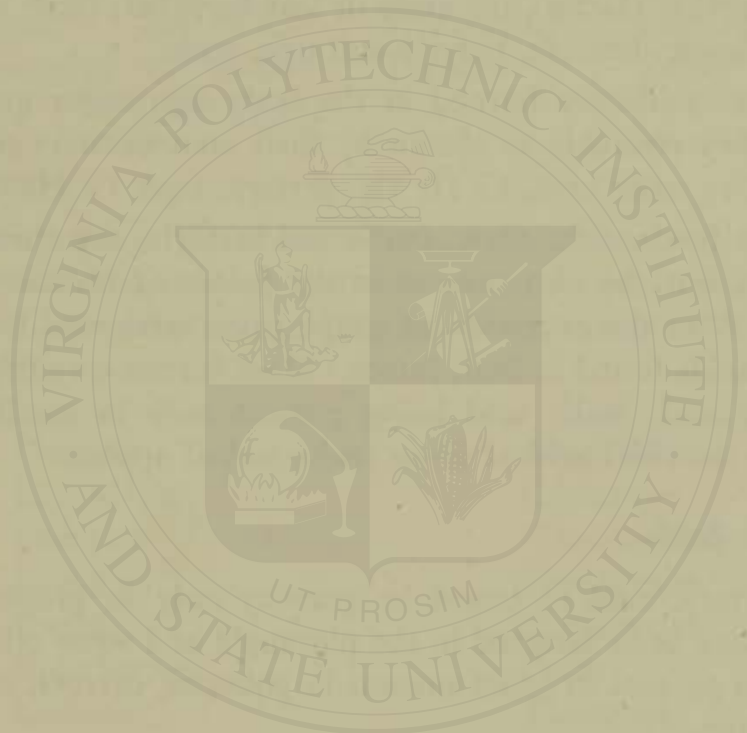




\section{The Acids of Foods}

There are three wholesome food acids; that is, acids which are found in natural foods and which when eaten are utilized the same as starch, sugar and dextrin, which they resemble in composition. These are citric, malic and tartaric acids. In addition to these there are sometimes found in foods other acids which are not utilized by the body and hence are not foods, and which with one exception (lactic) are more or less harmful; these are acetic, oxalic, tannic, benzoic, butyric and uric acids.

Food acids are burned in the body like sugar and starch, which they resemble as shown by their composition: citric acid, $\mathrm{C}_{6} \mathrm{H}_{8} \mathrm{O}_{7}$; malic acid, $\mathrm{C}_{4} \mathrm{H}_{7} \mathrm{O}_{5}$; tartaric acid, $\mathrm{C}_{4} \mathrm{H}_{6} \mathrm{O}_{6}$.

The food acids, citric, malic and tartaric, are chiefly found in fruits, but are also present in the juices of certain vegetable foods. With the exception of grape juice, citric and malic acids are the acids found in fruit juices. Most berries owe their acidity to citric acid, malic acid being present only in small amount. Malic is the chief acid of stone fruits and of apples.

\section{Tartaric Acid.}

Tartaric acid is found in quantity only in grapes, though traces have been detected in the pineapple and some other fruits. It is also present in small amount in potatoes, carrots, cucumbers and endive.

Underhill has pointed out that nephritis may be procuced by tartaric acid when given freely in the form of tartrates, such as Rochelle salts. This fact assumes considerable importance when considered in relation to the extensive use of baking powders which usually contain tartaric acid in large amount. It is true that the amount of tartaric acid, or tartrates, required to produce acute nephritis is considerable-much more than that ordinarily taken in the form of baking powder or even in a medicinal dose; nevertheless, this is no guarantee that profoundly mischievous results may not be produced by the long-continued intake of small doses of the acid, such as may occur in the free use of baking powders, especially in cases of nephritis. 


\section{Malic Acid.}

According to recent observations (Wise) malic acid is almost completely oxidized in the body, and large doses may be taken without producing any ill effect.

Malic acid is very widely distributed, being found in the following foods:

$\begin{array}{lrll}\text { Apples } & 0.21 \text { to } 1.81 & \text { Grapes } & \text { Greens } \\ \text { Pears } & 0.11 \text { to } 0.50 & \text { Quince } & \text { Beets } \\ \text { Cranberries } & 0.50 & \text { Strawberries } & \text { Asparagus } \\ \text { Cherry } & 0.56 \text { to } 1.54 & \text { Peach } & \text { Spinach } \\ \text { Currant } & 2.00 \text { to } 3.37 & \text { Watermelon } & \text { Lettuce } \\ \text { Gooseberry } & 1.7 \text { to } 2.6 & \text { Tomato } & \text { Celery } \\ \text { Plum } & 0.55 \text { to } 2.15 & & \end{array}$

\section{Citric Acid.}

Citric acid is the characteristic acid of lemons, and other citrus fruits, but it is at the same time found in many other fruits and some vegetables. The foods containing citric acid are as follows:

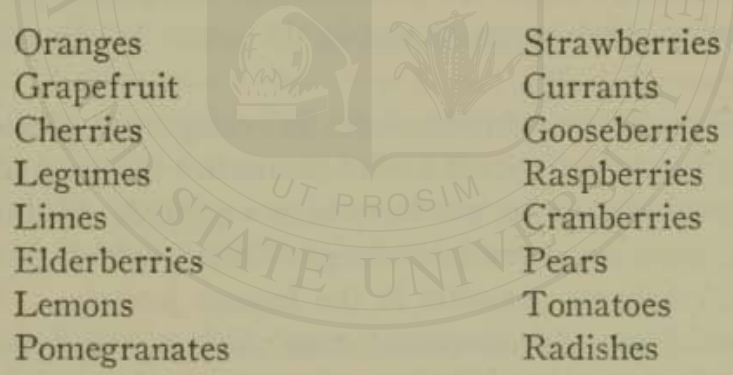

Salant and Wise, of the Bureau of Chemistry, have shown (1917) that citric acid, either in the form of lemon juice or citrate of soda, is oxidized in the body, none being found in the urine even when doses as large as one ounce of citric acid were administered.

\section{Phytic Acid.}

Phytic acid, which occurs in small amounts in bran in the form of phytin, is worthy of mention because of its laxative properties to which, it is claimed, the bran of cereals owes one of its most important uses. 


\section{Unwholesome Acids.}

The unwholesome acids which may be encountered in foods are more numerous than the wholesome. Fortunately, they may be easily avoided. Those most commonly met are acetic, oxalic, tannic, benzoic, butyric, and uric acids.

\section{Acetic Acid.}

Acetic acid is chiefly met with in vinegar, which contains 3 to 5 per cent. of this harmful chemical. It is formed by the fermentation of sugar. It is sometimes produced in the stomach. It may be recognized by its sour odor. It is a very highly volatile acid, and is always present when a sour odor is observed, as sometimes occurs in vomited matters and fermenting stools. It should be added, however, that the so-called "sour" or "acid" stomach is not often due to fermentation or to acetic acid, but is the result of an excess of gastric (hydrochloric) acid.

Acetic acid is harmful to digestion, as first demonstrated by Sir William Roberts. A teaspoonful of vinegar will at once arrest the process of salivary digestion in the stomach. Vinegar, as well as pickles, and all substances containing this acid, should be banished from the table. It is a chemical compound of use only as a reagent in the laboratory and in the arts. It is a poison, not a food.

When used freely in the form of vinegar or pickles, or in sauces, vinegar is an irritant to the stomach. Cases of ulceration of the stomach due to its use have been reported. Genuine cider vinegar is likely to abound in vinegar "eels," which it has been claimed may become parasitic in the human intestine.

Boix, of Paris, demonstrated some years ago by experiments upon animals that acetic acid has twice the power of gin to produce gin liver.

Lemon juice is more than a complete substitute for vinegar as an acid condiment. Vinegar is a useless and harmful chemical and should be excluded from the table.

\section{Oxalic Acid.}

Oxalic acid is found in minute amounts in many vegetable foods but in notable amounts only in a few. These are rhubarb or pieplant, sorrel, spinach, tea, cocoa and pepper.

All foods containing oxalic acid in any considerable amount should be parboiled and the boiling water rejected. 
A study of Table XXIV will show that only the first six articles named contain oxalic acid in dangerous quantities, viz., cocoa, sorrel, pepper, rhubarb, spinach and tea. Pepper is used only in very small quantities and has other noxious properties sufficient to condemn it. Cocoa and tea being used as beverages, easily introduce a harmful amount of the acid. A cup of tea or cocoa contains four times as much oxalic acid as the body normally produces in a day, as shown by the amount excreted in the urine. Twice this amount is regarded as pathological. So a single cup of cocoa or tea introduces into the body twice as much oxalic acid as is found in oxaluria, a diseased condition. And a cup at each of the three meals would load upon the body a dose six times as great. Evidently these drinks are harmful, if for no other reason, because of the oxalic acid which they contain.

In the use of spinach and sorrel, the oxalic acid should be removed by parboiling.

With the familiar pieplant, or rhubarb, the case is different. This plant is used solely for its acid flavor which, unfortunately, is chiefly due to the presence of oxalic acid of which it contains, as shown by the table, seventeen grains to the pound or more than one grain to the ounce. An ordinary serving would contain about three grains of the acid. This amount is not sufficient to produce immediate ill effects in ordinary persons, although cases of sickness have been reported. But the amount is more than forty times that with which the tissues and the kidney are normally required to deal, and this quantity has been known to bring on acute relapse in a case of chronic nephritis, a disease often present though unsuspected.

It is well known that degeneration of the arteries and resulting high blood pressure may be caused by the use of water containing such minute quantities of lead that the presence of the injurious metal is not noticeable and can only be detected by careful chemical analysis. Metchnikoff has shown also that premature old age with degeneration of blood vessels, heart and other vital organs is due to the daily absorption of small quantities of poisons from the colon. It is not to be supposed that the taking of a small quantity of rhubarb now and then will produce any very appreciable effect on either health or length of life but the same may be said of wine, the use of beer, tobacco, tea, coffee and every injurious substance that might be named. It is, however, of the highest importance that the character of this plant 
should be generally understood, otherwise it would be quite possible for a person who happened to be very fond of rhubarb to make use of it so frequently or freely as to suffer serious injury, especially in cases of disease of the kidneys.

The healthy body is able to utilize, that is, oxidize or burn up citric, malic, and tartaric acids, but the tissues are unable to oxidize oxalic acid, which consequently passes through the body unchanged like a mineral acid. In those morbid conditions in which oxalic acid is supposed to be a factor the amount of oxalic acid eliminated through the urine every twenty-four hours is only about double the normal amount. That is, oxalic acid is so injurious a substance that, if the amount ordinarily found in the tissues is doubled, highly pernicious consequences follow, among which are stone in the kidney, stone in the bladder and an extended group of distressing nervous symptoms which are included under the name of oxaluria. By the dietetic use of rhubarb it is easily possible to increase the amount of oxalic acid several times the amount which clinical observation has shown to be capable of producing grave disease. Of course, a single dose of pieplant would produce only temporary effect, but the daily use of pieplant, by exposing the tissues constantly to the influence of this poison, must certainly be followed by deleterious effects, since no one has ever undertaken to show that the oxalic acid found in pieplant is in any way different from oxalic acid derived from other sources.

Some years ago, Sir William Roberts, in experiments conducted for the purpose of determining the effects of alcohol and various other substances upon digestion, demonstrated that oxalic acid has a most pernicious effect upon salivary digestion. A solution consisting of one part of oxalic acid in ten thousand parts of water was found to destroy at once all action of the saliva upon starch. Nearly thirty years ago, the writer very carefully repeated these experiments and was able to verify in every particular the observations of Sir William Roberts. It is evident, then, that if pieplant is taken along with farinaceous foods, such as bread and other cereals, potatoes, etc., the normal digestive action of the saliva upon the starch, by which about half the work of starch digestion is normally accomplished in the stomach, must fail altogether. In other words, a very important part of the digestive work of the stomach is left undone when rhubarb is eaten. 
Those who recommend rhubarb as a food will doubtless reply to the above criticism that nearly all vegetables contain oxalic acid. This is not questioned; but it is important that the public should know that while it is true that nearly all vegetables contain this acid, the amount contained in most vegetables is exceedingly small. There are only three vegetables commonly eaten which contain any considerable amount; namely, sorrel, spinach and pieplant. In the preparation of sorrel and spinach, the oxalic acid is dissolved in the cooking water and by parboiling may be practically all removed. But if this were done with pieplant no one would care to make use of it for it is used solely and exclusively because of the acid which it contains. That is, in the case of sorrel and spinach, the oxalic acid may be readily removed without destroying the food value of these articles; whereas in the case of pieplant, removal of the oxalic acid would render it altogether useless and undesirable. The amount of oxalic acid contained in vegetables other than those mentioned is rarely so much as one-tenth the amount which these contain, and this small amount is left behind in the cooking water.

As regards the poisonous properties of oxalic acid we quote as follows from the United States Dispensatory:

Attention was first called to it as a poison by Royston in 1814, and the certainty and rapidity of its action have caused it to be largely used for suicidal purposes. Death has been produced by it in ten minutes. The minimum fatal dose recorded is one dram (60 grains).

When concentrated, it causes exquisite pain, followed by violent efforts to vomit, then sudden dullness, languor, and great debility, and finally death without a struggle. When dissolved in twenty times its weight of water, it possesses no corrosive and hardly any irritating power, but causes death by acting on the brain, spinal marrow and heart. It should be noted, however, that the acid, even in weak solution, always exercises a corroding or softening effect on the animal tissues.

The morbid appearances caused by oxalic acid are various. In a dissection reported by Christison, the mucous coat of the throat and gullet could be easily scraped off. The inner coat of the stomach was pultaceous, in many points black, in others red, and that of the intestines similarly but less violently affected. In another case the whole villous coat of the stomach was either softened or removed, as well as the inner membrane of the esophagus, so that the muscular coat was exposed, and this coat exhibited dark, gangrenous appearance, being much thickened and highly injected. The stomach usually contains a dark fluid, resembling coffee grounds, consisting chiefly of altered blood. The tongue and mouth are sometimes white or spotted with white. 
After reading the above official description of the poisonous properties of oxalic acid, who would care to make this noxious substance a part of his bill of fare? That the toxic properties of oxalic acid are really present in pieplant is shown by the numerous cases of poisoning which have been reported in recent times. Here are a few :

The Journal of the American Medical Association published an account of ten cases of poisoning from eating canned rhubarb stems, the part usually eaten. Nine of the cases were in one family. Only one member of the family, who ate none, escaped serious illness. Two who had two helpings had convulsions. The timely use of the stomach pump saved worse results. Chemical analysis showed no poison present other than oxalic acid in ordinary amount.

A man of 41 , his wife, four children and servants were all taken with violent diarrhea a few hours after having eaten a dish of rhubarb leaves cooked with spinach. All recovered rapidly except the man. He developed acute nephritis during the following week.

Tibbles reports cases in which persons who had previously suffered from nephritis (Bright's disease) suffered relapses after eating rhubarb. It may be easily understood that persons whose kidneys have been damaged by an attack of nephritis may be unable to deal with this highly irritant poison even in small amount. There are many thousands of such persons. The victims of typhoid fever, small pox, and even scarlet fever, measles and diphtheria, rarely escape more or less serious injury of the kidneys.

According to Thompson, the pieplant is not used as a food in Germany, being there grown only as an ornamental plant. According to an English monthly, Illustrated Gardening, the same is true in France. In the opinion of the writer the pieplant should be relegated to the list of noxious weeds. It is certainly not a wholesome food plant.

The fact shown by Pierson and Dutcher that rhubarb stems prevent scurvy in guinea pigs proves nothing in favor of their use by human beings. Grass and various weeds quite unfit for human food will also prevent scurvy in pigs. 


\section{TABLE XXIV.}

\section{Oxalic Acid in Various Foodstuffs per 1,000 Parts (Esbach).}

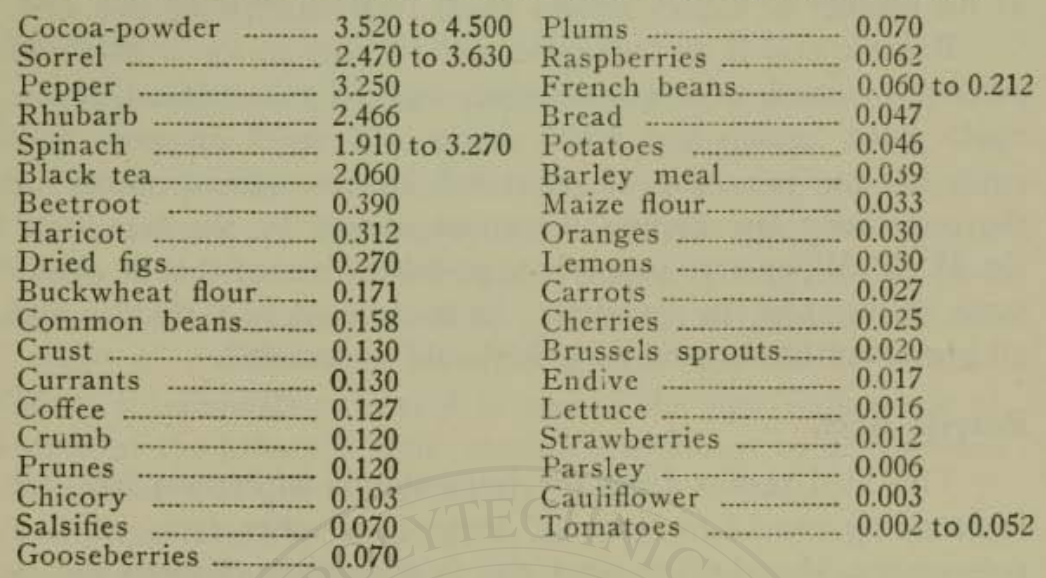

\section{Tannic Acid.}

Tannic acid, a well known astringent, is found in some green leaves used as greens, but in amounts too small to produce noticeable effects. It is also present in minute quantities in certain fruits. But tea, coffee and cocoa contain this harmful acid in large amounts. Tea, for example, contains tannic acid to the extent of one-eighth its weight. A single cup of tea contains seven to fifteen grains of this astringent drug which renders great service to the tanner of hides, but is obnoxious to the stomach and a hindrance to digestion through precipitation of the pepsin of the gastric juice.

Coffee contains tannic acid in still larger amount than tea, roasted coffee containing as much as 45 per cent. of caffeotannic acid, or nearly half its weight. The injurious effects of coffee upon digestion are thus distinctly worse than in the case of tea.

Cocoa contains much less tannin than either tea or coffee.

It is a mistake to suppose that the tannin of either tea or coffee may be avoided by care in brewing. Tannic acid is the most readily soluble of all the constituents of tea and so dissolves out first. The longer the tea is steeped, of course, the larger the proportion of the solid ingredients that will be dissolved.

\section{Benzoic Acid.}

Benzoic acid is found in certain fruits, especially cranberries, plums and prunes. The use of these fruits tends to render the 
tissue fluids and the urine acid. Persons with a tendency to acidosis or who suffer from gout, "gravel" or uric acid calculi of the bladder or kidney should avoid foods containing this acid.

Benzoic acid is an antiseptic, and for this reason it has been extensively used as a preservative, especially in canned fruits, apple cider, catsup and other sauces. A small amount-onetenth of one per cent.-is tolerated by the administrators of the pure food law though not countenanced by the law itself. Dr. H. W. Wiley very properly opposed the use of this unwholesome chemical in any quantity. Its use should be forbidden and all preserved foods containing it should be avoided.

\section{Butyric Acid.}

This acid results from the fermentation of certain fats. It is found in rancid or "strong" butter and other fats. It is a poisonous acid and often gives rise to acidosis in children and in diabetics when an excess of fats is eaten.

In starvation, the body is compelled to live upon its storedup fat, and the results are much the same as when an excess of fats or an exclusive fat diet is eaten. A certain amount of starch or sugar (2 to 3 ounces) is required daily to insure the complete combustion of fats and prevent the development in the tissues of the harmful butyric acid and other allied poisons.

Butyric acid in the food is highly irritating to the stomach glands, exciting an excessive secretion of gastric acid. Persons suffering from hyperacidity or diabetes should carefully avoid the use of strong butter or rancid fats. The butter used by such persons should be freshly prepared from sterilized cream.

Butyric acid is often produced in considerable quantity in the colon when Welch's bacillus or B. butyricus is dominant. There is much foul smelling flatus and the stools have a very strong rancid odor. In such cases the colon should be emptied by an enema twice a day, using sugar water-three or four pints, 2 ounces of malt sugar or milk sugar to the pint-and the flora should be changed as quickly as possible.

Rancid, strong smelling stools in an infant indicate the necessity for reducing the fats of the diet at once.

Boix, of Paris, ten years ago made experiments upon rabbits which demonstrated the toxic character of butyric acid. An animal weighing about nine pounds was given half a gram of butyric acid ( 7.5 grains) daily with its food. It died at the end of four 
weeks, having diminished in weight 40 per cent. Examination showed its liver to be in a state of atrophic sclerosis (gin liver).*

\section{Uric Acid.}

Uric acid is one of the waste or excretory products of the body. It results from the work of the living cells. It is, like other waste products, poisonous to the living tissues, and when it is not promptly and thoroughly removed from the blood by the kidneys it is often deposited in the tissues, giving rise to gout and many other grave disorders. An excess of uric acid is also a cause of renal calculi, so-called gravel and bladder stones. Failure to eliminate uric acid is one of the first indications of disease of the kidneys. This condition is shown by an increase of the axount of uric acid normally found in the blood.

It was formerly supposed to be a part of the duty of the liver to convert uric acid into urea, which is readily eliminated by the kidneys. It is now known, however, that uric acid is an end product of metabolism; that is, that it undergoes no further change in the body. The human liver is unable to convert uric acid into urea. This function is possessed by the livers of carnivorous animals, such as the dog and the members of the cat family; but the liver of man, as well as that of the chimpanzee, orang-outang and other members of the family of primates to which man belongs, do not possess the power to detoxicate uric acid by converting it into urea.

The significance of this fact will be at once apparent when attention is given to the work of Dr. Hall, of Manchester, who made a study of ordinary foodstuffs in relation to their uric acid content, and found that flesh foods of all sorts contain large amounts of this waste product. Beefsteak and lean meats generally were found to contain as much as 14 grains of uric acid to the pound, or 27 grains to 1,000 calories of food value. Sweetbread was found to contain five times as much, or 70 grains to the pound, more than 135 grains of uric acid to 1,000 calories. It will readily be seen that with a flesh diet enormous quantities of uric acid may be thrown into the circulation and thus a very heavy burden may be imposed upon the kidneys. The late Prof. E. G. Janeway called the writer's attention to the fact that the kidneys of the meat-fed house cat are usually found in an advanced state of degeneration.

- Le Foie des Dyspeptiques et en Particulier la Cirrhose par Auto-intoxication d'Origine Gastro Intestinale. By Emile Theodor Boix, Paris, 1895. 
Milk contains no uric acid. Sterile eggs are also free from uric acid; fertile eggs contain uric acid in small amount after the process of incubation has begun. Vegetable foods with few exceptions are practically free from uric acid. Beans contains a small amount 2.5 grains per 1,000 calories or less than one-tenth as much as beefsteak contains. This small amount can be practically removed by parboiling.

TABLE XXV.

Uric Acid in Foods (Hall).

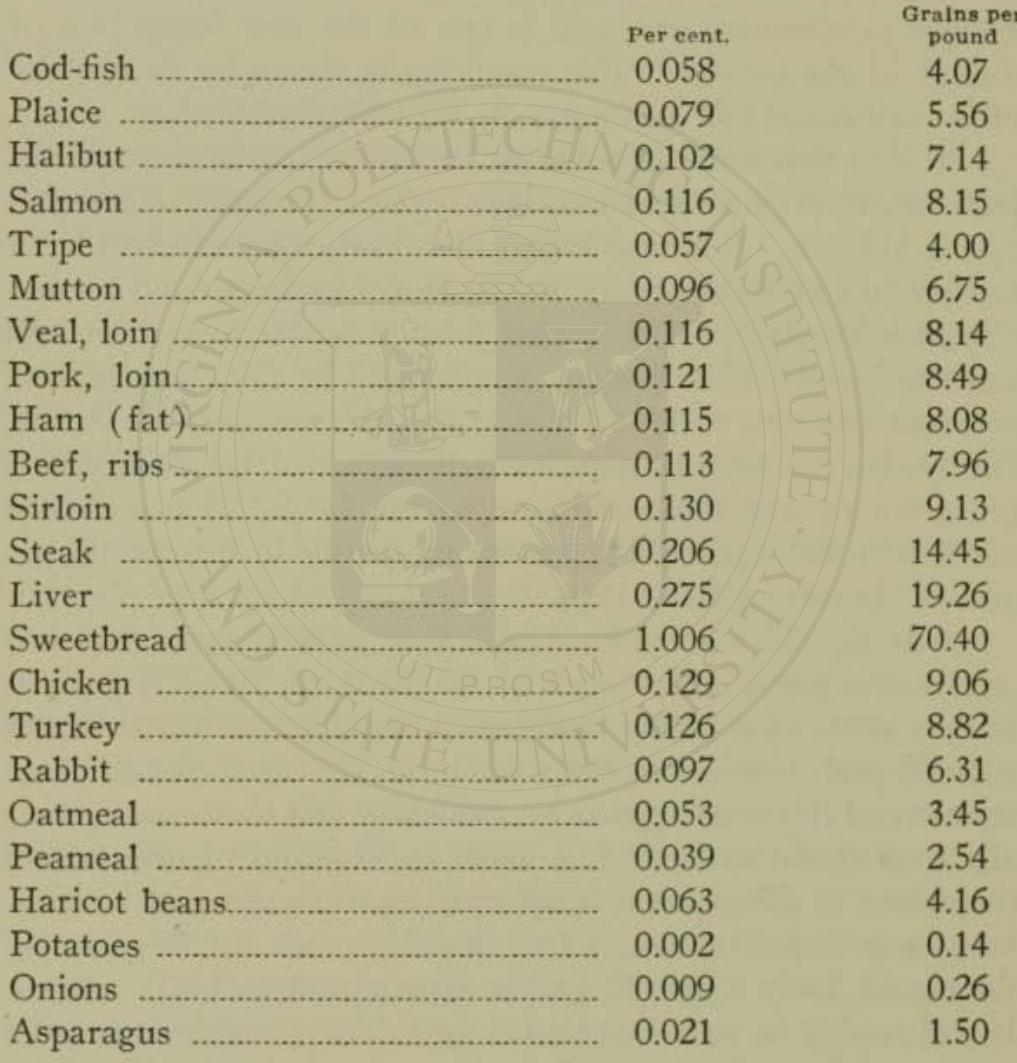

Lactic Acid.

While not a food, lactic acid is non-poisonous, rarely harmful, and renders real service in helping to maintain a healthy condition of the intestines, especially of the colon.

Lactic acid is produced in the body by the fermentation of sugars by certain bacteria. Two beneficent organisms, the 
B. bifidus and the B. acidophilus, take possession of the intestine within a few hours after birth. They produce lactic acid and no harmful substances. The lactic acid formed in the colon stimulates the colon to normal action. So long as these germs remain dominant in the intestine, other germs, especially the germs which cause putrefaction, cannot obtain a foothold. The stools are not putrid and the bowels move normally.

\section{The Toxic Effects of Uric Acid.}

While it is more than probable that many disorders for which uric acid is not responsible have been frequently charged to its account, it nevertheless cannot be denied that uric acid is a highly toxic excretory product and that the tissues should be kept as free as possible from it. Since it is now known that the human liver is not able to convert uric acid into turea or some allied non-toxic substance, it is clearly evident that one who wishes to live long and preserve his liver and kidneys intact to a good old age must eliminate uric acid from his dietary as fully as possible. There is no doubt of the truth of the statement by Tibbles that "the persistent consumption of foods containing a large percentage of purin bodies (uric acid) is apt to lead to their accumulation in the organism. . . A constant irritation of the kidneys by an excess of purins, especially uric acid, may result in chronic nephritis of the gouty variety, with other evidences of chronic gout. The retention of purins in the system is amongst the recognized causes of gout, rheumatic gout, uric acid gravel, uric acidaemia, migrain, neuralgia, sciatica, epilepsy, yascular diseases, and many other conditions of ill-health."

To avoid uric acid it is practically necessary to do nothing more than to eliminate meats from the dietary, for the amount of uric acid found in beans and other vegetable foods is too small to be of any significance.

\section{Mineral Acids}

Until comparatively recent times it was supposed that food acids, like the mineral or fixed acids, tend to acidify the blood and the tissue fluids, and on this account all acids were forbidden in rheumatism and other disorders in which acids were supposed to be present in the body to excess. It is now known that this is an error. The food acids, citric, malic and tartaric, are quickly burned up in the body like starch, sugar and dextrin, leaving 
behind the soda and potash with which they are combined, so that the actual effect of these food acids is to alkalinize the blood and tissue fluids.

In addition to the organic acids above mentioned, foods contain varying amounts of mineral acids, especially sulphuric and phosphoric acids, which are not oxidized or burned in the body and so tend to lessen the alkali reserve of the blood and tissue fluids. In vegetable foods, with the exception of cereals, these acids are associated with an amount of potash, soda and other alkalies much more than sufficient to neutralize the acids, so that they have no effect upon the tissue fluids; but in the case of meats of all sorts including fish and shell-fish and even eggs, the acids are in such great excess that when these foods are freely used the alkali reserve of the body is lessened, as is shown by the highly acid urine of meat eaters. An excess of mineral acids also exists in cereals although to a much less extent. Sansum and many others have called attention to the great injuries which result from a lowering of the alkali reserve, among which are arteriosclerosis, high blood pressure, nephritis and other grave chronic maladies, as well as numerous and profound functional disturbances.

These facts fully justify the exclusion of meats of all sorts from the dietary, the very restricted use of eggs, and care to avoid a diet too exclusively composed of cereals. A bread and meat diet, as McCollum has long contended, is highly objectionable. (For a fuller discussion of this subject, see Chemical Balance, Index.) 


\section{WHOLESOME AND UN- WHOLESOME FOODS}

There is probably no other animal that includes in its dietary so large a variety of substances as does man. In fact, the human race, taken as a whole, exploits the bills of fare of the entire animal world. There can be found scarcely a plant, a bird, a beast, a fish or a reptile eaten by any animal that is not also eaten in some form by some man. In addition, man has produced a great number of artificial foods some of which are denatured to such a degree as to make them unacceptable to many intelligent members of the animal kingdom. There are, indeed, few if any animals that could be induced to eat a spiced pickle or any one of a dozen common dishes which might be named,

And man essays this complex and varied bill of fare without a digestive apparatus adapted to so complicated and difficult a task. Like the man-like apes, the chimpanzee and the orang-outang, he has a simple digestive machine adapted to the digestion of fruits, nuts, soft grains, juicy roots, eggs and milk. His digestive system includes no mechanism corresponding to the gizzard of the barnyard fowl, the quadruple stomach of the bovine, or the seven-gastric-power digestive machine of the whale. He has only the simple, rather feeble stomach of the primate, the aristocrat of the animal kingdom, adapted to the digestion of the choicest and most delicate of nature's tidbits, to dine on the top shelf of Mother Nature's pantry, so to speak, and yet, with this delicate simian stomach, he does not hesitate to tackle the world's bill of fare, to do gastronomic stunts from which the hardiest beast of the forest might well recoil.

Under such treatment, it is no wonder that the human gastric machine has broken down, and that dyspepsia, constipation, and peristaltic woes of various description have become universal in civilized lands. Dinner pills and post-prandial sousings of mineral water have become a part of the national regimen, but even the stout odors of the modern perfumer cannot hide the disgusting flavor of my lady's breath which makes loud advertisement of the fact that there is an embargo in the large intestine with an unsavory accumulation of decaying food residues. 
A return to biologic living is the most pressing economic as well as the most necessary physiologic reform required at the present time.

To eat biologically is simply to eat scientifically, to eat aormally. This the average man seldom does for two reasons: First, he does not know how to do so, having lost largely his nutritive instinct, so that he has no safe guide, such as even the humblest of the lower animals possesses; and second, because wholesome food is in many places almost unattainable on account of the ignorance of cooks and caterers.

The purpose of this section is to present in as concise a form is possible the known facts concerning the individual foodstuffs which experience and scientific research have established.

The author has endeavored to avoid wasting space in commonplace descriptions merely for the sake of completeness. Most of the data concerning caloric value, reaction balance, and iron and lime content, will be found succinctly summarized in tables, thus making this technical data more readily available for reference than if crowded into the text of the book.

\section{The Calorie on the Bill of Fare}

The first bill of fare indicating the food value in calories of zach article served, was computed by the author and used at a dinner given by the faculty of the Battle Creek Sanitarium (1903) to the late Dr. H. P. Bowditch, professor of physiology at Harvard University. The idea was soon developed into the present system, which has now been in regular use at Battle Creek for nearly twenty years, and has been more or less completely zopied in some other institutions. This method provides simple mechanism for the quantitative regulation of the food intake which should come into general use in hotels and restaurants. The Childs Restaurants, of New York and other cities, have adopted the system in part and find it highly practical and useful. Table XLIV, prepared at the cost of much painstaking labor under the writer's supervision, furnishes the necessary foundation for the use of the system.

The calorie, as more fully explained in another chapter, is the modern unit of heat and of its equivalent in other forms of energy. The calorie is a useful measure of food value because food is oxidized or burned in the body, as in a furnace, though by a flameless mode of combustion. 
The calorie value of a food is not, however, the only measure of its nutritive qualities, for there are other food essentials beside food value. A substance may be a good fuel, while a very poor food or even a poison, as in the case of alcohol or gasolene. A food may have a high fuel value and yet possess a low food quality because of indigestibility. Again, a food may be easily digestible, as in the case of polished rice, and of high calorie value, and yet possess inferior merit as an exclusive food because of its lack of vitamins. Thus, in estimating the value of a foodstuff, we must take into account all its properties and not its calorie value alone.

Nevertheless, it is of first importance to keep in mind the calorie values of the foods eaten as well as their content of lime. iron, with other salts and vitamins.

\section{Method of Converting Percentages in Food Composition into Calories Per Ounce.}

As the composition of foods is usually given in percentage, while dietetic prescriptions are usually made in calories, it is useful to know how quickly to convert percentage figures into an equivalent expression of calories per ounce. The caloric value of protein and of carbohydrate is approximately 4 calories per gram, that of fats, 9 calories per gram. These figures allow a small margin for waste through non-absorption. To convert percentage values of protein and carbohydrates into equivalent calories per ounce, it is only necessary to multiply the percentage figures given by 1.136 , or approximately one and one-seventh. The calorie ounce equivalent of fats is found by multiplying the percentage figures by 2.55 , approximately two and one-half.

\section{Changes in Foods Caused by Cooking}

Man has been defined as a "cooking animal" and for ages the culinary art has been highly cultivated and made the means not only of utility but of harmful luxury. Through modern scientific research, we are coming to know that notwithstanding its great service to the human race the art of cookery has associated with it many perils, one of the greatest of which,- the most recently recognized,-is the fact that it destroys vital elements and so modifies the food as to greatly impair its nutrient value. The beasts of the forest, and to a large extent also the primitive savage, take their food directly from the hands of 
Nature, unsophisticated and uninjured, and as a result enjoy an immunity from disease and acquire a vigor and toughness of constitution which are unknown to civilized man. The chef of the future will display his finest talents, not in the compounding of complex combinations of foods with non-foods and poisons, into disease-breeding entrées and desserts, but in selecting and serving in wholesome and attractive ways the pure products of Nature's great food laboratories-the garden and the farm.

According to Professor Jordan, of the University of Chicago, the people of the stone age were not acquainted with the art of cooking and ate their food in the raw or natural state. Cooking utensils only made their appearance a few thousand years ago.

The cook stove is a necessity to meet the artificial needs of our conventional human life. In a primitive normal environment, the cook stove could be easily dispensed with. It is a wonderful convenience and very greatly enlarges our bill of fare, but is not at all a necessity provided the right selection of foodstuffs, those biologically suited to man's needs, can be made.

The use of fire in the preparation of foods dates from prehistoric times. Baking, roasting, boiling, are employed by the most primitive tribes in the preparation of foods. Long before metal vessels were in use, and even in the absence of stone vessels, boiling was done by dropping heated stones into water held in wooden vessels or even tightly woven baskets. Some of the wild tribes of India cook rice by placing it with a quantity of water in a hollow section of green bamboo, and dropping this into the fire. In whatever way cooking is done, its purposes are to change the consistency of the food, to develop palatable flavors and to render it more digestible. Cookery is, in fact, a sort of predigestion as regards the starch content of the food. When moisture and heat are applied to starch, it swells up, the granules rupture and the raw starch is converted into a simple form of dextrin known as amylo-dextrin, which gives to gelatinized starch or starch paste its adhesive properties. As ordinarily employed in cookery, starch is gelatinized at a temperature considerab'y below the boiling point. Different starches require different temperatures for gelatinization.

In cooking with water or steam, the cellulose is softened and the gums dissolved and pectose is gelatinized. This permits the 
food particles to fall apart. For this reason, cooked food is more readily soluble in the stomach and sooner passes out into the intestine.

The temperature required for the gelatinization of different starches is shown in the following table (Grindley):

Sats
Barley
Rye
Wheat
Rice $176^{\circ} \mathrm{F}$. $176^{\circ} \mathrm{F}$. $176^{\circ} \mathrm{F}$. $167^{\circ} \mathrm{F}$. $149^{\circ} \mathrm{F}$.
Maize
Potato

It is interesting to note that potato starch, which when cooked is digested by the saliva much more easily than the cereal starches, is also much more readily dextrinized by heat.

A higher temperature, such as that to which bread is subjected in baking, still further dextrinizes the starch, but no amount of heat will convert it into sugar, although starch as well as sugar is caramelized by a sufficiently high temperature.

It is evident, then, that the effect of cooking upon starch is to render it more readily digestible. This may or may not be an advantage. In the case of protein, digestibility is decreased except in the cases of whites of eggs. Lean meat, like the albumin of egg, is hardened by a temperature of $160^{\circ} \mathrm{F}$. or more. The white of egg exposed to heat just sufficient to curdle it is thereby rendered easily digestible, while the raw white of egg passes through the alimentary canal with little change, being practically indigestible. When white of egg is exposed to a higher temperature, as in boiling, the albumen is hardened to such an extent that it becomes difficult of digestion.

The same is true of meat and other albumins. The heat to which they are exposed in boiling, roasting, frying, etc., renders these nitrogenous substances less digestible than in their native state.

Fats through exposure to heat are likewise rendered less digestible. Emulsions exposed to heat tend to break down. The fat globules when heated run together, forming large masses which are less easily digestible and must be again emulsified in the process of digestion.

Sugars are not changed by cooking unless they are exposed 
to a temperature above $212^{\circ} \mathrm{F}$. At higher temperatures, the sugar is caramelized to some extent and its sweetness is lessened.

When cane sugar is boiled in the presence of acids, an inversion takes place which lessens its sweetening properties.

Exposure of foods to the boiling temperature destroys the antiscorbutic vitamin and may lessen the value of other vitamins present.

Boiling foods in water, if the water is thrown away, robs them of vitamins, soluble salts and other valuable elements.

It is thus apparent that the cooking of food has its disadvantages as well as advantages. It cannot be denied, however, that the bill of fare which man everywhere has adopted would be practically impossible without cookery. The cereals which constitute the staple food of both civilized and semi-civilized sections of the human race are in their native, dry state unsuitable for human sustenance. The same is true of most of the succulent roots which enter into our ordinary dietary.

Prolonged maintenance of the body in a state of health and fitness on a diet of raw foods is possible only when the foods are judiciously chosen. Such a bill of fare would necessarily consist of ripe fruits, nuts, tender shoots, succulent roots containing little or no starch, such as the turnip and the ground-nut and cereals in the milk state, with milk and eggs.

Eggs taken in a raw state could hardly be regarded as wholesome because of the indigestibility of the raw white of egg, recently discovered, and the increase of intestinal putrefaction when the raw white of egg is eaten.

Meat is digestible in a raw state even more readily than in the cooked state, but is readily accepted by no member of the primate family except when hard pressed by hunger.

According to Professor Snyder, 100 pounds of cabbage contain 7.5 pounds of solids, of which more than one-third, 2.5 to 3 pounds is lost when the cabbage is cooked in water.

Carrots cut in small pieces and cooked in water lose 20 to 30 . per cent, of their weight. Potatoes lose little if cooked in their jackets.

Spinach has a solid content of 10 per cent., of which nearly one-fourth is lost when the spinach is cooked in water and drained.

Celeriac has a solid content of 9.5 per cent., of which half is lost by boiling in water. 
TABLE XXVI.

Showing Percentage of Water and Solids in Various Articles of Food Before and After Cooking.

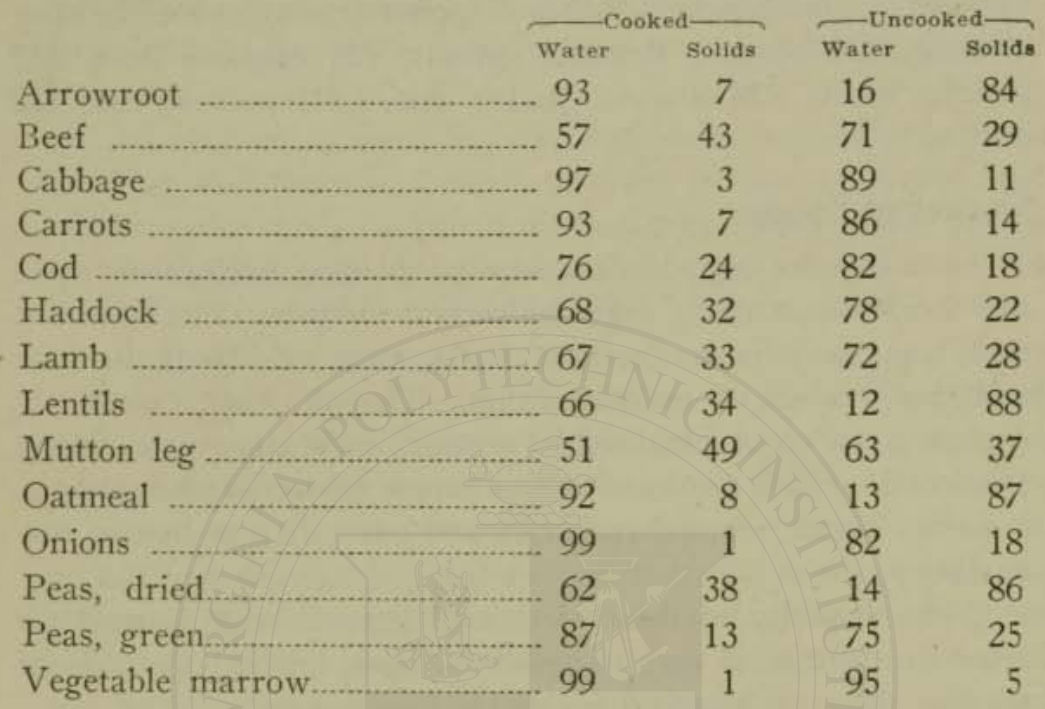

The following table (Grindley) shows the amount of water in raw vegetables and in the same vegetables after boiling:

\section{TABLE XXVII.}

Showing the Gain of Water by Vegetables in Cooking.

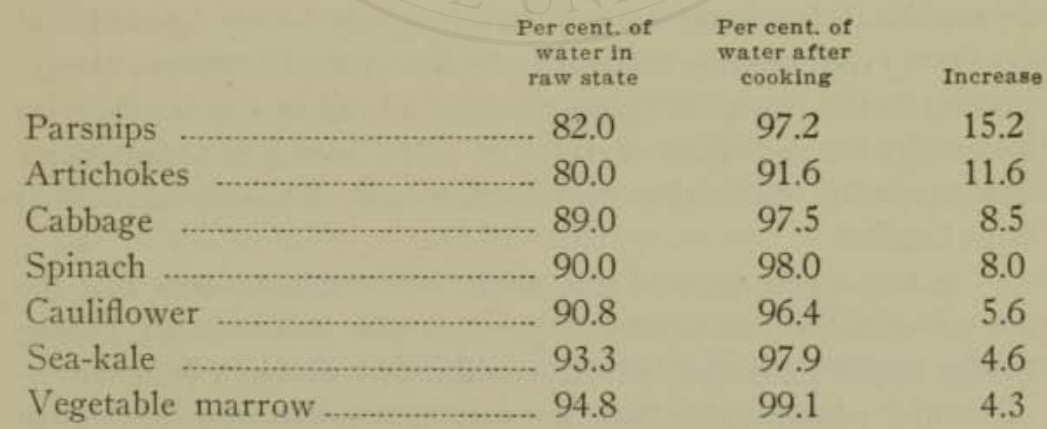

Curly greens, borecole, loses half the weight.

When boiled in water, rice, if the water is poured off, loses so large a proportion of its valuable nutrients that native Indian 
soldiers preferred the liquid drained off, leaving the residue for the British.

Vegetables in general are more weighty after cooking than before. If there is loss in weight it is because of the loss of nutrients. One hundred ounces of green artichokes weigh, after cooking, 336 ounces; Brussels sprouts, 121 ounces; leeks, 252 ounces; lentils, 238 ounces; Quaker oats, 1,110 ounces ; rice, 418 ounces.

\section{Uncooked Foods}

Some years ago, the practice of feeding exclusively upon raw foods was strongly urged by certain faddists. The argument used was that exposure to heat destroys the vitality of the food and thus lessens its nutrient value. The idea was extensively exploited and commercialized by certain adventurers under whose unscientific instruction hundreds of people subjected themselves to a dietary better adapted to horses and oxen than to human sustenance.

The majority of these victimized persons soon learned the error of their ways and returned to more rational methods of feeding, often after suffering more or less damage.

Not infrequently, however, persons who adopted the raw food diet found themselves appreciably benefited by the change, doubtless because their diet had previously been deficient in vitamins.

Another advantage of the raw food diet which may, perhaps, be one of its chief merits is the fact that it supplies to the colon a considerable amount of raw starch which, being digested and converted into dextrin and sugar by the unused ferments always present in the feces, furnishes the kind of nutriment necessary to encourage the growth in the colon of acid-forming or fermentative bacteria, thus combating putrefaction and encouraging normal bowel action.

It may fairly be said that while it is doubtless true that the art of cookery has rendered possible the existence of human beings under conditions which would otherwise have been impossible, and has probably been the means of preserving the human race under circumstances which might otherwise have caused its extinction, this art has nevertheless wrought much mischief through the unwise manner in which it has been employed. The art of cookery has been used not only to render food more digest- 
ible, but more often to lessen its digestibility and to transform the simple, wholesome products of Nature into noxious, diseaseproducing mixtures.

The fact that primitive man took his food in a raw or uncooked state is sufficient evidence that it is possible to subsist upon foods in the state in which they are provided by the hand of Nature.

In addition, it must be said that there is good ground for believing that there are certain qualities possessed by raw foods which render them an important and even indispensable part of the normal diet, even though one may not go so far as to exclude cooked foods altogether from his diet.

According to Hon. W. N. Beaver, for many years a magistrate in Papua, New Guinea, the natives of Kiwai formerly ate their rice raw. The Scotch Highlanders have from time immemorial eaten their oatmeal simply scalded.

A person who desires to live upon a raw diet, in arranging his bill of fare cannot base his selection upon the supposition that all raw foods are complete nutrients, but must possess a sufficient knowledge of the newer facts pertaining to nutrition to enable him to make such combinations of foodstuffs as will constitute an aggregate complete in all the elements required for perfect nutrition and in adequate quantities; in other words, a diet which supplies, in addition to a sufficient amount of fats, carbohydrates, salts and cellulose, a proper proportion of complete proteins and an abundant supply of the various vitamins essential to good nutrition. It is thus apparent that a diet consisting exclusively of fruits and nuts might not meet these requirements. Such a diet would meet all nutritive needs as regards proteins, fat and carbohydrates, but might be notably deficient in roughage, vitamins and salts. Certain nuts, if taken in sufficient quantity, would furnish the needed quantum of iron and lime, but there would still be a deficiency of cellulose and vitamins.

For a complete dietary, it would be necessary to add to fruits and nuts a liberal supply of green leaves in the form of greens, lettuce or similar foodstuffs. On such a diet, carefully selected, life might be well sustained for an indefinite length of time.

Jolles, of France, has shown that the blood contains ferments which he calls katalases, the function of which is to set free oxygen from the oxyhemoglobin. These ferments are found in healthy blood in large quantities, but they are greatly diminished 
in tuberculosis, nephritis, carcinoma. The intensity of oxidatior depends upon the amount of katalases present. It is possible that the reducing ferments which abound in raw fruit and vegetable juices may be of service to the body in conditions in which the oxidizing ferments are deficient in the blood.

There is some ground for believing that the remarkable in fluence upon nutrition exercised by the juices of fresh or un cooked fruits and certain vegetables is due to certain enzymes which are destroyed by cooking. Some of these enzymes very closely resemble those which are found in the body. For example Professor S. H. Vines reported in 1902 the discovery of ferments in plant juices capable of digesting albumin. The same investi gator later discovered that these proteolytic ferments are founc in pineapple juices, in the milk of the coconut, and in the juices of melons, cucumbers, tomatoes, grapes, pears, oranges, bananas lettuce, cabbage, green peas, onions, potatoes and turnips.

By means of cookery, grains and vegetables can be made tc furnish adequate quantities of the chief elements required tc satisfy our nutritive needs, and by supplementing these foodstuff: with greens and dairy products and an adequate supply of fresh uncooked foods in the form of fruit, celery, lettuce and other salad plants, it is possible to meet nutritive needs at a minimur of expense and inconvenience.

Finally, it is to be said that while we cannot easily dispense with cookery, we must take care to use the art in such a manne1 as to avoid the injuries which it is capable of producing, and musi not neglect to include in the bill of fare a sufficient amount of natural foodstuffs "untouched by fire," to make certain of sup plying to the body an ample amount of the subtle products which. are found in natural foodstuffs - such as vitamins-and which are damaged or destroyed by cooking. An exclusive diet of fresh or uncooked foods may be adopted for a change now and ther. with advantage. Taking such a diet for a day or two every twc or three weeks is an excellent means of stimulating intestinal activity and keeping the intestinal flora in good condition, and in some cases of obstinate constipation a continuous diet largely made up of fresh foods is found highly beneficial.

The gorilla, the chimpanzee and other anthropoids, thrive on raw foods and obtain marvelous strength and vigor. Doctor Akeley states that the gorilla subsists almost exclusively on bamboo sprouts. 


\section{Cereals}

The cereals are seeds for the most part derived from grasslike plants. The most important cereals are rice, wheat, oats, corn, rye, barley and millet, to which may be added buckwheat and the less known quinoa and kaoliang.

The cereals are among the oldest of foodstuffs used by man. Very likely they were first used in a half ripened, the so-called "milk" state, when they are very palatable and readily digestible. Some varieties of green corn are quite as palatable without cookery as with. In tropical countries cornfields are often robbed by apes and baboons, whose dietary is believed to be essentially the same as that of primitive man.

Cereals, like most other seeds, consist of three essential parts : (1) a germ which represents the future plant; (2) a store of nutrient material consisting of a considerable volume of starch, a smaller amount of protein and a still smaller quantity of fat. together with ferments for the digestion of the starch and protein and vitamins for the promotion of the growth of the new plant, with food lime, food iron and other salts; (3) a protective covering to preserve the stored nutrient material from injury by the elements and the attacks of bacteria and insects.

An interesting fact which has received little attention until very recent years is that the several essential elements named, all of which are essential not only for the proper nutrition of the plant but equally for the nutrition of the eater of the seed, are not homogeneously distributed throughout the seed but are very unequally placed. For example, the carbohydrates, starch and sugar are almost wholly found in the center of the kernel; the vitamins and salts are, on the other hand, found chiefly in the layers of cells placed just beneath the outer covering of the seed. Certain of the vitamins are found almost altogether in the germ and in the bran.

From the foregoing it is plain that if seeds are to be used as food the whole seed must be employed. The covering of the seed, or bran, and the germ contain precious elements which are essential for complete nutrition. Ignorance of this fact has resulted in the death of many thousands of persons annually in rice-eating 
countries. It is stated on good authority that until the importation of polished rice was forbidden not less than 15,000 people died annually from beri-beri in the Philippine Islands alone.

In general, the cereals are deficient in lime even when the outer covering or bran is not removed. The fat-soluble vitamin, one of the most important growth-promoting vitamins, the absence of which produces night blindness and other evidences of degeneration, even complete destruction of the eyes in rats and other animals when fed exclusively on a grain diet, is lacking in cereals except in the germ. By sprouting the grain, however, the amount of vitamins present may be increased to so great an extent as to supply the deficiency.

\section{The Protein of Cereals.}

Some years ago Rubner reported experiments which seemed to demonstrate that there is a great difference in the cereals as regards the nutritive value of their proteins. The proteins of corn were thought by Rubner to be much inferior to those of wheat. Recent observations made by Sherman, and later by Osborne and Mendel, have shown that there is little or no difference in the nutritive value of the proteins of wheat, corn, oats, rye and barley. Sherman showed that nine-tenths of the protein might be derived from any one of the cereals named provided the remaining tenth was obtained from milk or apple. The protein of milk is known to be very excellent in character, superior in fact to meats of any sort, and the protein of the apple appears to be about equal in value.

\section{The Iron Content of Cereals and Breads.}

It is first of all worthy of remark that while cereals are as a class poor in lime, they are comparatively rich in iron. A pound and a quarter of graham bread will supply a day's ration of iron, while four times as much would scarcely furnish the lime required for one day to make good the body's loss of lime salts. Even corn, which is so deficient in lime as to require 8 pounds for one day's lime supply, contains sufficient iron for one day's needs in a little more than a single pound.

The average iron content of all the cereals and breads is sufficient to supply in one ounce or in 100 calories more than one twenty-fifth of a day's needs.

Of all the cereals, the highest in iron content is the entire wheat, of which 11 ounces supply a day's iron ration. Barley, 
rye, oatmeal, and graham flour come next and in the order named, supplying three-fourths as much.

Graham bread contains three times as much iron as does white bread, 25 per cent. more than pumpernickel, 50 per cent. more than entire wheat bread, rye bread and soda crackers.

Graham bread supplies in 14 ounces a whole day's iron ration or four times as much iron as polished rice, white flour, or hominy, more than five and a half times as much as cornmeal, four and two-thirds times as much as farina, three times as much as macaroni, buckwheat flour or rye flour, and twice as much as pumpernickel, pearled barley, brown rice or tapioca.

Boston brown bread makes a better showing than any other of the breads because of the molasses in its composition, furnishing in a seven-ounce loaf iron enough for a day. As a practical source of food iron, oatmeal deserves special mention, 14 ounces supplying a day's ration.

The cereal foods which show so great a deficiency in iron as properly to put them in a list of foods only to be eaten when adequately supplemented by foods extra rich in iron, are white bread, cornmeal (new process), farina, hominy (toasted corn flakes are made from hominy grits), macaroni, buckwheat, polished rice (toasted rice flakes and puffed rice) and tapioca.

\section{The Lime Content of Cereals.}

Of the six great cereals, wheat, corn, barley, rye, oatmeal, and rice, the last named feeds many millions more of human beings than any of the others. The countless millions of the Orient are fed chiefly upon rice. Both polished and brown rice are in use throughout the densely populated countries of Asia, from Korea to Java. Of all the cereals and the several seeds which serve food purposes, rice contains the least lime, only onefourteenth of a grain ( 0.073 grs.) per ounce in brown rice and one-eighteenth ( 0.055 grs.) in polished rice. Wheat contains four times as much lime as brown rice, rye five times as much, and oatmeal six times. Corn is the only rival of rice in poverty of lime, having but a little more than one-fourth as much lime as has oatmeal.

Instinct seems to have informed the Orientals of the deficiency of rice in lime salts, for they invariably combine with it a liberal amount of legumes of some sort or of greens or fresh or salted and dried turnips, all of which foods are very rich in lime salts. 
It is true, as stated above, that wheat contains four times as much lime as rice, but this is only true of "entire" wheat, that is, of wheat, wheat meal, or graham flour which includes the bran and "shorts." Fine white flour contains less than half as much lime as does entire wheat and only one-sixth as much as does wheat bran. Its lime content is but little above that of rice and is just equal to that of corn and pearled barley, one-eighth grain of lime to the ounce $(0.122$ grs. $)$. More than 7.5 pounds of fine flour, whole corn or pearled barley, or 10.5 pounds of fine flour bread, are required to furnish one day's ration of lime. The polished rice in common use in this country is so poor in lime that 17.5 pounds barely suffice to furnish the 15.4 grains required to meet our daily needs.

Evidently a rice diet is highly deficient in bone-making material and must greatly impoverish the blood and tissues in lime content when adhered to for more than very brief periods.

The United States Government has prohibited the importation of polished rice into the Philippine Islands for the protection of the Filipinos from beri-beri and lime starvation, but no protection is afforded the loyal citizen of this country against the same danger. Every American citizen is permitted to indulge in lime starvation to the full extent of his ignorance and inclination.

The rice question is not, however, of such commanding importance in this country as in the Philippines, because rice is here used as a delicacy or a luxury rather than as a food staple, as in the Philippines. This may be due in part to the higher price of rice as compared with other grains, but is perhaps still more due to general lack of acquaintance with the many superior qualities of rice, a grain which aside from its deficiency in lime is undoubtedly the finest of all the cereals. Nevertheless, the consumption in this country of billions of pounds of rice annually, involving a deficiency of thousands of tons of lime in the national bill of fare, is one very real cause for the increasing prevalence of bone diseases and teeth decay in the United States, at least in certain sections.

A danger of identical character exists in certain sections of this country, especially in the South, where cornmeal is almost as important a food staple for large groups of the population as is rice in the Philippines and in South China and the Malay Archipelago. Corn pone is the chief item in the bills of fare of some millions of negroes and small white farmers in Alabama, 
Tennessee, Georgia, Kentucky, Louisiana, Arkansas, and the Carolinas. A pound of cornmeal per capita is probably not an overestimate of the amount daily consumed by the classes indicated. The 16 ounces of corn should supply at least 12 grains of food lime, but actually do furnish one and three-fourths grains ( 1.76 grs.), or about one-seventh $(0.15)$ the amount required. In view of this fact, it is no wonder that these poorly fed people present many evidences of physical deterioration.

It is still a question with the physiologists who are making a study of pellagra whether this disease may not be due in part, at least, to a deficiency of lime. The free use of molasses which contains more than a grain and a half of lime to an ordinary serving of one and one-fourth ounce, may to some degree mitigate the evils which might otherwise follow the use of a foodstuff so greatly lacking in lime salts.

The same may be said for the use of milk in connection with both rice and cornmeal. The combination of molasses or milk with cornmeal makes good its deficiency in lime, but milk adds also other essential elements and so is greatly preferable to molasses.

Still more deficient in lime is hominy, and also corn flakes which are made from grits, a corn product which represents the interior of the kernel wholly separated from the outer, limecontaining layers. Hominy is even more deficient in lime than is brown rice, containing, in fact, but little more lime than does polished rice. For a day's supply of lime one would have to eat more than 14 pounds of hominy or corn flakes. Instead of 12 grains of lime to the pound, as in milk; hominy and corn flakes contain only one grain.

Buckwheat flour is another defective food, containing but little more lime than polished rice, less than one-sixteenth of a grain per ounce ( 0.061 grs.). Fortunately, perhaps, the conventional buckwheat cakes served hot from the griddle as a breakfast dish are usually eaten either with molasses or maple syrup, botb of which are rich in lime, as is also malt honey.

\section{Breads Are Deficient in Lime.}

All the breads, with the exception of Boston brown bread and bread made from the entire rye meal, the pumpernickel of central Europe, are notably deficient in lime. Even graham bread contains only one-sixth the normal proportion of lime (which 
is three-fourths grain to 100 calories) and rye bread has scarcely half the ideal proportion.

As a class, the cereals are notably deficient in lime and must be supplemented by other foodstuffs rich in lime salts. Fortunately, such foods are not lacking. By the use of the nuts and fruits already mentioned as desserts, and by the regular addition to the bill of fare of legumes, greens and milk products, any deficiency may be easily made good.

But we must not close our discussion of the cereals without a further word concerning oatmeal, the richest of all the cereals in lime salts. An ounce of oatmeal supplies a little less than half a grain of lime $(0.425$ grs. $)$. In Scotland, where from very ancient times oatmeal has been the great food staple of the peasantry, it constitutes so large a proportion of the total food intake that it becomes a highly important source of lime salts. The Scotchman eats oatmeal not only in the form of brose, a dish resembling mush made by pouring hot water on oatmeal which is thus scalded but not well cooked as in this country, but also as bannocks, scones and other forms of bread.

Whatever the oatmeal lacks for supplying the full quota of lime is made up by the cabbage, greens, kale and buttermilk which are universally and freely used by the shepherd farmers of the Scotch Highlands. In the Highlands, a common practice is to scald the oatmeal with the water in which cabbage, kale or collards has been cooked. When thus prepared, the porridge, known as kale brose, is extra rich in lime salts and affords another example of the instinctive demand for mineral food elements made by the natural appetites of human beings who have not lost their normal instinctive guides through the cultivation of unnatural tastes.

The good results of this liberal supply of food lime are well shown in the tall and brawny figures of the Scotch Highlanders and doubtless accounts for the world-wide reputation for vigor and toughness enjoyed by these hardy grain feeders.

In the United States oatmeal is comparatively a new acquaintance. It was not until the Centennial Exhibition at Philadelphia where oatmeal was very adroitly exploited as "Avena" that this cereal came into general favor as a breakfast food in this country.

In more recent years, oatmeal has been largely displaced by toasted flakes and other breakfast foods made popular by adroit 
advertising. As eaten, the actual amount of the dry material in an ordinary serving of corn flakes or rice flakes is little more than an ounce and the amount of lime supplied is too small to cut any figure of importance in the lime ration.

An important cereal product which has long been used by farmers in the feeding of horses and cattle, but has only recently come into use by human beings, is wheat bran. While low in caloric value, as its chief constituent is cellulose, bran is a most valuable supplementary food, not only as a source of bulk tc stimulate the intestines, but as a source of lime and iron. Every ounce of bran contains three-quarters of a grain of lime, sufficient to balance one hundred calories of food principles. By the addi tion of sterilized bran in liberal quantity, the deficiency in lime of various cereal preparations, breads, mushes, etc., may be made up. Bran may also be added to potatoes and other vegetables which are deficient in lime.

Finally, it must be said concerning the cereals that while they are the world's staple foods for working animals as well as for working men, they are perhaps made to constitute a some. what too large proportion of the bill of fare in most civilized countries. As pointed out by Bunge many years ago, and con firmed by other authorities, the cereals contain an excess of the fixed acids, phosphoric and sulphuric, and on this account when used too freely they upset the nice balance between acids and bases or alkalies in the body, and produce a relative acidosis to which numerous ailments are attributed. This tendency may be successfully combated by the liberal use of fruits and vegetables especially potatoes, which contain an excess of bases, although greatly deficient in lime, a fact which must be borne in mind and which requires compensation by the addition of a suitable amount of foods rich in lime salts, such as milk, beans, lentils and greens.

\section{WHEAT.}

Wheat is one of the oldest of our cereal foods. It has come down to us from prehistoric times. According to Chodat, the ancestor of wheat was the wild emmer, a grass plant which is still found growing wild in Asia Minor.

Wheat is perhaps the most important of our American cereals, although in size the annual crop produced is much in ferior to corn. The whole grain contains about one-eighth its weight of protein, 70 per cent. starch and 2.4 per cent. cellulose 
It is deficient in certain vitamins; and hence should not be used as an exclusive food.

Analysis of Wheat and the Products of Roller Milling.

The gluten of wheat, which comprises almost the entire protein content, consists of about equal parts of gliadin, a vegetable gelatine, and glutenin. It is obtained by washing out the starch.

As produced by Nature, the wheat berry contains no elements which are not essential for nutrition. Rats fed on whole wheat flour thrive and produce young, while rats fed on white flour do not thrive and fail to produce young, or if they do, the progeny are feeble and do not thrive. Dogs fed exclusively on bread made from patent roller flour died on an average at three weeks, whereas dogs fed exclusively on whole wheat flour were hearty and thriving at the end of 30 days. Pigeons fed on fine flour bread soon lose weight and develop beri-beri. If the diet is continued they die, but if fed on whole grain, or whole grain with the addition of yeast, they recover. There can be no doubt that the outer covering of the grain, that is, the bran, is necessary for complete nutrition. Tibbles, an eminent English authority, says with reference to fine flour:

The miller and the baker have impoverished the flour and bread of some of the most important constituents of the wheat grain, viz., the vitamins, enzymes, activators, amino acids and lipoids, which only exist in the germ and subpericarpal layers of the bran of wheat. The removal of these substances does not matter very much in the case of well-to-do families, where fresh milk, animal foods and vegetables containing vitamins are constantly a part of the daily diet. But it matters greatly to the children of the poor, whose chief diet is white bread.

(United States Department of Agriculture.)

\begin{tabular}{|c|c|c|c|c|c|}
\hline Milling Products & $\begin{array}{l}\text { Water } \\
\text { per cent. }\end{array}$ & $\begin{array}{c}\text { Protein } \\
(\mathrm{N} \times 5.7) \text {, } \\
\text { per cent. }\end{array}$ & $\begin{array}{c}\text { Fat } \\
\text { per cent. }\end{array}$ & $\begin{array}{l}\text { Carbo. } \\
\text { hydrate, } \\
\text { per cent. }\end{array}$ & $\begin{array}{l}\text { Ash } \\
\text { per cent. }\end{array}$ \\
\hline First patent flour . & 10.55 & 11,08 & 1.15 & 76.85 & 0.37 \\
\hline Second patent flour ...... & 10.49 & 11.14 & 1.20 & 76.75 & 0.42 \\
\hline $\begin{array}{l}\text { First clear grade flour } \\
\text { Stright or standard }\end{array}$ & 10.13 & 13.74 & 2.20 & 73.13 & 0.80 \\
\hline $\begin{array}{l}\text { Straight or standard } \\
\text { patent flour }\end{array}$ & 10.54 & 11.99 & 1.61 & 75.36 & 0.50 \\
\hline $\begin{array}{l}\text { Second clear grade } \\
\text { flour }\end{array}$ & 10.8 & 15.03 & 3.77 & 69.37 & 1.75 \\
\hline "Red dog" flour ............. & 9.17 & 18.98 & 7.00 & 61.37 & 3.48 \\
\hline Shorts & 8.73 & 1487 & 6.37 & 65.47 & 4.56 \\
\hline Bran & 9.59 & 14.02 & 4.39 & 65.54 & 606 \\
\hline Entire wheat flour ......... & 10.81 & 1226 & 2.24 & 73.67 & 1.02 \\
\hline $\begin{array}{l}\text { Graham flour } \\
\text { Wheat ground in }\end{array}$ & 8.61 & 12.65 & 2.44 & 74.58 & 1.72 \\
\hline $\begin{array}{l}\text { Wheat ground in } \\
\text { laboratory }\end{array}$ & $\begin{array}{l}8.50 \\
8.73\end{array}$ & $\begin{array}{l}12.65 \\
27.24\end{array}$ & $\begin{array}{r}2.36 \\
11.23\end{array}$ & $\begin{array}{l}74.69 \\
48.09\end{array}$ & $\begin{array}{l}1.80 \\
4.71\end{array}$ \\
\hline Germ & 8.73 & 27.24 & 11.23 & 48.09 & 4.71 \\
\hline
\end{tabular}




\section{Bread Making.}

Bread is perhaps the oldest of all prepared foods. According to Pliny, the earliest Romans were wholly unacquainted with bread, but used instead a porridge prepared from parched grain. This simple mode of cooking cereals is still in vogue in widely separated parts of the world, as among the North American Indians and the wild natives of Mexico, who still show great preference for parched corn mixed with water, while the gofio of the Canary Islanders is another example of the same. The ancients prepared their bread from crushed cereals mixed with water, with or without the addition of oil. The art of bread making was known in ancient Egypt, but was known still earlier in Switzerland, as shown by the relics found in prehistoric lake dwellings, which include not only grinding stones for making flour but charred loaves of bread.

The sweetest and most wholesome bread is that made from the whole meal of the grain mixed with water, with perhaps the addition of a little salt, and baked in thin cakes or small loaves. Bread is made in this way by the native Mexicans, the wandering Arabs, tribes in northern India, and various other primitive people. The beaten biscuit of Virginia, famous for toothsomeness, is a water bread in which the dough is made to rise by air well beaten in with a hammer.

Yeast bread is not unwholesome when prepared from good yeast; in fact, the yeast may possibly add something to the proportion of vitamins contained in the bread. When eaten warm, however, raised bread is objectionable on account of the fact that the center of the loaf is in a pasty condition and in chewing is rolled into small masses which are not easily penetrated by the digestive fluids. Bread should always be allowed to stand a sufficient length of time to permit the center or crumb of the loaf to become friable so as to crumble when rubbed between the thumb and finger instead of forming a pasty ball.

Some alcohol is produced in the raising of yeast bread, but this is practically all driven off by the heat to which the bread is exposed during the process of baking. If bread is not sufficiently baked, the yeast and other ferments will not be killed, some alcohol will remain, and the result will be a continuation of the fermentation by which the alcohol will be converted into vinegar; in other words, the bread will sour. Bread, properly baked, will have a hard, friable crust on all sides. 
Recent observations by Wahl have shown that bread is improved by the addition of buttermilk or sour milk, the lactic acid present serving to dissolve the phosphates of the flour, to render the proteins more soluble, and to otherwise improve the quality of the bread. In European countries nut meats are added to bread with advantage.

Bread should always be wrapped with paraffin paper at the bakery and before it has had any opportunity for exposure to insects or contamination by the hands of workmen. Automatic wrapping machinery is now employed for this purpose in all firstclass bakeries.

\section{Baking Powders.}

The best baking powders are prepared from bicarbonate of soda and bitartrate of potash. In the chemical reaction which occurs between these two chemicals carbonic acid gas is set free. This, in penetrating the loaf, forms small cavities through the tenacity of the gluten and thus makes the bread light. The chemical combination of the constituents of the baking powder results in the formation of rochelle salts, a laxative drug. The use of baking powder is doubtless preferable to the old-fashioned soda or saleratus and sour milk method still much in use in the Southern states, but is more or less objectionable not only because it leaves an unusable chemical substance in the bread but because the alkalies present damage the vitamins, as pointed out by Funk.

Count Rumford long ago showed how to raise bread by the use of hydrochloric acid (C. P.) and bicarbonate of soda. The acid and soda combine, setting free the carbonic acid gas and leaving as a residue only a small amount of chloride of soda or common salt, the quantity being less than is commonly used as seasoning. The proper proportions are 80 minims of acid to a level teaspoonful of soda bicarbonate for two cups of flour. The dry soda is well mixed with the flour and the acid is added to the liquid, which should be cold. The ingredients are then mixed as speedily as possible and put at once into a hot oven. The amounts of soda and hydrochloric acid given above are equivalent to four level teaspoonfuls of baking powder.

Baking powders are superfluous as aids to good cookery. They are convenient, but non-essential. The very best and sweetest bread possible may be made without them. What could be more toothsome, for example, than the famous "beaten" bread of 
Virginia or the hoe cake of the South, a mode of bread making learned from the North American Indian. The tortillas of Spanish America and the unleavened cakes of the Arabs are other well known examples of toothsome bread made without yeast or baking powder and used by millions of people with entire satisfaction.

It cannot be claimed by any one possessed of a modicum of knowledge of human physiology that the daily use of a cathartic drug even in small amount can be otherwise than harmful. The use of baking powders may be, indeed, an insidious cause of constipation.

Says Professor A. E. Taylor, of Philadelphia:

We must not, however, be oblivious to the fact that a saline cathartic residue results from the reaction of every form of known baking powder now commonly employed.

Underhill has shown that rochelle salts cause nephritis in animals. It is reasonable to believe that the long continued use, even in small quantities, of a drug that in somewhat larger doses will cause inflammation of the kidneys in animals cannot be a wholesome practice. At least the avoidance of baking powder products by persons suffering from chronic nephritis may be insisted upon as a wise and reasonable precaution.

\section{Wheat Bran.}

One-fifth of the weight of wheat consists of bran, but this small portion contains three-fourths of all the salts and nearly all the cellulose of the entire berry. Whole wheat contains 4 grains of lime to the pound, fine flour only 1 grain, and lime 12 grains.

A pound of whole wheat flour contains 37 grains of potash. A pound of bran contains 119 grains of potash, while a pound of fine flour contains only 11 to 12 grains of this important element.

Bran contains one-twentieth of its weight of salts, onethirtieth of its weight of phosphate of potash, and 7 to 11 times as much salts as are found in fine flour.

Ordinary bran contains about one-third cellulose, 37 to 100 times as much as does fine flour. Another third consists of starch. In addition, there are about 3 per cent. fat and 12 per cent. protein. 
It is astonishing that we should habitually throw away such a large amount of most valuable food material. The evil consequences of this waste are simply enormous, really beyond estimate.

The loss of potash, according to Dr. Ross, an eminent English authority, predisposes to cancer, and is perhaps one of the leading causes of cancer. The loss of the cellulose which is necessary to give bulk to the food leads to constipation, a veritable mother of maladies. By the use of one-half to one ounce of bran, daily, regular bowel movement may be secured in the majority of cases of constipation. Many obstinate cases are relieved by adding simple exercises and the use of paraffin oil.

Decay of the teeth is often a result of loss of lime from the body. The teeth are robbed of their lime to supply the deficiency resulting from the use of fine flour bread, cane sugar, meat, and other foods deficient in lime. Half an ounce of bran taken daily with other food will aid in supplying the body with necessary mineral elements and thus help to check decay of the teeth and injury to other of the bone structures.

\section{How to Use Bran.}

Bran cannot be well eaten by itself. It must be combined with other foods. There are numerous excellent combinations which have been well tested and found satisfactory.

First of all, it is necessary that the bran itself should be of good quality. Ordinary bran is very dirty, abounding in germs of many sorts, and also weevil and other insects. To be wholesome as food, bran must be prepared from thoroughly sound wheat, which must be very well cleaned, brushed, and washed before grinding. The bran should then be sterilized so as to make sure that it is thoroughly free from bacteria. If prepared in the home, the bran should be carefully selected, thoroughly washed, and baked in an oven until well cooked.

This cooked bran may be mixed with different cereals in various proportions. Among the most practical combinations are bran and oatmeal, bran and cornmeal, and bran with rice. Bran may also be combined with purées of potato and other vegetables. The proportion should be about one part of bran by weight to three parts of oatmeal, corn, or rice, or equal parts by bulk. The steel-cut oatmeal is better for this purpose than rolled oats. As the bran has been thoroughly cooked in sterilizing, it does not require long cooking. The oatmeal and bran mixture is made 
very palatable by cooking six to ten minutes, and has a better laxative and antitoxic effect than when long cooked. Sterilized bran may also be added to ordinary graham flour to increase its richness in salts and cellulose, and may thus be used in the preparation of mush, gems, rolls, bread, crackers, etc. The very finest bran bread possible may be made by adding cooked bran to good spring wheat flour. This proportion should be about one part of sterilized bran to six of flour. Housewives nearly always have difficulty in making graham bread, for the reason that the diastase of the bran acts upon the starch, converting it into dextrine, making the loaf sour or heavy. This difficulty is obviated by the use of cooked bran, or by sifting out the bran of graham flour, and boiling or baking it, then returning it to the flour.

\section{The Anti-graham Campaign.}

One of the most meretricious of the many venial exploitations to which the public are at the present time being subjected is the campaign against the use of graham bread or flour prepared from the whole wheat. It is evident that this campaign is being carried on altogether in the interest of the millers, who for years have been exacting an enormous toll for their production of superfine, denatured flour which represents only a fraction of the nutritive value possessed by the whole wheat berry. The primary assumption of the millers is that they know more than does Nature concerning the nutritive needs of the human animal. Their object, of course, is not to promote the welfare of the public, but to enrich their own pocketbooks.

It is now nearly eighty years since Sylvester Graham, from whom graham flour and graham bread derived their distinctive names, started his nation-wide campaign in favor of whole wheat meal. His propaganda was urged with great earnestness, but after twenty years his disciples were few. In the writer's boyhood, graham flour could not be purchased west of Rochester, New York. It was only to be gotten by buying at the mill the various products obtained by grinding wheat and mixing these together in the proper proportions; but within the last 60 years the graham idea has rapidly extended and at the present time graham bread is obtainable at every bakery of any size in nearly all parts of the country. Graham bread is found upon tens of thousands of American tables daily. Besides, the people have become accustomed to the use of whole grain preparations in 
various other forms, such as wheat flakes, shredded wheat and oatmeal. It is quite too late to now come forward with scarecrow doctrines about indigestion, malnutrition and sundry other evils likely to result from the use of whole grain preparations. The people were fooled by these arguments for many years but now have their eyes open.

The purely mercenary motives of the propagators of these doctrines at the present time may be clearly seen in the fact that the same mills which employ skilled writers to instruct the public about the dangerous consequences likely to result from the use of graham flour because of the small percentage of bran which it contains employ other writers to extol the marvelous virtues of bran and the importance of making regular use of it daily and even at every meal. The public have learned by experience the value of bran as a substitute for the nauseous pills and mineral waters with which the American stomach has so long been persecuted.

A report that graham flour was the cause of illness and even deaths in Belgium during the war has been much exploited by the millers. There is good reason to believe that the real cause of the sickness was a cargo of spoiled wheat which was shipped from the West to Belgium after having been rejected as unfit for milling.

The wholesomeness of bran is clearly shown not only by the world-wide use from time immemorial of whole grain preparations, but by the general use of very coarse rye bread (pumpernickel) by many European nations for centuries before the war and of a still coarser bread during the war. Hindhede attributed the lowering of the death-rate in Denmark more than 25 per cent. chiefly to the use of bread made of rye flour with the addition of wheat bran. Mendel has shown that rats thrive on a diet in which meat has been replaced by wheat bran, and poultry raisers have reduced the mortality of incubator-hatched chickens from 40 per cent. to 4 per cent. by substituting bran and buttermilk for meat scraps and meat meal. 


\section{- CORN.}

Maize, or Indian corn, is distinctly an American product and has been justly termed "the Indian's gift to civilization."

The aborigines of America made corn their staple food. Said Dupratz, writing in 1763, "All the nations who inhabit from the sea as far as the Illinois and even further carefully cultivate the maize corn, which they make their principal subsistence."

The principal aboriginal uses of the several types of corn were, respectively, flint corn for hominy, sweet corn for parching, pop corn for popping, and flour corn for eating fresh in the roasting ear stage, for cooking and drying in that stage to preserve for winter, and for grinding into meal for various uses when ripe. Dent corn was not grown by northern tribes of Indians, but was grown by those in Virginia and south. Where it was grown it was also used for making meal.

In nutritive value, whole corn slightly exceeds wheat. It is less rich in protein than is wheat, and its protein, according to the observations of Rubner and others, is less valuable as a nutrient than is the protein of wheat. Its two proteins, zein and glutelin, are very unequal in nutritive value. Animals fed on zein by Osborne and Mendel did not thrive, while those fed on glutelin were well nourished. By the addition of milk, however, the deficiencies of zein are compensated so that a diet consisting of corn mush and milk is a combination well adapted to the nutrition of the body.

Dr. George K. Cherrie, a well-known naturalist who accompanied Mr. Roosevelt in his South American exploration, and who recently returned from his twenty-eighth expedition to the American tropics, reports that the diet of the natives of Venezuela is corn and goat's milk. That this diet is sufficient for complete nutrition is shown not only by the good health and great endurance of the natives of the country, but by the fact that $\mathrm{Mr}$. Cherrie himself lived for many weeks at a time on the native diet, and mentions no ill effects from the simple bill of fare.

A staple diet of cow's milk and corn would be a simple and effective solution of the problem of the high cost of living, at least so far as food is concerned. Our corn crop alone, if fed exclusively to human beings and dairy cattle, would more than suffice to feed our whole population of $100,000,000$ people. 
The Peruvians from the most ancient times have subsisted chiefly upon corn, as they do at the present day. The writer has in his possession an ear of corn recovered from an ancient tomb near Lima, Peru, which is believed by the authorities of the American Museum of Natural History to have been interred not less than 2,000 years ago. In appearance it does not differ from our modern hard corn.

TABLE XXVIII.

Showing the Composition of Corn Products.*

\begin{tabular}{|c|c|c|c|c|c|c|c|}
\hline Corn, whole grain & & & & & & & \\
\hline Corn, whole grain, ay & 10.8 & 10.0 & 4.3 & 71.7 & 1.7 & 1.5 & \\
\hline $\begin{array}{l}\text { Corn with low protem content... } \\
\text { Corn with high protein content. }\end{array}$ & 10.5 & 6.0 & 3.8 & 78.5 & & 1.2 & \\
\hline $\begin{array}{l}\text { Corn with high protein content. } \\
\text { Corn, white... }\end{array}$ & 10.5 & 12.9 & 4.4 & & & 1.4 & \\
\hline Corn, yellow & $\begin{array}{l}11.4 \\
11.9\end{array}$ & 10.7 & $\begin{array}{l}5.0 \\
4.8\end{array}$ & 68. & 2.2 & 1.5 & \\
\hline Sweet corn (matured) & 8.8 & 11.6 & 8.1 & 66.8 & 2.8 & 1.9 & 1,750 \\
\hline Sweet corn (green) .............. & 73.0 & 5.0 & & 19.5 & & & 446 \\
\hline Pop corn & 10.7 & 11.2 & 5.2 & 69.6 & $1.8^{\circ}$ & 1.5 & \\
\hline Hominy, fine & 11.0 & 9.4 & .7 & 78.2 & .4 & .3 & 1,8 \\
\hline $\begin{array}{l}\text { Samp, coarse } \\
\text { Cornmeal (whole grain ground). }\end{array}$ & 10.8 & 8.3 & .5 & 79.4 & & .3 & 1,7 \\
\hline unbolted & 12.0 & 8.7 & 4.7 & & & 1.3 & 1,85 \\
\hline bolted (w.1. & 12.0 & 8.9 & 4.9 & 72.0 & 1.2 & 1.0 & \\
\hline $\begin{array}{l}\text { Cornmeal, granulated } \\
\text { Cornstarch }\end{array}$ & 12.5 & 9.2 & 1.9 & $\begin{array}{l}74.4 \\
900\end{array}$ & 1.0 & 1.0 & 6 \\
\hline $\begin{array}{l}\text { Corn syrup ................. } \\
\text { Corn oil }\end{array}$ & 16.0 & & & 83.5 & & .5 & 1. \\
\hline orn oil ....... & & & & 100.0 & & & \\
\hline
\end{tabular}

\section{Cornmeal.}

The old-fashioned hoe cake and cornmeal mush, prepared from meal made from the entire grain, sometimes called water meal because ground on stones turned by the old-time water wheel, are the best modes of utilizing corn as food. New process cornmeal is robbed of much of its salts; and corn bread made in the modern fashion, with sour milk and saleratus or baking powder and a liberal admixture of lard, is still further deteriorated.

Old style cornmeal, mixed with water and a little salt and baked in thin cakes, the method still used in many parts of the Southern states, is the finest corn bread possible.

*Farmers' Bulletin 298, U. S. Dept. of Agriculture. 


\section{Cornmeal Mush and Milk.}

Hart and Steenbock, in experiments upon pigs, have shown that for the best results milk and cornmeal must be used in equal quantities. It is necessary to provide sufficient milk protein to balance the incomplete protein of corn. In the mixture named, the milk would furnish about 30 per cent. of the protein. This homely dish, long approved by common experience, is now shown to be a highly scientific combination.

\section{Corn Oil.}

Corn oil is prepared from the germ of the grain, which constitutes about one-tenth of its weight. The old process cornmeal contains the germ and the entire grain with the exception of the outer skin. The germ is removed by the new process, and the oil, which constitutes more than 4 per cent. of the whole of the grain, is separated and subjected to special processes of purification, the final product of which is corn oil, a wholesome vegetable oil which is as digestible as other varieties of vegetable oil although it is lacking in the fat-soluble vitamin which is found in butter, and hence is not a complete substitute for the dairy product.

\section{White and Yellow Corn.}

The chemical composition of white and yellow corn is essentially the same, although they differ in flavor because of the characteristic aromatic substances found in the oil. Hess has recently claimed that carotin, the coloring matter of yellow corn, contains the important fat soluble growth-promoting vitamin which is found in butter, from which it would appear that yellow corn has a somewhat greater value as a nutrient than has white.

\section{- Grits.}

Hominy and samp contain about 10 to 20 per cent. less protein and 10 per cent. more carbohydrate than the entire grain. They contain only one-fifth as much of salts, being almost wholly deficient in food minerals.

\section{Corn Starch.}

Corn starch is a preparation of corn from which all but the starch has been removed. It contains 10 per cent. water, the balance being carbohydrates in the form of raw starch granules. 


\section{- Corn Syrup.}

Corn syrup, or glucose, consists of corn starch which has been subjected to the action of a mineral acid and heat and thereby converted into a form of sugar. When thoroughly purified from the chemical substances used in the process of its manufacture, it is probably not unwholesome, although it cannot rightly be regarded as a complete substitute for the sugars and other carbohydrates found in the sweet juices of fruits and other saccharine foods.

\section{Pop Corn.}

Pop corn is one of the most wholesome of cereal foods. It is easily digestible and to the highest degree wholesome, presenting the grain in its entirety, and hence superior to many denatured breakfast foods which are found in the market.

Everybody is familiar with the method of popping corn. It is notorious, however, that the corn sometimes pops more readily than at other times. In order that it shall "pop" well it is necessary that the kernels should contain a certain amount of water. A good plan is to soak the pop corn in water over night, then allow to air dry until it no longer feels moist, the surface appearing dry. It is now ready to pop. A method of popping which produces particularly excellent results is the following:

Put into a large iron kettle half a pint of cooking oil of some sort. Heat nearly to boiling point. Drop in a pound of shelled pop corn prepared in the manner above directed, stirring gently. The corn pops at once and fills the kettle, making about six quarts. Honduras rice may be popped in the same way, and is very toothsome. Popped rice prepared in this manner is much used in China.

\section{Green Sweet Corn.}

This delicious vegetable, for which we are indebted to the North American Indian, is a product of our gardens during late summer and early fall. The ears are picked when the grain is in the milk state; that is, when the carbohydrates are still largely the soluble form of sugar and dextrin, which later are converted into insoluble starch for storage. Sweet corn contains about 13.5 per cent. of starch, 6 per cent. of sugar, and 5 per cent. of protein. It is usually prepared by boiling or roasting. Roasted ears are much sweeter than boiled for the reason that in boiling the sugar 
is largely lost in the cooking water. Steaming is a better mode of cooking than boiling, and the finer varietias, especially the yellow bantam and Mexican varieties, are entirely acceptable to the palate when eaten uncooked, and are also more wholesome.

\section{Toasted Corn Flakes.}

Toasted corn flakes are a breakfast food prepared from white corn grits by a process of which the following constitute the several successive steps:

1. Cooking at a temperature above the boiling point.

2. Drying.

3. Tempering by the addition of water or steam.

4. Milling by mills specially constructed for the purpose by means of which the grits are flattened out into large flakes.

5. Toasting in a traveling oven.

6. Salt and other flavorings added in the process of cooking.

This product, devised by the author many years ago, in an effort to provide palatable dextrinized cereal foods for the patients of the Battle Creek Sanitarium, was at first designated to be eaten dry as the best means of stimulating the action of the salivary glands and to encourage the practice of thorough mastication. Eaten with milk, corn flakes constitute an excellent breakfast food. It must be acknowledged, however, that when eaten moist with milk or cream they offer no better nutrient than the oldfashioned brose of the Scotch peasantry, or the cornmeal mush of our childhood days. It cannot be doubted that all our efforts to improve upon Nature's products by artificial manipulations are more or less futile and often result in a denaturing which lessens their value as foodstuffs.

\section{Gofio.}

The Indians made porridge from corn which had been parched and then pounded into a coarse meal. Corn and other grains are used in this way by the natives of the Canary Islands under the name of gofio.

The Tarahumara Indians of Mexico, who are the most enduring runners in the world, make long journeys at an amazingly rapid rate of speed, on a diet of parched corn. It is officially recorded that one of these Indians carried a government dispatch bag three hundred miles and back in five days. They are employed to run wild horses and deer. 


\section{OATS.}

The oat has for hundreds of years been the staple food of the hardy natives of the Scotch Highlands, but it is only within comparatively recent years that oatmeal has become a common article of diet in this country, and still more recently that its use has become common in other parts of the British Islands than Scotland and Ireland. Even at the present time oatmeal porridge is rarely found as a component of dietaries in Continental Europe. When Johnson issued the first edition of his dictionary, oatmeal was so seldom used in England that he felt justified in defining the oat as a food for men in Scotland and for horses in England. When chided by a Scotchman for the opprobrious suggestion implied in his definition, Johnson rather testily retorted, "And is it not the truth?" Whereupon the Scotchman, after a moment's hesitation, admitted the statement to be a fact, but added, "And where do you find such foine horses as in England and such foine men as in Scotland?"

As a human food, the oat is far superior to corn. It is rich in lime, which is almost altogether lacking in corn, besides containing considerable fat. Its nutritive value is so great that a single ounce of oats represents an amount of energy equivalent to more than 150 foot tons, which is more than double the energy represented in an ounce of lean meat, nearly double the energy value of bread and more than four times that of the potato. Prepared in the form of brose and bannocks, the oat is still the staple article of diet of the Scotch Highlander; and when supplemented with buttermilk and potatoes, it forms, from a nutritive standpoint, an ideal diet for securing the fullest degree of physical development and maintaining a high degree of energy and endurance.

A very old writer, Sinclair, tells us in his "Code of Health" that "In their ancient predatory incursions into England . . . the Scottish warriors fastened to their saddles a bag filled with oatmeal and a plate of flat iron, upon which they baked their grain into cakes. Laying this iron plate, called a griddle, upon a fire, they spread upon it their meal, made with water into a thin paste, and thus converted it into bread. They could carry a sufficient supply of this for thirty days' consumption, which gave them great advantages over an army whose wants were more numerous." 
The brose of the Scotch consists of oatmeal stirred into boiling water until the mixture thickens, then removed from the fire and served at once. The meal prepared in this way is scalded rather than cooked. It is, nevertheless, highly palatable. After an experience of some years, the writer is prepared to recommend this as by far the best method of preparing oatmeal porridge. Cooked in the ordinary way until it becomes a slimy pasty mass, oatmeal is constipating; but prepared after the Scotch manner, the porridge contains a sufficient amount of uncooked starch to produce a laxative effect. The raw or imperfectly cooked starch being more slowly acted upon by the digestive juices is able to reach the colon before absorption takes place. Starch in the colon supplies the nutrient material necessary to encourage the growth of acid-forming bacteria and the production of acids, thereby preventing putrefactive changes with the formation of poisons and encouraging intestinal activity. The food residues are thus promptly discharged from the body instead of being retained to undergo putrefaction and produce autointoxication.

The bannocks of the north of England consist of thoroughly kneaded oat dough rolled into thin cakes and baked on an iron plate suspended over the fire.

\section{RICE.}

This cereal feeds more human beings than any other. It is the staple food of hundreds of millions of human beings in India, Burma, China, Japan, and other Oriental lands. Its nutritive value is high and its productivity great. Many varieties are grown, some requiring submergence, others (upland rice) adapted to dry soils.

Rice takes the place of bread, potatoes and breakfast cereals, all three in one, in the Oriental diet. The Chinese coolie works long hours on rice; the Japanese jinriksha boy carries his fare long distances at the rate of six miles an hour on a diet of rice and refuses to substitute the meat which contact with modern civilization has introduced to Japan, because it destroys his endurance. The toiling millions of southern Europe have within recent years come to appreciate more and more the value of rice, and in this country, where wheat and barley have been grown from remote ages, rice is steadily gaining ground in popularity through its greater digestibility and energy-supporting properties. 
Rice is a highly emollient food. It is sometimes tolerated by the stomach in cases in which most other foods are rejected. It is less acid than other cereals.

The rice diet, described elsewhere, is of special value in cases in which there is great irritation of the tissues of the mouth esophagus or stomach and in acute disorders of the kidneys.

Dr. S. A. Knapp, of the United States Department of Agriculture, himself a sufferer from impaired digestion, seeking for a diet which might afford him relief from the distress of indiges. tion, recalled the fact that rice eaters while performing prodigies of work complained of hunger between meals, due to the fact that rice leaves the stomach within an hour and so gives this organ an opportunity for rest. Dr. Knapp remarks: "This was precisely what I wanted-energy with hunger. I had it as a child and lost it somewhere in a busy life. Rice-eating nations have energy with unimpaired digestion." Dr. Knapp also calls attention to the fact that the Japanese soldiers subsisting on a ration of rice were able to outmarch all the armies of European nations in the advance upon Peking at the time of the Boxet uprising. "With full equipment they advanced all day at double time, and repeated it until even the Russians fell behind exhausted."

Rice is low in protein, containing only a little more than half the amount of protein contained in wheat, but its protein is of a superior quality so that its actual value is, as a nitrogenous nutrient, not materially less than that of wheat. Rice contains a smaller amount of salts than any other of the cereals. The amount of lime and iron which it contains is almost nil.

\section{Polished Rice.}

In the form in which rice is used in this country, it is not only lacking in salts but in vitamins, and hence should be regarded as a deficiency food which should never be relied upon as a chief nutrient. When polished rice is used, it is well to take at the same meal a quantity of wheat bran. This may even be mixed with the rice with advantage. It detracts from its appearance but completely restores the wholesomeness which is lost by the polishing of the rice. A good rule is to mix with the rice about half its volume of sterilized bran. The bran should be mixed with the rice before it is added to the boiling water. In a 
similar way bran should be added to new process cornmeal. Oatmeal is also improved by the addition of bran.

The addition of greens to rice is a most appropriate and satisfying food combination. The greens supply the lime, iron and vitamins which the rice lacks, and in ample quantity. It also furnishes an excellent quality of roughage. The Chinese, Japanese and East Indians eat liberal quantities of greens with their rice, as do the Kaffirs with their "mealies."

It would be to the advantage of the people of this country if the manufacture and sale of polished rice were forbidden. The Filipinos are in this respect better protected from disease than are the people of the United States.

Brown rice, that is, rice from which the bran has been removed by melting and polishing, is now available. It is rich in vitamin B and iron and superior in flavor as compared with polished rice.

The great attention which has been given to the development of the rice industry in India in recent years is well shown by the fact that in the rice exhibition held in Bengal in 1906-7, nineteen distinct classes of rice were exhibited, among which were drought-resisting varieties of rice, flood-resisting rice, rice which can be grown on hillsides without terracing, autumn-maturing rices which produce fine grain, winter rices which grow on salt lands without protection by embankments, rice producing four to six grains in each head, rice the whole plant of which is fragrant, black rice, the leaves of which are also black, rice which may be eaten without boiling, wild rice, rices which mature in three months, and rice which requires nine months to produce a crop.

Wild rice grows in great abundance in shallow lakes in many parts of the United States. It was a staple food of the American Indian in pre-Columbian days and is still used by them. It is a delicious cereal. It is gathered in boats, then dried and slightly roasted to insure keeping.

\section{BARLEY.}

Barley has been so long used in making beer that its value as human food seems to have been largely forgotten, at least in English speaking countries. Barley is believed to have been the 
first of the cereals to be used as human food. Barley cakes are mentioned in the most ancient accounts of the eating habits of man.

In composition barley much resembles wheat, although it contains a smaller percentage of protein. The protein of barley, however, is sufficient in amount and is much superior in quality to the protein of corn and beans.

Barley thrives in a greater diversity of climates than any other cereal. It flourishes at the equator and grows equally well in the arctic climate of northern Russia. Barley is a staple food in Siberia and among many of the tribes of central Asia.

Hindhede, the eminent physiologist of Copenhagen, conducted a series of investigations to determine the practical food value of barley and concludes that this cereal "is too valuable as a human food to be used in these hard times (during the war) as a fodder for pigs and in the production of beer." In Hindhede's feeding experiments the diet of his subjects consisted exclusively of barley, margarine, and sugar. The results showed most conclusively its ability to largely replace other grains as a cereal food.

Its chief drawback is the fact that to most people it is less palatable than wheat. The bread made from barley is much inferior to that which may be made from rye or wheat, and it accordingly falls into disuse in countries where these grains can be grown. Fairly good bread can be made from it by the addition of a proportion of wheat flour.

\section{Pearl Barley.}

Barley is little used in this country as a food grain except in the form of pearl barley, which is frequently used as an invalid dish. In the process of pearling the tough outer coat of the grain is removed.

\section{RYE.}

Rye contains a certain proportion of gluten and is capable of being made into a nutritious bread, the pumpernickel of the Germans, a sour dough bread in which the whole grain is represented. Although coarse, and to the American palate rather unpalatable, this black bread is nevertheless far more wholesome than the superfine white bread in general use, not only because 
of its marked laxative properties but because of the large proportion of lime and iron, together with a rich store of vitamins which it contains.

\section{MILLET}

Although largely used throughout Asia and in some parts of Africa, millet is little known as a food cereal in either the United States or Europe. Its composition is very similar to that of barley. It contains nearly twice as much cellulose as does wheat. Recent experiments by Osborne and Mendel indicate that millet contains a richer store of vitamins than any other of the cereals. It is, indeed, the only cereal which is able to support animal life and development for a considerable length of time when used as an exclusive dietary. It is probable that this cereal will ultinately fill a more important place in the world's bill of fare than it does at the present time. Mixed with a proportion of wheat flour, millet flour, especially a flour prepared from proso millet, is said to make a fairly good bread.

\section{QUINOA}

Quinoa, a cultivated pigweed, was a staple food of the natives of Mexico and South America before the conquest of these regions by Cortez. It is said to have produced prodigious crops. The seed is rich in protein, of which the flour contains 19 per cent. with 60 per cent. of starch and 5 per cent. of fat. The amount of cellulose contained in the whole seed is greater than that of any other food cereal.

The green leaves when cooked resemble the common lamb's quarters in flavor. The seeds are cooked like oatmeal after soaking in cold water for twenty-four hours to remove a bitter flavor.

\section{KAOLIANG}

The sorghum grain, long used in India and nothern Africa, known for more than 50 years in this country but thus far little used, is worthy of more attention than it has heretofore received.

\section{- BUCKWHEAT}

Flour prepared from buckwheat is chiefly used for griddle cakes. When well prepared and made without contamination by burned fat, the griddle cake is not unwholesome; but as ordinarily 
prepared, buckwheat cakes are highly productive of indigestion. Buckwheat contains more carbohydrate than most other cereals, nearly 78 per cent., with a protein content of 6.4 per cent.

\section{BREAKFAST CEREALS}

The manufacture of breakfast cereals in the United States really originated with the late Ferd. Schumacher of Akron, Ohio. Mr. Schumacher told the writer that as an apprentice in a pharmacy in Hamburg, Germany, when a boy, one of his duties was to prepare the oatmeal. This was done by hulling the grain by putting it through a set of millstones made for the purpose and turned by hand, then reversing the stones and putting the grain through a second time to convert it into grits. On coming to this country he found that the oatmeal in use here was imported and sold only in small packages in drug stores for use by invalids. He set to work to devise means for manufacturing oatmeal on a commercial scale. From a small beginning, he gradually developed a great industry, and by carrying on a vigorous educational campaign developed a demand for oatmeal which finally grew so great as to make the grain a food staple.

\section{Ready-to-Eat Breakfast Foods.}

Carlsbad, the famous Bohemian watering place, has for a sentury or more owed its popularity in part, at least, to the zwieback (provincial German for twice baked) of which visitors to the resort were expected to make liberal use as a part of the zure. From Carlsbad the use of zwieback, which is simply well toasted bread, was introduced into this country through the water zures, which at a certain period beginning more than a hundred years ago enjoyed a considerable degree of popularity. The zwieback was sometimes ground to render it more easily eatable, but neither zwieback nor "rusk" was commercially obtainable in this country.

When a medical student in New York City in 1874-5, after having made a special study of foods for two or three years, the writer became convinced that there existed a widespread need for ready-cooked foods, at least, ready-to-eat cereals. A year or two later, after becoming superintendent of the Battle Creek Sanitarium, the idea became concrete in the production of granola, which consisted of a mixture of oatmeal, cornmeal and wheat 
meal made into cakes with water and exposed to a temperature sufficient to dextrinize the starch, with the idea of thus rendering it more readily digestible. The product was ground to give it a granular form convenient to eat with milk, cream or fruit juices.

The product became the forerunner of several other similar products in which the idea of dextrinizing the starch content of cereals was developed in various ways. This was considered important for the reason that certain forms of indigestion were found to be relieved by the use of dextrinized foods. The reason was not then wholly clear, although it was observed that digestion was aided by the pre-digestion of starch. We now know that undigested cooked starch absorbs the pepsin of the gastric juice, leaving the gastric acid free, and thus giving rise to acidity. Pavlov also showed that dextrin is strongly peptogenic, that is, acts as a natural gastric stimulant.

\section{Toasted Cereal Flakes.}

The writer early in his medical experience became convinced that indigestion and decay of the teeth were encouraged to a marked degree by failure to use the teeth sufficiently in the thorough mastication of the food. He accordingly made it a practice to require his patients to begin each meal by chewing slowly a small slice of dry zwieback. One day a patient came into the office with a complaint that our prescription had broken her teeth, for which she jokingly demanded compensation. The impracticability of zwieback as a dry food in several classes of patients was at once apparent. Patients with artificial teeth, with sore teeth or diseased gums, and patients without teeth, needed something which they could chew and comminute without running the risk of injury to their teeth or other inconvenience. Experiments were begun to produce toasted or dextrinized cereals in a form which, while dry and crisp, could be properly offered to such persons and eaten by them without the addition of milk or cream, which would destroy their value as a dry food capable of stimulating an abundant flow of saliva, experiments made about the same time by the writer having demonstrated dryness to be one of the most efficient means of stimulating the activity of the salivary glands.

After some months of experimentation, the writer developed the process for making toasted cereal flakes which is now widely used in the manufacture of toasted corn flakes, toasted rice flakes, 
wheat flakes, etc. The first flakes made were wheat flakes and were known as Granose, and later as Toasted Wheat Flakes. Various imitations were known as Malta Vita, Force, Vim, Egg-O-See, etc. Later, toasted rice flakes, and other cereal flakes were developed.

The writer has been glad to see that these dextrinized products have been so generally adopted as components of the American breakfast. Of the score or more different brands of these flake cereals now on the market, probably not less than 50 to 100 carloads are used daily by the people of the United States alone, and their use is gradually extending to every other civilized country of the globe. The only objection that can be offered to these dextrinized cereals is the fact that they are deficient in cellulose, vitamins, iron and lime. Vitamins and lime are, however, added in the milk with which they are served, and the addition of a tablespoonful of bran to the morning dish is a most convenient method of making use of this important laxative element which, through the pernicious ingenuity of the American miller, has been until recently almost altogether excluded from the national bill of fare. The bran supplies iron but not enough. Spinach or other greens should be made a part of the daily menu when corn or rice is freely used.

The argument offered by some critics that malted and dextrinized foodstuffs weaken the stomach by lessening the work required of it, though theoretically plausible, is not conclusive. There is a physiologic antagonism between the salivary digestion of starch, which takes place first in the mouth and later in the stomach, and the gastric digestion proper, which is carried on by the acid gastric juice. The more gastric juice in the stomach, the more active gastric digestion, the sooner the action of the saliva upon the starch ceases after the food enters the stomach; and on the other hand, the less complete the digestion of the starch, the more gastric digestion is interfered with through the absorption of pepsin by the undigested starch, already referred to, and also through the incomplete disintegration of the food, a process which is greatly facilitated by the action of the saliva, rendering the starch soluble. When the food is dextrinized, the digestion of starch, being more complete, does not interfere with gastric digestion but, on the other hand, stimulates the gastric glands to greater activity and so promotes the work of the stomach instead of hindering it. 
The writer thinks it important to add, however, that he would by no means advocate exclusive use of predigested foods or of predigested starch for the reason that it is desirable to send down into the colon daily a certain amount of undigested starch to serve as nutrient material for the protective germs which discourage putrefaction and encourage bowel action by the production of acids through fermentation. Raw starch is better for this purpose than cooked.

\section{SESAME SEED MEAL}

This oleaginous seed is much used in India and the Orient, as well as in all parts of Africa. Dr. Thompson thus describes this valuable plant and its preparation:

The plant grows "to a height of three to six feet, giving off blossoms at the beginning of each stalk or branch. The seed pod is about an inch in length, a quarter of an inch in diameter, and has four cells running lengthwise of the pod, which contain the seed. The plant is raised in connection with millet in the gardens of the natives. The name is Sesamum Indicum. It is grown a good deal in India.

"When the seed is ripe, the natives cut the plant off near the ground, tie it in bundles, and stand the bund'es up where they can get perfectly dry. A cloth is then spread on the ground and the bundles are beaten with sticks to rattle the seeds out. The seed is moistened with warm water and rubbed between the hands to remove the outer hull. The seed is commonly black in color, but some varieties are nearly white. The seed is next roasted, which is the most particular part of the process. A little salt is added and the seed is ground between two stones as the natives grind their other grains."

The writer witnessed the process described in operation on quite an extensive scale in Cairo, Egypt, some twenty years ago. The ground, roasted seed was used as a meal and also in the form of a paste or butter much resembling the peanut butter now in general use in this country. 


\section{Vegetables}

Vegetables are chiefly useful because of the carbohydrates which they furnish, together with an abundance of the alkaline salts, vitamins, and cellulose. Only one class of vegetables, the legumes, is rich in protein. A comparatively few vegetables contain carbohydrates in large quantities. For the most part, vegetables, especially green vegetables, contain a large amount of water.

The carbohydrates of vegetables, when abundant, usually exist in the form of starch which possesses the property of being more easily digestible than starch from any other source. Certain vegetables are rich in sugar, which is, for the most part, in the form of cane sugar, the sugar of roots, stems, and leaves.

\section{Uncooked Vegetables.}

Green vegetables, such as lettuce, parsley and other salad vegetables, may be eaten without cooking and may be advantageously utilized in this way because of the value of the vitamins which they contain, some of which are wholly or in part destroyed by cooking.

It is hardly possible to overemphasize the importance of taking a portion of the food in uncooked form. The primitive ancestors of the human race subsisted wholly upon the natural products of the forest, as do a few wild tribes even at the present day. As an exclusive diet, uncooked food must be selected with the greatest care; and such a diet is hardly possible without the addition of milk, eggs or meat, at least for long periods.

The best foods for use in uncooked form are lettuce, celery, green salads, Chinese cabbage, radishes, turnips and green sweet corn. Wheat, in the milk state, may be used in like manner. The turnip, and even the carrot, properly prepared may be utilized in the absence of other more suitable raw foods. Recent experiments by Langworthy have shown that raw potato starch is almost wholly indigestible, although cooked potato starch digests more quickly than any other form of starch known. The starch of the unripe banana, like that of the potato, appears to be unusually 
difficult of digestion. The starch of raw cereals, on the other hand, has been shown to be quite readily digestible.

\section{Roots and Tubers.}

Most plants store up in the seed a supply of nutriment to be utilized in starting the growth of the new plantlet. The plant gathers its nourishment through its roots and leaves. The leaves constitute both the lungs and the stomach of the plant. These organs must be provided before the plant can begin an independent existence. Certain plants, because of peculiar habits, provide an additional store of nourishment in fleshy, succulent roots, as in the beet, turnip, parsnip, and carrot; or in fleshy underground stems, as in the potato, the sweet potato, the yam, the taro, dasheen and yautia.

\section{Root Starches.}

The idea that root vegetables, particularly starchy roots such as the potato, are inferior foodstuffs and indigestible, is a serious error which somehow has attained wide currency. The fact is that root starches when cooked are by far the most easily digesible of all foodstuffs.

Some years ago, Professor Grierson, an eminent German physiologist, made a careful study of the digestibility of starches from various sources. He found that the starch of rice, wheat and corn require two hours for digestion; the starch of oatmeal, eighty minutes; while arrowroot digests in thirty minutes and potato starch is completely digested in ten minutes. In other words, the starch of wheat, corn and rice requires twelve times as long for digestion as does the starch of potatoes; and the starch of oatmeal, eight times as long.

No doubt the substitution of cereal breakfast foods for the old-time griddle cakes, fried potatoes, fried pork and other indigestibles has been in the interest of the conservation of the American stomach, but there is much reason to believe that the Irish potato, steamed or baked, might well fill a larger place in the national bill of fare, especially as a breakfast food.

A baked potato digests in much less than half the time required for the digestion of a slice of bread and affords nutritive substances in some respects superior to those provided by wheat. The housewife adds potato to her bread sponge because it encourages the growth of the yeast plant and so makes the bread rise 
better. The growth-promoting vitamin which the potato supplies in great abundance is as active in encouraging the growth of children as in helping bread to rise. It is equally useful to adults in maintaining good nutrition and in promoting the repair of the tissues.

The potato and other root vegetables are also superior to cereals in the fact that they supply an abundance of organic alkalies, compounds of soda and potash, which are of the greatest service in neutralizing the acid wastes of the body and promoting the purification of the tissues.

The sedentary habits and the free use of meat and cereals in the diet of the average American produce a widespread tendency to acidosis, which is a common cause of premature senility, hardening of the arteries, Bright's disease and other degenerative processes. If the average American would eat four or five times as much potatoes as he now eats, and an equivalent amount less of cereals and cane sugar, the result would be a notable lowering of the death rate and a marked increase in physical fitness and freedom from crippling disease.

\section{The Iron Content of Vegetables.}

Vegetables are like fruits in their small average content of iron per ounce ( 1.46 per cent. of ration) but relatively large content per 100 calories. In this respect they are even more remarkable than fruits. Vegetables are also like fruits in the fact that their small nutrient value renders possible a bulky intake, so that in practical use fresh vegetables become a highly important source of food iron. The average iron ration percentage value for nineteen common vegetables is 10.4 per 100 calories.

The vegetable which provides the most iron in an ordinary serving is the potato, which in a small serving of three ounces provides one-twelfth of a day's requirement. The Irish farmer, who daily eats several pounds of potatoes, evidently gets a full supply of iron, notwithstanding the fact that his chief article of food aside from the potato is buttermilk, which contains very little iron. The Scotch Highlander finds an equally fortunate food combination in buttermilk, supplemented by oatmeal brose, bannocks and scones.

Brussels sprouts and cabbage each furnish in a 5 ounce serving one-tenth of a day's supply of iron; and beet roots, carrots and turnips provide in a small serving, which in many 
cases might easily be doubled, more than half as much iron as a serving of potatoes.

The vegetables which contain so small an amount of iron as to be of comparatively little value as sources of food iron, named in the order of their inferiority are, beginning with the cucumber which contains least of any of the common vegetables, eggplant, turnip, and sweet potato. The last named vegetable contains less than half as much iron as the Irish potato.

Onions have a special claim to notice because of the high value of the iron compound they supply which, according to Stokless, contains more iron even than the hematogen of egg yolk, discovered by Bunge, and is equally well utilized by the body. So, at last, this often rejected, and by many much despised, vegetable is likely to be accorded a more honorable place in the list of choice comestibles than it has heretofore enjoyed. It must not be forgotten, however, that not a little culinary skill is required to eliminate the offensive essential oil of the onion, which is by no means its chief attraction, although its most conspicuous feature. A peculiar saccharine substance which reveals itself only after the rank, blistering aromatic oil has been driven away by heat gives to the onion its valuable flavoring quality.

\section{The Lime Content of Vegetables.}

In studying vegetables with reference to their lime content, one is struck by the fact that among a dozen commonly used vegetables, omitting greens there is not a single one in which the lime salts are largely represented. The average lime content is one-tenth grain to the ounce, almost the same as that of cereals. Lime is found, however, in notable quantities in certain vegetables.

Parsnips, onions, string beans and kohl-rabi are comparatively rich in lime, supplying in an ordinary serving, respectively, $7.0,3.1,7.2$ and 12.2 per cents. of the total day's needs.

Because of their bulky nature vegetables may be eaten in much larger quantity than the concentrated cereals, and so a liberal intake of lime may be made in eating vegetables in which the percentage of lime is comparatively small. The turnip, for example, contains less than 0.4 of a grain of lime to the ounce, but an ordinary serving is 4.5 ounces, which supplies more than one-tenth (11.4 per cent.) of a day's lime ration.

Hence nearly all the vegetables may supply a considerable 
amount of food lime, when in their natural state, or when cooked by steam or oven heat; but if boiled, when removed from the water in which they have been boiled they leave behind a large part of their content of lime and other food salts. Vegetables should always be steamed or baked rather than boiled, so as tc preserve the so-called inorganic elements, which are as essential to animal and human life as to the lives of plants.

Of all the vegetables, the eggplant contains the least lime, sc little in fact as to be hardly worth noting. The amount found in an ordinary liberal serving is only one-tenth of a grain. Next in order of deficiency is our most commonly used vegetable, the Irish potato, which presents a lime content of only one-twelfth of a grain (.086 grs.) to the ounce, requiring the impractical amount of 11 pounds for a full lime ration.

The sweet potato contains a little more lime than the Irish potato but is still very deficient in this essential element. Evi dently the potato cannot be safely used as a staple without sup plementing it by other foodstuffs, which are rich in lime. Hind hede, who has devoted his life to food research and with most extraordinary benefit to his country and the whole civilized world has shown that human life and energy may be well sustained for an indefinite time on a potato diet. A private letter recently re ceived from Professor Hindhede by the writer states that in his potato-feeding experiments then still in progress he found it necessary to add to the potato very liberal quantities of greens. The greens supply not only a sufficient amount of lime, but alsc. the essential fat-soluble vitamin.

\section{THE WHITE POTATO}

One of the most important discoveries made by Sir Walter Raleigh was the white potato, which he found on Roanoke Island from which he sent to England in 1586 the first cargo of this valuable vegetable. Next to the cereals the potato is the most important food staple raised in this country. In certain European countries it holds a much larger place in the national dietary than in this country.

When first introduced in Europe, the potato met with great opposition. It was accused of being the cause of leprosy. In more recent years, the potato has been suspected of being the cause of indigestion and of rheumatism. All of these objections 
are equally unfounded. The potato is one of the most digestible of foods, and has a much higher nutritive value than is generally supposed, a single ounce affording more than twenty-four calories of nutrient material, or one-third as much as an equal weight of bread. Its chief food principle is starch, but it contains also a notable amount of protein which is of exceptionally high value. It has, indeed, been demonstrated by Hindhede in a very extended research that the potato is capable of serving as an exclusive source of protein. Hindhede's subject, a young man, lived on potato and margarine as an exclusive diet for three hundred and nine days, and during this time did heavy work.

A second research was undertaken by Rose and Cooper, in the laboratory of Columbia University. This study showed the protein of the potato to possess a high nutritive value, and fully capable of meeting the protein requirements of the body when taken in the quantity of about three pounds daily by a person weighing one hundred and ten pounds. The only other articles of food eaten were butter and sugar, the latter in very small quantities, and the daily food intake was 2000 calories. The water in which the potatoes were boiled was always used.

It is evident, then, that even with a dietary of which the potato forms a very substantial part there need be no apprehension of a deficiency of protein.

The potato is rich in soda and potash, salts which make it valuable as a means of combating a tendericy to acidosis. It would doubtless be well for the American people if they would substitute potatoes for a considerable proportion of the cereal breakfast foods which they now eat. The excess of acids contained in cereals, and especially the great excess of acids found in meats of all sorts, tends to encourage the development of arteriosclerosis, or hardening of the arteries, and premature old age.

The alkaline salts are found in greater abundance in the potato than in any other staple vegetable food. The potato furnishes forty times as much of these alkaline elements as do some of the cereals. Human beings require the potato and green vegetables to furnish these salts for the same reason that the horse and the ox require grass.

The potato is, however, lacking in lime, and for this reason should be supplemented by spinach, or other greens and milk.

The potato is rich in antiscorbutic and antineuritic vitamins. 
After the potato was introduced and became generally used in Europe, the great epidemics of scurvy which had previously prevailed ceased to appear. Potato soup or porridge should be used with bottle-fed infants when sterilized milk is employed and orange juice is not obtainable. A larger use of the potato would be of immense profit to the American people.

Since Count Rumford (Benjamin Thompson, a New England Yankee) taught the Bavarians how to make potato soup, this vegetable has steadily increased in popularity in Germany, where it holds a large place in the national bill of fare.

The peasantry of Ireland afford, also, a good example of the high nutritive value of the potato which, with the addition of buttermilk and oatmeal, has shown its ability to develop a vigorous and long-lived race.

The poor keeping qualities of the potato is one of the drawbacks to its greater use as a nutrient. The new methods of dehydration promise, however, to overcome this difficulty in this country, as they have in Germany, where many thousands of tons of potatoes are annually preserved by drying for the feeding of both human beings and domestic animals. Dried potatoes seem to have all the value of the fresh vegetables except in one particular; the process of drying appears to impair the value of the vitamins, particularly the antiscorbutic vitamin.

\section{Potato Poisoning.}

An outbreak of potato poisoning occurred in Glasgow in 1917 which was traced to sprouted potatoes. Solanin is found in very minute quantity in all potatoes, about one part in ten thousand, in which quantity, however, it is not harmful and is even supposed to give the potato a characteristic, agreeable flavor. Very small and half-grown potatoes contain much more solanin than do fully mature ones. Potatoes which have sprouted or which have grown on the surface and been partially exposed to sunlight, contain a harmful amount of solanin and should not be eaten.

Several cases of poisoning from potatoes have occurred in which the potatoes became contaminated after having been scrubbed and pared, by being left to stand exposed amid unsanitary conditions. The potato affords an excellent culture medium for various forms of dangerous bacteria. 


\section{Potato Cookery.}

The manner of cooking the potato is a matter of much importance. If peeled and soaked in cold water for some hours before cooking, 25 per cent. of the protein and a large share of its salts are dissolved by the cooking water and wasted if the water is thrown away. This method is very suitable for preparing soups when the water is used, but is otherwise most wasteful. If peeled potatoes are plunged into cold water and brought at once to the boiling point, the loss is about two-thirds as great. If the peeled potato is plunged directly into boiling water the loss is still further reduced about one-third. When boiled without peeling, the loss of protein is very small, only about one per cent., and the loss of mineral matter about three per cent. When boiled in this way the potato should be removed at once from the water when done. By drainage and evaporation a sufficient amount of water is removed to render them dry and mealy; whereas if allowed to remain in the water they become soggy. Mealiness is due, not to the swelling of the starch grains, as is generally supposed, but to the separation of the cells in cooking under the influence of heat and moisture which soften and dissolve the cellulose framework.

The best method of cooking the potato is by baking. As soon as sufficiently baked, they should be removed from the oven and their jackets should be broken by squeezing so as to allow the steam to escape.

It has been experimentally proved that 5.5 ounces of potatoes will pass through the stomach in 2.5 hours. Mealy potatoes digest in less time than waxy ones, and mashed potatoes, sooner than unmashed.

The question is often asked, "Should potato skins be eaten?" The skin of the potato is good roughage, but the popular notion that the potato skin contains important nutritive material, especially vitamins, is erroneous. Experiments made with animals show that potato skins have no protective action against scurvy. This is true of boiled and steamed potatoes as well as of baked potatoes. Any antiscorbutic properties which the potato skin may possess in the raw state are destroyed by cooking. 


\section{THE SWEET POTATO}

While somewhat more nutritious than the Irish potato, the sweet potato, on account of its more pronounced flavor, is not so universally liked. It grows best in warm countries and its use is chiefly confined to the Southern states and semi-tropical countries. It is subject to different kinds of rots and does not keep as well as the Irish potato. The sweet potato contains 25 to 30 per cent. of carbohydrates, of which about two-thirds is starch. The balance is chiefly cane sugar, of which some varieties contain as much as 10 per cent. It would be more largely used if it had better keeping qualities. The southern varieties are sweeter and keep better than Jersey sweet potatoes. It is probable that drying processes will soon be employed as a means of preserving this valuable vegetable.

\section{THE DASHEEN}

The dasheen is a highly valuable tuber which has been introduced into this country from South America by the United States Department of Agriculture. It is botanically allied to the elephant ear and the dahlia, the tubers of which are also edible. The dasheen is richer in starch and protein than is the potato. When properly cooked it is highly palatable, and being exceedingly prolific and growing in soil not adapted to either white or sweet potatoes it may be regarded as a valuable addition to our list of foodstuffs. Like the sweet potato, the dasheen requires great care in keeping. It is not likely to take the place of the potato, but is a palatable and wholesome esculent and is perhaps the most easily digestible of all the tubers grown in this country, closely resembling in this particular the celebrated taro.

\section{THE TARO}

The taro is a tropical tuber much used in the Hawaiian Islands and certain parts of India, China, and Japan. It resembles the sweet potato in appearance, and has about the same nutritive value. It is eaten by the Hawaiians as poi, a sticky, doughy mass which is allowed to ferment before using. Attempts have been made to introduce the dried taro in the form of flour into this country as a substitute for other farinaceous foods, especially for use by invalids. It is less palatable than corn starch, but as a nutrient is doubtless superior. 


\section{SAGO}

Sago is a form of starch prepared from a variety of palm tree known as the sago palm. A traveller in the South Sea Islands describes the simple method employed by the Kia Kias in the preparation of sago, which forms a staple article of food in the South Sea Islands:

The palm from which the sago is derived is indigenous to their jungles, and we are told that one large trunk of say, two-foot thickness and twenty-four length will supply food for four persons for a year. When sago is to be prepared a palm is felled and the pithy center is scraped from it, macerated with pestles, and soaked in water. The water dissolves the starch content and, when evaporated, leaves the starch ready for immediate consumption.

The moist starch is molded into cakes which are dried bone-dry, and in this form it seems to keep indefinitely. In preparation for eating, the cake is simply softened with water and toasted over a fire until cooked sufficiently to suit the individual taste.

\section{THE CASSAVA}

This is a tuberous root which grows from 10 to 12 inches in length, often 3 to 4 inches in thickness and in clusters of 4 to 8 . This vegetable is little known in the United States but is much used in Cuba and other portions of tropical America. It is now grown to a limited extent and used in Florida, especially in the vicinity of Tampa, chiefly by Cubans who have recently come to this country in great numbers in connection with the cigar business.

According to Turner, the native tribes of Africa prepare a poisonous species of the cassava for food by the following primitive method:

The fresh root is rubbed through a perforated grater or more commonly is scraped with a snail's shell into a coarse powder; this is dried in the sun, is afterwards placed in a kaffir pot over the fire and continually stirred during the roasting, in the way that coffee beans are treated. This process dispels all hydrocyanic acid. After roasting in this manner it can be stored, and will remain good for a long time -one to two years.

When properly prepared the cassava is a very palatable and highly nutritious vegetable. Its use in this country should be encouraged.

Cassava bread is prepared from the kassabi root which grows 
everywhere in tropical South America, Central America and the West Indies. Bread prepared from the kassabi root is a sort of hardtack with a slight potato flavor. It is prepared without yeast, salt or shortening. It is slightly laxative and is largely used in preference to other breads on that account. Cassava bread is said to be the oldest form of bread used on the American continent, the tortilla prepared from corn being a later invention. It is the food of millions of people in South America and is to a small extent imported to this country and is nearly always obtainable in certain stores in New York.

\section{TAPIOCA}

Tapioca is prepared from cassava starch by heating while moist on a hot iron plate with constant stirring. A part of the starch is cooked and forms a paste which agglutinates the mass which when dry forms small semi-translucent pellets.

\section{THE YAUTIA}

This is a tuber which resembles the taro, native to the West Indies and largely grown in Porto Rico. It is prepared for the table in the same manner as yams or sweet potatoes. As served in the native hotels of Porto Rico the writer found this vegetable quite insipid and by no means equal to the potato.

\section{THE ONION}

The principal bulb used as food in this country is the onion, of which there are more than 70 varieties. The characteristic flavor of the onion is due to an oil containing allyl sulphid, which is excreted through the lungs when this vegetable is eaten, giving the breath a characteristic odor.

The onion also contains a peculiar form of sugar to which the sweet flavor of roasted onions is due. The onion is much used as a food in Egypt where it is soft and sweet, in strong contrast with the hard pungent product of other lands. Herodotus tells us that nine tons of gold were spent in the purchase of onions for the workmen who built the pyramids.

The white onion is preferable to the red and yellow varieties, having a milder flavor. Young onions are better than mature ones for the same reason. The best method of cooking onions is 
by roasting in the ashes or in a very hot oven. To roast in the oven they may be buried in sand. When prepared in this way, the high temperature to which they are subjected volatilizes the greater part of the essential oil to which the odor is due and thus removes one of the chief objections to the use of this vegetable. Bunge, many years ago, called attention to the fact that the onion affords a very excellent form of food iron, and on this account may often be eaten freely with advantage by persons suffering from anemia.

A very sweet onion, closely resembling the Egyptian variety, is now grown in the Coachella valley of California.

\section{THE GARLIC}

A member of the onion family much used in Oriental and other tropical countries. Notwithstanding the general prejudice against this vegetable on account of the disagreeable character of its odor when present in excess, it must nevertheless be admitted that when properly used it does increase the palatability of certain salads, soups and other dishes, and a liking for it may be easily cultivated.

\section{THE LEEK}

A relative of the onion much used as a flavor by the Scotch and in some parts of England. It was an ingredient of the famous cock-a-leekie soup described by Sir Walter Scott and of the "Herby" pie of Cornwall, which according to Sir Charles Kingsley "diffuses through the ambient air a delicate odor of mingled guano and polecat." This highly aromatic vegetable has never found favor in this country, but is perhaps worthy of greater consideration.

\section{LILY BULBS}

The Chinese and some other Orientals make considerable use of the lily bulb, which is boiled or roasted and eaten with sugar or syrup. It is a wholesome food, free from sugar and starch and is beginning to be used in this country, especially by diabetics.

\section{THE WATER CHESTNUT}

The water chestnut is a tuber which grows in damp or swampy ground. It is small, irregularly shaped and covered with a brown skin. It has a white, brittle flesh which much resembles the chestnut in flavor. 


\section{Green Vegetables}

This class of food products contains a smaller amount of nutrient material than any other; nevertheless vegetables are essential to a biologic diet because of the valuable salts, cellulose and vitamins which they contain. They are nearly always spoiled in the cooking. When cooked in hard water the nutritive material is hardened and its digestibility diminished by the lime and magnesia salts of the water.

Analysis of the water used in boiling vegetables shows that it contains a large part of the nutritive salts and vitamins they are able to furnish. On this account vegetables should be steamed or baked rather than boiled, or if boiled the water in which they are cooked should be utilized in soups or porridge so that the important nutritive principles which they contain shall not be lost. Fresh vegetables should be used much more freely than is customary in this country.

\section{THE CABBAGE}

At the head of the list of green vegetables should be placed the cabbage, which has been used from the most ancient times. The cabbage was the Emperor Pompey's favorite dish. This garden vegetable is chiefly valuable for the stores of lime and alkaline salts which it contains, together with a considerable amount of the valuable antiscorbutic vitamin. These constituents are largely lost in boiling, however, so the vegetable should be eaten either raw or steamed. The Scotch save the water in which cabbage has been cooked and cook oatmeal in it, a dish which they call kale brose, a wise economy.

Cabbage may be eaten raw by many persons who are unable to eat cooked cabbage; in fact, raw cabbage is often tolerated by persons whose digestion is considered very delicate. When properly chewed, this excellent vegetable is very little likely to disagree. Some years ago the writer conducted a research for the purpose of determining the reason for this difference between raw and cooked cabbage. One experiment consisted in the inoculation of equal quantities of raw cabbage and cooked cabbage 
with a definite amount of colon germs. Later examination showed that while the bacteria had greatly multiplied in the cooked cabbage they had actually diminished in number in the raw cabbage. The greater readiness with which cooked cabbage undergoes bacterial changes in the intestine may be the cause of the disturbance noticed in some persons.

\section{Sauerkraut.}

This is a preparation of chopped cabbage which has been permitted to undergo lactic acid fermentation. Sauerkraut serves the same purpose for the peasantry of eastern Europe and western Asia that is served by the silo of the modern dairy farm, in which greenstuffs of various sorts are preserved by being made to undergo lactic acid fermentation. Sauerkraut supplies the Russian peasant with food lime and food iron during the long winter months when fresh vegetables are not obtainable. Sauerkraut is little used in this country except by foreigners, but it deserves a larger place in the national bill of fare.

The cabbage is a valuable source of iron, vegetable salts, and vitamins. In addition, it is a valuable means of giving the alimentary mass the necessary bulk to encourage peristaltic action.

When properly made sauerkraut is a most wholesome foodstuff. The following is a well tested method:

Select mature cabbage. Remove green and wilted leaves, also remove the core of the cabbage. Shred quite fine. Place a layer two inches thick in a small crock and pound until juice appears, then put into a larger crock or the permanent container. Add salt in the proportion of two ounces for each five pounds of cabbage, sprinkling in evenly as each layer is added.

When the crock is full, place a clean cloth over the top of the cabbage, then over this press down a clean board of such size that it will fit inside the crock rather loosely. Put one or more stones upon the cover. The weight should be sufficient to cause the juice to cover the cabbage within a few days.

Set the container aside in a room of ordinary temperature to ferment. When scum forms upon the surface, remove it. The scum consists of a micro-organism which if permitted to remain, will destroy the acid and cause decay of the cabbage. It is this that often develops a bad flavor and odor in sauerkraut.

The process of fermentation is shown by the rising of bubbles to the surface of the liquid. When the bubbling ceases, fer- 
mentation is completed, which will be at the end of eight or ten days in a warm room; in a cold room, two to four weeks.

In examining with reference to fermentation, tap the side of the container to loosen the bubbles adhering to the inner surface.

When the fermentation is finished, that is, when bubbles cease to rise, skim carefully and pour on melted paraffin wax to the depth of a quarter of an inch. The paraffin should be hot enough to cause the brine to boil as it is poured on. The layer of paraffin should be sufficiently thick to tightly seal the container and should not be disturbed until the sauerkraut is to be used. Paraffin is not necessary if a very cold place is available for storage.

\section{Composition of Satrerkraut.}

A reliable German authority gives the following as the chemical composition of sauerkraut:

\begin{tabular}{l|r}
\hline & $\begin{array}{r}\text { Per cent. } \\
\text { Water }\end{array}$ \\
Protein & 1.60 \\
\hline Fat & 0.35 \\
Lactic acid & 1.59 \\
Dextrose & 0.65 \\
Mannose & 1.00 \\
Crude fiber & 1.00 \\
Ash & 2.90 \\
Sodium chlorid (common salt) & 2.00
\end{tabular}

\section{BRUSSELS SPROUTS}

A curious member of the cabbage family in which instead of one large head the plant bears many small heads. Brussels sprouts may be regarded as superior to ordinary cabbage on account of the larger amount of food iron present, as indicated by the green color due to chlorophyl.

\section{CAULIFLOWER}

Another remarkable member of the cabbage family of which the flower is the edible part. Its chief value is in the salts and vitamins which it supplies. 


\section{BROCCOLI}

A variety of cauliflower which is distinguished by the pe culiar form and coloring of its edible flowers.

Broccoli sprouts, a recent development, consist of flowering sprouts which spring from the axils of the leaves. The purple leaved variety is especially esteemed.

\section{KALE}

A plant of the cabbage family which does not form a head, but has long, curled, dark green leaves which are rich in lime, iron and vitamins. Kale is a good winter salad, but it is best used as greens. The thick leaves are not damaged by frost, but become more tender by repeat d freezing an so may be left in the garden during the winter and gathered as needed for use.

\section{THE PAI TS'AI OR CHINESE CABBAGE}

The pai ts'ai (pronounced pi-tsi) is a relative of the turnip It forms a cylindrical, rather loose head with greenish outes leaves and a white, very tender and pleasantly flavored heart. It matures best in autumn and should be sown in midsummer in rich friable soil and must be well watered and kept growing steadily. Use like cabbage.

The percentage composition of the fresh raw cabbage is as follows : protein, 1.2 ; sugar, 1.4 ; fiber, 0.5 ; ash, 0.5 ; water, 96.4

After extraction by thrice boiling, the composition is: pro tein, 1.07 ; sugar, 0.05 ; fiber, 1.16 ; ash, 0.24 ; water, 95.30 .

\section{PALIM CABBAGE}

The terminal bud of several varieties of palms is a very toothsome vegetable which somewhat resembles cabbage in tex. ture and has, when raw, a delicious flavor suggesting that of the chestnut, and when cooked a delicate meaty flavor. Xenophon mentions palm cabbage as being a favorite among the young Greeks, who, he says, greatiy enjoyed the "brain" of the palm as well as its luscious dates.

The palm cabbage contains a very small amount of carbo. hydrates and is suited for use by diabetic patients, for whose benefit the millions of cabbage palms growing in the swamps of Florida ought to be utilized. This vegetable, canned and sold 
under the name, "The Heart of the Palm," may be obtained from certain dealers in delicatessen who get their supply from Union, a group of islands of the South Pacific.

\section{CELERY}

This vegetable is usually eaten raw. Its nutritive value is very low, only about five calories to the ounce, but it is rich in vitamins, as has been proven by McCollum in feeding experiments. It is also rich in food salts. Its flavor is appetizing and its free use is to be commended. It may be eaten cooked.

\section{LET'TUCE}

The lettuce is a very old-fashioned plant. Its mildly flavored, juicy leaves have made it a great favorite from the most ancient times.

Augustus Caesar was very fond of lettuce and attributed to its use his recovery from a serious illness. It is said that he afterward held this plant in such high esteem that he erected a statue and dedicated an altar to it.

In the time of Domitian, the Romans served lettuce with sggs, as we do at the present time-probably one of the most ancient of eating customs. It was served with the last course, so as to stimulate the jaded appetites of the feasters.

It is strange that this estimable plant did not reach the western world until the time of Queen Elizabeth, when it was first grown in England.

The lettuce furnishes valuable salts and vitamins and supplies bulk to aid bowel action. It is especially to be recommended to those who have a tendency to overfatness. It should be used by such persons very freely, and with lemon juice only, oil being svoided. A large plateful should be eaten at the beginning of the meal.

\section{ASPARAGUS}

A highly toothsome green vegetable, valuable for its salts and vitamins, as well as for the large amount of cellulose which it contains.

Recent analyses of asparagus show that the raw vegetable contains 10 per cent. of solids, of which 70 per cent. is soluble in cold water and 80 per cent. in boiling water. The composition is as follows: 


\begin{tabular}{lll} 
Water & Per cent. \\
Sugar & \\
Protein & \\
Fiber & \\
\hline Fat & \\
Mineral matter & \\
\hline
\end{tabular}

Green asparagus contains less fiber than white and yields more soluble matter when boiled. The asparagus tip is especially rich in vitamins.

Asparagus is botanically related to the onion. If the tender shoots are left in water a few days a marked onion odor develops. An oil may be extracted from raw asparagus shoots which contains sulphur, and it is to this that is due the peculiar odor observed in the urine after eating asparagus.

It is evident that asparagus should be steamed rather than boiled. After boiling for a few minutes the amount of nutritive material left is too small to be of any real value, at least fourfifths of all the nutrients having been dissolved by the boiling water.

\section{EINDIVE-WHITE CHICORY}

The blanched leaves are the edible part. It is fairly rich in salts but of little nutritive value.

\section{OKRA}

The edible part is the large unripe seed pod, which contains a large amount of pectose which jellies when the pods are cooked in water, on account of which it is largely used in preparing a dish called gumbo. The pods may be boiled and eaten like asparagus.

\section{THE ARTICHOKE}

This plant is a variety of thistle of which the undeveloped flower buds are cooked and eaten. The plant is said to contain inulin instead of starch, on which account it is recommended as a food for diabetics.

\section{ICELAND MOSS}

Iceland moss is a lichen which grows in the Arctic regiors. It is much used by the Laplanders. It contains no starch, and whatever food value it has is doubtless due to the cellulose and vitamins which it contains. Reindeer moss is also a lichen. 
The lichen is a curious partnership between an alga and a iungus. The alga forms food, which the fungus cannot do, and the thread-like fungus which entwines the alga supplies water; thus these two distinct species of plants thrive in partnership although neither is able to live alone.

The use of the lichen as a food for human beings has been known from the most ancient times. According to A. A. Hansen, "it has been almost definitely demonstrated that the manna which was such a boon to the children of Israel was the manna lichen, lecanora esculenta. During certain seasons of the year, this plant dries and hardens, forming warty grains which fall in showers from the mountain sides into the valleys. These are used as food in Algeria, southwest Asia and the Steppe region. The grains form an acceptable substitute for corn."

Iceland moss is used in northern regions for bread. After soaking in water to remove a bitter principle, the moss is dried and ground to a fine flour which is mixed with an equal amount of wheat flour and made into loaves of an excellent bread.

Reindeer moss has been used in Sweden in like manner for bread making. This remarkable lichen grows in large tracts in northern Minnesota and in many places along the north shore of Lake Superior. Its food value is said to be three times that of the potato. Millions of reindeer, musk oxen and other antelopes thrive upon this hardy plant, which they dig out from beneath the snow and the ice of the far north. Reindeer moss may sometime become an important source of human sustenance.

\section{IRISH MOSS}

This is an alga which has long been in use, especially as a delicacy for invalids. It is a seaweed which grows along the Irish coast and the coast of France. It has little, if any, food value and is much less used than formerly.

\section{AGAR-AGAR}

Sometimes called Ceylon moss, also known as Japanese or Bengal isinglass, obtained from a species of seaweed that grows along the coast of Japan and Ceylon.

Agar, taken in its crude state, is an excellent remedy for constipation, especially when its use is combined with paraffin oil as a lubricant.

Agar is also most valuable for all culinary uses for which 
gelatine is commonly employed. It makes much finer jellies than does gelatine, and is free from every objection which can be offered against the animal product. Being pure cellulose (hemicellulose) it cannot undergo putrefaction, and so may be used when most animal products are forbidden.

Agar is superior to animal gelatine not only in the fact that its flavor is not reminiscent of hoofs, horns, fragments of hide and sundry other slaughter-house remnants, but also because it is more convenient in use. Agar melts at the temperature of boiling water, and congeals or jellies at a temperature above $120^{\circ} \mathrm{F}$., and so does not require an ice box to harden or set even on the hottest summer day. It may be used for any purpose for which animal gelatine is used.

\section{MUSHROOMS}

The mushroom is practically of no value as a nutrient, as the amount of digestible protein it aftords is seldom more than 3 per cent., with an equal amount of carbohydrates. Whatever value it has is due to the meat-like flavor which it imparts to soups and other dishes. It is highly indigestible, and is of very doubtful value as an article of food. The mushroom flavor, which is so highly relished by many persons, can now be obtained without the accompanying risk by the use of certain dried yeasts and yeast extracts.

Kakowski has shown experimentally that the eating of mushrooms, even those which are regarded as entirely wholesome, produces harmful effects in cases of nephritis.

\section{YEAST AND YEAST EXTRACT}

Certain yeasts and molds, like the mushroom, are rich in highly soluble substances which closely resemble in flavor the osmazomes of meats. A species of mold is thus the source of the famous soy sauce of Japan. German research has recently perfected a yeast which has a rich mushroom or meaty flavor and, added to soups, broths and vegetables, renders them highly palatable. This yeast is, besides, extraordinarily rich in vitamin B.

An extract (Savita) is prepared from this yeast which presents all the properties in a highly concentrated form (ten fold) and is very rich in food iron. This product readily replaces meat extracts in all culinary processes in which meat flavors are desired. 


\section{GREENS}

We are only just beginning to appreciate the value and essential importance of greens as a staple constituent of the daily bill of fare. In this respect civilized people are far behind the rest of the world. The Chinese and Japanese, in fact, all Oriental people make great use of greens, as do savage or primitive tribes in all parts of the world. Dr. Turner tells us that "few people can compete with the Kaffir woman in making 'a spinach,' for which purpose the young shoots of innumerable plants are used, among others the sprouts of the pumpkin, the sweet potato, mandioca, and numerous wild plants." The North American Indians made use of purslane, pigweed, and many other common weeds as greens. The recent studies of the green leaf and of its dietetic uses have revealed many most interesting and important facts.

The most marvelous chemistry known to man is that which is wrought out in the secret laboratory of the green leaf. The sun is the alchemist, the chlorophyl grain the reagent. Here, in this simplest of laboratories, are formed all the marvelous products of the vegetable kingdom. Here are produced the subtle elements from which come all the curious gums and resins, oils and essences, all the delicate aromas, - the attar of the rose, the fragrance of the lily, the delicate scent of the violet, the penetrating odors of the leek and the garlic, the soporous smell of the poppy, all the deadly poisons of the nightshade and hemp, upas tree and bitternut, all the rainbow tints of leaf and flower, all the proteins and starches and fats of foods, the grain and fibre of all woods, and the bark of tree and bush and twig in all the forests of the world.

Cholorophyl was once supposed to be a single color, but now we know there are four, two greens and two yellows; and from these are compounded all the rest of the numberless tints that beautify the world of flowers in field and glen.

But the end is not yet. In the wonderful green leaf, itself the scene of marvelous activity in growth and development, is produced a substance that imparts to the young of animals the power to grow. Here is the explanation of the marvelous growth of calves, colts, rabbits, guinea pigs and antelopes. They seem almost to grow and expand before one's very eyes while he stands gazing in wonder and surprise. 
Without this subtle vitamin which promotes growth and development, no animal cou'd grow to maturity. Without it the reproduction of animal life would soon cease, and the world become waste and void.

It is for this reason that green vegetables, and especially greens, are so important an element of the dietary. Cabbage, lettuce, spinach, cucumbers, greens of all kinds-some of these should be a part of every meal.

Here, too, is found the explanation of the importance of milk and dairy products in Nature's scheme of animal nutrition. In her peregrinations about the pasture, the cow gleans from grass and tender buds and twigs the precious vitamins, and gives them back in her generous lacteal fluid at milking time.

Nature has made more lavish provision for our sustenance than we appreciate. Our perverted instincts and erroneous dietetic habits have led us to overlook and ignore some of Nature's finest contributions to our welfare. We quite agree with the sentiments expressed by the writer of the following paragraphs, one of the experts of the United States Agricultural Department:

What we call weeds are no more so than other plants that we term vegetables. Weeds are vegetables, and our so-called vegetables were once upon a time no more than weeds. The classification results from a matter of habit. We are slaves of habit, and because we are so it has not occurred to us that we could eat anything but just the old list of vegetables our ancestors have eaten for generations. But now we are having our eyes opened and are beginning to peer into fence corners and back yards and wild pastures for new and wonderful foodstuffs that we have heretofore regarded as just weeds. It is a bit mortifying that because of the preconceived idea we have let most nutritious and valuable foodstuffs go to waste under our very eyes, while perhaps we were wailing that we had little to eat and that vegetables were too expensive and so on.

Among the plants thus neglected, but which, if properly improved and marketed, would enrich truck farmers, are yellow dock, dandelions, milkweed, golder thistles, mallows, purslane (recommended by Thoreau), poke-root, red clover, sorrel, hop shoots, yarrow, leek, and lupines.

All green leaves contain iron, lime, vitamins and other useful elements and may be used as greens when sufficiently tender for the purpose, provided no objectionable flavors or other noxious substances are present. Even grass and alfalfa have been used in emergency. The Chinese make use of a great number of 
greens which form a part of every day's bill of fare. The greens most commonly used in this country are spinach, New Zealand spinach, chard, dandelion, endive, mustard, turnip tops and let tuce. Lambs' quarters, a variety of pigweed, red root, purslane and narrow leaved dock are other excellent greens which may be cultivated or gathered from fields or fence corners. The accom panying table shows the remarkable amount of lime and iron found in each. (Table XXIX.)

Through the courtesy of Dr. David Fairchild, head of the Plant Introduction Bureau of the United States Department of Agriculture, the writer recently received several plants of the Abyssinian rumex, a relative of our native dock, which promises to be a most valuable addition to our own list of garden greens This plant produces in abundance large tender leaves during the whole season. The stems are tender as well as the leaves. The presence of oxalic acid in considerable quantity is indicated by a distinct acid flavor, but this may be easily removed by parboiling.

\section{Greens as Roughage.}

Greens contain a very considerable amount of cellulose, and are thus an excellent sort of roughage. The amount of carbo hydrates, protein and fats which they present is so small as to be almost negligible. But they are invaluable as the best sort of roughage, a source of the fat-soluble vitamin, one of the most precious of all vitamins, being an activator of growth, and lastly. a means of supplying to the tissues in a convenient and agreeable way the essential requirement of iron and of lime.

\section{The Iron Content of Greens}

Next to legumes, the greens show the highest iron content per ounce of any class of foodstuffs, the average being more than 6 per cent. of the daily ration of iron. That is, approximately 16 ounces of a mixture of the several greenstuffs named in the table furnish iron sufficient for one day. This is, of course, more than one could conveniently eat; but a quarter or even a third of this amount may be easily eaten.

Of the ten most valuable greens, the ones richest in iron are mustard greens, spinach, turnip tops, red root, dandelion and dock.

One of the best of all the greens is spinach, which supplies in fifteen ounces of raw material iron sufficient for a day. Dock and 
red root are so richly stocked with iron that only seven and a half ounces are needed to meet a day's requirement. An ounce and a half of dried spinach leaves will furnish iron sufficient for one day. The same amount of iron is supplied by half an ounce of the dried leaves of a common garden weed, the red root. An ounce of fresh spinach contains as much iron as an equal weight of ordinary beefsteak, and of a better quality.

It is important to remark about foods in general and of greens in particular that care must be exercised in cooking to prevent the loss of the lime and iron salts and the equally valuable vitamins. Boiling in water and drawing off and discarding the water in which the greens have been cooked, results in the loss of a small part of the useful elements of greens. On account of the considerable amount of oxalic acid found in spinach, this vegetable should be parboiled for two or three minutes even at the risk of losing a small portion of its salts.

Prolonged cooking at a high temperature is also damaging, and the same seems to be true of drying. It is important to make use of greens as soon as possible after gathering from the garden, while fresh and crisp. When wilted they become less tender. Canned greens are not equal in flavor to freshly gathered material but are nevertheless invaluable additions to the diet when fresh are not obtainable.

It is claimed for the newly perfected methods of preparing evaporated vegetables that the process does not destroy the vitamins, although they may be somewhat damaged.

Hindhede, the eminent physiologist of Copenhagen, wrote the author some time ago (1919) that he had kept a man in good health for twenty-three months on a strict diet of bread and potatoes with an abundance of greens. Without the aid of the greens, a person would soon become anemic on such a diet.

We learn from a friend who has lived many years in Japan that the Japanese peasant always eats greens with his rice.

\section{The Lime Content of Greens.}

There seems to be in man as well as in lower animals an instinctive recognition of the important position held by these coarse and relatively innutritious products in Nature's scheme of nutrition. The craving for greenstuffs experienced by nearly everyone as spring approaches is the natural consequence of the widespread lime starvation imposed by a winter dietary from 
which foodstuffs rich in lime are largely excluded. It is true that this deficiency in lime, almost universally characteristic of winter dietaries, is not necessary, and may be easily prevented by a proper selection of readily accessible foodstuffs; but, unfortunately; the fundamental facts respecting the lime content of foods have only recently been brought to light through the researches, most laborious and painstaking, of the eminent Professor Bunge of Switzerland, and more recently by Dr. Sherman, of Columbia University of New York. These facts are as yet practically unknown to the general public although of vital importance.

In proportion to their nutritive value, there is no class of foodstuffs so rich in lime as are greens, as shown in Table XIV. For example, polished rice contains one part of lime in 2,000 calories; cornmeal, one part to 900 calories; beans, one to 600 ; whole wheat, one to 400 ; hazelnuts, one to 100 ; while in asparagus we find one part of lime to 30 calories; in cabbage, one to 24 ; cucumbers, one to 50 ; dandelion, one to 25 ; and in chard and celery, one to 12 .

Considered from the standpoint of bulk and weight, the situation is decidedly different. With a few exceptions, the lime content per ounce is not greater in "greens" than in many other vegetables. Notable exceptions are, chard, with a grain to the ounce; mustard greens, with 3 grains; paprika, nearly 1.5 grains; turnip tops, 2 grains, and watercress with more than a grain.

As will be seen by a careful study of Table XIV, there is a marked difference between the different species of greenstuffs. Brussels sprouts, cucumbers, lettuce and romaine contribute to the diet no larger amounts of lime salts than string beans, green peas, onions, pumpkins and squash. Lettuce, cucumbers and celery are of no more value as sources of lime in the quantities eaten than are potatoes, their content of lime being very low. The really valuable greens, as sources of lime, are mustard greens, turnip tops, chard, collards, cabbage greens, kale, spinach, endive and dandelion. Ordinary servings of three or four ounces of these greens will yield from 13 to 60 per cent. of the daily lime requirement, as shown in the table.

It is clearly evident that greens are dependable sources from which lime salts may be obtained in almost any quantity desired. The small ordinary serving of 3 ounces may be easily doubled if occasion demands, as when it is necessary to replenish the de- 
pleted body its store of lime in a demineralized patient. By feeding greens twice a day and doubling the size of the serving, at the same time taking care to include in the bill of fare as many foods rich in lime as possible, one may easily increase his lime intake to three or four times the normal requirement, and thus rapidly make good the deficiency in salts which is not infrequently the fundamental fault in cases of chronic disease.

Among other excellent greens which may be mentioned are milkweed shoots, golder thistles, poke shoots, cowslip, red clover, yarrow, hop shoots, mallows. Purslane, now a neglected garden weed, was boiled and eaten in every cottage in the time of Charlemagne. It was a favorite of Thoreau and was presented to the early settlers of Massachusetts by the Indians who gathered it from their corn fields.

The tender leaves of the alfalfa have been used as greens with some success. The youngest leaves are selected, freed from stems, parboiled, well boiled, and made into a purée.

The root of the poke root is poisonous but its young shoots may be served when cooked like asparagus, which they much resemble. The young leaves make excellent greens resembling spinach. They should be twice scalded or parboiled.

Witloof is a variety of chicory. It is planted in the late spring or even as late as June, to produce roots to be placed in the cellar in the late fall for winter forcing. The roots may be purchased in the late fall from gardeners. Serve as an uncooked salad.

The archeologists tell us that they find among the relics of ancient dwellings evidence that our primitive forbears made use of a vast number of plants which we now ignore, and some of which might be restored to our bill of fare with advantage. More than a hundred such plants, including several excellent greens, have been described by an English author, among which may be named the red poppy, shepherd's purse, cuckoo flower, hawthorn buds, Alexanders, goat's beard, sow thistles, nettle, borage, marjoram, couch grass and nipplewort. 
TABLE XXIX.

Lime and Iron Content of Greens.

\begin{tabular}{|c|c|c|c|c|}
\hline & \multicolumn{2}{|c|}{ LIME } & \multicolumn{2}{|c|}{ IRON } \\
\hline & $\begin{array}{l}\text { Grains } \\
\text { per oz. }\end{array}$ & $\begin{array}{c}\text { Per cent. } \\
\text { of day's } \\
\text { ration in } \\
1 \text { oz. }\end{array}$ & $\begin{array}{l}\text { Grains } \\
\text { per oz. }\end{array}$ & $\begin{array}{c}\text { Per cent. } \\
\text { of day's } \\
\text { ration in } \\
1 \mathrm{oz} \text {. }\end{array}$ \\
\hline Cabbage greens & 0.65 & 4.2 & 0.008 & 3.4 \\
\hline 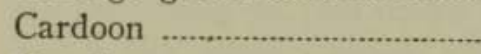 & 0.93 & 6.0 & 0.033 & 13.2 \\
\hline Chard ............................. & 0.92 & 6.0 & 0.010 & 4.7 \\
\hline 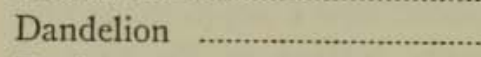 & 0.64 & 4.2 & 0.012 & 5.1 \\
\hline Dock, narrow leaved... & 1.00 & 6.7 & 0.028 & 13.4 \\
\hline 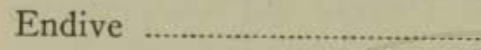 & 0.99 & 6.4 & 0.018 & 7.2 \\
\hline Imperial kale & 1.30 & 8.4 & 0.019 & 7.6 \\
\hline Lamb's quarters ... & 1.90 & 12.5 & 0.009 & 4.3 \\
\hline Lettuce & 0.26 & 1.7 & 0.003 & 1.3 \\
\hline 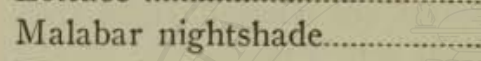 & 1.42 & 9.2 & 0.013 & 5.2 \\
\hline Mustard & 3.01 & 19.6 & 0.021 & 9.2 \\
\hline Portulaca flora & 1.45 & 9.4 & 0.023 & 9.2 \\
\hline Purslane ....................... & 0.88 & 5.8 & 0.008 & 3.9 \\
\hline Redroot $\ldots \ldots \ldots$ & 3.24 & 21.6 & 0.028 & 12.9 \\
\hline Rocket salad & 1.32 & 8.6 & 0.018 & 7.2 \\
\hline Romaine & 2.76 & 1.8 & 0.053 & 22.7 \\
\hline Spinach & 0.41 & 2.7 & 0.016 & 6.8 \\
\hline Spinach, mountain & 1.27 & 8.5 & 0.008 & 3.9 \\
\hline Spinach, New Zealand & 0.48 & 3.2 & 0.009 & 4.1 \\
\hline Turnip tops & 1.05 & 7.0 & 0.010 & 4.7 \\
\hline Watercress & 1.15 & 7.5 & 0.008 & 3.6 \\
\hline
\end{tabular}

The most popular of all greens is spinach, a native of Persia where it is known as ispany, hence its Latin name spinachia. Rhazes tells us that spinach was cultivated in ancient Babylon and Nineveh. It grows wild in Asia Minor and has long stems and narrow leaves with points like thistles. The cultivated plant has rounded thick leaves which Olivier de Serres trained to gigantic size. There is also the cabbage spinach of Milan.

The composition of spinach is:-Water, 92.32 ; nitrogenous matters, 1.34 ; fats, 0.17 ; starch, 4.77 ; cellulose, 0.87 ; ash, 0.55 . 


\section{Salads.}

In the Middle Ages, the salad was in high repute, and its value as a food accessory seems to have been very well appreciated. John Evelyn wrote a discourse on "sallets," and Chaucer and others dwelt upon the virtues "of the blood-cooling sallet." The cabbage, centuries before it acquired robustness, rotundity and variety through the ingenious tutelage of the old Dutch gardeners, grew wild on the shores of the Mediterranean, whence it was gathered to garnish the tables of the rich epicures of Rome.

The salad is deserving of more attention and better treatment than it receives in these degenerate days. It should be given the place of honor in the table service and should appear at two daily meals instead of one, and at the beginning of the meal instead of at the end. Thus treated, the salad, composed of fresh garden products rich in lime, iron, cellulose and vitamins, may become a valuable corrective and regulator and perhaps an efficient means of reforming our wretched modern dietary.

Among the plants most commonly used as salads are lettuce, cabbage, Chinese cabbage, cress, cucumber, endive, tomato, onion, beet root, cardoon, peppergrass, chevril, borage, burnet, mint, parsley, nasturtium and sorrel. 


\section{Root Vegetables}

This class of vegetables is characterized by a low content of fat and protein. Recent observations have shown that the protein of vegetables is probably superior in general to that of cereals.

As regards carbohydrates there are two classes, those which contain carbohydrates in the form of insoluble starch and those in which the carbohydrates are in the form of sugar or dextrin and hence soluble. The last mentioned class of vegetables are of special value in the feeding of diabetic patients, since by boiling two or three times they may be freed from their carbohydrates In this class are the turnip, beet, parsnip, cabbage, egg plant and spinach. The chief value of most vegetables lies in their rich store of alkaline salts and the valuable vitamins which they contain.

Steenbock and Gross have recently reported experiments which show that the carrot, the artichoke and dasheen are more satisfactory as sources of vitamins than are the sweet potato and sugar beets.

\section{THE BEET}

There are numerous varieties of beets, which differ much in flavor, form, and nutritive value. The ordinary beet contains about 8 to 10 per cent. of carbohydrates. Young beets are often eaten whole, the roots and tops being boiled together, and in this form are an exceedingly wholesome and valuable addition to the bill of fare. Mature beets are best prepared by steaming or baking. Boiled beets are commonly served with vinegar, which renders them more or less unwholesome. Lemon juice should be used instead of vinegar, for which it is more than a complete substitute since it is not only a wholesome food instead of a poison, but supplies vitamins and aids digestion and assimilation instead of hindering these processes.

\section{THE PARSNIP}

The parsnip has been cultivated and regarded as a valuable foodstuff for more than two thousand years. It is even riche1 than the beet in carbohydrates. In nutritive value it exceeds all the common root vegetables. 


\section{THE CARROT}

The carrot, like the parsnip, is an old-fashioned vegetable. It is much more palatable and edible when young than when mature. Its nutritive value is about the same as that of the beet. Its yellow color is due to carotin, a coloring matter which Hess has recently shown to be associated with the highly important fat-soluble vitamin which is essential for growth of young animals and for the maintenance of health in adult animals. Steaming is the proper method of cooking the carrot. When boiled in the usual way, the carrot loses a large portion of its nutrient value.

The carrot has long enjoyed a great reputation as a vegetable antiseptic. At Vichy, carrots are served in some form at every meal and are believed to be of value in combating intestinal putrefactions. Metchnikoff noticed that by feeding carrots to animals he could make their stools nearly free from bacteria.

Dr. Mary S. Rose and others have shown that on a diet consisting chiefly of carrots the calcium balance may be maintained; that is, the daily intake of lime may be made to equal the daily loss. The amount of carrots eaten daily must be considerable.

\section{Pigmentation of the Skin from the Use of Carrots and Squashes.}

Hess and others have called attention to the fact that the yellow coloring matter of the carrot, carotin, may be deposited in certain skin areas, particularly the palms of the hands and the soles of the feet when carrots are freely eaten. Hashimoto, of Japan, states that the same coloration may result from the free use of the squash and other yellow vegetables, as well as the eating of large quantities of "maikan," a Japanese orange and "laver," another Japanese vegetable.

No harm has been shown to result from this pigmentation, which appears to be confined to the superficial layers of the skin. The pigmentation is in some cases observed to be quite prominent in the nasolabial folds of the face.

\section{THE TURNIP}

This root, like the other common food roots, has been used from the most ancient times, and like them is found growing wild in most European countries. There are many varieties of this vegetable, the best of which is the swede. The turnip contains 
less carbohydrates than beets, parsnips or carrots, and no starch, for which reason it is much used for diabetics after removing by repeated boilings the sugars and other soluble carbohydrates which it contains. The turnip is richer in lime than other roots, and the tender tops may be used as a green or salad. Turnip tops contain more iron than any other green except red root and dock.

\section{SALSIFY-VEGETABLE OYSTER}

A root which somewhat resembles the parsnip and has about the same nutritive value as the carrot. When cooked, it has a flavor somewhat resembling that of the oyster. The leaves or tops may be eaten as a salad or served as greens. In the black saisify the principal carbohydrate is inulin, which makes it a valuable food for diabetics.

\section{THE RADISH}

A highly succulent root which has a pungent flavor, due to an essential oil resembling the oil of mustard. The oil is found chiefly in the skin, which should be removed when this vegetable is eaten raw, as the essential oil is irritating and while temporarily stimulating is in the end damaging to the gastric glands.

\section{THE HORSE RADISH}

This root contains a highly pungent and very acrid essential oil, on account of which it is used as a condiment. The oil of horse radish is highly irritating and its presence marks this vegetable as a poisonous weed rather than a food. It should never be eaten.

\section{KOHL-RABI (TURNIP CABBAGE)}

This is a curious vegetable in which the stored material is placed in an enlargement of the stem just above the ground instead of in the underground root. In flavor the kohl-rabi resembles both the turnip and the cabbage, but when young and tender is more delicate. Its store of carbohydrate is small, only 4 per cent. and its total nutritive value is also small. It is chiefly valuable for its alkaline salts. 


\section{THE JERUSALEM ARTICHOKE}

The Jerusalem artichoke is a member of the sunflower family. It got the name artichoke because it tastes like the true artichoke, a different plant. It is a native of America and was much used by the aborigines, who carefully cultivated it in all parts of North America. It was cultivated in Italy nearly 300 years ago, where it was known as girasole or sunflower artichoke. The word girasole was easily corrupted to Jerusalem, and the bulbs became known as Jerusalem potatoes from which was made Palestine soup. Hopkins reports that the Jerusalem artichoke may satisfactorily take the place of the potato when necessary, although it is probably less well utilized.

The artichoke produces prodigious crops. Such enormous yields as 20 to 40 tons to the acre have been reported, which is many times the yield of the potato or any other root crop.

The artichoke should be steamed rather than cooked in water as this prevents loss of flavor and aroma. A still better plan is to bake in a hot oven in a covered dish with a little butter and salt. No water should be added. This bulb is worthy of more consideration than it has heretofore received.

Colin finds that the inulin of the Jerusalem artichoke is during the winter converted into sugars which in the spring are utilized in starting the new growth. In the use of artichokes by diabetics note should be taken of this fact.

\section{CELERIAC}

This vegetable has a root similar to that of the turnip. It has the flavor of celery and is cooked and served like the turnip. It is deserving of more attention than it has received up to the present time. 


\section{Vezetable or Garden Fruits}

Among the most commonly used vegetable fruits, or fruits produced by annual plants, are the tomato, the cucumber, the chayote, the eggplant, the citron, watermelon, muskmelon, squash, pumpkin and vegetable marrow.

\section{THE TOMATO}

The tomato is one of the best and most useful of our garden fruits. It was introduced to Europeans by Sir Walter Raleigh, who first discovered it on Roanoke Island. It was long cultivated as an ornamental plant, but has only been extensively used as a food during the last seventy years. There are people still living who first knew the tomato as a show plant in the flower garden, then known as the love apple.

For a long time after the tomato began to appear on American tables it was suspected of being the cause of cancer, though without the slightest ground for such a prejudice, and was avoided by many people on this account.

Steadily the tomato has won its way into popular favor until now there is no garden vegetable so largely and universally used in this country as is the tomato, with the exception of the potato. The same is equally true in Mexico, Italy, and several other countries.

The tomato contains nearly twice as much iron as does milk, and five times as much as egg white; in fact, it is richer in iron than apples, pears, oranges, grapes, melons, cucumbers and peaches, and contains more than half as much as onions, parsnips, turnips, radishes, sweet potatoes, kohl-rabi, eggplant, carrots, celery, cauliflower, chestnuts, beets and bananas. Of lime, the tomato contains more than apples, bananas and rice, and as much as hominy, corn flakes and eggplant, and more than three times as much as beef, mutton or fish.

The tomato is also rich in useful potash salts, of which it contains more than most fruits and many other vegetables. The tomato owes its acid flavor to citric rather than to oxalic acid as was formerly supposed. 
Givens has shown that raw tomatoes may be dried at a temperature of $60^{\circ} \mathrm{C}$. $\left(140^{\circ} \mathrm{F}\right.$.) without destroying the antiscorbutic vitamin. The vitamins are also uninjured by canning.

\section{Vitamins in Tomato Juice.}

Hess has shown that the juice of canned tomatoes is a most valuable source of vitamins. Its superlative value is due to the fact that it contains all three of the food essentials, or vitamins, while most fruit and vegetable juices are deficient in one or more.

Hess recommends that the juice of canned tomatoes should be given to infants instead of orange juice as being less expensive. The average infant requires about one ounce daily.

Observations by Mendel showed that one-tenth of a gram (one grain and a half) of dried tomato contains a sufficient amount of the fat-soluble vitamin to take the place of butter in the dietary of growing rats and to bring them quickly to full maturity. An equivalent amount for a human being would be about one-half ounce of dried tomato per day or four to six aunces of fresh tomatoes.

\section{THE CUCUIMBER}

One of the first cultivated plants, the cucumber, has been known for more than 2000 years. The cucumber is still largely ased in Egypt, where it is almost a food staple and is much eaten by children and even infants as well as by adults, thus proving its essential wholesomeness. In this country the cucumber is usually spoiled by pickling before it appears upon the table. Fresh cucumbers, well masticated, are wholesome and valuable as sources of vitamins and salts which help to maintain the alkalinity of the blood, and also furnish roughage.

\section{THE EGGPLANT}

The eggplant contains, in small quantities, a poisonous alkaloid and an irritating substance, both of which may be removed by parboiling for two or three minutes before cooking. Prepared in this way, it is a wholesome plant and a valuable source of food alkalies and vitamins.

\section{THE WATERMELON}

A native of ancient Egypt and of America. It was discovered by Sir Walter Raleigh when he discovered Roanoke Island in 1584. A very valuable fruit on account of the large amount 
of sugar found in its juice. When eaten in quantity, the pulp should be rejected as the total food value is in the juice.

\section{THE MUSKMELON}

All the different varieties of this excellent melon are wholesome. It is richer in alkalinizing elements than any other vegetable food and hence may be freely eaten with advantage by persons who have a tendency to acidosis.

\section{THE SQUASH AND VEGETABLE MARROW}

The several varieties of squashes are chiefly valuable for the vitamins and alkali-forming elements which they furnish.

The vegetable marrow is a wholesome and valuable garden fruit with properties similar to those of the squash, though its nutritive value is less.

\section{THE PUIMPKIN}

This gigantic fruit is a contribution from our Indian predecessors, who cultivated it in their corn fields. It is a coarse, crude product, but when properly prepared is a wholesome and grateful addition to the bill of fare and yields a good share of useful food salts and vitamins together with a minimum of nutriment in the form of starch, dextrin and sugar.

\section{THE CHAYOTE}

This new garden vegetable, recently imported from Mexico, is a relative of the cucumber. It grows on a vine trained upon a trellis, and when fully matured is about the size of a small apple. The chayote, like the cucumber, is somewhat lacking in flavor, but when cooked and served with some suitable sauce is an acceptable addition to the bill of fare in early winter. It is sold in the New Orleans markets. 


\section{Legumes}

There are many species and many varieties of legumes. The bean and the lentil have been known from very ancient times. Many kinds of beans were cultivated by the aborigines of this country. The lentil has been a staple food in the Orient from remote ages. The soy bean has been the meat and milk of China for thousands of years.

With the exception of the soy bean, beans, peas and lentils are much alike in composition. The soy is richer than other legumes in fat and protein.

All the legumes are very rich in lime, iron and vitamins.

Legumes differ from most other vegetable foods in the fact that they contain an appreciable proportion of purin bodies, which are converted in the body into uric acid. This amount is small, however, compared with that contained in meat. Dried beans contain only 4 grains of uric acid (xanthin) to the pound, whereas dried meat contains 56 grains.

The protein of beans is inferior in quality to that of the cereals, potatoes and nuts, as well as to that of meat, milk and eggs, being incomplete. This is not true of the soy bean, however, which furnishes a protein of high quality.

\section{Digestibility of Legumes.}

The comparative indigestibility of the protein of vegetables has been urged as an argument in favor of flesh foods and as an objection to the use of vegetable foods. The evidence is accumulating, however, that the discrimination against vegetables in this regard has been unfair and based upon inaccurate observation. Lean meat consists of the soft pulp of the muscle tissue proper held together by a framework of connective tissue. The connective tissue is completely digested and dissolved by normal gastric juice. Thus lean meat is well broken up in the stomach by gastric digestion, even though it may be swallowed in masses and without sufficient mastication. The nutritive substance of vegetable foods is imprisoned in a meshwork of cellulose which is not acted upon by either the saliva or the gastric juice and hence cannot be dissolved in the stomach. The consequence is that if 
vegetable food is not thoroughly reduced to a paste in the mouth but through hasty eating is swallowed in masses, quite a common practice, the necessary result is incomplete and tardy digestion. Only that portion of the starch with which the saliva can come in contact will be digested and only such portions of protein will be dissolved as are brought in contact with the gastric juice, so a considerable portion will escape digestion altogether.

Dr. Wait, an expert employed by the United States government in the study of foods, some years ago published a most interesting account of experiments by which he showed that legumes, when properly cooked, have a very high degree of digestibility. Cooking in hard water impairs their digestibility, but when cooked by the Japanese method the results are most excellent. For example, the Japanese to $f u$, which is prepared from the soy bean, has a digestibility for protein of 97 per cent., and for carbohydrates of 88 per cent.

It should be noted that cooking in hard water hardens the protein of legumes and lessens its digestibility.

Experiment has shown (Adkins) that the digestibility of beans may be increased as much as 15 per cent. by sprouting. This increase of digestibility disappears, however, if the sprouted beans are dried. After sprouting they must be kept moist until used.

\section{Alkalies to be Avoided.}

The common custom of cooking beans with soda or saleratus to soften the seed coverings is most objectionable. Alkalies exercise a powerfully destructive effect upon vitamins; hence the practice referred to cannot be too strongly condemned.

\section{The Iron Content of Legumes.}

The product of a most extraordinary class of plants, the legumes (beans, peas and lentils) are the richest of all vegetable foods in food iron. The lentil, richest of all, contains exactly the same amount of iron per ounce as the egg yolk, a percentage so great that a full day's ration is supplied by six ounces of the raw material. The lentil has been serving the human race as a blood builder from the most ancient times.

Beans, both navy and lima, are almost equally rich in the blood-making element, seven ounces being sufficient for a complete iron ration for a day. 
Peas furnish not quite two-thirds as much iron as do lentils, but nine ounces suffice for a day's ration. Even green peas are equally well stocked with iron if the estimate is based upon food values, one hundred calories furnishing one-ninth of a day's requirement of iron.

The ruddy and well-nourished condition of the famous Hebrew children who insisted on sticking to their accustomed fare of "pulse," rejecting the juicy venison and mutton chops of the king's table, must have been due, then, to the liberal ration of iron which they received rather than to a high protein intake. Peas and lentils are just as good for the children of the present generation as for those of Nebuchadnezzar's day.

\section{The Lime Content of Legumes.}

The legumes furnish in small bulk so large a store of lime that they are most useful in balancing up the bill of fare. This may be clearly seen by reference to Table XIII, which shows us that an ounce of beans contains a grain of lime. An ordinary serving represents about an ounce and a quarter of dry beans, which would supply 8 per cent. or one-twelfth of a day's ration.

Peas have a lime content about half that of lentils, and two-thirds as much as beans. When made a staple of the bill of fare, the size of servings may be increased to two or even three times the ordinary, making the legume supply nearly one-fifth of the day's ration of lime.

Legumes, particularly lentils, are deserving of a larger place in the dietary than has heretofore been accorded them. Their use has been restricted somewhat, perhaps, by the fact that much more time and skill are needed to render them an attractive feature of the bill of fare than is required for cereals and vegetables. Many breakfast foods are ready to serve straight from the carton. Oatmeal may be served in ten minutes if the water is already hot. Potatoes go into the oven and out again in half an hour, and other vegetables take care of themselves in a fireless conker. But beans need several hours' exposure to full cooking heat under carefully regulated conditions to render them acceptable to both the palate and the stomach; and so the bean is not popular with the cooks except perhaps in the vicinity of Boston, unless supplied in tins ready cooked from the grocer.

The solution of the bean problem will be found in the pressure cooker, the next improvement needed to equip the complete 
modern kitchen. At a temperature $8^{\circ}$ or $10^{\circ} \mathrm{F}$. above the boiling point of fresh water beans cook very quickly, in an hour easily, and they are improved in flavor also. And still further, the tough woody hulls are so softened that they occasion less disturbance in the intestinal tract, being readily broken up.

A word of caution must be said in relation to the use of legumes. The elaborate feeding experiments of McCollum and others have shown that the protein of beans is of inferior quality and by no means a substitute for the fine protein of milk. This fact is important in this connection because of the possible injury in making the diet consist so largely of beans as to overburden the body with the elimination of unusable protein. The proteins of peas and lentils are superior to those of beans; hence it is advisable to utilize these legumes more largely than beans as a source of food lime and its associated protein. Beans should be supplemented by cottage cheese or nuts, one to three ounces.

When the soy bean has become well established in this country as a food product it will be doubtless recognized as a superior source upon which to draw for extra supplies of a fine quality of vegetable protein.

\section{BEANS}

Beans and other pulses are among the most ancient of cultivated foodstuffs. The ancient Egyptians worshiped the bean because of its resemblance in shape to the kidney and to the male sex gland. They refused to eat this legume, fearing that in so doing they might be consuming a human soul consigned by fate to expiate its sins by a term of life in this humble plant.

The old Romans used beans in their religious services, and threw black beans on the graves of their deceased friends as a part of the burial service. At Roman feasts in memory of the dead, beans were cast upon the altar fire. It was their belief that the odor of burning beans would drive away evil demons.

This ancient superstition in relation to beans still survives among the modern descendants of the Romans. In Italy, it is at . the present day customary to eat beans on the anniversary of the death of a friend and to distribute the seeds among the poor.

An ancient Jewish ceremonial law forbade the use of beans by the high priest on the day of atonement, because of the belief that the eating of beans caused drowsiness.

Pythagoras thought the black spot on the bean to be an 
mblem of death, and warned his followers to avoid eating beans. A relic of these old superstitions still survives in some of the zountries of England, where it is commonly believed that sleeping in a bean field will cause bad dreams or even insanity.

Beans were among the few vegetables cultivated by the Indians when this country was discovered by Europeans. They planted pole beans with their corn, and had developed varieties of the legume from which many of our modern beans are derived.

The kidney or haricot bean occurs in many varieties, white, red, navy bean, etc.

Broad beans, lima beans, butter beans and frijoles are other varieties the characteristics of which are so well known as to require no description.

The bean is the most strongly basic or alkaline of all foods and so may be used advantageously with such "acid" foods as oatmeal and other cereals.

\section{The Mung Bean.}

The composition of the mung bean is very similar to that of the ordinary bean of this country. According to Church, the percentage composition is: water, 11.4 ; ash, 3.8 ; protein, 23.8; fat, 2.0 ; fiber, 4.2 ; carbohydrates, 54.8 . It is the best bean for sprouting.

\section{The Adsuki Bean.}

Another variety introduced into this country from Japan is the Adsuki, a red bean which closely resembles the mung in composition. It is the favorite bean of Japan, where it commands the highest price. It has a delicious flavor, being wholly free from the beany taste characteristic of the American varieties of this legume. Tests made by the Plant Introduction Bureau of the Agricultural Department show that this bean can be readily grown in this country and it will doubtless in time achieve high rank as a staple foodstuff.

\section{String Beans.}

The unripe bean pod with its contents, so-called snap beans, when properly cooked and served, is not only very palatable but a highly valuable vegetable dish for which we are indebted to the American Indian, from whom Europeans learned its value. 


\section{Shelled Beans.}

This is a very excellent and easily digestible vegetable when properly cooked and served. The relative proportions of protein, carbohydrates and salts are about the same as in the ripe bean, but the quantities only half as great on account of the large amount of water present.

The hahto bean, a Chinese variety, which is about half the size of the lima bean, is superior to all other beans for use as a green vegetable. In cooking it requires only the addition of a little salt, since it contains a considerable percentage of a very digestible fat. The flavor developed in the cooked bean is most delicious. This choice vegetable has recently been introduced into this country by the United States Department of Agriculture, from whom information can be obtained concerning seed. It is quite hardy and ought to be planted in every garden.

\section{Succotash.}

Succotash is a dish invented by the American aborigines, by whom it was known as "sawquetash." It consists of a mixture of shelled beans and green corn cooked together.

\section{THE SOY BEAN}

The soy bean has been used in China since 2838 B. C. It was introduced into France in 1740, into England in 1790, and into this country not until a century later. This bean requires a long season and therefore does best in the Southern states. It is quite hardy, however, and some varieties have been found which mature as far north as Canada.

The soy is the best of all the beans. It differs decidedly from other beans in its composition. It contains 40 per cent. protein, practically no starch, and nearly 20 per cent. fat, giving it characteristics approaching those of the peanut more closely than those of ordinary beans.

More than one thousand varieties have now been tested by the United States Department of Agriculture. Twenty varieties are at the present time being handled by growers and seedsmen in this country. The green and yellow varieties are best adapted for food. The black and brown are chiefly grown for forage.

In China, the soy bean is very little used in the manner in which beans are used in this country. Instead, according to W. J. Morse, of the United States Department of Agriculture, 
"the beans are soaked in water and roasted, the product being eaten after the manner of roasted peanuts. This method of preparing the beans is improved by soaking the beans for about twelve hours in a 10 per cent. salt solution, boiling slowly for about thirty minutes, and then roasting to a light-brown color. The yellow-seeded and green-seeded varieties are preferable, as they make a product of better appearance.

"When soy beans are three-fourths or more grown, the seed makes a most pa'atable and nutritious green vegetable. As such it may be used much as is the green pea or the lima bean. The pods are somewhat tough and not desirable to eat. The green beans are rather difficult to shell, but after cooking in the pods for about five minutes they shell out very easily. These beans may also be canned, like green peas, and they thus make an excellent green vegetable for the winter."

This bean not only contains a large amount of protein, more than is found in the same weight of beef, but its protein is of a particularly fine quality. Heretofore, the casein of milk has enjoyed a unique reputation as a protein of finest quality, but now "the protein of the soy bean appears to be as valuable as the casein of milk."

The soy bean is a highly valuable food for persons suffering from diabetes. It contains very little carbohydrate, only about 6 per cent, and is practically wholly free from starch when allowed to become thoroughly ripe before harvesting.

In view of the shortage of milk, which is likely to increase, it is gratifying to know that a protein has been discovered equally valuable as casein and one which may be produced in unlimited quantities.

For the promotion of growth, it is not only necessary for the food to contain complete protein in proper amount, but it must also contain a sufficiency of the two vitamins, designated as fatsoluble $A$ and water-soluble $B$. Osborne and Mendel demonstrated that the soy bean contains an adequate supply of both fat-soluble and of water-soluble vitamins, in which respect it is superior to all seeds heretofore examined, with the possible exception of flaxseed and millet.

The soy bean is destined to become one of the great food staples, not only of this country but of the world. It is capable not only of supplying the essentials for growth and maintenance, but may also act as a complement to other imperfect foods, such as corn for example, in combination with which it has shown most excellent experimental results. 


\section{Composition of the Soy Bean.}

The following table is compiled from the analyses published by the United States Government and by Bailey and Street, (Journal of Industrial and Engineering Chemistry, 1915):

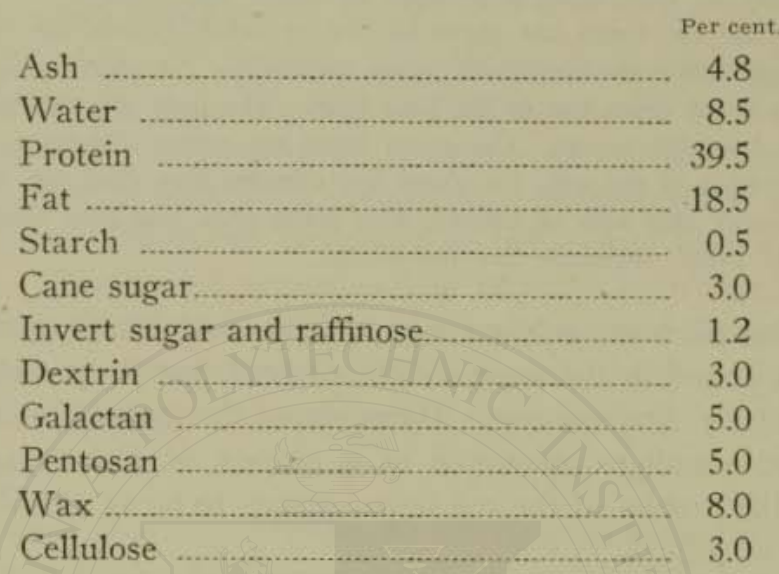

The above analysis clearly shows the soy bean to be a most remarkable food product. Its composition is in some respects more like that of a nut than that of other legumes. In this respect it very much resembles the peanut. Its protein content, nearly 40 per cent., is higher than that of any other foodstuff. Even lean meat affords but half as much. Its high percentage of fat gives it a very high fuel value and makes it a rich source of oil for various industrial purposes as well as for food.

The soy fills the place of meat as well as milk in the dietary of many millions of sturdy Orientals. Since the composition of the soy has been understood, it has been much used as a food for diabetics. It is evident from the above that it contains little which can be objectionable in diabetes. The small amount of dextrin and sugar may be easily removed, when necessary, by parboiling.

Experiments by Holmes, of the office of Home Economics, United States Department of Agriculture, have shown that the well cooked soy bean (cooked for two hours under steam pres. sure) is very easily digestible, and is an exceptionally wholesome article of food, superior to most other legumes.

The soy bean may not become really popular until the pressure cooker comes into general use, which may be some time. In 
the meantime, while the patent pressure cooker is coming, any resourceful housewife may improvise a perfectly good and satisfactory pressure cooker from inexpensive materials close at hand. Get a stone jug or jar that can be hermetically closed. The little stone jars in which apple butter is sometimes sold are well adapted to the purpose. After soaking the beans over night put into the jug with a little salt and enough water to cover, seal up tight and secure the cover well, remembering that the pressure will be from within. Set the jug in a saturated solution of common salt, place over a hot fire and boil for one to two hours. The salt solution boils at a temperature above $220^{\circ} \mathrm{F}$. and so the beans are exposed to a higher temperature than in ordinary boiling. This method is equally good for greens, cabbage, and ordinary beans. Cooking at the higher temperature not only softens the cellulose and so renders the foodstuffs tender, but greatly improves the flavor.

\section{Soy Bean Milk.}

In Japan infants are sometimes nourished artificially on milk prepared from the soy bean by methods for a long time kept secret. Soy milk is now well known and quite extensively in use in France and England, and likely soon to be introduced into this country. The writer has made, experimentally, a quite palatable milk from the soy bean.

The beans may be prepared either by grinding to a fine flour or by soaking over night and crushing to a pulp between rollers. The dry meal is most convenient. Add to the dry meal sufficient water to a little more than saturate. Allow to soak for two hours. Add five times the quantity of boiling water. Boil for ten minutes, stirring. Filter through a fine cloth. The milky liquid thus obtained has properties very closely resembling cow's milk. It is rich in protein and fats. The fat is well emulsified and easily digestible, and the protein very closely resembles that of milk, being what is known to chemists as a complete protein which may replace the casein of milk as a food. The soy milk is rather deficient in salts, both lime and iron. It contains vitamins but less than milk, and it contains no carbohydrates. To be used as milk it should be sweetened by the addition of 2 per cent. of cane sugar or 4 per cent. of milk sugar. To suit most palates a little salt should be added also. The flavor is different from that of cow's milk, but it is wholesome and not unpalatable and may to a 
considerable extent take the place of cow's milk by persons who are sensitized to milk or when milk is not available. A similar preparation may be made from peanuts.

Soy bean milk, according to Adolf and Kiang, has the following percentage composition :

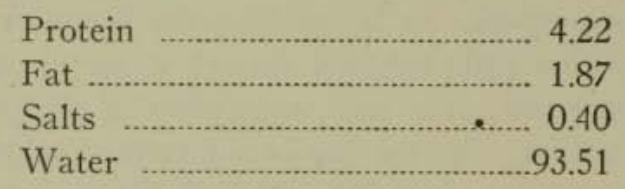

The composition of the milk will vary, of course, with the amount of water used in its preparation. It will be noted that soy milk contains no sugar and is very poor in salts, containing only half the amount of salts found in cow's milk. All these may be easily added, however, and so soy milk is a possible resource for lacteal food in case of scarcity of the bovine product; but it seems to the writer doubtful that soy milk will ever be able to compete with cow's milk as a nutrient for human beings, and especially in the feeding of infants.

\section{To Fur.}

This is a cheese-like preparation made from the soy bean, much used in China. The method is as follows:

Prepare soy bean milk. While it is boiling add one-fifth its volume of a one-half per cent. solution of citric acid, stirring. Strain through a cloth to separate the curds.

A to $f u$ omelet is more palatable and more wholesome than ordinary egg omelet. It is made as follows: Four ounces of to $f u$, one rounded tablespoonful of graham flour, milk sufficient to give the mixture the consistency of pancake batter. Add one well beaten egg. Cook in the usual way. This to fu or soy bean omelet is more wholesome than the ordinary egg omelet and is more easily digestible. Made without the addition of flour the to $f u$ omelet is a valuable dish for diabetics.

The following is the percentage composition of to $f u$ as given by Adolf and Kiang:

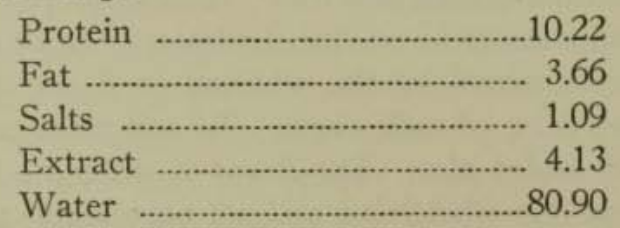


To $f u$ is used in soups and salads and in a great variety of combinations with other foodstuffs. It is one of the fundamenta? things in Chinese and Japanese cookery.

\section{Soy Sauce.}

Soy or shoyn sauce is a dark-brown liquid prepared from a mixture of cooked and ground soy beans, roasted and pulverized wheat (barley is sometimes used), salt and water. This mass is inoculated with a culture known as rice ferment (Aspergillus oryzae) and left in cakes to ferment from six months to a year, or sometines longer.

In odor and taste this sauce suggests a good quality of meat extract, though perhaps more salty and a trifle more pungent.

\section{Soy Bean Sprouts.}

Several species of beans are sprouted and used as a garden vegetable by the Chinese. Soy beans are used to a very considerable extent for this purpose, as these sprouts are larger and firmer than those of most other legumes. Bean sprouts may be used as a home winter vegetable, for the dried beans are sprouted easily in a short time under proper conditions of heat and moisture.

Mr. Shih Chi Yien, of Foochow University, from whom the above quoted paragraphs are borrowed, gives the following directions for preparing bean sprouts, for which purpose either yellow or green soy beans or the mung beans may be used.

The beans must be thoroughly washed as a preparation for growing the bean sprouts. Then they are poured into a big vessel which is about three feet high and one foot and a half in diameter. In the bottom of the vessel a small hole is made for draining the water off from the beans. The vessel is covered with a straw cover to keep out the light.

The beans must be moistened at least three times each day in the summer time and twice in the winter time. They are kept in the vessel about three days in the summer and fifteen days in the winter. At the end of the time, the sprouts are fully grown and they are taken to the market for sale.

Another method of sprouting is to spread the beans one layer thick on a wet cloth and cover with a heavy wet, dark colored cloth which must be kept damp by frequent sprinkling.

Mr. Morse of the Bureau of Plant Industry, United States Department of Agriculture, has kindly supplied us with the following information respecting the composition of soy bean sprouts: 
Composition of Soy Bean Sprouts.

Water

Nitrogenous materials

Fat

Carbohydrates

Mineral Salts

Composition of Mung Bean Sprouts

Water

Nitrogenous materials (protein)

Fat

Carbohydrates

Bean sprouts are used as a green vegetable at all seasons of the year. In this country they would be especially useful in the winter season because of their richness in vitamins. They have been much used in combating beri-beri and other deficiency disorders. Their use in this country should be encouraged.

The sprouts may be served raw as a salad or cooked as a green vegetable. They are a constituent of the famous Chinese dish, chop suey.

\section{THE PEA}

The garden pea has been known in Europe from prehistoric times. There are many varieties, which differ in mode of growth and appearance and to some extent in flavor, but are practically identical in composition. When mature and dry, the pea contains about 25 per cent of its weight of protein and 62 per cent. of carbohydrates. The protein of the pea is superior in quality to that of the bean, a fact which should lead to the more extended use of dried peas.

\section{Green Peas.}

A very delicate and delicious vegetable containing a considerable amount of sugar, which in the ripe pea is converted into starch. Both the protein and the carbohydrate is much smaller in amount in the green pea than in the ripe pea. Canned green peas are a valuable addition to the dietary in the cold months. 
Care should be taken to avoid peas which have been colored with copper or sweetened with saccharine.

\section{CHICK PEA-GARBANZAS}

This excellent legume is known in the Orient as the Egyptian pea, and has there been used from the most ancient times. It is little used in this country, but is cultivated and highly valued in Mexico. Garbanzas are imported from Spain, Algiers and Mexico. Those from the last named country are much superior in quality to other varieties.

\section{LENTILS}

Lentils have been used as a food from prehistoric times. The red pottage which Jacob gave Esau in exchange for his birthright was prepared from the red or Egyptian lentil which has been a staple article of food in Egypt for thousands of years and has in recent times been introduced into this country. Lentils are much used in India as a supplement to the rice diet which is widely used in that country. The lentil contains somewhat more protein than either beans or peas and does not produce offensive latulence as do beans, because of the absence of sulphur. Lentils are best served in the form of soup. They are a highly valuable regetable, rich in iron and lime, and should be more widely used.

Poisonous Legumes. Certain oriental legumes are known to be poisonous. "Java peas" contain prussic acid, and sometimes in so great quantities that one pound contains enough to kill thirty persons. Burmah beans and beans from Madagascar and the Cape are likewise sometimes poisonous from the same cause.

\section{DRIED VEGETABLES}

Within recent years processes have been perfected whereby vegetables may be preserved by drying with comparatively little change in their flavor and nutritive qualities. It is necessary, however, that the drying should be accomplished at a low temperature to avoid destruction of some of the valuable vitamins which abound in most vegetables. 


\section{Fruits}

Fruits may justly be regarded as among the most refined, as well as the most delicious, of natural food products. Their attractive aroma, due to the presence of essential oils, their tasty and refreshing juices, together with their valuable sugars, vitamins, salts and acids, give to them a very important place in the category of useful foodstuffs. With few exceptions, fruits contain practically no fats and only minute quantities of protein, rarely more than one-half of one per cent. to one per cent.

The carbohydrates of fruits are chiefly in the form of sugar, dextrin and acids, and are for the most part ready for immediate absorption.

The absence of fat and the presence of minute quantities of protein, and the fact that the sugar is generally in a form in which it can be immediately assimilated, render fruits, of all foodstuffs, the least taxing to the digestive organs. The nutritive material which they contain being ready for immediate use accounts for the almost immediate refreshing effects experienced by a fatigued person from the drinking of fruit juices and for the great popularity of fruit juices of all sorts, especially in seasons of the year when the depressing effects of exercise are most generally felt.

Edible fruits occur in greater variety than does any other form of food. This is especially true in the tropics. More than 300 different edible fruits are known.

Of the three acids found in fruits, citric and malic acid are the most common. Tartaric acid is found almost exclusively in grapes. Varying quantities of dextrin and small quantities of starch are sometimes found in fruits, especially in unripe fruits. The sugar of fruits usually consists of a mixture of about equal parts of dextrose, or grape sugar, and levulose, or fruit sugar, sometimes called fructose. Cane sugar is found in large proportion only in a very few fruits. 
TABLE XXX.

Showing the Amount of Sugar in Common Fruits.

\begin{tabular}{|c|c|c|}
\hline & Per cent. & $\begin{array}{c}\text { Calories } \\
\text { per ounce }\end{array}$ \\
\hline Apples, preserved. & 6.25 & 7.1 \\
\hline Apricots & 8.78 & 10.0 \\
\hline 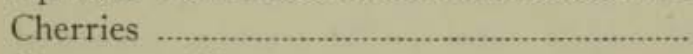 & 10.00 & 11.6 \\
\hline Currants, white ................ & 6.40 & 7.3 \\
\hline Figs (fresh) ...................... & 11.55 & 13.2 \\
\hline Grapes, hot-house ..... & 17.26 & 19.7 \\
\hline Grapes, preserved. & 16.50 & 19.1 \\
\hline 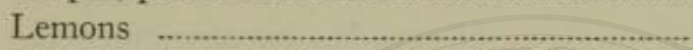 & 1.47 & 1.7 \\
\hline 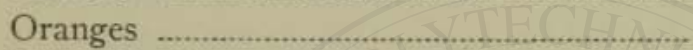 & 8.58 & 9.8 \\
\hline Pears, fresh & 7.84 & 9.0 \\
\hline Pears, preserved ... & 8.78 & 10.0 \\
\hline Pineapples ............ & 13.31 & 15.2 \\
\hline Plums ........................ & 1.99 & 2.1 \\
\hline Raspberries & 7.23 & 8.3 \\
\hline Strawberries & 5.86 & 6.7 \\
\hline
\end{tabular}

The amount of cane sugar and other sugars found in fruits together with the amount of acid and the caloric value is shown in Table XXXII.

\section{TABLE XXXI.}

In the following table there are given the proportions of sugar and pectin in some common fruits (Watson):

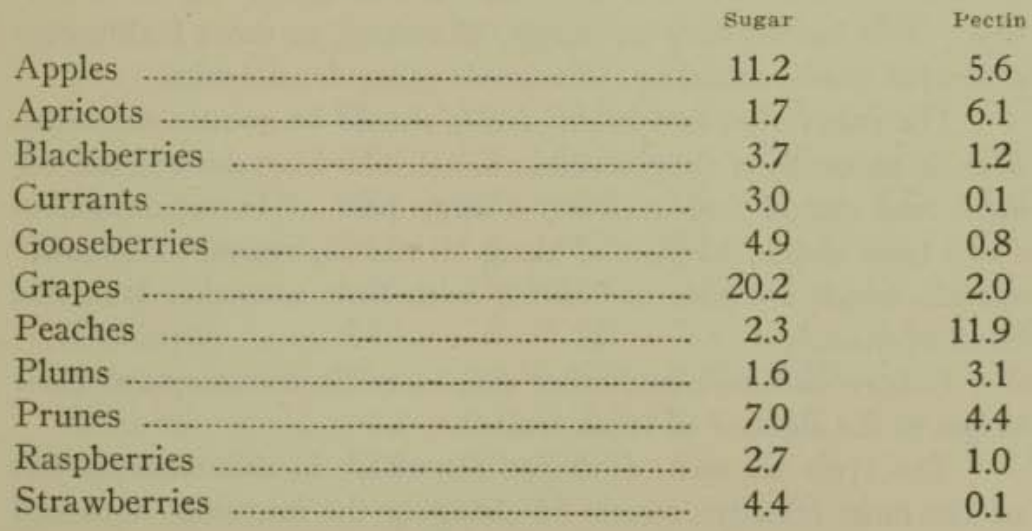


All ripe fruits are easily digestible in a raw state when properly masticated. Cooking softens the cellulose, coagulates the pectin or jellifying principle of fruits, and renders them somewhat more digestible but lessens their nutritive value by destroying in part the vitamins which give to them one of their chief nutritive values. The antiscorbutic, or scurvy-preventing vitamin, may be wholly destroyed in the cooking of fruits. On this account, fruits should be largely eaten in the fresh or uncooked state. Drying also injures this vitamin.

Fruits are worthy of a dignified place in the dietary. They are usually regarded as a luxury to be indulged when convenient or when they can be afforded, whereas they should be considered an essential part of the dietary. Nature has so distributed the various items which make up a complete biologic ration that it is necessary to include in our list of edibles all parts of the plantleaves, roots, seeds and fruits-in order to be certain that nothing is missed. This has been clearly shown by McCollum's classical experiments. Fruits supply in more palatable though less concentrated form much the same food essentials that are found in roots, viz., carbohydrates, roughage, and especially the alkaline salts of which we need a considerable amount to neutralize the acid wastes of our bodies which are greatly exaggerated by a sedentary mode of life and a high protein diet.

Ripe fruits require little work of the digestive organs. Their sugars, acids, salts and vitamins are all ready for immediate absorption, while the amount of fat and proteins present are so small that they are quickly disposed of. For this reason, fruit alone of all natural foodstuffs, may be eaten at any time when the stomach is not overfilled, without disturbing the digestive process. This license does not apply, of course, to those highly concentrated foods, dates, figs, raisins or other dried fruits.

The cultivation and use of fruits should be greatly extended. Man is naturally a frugivorous animal, like his relatives the big apes, and should find in fruits a large part of his nourishment. Fruit trees should be planted along all our highways, those hardy species which are able to flourish with little attention from the hand of man.

Scurvy, beri-beri, and other deficiency diseases may owe their origin to the absence of fresh fruit.

The fruit regimen, described elsewhere in this work, is one of the most effective means of changing the intestinal flora and 
FRUITS

TABLE XXXII.

Showing the Percentage Composition of the Edible Parts of Fruits

\begin{tabular}{|c|c|c|c|c|c|c|c|}
\hline & Water & Proteid & $\begin{array}{l}\text { Carbo- } \\
\text { hydrates }\end{array}$ & Ash & $\begin{array}{l}\text { Cellu- } \\
\text { lose }\end{array}$ & Acids & \\
\hline Apples & 82.5 & 0.4 & 12.5 & 0.4 & 2.7 & 1.0 & 15.4 \\
\hline Apples (dried).... & 36.2 & 1.4 & 49.1 & 1.8 & 4.9 & 3.6 & 59.8 \\
\hline Apricots & 85.0 & 1.1 & 12.4 & 0.5 & & 1.1 & 15.5 \\
\hline Bananas ...................... & 74.0 & 1.5 & 22.9 & 0.9 & 0.2 & the & 27.9 \\
\hline Blackberries & 88.9 & 0.9 & 2.3 & 0.6 & 5.2 & & 3.7 \\
\hline Cherries ....... & 84.0 & 0.8 & 10.0 & 0.6 & 3.8 & 1.0 to 1.5 & $513 . \mathrm{C}$ \\
\hline Cranberries ........ & 86.5 & 0.5 & 3.9 & 0.2 & 6.22 & 2.0 to 2.5 & 56.0 \\
\hline Currants .............. & 85.2 & 0.4 & 7.9 & 0.5 & 2.7 & 1.5 & 11.4 \\
\hline Currants (dry). & 27.9 & 1.2 & -64.0 & 2.2 & 1.7 & $\ldots .$. & -74.5 \\
\hline Dates (dried)... & 20.8 & 4.4 & -65.7 & 1.5 & 5.5 & $\ldots .$. & -80.1 \\
\hline Figs (dried).... & 20.0 & 5.5 & -62.8 & 2.3 & 7.3 & 1.2 & -76.7 \\
\hline Figs (fresh) ... & 79.1 & 1.5 & 18.8 & 0.6 & & & 23.2 \\
\hline Gooseberries . & 86.0 & 0.4 & 8 & & 2.7 & 1.5 & 11.4 \\
\hline Graj & 79.0 & 1.0 & 15. & & 2.5 & 0.5 & 19.1 \\
\hline Greengages ... & 80.8 & 0.4 & 13.4 & 0.3 & 4.1 & 1.0 & 13.0 \\
\hline 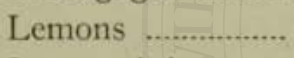 & 89.3 & 1.0 & 8.3 & 0.5 & $\cdots$. & $\ldots$ & 11.0 \\
\hline Lemon juice... & 90.0 & 0.0 & 2.0 & 0. & .... & 7.0 & 6.3 \\
\hline Melons & 89.8 & 0.7 & 7.6 & & 1.0 & $\ldots$. & 9.5 \\
\hline Mulberries ....... & 84.7 & 0.3 & 11.4 & 0.6 & 0.9 & 1.8 & 14.4 \\
\hline Nectarines & 82.9 & 0.6 & 15.9 & 0.6 & & $\ldots$. & 18.8 \\
\hline Olives .............. & 67.0 & 2.5 & 5.7 & 4.4 & 3.3 & & 9.4 \\
\hline Oranges ................ & 86.7 & 0.9 & 8.7 & 0.6 & 1.5 & 1.0 to 2.5 & 512.1 \\
\hline Orange juice...... & 85.0 & 0.0 & 10.8 & 0.0 & $\ldots$. & 1.9 & 15.3 \\
\hline Peaches ................ & 88.8 & 0.5 & 5.8 & 0.6 & 3.4 & 0.7 & 7.0 \\
\hline Pears ................. & 83.9 & 0.4 & 11. & 0.4 & 3.1 & 0.1 & 13.7 \\
\hline Pineapples & 89.3 & 0.4 & 9.7 & 0.3 & & $\ldots$. & 11.5 \\
\hline Plums ................ & 78.4 & 1.0 & 14.8 & 0.5 & 4.3 & 1.0 & 15.3 \\
\hline Pomegranates ....... & 76.8 & 1.5 & 16.8 & 0.6 & 2.7 & & 20.9 \\
\hline Prunes (dried).... & 26.4 & 2.4 & -66.2 & 1.5 & $\ldots$. & 2.7 & -70.0 \\
\hline Prunes (fresh).... & 80.2 & 0.8 & 18.5 & 0.5 & $\ldots$. & $\ldots$. & 22.2 \\
\hline Raisins & 14.0 & 2.5 & -74.7 & 4.1 & $\ldots$. & $\ldots$. & -88.2 \\
\hline Raspberries ......... & 84.4 & 1.0 & 5.2 & 0.6 & 7.4 & 1.4 & 8.0 \\
\hline Strawberries ......... & 89.1 & 1.0 & 6.3 & 0.7 & 2.2 & 1.0 to 1.2 & 9.0 \\
\hline Tom & 94.3 & 0.7 & 3.7 & 0.4 & 0.5 & 0.6 & 5.0 \\
\hline Watermelons . & 92.9 & 0.3 & 6.5 & 0.2 & $\ldots .$. & .... & 7.8 \\
\hline
\end{tabular}


lifting from the liver and kidneys an enormous burden of onerous and destructive work in poison elimination. The free use of fruit is a most effective means of combating intestinal autointoxication, constipation, a condition of demineralization, and deficiency disorders such as chronic scurvy.

John Hunter advocated the free use of apples as the best remedy for gout. The great naturalist, Linnaeus, cured himself of a severe attack of sciatica by eating freely of strawberries. Tissot tells of a regiment of soldiers suffering from dysentery who were quickly cured after encamping close to a vineyard full of grapes.

The medical history of the Civil War recounts a similar experience by a regiment of soldiers from which a scourge of dysentery disappeared very quickly after their camp was located near an orchard of ripe peaches.

\section{Fruit Acids.}

The acids found in fruits are malic, citric and tartaric. These acids are carbohydrates and like starch and sugar are burned in the body, having a fuel value about half that of sugar. Fruit acids are found in foods in combination with soda or potash, usually the latter. When taken into the body the acid parts are oxidized or burned, leaving the base, or alkali, behind. On this account, the effect of these food acids is to increase the alkalinity of the fluids of the body, a matter of much consequence since by this means the acid waste products of the body are neutralized, and the tendency to acidosis always present is overcome. Citric acid and malic acid appear to be the most easily burned or utilized by the tissues. Tartaric acid is less readily utilized.

Citric and malic acids are the common acids of fruits, the last named being most abundant except in berries and in fruits of the citrus family, in which citric acid predominates. Grapes contain tartaric acid only when ripe, the malic acid present in green grapes being changed to tartaric acid in the process of ripening. Apples and cherries contain malic acid chiefly. Cranberries contain 0.05 per cent. of benzoic acid, which is also present in plums and prunes. Citric acid is found in tomatoes.

Many people have noticed the marked benefit from the use of acid fruits. The writer has not infrequently been told by persons suffering from dyspeptic disorders that the juice of a lemon 
taken soon after meals has sometimes given relief. Others have been benefited by apple juice and others by moderate acid fruits of other sorts.

The nature of this beneficial effect of fruit was not understood until explained by experiments by Pavlov and others, which demonstrated: first, that the acids of fruits stimulate the stomach to produce gastric acid, which is absolutely essential for good digestion; and second, that the acids of fruits are able, to some degree, to take the place of the natural acid of the stomach when this acid is absent.

These facts emphasize the importance of acid fruits, including the tomato- $\mathrm{a}$ vegetable-fruit-as an aid to digestion in achylia, a condition in which there is deficiency or absence of hydrochloric acid, the normal acid of the gastric juice.

\section{Disinfection of Fruits.}

Raw fruits and vegetables as received from the market are always open to suspicion of being contaminated with bacteria. In fact, this is true of most raw foodstuffs. All kinds of fresh fruits are infected with germs, yeasts and molds, which speedily develop and destroy the fruit unless prevented from so doing by means of cold storage. These germs may be derived from the garden soil, the hands of the pickers, the receptacles in which they are transported, or the markets where they are exposed for sale. In the summer time the germs grow rapidly while the fruit is being transported to market, or while exposed in the market, or even after purchase by the consumer. Flies are a common source of infection.

Thus fruit, eaten without disinfection, may easily become a source of mischief, as germs may grow in the stomach or intestine when swallowed with the food. In this way the average individual is constantly collecting germs of various sorts and every adult carries in his colon a vast assemblage of bacteria, the so-called intestinal flora.

Such vegetables as lettuce, celery and radishes and fruits which like strawberries grow in, on or near the ground are liable to carry not only germs but the embryos of various intestinal parasites.

The use as fertilizer, of "night soil," gathered by city scavengers and largely disposed of to gardeners in the neighborhood of large cities, very naturally facilitates the distribution of intestinal 
parasites of various sorts. Some years ago Metchnikoff called attention to the danger of infection from this source.

Soil may become infected with $B$. botulinus by means of stable manure. The owner of a small garden for eight years canned string beans grown in his own garden, with excellent success. There was no spoilage and no sickness due to the use of the beans. In 1918 animal manure was used as a fertilizer. The beans raised were canned by the same process employed before, but instead of keeping as before, they spoiled, and some of the cans were found to contain the deadly poison produced by the B. botulinus.

Snails, worms and insects gather typhoid and other germs from the soil and in crawling over the leaves of vegetables deposit a trail of germs with fecal matters upon the surfaces which they traverse.

A fresh chloride of lime solution, half ounce to two gallons, will destroy the germs without impairment of the food in flavor or any other particular. A large stone jar is half filled with the solution. Fruit, lettuce, or other food, after thorough washing, is immersed in the disinfectant and allowed to remain for five minutes. The solution should be kept covered and will be effective for several days.

At the Battle Creek Sanitarium, this method is in daily use for the disinfection of all fresh, uncooked fruits and vegetables used in the feeding of the great family of 2,000 to 3,000 persons who are served in the several capacious dining rooms of the institution.

Since this practice was introduced about 20 years ago, outbreaks of "bowel trouble," which formerly occurred with considerable frequency during the summer months, have wholly ceased.

This disinfecting all fresh foods will no doubt in time become general. It is the only means of protection against certain intestinal parasites which are very difficult to dislodge after they once become established.

\section{Fruit Seeds.}

The seeds of fruit consist chiefly of cellulose. Small seeds such as are found in figs, blueberries, raspberries and most other seedy fruits are entirely harmless and are perhaps to some degree beneficial through increasing the bulk of the food. Large seeds. 
as those of the apple or grape and the cherry, if taken in considerable quantity might prove burdensome to the intestine and on this account ought to be discarded. It should be mentioned, however, that the danger which many people apprehend from the entrance of these seeds into the appendix is largely imaginary. Seeds are sometimes found in a diseased appendix, but their presence is purely accidental; they are not the cause of the disease but a consequence of it. When the appendix becomes diseased in such a way that the valve which guards its mouth no longer operates, remaining open, seeds and other foreign bodies may find their way into the appendix and possibly may thus become a source of injury and danger, although the danger from this source must be quite remote.

\section{The Fruit Diet.}

By making a proper selection of fruits it is possible to construct a dietary quite capable of sustaining life for an indefinite period. Bananas, figs, dates, raisins and prunes contain an abundance of nutritive material in sufficiently concentrated form to be readily available as suitable foodstuffs. The only element lacking is fats and these may be readily obtained from such fruits as the avocado or alligator pear of the tropics and by the addition of nuts to the bill of fare. By a combination of fruits and nuts a very complete and luxurious bill of fare may be provided.

For entire safety, however, it is better to add milk to the above bill of fare. A pint of milk a day will insure the proper intake of food lime and of the complete proteins which are necessary for the repair of the blood and of the tissues.

Contrary to the opinion formerly held by physicians, fruits and fruit juices are not only not injurious to persons suffering from gouty or rheumatic affections, and even in cases of acidosis, but are highly beneficial.

Fruit juices of all sorts contain in addition to vegetable acids alkaline substances which neutralize acids and aid in the elimination of uric acid. Pure uric acid dissolves with great difficulty but the urate of soda or potash is readily soluble. Soda and potash are present in fruits in considerable quantities in combination with other acids. When fruit is eaten the acids are utilized or burned in the body leaving the soda behind. It combines with the uric acid and thus aids its elimination. The popular idea that the acids of fruits are unwholesome for persons 
suffering from gout and other uric acid disorders is a very mischievous error. The fallacy of this teaching has been pointed out for many years by scientific writers on dietetics, but like many other popular fallacies this erroneous notion is very hard to eradicate.

\section{The Germicidal Properties of Fruits.}

More than twenty years ago Kitasato and Van Ermenger demonstrated experimentally that citric acid and other fruit acids are possessed of active germicidal properties. The typhoid bacillus and also the bacillus of cholera were destroyed by a one-half of one per cent. solution of citric acid. Malic acid, the acid of the apple, was found equally efficient. In making a direct application of this discovery, the writer succeeded in sterilizing the stomach of a patient, a physician, whose stomach fluid showed more than $5,000,000$ aerobes and 2,500,000 anerobes per cubic centimeter The stomach was found completely sterile after a meal consisting of 10 ounces of strawberries and 9 ounces of water. Half a pint of orange juice given half an hour before the meal has been found of very great value in similar cases. The idea of using fruit juices in cases of gastro-intestinal autointoxication is, however, by $\mathrm{nc}$ means new.

Dr. Dwight more than a century ago reported cases of sick headache successfully treated by giving the patient a glassful of apple cider before each meal.

The character of the food certainly makes a very marked difference in cases of this sort. For example, in the case of a meal consisting of scraped beef prepared and cooked in the usual way bacteriological examination of the stomach fluid showed nearly $24,000,000$ bacteria per ounce, although after a sterile meal no bacteria at all were found. In the same case a test meal of cheese gave $280,000,000$ bacteria to the ounce of stomach fluid These observations led the writer many years ago to adopt in the treatment of cases of gastro-intestinal disease a strict antiseptic or rather an antitoxic or atoxic dietary, from which meat and eggs are wholly excluded.

Later, after many experimental efforts, the fruit regimer was devised. In this diet fruit is made the exclusive food with bran in liberal quantities, as explained in detail elsewhere. This regimen has proven to be of great service in the treatment of constipation (see index). 


\section{The Iron Content of Fruits.}

We are so accustomed to think of fruits solely as sources of sugars and acids that we are naturally surprised at the discovery that these choice comestibles are really most valuable sources of food iron. It is true the average iron content per ounce is small. less than 2 per cent. of the daily ration (1.74); but then the food value is also low on account of the large percentage of water so that the average percentage of the ration of iron is more than three times as great (5.6). One can eat a larger bulk of fruit without risk of injury than of any other class of foodstuffs.

Besides, there are certain fruits which are exceptionally rich in iron, containing on the average twice as much iron as the general run of fruits. Of less than a half dozen fruits in this class, dates, figs, Zante currants, prunes and raisins, the average percentage of the day's ration per ounce is 5.2 and per 100 calories 6.8 , considerably higher than the averages for all foodstuffs which are, respectively, per ounce, 3.25 and per 100 calories 6.38 .

It will be readily seen how the fruits named may be usefully employed in balancing the ration for iron. A quarter of a pound of dates supplies nearly one-fourth of a total day's iron ration. and at the same time furnishes a substantial amount of energy to support muscular work. Two ounces each of figs, olives and prunes, and an ounce each of raisins, dates and currants would supply half a day's requirement of iron and one-third of a liberal day's food supply.

Taking into account the large amounts which may be eaten with impunity, together with their other good qualities, fruits may well be regarded as a most valuable source of food iron The strawberry and the raspberry are especially noteworthy.

\section{The Lime Content of Fruits.}

A characteristic of fruits in general is their small lime content. The average amount of lime in 28 of our commonly used fruits is one-eighth grain per ounce, requiring 123 ounces for one day's supply of lime (15.4 grains), more than is demanded of any other class of foods with the exception of meats, which require the consumption of 270 ounces, more than 17 pounds, an impossible amount for anyone but an Eskimo.

It is important to observe, however, that there are some very noteworthy exceptions to the general rule of low lime content in 
fruits. Zante currants are rich in lime, containing half a grain to the ounce, or four times as much as the average of all fruits. The olive affords three-quarters of a grain to the ounce and the fig, which has been a choice food staple for millions in the Orient from remote ages, supplies a whole grain of lime to each ounce, or a day's lime ration in less than one pound of the dried fruit, and one-third as much in the same quantity of figs fresh from the tree.

Fruits are thus seen to be a really practical means for replenishing as well as maintaining the lime supply of the body, a need which our ordinary bills of fare wholly ignore.

And fruits have the advantage not only of being a most agreeable form of aliment but a most reliable source of lime, since they are not likely to be robbed of their mineral constituents by the misguided efforts of those experts in denaturing and sophisticating foodstuffs, the manufacturers, canners and cooks.

It is well to note the fact that quite a number of our most commonly used fruits are very deficient in lime salts. For example, the apple contains so little lime that one would have to eat 22 pounds daily to get his supply if dependent on this source alone. The banana is almost equally deficient, requiring 17 pounds for a day's ration. The tomato yields a day's supply in 14 pounds, the watermelon in an equal quantity, the pear and the peach in 10 pounds and the cantaloup in 9 pounds. In other words, the proportion of lime in these fruits is so small that it may be ignored in estimating the lime content of bills of fare.

\section{The Ripening Process in Fruits.}

In Mexico, ripe fruit is said to have been cooked in the sun (cocido in el sol). The Arabs have a similar saying. The changes which take place in the cooking of green fruit are about the same as those which occur during ripening, though much less extensive. Cooking renders green fruit digestible, and ripening does the same, but also involves many other changes. The Experiment Station Record tells us that "the apple fruit, considered as a living organism, has first a period of growth during which the dry matter, principally starch, is continually increased. A second or ripening period follows, during which starch is changed into sucrose (eane sugar). This in turn is gradually changed into invert sugar, with a gradual decrease of malic acid and of carbohydrates and an accompanying respiration of carbon dioxid. 
These changes are largely due to the activity of oxidizing enzymes, the oxygen of the air being drawn upon in the process.

The changes progress much more rapidly after picking, at high temperatures, and in the short-season varieties. Soon after picking, apples should be placed in cool storage to retard the processes above mentioned. Respiration continues during the storage period, becoming ultimately less active. Transpiration continues during the life of the apple. The third period is that of disintegration.

\section{Arsenic in Fruit.}

The extensive use of arsenic for the spraying of fruit trees has led to an investigation of the possibility of contamination of the fruit with this poisonous chemical. Careful analyses have shown that spotted apples often contain considerable quantities of arsenic. As much as three-tenths of a milligram, nearly one two-hundredth of a grain, of arsenic has been found in a single apple. Arsenic has also been found in the paper with which apples are wrapped. This is a matter which should receive serious attention by apple growers and shippers.

\section{THE APPLE}

The apple tree thrives in all temperate regions along with the oak, and has been known and appreciated from prehistoric ages. There are about 1,500 cultivated varieties of the apple, but of this great number not more than 50 are of commercial importance.

The apple contains about one-seventh its weight of solids, of which the main part is sugar and protein. The acid of apples is chiefly malic, the percentage of which varies greatly in different varieties of this valuable fruit.

\begin{tabular}{|c|c|}
\hline & $\begin{array}{l}\text { Per cent, of Acid } \\
\text { (Malic) }\end{array}$ \\
\hline Baldwins & 0.60 \\
\hline Maiden blush (green) & 1.68 \\
\hline Early ripe (green) ......... & 1.09 \\
\hline Gravenstein (green) ....... & 1.27 \\
\hline Sweet bough (green)..... & 0.21 \\
\hline Sweet bough (ripe) ....... & 0.13 \\
\hline 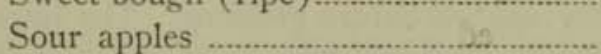 & 0.62 \\
\hline 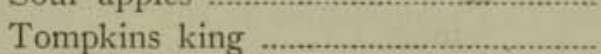 & 0.41 \\
\hline 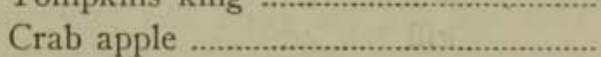 & 0.78 \\
\hline 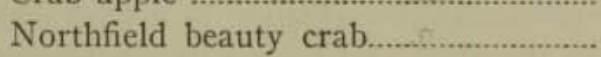 & 1.81 \\
\hline Sweet cider & $0.40-0.56$ \\
\hline
\end{tabular}


The presence of pectin gives to the juice of apples the property of jellying when concentrated by heat.

Apples and apple juice are of great dietetic value. Taken freely they are laxative, especially when taken on an empty stomach.

The apple is a universal favorite as a luxury and is by no means of small value as a food. Its fine flavor and appetizing acid give it pre-eminence as a peptic stimulant. Recent observations in feeding experiments seem to indicate that its protein, though small in amount, is particularly fine in quality.

As is true of other fruits, some persons are sensitized to the protein of apples and cannot even endure their presence, although such cases are rare. One such example is mentioned by Soyer, who relates that Ulaislas, king of Poland, in the presence of apples became so distressed and terrified that he was compelled to flee, a circumstance which at that time was most mysterious and unaccountable on rational grounds.

\section{Apple Juice.}

Unfermented apple juice is exceedingly wholesome. The ugreeable acid flavor is due to malic acid, which is an excellent antiseptic or disinfectant for the stomach and intestine. It is a very highly valuable remedy in gastric and intestinal catarrh, so-called biliousness, jaundice, constipation, and in cases in which the kidneys and the liver are disordered, in emaciation, loss of appetite, and general malnutrition. It may be taken in quantities of one quart daily with advantage. The best times for taking are one-half hour before each meal and just before retiring at night

Freshly prepared apple juice is one of the best of all fruit juices and is even superior to grape juice. Malic acid is a milder acid than the tartaric acid of grapes and is more easily utilized by the body. Freely used, apple juice is a laxative and, combats any tendency to acidosis. Persons subject to gout and other disorders due to chronic uric acid poisoning may make a liberal use of apple juice to advantage.

Apple juice should be prepared from selected apples, which should be thoroughly washed before grinding. The juice may be preserved by heating to a temperature of $165^{\circ} \mathrm{F}$. for fifteen or twenty minutes. This will kill the yeast and other organisms which give rise to fermentation. Heating to a high temperature impairs the flavor of the juice, and lessens its wholesomeness. 
The common practice of adding a small percentage of benzoic acid to apple juice to prevent fermentation is highly objectionable and cannot be too strongly condemned.

\section{THE PEAR}

The pear, at its best, is a most delectable product of Nature's food laboratory. It is a sweet fruit, containing less acid than slmost any other fruit. The following table shows the small amount of acid found in those varieties which contain the most:

\begin{tabular}{l|c} 
Kieffer & $\begin{array}{c}\text { Per cent. of A } \\
\text { (Malic) } \\
\text { LeConte }\end{array}$ \\
Bartlett & .50 \\
Angonleme & .28 \\
Seckel & .30 \\
\hline Winter Nelis & .18 \\
\hline & .06 \\
\hline & .20 \\
\hline
\end{tabular}

\section{THE QUINCE}

The quince, like the apple, has come down to us from the nncients. Its flesh is too hard for use in a raw state and is hiefly employed for marmalade and preserves.

\section{THE PEACH}

In its best state, the peach is one of the most delicious of all our orchard fruits. The finest varieties are rich in sugar ( 6 per cent.) and contain little acid. This fruit, even when ripe, contains considerable cellulose and thus is valued as a laxative.

The nectarine is a variety of the peach which has a smooth skin.

The apricot is another member of the peach family. It differs from the peach in containing about twice the amount of sugar and still less acid.

\section{PRUNES AIND PLUIVIS}

Of these fruits there are many varieties, some of which are sweet while others contain a considerable amount of acid. The prune when dried is nearly as rich in sugar as the fig.

Prunes soaked in water for twenty-four to forty-eight hours 
and freely eaten have a decided laxative effect. They are much used for this purpose by eminent German physicians.

A research conducted by Dr. Blatherwick, of the Sheffield Scientific School of Yale University, some years ago in the laboratory of the Battle Creek Sanitarium showed that prunes contain so large an amount of unusable acid that they tend to acidify the arine. This acidity was later found to be due to benzoic acid. it is the only possible objection to their use. When eaten in connection with fresh vegetables in liberal quantities this objection would probably not hold good as the small amount of benzoic acid would be neutralized; but if eaten freely in connection with a cereal diet the urine might be rendered so highly acid as to be decidedly injurious in some cases, especially in cases in which the bladder or kidneys are diseased.

Prunes and plums should be avoided in cases of irritable bladder and whenever there is a tendency to acidosis.

\section{THE CHERRY}

The cherry is one of the most highly valuable of our orchard iruits, and is much appreciated because of its early appearance. it contains more than 10 per cent. of sugar, and has a nutritive value higher than that of the apple.

"According to Pliny," says Fernie, "cherries were first brought to Rome by Lucullus after his great victory over Mithridates, 89 B. C. The cultivated cherry disappeared in this country (England) during the Saxon period, and was not re-introduced until the reign of Henry the Eighth.

"Cherry stones have been found in the primitive lake dwellings of western Switzerland. There is a tradition that Christ gave a cherry to St. Peter, admonishing him not to despise little things.

"In France, soup made from cherries and taken with bread, is the common sustenance of the wood-cutters and charcoal burners of the forest during the winter."

The cherry is one of the most nutritious of the tree fruits. Some varieties are very rich in sugar, while others present a large percentage of citric acid. Its iron content is greater than that of egg white, milk, pears and apples, and equal to that of the tomato.

\section{THE PERSIMIMON}

The native persimmon, which grows wild in the south and occasionally occurs as far north as Michigan, is a very sweet and 
palatable fruit when fully ripe. The Japanese variety is much larger than the American and is a very luscious and wholesome fruit.

\section{THE OLIVE}

This is another very ancient fruit, now produced extensively in this country, particularly in California. As picked from the trees, the olive is exceedingly bitter in taste. The bitter flavor is removed by immersion in running water for several weeks, or by placing for some hours in an alkaline solution.

Green, pickled olives are inferior in food value. The ripe olive is highly nourishing. It is chiefly sold in this country in bottles or cans, being preserved by heating like other canned fruit. A few cases of poisoning have occurred from the use of olives infected with the $B$. botulinus, which gives rise to botulism, a serious, sometimes fatal, form of food poisoning. Canned olives which have an unpleasant odor when opened should be rejected. Poisoning is not likely to occur from the use of olives canned in tin.

The olive is usually eaten in the ripe state in Oriental lands where this oleaginous fruit is at home. In this country, however, the green olive has been the general favorite as a relish until recent years have seen the beginning of a change in the taste of the American public and the ripe olive is now growing in favor, as it well deserves to do. The green olive is practically indigestible and of little value as a food.

According to Gautier the green olive contains little fat but much mannite, which in the process of ripening is converted into fat.

\begin{tabular}{|c|c|c|}
\hline & $\begin{array}{l}\text { Green ollve } \\
\text { (Gautier) } \\
\text { Per cent. }\end{array}$ & $\begin{array}{l}\text { Ripe ollve } \\
\text { (Atwater) } \\
\text { Per cent. }\end{array}$ \\
\hline Water & 73.40 & 64.7 \\
\hline Protein ......... & 0.76 & 1.7 \\
\hline Fat & 14.48 & 21.0 \\
\hline Extractives & .. 8.04 & 3.5 \\
\hline Cellulose .... & ... 0.90 & $\ldots \ldots$. \\
\hline Ash & 0.42 & 2.7 \\
\hline
\end{tabular}

The Scientific American Monthly gives us the following inieresting information concerning the production of ripe olives:

Most of our ripe olives originate in California, where three pickings are made during the season in order to obtain fruit of equal ripe- 
ness. At the factory the fruit is first sorted, graded for size, and is then placed in an alkaline solution, usually sodium hydrate, the strength of which varies, but probably averages one and one-half per cent. At the end of six to eight hours this lye is drawn off, the olives exposed to the air to brown them by oxidation, and the operation repeated until an examination shows that the lye has penetrated to the pit. Clear water changed twice daily is now used until the lye and bitterness are removed, this requiring from four to eight days. The next treatment consists of a series of brine solutions, beginning with 1 per cent. and increasing the strength at intervals of about two days until approximately 4 per cent. is used. At this point they are ready to be put into glass cans and sealed.

Some packers allow the olive to remain in the weak brine long enough to produce an acid flavor by fermentation and others endeavor to retain the natural color rather than to induce oxidation. If it is intended to hold the olives in bulk the strength of the brine is in creased until 10 or 12.5 per cent. is reached, the stronger solutions being necessary if they are to be carried through the summer.

To be sure the process as outlined is modified in practice to satisfy the conditions, taking into consideration varieties of fruit, tem perature, etc. As the olives are packed the soft and defective ones are discarded and the containers are filled with three per cent. brine at 2 temperature of $175^{\circ}$ or $180^{\circ} \mathrm{F}$. The air is then exhausted while the temperature is raised to $185^{\circ} \mathrm{F}$., when the containers are sealed ready for processing. Processing consists in cooking in the bottle from fifty to sixty minutes, depending upon the size of the olive and the size of the container. In some factories $240^{\circ} \mathrm{F}$. is reached. If a lower temperature is used the time is increased.

\section{THE GRAPE}

The grape is one of the most valuable of fruits and has been in use for ages. When partially ripe, the grape contains both tartaric and malic acids, but the malic acid is changed to tartaric as the process of ripening continues.

The varieties differ in sweetness, the sugar content ranging from 13 to 25 per cent. The acid content also varies.

Zante currants are prepared from the Corinth grape, a small black variety which has a thick skin and is seedless.

\section{Grape Juice.}

Grape juice has now become a very extensive article of commerce. It is preserved by bottling and pasteurizing. In Oriental countries, the juice of sweet grapes is concentrated in the sun to the consistency of syrup. This grape syrup is highly prized as 
an article of diet throughout the Orient and has been in use from the most remote times.

\section{The Composition of Grape Juice.}

The following table shows the percentages of tartaric acid and other solids found in commercial grape juices, the solids consisting almost wholly of levulose and dextrose, the sugar added for sweetening having been inverted by the acids present:

\begin{tabular}{lcc} 
& Solids & Tartaric acid \\
Welch's $\ldots \ldots \ldots . .19$ & 19.00 & 1.00 \\
\hline Armour's Top Notch & 14.51 & 0.93 \\
\hline Duff's & 15.37 & 1.07 \\
Fremont & 13.99 & 1.08 \\
\hline Liggett's & 15.13 & 0.86
\end{tabular}

The above analyses, with the exception of Welch's grape juice, were made by the Canadian Inland Revenue Department.

\section{BERRIES}

The characteristic of the berry is its seedy pulp. The seeds are indigestible but of value as roughage.

The acid of berries is chiefly citric acid, with a small amount of malic acid.

Of the dozen or more edible berries which grow wild or in zultivation scarcely half a dozen are in such general use as to be important commercially.

\section{The Strawberry.}

The strawberry, with its delicious aroma and its delicate sweet-acid flavor, is one of our choicest and most popular fruits. It is at the same time a very useful and wholesome food. Its high water content reduces its nutritive value below that of most fruits, placing it on a footing with the orange, the muskmelon, string beans and turnips; but it is unusually rich in lime and iron, not only as compared with other fruits but with most other foodstuffs. Here are a few interesting comparisons:

The strawberry contains one-fourth grain of food lime to the ounce, which is more than any other fresh fruit except figs and raspberries. Its lime content is six times as great as that of the apple, five times the banana, four times the tomato 
and watermelon, and double or more than that of the blackberry, blueberry, cherry, cranberry, grape, muskmelon, peach, pear, pineapple or plum. Its lime content is exceeded among fruits only by dried fruits, especially figs, dates, currants, raisins and prunes.

Notwithstanding its high water content, the strawberry presents a higher proportion of lime than any cereal food except oatmeal and entire wheat and rye. It affords, in equal weight, five times as much lime as does rice, four times as much as hominy, and three times as much as white bread.

The richness of the strawberry in iron is still more noteworthy. With the exception of the raspberry and fresh figs, the strawberry excels all other fresh fruits in richness in food iron. It contains four times as much iron as does the orange and nearly three times as much as grapefruit, apples, apricots, cantaloup, muskmelon, grapes, peaches, pears or watermelon; twice as much as do tomatoes and cherries, and 50 per cent. more than raspberries, currants, cranberries, bananas and blackberries.

A pint of strawberries contains as much food iron as a pound loaf of white bread and one-fourth more than an equal weight of cornmeal.

Strawberries are richer in iron than many fresh vegetables, affording more than celery and lettuce, as much as sweet corn, cauliflower and cabbage, only a little less than string beans, cabbage and brussels sprouts, and one-half more than turnips, squash, radishes, eggplant, carrots, parsnips, beets, onions and sweet potatoes, and four times as much as cucumbers. It is only excelled as a source of food iron by two vegetables - fresh lima beans and green peas. The strawberry has great value, when freely used, as a protection against the loss of lime or of iron, and it is also invaluable as a source of organic bases which are needed to neutralize the acid wastes of the tissues, thus preventing the development of acidosis and its numerous accompanying evils.

This excellent fruit contains 7 per cent. of sugar and 1.5 per cent. of acid, chiefly citric.

Walton in his "Complete Angler" quotes Dr. Boteler as saying, "Doubtless God Almighty could have made a better berry, but He never did." And most people will agree with the doctor in his opinion.

The strawberry is a hardy plant and thrives in the far North. 
The Laplanders mix the berries with reindeer cream and dry in the form of a sausage for use during their long winter. The liberal use of fresh strawberries has been found an excellent remedy for sprue and pellagra.

\section{The Currant and the Gooseberry.}

These are two excellent berries rich in both sugar and acid, which is for the most part citric.

\section{The Cranberry.}

This very popular berry contains about 4 per cent. of sugar and half as much acid, including one-twentieth of 1 per cent. of benzoic acid, the balance being citric acid.

The cranberry should be avoided by persons who have a tendency to acidosis or highly acid urine.

\section{The Huckleberry or Blueberry.}

This excellent fruit flourishes in a wild state over a large territory extending from the Southern states to the far North. Recent and very successful attempts have been made to cultivate the blueberry in Florida and elsewhere. The blueberry is one of the most laxative of berries on account of the very large amount of cellulose found in its thick skin and numerous seeds.

\section{The Blackberry and the Loganberry.}

The blackberry is a richly flavored fruit containing a little less than 2.25 per cent. of sugar and 5 per cent. of cellulose, which gives it laxative properties. It grows wild in great quantities in certain parts of the country. Cultivated varieties are larger and finer in appearance, but not superior in flavor to the wild berry, which is much sweeter than the cultivated.

The loganberry is simply a red blackberry, a sport.

Commercial loganberry juice consists of the pure juice of the loganberry with the addition of cane sugar. A pint of loganberry juice contains about six ounces of cane sugar and a quarter of an ounce of fruit acids, of which three-fourths is citric acid, the remainder malic acid.

The following is the composition of loganberry juice, according to an analysis made by the Columbus Laboratory of Chicago: 
Solids

Reducing sugars, invert.

Cane sugar

Citric acid

Malic acid

Pectose, color, etc.

Protein

Ash

\section{The Raspberry.}

Now produced in many varieties, is somewhat finer in flavor than the blackberry. It contains a little more sugar and less acid than the blackberry.

\section{The Mulberry.}

This fruit, though highly palatable and rich in sugar, is little used in this country but in Daghestan is greatly prized for its richness in sugar. The dried Daghestan mulberry is so sweet it melts in the mouth like a small lump of sugar.

\section{Wild Berries.}

The numerous berries which grow wild are worthy of larger consideration than they have heretofore had in the national dietary. They are particularly valuable because of their richness in vitamins and the large amount of cellulose, which gives them laxative properties which are almost universally needed. Among the best of these may be enumerated the whortleberry, blackberry, barberry, raspberry, dewberry, cranberry and strawberry, which is the queen of them all. The elderberry, service berry, cloud berry and the June berry are also worthy of mention.

\section{CITRUS FRUITS}

\section{The Orange.}

The-orange is one of Nature's finest-gifts-to-man. Orange juice contains pre-digested food in a most delicious and attractive form, ready for immediate absorption and utilization.

The amount of food contained in a single large orange is about equivalent to that found in a half slice of bread, but it differs from bread in that it needs no digestion, while bread, before it can be used in energizing and strengthening the body, must undergo digestion for-several hours. It is for this reason 
that oranges are so refreshing to an exhausted or feeble person. The sweeter the orange, the greater its food value.

A pint of buttermilk has a food value of 176 calories, onefourth less than that of an equal quantity of orange juice. A pint of oysters affords the same number of food units as a pint of buttermilk and hence has a food value inferior to that of orange juice. Even full milk is not so much superior to orange juice in nutritive value as one might expect. A pint of orange juice contains nearly the same number of food units as threefourths of a pint of milk. Numerous green vegetables have a nutritive value no greater than that of orange juice.

But the energy value of the orange, which for an ordinary sized orange amounts to 75 to 100 calories, is by no means its only value. Orange juice is rich in salts, especially in lime and alkaline salts, which counteract the tendency to acidosis that is always threatening sedentary people, hearty meat eaters, and those advanced in age. The free use of orange juice is a valuable means of combating the inroads of "Father Time," and is also an excellent means of antidoting, to some extent, the bad effects of an indoor or sedentary life.

One or two oranges taken at bedtime and on rising in the morning are an excellent means of stimulating bowel action. Oranges may be taken between meals with great benefit by feeble persons and those suffering from constipation. The delightful flavor and general stimulating influence of orange juice excites peristaltic activity, and so tends to prevent the accumulation of food residues in the colon which leads to putrefaction and autointoxication.

As a food in fever cases, nothing could be more perfectly suited to the requirements of the patient's condition. The fever patient needs water to carry off poisons which are burning him up and against which his cells and organs are struggling. Four to six quarts of water are needed daily to quench the fever's fires and aid elimination through thelskin and kidneys.

Fruit acids aid in satisfying thirst, and their agreeable flavor makes it possible for the patient to swallow the amount of liquid needed. The intense toxemia from which the fever patient suffers and the lack of saliva coats his tongue and often destroys his thirst for water as well as his desire for food. The agreeable flavor of orange juice aids greatly in overcoming this obstacle.

Another special and valuable property of orange juice is the 
small amount of protein or albuminous matter which it contains. Fever patients have little gastric juice and very small digestive power, and so need to take food which is ready for absorption and immediate use. Foods poor in albumen are also needful in fevers because they do not leave residues to undergo putrefaction in the colon, as do meat, eggs and numerous other foods.

Orange juice contains less than 1 per cent. of protein or albumin so that a patient may take three or four quarts of the juice without getting an excess of protein which might easily become a source of great injury.

Two quarts of orange juice with an equal amount of barley, oatmeal or rice gruel make an ideal daily ration for a patient battling with typhoid fever.

Another class of cases in which orange juice is almost indispensable is found in those most unfortunate and suffering of mortals-bottle-fed babies. Usually fed on pasteurized or sterilized milk, these unhappy little ones seldom fail to show marked evidence of malnutrition. They are, indeed, not infrequently victims of scurvy, rickets or pellagra. The investigations of Funk, McCollum and many others have shown that the emaciation, weakness, arrest of growth and general malnutrition in such cases is due to absence from their food of the essential known as vitamin.

A few years ago the fortunate discovery was made that orange juice contains element capable of supplementing the diet of puny, bottle-fed babies, rosulting in immediate resumption of growth and a speedy return to health. This remarkable transformation occurs not only in human infants, but in young animals upon whom the orange juce feeding experiment has been repeated.

Every infant fed on starilized milk or artificial infant foods, in fact, every infant fed ffom a nursing bottle not doing well should receive daily half an ounce to two ounces of orange juice, according to age, to supply necessary vitamins. Older children not doing well should recepve two to four ounces of orange or tomato juice daily.

The acid of orange juice and the sugars it contains aid digestion by stimulating the gastric glands to increased activity. It is also an appetizer of the first quality.

On the whole, orange are probably capable of serving more useful purposes in the ecofomy of the body than any other juicy 
fruit. As people become better educated in dietetics, oranges will be more and more appreciated and more freely used.

\section{The Lemon.}

There are more than sixty varieties of the lemon, including the lime and sweet lemon. Formerly the best lemons came from Sicily, but at the present time the finest lemons grown are produced in the United States. In southern California and Florida the enterprise of American horticulturists has brought the fruit and its culture and handling to a very high state of perfection.

The lemon rind contains an essential oil which is useful for some purposes, especially for flavoring, but the real value of the fruit is found in the two ounces of acid juice which the average lemon contains.

The composition of lemon juice varies considerably, according to the time of picking and the length of time the fruit has been kept, together with the manner of keeping. The chief constituent of lemon juice is citric acid, of which the average lemon contains about 7.5 per cent. A lemon picked in April contains only about two-thirds as much acid as one picked in November. From April on, the percentage of acid steadily diminishes until by July there is little left, the citric acid being converted into sugar (dextrose) and carbondioxide. This is due to the vital activities of the living cells of the fruit. If oxygen is excluded, by varnishing the fruit, the change may be prevented, as also to a large extent by cold storage.

Lemon juice also contains a notable amount of salts ( 2 to 3 per cent.) chiefly potash and phosphorus.

The percentage of potassium in lemon juice is greater than in either apple juice or grape juice. This abundant and unusual supply of salts makes the lemon an important source of this highly essential food principle, and brings clearly to view the fact that the nutritive effect of lemon juice is that of an alkali instead of that of an acid, as superficial knowledge would suggest.

It thus appears that the lemon is worthy of a much larger place in our national dietary than it has heretofore enjoyed.

All fruits are valuable as a means of counteracting the tendency to excessive accumulation of acids through the large use of cereal breakfast foods and excessive meat consumption, which are current practices in this country; but the lemon has the advantage that it is accessible at all seasons of the year. It may 
be readily obtained when most other fruits are difficult to procure.

But the claim of the lemon to a prominent place in the national dietary does not rest alone upon its valuable store of alkaline salts. It has long been known that the juice of lemons contains something of such extraordinary value as a food that without it the diet of soldiers, seamen, and other bodies of men who are compelled to live for long periods of time upon a restricted diet is almost certain to prove so inadequate as to give rise to very grave and often fatal disease. Outbreaks of scurvy, especially among soldiers and sailors, have been recognized for hundreds of years.

The lemon, of all fruits, seems to possess the highest scurvypreventing value. It was formerly thought that the sour lime of the West Indies was especially valuable for this purpose, but Alice Henderson Smith has demonstrated that the West India sour lime has only one-fourth the scurvy-preventing value of the lemon. This observer has also proven that the so-called lime juice used many years ago as a preventive remedy was prepared from lemons and sweet limes and not from the sour lime. This property of the lemon is found to be due to a subtle substance present in lemon juice in minute quantities, known as the antiscorbutic vitamin. This vitamin is also found in many fresh fruits and vegetables, particularly in the tomato and the potato, but the juice of the lemon and of the orange, especially that of the former fruit, has been shown, not only by clinical experience but by crucial laboratory experiments, to possess this property in a very high degree. This property is not associated with the acid of the lemon, for it is found to be present even after the acids have been removed, and is not possessed by the citric acid of the laboratory which may be purchased at drug stores in crystalline form.

It is believed by observing medical men that scurvy in mild and obscure forms is very widely prevalent in this country and probably in all civilized countries.

The conventional breakfast, consisting of coffee, white bread or toast, bacon, or griddle cakes with syrup, and, perhaps the addition of pickles or doughnuts, is almost altogether deficient in vitamins, and is an excellent formula for producing scurvy.

The substitution of lemon squash or hot lemonade for tea or coffee, or even any of the various cereal substitutes for coffee, would initiate marvelous physical improvement in thousands of 
families which are now languishing under various forms of malnutrition.

Lemon juice should be universally substituted for vinegar as an acid flavoring for salads and other vegetable dishes. It has long been used for this purpose by the best French cooks, and for many years has been gaining in favor in this country.

As an appetizer, lemon juice has no superior. It stimulates the flow of saliva, and in like manner causes the gastric glands to pour forth the gastric juice which both disinfects and digests the food.

Lemonade prepared by the addition of the juice of one small lemon to a quart of water, with three or four ounces of malt sugar, is an excellent beverage for use in fevers. Two or three quarts may be given daily with advantage.

Lemonade may be freely used in rheumatic fever as well as other fevers, since the potash salts present serve to alkalinize the blood and other tissue fluids, as already explained.

\section{The Lime.}

This citrus fruit, allied to the lemon, possesses a characteristic flavor much liked by most persons, but on the whole ranks below the lemon in dietetic value, chiefly because less active as an antiscorbutic.

\section{TROPICAL FRUITS}

There are many tropical fruits which, with our improved methods of transportation, will probably in time become as popular in the United States as they are in the Central American countries. The mango and the avocado, or (alligator pear, and the various varieties of the sapota are excellent fruits which might be added to the rather limited list of those grown in the United States.

\section{The Banana.}

A native of Asia, the banana is now grown in most tropical countries, and is one of the most productive of all food plants. Its food value is a little greater than that of the potato. Its protein content is small, one and a half calories to the ounce. It also contains very little fat. When ripe, it affords a large amount, more than 25 calories to the ounce, of an easily digestible carbohydrate. 
The popular idea that the banana is difficult of digestion is the result of the use of the fruit in an immature state. The banana is never allowed to ripen on the plant which produces it. It is always picked when it has attained full size but is not yet mature. The fruit is not fully ripe until the skin has become, in part at least, brown in color. During the change from yellow or red to brown, the starch, of which the green banana contains a large amount, is converted into dextrin and sugar, of which the ripe banana contains nearly 25 per cent. When thoroughly ripe, the banana is one of the most easily digestible of foods. When to be eaten by invalids or infants, it is well to convert the pulp into a pürée by passing through a colander, after which it may be rendered very palatable and inviting to the eye by beating with an egg beater.

The banana has a higher nutritive value than almost any other fruit which can be eaten fresh. Pound for pound, it supplies as much nutriment as the potato and is the staple food for thousands of rubber gatherers who perform prodigious feats of muscular exertion and endurance with almost no other sustenance.

A well ripened banana contains almost no raw starch. Myer and Rose report that when bananas not well ripened are eaten a considerable amount of starch is found in the stools. This fact emphasizes the importance of taking care to select well ripened fruit, as indicated by the dark brown color of the skin, especially when feeding bananas to children. The raw starch is of course not objectionable, but its presence is evidence of the indigestibility of the unripe banana.

The banana is fairly rich in vitamin A. It is also a good source of vitamin $\mathrm{C}$, antiscorbutic, if eaten raw or baked with the skin on (Eddy and Kellogg). With the addition of milk, the ripe banana forms a complete food. An excellent food for infants is one part ripe banana pulp well mixed with three parts of milk.

Humboldt first called attention to the enormous amount of food which may be produced by a single acre of bananas. Competent authorities have estimated that the banana yields per acre three or four times as much food material as is produced on the average by wheat, potatoes or corn. 


\section{Banana Flour.}

Banana flour is made by drying and grinding the mature but unripe fruit, the starch of which has not yet been changed to sugar. Banana flour is an easıly digestible, highly nutritir,us product and is much used in the tropics. Stanley in his journeyings in central Africa depended upon banana flour as his staple food. Mixed with wheat flour it may be made into very palatable cakes. Prepared in the form of gruel it affords a light and easily digestible form of nutriment for feeding certain classes of invalids, particularly when it is desired to combat intestinal toxemia.

\section{Banana Figs.}

When dried, the ripe banana becomes a sweet leathery product somewhat resembling a dried fig in consistency but less sweet and much less palatable. Various attempts have been made to popularize banana figs in this country, thus far without success. In this highly favored part of the world so many attractive foodstuffs are available it is doubtful whether this very wholesome, if not highly attractive foodstuff, will ever come into general use.

\section{The Fig.}

This is one of the oldest cultivated fruits. It is generally eaten in the dried state, its content of sugar being sufficiently great to preserve it. The ripe fig eaten fresh from the tree is one of the most delicious of fruits. Eaten either fresh or dried, the fig is highly laxative on account of the large amount of cellulose which it contains, due to its tough skin and great number of small seeds.

The dried fig has a nutritive value double that of lean beef. This excellent fruit is too little appreciated and used in the United States. In many parts of the Orient, particularly around Smyrna and in southern Greece, the dried fig is one of the staple articles of food of the peasantry. Coarse bread, ripe olives, and dried figs furnish a complete meal for the Greek sailors who man the ships of the Mediterranean. The fresh, undried ripe fig is a most excellent fruit but quickly perishable. The purple fig of California is particularly valuable as a laxative, and if properly prepared and preserved without the addition of cane sugar would be a most valuable addition to the national bill of fare.

The dried purple fig soaked for 48 hours in cold water and eaten freely twice a day is an excellent remedy for constipation. 


\section{The Date.}

The date, like the fig, has come to us from prehistoric times. Until recently its culture was wholly confined to the Orient. Its cultivation has in late years been undertaken in the Coachella valley and neighboring parts of the Colorado Desert in southern California. The date affords nearly three-fourths ( 70 per cent.) its weight of sugar, besides a considerable amount of protem, which sometimes rises as high as 7 per cent.

Recent studies of the sugar content of the date have shown that while cane sugar is always present in the immature date it generally disappears to a large extent in the process of ripening, so that the fully ripe date contains almost exclusively invert sugar; that is, the sugar usually found in fruits-dextrose and levulosein about equal parts. The Deglet Nur, grown in Algeria and California, is practically the only well known date which contains cane sugar. Professor Vinson, of the University of Arizona, found in the Deglet Nur 41.4 per cent. of cane sugar and a little more than 4 per cent each of levulose and dextrose. The Fard and most other commercial dates grown or sold in the United States are of the invert sugar varieties. Most dates eaten contain not more than 10 per cent. of cane sugar, with a total sugar content of about 70 per cent.

It is gratifying to note that the date industry, especially in the Coachella valley, is making rapid advancement, and it is to be hoped that in time this superior fruit may be produced in sufficient quantity to supply our home market. It is particularly important that the growth of invert sugar dates should be encouraged because of the superior value of the invert form of sugar, due to the fact that it is ready for immediate absorption and utilization, whereas cane sugar requires a somewhat prolonged digestive process to prepare it for absorption.

It is believed by many eminent authorities that the free use of cane sugar in this country may be one of the causes of the increasing prevalence of diabetes, and Sherman has called attention to the fact that the large use of cane sugar in the form of confectionery and otherwise results in extensive lime starvation, which affects probably half the population of the United States. To this may be attributed the dental caries which is becoming almost universal in this country even in children, as well as other forms of disease of the bony structures of the body. The 
substitution of dates and other sweet fruits for the present free use of cane sugar would unquestionably greatly improve the health of the American people. The sugar in the date is not only a natural sugar, which is ready for immediate absorption, but in its natural state the sugar of the date is associated with the lime. iron, vitamins and other essential food constituents which are altogether lacking in cane sugar.

The dates of commerce should be prepared for use by washing. This rule should, in fact, apply to all sorts of dried fruits, especially those imported from the Orient. The latter, in addition to washing, should be subjected to the disinfecting action of a 2 per cent. solution of peroxide of hydrogen, in which the fruit should be soaked for four or five minutes after washing. "After disinfecting, the dates should be dried if not used at once. American grown dates are clean and need no disinfecting.

\section{The Guava.}

This fruit, a native of the tropics, is now grown quite extensively in Florida and southern California. It has a rich, peculiar flavor which by many is greatly relished. The red, or strawberry, guava grown in southern California is very palatable. The guava has a nutrient content of about 20 per cent., the greater part of which is sugar.

\section{The Ilango.}

This highly palatable tropical fruit is now produced in considerable abundance in southern Florida. As this fruit becomes better known, it will be more and more appreciated as a valuable addition to our growing list of fruits.

\section{The Pawpaw.}

This wonderful product of the tropics, known in Mexico as the melon sapote, is one of the most remarkable of fruits. The pawpaw, when mature, is nearly as large as a muskmelon, and has a rich, sweet pulp of such consistency that it may be eaten with a spoon. It contains papayotin, a digestive principle resembling pepsin and pancreatin.

\section{The Papaw.}

The papaw, though similar in name to the pawpaw, is very different. In flavor it somewhat resembles the banana and is much appreciated by those who have cultivated a liking for it. 
This fruit now growing wild in many parts of the United States should be cultivated and improved and may sometime become a very valuable orchard fruit. Its composition is, protein, 5.2 per cent.; fat, 0.9 ; carbohydrate (sugar), 6.8 ; ash, 0.5 ; water, 76.6 .

\section{The Pineapple.}

This luscious fruit is grown in Central America, Porto Rico, the Sandwich Islands, the Philippines, the West Indies and in the southernmost part of Florida. When ripened before packing, this is one of the most delicious of fruits, affording nearly 25 per cent. of its weight of sugar and one-half of 1 per cent. of citric acid.

As found fresh in the markets, the pineapple is usually somewhat inferior in quality, having been picked before fully ripe. Although the pineapple contains a ferment which has an action upon proteins resembling that of pepsin and pancreatin, it is rather indigestible unless cooked or eaten in its fully mature state.

\section{The Pomegranate.}

This ancient fruit, formerly little known in this country because of the difficulty of importing it, is now produced in considerable quantity in California and certain Southern states. Its leathery rind encloses a mass of highly delicious pulp, the juice of which contains more than 13 per cent. of sugar and one-half of 1 per cent. of citric acid.

\section{The Avocado, or Alligator Pear.}

This is a remarkable tropical fruit, a native of Mexico, now cultivated in Florida and southern California. The tough green or reddish rind of this fruit encloses a thick yellow pulp which has the consistency of chilled butter and contains besides 2 per cent. of protein and 6 per cent. of sugar, 15 or 20 per cent. of highly digestible, emulsified fat, or as much as rich cream. This is indeed a very precious characteristic which will render it invaluable as a fat-building food when the supply becomes more regular and abundant. The United States Bureau of Plant Introduction promises to supply in the near future different varieties of the avocado which will yield fruit in every month of the year. The avocado is the meat of the Guatemalans who do the hardest work on a diet of tortillas (corn cakes) and avocados.

The avocado supplies not only a remarkably fine quality 
of fat, but a protein probably about equal to that of milk in its nourishing value.

Still another superior quality of this fruit is its richness in vitamins, one of the latest finds about it. When fed to rats, the avocado was found to promote growth in a most remarkable way. It lacks vitamin $\mathrm{C}$.

It is evident that the avocado is a most valuable addition to our food supply. The tree which produces it grows rapidly and begins to bear early. It flourishes in southern Florida in the vicinity of Miami, and in certain sheltered spots in southern California. Lower California is an ideal place for the growth of this as well as other tropical plants. If ever Uncle Sam finds some way to add this strip of tropic sunshine to his dominions, it will become the market garden of the whole country.

The soft pulp of the avocado may be easily worked into an emulsion, which by the addition of water becomes a delicious and nourishing milk which ought to prove a good food for babies and invalids.

The avocado lends itself admirably to the preparation of salads. With the addition of a little salt and lemon juice it makes an excellent salad. Some varieties are so rich in fat that the fruit may serve as an excellent table substitute for butter. The Guatemalan avocado, now cultivated in this country, contains as much as 30 per cent. of a most delicious and digestible fat.

The avocado begins to bear three years from the seed, producing fruits which weigh from a pound to two and one-half pounds each. An acre of land planted to avocado trees will produce from 600 to 1,000 pounds of fat, mutch more than can be produced by any other crop known.

\section{The Karob.}

The karob is a fleshy pod having a sweet flavor somewhat similar to the date, produced by a tree resembling the locust. It is cultivated to some extent in Egypt and other tropical countries. The fruit is commonly known as St. John's bread.

The mesquite tree of the Colorado desert, western Texas, Arizona, and northern Mexico produces a somewhat similar fruit having a sweetish flavor. It has been much used by the Indians for food and is greatly relished by cattle and mules. 


\section{DRIED FRUITS}

Dried fruits are highly valuable, though to some extent depreciated by the process of drying. It has been shown that the ordinary processes of drying fruits destroy in large part, and sometimes entirely, the rich store of vitamins present in fresh fruits. Ordinary dried fruits are of little or no value as antiscorbutics. In most other particulars dried fruits are nearly as valuable as fresh. When carefully dried by the most up-to-date methods, some fruits retain a considerable part of their vitamins, and when soaked in water recover almost their original freshness in appearance and flavor. The prune and the blue California fig are much more efficient as remedies for constipation, as well as more palatable, when prepared by simply soaking for 24 to 48 hours in cold water and without cooking. Fresh milk, with tomatoes, potatoes and other foods rich in vitamins, should be liberally used with dried fruits. Drying does not destroy the vitamins of the tomato.

The following table shows the composition of the dried fruits in ordinary use :

TABLE XXXIII.

Percentage Composition of Dried Fruits.

\begin{tabular}{|c|c|c|c|c|c|c|c|c|}
\hline & Water & Protein & Carb. & Fat & Acias & Fiber & salts & $\begin{array}{l}\text { atortes } \\
\text { per oz. }\end{array}$ \\
\hline olec & 28.1 & 1.6 & 57.6 & 2.2 & 3.6 & 4.9 & 2.0 & 85.2 \\
\hline pricots & 29.4 & 4.7 & 56.0 & 1.0 & 0.3 & 7.5 & 2.4 & 81.2 \\
\hline ananas ......... & 29.2 & 5.3 & 55.5 & & .... & 2.1 & 3 & 69.5 \\
\hline ha flour.... & 9.7 & 3.1 & 83.4 & & .... & 0.7 & 2.6 & \\
\hline urr & 17.2 & 2.4 & 74.2 & 1.7 & & & 4.5 & 93.5 \\
\hline ate & 20.8 & 4.4 & 65 & 2.8 & & 5.5 & 1.5 & 98.8 \\
\hline$g$ & 18.8 & 4.3 & 67.3 & 0.3 & & 7.3 & 2.4 & 90.0 \\
\hline & 16 & 2.8 & 66.0 & 5.4 & & 6.9 & 2.4 & 92.4 \\
\hline & 21.0 & 2.1 & & & 2.7 & 2.3 & 2.3 & 83.6 \\
\hline & & 2.6 & & 3.5 & $\ldots$. & 2.5 & 3.4 & 92.0 \\
\hline Kaspderries. & 8.1 & 6.5 & 50.0 & 1.8 & 6.0 & 25.0 & 2.6 & 69.1 \\
\hline
\end{tabular}

Certain fruits, particularly figs and raisins, are generally dried in the sun. This method, however, exposes the fruit to contamination by dust and insects. The modern improved methods of artificial drying afford a much cleaner and more uniform product. The great fruit industry of California has stimulated 
invention to such an extent that at the present time the art of drying fruit has attained a very high degree of perfection, and has developed the enormous industry which produces annually more than half a billion pounds of dessicated fruits.

Dried fruit has the advantage that it retains most of the properties of the fresh fruit, occupies less bulk, and with proper precaution may be preserved for a considerable time.

A bushel of fresh apples weighing fifty-five pounds, in the process of drying loses more than forty pounds of water. The loss of water in the drying of some other fruits is even greater. The fruits for which the greatest demand in the dried state has existed heretofore are the apple, peach, apricot, raisin, date and fig; but there is an increasing demand for the dried banana and banana flour, two excellent products which present a choice quality of nutritive material in easily digestible form.

The bleaching of dried fruits by means of the fumes of burning sulphur has been generally condemned by hygienic authorities because of the considerable amount of suiphite left in the bleached products. The Federal Government and several States have passed laws restricting the amount of sulphite permissible. A process has recently been perfected, however, by which this objection is overcome. It has been found that by the use of peroxid of hydrogen the injurious sulphite may be converted into a harmless compound, so that the sulphur bleaching process may now be applied for the disinfection and preservation of foodstuffs as well as bleaching and may be employed for the treatment of nuts, sirups, gelatin, potatoes and other foodstuffs.

\section{CANINED FRUITS}

The preservation of fruits by canning has greatly enriched our winter and spring bills of fare and provided a useful supply of appetizing carbohydrate foods at a season when, in former times, the dietary often became monotonous.

When canned in tin, the organic acids of fruits are likely to attack the container and may thus become contaminated with lead from the solder. After a long time, the acid may even attack the tin. This difficulty has been largely overcome in recent years by the application of enamel to the inside of the can. Glass fruit cans are evidently much better adapted to the preservation of fruits than tin containers. 
It is to be remembered, also, that the antiscorbutic vitamin is likely to be greatly imparred, even destroyed, by the heat employed in processing canned fruit. Fortunately, the temperatures employed in the canning of fruits are not so high as those required for vegetables, and on this account the vitamins are less damaged. The juice of canned tomatoes has been found to be an excellent antiscorbutic.

Canned fruits as well as other foods which contain preservatives, such as benzoate of soda, are unwholesome and should never be used. The following table shows the average composition of canned fruits in general use:

TABLE XXXIV.

Showing the Percentage Composition of Canned Fruits.

\begin{tabular}{|c|c|c|c|c|c|c|}
\hline \multirow[b]{2}{*}{ Apples, crab. } & \multirow{2}{*}{$\begin{array}{l}\text { Water } \\
42.4\end{array}$} & \multirow{2}{*}{$\begin{array}{c}\text { Protein } \\
0.3\end{array}$} & \multirow{2}{*}{$\begin{array}{l}\text { Fat } \\
2.4\end{array}$} & \multirow{2}{*}{$\begin{array}{c}\text { Carb. } \\
54.4\end{array}$} & \multicolumn{2}{|c|}{$\begin{array}{r}\text { Calorles } \\
\text { Salts per oz. }\end{array}$} \\
\hline & & & & & .5 & 70 \\
\hline Apple sauce. & 61.1 & 0.2 & 0.8 & 37.2 & .5 & 44 \\
\hline Apricots & 81.4 & 0.9 & & 17.3 & .4 & 21 \\
\hline Blackberries & 40.0 & 0.8 & 2.1 & 56.4 & .7 & 72 \\
\hline Blueberries & 85.6 & 0.6 & 0.6 & 12.8 & .4 & 17 \\
\hline Cherries .... & 21.0 & 1.1 & & 77.2 & .7 & 91 \\
\hline Peaches & 88.1 & 0.7 & 0.1 & 10.8 & .3 & 14 \\
\hline Pears ............. & 81.1 & 0.3 & 0.3 & 18.0 & .3 & 22 \\
\hline Pineapples ... & 61.8 & 0.4 & 0.7 & 36.4 & .7 & 45 \\
\hline Prune sauce & 76.6 & 0.5 & 0.1 & 22.3 & .5 & 27 \\
\hline Strawberries & 74.8 & 0.7 & $\ldots .$. & 24.0 & .5 & 29 \\
\hline Tomatoes ..... & 94.0 & 1.2 & 0.2 & 4.0 & .6 & 6 \\
\hline
\end{tabular}

\section{FRUIT JUICES}

The value of fruit juices is as yet much too little appreciated. Fruits differ from practically all other foods in the fact that their nutritive elements exist in the soluble forms of sugar, dextrin and acids, and hence are almost exclusively found in their juices. The dry pulp left after the expression of the juice contains almost nothing except cellulose, a little protein and the major part of the salts.

Fruit juices of all sorts are exceedingly wholesome. They contain all the valuable properties of the fruits from which they are prepared with the exception of cellulose. The popular preju- 
dice against acid fruit juices on the ground that they encourage the development of rheumatism is entirely without scientific basis. Fruit juices, like fruits, are rich in the salts of sodium and potassium and increase the alkalinity of the blood and tissue fluids.

The aromatic oils of fruit juices which give to them their characteristic flavors are appetizing and thus promote digestion. It is probable that many persons who are in the habit of using light wines value them for their fruity flavors rather than for any effects experienced from the alcohol present. The personal introduction of grape juice and of other fruit juices in recent times has no doubt contributed in no small degree to the success of the prohibition movement. An effort should be made to replace the wine and beer of ante-prohibition days by fruit juices of many different sorts.

By means of a recent invention it is now possible to retain in fruit juices, in concentrated form, the aroma which was formerly lost in the processes of canning and condensation.

Fruits and fruit juices are for the most part eaten as luxuries rather than as food staples. As a matter of fact, fruits and the juices of fruits are worthy of a more dignified position on the bill of fare. A half pint of grape juice has a food value of 200 calories, fully equal to that of a like quantity of milk, though of a different sort, consisting of sugars with practically no protein or fat.

Fruit juices, even much diluted, as in the form of lemonade, orangeade and similar drinks, contain an amount of nourishment worth considering. For example, as ordinarily made, a glass of lemonade (six ounces) affords 85.2 calorics. In this case, the greater part of the calorie value is due to the sugar employed for sweetening. Cane sugar may be taken in this diluted form with less injury than in any other way.

The establishment of prohibition has made of grape juice a national beverage. Other juices are equally valuable. Loganberry juice is rapidly gaining in favor and orange juice now appears on the bill of fare of every first-class hotel or restaurant. The amount of food material found in fruit juices is much larger than is generally supposed. This will readily appear by reference to the accompanying table, in which it will be seen that the juices of the blackberry, grape, pear, raspberry and the strawberry, and even the pineapple, all equal or exceed whole milk in nutritive 
value; in fact, with the exception of lemon and orange juice, fruit juices in general are practically equal to milk in the actual amount of nutriment which they supply. Of course, none of these juices could take the place of milk as a food for the reason that the nutriment which they furnish is almost exclusively carbohydrate. There are conditions, however, in which exclusive carbohydrate feeding is desirable, as in certain cases of acidosis. In such cases fruit juices become a valuable resource.

\section{TABLE XXXV.}

\section{Composition of Fruit Jurice.}

\begin{tabular}{|c|c|c|c|}
\hline & \multicolumn{3}{|c|}{$\begin{array}{l}\text { Calories per Ounce } \\
\text { Protein }\end{array}$} \\
\hline Apple juice & 0 & 0 & 17.0 \\
\hline Blackberry juice & 0 & 0 & 30.3 \\
\hline Blueberry juice.. & 0 & 0 & 18.9 \\
\hline Grape juice ............. & 0 & 0 & 23.8 \\
\hline Orange juice ........ & 0 & 0 & 15.1 \\
\hline Peach juice ............. & 0 & 0 & 20.8 \\
\hline Pear juice ............... & 0 & 0 & 25.6 \\
\hline Pineapple juice & 0 & 0 & 21.8 \\
\hline Raspberry (red) juice.... & 0 & 0 & 23.7 \\
\hline Raspberry (black) juice. & 0 & 0 & 29.4 \\
\hline Strawberry juice & 0 & 0 & 24.5 \\
\hline Lemon juice & 0 & 0 & 11.4 \\
\hline
\end{tabular}

It should be noted that the figures of the above table represent fruit juices as they are usually served and include a small addition of cane sugar to the natural juice. This is true in practically all instances except apple, orange and lemon juice.

Fruits usually contain the antiscorbutic vitamin but are, however, rather poor in other vitamins. This important fact, to which attention has been called, emphasizes the necessity for accurate information in the making of bills of fare for the sick, since it may frequently happen that the patient is already suffering from a deficiency of some one or all of the three vitamins known to be essential to good nutrition. For example, a person attempting to live on a diet of fruits and nuts would suffer from lack of the water-soluble $\mathrm{C}$, antiscorbutic vitamin, if the bill of fare was largely made up of dried fruits such as figs, dates and 
raisins with nuts, even though the supply of carbohydrates, fats and protein might be very ample.

On this account it is well always to include with the fruit and nut diet a liberal allowance of oranges to supply water-soluble vitamins $\mathrm{B}$ and $\mathrm{C}$, and greens, carrots, butter or tomato, to supply the needed fat-soluble $\mathrm{A}$.

The tomato, either fresh or dried, is always a most safe addition to the bill of fare because it is rich in all the vitamins. Dried tomato contains twice as much of the fat-soluble vitamin as does the same weight of butter.

\section{TABLE XXXVI.}

\section{Showing the Composition of Commercial Lime Juices.}

The following analyses were made by the Canada Inland Revenue Department:

\begin{tabular}{|c|c|c|c|c|}
\hline & Sollas & $\begin{array}{l}\text { Citr } \\
\text { Pres }\end{array}$ & $\begin{array}{l}\text { c Acid and } \\
\text { ervatives }\end{array}$ & \\
\hline Blackwood's .... & 8.8 & 7.6 & Salicylic acid & \\
\hline Dalton's .......... & 7.2 & 7.1 & 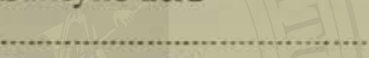 & \\
\hline Dominica ........ & 10.6 & 8.1 & Benzoic acid & \\
\hline Eaton's .... & 8.7 & 6.9 & Salicylic acid & \\
\hline Howard's ... & 6.7 & 6.6 & & \\
\hline Montserrat & 9.2 & 8.0 & & \\
\hline Olympia .... & 7.8 & 7.3 & Benzoic acid, none & \\
\hline Rose's ..... & 8.6 & $\begin{array}{r}7.9 \\
P\end{array}$ & $\begin{array}{l}\text { Benzoic acid, none, or } \\
\text { ous acid }\end{array}$ & r sulphur- \\
\hline 1 & & 6.9 & 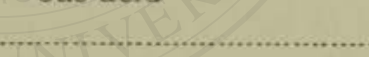 & \\
\hline Sovereign & 8.0 & & $\begin{array}{l}\text { Salicylic acid, benzoic a } \\
\text { or sulphurous acid }\end{array}$ & acid, none, \\
\hline Sterling & 7.3 & & $\begin{array}{l}\text { Benzoic acid, salicylic } \\
\text { none }\end{array}$ & acid, or \\
\hline & 9.3 & 7.7 & Sulphurous acid & \\
\hline hite Sta & 7.9 & 6.9 & & \\
\hline
\end{tabular}




\section{Nuts}

The nut is one of the oldest and best of Nature's products intended as food for man. The paleontologists tell us that early man was a nut eater as are the gorilla, the orang-utan, and the chimpanzee, his modern prototypes. The chestnut has been from the most ancient times a staple article of food in northern Italy. The early inhabitants of Great Britain were acorn eaters, as were the ancient Greeks.

In modern times nuts have been used as a luxury rather than as a staple article of food; but as the public become better informed respecting the high food value of nuts, especially in view of the steadily rising cost of flesh meats, the nut is certain to gain appreciation, and the writer has no doubt that some time in the future nuts will become a leading constituent of the national bill of fare and will displace certain foodstuffs which today are held in high esteem but which in the broader light of the next century will be regarded as objectionable and inferior foods.

Botanically, a nut is a fruit; but nuts differ so widely both in composition and appearance from the foods commonly called fruits that they are properly classed by themselves.

In nutritive value the nut far exceeds all other food substances; for example, the average number of food units per pound furnished by half a dozen of the more common varieties of nuts is 3231 calories while the average of the same number of varieties of cereals is 1654 calories, half the value of nuts. The average food value of the best vegetables is 300 calories per pound and of the best fresh fruits grown in this country 278 calories. The average value of the six principal flesh foods is 810 calories per pound or one-fourth that of nuts.

Recent studies of the proteins of nuts by Osborne and Harris, Van Slyke, Johns and Cajori, have demonstrated that the proteins of nuts are at least equal to those of meat.) This has been shown to be true of the almond, English walnut, black walnut, butternut, peanut, pecan, filbert, Brazil nut, pine nut, chestnut, hickory and coconut; that is, of practically all the nuts in common use. 
The superior nutritive value of nuts is clearly shown by the following table:

TABLE XXXVII.

Composition and Food Value of Edible Portion of Nuts.

\begin{tabular}{|c|c|c|c|c|c|}
\hline & $\begin{array}{l}\text { Protein } \\
\text { Per cent. }\end{array}$ & $\begin{array}{c}\text { Fats } \\
\text { Per cent. }\end{array}$ & $\begin{array}{c}\text { Carbo- } \\
\text { hydrates } \\
\text { Per cent. }\end{array}$ & $\begin{array}{c}\text { Ash } \\
\text { Per cent. }\end{array}$ & $\begin{array}{l}\text { Food value } \\
\text { per pound } \\
\text { calories }\end{array}$ \\
\hline Acorns ... & 8.1 & 37.4 & 48.0 & 2.4 & 2,718 \\
\hline Almonds. & 21.0 & 54.9 & 17.3 & 2.0 & 3,030 \\
\hline Beechnuts ... & 21.9 & 57.4 & 13.2 & 3.5 & 3,263 \\
\hline Brazil nuts.... & 17.0 & 66.8 & 7.0 & 3.9 & 3,329 \\
\hline Butternuts ................. & 27.9 & 61.2 & 3.4 & 3.0 & 3,371 \\
\hline Chestnuts, fresh & 6.2 & 5.4 & 42.1 & 1.3 & 1,125 \\
\hline Chestnuts, dried.... & 10.7 & 7.0 & 74.2 & 2.2 & 1,875 \\
\hline Coconuts & 5.7 & 50.6 & 27.9 & 1.7 & 2,986 \\
\hline Coconuts, shredded... & 6.3 & 57.3 & 31.6 & 1.3 & 3,125 \\
\hline Filberts .... & .15 .6 & 65.3 & 13.0 & 2.4 & 3,432 \\
\hline Hickory nuts............... & 15.4 & 67.4 & 11.4 & 2.1 & 3,495 \\
\hline Litchi nuts......... & 2.9 & 0.2 & 77.5 & 1.5 & 1,469 \\
\hline Peanuts, raw........ & 25.8 & 38.6 & 24.4 & 2.0 & 2,560 \\
\hline Peanuts, roasted... & 30.5 & 49.2 & 16.2 & 2.5 & 3,177 \\
\hline Pecan nuts.................. & 11.0 & 71.2 & 13.3 & 1.5 & 3,633 \\
\hline Pine nuts or piñons.... & 14.6 & 61.9 & 17.3 & 2.8 & 3,364 \\
\hline Pistachios, kernels... & 22.6 & 54.5 & 15.6 & 3.1 & 3,010 \\
\hline Black Walnuts & 27.6 & 56.3 & 11.7 & 1.9 & 3,105 \\
\hline English Walnuts ....? & 16.7 & 64.4 & 14.8 & 1.3 & 3,305 \\
\hline
\end{tabular}

Observation seem to show that, in general, the proteins of oily seeds are complete proteins.

Cajori's research has also shown the presence of growthpromoting vitamins in abundant quantity in the almond, English walnut, filbert, pine nut, hickory, chestnut and pecan.

That the nut is appreciated as a dainty is attested by the frequency with which it appears as a dessert and the extensive use of various nuts as confections. That nuts do not hold a more prominent place in the national bill of fare as food staples is due chiefly to two causes: first, the popular idea that nuts are highly indigestible; and second, the limited supply.

The notion that nuts are difficult of digestion has really no foundation in fact. The idea is probably the natural outgrowth 
of the custom of eating nuts at the close of a meal when an abundance, more likely a super-abundance, of highly nutritious foods has already been eaten, and the equally injurious custom of eating nuts between meals.

Neglect of thorough mastication must also be mentioned as a common cause of indigestion following the use of nuts. Nuts are generally eaten dry and have a firm hard flesh which requires thorough use of the organ's of mastication to prepare them for the action of the several digestive juices. It has been experimentally shown that nuts are not well digested unless reduced to a smooth paste in the mouth. Particles of nuts the size of small seeds may escape digestion. Nut paste or butter is easily digestible.

Delicious nut butters may be prepared from true nuts such as the almond, filbert, and pine nut, by blanching and crushing, without roasting. Peanuts require steam roasting. Over-roasting renders the nut difficult of digestion.

According to the New Zealand Dairyman, more than 50,000 tons of nut butters are produced in England every year. Peanut oil, palm kernel oil and coconut oil are the principal raw materials used. In face of vanishing meat supplies it is most comforting to know that meat of all sorts may be safely replaced by nuts not only without loss, but with a decided gain. Nuts have several advantages over flesh foods which are well worth considering.

1. Nuts are free from waste products, uric acid, urea, and other tissue wastes which abound in meats.

2. Nuts are aseptic, free from putrefactive bacteria, and do not readily undergo decay either in the body or outside of it. Meats, on the other hand, as found in the markets are practically always in an advanced stage of putrefaction. Ordinary fresh dried or salted meats contain from $3,000,000$ to ten times that number of bacteria per ounce, and such meats as Hamburger steak often contain more than a billion putrefactive organisms to the ounce. Nuts are clean and sterile.

3. Nuts are free from trichina, tapeworm, and other parasites, as well as other infections due to specific organisms. Nuts are in good health when gathered and usually remain so until eaten.

In view of these facts it is interesting to know that in nuts, the most neglected of all well-known food products, we find the assurance of an ample and complete food supply for all future 
time, even though necessity should compel the total abandonment of our present forms of animal industry.

Another of the great advantages of the nut is that with few exceptions it may be eaten direct from the hand of Nature without culinary preparation of any sort. Indeed, the common custom of offering nuts as dessert is an acknowledgment that in the nut the refined chemistry of Nature's laboratory permits of no improvement by the clumsy methods of the kitchen.

The superior food value of nuts will appear from the following:

\section{One Pound of Walnut Meats Equals in Food Valuse}

\begin{tabular}{|c|c|}
\hline \multicolumn{2}{|l|}{$=1$} \\
\hline Beef loin, lean... & 4.00 \\
\hline Beef neck, lean... & 9.50 \\
\hline Beef ribs, lean....... & 6.50 \\
\hline Chicken, broilers... & 10.00 \\
\hline Eggs & 5.00 \\
\hline Evaporated cream. & 4.00 \\
\hline Fowls & 4.00 \\
\hline Frogs' legs................ & 15.00 \\
\hline Ham, lean ........ & 3.00 \\
\hline Lobsters ... & 22,00 \\
\hline Milk ........................ & 9.50 \\
\hline Mutton leg, lean... & 4.20 \\
\hline Oysters & 13.50 \\
\hline Red Bass ................... & 25.00 \\
\hline 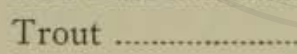 & 4.80 \\
\hline & 5.50 \\
\hline
\end{tabular}

Every highway should be lined with nut trees. Many nut trees will grow on land unsuited to ordinary farm crops. The piñon flourishes on the bleak and barren peaks of the rockies.

A few nut trees planted for each inhabitants would insure the country against any possibility of food shortage. A row of nut trees on each side of our $3,000,000$ miles of country roads would provide protein for a population of 100,000,000.

Nuts should be eaten at every meal and made a substantial part of the bill of fare. So long as the nut is regarded as a dainty, suitable only for dessert, the demand will be limited. 
But as its merits come to be appreciated it will be in greater demand and the supply will rapidly grow in volume.

\section{The Lime Content of Nuts.}

In proportion to their weight, nuts contain more lime than any other class of foodstuffs except legumes, the average being more than one-third grain to the ounce. $(0.370$ grs. $)$. Certain nuts are surprisingly rich in lime. For example, the almond offers nearly one and a half grains of food lime to the ounce, while the hazelnut or filbert affords one and three-quarters grains of lime to the ounce, or 11.3 per cent. of a day's ration of lime. The pecan and the walnut are also fairly rich in lime, as well as the peanut.

An ounce and a half each of almonds and hazelnuts or filberts will supply one-third the total lime requirement for a day. In general, this addition to the ordinary bill of fare would be quite sufficient to insure against any serious deficiency of lime.

By a combination of the proper nuts and fruits, the most delicious of all food combinations, the total needed supply of lime, or at least the major part of it, may be readily secured.

Here is a convenient example which may well serve as a model supplementary lime ration for general use. Take at one meal a large orange and an ounce and a half of almonds. At another meal, an ounce and a half of filberts and two ounces of figs. These nuts and fruits would supply 50 per cent. of the necessary lime, and by adding for a third meal two oranges and two ounces of walnuts more than three-fifths of the total lime requirement would be furnished.

Evidently there is no need of lime starvation if one only knows how to make a right selection of foods.

\section{The Iron Content of Nuts.}

The almond, hazelnut, chestnut, peanut, pecan and walnut all contain a rich store of iron, the average iron content expressed as per cent. of the iron ration being 4.79 , more than two and one-half times that of fruits (1.74), three times that of vegetables (1.46), greater than that of cereals and even superior to average meats. It is true that the extraordinarily high food value of nuts renders them less available than fruits as prime sources of iron, for one would have to eat 5,000 calories of chestnuts or 
walnuts or more than 4,000 calories of pecans or peanuts to get a day's ration of iron ; but three-quarters of a pound of almonds or hazelnuts would supply the needed quantum of iron with an energy intake of 2,500 calories, on account of their unusually rich store of iron.

It is worth while to know that vegetable milk prepared from almonds, by adding five parts of water to one part of blanched almonds made into a smooth paste, supplies two and a half times as much iron as does cow's milk in equal quantity, and furnishes the same amount of protein. It is worth noting just here, also, that the protein of the almond is, like that of milk, a complete protein; that is, a protein out of which human tissues may be readily formed, which is by no means true of all vegetable proteins. Such a milk, however, would be somewhat deficient in lime, a lack which could be supplied by lentil soup.

A product commercially known as Malted Nuts, prepared from almonds or peanuts, has been found of very great service in meeting the needs of infants and some classes of invalids for an easily digestible liquid nourishment to take the place of milk when a substitute is needed.

Of the nuts which grow in this country, the most important are the almond, the English walnut, the pecan and the peanut. The native black walnut, the hickory nut, the piñon, the hazelnut, and the beechnut are all valuable nuts which, by cultivation, might furnish enormous additions to our food supply. This is particularly true of the black walnut.

Among the imported nuts, the most important are the Brazil nut, the coconut, the pistachio, and the recently introduced litchi nut.

A study of the relative protein content of nuts, milk and meat shows that, pound for pound, the almond, beechnut and walnut contain on an average as much protein as does meat and five times as much as is found in milk, and protein which from rat feeding experiments appears to be of equal value. The chestnut, the chinquapin, the filbert, the hickory, pecan and pine nut contain on an average as much protein as is found in fish, while the butternut, the peanut and pignolia contain twice as much or 50 per cent. more than is found in the best cuts of meat.

The following table shows the number of pints of milk equaled in protein content by one pound of nuts, together with 
the amount of nuts (ounces) needed to supply sufficient protein to equal one pint of milk, an amount amply sufficient to supplement the proteins of an ordinary mixed ration in the absence of meat :

TABLE XXXVIII.

\begin{tabular}{|c|c|c|}
\hline & $\begin{array}{l}\text { Pints (lbs.) } \\
\text { of milk equal in } \\
\text { protein content to } \\
1 \text { pound of nuts }\end{array}$ & $\begin{array}{l}\text { A mount of nuts } \\
\text { (ozs.) equal } \\
\text { in protein to } \\
1 \text { pint of millk }\end{array}$ \\
\hline Almonds & $\ldots .6 .4$ & 2.6 \\
\hline Beechnut .................... & 6.6 & 2.4 \\
\hline Butternut .......................... & .... 8.5 & 1.9 \\
\hline 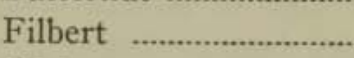 & $\ldots .7$ & 3.4 \\
\hline Hickory nut................ & .... $\quad 4.7$ & 3.4 \\
\hline Pecan & 3.0 & 5.3 \\
\hline Peanut & ..... 7.8 & 2.0 \\
\hline Pignolias & ..... 10.3 & 1.6 \\
\hline Piñon & .... 4.4 & 3.6 \\
\hline Walnut (California) & 5.6 & 2.9 \\
\hline Walnut, black................ & 8.4 & 1.9 \\
\hline
\end{tabular}

Coward and Drummond have shown (Biochemical Journal. 1920), that the fat-soluble A is present only in very small percentage in nuts (Brazil, Barcelona, peanut, walnut, almond, butternut). In general the fat-soluble A vitamin is very sparingly found in seeds and is abundant only in the green parts of the leafing plant, in green shoots and in dairy products.

\section{The Almond.}

The almond perhaps deserves to be placed at the head of the list of all edible nuts. It is one of the finest of all the products of the vegetable kingdom. Until recently the almond was produced almost exclusively in Oriental countries bordering on the Mediterranean. In recent years the culture of the almond has been greatly developed in California and to some extent in Florida. The so-called Jordan almond is the largest and best flavored of all the numerous varieties of the almond. The name Jordan does not refer to the Jordan river but is a corruption of an original word meaning garden, which was applied to this variety of nut for the reason that it was first raised only in gardens. The almond contains a protein very similar to that 
found in milk, which is soluble in warm water. It also contains an emulsifying principle. Because of these characteristics a very delicious milk may be prepared from the almond, the composition of which is not very different from that of ordinary cow's milk. The almond contains no starch but some sugar. About 50 per cent. of the nut consists of a fine quality of oil which is easily digestible.

The almond has the advantage over many other nuts in the fact that the astringent, leathery skin with which it is covered may be easily removed by a simple process of blanching.

An ounce of almonds blanched and slightly roasted, or crushed and served as a nut butter, is a most wholesome addition to any meal and may be used once or twice a day with advantage.

\section{Almond Milk.}

Almond milk has long been used in Oriental countries as a substitute for mother's milk. The writer has made use of almond emulsion for more than thirty years. The almonds are prepared by blanching, then grinding the kernels to a paste. This is then diluted with water or with whey, prepared by the addition of one or two drams of calcium lactate to a quart of milk. Commercial almond creams are now prepared which are ready for use by the addition of water or whey. Professor Mowl of Vienna, Epstein, Raudnitz, and others, have reported excellent results in the treatment of 100 young infants suffering from diarrhea, also several cases of diarrhea and intestinal toxemia in adults.

Fischer found that almond milk is much more easily digested than cow's milk and recommends the use of almond milk in peptic ulcer and hyperacidity, and Von Noorden also recommends the use of almond milk in intestinal infections, putrid diarrhea and in cases of hyperacidity.

According to Mowl, under the use of almond milk in children suffering from diarrhea, the stools became normal, brown yellow in color and a slightly sour odor, resembling the stools of breastfed infants. Thin, liquid stools with toxic symptoms, soon disappeared. Almond milk was in many cases used as a substitute for mother's milk when the supply of mother's milk was insufficient. It was found that almond milk is free from the disadvantages of cow's milk and could be used as a substitute, or complement of mother's milk with entire success. Almond milk was found to be preferable to milk-whey mixtures, malt soup, diluted 
milk and meal mixtures. The cause of its superiority was thought to be the elimination of caseine, which was regarded as the injurious element of cow's milk. In certain cases, it seemed necessary to wholly eliminate caseine from the diet, in order to affect a cure of the intestinal infection.

Children were found to thrive on the almond milk diet, increasing in weight and improving in all particulars. The utilization of nitrogen and fat was studied and found to be excellent.

The calory value of the almond milk employed by Mowl was the equivalent of that of mother's milk.

\section{The Hickory Nut.}

The meat of the shellbark hickory nut is a most delicious morsel. It is richer in fat than any other nut with the excepion of the pecan, a relative of the hickory which contains two-thirds :ts weight of easily digestible oil, with 15 per cent. protein and 11 per cent. carbohydrate. A pound of hickory nut meats is equal in nutritive value to more than 4 pounds of average meat.

The Indians made hickory milk by pounding the nuts, casting the mass into boiling water and passing through a fine strainer. The nut cream thus made was used in preparing hominy and corn cakes. Black walnuts were treated in the same way. Hickory milk sweetened with maple sugar was used as an infant food.

The new method of grafting improved varieties of hickory nuts upon hardy wild or seedling stock promises to greatly increase the production of this choice nut.

\section{The Pecan.}

The pecan, a near relative of the hickory nut, native to our Southern states, contains 4 per cent. more fat and 4 per cent. less protein than the hickory nut. In food value, a pound of pecan meats exceeds the hickory nut by 200 calories, and is perhaps the most highly nutritious of all the natural products of the vegetable kingdom.

\section{The Walnut.}

The native black walnut is perhaps the finest food producing tree indigenous to this country. The fat of walnut meats equals nearly three-fifths its weight, and the protein content is nearly 28 per cent, giving a nutritive value three times that of fat meat. A pound of walnuts, in fact, contains nearly 50 per cent. more 
protein than the same quantity of beef, besides two-thirds as much fat as a pound of butter.

This fine nut is deserving of more consideration than it has thus far received. No investment could be more certain of a good return than the planting of a walnut orchard in suitable soil. It is a hardy tree and its wood is sufficiently valuable to pay the full cost of production.

The walnut is also a fine shade tree. It might well replace many of our shade and ornamental trees in city streets and parks which produce nothing but leaves.

\section{The English Walnut.}

This excellent nut is now grown in great quantities in California, and some hardy strains are being grown in the Eastern states. (It is less highly flavored than the black walnut and has less than two-thirds as much protein.

\section{The Butternit.}

This relative of the black wa ${ }^{1}$ nut produces a very rich nut containing barely 3 per cent. of carbohydrate, less than any othe: nut, which renders it a valuable food for diabetics.

\section{The Pine Nut.}

There are numerous varieties of pine nuts. The most com mon in this country is the piñon, which grows wild and produces great quantities of small but delicious nuts on the rocky slopes of the coast range in California, where it still forms an important element in the diet of certain tribes of Indians. Nut pines of various sorts grow in South America, Spain, and on the west coast of Africa, where there are great forests of nut pines which produce an annual crop large enough to supply fat and protein to whole provinces. Some varieties of the pine nut produce seeds as large as chestnuts and somewhat resembling the kernel of the chestnut in appearance and in chemical composition.

As found in the market, shelled pine nuts are dirty and quite unattractive in appearance; but the nut meats may be easily cleansed by washing first with ordinary water then with water containing half of one per cent. of peroxide of hydrogen. This will not only cleanse but disinfect the nuts, destroying germs of any sort with which the kernels may have become infected in their peregrinations from the distant forest to the dinner table. The 
nut meats may be quickly dried by exposure to the heat of an oven. The flavor of the nuts is improved by very slight roasting.

\section{The Chestnut.}

The cultivated chestnut is produced in enormous quantities in some parts of Europe. A wild variety of chestnut grows in some parts of the United States, but is rapidly disappearing under the influence of a recently developed blight.

In composition the chestnut is much like the acorn, containing little fat, a moderate amount of protein and a large amount of starch. It is usually eaten after boiling or steaming and roasting. A kind of cake which is used as bread and various other dishes are prepared from chestnut meal in Italy, where a large and highly valuable variety of the chestnut is very widely grown and constitutes quite a proportion of the sustenance of the people of the northern provinces.

\section{The Chinquapin.}

This nut is a sort of dwarf chestnut, being about half the size of the latter. It is, however, sweeter in flavor, and the tree on which it grows is more hardy than the chestnut.

\section{Filberts, Cob Nuts and Hazelnuts.}

These closely related nuts are rich in fat and protein of very fine flavor and rank close to the almond in food value. Filberts are grown in most European countries and especially in Spain. The cob nut grows in Ireland and the hazelnut is a common wild bush in many parts of this country. A fine nut butter may be made from blanched filberts.

\section{The Beechnut.}

A delicious nut, the product of the beech tree. It is characterized by its exceeding richness in oil, of which it contains about 60 per cent. Although the beechnut has a very delicious flavor and is a very choice nut it is comparatively little eaten by human beings because its small size renders it difficult to collect and prepare for food. The nut grows abundantly in certain countries, particularly in Silesia, where the oil is used as a substitute for butter. 


\section{The Acorn.}

The acorn is a farinaceous nut, the product of the oak tree. In ancient times it was very extensively used for food in the British Islands and in other parts of Europe. The acorn is still extensively used as a food in certain parts of Turkey. More than 300,000 Indians living in California when first visited by white men had thrived for hundreds of years on a diet of which the acorn was the staple.

The nuts are prepared for use by first burying them in the ground for a certain time during which changes take place which destroy the tannic acid which gives to most varieties of the acorn an unpleasant bitter flavor. Another method of preparation is to - grind the nuts into a coarse meal, then spread on sand and leach for several hours by pouring on water and allowing it to soak away. Another method was boiling with hickory ashes.

There is reason to believe that the acorn was at one time the chief source of carbohydrate for primitive man in some of his forest abodes.

\section{Percentage Composition of the Acorn.}

\begin{tabular}{l|r|r} 
& Leached & Unleached \\
Water & 11.34 & 8.7 \\
Protein & 4.48 & 5.7 \\
\hline Fat & 19.81 & 18.6 \\
\hline Carbohydrate & 62.02 & 65.0 \\
\hline Fiber & 2.06 & 6.6 \\
\hline Ash & 0.29 & 2.0 \\
\hline
\end{tabular}

The acorn contains besides starch, tannic acid and oil a peculiar sugar, quercin.

\section{The Ground Nut.}

A native of this country which was eaten to considerable extent by the North American Indians in aboriginal times. It is nutritious and wholesome but not at present produced in quantities sufficient to give it more than a very minor place in the world's bill of fare. 


\section{The Brazil Nut.}

This is a large South American nut with a rich white meat containing more than 60 per cent. of fat, 17 per cent. of protein, with scarcely more than a trace of sugar making it an excellent food for diabetics.

\section{The Paradise Nut.}

Somewhat resembles the Brazil nut, though finer in quality. Little used in this country.

\section{The Coconut.}

This highly valuable nut is the fruit of the coconut palm tree which grows in many tropical countries, especially India, West Indies, China, Japan, and the South Sea Islands. Within recent years the coconut tree has been grown to some extent in Florida. In tropical countries the coconut is chiefly eaten when in a green state. The nut is cut open, the water which it contains is used as a beverage, and the meat of the nut, which is at this stage of its development soft and creamy, is eaten with a spoon. In the ripe state the nut is rather indigestible unless very thoroughly chewed. It is very rich in wholesome fats and oils, which may be prepared in the form of cream and butter, and form very acceptable substitutes for dairy products, although lacking in the valuable vitamins which are supplied by butter from cow's milk.

Nut margarine and other coconut fats are prepared from the dried coconut meats. They are not a complete substitute for dairy butter, but are more easily digestible and more wholesome than lard and other animal fats and may be used safely if care is taken to include in the daily dietary liberal amounts of cottage cheese, skimmed milk or greens to supply the necessary vitamins which are deficient in most fats, whether of animal or vegetable origin, with the exception of butter.

\section{Coconut Butter.}

A very good butter may be prepared from the coconut by grinding the white meat as fine as possible, soaking for an hour in hot water, then straining through cloth to separate the pulp and collecting the cream which rises as the milky liquid cools while standing for an hour or two. The thick cream which rises to the top is worked like butter, which it resembles in consistency. 
The protein of the coconut has been shown by various experiments to be a complete protein and hence capable of taking the place of meat and milk proteins.

Jansen, of the Public Health Service of the Netherlands Indies, showed by experiments upon men and rats that growth and physical vigor may be maintained by a diet of rice and the coconut press cake remaining after the oil has been removed. In this country, coconut press cake has been used successfully for feeding cattle, and Cajori has shown its protein to be equal to that of meat.

\section{The Cashew Nut.}

A native of the West Indies, also grown extensively in certain parts of India. The seed of this curious nut is enclosed in a shell which contains a poisonous and highly irritating volatile oil. This is driven off by roasting the nuts. Thus prepared the nut has a highly delicious flavor and is easily digestible and wholesome.

\section{The Peanut.}

Though usually classed with nuts, the peanut is really a legume and botanically should be classed with beans and peas In chemical composition, however, it much more closely resembles a nut than a bean. The peanut is a native of Central America. It is now grown, however, in all parts of the world. It is used extensively as a food in various parts of Africa, where it is known as the monkey nut.

Peanut oil has been extensively used as a substitute for butter and in the adulteration of olive oil. It must be remembered, however, that these vegetable oils do not contain the fatsoluble vitamins which abound in butter and which are essential for good nutrition.

Evidence is constantly accumulating to show the great food value of the humble peanut. Dietary studies made by the office of home economics of the United States Department of Agriculture have shown that the peanut is not only easily digested but that the fat and protein which are its chief constituents are of a very superior character. The oil of the peanut is easily digested and the protein has been shown to have about twice the value of the proteins of wheat. It is well known that experimental animals cannot be brought to normal development on a diet of bread alone, but bread consisting of three parts of whieat flour and one of peanut flour will secure normal growth. 
A study by Eddy and Eckman of the value of peanut protein as compared with meat protein, led them to the following conclusion:

"When the protein supplementing power of peanut flour is compared with that of muscle protein by feeding rations so constituted as to contain only about 10 per cent of protein, 6 to 7 per cent of this protein being contributed by wheat flour and the rest by peanut flour or meat residue, respectively, and when these rations are further supplemented with 3 per cent of butter fat, 4 per cent salts and brought to nearly equal caloric value per gram; the peanut flour proves slightly superior to meat as a growth producer and markedly superior for promoting reproduction." (Sherman's "Food Products," pages 400-402).

Prof. J. Russell Smith (Columbia University) considers the peanut "one of the most valuable of foods. It is nearly as nutritious as cheese, contains per pound more protein than a pound of sirloin steak, plus more carbohydrate than a pound of potatoes, plus one-third as much fat as a pound of butter-an amazing total. Excluding shells, a pound affords more nourishment than a pound of sirloin steak added to a pound of white bread."

A bushel of peanuts contains 21 pounds of meats and 9 pounds of shells. As an average of 34 bushels can be produced to the acre, one acre of land will yield 714 pounds of shelled peanuts. One bushel of peanuts yields a gallon of fine edible oil which may take the place of olive oil for table use. A field of peanuts 200 miles square $(25,000,000$ acres) would furnish a year's supply of food fat for the United States.

\section{Peanut Butter.}

Many years ago (1893) it occurred to the author that one of the objections to the extensive dietetic use of nuts might be overcome by mechanical preparation of the nut before serving so as to reduce it to a smooth paste and thus insure the preparation for digestion which the average eater is prone to neglect. The result was a product which was called peanut butter and sprang into public favor with surprising readiness. Several years ago a wholesale grocer of Chicago stated that the firm's sales of peanut butter amounted on an average to a carload a week. It is safe to estimate that no less than one thousand carloads of this product are annually consumed in this country.

\{ The ordinary method of preparing peanut butter renders the 
product more or less indigestible by setting free and decomposing some of the oil which constitutes nearly 50 per cent. of the peanut. Most peanut butters are objectionable on this account.

\section{Flour from the Peanut.}

It has been found that by special treatment of the press-cake obtained after the extraction of the oil from the peanut an excellent flour, containing only 5 per cent. oil, may be made. The flour contains a high percentage of an excellent protein.

\section{Peanut Miilk.}

The protein of the peanut is quite soluble in hot water, and the fat being in a finely divided state it is easy to prepare from peanut butter a very palatable cream or milk by the simple addition of hot water.

\section{Malted Nuts.}

Nearly thirty years ago (1892-3) the writer, in seeking to supply the needs of invalids unable to eat cow's milk, conceived the idea of combining malt digested cereals with nuts to prepare a substitute. The result finally reached after many experimental efforts was the product known as "Malted Nuts," which much resembles malted milk in appearance while having a delicate and very agreeable nutty flavor. This product has been produced in considerable quantities and has come to be somewhat widely used. The following is the analysis made by Atwater, as published by the United States Department of Agriculture: water, 2.6 per cent; protein, 23.7 ; fat, 27.6; carbohydrate (malt sugar and dextrin), 43.9; ash, 2.2. Calories per ounce, 140.

It was soon discovered that "Malted Nuts" had certain characteristic properties which rendered it of special value as a nutrient. One of these is its richness in food iron of which it contains double the amount found in ordinary beefsteak.

Another of the characteristic qualities of "Malted Nuts" is its influence upon the milk secretion in nursing mothers. This discovery was first made by a mother who by its use was able to double her milk production and to nurse the child of a neighbor in addition to her own. Later, Dr. Hoobler of Detroit, superintendent of a lying-in hospital, independently undertook an extended series of experiments, the interesting results of which, largely attained by the use of "Malted Nuts," were reported in 
the Journal of the American Medical Association for August 11, 1917. The purpose of the research was to determine the influence of diet upon the milk production of nursing mothers. It has long been known that a simple increase of food or of fat has no other effect than to make the mother fat without increasing the flow of milk. Dr. Hoobler's experiments had for their purpose the determination of the influence of individual foods and specially arranged dietaries upon the production of milk. Studies were made of the effects of meat, eggs, cow's milk, a strictly vegetable dietary (fruits, grains and vegetables) and nuts.

The influence of the diet was judged by the following points:

1. The amount of milk produced.

2. The food value of the milk.

3. The effect upon the mother in loss or gain of weight.

It was found that a diet consisting largely of nuts ( 50 per cent.), was far superior to any other dietary and in every particular. The amount of milk was larger than the average (14.8 per cent), the food value was greater (30 per cent.), and the mother did better. It was noted that the mothers "took the diet readily and in fact enjoyed it."

"Malted Nuts" has been demonstrated practically the best substitute for milk which has thus far been found. It is relished by bottle-fed infants and "agrees" when cow's milk is not tolerated. It is also of value in cases of adults who cannot take milk without suffering from headache, hives, or other evidences of sensitization. When used as an exclusive food for a bottle-fed infant, an ounce of orange or tomato juice should be given the infant daily. It is also well to add daily a few ounces of lentil or potato soup.

\section{Nut Meats-Protose.}

Nut meat of excellent flavor and superior to meat in wholesomeness, may be prepared as follows:

To a suitable quantity of wet gluten (made by washing the starch out of wheat flour) add peanut meal with a little salt in sufficient quantity to produce a mass having the consistency of a thick mash. Bake in a thick layer in a dripping pan till slightly brown. Serve either with or without brown gravy. This preparation is an excellent entrée and fully supplies the place of a meat dish.

Many years ago (1899) by request of the Assistant Secretary 
of the United States Department of Agriculture, experiments were undertaken by the writer to find a vegetable substitute for meat. The result was the production of "Protose," a nut preparation, which to a considerable degree resembles meat in appearance, taste and odor, having a slight fibre like potted meat. Some hundreds of tons of this and kindred nut foods have been made and used and have proved to be more than complete nutritive substitutes for meat.

"Protose" is a vegetable meat which has much the appearance, flavor, composition, and even the fiber of potted meat. This product has the further advantage of being wholly free from parasites, bacteria and bacterial products. It does not readily become putrid as meat, either in or out of the intestines. Protose contains more food iron than does beef and seven times as much lime. It is also rich in vitamins, in which meat is deficient, and is more easily digestible.

The superiority of vegetable meat (Protose) to ordinary flesh meats is clearly shown by the following table showing the calories per ounce and pound of protose and various meats:

\begin{tabular}{|c|c|c|c|c|c|c|c|c|}
\hline Protein & $\begin{array}{c}\text { Protose } \\
23\end{array}$ & $\begin{array}{l}\text { Veal } \\
17.8\end{array}$ & $\begin{array}{c}\text { Mutton } \\
\text { (leg) } \\
18.7\end{array}$ & $\begin{array}{r}\text { Ordina } \\
\text { steak } \\
22.0\end{array}$ & $\begin{array}{c}\text { Chicken } \\
14.5\end{array}$ & $\begin{array}{c}\begin{array}{c}\text { Fish } \\
\text { (bass) } \\
10.6\end{array}\end{array}$ & $\begin{array}{c}\text { Oyster } \\
6.8\end{array}$ & $\begin{array}{c}\text { Egg } \\
15.2\end{array}$ \\
\hline Fat ...... & 16 & 15.8 & 26.3 & 8.4 & 3.6 & 2.0 & 3.4 & 27.0 \\
\hline Carbohydrates & 10 & & & & & $\ldots . .$. & & \\
\hline Total per $1 \mathrm{~b}$. & 784 & 538 & 686 & 486 & 290 & 202 & 163 & 675 \\
\hline Iron gr. per lb. & .3984. & .1566 & .1645 & .1936 & .1270 & .464 & .299 & .3904 \\
\hline Lime gr. per 1 & 6.6 & 0.5 & 0.6 & 0.7 & 1.0 & 2.0 & 1.4 & 7.2 \\
\hline
\end{tabular}
tolene."

Another nut meat prepared from peanuts is known as "Nut-

\section{The Litchi or Chinese Hazelnut.}

This nut differs from all other nuts in the fact that it consists almost exclusively of a single food principle, sugar or carbohydrate. And the sugar is of the finest sort, like that found in honey rather than cane sugar. This nut also contains a small amount of citric acid, sufficient to give character to its flavor, which is very delicate and pleasing.

The litchi contains only a trace of fat, and a minute amount of protein.

With the exception of the chestnut, the acorn, and certain pine nuts, nearly all nuts are rich in protein and fat, often con- 
taining half their weight or even more of fat. But here is a nut which is pure carbohydrate, and of a sort which requires no work of the digestive organs, being already digested by the ferments of the plant, and ready to be absorbed and utilized at once. It is claimed that the litchi nut will easily produce half a ton of sugar to the acre, and with almost no care, and no cost for manufacture. $8,000,000$ acres planted to litchi nuts would thus furnish the $4,000,000$ tons of sugar which the American people annually consume, better sugar than the cane sugar of commerce, and at far less cost. We are only just beginning to appreciate the value of nuts as nutrients.

The nut may even protect us against a milk shortage. China has gotten along for many centuries without the cow by feeding motherless babies with milk made from the walnut. The almond. the peanut and the soy bean have all been used successfully as substitutes for cow's milk.

\section{Nust Meals.}

Nut meals of various sorts may be made by grinding carefully prepared nut meats in a hand mill set to grind a very coarse meal or samp. Nuts which can be blanched, such as the peanut, almond and the filbert, the latter less easily than the first named, make much the best meal. The shelled peanut is on the whole the best for this purpose. The peanut should be blanched and carefully assorted, then cooked and dried, after which they may be ground to a moderately fine meal which may be used in many ways. Some favorite uses are the making of soups, as an addition to cakes, as a substitute for butter in pie crusts and other shortenings. Almonds, hazelnuts and pine nuts are made into butter without cooking.

A fine flour of excellent quality is now made from the peanut after extraction of the oil.

\section{Nut Creams}

By combining a nut emulsion with soluble carbohydrates, a nut cream rather closely resembling the dairy product may be prepared. Almonds, filberts, cashew nuts and peanuts, are best suited to this use. 


\section{Animal Foods}

All food comes originally from the vegetable kingdom. The chlorophyl grain of the green leaf is the mystic laboratory in which the lifeless inorganic elements of the mineral world are endowed with life. The green parts of plants are factories in which are woven the living fabrics known as organic substances, of which the actinic rays of the sun constitute the warp while nitrogen, carbon, hydrogen, oxygen, calcium and other elements which enter into the composition of living things form the woof. Each little chlorophyl grain is a trap which captures sunshine and stores it up in the form of carbohydrates, protein, fats and other food principles to serve as fuel to feed the vital fires which burn within the bodies of animals. Animals cannot originate food; they only utilize food principles which have been produced by plants. In passing through the bodies of animals, food materials undergo changes of form, but are not produced.

Plants are food producers; animals, food users. Food is to the animal what fuel is to the lamp, the stove or the locomotive.

In the economy of Nature, while it is true that muscle tissue is largely used as food by animals, it is clearly evident that its first and primary purpose is to serve for force transformation through food consumption. The only animal substances specifically prepared by Nature as food for animals are milk and the yolk of eggs, both of which are intended to be used by the infant animal until able to gather food for itself.

In the yolk of the egg is found not only fat, protein and a small amount of carbohydrate but the lime, iron and other salts and vitamins-everything which the young chick needs for the building of blood, flesh and bones. It is a little luncheon provided by Nature to support the young creature during its period of helplessness. Milk is supplied day by day and ho'rr by hour to meet the current demands of the young mammal.

The yolk of egg and milk are exactly adapted to the nourishment of the young of the species of animals producing them. They are not so well adapted for use by adults of the same species nor by the young of other species. 
It has long been believed that certain plants are by nature carnivorous; but it appears from recent observations made by $\mathrm{Du}$ Bois that this belief is a myth. Plants do not secrete ferments capable of digesting animal matter. The supposed digestion which has been observed to take place in connection with the insecteatching parts of the insectivorous plants is simply a decomposition due to the action of microbes. The products of this decomposition may be absorbed by the plants, but the latter have no part in the digestive process.

\section{Special Characteristics of Animal Foods.}

Milk and eggs are anima foods especially adapted not only to meet the needs of young animals in general but to suit exactly the nutritive requirements of infant animals of the particular species to which the aninal producing the milk or the egg belongs; that is, cow's milk is exactly suited to meet the needs of calves. Goat's milk is different and is adapted to kids. The milk of the dog or the seal is still different, and is precisely adapted to the young of these animal. A young animal may be raised on the milk of an animal of another species, but not without considerable difficulty and risk, and not infrequently the experiment is a failure. Of the 300,000 infants who die in the United States annually not less than nine-tenths are bottle-fed infants, a fact which bears eloquent testimpny to the inferiority of cow's milk to human milk for human infants.

The yolk of an egg is a complete foodstuff, and is easily digestible; whereas, the white of egg in a raw state is almost totally indigestible in the alimentary canal of human beings. Eggs are poisonous to some people. Cooking, however, to a large degree lessens this toxic property of eggs.

Of the three sorts of animal foodstuffs, milk, eggs and flesh, milk is the only one that contains any appreciable amount of carbohydrates. In cow's milk about one-third of the total solids consists of lactose, or milk sugar, a wholesome carbohydrate, although one which is utilized with much greater difficulty than any other of the sugars. Cow's milk is very deficient in iron, while flesh foods are equally deficient in lime. The yolk of the egg is well supplied with both lime and iron. Milk and egg yolks furnish a rich supply of vitamins. Meats are very deficient in vitamins for the reason that in animals the vitamins are chiefly stored in the liver and kidneys, just as the lime is almost wholly 
stored in the bones, leaving the lean flesh, the part commonly eaten as food, almost wholly deficient in vitamins as well as lime, two highly important food principles.

Food iron, another essential food principle, is present in meat, but in not nearly so large an amount as is generally supposed. Most of the iron contained in the body of an animal is found in its blood. In the slaughtering of an animal most of this is lost, the amount left being so small that an ounce of beefsteak contains no more iron than the same weight of garden greens such as spinach, and only half as much as is found in the same weight of certain greens, as red root or dock. Graham bread, the date, the fig, and a score of other common vegetable foodstuffs, contain as much or more iron as does meat, and iron of better quality (Sherman).

The only particular in which all animal foods are superior to most vegetable foods is in the amount and quality of the protein which they supply. The protein of milk, eggs and meat is necessarily complete protein; that is, it contains all the elements (amino acids) essential for the building of animal tissues.

Most vegetable proteins are incomplete, although there are many vegetable products, such as almonds, peanuts, soy beans, and probably many more not yet discovered which contain complete proteins.

The protein of grains and of most of the common staples which have been selected for human consumption are incomplete, and it is probable that this is the principal biologic reason why meat has come to be considered an essential part of the dietary in cases in which grains are the chief food staple. The lack of knowledge of this fact has been the principal cause of failure in some attempts to live on a diet consisting exclusively of vegetable foodstuffs. It is possible, however, to select from the vegetable kingdom a complete and satisfactory diet, as has been abundantly proven by McCollum, Hindhede and others. It must be admitted, however, that the present supply of available vegetable foodstuffs containing complete proteins is not large enough to furnish the great population of the world with a sufficiency of this essential element.

We are glad to be able to say at once, however, that it is not on this account necessary to resort to the use of flesh foods, for, as several investigators have abundantly proven, milk is even better adapted than is meat to complement the proteins of 
vegetable foodstuffs. Milk not only supplies its own complete proteins, but furnishes the elements necessary to render complete the proteins of other foodstuffs to which it is added.

Eggs also are an important and competent source of com. plete proteins. And so we are not compelled to resort to the use of flesh foods for our essential supply of complete proteins. although it is important that care should be taken to make certain that the dietary is not deficient in this essential element. This may be accomplished even when the dietary is otherwise exclu. sively vegetable by the inclusion in the daily bill of fare of milk to the amount of 16 to 20 ounces. With a diet of cereals, such as corn, the addition of 10 per cent. of milk protein (Sherman) is found to be sufficient to insure perfect nutrition.

\section{MEATS}

Flesh foods are not the best nourishment for human beings and were not the food of our primitive ancestors. They are secondary or second-hand products, since all food comes originally from the vegetable kingdom, being the product of the magic chemistry of the chlorophyl grain. There is nothing necessary or desirable for human nutrition to be found in meats or flesh foods which is not found in and derived from vegetable products.

For these reasons we think it not necessary to devote more than limited space to the description of meat products, especially as these inferior foods receive in most works on foods and dietetics a very liberal and undue amount of attention.

The World War made clear to all competent authorities on human feeding that it is important to disabuse the minds of the public of the erroneous but deep seated notion that meat is an essential article of food and to lessen the consumption of flesh foods.

The International Scientific Food Commission which met in London, Rome and Paris during the World War was without doubt the most authoritative body which ever considered the sub. ject of human nutrition. At its Paris meeting the question of a minimum meat ration was discussed by the commission, but it was decided to be unnecessary to fix a minimum meat ration "in vieu of the fact that no absolute physiological need exists for meat since the proteins of meat can be replaced by other proteins of animal origin, such as those contained in milk, cheese and eggs as well as by proteins of vegetable origin." 
The United States Department of Agriculture sends out a circular prepared by C. F. Langworthy and Caroline L. Hunt, in which we are informed that "it is of course possible to eat meat dishes less frequently (than once a day), or to omit meat from the diet altogether, for it has been determined that all the necessary protein and energy may be obtained from other materials, if one so desires and the diet is so arranged that it remains well balanced."

Recent writers on nutrition* call attention to the fact that "Of late there has been a distinct reaction in the meat-eating of the wealthier classes, and one sees less meat and more vegetable habits as they progress upward in the scale of civilization, and find they need less of the stimulating qualities of animal protein; and, because, also, on account of their sedentary habits, people find that the ingestion of considerable quantities of animal protein, with the consequent increase in intestinal putrefaction, gives rise to symptoms of toxemia, which have assumed a very definite place in the pathology of disease."

And yet we are also told that meat constitutes one-sixth of the total food consumed in this country. A large expenditure for an unnecessary foodstuff. We will in time learn to do without it.

"We could entirely dispense with meats without suffering any ill effects whatever," says McCollum.

\section{The Iron of Meats.}

Meats, fish, flesh, and fowl contain a considerable amount of iron, but it is of an inferior character. An ounce of lean beef contains one-fourteenth of a day's iron supply, but of medium fat beefsteak one must eat a pound and a half to get a day's iron ration. And if fish is the sole source of supply, ane is obliged to eat seven and three-quarters pounds to meet his daily iron need. Such quantities of meat would burden the body with a great excess of protein, two to five times the amount needed and safe.

Blood is naturally rich in iron because of the hemoglobin, the special iron-containing element of all animal life. An ounce of blood contains exactly the amount of iron which replaces the daily loss of the body. The red color of meat is due to the blood which it contains, and this is the source of its iron content.

These facts would seem to support the popular idea that red

* "Nutrition and Clinical Dietetics," by Carter, Howe and Mason. Lea and Febiger. 
meats possess superior value as blood-making foods; yet science does not sustain this view, but supplies abundant evidence to the contrary.

Many years ago when the writer was a medical student at Bellevue Hospital Medical College a patient was one day brought before a small class of special students of which the late Professor E. G. Janeway was the teacher. The patient was suffering from a very pronounced form of anemia. Under advice of his physician he had been making daily visits to one of the big city slaughter houses to drink a tumblerful of the warm blood caught as it gushed from the cut throat of a slaughtered animal. This he had done for several weeks, as many others were doing at that time, but without any apparent benefit. In each glassful of blood the patient had taken six times as much iron as his body needed to make good the daily waste, but no change for the better had been observed; and Professor Janeway remarked that this was the usual disappointing result.

The drinking of blood never became a popular fad, as might have been the case if it had been less repulsive; but the false and useless notion still survives in the use of beef juice and a whole tribe of loathsome nostrums consisting chiefly of blood.

Although the idea that red meat makes red blood is wholly without scientific foundation it is still a common practice of physicians to urge anemic and debilitated patients to eat freely of red meats, roast beef and beefsteak, and to make free use of beef juices. "Salisbury steaks" are now seldom seen or heard of, but beef powders and other meat preparations have in a measure taken their place.

The absurdity of these popular practices becomes fully apparent when the fact is known that science has demonstrated that the iron of the blood and of meat is no more easily assimilated and made into blood than is the organic iron of eggs, milk and vegetable foods. In summing up the evidence upon this subject Sherman says that the iron of meats is "of distinctly lower nutritive value than the iron compounds of milk, eggs and foods of vegetable origin."

To this Sherman adds: "In an experimental dietary study made in New York City it was found that a free use of vegetables, whole wheat bread, and the cheaper sorts of fruits, with milk but without meat, resulted in a gain of 30 per cent in the iron content of the diet while the protein, fuel value and cost remained prac- 
tically the same as in the ordinary mixed diet obtained under the same market conditions." And it is to be remembered that the iron from vegetable sources was not only more abundant, but superior in quality.

\section{Lime Deficient in Flesh Foods.}

Surprising as the fact may seem, butcher's meat stands near the head of foods poorest in lime salts. Only the tomato, the apple, polished rice, cornmeal, buckwheat flour, hominy and the banana contain less lime than does average lean beef, with its content of one-fourteenth $(0.007)$ of a grain per ounce. The explanation is found in the fact, well known to physiologists that the lime content of an animal body is almost wholly contained in the hard tissues, the bones, less than one per cent. of the total being found in the soft parts. Blood contains even less than lean meats-only one-twentieth of a grain to the ounce.

It seems to the writer quite evident that the flesh of animals was not designed by Nature for the food of other than carnivorous animals, if indeed it was originally designed for them. And other food essentials besides lime are lacking in the flesh of animals. The vitamins are present only in small and inadequate amounts in animal flesh, but are found in abundance in the marrow of bones and the liver. Carnivorous animals eat the bones and the liver as well as the fat and lean tissues of the soft parts. Men, when compelled to live upon a carnivorous diet, likewise find it necessary to eat bones, bone marrow and liver, as pointed out by Stefansson, the eminent Arctic traveler.

Fish contains a larger percentage of lime than does the flesh of warm-blooded animals, nearly twice as much in fact, but the lime content of fish is only about equal to that of white bread, which is very poor in this element.

It is interesting to note in this connection the fact that in vegetable foods the iron and lime are associated with the protein content while in flesh foods the iron is almost exclusively confined to the blood and the lime to the bones. Animal fat, like other fats, is wholly lacking in lime, hence fat meat contains less lime than does lean meat.

It is evident, then, that a high protein diet, especially a meat diet, is contraindicated in conditions characterized by a deficiency of lime. Indeed, there is a reason to believe that such a diet, by in- 
creasing the acid wastes of the body, may cause an undue loss of lime and so aggravate a condition which needs to be relieved.

\section{The Acids of Meats}

Meats are not only deficient in lime, but they contain a great excess of mineral acids which are not oxidized in the body and which reduce the alkali reserve of the blood and tissue fluids and produce a highly acid urine. Sansum, Newburgh and others have shown the harmful results which follow the use of meat because of this acid excess. Newburgh has shown that a diet of bread and meat causes nephritis in rabbits. Sansum has shown that a meat diet increases the acidity of the urine to a notable degree and likewise raises the blood pressure in cases of vascular renal disease. The experience of the Battle Creek Sanitarium has demonstrated that the elimination of meats from the dietary lessens the acidity of the urine and lowers the blood pressure when it is above normal.

\section{$0+$ Beef.}

The lean flesh of the ox and other animals consists almost exclusively of muscular tissue. There is usually more or less fat mingled with the muscular fibers, and in fat animais the muscular tissue is often thickly overlaid with fat.

The popular idea that beef is the most strengthening of foods has no scientific foundation. In order to enter into the composition of the body, meat, like gther foodstuffs, must be reduced to amino acids, the building stpnes which are identical whether of vegetable or animal origin.

Suet, the fat of the ox, has a higher melting point than the fat of other animals except the sheep, as well as of most vegetable fats, and hence is less digestible.

Beef is not infrequently infected with the young of a variety of tapeworm, being the principal source of infection by tapeworm.

As offered for sale in the markets fresh meat is always swarming with putrefactive bacteria, among which is the deadly Welch's bacillus, the cause of gas gangrene. These bacteria are present in countless numbers, the count usually reaching 15,000 ,000 to $100,000,000$ to the ounce. Milk containing one-tenth as many harmless sour-milk germs or water containing onethousandth part as many germs of any sort would be condemned at once. Meat bacteria are a cause of appendicitis and colitis. 


\section{Mutton.}

Mutton is equally as digestible as beef and equally nourishing. It is perhaps somewhat less subject to disease than is the flesh of the ox, but from a hygienic and nutritive standpoint the difference between the flesh of the sheep and the ox is immaterial.

\section{Goat.}

The flesh of the goat is much like that of the sheep. It is more highly flavored and generally less well liked by meat eaters.

\section{Venison.}

The flesh of the deer resembles that of the sheep and the goat though differing in flavor. In general, so long a time elapses after the death of the animal before it reaches the consumer that its flesh has reached an advanced stage of decomposition. It is this which gives to the flesh of wild animals its high or gamey flavor. Such flesh is reeking with infectious germs and is most unfit for human food.

\section{Pork.}

Pork is generally recognized as being less wholesome and less digestible than most other meats. Its use was forbidden to the Hebrews by Moses, and the injunction of the great law-giver is strictly observed by Mohammedans as well as by orthodox Hebrews, even at the present day.

Swine are much more likely to be infected by parasitic diseases which are transmissible to human beings than are sheep and beeves for the reason that they are omnivorous. Their scavenger instinct even leads them to consume putrefying dead animals. The country slaughter-house always has a pig pen behind it into which the offal of slaughtered animals is thrown to be eaten by swine which are later themselves butchered and sold for consumption by human beings. Not infrequently hogs are fattened on dead horses and other animals which have died of themselves or have been killed for the purpose. It is well recognized that the flesh of hogs fattened upon the flesh of other animals has a strong and offensive flavor resembling that of the dog, lion and other carnivorous animals. The scientific stock raiser insists that hogs should be fed exclusively upon clean, wholesome vegetable foods. There is certainly no doubt that the flesh of hogs fed upon flesh is much inferior to that of animals fed upon -a clean vegetable 
diet. From which it is an easy inference, which seems to be over. looked by most writers on dietetics, that if flesh eating will have the effect of rendering the tissues of a hog inferior and reeking with bad odors and flavors, flesh eating by man must produce a similar effect upon his tissue. Can tainted tissue be healthy?

\section{Fowls.}

The flesh of fowls, particularly chicken, is highly esteemed by meat eaters because of its flavor, and many persons suppose the flesh of fowls to be more wholesome than beef, mutton and other meats. As a matter of fact, there is practically little difference in the wholesomeness of the different sorts of meats, fish, flesh or fowl. The principal objection to meats of all sorts is the fact that the undigested residues readily undergo putrefaction in the colon, giving rise to autointoxication. All meats contain when eaten putrefactive bacteria which readily grow in the colon, producing toxins, ptomains and autointoxication as the result of the absorption of these putrefaction products. White meat of fowls, once supposed much more wholesome than red meats, is now known to have no superiority whatever over beef, mutton and other red meats. Von Noorden has shown by careful analysis that the amount of uric acid and other extractives found in white meats is as great as that found in red meats and sometimes greater.

Fish.

Professor Dujardin-Beaumetz, an eminent French physician, many years ago called attention to the fact that fish are less wholesome than other meats for the reason that they so readily undergo putrefaction. Within a very short time after a fish is removed from the water, the putrefactive processes are far advanced. This is probably because most fish are carnivorous so that their bodies are continuously charged with the products of putrefaction. It is probably this fact, also, which gives rise to the peculiar fishy flavor which is characteristic not only of fish but of birds which live largely upon fish. An eminent authority on dietetics calls attention to the fact that "people who subsist largely upon a fish diet have a peculiar rancid odor." Skin diseases are often aggravated by a fish diet, and not infrequently nettle rash and other skin eruptions occur whenever fish is eaten. Certain forms of asthma have been traced to the eating of fish, also certain parasites, to which fish are very subject. 


\section{Sea Foods.}

Lobsters, crabs, oysters, and other sea foods are all scavengers in their habits, subsisting largely upon the animalculae in the ooze and slime which cover the stones and weeds found in the waters where these creatures thrive. Lobsters and crabs are notoriously hard of digestion.

\section{Oysters.}

The oyster has been thought to be one of the most innocent of foods. Raw oysters, stewed oysters, oyster broths, and other preparations of oysters are not infrequently recommended by physicians for the use of persons whose stomachs are supposed to be too delicate to digest ordinary food. But the oyster does not deserve its good reputation. Beaumont's experiments showed that stewed oysters are difficult of digestion, remaining in the stomach for three hours, whereas boiled rice leaves the stomach within an hour; stewed or cooked fruit, such as baked sweet apples, nearly as soon.

The idea that the oyster aids digestion by virtue of its own gastric secretions is a most ridiculous and fanciful theory.

If the oyster lover would take a look with a microscope at the juice of the oysters which he swallows with so much complacency he would hesitate before risking the slimy morsel down his throat. The oyster is a scavenger; he dines on germs. His body is covared and filled with bacteria of various sorts, and the slimy juice in which he is immersed is simply alive with wriggling germs of various descriptions.

A few years ago a paper by Hindman and Goodrich, of the University of Washington, published the results of an extended study of the bacteria of oysters and oyster juice, which showed irom $100,000,000$ to $200,000,000$ bacteria to the teaspoonful of juice. These bacteria were not of the innocent kind such as those which produce sour milk, but were gas-producing organisms of the sort which cause putrefaction. These pernicious organisms were found in great abundance, in one series in 23 out of 24 specimens. In another series the gas-producing organisms were found in all but one of 22 specimens.

The raw oyster, from a sanitary standpoint, is certainly one of the most unclean things that comes upon the table and may rightly be regarded as quite unfit for human consumption under conditions other than those of emergency and dire necessity. 
Typhoid fever epidemics and outbreaks of other intestinal disorders have in many instances been traced to the use of oysters gathered from sewage-polluted waters.

\section{Meat Extractives.}

According to König, lean meat is 75 per cent. water, 16 to 24 per cent. protein, 0.5 to 3 per cent. fat, 0.8 to 1.9 per cent. ash, 0.5 per cent. extractives.

According to Gautier, meat solids contain 9.6 per cent. of hemoglobin and blood albumin, and 13.7 per cent. extractives.

The extract of meat contains a large variety of tissue poisons, among which are xanthin, (uric acid) adenin, tyrosin and leucin, besides 10 or 12 per cent. of creatin and creatinin, together with ignotine, novaine, carnitine, vitiatine, oblitine, carnic acid, and numerous other waste products which have been recently isolated by Kutscher.

Roger, the eminent pupil and successor of the famous Bouchard, says of meat extracts: "The toxicity of extracted matters is real. Extracts of meat contain 22 per cent. to 25 per cent. of salts of potash and 10 per cent. to 15 per cent. extracted matters. Their ingestion, even in moderate doses, produces intestinal disturbances, notably diarrhea. Dogs fed with extracts of meat die more rapidly than dogs which receive nothing at all. A person in health may use extracts of meat in small quantities, but it is necessary to prohibit meat extracts for invalids."

\section{The Poisons of Meat.}

The tissues of all animals contain poisonous materials, the products of the vital activities which are constantly taking place in all living beings. The activities of the tissues result in the formation of special toxins, fatigue poisons and toxic residues which are more or less imperfectly removed from the tissues by the blood during life and carried to the kidneys and other excretory organs to be discharged from the body. The formation of these poisons is in life so rapid that it is only necessary that their elimination should be suspended a short time for fatal results to develop. On the death of an animal, as soon as the heart ceases to beat the removal of these toxins from the tissues ceases; but the development of toxic matters continues for some time after, until the final moment of actual tissue death when rigor mortis occurs. It is consequently evident that the amount of these toxins 
found present in the tissues of a dead animal is much greater than that which is found during life. The character of these poisons is to a considerable degree well known. Many of them have long been familiar through the examination of the urine, which is simply an extract of the tissues. In the words of Professor Halliburton, the eminent English Chemist, "beef tea is simply an ox's urine in a tea cup."

While it is no doubt true that the chief objection to the use of meats of various sorts as a human foodstuff is the enormous quantity of toxins produced by the growth of putrefactive bacteria in the intestine, it should not be forgotten that meats when eaten contain a considerable amount of toxins which are in part the result of putrefaction. This is always true, since meat, as eaten, is always in a state of beginning decomposition. This is inevitably the case for the reason that within a few hours after death the tissues of an animal are found swarming with putrefactive flora, even when the carcass has been kept in a refrigerator. Cold storage delays but does not prevent putretaction.

The amount of these substances which is found in beef and other meats must be very much greater than exists at any time during the life of the animal. According to the late Prof. Gautier, the world's greatest authority, these substances are found in meat broth in the proportion of about one per cent. or about one-half of the total solid constituents. These extractives, according to Gautier, when taken into the stomach produce effects "comparable to those of the caffein and thein of tea and coffee."

Says Gautier with further reference to these extractives: "We must not forget that these bases are poisonous." The observations of Gaucher, cited by Gautier, have clearly proven this. A guinea pig weighing one pound died after receiving several subcutaneous injections of sarcin, a constituent of meat extract The animal grew thin and passed a deep yellow albuminous urine and died at the end of fifty days. Another guinea pig died as the result of subcutaneous injection of one and one-half grains of creatin, always found in beef tea. Post mortem examination showed death to be due to nephritis.

According to the analyses of Gautier, a quart of beef tea contains enough creatin to kill nine guinea pigs, besides potash salts, purin bodies and other substances even more toxic than creatin. When to this we add the fact that the nutrition value of meat broths is practically nothing, according to Gautier only 
one calorie to the ounce, we find ample justification for the assertion of Legendre that bouillon is "a veritable solution of poisons," and the caution of Roger that meat broths and extracts if used at all should be taken only by healthy persons and should be prohibited for invalids.

The meat extracts in general use are, if possible, still more objectionable than freshly prepared broths. Fully 20 per cent. of these extracts consist of poisonous substances. So long ago as 1871, P. Muller observed that the addition of an ounce of meat extract to his daily diet produced diarrhea, an evident defensive effort. Two-thirds of an ounce of Liebig's extract of beef added to the regular diet of a dog weighing fourteen pounds produced diarrhea on the sixth day and death from collapse on the ninth.

Meat extracts of all sorts are concentrated preparations of the toxins of meat. They have no nutritive value and their use cannot be too strongly condemned. Meat extracts are, according to Gautier, fifty times as toxic as broth made from two pounds of beef and five pounds of water.

\section{Viscera Highly Objectionable.}

The viscera of animals must be looked upon as the most objectionable form of flesh food. All the viscera contain xanthin or uric acid in large amount. Gautier says of the spleen: "This flesh is only a very bad aliment"; and of the kidneys, "these organs are bad when they come from old or carnivorous animals." The fact that the kidney of the carnivorous animals is always so bad as to be condemned as food naturally leads one to the conclusion that a flesh diet is not conducive to the health of the kidneys. Sweetbreads, according to Hall, contain 70 grains to the pound of potential uric acid. Blood has from the earliest ages been considered unfit for use as food by the most enlightened nations. The orthodox Hebrew still obeys the injunction given, according to the Bible, to Noah: "The blood thereof which is the life thereof thou shalt not eat of it."

\section{High Meats.}

When an animal is alive, its muscular tissues are almost as tender and fragile as jelly; but after death stiffening or rigor mortis occurs as a result of the coagulation or clotting of the muscular tissues, the tissue becomes tough and it never becomes tender again until putrefaction occurs. It is for this reason that 
meat is allowed to hang for some time after killing to ripen, in other words, to decay.

Experiments reported by Farger and Walpole (Journal of Physiology and Pathology) show that putrid meat contains many poisonous substances, some of which cause rise of blood pressure. It is for this reason that the up-to-date physician forbids the use of meat by persons suffering from apoplexy or arteriosclerosis or from high blood pressure. This restriction is necessary not only because of the poisons which meat always contains from putrefaction which has already taken place, but because of the further putrefaction of undigested remnants of the flesh which takes place in the lower part of the small intestine and the colon.

Gaspard demonstrated in 1822 the toxic nature of putrefying substances. He showed that putrid substances contained poisons which were much more deadly than the excretory products of the body.

Bailey (Fitch, "Dietotherapy") very wisely calls attention to the danger of eating high meats as follows:

The habit of hanging game until it gets high is quite objectionable and decidedly unhygienic. When it is hung up for this purpose and allowed to become malodorous, putrefaction is already well under way. This process of decay not only leads to decomposition and the development of toxins, but is a genuine hotbed for micro-organisms which may cause gastric troubles and enteric disorders. Such practices, which are bound to endanger the health even of the most robust, should be condemned.

High game, on account of the toxic substances it contains, is even more dangerous to the circulatory system; the toxins contract the small blood vessels and thereby put extra work upon the heart. For this reason, in critical cases of cardiac affection and high blood pressure and in Bright's disease, it should not be eaten.

When meat is stale or game high it is positive evidence that putrefactive changes have taken place by bacterial action, whereby certain alkaloidal substances of the nature of ptomains and leucomains of an exceedingly poisonous nature are formed. When such food is ingested and absorbed, serious and even fatal results often follow.

The eating of putrid flesh is always dangerous; in certain cases immediately so; in others, the injury appears later. Decaying flesh is always infested with germs which produce gas gangrene, suppuration, peritonitis, pneumonia, abscess and other deadly disorders. When introduced into the intestine, these germs fix themselves upon the intestinal wall and become permanent inhabitants which are even more difficult to get rid of than Canada 
thistles in a pasture lot. They take deep root and spread. They invade the appendix and cause appendicitis. They cover the walls of the colon and cause colitis. They work through the wall and produce adhesions, even inflammations, of kidney and bladder. They work slowly upward in the small intestine and finally reach the gall bladder, causing gallstones. They may be the cause of gastric or duodenal ulcer. They become a serious menace to the entire body through the poisons which they form, some of which are as deadly as the virus of snakes. The writer has met many cases in which a single meal of cold storage chicken or duck or other game, long dead and neglected to be buried, has been the cause of many years of misery and invalidism.

Pezard, a French investigator, has shown that an exclusive flesh diet in cocks causes degeneration and atrophy of the genital glands with the usual effects of castration. Chalmers Watson made similar observations.

\section{Putrefaction Products in Meat.}

The popular practice of keeping meat until it becomes prime, or ripe, is in the highest degree reprehensible. It is important that the fact should become well known and understood that this so-called process of ripening is in no way different from the process of putrefaction. It is, in fact, putrefaction pure and simple. Whether the carcass of a dead cow hangs in a meat market or lies neglected in the corner of a pasture lot, the changes which take place in the tissues of the animal are precisely the same. Decomposition begins within a few hours after death, and progresses more or less rapidly according to the temperature to which the body of the animal is exposed. At a low temperature $\left(40^{\circ} \mathrm{F}\right.$.) decomposition proceeds slowly, but nevertheless it proceeds. The bacteria which develop at a low temperature are somewhat different from those which grow at a high temperature, but toxic products are the result in both cases. Says Roger:

Flesh and meats kept for a length of time undergo the influence of bacterial growths, changes which render them toxic. . . The digestive tube of man is much more sensitive to these poisons than that of animals. . . . Meats which are slightly putrid produce in human beings gastro-intestinal disorders, while perfectly well tolerated by carnivorous animals, and even by animals which are not naturally carnivorous.

The worst effects, however, are the more remote consequences which result from the continued introduction in the intestine of these poisons and the germs which produce them. To infections 
of this cause must be attributed, in the writer's opinion, a large portion of the great number of cases of intestinal autointoxication which are now recognized in every civilized country. Professor Brouardel, an eminent French physician, reports a case in which a number of persons suffered seriously after eating of stuffed goose. One of the persons, a woman suffering from chronic disease of the kidneys, died, evidently because the kidneys were not able to cope with the great quantity of enterotoxins which entered the blood from the intestine. There can be no doubt that the lives of many persons suffering from chronic renal and liver diseases are cut short in a similar way. The writer recalled a number of cases in which persons suffering from chronic renal disease have by a single indiscretion in eating a hearty meal of meat brought on a fatal relapse.

Sausage often contains great numbers of ptomains and microbes; hence if not well cooked it is very likely to cause serious poisoning. Sausage contains all forms of noxious substances. (Roger.)

If sausage does not always contain great numbers of bacteria and great quantities of bacterial poisons, it is only because antiseptics of some sort have been used. Muller reports 265 cases of poisoning from sausage, in which the symptoms generally began after an incubation period of about eighteen hours. The first symptoms were weakness, exhaustion, nausea, vomiting, diarrhea, dryness and burning of the throat. Two or three days later worse symptoms appeared; as vertigo, staggering gait, difficult respiration, double vision, difficulty in swallowing, hoarseness, clammy skin, paralysis of the legs, collapse and death. In some epidemics one-third of the cases are fatal.

According to Roger, "decomposing meats (comprising practically all butcher's meat), are full of toxins and bacteria which find in the intestines excellent conditions for their development."

The poison produced by the bacillus botulinus is extremely deadly. Less than one ten-thousandth part of a drop of a culture of this germ was found sufficient to kill a mouse.

"For efficient destruction of the bacteria found in them, meats must be heated to a temperature of $248^{\circ} \mathrm{F}$. for two hours." (Roger). This is seldom done, in fact, it is quite impracticable in the ordinary cooking of meat.

"Fish are still more likely than the flesh of other animals to give rise to poisoning." (Roger). This fact has long been 
known. It was on this account that Alexander the Great forbade his soldiers to eat fish. The tissues of some fish contain a poison similar to curare, the arrow poison of certain savage tribes. Certain fish have poison glands, so that their bite is as poisonous as that of a venomous snake. Roger gives a long list of poisonous fish. The late Professor Brieger, as well as others who have made a careful study of this subject, isolated numerous ptomains which are found in the flesh of fish. Even in a fresh state it is known that sturgeon meat contains various toxic alkaloids. In Russia, where many persons live largely on sturgeon during Lent, poisoning from this source is often observed. Anrep reports the death of five persons from the use of salted sturgeon. Fish become poisonous at certain times or in certain places because of the noxious character of the food on which they live. This is the case with fish living in the vicinity of coral reefs. Cases of poisoning have been noted in France from the use of sardines, salmon and codfish. The so-called red cod owes its color to a chromogenic microbe which is associated with poison-producing bacteria.

Virchow reported an epidemic in which nineteen persons were made sick, four fatally, by eating mussels. Mussels habitually gather poisons from the waters in which they live.

According to Roger, shellfish are a dangerous diet. He says: "Even healthy mussels are not a good food. Their prolonged use seems to be capable of producing chronic poisoning and cirrhosis of various organs. These effects have been observed in the natives of Terra del Fuego."

Typhoid fever germs have many times been found in oysters, and numerous epidemics of typhoid have been traced to their use. Simon reports a case of poisoning through eating of lobsters. Rapin reports an epidemic of severe illness from eating crabs. One of the sufferers died at the end of twenty-three days from symptoms resembling typhoid fever.

All of these creatures-oysters, clams, mussels, lobsters, crabs -are scavengers and quite unfit for food.

All so-called food animets are liable to infection. According to Littlejohn, in a paper read before the National Tuberculosis Conference of London, based upon the conditions observed in England, 20 to 30 per cent. of cattle are tuberculous. Old milch cows ( 10 to 15 years) are infected to the extent of 75 per cent. Pigs are infected to the extent of 4 to 5 per cent. Birds, includ- 
ing turkeys, ducks and pheasants, are infected with avian tuberculosis. Sheep, goats and rabbits are rarely infected naturally, but rabbits are very susceptible to experimental infection. Horses are tuberculous only to the extent of one per cent.

In the middle states of this country cows are believed to be generally infected to the extent of about 10 per cent. The carcasses of these tuberculous cattle are usually placed on sale in the meat markets along with other meats.

\section{The Bacteria of Meat.}

According to Weinzirl (Amer. Jour. Pub. Health, 1914): The standard of $1,000,000$ bacteria per gram, advocated by some as a limit, is apparently too low, since it would condemn nearly all samples of hamburger, when showing no taint or other evidence of putrefaction. Samples of other market meats, all of which would otherwise pass inspection, often exceed this limit.

A bacterial standard of $10,000,000$ per gram is the limit proposed, on the basis of which 50 per cent. of the market samples of hamburger would still be condemned. This is justified on the following grounds: (a) much of it is actually spoiled when it reaches the consumer or is to be cooked; (b) meat teeming with $10,000,000$ per gram is potentially rotten and soon will be actually spoiled under ordinary methods of handling; (c) the fact that markets are prone to add sodium sulphite to hamburger, even though the dealer knows it to be contrary to law, indicates that something is wrong with the hamburger; (d) and finally, if hamburger were made from wholesome meat in the first place, then properly iced, it is believed that the bacterial content could readily be held within the $10,000,000$ limit.

Ten million bacteria per gram may be said to be equivalent to $300,000,000$ bacteria per ounce. The significance of this will be appreciated when it is remembered that certified milk is not permitted to contain more than 10,000 bacteria per gram, or 300,000 per ounce ; that is, marketable hamburger steak may contain a thousand times as many bacteria as certified milk.

Milk which contains as many as 200,000 bacteria per cubic centimeter, or 6,000,000 per ounce, is regarded as unmarketable, and is promptly condemned wherever a milk inspection service is maintained, while at the same time hamburger steak containing fifty times as many bacteria is sold and eaten without protest.

It is to be further noted that the bacteria of milk consist almost wholly of harmless lactic acid-forming organisms, or sour milk germs, while the bacteria of meat consist almost exclusively of putrefactive organisms such as the Welch bacillus, the cause of gas gangrene, the bacillus putrificus, the bacillus proteus and 
other bacteria which are usually present in carrion or putrefying animal bodies.

An unpleasant fact which meat-eaters should become acquainted with is that the bacteria of meat are ordinary colon or manure germs. The meat becomes contaminated in the process of slaughtering. The hair and skin of an animal are, of course, infected with their own excreta. In slaughtering, the butcher's hands and instruments become soiled and in the skinning of the animals, and contact with the hands and the killing-floor or platform, contaminates the surface of the warm flesh from which the skins are removed and the bacteria spurts through the carcass with great rapidity. Every one is familiar with the quickness with which warm milk will sour if put into a sour pan, or if a few drops of sour milk are added to it: Manure germs spread through the warm flesh of a slaughtered animal with almost equal rapidity, so that at the end of 24 or 48 hours after killing, the whole carcass is swarming with bacteria. This statement is based upon the results of careful bacteriological examination and can be verified by any bacteriologist.

To prevent the contamination of flesh meats with manure germs, it would be nccessary that the slaughtering process should be conducted with the same aseptic precautions with which surgical operations are performed. So long as butchering is done in the present fashion, it will continue to be no exaggeration to say that ordinary steaks and chops are the filthiest things which ever appear upon our tables.

According to Bailey "the bacillus putrificus coli is present in the colon of animals and contaminates all meats. The spores of this bacillus are killed by exposure to a temperature of $224^{\circ} \mathrm{F}$. for 15 minutes; $230^{\circ} \mathrm{F}$. for 10 minutes; $240^{\circ} \mathrm{F}$. for 5 minutes. In the process of canning meats, if these temperatures are not maintained, the meat in the interior of the can is not sterilized. This gives the bacilli an opportunity to develop and decompose proteins and other organic compounds. The sulphur generated by decomposed proteins forms a sulphid of iron which blackens the tin, while the generated gas may cause the end of the can to be "blown" or "swollen."

A research carried on by the Meat Committee of the Food Investigation Board of Great Britain has shown that the fungus which produces black spot on cold storage meats will grow at a 
temperature 9 degrees below freezing. Numerous other molds have been found which grow well at the freezing point.

It remains further to be said that all fresh meat is eaten in a state of decay. The process may not have proceeded so far that the dull human nose can discover it, but a carrion bird or a carrion fly can smell it from afar and various scavenger creatures will quickly show their appreciation of the unsavory tidbit if the arch scavenger man does not get the start of them by an hour or a day. Indeed, the putrefactive bacteria always found in meat in countless numbers, smoked and salted meats as well as fresh meats, are scavenger parasites which play in nature the important role of reducing back to the soil the dead bodies of animals. Another and quite different class of bacteria performs this office for plants.

Dr. C. E. Roderick, bacteriologist of the laboratory of the Battle Creek Sanitarium, recently made, at the request of the writer, an extended study of the bacteria of meat and of the fresh droppings of animals. Specimens of meat were purchased from seven different markets. The bacteria found were identical with those found in fresh manure and in several instances the bacteria in meat exceeded in number those of fresh manure. It should be said that the meats examined appeared to the eye and the nose to be in as good condition as usual, and would have been sold to some one else and eaten if they had not been collected for examination. Care was taken to make the examination at once after collecting the specimens so as to allow no time for further putrefactive changes. The figures given show the maximum number of manure germs per gram found in meats and in fresh manure droppings, also in oyster juice and limburger cheese. The higher figures were the most frequently observed and of course are of chief interest. The various species of putrefactive and other bacteria found in the human colon and the colons of animals were all present in meat together with some other species due to air contamination.

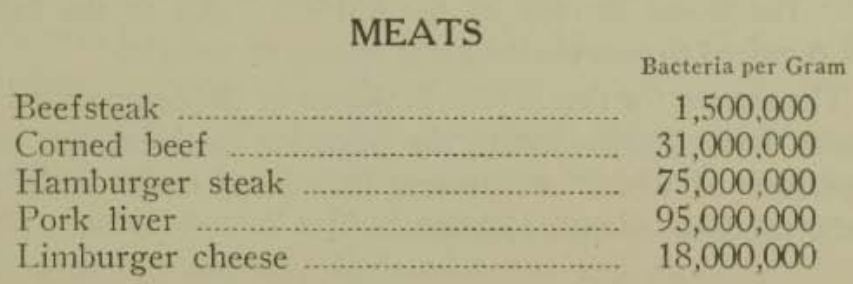




\section{DROPPINGS OF ANIMALS}

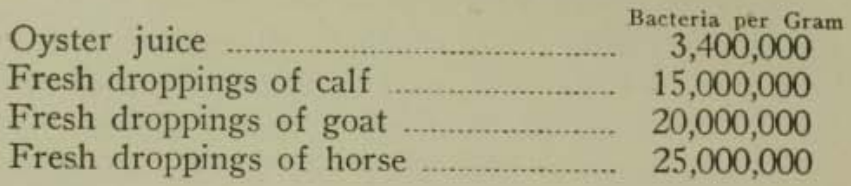

A comparison of the above figures shows an average bacterial content of the meats examined nearly three times as great as that of the animal droppings, and the same kind of bacteria, viz., B. coli, Welch's bacillus and various putrefactive bacteria. The species of bacteria, especially Welch's bacillus, are not destroyed by ordinary cooking temperatures.

\section{Acidosis from a Flesh Diet.}

Gautier calls attention to the fact that oxalic acid and other poisonous acids are among the products of the oxidation of protein in the body. "On a flesh diet these toxic bodies accumulate and acidify the blood, excite the heart and intoxicate the subject, disturbing the functions of the skin, lungs, liver or kidneys." "Oxybutyric and other oxyacids," says Gautier, "appear to be derivable from a direct or indirect splitting up of the albuminoids. . . Oxybutyric acid and oxalic acid are toxic, acidifying the fluids of the body and preventing the action of the oxidizing ferments which require an alkaline medium."

\section{Effect of Flesh Eating on Animals.}

Hunter, in experiments to determine the effects of meat eating upon animals (rats), found that a flesh diet, as compared with a bread and milk diet, caused a great concentration of the urine with strong odor; the stools were dark colored. Examination of the animals after death showed in the meat-fed animals hypertrophy of the kidneys, which was very pronounced in the second generation. The effects produced were like those caused by toxins and were considered to be the result "of a special strain on the functional activity of the kidneys," due to the increased work required to excrete the great excess of protein.

The reason for this is clearly shown by the composition of the extracts derived from meats, the so-called meat extract, some of the poisons of which are present in normal tissue while others are the products of putrefaction. 


\section{The Pernicious Effects of a Meat Diet Experimentally Proven.}

For several years, Dr. L. H. Newburgh, a professor in the Medical Department of the University of Michigan, assisted by Sarah Clarkson, M..S., has been engaged in a research to determine the effects of a high protein diet or, to be more specific, the effect of the feeding of meat to rabbits.

The first report of the results of these experiments was published by Dr. Newburgh more than two years ago at the meeting of the American Medical Association held at St. Louis last May (1922). Dr. Newburgh presented a report of the further results which he has obtained in the most interesting study which shows beyond any chance for controversy that the free use of animal proteins-meat-is a very active cause of degeneration of the arteries, or arteriosclerosis. The meat was given to rabbits in the form of bread made of wheat flour and meat powder. The rabbits were divided into two groups, one of which received bread containing 36 per cent of protein (chiefly meat) and the other 27 per cent. The bread for the latter group was made of equal parts of flour and wheat bran, with which the lean beef powder was mixed. The rabbits were healthy animals, having been raised out-of-doors. In order to make sure that the diet alone was the cause of the results obtained, a group of rabbits was kept in the laboratories ynder the same conditions as those receiving the experimental diets, but were given no meat.

Of ten animals fed on bread containing chiefly meat, two, or 20 per cent., showed disease of the blood-vessels within four to eight weeks. Of six animals that lived from ten to sixteen weeks on the same diet, four, or 66 per cent,, showed disease of the blood-vessels. Of eight other animals that lived from eight to thirty-six weeks on the same diet, every one "showed marked and extensive atherosclerosis; that is, all these animals showed the blood-vessels extensively hardened with chalky deposits.

Summarizing the results, it appears that of twenty-four animals fed on bread containing about one-half its weight of lean meat, fourteen, or $58 . .3$ per cent, developed in less than eight and a half months, disease of the blgod-vessels, or arteriosclerosis.

Of the animals fed on bread containing about one-third its weight of lean meat, out of eleven animals that lived more than six months, eight showed typical arteriosclerosis, and in seven the changes in the arteries "were advanced and extensive." 
Not one of the animals kept in the laboratory under the same conditions as the experimental animals, showed any evilence of disease of the arteries, although two-thirc's of them lived more than six months.

It thus appears that the larger the amount of protein reseived by the animals and the longer the duration of the experiment, provided the animals lived, the greater was the destructive effects upon the arteries.

It is notable that the amount of meat eaten by the rabbits vas no greater than that which/is eaten by many persons habitually, at least by those who try to follow the teachings of such misleading writers as Woods Hutchinson, the number of which, let us hope, is not very great. Indeed, the proportion of protein given to the rabbits was not much greater than that required in the Atwater ration.

It is interesting to note in this connection, the fact that Atwater himself died of arteriosclerosis after having lived for two or three years a completely helpless, imbecile paralytic. It is also to be remembered that Hall, Peary and Shackleton, three .oted Arctic explorers who adopted the meat diet of the Eskimos, died early and apparenfly with diseased blood-vessels.

The fact that meat produces changes in the blood-vessels of ubbits, is especially significant, as pointed out by Dr. Newburgh, hecause "spontaneous atherosclerosis (arteriosclerosis) is a very rare clisease in rabbits."

In the discussion which followed Dr. Newburgh's paper, Dr. Lyon remarked as follows:-

"I think anatomic and zoölogic investigations in human beings would establish the fact that men have had a rather high orotein diet for many generations and have become accustomed to it; whereas, the rabbit is one of the most strictly herbivorous mammals known. It is even more strictly herbivorous than many of the true rodents. The real origin of the rabbits is probably irom some preungulate ancestor. It seems to me that it might be advisable to try the experiments on an animal which has a diet much more like that of human beings than a rabbit.

In closing the discussion of his paper, Dr. Newburgh fully answered the doctor's criticism by reference to the observations of Dr. Fox, remarking, "Dr. Fox, of Philadelphia, has examined all of the animals that have died in the Philadelphia Zoological 


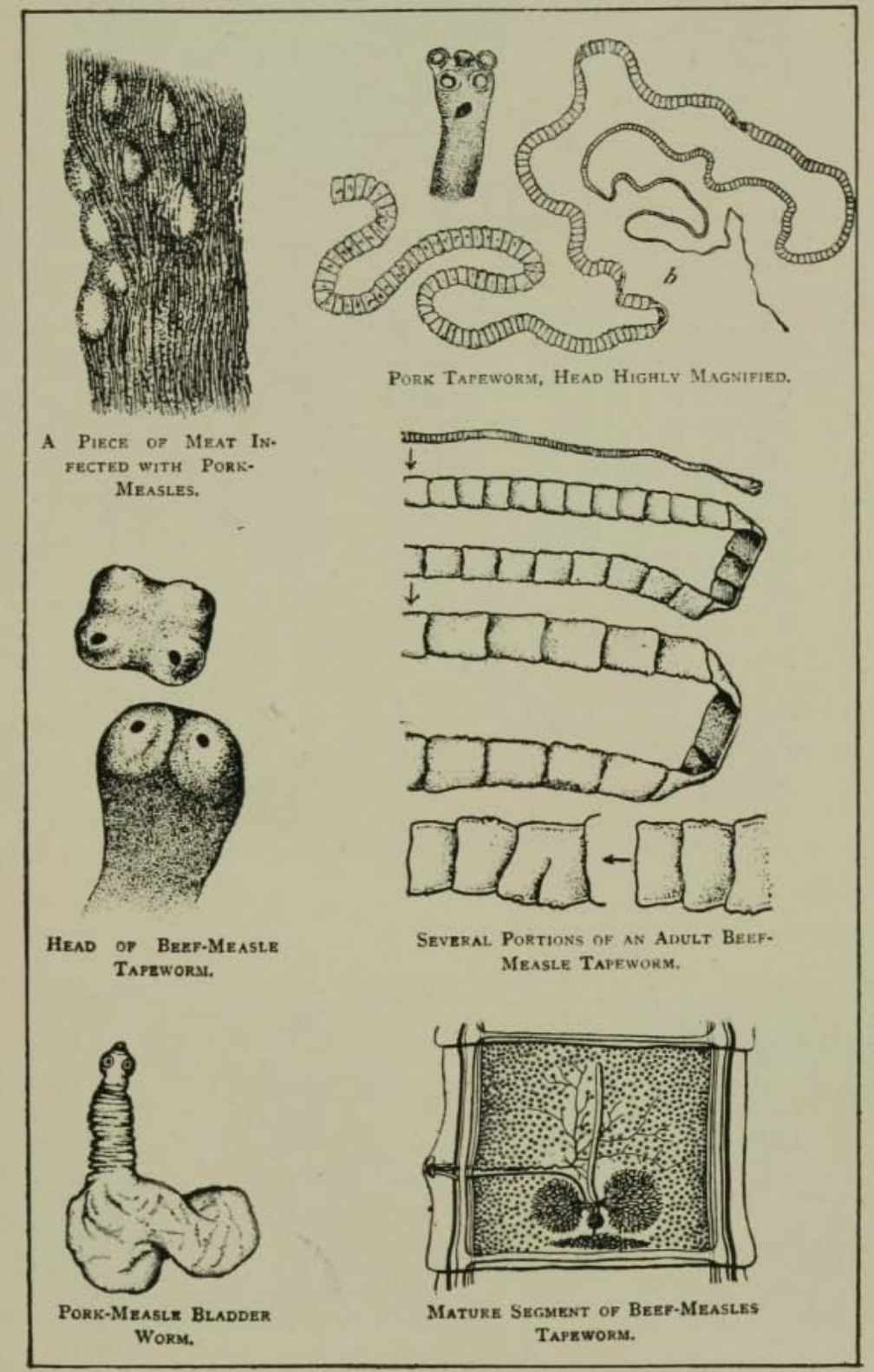



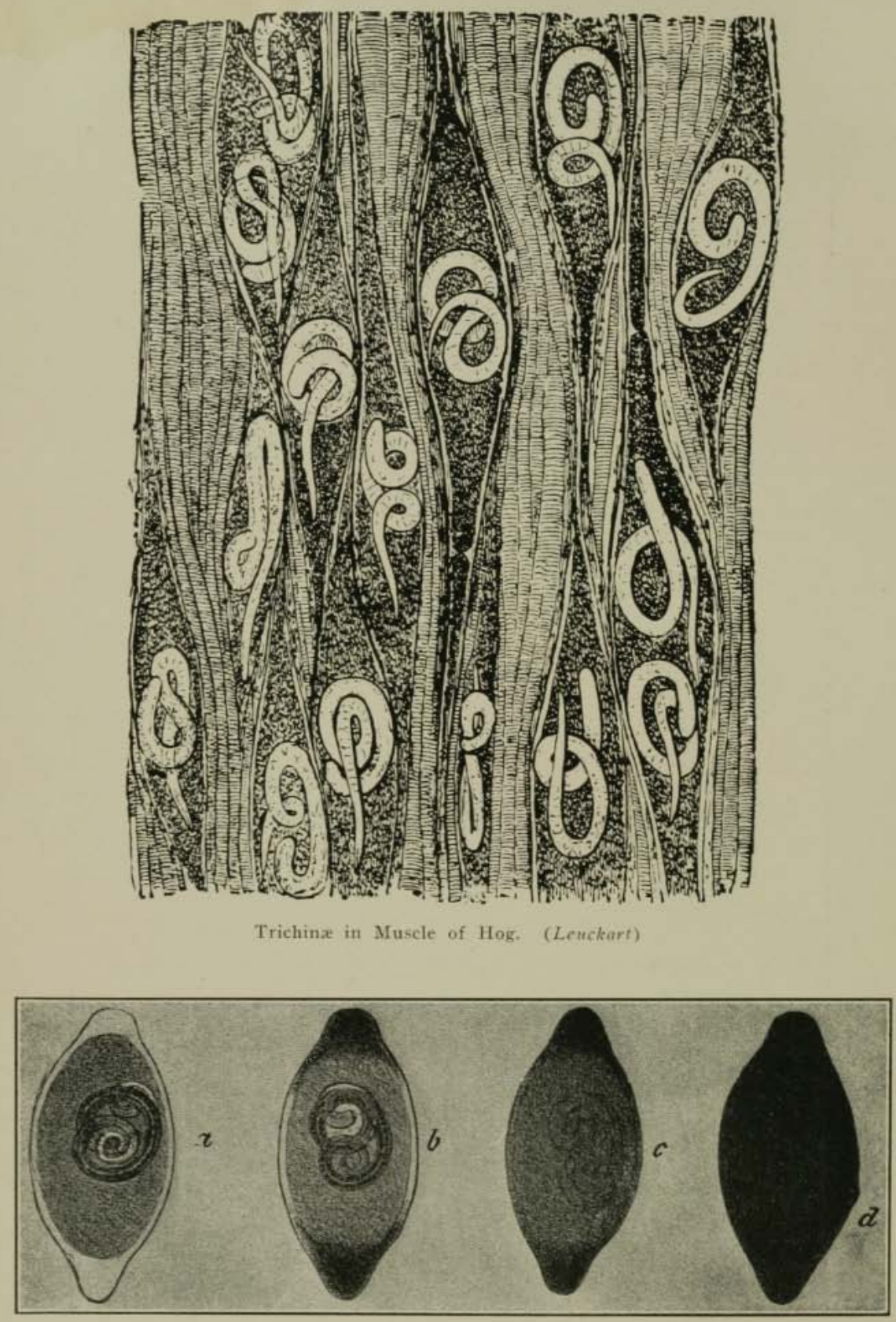

Trichina in Capsules Undergoing Calcification. (a) Not calcified. (b) Calcification beginning. (c) Calcification further advanced. (d) Calcification completed. 
Garden. His conclusions are of interest in this connection. He pointed out that the carnivora had chronic vascular and renai lesions, and that they were practically the only ones that had such lesions. I wrote to Dr. Fox, asking him whether I was justified in assuming that definite relationship existed between the carnivorous diets and these chronic lesions of the arteries and kidneys. In his reply, he stated that this certainly was true, that the meat-eating animals showed a high incidence of chronic disease of the arteries and the killneys as compared with a'l the other animals on which he had performed necropsies."

Dr. Lyon was right about the hog. He will eat anything given him, including dead horses, even dead hogs, any sort of carrion he finds lying about, for he is a natural born scivenger.

Dr. Lyon is also, we are sorry to say, correct in his claim that the diet of the hog is "much/more like that of human beings than that of the rabbit." But this is just the point of interest. Should a man eat like a hog of like a rabbit? Or, if not like a rabbit, is there some other animal, such as the chimpanzee, for example, whose natural diet furnishes a safe model for man to follow? The comparative anatomists tell us that man belongs to the family of primates, with the big apes, which clearly indicates him to be a low-protein feeder, and entitles him to the same immunity from afteriosclerosis which Dr. Fox has shown to be enjoyed by the non-flesh-eating animals; but, of course, if a man adopts the diet of a carnivorouts animal, he must expect to suffer the natural consequences of high protein feeding, one of which is early senility through degereation of the blood-vessels and the kidneys. As regards immunity through long use of the high protein/diet, it is clearly evident that if carnivorous animals have not developed such an immunity, it would be futile to expect a natural low protein feeder like iman to develop immunity, no matter how long or to what extent he may have practised meat-eating.

\section{Parasites of Meat.}

Ambrose Paré, the famous French surgeon, long ago gave the advice that meat should be boiled to kill parasites, but his warning has usually been neglected.

According to Professor Stiles, of the United States Public Health Service;- 
Every animal used for food has in its intestines either protozoa, round worms, flukes, or tapeworms. Some of these parasites, especially certain protozoa, are mere scavengers in the intestinal tract, but many of the infections undoubtedly do more or less harm to their hosts. Accordingly, academically these animals are diseased. . .

If all animals harboring animal parasites are to be construed as diseased in the sense of the food laws, as they are undoubtedly diseased from the abstract academic point of view, it is clear from the zoologic point of view that no meat can be sold legally.

The professor's view is that although an animal may be diseased it should not be condemned as unfit for food unless it is diseased in such a way as to cause disease in the eater. In illustration he adds: "When a meat dealer sells pork he is selling a product which in its raw state is dangerous to health for the reason that it frequently contains certain disease germs, trichinae, the absence of which cannot be guaranteed by any practical and proper method of meat inspection known. If the pork is properly cooked or otherwise prepared, these germs are killed, are rendered harmless and do not per se render the pork diseased (as food) for if dead they cannot produce disease."

The record of the Bureau of Animal Industry shows that in the year 1908 the meat and meat-food products condemned amounted to nearly three-fourths of one per cent. of the total amount examined.

\section{Canned Meats Usually Not Sterile.}

The generally accepted belief that canned meats are necessarily sterile because they do not spoil, has been completely exploded by the recent report of the British "food investigation board" on the bacteriology of canned foods. Of 323 cans examined, 246 were found to contain living bacteria. The most surprising thing was the fact that less than 30 per cent. of the cans which appeared to be perfectly sound were found, on examination, to be really sterile. Of such cans, apparently good, and which would have been sold and eaten if they had not been subjected to the examination, 36 per cent. of the sardines, 63.6 per cent. of the meats, and 100 per cent. of the crabs were found not sterile, and were rejected as unfit for food (Jour. A. M. A., for March 24, 1923).. Since all fresh,smoked, dried and salted meats are infected, and 30 per cent. of all canned meats, it is evident that the only safe course is to discard meats from the dietary. 


\section{Flesh Meat Not Essential.

Half the people of the world eat very little meat. According to Report No. IOO of the United States Department of Agriculture, the total production of the principal meats, beef, mutton and pork, of the whole world, excluding China, is about 50,000,000,000 pounds annually, which makes the per capita consumption of meat for the people of the wotld, excluding China, 11 pounds per annum, or less than 9 pounds if China is included.

Evidently, meat plays really a small part in the world-wide scheme of human nutrition.

Professor Alonzo E. Taylor contributed some years ago (1911) to the Popular Science Monthly an article on "Vegetarianism" in which he says, with reference to a non-flesh dietary:

Is a vegetarian diet physiologically correct, adapted to the best purposes of a normal life, endurance and longevity? We receive a reply couched in no uncertain terns. Yes, a properly selected and prepared vegetarian diet meets completely the highest requirements of the diet.

In confirmation of the above, Professor Taylor adds: "Purely physiological and chemical data, abundantly sustained by laboratory researches and animal experimentation, confirm as well as elucidate the now widely-made human experience that a properly selected and prepared vegetarian diet is a complete diet for all conditions and periods of life/ beyond the lactation term of iniancy."

After admitting the entire adequacy of a non-flesh dietary, Professor Taylor suggests that such a dietary is not practical at the present time for the reason that "the area of the earth's surface now under cultivation could not, with the present methods of agriculture, dependably produce enough plant albumin to meet the present needs of the population."

The last statement must have been made without consideration, since it requires only a very simple calculation to show that the more than $4,000,000,000$ bushels of cereals produced in this country every year contain more than five times enough albumin to supply 2.2 ounces daily for every man, woman and child composing the $115,000,000$ of our present population. With this fact established, it cannot be denied that Professor Taylor has clearly shown not only that a non-flesh dietary is adequate to meet all human needs, but also that flesh foods might be at once, and perma- 
nently, eliminated from our nafional bill of fare without risk of injury to the national health.

We quote the following from "Food in War Time," by Dr. Graham Lusk, the eminent physjologist:

If one takes milk with other foods, meat may be dispensed with. Thus Hindhede advocates as ideal a diet consisting of bread, potatoes, fruit and a pint of milk. Splendid lealth, both of body and mind, the peasants' comparative immunity to indigestion, kidney and liver disease, as well as an absolute immufity to gout, is the alluring prospect held out by the following dietary

Graham bread
Potatoes
Vegetable fat
Apples
Milk

This bread-milk-potato-fruit diet gives a very excellent basis for wholesome nutrition.

The question is, is meat necessary? The description of the Italian dietary answers this in the negative.

Mr. Chester Holcomb, in his work "The Real Chinaman," thus describes the ordinary diet of the Chinaman:

Their daily food consists of rice steamed, cabbage boiled in an unnecessarily large amount of water, and, for a relisn, a tew bits of raw turnip, pickled in strong brine, When disposed to be very extravagant and reckless of expense, they buy a cash worth of dried watermelon seeds and munch them as dessert. . . The description answers with entire accuracy for the food consumption of the great masses of the Chinese people-not for the beggars or the very poor, but for the common classes of ipdustrious workingmen and their families, whether in the great cities or in the rural districts. . . The only luxuries of which they dream are an ounce or two of meat at very rare intervals with their invariable food of rice and cabbage. . . .

In a consular report by Horton, in 1908, the diet of the native of Greece is thus described:

At night the family dines on a few cents worth of rice, boiled together with wild greens and olive oil, and bread, or wild greens boiled in olive oil and eaten with bread or some similar inexpensive dish. Meat is eaten by the laboring classes as a general thing three times a year, Christmas, Easter and the so-called "Birth of the Virgin." Such a family as I am describing, the average laboring man's family of Greece, rarely if ever see such things as butter, eggs and milk.

According to Tibbles, "it has long since been stated that no long lived person is a large consumer of meat; on the other hand, 
that a small vonsumption of neat tends to longevity, providing the amount consumed does not fall so low as the amount of protein disintegrated and excreted during starvation."

This will never happen if one eats the ordinary amount of bread and adds a glassful of milk each day.

Sir Herman Weber says: "Few people know how little food is necessary in advanced age to maintain bodily health. Few people who live to more than eighty are large eaters of meat."

William Cullen Bryant, one of the most notable literary lights of the nineteenth century in this country, wrote in 1837 to his friend, the Hon. John Bigelow, to whose son, Mr. Poultney Bigelow, the historian, we are indebted for the information, "I accustom myself to the greatest simplicity of diet-renouncing tea, coffee and animal food" (flesh food), a practice which he followed for many years until he died, still in full vigor of health and usefulness, not from disease but as the result of an accident. The Hon. John Bigelow, friend of Carnegie and a distinguiched diplomat, was a flesh-abstainer for many years before his death, as is also his son, well-known as historian and litterateur.

From the above paragraphs, it is evident that it is possible for human beings to be sustained in health and vigor with the practical exclusion of meat and even eggs from the dietary. It is important to note, however, that grains enter very largely into these meatless dietaries. These furnish an adequate supply of protein if supplemented by a daily half pint of milk or a handful of nuts.

Greens are also an essential part of the biologic fare, with potatoes and other tubers and roots. These supply lime, iron and alkaline salts which are essential to good nutrition.

E. V. McCollum states "that the greatest thing that can be done to raise the standard of health in this country is to change the type of diet in most homes by decreasing the consumption of meat and increasing the consumption of milk and green vegetables."

The late Dr. Pavy, the most eminent authority on dietetics of his time, writing so long ago as 1874 , remarks:

"Many people seem to look upon meat almost as though it formed the only food that really nourished and supplied what is 
wanted for work." In disproof of the correctness of this popular view, Dr. Pavy refers to the report of Dr. E. Smith, according to whom "it is not uncommon to find, amongst the agricultural laborers of Scotland, that no meat is consumed, oatmeal and milk forming the staple articles of diet. Further, Dr. Guy, from his observations in the case of English prisons, gives as one of his deductions 'that we possess conclusive evidence of the sufficiency of a diet from which meat is wholly excluded, and even of a diet consisting wholly of vegetable matter.'"

Pavy further states that "animal food certainly taxes the stomach more than the ordinary forms of vegetable food that we consume, as is well known by those who have weak digestive power."

According to Liebig, "A bear kept at the Anatomical Museum of Giessen showed a quiet, gentle nature, so long as he was fed exclusively on bread, but a few days' feeding on meat made him vicious and even quite dangerous. That swine grow irascible by having flesh food given them is well known-so much so, indeed, that they will then attack men."

Baeltz, a German army surgeon, made an interesting observation on the Japanese laboring man, which he thus described:

I had two jinrikisha-men; two powerful young fellows, one twenty-two, the other twenty-five years of age. They had followed the same calling for years. I provided their food, which was exactly measured, what they ate, and what they drank; and the chemical composition of the food was ascertained by recognized means. These men received definite instructions:-Every day for three weeks they had to drag me, a man weighing $80 \mathrm{kgs}$. (176.4 lbs.), a distance of $40 \mathrm{kms}$. (24.84 miles), running all the time. That appears to be a fairly arduous task-but not more so than that which these men would have willingly undertaken. But for my purpose it was quite enough; for we considered a walking expedition of $40 \mathrm{kms}$. as something quite respectable; but for a man to run $40 \mathrm{kms}$. every day throughout a sunny August is rather more than one would usually expect of us.

Now the men, during the experiment, kept to their usual diet, which contained fats amounting to less than half that proposed by Voit, while the contained protein fluctuated from between 60 to 80 per cent. of his postulate. Carbohydrates, on the other hand, were provided in exceedingly large quantities, in the form of rice and potatoes, barley, chestnuts, lily-roots and other foodstuffs peculiar to the country. After fourteen days I weighed the two men. One had not made any change, while the other had added half a pound to his weight. After the fourteenth day I told the men I wished to give 
them meat. They were delighted, for meat is a luxury to them. I therefore substituted for a part of the carbohydrates a proportionate quantity of protein-not quite so much as Voit demanded, but a considerable amount. The men ate it with avidity; but after three days they came and asked me to discontinue the meat, and to give it to them only upon the conclusion of their probation, because they felt so fatigued, and could not run so well as before.

This is certainly most convincing evidence of the great endurance which may be supported by a meatless dietary. But the suggestion may be offered that the Japanese are a vegetarian race and have been such for hundreds of years and that racial characteristics may be a dominating factor. The answer to this will be found in a special research by Professor Irving Fisher of Yale University an account of which was published in the Yale Medical Review and later in Munsey's Magasine. The purpose of the experiment was to determine the comparative endurance of flesheaters and flesh-abstainers.

The flesh-eaters were largely men in training for athletic contests at $\mathrm{Yale}$; the flesh-abstainers were such Yale students as I could find who did not use meat, or used it very sparingly, and nurses and physicians of the Battle Creek Sanitarium. Forty-nine subjects were tested. The results of the comparison were certainly surprising, and showed almost conclusively that those who used low protein and little or no flesh, not only had greater physical endurance, but far greater than those who were on a so-called "training diet."

Very few of the flesh-eaters for instance, could endure holding their arms horizontal for more than a quarter of an hour, where it was common among the flesh-abstainers to hold them for more than an hour, and without as much pain or discomfort as the meat-eaters experienced in half the time. In one case a flesh-abstainer held his arms out for three hours and twenty minutes, and then stopped merely because he had reached a round number of minutes-two hundred.

One of the most severe tests was in deep knee-bending, or "squatting." Few of the meat-eaters could "squat" more than three hundred to four hundred times, and it was almost impossible for them to walk down the gymnasium stairs after the test without falling, so weakened were their thigh muscles. On the other hand, one of the Yale students who had been a flesh-abstainer for two years did the deep knee-bending eighteen hundred times without exhaustion, after which he ran without difficulty on the gymnasium track and walked several miles. Another subject, a nurse at the Battle Creek Sanitarium, did the deep knee-bending twenty-four hundred times; after which he proceeded with his regular work without serious inconvenience. Another nurse has recently accomplished the surprising 
record of five thousand times. One remarkable difference between the two sets of men was the comparative absence of soreness in the muscles of the meat-abstainers after their tests.

Bunge maintains (Science) that the great waste of energy when meat is eaten is the result of over-stimulation of the liver which results in over-production of catalase, an oxidizing ferment which causes excessive oxidation or heat production.

Bunge twenty' years ago called attention to the fact that "the normal food of the adult should be furnished by the protein and carbohydrates in the proportion met with in the cereals." $\mathrm{He}$ also mentioned the fact that Bavarian laborers who do the hardest work live on a diet of flour and fat, and also refers to the investigations of Panum and Buntzen, from which it appears that "even a carnivorous animal can be nourished on cereals and fat." These investigators kept a dog in good health for months on an exclusive diet of oatmeal and butter, and without loss of weight.

The chemical changes which occur in muscle tissue during work afford a clear explanation of the diminished endurance of meat eaters. either animals or men, when compared with vegetable feeders.

During contraction, lactic acid is produced in the muscle tissue. While the muscle is relaxing, the acid is neutralized by the alkalies present in the tissue fluids. This enables the muscle to contract again. Later, after relaxation, the lactic acid is burned up by the oxygen of the blood stream. Fatigue begins as soon as the lactic acid begins to accumulate in the muscle.

It is evident that if the tissue fluids are only slightly alkaline, fatigue will occur sooner than if they are more strongly alkaline. The tissue fluids of flesh eaters are much less alkaline than those of vegetable feeders, as shown by the fact that the urine of flesh eaters is always highly acid. This is one of the reasons why mixed feeders invariably show themselves inferior to flesh abstainers in tests of endurance.

\section{Raw Beef not an Antiscorbutic}

It was long believed that the chief cause of scurvy was the use of salt meats, and the befief is still generally held that fresh meat is an antiscorbutic, that is, that the feeding of fresh meat will prevent scurvy.

Vitamin studies recently made by Dutcher and others have demonstrated that watery expracts of raw, lean meat will not pre- 


\section{ANIMAL FOODS}

vent scurvy in guinea pigs and will not prolong the lives of these animals when fed on a diet insufficient to prevent scurvy. Orange juice, on the other hand, was found to prevent scurvy when fed either with or without beef extract.

The excuses for the use of flesh foods under any conditions are rapidly disappearing one by one. In fact, at the present time there is left no apology whatever/for the use of flesh under any circumstances except the absence of better food.

A well known editorial writer recently (1921) said in a leading newspaper:

Growth of understanding, also, regarding the human diet is bound to lead to the use of smaller meat rations as a measure of health. When people learn that individuals do not require large quantities of flesh foods and that eating much meat commonly leads directly to illness, the United States may cease to pride itself on being the greatest meat-eating nation.

In spite of government efforts towards enlightenment regarding the care of farm animals during the last sixteen years approximately $1,475,000$ cattle have died annually from disease and $1,500,000$ from exposure. In 1914, 7,000,000 hogs died from cholera.

According to Armsby, only 3 per cent. of the corn fed to a steer can be recovered from the edible portion of the carcass of the animal; that is, for every hundred pounds of corn fed to a steer, only three pounds of actual food material can be recovered.

Of food fed to a dairy cow, 18 per cent, or six times as much food, is recovered as in the case of the steer.

According to Prof. J. Russell Smith, of Columbia University, in a notable work "The World's Food Resources," in the United States and Canada, but especially in the United States, the chief object of agriculture is not to feed men, but to feed beasts. This was true even in the food crisis of 1918 . We now raise altogether about $5,191,000,000$ bushels of grain, as follows:

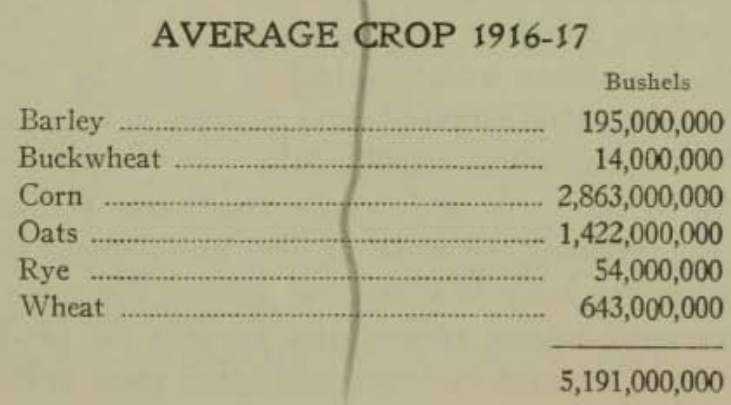


Of this great total the American people ate less than $550,000,000$ bushels of wheat when unrestricted $180,000,000$ bushels of other grains, and the total export of grains, $341,000,000$, brought the amount used for human food up to about $900,000,000$. The rest, $4,300,000,000$, went to our four-footed brethren, who outnumber us and whose food requirements, because of their greater size, are several times our own.

In addition to the grain, they get all of the $85,360,000$ tons of hay grown on $54,618,500$ acres. (More than 10 per cent. of the half billion acres under cultivation in the United States). They also roam over millions of acres, eating all the grass. It is therefore plain that more than four-fifths of the produce of the American agriculture, even in 1918 , went to feed the beasts.

\section{Special Contraindications of Flesh Foods.}

Albu, an eminent German authority, so long ago as 1901 (Berlin Klin. Woch.) recognized the injurious effects of a flesh diet, especially in maladies dependent upon intestinal putrefactions, and prohibited its use in the following: neurasthenia, nervous disorders of the stomach, hyperacidity of the stomach, mucous colitis, chronic constipation, obesity, exophthalmic goiter, renal diseases, affections of the skin, disorders of metabolism. diseases of the blood, diseases of the gastro-intestinal tract, pruritis, furunculosis, urticaria, erythema nodosum, and the various forms of eczema.

While flesh foods are unnecessary in any case when other food is available, there are many diseases in which the use of nesh foods of any sort is particularly objectionable because likely to encourage the disease and interfere with the efforts of Nature to accomplish a cure. Among these diseases may be mentioned particularly the following:

All conditions in which there exists a state of autointoxication, because the putrefaction of remnants of flesh foods in the colon is the most active cause of intestinal toxemia.

Constipation, because of the paralyzing effect of putrefactive poisons upon the muscular walls of the colon.

Colitis, a common malady which is caused by the infection arising from the putrefaction of food residues in the colon. Meats contain the bacteria which cause colitis.

Fevers of all sorts and febrile conditions because of the necessity for lessening the work of liver and kidneys as much as possible when the body is struggling against the toxic influence of a temperature-raising poison, also because the production of gastric juice is lessened in fever and hence meat is likely to be 
iess well digested and certain to undergo putrefactive changes.

Hyperthyroidism or Grave's disease, and hypothyroidism or myxedema and other diseases of the thyroid, all of which are greatly aggravated by the poisons resulting from a meat diet and are often due to this cause.

Bright's disease, stone in the kidney or bladder, and other diseases of the kidney, for the reason that the kidney is the chief means of elimination of the poisons arising from meat eating, and the crippled kidney is unable to do the extra work imposed by a meat diet and so is made worse by it. The work of the kidneys is doubled and tripled by flesh eating.

Gout, rheumatism and all rheumatic affections, which are aggravated and often caused by a meat diet.

Pigmentation, liver spots, since the putrefaction of meats in the colon is the source of the brenzcatechin and other poisonous pigments which, the crippled suprarenal capsules being unable to destroy them, are deposited in the skin.

Acidosis is increased by a diet rich in fat and protein, hence in this condition and in diabetes and other maladies in which acidosis is likely to occur, meat should be avoided.

Even in pellagra meat may be discarded with advantage. Goldberger has shown that an increase of milk in the diet is the most efficient method of combating this disease.

The same may be said with reference to the use of meat in cases of sprue and beri-beri, maladies which are very common in Oriental countries and are often brought to this country by travelers or returning missionaries, Many cases of sprue have been successfully treated at the Battle Creek Sanitarium without meat.

High blood pressure, arteriosclerosis, myocarditis, apoplexy and paralysis are closely allied affections in all of which a nonflesh dietary is most necessary.

Cirrhosis of the liver is a crippled condition of a highly important vital organ which demands a fleshless bill of fare as a precaution against a fatal toxemia.

Gallstones, gall bladder disease, jaundice, and hepatic abscess are all conditions which demand the prohibition of meat as a protection of the most important defense of the body against poisons of all sorts, and especially against the poisons produced by germs in the intestines.

Dropsy is an indication of a crippled heart or kidneys in 
most cases and requires special care to avoid the intestinal poisons produced by a meat diet.

Cancer is a meat-eater's disease. It is common among meateating races of men and animals, rare among flesh-abstainers (Williams, Hoffman). A fleshless diet may not cure cancer, but will delay its progress (Bulkley).

Chronic cystitis and disease of the prostate gland are aggravated by a meat diet because of unfavorable effects on the urine.

Ordinary or secondary anemia does not require meat when egg yolks and garden greens and a score of other foods rich in iron are available, and do better without meat than with it. Pernicious anemia is made much worse by a meat diet. The iron of meat is inferior in quality (Sherman, Chemistry of Food and Nutrition).

Tuberculosis is aggravated by meat diet. Milk and greens are of special value in this disease, and when these are provided there can be no reason for the use of meats. Meat is harmful because it adds to the already heavy burdens of the liver and kidneys, and fails to supply the lime which is so important as an aid to recovery. Any amount of protein required may be obtained from milk and egg yolks; but it is now well known that an excessively high protein diet is injurious in lung tuberculosis; so that milk and eggs as well as meat may be used to excess. This is especially true of eggs.

Gastric catarrh, like catarrh of the colon (colitis) and of the small intestine (enteritis), requires disuse of meat. A diet of buttermilk aids in starving out the bad flora (Debove).

Bronchial catarrh and asthma are often cured by a change from a high to a low protein ration.

Chronic appendicitis is a meat-eater's disorder. Appendicitis, both acute and chronic, is practically unknown among the vegetable eating tribes of Africa (Senn, Cole).

Skin disorders such as chronic eczema and psoriasis, often incorrigible under every other mode of treatment, rarely fail to yield promptly to a dietary from which meats are rigidly excluded (Bulkley).

Neurasthenia, insomnia, migraine, insanity, epilepsy, and many other nervous disorders are now known to be either due directly to the influence of toxins derived from the colon and circulating in the blood, or at least greatly aggravated by such poisons. The adoption of a fleshless diet certainly exercises a most favorable influence on this class of disorders. 
Inebriety requires the total exclusion of flesh foods from the dietary. The effect is often most striking, causing the disappearance of the alcoholic appetite within a few days, or at least so mitigating it as to render control possible (Booth).

Parasitic infections such as malaria and syphilis are favorably influenced by the fleshless regimen. Sanderson, the famous elephant hunter of India, informed the writer that after abstaining from flesh foods he remained free from fever in the jungles, although before he was always attacked with fever whenever he visited the jungle.

Gastric and duodenal ulcer require avoidance of meat because of the fact that flesh foods very probably play an active part in the causation. "Gastric ulcer is a meat-eater's disease."

Hyperchlorhydria or hyperacidity is greatly aggravated by a meat diet, which stimulates the gastric glands to secrete an excess of acid (Pavlov).

In hypochlorhydria and achylia flesh foods are contraindicated because a highly active gastric juice is essential for the digestion of meats. Flesh foods are of all foodstuffs most out of place in cases in which the normal acid of the gastric juice is absent or greatly deficient.

Meats of all sorts should be excluded from hospital bills of fare. The patient who has undergone a surgical operation needs essentially the same diet as a fever patient until and after he reaches convalescence. The common use of beef tea, meat extracts, meat soup stocks and like preparations is in the highest degree unjustifiable in the light of modern knowledge of organic and physiologic chemistry. Such preparations only add to the burdens of the liver and kidneys, which in the surgical patient are always heavily taxed, and lessen rather than improve chances for recovery. Constipation, usually very pronounced in surgical cases, renders a meat diet particularly objectionable because of the aggravated toxemia induced thereby and is rendered still more obstinate through the paralyzing influence of the ammonia and other putrefaction poisons produced.

\section{EGGS}

The eggs of birds, especially of domestic fowls, are universally used as food by human beings as well as by the anthropoid apes and various other animals. The eggs of the common domestic fowl and the guinea hen are the most delicate and agreeable. 
The eggs of the duck and goose are richer in fat and of stronger flavor.

The eggs of sturgeon and gther fish, known as roe, are much used in some countries.

The egg is a highly nitrogenous food. The whole egg affords 15 calories of protein to the ounce and 31.7 of fat, a total of 46.7 calories, or two-thirds that of porterhouse steak and more than double that of milk.

A hen's egg of average size weighs a little less than two ounces. Approximately, the egg consists of one part shell, six parts white, and three parts yolk.

\section{Weights of Eggs and Constituents (Average)}

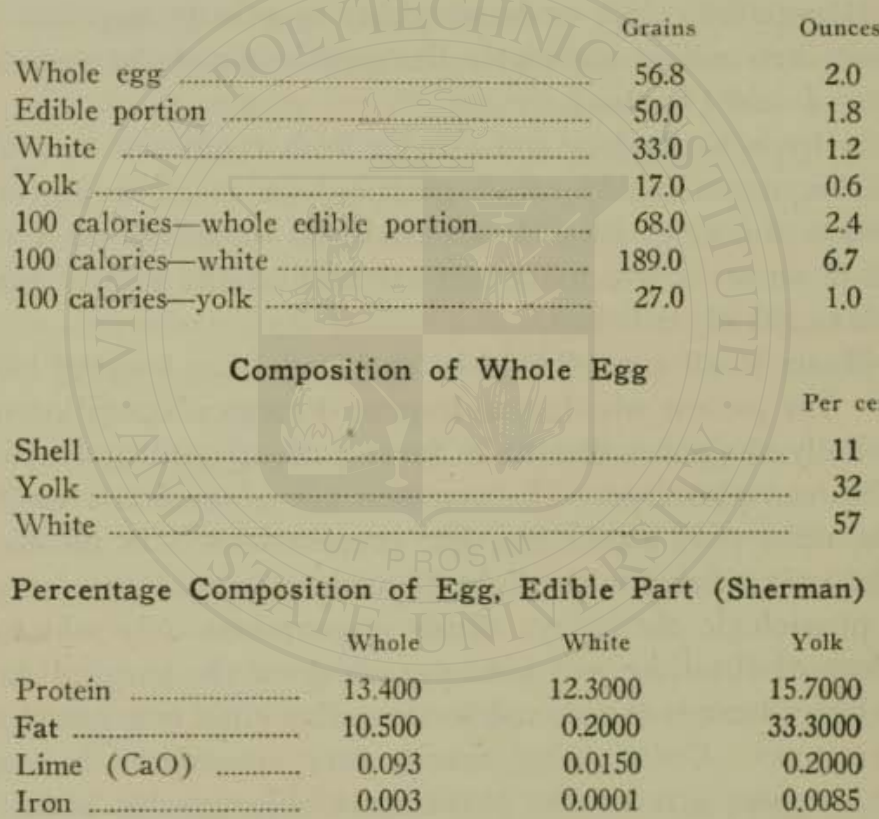

A single egg furnishes about 30 calories of protein. It is evident, then, that four or five eggs supply protein sufficient for an ordinary day's ration. But it is to be remembered that cereals are rich in protein and all vegetables contain some of this element, while milk contains an excess, and supplies in one-half pint more protein than does an egg. A dietary which includes two glassfuls of milk with a sufficiency of other foods not of animal origin will afford an abundance of protein without the addition 
of eggs. Even if neither meat nor milk is included in the bill of fare, not more than two eggs at most will be needed to supply ali the supplementary protein required for one day.

Milk lacks iron and so is not a complete food; but on account of its great excess of protein, the egg is less suited to serve as an exclusive diet, and should not be too freely used. Its proper place in the dietary is to round out a diet otherwise deficient in complete proteins, vitamins and lime.

As a source of complete proteins, the egg is inferior to milk because less digestible and also because very liable to be rendered unfit for food by putrefaction. Milk ferments or sours when stale, while eggs putrefy. A stale egg is certainly one of the most disagreeable as well as most dangerous of unwholesome foodstuffs, while sour milk is actually more digestible and really more wholesome for most persons than is fresh milk. This property of milk is due to its carbohydrate, sugar of milk, an element which in eggs is lacking.

\section{Egg Yolk.}

Egg yolk is a very remarkable substance prepared by Nature for food, and henee contains all the elements necessary for complete nutrition, including a rich store of lime and iron and other salts, with an abundant supply of vitamins. Nothing is forgotten. The yolk is the sole food of the young chick while it is undergoing-development in the shell, and when it leaps out of its prison house it is fully equipped to enter upen its life eareer, and to thrive upon the foodstuffs which it finds ready at hand. It does not have to be nursed or brought up on a bottle. It needs only to eat, exereise-and-grow. The yolk of the egg has -supplied-it with everything needful for its complete life equipment.

It is sometimes well to advise the use of egg yolks alone. excluding the white when the object is to increase the intake of lime salts. The writer has followed this plan for many years.

To insure the utilization of eggs it is highly necessary that they should be properly cooked. Raw eggs are indigestible. Half cooked eggs are half digestible. Fried eggs and omelets are hard of digestion, and the undigested fragments which appear in the stools carry away a considerable part of the precious lime which should be retained and absorbed. This difficulty may be obviated by cooking the egg at a low temperature, $150^{\circ}$ to $160^{\circ} \mathrm{F}$. Cooked in this manner, the egg is soft and jelly-like or coddled, and is 
presented to the stomach in most digestible form. When egg yolks alone are eaten, they may be separated either before or after cooking. Hard-boiled yolks are easily digestible, which is not true of the whites.

\section{Egg White.}

Raw white of eggs has long been regarded as among the most easily digestible of all food substances. This idea was chiefly based upon the observation by Beaumont in his experiments upon Alexis St. Martin. Beaumont noted that raw egg white left the stomach very quickly, in less time in fact than any other foodstuff which he investigated. More recent investigations, however, by Pavlov and others have shown that this interpretation of Beaumont's observation is incorrect. Pavlov, for example, showed that the raw white of egg does not stimulate the flow of gastric juice. In this respect white of egg differs from other proteins. The effect of which is to cause the stomach to pour out an abundant supply of a highly acid gastric secretion.

The effect of raw white of egg on the stomach as noted by Pavlov was the same as that of water. Abderhalden discovered another peculiarity of raw white of egg. He found that this form of albumen is not readily acted upon by pepsin. Okada showed that the intestinal fluids, both the bile and the pancreatic juice, are indifferent to white of egg. Very little bile enters the intestine when white of egg is introduced and trypsin, a digestive principle of the pancreatic juice which acts vigorously upon other protein, has no effect whatever upon raw egg white. Other investigators have confirmed this observation. A still further very remarkable observation made by Vernon, Hetin and numerous other investigators shows that the raw white of egg not only is not acted upon by the digestive fluids but that it hinders the digestion of other substances.

Steinitz noted that raw egg white caused vomiting and diarrhea in dogs. Five egg whites given to a fifteen pound dog caused very severe diarrhea. The stools were not only loose but had an extremely offensive odor and contained a great deal of mucus, indicative of very active putrefaction and marked infection. Even when small amounts of raw white of egg are fed, the egg albumen can be recovered from the stools unchanged.

These peculiarities of raw egg white disappear when it is cooked at a temperature of $160^{\circ} \mathrm{F}$. At this temperature the egg 
white is thoroughly coagulated but remains soft and jelly-like. Egg yolk on the other hand was found to be very easily digestible when eaten either raw or cooked. Careful experiments have shown that raw egg white has no food value when introduced into the colon and hence it should not be used in rectal feeding. Another objection to the use of raw egg white is the readiness with which this substance is absorbed into the blood, producing anaphylaxis.

The peculiar effect seen in persons who are sensitized against egg is said to be due to ovo mucoid which the egg contains. Egg white contains also three other substances, ovo globulin, ovo albumin and con albumin. Albumin is shown to be the cause of diarrhea. The amount of white of egg required to give rise to anaphylaxis is extraordinarily small. This effect was produced in guinea pigs by less than one-millionth of a grain of egg white.

From the above facts it is evident that the practice of giving raw eggs to invalids with or without milk feeding and the use of raw white of egg in the feeding of babies are certainly of less value than has been supposed and are really more or less dangerous procedures.

Liquid albumin retards the digestion of coagulated albumin, hence partially cooked egg white digests very slowly. This is true with reference to both gastric digestion (Linoissier) and pancreatic digestion (Delezenne).

According to Bayliss, the eminent professor of physiology in. the University of London ("The Physiology of Food and Econamy in Diet"), raw white of egg contains some substance which, even in very small amount, hinders the action of the digestive fuids. Cooking destroys this substance; but at the same time long cooking renders the white of egg almost insoluble in the digestive juices, just as large lumps of ice melt less rapidly than finely pulverized ice. White of egg has for centuries been considered almost indispensable for the feeding of the sick and especially the feeding of convalescent surgical cases. Many a nurse will feel that without the time-honored eggnog her ward patients will certainly starve. But raw eggs should be dropped from the hospital bill of fare and sick room dietaries. In fact, the egg in any form is under suspicion and must be used in moderation and with caution to make sure that it is perfectly fresh and free from infection. 
Recent experiments by Rose and MacLeod seem to indicate that the raw white of egg is well digested if lightly beaten.

Prefesser Linossier, a member of the Academy of Medicine of-Paris, holds that there is in eggs a toxalbumen to which some persons are susceptible either by heredity or through acquirement as the result of disorder of the liver or intestines. This poison, according to Linossier, is destroyed by heat. Hence, eggs should never be eaten raw, but should be cooked, both white and yolk, to the point of coagulation or curdling. Further cooking lessens digestibility.

\section{The Cooking of Eggs.}

According to Penzoldt, the time required for the gastric digestion of eggs cooked in different ways is as follows:

Lightly boiled
Poached $\ldots \ldots$
Hard boiled
Omelet

When boiled two minutes the white of egg next to the shell is coagulated; the yolk remains fluid. In a three-minute egg the white is coagulated and the yolk is thickened. A ten-minute egg is hard boiled.

A good way of boiling eggs is to drop three eggs into a quart of boiling water. Remove at once from the source of heat. In ten minutes the eggs will be found to be uniformly soft boiled, or jellied.

The greenish black color which is sometimes seen on the surface of the yolk of a hard boiled egg is due to the formation of sulphide of iron (Tinkler). If the egg is cooled at once after boiling, the chemical change does not occur.

\section{Influence of Food on Eggs.}

Eggs are greatly influenced by the food of the fowls by which they-are-laid. Meats and putrefying substances give to eggs a strong flavor. Milk, sunflower seeds, and other clean and wholesome foods insure a very superior flavor.

When chickens have plenty of green feed and yellow corn the yolks have a much deeper color, due to the presence of carotin, which is often associated with the fat-soluble vitamin. 


\section{Eggs and Biliousness.}

Many persons know by repeated experiences that the free use of eggs produces a well defined physical condition which is commonly termed "biliousness" although, very likely, connected with the bile or bile-making function of the liver only in a remote-way. "Biliousness" is really acute autointoxication. Its cause is the putrefaction of undigested and unabsorbed protein in the colon and the absorption of the poisonous products of bacterial action. Eggs encourage putrefaction, milk does not.

If the eggs happen to be even a little stale, the putrefaction and autointoxication are greatly intensified.

Doctor Tissier of the Pasteur Institute has recently shown that eggs are often infected with the B. paracoli or paratyphoid bacillus. The white diarrhea of fowls is caused by this germ and is always present in eggs laid by fowls suffering from this very common disorder.

\section{Eggs and Acidosis}

When burned in the body, eggs, like meat, leave a strongly acid residue which lessens the alkali reserve and so tends to cause acidosis. On this account eggs must be used very sparingly, if at all, by persons suffering from arteriosclerosis, high blood pressure or nephritis.

\section{The Food Iron of Eggs.}

The iron of the egg is contained almost wholly in the yolk, where it is stored up for use by the developing chick in making its first outfit of red blood cells. The proportion of iron found in the yolk is one hundred times as great as that found in the white.

Professor Bunge found in the egg yolk an iron compound so closely resembling the hemoglobin of the blood corpuscles as to be easily converted into it, and so he gave to the compound the name of hematogen, which means, in simple terms, generator of blood. Hematogen contains 0.3 per cent. of iron and yet all the ordinary laboratory tests for iron fail to reveal its presence. This remarkable fact clearly showed that food iron is a quite different thing from the ordinary metallic iron of the laboratory. The deft hand of Nature working in her secret laboratories, has, through the agency of vital activity, lifted the cold inert iron of the mine and the foundry into the realn of organic life, and has endowed it with marvelous properties by enormously increasing its natural 
affinity for oxygen. Ordinary rusting proceeds very slowly. When the oxygen hunger of iron is fully developed, as in the hemoglobin of the blood, this oxygen absorbing propensity operates with lightning rapidity. All the blood in the body, about five quarts, rushes through the lungs every minute or two, and yet the stream is not so rapid that the subtle hemoglobin does not have time to snatch for each tiny red cell its full load of oxygen.

It is well worth while to remember that when eggs are used as a source of food iron, to enrich the blood in anemia, it is the yolk only and not the whole egg that is useful.

As a source of food iron, the egg fills an important place in the dietary, and one that milk cannot fill because of its deficiency in iron. Two yolks contain as much food iron as a five ounce serving of meat, four times as much as an equal weight of meat, and eight times as much as the same weight of fish.

It is to be said, also, that the iron of the egg yolk is superior to that of meat, being especially prepared by nature to serve as food iron, while the iron of flesh is practically identical with that derived from the dead blood cells of the body, or waste iron which is in large part excreted by the liver.

Here again the green plant shows its superior value as a supplementary nutrient. An ordinary serving of spinach supplies as much food iron as three or four egg yolks and a serving of red root or dock greens as much as half a dozen eggs.

\section{The Lime Content of Eggs.}

An egg yolk of average size weighs half an ounce and so contains a little more than two-fifths of a grain of lime ( 0.42 grain) or 2.7 per cent. of a day's ration.

A comparisen of the lime-content of the whole egg, the white and the yolk is both interesting and instructive. The egg yolk contains, per ounce, nine times as much lime as the white. The figures for the whole egg represent the combined compositionof the yolk and white.

It-is-evident that eggs, and especially-the egg yolks, are a most valuable source of food lime, but this is not true of the white taken-by-itself, which contains less lime per ounce than most of the cereals, scarcely more than rice, about the poorest in lime of all the cereals.

As a source of lime, the egs is again inferior to mill, which supplies a much larger amount of food lime than the same weight 
of eggs. The lime content of a glassful of milk equals that of three or four eggs.

The lime of the egg is mostly found in the yelk, although the unhatched chick absorbs considerable lime from the egg shell. An ounce of egg yolk furnishes more lime than an ounce of milk, but more than ten yolks are required to supply as much food lime as a half pint of milk.

It must be remembered, also, that the green leaf is a most valuable source of food lime. An ounce of chard nearly equals two egg yolks in lime content, and even celery furnishes as much lime as the same weight of yolks, while an ounce of turnip tops supplies as much lime as the yolks of four eggs, and an ounce of mustard greens as much as half a dozen yolks.

From the above, it will be plainly apparent that while the egg is valuable as a source of protein, lime and iron, it is not essential. Milk will supply equally good lime and protein, and the green leaf is a still richer source of food iron.

It is not te-be-forgotten-that the egg is also a source of valuable vitamins, especially the antineuritic or beri-beri preventing vitamin and the antirachitic or fat-soluble vitamin. Both of these food essentials are supplied, however, in rich abundance by the green leaf.

\section{Egg Substitutes.}

Egg substitutes are generally unsatisfactory and sometimes unwholesome. They are chiefly used by bakers. The best are made from the casein and albumen of skimmed milk, which are mixed with flour and dried. Others are made from the blood of slaughtered animals and are most unwholesome.

\section{Preserved Eggs.}

There is no known means of preserving eggs without deterioration except refrigeration. The Chinese have for ages employed a process of preservation which is not likely to become popular in this country. Fresh duck's eggs are kept in an infusion of tea, lime, salt and wood ashes for half a year, then drained and coated with rice hulls. Eggs thus treated are known as pidau. Another method of preservation is to encase the egg with a thick layer of plaster of Paris. Eggs preserved in this way have a strong flavor of ammonia, and are of course in a state of decay much like that of limburger cheese. 
The popular method of preserving eggs by means of immersion in silicate of soda or water glass is far from satisfactory. Such eggs are not always unwholesome, but they lack the properties of strictly fresh eggs.

In hot weather, eggs will keep perfectly fresh without refrigeration not more than three or four days. A low temperature is nearly as necessary for the keeping of eggs as of milk.

\section{Bad Eggs.}

Eggs are not eaten in this country unless supposed to be fresh or well preserved. The decayed egg is rejected at once. The Chinese gourmand, however, has a great penchant for stale eggs. His highly cultivated palate delights in the pungent aroma of an egg two or three years advanced in putrefaction. The American turns in disgust from the stale egg, but curiously enough, requires in his beefsteak the same condition that the Chinaman demands in his egg.

It was formerly supposed that if the fresh egg were kept free from contact with bacteria it would remain sterile, but since the investigations of Rettger it is known that quite a large proportion of all eggs show bacteria when carefully examined. This is especially true of the yolk of the egg, which is infected much more often than the white. According to observations made by the United States government, one egg in seven, on the average, is infected with harmful bacteria, which are only destroyed by very thorough cooking.

Within a few years an infectious disease due to a germ known as the $B$. pullorum has spread rapidly over the country. This disease affects the ovaries of fowls, and eggs laid by infected hens contain the $B$. pullorum, which when fed to rabbits in experimental work has produced the same disease in them. The $B$. pullorum has been shown to be a cause of diarrhea.

Although the Chinese eat with seeming impunity the pidau, or ancient egg, which is known to be swarming with bacteria, there is no evidence that it is harmless. Eating decayed eggs may inoculate the intestine with bacteria that gradually break down the resistance of the intestinal mucous membrane and finally produce infection, besides flooding the body with toxins which the kidneys must eliminate to their certain detriment.

Doctor Rettger, who has investigated the subject, has found that eggs are often badly infected, showing a high bacterial count 
and much ammonia from putrefaction, notwithstanding the fact that they pass the candle test well and give no great evidence of decomposition. New and better standards for examination of eggs, milk and meat are urgently called for.

M. E. Pennington, of the United States Department of Agriculture, some years ago (1911) made a careful study of eggs, especially with reference, to the presence of bacteria. Here are some of the facts discovered:

The fresh, sound, shell egg is for practical purposes free from bacteria, though it is not always sterile. It is free from organisms of the colon group so far as we know. "Stuck spots," "mold spots," definite "blood rings," "white rots," eggs with a pronounced odor, "grass eggs" (those having a greenish color in the egg white), "musty eggs," and "sour eggs," that is, eggs having a pungent quality which is difficult to describe but which is recognized by the trained sense of smell, are ordinarily possessed of a high bacterial content. "Grass eggs," "musty eggs," "sour eggs," and eggs having a pronounced odor cannot always be excluded by the candler. The others can and should be. The great majority of "seconds," "heated eggs," dirty shell eggs, and cracked eggs (not those from which the contents are escaping) show but few bacteria present when studied in the producing section. When cracked-shell eggs are kept, even for a few days, they frequently become infected with molds and bacteria. Cracked eggs, or "checks," as they are called in the trade, dirty shell eggs, and "seconds," which signifies a grade including undersized, soiled, "checked," and stale eggs, are commonly used by egg breakers. No "spot" eggs of any sort are used by reputable breakers.

Pennington, to whose work we have above referred, and Kossowitz, of Vienna, have found that fresh eggs often contain molds, yeasts and various other micro-organisms. Pennington found only 12 per cent. of the eggs examined entirely free from bacteria. Kossowitz found most eggs sterile when first laid, but discovered that infection readily occurs through penetration of the shell by various bacteria if the eggs by careless handling are exposed to contamination.

\section{Candling of Eggs.}

Eggs, even when known to be fresh, should be candled before using, as eggs are sometimes infected before they are laid and are also in other ways defective. The Bureau of Chemistry of theUnited States Department of Agriculture has prepared an egg chart which is shown herewith, together with an explanation of: the same. 
The popular notion that dark colored eggs are richer in fat than others is wholly without foundation.

Non-fertile eggs are generally considered of better flavor and are believed to keep safer than fertile eggs.

\section{Egg Poisoning.}

Some persons are sensitized to eggs and cannot eat even a small fraetion of an egg witheut stffering -most-unpleasant censequences. Nausea, vomiting, purging, headache, and a distressing nettle rash or urticaria are a few of the symptoms which result from the use of eggs by sensitized persons. Similar symptoms have been observed to follow the use of milk and of various other foodstuffs in different persons.

Lemoine, an eminent French authority who has recently made a careful study of this subject, tells us that raw egg albumin and the lean flesh of animals in a raw state contain poisons which damage the kidneys. To the effects of this poison is due the appearance of albumin in the urine when raw eggs are eaten freely. These poisons may be destroyed by the gastric juice and by thorough cooking. The action of normal gastric juice upon raw egg albumen is so slow that its use is hazardous since, in many cases of chronic nephritis, achylia exists. Nephritics should use eggs sparingly and never raw.

In a case recently observed by the writer, the symptoms of poisoning occurred only after the eating of the yolks of eggs, although in the majority of cases the unpleasant symptoms follow the use of the whites rather than the yolks.

Lemoine states that the effects of food poisoning may be avoided in many cases by taking a very small quantity of the objectionable food half an hour before the meal is eaten. It is also possible to overcome this special susceptibility by taking the objectionable substance in very small but gradually increasing doses. In the course of some weeks the amount may be gradually increased to the quantity usually taken at an ordinary serving. When the susceptibility is overcome in this way the effect, however, is often not permanent, the susceptibility returning after a time if the use of the particular article is discontinued. 
2. 3
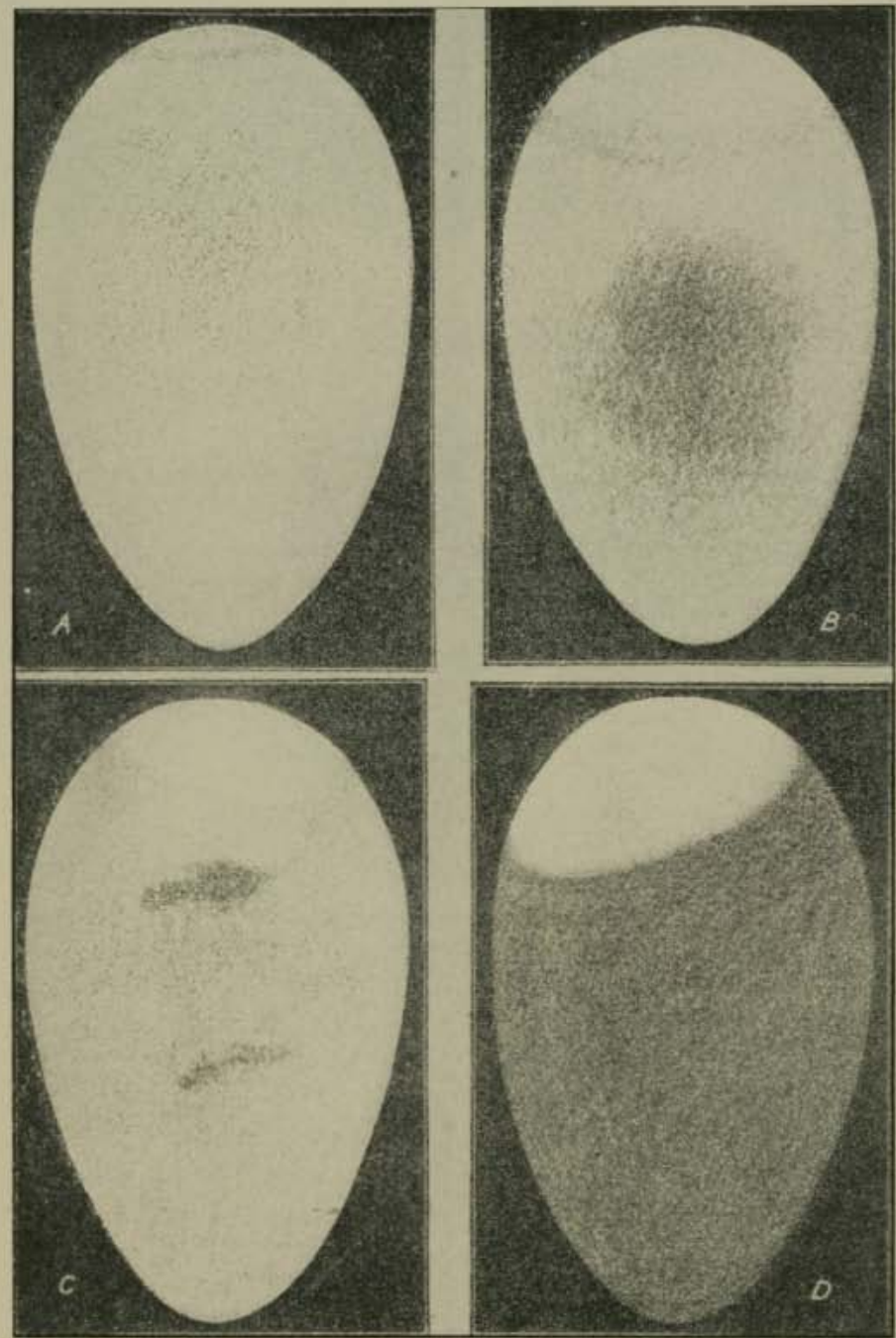

APPEARANCE OF EGGS WHEN CANDLED

A Fresh Egg; B Stale Egg: C Egg Showing Fungous Spots; D Rotten Egg. (Yearbook U. S. Dept. of Agric,, 1911.)

$$
\therefore
$$



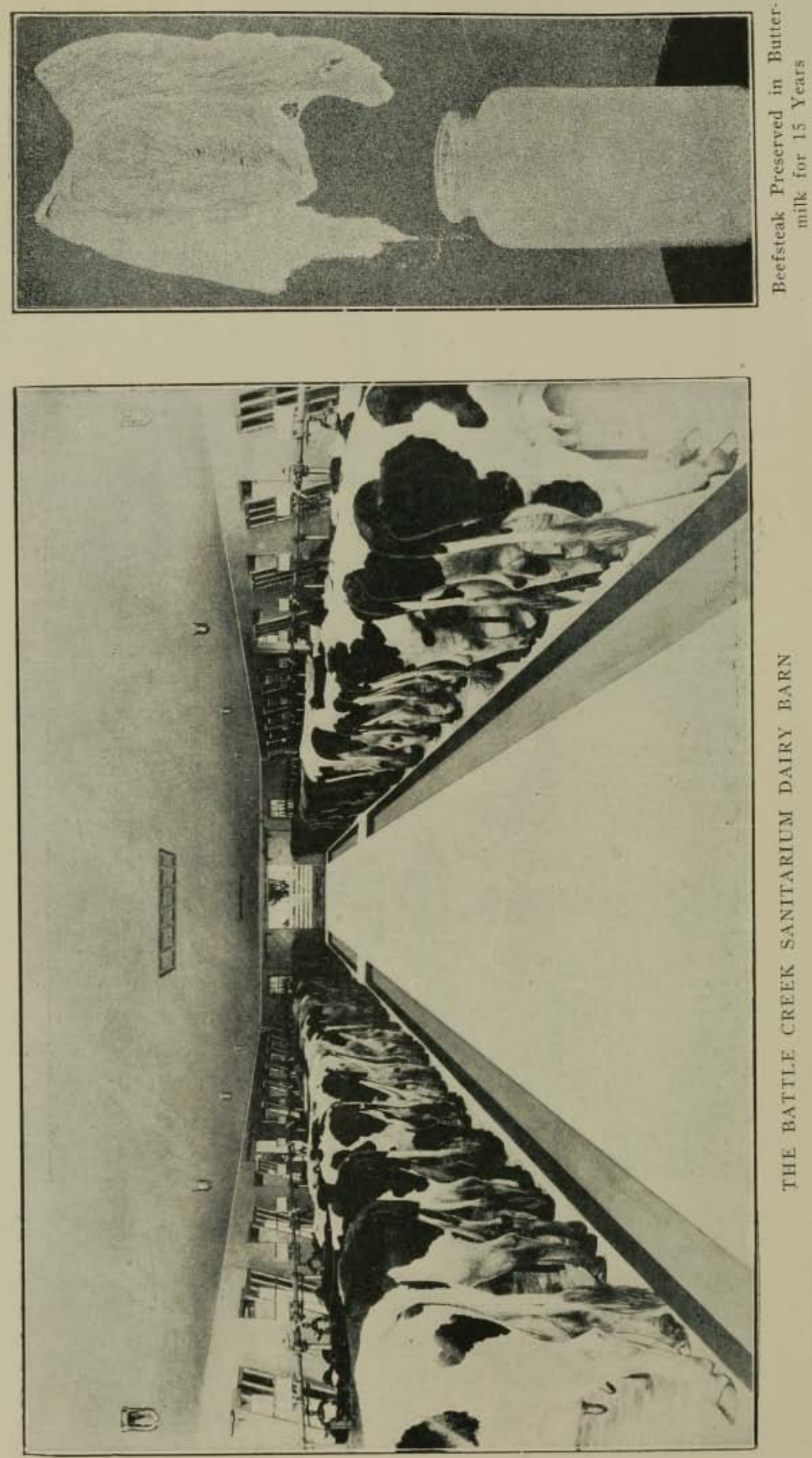


\section{When Eggs Should Not Be Eaten.}

Claude Bernard observed that albumin appeared in the urine after the eating of a considerable quantity of white of egg. On this account many physicians forbid the use of egg by persons suffering with disease of the kidneys. Recent investigations lead to the conclusion that the healthy kidney never permits the passage of albumin and that when albumin appears in the urine it is evidence that the kidneys have been damaged. Persons suffering from Bright's disease, or who have albumin in the urine, or arteriosclerosis, should avoid the use of eggs, or at least should use eggs very sparingly, eating the yolks only.

Persons who are subject to so-called bilious attacks, sick headaches, or who have bad breath or other marked symptoms of autointoxication, would do well to avoid the use of eggs because they encourage the development of putrefactive flora in the colon. This is especially true of the white of egg. Egg white, when raw, leaves the stomach quickly but is not well digested, and soon finds its way to the colon, where it readily undergoes putrefaction. When boiled, egg white is digestible, but digests slowly when hard boiled, and when not well masticated, as is generally the case, it is likely to reach the colon and there undergo putrefactive changes. The yolk of egg is readily digestible, even when hard boiled, and so is greatly to be preferred to the whole egg in cases in which it is desirable to restrict putrefaction as much as possible; but even yolks should be eaten sparingly. There is rarely ever real necessity for the use of eggs, for protein of equal if not superior quality may be obtained from milk, the soy bean and from nuts, including the humble peanut.

Cholesterol, a resinous substance of which gallstones are formed, is normally present in the blood in the proportion of one part in 2,000 parts of blood. The blood of a person of ordinary size contains about 20 grains of cholesterol. Laroche has shown that in persons suffering from gallstones the amount of cholesterol in the blood is considerably increased. A single egg contains about 4 grains of cholesterol. A couple of eggs would thus increase the amount of cholesterol in the blood to the extent of 40 per cent. From these facts it appears that persons who have undergone operations for the removal of gallstones, or are believed to be suffering from this disease, should carefully avoid the use of eggs. 


\section{MILK}

Milk is a special food product prepared for their young by mammals or warm-blooded animals. The milk-forming glands, which with rare exceptions are developed only in the females of mammals, are ordinarily active only subsequent to the giving birth to offspring. Occasionally, however, the mammary glands become active in females that have never given birth to young, and in rare instances the milk glands have shown a certain degree of activity in males.

Milk is nearly a perfect food, containing not only protein and fat as do other animal foods, but also carbohydrates, besides salts and vitamins. It is deficient only in iron.

TABLE XXXIX.

Percentage Composition of Cow's Milk and Milk Products.*

\begin{tabular}{|c|c|c|c|c|c|}
\hline & Water & Proteins & Fats & Sugar & Salts \\
\hline Butter & 6.0 & 0.3 & 91.0 & ...... & 2.7 \\
\hline uttermilk & 90.6 & 3.8 & 1.2 & 3.3 & 0.6 \\
\hline heese ...... & 36.8 & 33.5 & 24.3 & ........ & 5.4 \\
\hline ottage chees & 72.0 & 20.9 & 1.0 & 4.3 & 1.8 \\
\hline eam ........ & 66.0 & 2.7 & 26.7 & 2.8 & 1.8 \\
\hline ilk ........... & 86.8 & 4.0 & 3.7 & 4.8 & 0.7 \\
\hline . & 88.0 & 4.0 & 1.8 & 5.4 & 0.8 \\
\hline hey & 93.6 & 0.8 & 0.2 & 4.65 & 0.7 \\
\hline
\end{tabular}

Cow's milk contains about one-eighth its weight of solids, of which approximately one-third or 4 per cent., is fat, 4 per cent. casein and other proteins and 4.5 per cent. lactose or milk sugar. To these are added 0.6 per cent. of lime and other salts. The milk of each species of animals is exactly adapted to the use of the young of the species. In the milk of rapidly growing animals the amount of protein and salts is much greater than in that of animals that grow slowly.

The milk of the cow enjoys in this country almost exclusive favor, but is by no means the universal lacteal food. Milk of various animals is used in different countries. Among the ancient Egyptians the milk of dogs was employed as well as that of the

*Compiled from various sources. 
cow. In various parts of Europe the milk of the goat and the ewe is used quite extensively, and to some extent also the milk of the ass. The milk of the camel is used in Arabia and Persia, of mares in Tartary, of the buffalo in Africa and China, of the yak in Tibet, of the reindeer in Lapland, and the llama and vicuna in South America.

The amount of milk produced in the United States yearly is about $87,000,000,000$ pounds, representing $11,250,000,000$ pounds of dry food substance, which is produced by $24,000,000$ dairy cattle, each of which requires daily 17 pounds of digestible food to produce 456 pounds (equivalent to 1800 quarts) of milk yearly, 13 pounds of dry digestible foodstuff for each pound of dry milk. To support these dairy cattle requires the product of not less than $48,000,000$ acres of land. There can be no doubt that, as meat production diminishes, as it is certain to do, milk production will likewise decrease. There is even at the present time a notable shortage of dairy products and the average per capita production will undoubtedly continue to decrease from the same causes which inevitably lead to a lessened meat production. Some other source of the complete protein needed to supplement the incomplete proteins of cereals and roots must be provided. Fortunately, Nature has supplied us with this all-essential foodstuff in nuts. This is a vitally important fact which sometime will save the race from protein starvation.

If in the case of adults it needs to be supplemented by other foodstuffs, cow's milk, when properly modified, is for the young infant a nearly perfect food. It contains in excellent proportions all the elements needed by the growing child. This is not true of any other substance known.

The fuel element is represented in milk by fat and sugar of milk. The fat is of a sort easily ntilized by the body.

\section{Why Milk Sours While Meat Putrefies.}

The sugar of milk is a special product well adapted to the needs of the body, far superior to cane sugar and free from the unwholesome properties of the products of the sugar cane. It is found nowhere in Nature except in the milk of animals. Milk sugar is slowly digested and is absorbed only one-fourth as rapidly as malt sugar. This enables it more easily to reach the lower intestine, where it is converted into lactic acid and so prevents the putrefaction to which modern science has traced a great number of the maladies of both infants and adults. 
It is due to the presence of lactose that milk sours while meat putrefies. Several years ago (1908), the writer placed in a jar of buttermilk a raw beefsteak with no antiseptic of any sort. The beefsteak is still intact, thanks to the antiputrefactive properties of milk sugar and the acid-forming bacteria it feeds. One reason for this antiputrefactive property of milk is that in the presence of sugar even highly active putrefactive organisms produce harmless acids instead of noxious toxins and ptomaines. This is a most beneficent provision of Nature whereby the normal food of the young infant is kept in a wholesome state while undergoing digestion and absorption in the intestine.

\section{The Special Value of Milk Proteins.}

Milk is of special value in the dietary of human beings because of the fact that its protein is of very superior value. It is not only complete, but is capable of supplying all the elements required for building or repairing the living structures of the body, and is so rich in certain essential elements that it is able to make good the deficiencies in cereals and vegetables. In other words, the proteins of milk may be regarded as super-complete. Experiments have shown (Caspari) that milk protein is superior to meat protein.

It is evident, then, that milk is a foodstuff of high value, since it not only makes available to their fullest value the enormous stores of protein found in wheat, oats and other cereals, but is also able to completely replace flesh-foods and even eggs in the dietary (Sherman).

McCollum considers milk of greater importance than meat in the national bill of fare and attributes to milk rather than to meat the physical superiority of certain meat-eating nations. This writer calls especial attention to the fact that meat-eating nations are also milk-eating. In view of this highly interesting result of recent scientific inquiry it is evident that the dairy interests of the country should be encouraged rather than beef and pork production.

In the use of milk it is important to remember that milk is a perfect food only for young animals of the particular species by which it is produced; that is, cow's milk is exactly adapted to calves, goat's milk to kids, sheep's milk to lambs, and human milk to human infants. The milk of most lower animals differs very widely from human milk in various important particulars, especi- 
ally in containing a much larger amount of protein and a smaller amount of sugar. It is a curious fact that the milk of the mare and the ass approaches very closely in composition to that of woman, having approximately the same amount of protein, sugar and of salts. The milk of mares and asses is, however, deficient in fat, containing only one-third as much as does woman's milk.

Schlossman and Moro have shown that the proteins of human milk and of blood are identical biologically. Says McCollum:

We could entirely dispense with meats without suffering any ill effects whatever, but if we permit the use of milk, even in the diet of adults, to fall much below the present consumption its effects will soon become apparent in our national efficiency.

\section{Milk Rich in Vitamins.}

Another notable quality of milk is its richness in vitamins. These remarkable and magic-working substances are, according to Funk, the discoverer, produced only by vegetables. Each plant produces its own sort of vitamins. The vitamins of milk are not produced by the cow, but are only collected by her.

It should be mentioned, however, that the full value of milk is possessed only by clean milk as it flows from the cow, and not by milk which has been boiled or pasteurized, or doped with alkaline or antiseptics, which destroy the vitamins and deprive the milk of one of its most unique and valuable properties.

\section{Influence of Food of Cow upon the Vitamins of Milk.}

Every farmer knows that both the quality and the quantity of milk produced are influenced by the food of the cow. Scientific research and practical experience have developed in recent years the science of animal feeding to a high state of perfection. The up-to-date farmer knows just what and how much to feed to produce the most milk containing the highest percentage of butter fat. Richness in fat, is not, however, the quality of greatest importance. Of far greater significance is the vitamin content of the milk. Recent investigations have shown that cow's milk varies greatly in the amount of this most essential food constituent. Cows fed upon fresh grass or other fresh green food produce milk rich in vitamins, while the milk of cows fed on dry hay is deficient. Since milk is often the exclusive food of young infants for several months during the most critical development period of their lives, this becomes a matter of greatest importance. 
Infants fed on milk from hay-fed cattle do not grow at the proper rate and may be dwarfed and weakened for life, not only in stature but in other vital respects, even in the development of important internal structures.

Milk inspection should not stop with sanitary conditions and the health of the cow, but should include inspection of the cow's food supplies with reference to the infant's needs. Vitamins are produced only by plants; and if green stuffs are lacking in the food of the cow, they will be lacking in the milk, and the milk fed infant will suffer.

The feeding of sprouted grains to cows is an excellent means of increasing the vitamin content of their milk. Bottle-fed babies always require orange or tomato juice to insure an ample supply of vitamin, even when fresh, unpasteurized milk is used.

\section{Milk Rich in Salts.}

Cow's milk is very rich in salts, containing four times as much of these mineral elements as does mother's milk. Milk contains more lime than is found in lime water. Note the contrast in this regard between milk and beef. Meat supplies only half a grain of lime to the pound, although containing twice as large an smount of solids as does milk. The reason for this is obvious. Milk is a substance provided by Nature as an exclusive food for a growing animal, and so must furnish lime for the bones as well as protein for the muscles. Meat represents but a fraction of the original foodstuff. (When corn or other food is eaten by an ox the several elements are separated, each going to form its own tissues-fat to fat, muscle to muscle, and lime to the bones. So to get back the whole assortment of food principles fed to an animal one must eat it entire body, the whole ox, or the whole hog, bones and all. This being impossible, kind Nature has supplied us in milk with bones, muscles, brains, nerves, every bodily structure in solution and in attractive form. Milk, in other words, is liquid flesh and bones with the tissue wastes, putrefaction products, parasites and other objectionable features left out.

\section{The Lime Content of Milk and Cheese.}

This group of foodstuffs is of unrivaled value as a source of food lime. All dairy products with the exception of butter are very rich in salts of lime. Pure fat is, of course, free from lime, 
which is chiefly associated with the protein or casein of the milk. But butter is not pure fat. It retains a portion of the buttermilk and so has a smali lime content, which is, however, too insignificant to deserve consideration in the planning of a balanced bill of fare.

Milk, in some form, is one of the most convenient and reliable of all sources of food lime. Whole milk contains threequarters of a grain of lime to the ounce. This is only half the amount found in almonds and filberts; but while these nuts can be utilized only to the extent of a few ounces daily, the milk intake may be raised to several quarts.

A pint of milk contains 12 grains of food lime of finest quality and 20 ounces of whole milk will supply a whole day's ration of food lime. Skimmed milk is a little richer in lime than whole milk, a fact which in itself condemns the very common waste of skimmed milk in connection with creameries. Considering the great lack of lime in the national dietary it is a serious question whether the feeding of skimmed milk to domestic animals should not be discontinued. The $87,000,000,000$ pounds of milk produced annually in this country contain lime enough to supply $200,000,000$ persons.

We are rapidly becoming a toothless nation because of the lack of lime in our national bill of fare. This deficiency may be easily made up by the proper utilization of our dairy products and a suitable selection of foodstuffs. Cattle and hogs can easily obtain their lime from grass and other foodstuffs, to the utilization of which their digestive organs are specially adapted, so that they can consume the large bulk necessary.

In the use of milk, man adapts to himself the choice lime salts which the mother cow has laboriously garnered from the fields and meadows and prepared in concentrated form for the feeding of her calf. Associated with the lime in whole milk is found also a choice collection of vitamins which promote growth, so that milk not only furnishes the material needed for building and maintaining the body frame work, but supplies an activating hormone which insures the proper utilization of the building material at hand.

It is interesting to note that a tablespoonful of milk supplies nearly the same amount of lime as an egg yolk, while a little more than half a pint of skimmed milk ( 9 ounces) supplies as much lime or bone building material, as a dozen eggs. 
While skimmed milk contains more lime than whole milk the vitamin content is much less, a large portion being held in solution by the butter fat which has been removed. But the chief values of milk are left after the fat has been removed, and the great economic waste and physiologic danage which have resulted from the past failure to utilize this by-product of our great dairy industry should be stopped as speedily as possible.

Buttermilk has a lime value almost equal to that of whole and skimmed milk, a pint and a half affording a full day's supply of lime. Sour milk commonly sold as buttermilk and under various trade names is fermented or soured skimmed milk and hence has the same lime content as skimmed milk.

Cream is not by any means the equivalent of milk as a source of lime. An ounce of cream contains only two-thirds as much lime as the same quantity of whole milk. Nearly a quart of cream is necessary to supply a day's ration of lime on account of the larger amount of fat present.

But it is in cottage cheese that we find the richest and readiest means of increasing our lime intake. The solids of milk constitute one-eighth of its bulk and weight. Consequently, the elimination of the greater part of the water in the process of cheese making results in a great concentration of the lime content. Ordinary cheese has a lime content of 5.7 grains per ounce, or eight times the amount in whole milk, more than any other foodstuff. An ounce of average cheese supplies as much lime as seven yolks or five entire eggs, five pounds of beefsteak, or half a peck of potatoes.

Old cheese is not easily digested by many persons. The butyric acid which it contains in considerable amount when old and strong, excites the gastric glands to secrete an excess of hydrochloric acid, and so gives rise to heartburn, a common symptom following the free eating of cheese. There are in old cheese various more or less toxic products, the result of the activities of the numerous molds, yeasts and germs which co-operate in the production of cheese, to say nothing of the "mites," "skippers" (maggots) and other scavengers which are usually found in "ripe" specimens of cheese.

In view of these discouraging facts, it is pleasant to find that the simple cottage cheese which the farmer's wife prepares on short notice in her own kitchen from the well skimmed sour milk of her milk house, possesses all the good qualities of ordinary 
cheese while free from all its objectionable features. The only inconvenience is that it must be freshly prepared unless kept in an ice box. Cottage cheese supplies 4.2 grains of lime per ounce, and hence three and three-fourths ounces of cheese will furnish a day's lime ration.

Yogurt cheese, a cream cheese prepared by a process modified by the writer from that employed in making the famous camembert cheese, omitting the green mold and using pure cultures of the $B$. acidophilus, has all the advantages of cottage cheese and will keep for months if in a cool place. This dairy product is a complete and satisfactory substitute for ordinary cheese for all but those who have developed a connoisseur's appetite for the putrescent aromas of limburger and gorganzola.

Even whey, a by-product of cheese making which usually goes down the sewer, possesses no mean value as a source of nutritive lime salts. There are in the whole category of foodstuffs few, in fact, which are so rich in lime as is whey, which contains more than one-fourth grain to the ounce. The lime content of whey is greater than that of any cereal, three times as great as that of egg whites, and four times as much as that of cornmeal or rice. A large glassful of whey contains as much food lime as a large serving of rice. The same amount of whey contains as much lime as two pounds of beefsteak and a pint is equal in lime content to two-thirds of a dozen eggs. Three pints a day, taken as a beverage in place of beer, for example, would supply nearly a day's lime ration, as much as would be furnished by a fifty gallon cask of beer.

\section{The Iron Content of Milk.}

The milk of all animals is notably deficient in iron. The milk of woman contains only a trace of iron for the reason that in human infants, as well as in the young of all animals that have a comparatively long nursing period, iron is before birth stored up in large amount in the liver. The liver of the new born child contains iron enough to furnish an adequate supply for blood-making until the appearance of teeth enable it to make use of iron-containing foods. The same is true of the calf. It is to be noted, however, that in the case of the calf the possession of teeth enables the young animal to begin securing supplies of iron from other sources than its maternal food within a few days after its birth. On this account cow's milk contains but a very small pro- 
portion of iron, less than one thirtieth of a grain to the quart, or one-third as much as breast milk.

Evidently milk is a food naturally adapted to very young animals whose livers are able to supplement the iron of the food intake. An adult fed upon milk exclusively soon becomes anemic because of the exhaustion of his small reserve store of iron. It is not possible to obtain a full ration of iron from milk alone without taking a great excess of food. An ounce of milk contains less than half of one per cent. of a day's ration of iron $(0.47$ per cent.), so that more than thirteen pints, or six and one-half quarts of milk are barely sufficient to furnish one day's iron supply. This represents a food intake of 4,400 calories, or nearly twice an ordinary day's ration. For this reason, milk feeding should always be accompanied by an abundant supply of greenstuffs and fruits rich in iron but with a small food value, such as spinach and other greens.

When man lived in his nornfal primitive state, subsisting upon his natural or biologic bill of fare, there was no occasion for the use of milk by adults. Everything he needed was supplied to him by the vegetable kingdom, as is still true respecting some primitive forest-dwelling men but in his present artificial, so-called civilized state man has so far departed from his normal environment and has changed and denatured his food supply to such an extent that his diet has become deficient in many particulars and dangerously unbalanced. This situation has created a pressing need for certain things which are found in milk in rich abundance, especially lime, complete protein, and growth stimulating vitamins. Hence, notwithstanding its deficiency in iron, cow's milk and dairy products are highly important articles of food and render invaluable service in balancing the bill of fare by contributing essential salts and vitamins which the cow collects from the wild grasses of the pasture and other foodstuffs rich in lime and vitamins.

Fresh cream cheese and cottage cheese contain nearly four times as much iron as does milk, and thus, when freely used, make a distinct contribution to the iron intake.

\section{Dried Milk.}

Milk powder, or dessicated milk, consists of either whole milk or skimmed milk which has been reduced to a powder by removal of the water content by either one of several processes. 
The best process devised up to the present time consists in forcing the milk, in the form of a fine spray, into a heated vacuum chamber. The water contained in the minute droplets of milk is at once vaporized, leaving the solids to fall to the bottom in the form of a fine powder. The addition of water to powdered milk in the proportion of one part of milk to seven or eight parts of water restores the milk to a condition almost identical with that of fresh milk.

Dried milk possesses all the nutritive properties of fresh milk except that it is deficient in the scurvy-preventing vitamin, and so should be supplemented by orange or tomato juice.

The experiments of Chick and others have shown that the value of the antiscorbutic vitamin of milk is reduced at least one-half by drying, twice as much dried milk as raw milk being required to prevent scurvy in guinea pigs and monkeys.

\section{Cream.}

Cream consists of the fat of the milk with a certain proportion of casein and a small amount of salts and milk sugar. The fat of cream is in a state of emulsion and for this reason more readily mixes with the digestive fluids and is usually more quickly digested than is butter, oils or other animal or vegetable fats.

\section{Butter.}

Butter is the most important of all the animal fats. It is superior to vegetable fats in the fact that it is rich in vitamins which are derived from the milk. Vitamins are for the most part absent from vegetable fats. They are sparingly found in olive oil, cotton-seed oil, corn oil, peanut oil, and are absent in lard and found only to a small extent in other animal fats, with the exception of cod liver oil, owing to the fact that vitamins are stored in the animal body in the liver, kidneys and other glands. Cod liver oil contains vitamins, which at last explains the benefit Iong and widely attributed to this special oil in cases requiring improved nutrition. It is to be noted, however, that ordinary cream and butter possess all the advantages of cod liver oil in this particular besides being palatable instead of repulsive. When oleomargarine, nut margarine, vegetable oils or any other fat is used as a substitute for butter, free use should be made of milk to make certain that the body receives daily the due supply of 
vitamins, without which all the vital processes will languish and grave diseased conditions, which may even lead to death, are certain to follow.

\section{Butter Color.}

Good butter has a natural and decided yellow color. There is reason for believing that pale butter, which requires the use of annatto or some other coloring matter to give it the usual color, is lacking in vitamins, since Stenback and Bontwell have shown that the fat-soluble A vitamin, the absence of which gives rise to rickets, is often, if not always, associated with the yellow color found in yellow corn, carrots and green herbage.

The milk of cows contains more butter in the winter than in the summer, but winter butter is pale because winter food is largely lacking in the yellow pigments, carotin and xanthophyll, which abound in meadow grass, in which the pigments are associated with chlorophyl.

\section{Cheese.}

Recently-made cheese, particularly cream cheese and cottage cheese, is wholesome food. The so-called ripening of cheese in no way improves it, but rather develops objectionable qualities. The ripening process is carried on by means of molds, yeasts and bacteria which are present in the milk or added to it in the process of manufacture. Cheese cannot be made from sterilized milk without the addition of bacteria. Fresh cheese, particularly cottage cheese, is a valuable addition to the dietary as a source of lime. As a rule, the food contains a sufficient amount of protein without the addition of cheese, even in the absence of meat, but lime is very frequently deficient and may be conveniently added in the form of cottage cheese. Four ounces of cottage cheese will supply a day's ration of food lime.

\section{Cheese Bacteria.}

Professor Adametz made some years ago an interesting study of cheese, in which he identified 19 varieties of bacteria which are ordinarily active in the ripening of various sorts of cheese. Some of these bacteria are furnished by the milk, others are derived from the vessels in which the milk is kept, still others from the air and from the rennet ferment added to the milk in the process of cheese making. In certain cases molds or other 
fungi are added, as in the case of camembert cheese. Adametz estimated the number of bacteria in cheese to be about $25,000,00 \mathrm{C}$ germs to the ounce. Roquefort cheese is made by adding to the curd of milk mold from a dough made from barley flour, with which yeast and sometimes vinegar have been mixed. The mold which forms on this dough when allowed to stand is rubbed into a powder and added to the milk. Gorgonzola and stilton cheese are made by the same process. The mold consists of several varieties of fungi, one being the common green mold frequently found in bread and other food kept in warm moist places. Einhorn has shown that molds sometimes take up their abode in the stomach, form colonies on the mucous membrane, and thus become a cause of grave disease.

Cream or cottage cheese, when freshly made, is more wholesome than meat and more nutritious. According to Williams, 20 pounds of cheese contain as much nutrient as the carcass of a sheep weighing 60 pounds. Cheese contains both more fat and more protein than beef and less than half as much water. The energy value of an ounce of cheese averages about 135 calories, while that found in an ounce of porterhouse steak is 70 . Even cottage cheese made from skimmed milk has more than half the food value of a round or sirloin steak of the same weight. Cheese is also rich in all the essential vitamins, which are lacking in meat, and contains a rich store of food lime, which is almost altogether absent in meat. The dietary of the average American will be greatly improved by discarding meats and substituting cottage cheese or cream cheese.

Since the ripening process in cheese-making is really a process of decomposition due to the action of bacteria, yeasts and molds, all ripened cheese contains considerable quantities of decomposition products.

Examination of a limburger cheese in the bacteriological laboratory of the Battle Creek Sanitarium (Roderick), showed 18,000,000 bacteria per gram $(540,000,000$ per ounce). The bacteriologist noted that the bacteria were of the same character as those found in the human intestine.

Indol is usually found in limburger cheese and may occur in camembert cheese. Carbolic acid, a common product of putrefaction, is found in limburger. The older the cheese, that is, the longer the "ripening" process has continued, the larger the amount of toxic products present. During the early stage of the ripening, 
the growth of putrefactive organisms is prevented by the presence of milk sugar, but this disappears after the first few days as the result of the action of lactic acid bacilli upon lactose, and the decomposition changes in the milk protein, or casein, to which the softening of the cheese is due, proceeds at a rate depending upon the temperature to which the cheese is exposed.

Ordinary cheese, especially old cheese, such cheeses as camembert, roquefort, limburger and other varieties of highly flavored well-ripened cheese, are wholly unfit for human food and are positively dangerous for use by persons suffering from diseases of the liver or kidneys. It should be remembered that every person suffering from disease of the gallbladder or who has had one or more attacks of jaundice, has a more or less damaged liver. In fact, in all cases of chronic intestinal toxemia, the integrity of the liver is more or less impaired.

A person with sound digestion and a liberal supply of gastric juice may be able to eat a moderate amount of cheese with impunity, but persons suffering from achylia, and hence lacking the protective, disinfecting influence of the gastric juice, and even healthy persons who eat very freely of well-ripened cheese, are likely to suffer from intestinal autointoxication. The bacteria present in the cheese find in the intestine conditions favorable for development and by their rapid growth may give rise to diarrhea and even symptoms resembling cholera morbus. The writer has encountered several cases of this sort, one of which ended fatally.

\section{Skimmed Milk.}

Milk which has been skimmed, especially separated milk, has been deprived of one of its most precious constituents, a highly valuable fat. Butter fat as present in milk is in a state of fine emulsion, a condition which greatly aids its digestion and assimilation, and contains a very precious vitamin, fat-soluble $\mathrm{A}$, or antirachitic (anti-rickets) vitamin.

The suggestion of some writers that skimmed milk may be used in place of full milk as a measure of economy, provided that margarine or some other fat replaces the fat removed, is bad advice. Skimmed milk has a considerable nutritive value, but it lacks the growth-promoting power of full, fresh milk, and on this account cannot wholly take its place. When skimmed milk is used, it should not be used as a substitute for full milk but as an addition to the regular daily supply of full milk which every child 
should have. It may also be used as an added source of lime salts. It may be employed in cooking as in the preparation of bread, puddings, etc.

Skimmed milk may be used without detriment in connection with a liberal supply of butter. Used in this way, the skimmed milk now thrown away or fed to pigs, if fed to human beings, would be worth more to the country than all the meat produced by the live stock industry.

\section{Canned Milk.}

Sekina, in experiments on white mice, found that canned milk in time gave rise to anemia and beri-beri. The mice did well for 100 days, then rapidly failed. The addition of iron and the water-soluble vitamin quickly restored them to the normal condition. Canned milk and condensed milk are thus shown to be unsuited to serve as staple foods for infants because of the lack of iron and the water-soluble B.

Canned milk may be advantageously used in connection with other foodstuffs as a source of complete protein, but should never be used as the chief food for any length of time unless supplemented by orange or tomato juice to supply vitamins and purée of spinach to furnish food iron.

\section{TABLE XL.}

Average Percentage Composition of Condensed Milks (Mohan).

\begin{tabular}{|c|c|c|c|c|c|c|}
\hline & $\begin{array}{l}\text { Solins } \\
\text { Per } \\
\text { eent. }\end{array}$ & $\begin{array}{l}\text { Ash } \\
\text { Per } \\
\text { cent. }\end{array}$ & $\begin{array}{l}\text { Fat } \\
\text { Per } \\
\text { cent. }\end{array}$ & $\begin{array}{c}\text { Protein } \\
\text { Per } \\
\text { cent. }\end{array}$ & $\begin{array}{c}\text { Lactose } \\
\text { Per } \\
\text { cent. }\end{array}$ & $\begin{array}{c}\text { Cane } \\
\text { sugar } \\
\text { Per } \\
\text { cent. }\end{array}$ \\
\hline Condensed whole milk......... & 72.6 & 1.6 & 10.0 & 8.0 & 12.0 & 41.0 \\
\hline Condensed skimmed milk.... & 70.0 & 2.0 & 1.0 & 10.5 & 14.5 & 42.0 \\
\hline Evaporated milk.................... & 26.3 & 1.6 & 7.9 & 7.7 & 9.1 & ....... \\
\hline Whole milk powder............ & 96.3 & 5.6 & 26.8 & 32.0 & 31.9 & \\
\hline Skimmed milk powder.. & 91.7 & 6.9 & 1.7 & 33.8 & 49.3 & \\
\hline
\end{tabular}

Cane sugar to the amount of 16 to 19 pounds is usually added to 100 pounds of raw milk. The condensing is done by heating for about 2 hours at $140^{\circ} \mathrm{F}$. Condensed milk contains too much cane sugar to be suitable for use as an infant food.

\section{Buttermilk.}

Buttermilk and sour milk have essentially the same composi- 
tion. The sour flavor is produced by harmless lactic acid-forming bacteria. Buttermilk contains essentially everything which whole milk contains except the fat, which is reduced to about one-fourth of the ordinary amount. Buttermilk is more easily digestible than sweet milk. The acid-forming bacteria found in ordinary sour milk are of no particular value in changing the intestinal flora for they are unable to live and grow in the intestine. When buttermilk is desired for correcting biliousness or changing the intestinal flora, it should be prepared with the B. acidophilus. A quart or better, three pints of acidophilus buttermilk taken daily for a week or two will usually produce a marked change in the character of the stools, causing the disappearance of the putrid odors through the suppression of putrefactive processes in the intestine.

Buttermilk ice cream is greatly to be recommended in place of ordinary ice cream. It contains but a small proportion of the fat found in ordinary ice cream and hence is much more easily digestible. Buttermilk ice cream is also practically free from any danger of tyrotoxicon poisoning, which occasionally occurs from ice cream made in the usual way.

\section{Whey.}

The fluid residue left in the process of cheese making contains the milk sugar and much of the mineral matters of the milk, the casein or protein and fat having been removed. Whey is also rich in vitamins. It may be freely used to great advantage by persons who have long subsisted upon a dietary insufficient in lime. The composition of whey is as follows (Hutchinson):

\begin{tabular}{|c|c|}
\hline Water & 93.64 per cent. \\
\hline Protein & 0.82 per cent. \\
\hline Fat ………................ & 0.24 per cent. \\
\hline Milk sugar & 4.65 per cent. \\
\hline Mineral matters, lime, etc.......... & 0.64 per cent. \\
\hline
\end{tabular}

The whey cure has long been practiced at various health resorts in Europe. The whey is not used alone, but in connection with other foodstuffs, particularly with a mixed diet from which flesh foods are largely excluded. The patient usually begins by taking a tumblerful of whey night and morning. The amount is increased from day to day until 10 tumblerfuls are taken daily. The whey is taken either warm or cold, plain or aerated. It is 
recommended by Gautley as a substitute for milk in feeding typhoid fever patients. The caloric value of whey is small, only 7 calories per ounce. By the addition of malt sugar or cereals, the food value of whey may be increased to any desirable extent. By adding 3 ounces of malt sugar or lactose to a pint of whey, the energy value is increased to 25 calories to the ounce, or 400 to the pint, a good carbohydrate food.

\section{Modified Milk.}

When employed in the artificial feeding of infants and in very many cases in the feeding of invalids, cow's milk must be modified. Ignorance of this fact and of the proper method of feeding milk is responsible for the death annually of a great multitude of artificially fed infants. Of the 2,500,000 infants born in the United States anmually not less than 250,000 die as the result chiefly of improper feeding. The mortality of bottlefed infants is more than four times that of breast-fed infants. Cow's milk differs very decidedly from mother's milk. It contains four times as much lime and three times as much protein and only two-thirds as much sugar. Protein and fat are the elements of cow's milk which are the greatest source of trouble to the human infant.

Cow's milk contains a large amount of protein and lime to support the rapid growth of the calf which attains puberty at the end of two years, about one-seventh of the time required for the human infant to reach the same stage of development.

Various formulas have been devised and recommended for the modification of cow's milk in artificial feeding. The most of these are more or less complicated. Recent experience has shown that a very simple method is much superior to the complicated measures which have been developed. It is only necessary to add two things, water and milk sugar or malt sugar, to render cow's milk suitable for the use of very young infants. A good formula is equal parts of full milk and boiled water with an ounce of malt sugar for each pint of water added to the milk.

\section{Milk Must Be Clean.}

The chief reason assigned for the pasteurizing or sterilizing of milk is the presence in the milk of large or small quantities of filth whieh should have-been-left in the-otable-or the barnyart. Combe and others have shown that the germs associated with this 
putrefying filth are the most prolific source of diarrheas and other intestinal disorders which annually carry off so many thousands of infants during the summer months. These same putrefactive germs are likewise the cause of intestinai toxemia or autointoxication.

As received from the cow, milk may or may not contain bacteria. In general, the milk of a healthy cow, if removed from the animal with sufficient care, will be found to be absolutely free from bacteria and if put into a proper container will keep without spoiling for an indefinite period. Certified milk must contain less than 10,000 bacteria to the cubic centimeter (about one-fourth of a small teaspoonful). Ordinary commercial milk contains from 100,000 to several million bacteria to the cubic centimeter and is absolutely unfit for use. The number of bacteria found in milk rapidly increases. For example, in a specin:en of milk examined 24 hours after milking the number of lacteria had increased from 100,000 to $5,600,000$.

By the exercise of sufficient care in dairy management the number of bacteria count may be kept below 1,000, and such harmful bacteria as Welch's bacillus, and other putrefactive organisms, as well as the specific organisms of typhoid, tuberculosis and other infectious organisms may be practically excluded. The cost of clean milk will, of course, be greater than the dirty product now offered to the public; but the superior value of clean milk will far more than balance the extra cost. The model (lairy of the Battle Creek Sanitarium has for years demonstrated the practicability of producing clean milk on a large scale at a reasonable cost. In this the bacterial count is often kept below 1,000 even in summer weather.

\section{Sour Milk.}

The souring of milk is due to the development of lactic acid through the growth of acid-forming bacteria. There are many varieties of acid-forming bacteria which readily find access to milk, and as they grow rapidly at ordinary temperatures milk will naturally sour within a few hours after milking unless the temperature of the milk is at once lowered to a point at which the growth of the bacteria is prevented.

The $B$. acidophilus is a special acid-forming organism which not only grows and thrives in milk but is also able to live in the colon of animals. This is known to be true of the B. acidophilus, 
which is by some thought to be a variety of the B. bulgaricus. It is for this reason that buttermilk prepared with the B. acidophilus is to be preferred to ordinary buttermilk since ordinary buttermilk or sour milk germs will not grow in the colon and hence are of no value in combating putrefaction.

\section{Bitter Milk.}

The bitterness of milk may be due to the fact that the cow has eaten substances having a bitter flavor. A bitter flavor also results from changes in the milk produced by certain germs. Such milk should be rejected.

\section{Slimy and Colored Milk.}

Slimy or ropy milk owes its peculiarity to the growth of a special micro-organism which causes viscosity.

There are various germs which color the milk through the production of characteristic pigments.

\section{Bacteria on Milk Bottles.}

The city health department of Baltimore, Maryland, made a study of the bacteria deposited on the caps and rims of milk bottles. In the handling of milk bottles by milk distributors and their exposure to city dust in milk wagons and while waiting upon doorsteps to be taken in, germs of various sorts, some dangerous to life, are deposited in considerable numbers. Germs, even very dangerous germs, are sometimes conveyed to the lips of milk bottles by the hands of milkmen. Other germs, such as typhoid and dysentery germs, are deposited by flies. Contamination may occur through contact of animals with milk bottles. Cats and dogs often lick the mouths of milk bottles while standing on the doorstep.

The examinations made of numerous milk bottles showed various sorts of dangerous germs and indicated that there is a real risk in the use of milk from containers in themselves thoroughly clean and sanitary. The milk becomes contaminated by contact with the lip of the bottle when it is poured from the bottle. The cap and lip of the milk bottle should be very carefully wiped with a cloth dipped in boiling water or peroxide of hydrogen before the milk bottle is opened. The importance of doing this should be known to every housewife and should never be forgotten. 


\section{Infections Due to Unclean Milk.}

Milk must be free from the germs of disease. In addition to the common organisms which give rise to putrefaction and with which the milk becomes contaminated through careless dairy methods, milk may contain germs of various specific diseases such as tuberculosis, typhoid fever, diphtheria, scarlet fever, sore throat, Malta fever-maladies originally derived from human beings suffering from the above named disorders and with the germs of which the milk, by direct or indirect contact, becomes contaminated.

Milk may also communicate to human beings various disorders which originate in cattle, but which may be communicated to human beings by making use of the milk of sick animals, such as foot and mouth disease, milk sickness, gastroenteritis, anthrax, cowpox, rabies, actinomycosis and perhaps other maladies.

\section{Infected Milk a Cause of Tuberculosis.}

Modern research has shown that bovine tuberculosis is communicable to human beings. According to Rosenau, it must be conceded that not less than 5 to 7 per cent. of all cases of human tuberculosis are due to infection from the use of infected milk or the flesh of tuberculous animals.

A careful examination of the mortality tables published by the United States Census Bureau shows that not less than 3,000 children die annually as the result of infection with bovine tuberculosis, not less than 60,000 children are constantly suffering from bovine tuberculosis contracted chiefly through the use of diseased milk.

The New Jersey Tuberculosis Commission found 16 per cent. of the dairy cattle in that state suffering from tuberculosis. In some parts of Germany 30 per cent. of all cattle were found to be infected with this disease. An investigation made of the milk supply of the District of Columbia showed that 15 to 25 per cent. of all the cows furnishing milk to that community were infected.

Tubercle germs are not readily killed by dairying processes. Schroder killed guinea pigs by infection with germs found alive in butter five months after it was churned. Tubercle germs have been found in great numbers in cheese and ice cream. Morgenroth even found tubercle germs in 9 out of 28 samples of oleomargarine purchased in the open market. 


\section{Injurious Effects of the Sterilizing or Pasteurizing of Milk.}

The public has been taught to place too much faith in sterilized or boiled milk. It is true that pasteurization or boiling of milk destroys certain specific disease-producing organisms such as those of typhoid fever, tuberculosis and diphtheria, but these processes at the same time destroy certain highly essential, vital properties of milk, and as has been long known, fail to destroy the spores of putrefactive organisms, which probably are, on the whole, the cause of far greater mischief and many more deaths than the organisms which give rise to tuberculosis, typhoid fever and other specific organisms. If left to itself, cow's milk does not decay but sours. Boiled milk rots. The acid-forming organisms which find their way into the milk from the air thus exercise a protective influence, preventing the toxemia which results from intestinal putrefactions. When an infant is fed upon sterilized milk, the stools, which are naturally slightly acid, quickly become foul smelling through putrefaction and the infant is thus exposed to highly potent disease-producing influences against which it is protected when fed upon natural, clean milk. A temperature of $240^{\circ} \mathrm{F}$. for half an hour is required to destroy the spores of putrefactive germs and even such milk is likely to promote putrefactive processes in the intestine, especially in the case of young children. It is thus apparent that pasteurization and boiling of milk should be regarded only as makeshifts which mitigate to some degree the evils resulting from the use of milk contaminated with barnyard filth. The movement to provide certified milk should be everywhere encouraged.

Whenever pasteurized or sterilized milk is used, free use should be made daily of orange juice, tomato juice, potato soup or some other foodstuff rich in vitamins.

The boiling of milk modifies in a harmful way nearly all its ingredients and considerably reduces its nutritive value. Rats fed on boiled milk grow to only half their normal size.

Scurvy sooner or later appears in babies exclusively fed on pasteurized or boiled milk. The subtle alchemy by which milk is prepared in the laboratory of Nature is upset by the crude process of cooking. Boiled milk will sustain the life of rats but it will not enable them to grow to full development, and reproduction fails altogether. 
Another defect of the pasteurizing process is found in the fact that it is not absolutely certain. Cases of tuberculosis have occurred in calves fed on the pasteurized milk of tuberculous cows.

Another matter of much significance which must be borne in mind in relation to pasteurized milk is the rather surprising fact that, if not handled with very great care, pasteurized milk is likely to develop within a short time more bacteria of a dangerous type than are found in ordinary raw milk. The reason for this is that most of the bacteria found in ordinary raw milk are of the acid-forming sort; that is, they are of the kind commonly known as buttermilk or sour milk germs, so-called friendly or protective germs. So long as these bacteria are dominant, the growth of putrefactive organisms is prevented.

Boiling, if thoroughly done, may destroy the germs of typhoid, tuberculosis, and other infectious diseases. It does not, however, destroy the spores of the germs which produce putrefaction and consequently, if boiled milk is allowed to stand for a few days these undestroyed spores develop and putrefactive changes take place.

It should be noted, also, that in the boiling of milk the germs which produce souring are destroyed. Consequently boiled milk is less likely to sour than unboiled, but instead may undergo putrefactive changes, and the same thing that happens in the laboratory test tube or the milk bottle may happen in the intestine; that is, boiled milk is more likely to undergo putrefaction in the intestine than is raw milk.

Among the numerous dangerous organisms derived from stable filth and found in unclean milk is the highly virulent Welch's bacillus, the cause of gas gangrene. Estey found this germ present in all but two of a large number of samples of market milk obtained in Providence, Rhode Island. It is often present in commercial milk.

In a recent bacteriological study of milk, Roderick of the Battle Creek Sanitarium laboratory found Welch's bacillus present in 54 per cent. of 470 specimens of commercial milk examined. This dangerous germ being a spore bearer is not destroyed by pasteurizing or even by boiling. Scrupulous care in dairy practice is the only possible protection against this highly dangerous organism against which every housewife should be warned.

It is evident that pasteurization does not solve the milk prob- 
lem. When scientifically done, pasteurization does mitigate some of the evils associated with unclean milk but the only true solution for the problem is clean milk.

It is also known that boiled milk is less easily digestible than raw milk. Hence it is important to emphasize the fact that milk should be produced in such a way as to be in the highest degree possible free from bacteria and other impurities of every sort. In an experiment in feeding of calves with boiled milk between 80 and 90 per cent. of the calves died, and there can be no doubt that thousands of human infants have lost their lives from the same cause. Whenever boiled or pasteurized milk is used for feeding infants or young children or as an exclusive diet for invalids, orange or tomato juice must be freely given to supply the deficiency of vitamin C. It is, in fact, safer to use orange juice for all bottle fed infants even when certified milk is used for the reason that raw cows' milk is often deficient in both B and C vitamins.

\section{Certified Milk.}

Certified milk is milk produced under special conditions which are calculated to insure freedom from disease and germs. The following are the requirements as laid down by the American Association of Medical Milk Commissions:

(a) Certified milk shall be produced by a trustworthy dairyman in accordance with a code of requirements prescribed by a medical milk commission. The dairyman shall enter into a legal contract with the commission, in which he shall agree to comply with all its requirements.

(b) Certified milk shall be obtained from healthy, tubercul in tested cows under veterinary inspection; all persons who directly or indirectly come in contact with the milk shall be under medical supervision and the milk itself shall be subjected periodically to bacteriological, chemical and other tests.

(c) Certified milk shall be free from harmful germs and shall contain relatively few of the common bacteria. It should not contain more than 10,000 bacteria per cubic centimeter; this average should be based upon bacteriological examinations covering a period of ninety days, and the counts should be made at least once a week during this time.

(d) Certified milk must be bottled at the point of production, rapidly chilled, kept cold and delivered promptly to the consumer. After it is once chilled, the temperature of certified milk should at no time go above $45^{\circ} \mathrm{F}$, or below $32^{\circ} \mathrm{F}$. 
(e) Certified milk shall be normal milk; that is, neither heated, frozen nor altered in any way except strained and cooled.

(f) Certified milk shall be of uniform quality and contain not less than 3.8 per cent, nor more than 4.2 per cent, of fat, unless it is labeled otherwise, in which case it shall not vary more than 0.2 per cent, from the amount stated on the label.

(g) Certified milk shall not be subjected to the action of heat; shall not be subjected to the action of any preservative whatever except cold; shall not be subjected to the action of light, electricity, pressure, or any special force or agency of any kind for any purpose; no substance of any kind shall be added to the milk for any purpose; and no part of the milk shall be removed.

Pastures or paddocks to which cows have access shall be:

(a) Free from marsh or stagnant pools.

(b) Crossed by no stream which might have easily become dangerously contaminated.

(c) A sufficient distance from offensive conditions to suffer no bad effects from them.

(d) Free from plants which affect the quality of the milk deleteriously.

It is highly desirable that associations for the production of certified milk should be formed in all parts of the United States so that safe milk may be provided, especially for children and invalids. As a matter of fact, the entire milk supply of the country ought to be produced under certified conditions. Milk is wholesome only when clean, and it is to the highest degree absurd to accept the ordinary commercial product as it reaches the average consumer at the present time. Water containing onethousandth part as many germs as are usually found in milk would be at once condemned as unfit for use.

The importance of clean milk can scarcely be exaggerated and cannot be too strongly insisted upon. Notwithstanding all that has been spoken and written on this subject within the last 25 years, the apathy of the general public in reference to the character of the milk they use is truly amazing. This apathy and the ignorance of which it is the result are responsible for thousands of deaths annually and for an enormous amount of morbidity not only in infants but in older children and adults.

The importance of thorough cleanliness as a condition of healthy human life is only just coming to be appreciated. During the long ages of savagery from which the human race has only recently begun to emerge, we acquired a great number of filthy practices which as yet we have only in part eliminated. In the savage state our vital resistance was so high we were able to 
maintain good health notwithstanding our intimate contact with pernicious bacteria of various sorts because of the high resistance of our tissues; but under the conditions imposed by civilized life, especially house dwelling and pernicious habits in eating, drinking, etc., our vital resistance has been greatly reduced; so we have become a prey to a great number of micro-organisms which to our tougher forebears of prehistoric times were innocuous.

In very recent times we have learned the importance of clean water, and by applying this knowledge we have practically eliminated typhoid fever and other water-borne diseases from the mortality tables. The slightest taint or odor in water or the slightest suspicion of filth contamination leads to an appeal to the health authorities, and a report that the water submitted for examination contains one or two hundred colon bacteria per cubic centimeter will lead to its prompt condemnation as unfit for use.

No one has ever offered any reason for believing that colon germs in milk are any less unwholesome than in water; and yet average commercial milk contains a hundred times as many bacteria as would be regarded as sufficient to condemn water as quite unfit for use.

The pasteurizing and sterilizing of milk certainly mitigates the evils of unclean milk to some degree; but it is a mistake to accept pasteurization as the solution of the clean milk problem. Pasteurization lessens the liability of tuberculous infection, but this is about the only real service it renders and in this it is by no means 100 per cent. efficient. Dr. Schloss of the Harvard Medical School, from a very extended experience in connection with the infants' hospital, insists that ordinary pasteurization does not adequately protect against tuberculosis and requires that all milk given to infants and young children shall be boiled three minutes. Mr. C. W. Barron has also demonstrated the inefficiency of pasteurization as a protection to calves. The only real protection against tuberculosis must be sought in thorough and continuous testing and inspection of dairy cattle by qualified experts.

So far as typhoid is concerned, cases of this disease are now becoming so rare that protection against it is scarcely needed. About the only thing that pasteurization does is to destroy the bacteria which cause the souring of milk and so to increase its keeping properties. But even this has its disadvantages, for Esty has shown that the mischievous B. Welchii which is generally found in market milk does not germinate in raw milk but germi- 
nates rapidly in sterilized milk and sporulates in the intestine. Within two days after giving a guinea pig milk infected with $B$. Welchii spores were found in the feces.

It is evident, then, that aside from partial protection against B. tuberculosis, pasteurization affords little advantage, from a hygienic standpoint, for ordinary milk-souring germs are not at all dangerous or even unwholesome, while, on the other hand, pasteurizing milk enables B. Welchii to germinate and multiply, and sometimes to such an extent as to make the milk a rich culture of this pathogenic organism.

It seems to the writer high time that more attention should be given to the character of the bacteria of milk, rather than to mere numbers. The millions of acid-forming organisms in buttermilk are harmless, whereas a few thousand B. Welchii are not only an evidence of gross contamination but also a menace to health since this organism easily becomes domiciled in the intestine and flourishes amazingly under the anerobic conditions found in the colon, producing spores as well as vegetative forms.

The dangerous character of this organism has unfortunately not been fully appreciated until recently. Wright, West and others had erroneously concluded that B. Welchii produces no toxin, either exo- or endo-, and this led to its being grouped among harmless organisms; but the researches of Bull and Pritchett in 1917 and the later study of the pathogenicity of the organism by Esty (1920), have clearly shown the older views to be erroneous, and it is now well established that B. Welchii is highly pathogenic, although non-virulent strains are often met.

Bull and Pritchett showed that B. Welchii produces a specific bacterial toxin which may be separated from the bacteria. This highly virulent toxin Esty has shown to be similar to the toxin of the diphtheria bacillus.

The highly dangerous character of Welch's bacillus will be recognized when it is recalled that this organism is the cause of gas gangrene, one of the most formidable complications of wounds with which the military surgeon has to contend. Experimentally, the organism shows itself to be highly active. So small a quantity of culture as 2 c.c. injected into the abdomen of a guinea-pig causes death from gas gangrene in 12 to 30 hours. A still smaller dose, 0.25 c.c. (four minims), injected into the peritoneal cavity, caused the death of a 607 -gram guinea-pig (Esty), and 0.1 c.c. killed a 400-gram guinea-pig. 
As already noted, some strains of B. Welchii are not virulent. This fact was first pointed out by Herter, who noted that strains of $\mathrm{B}$. Welchii obtained from the droppings of cows were less virulent than those from meat eating animals. Esty found of 9 strains obtained from human sources, all were pathogenic. Of 10 strains from the cow, 40 per cent. were pathogenic, and of 11 strains from milk 8 , or 72.7 per cent., were pathogenic. The conditions found in the colon of a meat eater or a mixed feeder, such as man, are particularly favorable for the development of B. Welchii.

Observations made by Esty as regards the effects of heat upon B. Welchii showed that some strains survive $100^{\circ} \mathrm{C}$. $\left(211^{\circ}\right.$ F.) for 30 to 40 minutes. Roderick has confirmed these results.

These facts show most conclusively the utter futility of pasteurization which seldom exceeds $160^{\circ} \mathrm{F}$, as a means of rendering unclean milk safe.

In view of these facts it is evidently important that milk inspection should take account of the frequency and extent of infection with B. Welchii. Esty found B. Welchii in nearly every sample of pasteurized milk examined in Providence, R. I. Roderick found B. Welchii present in 54 per cent. of 470 samples of market milk examined in Battle Creek. The organism is never found in freshly drawn milk protected from contamination. Its presence is wholly the result of lack of cleanliness. Most dairies are badly infected with $\mathrm{B}$. Welchii. Esty found the organism everywhere not only on all parts of the cow, but on the walls, ceiling and floor of barns, in the milk pails and containers, in the stable air and barn dust and even on the milker's hands. That milk may by sufficient care be kept free from B. Welchii has been many times demonstrated. It is only a matter of painstaking cleanliness as has been abundantly shown in the experience of the Battle Creek Sanitarium dairy.

The number of $\mathrm{B}$. Welchii present in raw milk may be considered, then, as a measure of the amount of filth contamination, while the total bacteria count is more an indicator of the rate at which the milk was cooled and the temperature at which it has been kept. Certainly it is much more important to determine the extent of filth contamination than of the heat exposure, for the reason that the bacteria which grow in raw milk are acid formers and not of the dangerous sort.

The examination of milk for the presence of B. Welchii is 
not a difficult procedure. The test is a simple one. A portion of milk is boiled in a test tube with a little litmus solution and incubated at $37^{\circ} \mathrm{C}$. for 24 to 48 hours. When $\mathrm{B}$. Welchii is present a stormy fermentation occurs. Gas bubbles rise to the top of the tube, separating the masses of curd. The litmus is reddened and a strong odor of butyric acid is present.

The presence of $\mathrm{B}$. Welchii always means filth and should lead to prompt inspection of dairies, creameries and handlers and prohibition of sale if the contamination is not eliminated. That such elimination is possible is proven by the fact that this organism is rarely found in certified milk and then only when the count has suddenly gone up through some accident or neglect. It must be remembered, however, that neglect of prompt cooling will not increase the number of B. Welchii for the reason that practically only spores are found in milk and these do not grow in raw milk, the organism being an obligate anerobe.

Interest in clean milk is heightened by the importance now being attached to changing the intestinal flora. As pointed out some years ago by Burnett, in the war against pernicious intestinal bacteria the chief enemy to be overcome is B. Welchii. This organism is always found more or less abundant in proportion to the intensity of the toxemia. As pointed out by Herter many years ago, there are two types of intestinal toxemia; the "indolic type" in which the dominant organism is B. putrificus and the "butyric type," in which B. Welchii is dominant. In the most severe cases, both organisms are present, forming a third or mtxed type.

At the outset of its independent life, nature supplies every mammal with a stock of acidophile organisms to keep its alimentary canal free from putrefactive processes and the resulting toxic products. So long as an infant receives only mother's milk, it usually remains in health. Its stools are frequent, of slightly acid odor, free from mucus, and yellowish in color. Soon after weaning, infants fed cow's milk begin to show symptoms of disturbance. The stools are less frequent. They often become foul smelling, dark colored, and show more or less mucus. These are the first evidences of infection with $\mathrm{B}$. Welchii and allied organisms. If nothing is done to change the situation, the infection will steadily increase from year to year, giving rise to a succession of maladies and miseries of which constipation, headaches, nervous prostration, insomnia, skin disorders, colitis, appendicitis, rheuma- 
tism, Bright's disease, arteriosclerosis, so-called neuritis (neuralgia), with manic depressive insanity, are only a few common examples.

There is no way in which intestinal toxemia with all its dire consequences can be successfully combated except by changing the intestinal flora, that is, by getting rid of B. Welchii and establishing the dominance of acidophile organisms (B. acidophilus). This cannot be done efficiently so long as fresh importations of $\mathrm{B}$. Welchii are being constantly made with the food intake. It is of especial importance that sick infants and children that depend much upon cow's milk as well as adults who take the "milk cure," must be provided with milk free from $\mathrm{B}$. Welchii.

Practically the whole poptulation, not only of the United States, but of every civilized country, is suffering from the use of unclean milk. The bacteriologists have shown us the facts, and it is now the duty of the Boards of Health and milk commissions to inform the people by a campaign of education which will open their eyes to the necessity for using more milk as well as cleaner milk.

A recent bacteriological examination of market meats made by Roderick of the Battle Creek Sanitarium bacteriological laboratory showed that all meats are rich cultures of the colon group of bacteria. Roderick found in apparently fresh beefsteak, 1,500,000 bacteria per gram; in corn beef $31,000,000$; in Hamburger steak $75,000,000$ and in pork liver $95,400,000$. Examination of the droppings of calves, cows, goats and horses showed $15,000,000$ to $80,000,000$ bacteria of the same sort found in meat. That is, some meats actually contain more manure germs than does fresh manure.

In view of these facts, the producer of the dirtiest milk may feel proud of his product as compared with that of the butcher and the packer. The conditions under which the dirtiest milk is produced are clean compared with those which exist in the average slaughter house. In the process of slaughtering, all meats are freely infected with manure germs and within twenty-four hours every carcass is swarming with $\mathrm{B}$. Welchii and other putrefactive organisms, and the bacteria increase continually even in cold storage until the meat is eaten.

The dairy industry rather than the packing industry needs encouragement. There is no other essential American industry so poorly paid and so much in need of and deserving of encourage- 
ment as the dairy industry. The country's great food need at the present time is more milk and cleaner milk. As soon as the public become convinced of these facts, they will be quite willing to pay the higher price which clean milk will cost. Clean milk at a dollar a quart would be preferable to beefsteak at ten cents a pound.

The accompanying cuts show the dairy of the Battle Creek Sanitarium, which supplies milk free from Welch's bacillus. Every dairy should do the same. Our milk supply should be as clean as our water supply.

\section{A Person May Be Sensitized to Milk.}

Another point to which attention should be called in the interest of both infants and invalids is the fact that certain persons become sensitized to milk as well as to other forms of protein, and in a person who is sensitized even the smallest amount of milk may give rise to dangerous or even fatal symptoms. Many infants die annually from this cause. This fact should be borne in mind in changing the infant from the breast to bottle feeding. The milk should first be given in very small quantities, a teaspoonful in a half glass of water, the proportion being gradually increased until the proper dilution is reached. The same method should be pursued with individuals who have learned by experience that unpleasant symptoms are noted after the use of milk. The adult or infant who is sensitized to milk may be cured by the administration of milk in graduated proportions, beginning with extremely small doses. Such a case requires the personal care of a physician.

According to Weill, of Paris, the intolerance for milk shown by infants and many adults may be overcome by the hypodermic injection of milk. The first dose should be very small, eight or ten drops. At the end of an hour, four times this dose may be administered, and three hours later, ten times the original dose. The milk should be sterilized by boiling fifteen or twenty minutes.

Another method which has been employed with success is to take the milk in graduated doses, beginning with very small quantities, say a teaspoonful or even half as much, diluted with water. In the course of a few weeks the amount may be increased to half a glassful or even a glassful at each meal. Another method is to take a small quantity of milk, say one or two teaspoonfuls, half an hour or an hour before a meal at which a larger quantity of milk is taken. 


\section{Medical Uses of Milk.}

Milk is not only useful as a nutrient for healthy persons, but by proper management, may be made to play a highly important role as a curative agent. For example, there is no better means of inducing a rapid gain in flesh than by milk feeding. There are many other medical uses of milk in the form of the milk regimen. The free use of milk is especially useful to cure as well as to prevent lime starvation.

\section{How to Eat Milk.}

Milk must be eaten, not swallowed as a beverage. It must be chewed. All foods need to be masticated. The calf and the nursing infant chew the milk which they draw from the maternal font. The movements of the jaws and the sucking movements executed by an infant in nursing induce an abundant flow of saliva which, mixing with the milk, properly dilutes it and to a high degree promotes digestion. Milk when swallowed rapidly as a beverage is likely to form in the stomach large and hard curds which are very slowly digested. Many persons who suffer from taking milk in this way imagine themselves to be unable to take milk and so abandon its use. The writer remembers a man to whom he had recommended the liberal use of milk. He protested that he was absolutely unable to use it at all and stated that on the last occasion on which he had taken milk he had nearly lost his life. A few hours after hastily swallowing several glasses of milk he experienced a sensation of suffocation, was then nauseated and on attempting to vomit experienced a choking sensation. On reaching his finger down his throat he felt a mass which he seized and to his astonishment drew out a rope of milk nearly a yard in length. The milk had formed in his stomach one large, hard curd which he was certainly very fortunate in being able to get rid of so easily. The famous English surgeon, Dr. Lawson Tait, told of a case in which he was obliged to perform a surgical operation to remove a similar mass of curds which had lodged low down in the intestine.

Milk should be sipped slowly and with a sucking movement or taken through a straw so as to secure a liberal admixture of saliva. By this means the formation of hard, indigestible curds will be prevented.

Milk must be taken in right quantities and in right combinations. It cannot be denied that milk digests better when taken by 
itself or in very simple combinations than when mixed with a large variety of other foodstuffs. In some instances, also, a large quantity of milk is more easily digestible than a small quantity. When the stomach produces a large amount of highly acid gastric juice, as is usually the case with persons who have been accustomed to a hearty meat diet, the curds formed when a small amount of milk is taken will be large and tough, whereas if a larger amount of milk is taken, the curds formed will be smaller and softer. Hence, the proper remedy in many cases in which a person complains that he cannot take milk is to take more milk.

As already remarked, the taking of milk with meat is perhaps the worst of all dietetic combinations. The reason for this was made clear by Pavlov, the eminent Petrograd physiologist, who showed that meat requires a highly acid gastric juice for its digestion and that the stomach produces this sort of juice when meat is eaten, while milk demands and calls forth a juice lower in acid.

When milk is largely used as a nutrient, the remainder of the diet should consist chiefly of fruits and vegetables for the reason that milk contains an excess of lime and is deficient in the potash and soda which are necessary for perfect human nutrition. The last named elements are abundant in fruits and vegetables, particularly the potato, which is also very rich in salts of potash. A diet consisting exclusively of milk and cereals is less satisfactory. Such a diet often gives rise to scurvy in infants. Cereals are deficient in the alkaline elements which are needed to neutralize the acid products developed in the body.

In the use of milk, especially when it is freely taken, it is well to remember, also, that one may easily by this means ingest an excess of fats. The milk of certain breeds of dairy cattle is exceedingly rich in fat. The use of such milk in some persons, and especially in infants and young children, gives rise to symptoms which are sometimes denominated as biliousness, but which are not directly connected with the liver, being due to putrefactive changes set up in the intestine by the presence of an excess of fat. Breeders of dairy cattle have labored to produce strains of milch cows which produce milk containing a large amount of fat because they are more profitable, but for table use milk containing only a moderate amount of fat is preferable.

Milk is very little used by the Chinese, who from the most remote times have had a prejudice against this article of food, 
believing that by using milk one would acquire the characteristics and qualities of the animals from which the milk was obtained, just as certain primitive tribes refuse to eat pigs, fearing that their eyes will become small like those of the pig. The Chinese have been able to dispense with milk because of the superior virtues of the soy bean, from which they prepare an excellent milk and also a palatable cheese, and because of the very large use of greens.

It must be recognized, however, that the greatest value of milk as a food for adults is to be found in its use as a supplementary or complementary food; that is, as a means of rendering biologically complete a dietary otherwise consisting exclusively of the products of the vegetable kingdom. A strictly vegetarian diet is likely to be incomplete unless formulated with extraordinary care and scientific wisdom; but almost any group of vegetable foodstuffs which may be selected will prove satisfactory as nutrients if supplemented with a daily pint of good milk.

The Africans, unlike the natives of the far East, make large use of milk. This may be due to the fact that they have abundant pasturage whereas the Orient has long been, in many parts at least, over-populated.

According to Simmond, the Kaffir often subsists almost exclusively on a diet consisting of sour milk with the addition of a little millet. One meal a day of this food suffices to keep him in vigorous health.

Among the primitive tribes of Africa, according to Dr. Turner and Rev. Bryant, milk is practically always permitted to sour before using. The Zulus pour the milk when still warm from the cow into a gourd kept for the purpose. Loosely stoppered, the gourd is placed in the sun. At the end of 48 hours the milk is clotted and the whey is drained off. The gourd is filled with fresh milk. The next morning the milk is ready for use. The whey is drained off and the curds shaken out. Once a week the gourd bottle is thoroughly washed and scalded. The amasi (amafi of the Kaffirs) thus prepared is closely akin to the yoghourt of the Bulgarians.

\section{Milk a Cheap Food.}

Even at its present high price which is very likely to be higher rather than much lower, milk is a comparatively cheap and economic food. A pint of milk is equal in food value (calor- 
ies) to a pound of codfish, a half pound of lean beef or veal, a quart of oysters, three pints of beef juice, or three and a half quarts of bouillon.

Ten cents will buy in the form of milk more than twice as much food as in the form of beefsteak, ten times as much as in the form of oysters, three to six times as much as is supplied by ten cents worth of eggs. Milk is really by far the cheapest of our ordinary animal foods.

Milk, even at the price of certified milk, is a cheaper food than beefsteak. For example, a pound of steak at 40 cents supplies 450 to 650 calories, or 14 calories for one cent; while a quart of certified milk at 35 cents supplies 650 to 740 calories, or 20 calories for one cent, a difference of more than 40 per cent. in favor of the milk, to say nothing of the very great superiority in quality.

When we consider the amount of tissue-building material which may be produced on a given area of land, the economy of milk as a foodstuff becomes still more apparent. According to Professor Henry, late Dean of the Agricultural Department of the University of Wisconsin, 100 pounds of digestible food when fed to animals produces the following quantities of actual foodstuff :

\begin{tabular}{l} 
Milk \\
Eggs \\
Beef \\
Mutton \\
$\begin{array}{l}18.0 \\
5\end{array} \quad 2.6$ pounds \\
2.6 pounds \\
\hline
\end{tabular}

It is evident from the above that the cow is more than six times as efficient a food transformer as is the steer. Feeding corn to steers wastes 97 per cent. of the corn. 


\section{TABLE XLI.}

\section{Percentage Composition of the Milk of Various Animals.}

The following table compiled by Voltz from various sources, shows the great differences in the composition of the milk of different animals :

\begin{tabular}{|c|c|c|c|c|c|c|c|c|}
\hline & Water & Solids & Fat & Casein & $\begin{array}{c}\text { Total } \\
\text { protein }\end{array}$ & Sugar & $\begin{array}{l}\mathrm{Ca} \\
\text { Assh }\end{array}$ & $\begin{array}{l}\text { lories } \\
\text { er oz. }\end{array}$ \\
\hline Human & 87.58 & 12.42 & 3.74 & 0.80 & 2.01 & 6.37 & 0.3 & 19 \\
\hline Cow ...... & 87.80 & 12.20 & 3.40 & 2.70 & 3.40 & 4.70 & 0.7 & \\
\hline Buffalo & 82.30 & 17.70 & 7.70 & & 4.80 & 4.40 & 0.8 & \\
\hline Zebu ..... & 86.13 & 13.87 & 4.80 & & 3.03 & 5.34 & 0.7 & \\
\hline Llama .... & 86.55 & 13.45 & 3.15 & 3.00 & 3.90 & 5.60 & 0.8 & \\
\hline Camel .... & 87.60 & 12.40 & 5.38 & 2.98 & & 3.26 & 0.7 & \\
\hline Goat ... & 86.50 & 13.70 & 4.00 & 3.60 & 4.60 & 4.30 & 0.8 & \\
\hline p ....... & 81.50 & 18.50 & 7.00 & 4.30 & 5.60 & 5.00 & 0.9 & \\
\hline Reindeer & 67.70 & 32.30 & 17.10 & & 10.90 & 2.80 & 1.50 & \\
\hline Mare ...... & 90.58 & 9.42 & 1.14 & & 2.50 & 5.87 & 4.36 & \\
\hline Donkey .... & 90.12 & 9.88 & 1.37 & 0.79 & 1.85 & 5.19 & 0.47 & \\
\hline Elephant .......... & 67.85 & 32.15 & 19.57 & ...... & 3.09 & 8.84 & 0.65 & \\
\hline Hippopotamus & 90.43 & 9.57 & 4.51 & & & & & \\
\hline Rabbit ................ & 69.50 & 30.50 & 10.45 & ...... & 15.54 & 1.95 & 2.56 & \\
\hline Guinea pig & & 58.89 & 45.80 & & 11,19 & 1.33 & 0.57 & 13. \\
\hline Dog …............ & 77.00 & 23.00 & 9.26 & 4.15 & 9.72 & 3.11 & 0.91 & \\
\hline Cat & 81.6 & 18.5 & 3.33 & 3.11 & 9.53 & 4.91 & 0.59 & \\
\hline ig & 82.37 & -17.6 & 6.4 & ...... & 6.09 & 4.04 & 0.59 & \\
\hline Blue w & 50.47 & 39.53 & 20.00 & & 12.42 & 5.63 & 1.48 & \\
\hline
\end{tabular}

\section{Relation of Diet to Growth}

The influence of the composition of the milk of animals of different species upon the growth of their young is well shown in the following table:

\begin{tabular}{|c|c|c|c|c|c|}
\hline \multirow[b]{2}{*}{ Man } & \multicolumn{2}{|l|}{$\begin{array}{l}\text { Time in days } \\
\text { required for the } \\
\text { newborn animal }\end{array}$} & \multicolumn{2}{|c|}{00 parts of milk contain } & \\
\hline & $\begin{array}{c}\begin{array}{c}\text { to double e t } \\
\text { weight } \\
1 \\
180\end{array} \\
1\end{array}$ & $\begin{array}{c}\text { Protein } \\
1.6\end{array}$ & $\begin{array}{l}\text { Ash } \\
0.2\end{array}$ & $\begin{array}{l}\text { Calcium } \\
.021\end{array}$ & $\begin{array}{r}\text { phoru } \\
.022\end{array}$ \\
\hline Horse & $\ldots 69$ & 2.0 & 0.4 & .086 & .0 \\
\hline Cow . & 47 & 3.5 & 0.7 & .114 & .0 \\
\hline Goat & 22 & 3.7 & 0.78 & .143 & 1 \\
\hline Sheep & 15 & 4.9 & 0.84 & .178 & \\
\hline Swine & 14 & 5.2 & 0.80 & .178 & \\
\hline Cat ... & 9.5 & 7.0 & 1.02 & & \\
\hline Dog & 9 & 7.4 & 1.33 & .321 & \\
\hline Rabbit & 6 & 10.4 & 2.50 & .636 & \\
\hline
\end{tabular}

Goat's Milk

The claim made for goat's milk that it is more digestible than cow's milk is not justified by the facts. It contains more casein than does cow's milk, more than four times as much as does hu- 
man milk, more fat and less sugar. It is sometimes cleaner, which is the best and perhaps the most that can be said in favor.

Goat's milk has the disadvantage that the goat is a carrier of germs of a disease known as Malta fever, or Mediterranean fever. This infection is very common among goats in the Mediterranean region and the germs are found in abundance in the cheese made from the milk of infected animals. This cheese is now being eaten not only in the Mediterranean region but in different parts of the world to which it is shipped. It is on this account important that the facts should be known. For the present at least imported cheese prepared from goat's milk should be avoided as incurring risk of infection.

The goat appears to be immune to the germ or is at least only very slightly inconvenienced by it. Little or nothing is being done to suppress the disease in the region where it prevails for the reason that the goat herders, having become themselves immune, cannot be made to believe in its existence.

Several outbreaks of Malta fever have occurred in this country. If goat's milk is used, great care must be taken to make certain that it is from a herd of healthy animals.

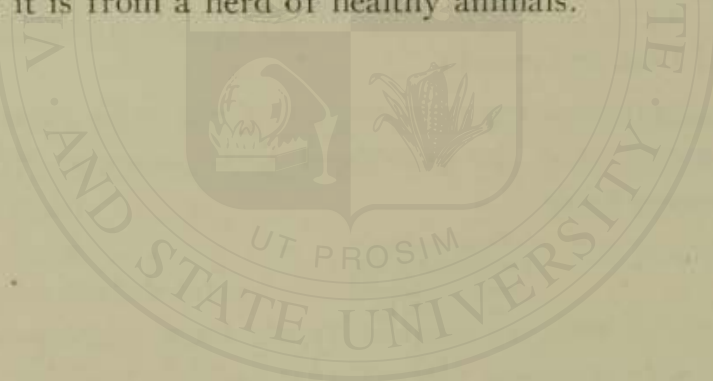




\section{Condiments}

Man is the only animal that deliberately commits suicide by self-poisoning. He is the only one that spoils his food before he eats it. The average man suffers constantly from chronic poisoning. He doses himself with poisons of various sorts which in the aggregate make a per capita dose of more than fifty grains of poison every twenty-four hours for every man, woman and child in the United States. He begins the day with a poison dose in the form of coffee to wake him up. After breakfast he smokes a cigar to settle his stomach and quiet his nerves. Before dinner he swallows half an ounce of whiskey or bitters to get an appetite. He finds an afternoon cup of tea necessary to cure after-dinner stupor, and at night needs an opiate to get to sleep, and in the morning a cathartic to move his bowels. With his other poisonings he deliberately spoils his food by putting into it toxic substances, the excretions of plants, which by means of acrid, biting and burning flavors are labeled by Nature as belonging to the poison class and unfit to be eaten. These products, used only for their flavoring properties, having no food value, are known as condiments.

There are two classes of condiments: first, those which contain irritating but non-volatile oils, like mustard, pepper, cayenne, capsicum, horse-radish, ginger, and spices; second, those which contain irritating volatile oils, like mint and thyme. To these may be added common salt, although this is by some regarded as a food.

Condiments of the first class stimulate the activity of the stomach, causing it to empty somewhat quicker but lessening the secretion of gastric acid. Condiments of the second elass lessen the activity of the stomach and diminish secretion. It should be added at once, however, that condiments of the first class, although they at first increase the contractile activity of the stomach, later produce an opposite effect. Consequently, the effect of condiments is the opposite of what it is popularly supposed to be, depressing and hindering digestion rather than aiding it. The ultimate effect of artificial stimulation is always to depress and lessen function. The effect of such stimulants upon a weakened 
organ is precisely the same as that of a whip when applied to a tired horse. The forced action produced inevitably has the effect of still further lessening an already depleted store of energy and so hastening the time when complete failure will result.

Condiments of all sorts should be rigorously excluded from the table. The essential oils found in condiments are all irritant poisons. When applied to the skin in concentrated form they produce irritation or blistering, inflammation and even serious destruction of tissue if the contact is prolonged. The effect upon the stomach is similar.

Various condiments are the chief ingredients of the curries of the East, the effects of which, in producing gastric catarrh and hemorrhoids with other forms of disease of the rectum, has long been well known. A moment's thought will make clear how these irritative substances are particularly injurious to the terminal portion of the alimentary canal. When pepper, mustard, and similar substances enter the mouth they are very largely concealed by the food, but when the food has been digested and absorbed the condiments, which are in large part left behind, are concentrated and, remaining some time in contact with the mucous membrane of the rectum, are quite capable of setting up a serious irritation similar to that which is produced by concentrated mixtures of condiments upon the skin and other sensitive parts.

Through absorption into the blood, the poisonous oils of condiments are brought into contact with every cell and fiber of the body. The first effect is produced upon the delicate walls of the blood vessels. A defensive effort causes these to become thickened, a change known as arteriosclerosis, because the primary thickening is followed by hardening through a deposit of chalk. The eminent Professor Huchard, of Paris, the world's greatest authority upon this subject, showed that mustard and other condiments when administered to animals may produce changes in the blood vessels within a few months' time.

The same poisonous effects produced upon the blood vessels are also produced in the kidneys, the delicate cells of which, being brought in contact with the poisons in more concentrated form through their elimination by way of the urine, are made to undergo degenerative changes through which their efficiency is impaired and Bright's disease and other disorders produced.

Boix fed half a gram ( 7.5 grains) of pepper to a rabbit daily. The animal died at the end of twenty-seven days. The 
liver was found sclerotic and its cells degenerated. The kidneys were greatly congested.

\section{The Use of Condiments as Preservatives.}

The preservative influence of the essential oils found in various condiments, particularly in cloves and cinnamon, has long been known. Recent experiments show that 2 to 5 per cent. of an alcoholic extract of cloves or cinnamon, or 4 per cent. of scraped horseradish, will prevent the development of bacteria in tomato pulp. Mustard and anise seed have a similar effect. These preservatives have been much used in recent years as substitutes for benzoic acid, salicylic acid and other preservatives, the use of which has been excluded by the pure food laws. In the opinion of the writer there is no advantage in exchanging salicylic acid for oil of cloves or any other of the essential oils mentioned. As a matter of fact the salicylates are probably less harmful than these acrid oils. The use of these chemical preservatives is not necessary, and since all of them are alike harmful their use in the manufacture of foods should be forbidden by law.

\section{The Taste for Condiments Unnatural.}

The taste for condiments is altogether an acquired one. Condiments of all sorts are highly repulsive to infants and to all persons whose tastes have not become vitiated. There is no place for these poisonous products in the biologie dietary.

That the taste for pickles, which has become quite generalized among civilized people, is an acquired and unnatural appetite, is well shown by the following story told by George Kennan in his delightful book, "Tent Life in Siberia":

- One evening, soon after we left Shestakova, they (Korak drivers) happened to see me eating a pickled cucumber; and as this was something which had never come within range of their limited gastronomical experience they asked me for a piece to taste. Knowing well what the result would be, I gave the whole cucumber to the dirtiest, worst-looking vagabond in the party, and motioned him to take a good bite. As he put it to his lips, his comrades watched him with breathless curiosity to see how he liked it. For a moment his face wore an expression of blended surprise, wonder and disgust, which was irresistibly ludicrous, and he seemed disposed to spit the disagreeable morsel out; but with a strong effort he controlled himself, forced his features into a ghastly imitation of satisfaction, smacked his lips, declared it was "akhmael nemelkin"-very good, and handed the pickle 
to his next neighbor. The latter was equally astonished and disgusted with its unexpected sourness, but rather than admit his disappointment and be laughed at by the others he also pretended it was delicious, and passed it along. Six men in succession went through with this transparent farce with the greatest solemnity; but when they had all tasted it, and all been victimized, they burst out into a simultaneous "ty-e-e-e" of astonishment, and gave free expression to their long-suppressed emotions of digust. The vehement spittings, coughing, and washing out of mouths with snow which succeeded this outburst proved that the taste for pickles is an acquired one, and that man in his primitive state does not possess it.

Pickles are unwholesome not only because of their utter indigestibility, but because the acetic acid (vinegar) with which they are saturated is a gastric poison, arresting at once the action of the saliva and giving rise to gastric catarrh and sclerosis of the liver.

Schibkow has shown that acetic acid is an active poison, behaving like a mineral acid. In some cases death was caused by a few drops. The poisonous effects, when applied to the mucous membrane, began with a 12 per cent. solution. The pulse was slowed and the temperature lowered. The vapor of acetic acid, when inhaled, caused the death of animals either during the inhalation or a short time after.

\section{CHLORID OF SODIUIM-COMIMON SALT}

For centuries the practice of adding chlorid of sodium or common salt, to many foodstuffs, particularly cereals, meats and vegetables, has been current among civilized people, though by no means universal among primitive tribes. The use of sodium chlorid seems to have been, for the most part, unknown to the North American Indians at the time of the discovery of America, and Indian tribes living remote from civilization, as in the Hudson Bay region and perhaps in a few other isolated localities, still thrive on a saltless diet. The Indians of the Hudson Bay region, when compelled to eat salt beef soak the meat over night in an abundance of water, turn off the water, then add fresh water and boil for an hour, and then again for a third time place the meat in fresh water to continue the cooking, thus getting rid of the salt flavor of the meat as thoroughly as possible. Millions of natives of Central Africa never taste salt. The great herds of cattle on the western plains are fattened without salt. The Eskimos eat their meat without salt.

Dr. Benjamin Rush, one of the most eminent physicians who 
has lived in modern times, one of the signers of the Constitution of the United States, who made a careful study of the habits of the American Indians a hundred and fifty years ago, said of salt: "Although the interior parts of our continent abound with salt springs, yet I cannot find that the Indians used salt in their diet till they were instructed to do so by the Europeans."

Among civilized people the excessive use of salt is almost universal. One result of this excess is overwork of the kidneys. Another ill effect is overstimulation of the gastric glands, which are thus led to produce an excess of gastric acid which is formed from salt. The use of salt as an addition to foodstuffs is due to the desire to give to them a more piquant taste. The natives of India use curry, a hot condiment, with their rice instead of salt. Bunge believed that salt is necessary with vegetable foods to take the place of the excess of potash which they contain; but Renmerich and Kurz, by experiments upon dogs, have disproved this theory, showing that the use of foods containing potash salts does not drive out the soda salts but that they are retained by the body in normal proportion.

A story which seems to have originated with an old writer, John Marshall, and which has been repeated by many authors, tells us that the ancient laws of Holland required that criminals should be given bread without salt until they were devoured by worms which developed in their intestines. The story has been in modern times wholly discredited. Numerous persons have discarded salt wholly and lived entirely without it without suffering any injury. Within recent years it has become the custom of physicians to place persons suffering from epilepsy upon a saltless diet. The salt-free regimen has also been used in other disorders. These persons when under treatment have discarded salt entirely from their dietary, except so far as it is contained in natural foodstuffs, for months at a time and without injury.

Linossier showed in 1900 that three parts of salt added to one thousand of gastric juice retarded the digestion of albumin to the same extent as a 40 to 50 per cent. diminution in the amount of pepsin. Three parts of chlorid of sodium to one thousand of gastric juice is practically the proportion of salt found in the gastric contents after an ordinary meal. From this it is evident that while the intake of an excessive quantity of salt may increase the amount of hydrochloric acid produced by the stomach the digestive activity of the gastric juice is diminished. 


\section{Salt Hunger.}

Persons who have been accustomed to make use of salt, especially those who use it freely, miss it greatly when deprived of it. Such persons especially crave salt with vegetable foods such as potatoes and many desire salt with cereals. Few persons, however, go so far in their liking for salt as to demand its use in connection with fresh fruits, though it is often eaten with nuts and frequently with the cataloup or musk melon, a vegetable fruit which is possessed of ample flavor to commend it to the palate without addition of any sort.

Bunge, of Basle, advanced the theory that salt is craved with vegetables for the reason that chlorid of sodium is found only in small quantity in these foodstuffs, while potash is present in large amount. This theory has not been universally accepted by physiologists, and Carlson tells us that "It is still an open question whether any animal, man included, actually needs sodium chlorid in addition to the salts present in all natural foodstuffs. Smith, the veterinary physiologist, maintains that both carnivora and herbivora obtain in their natural diet a sufficiency of salts, although there is a general impression that the wild herbivora long for sodium. It is quite certain that under conditions of domestication horses can be kept in perfect health without receiving any sodium chloride other than that contained in the food, and the amount of this in vegetable substances is very small.' "

The theory that wild animals, especially grazing animals, require salt has been many times shown to be wholly erroneous. The cattle men on the Western plains who raise enormous herds of cattle do not feed salt to their animals. It is well known that the finest oxen raised in Great Britain are not given salt.

Some twenty-five years ago the writer placed in his small park a pair of deer which have at the present time become quite a little herd. These animals have never eaten salt. On one occasion a new caretaker placed a pan of salt in the enclosure in which they are confined, but they refused to touch it, although they had free access to it for two or three weeks before the error was discovered, it having been intended from the start to rear these deer without salt. On one occasion, when Mr. Ernest Thompson Seton was a guest at the Battle Creek Sanitarium, he was invited to see these deer, which he was informed refused to eat salt. He was sure that all deer would eat salt, and was invited 
to test the deer by offering them salt, which he did. To his great surprise not one could be induced to touch it.

The Tlascalans, one of the tribes of ancient Mexico, were found at the time of the conquest, according to Prescott, to have been for some reason deprived of salt for more than half a century. Prescott adds that it required the lapse of several generations after the conquest to reconcile them to the use of salt with their food. (Conquest of Mexico," by Prescott, Vol. I., p. 466.) The same author describes these people as "a bold and hardy peasantry."

The Daramas in southwestern Africa "never take salt by any chance."

Dr. Achard and Professor Richet, of Paris, have in recent years made extensive investigations by which they have shown that while salt is found in the tissues of all animals, it is also found in all vegetable structures and in sufficient quantity in ordinary foodstuffs to supply the needs of the body.

According to these observers, the amount of salt found per 1,000 parts of animal tissues is for different animals as follows:

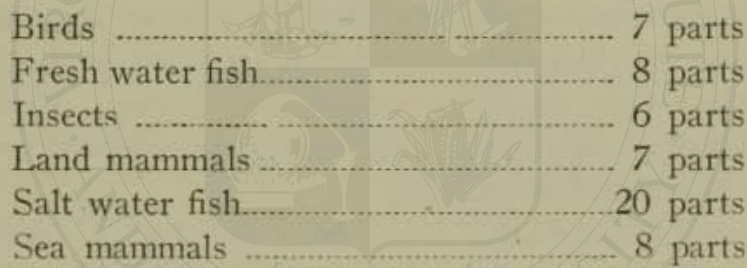

Human blood contains 8 parts of sodium chlorid to 1,000 parts.

The salt leaves the body chiefly through the kidneys and the skin.

The average person takes about two-thirds of an ounce of salt daily. Of this about one-eighth is lost through the skin, most of the remainder through the kidneys. In fasting, salt almost entirely disappears from the urine. Observations on the Italian faster, Succi, showed that after fifteen days of fasting, his urine contained only 3 grains of salt in twenty-four hours, or about one one-hundredth part of the amount frequently found in the urine.

The use of chlorid of sodium raises the blood-pressure. When salt is removed from the body by profuse sweating or by copious water drinking the blood pressure falls. 
Richet, an eminent French scientist, has shown that the food required for a day's ration contains naturally half a dram of salt (30 grains). Numerous experiments have shown that this amount of salt is exactly the amount which the body requires to make good its loss of salt, and that no more is actually needed. In fact, Richet claims that the amount actually required by the body is even less than half a dram. More than this, as this distinguished scientist says, is luxury and not necessity.

Dropsy is produced in the great majority of cases by the excessive accumulation of salt in the tissues; the kidneys being unable to eliminate salt, it is pushed out into the tissues and of course must be held in solution by water. For every half ounce of salt retained nearly four pounds of water will be retained to hold it in solution. This is shown by the rapid increase in weight when the kidneys cease to eliminate salt.

Javal and Widal have shown that the retention of one-third of an ounce of salt may give rise to edema.

Many observations have shown that the kidneys fail to remove chlorid of sodium at the usual rate in various diseases, particularly in pneumonia, pleurisy, Bright's disease, some forms of heart disease, erysipelas, typhoid fever, jaundice, cirrhosis of the liver, scarlet fever, smallpox, edema and dropsy. Hence, it is evident that in these disorders salt should be withheld or at least very greatly reduced in amount.

By careful observation it has been found that a marked retention of salt occurs before dropsy or edema makes its appearance. Several cases which have come under the writer's observation are of interest in this connection. The urine of a patient suffering from diabetes in a moderate degree was found to contain no chlorid of sodium whatever, although the amount taken in the food must have amounted to at least one or two drams. Several examinations made within twenty-four hours showed not a trace of chlorid of sodium in the urine. This was the first indication that the patient's condition was serious. In another twenty-four hours the patient was dead, in spite of all that could be done to prevent a fatal termination. The failure of the kidneys to eliminate chlorid of sodium was evidence that these organs had ceased to do efficient work, and the rapid accumulation of poisons in the body resulted in the patient's death, the kidneys evidently being so far damaged that it was impossible to restore them to normal activity. 
A patient suffering from cardiac disease was found to be eliminating through the kidneys only a very small amount of chlorid of sodium, less than one grain. This patient likewise died a few hours later. The kidneys were found in an advanced state of degeneration.

Many similar cases might be cited, including a number of cases in which the withdrawal of salt has been followed by the disappearance of the dropsy and other unpleasant symptoms.

If a person eats one-fourth ounce of salt and only eliminates one-eighth ounce, salt will necessarily accumulate in his body, and for each eighth of an ounce that is retained, he will have to retain one pound of water to dilute it. If one stops eating salt, he continues to eliminate it at the rate of an eighth to a quarter of an ounce daily. This causes the elimination of an equivalent amount of water or one to two pints or pounds, and so the person loses in weight. This is what happens in dropsical persons who are put on a saltless diet.

Loufer reports the case of a young girl who suffered from anemia, emaciation, and dyspepsia as the result of the excessive use of salt.

\section{Poisonous Effects from Salt.}

The idea that salt is a harmless substance is a grave error. Many persons are doubtless greatly damaged by the free use of salt. Agricultural authorities tell us that two or three pounds is a poisonous dose for a horse and four or five pounds are equally fatal to a cow. A half pound of salt will kill a hog.

An ounce of salt taken in a quart of water produces a decided laxative effect but the use of salt as a laxative is a pernicious practice.

The effects are no different from those produced by the habitual use of mineral water, the laxative effects of which are in large part due to the salt which they contain.

If to a dog is administered one two-hundredth of its weight of salt (a little more than 3 ounces for a dog weighing 40 pounds), the animal falls into convulsions and dies.

Dr. C. Heman Borrow, who spent some years in China, states that in that country "the drinking of a saturated solution of salt is a common mode of committing suicide, and there is none more difficult to treat. In only one case did I succeed in securing recovery. Salt is taken for suicidal purposes sometimes in a com- 
mon saturated solution made with water as the solvent, and sometimes the brine from salted kraut. . . The amounts taken vary with the determination of the person taking it, but usually are from one to three rice bowlfuls-half a pint to a pint."

A young woman died after taking a half pound of salt as a vermifuge.

While salt may be used in minute quantities with little if any injury, the amount commonly used is greatly in excess even of reasonable gustatory requirements and should be reduced at least three-fourths.

Tibbles, an eminent English authority in dietetics, tells us that with a diet consisting of cereals and legumes one-half to one dram of salt is sufficient, and with a diet consisting chiefly of rice a fraction of this quantity is ample. He further adds that very little salt is needed with an ordinary mixed diet; whereas, most people take 20 to 30 grams (two-thirds of an ounce to an ounce) in one way or another. Evidently, the use of salt is merely a concession to an artificial appetite not required except in the case of those who have cultivated a taste for the saline condiment.

According to Gautier, the amount of salt consumed by most persons is at least five times this amount. It is evident, then, that the addition of salt to the foodstuffs is not a physiologic necessity, but is an acquired habit, which is also shown by other facts to which reference has been already made. The excess must be eliminated through the kidneys and skin, imposing unnecessary work upon these emunctories. The free use of salt has been shown by Debove and others to be a serious injury to the digestive process.

Vilhjalmar Stefansson, the Arctic explorer, states that salt is not only not used but is greatly disliked by the Eskimo. He says :

Most people are in the habit of looking upon the articles of our accustomed diet, and especially upon salt, as necessities. We have not found them so. . . . Salt I have found to behave like a narcotic poison-in other words, it is hard to break off its use, as it is hard to stop the use of tobacco; but after you have been a month or two without salt, you cease to long for it, and after six months I have found the taste of meat boiled in salt water distinctly disagréeable. In the case of such a necessary element of food as fat, on the other hand, I have found that the longer you are without it the more you long for it, until the craving becomes more intense than is the hunger of the man who fasts. Among the uncivilized Eskimo the dislike of salt is so strong that a saltiness imperceptible 
to me would prevent them from eating at all. This circumstance was often useful to me later in our travels about Coronation Gulf, for whenever our Fskimo visitors threatened to eat us out of house and home we could put in a little pinch of salt, and thus husband our resources without seeming inhospitable. A man who tasted anything salty at our table would quickly bethink him that he had plenty of more palatable fare in his own house. "My Life with the Eskimo," pp. 69, 70.

The Highlanders of Nepaul refuse to eat salt as do also the Kamschatdales.

The native tribes of Siberia have no use for salt.

The Bedouins consider the use of salt ridiculous. The same is true of the Bushmen of South Africa,

\section{Harmful Effects from the Excessive Use of Salts}

In the case of a man who died after taking a large dose of salt marked changes were found in the kidneys, which leads to the conclusion that the habitual use of salt in excess may lead to chronic disease of the kidneys. Bunge called attention to this important fact may years ago. Says Bunge:

The habit of drinking spirituous liquors, which moreover is reckoned one of the causes of chronic nephritis, also brings about the immoderate use of salt. Thus one sin against Nature leads to another. These are questions to which I would direct the attention of practitioners.

There is no organ in our body so mercilessly ill treated as the kidneys. The stomach reacts against overloading. The kidneys are obliged to let everything pass through them, and the harm done to them is not felt till it is too late to avoid the evil consequences.

According to Torindo, the excessive use of salt is a cause of Bright's disease. It is well known that salt increases the amount of albumen in cases of this disease.

M. Achard, an eminent French physician, also Professors Strauss, Weidall, Lemierre, and Javal, have shown that in cases of albuminuria the use of salt increased the albumen; by withholding salt entirely, the albumin was made to disappear. Other authorities also completely withhold salt from the food in all cases of albuminuria as well as in cases of latent nephritis in which albumin may not be present.

The great importance of this discovery can only be appreciated when one considers the enormous amount of salt which is used by many persons in this country. In France and most continental countries salt is used in very much smaller quantities dietetically than in this country. 
Recent discoveries which connect the use of salt with Bright's disease of the kidneys will certainly command the thoughtful attention of thousands of physicians who, perhaps, have never before given this matter any serious attention. Salt should be used in exceedingly small quantities, if at all. One can easily accustom himself to the disuse of salt without any great inconvenience and certainly without any risk to health.

\section{The Salt-Free Diet.}

Natural foodstuffs contain sufficient salt to meet all the natural requirements of the body, 30 to 60 grains daily, (Tibbles), as will be seen by reference to the following table based chiefly upon a table by Strauss:

\section{TABLE XLIII.}

\section{Amount and Percentage of Sodium Chlorid in Foods.}

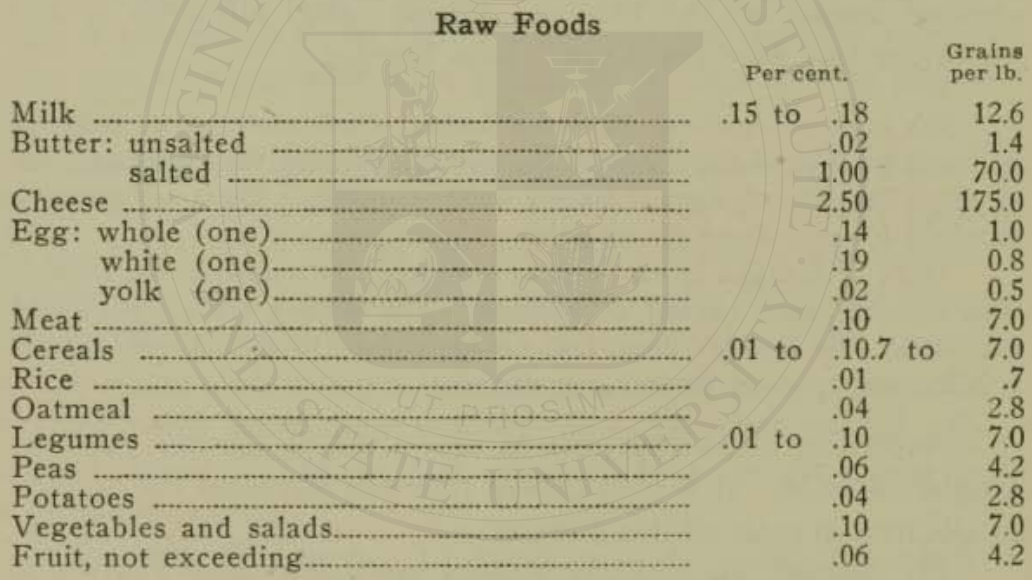

\section{Cooked Foods}

Poached eggs (one).

Buttered eggs and omelets (one)

Roast beef

Beefsteak

White bread

Brown bread.

Mashed potato

Asparagus

$\begin{array}{rrr}2.4 \text { to } 2.70 & 3.3 \\ 1.9 \text { to } 2.80 & 19.0 \\ & 300.0 \\ .48 \text { to } .70 & 210.0 \\ & .75 & 50.0 \\ .50 \text { to } .91 & 52.5 \\ 2.70 \text { to } 3.50 & 245.0\end{array}$

By reference to the table it will be seen that while milk contains more salt than most other natural foods it contains much less than an equal amount of any of the ordinary cooked foods. 
For example, a quart of milk contains 25 grains of sodium chlorid, while the same weight of bread contains two or three times as much, ordinary salted butter four or five times as much; and cooked vegetables usually contain from five to twenty times as much salt as does milk. A milk diet is thus much superior to the ordinary mixed diet of cooked foods when salt is to be avoided, but is decidedly inferior to natural foodstuffs eaten raw or cooked without salt.

The excessive use of salt is without doubt among the causes of several serious conditions. It is particularly harmful in pleurisy, in fevers, in Bright's disease, and has been shown to greatly aggravate the symptoms of epilepsy.

Withdrawal of salt by a salt-free dietary has been found of very great value in the treatment of general dropsy and abdominal dropsy when due either to disease of the kidneys or the heart.

Not infrequently when salt is withdrawn in cases of dropsy the excess of fluid disappears almost wholly within a few days.

In obesity, a salt-free diet accelerates the loss of weight.

The elimination of salt is also a valuable means of reducing high blood pressure.

The salt-free diet is especially useful in chronic parenchymatous nephritis with dropsy or edema. It is important to discover the beginning of edematous accumulations in the viscera before external edema develops. Careful comparison should be made between the intake and output of chlorids. If the amount of chlorids found in the urine is considerably less than that of the food, in the absence of profuse perspiration, it may be taken for granted that retention of sodium chlorid is taking place.

It should be remembered, also, that retention of chlorid of sodium may occur with edema, the so-called, dry retention. In this condition there is usually an increase of arterial pressure, vomiting, dyspnea, Cheyne-Stokes breathing, diarrhea, and convulsions of an epileptiform character may occur.

Leading European medical journals have reported obstinate cases of eczema in which a cure has been effected by the adoption of a diet free from salt or nearly so.

Salt should be eliminated in diabetes, in syphilis, as well as in dropsy from disease of the heart, liver and kidneys.

Mayer mentions salt as one of the causes of war edema.

The excessive use of common salt raises arterial pressure 
very readily in subjects already suffering from arteriosclerosis, a rise which quickly subsides on the free excretion of salt. The rise of arterial pressure after meals has been attributed to the use of salt.

Tuberculous ascites is treated by Alwens with a diet almost free of salt. He gives gruel, rice, cooked fruit, eggs, milk and non-salted butter with two-thirds gram of table salt for a few days, alternating with a normal diet for a few days. In a large number of cases the ascites disappeared completely after five to six weeks. Unfavorable cases are those in which the process is active and intense and cases with serious complications which influence the nutrition and distribution of water in the body.

It is worthy of note that Dopter has observed that the course of scarlet fever is greatly modified for the better when salt is eliminated from the diet. If nephritis has appeared, which is often the case in severe attacks, it often disappears very promptly when the patient is put on a saltless regimen. These observations have been confirmed by Peter, of Paris.

The saltless diet not only lessens the severity of this disease and lessens the danger of serious complications, but shortens its course. The same is true of all the other infectious diseases and particularly of the exanthems, measles and small pox.

In pneumonia, as well as in typhoid and other fevers, there is a tendency to salt accumulation which seems to be due to the influence of the poisons upon the body cells and not alone to the inability of the kidneys to eliminate the chlorine salt. On this account, the use of salt should be reduced to a minimum if not entirely eliminated in all febrile disorders.

In the salt-free diet the amount of salt should never be permitted to exceed 30 grains daily. In general, the salt-free diet is preferable to the milk diet. Better results are obtained and the patient is better pleased because of the larger variety of food permitted.

To make the saltless fare acceptable to the patient, care must be taken to give the food flavor when salt is omitted by means of lemon juice and other simple and wholesome flavors, particularly raisins and other fruits.

\section{Borax versus Salt}

The antiseptic properties of borax and slight toxicity of this drug, when compared with many other antiseptic substances, have 
led to its extensive use as a preservative of foods. It has, indeed, been advocated as a substitute for salt in the preservation of butter. While it cannot be doubted that the use of very small quantities of borax or boracic acid might prove less injurious than large quantities of salt, it must not be forgotten that all these antiseptic substances are toxic and that the desirable thing is to dispense with them as fully as possible. Salt is a normal constituent of the body, and while it has been clearly shown that the addition to the food of mineral salt is unnecessary and that large quantities of salt do much harm, it is also a fact that salt has been longer tested as a food preservative than any other substance. Boracic acid is a substance entirely foreign to the body. There is no evidence that it can be of any service whatever in the bodily functions. If used for the preservation of butter, the safest thing would be to remove it by careful washing before the butter is used. The adoption of this method in relation to salt would lessen the injury from this mineral. Indeed, this method has long been practised in the management of cases of Bright's disease. The salt is held in solution in the water or buttermilk left in the butter, and hence may be easily removed by washing.

\section{ALCOHOL}

Fifty years ago nearly all medical authorities taught that alcohol was a food, a stimulant, a remedy of the highest value; that it was indispensable in the treatment of collapse, surgical shock, in blood poisoning, in pneumonia, in lung tuberculosis, in weakness from whatever cause, as a preventive in exposure to contagion, a protection from fevers, a support in heart weakness from hemorrhage or other cause; in fact, alcohol was the one and universal remedy, first on the list of emergency supplies, the biggest item next to foods in the hospital expense bill, the most frequent prescription of the medical practitioner, and the prescription which he most often took himself. The use of alcohol as a remedy was most emphatically endorsed by scientific men and supposed to be backed up by scientific evidence.

Today all this is changed. Laboratory researches conducted by the aid of instruments of precision have been brought to bear upon the study of alcohol and its effects upon the human body, and the result has completely upset and reversed the old beliefs and the old teachings. 
Every function of the body has been subjected to the minutest scrutiny; every bodily activity and energy has been calipered with the finest accuracy. With a normal man before him, measured, calipered, tested and charted in every conceivable way, the modern laboratory physiologist has made a study of the influence of alcohol upon the human body, its tissues and its activities. The result has been the demonstration that alcohol is not a food and that this drug damages every tissue and impairs every function of the body; that it is a universal poison; that it is of no essential assistance to the body under any circumstances; that it is not capable of increasing strength or endurance or vitality one iota, but does the opposite.

In doses so small as one twenty-five hundredth of the body weight, that is, one ounce for a man weighing one hundred and fifty pounds, alcohol shrivels the nerve cells and impairs every mental function.

By most careful measurements, it has been found that under the influence of alcohol the fires of the body burn low, the amount of oxygen consumed is less, and the tissue activities are slow.

Alcohol is not a stimulant or tonic in any sense of the word. It is a depressing agent, an anesthetic, a narcotic: it is the mother of many anesthetics.

The old idea that alcohol strengthens the heart and hence is just the thing to use in case of fainting, shock, or collapse, has been shown to be utterly fallacious. According to Professor Kronecker of Berne, Switzerland, a two per cent, solution of alcohol (Bavarian beer), will paralyze a frog's heart. Ordinary beer and hard cider contain two or three times as much alcohol; wine, five to ten times as much; brandy and whiskey, twenty to twentyfive times as much. Hence, the paralyzing effect of these strong liquors is proportionately greater.

\section{Alcohol Lessens Vital Resistance}

Animals under the influence of alcohol are a more likely prey to germs. For example, pigeons, which are ordinarily proof against the bacteria which produce malignant carbuncle, under the influence of small doses of alcohol are easily infected and die.

When nearly fifty years ago the writer was walking the wards of Bellevue Hospital as a pupil of the Senior Flint, that most eminent therapeutist of his time was an enthusiastic advocate of the use of alcohol as a supporting agent in all conditions of vital 
exhaustion. Following Todd, Doctor Flint led the medical profession in this country to adopt the whisky bottle as a mainstay in the treatment of typhoid fever, pneumonia and most other actite febrile disorders, and for a whole generation this practice was current in this country in the treatment of fevers of all sorts. Even so late as the Spanish-American war, alcohol was relied upon as a chief remedy in the treatment of typhoid fever and pneumonia in the army.

Sir Thomas Barlow, who was one of the early dissidents from this practice, in commenting upon the great change which has taken place in the opinion of the medical profession in relation to the necessity for alcohol as a remedy, remarked that when the Temperance Life Insurance Society of England was formed, some of the men who constituted the first Board of Directors had actually been refused insurance by other companies because they were total abstainers; and when the Temperance Hospital of London was founded later, threats were made that if any deaths occurred in the institution in cases in which alcohol had not been administered, a coroner's inquest would be demanded.

The National Standard Dispensatory still recommends alcohol in the treatment of typhoid fever and as a circulatory stimulant in collapse. But there is reason to believe that in the future such pernicious statements will cease to appear in standard medical literature.

Several years ago, at its annual meeting, the American Medical Association, the world's largest body of organized scientists, adopted the following preamble and resolution:

"Whereas, We believe that the use of alcohol is detrimental to the human economy, and whereas its use in therapeutics as a tonic or stimulant or food has no scientific value,

"Resolved, That the use of alcohol as a therapeutic agent should be discouraged."

According to Professor Woodhead, experiments have been made with various animals which demonstrate that immunity cannot be established in an animal which is under the influence of alcohol.

The death rate under the old method of treating typhoid fever and pneumonia was from twenty to thirty per cent. Since the use of alcohol has been lessened, and water and other physiologic remedies have been substituted, the mortality from typhoid fever 
has been reduced to five per cent. In one series of twelve thousand cases the mortality was scarcely three per cent.

\section{Alcohol Lessens Nerve Sensibility}

Nervous impressions travel over nerves in a healthy person at the rate of ninety-one feet per second; but under the influence of alcohol the rate of transmission may be as low as thirteen feet per second.

Smiedeberg, more than twenty years ago, pointed out the fact that under the influence of alcohol "the finer degrees of observation, judgment and reflection disappear," and that all the effects produced by alcohol are really those of a sedative or paralyzing agent. Benedict, of the Carnegie Nutrition Laboratory of Boston, has shown that the depressant toxic effects of alcohol are produced by ordinary beverage doses, and that not alone the higher faculties are affected, but the automatic reflexes, including those which control the circulation and other vital functions. Indeed, the carefully conducted researches of Benedict and Wells showed that the reflexes are much more sensitive to the effects of alcohol than the higher faculties, and are the first to show its influence.

\section{Alcohol Hinders Digestion}

It has long been known to physiologists that the administration of alcohol excites the stomach and causes an increased flow of gastric juice. But Radzikowski, the famous Russian investigator, has shown that the gastric juice thus produced by the action of alcohol upon the stomach is absolutely worthless as a digestive agent since it contains no pepsin, which is one of the two essential principles required for digestion. Alcohol, then, only induces the stomach to pour out an acid liquid which has no digestive power. Professor Chittenden, the eminent director of the Sheffield Scientific School of Yale University, in experiments upon a dog, found that strong alcohol produced an abundant flow of gastric fluid, but he also observed that an equal quantity of simple water produced an equal amount of gastric juice. Further investigation showed that the gastric juice produced by the administration of water was possessed of much more powerful digestive properties than the gastric juice produced by the administration of alcohol.

Other investigators, especially Haan, have shown that the influence of alcohol in stimulating the formation of acid by the stomach glands, is a temporary effect which rapidly disappears, so that the ultimate effect of the administration of alcohol is not only 
to hinder the formation of pepsin, but also to diminish the acid secretion as well. Large doses of alcohol cause the mucous glands to pour into the stomach a large amount of alkaline fluid which completely upsets stomach digestion.

The reason for this, according to the late Lauder Brunton, is that alcohol blunts the sensibility of the gastric nerves, so that the stomach fails to respond in a normal way to the natural stimuli of the foodstuffs. The only natural stimuli of the stomach are those which are found in normal food and water. It is thus apparent that alcohol does not aid digestion, but, on the contrary, hinders it.

The poisonous effects of alcohol have been well shown by the striking experiments of Doctor Stockard, of the Cornell Medical College, to determine the hereditary effects of alcohol. It was found that in the case of guinea pigs, if one parent was of normal heredity and the other from grandparents to which alcohol had been given, numerous defective offspring resulted. The descendants of inebriate guinea pigs were found to be dwarfed, weakly, malformed, sterile, and few survived more than a few days.

\section{Alcohol is a Discredited Drug}

The verdict of modern science respecting the use of alcohol in disease may be briefly summed up as follows:

(1) Atcohol never, under any conditions, increases the vital energy of the body, but, on the contrary, decreases it in a marked and uniform manner, through its poisonous influence upon the living cells.

(2) Alcohol is never a tonic or stimulant. It is always a narcotic, interfering with the bodily functions and lessening the nerve tone and vital energy.

(3) Alcohol always diminishes, never increases, the energy of the heart, and hence is detrimental rather than beneficial in cases of shock, collapse, fainting, etc.

(4) Alcohol increases the liability to infectious disease, and prevents the development of immunity.

(5) Alcohol does not aid digestion, but actually hinders it, especially in cases in which the digestion is already weak or slow; hence its use in connection with meals is absolutely unscientific and irrational. 


\section{Tea and Coffee}

The annual consumption of tea and coffee in the United States amounts to more than 10 pounds of coffee per capita, and one pound of tea. (U.S. Crop Reporter). pounds.

In 1921 the per capita consumption was increased to 12.5

Besides these, there are used considerable quantities of the cacao bean in the form of chocolate and large quantities of caffein-containing drinks of the Cola type.

According to Thompson, one-third of the total coffee produced in the world is consumed by the people of the United States.

Coffee contains 3 to 9 grains of caffein per ounce; tea, 6 to 8 grains; maté, 5 grains; cocoa, more than 4 grains of theobromine, a close relative of caffein, and Coca Cola, 4 to 5 grains per ounce of the syrup.

Guarana, a South American herb much used as a beverage, contains 17 to 22 grains to the ounce.

Mallet has shown that one cup of black coffee (about two ounces) contains one and three-quarters grains of caffein and the ordinary breakfast cup of coffee and hot milk about two and two-thirds grain of caffein. Dr. H. W. Wiley's analyses showed two grains of caffein in the coffee served at cheap restaurants, and four grains in that served at first-class hotels.

\section{Tea and Coffee are Poisonous Drugs.}

Caffein is recognized as a poison by all qualified authorities. The National Standard Dispensatory says that in large doses caffein is "capable of producing marked increase of the reflexes, cramps in the extremities, staggering gait and convulsions of a tetanic character."

The renowned chemist, Lehmann, showed long ago that caffein is a highly poisonous substance. He found that so small a quantity of caffein as 2 to 10 grains "will produce the most violent excitement of the vascular and nervous systems-palpitation of the heart, extraordinary frequency, irregularity, and often intermission of the pulse, oppression of the chest, pains in the 
head, confusion of the senses, singing in the ears, scintillations before the eyes, sleeplessness, erections, and delirium."

Prof. Lehmann further says:

The above-named results were yielded by experiments instituted on myself and several of my pupils with pure caffein. Five persons, after taking from 5 to 10 grains of this substance, were unfit for any business during the next day.

The following table shows the amount of caffein found in various caffein-containing preparations:

Per cent.

Cocoa (theobromine) $\ldots \ldots \ldots \ldots \ldots \ldots . . .1 .00$
Coco Cola
Coffee (roasted)
Kola
Maté
Tea

As ustully prepared, tea and coffee both contain about the same amount of caffein. Dry tea contains about one-third as much caffein as does the same weight of coffee. Cocoa contains about the same amount of caffein as does coffee. Cocoa nibs also contain caffein,

\section{Chronic Caffein Poisoning.}

From the above, it will readily appear that the amount of caffein contained in tea and coffee and their congeners is quite sufficient to produce toxic effects.

In view of the fact that the average daily per capita dose of caffein by the people of the United States is nearly 2.2 grains it becomes evident that the tea and coffee habit is a grave menace to the health of the American people.

The increasing consumption of tea and coffee in this country, due in part, perhaps, to the prohibition of alcoholic beverages as well as to the extensive advertising campaign organized by the producers and distributors of these drugs and the highly deceptive and widely scattered announcements of the claims of cola manufacturers concerning the virtues of their pernicious concoctions, renders necessary the warning of the public as to the dangers arising from chronic caffein poisoning.

Caffein produces a kind of stimulation which forces the cells 
of the body to do more work than they would naturally do, but without repaying them by replacing the energy which they expend.

Such stimulation of the cely is unnatural and always harmful. The cell is much like a storage battery. It must be charged before it can discharge. When a batter is fully charged, its voltage is high; it can overcome the maximum amount of resistance, and can do the maximum amount of work. The same is true of the cell. During rest, the cell stores up energy. A rested cell is full of "energy granules," its store of force. The body cells, when rested and well stored with energy, manifest their readiness for work by a feeling of fitness, which is sometimes called "pep." Activity is the natural overflow of accumulated animal spirits, a full charge of vital energy. No stimulus is needed to coerce the living cells to give off the energy with which they are brimming over.

When one feels unfit, the condition that seems to call for stimulation, it is most likely becouse the cells are lacking in stored energy, like a run-down battery. They need charging by food intake or rest, or by both.

The cells may be well chafged, but rendered inert or sluggish by stupefying poisons absofbed by smoking or liquor drinking.

Caffein destroys the sense of fatigue, but does not "rest you in five minutes," as the advertisements of poisonous caffein soda fountain drinks declare.

Caffein does not in any way whatever repair the vital losses which result from work. It only increases the losses. This is clearly shown by the fact first pointed out by Dr. Edward Smith, the eminent English authority on foods. Dr. Smith demonstrated that work under the influence of caffein seems easier than without it, but that it is followed by an increased sense of fatigue.

In his two most interesting books, "Across Greenland" and "Eskimo Life," Nansen takes a strong stand against tea and coffee as well as alcohol and tobacco. "After having tried the coffee extract two or three times in the afternoon and evening, and found that, though it cheered them up for a time, they got little or no sleep at night, they first restricted themselves to a morning cup, and then tabooed it altogether.

"My experience leads me to take a decided stand against the use of stimulants and narcotics of all kinds, from tea and coffee to tobacco and alcoholic drinks. It must be a sound principle 
that one should live in as natural and simple a way as possible, and especially when the life is a life of severe exertion in an extremely cold climate. The idea that one gains by stimulating body and mind by artificial means, betrays, in my opinion, not only ignorance of the simplest physiological laws, but also a want of experience by observation."

Dr. H. H. Rusby, dean of the College of the City of New York, and joint author of the "National Standard Dispensatory," says: "It is nevertheless true that caffein is a genuine poison, both acute and chronic. Taken in the form of a beverage, it tends to the formation of a drug habit quite as characteristic, though not so effective, as ordinary narcotics. While not cumulative in substance, it is so in effects, permanent disorders of the cardiac function and of the cerebral circulation resulting from its continued use."

\section{Coffee Raises Blood-Pressure.}

The effects of caffein upon blood pressure are well known. It is, indeed, one of our most dependable drugs for raising blood pressure in cases of shock, which fact alone is sufficient to condemn its habitual use as a beverage. The average coffee drinker is probably not aware of the fact that in drinking an ordinary "good" cup of coffee he is administering to himself a medicinal dose of caffein.

In a paper read before the Massachusetts Medical Society, Dr. Bullard gave an analysis of the various symptoms of teapoisoning, obtained from the study of a large number of cases. He arrives at several important conclusions: (1) that the action of tea is cumulative, and is most pronounced on the young and those in a depressed physical condition, although persons otherwise healthy not infrequently show poison symptoms; (2) that, as a rule, in adult women the average amount needed to cause poison symptoms was a little less than five cups daily; and (3) that chronic tea poisoning is a frequent affection whose most common symptoms are loss of appetite, dyspepsia, palpitation, headache, vomiting and nausea, combined with nervousness, and hysterical and neuralgic affections, frequently accompanied by constipation and pain in the region of the heart.

The writer has in many cases seen the blood pressure drop 20 to 40 points in cases of high blood pressure, after the disuse of coffee, and with a notable improvement in health. 
Tea, coffee, and the whole family of caffein-containing drugs must be regarded as poisons which especially affect the heart and blood vessels. The increasing use of these drugs may well be charged with responsibility in part at least for the notable increase in the death rate from diseases of the heart and blood vessels which has occurred within the last thirty years, making this class of maladies the chief cause of death in this country at the present time.

The amount of caffein contained in tea and coffee as ordinarily used is practically the same, the chief difference being in the fact that coffee contains in addition a considerable amount of smoke poisons.

"Coffee heart" has become a familiar term with life insurance examiners, who know well the damaging effect of this heart poison.

\section{Tea, Coffee, and Uric Acid.}

The evil effects of uric acid have long been recognized. It is probable that uric acid is not responsible for all the mischief attributed to it by Haig and others some years ago; but it is an excretory poison which the kidneys must eliminate. The very first indication of failure in the efficiency of the kidneys is an increase of uric acid in the blood. Anything that tends to increase the amount of uric acid in the blood and urine tends to overwork the kidneys and induce their premature failure and also to encourage degeneration of the arteries (arteriosclerosis), heart, liver and other organs.

It is a fact well known to chemists that caffein is very closely related to uric acid. Both these poisons belong to the same chemical family, the xanthin group. Their effects upon the body are the same.

Professor Mendel and his students made a careful study of the effects of tea and coffee upon the uric acid of the urine. With reference to the results, Professor Mendel says:

"The addition of a strong coffee infusion to a purin (uric acid) free diet causes a marked increase in the excretion of uric acid. The analyses showed that the amount of uric acid after tea drinking was doubled." There is more uric acid (xanthin purin) in a cup of coffee than in the same quantity of urine.

The damaging effect of this increase of uric acid will be better appreciated when the recently ascertained fact is recalled 
that the human liver does not possess the ability to destroy or detoxicate uric acid, which is an important function of the liver in all carnivorous animals.

\section{Coffee Cripples the Liver.}

Tibbles, an eminent English authority on diet, calls attention to the pernicious influence of caffein on the glycogenic function of the liver, which leads him to condemn the use of coffee altogether. This observation suggests that the coffee habit may be one of the causes for the notable increase of diabetes in this country in recent years.

\section{Other Coffee Poisons.}

Tea and coffee contain a notable amount of tannin. This is particularly true of tea. Ordinary tea contains 15 per cent. of tannic acid. Assam tea contains 32 per cent. Tannic acid is so highly soluble that it is impossible to make a decoction or infusion of tea which is not strongly charged with this astringent drug, the effects of which upon the stomach are highly pernicious. Tea could easily be used as a substitute for oak bark in the tanning of leather. There is no foundation for the prevalent belief that the tannin may be avoided by making the infusion quickly.

Another point of importance which has recently been brought out by Dr. L. F. Sayre, head of the School of Pharmacy of the University of Kansas, is the fact that the process of roasting coffee develops certain incomplete combustion products, phenolic in character, or substances allied to carbolic acid, including creosote, pyridine, and a long series of chemically related poisonous substances.

All of these substances, together with certain oils and caffein, give to tea and coffee their toxic properties.

Burmann has shown that the volatile principle of coffee damages the hemoglobin of the blood, depresses the central nervous system, causes irregular heart action, and produces dyspnea.

According to Potter, roasted coffee contains besides, pyridine (one of the poisons of tobacco smoke) and furfuryl, a substance so poisonous that so small a dose as 1.5 grain will produce persistent headache, and 7 or 8 grains will kill a rabbit. An extensive investigation made by Sayre showed that all roasted coffees contain these poisons. The observations by Dr. Sayre with reference to the poisonous effects of the volatile products resulting 
from partial combustion found in roasted coffees were verified by Dr. Hamilton, head of the biological laboratory of Parke, Davis \& Company.

Dr. Sayre calls attention to the fact that the starches and sugars of coffee when roasted are in part converted into furfuraldehyde, one of the constituents of bad whiskey.

Roasting, also, according to Dr. Sayre, converts tannic acid, always present in coffee, into catechol, which is more poisonous than carbolic acid, and pyrogallol, which is still more poisonous.

The phenolic substances found in roasted coffees by Dr. Sayre have been shown by the experts of the Lederle Laboratories of New York to have a decidedly harmful effect upon living tissue cells cultivated outside of the body. This most delicate biologic test is a convincing and unanswerable proof of the damaging character of all roasted coffees.

Evidently not wishing to be considered as an enemy of the coffee business, Dr. Sayre naively says, "We should not condemn coffee simply because its toxic principle has been identified more certainly." Certainly not. Coffee is to be condemned not because its toxic principles are known, but because of the bad effects of these toxic principles, which have been clearly demonstrated, not only through the scientific experiments of Dr. Sayre and other experts, but by the clinical observations of hundreds of physicians and thousands of unhappy victims of the coffee habit.

\section{Some Poisonous Effects of Tea and Coffee.}

According to Dr. Wm. N. Leszynsky, of New York, coffee is particularly poisonous to children, over-exciting the brain and producing functional disturbances. He says, "I have often seen night terrors, insomnia, tremulousness disappear after the withdrawal of coffee." He attributes to the use of coffee arrest of physical development, and tells of a boy six years of age who suffered from acute coffee poisoning, the symptoms of which were "active delirium, widely dilated pupils, tremor in the facial muscles and the extremities, and severe tachycardia, the pulse rate being two hundred beats a minute." The child also had hallucinations. These symptoms were produced by eating half an ounce of coffee beans.

Doctor Leszynsky also states that the transitory sensation of well-being which is experienced by many susceptible persons after taking a cup of coffee "is soon followed by a feeling of apprehen- 
sion, general tremulousness, and indigestion." $\mathrm{He}$ asserts that the habitual use of coffee in such persons "invariably leads to persistent functional disorder of the nervous system, as well as disturbances of digestion."

Dr. Gilman Thompson, professor in Cornell University Medical College, of New York City, asserts that the use of coffee to produce wakefulness at night "soon results in forming a coffee or tea habit in which the individual becomes a slave to the "beverage" and when deprived of it, "suffers from languor, prostration and restlessness and craving." Two or three cups three times a day produce "muscular tremors, nervousness, anxiety, apprehension, palpitation of the heart, vertigo, heartburn, dyspepsia, constipation, insomnia, and emaciation."

Doctor Thompson well suggests that coffee users, by suddenly stopping the drug, may discover the ill effects which have been produced. The degree of craving experienced is an evidence of the damage which has been done and of the influence which the drug has obtained over the system.

Gautier, the great French authority, says that coffee produces nervous excitement, insomnia, hallucinations, pain in the heart, distressed breathing, weakness of the muscles, disorders of the circulation. He says, "One may become a caffeis (coffee drunkard) just as one may become an alcoholic or morphia maniac,"

Here are some of the poisonous effects as described by Dr. John V. Shoemaker, dean of the Medico-Chirurgical College of Philadelphia, and professor of materia medica, as described in his great work on "Materia Medica and Therapeutics."

Caffein paralyzes the absorbing power of the convoluted tubules (of the kidney). Small doses cause irritation of the digestive tract, venous congestion and hemorrhoids.

From a dose of twelve grains (three or four cups of ordinary coffee) Doctor Pratt experienced restlessenss, sleeplessness, mental depression and tremor.

Zenetz, an eminent German physician, called attention to the dangers of caffein, asserting that three grains two or three times a day (two ordinary cups) cause rise of blood-pressure (leading to apoplexy), constriction of the chest, dyspepsia, restlessness. Zenetz has seen death result from five grains two or three times a day. The cause of death was tetanic contraction of the heart.

Miller, Rehfuss and others, have shown that tea and coffee cause trembling of the hands. This effect of coffee is well known 
to coffee users. Trembling of the hands may be produced by a single cup of coffee in susceptible persons. This fact alone is sufficient evidence of the dangerous character of this drug. If a certain baker's bread or a particular brand of tomatoes were found to produce such effects, it would be discarded at once. Evidently this is the proper thing to do with coffee.

According to Tibbles, tea deadens the sensation of hunger, inhibits peptic and salivary digestion and causes indigestion, atony and catarrh of the stomach. The following bad effects from the use of tea are also noted by Tibbles: "Insomnia, palpitation of the heart, flushing of the face, muscular tremors, and other signs of nervous irritability.

"Rivers found that an excess of caffein has a very transitory stimulating effect, and may be an accelerator of fatigue, and 'experiments suggest that caffein is a dangerous stimulant in cases of prolonged fatigue or in that tendency to fatigue which characterizes neurasthenia.' Smoking tea in cigarettes is probably the most injurious mode of consuming it, the nervous symptoms being much increased."

Professor Kronecker, an eminent physiologist of Berne, Switzerland, denounces coffee as one of the poisons which is blighting the human race, and urges strongly that tea and coffee, which as he says contain known poisons, should be discarded.

The relief from the sensation of fatigue which follows the taking of coffee is highly deceptive. It gives to the taker the impression that the agreeable beverage has wholly removed the exhaustion of which the sense of fatigue was the signal, and consequently that effort may be continued without injury. This is altogether untrue. As a matter of fact, this effect of tea and coffee in dissipating the sensation of fatigue is one of the greatest dangers which accompany these beverages. They do not cure fatigue but only hide it by benumbing the fatigue centers so that they are not able to sound the warning signal which indicates that effort should cease, to afford opportunity for repair of the wasted energies. Experiments conducted by Rivers clearly demonstrated that the repeated use of caffein produces the "special tendency to fatigue which is characteristic of neurasthenia."

\section{Tea and Coffee Neurasthenia.}

The widespread use of tea and coffee in England and America and in other civilized countries is unquestionably a prolific cause of neurasthenia, especially in women who on the whole seem to 
be more susceptible to these drugs than are men, and who are more addicted to their use.

A man or woman who cannot begin a day's work in comfort without one or two cups of coffee, or who suffers from headache or nervousness when deprived of the accustomed beverage, is a tea or coffee neurasthenic and as much a drug habitué as a person who is addicted to the use of opium or cocaine.

Coffee is without doubt a leading string to other drug habits, particularly the use of alcohol and tobacco. Claude Bernard, an eminent French physiologist, noted that the victims of the opium habit were largely found to be persons who had been much addicted to coffee.

\section{Coffee Insomnia.}

Thousands of men and women are suffering from sleeplessness, nervousness, chronic fatigue, indigestion, hardening of the arteries, constipation and various other disorders which are caused, perpetuated and continually aggravated by the habitual use of coffee.

Brillat de Savarin, an eminent French jurist and author of the celebrated work, "Physiologie du Gout" (Physiology of Taste), thus strongly condemned the use of coffee:

Every father and mother should strictly forbid their children to take any coffee, unless they wish to see them shriveled up, bony and withered little things before they are twenty years old.

Savarin tells how that having to do some important work for the Minister of Justice he had to sit up all night. In order not to fall asleep he drank two cups of coffee. He then found he did not have to do the work, and tried to go to sleep. He said, "After being two hours in bed, I felt more wide awake than ever. I was in a very lively state of mental excitation, and I pictured to myself my brain as a mill of which the wheels were constantly going without having anything to grind. I therefore passed the night without sleeping and without feeling sleepy for a single minute. I arose the next morning and passed the whole day in the same state. . . . Finally, on retiring to rest the second day at my accustomed hour, I concluded I had been forty hours without closing my eyes."

This experience was sufficient to convince the jurist that coffee was a nerve poison. He accordingly renounced its use forever. 
Tea drinking was mentioned by the Irish Commissioners for Lunacy as a cause for the increasing insanity among the people of Ireland, one of the late results of chronic insomnia and neurasthenia.

\section{Tea, Coffee and Indigestion.}

Chronic indigestion is a very common result of the use of either tea or coffee. Caffein overstimulates the acid-forming glands of the stomach, causing hypcracidity and delay of food in the stomach. There are strong reasons for believing that the free use of coffee causes gastric ulcer, and there are eminent authorities who insist that the use of coffee causes cancer of the stomach. The production of cancer is attributed both to the caffein and to injury from swallowing the beverage at an excessively high temperature.

The tannic acid of tea precipitates pepsin, thus causing indigestion and hyperchlorhydria.

Sick headache, nervousness, trembling of the hands and insomnia are among the most common of the evil results of the use of tea and coffee.

Roberts found that tea destroys the action of the saliva upon the food and prevents the digestion of starch in the stomach, a serious hindrance to digestion. Tea drinkers are thus certain to suffer from indigestion, through which all the other bad effects of tea are aggravated.

The National Standard Dispensatory, a recognized authority, says concerning the habitual use of coffee: "When taken for a long period of time, it often produces indisposition and acidity of the stomach, due to hypersecretion of gastric juice. In other persons, it tends to produce biliousness, and in still others palpitation of the heart and tremors."

\section{Decaffeinated Coffee (Kaffee Hag).}

Certain coffee preparations recently introduced are claimed to be free from caffein, but to possess all the other properties of ordinary coffee. Unfortunately, such undue stress has been laid upon caffein as the one poison of coffee that many persons are likely to be beguiled into the use of these preparations, which are only a little less injurious than the ordinary preparations of the coffee bean. It is true that decaffeinated coffee contains caffein only in minute quantities, but in other respects it is like other 
coffees. Being roasted, it must contain pyridine, catechol and other phenolic bodies more poisonous than carbolic acid, together with various other toxic smoke products-poisons which in small quantities kill frogs, guinea pigs and rabbits, and have been shown to cause headache in minute doses as well as disturbances of respiration and heart action, and to produce destructive effects upon the blood.

These unwholesome properties are certainly quite sufficient to render decaffeinated coffee unworthy of recommendation as a beverage. Nature has supplied us with pure water, with a great variety of fruit juices and wholesome and harmless flavors quite sufficient to meet all our needs. Tea, coffee and their congeners are all substances which are unattractive in flavor, and the taste for which must be acquired. Even then, the majority of users of these drugs find it necessary to conceal their natural flavors by means of sugar, cream and other more agreeable substances.

Coffee and tea are particularly objectionable for children because they lessen the time spent in sleep and thus diminish the opportunity for growth. In adults these drugs in the same way lessen the opportunity for tissue repair and so hasten the beginning of old age processes and lessen the length of life.

A report of the Connecticut Agricultural Experiment Station states that vacuum coffee contains the same amount of caffein as does ordinary coffee. "George Washington Coffee" contains about four times as much as ordinary coffee.

\section{Maté.}

Efforts have recently been made to introduce into the United States the use of maté, a caffein-containing product of South America. Maté consists of the powdered leaves of a variety of holly, a native of Paraguay. It contains caffein, but in somewhat less amount than tea.

Maté was found in use by the Indians when discovered by the Spaniards. It was introduced into England more than a century ago, but was unable to compete with tea as a mild intoxicant. Its properties are essentially the same as those of tea and coffee.

\section{Coca Drinks.}

A prominent medical journal published during the war the following warning against gaffein-containing drinks: 
We understand that the govefnment is interested in the sale of caffein drinks to the soldiers, which are admitted to have done very great harm to them, lowering the morale and injuring the health of the victims. They are conceded to have encouraged crime and immorality.

But is there not an inconsistency involved in this crusade against caffein drinks, so long as the meh are provided with coffee? Why should young and vigorous men in the service be habituated to a powerful stimulant which they do not need, and which is known to be pernicious, while at the same time caffein drinks are prohibited outside the camps?

\section{Caffein the Cause of Goiter.}

Dr. von Wedekind, of the United States Navy, writing to the Journal of the American Medical Association in 1912, called attention to the remarkable frequency of goiter in young men applying for enlistment in the navy. Among the questions asked these young men was, "What beverages do you drink?" and in every case the reply was "Coca-cola." These, with previous observations, led to the conclusion that caffein may be a cause of goiter. It is well known that caffein is the active constituent of Coca-cola.

The Mellon Institute of Pittsburgh, one of the great research laboratories of the country, has made a study of the effects of coffee and strongly condemns its use, calling attention to the special dangers arising from its use by persons past the prime of life.

"The human body with advancing age has a marked tendency to become more sensitive to stimulants such as caffeine, and the excitement of the nervous centers is less well borne in senescence than in the prime of life. With age comes increased nervous irritability and the need for more repose and sleep." The use of the stimulants, tea and coffee, by old people is thus highly injurious. It is evident that the same objections apply to young people as well as old, the only difference being that in young persons the bad effects are not so noticeable because of the greater vital reserve which enables the young to digress the laws of health with apparent impunity, but of course only at the expense of the precious vital reserve, the loss of which is felt in later years.

Professor Oliver T. Osborne of Yale University says of the effects of coffee on aged persons, "The action of caffeine is to increase general nervous irritability, cause polyuria, and especially to stimulate the thyroid and parathyroids to abnormally increased 
activity, with the result of more nervous irritability and muscular irritability and trembling. Caffeine often raises the pressure, where such an increase of blood pressure is not needed."

Dr. Albert Merrell, Professor of Chemistry, Pharmacy and Toxicology, and Member of the State Board of Health of Missouri, in his "Digest of Materia Medica and Pharmacy," says, "Experiment has proven that upon animals the action of caffeina, theina and theobromia is identical in kind, though the latter (theobromine) is poisonous and fatal in much smaller doses."

\section{Soda Fountain Drinks Containing Caffein.}

Dr. Wiley has shown that coca drinks contain practically as much caffein as does the same amount of coffee. They are simply flavored decoctions of caffein.

There are mischiefs in the soda fountain preparations which render them even more dangerous than coffee. These are, first, the fact that they are always ready and accessible at any hour of the day and night. Second, that they are generally supposed to be not only innocent of possible harmful effects, but through most alluring advertising are presented to the public as possessing most remarkable beneficial properties. As a result, not only adults, but children are induced to use caffein in the form of a soda fountain beverage, and in larger quantities than coffee would ever be used. In other words, the public are beguiled into a "dope" habit. Cola drinks are an insidious poison, the sale of which should be prohibited by law. Dr. Wiley's campaign against this nefarious traffic was wise and timely, and should have had the support of all medical men.

\section{Coffee Substitutes.}

Most coffee substitutes consist of cereals in some form combined with molasses and roasted until a part of the starch and sugar are caramelized. The process of roasting which is always employed develops in these substitutes poisonous phenolic and other smoke products the same as are produced in ordinary coffee. These substances may be present in such quantity as to cause nausea and vomiting. Of course, caffein is not present in cereals. But cereal coffees charged with smoke poisons may be the source of as much harm as that which usually results from ordinary coffee. 


\section{Tobacco}

Smoking after or in connection with meals has become so common a practice that a brief reference to this pernicious habit

- seems proper in a work devoted to nutrition and dietetics. Tobacco is by no one claimed to be a food, although value has been claimed for it on the ground that by its use the consumption of food is lessened. This means nothing more than that by its poisonous effects the activities of the body cells are interfered with, certainly not a useful property.

\section{The Effects of Tobacco.}

Tobacco smoking produces a sort of comfort or solace because of its narcotic or sedative effect upon the nerve cells, especially the cells of the higher cerebral centers which are concerned in thought. The continued use of tobacco, however, as has been pointed out by Tibbles, "causes degeneration of the same cells (brain cells), and thus leads to loss of power of thought, enfeeblement of the attention or power of concentration, and deterioration of the memory."

Rivers demonstrated by means of the ergograph that tobacco has a most unfavorable influence upon capacity for muscular work. Lombard, professor of physiology of the University of Michigan, demonstrated the same toxic influence of nicotine upon the muscles. He demonstrated that a single cigar lessens muscular power for many hours.

The tobacco habit leads to serious impairment of the movements and secretions of the stomach and intestines and gives rise to dyspepsia, gastric catarrh, and impaired nutrition. Under the influence of this drug the body cells act with less vigor, metabolism is reduced, and all the life processes are slowed.

\section{Tobacco a Heart Poison.}

The first effect of a cigar is to raise the blood pressure and slow the pulse, but this effect is later followed by a quickening of the pulse. The pulse of the old smoker becomes irregular, and myocarditis and other changes in the heart are produced. According to Tibbles, "the juice from 22 pounds of tobacco yielded 
2.2 pounds of nicotine, two-thirds of an ounce of nicoteine, onesixth of an ounce of nicotinine and 15 grains of nicotelline," all deadly poisons.

Angina abdominis, a form of angina affecting the vessels of the abdominal organs which is characterized by crises of very severe abdominal pain, has been shown to be one of the consequences of smoking.

French tobacco contains 7 or 8 per cent. of nicotine, which is the principal poison of tobacco. In Kentucky and Virginia tobacco 6 to 7 per cent. of nicotine is found. In smoking the nicotine is partially destroyed, 50 per cent. of it remaining. The smoke contains besides nicotine, pyridine, hydrocyanic acid, ammonia, carbon monoxide and numerous other poisons, some twenty or more.

The highly poisonous effects of carbon monoxide are well known. Carbon dioxide, or carbonic acid gas, is a comparatively harmless poison. It is, of course, irrespirable, and in pure or rather concentrated form will produce asphyxia; but carbon monoxide is deadly in minute doses. It is found in considerable quantity in cigarette smoke, as shown by Hartridge (Journal of Physiology, 53). This investigator has shown that heavy smokers are in a state of chronic carbon monoxide poisoning.

We are glad to be able to quote the following keen and thoroughly just criticism of the tobacco habit from so competent a medical authority as the New York Medical Journal (Feb. 2, 1918) :

Psychical Pharmacology of Nicotinism.-Of the three principal narcotics used in this country, nicotine for several reasons leads in popularity. One of the reasons is that nicotine most quickly satisfies the longing of the storm tossed "mental" and of the inept neuron. Tolstoi said that after the first whiff from his pipe he no longer felt the prick of conscience. To be able to banish care is, to a jaded mentality, the one supreme requirement of a narcotic. Nicotine exalts the ego. The nicotine-enthused and flattered ego feels like advertising its way of taking a narcotic. The user actually feels that everybody likes, or at least ought to like, everything that goes with his use of nicotine. To him it is entirely unbelievable that a sane person can object to any feature of its use. The grandeur intoxication prompts the customary good-natured obtrusion of the practices of nicotinism into the presence of others. As care flees away in the intoxication, there comes in its stead a feeling of being at peace with the world. The narcosis grants the peace, but the condition of peace always is that the use of nicotine is not to be interfered with. Because 
the narcotic effect is upon the ego itself, inflated ideas as to personal estimation of the meaning of the intoxication and resentment to any and all criticism of nicotine are very acute.

The reactions of public opinion to these features of the narcosis are favorable to the public use of nicotine. This accords with the psychological law, that the mind is likely to be captivated by that which presents a bold front of assumed sincerity. Public opinion does not give the matter study before it sanctions the public use of nicotine, but the use forces itself upon the public, and as non endorsement of the narcotic act means a fight, the peace-loving instinct in man surrenders to nicotine. The medical matter involved is that the end results of nicotine narcosis are never as good as the non-narcotized man would have been capable of. Besides its use violates every principle of scientific medicine.

The tobacco habit has obtained almost a strangle hold upon society in every civilized land and is rapidly invading the countries just emerging from a less sophisticated and possibly less culpable heathenism. Sometime the hold will be broken and this pernicious drug habit, a gigantic curse of modern civilization, will be cast off as a handicap which a degenerating race can no longer tolerate. Already, forces are at work which within a quarter of a century, if not sooner, will so discredit the delusive drug that its condemnation by sane and intelligent men and women will be universal. One of the most important of these agencies by which this

good work will be accomplished we believe to be "The Committee of Fifty to Study the Tobacco Problem," which has initiated and is carrying forward important researches on all phases of the tobacco question as related to human welfare, physical, mental and moral, by the aid of scientific experts of such national and international standing that their findings, when published, and the ultimate verdict when rendered, will be above and beyond dispute or refutation. A similar authoritative scientific inquiry on the alcohol question laid the foundation for prohibition and the overthrow of the saloon; and a like inquisition followed by a nationwide educational campaign will in due time abolish the smoke nuisance, a thing too impudent and offensive, to say nothing of its insidious destructiveness, to be tolerated by a decent and rational society. 


\section{Poisoned Foods}

Ptomains are poisons produced by the decomposition of protein. They are particularly encountered in decomposing animal foodstuffs, and especially in flesh that has been too long kept after the slaughter of the animal. Some of the ptomains are highly virulent poisons. The most deadly are likely to be found in sausage, hamburger steak, game, and chickens, turkeys, ducks and geese which have been long in cold storage.

Ptomains are produced in the early stages of decay and so may be present before there is any taint of decomposition perceptible to the senses.

Müller calls attention to the fact that so-called ptomaine poisoning is usually due to infection of the slaughtered animal with paratyphoid (paracoli) bacteria or other similar infections. $\mathrm{He}$ recommends that the term ptomaine poisoning should be abandoned.

It is interesting to recall in this connection that Tissier has shown that the paracoli (paratyphoid) is the cause of the colitis. It is also known that white diarrhea, a common disease in chickens, is due to the same germ, and eggs are frequently infected by it and may thus become a cause of colitis.

All meat becomes infected with colon germs during the process of slaughtering. It is consequently evident that all meats are open to suspicion, and that the disuse of meats would eliminate an active cause of disease.

Oysters and mussels may contain deadly ptomains when fresh, doubtless because they are scavengers and subsist upon the offal which they find in the water, especially about river mouths.

There are more than 60 different ptomains known, of which may be named, cadaverine, putrescin, butylamin, amylamin, neuridine, saprin, sperium, mydalein, cholin, neurin, muscarin, betain, gadinin, mytilotoxin, callidin, parvolin, corrindin, morrhuin, asellin, typhotoxin, tetanin, spasmotoxin, telanotoxin, pyocyanin, tyrotoxicon.

This is certainly a formidable array of deadly agents, nearly all of which may be escaped by avoiding flesh foods, which under all ordinary circumstances must be regarded as wholly unnecessary additions to the bill of fare. 


\section{Food Toxins.}

Besides ptomains, there may occur in food other deadly poisons known as toxins. These are the result of the growth in animal tissues, either before or after slaughter, of germs which cause specific infections. One source of food toxins is a germ which gives rise to botulism. This germ grows readily in fish and meats which have not been properly processed in canning. It has also been found in spoiled canned beans, spinach, and ripe olives canned in glass.

Flesh foods are especially liable to contamination with poisons which may be produced by the action of either specific germs or the ordinary organisms which produce putrefaction. Sausages, potted meats, fish, oysters, lobsters, eels and canned meats which have spoiled in the can or been allowed to stand after opening, often contain poisons which produce very severe and often fatal illness.

Meat poisoning often results from the ingestion of Gärtner's bacillus in cold storage meats. It most frequently occurs as the result of eating pork, veal, fowl, beef or ham.

Fish poisoning may result from the use of fish that are partly decomposed or diseased, or which have spoiled in the can, either before or after opening, or it may result from the use of fish of certain species which are always poisonous. Fish sometimes become poisonous through feeding on decomposing corals and other poisonous substances.

Poisoning often occurs as the result of eating shellfish which contain a special poison, mytilotoxin. Shellfish are scavengers and thrive in the vicinity of the outlets of rivers which receive the sewage in large cities. Typhoid fever and other germs are found in the intestines of shellish. Oysters strain the water through their gills and capture great numbers of bacteria and other organisms as well as particles of decomposing matter. The poison found in these creatures is chiefly concentrated in their livers.

Oysters and shellfish of all sorts are always swarming with bacteria. Oyster juice is fairly alive with the wriggling bacilli. Raw shell fish are too unclean to be given a place in any stomach except that of a scavenger.

Other virulent poisons have been found in the flesh of animals which when slaughtered were suffering from pyemia, septicemia or specific enteritis. 
The flesh, milk, and dairy products from cows infected with foot and mouth disease, particularly cheese, butter, and milk, may give rise to the same disease in human beings.

Acute poisonings from animal foods are frequently seen in the sickness which follows the use of herring, pickled fish advanced in decay, particularly stale oysters, salted sturgeon and tainted horse flesh.

The flesh of hunted animals often becomes poisonous through the entrance of the $B$. coli into the blood before death.

Cold storage meats sometimes become poisonous from the slow decomposition which takes place even at temperatures only a little above freezing.

Rouennaise ducks are rendered poisonous by the peculiar way in which they are killed. They are killed by a puncture of the medulla, which causes death without loss of blood. They are afterwards beaten, causing absorption of intestinal bacteria and poisons which are diffused throughout the body.

\section{Hamburger Steak.}

Hamburger steak is usually prepared from odds and ends, trimmings of meats which have often been accumulating for several days. The retail butcher keeps under his counter a receptacle into which these scraps are thrown. When a sufficient amount has been collected, the mass is worked over and served up in a form which offers no suggestion of its miscellaneous origin. Meat inspectors report that not infrequently the butcher neglects to scour and disinfect his collecting receptacle and in consequence it is often found with fragments of putrid meat clinging to its interior, and in hot weather are found swarming with maggots.

In view of these facts it is evident that Hamburger steak cannot be too strongly condemned as an article of food for human consumption. According to Weinzrl and Newton who some years ago (1914) made an extensive study of Hamburger steak and other meats with reference to their bacterial content, steak practically always contains $1,000,000$ bacteria to the gram, or $30,000,000$ to the ounce even "when showing no taint or other evidence of putrefaction," and half the samples contained 10 times as many bacteria, or $300,000,000$ to the ounce.

According to these authors, fully half of all the Hamburger steak offered for sale "is potentially rotten." As a matter of fact it is evident from the above statements that all Hamburger steak 
is potentially rotten since the difference between the best and worst samples is only one of degree. Putrefactive bacteria grow so rapidly that their numbers may easily multiply 10 fold or even more within 24 hours under favorable conditions.

\section{Moldy Meat.}

The average meat-eater is probably not aware that the meat he consumes at the breakfast table often belongs to the longneglected-to-be-buried type. The fact is that meat kept long in cold storage grows such a prodigious crop of mold, bright green, black and other colors, that it has the appearance of being covered with a flourishing beard.

The Food Investigation Board of Great Britain has been making a study of the fungi of frozen meat. Special Report No. 6 contains information concerning "the black spot and other types of fungi found on chilled and frozen meat. Black spots on the surface of beef and mutton brought from the Argentine and New Zealand and some other countries are commonly found upon arrival."

These black spots are produced by molds of fungi and indicate that the meat has been stored for a long period before shipping. The spots are frequently so numerous that they overlap one another and the fungus threads penetrate below the surface of the meat. Experiments have shown that these fungi grow at temperatures 10 or 12 degrees below freezing.

The writer was recently informed by a Boston meat dealer that hotel chefs preferred meats that were covered with slime, doubtless because such meats are much more tender, being farther advanced in putrefaction.

Edelmann, Mohler and Eichhorn, in their Text-Book of Meat Hygiene, quote Marxer as authority that "meat should be considered as putrefactive if one gram contains over a million organisms of any kind, or proteus bacteria in large numbers."

According to Weinzrl and Newton, a standard of $1,000,000$ bacteria per gram (30,000,000 per ounce) "would condemn nearly all samples of hamburger, when showing no taint or other evidences of putrefaction. Samples of other market meats, all of which would otherwise pass inspection, often exceed this limit."

It is evident that whether the standard be $1,000,000$ or $10,000,000$, it is a question of degree of rottenness, since the lower standard named means not less than $30,000,000$ bacteria 
per ounce, and bacteria of the most objectionable class, including B. Welchii, the terrible organism of gas gangrene. Hamburger steak is carrion, and quite unfit for food except by a turkey buzzard, a hyena, or some other scavenger.

\section{Poisons in the Flesh or Milk of Sick or Overworked Animals.}

The flesh of a horse which was killed in the effort to break him was fed to hogs. The hogs sickened and died. (Kahnert).

The flesh of a deer caught in a trap and killed by its efforts to escape was the cause of sickness in a large number of persons who ate of it. The infant of an overworked mother showed marked symptoms of poisoning which disappeared when the child was nursed by another woman.

It has long been known that the flesh of overworked animals undergoes putrefaction very rapidly after death. It is even possible that in such cases the tissues of the animal may be pervaded by bacteria during life on account of the greatly lowered resistance which results from overwork. It should be generally known that the flesh of an animal subjected to violent exertion or overwork before killing is absolutely unfit to eat. Without doubt, many such animals find their way to the market.

Some years ago the people of Boston were very much disturbed by the discovery that animals which had been condemned by the State Board of Health because of infection with tuberculosis were sold in their markets. Investigation showed that such animals were regularly disposed of for food purposes. The defense of the board was that the same practice prevailed in other states and was recognized as legitimate by the United States Department of Agriculture. This repulsive practice seems to be general.

A case was reported by Bollinger in which sixteen persons were made sick by eating sausage made from the body of a pig which had received arsenic as a remedy.

It should always be recollected that fish are scavengers, and when caught in waters near the mouths of sewers are very likely to be infected by the sewage which they greedily consume as food.

Poisonous substances are found in the flesh of animals which have suffered from apoplexy, especially hogs, animals which have died of indigestion and which have been affected with dropsy or infectious disorders. Poisons of this sort are not destroyed by 
cooking and have given rise to symptoms in human beings resembling cholera or typhoid fever. Of more than one hundred people who ate of a cow that had died of puerperal fever half were made very sick.

The flesh of animals which have suffered from disease of the lungs, from enteritis, peritonitis, nephritis, and hepatic affections; always contain poisonous substances and are wholly unfit for food.

Dr. Weseberg reported some time ago an epidemic of meat poisoning in which sixty-three persons became seriously ill as the result of eating the flesh of a cow which had been killed because of pericarditis resulting from an accident. The persons who became ill had eaten of minced meat prepared from the animal and also portions of the cooked liver. The symptoms resulting were violent headache, severe abdominal pain, dizziness, muscular weakness, diarrhea and vomiting. Bowel discharges were highly offensive. Mice inoculated with portions of the meat died within one to three days, with symptoms similar to those shown by the persons who had eaten of the same flesh.

When the fact is recalled that all the flesh sold in the market, and practically all that is eaten, is swarming with the bacteria of putrefaction, the wonder is that the number of persons who suffer from acute symptoms of poisoning after eating flesh is so small.

\section{Milk from Tuberculous Cows Poisonous.}

When it is recalled that in the most favored portions of the United States 5 to 10 per cent. of all the milch cows are tuberculous, while in many states the proportion of infected animals reaches 25 per cent., some idea may be formed of the enormous mischief done by the use of the milk of tuberculous cows. Milk should be regarded as unsafe for use as food unless known to be produced by cows proven by federal inspection to be free from tuberculous disease.

Roger insists that too much precaution cannot be taken against the use of the milk of tuberculous animals, since the cooking of milk does not render it harmless. The researches of Calmette and Breton demonstrate that the ingestion of dead germs may produce a fatal cachexia. Even though it contains no bacilli, the milk of tuberculous cows should be rejected. When such milk is given to young rabbits or injected under the skin of 
guinea pigs, the animal falls promptly into a state of cachexia and marasmus. The experiments of Pasq. de Michele show that such milk contains poisons which are not destroyed by boiling or by digestion.

\section{Poison in Clams.}

Soper says: "It is not unusual to see persons digging hard clams close to the mouths of sewers. After a very short period in market this form of shellfish is often eaten raw. Hard clams are particularly in favor during those warm months when oysters are out of season and diarrheal diseases are most common. It would not be at all surprising if many outbreaks of diarrheal disease at seaside summer resorts were traceable to hard clams, taken from nearby polluted sources.

\section{Typhoid from Oysters.}

An investigation made by Klein and Boyce of typhoid fever epidemics in Brighton, England, traced the disease to the use of oysters. Sewage bacteria were found both in the oysters and in the mud. The proportion of cases definitely traced to the use of oysters was in 1894, 38 per cent.; in 1895, 34 per cent.; in 1896, 32 per cent; in 1897, 31 per cent. In recent epidemics in New York and Chicago more than eighty per cent of the cases were traced to the use of infected oysters. These facts ought certainly to condemn the oyster as a food under any condition except the absence of any more wholesome sustenance.

\section{Poisonous Ice Cream.}

The unwholesomeness of ice cream, on account of its low temperature and depressing influence upon the activities of the stomach, has long been appreciated, but the great danger of infection from this source has within the last few years been made clear by bacteriological studies conducted in the biological laboratory of the city of Philadelphia by Drs. Pennington and Walter. Physicians constantly meet cases of disease of the stomach, both acute and chronic, which are directly traceable to the use of ice cream. This matter is especially important in view of the large amount of ice cream consumed by young children and invalids and its extensive use in connection with soda water.

It was found by Drs. Pennington and Walter that the streptococcus, a pus-forming organism which is always found in ice 
cream, is the principal cause of the acute disorders directly traceable to its use. Escherich, Kaiser, Petroschky and others have called special attention to the dangerous character of the streptococcus found in milk, and attributed to this microorganism a large part of the infant mortality which occurs in the summer time. Heynemann found that the streptococci of milk readily take on the highest degree of virulence, especially in persons whose resistance is low. There are many such persons who show signs of bacterial infection whenever they eat ice cream. In the special research above referred to streptococci were found in 80 per cent. of the samples examined. Of 50 per cent. of the samples examined, all but three contained more than 500,000 microorganisms per cubic centimeter $(15,000,000$ per ounce).

Ice cream is usually made from stale cream. It is cheaper, and it is claimed makes better ice cream than fresh cream, because it is near the souring stage. The pasteurization which ice cream makers give the cream rather encourages the growth of bacteria by the increased temperature, as the presence of fats protects the microorganisms from the heat, so that a higher temperature is required than that used in pasteurization to kill the bacteria of milk. It is more than probable that the cheap ice cream which is sold in the summer time is largely responsible for the wide prevalence of diarrheal disorders at this season.

\section{Cheese Bacteria.}

Professor Adametz made some years ago an interesting study of cheese, in which he identified nineteen varieties of bacteria which are ordinarily active in the ripening of various sorts of cheese. Some of these bacteria are furnished by the milk, others are derived from the vessels in which the milk is kept, still others from the air and from the rennet ferment added to the milk in the process of cheese-making. In certain cases molds or other fungi are added, as in the case of camembert cheese. Adametz estimated the number of bacteria in cheese to be about $25,000,000$ germs to the ounce. Roquefort cheese is made by adding to the curd of milk, mold from a dough made from barley flour, with which yeast and sometimes vinegar have been mixed. The mold which forms on this dough when allowed to stand, is rubbed into a powder and added to the milk. Gorgonzola and stilton cheese are made by the same process. The mold consists of several varieties of fungi, one being the common green mould frequently 
found in bread and other food kept in warm moist places. Einhorn has shown that molds sometimes take up their abode in the stomach, form colonies on the mucous membrane, and thus become a cause of grave disease.

\section{Cheese Poisoning.}

Cheese poisoning is due to tyrotoxicon. This was discovered by Vaughan forty years ago (1883). A large number of cases of cheese poisoning, some three or four hundred in all, had then been reported to the Michigan State Board of Health. At the suggestion of the writer, at that time a member of the Board, Dr. V. C. Vaughan, of the University of Michigan, was asked to make a special research for the purpose of ascertaining if possible the cause of the poisoning. After some months of careful study of the subject, Dr. Vaughan announced the discovery of tyrotoxicon, a ptomain which in minute doses produces vomiting and purging, pain in the region of the stomach, feeble heart action, cyanosis, and in larger doses speedy death. Cheese containing this poison does not differ in appearance from ordinary cheese, and to the taste and smell of human beings gives no indication of being unwholesome, the distressing symptoms appearing some hours after it has been eaten. But cats and dogs, which readily eat ordinary cheese, at once recognize the poisoned cheese and refuse to eat it. Cats die quickly in convulsions when injected with small doses of tyrotoxicon.

\section{Poisons in Plant Foods.}

Plants sometimes absorb poisons from the earth. Paul Bert watered radishes with water containing strychnia. The radishes accumulated so much strychnia that they killed the animals to which they were fed.

Poisonous mushrooms may cause death from the muscarin which they contain. The poisonous mushroom is much used in Siberia for preparing an intoxicating drink. This form of intoxication is so pleasing to those addicted to it that, according to Roger, "The poor do not hesitate, it appears, to drink the urine of the rich in order to enjoy the same pleasures."

\section{The Poisons of Molds.}

Certain molds, particularly black and orange molds, produce poisons which cause vertigo, colic, sweating and even coma, symp- 
toms similar to those produced by the poisons of the mushroom. These poisons are destroyed, however, by oven heat.

M. Pietro, an Italian investigator, in the study of pellagra made the discovery that the common green mold known as pennicilium glaucum, produces a highly toxic substance in its spores. This toxin is active when introduced into the stomach or under the skin. Dogs, rabbits and guinea pigs are sensitive to its effects as well as human beings. The poison of green mold produces muscular trembling, paralysis, spasms and other symptoms which in many respects resemble those present in pellagra.

Moldy grain and flours prepared from such grain may give rise to poisoning from the specific poisons developed by molds.

\section{Lathyrism.}

This is a form of poisoning produced by the chick-pea. It has sometimes occurred as the result of eating flour adulterated with the chick-pea. The disease is also known as lupinosis.

\section{Ergotism.}

This is a form of poisoning due to the eating of grain, particularly rye, infected with "smut." In the Middle Ages severe epidemics of this disease, then known as St. Anthony's, occurred at various points in Europe. In recent times epidemics have occurred in Russia.

\section{Actinomycosis.}

This is a parasitic disease which sometimes rather resembles cancer in its gross appearance. It may give rise to abscesses in bones, in the brain, or in the liver. Its origin is not well known, but probably comes from contact with cattle.

\section{Infected Vegetables.}

Melec has shown that radishes grown in contaminated soil showed infection after 28 to 37 days; lettuce, after 21 days. The bacteria were found only on the outside of the plants, and were not removed by ordinary washing. This shows the necessity for disinfection with peroxide of hydrogen or hydrochloric acid or chlorine. (See Index.)

\section{The Germs of Fruit Skins.}

Professor Ellen H. Richards, of the Massachusetts Institute of Technology, made some years ago a study of the condition of 
fruit found on open air fruit stands. A pint of various fruits taken at random from a stand were washed and a bacteriological examination made of the washings. $140,000,000$ germs were obtained from this single pint of fruit. Fruit thus exposed to street dust is certain to collect a great number of germs derived from the excreta of animals and other dangerous sources, and is quite unfit for food. Market fruit should be disinfected by washing and soaking five minutes in a solution of fresh chloride of lime, an ounce to the gallon.

\section{Infection from Flies.}

Fruits and other foods may become a source of infection through the medium of flies. Flies are in the summer always liable to be carriers of infection. Epidemics of typhoid fever have not infrequently developed through the medium of flies. Hence, these pests should be carefully excluded from kitchens, pantries and dining rooms, and should never under any circumstances be allowed to come in contact with food.

Ladd reports the results of an examination of grapes in which "the grapes from the outside of the cluster were found to bear colon bacilli, approximately 100 colon bacilli per grape

\section{Botulism.}

The increasing prevalence of this disease, outbreaks of which are now frequently appearing in different parts of the country, renders it necessary to be sharply on the lookout to prevent serious or even fatal poisoning. This duty is especially incumbent upon proprietors, dietitians and chefs of hotels, restaurants, boarding houses and other eating places, and especially hospitals, and other institutions where canned vegetables are much used.

Botulism is a disease due to poisons produced by sporeforming organisms of which there are two types, A and B. The germs themselves do not grow in the human body; hence the effects are produced by the poisons already developed in the spoiled food, the eating of which is the cause of the disease. In former times attacks were usually the result of eating spoiled salmon, sardines or some other sort of canned fish or meats. More recently, attacks have resulted from the use of canned beans, beets, olives or spinach. The cans are usually bulged and the contents have an unpleasant flavor and offensive odor. An offensive odor is not always present, however. 
The cause of botulism appears to be lack of cleanliness, the use of decomposing materials, and insufficient processing. If the goods are processed at a sufficiently high temperature $\left(240^{\circ} \mathrm{F}\right.$.) the spores as well as the germs which produce them will be destroyed. When the cans are not heated at a sufficiently high temperature, the spores are left viable and later develop, producing the highly virulent poison characteristic of $B$. botulinus. Fortunately, thorough heating at the boiling point will destroy the poison. In the outbreaks which have occurred, the foods have been eaten cold or have been insufficiently heated. When cans are bulged or "flip," the goods should be promptly rejected no matter whether an offensive odor is present or not. The slightest taint should always lead to the rejection of canned goods; but even when no evidence of spoiling is present it is necessary for entire safety to subject canned vegetables of all sorts to thorough boiling before serving.

Dr. Robert Graham has recently shown that $B$. botulinus is often present in flour, though fortunately not in a state dangerous to human life.

When an attack of botulism occurs, one of the first symptoms is double vision. Headache, more or less gastric disturbance and a peculiar feeling in the throat with difficulty in swallowing are characteristic symptoms. Drooping of the eyelids is another prominent symptom, and great prostration in severe cases. Sometimes there is a disappearance of the symptoms shortly after the beginning of an attack. The patient may feel well enough to resume his usual duties; but after twenty-four hours a relapse may occur. Fever is usually absent.

The symptoms of botulism may be so mild as to be hardly noticeable. In other cases the attack may prove rapidly fatal.

At the present time more can be accomplished by prevention than by curative measures after an attack has developed.

The disease is very frequent among fowls, cattle and horses, among which it is known under various names, such as "limber rock," "blind staggers," "corn stalk disease," "forage poisoning," etc.

\section{Parasitic Infection of Foods.}

Certain foods, particularly animal foods, are liable to become injurious to health through the presence of parasites. Among the parasites which may be present in foods, either in the form of ova or in the young or adult state, are the following: 
Ameba coli, which gives rise to dysentery.

Coccidium oviforme, derived from the eating of infected green vegetables or contaminated drinking water and the handling of domestic pets, which are likely to carry this parasite.

Liver fluke, (distoma hepaticum), derived from aquatic plants. The infection occurs through drinking water. The parasite is found in the gall-duct and the gall-bladder.

The blood fluke, (bilharzia), found in the urine. Probably derived from drinking water. It occurs especially in Egypt and is often fatal.

Whip worm, (ascaris), a common parasite in the tropics, derived from water or infected raw vegetables.

Pin or thread worm, (oxyuris), infects the lower extremity of the small intestine. Descends to the colon and rectum. Enters the body with raw fruit or other uncooked foods.

Pork tapeworm, (tenia solium), a parasite derived from raw or uncooked pork (measly pork).

Beef tapeworm, (tenia saginata), a parasite derived from the eating of raw or undone beef (measly beef).

Tenia echinococcus, a parasite which is found in the intestines of dogs. It is communicated to human beings by the handling of dogs or the eating of infected raw, green vegetables.

Bothriocephalus latus, a tapeworm derived from fish, common in northern Europe but rare in the United States.

Hookworm, derived from infected water or through abrasion of the skin, especially of the feet.

Filaria sanguinis, a parasite which gives rise to elephantiasis. It is derived from impure water.

Trichina spiralis, a parasite derived from pork which develops in the muscles. The hogs in this country are so generally infected that the United States government has issued repeated warnings against the eating of pork which has not been thoroughly cooked. The disease has become so widespread that no attempt is being made to control it, and there is no inspection of pork sold for consumption in the United States.

\section{Adulterated Foods.}

In general, wholesome foods are little adulterated. The pure food law has, in fact, almost wholly suppressed the adulteration of foods of any sort in this country. The articles most likely to be adulterated are mustard and spices, pickles, coffee, tea and 
cocoa. Butter is sometimes adulterated with oleomargarine, lard, beef tallow, fats, and oils. Olive oil is frequently adulterated with cottonseed oil, and strained honey is sometimes adulterated with glucose. None of these adulterations, however, are particularly harmful. The most harmful adulterations are those which consist of the use of preservatives, such as sulphurous acid, sulphites, sulphates, salicylic acid, benzoic acid and formaldehyde in foodstuffs. These preservatives are often added to canned foods. All of them are harmful. Boric acid and borax, which are frequently used, have been shown to be injurious to digestion. Copper, iron and nickel are sometimes used to improve the color of pickles, green peas and other vegetables. Zinc occurs in dried apples as the result of using galvanized iron pans in drying. Iams and jellies are adulterated with aniline colors and with agar, turnips, apple or gooseberry pulp.

\section{Benzoate of Soda.}

Benzoic acid is much more toxic than benzoate of soda, just as hydrochloric acid is more poisonous than sodium chlorid or common salt. It should be noted, however, that the experiments on animals which seemed to show the benzoate of soda to be harmless cannot be taken as evidence of the effects in man, who is much more susceptible to these poisons than are lower animals.

The facts now known concerning the poisonous properties of benzoic acid and benzoate of soda are certainly sufficient to condemn its use as a preservative. It is to be hoped that the federal pure food department will soon see its duty to prohibit the use of this drug in foodstuffs, especially as it is so often used to conceal the inferior character of the raw material used; and further, in view of the fact that it is almost exclusively used in the preservation of hot sauces which are an unnecessary and often highly injurious addition to foods.

\section{Toxicity of Certain Foodstuffs.}

Recent experiments by Cook on the toxicity of foodstuffs have given some most interesting results. Frogs and gold fish were the subjects used. Cane sugar, the acetic acid of vinegar, the potassium oxalate of rhubarb, alcohol and Rochelle salts, the residue of baking powder, all were found to be toxic. Sodium benzoate was found to be much more toxic than oxalic acid or potassium oxalate of rhubarb. Cane sugar was found to be three times as toxic as grape sugar. 


\section{Keeping the Soil Clean.}

One of the advantages of cookery is the fact that it lessens the danger from infection with disease-producing bacteria. Fortunately, cooking at ordinary temperatures will destroy most of the bacteria which produce disease and in some cases cookery destroys the poison produced by the germ as well as the germ itself. Consequently, the cooking of food is in no small measure a protection against infection by disease-producing germs, such as those of typhoid fever, tuberculosis, cholera and various waterborn diseases. There are a few germs, however, which are not destroyed by ordinary cooking for the reason that they produce spores which are highly resistant to heat. Some of these spores resist a temperature as high as $240^{\circ} \mathrm{F}$. for a few minutes without being destroyed and for their destruction require heating for 40 minutes at the high temperature named. Of germs of this class which are dangerous to human life, the bacteriologists especially mention four - the bacillus which produces tetanus, or lockjaw; Welch's bacillus, which causes gas and putrefaction and gives rise to gas gangrene in infected tissues; the $B$. botulinus, which causes botulism, and the anthrax bacillus, which causes malignant postule.

The tetanus bacillus usually finds access to the body through wounds. The bacillus of gas gangrene is likewise due to infection of the tissues with Welch's bacillus through a wound of some sort, usually either the bite of animals or a wound received in battle. Welch's bacillus, however, finds its entrance to the body through the mouth with certain foods. It is always present in meats, which as obtained from the butcher shop are infested with putrefactive bacteria of various sorts, among which is the gangrene bacillus. These bacteria, when introduced with the food, find in the colon a favorable opportunity for development and are the chief cause of the putrefactive changes which occur in the colon and which give rise to gas-forming and the development of offensive odors. This bacillus is thus largely responsible for the intestinal toxemia or autointoxication which is always present in cases of chronic constipation, although the characteristic symptoms which will result from various kinds of damage to the body are sometimes slow in developing.

These most pernicious organisms, some of which are the most deadly with which the human organism comes in contact, find 
in the colon of animals the most favorable place for their development. The bacillus of lockjaw apparently grows with a special luxuriance in the colon of the horse. It is thus evident that the use of manure as a means of fertilizing the soil is also a source of infection of the soil. It is for this reason that stepping on a nail is a common cause of tetanus. Either the nail or the unprotected foot is very likely to be more or less soiled with dirt, and if the earth has been infected, through fertilizing with animal manure, the tetanus germs are very likely to be present.

A recent experience shows that soil may become infected with $B$. botulinus, the cause of botulism, in a similar manner. The owner of a small garden for eight years canned string beans grown in his own garden, with excellent success. There was no spoilage and no sickness due to the use of the beans. In 1918 animal manure was used as a fertilizer. The beans raised were canned by the same process employed before, but instead of keeping as before they spoiled and some of the cans were found to contain the deadly poison produced by the B. botulimus.

Since the scores of cases of botulinus poisoning from ripe olives canned in glass and from spinach, an investigation has been made of the soil in the vicinity of the canning factories in California from which the infected products came and it has been found to be infected with the botulinus bacillus. The real source of infection is thus clearly seen.

All agricultural authorities warmly advocate animal manure as fertilizer. Doubtless, this method of fertilizing the soil has distinct advantages. However, the dangers which arise from infection of the soil with the pernicious bacteria found in animal manure must be given consideration. It is, indeed, not unreasonable to suggest the advisability of discouraging the use of animal manures for use in vegetable gardening; or at least if such manures are used should they not be first subjected to a process of sterilization which will insure the destruction of the dangerous germs which are always present? That manure is not necessary for the enrichment of the soil is clearly shown by the fact that natural soils are found which are as rich as could possibly be desired in plant food of the finest sort.

When we are taking such precautions to avoid contamination through drinking water, insisting not only that the water should be pure but also that the individual drinking cup shall be used so as to make certain that the vehicle as well as the water itself 
is clean; when we are taking care to see that food is protected from flies, that our bread is wrapped in paraffin paper, that milk is pasteurized or certified and delivered in aseptic containers; and while we are even taking the precaution to inspect the people who handle foodstuffs in any way, such as grocers, bakers, cooks or waiters; when we take such great pains in so many ways to make sure that our food is kept clean after it is produced, why should we permit it to be produced under conditions which almost absolutely insure contamination with the dirtiest kind of dirt and infection with the most deadly of known germs?

There are many excellent fertilizers besides animal manure and there are well-known methods of improving the fertility of the soil without the addition of either manure or artificial fertilizers of any sort. It seems more than likely that the sanitarian of the future will extend his inspection to the soil in which food is grown, thus cutting off at its source one of the most common, most certain and most deadly sources of food contamination.

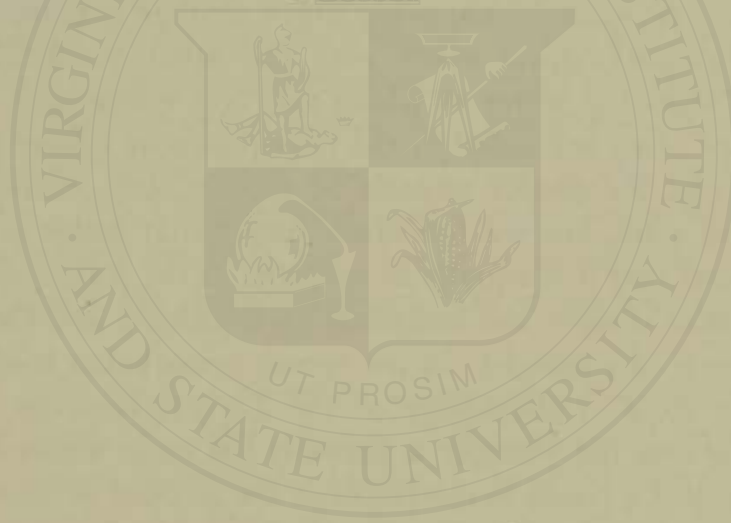




\section{Water Drinking}

Claude Bernard, the eminent French physiologist, many years ago pointed out the fact that all life is under water. The work of the brain, heart, liver and every other vital organ is performed in a water medium. The blood cells float in a liquid stream, and the muscle cells and gland cells - all the living elements of the body-are submerged in water as truly as the fishes which swim in the sea.

So it is literally true, as pointed out by Bernard, that the difference between water breathing animals and air breathing animals is more in appearance than in fact. These animals differ really only in their method of obtaining air. Warm blooded animals obtain air directly from the atmosphere, whereas cold blooded or water breathing animals, requiring much smaller quantities of air, are able to satisfy their needs with the small quantities of air obtainable from the water in which they are submerged.

We are often reminded of the importance of taking food and of taking just the proper amount of protein, fats, carbohydrates and mineral salts, but we too often forget that water is really a food also and that it is equally as essential as the solid constituents of foodstuffs. Roger, the successor of Bouchard and the leading living French physiologist, in his recent remarkable book, "Physiologie du Foie" (Physiology of the Liver), remarks, "It is a general law that the younger a tissue is the more active it is, and the more active a tissue is the larger is the amount of water which it contains."

The amount of water in the body gradually diminishes with age and with lessened activity. The water content of the body of a new-born babe is higher than at any later period of life, being 82.5 per cent., more than four-fifths the body weight. So tenacious is the body of its water content even temporary starvation and deprivation of water does not greatly diminish the proportion of water present in the tissues. The tissues actually produce water out of their own substance.

There is an important relation between food and the water content of the body. The amount of water in the tissues is in- 
creased to a marked degree by carbohydrates, that is, starch, sugar and dextrin, and to a much greater extent than by a nitrogenous diet or a diet rich in fats. Zuntz showed that for every ounce of carbohydrates retained in the body in the form of glycogen four ounces of water are retained in combination with the carbohydrate. Roger observed that the larger the amount of fat contained in an organ the less is its activity.

These facts emphasize the importance of giving the same attention to supplying the tissues with water as with solid foodstuffs. Many people suffer from headaches, malaise and other discomforts because of their neglect to supply the body with water with which to wash away the waste elements through the kidneys.

A simple experiment will show the important relation of water drinking to the kidney function. If a quart of water (five tumblerfuls) is swallowed within a short period, say 15 or 20 minutes, within an hour or two almost the entire quantity (four-fifths) will be passed out through the kidneys. While this large quantity of urine is less concentrated than usual, it will nevertheless contain a larger amount of waste matter than would have been excreted by the kidney in the same length of time if the water had not been taken. It is evident, then, that copious water drinking is a means by which the body may be given an internal bath. The internal bath is far more important than scrupulous grooming of the skin.

The daily loss of water is about four and one-half pints. Ordinary food, as eaten at the table, contains water to the extent of about one-half its weight. About 12 ounces of water are produced in the body by the reactions which occur in the processes of digestion, assimilation and dissimilation. To supply the daily loss, at least two to four pints of water should be taken daily in addition to that contained in the food. When food is not eaten, more water is needed. When water is taken in insufficient amount, one result is to diminish the volume of the blood. The effect of this is to lower the blood pressure. Waterdrinking is important as a means of raising the blood pressure in persons in whom the blood-pressure is too low.

Hunt (Cambridge) has shown that in a hot atmosphere as much as six liters (quarts) of water may be needed to neutralize and carry off the enormous amount of heat produced and absorbed. The body's store of water is mostly in the muscles, 
which give it up when needed but replace it slowly. This stored water should never be drawn upon more than is absolutely necessary.

Certain animals, as the camel, are able to go long periods without drink, but only because they are able to store up large quantities of water. Camels often travel 200 or 300 miles with heavy loads, going as long as 15 days without water, and instances are on record in which waterless stretches of more than 600 miles have been covered. After having been deprived of water for several days, the camel readily swallows 40 gallons of water, and its ordinary drink is 7 or 8 gallons. The sheep of the Nebo national forests in Utah pass the four and one-half months of summer without water, and thrive on the small quantity of moisture found in the scanty herbage which grows in that dry, rocky region. The American prong horned antelope lives contentedly in the desert of the southwest notwithstanding the absence of water. (Hornaday).

Human beings differ from these desert bred animals in that they require a large amount of water. The water content and daily need of the body depends upon age as well as weight. An infant needs four times as much water as an adult in proportion to its weight. That is, a child weighing 35 pounds needs as much water, daily, as an adult weighing 140 pounds. Restriction of water hinders growth and development. Great care should be taken to supply infants and school children with water at frequent intervals.

Many people never drink except at meals, a time when copious drinking is not advisable. Two or three hours after eating is the best time for water drinking, although it is perfectly proper for a person to drink whenever he feels thirsty, since thirst is an expression of the need of the tissues for water.

It is wise to form the habit of taking water at regular times. A very good plan is to drink a glass of water on rising in the morning, on going to bed at night and every time the bladder is evacuated. A sense of dullness or weariness is often at once relieved by drinking a glass of cool water. Ice water is not objectionable if it is sipped slowly, but ice cold water should never be swallowed rapidly. A large quantity of ice water taken into the stomach suddenly may stop digestion for an hour or two.

Every person should take daily two or three pints of water in addition to the water contained in ordinary foodstuffs, which has been estimated to be about one pint. 
In some up-to-date business houses the excellent practice has been established of supplying water to each employee six times a day, a capital means of increasing efficiency.

\section{Tests for the Purity of Water.}

A critical examination of water with reference to its purity and fitness for use as a beverage or for culinary purposes requires the services of expert chemists and bacteriologists. There are a few tests, however, which are of considerable value and may be employed by a person who has such a knowledge of chemistry as may be obtained in the high school department of the public schools.

The following table (Notter and Firth) gives the method of testing for some of the most common chemical impurities likely to be found in drinking water.

\section{Reagents Necessary for Qualitative Examination of Water.}

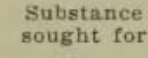

Reaction

Lime

Ammonia

Iron

Hydrogen sulphid

Lead or copper
Reagents to be used, and effects

Litm us and tumeric powders; usual red or brown reactions.

Oxalate of ammonium; white precipitate.

Nessler's solution; a yellow color or a yellow brown precipitate.

Red and yellow prussiates of potash and dilute $\mathrm{HCl}$; blue color.

A salt of lead; black precipitate.

Ammonium s u $1 \mathrm{ph}$ id; dark color, not cleared up by $\mathrm{HCl}$. sium bichromate give

Remarks

Usually neutral. If acid, and acidity disappears on boiling, it is due to carbonic acid. If alkaline, and alkalinity disappears on boiling, to ammonia (rare); if permanently alkaline, to sodium carbonate.

Six grains per gallon ( 9 per $100,000)$ give turbidity; sixteen grains (23 per 100,000 ) considerable precipitate.

If in small quantity, several inches in depth of water should be $100 \mathrm{ked}$ down through on a white ground.

The red for ferrous and the yellow for ferric salts.

When the water is heated the smell of hydrogen sulphid may be perceptible.

Place some water $(100$ c. c.) in a white dish, and stir up with a rod dipped in ammonium sulphid; wait till color produced, then add a drop or two of hydrochloric acid. If the color disappears, it is due to iron; if not, to lead or copper.

One-tenth grain per gallon gives an immediate tur- 
a turbidity

Zinc

Render water slightly ammoniacal; boil; filter; a few drops of potassium ferrocyanid give a haze to white precipitate; hydrogen sulphid gives a white precipitate. bidity; one-twentieth grain per gallon after one minute; one-fiftieth grain per gallon after half an hour.

The filtrate must be quite clear before the ferrocyanid is added.

This reaction is not available if iron be present or if the water be acid or alkaline.

Water obtained from uncertain sources should always be boiled; indeed, this precaution is a wise one under nearly all circumstances when traveling.

Very hard water should be boiled for a long time to eliminate, so far as possible, the lime which it contains

\section{Mineral Waters.}

Mineral waters are unwholesome. Their use should be avoided. Saline waters, which are usually taken for their laxative effect, when long used cause colitis. This was pointed out by Von Noorden and other clinicians some years ago. These waters also disorder the stomach. So-called calybeate waters, supposed to be beneficial to the blood, often contain so little iron that they can produce no appreciable effect unless taken in quantities amounting to some gallons daily. The same is true of waters supposed to be beneficial in certain maladies because of the presence of lithia. The amount of lithia in these waters is so small that one would need to drink a barrelful to get a single medicinal dose. More than this, it has been shown that lithia is absolutely worthless as a remedy for gout and other uric acid disorders for which these waters are recommended; and it is doubtful if mineral iron can replace the organic iron of the blood.

Some bottled waters are valuable and highly to be recommended because of their unusual purity and freedom from minerals. These are not to be classed with mineral waters.

\section{Distilled Water.}

Water which has been purified by double distillation and rendered palatable by aeration is a perfect beverage." Sucin water is in no respect inferior to water from any natural source and is absolutely pure and not liable to contamination.

The idea set afloat by an impractical English physician some 
years ago that distilled water is not only unwholesome but actually dangerous, is wholly without scientific foundation. It is true that the body cells are adapted to a saline medium. The fluids of the body which bathe the cells and tissues contain about four-fifths of one per cent. of salt and other mineral substances. When these cells are exposed to distilled water they swell up and break into fragments. But Nature has provided against any such catastrophe. When water is taken into the stomach, it always finds there a sufficient amount of saline liquid to give to it a degree of salinity sufficient to protect the body cells from any possible harm.

The use of distilled water or absolutely soft water is not essential to health. No evidence has been offered that moderately hard water, that is, water containing a few grains of lime salts to the gallon, is in any way prejudicial to health. It is barely possible, in fact, that the lime of hard water may under some circumstances render the body service by supplementing a deficiency of lime in the food.

It has been noted, however, that stones in the bladder and kidney stones are much more common in districts where only very hard water is obtainable than in regions where an abundant supply of soft water is available.

\section{Purification of Water With Chlorin.}

Freshly prepared chlorid of lime is added to the water and thoroughly mixed with it in proportion of 5 to 8 pounds of chlorid of lime, or "bleach," to $1,000,000$ gallons of water. When liquid chlorin is used, which is the best practice, only one or two parts per $1,000,000$ are used. The residues left in the water when hypochlorite of lime or liquid chlorin is employed are harmless. The chlorid of lime gives to the water a very slight degree of hardness. This is a very practical emergency method which may be applied at once to water known or suspected to be contaminated. Either chlorid of lime or chlorinated soda may be used. The preparation must be fresh.

This method is now used in treating the water supply of many cities, and has in some cases quickly reduced the death rate from typhoid fever to one-fifth the original rate.

The old methods of filtration cannot be relied on as a means of rendering water safe for drinking except as regards animal parasites. Modernly constructed filter beds greatly reduce the 
number of bacteria, but do not destroy them all. Chlorination is the only safe method, and fortunately is so easily available and so thoroughly efficient and practical that it may be utilized on short notice and under almost any conditions. By the installation of automatic machinery for chlorination, many dangerously polluted public water supplies have been rendered safe, and many typhoid epidemics have been promptly arrested.

It is well for travelers to carry with them a supply of freshly made chlorid of lime (not calcium chlorid) for use when water is not known to be pure. One grain in a quart of water will in a few minutes destroy all bacteria that may be present. This quantity is, in fact, sufficient to sterilize three gallons of water in half an hour. It is a good plan to put a grain of the chlorin salt into the water bottle whenever it is filled so as to make sure of a supply of pure water whenever needed.

A stronger solution, say a teaspoonful to the quart may be used for disinfecting fruits and other raw foods.

A convenient method of rendering suspicious water safe is by the addition of a drop or two of ordinary tincture of iodine to a quart of water in a glass pitcher or a thermos bottle. After half an hour the water may be used with perfect safety. The amount of iodine is too small to do any harm, or to produce any unpleasant flavor. 


\section{Medical Dietetics}

Regulation of the diet is perhaps the most important of any single measure in the treatment of chronic disease. As Professor Chittenden, the eminent head of the Sheffield Scientific School, has well said: "It is one of the axioms of physiology that the majority of the diseases of mankind are due to, or connected with, perversions of nutrition." Food supplies the materials necessary for the repair of the body cells; food furnishes the materials upon which the body cells work in their various activities, and food also acts as a vital stimulus to the cells. By regulating the food supply in kind and in amount, and by modifying its form, every tissue and function may be profoundly influenced.

It is important to remember that a large part of our daily diet is comparatively new to the human stomach. The development of the art of cookery has led to the use of many substances with which the primitive stomach was wholly unacquainted and has introduced into the dietary a great number of artificial foods, many of which are by the processes of preparation more or less modified. Man no longer takes his sustenance from the hand of Nature, gathering it, often at the cost of much physical exertion, from forest and plain, hillside and valley, but receives it at his door from the hands of the grocer, the butcher, the miller, the baker and a hundred other purveyors of food and culinary accessories. Instead of taking our food supply in the natural state in which it is furnished to us by Mother Earth, or made more acceptable by simple cooking processes, we require it to be sophisticated and in a multitude of ways denatured and even poisoned by an army of caterers, cooks, confectioners and food adulterators, thus exposing our bodies to an indefinite number of damaging influences which our primitive ancestors escaped.

Clinical experience has demonstrated that a large share of all chronic disorders to which civilized man is subject are the result of erroneous habits of life, and there can be no question that wrong eating habits are, of all our habitual errors, most active in the production of the disorders of nutrition which characterize all forms of chronic disease. It is evident, then, that in dealing 
with these disorders the first thing to be done is to correct errors in uutrition by so regulating the dietary as to eliminate faulty habits, cultivate normal or biologic appetites and train the digestive organs to normal activity.

Among the illustrious examples of the benefits arising from such a correction of faulty dietetic habits may be mentioned the case of Professor Huxley, who, after describing himself as suffering from want of energy and "life-long depression," by renouncing the use of alcohol and tobacco and greatly lessening his intake of food, freeing himself, as he said, "from gross intemperance," recovered his former vigor and enjoyed as good health as ever in his life.

Until very recent years, almost nothing was known about the therapeutics of diet. The whole subject, like most other medical matters, was based on empiricism. Today, thanks to the labors of Pavlov, Rubner, Voit and Pettenkofer, Bayliss, Atwater, Benedict, Chittenden, Osborne, Mendel, Cannon, McCollum, and scores of other modern investigators, we have a really scientific basis for dietetics; and as a result such wonderful victories in the battle against disease are daily being won as a century ago would have been regarded as truly miraculous. Indeed, the old materia medica of the last generation is rapidly falling into innocuous desuetude, having been supplanted by a rational and efficient system of materia alimentaria.

The purpose of this section of this work is to make practical application to individual diseases and diseased conditions of the scientific facts which have been presented in the foregoing pages. If the reader misses many of the familiar formulas usually found in works on medical dietetics, let him not be disconcerted and imagine himself on untried ground; but rather, let him be assured that every suggestion or recommendation has behind it not only a sound physiological fact, but also long and thorough practical tests at the Battle Creek Sanitarium, where many thousands of intelligent men and women are annually fed in harmony with the principles herein set forth and in numerous other medical institutions as well as by hundreds of physicians engaged in private practice. The same methods are likewise in extensive use in a more or less complete form in many other sanitariums and hospitals which are served by dietitians trained in the Battle Creek Sanitarium School of Home Economics, from which hundreds of 
expert dietitians have gone out to teach and practice the principles of biologic living and scientific dietetics.

\section{COMIVION DIETETIC ERRORS}

Before considering the dietetic treatment of individual diseases we shall first give attention to some general considerations which apply to nearly all disorders, especially the question of intestinal toxemia or autointoxication, common errors in diet, and some of the more important tests for the functions of digestion, nutrition, metabolism and excretion, by means of which the regulation of the dietary may be scientifically directed.

The many forms of indigestion, as well as most of the disorders of nutrition, are due to errors in diet. Attention has already been called in the preceding chapters to numerous common faults and erroneous notions in relation to human feeding, but it will perhaps be profitable again to briefly sumnarize those errors in diet which are most common and the correction of which is highly essential in the treatment of nutritive disorders.

\section{Overeating.}

An excessive intake of food is much more common than a deficiency. The average person has little appreciation of the fact that there is no definite relation between the bulk and weight of food and its nutritive value. For example, a pound of almonds has more food value than 20 pounds of cabbage, cauliflower or eggplant, or 15 pounds of beets, carrots or string beans.

So it is possible for one to overeat by unduly distending the stomach with bulky food, such as coarse vegetables or juicy fruits, while the quantity of nutriment absorbed may really be insufficient to nourish the body. On the other hand, one may easily take a great excess of nourishment, although the actual weight and bulk of the food consumed be small.

On this account it is highly important that every one should learn how to estimate the actual values of foodstuffs as expressed in calories. This may be easily done by a careful study of the extensive tables presented elsewhere in this work.

The general bad effects of overeating are an excessive gain in weight, dullness after meals, drowsiness, insomnia, indigestion. It is to be noted, also, that overfed people are more likely to suffer from cancer than the underfed. According to Williams, 
cancer is almost unknown among the poorly-fed Hebrews of East London, but is very common among the wealthy Jewish bankers of Hyde Park. Bright's disease, arteriosclerosis, high blood pressure, and apoplexy are also among the consequences of overfeeding. In time, the stomach breaks down from overwork; flatulence, hyperacidity, disorders of the liver and gall bladder and hemorrhoids are among the common results.

It is always to be remembered that what would be only a sufficiency or even not enough for a hard working laborer might easily be a great excess for a man of sedentary habits. The daily ration for persons of different occupations has been considered in a previous chapter.

The method of serving the food in successive courses, and especially the plan of arranging the bill of fare in such a way as to encourage eating to excess by presenting the most highly palatable dishes last, is productive of great mischief. Desserts consisting of rich dishes, such as ice cream, pastry and confectionery, are on this account to be condemned. Persons who are addicted to overeating should adopt the plan of eating at the beginning of the meal a considerable quantity of bulky food, such as spinach, cabbage, turnips, carrots, lettuce, etc. By this means, the sense of satiety may be induced before an excess of nourishment has been swallowed.

Special injuries may result from an excess of particular food principles: for example, an excess of fat leads rapidly to obesity and induces so-called "biliousness" by causing too long retention of foodstuffs in the stomach and of food residues in the colon, thus promoting intestinal putrefactions. Intestinal toxemia induced in this way is the explanation of the injury suffered from eating an excess of fat, rather than "clogging" of the liver, the popular explanation.

Persons whose stomachs do not produce a sufficient quantity of gastric juice and those suffering from intestinal toxemia, the result of chronic constipation, should especially avoid an excess of fat. The oil enema, sometimes employed for the relief of constipation, may do harm by promoting intestinal putrefaction and by interfering with gastric digestion. On this account, paraffin oil should be used for this purpose rather than olive or linseed oil, for the reason that it is non-absorbable, has no effect upon the digestive processes, and does not undergo either fermentation or putrefaction. 
Less injury, perhaps, is likely to follow from an excessive intake of starch than from any other quantitative error in diet, as it taxes the digestive process less than any other food principle and produces less disturbance of metabolism. This cannot be said, however, of the carbohydrate, sugar, the reverse being true; especially in relation to cane sugar, the excessive use of which not only induces disorders of digestion, particularly hyperacidity, but is very probably one of the chief causes of diabetes as well as of chronic gastric catarrh. The free use of cane sugar also leads to lime starvation, as elsewhere explained. Malt sugar is much less likely to produce gastric or nutritive disturbances; and the free use of milk sugar, because of its slow absorption, may become a valuable means of changing the flora by promoting the growth of acid-forming organisms in the colon.

Eating an excess of protein is one of the most grievous of all dietary faults. It may be rightfully regarded as one of the leading causes of chronic disease, through the intestinal toxemia which results from the putrefaction of undigested residues of meat and other proteins in the colon and the absorption of indol, phenol and other putrefaction products. Bright's disease, rheumatism, cancer, gastric ulcer, skin disorders, neurasthenia, insomnia and a long list of chronic disorders directly or indirectly due to intestinal toxemia are among the evil results of a high protein diet.

The researches of Rubner, Chittenden, Sherman, Folin and others supply a scientific basis for the view that one calorie of protein for each pound of normal body weight is sufficient to supply all the nutritive needs of the average individual. This amount of protein will be well supplied by a pint or even by half a pint of milk, according to the size of the individual, even though eggs as well as meat are excluded from the dietary. A few ounces daily of soy beans, almonds or other nuts will also fully meet the demand. If meats are discarded from the bill of fare, and eggs and milk are used in moderation, the ordinary mixed diet will usually furnish a proper balance of protein.

Injury may result, as shown by Bunge and as pointed out elsewhere in this work, by making the bill of fare consist too largely of cereals, such as are supplied by ordinary breakfast foods. Cereals introduce into the body an excess of mineral acids, and when used in excess may lead to a preponderance of acids in the body, inducing a mild form of acidosis, with lowered 
vital resistance and impaired nutrition. If the cereal intake of the average individual were reduced one-half by substituting an equivalent amount of farinaceous material in the form of potatoes and other vegetables, the results would be a great improvement in our national health. Potatoes are rich in alkaline salts, which neutralize the excess of acid found in cereals as well as those produced by the cell activity of the body. These useful salts are also found in fruits. The preponderance of acids found in meats is four times as great as that found in cereals, hence the injury from a heavy meat diet is so much the greater.

\section{Underfeeding.}

Underfeeding is most frequently observed among the women and children of the poorer classes. Invalids, also, through lack of appetite or ignorance of food values, frequently suffer from lack of nourishment. An adult person of average size who is taking less than 1,800 calories daily is almost certain to be underfed, unless confined to bed.

Underfeeding is naturally accompanied by a loss in weight, the degree of which is a fairly accurate means of determining the measure of the food deficiency. The underfed individual also suffers, often to a very extreme degree, from a deficiency of vitamins. Famine is almost universally accompanied by scurvy and other deficiency disorders.

Modern studies of metabolism have shown that at least two or three ounces of carbohydrates (starch and sugar) must be taken daily to insure the proper utilization of other foodstuffs and to prevent acidosis. A normal dietary contains six or eight times this amount of carbohydrates.

The amount of protein eaten is rarely too small, although a dietary of exclusively vegetable origin may easily be deficient in complete proteins unless very carefully selected. The addition to a dietary of ordinary cereals or other vegetable foodstuffs of four to eight ounces of milk will insure an ample amount of complete proteins to meet the needs of the body.

\section{Deficiency Diet.}

Bills of fare notably lacking in vitamins are very commonly found in prisons, almshouses, hospitals for the insane and feebleminded, college dormitories and boarding-houses, also in many private homes. A breakfast consisting of toast and coffee, bacon, 
griddle cakes and syrup, a very common menu, is almost altogether lacking not only in vitamins but in iron, lime and cellulose-elements highly essential to a complete dietary. Canned foods, particularly canned meats and vegetables, as well as dried fruits, are likely to be deficient in vitamins. Canned tomatoes and canned fruits are processed at a sufficiently low temperature largely to prevent the destruction of the vitamins, which are also preserved to a notable extent by fruit acids.

Reducing diets, such as are recommended for combating obesity, are almost always deficient in vitamins. Reduction in the amount of food eaten necessarily reduces the vitamins along with the other food constituents; and because of it, many persons on a special fat-reducing regimen have been greatly injured by the lack of these food essentials. A reducing diet should always comprise an abundance of foods rich in vitamins. The lack of this important food principle in the so-called light diet of hospital wards and the ordinary fever diets in common use has no doubt been responsible for delayed convalescence and various untoward symptoms and disappointing failures in thousands of cases in hospital and private medical practice.

\section{Irregular Meals.}

Irregularity in the times of eating is highly injurious for numerous reasons which become at once apparent when one recalls some of the simple facts relating to the physiology of gastric digestion. The stomach empties itself normally in about four hours. When an interval of five hours is allowed between meals, it has an hour in which to completely empty and disinfect itself, as well as to recruit the energies of its gland cells in preparation for the work of digesting the next meal. This is essential for good digestion.

There is a close relation between the taking of food and the normal rhythmic activity of the colon. During the taking of a meal the food residues are moved forward in the colon four times as far as during the hour preceding the meal. When a meal is omitted, this physiologic stimulation of the colon is lacking, and the result may be the failure of the colon to evacuate its contents at the normal time, leading to distention of the colon and stasis or stagnation, which may become the starting point for chronic constipation. Thus regularity of meals is necessary in order to secure normal rhythmical action of the colon in the evacuation of food residues and other body wastes. 
When the interval between meals is shortened, which is likely to occur when meals are taken at irregular hours, the result will be the reception of fresh food into the stomach before the digestion of the previous meal is completed. This deprives the stomach of rest and so prolongs the stay of the digesting foodstuffs in the stomach as to produce not only exhaustion of the organ but undue congestion of the gastric mucous membrane from too long contact with the gastric juice and digesting foodstuffs. The ultimate result will be gastric catarrh, slow digestion, together with other disorders affecting the later stages of digestion.

Late suppers are particularly injurious. They not only lead to impaired sleep, but take away the appetite for breakfast the next morning because the stomach is exhausted. Thus it is very common for the late diner to suffer from heaviness, headache and a feeling of malaise and inefficiency the next morning.

The heavy meal usually eaten at night by city dwellers is, in the writer's opinion, chiefly responsible for the obstinate insomnia so frequently encountered, especially among persons active in business or society.

\section{Hasty Eating.}

Insufficient mastication is a fault which has become almost universal because of the general use by civilized people of foods which by cooking have been rendered soft or semi-liquid, and hence receive little or no chewing to prepare them for swallowing.

All foods, whether solid or liquid, should be chewed, and it is important to take at each meal a sufficient amount of dry, hard food, not only to exercise the teeth but to stimulate the salivary glands to produce an adequate amount of saliva, which when thoroughly mixed with the food by mastication renders material aid in its digestion.

Burton says in his "Anatomy of Melancholy":

Crato adviseth his patient to eat but twice a day, and that at his set meals ... and to put seven hours' difference between dinner and supper. Which rule, if we did observe in our colleges, it would be much better for our healths, but custom, that tyrant, so prevails that, contrary to all good order and rules of physic, we scarce admit of five. Moreover, that which he doth eat must be well chewed, and not hastily gobbled, and by all means to eat no more than he can well digest.

Crato's advice to put seven hours between the meals may be advantageously followed by many sufferers from gastric disorders. Some stomachs need even more time for rest and clearance. 


\section{Drinking at Meals.}

The general custom of drinking several glasses of water or several cups of tea or coffee or other beverages with meals is a common cause of indigestion. One glassful of water or other liquid at a meal is quite sufficient to supply all dietary needs. Water-drinking does not interfere with digestion by diluting the gastric juice, but by overstimulating the glands of the stomach, causing them to produce an excess of gastric acid.

\section{Bad Food Combinations.}

Much stress was formerly laid upon the making of proper combinations of foods. The mixing of fruits with vegetables and of acids with milk were particularly considered to be highly productive of indigestion. Later experience, however, has shown that while some attention should be given to food combinations there is comparatively little ground for anxiety upon this point, provided the foodstuffs eaten are wholesome in character and are thoroughly masticated. Wholesome foods harmonize with one another in the liquid state or when reduced to a smooth pulp by thorough chewing. It is, of course, essential that the foodstuffs which enter into a menu should be so selected as to secure a balanced bill of fare; that is, one would not combine eggs with beans because both are rich in protein, or olive oil with nuts for the reason that nuts are rich in fat. Neither is there any occasion for the addition of sugar to oatmeal and other breakfast cereals or syrup to griddle cakes, because these foodstuffs consist chiefly of starch, which by the process of digestion is all converted into sugar. Cream or milk and fats are the proper accessories for cereal foodstuffs rather than sugar, the free use of which with breakfast foods is a very common cause of indigestion.

There are, however, certain unphysiologic combinations which should be noted, such as drinking freely with liquid foods, the addition of cream to pastry. Meat and milk is an objectionable combination, because both are highly nitrogenous foods and because the highly acid gastric juice evoked by meats produces hard, indigestible curds which remain long in the stomach.

\section{Highly Seasoned Foods.}

Foods which are highly seasoned are overstimulating to the appetite and lead not only to overeating but to overstimulation of the digestive organs, the result of which is hyperacidity, which 
is later followed by degeneration of the secreting glands and a deficiency of gastric acid. Gourmands are certain to suffer sooner or later from their gastronomic abuses, being especially subject to gastric ulcer and cancer.

\section{The Use of Condiments.}

Mustard, pepper, pepper-sauce, horse-radish, cayenne, capsicum and other hot and irritating substances have no proper place in the bill of fare. These substances all owe their properties to highly poisonous essential oils which in the pure state readily raise blisters upon the skin, and when taken internally produce irritation of the delicate structures with which they come in contact. Though certain condiments may at first stimulate the stomach to increased activity, the ultimate result of their use is lessened gastric activity and irreparable injury to the stomach, liver, kidneys and other vital organs, including the heart and blood vessels.

\section{Insipid Foods.}

Foods which are lacking in flavor, while much less positively injurious to the body than those which are highly seasoned, are nevertheless objectionable for the reason that they are deficient in the flavoring substances which Nature places in normal foods to render them attractive to the palate, and which through the nerves of taste call forth the glandular and other activities concerned in the process of digestion. To be well digested, food must be attractive. It should please not only the sense of taste but the sense of smell and the sense of sight. This has been clearly shown by the classical experiments of Pavlov.

\section{A Monotonous Diet.}

A monotonous diet is objectionable, not simply because it fails to gratify the sense of taste but because it is likely to be deficient in some of the elements essential to good nutrition. To make sure that the body is abundantly supplied with all the subtle principles which it needs to repair its tissues and sustain its varied activities, a considerable variety in the bill of fare is required. The number of kinds taken at any one meal should not be too great, but they should be varied from day to day. 


\section{Indigestible Foods.}

Such indigestibles as pickles, green olives, rich pastry, preserves and fried foods present to the digestive organs a difficult or even impossible task, since they remain long in the stomach, irritating its walls and exhausting its secreting glands and sometimes causing injury through mechanical irritation.

\section{Cold Foods.}

Very cold foods, such as ices, ice cream, and ice water, are questionable as elements of a meal. If taken at all, they should be in limited quantity and so slowly swallowed that before reaching the stomach they will acquire a temperature near that of the body.

\section{Hot Foods.}

Very hot foods are objectionable for the reason that excessive heat produces the same injurious effects upon the mucous membrane of the stomach as upon the skin. The mucous membrane, in fact, appears to be less able to endure high temperatures than the skin, being more delicate in structure and less accustomed to such contacts. The use of hot food is an unnatural habit and one which is without doubt more or less detrimental to the health. Dr. William Mayo, the eminent surgeon, has for years maintained that the taking of hot foods and liquids into the stomach is a cause of gastric cancer.

\section{Eating When Exhausted.}

When the nerves or muscles are in a state of exhaustion as the result of effort, all the bodily activities are slowed down. The taking of a hearty meal under such conditions is often attended by bad results. Digestion fails for lack of a sufficient amount of active gastric juice, and acute indigestion is a frequent consequence. Rest, especially sleep, is indicated rather than food, unless the exhausted person has been fasting for some time. In such cases the most suitable nourishment will be found in a solution of honey or malt sugar in water or diluted fruit juice. Nutriment of this sort is quickly absorbed and immediately appropriated, requiring little or no action of the digestive organs. An exhausted person, after resting for an hour or two, may take simple food in liberal quantity. Eating heartily when one is tired out by either mental or muscular effort is likely to be followed by indigestion, malaise and incapacity for work. 


\section{Misinformation and Pseudo-scientific Teaching.}

The popular newspaper and magazine literature of the day is filled with dangerous misinformation about diet and other matters pertaining to health. Articles by a chemist of Philadelphia, claiming to be based upon scientific experiments, have been particularly misleading. This writer would have us believe that he has demonstrated that bolting meat is harmless, that drinking freely of water at meals aids digestion instead of hindering it, and that drinking large quantities of ice water at meals does not involve risk of indigestion.

The experimenter draws his conclusions from the fact that he was able to subject the stomachs of healthy men to these various abuses without immediate apparent injury. This is the same old argument with which we are so familiar. It has been brought forward in support of liquor drinking and tobacco using, the tea and coffee habit, the breathing of bad air, neglect of exercise, neglect to breathe properly, neglect of the bowels, and any number of other unhygienic practices that might be mentioned. Within a week of this writing the writer received a letter from a man who maintained that infrequent movement of the bowels is conducive to health, and offered as proof the fact that he enjoys good health though moving his bowels but once in two weeks.

These experiments simply demonstrate how much abuse a healthy stomach is able to endure before it surrenders to disease. Fortunately, we are all born with a certain margin of safety. The first violations of biologic law may not give rise to any tangible ill effects; the bodily functions are performed as usual. The individual therefore thinks himself invulnerable, perhaps priding himself upon the fact that he can do with impunity things which are considered harmful. He is unaware of the fact that a hidden damage is being done, the effects of which may be irreparable. His margin of safety is being consumed and when it is wholly exhausted he will begin to realize the harm which has been done; but it will then probably be too late to repair the injury.

Because it seems to make very little difference to a healthy stomach whether bread is stale, and hence quickly digested, or new and slowly digested, it cannot be assumed that it makes no difference to any stomach. The new bread, which only causes the healthy stomach to empty a little more slowly, when taken into a stomach which always empties slowly, even when supplied 
with the very best of food, may upset the stomach altogether and enormously lengthen the time required for digestion. This principle applies to all sorts of unhygienic practices. Infractions of the laws of good digestion which are apparently followed by no ill effects whatever in healthy persons make the difference between comfort and discomfort, good digestion and no digestion at all in persons whose digestive powers are impaired.

It is true that husky lumberjacks "bolt their three square meals each day," along with (until very recently) a lot of bad whiskey, besides doing many other equally unwholesome things. But it must not be imagined that even the lumberjack can go on doing this forever with immunity from stomach and other troubles. Some of the worst cases of gastric disorder the writer has ever encountered were those of men who had spent a few years of their lives in lumber camps and whose stomachs had been subjected to the sort of treatment described above as innocent. Cancer, gastritis, ulcer of the stomach, and other gastric disorders are common among these men, notwithstanding the great advantages which they derive from their out-of-door life and vigorous bodily exercise, which should give them supreme health and high resistance.

Professor Pavlov showed that taking a quantity of water into the stomach gives rise to the production of an increased amount of gastric acid. The increase is not so great as to cause any serious disturbance in a healthy stomach, but in the case of a stomach which is already making an excess of gastric acid the drinking of a quantity of water may be the means of greatly increasing the difficulty. Thus it is an easy inference that the habitual use of liberal quantities of water in connection with meals, like other gastric stimulants, will in time produce pronounced hyperacidity.

A glass of water at meal times is not at all harmful for the ordinary person, but the practice of drinking water to wash the food down is very injurious. Those whose stomachs are already producing an excess of acid, however, should avoid all water drinking at meals as well as the use of broths and thin soups.

In the feeding of milk to many hundreds of patients subjected to the milk regimen experience has shown the taking of milk slowly to be so important that the patient is invariably required to draw it through a straw or a small glass tube. In this way a person may take as much as six quarts of milk a day without difficulty. 
A unity of function characterizes the whole alimentary tract. All the various processes of digestion are linked together. When food is taken into the mouth and the muscles of mastication begin to work, activity begins all along the alimentary canal. While the food is being sipped or chewed, the food residues in the colon move forward toward the exit four times as rapidly as at other times. Consequently, sipping, chewing and tasting the food are most important natural means of encouraging efficient bowel action. An infant or young animal always sips its food with a sucking action. This is important for the reason that the suction force exerted on the salivary glands increases the flow of saliva. This is easily demonstrated by experiment.

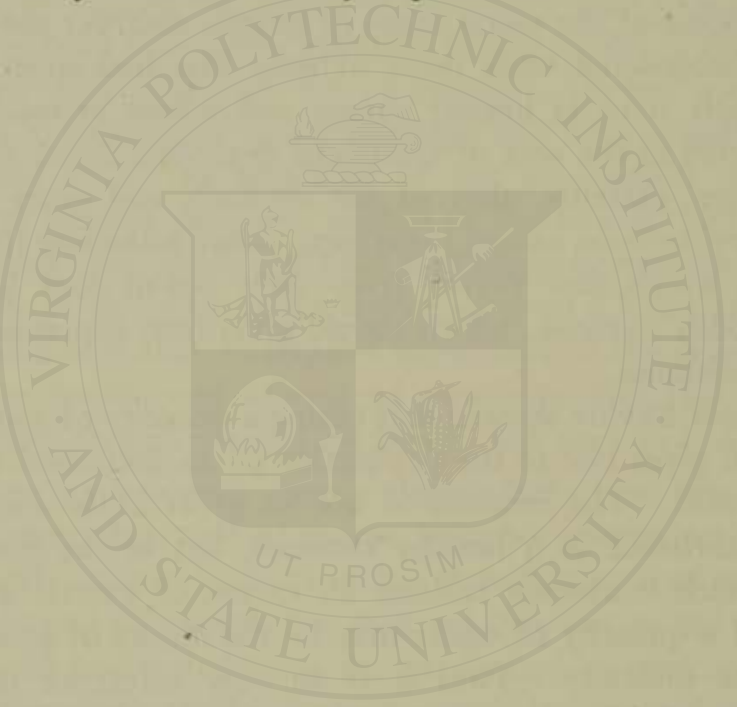




\section{Intestinal Toxemia--Auto- intoxication}

Miller, of Berlin, made an exhaustive study of the microbes of the mouth and showed the dominant role which they play in caries of the teeth and various other diseases of the teeth and gums. When the author, some thirty years ago (1893), established a laboratory for the study of the bacteria of the stomach, with the expert assistance of Professor Novy, of the University of Michigan, it was generally believed that bacteria were always present in the stomach since it was known that they existed in the mouth and were swallowed in great numbers with the food. These studies were begun by giving a sterile test meal to healthy persons, great care being taken to avoid accidental contamination by thorough cleansing of the mouth and the use of sterilized stomach tubes. The expectation was that many bacteria would be found and it was intended to make a study of these, which might be termed the normal bacteria, for subsequent comparison with the bacteria in pathological cases. After ten days' work, during which time a considerable number of cultures were made, no bacteria at all had been found. Various culture mediums had been employed and the conditions essential for encouraging the growth of anaerobic as well as aerobic species had been supplied, but without result. The character of the test meal was then changed. It was no longer made sterile as before, and the result was that some of the cultures showed growths, although the majority of them still remained sterile.

So far as the writer knows, this was the first demonstration of the fact that the healthy stomach carries on its work without the aid of bacteria, and that it is even capable of sterilizing nonsterile food substances which may be introduced into it. Since then, more than twenty thousand bacteriological examinations of stomach fluids have been made in the clinical laboratory of which the writer has charge. Of these, the great majority have been found sterile. Numerous other investigators have obtained similar results, and it may be considered as now thoroughly established 
that the healthy stomach carries on its work without the aid of bacteria, and that their presence in the stomach fluid obtained after a test meal is an evidence of the presence of a pathological state. Normal gastric juice destroys bacteria, or at least inhibits their growth, so that the process of gastric digestion is carried on without the aid of microbic activity. When the gastric secretion becomes deteriorated in character so that it no longer destroys bacteria or inhibits their growth, various pathologic processes are set up in the stomach, which extend to the intestine and to the liver and other adjacent organs, resulting in serious derangement not only of the digestive functions but of the system at large.

Later the study of the bacteria of the small intestine occupied the attention of bacteriologists. Roux, a pupil of Pasteur, showed the fallacy of his master's contention that microbic intervention is necessary for the maintenance of plant life by causing beans to grow in a thoroughly sterile medium. Nuttall and Thierfelder showed that guinea pigs brought into the world aseptically by Caesarian operation lived when fed upon sterilized food, although they did not grow so rapidly as the control animals. Experiments made upon chickens and other animals indicated that animals fed upon sterilized food did not thrive so well as those fed upon ordinary food. Subsequent researches have made it clear that the reason animals fed upon sterilized diet do not thrive is not on account of their being deprived of the beneficent influence of microbes but because the vitamins of the food have been impaired or destroyed.

Dr. Levin, of Stockholm, accompanied the Natthorst expedition to the Arctic regions in the summer of 1898 for the purpose of studying the bacteria of that region. In obtaining each specimen he passed through his apparatus 20,000 liters (more than 5,000 gallons) of air. He found practically no bacteria in the air. In sea water, snow, and melted ice he found on an average one organism in a little more than one-third of an ounce. In 12 samples of brown mud he found only a single microbe. The intestinal contents of the polar bears, seals, eider ducks and other birds, as well as sharks, sea urchins, sea anemones and crabs, were nearly always sterile. His examinations were made both by culture and by staining, so as to exclude the presence of dead bacteria. These results agree with those of Nencki and also Nuttall and Thierfelder, whose experiments showed that animals are able to live without the presence of bacteria in their intestines. 
The fact that only a minority of these animals show bacteria in the intestines clearly indicates that their presence in any part of the alimentary canal is due to accidental infection and is not essential to the well-being of the animal organism.

Conclusive as are the observations of Levin in the Arctic regions as indicating beyond the shadow of a doubt that microbes are not essential to the most vigorous animal life, we may still call attention to the exhaustive researches of Adami, who studied the contents of different parts of the intestinal tract from the stomach to the rectum, and found the contents of the duodenum and the upper part of the small intestine to be practically sterile. In fact, in healthy conditions the entire small intestine was found free from bacterial development, with the exception of harmless lactic-acid-forming organisms. Bacteria capable of producing putrefaction were found only in the large intestine or at the extreme lower part of the small intestine. In pathological conditions, however, the putrefactive bacteria which ordinarily exist only in the large intestine are found higher up, in the small intestine, in some cases occupying the entire intestine and even the stomach and biliary passages. This general infection of the intestine exists in both acute and chronic gastro-enteritis and in various other pathological conditions of the stomach and intestine.

Escherich made an interesting study of the bacteria of the intestine in infants. His observations showed the intestinal contents of an unborn child to be absolutely sterile. Within eight or ten hours after birth the B. bifidus and other lactic-acid-forming bacteria make their appearance. So long as the nourishment of the child consists exclusively of mother's milk the intestine is dominated by acid-forming bacteria, the stools are free from putrescence, no indol is found in the urine, and there is no evidence of the slightest putrefaction in any part of the intestine. When fed upon cow's milk, however, the intestine often becomes infected and the stools offensive, and bacteriological examination shows the presence of the putrefactive organisms which are always found in market milk. Tissier, of the Pasteur Institute, has confirmed and extended these observations. He found that while Welch's bacillus, B. putrificus and other organisms make their appearance their increase is prevented by B. bifidus and other acid-formers which constitute about seventy-five per cent. of the total bacterial inhabitants of the intestine.

Later studies have shown that while in early infancy the 
B. bifidus is the dominant organism and protects the colon from the invasion of putrefactive bacteria, another, stronger-growing acid-forming bacillus, the $B$. acidophilus, makes its appearance as the child advances in age. And this organism, if encouraged by a biologic or non-putrefactive diet and proper care of the colon, is capable of preventing the putrefaction of the body wastes and food residues in the colon, thus protecting the body against the numberless evils which result from the absorption of putrefactive products from the colon, the chief cause of intestinal toxemia or autointoxication. The loss of this most important defense against disease is one of the greatest misfortunes a human being can suffer, and yet there are few among civilized people who do not undergo life-long injury from this cause. The maintenance of normal intestinal flora is one of the chief essentials of a biologic life; and the restoration of the normal flora by securing the dominance of the B. acidophilus in the colon is one of the chief objectives in the rational dietetics the principles of which we have undertaken to set forth in this work.

Bouchard was one of the first to call attention to the rôle played by bacteria in the production of toxins in the colon, resulting in a condition which he termed autointoxication. $\mathrm{He}$ showed by experiments on rabbits that solutions of fecal matter were highly toxic in character and that the toxicity was increased in proportion to the degree of putrefaction. Thus, the fecal matters of a meat-eating animal or a meat-eating man were shown to be twice or more as toxic as the feces of a non-flesh-eating man or animal. Bouchard estimated that in some cases half the entire bulk of the feces consisted of bacteria.

Charrin of Paris, Combe of Lausanne, Tissier, Escherich and numerous other European bacteriologists, as well as Herter in this country, have made exhaustive studies of the different species of bacteria which are produced in the intestine and of the toxins which they generate and the effects they produce upon animal organisms.

Prof. A. B. Macallum, of McGill University, considers the absorption of poisons by the colon one of the chief causes of disease. He says (Science, 1923):

"The mucous membrane is the first line of defense of the body. It is exposed more than is any other structure of the body to the attacks of bacteria and to the toxic products of putrefaction.

"That the cells constituting this membrane, battling against 
hosts of bacteria and subject to the action of the products, many and varied, of putrefactive fermentation, may finally fail to 'play up' must naturally be expected. Then pass into the circulation some or all of those elements which the cells in their fully normal condition do not absorb, or if they do absorb, do not transfer to the underlying tissues.

"It is not too much to say that in this failure on the part of the epithelial cells an explanation may be found of the origin of a large number of diseases of the body. It is my firm conviction that arteriosclerosis, hepatic cirrhosis, acute yellow atrophy of the liver, nephritis in some of its forms, angina pectoris, senile dementia and dementia praecox are all due to failure, more or less pronounced, on the part of the intestinal epithelial cell to play its normal part.

"The intestinal mucosa must then be regarded as the great portal to disease of the body. This portal may be completely closed as it is in the normal healthy condition of the mucosa or it may be gradually or suddenly pushed open and disease, chronic or acute, may result. If this portal could be kept closed always old age might be indefinitely postponed and bodily vigor maintained for a much longer period than it is now.

"It is probable that excess in diet, especially in its protein constituents, may task the capacities of the epithelial cells and diminish their power to react normally, with the result that they allow to pass through them, and to the underlying tissues and the circulation the products of intestinal putrefaction which in their healthy, vigorous condition they do not permit to enter them. This would explain some of the pathological results of a high protein diet. It is also very probable that the primary and most important action of vitamins in diet is on the epithelial cells, with the glandular structures below them."

The whole alimentary canal is highly sensitive to acids, which raise the tone of the muscular walls of the intestine and produce active peristalsis, whereas the alkaline products of putrefaction have the opposite effect, producing paralysis. The highly acid gastric juice produces strong peristaltic waves in the stomach and high tone or gradient of its muscular walls. The gastric acids are neutralized in the duodenum so as to lower the gradient and permit the food to pass onward from the stomach into the intestine. The interesting gradient theory which has been put forward 
by Alvarez and his associates helps to a clearer understanding of the physiology of the intestine, although Alvarez seems to have overlooked the significance of the opposing relation of acids and alkalines to the muscular tone of the intestine. The gradient is lowered in the small intestine by the alkaline bile, pancreatic and intestinal juices; but when the colon is reached, it is necessary that the gradient should be raised again in order to secure expulsion of the food residues and wastes. Nature has made provision for this by supplying the B. acidophilus, which by the fermentation of carbohydrates produces lactic acid which, as long ago pointed out by Adolph Schmidt, is the normal intestinal stimulant. When lactic acid is present in sufficient amount, the food residues in the colon are rapidly moved forward toward the exit and the colon is emptied so frequently that there is not time for the development of putrefactive changes even if this were possible in the presence of an aciduric flora. The gradient rises so high in the colon that peristaltic waves starting at the middle of the transverse colon travel backward toward the cecum.

Herter, as well as others, has shown that the number of these pernicious, toxin-producing organisms present depend largely upon the character of the food-the larger the amount of protein food, the larger the number of organisms. The feces of the lion, tiger, wolf and cat show enormous numbers of these pathogenic organisms, while the fecal matters of the buffalo, goat, camel and elephant were found practically free from virulent organisms. This shows, as Herter pointed out, that vegetable feeders possess a distinct type of intestinal bacteria. When the feces were inoculated into broth, he found mercaptan was produced by the feces of carnivorous animals but that none resulted from the feces of herbivorous animals. Emulsions of carnivorous feces inoculated into guinea-pigs gave rise to edema, hemorrhages and destruction of tissue. Gas and butyric acid were formed. None of these pathological effects were produced by the feces of herbivora.

An interesting clinical fact in harmony with the above observation was mentioned by Herter; namely, that carnivorous animals frequently suffer from pernicious anemia and other forms of anemia, while these diseases very rarely occur in herbivorous animals unless associated with intestinal parasites.

Langer has shown that in breast-fed infants the colon bacillus develops feebly, while in older persons it grows with vigor. This would seem to indicate that the bacillus becomes more viru- 
lent in time or that the infant intestine is a less favorable habitat than that of the adult. By means of a biologic diet, the adult colon may be rendered as inhospitable a place for the mischievous colon bacillus as is that of the normal nursing infant.

Another interesting fact to which Herter calls attention is that in the feces of healthy young persons disease-producing bacteria are comparatively few in number, and that there is a close relationship between the decrease of vital resistance incident to advanced age and the increase of putrefactive bacteria and their products found in aged people.

Of the three great food principles which constitute the digestible portions of our foods, carbohydrates are chief in importance in relation to the bacteriology of the intestine. No matter how favorable other conditions may be, bacterial growth cannot take place without a food supply. Carbohydrates, that is, starch, sugar and dextrin, constitute the natural and necessary food supply for acid-forming bacteria, the harmless character of which has already been referred to. The lactose, or milk sugar, of the nursing infant's food supply thus encourages the growth of the acid-forming organisms which are found present within a few hours after birth and which, as Escherich has shown, are a protection against the harmful putrefactive organisms which appear later. The proteins, on the other hand, are the natural and necessary food supply of the putrefactive and pathogenic poisonforming organisms which grow in the lower part of the intestine, where the absence of air and the presence of retained and undigested and unabsorbed protein substances furnish conditions in the highest degree favorable for the development of this class of organisms.

The classical experiments of Fischer have shown us that the process of digestion of proteins reduces these complex compounds to simple crystalline bodies (amino acids) which, when absorbed by the intestinal mucous membrane, are reconstructed into a new albumin molecule-human albumin, as it has been called. Putrefactive bacteria are likewise capable of breaking up the albumin molecule, and produce the very same amino acids, fatty bodies and other substances as do the digestive fluids of the stomach and the intestines. But the putrefactive bacteria do not stop with the formation of these useful products, which at least resemble those formed in animal digestion, but go farther, breaking up the crys- 
talline substances which the body uses in building up its own kind of albumin, converting them into substances of a highly toxic character.

\section{Poisons Produced by Putrefactions in the Colon.}

According to Nencki, MacFayden and other investigators, not less than one-seventh of the total amount of protein eaten is ordinarily destroyed by putrefaction, thus occasioning a very considerable loss to the body. But this loss is a matter of small consequence compared with the mischiefs which result from the poisons into which this one-seventh of the nitrogenous food supply is converted instead of being transformed into human albumin adapted to the nourishment and repair of the tissues. Amorig the first substances produced by bacteriological action through the breaking up of the crystalline products of normal digestion are the so-called aromatic bodies. From the normal tyrosin is formed cresol and phenol, while from tryptophan are formed the wellknown malodorous skatol, indol and pyrrhol, to which the characteristic odors of putrid feces are due.

Of the various ptomains which are formed the best known are putrescin, cadaverin, parolin, puridin and collidin. These ptomains have been found in the stools of persons subsisting upon an ordinary mixed diet by Roos, Garcia, Baumann and others.

Bouchard, more than thirty years ago, showed the enormous difference which may exist in the character of the fecal matters. In one case he found a sufficient amount of toxic material in the extract obtained from 17 grams (less than two-thirds of an ounce) of fecal matters to kill a rabbit weighing one kilogram (two and one-fifth pounds). After changing the intestinal flora, the extract of 200 grams, or twelve times as much of fecal matters, was required to kill an animal weighing one kilogram.

That these ptomains are not only present in the intestinal contents but that they are absorbed and circulated throughout the body and thus brought into contact with all the tissues is clearly shown by the fact that they have been recovered from the urine. Numerous observers have found cadaverin, putrescin and other ptomains in the urine of subjects in various forms of disease. Salkowski found indol and phenol in the urine of starving dogs. Observations made by Muller on the faster, Cetti, found both indol and phenol. It was observed that the phenol increased after the fifth day. Indol was also found in the urine of the faster, 
Succi, and the professional faster, Breithaupt. Ernst and others have shown that in these cases phenol, indol and skatol are formed by the putrefaction of bile, the pancreatic juice and the intestinal juice. According to Muller, the putrefactive activity of bacteria is increased during starvation because of the absence of lactic acid in the intestinal contents and the frequent occurrence of hemorrhages. The stools of new-born infants contain none of these poisons, for the reason that putrefaction has not as yet begun in the sterile intestine of the new-born, although it is true that they may be found in small amount in the urine of a new-born infant, having been derived from the blood of the mother through the placenta. The pancreatic juice seems to be particularly prone to putrefaction. The intestinal mucus also putrifies readily, and the coloring matters of the bile are decomposed by bacteria, by which they are converted into toxic substances.

Phenol (carbolic acid) appears to be formed only in the large intestine. At least this is the opinion held by the majority of authorities. It appears in the urine whenever stasis of the colon occurs, sometimes in cases of obstruction increasing to as much as seventy times the ordinary amount. Brenzcatechin, hydrochinon and alcapton are three curious coloring matters of a toxic character which are formed by the putrefaction of protein, and which sometimes are found in the urine in such quantities as to give it a brownish or even a blackish color.

But by no means all the poisons produced by putrefaction processes in the intestine appear in the urine. Through the action of the intestinal mucous membrane, the liver and various antitoxic glands, a considerable portion of the toxic substances is either destroyed or so changed that their toxicity is very considerably decreased.

Animal parasites are nearly always present in dogs and other carnivorous animals, and are not infrequent in the intestine in man; but the parasites found in the human intestine rarely do much injury, though they sometimes encourage infection by typhoid bacteria by wounding the intestine, and occasionally give rise to appendicitis.

\section{Intestinal Antiseptics Harmful.}

An investigation conducted by Nicate and Schulz (Berlin Klin. Woch., June, 1900) shows that intestinal antiseptics diminish the ability of the intestine to destroy bacteria. After a dose 
of castor oil and calomel, cholera germs were found in abundance in the stools of dogs to whom cultures of this micro-organism had been given, while before the calomel was given no bacteria were found, although cholera germs in quantity had been introduced into the intestine in such a way as to avoid action by the gastric juice. This experiment indicated that the intestine has in some way the power to destroy bacteria, probably, as suggested by Buchler, by means of a special ferment, and that the action of calomel interferes with the action of this protective process.

The observation that disease germs are destroyed in the normal intestine has been confirmed by Eiskamp and Park (Jour. of Infectious Diseases, Jan., 1921), who noted that the pathogenic organisms disappeared in the intestine in two to seven hours.

\section{Gastric Toxicity.}

It was long ago discovered that the secretions of the stomach are more or less toxic. Among the observations made was the fact that a hypodermic injection of a solution of morphia or similar poisons is quickly followed by the excretion of the poison into the stomach. For example, half an hour after the injection of a quarter of a grain of morphia one-half of the quantity has been obtained from the gastric contents withdrawn by a stomach tube.

Roger found in experiments upon dogs and rabbits that when intestinal putrefaction is active the toxins absorbed are excreted into the stomach in large quantities. M. Borri conducted a series of experiments for the purpose of determining the degree of toxicity of the gastric contents obtained after a test breakfast in normal and diseased conditions. The results showed that in hyperacidity toxicity is very much increased. That this is not due to the acid was proven by the fact that the toxicity was increased even when the acid was neutralized. Toxins were also increased when motility of the stomach was diminished. The toxicity is also increased in gastritis.

These observations furnish a rational explanation for the headache, sense of weight and pressure in the head, mental inertia, general feebleness and other symptoms which accompany disturbance of gastric motility and other forms of indigestion; they also show why gastric lavage usually gives such great relief in cases of gastric disorders accompanied by slow emptying of the stomach. 


\section{Tests of the Nutritive Functions}

The purpose of this short chapter is not to present the technical details of medical examinations in relation to digestion, but briefly to describe the several physical examinations and laboratory tests which may be utilized in the investigation of gastric and intestinal disorders.

\section{Examination of the Mouth.}

Every examination of the digestive functions should begin with the mouth. It should include, to be complete, an inspection of the tongue, examination of the teeth by a competent dentist, an X-ray examination of the teeth and a bacteriological and chemical examination of the saliva. The mouth has formerly received much less attention than it deserves, and we still lack much of knowing all that needs to be known concerning the various conditions found in the mouth.

\section{Significance of the Coated Tongue.}

The tongue naturally received first attention in the examination of the mouth. Old medical writers attached great significance to the various coatings and other appearances presented by the tongue, but modern medical researches have shown most of these views to be erroneous. Many years of observations have convinced the writer of the truth of the suggestion of the eminent French physician, Lasegue; "La langue ne denonce pas la maladie, mais l'etat du malade qui en est atteint." (The tongue does not indicate the disease, but the state of the patient).

To understand the significance of the coating of the tongue, it is necessary to remember that the mouth, next to the nasal cavity, is of all parts of the body most exposed to contact with germs and micro-organisms of all sorts. The warm, moist cavity of the mouth affords an ideal incubating chamber, in which the numerous bacteria swept in with each incoming breath may grow and develop luxuriantly unless prevented from doing so by some special provision. The only reason why the tongue and all the 
mucous surfaces of the mouth are not always thickly coated is because the saliva, under normal conditions, prevents the growth of the various yeasts, molds and other organisms, which a microscopic examination shows to be present in countless numbers in the material scraped from a coated tongue.

The studies of Metchnikoff, Wright, and others have shown that the blood contains alexins, opsonins and other suble agents which hinder the growth and development of bacteria. Under normal conditions, the white cells of the blood, or leukocytes, of which the blood of a person of ordinary size contains more than $30,000,000,000$, are constantly active in destroying the bacteria which are continually finding their way into the blood stream; and thus, so long as an individual remains in perfect health, the blood is kept free from these invading enemies of the body. Although countless millions of these organisms daily find their way into the blood from the intestine, especially the colon, they are destroyed as rapidly as they enter, and so do not obtain a foothold within the body.

The saliva is formed from the blood and for a time after being freshly secreted it possesses the same germ-resisting properties exhibited by the blood. The same is true of milk when freshly produced and still warm from the body of the cow. Small numbers of typhoid fever germs introduced into such milk rapidly disappear. The freshly secreted saliva contains not only the same opsonins and alexins found in the blood but also great numbers of white blood cells. As it pours into the mouth from the salivary ducts, it floods the tongue and teeth and other mouth surfaces, coming in contact with any bacteria which may have been lodged in the mouth and so preventing their development; and the white cells, though outside the blood vessels, may continue their work of capturing bacteria. And thus the mouth under normal conditions is kept clean.

When the mouth is dry, as in fever, when the salivary glands are paralyzed and produce very little saliva, the tongue becomes thickly coated by the growth of bacteria, molds, yeasts and other fungi. Persons who sleep with the mouth open have the tongue coated in the morning because the surfaces are dried and no longer bathed with the germ-resisting saliva.

But there is still another and much more common cause of coating of the tongue. The power of the blood to deal with germs and germ toxins is necessarily limited; hence, in autointoxication, 
when the body is flooded with putrefaction products, and virulent bacteria swarming in countless myriads in the alimentary canal find their way into the blood in unusual numbers, the germdestroying properties of the blood and saliva are exhausted. The same condition of the blood may result from germs and germ poisons derived from other sources than the alimentary canal; thus a coated tongue is practically always found present in fever, no matter what the cause of infection. A coated tongue may be regarded as practically always an indication of a toxic condition and lowered resistance to bacteria, as the result of which the saliva has been rendered incapable of affording the mouth the natural protection which it gives in conditions of health.

When the tongue is thickly coated, the breath has a bad odor and not infrequently a characteristic fecal odor. In such cases, there will always be found very pronounced intestinal stasis, the food residues and other body wastes being retained in the colon for days, instead of being promptly dismissed from the body.

\section{The Teeth.}

As a result of bacteria in the mouth the teeth undergo decay and the gums become the seat of bacterial growth. These pernicious organisms even penetrate to the roots of the teeth, producing abscesses and infections which may insidiously attack almost every organ and structure in the body. Rheumatism, neuritis, disease of the eyes, even gastric ulcer, duodenal ulcer, gall bladder disease and appendicitis, may find their origin in these infections of the mouth.

A thorough examination of the teeth should include not simply inspection by a dentist but also a careful X-ray examination.

In a special study of the teeth as related to digestion, made more than twenty years ago, the writer found that there exists a definite correlation between the state of the teeth and the function of the stomach, showing very conclusively a close relation between certain gastric disorders and the condition of the teeth. Some of the facts noted may be of interest. Of the one hundred cases examined, only two had perfectly sound teeth. Of persons suffering from a deficiency of gastric acid (about one-half the total number of cases studied) the average number of sound teeth was ten, whereas in the less advanced cases of digestive disorder the 
average number of sound teeth was twenty. Of the persons suffering from simple dyspepsia, none had been compelled to resort to the use of artificial teeth, whereas among the fifty-one persons suffering from a deficiency of gastric acid twelve were dependent upon artificial teeth, five having upper sets only and seven having both upper and lower sets. Among the persons suffering from infection of the stomach or gastric catarrh, the number of sound teeth was only about one-half as many as in persons not so affected.

\section{The Saliva.}

The saliva should be included in a thoroughgoing examination of the mouth. A bacterial examination will give some idea of the state of the alimentary canal as regards bacterial infection. If the mouth is badly infected with putrefactive germs and disease-producing organisms, especially if the colon bacillus is found present, it may be taken for granted that the whole alimentary canal, with the possible exception of the stomach, is infected with pernicious bacteria.

More important still is a test of the digestive properties of the saliva. Some years ago (1890) the writer began the systematic study of the digestive power of the saliva, and soon discovered that wide differences existed in the digestive activity of the saliva produced by different persons. The plan of examination adopted was that devised by Sir William Roberts in his study of the effects of acids upon salivary digestion, the technic of which need not be given here. This method was employed in testing more than fifty thousand salivas. Very great differences were found in the digestive power of the saliva. In a few cases it was found to be almost inactive. Gum chewing rapidly depreciates the activity of the saliva.

While the great physiologist, Magendi, certainly was wrong in his supposition that the saliva is "the principal solvent of alimentary matter," it has recently been shown that cooked starch which has not been acted upon by the saliva interferes with gastric digestion by absorbing the pepsin, leaving hydrochloric acid in excess and so giving rise to hyperacidity.

The action of the saliva in liquefying the starch of the food is a necessary aid to its disintegration, thus preparing it for the action of the gastric juice and succeeding digestive fluids. 


\section{The Ewald Test Breakfast}

The test breakfast of Ewald introduced a new era in the treatment of diseases of the stomach. This breakfast consists of two ounces of bread with eight ounces of water. At the end of an hour from the beginning of the breakfast a stomach tube is passed into the stomach and its contents evacuated, filtered, and examined for total acidity, the amount of free hydrochloric acid present, the amount of combined and fixed acid, the digestive activity, as shown by the digestion of egg albumin in Mett's tubes. Tests are also made for the rennet ferment, for lactic and other organic acids. A bacteriological examination is also made.

\section{The Fractional Test Gruel Meal}

The patient is directed to take no food or drink after $9.30 \mathrm{P}$. M. on the evening before the test. At 9.30 A. M. sitting up in bed or propped in an easy chair, he swallows the tube. He should be instructed to drop the bulb to the back of the tongue, and then to swallow; thereafter occasional swallowing movements are made, but should be interspersed with deep breaths through the nose, with a view to obviating the retching sensation. When the bulb has passed the cricoid level its downward progress becomes easy and is largely involuntary, and it is only necessary to "pay out" the tube gradually until the fundus mark is at the level of the teeth. Then syringe is attached to the tube, and, with gentle suction, the whole of the fasting juice is removed and measured. With the tube in position, the patient then drinks one pint of oatmeal gruel (or in the case of women slightly less), made according to the following recipe: Two tablespoonfuls of breakfast oatmeal are stirred with two pints of water and boiled slowly down to one pint and strained through muslin; salt can be added to taste unless chloride estimations are to be made. The patient should be encouraged to read a book throughout the period of the test. At intervals of a quarter of an hour, until the stomach is empty, specimens of 10 to 15 c.c. are withdrawn. If there is delay up to three hours, it is usual to withdraw all the remaining residue and to measure its amount. After withdrawal of each specimen a few c.c. of air are blown down the tube to clear it. The mucus, bile, and food content, and the presence or absence of blood in each specimen, are noted by naked-eye inspection. Sometimes it may be necessary to examine a sample from the fasting specimen under the microscope for blood, pus, or debris. The free and total acidity in 5-c.c. samples from each specimen are determined, and the results plotted on the standard chart.

The time of disappearance of starch in the later specimens is determined by the addition of a few drops of iodine solution. This indicates the rate of evacuation of the meal, but withdrawal of further samples may be necessary to demonstrate the presence or absence of continued secretion." 


\section{ADVANTAGES OF THE FRACTIONAL METHOD}

1. Permits accurate study of fasting juice, also mucus, bile and cell contents of juice.

2. Makes possible a graphic of the different stages of digestion and bile regurgitation, indicating pyloric reaction and duodenal reflux.

3. Accurately determines emptying time of stomach.

4. Permits the study of neutralization and of the functions of the pylorus.

5. Permits study in disease of food residues in fasting specimen and noting of blood, pus, odor and other characteristics of gastric contents.

\section{Fasting Juice}

The average is about 54 c.c. Varies from 10 to 150 c.c. Bile is present in 40 per cent of fasting specimens. Free acidity ranges from 0 to 22 .

The amount of fasting juice is less with a salt-free diet. Bile is present in about half the cases. It is sometimes absent in the first syringeful and present in the second, showing it to be due to reflux or aspiration.

\section{Acidity}

Increases steadily to a maximum at an hour and a quarter. The difference between free and total acidity is usually 10 to 15 per cent. Neither hyperchlorhydria nor achlorhydria should be regarded as pathological findings. They may occur in healthy persons.

\section{Bile Regurgitation and Secretion of Mucus}

Duodenal reflux is a natural occurrence and prevents hyperacidity. It is most frequent in cases of hyperchlorhydria. It is frequently found in the fasting stomach and in the later stages of digestion. The more mucus present the less the acidity.

\section{Rate of Emptying}

The emptying time with the gruel meal is two hours. Hypochlorhydria or achlorhydria is most common in sedentary people and is accompanied by rapid emptying. Hyperchlorhydria is most frequent in more vigorous and active men and is associated with delayed emptying. The barium meal is much slower in leaving the stomach. 


\section{The Water Meal.}

A pint of warm water is introduced into the stomach before breakfast and small quantities withdrawn for testing at intervals of fifteen or twenty minutes.

Pavlov showed many years ago that water in sufficient quantity stimulates the acid secreting glands. The degree of acidity found may equal that of the normal juice.

The water meal also gives information about the presence of mucus or regurgitated duodenal contents.

\section{Tests for the Chemical Secretion.}

The small gastric tube having been introduced into the stomach, a tes: meal is introduced through the tube, care being taken that no food comes in contact with the mouth. Various meals may be used, as simple water, animal or vegetable extracts, milk, purées of cereals or vegetables, oil, or combinations. Fluid is withdrawn at intervals for examination.

\section{Test for the Psychic Secretion.}

A small tube is introduced into the empty stomach and the subject is then allowed to see, smell, and taste food, but to swallow none. Samples of the gastric secretion are withdrawn at intervals of fifteen minutes for examination.

\section{Test for the Condition of the Gastric Glands.}

Taylor calls attention to the fact that if acid protein is given in cases of achylia ferments are very often formed. If ferments are not formed, it is concluded that the glands are degenerated; hence this test should be applied in every case in which ferments are absent, by the giving of acid protein (protein $+\mathrm{HCL}$ ). In cases in which acid protein causes the ferments to appear in the gastric fluid when previously absent it should be used with each meal as an aid to the glands until the ferment-forming function is restored. The continuous use of acid protein (acidone) is necessary when no hydrochloric acid is formed.

\section{The Barium Meal.}

Soon after the discovery of the X-ray it became evident that valuable information might be obtained by the use of a test meal containing substances opaque to the X-rays. First, nitrate or carbonate of bismuth was employed for this purpose. Later, 
barium was substituted. For the efficiency of this plan of examination, the world is largely indebted to Dr. James T. Case, of the Battle Creek Sanitarium, who has contributed much to the perfecting of the technic of this valuable method.

The digestive apparatus has two distinct classes of functions. One of these is concerned with the transformation of the food from insoluble or non-absorbable substances into soluble and absorbable products which may be utilized by the body in building and repairing the vital machinery and in replenishing the vital energies of the body. This work is done chiefly by the aid of the digestive juices, which are formed by glands placed at various points along the digestive canal, chiefly in its upper part.

A second class of functions consists of mechanical treatment of the foodstuffs by which they are: (1) reduced to a soluble or pultaceous state; then (2) mixed with the digestive juices; (3) spread out upon the intestinal wall so as to aid in absorption; (4) moved from one part of the alimentary canal to another, constantly in the same direction; and (5) when reduced to the unusable residues discharged from the body.

The Ewald meal and chemical examination of the stomach fluid give us information concerning the first class of functions -the action of the digestive juices upon the food. The barium meal gives information concerning the second class of functions, which are executed by the muscular walls of the intestine. The information obtained by the aid of the barium meal is of even greater value than that secured by the test breakfast; but for complete information it is necessary that both methods be employed. It is further to be noted that the barium meal not only advises us concerning the stomach, but visualizes the entire alimentary canal from mouth to anus, giving information concerning the conditions of the small intestine, the colon, the gall bladder and the liver, which facts are of even greater importance than those elicited concerning the stomach by the Ewald meal. The following is a brief resumé furnished by Dr. J. T. Case of the principal conditions concerning which information may be obtained by the opaque (bismuth or barium) meal:

Ulcer, cancer or tumor of the stomach, cardio-spasm, spasm of the esophagus, spasm of the pylorus, distortions of the stomach, cysts and tumors of the pancreas, adhesions of the pylorus, ulcer of the duodenum, distortions, obstructions and adhesions of the duodenum, gallstones, adhesions of the gall bladder, loca- 
tion and changes in the liver, abscess in or about the liver, tumors, cancer, ulcers and other changes in the small intestine, incompetency of the ileocecal valve, adhesions of the intestine, adhesions of the colon, disease of the appendix, dilatation and adhesions of the cecum, cancer of the colon, tuberculosis, syphilis, atony, colitis and spasm of the colon.

\section{The Motility Test.}

The rate at which the undigested foods and residues are moved along the alimentary canal is termed its motility. The barium meal affords an exact and scientific method of studying the motility of the alimentary tract. Another very simple and high practical method, affording final results which agree very exactly with those obtained by the barium meal, is the following:

Two capsules, containing together about 10 grains of carmine, are swallowed with the breakfast. In place of carmine a teaspoonful of bone black or finely pulverized charcoal may be used. The carmine or the charcoal mingle with the food, giving it a characteristic color which can readily be recognized in the stools. After the administration of the carmine or charcoal the stools are carefully watched and the exact time noted when the color first appears. Further observations are made until the color disappears, the time of which is also recorded. The time of the first appearance of the color has long been in use as a means of determining motility, but the writer's experience has been that this is not the true measure of the motility rate, but that the motility time is marked by the disappearance of the color. This time is found to correspond very accurately with the results obtained by the use of the barium meal. Some thou'sands of observations made under the writer's supervision indicate that the motility period may vary from eight or nine hours to several days.

It is found that persons whose bowels move once a day usually have a motility period of 50 hours or more. When the bowels move thoroughly three times a day, the motility period is reduced to 16 to 24 hours.

The normal motility rhythm is in the writer's opinion the following:

The breakfast residue is discharged some time between supper and bedtime; the dinner residue before breakfast the next morning or not later than soon after breakfast; the supper residue after breakfast or not later than soon after dinner the 
following day. When this normal motility rate is maintained, the time which the food residues remain in the body will be; for breakfast, 13 to 15 hours; for dinner, 17 to 20 hours; for supper, 15 to 20 hours.

When the body wastes are dismissed in this prompt and regular manner, there is no time for putrefaction; wild bacteria cannot obtain a foothold, the normal acid-forming flora of the intestine is dominant, and the vital machinery is not crippled by the absorption of the enormous quantities of high toxic substances which are constantly pouring into the blood when the food residues and body wastes are retained so long in the colon that they become putrid.

This is the normal or ideal alimentary cycle, but it is by no means realized in ordinary experience. The colon of the average man is in the condition of a congested railway system. The right of way is choked, and if bowel movements occur, they are belated, - two or three days in arrears; the residues of half a dozen or more meals being packed away in the colon undergoing putrefaction and poisoning the body.

The motility test is invaluable in connection with diet and treatment for changing the intestinal flora, and should always be used in connection with this method as a means of checking the results obtained, in addition to careful bacteriological studies.

\section{Examination of the Gall Bladder.}

The X-ray examination of the gall bladder region often gives information of great value concerning the condition of the gall bladder. Gallstones are sometimes seen. A certain proportion of cases, however, remain obscure, and in these some help may be gotten by the use of the Metzer-Lyon method of obtaining specimens of bile, which is as follows:

The test is made before breakfast, 12 hours after the last meal. A duodenal feeding tube is introduced while the patient is in a sitting position. The patient is then placed on the right side, with the left limb drawn up over the right, so as to get the gall bladder region as low down as possible. The position aids the tube in entering the duodenum so as to bring the bulbous end of the tube near the mouth of the bile duct. The tube will usually reach the duodenum in 10 to 60 minutes. When it is certain that the bulb is in the duodenum some of the duodenal fluid should be collected. The first fluid obtained should be a "pearly fluid" with 
little mucus and alkaline in reaction. If the duodenum is diseased, many germs will be present and the fluid will be turbid.

The next step is to inject with a small syringe through a tube one and two-thirds ounces of a 25 per cent. solution of magnesium sulphate. This will cause relaxation of the bile ducts and opening of the common duct, with discharge of bile from the gall bladder. By gentle suction on the syringe, some of the fluid may then be withdrawn, after which the tube is disconnected and hung over the side of the couch to drain. The first liquid will be clear duodenal fluid, bile and sulphate solution mixed. In 10 to 30 minutes the bile will become darker and more viscid. This is gall bladder bile. If the quantity is large,--more than two and twothirds ounces-there is probably stasis in the gall bladder. After a time the bile again becomes thinner and lighter in color, coming direct from the liver.

If no bile can be obtained, the common duct may be obstructed. If only clear, light-colored bile is obtained, there may be obstruction of the cystic or gall bladder duct.

The benefit which sometimes results from this mode of gall bladder drainage is probably due to the getting rid of a quantity of stale and concentrated and hence highly poisonous bile.

By far the most dependable test for the liver and gall bladder is that of Graham, which often discovers lesions, which other methods of examination fail to disclose. The details of this procedure are too technical for description here.

\section{Examination of the Stools.}

The chemical and microscopic study of the stools is of great importance, not only as a means of investigating the residual products of digestion but also for the purpose of detecting the presence of animal parasites, tapeworms, the amoeba of tropical dysentery, etc.

Chemical and macroscopic as well as microscopic examinations are also made for undigested food, parasites, blood, mucus, and other evidences of pathological conditions.

The examinations are repeated until the flora is changed and the stools lose their putrid character and become in other respects normal.

Some of the most significant indications in relation to the stools are the following:

The Color. Very light or clay-colored stools signify the 
absence of bile. Pancreatic digestion may be deficient also. Such stools are usually very foul smelling.

Dark-colored stools denote putrefaction. The stools of a healthy breast-fed infant are light yellow in color and nearly odorless. When they become brown or dark, the child is sick and toxic. Black stools may indicate blood from hemorrhage.

The normal stool is of a yellowish color and soft consistency. The so-called "well-formed stool" is an indication of chronic constipation. It occurs when the bowels move but once daily, a form of constipation usually mistaken for an indication of health.

The Odor. This is most important. Normal stools have a slightly sour odor or a very faint fecal odor, but no suggestion of putridity. A putrid, strong, ammoniacal or rancid (butyric) odor is a certain sign of putrefaction; and when putrefaction is present, there are also infection, colitis and autointoxication.

Lumpy stools indicate a spastic state of the colon, probably due to colitis.

The presence of mucus is a certain sign of colitis.

Frothy stools with a sour odor indicate very active fermentation.

Gas or flatulence accompanying the stool indicates the presence of the pernicious Welch's bacillus and other gas-forming organisms, especially the colon bacillus.

\section{Bacteriological Examination of the Stools.}

Most important of all is the bacteriological examination of the stools by modern methods. Examination of the stools with reference to typhoid and other intestinal infections is of course important, but of far greater importance is examination to determine the character of the dominant flora present in the intestine, particularly in the colon. The normal flora of the colon is fermentative, or acid-forming. The dominant organism present is $B$. acidophilus, a germ which produces lactic acid and does not produce gas. This germ appears as the dominant flora in very young healthy children. It is also found in the colons of chickens, monkeys, rats and other animals when fed on food rich in carbohydrates and given no meat.

In adults on a mixed diet the $B$. acidophilus is found in the stools only in very small numbers if at all. The dominant flora is putrefactive. That is, the food residues are of such a sort that they undergo putrefaction in the colon. Putrefaction is encour- 
aged by the constipation generally present in the average American. A bacteriological examination shows the presence of Welch's bacillus, a common putrefactive germ and the organism which causes gas gangrene. The colon bacillus is also present in large numbers with various other putrefactive and poison-form. ing germs.

By means of bacteriological cultures, the character of the flora may be readily determined. The presence of large quantities of gas shows the presence of Welch's germ. Small gas bubbles in the Veillon tube indicate the colon bacillus. The absence of gas and characteristic colonies show the presence of $B$. acidophilus, the normal acid-forming flora. As the flora improves under proper feeding, the gas gradually diminishes until it disappears when the flora is changed, and the B. acidophilus becomes dominant.

The accompanying cuts show the appearance of the Veillon culture tubes before and after changing the flora. Litmus milk and plate cultures are also made.

\section{The Blood.}

The normal blood count per cubic millimeter is $5,000,000$ red cells and about 7,000 white cells. The total number of red cells in the body of a man of average size is $25,000,000,000,000$. The absorbing surface presented by the red cells is about 82 square rods, more than one-half acre. It is the function of the red cells to absorb oxygen, which is taken up and concentrated by the hemoglobin, an organic iron compound to which the red corpuscles owe their color. The blood of a well-developed man contains about 40 grains of iron.

In the words of the old prophet, "The blood is the life." The blood was held as sacred by the ancients because it was believed to be the seat of the life principle of the body. The modern physiologist recognizes the fact that the blood is the vitalizing element of the body, that it creates and maintains the body; in other words, that it is in a literal sense the life of the body.

It has long been known that oxygen is carried by the red blood cells. It has been supposed, however, that the transport of the carbon dioxide was chiefly accomplished by the serum or plasma of the blood. Recent observations by Joffe have shown that at least two-thirds of the carbon dioxide is carried by the red blood cells. It is now known that the red blood cells carry 
oxygen from the lungs to the tissues and carbon dioxide from the tissues back to the lungs. The little burden bearers are always loaded.

In anemia the blood iron is reduced and the blood cells may also be reduced in either greater or lesser proportion than the coloring matter of the blood. In a person whose hemoglobin is 50 per cent., for example, the blood contains only half the normal amount of iron, which means that the vital resistance, endurance, and ability to cope with disease are proportionately reduced. His stream of life is at a low ebb. When the white cells are reduced, the ability to resist the invasion of bacteria is depreciated, since it is upon the white cells that we depend for defense against these invisible enemies. Whenever infection occurs, that is, whenever disease-producing germs find entrance to the body, the white cells usually increase in number very rapidly. This is simply the rallying of the defenders of the body to combat the invading enemy. For example, when an attack of appendicitis is developing, the white blood cells may within a few hours increase from 7,000 per cubic millimeter to 20,000 or even more. At the beginning of an attack of pneumonia the increase may reach 100,000 , many times the original number. In general, an increase of the white cells in the blood indicates bacterial infection.

The white cells are usually increased after meals, doubtless because the number of bacteria entering the blood from the intestine is also increased after eating, requiring a greater number of white cells to deal with them.

\section{Chemical Examination of the Blood.}

Chemical examination of the blood is of the highest importance. Though not as yet in general use, this method, thanks to the new and exact technic of Folin, is now thoroughly practical and its use is most desirable, almost indispensable, in certain classes of disorders. This is, indeed, the most exact means of determining the degree of efficiency of the kidneys. It is the function of the kidneys to remove waste matters from the blood. These toxic substances taken collectively are termed non-protein nitrogen. By drawing a few drams of blood from a vein by the aid of a suitable syringe and a fine needle the exact amount of non-protein nitrogen present in a given quantity of blood may be readily determined. By this means it is possible to ascertain the amount of waste matters left in the blood by inefficient kidneys. 


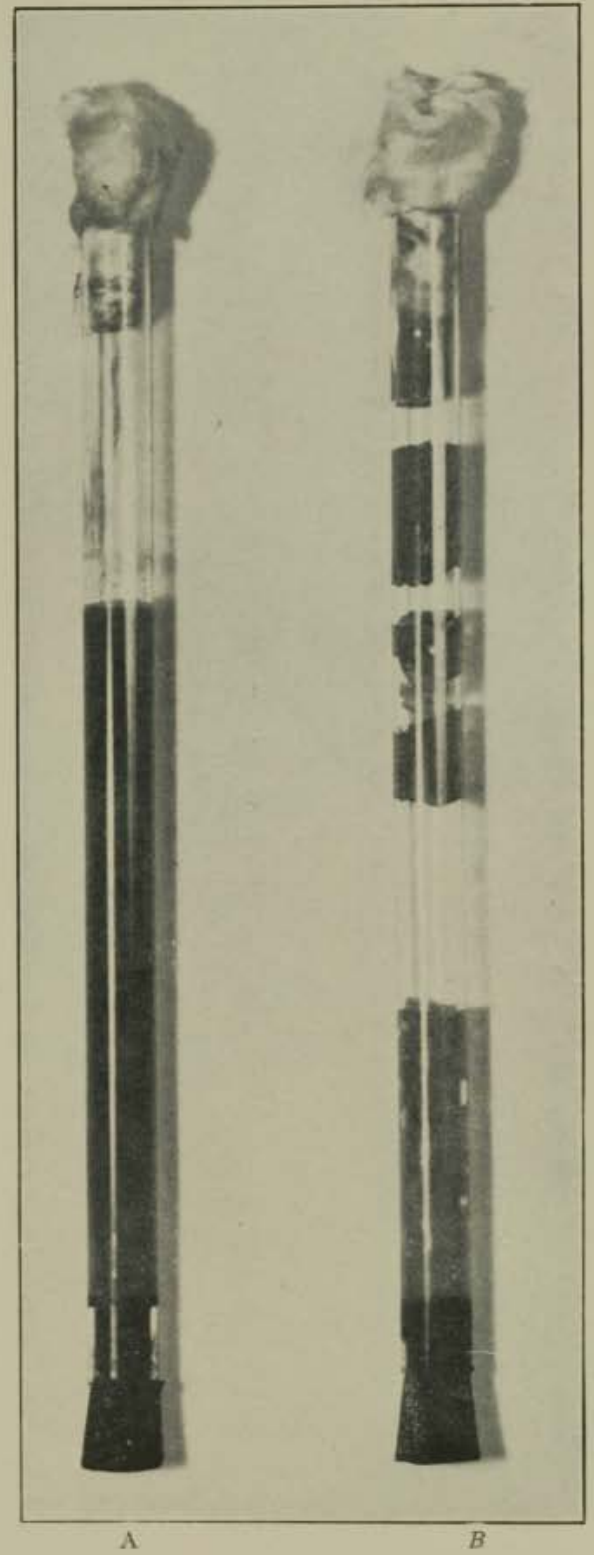

Veillon Tube Cultures Showing. A. Flora Changed, No Gas; B. Putrefactive Flora, Weleh's Bacillus, Much Gas 


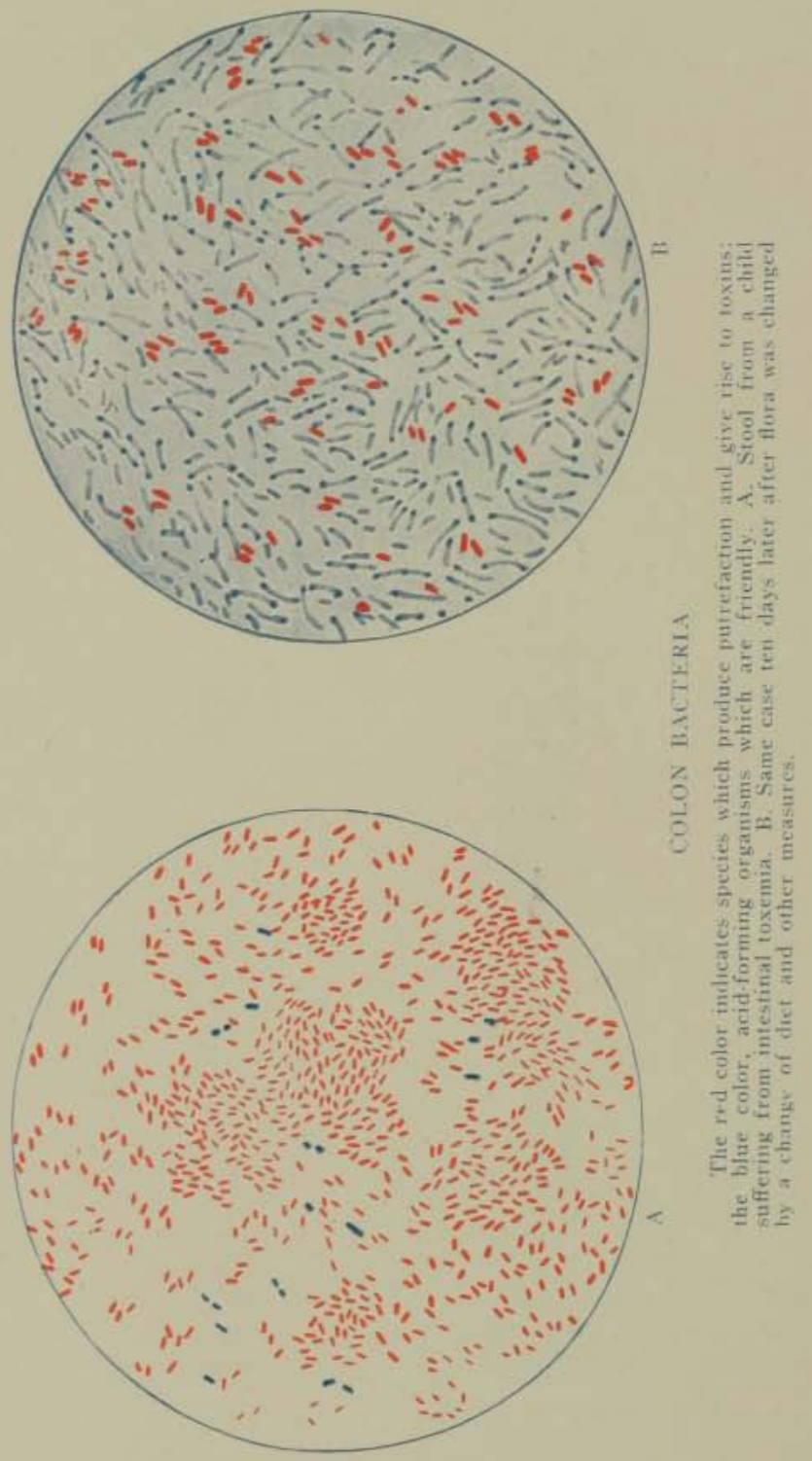


The normal amount of this substance is 28 to 32 milligrams (onehalf grain) per 100 cubic centimeters (three and one-third ounces) of blood. When the amount of non-protein nitrogen is much greater than this, there is reason to suspect a considerable degree of renal inefficiency; that is, the kidneys are not able to remove from the blood the waste matters which it is their duty to extract from it.

The determination of an increase in the amount of uric acid present in the blood is the best means of detecting the earliest indications of failure of the kidneys to perform their work efficiently. An increased amount of creatinin in the blood indicates that disease of the kidneys is far advanced.

A knowledge of these conditions is of the utmost consequence, as it shows a necessity for the establishment of a rigorously low protein diet, which might not otherwise be considered justifiable, but is absolutely required for the prolongation of the individual's life.

Sugar is normally found in the blood in the proportion of about one part in one thousand. When the sugar is increased considerably beyond this amount it appears in the urine and the patient is said to have diabetes. Examination of the blood, however, often shows a marked increase of blood sugar when it has not yet appeared in the urine; hence, an increase of blood sugar is a most important symptom of the approach of diabetes.

\section{Waste Constituents of Normal Blood.}

\begin{tabular}{|c|c|}
\hline Non-protein nitrogen (urea, uric acid, etc.). & $\begin{array}{l}\text { Mgms. per } 100 \mathrm{ccm} . \\
\quad 25-32\end{array}$ \\
\hline Urea & $8-15$ \\
\hline Uric acid .... & 2- 4.5 \\
\hline Creatinin ......... & $1-2.5$ \\
\hline gar ................... & $80-120$ \\
\hline
\end{tabular}

The so-called Wassermann test and various other serological tests are important as means of indicating the presence of syphilitic and other infections, which may be recognized by changes in the blood.

\section{The Urine.}

The urine is an extract of the tissues. It represents to a large extent the acid wastes of the body, while the bile represents the alkaline wastes. The quantity of the urine, its specific grav- 
ity, the amount of urea, uric acid and other substances, are all highly important in measuring the tissue changes which are taking place. The amount of ammonia, indol and other putrefaction products, also the presence or absence of albumin, casts and other abnormal elements, are most important means of judging of the kind and extent of morbid changes which may be taking place in the body.

A high protein diet is necessarily accompanied by a great increase in the amount of urea and uric acid. Folin has shown, in fact, that urea represents the excess of protein which has been eaten, the only use for protein being to make good the wear and tear of the living tissues. Any amount beyond the small quantity required to accomplish this, instead of being built into tissue, is directly carried out of the body as so much waste matter. It will thus be readily seen how great a burden is imposed upon the kidneys by the extra work required of them in the elimination of surplus protein eaten in the form of steaks and chops.

Still another extra burden thrust upon the generally overworked kidneys when an excess of protein is eaten is the necessity for the elimination of the indol, skatol, and other poisonous products resulting from the putrefaction in the colon of the undigested residues of meat and other food substances rich in protein. These putrefaction products are probably the source of the ammonia which is found in the urine in quantities closely proportional to the amount of meat eaten.

\section{Tests of the Urine.}

Diabetic patients, when possible, should be instructed in the methods of examining the urine, so that they may be able to note the effects of diet and to detect at once the appearance of threatening symptoms in their own cases. The following directions for testing the urine are borrowed from the author's work on diabetes*:

\section{Collection of the Specimen.}

Use for the purpose only glass jars or bottles which have been thoroughly cleaned and scalded with boiling water. During the collection keep the jar well covered and in a cool place. The

- "The New Method in Diabetes," John Harvey Kellogg, M. D. Modern Medicine Publishing Company, Battle Creek, Mich. 
specimen to be analyzed should cover a period of twenty-four hours, and should be obtained in the following manner:

Empty the bladder at the usual time of rising in the morning, say $7 \mathrm{~A}$. M. This is to mark the beginning of the collecting period; hence this passage is not to be saved. Then carefully save all the urine passed until $7 \mathrm{~A}$. M. the next day, taking care to completely empty the bladder at this time. If the quantity is so large as to require several containers to hold it, the whole of the twenty-four-hour collection should be poured into one vessel large enough to hold it all, so as to secure thorough mixing. Note the total quantity, being careful to measure very accurately. If it is desired to send to the laboratory a specimen for complete analysis, fill a perfectly clean bottle or jar, holding at least one pint, with the mixed urine, seal properly and label with the name and the total quantity of urine passed during the 24 hours. A half dram of toluol may be added to preserve.

\section{Metabolism Tests.}

In the term metabolism is summarized the total vital activities of the body. All these activities depend upon the consumption of oxygen, just as does the combustion taking place in a furnace. The amount of steam produced, and thus the amount of energy set free from the coal in a boiler plant or a locomotive, is determined by the amount of oxygen admitted to the boiler furnaces. In like manner, the amount of oxygen consumed in the body is an accurate measure of the amount of energy produced. Each cubic foot of oxygen burned means the liberation in the body of energy equivalent to 160 foot tons. Exposure to cold and muscular activity increase the rate of oxygen consumption and of energy expenditure.

By means of recently devised apparatus, it is now possible to make very exact measurements of the amount of oxygen consumed and hence to determine accurately the rate of energy production in the body. The application of these tests has shown that the rate of metabolism is increased in certain diseases and sometimes to a very high degree. This is particularly true of hyperthyroidism, or toxic thyroid, commonly known as exophthalmic goiter. There is also increase of metabolism in various other serious disorders, as in advanced cases of heart disease, pernicious anemia, in fevers, in hysteria, in arteriosclerosis and in nervous diseases accompanied by extreme nervous tension. 
The metabolism rate is commonly diminished in aged persons, and in certain diseases, as chlorosis, anemia, cachexia, Bright's disease, myxoedema, malnutrition and malignant diseases.

\section{Test for Acidosis}

The condition of acidosis is often indicated by a sweetish or sweetish-sour odor of the breath, due to the presence of acetone. Acetone may also be found in the urine. The most accurate means of measuring the amount of acidosis present is by determination of the $\mathrm{CO}_{2}$ tension of the residual air of the lungs. This is done by the use of a special apparatus, into which the patient breathes. The carbon dioxide is carried in the blood in loose combination with soda salts, which normally abound in the blood. When abnormal acids are present in the blood, they combine with the soda and thus prevent the taking up of the carbon dioxide from the tissues, where it is produced by the vital combustion continually taking place, in which oxygen combines with carbon in connection with muscular work and other vital changes. The minimum normal $\mathrm{CO}_{2}$ tension is 34 . When the tension falls below this it is evident that the supply of oxygen to the tissues is insufficient, and acids are accumulating. In high health the tension is 40 to 45 . Inability to hold the breath thirty seconds or more suggests acidosis.

\section{The Body Weight.}

The body weight is a simple and very practical metabolism test which should have more attention than it commonly receives. Rapid loss of weight may be due to excessive metabolism. This condition is often the result of worry, suspense, or anything which increases nerve tension. The increased expenditure of energy is due to an increase of the so-called muscle tonus, which in health and with the body in a state of rest, accounts for 30 to 50 per cent. of the total energy expenditure, but which may be increased 100 per cent. or even more as the result of tension from exciting emotions, as well as exercise of the muscles.

\section{Strength Tests.}

Tests of strength are, when properly interpreted, a valuable means of determining general neuro-muscular conditions. Muscular effort is the result of the combined activity of nerves, nerve centers and muscles-the so-called neuro-muscular mechanism. 
Weakness may be the result of an impaired condition of either one of the three sources named-an exhausted nerve center, an impaired nerve conductor, or a muscle exhausted because saturated with fatigue poisons. Exhaustion of the muscle may be quickly removed by the application to it of simple massage while an exhausted nerve center may be restored only by the renewed accumulation of energy in its cells and a damaged nerve conductor must be repaired. A muscle which has become definitely weakened by disuse requires graduated exercise.

More than thirty-five years ago (1884-5), the writer began experiments for the purpose of devising means for measuring the individual groups of muscles of the body. After ten years' effort an apparatus was constructed, known as the universal dynamometer, which has now been in extensive use for more than twenty-five years. By means of this device the strength of each of the large groups of muscles in the body may be accurately determined. The results are then placed on a percentile chart and a graph made, showing the relation of the strength of each group of muscles in the body to the strength of corresponding groups in a normal man of the same height.

\section{Tests of Heart and Circulation.}

The heart muscle expends in its activities about 10 per cent. of the total energy output of the body when in a state of rest. The strength of the heart and its powers of endurance vary within almost as wide limits as does the strength of other muscles, though of course complete paralysis of the heart muscle cannot exist without immediate death. No dynamometer has yet been devised by which the strength of the heart can be measured, but useful information concerning its strength may be obtained by the use of the sphygmomanometer, an instrument for obtaining the blood pressure.

The method is simple. The blood pressure is taken, both systolic and diastolic, first with the patient lying, and then standing, and finally standing after gentle exercise for ten minutes. The pulse rate is also noted in both the lying and standing positions and in the standing position after exercise. The difference between the diastolic pressure and the systolic pressure is known as the pulse pressure. Both the diastolic and the systolic pressure normally rise when a person changes from the horizontal to the vertical position, the diastolic rising more than the systolic. 


\section{Regimens and Dietaries}

While there are many special feeding indications for individual diseases, most of the hundreds of different maladies and morbid conditions which are encountered by the physician and the nurse may be grouped as regards dietetic requirements into a small number of classes; and most of the requirements may be met by some one of the following dietaries or regimens or a combination of two or more. Some of the dietaries described, for example, the laxative and the antitoxic dietaries, are applicable, with modifications, in most cases.

\section{All Dietaries Must Supply}

\section{Lime, Iron and Vitamins.}

In arranging bills of fare, it is important to remember that certain elements must be found in every dietary which is to be followed for more than a few days. This is especially true as regards vitamins, lime, iron, and the alkaline salts. Hence, no matter what other food constituents may be reduced, the supply of iron, lime and vitamins must be kept up to the normal. It is especially important to bear this in mind in the treatment of cases of fever, obesity, peptic ulcer, and surgical cases.

\section{THE FRUIT REGIMEN}

(See also page 987)

The fruit cure has been practiced in various ways. The most familiar is the so-called "grape cure." The patient takes ten to fourteen pounds of grapes a day and a few pieces of zwieback.

Linnaeus tells us of the cherry cure, which he himself employed with advantage.

During the Civil War the diarrhea and dysentery from which soldiers suffered were relieved during the peach season when the soldiers had access to peach orchards.

\section{How Fruits Differ.}

Fruits do not stimulate the bowel by irritation, but by the bulk that they furnish because of the cellulose which they contain 
and through their acids and sugars. Fruits differ in these properties. Apples and pears contain almost no cellulose, bananas contain practically none and are little laxative. Cherries, grapes, apricots and peaches contain more cellulose and are therefore more laxative. Prunes contain a considerable amount of cellulose and are highly laxative. Raisins are also laxative, as are figs. Dates are less laxative, but when freely used (one-quarter pound three times a day) greatly aid the change of flora. Huckleberries contain the largest amount of cellulose of any food, and are very highly laxative. Raspberries and blackberries are laxative, but less so than huckleberries.

Fruits that contain much acid are prohibited in cases of hyperacidity, and sometimes in chronic gastric catarrh without hyperacidity, and also in achylia when the gastric mucous membrane has become hypersensitive to organic acids. In such cases fruits free from acids should be taken, such as bananas, pears, white cherries, very sweet peaches, cantaloupe, watermelon and casava melon.

\section{The Daily Routine.}

At the start, the patient should take a carmine capsule and note the time when the color appears and disappears. The character of the stools should also be noticed, especially the color and the odor. Putrescent stools are generally dark in color, either black or brown, and have an ammoniacal, rancid or putrescent odor.

With the fruit regimen the patient takes four meals a day. Each meal consists of fruit-any kind of fruit which agrees with the patient-one-half ounce of bran and one ounce of paraffin oil. If desired, one ounce of brose (a mixture of equal parts by measure of oatmeal, cornmeal and sterilized bran) made into a porridge may be added twice a day. Roughage may be taken in the form of fig bran. For persons who cannot take bran, or think they cannot, agar-agar may be used instead. The bran or agar-agar should be divided into six or eight equal parts and distributed through the meal, so that it may be well mixed with the other foodstuffs. Paramels and psyllium seed are highly efficient laxatives. Two or three ounces of milk sugar or lacto-dextrin should be taken three times a day for speedy results. The lactodextrin may be taken before meals or three hours after eating. 
After three or four days of the fruit regimen the stool generally becomes nearly odorless and consists chiefly of bran. The fruit regimen should be continued until this change occurs, unless the patient is very feeble, in which case the diet may be changed and the patient allowed to take a more liberal bill of fare for a few days, when the regimen may be repeated.

In exceptional cases the diet must be continued for ten days or two weeks. When this is necessary, no serious inconvenience will be experienced by the patient, provided he is kept at rest.

In the case of very feeble or emaciated patients the fruit regimen may be used for three or four days, and then followed by the milk regimen, which not only serves to perfect and establish the change in the intestinal flora but secures a very desirable gain in flesh, often at the rate of half a pound a day or even twice as much.

Thin persons should rest while taking the fruit regimen, so as to avoid undue loss of flesh. It is not necessary to remain in bed, but the horizontal posture should be maintained; if not in bed, on a cot or a sofa, and always in the fresh air.

By the addition of lettuce, celery, and in some cases salads of carrots, turnips, cabbage, and palm cabbage when available, valuable vitamins are supplied as well as nutrient material-elements of the greatest value in rebuilding the diseased and deteriorated organism.

An especial advantage of the fruit regimen is that it is so simple that it may be employed by anyone, at almost any time and in almost any place. For good results it is only necessary that the rules here laid down should be carefully followed.

By this regimen all the good effects claimed by the advocates of fasting may be obtained, without the dangers and inconveniences of total abstinence from food.

Evidence that the Intestinal Flora is Changed: When the flora is changed, intestinal gas disappears, the stools are no longer foul or strong smelling, they are lighter in color unless colored by spinach or other food, they usually have a slightly acid odor, chemical tests show acids dominant, and a bacteriological examination shows absence of Welch's bacillus and other putrefactive and gas-forming bacteria, very few colon bacilli, and an abundant growth of acid-forming bacteria, especially the $B$. acidophilus.

Keeping the Flora Changed. After the flora has been changed, 
it may be easily kept in a nearly normal condition by close adherence to an antitoxic diet and once in three or four weeks taking 2 or 3 ounces of milk sugar or lacto-dextrin three times a day for two or three days. This should be done whenever the stools become foul smelling or when intestinal gas is troublesome. The continuous use of acidophilus buttermilk is an excellent means of keeping the flora changed. Half a pint to a pint at each meal is required for best results.

\section{The Green Regimen.}

In certain cases of hyperhydrochloria the stomach is exceedingly sensitive to fruit acids, so that the fruit regimen gives rise to pain even when bicarbonate of soda and small quantities of olive oil are employed, as elsewhere suggested. In such cases fruits may be eliminated altogether and the dietary may be made to consist exclusively of green vegetables, such as lettuce, celery, cucumbers, green corn, cabbage, carrots, turnips, and cooked greens of all sorts, spinach (parboiled), dandelion, beet tops, endive, etc. Brose (equal parts by measure of oatmeal, cornmeal and cooked bran) cooked for five to ten minutes may be used twice a day. Otherwise the regimen is managed exactly like the fruit regimen.

In many cases purées of sweet fruits such as bananas, dates, soaked figs or raisins, and sweet apples, pears or white cherries may be carefully added to the regimen; and later, acid fruits.

Carrots, both raw and cooked, are of special service. Metchnikoff found the stools of rabbits nearly sterile when the animals were fed on carrots.

\section{When the Tongure Remains Coated.}

In certain cases the tongue remains coated after the fruit regimen or the green regimen has been closely followed for several days. This is an indication that the normal state of the blood has not been restored. This may be due to an excessive accumulation of toxic matters in the body, or the cause may be an adherent or pouched cecum in which food residues accumulate and putrefy because the crippled cecum is not able to empty itself. In such cases the systematic use of the enema is very necessary. An enema consisting of three pints of water at a temperature of about $90^{\circ} \mathrm{F}$. should be slowly introduced into the colon at bedtime. In cases of spastic constipation the temperature should be 
$115^{\circ}$ to $120^{\circ} \mathrm{F}$. Five minutes should be occupied in getting the water in the colon, so as to give time for it to reach the cecum and thoroughly flush the head of the colon. There are sometimes adhesions of the pelvic colon which prevent efficient bowel action. In such cases a simple surgical procedure may be needed.

If necessary, repeat the enema so as to make sure that the colon is thoroughly emptied. Continue this practice as long as necessary.

\section{Disorders in Which the Fruit Regimen is Indicated.}

In high blood pressure, whether due to degeneration of the arteries, to renal disease or to fully developed cardio-vascularrenal disease with or without dropsy, the fruit regimen is capable of rendering valuable service. The urinary acidity is high and the diminished activity of oxidation leads to a continued tendency in the direction of acidosis.

The large amount of acid salts contained in fruits in which an organic acid (citric, malic or tartaric) is combined with potash and soda to alkalinize the tissues, or at least to neutralize the acid products present, renders this class of foods, especially juicy fruits, most useful.

The fruit regimen is of special service in cases of parenchymatous nephritis.

The fruit regimen is often highly successful in cases of dropsy. In dropsy there is an accumulation in the tissues of fluid charged with salt and urea. A diet free from salt has been found to be one of the most efficient means of relieving dropsy and edema. The fruit regimen is salt-free and almost nitrogenfree and so rapidly clears the tissues of salt and urea.

The fruit regimen is especially adapted to the treatment of obesity. Notwithstanding the fact that a sufficient bulk of food is taken to afford the patient a sense of satiety, or to satisfy the appetite, the actual amount of nourishment is not more than half or two-thirds the maintenance ration.

In intestinal toxemia, which is commonly indicated by a coated tongue, bad breath and dingy or pigmented skin, very foul stools, and the other well-known symptoms of this condition, the fruit regimen is almost a panacea. In all cases in which there is no serious mechanical obstruction to bowel action present, such 
as adhesions of the pelvic colon, the fruit regimen may be expected to accomplish the result desired-cases being very rare indeed in which the most sanguine expectations are not realized. Almost invariably within three or four days after the beginning of the regimen the stools will lose their foul odor, the tongue will be found very much less coated, the breath less offensive, and in a few days more the symptoms will be very greatly ameliorated.

The fruit regimen so greatly facilitates the attainment of the results expected of the milk regimen that it serves a highly useful purpose as an introductory measure. In many cases in which the milk regimen is indicated the patient has a coated tongue, a foul breath and is obstinately constipated. It is the writer's custom in dealing with such cases to employ the fruit regimen for three or four days before beginning with the milk. The tongue clears off, the appetite is improved, the bowels become more active, and thus the patient is prepared to take the milk with relish and to digest and utilize it.

\section{THE MILK REGIMEN}

The value of milk as a food remedy has been recognized from the most ancient times. Hippocrates especially commended it as a remedy for phthisis and for gout. He was perhaps the first to recommend the addition of water to milk (hydrogala) as a means of increasing its digestibility.

Aretœus, a Greek physician who wrote an extended treatise on acute and chronic diseases in the second century of our era, recommended as a remedy for phthisis the free use of milk, directing that it be prepared by adding a fourth part of water and then boiling it until the mixture was-reduced to one-half its original bulk.

Galen made use of cow's milk, milk of the goat and sheep, and even woman's milk. He very highly commended milk as a remedy for various diseases, and refined the method by requiring that the cow producing the milk should be fed upon certain special herbs.

The ancients evidently attributed to milk specific healing properties which their keen powers of observation had enabled them to discover.

Hoffman especially recommended the milk of cows fed in pastures which abounded with lotus and polygonum. As the 
patient improved in health, he was allowed to sail down the Tiber and use milk from the cows of Strabiø.

Health resorts in which the milk cure was practiced existed even in those days. Each invalid was given a goat, an ass or a cow as his source of milk supply, and it was part of the duty of the patient to lead his animals to graze in pastures supposed to be best adapted to his particular case and to take the milk warm, direct from its source.

The ancients often made use of woman's milk as a remedy, and it is still used in some parts of Africa in feeding fever patients. Mr. Poultney Bigelow relates a personal experience of this sort when sick with a fever on the pest-ridden Gold Coast. This is very interesting evidence of the appreciation of the special curative properties of milk by primitive people.

Sir John Sinclair, in his "Code of Health and Longevity," mentions the use of sour milk as common in Scotland and Ireland. The milk was prepared by permitting it to sour in a vessel placed in warm water, after which the whey was turned off and the curds placed in a "plunge churn," where they were churned for some time.

Sydenham and other eminent physicians of his time made large use of milk as a remedy.

Van Swieten especially recommended milk as a remedy for gout, declaring that the victims of this disease would find themselves wholly free from pain so long as they lived upon an exclusive diet of milk.

In modern times the use of milk as an exclusive diet was brought to the attention of the profession by Philip Karell, of Petrograd, physician to the Emperor of Russia. A paper written by this sagacious physician was translated by Carrick, at that time physician to the British Embassy at Petrograd, and was published in this country in 1870 .

Karell was perhaps the first to systematize the use of milk and to insist upon its use in small doses. He made use of the remedy especially in the treatment of obesity and dropsy, though he commended it in many other chronic diseases. On this account he employed small quantities, generally not exceeding two to four pints daily, the object being to reduce flesh or to promote the absorption of dropsical fluids. He said: 
1 have, after fruitlessly trying all sorts of remedies in many chronic and obstinate diseases, at last succeeded in thoroughly bringing the alimentary canal, that seat of so many diseases, under my control. I did this by administering milk according to a new method.

Karell's method consisted in giving one glassful ( 7 ounces) of milk four times a day at intervals of four hours. After a few days the amount of milk was slightly increased, but always remained much below the normal requirement of liquids and solids until the end desired, the reduction of fat or dropsy, had been attained.

Among the specific virtues of milk are:

1. Its richness in lime salts; an ounce of milk contains more food lime than a pound of beefsteak and nearly as much as a pound loaf of bread.

2. Richness in vitamins, supplying every sort needed by the growing animal, anti-rachitic, anti-beriberi, and anti-scorbutic.

3. Protein of exceptional quality which is not only capable of supplying all the elements needed for repair of living cells, but is capable of supplementing the deficiencies of the incomplete proteins of vegetable origin.

4. A considerable proportion of lactose, a milk sugar which by its slow absorption is able to reach the colon, where it serves a most useful purpose in encouraging the growth of $B$. acidophilus, the protective intestinal flora, thus suppressing the putrefactive processes which give rise to autointoxication.

5. The fats of milk are in a state of fine emulsion and thus well prepared for the action of the digestive juices.

\section{Technic of the Milk Regimen.}

First of all, it must be emphasized that simply feeding milk is not what is meant by the milk regimen. The difference is a vital one. Milk feeding is often a failure in cases where the milk regimen succeeds admirably.

The first essential is that the milk should be taken in large amount, so large as greatly to exceed the needs of the body, and thus fill the alimentary canal with material which will promote the growth of friendly bacteria.

A second essential is that the milk shall be taken often. A half pint every half hour is the usual plan. This makes it possible to take into the stomach the five or six quarts of fluid for 
one day's ration. It is also important to maintain a constant stream of fresh material passing along the alimentary canal, so that a considerable portion may reach the colon undigested and unabsorbed. It is especially desirable that a sufficient amount of milk sugar should reach the colon unabsorbed to maintain in the colon a state of acid fermentation, thereby preventing putrefaction and changing the intestinal flora. This is, indeed, in many cases the chief benefit derived from the milk diet. An important fact pointed out by Magnus-Levy is that "the absorption of the various forms of sugar in the intestine proceeds with unequal velocity. This observation, which has been made repeatedly on animals, Weinland has found to hold good for man (Nagano); maltose and dextrose disappeared completely in one hour, whereas only 26 per cent. of milk sugar, present originally in equal quantity and in the same concentration as the maltose and dextrose, was absorbed during the same period."

To encourage the acid fermentation in the colon it is well to give at each alternate feeding acidophilus buttermilk in place of sweet milk; or equal parts of sweet milk and milk soured by the $B$. acidophilus may be taken at each feeding.

Milk ferments but does not putrefy. This fact has been established by Hirschler, Rovighi, Schmitz, Eisenstadt and many other observers. The fact that milk also "arrests or diminishes intestinal putrefaction accounts for its absence in milk diet" (Czerny). As a matter of fact, however, milk may encourage putrefaction if constipation coexists. This is due to the fact that undigested curds remain in the colon after the sugar of milk has been wholly absorbed, so that its protective influence in supporting acid fermentation is lost. This makes bran or agar and paraffin oil an essential part of the milk regimen to insure frequent and efficient action of the colon.

Feedings begin at 7:00 A. M. and end at 7:30 P. M.-twentysix feedings in all. At 10:00 A. M. and 4:00 P. M. the milk is omitted and a meal of fruits is taken instead. The purpose of this is to encourage bowel activity, since very free and frequent bowel movement is essential to success. The disappointing results often encountered in the use of the milk diet are chiefly due to the constipation which is likely to be produced in many persons, the natural result of which is intestinal toxemia and an aggrava. tion of the very symptoms for which relief is sought. 
Two ounces of sterilized wheat bran or an ounce and a half of agar-agar should be taken in half ounce doses, in connection with several of the milk feedings, preferably at 7:00 A. M., 11:00 A. M., 3:00 P. M. and 7:00 P. M.

In cases in which the colon is badly crippled-that is, when there is obstinate constipation-the use of the Russian paraffin oil in doses of one to three tablespoonfuls four times a day is necessary to secure the activity required for a rapid and efficient change of the flora.

When the period of exclusive milk feeding is ended, a laxative and strictly antitoxic diet must be adopted.

It should be remembered that the chief advantages of the milk regimen as a means of changing the intestinal flora lie (1) in the large amount of milk sugar that by this means is carried into the colon and there, fermenting, produces lactic acid and so prevents the growth of the putrefactive bacteria; (2) in the frequent bowel actions induced. The soft curds, undigested and unabsorbed, by their bulk as well as by their acidity, stimulate peristalsis and cause several bowel movements daily.

The bowels should move four or five times a day, the enema being used if necessary. No laxatives other than bran or agar and paraffin should be used. If proper bowel action cannot be secured by these means, the milk regimen must be suspended at once.

If the patient is emaciated, so that a gain in flesh is one of the objects of treatment, complete rest should be maintained during the first week-not necessarily in bed, but on a cot, or in a wheel-chair a part of the time if more agreeable.

During the resting period the patient should take a few deep breaths four or five times every hour when awake, to counteract the ill effects of lying in bed. The position should be changed occasionally. When lying on the back, a cushion should be placed at the small of the back. Sand bags are useful as props.

The patient must spend every possible moment out of doors, day and night.

\section{Amount of Milk to be Taken.}

The amount of milk taken daily must depend upon the capacity of the patient. Much more is necessary than actually needed for nourishment. The ratio to body weight is about one and one- 
half ounces of milk to each pound of body weight. The following table shows the aggregate amounts for different sized feedings :

\begin{tabular}{|c|c|c|}
\hline \multirow{2}{*}{\multicolumn{2}{|c|}{ At each feeding }} & Total \\
\hline & & 6 pints \\
\hline $41 / 2$ & “ & $63 / 4 \quad \cdots$ \\
\hline 5 & “ & $71 / 2$ \\
\hline $51 / 2$ & “ & $81 / 4$ \\
\hline & $"$ & 9 \\
\hline $61 / 2$ & unces & $93 / 4$ pints \\
\hline 7 & “ & $10^{1 / 2}$ " \\
\hline $71 / 2$ & “ & $111 / 4$ \\
\hline 8 & “ & 12 \\
\hline
\end{tabular}

The total food value in calories of the full regimen ( 6 quarts) is not far from 3600 calories, of which nearly one-half is fat, one-fourth casein and one-fourth milk sugar; that is, 1,700 calories of fat, 830 of protein and 1,100 of carbohydrates.

The fat and milk sugar are more completely utilized than the casein. The body can store up almost any amount of sugar and fat in the form of adipose tissue and glycogen, but only a very limited amount of protein. This is especially true while the patient remains in a state of rest. Protein cannot be retained unless it is utilized by entering into the composition of the living tissues of the body - the glands, nerves, blood, and muscles. Not more than 200 or 300 calories per day can be used for this purpose, so that several hundred calories of casein must be lost in the stools. The loss is not complete, however, for the soft, slightly acid curds have a highly beneficial effect upon the diseased surfaces of the colon, suppressing the growth of pernicious bacteria and gathering them up and sweeping them away by stimulating intestinal activity.

In the exceptional cases in which a larger amount of milk is needed, an extra quart may be added by giving feedings at 8:00, $8: 30,9: 00$ and 9:30 P. M. These extra feeding hours may also be utilized to make up the full amount needed in cases in which the patient is not able to take so much as 8 ounces at each feeding.

In very exceptional cases feedings may be given at midnight, 2:00 A. M., 4:00 A. M. and 6:00 A. M. It is better, however, in general to allow the stomach an opportunity to rest during the 
night. Within about eight hours after the last feeding the stomach and small intestine should be empty of food, thus giving the digestive tube and its secreting glands an opportunity for complete rest during five or six hours. Glands, like muscles, need rest to enable them to replenish their stores of energy.

One of the chief benefits derived from the milk regimen is the change of flora which is produced by it. This requires the ingestion of 8 to 10 ounces of milk sugar. When the full six quarts of milk are taken this amount of lactose will be assured. In many cases the addition of 3 or 4 ounces of milk sugar or lactodextrin is an advantage, and may be added in proportion as the amount of milk is diminished.

\section{How the Milk Regimen Differs from the Milk Diet.}

There are several important particulars in which the milk regimen differs from the milk diet, or ordinary milk feeding, and these points are so important that we shall give brief attention to each one, and endeavor to make clear why the milk regimen may be expected to succeed in cases where the so-called milk diet has failed.

Cases are, indeed, very rare in which the milk regimen can not be employed with success, even though the patient's former experience may have been such as to justify the expectation of failure. About the only cases, in fact, in which the milk regimen is positively contra-indicated are those in which the individual has been sensitized to milk protein.

The milk regimen differs from ordinary milk feeding, especially in the following particulars:

1. It is a systematized method. In ordinary milk feeding, it is a common practice with physicians to say to the patient: "Take all the milk you can at meals and between meals." That is, the milk is simply added to the ordinary bill of fare. This method occasionally succeeds fairly well in securing a gain in weight when this is the chief object sought, but not infrequently, perhaps in a majority of cases, the patient soon finds his digestion upset. The appetite disappears, the bowels become inactive, the tongue coated, and pronounced symptoms of intestinal toxemia appear.

When taken in this desultory manner, milk is likely to promote rather than combat putrefaction in the colon. In cases in 
which a normal or excessive amount of free hydrochloric acid is produced by the stomach, large tough curds are likely to be formed which, finding their way into the colon, furnish a favorable medium for the development of the putrefactive organisms that abound in this part of the alimentary canal. This is particularly apt to occur if the milk is taken between meals, for then it enters the stomach at a time when a large amount of highly acid gastric juice is present, a condition favorable to the production of large indigestible curds.

Intestinal putrefaction increases the constipation and resulting toxemia that destroy the appetite and give rise to malaise, headache, and other distressing symptoms. Thousands of persons have learned from experience that milk, when taken in connection with other foods, produces the disagreeable symptoms here mentioned; but when taken in sufficient quantity and as an exclusive article of diet, these symptoms do not appear, for the following reasons:

(a) The quantity of milk taken is so great that the lime and casein present neutralize the acid of the gastric juice and diminish the intensity of the action of the rennin, so that the curds formed are soft and friable and easily broken up.

(b) When milk is taken in connection with an ordinary diet, the quantity is so small and its movements along the intestine so slow that the milk sugar present is all absorbed before it reaches the colon. Thus the undigested curds present are not protected from the action of putrefactive bacteria, but are left to undergo decomposition. When taken in the quantity prescribed by the milk regimen, the whole alimentary canal is flushed with milk, in which the lactic acid fermentation begins before the colon is reached; and the movement toward the colon is so rapid that a considerable portion of the milk sugar taken reaches the colon. The twelve pounds of milk usually taken by the patient on a milk regimen contain about half a pound of milk sugar. A suffcient amount of this will reach the colon to maintain acid fermentation by which the growth of the putrefactive organisms is prevented.

Bienstock, Tissier, and Martelly have shown that when sugar is present to the extent of 1 per cent. putrefaction and toxemia are prevented. Careful chemical analysis of the stools of patients taking the milk regimen shows the presence of sugar to the extent of 1 per cent. In a case in which a fistulous opening existed in 
the intestine just above the ileocecal valve the amount of lactose found present in the intestinal contents while the patient was taking the milk regimen was more than 2 per cent. That is, the food residues of the small intestine which were just ready to enter the colon contained an amount of sugar amply sufficient to protect the contents of the colon against the action of putrefactive bacteria.

2. The frequency with which the milk is administered plays a highly important part in the success of the milk regimen. Each time the milk is taken a new impetus is given to the peristaltic activity of the stomach and the intestine. Fresh food entering the stomach sets up active peristalsis the whole length of the alimentary canal. This serves an important purpose in getting a sufficient quantity of lactose into the colon to support the active growth of acid-forming bacteria. Carmine given at the seven o'clock feeding to a patient on the milk regimen will usually make its appearance in the stool within 14 hours, and may disappear within 25 hours.

3. Peristaltic activity is increased with the milk regimen and decreased with the ordinary milk diet. When used in the ordinary way, milk is generally found to be constipating. Some persons note laxative effects from the use of buttermilk, although this is not uniformly the case. Boiled milk is almost universally regarded as constipating. There are two reasons for this :

(1) When milk is well digested, no residue is left behind to stimulate peristaltic activity.

(2) If it is not well digested, the putrefaction of the curds that pass into the colon will cause the production of ammonia and other putrefaction products, the effect of which is to paralyze the colon and produce constipation.

The older writers on the milk diet call attention to the fact that patients taking the milk cure are constipated, and even refer to the constipation as a good indication. Some modern advocates of the milk diet have expressed the same view. One writer even goes so far as to recommend that the "call" for evacuation of the bowels should be resisted and postponed so as to secure a large movement the next day! Nothing could be more absurd. The bowels should always move three times a day, and a person on the milk regimen should have at least four bowel movements daily. A steady stream of sour milk must pour along the intestinal canal. In this way the old flora or meat bacilli (Herter) and 
other "wild" bacteria, may be driven out and the new protective, acid-forming flora may be established.

The employment of special means for increasing intestinal activity has been found to be highly advantageous. It is for this reason that bran or agar-agar, with white refined paraffin oil in some form, should be employed in sufficient quantity to insure vigorous peristaltic activity. In the exceptional cases in which the bowels move too frequently the quantity of milk must be lessened.

4. It has been found decidedly advantageous to allow the patient two meals of fresh fruit, one in the forenoon, the other in the afternoon-at 10:00 A. M. and 4:00 P. M. The usual glass of milk is omitted and the patient is allowed to take, instead, fruit of any kind for which he may have a fancy. Fresh fruits or dates are, however, preferable, particularly strawberries and juicy fruits of all sorts. Great care must be taken to masticate the fruit very thoroughly. Lettuce and spinach may be added to the fruit meal without any disadvantage in most cases and with very great advantage in some. These foods require almost no digestive activity, while they supply vegetable acids together with carbohydrates, which aid in establishing the acid-forming bacteria in the colon, as well as vitamins, iron and various salts that promote nutrition. The alkaline salts furnished by fruits are particularly valuable to combat the slight tendency of the milk regimen to disturb the equilibrium of the body fluids as regards the exact balance of acids and bases.

In cases of anemia, the addition of purées of spinach or other greens is important to supply food iron for the blood.

\section{Practical Suggestions.}

When a gain in flesh is one of the chief objects desired to be obtained by the use of the milk regimen, the patient should take very little or no exercise during the first week. It is not necessary, however, that he stay in bed, as some have insisted, but he must avoid active exercise. Even moderate exercise may increase metabolism from 25 to 50 per cent. The energy thus consumed in muscular activity diverts a definite amount of nutritive material which has been digested and absorbed, and consumes instead of storing it. Generally, the patient may read, chat and occupy himself, and may be entertained with music but should avoid excitement and should sleep as much as possible. 
Care should be taken to see that while the patient lies in bed the trunk is properly supported by a firm cushion placed at the small of the back. When this precaution is neglected, patients lying long in bed often suffer from backache due to lack of back support, and sometimes sublaxation, or strain, of the sacro-iliac synchrondrosis, the joint formed by the junction of the sacrum and the lateral bones of the pelvis.

It is also wise to lie with the body inclined toward the right side. This position facilitates emptying of the stomach, especially when it is dilated or prolapsed; but if nausea is present, the patient should lie upon the left side, to prevent drag upon the cardiac orifice (Brunton).

Sand bags weighing ten to twenty pounds are of great service in supporting the patient in a position of complete relaxation and rest. More than half the internal work of the body is due to muscle tension. Lying in bed is often far from being the complete rest that one naturally conceives it to be. The evidence of this is seen in the fact that the patient often becomes uncomfortable and requires frequent change of position. The chief cause of discomfort is undue muscle tension, due to unconscious muscular effort in mantaining the body in position. That this is considerable is shown by the instant relief and sense of comfort experienced when sand bags or other suitable props are properly applied.

The tendency to slip down in bed, if the head and shoulders are raised even a little, is unconsciously antagonized by involuntary muscular action involving waste of energy. This may be easily prevented by a twenty pound sand bag placed against the buttocks.

Absorption of the digested food material from the intestine may be promoted by application of pressure upon the abdomen. In the majority of cases in which the milk regimen is employed the patient will be found to have very relaxed abdominal muscles, and in consequence the intra-abdominal pressure is much diminished if not altogether absent. This greatly hinders the absorption of the digested foodstuffs. By the application of a sand bag or a shot compress to the abdomen the necessary pressure may be secured and absorption thus greatly facilitated. Or, instead of applying weight to the abdomen the patient may obtain essentially the same result by lying upon the face, so that the abdomen will 
be compressed by the weight of the body. By lying over a cushion or a pillow the effect of the prone position may be greatly increased.

The care of the mouth is important. It should be well cleansed by rinsing with water after each feeding, and the teeth brushed and polished, preferably with the paper polisher (Hygos).

\section{Results of the Milk Regimen.}

The tongue becomes clean, the breath sweet, the stools are no longer offensive, and usually have a yellow color and little odor or a slightly sour odor. This change is generally observed within a week or ten days, and even sooner when the bowels move very freely. The skin clears, the blood improves, the patient gains in flesh, often a pound a day, and the old depression, headache, mental dullness and other miseries are replaced by a sense of energy and well-being. The transformation of the patient from a poor, emaciated, despairing invalid to a plump, rosy cheeked, bright, forceful person is often so rapid as to seem almost miraculous.

This rejuvenating process sometimes continues for two or three months, if the regimen is faithfully carried out and modified to meet the patients changing needs. A gain of 25 to 30 pounds in six or eight weeks is not uncommon. Sometimes a gain of 40 pounds is made in as many days. This is easily accounted for by the large amount of lactose in the ration. One ounce of sugar absorbed in addition to a full ration may cause an increase of flesh amounting to four ounces. Fats only add their own weight.

\section{SUPPLEMENTARY FEEDING}

While, in general, eating between meals is to be discredited and condemned, supplementary feedings at stated times between regular meals are sometimes useful, particularly in cases in which the amount of food which can be taken at meals is so small that the required number of calories cannot be taken at three meals, and in cases in which it is desirable to increase the food intake to the maximum limit. Supplementary feeding is also found advantageous in cases in which the patient's stomach empties too rapidly, giving occasion for the development of annoying hunger pains. In all cases when supplementary feedings are employed, care 
should be taken to make sure that the food taken both at the regular meals and the supplementary meals is of such a character that it will pass out of the stomach quickly-say within an hour or two. To insure this it must be taken in the form of purées. It should be well chewed, whether mastication seems necessary or not, and should contain little fat. Fats pass from the stomach very slowly and on this account are not suited to supplementary feeding.

Among the foodstuffs best adapted to supplementary feeding are juicy fruits and fruit juices. The list of fruits should not include, however, such substantial foods as dates, figs, raisins or bananas. Peaches, apricots, plums, apples, pears, melons of all sorts (rejecting the pulp) may be taken at any time when the stomach is not already filled with food. Such foodstuffs require practically no work on the part of the digestive organs. Almost their sole nutritive constituents are acids and sugars. These are ready for immediate absorption and furnish to the heart and other muscular structures material ready to be utilized at once. Malt sugar also requires little digestive work to prepare it for immediate assimilation.

Milk sugar may also be employed for supplementary feeding with advantage in cases in which it is desirable to change the intestinal flora.

Whey is another supplementary food of value. Rice jelly and agar jelly prepared with fruit juices, also fruit ices, may be employed in this way. Ices and all cool foods should be eaten very slowly, so as to avoid disturbing digestion by the chilling of the stomach.

It is useful to bear in mind that the food value of an ounce of sugar is about 100 calories. An ounce of dry starch or flour has practically the same value. An ounce of grape juice has a caloric value of one-fourth as much, an ounce of whey 8 calories, an ounce of tomato broth 3 calories.

By means of supplementary feedings, especially by the use of malt sugar and milk sugar, it is easy to introduce 800 or even 1,000 calories of food in addition to the regular meals, thus securing a rapid gain in flesh.

Vegetable broths, such as savita broth, bean broth, tomato broth and a broth prepared from the unripe soy bean (Hahto or 
Easy Cook), are excellent for supplementary feedings when the desire is to increase the supply of vitamins and organic salts. When taken with the regular meals these bulky liquid foods fill up the stomach and prevent the patient from taking the needed quantity of proteins, fats and carbohydrates. Eggnog, beef juice, beef tea, animal broths, bread and milk, bread and butter, sandwiches, cake, pie, and similar foods, and all solid foodstuffs are wholly unsuited for supplementary feedings.

\section{METHOD OF CHANGING THE INTESTINAL FLORA}

The intestinal flora is the bacterial vegetation of the intestine, just as the flora of a country consists of the plants-trees, shrubs, weeds, flowers, mosses and other growths which its soil produces.

\section{Why the Intestinal Flora Needs to be Changed.}

When a physician is called to attend a young infant, one of his first inquiries is respecting the character of the stools. The well-trained nurse always has the infant's napkin ready for exhibition when the doctor is expected.

A normal infant's stool is yellow in color, soft in consistency, and nearly odorless or perhaps of a slightly sour odor.

If the stool is dark in color and putrid, ammonical, rancid, or otherwise foul smelling, no other evidence is needed that the child is ill and suffering from the absorption from the colon of putrefactive products, the result of intestinal stasis.

Careful examination of the stools is as important in the treatment of adults as of infants. The significance of a dark colored, putrid, strong-smelling stool is essentially the same whether the patient is an adult or an infant. Such a stool means colonic stasis or delay in the colon and the putrefaction of the colon contents, with the absorption of the products of putrefaction and resulting toxemia. All physiologists agree that, normally, there is no putrefaction in the human intestine. Only when delay occurs is there opportunity for putrefaction. Putrefaction is, for the most part, due to the development of spore-forming organisms which require an incubation period, so that twenty-four hours or more are required for the development of active putrefaction in a freshly inoculated medium.

The food leaves the stomach in about four hours after it is eaten, and in four hours more the food residues have completely 
passed out of the small intestine and have reached the transverse colon; the process of digestion is completed and the utilizable portions have been absorbed. There is nothing left but unusable residues and excretory products and body wastes which ought to be dismissed from the body in six or eight hours more, or in less than twenty-four hours from the time the food was eaten. This is what takes place when the normal intestinal rhythm is maintained; that is, when three complete bowel movements occur daily.

When the bowels move but once a day, the motility period of the alimentary canal is fifty to fifty-four hours, often longer. In quite a large percentage of cases the stasis is not confined to the colon. Because of incompetency of the ileocecal valve, the stasis may extend to the small intestine and even the stomach.

Savages and the big apes move their bowels three times a day and escape intestinal toxemia; but among civilized people a faulty diet, lack of exercise, bad postures, neglect to attend promptly and regularly to the evacuation of the bowels, the use of laxative drugs, mineral waters and other disturbing influences and conditions connected with modern life, have made constipation almost universal, and as a result chronic intestinal toxemia and a host of chronic ills growing out of this condition including premature senility, have likewise become nearly universal.

As a natural consequence of chronic stasis, the flora of the adult colon is entirely different from that of the infant. The flora of the healthy nursing infant consists mostly of acid-forming organisms, especially the $B$. bifidus and the $B$. acidophilus as pointed out by Dr. Tissier of the Pasteur Institute many years ago.

The flora of the average adult living on a mixed diet is largely made up of putrefactive organisms, the $B$. Welchii, B. putrificus, with $B$. coli and streptococci. The same sort of flora, as Torrey and Distaso have pointed out, is found in the dog and for the reason that the diets of the $\operatorname{dog}$ and of the average man are much alike.

The suppression of intestinal putrefactions by means of antiseptic drugs was offered as the solution of the problem of intestinal asepsis soon after Bouchard's great work appeared and various agents, chiefly coal tar products, were for some years widely used; but the results were most disappointing. Laboratory tests showed the utter futility of this method. The admin- 
istration of B-napthol for several days failed to lessen the number of bacteria (Stern). But complete sterilization of the intestine by chemical means would be harmful rather than beneficial for the reason that it destroys the protective flora as well as the putrefactive.

Poehl, of Petrograd, was the first to call attention to the remarkable influence of diet upon the intestinal flora. He noted that a diet of buttermilk quickly caused the disappearance of putrid stools in children.

Tissier later called attention to the antagonism between lacticacid-forming organisms and bacteria which produce putrefaction.

Bienstock showed that the $B$. coli produces harmless acids instead of toxins when abundantly supplied with carbohydrates.

Sittler showed some years ago that when infants were fed maltose and dextrin in the form of malt soup B. acidophilus appeared in the stools in abundance.

The notable work of Herter and Kendall, published in 1910, and the more recent work of Torrey, Rettger and others, have clearly demonstrated the readiness with which the intestinal flora may be changed by controlling the diet. Indeed, experiments by Metchnikoff and extensive clinical observations by Combe had previously established the same fact.

The writer became interested in the question of suppressing intestinal putrefactions nearly thirty years ago, when Metchnikoff first called attention to the pernicious influence of colon bacteria upon health and longevity. Beginning some twenty-eight years ago, the author employed with a considerable measure of success, cultures prepared from sterilized milk with various lacticacid-forming organisms obtained for the purpose from Professor Conn, of the Connecticut Agricultural Experiment Station.

Some twenty years ago, the idea occurred to the writer that if buttermilk rendered service when taken in at the upper end of the alimentary canal it ought to render still greater service if applied directly to the colon, where its services are most required. The method was found somewhat inconvenient, however, on account of the enormous quantities of gas sometimes produced in the colon, which gave the patient much distress.

Some sixteen years ago (1911), opportunity arose to spend a few weeks in the laboratory of Dr. Distaso, at the Royal Institute of Public Health, London. Dr. Distaso was at this time making a 
study of the intestinal flora in persons upon whom Sir Arbuthnot Lane had operated for short-circuiting or removing the colon. At the same time there was an opportunity to observe the operative work of Lane and the immediate and remote results, as seen in his clinic. The writer became convinced that for a thoroughgoing change of the intestinal flora, it was necessary not only to implant protective cultures in large quantities, but to make a radical and permanent change in the dietary, eliminating foodstuffs calculated to promote putrefaction; and lastly, perhaps most important of all, to increase the motility of the intestinal tract so as to dispose of the food residues and body wastes so rapidly as to leave no time for the development of putrefactive changes.

This led to the establishment of a plan of treatment in which these three factors were combined, namely:

1. A radical change of diet, eliminating putrescible material and supplying carbohydrates in a form suitable to reach the colon (raw starch, dextrin and later lactose).

2. Increased intestinal activity, so as to secure three bowel movements a day by the combined use of bran or agar and mineral oil.

3. Flushing the alimentary canal with an active whey culture of acid-forming organisms (B. bifidus and later acidophilus), by the administration by mouth of a half pint to a pint of whey culture, and the injection of a similar amount of culture into the colon after administering a warm enema.

At once more definite and reliable results were obtained than before. This method has now been extensively used at the Battle Creek Sanitarium for ten years, and has been employed in thousands of cases with excellent success.

The intestinal flora may be favorably influenced by any one of these three measures, but for definite and rapid change of the flora all three of the methods must be employed simultaneously. We will consider each of the three distinct methods.

\section{The Low Protein Diet.}

It is a matter of common observation that carbohydrates (starches and sugars) ferment, while proteins putrefy. Although both changes are due to bacterial action, there is a most important difference between the classes of microorganisms involved in the two processes. The organisms that give rise to fermentation pro- 
duce acids-chiefly lactic or acetic-that are harmful only in very large quantities. In the minute quantities in which they can be produced in the intestine they are usually entirely harmless. On the other hand, the microbes that give rise to putrefaction produce deadly poisons and toxins which are highly injurious in very minute quantities.

Bacteria, like other plants, require soils favorable for their development. It is evident that the most effective way of suppressing the growth of poison-forming organisms in the intestine is to reduce to a minimum the amount of protein in the diet.

The marked difference in the character of the stools of a vegetable-eating animal like the sheep and those of a carnivorous animal like the dog is clear evidence of the influence of a high protein diet in promoting putrefactive changes in the intestine. The same difference is observed between the stools of a flesheating man and those of a flesh-abstainer. When meat and eggs are eaten freely, considerable of the proteins escape digestion, find their way into the colon and there undergo putrefactive changes. If, on the other hand, the protein of the diet is reduced to a minimum, so that the protein residues that reach the colon are small in quantity, the putrefactive changes are slight and the number of bacteria, with their toxins and endotoxins, is notably lessened.

By a change of diet it is not only possible to change the bacterial vegetation of the intestine, but also to change the action and the nature and influence of the colon bacillus and its congeners so that they no longer produce pernicious effects, but actually become a means of protection and defense. In other words, the wild bacteria that in various ways, as already pointed out, find their way into the human intestine and give rise to putrefaction and other bacterial changes whereby poisons in large quantities are produced may be either eliminated or changed to harmless acid formers. The researches of Bienstock and Kendall have shown beyond question that these poison-forming bacteria cease to produce poisons when they are adequately supplied with carbohydrates of a kind that they can utilize. Kendall found that even most virulent disease-producing bacteria, like the diphtheria bacillus, in the presence of sugar cease to produce toxins. The same is found to be true of the colon bacillus and of other pernicious organisms that grow in the human intestines.

How to get the required amount of carbohydrates into the 
colon is the problem. Cane or malt sugar and cooked starch are so quickly digested and so completely absorbed in the small intestine that it is by no means easy to get any amount of them into the colon. Raw starch digests less quickly, and milk sugar is much more slowly absorbed than other sugars. Practically only three carbohydrates can be made to reach the colon without undergoing digestion and absorption-raw starch, dextrin and milk sugar. Raw starch digests so slowly that when taken freely considerable quantities will reach the colon and there be acted upon by amylolytic bacteria which are always present; sugar is thus produced, supplying to the colon bacillus and other harmful bacteria the material necessary for changing the action of these organisms, so that they become protective instead of destructive, through the harmless acids which they produce.

Sugar of milk in considerable quantities ( 3 ounces three times a day) in connection with means for stimulating intestinal activity may also be made to reach the colon because of the great slowness with which it is digested and absorbed in adults. Milk sugar remains much longer in the alimentary canal than does cane sugar. By means of the milk regimen, it is possible to introduce lactose or milk sugar into the colon in almost any desired quantity. Milk sugar and dextrin may be introduced directly into the colon.

When the colon is thus supplied with carbohydrates, fermentation takes the place of putrefaction and the acids produced act as normal stimulants to the colon, encouraging its frequent and normal emptying.

The milk regimen usually changes the intestinal flora, which is, in many cases, no doubt, the chief benefit derived from its use.

Combe, a recognized authority in the treatment of autointoxication, more than twenty years ago laid down the following among other rules in relation to diet.

Nitrogenous foods: 1 . Diminish as much as possible the nitrogenous foods from which the intestinal bacteria derive their nourishment. 2. Absolutely prohibit those nitrogenous foods (meats) that are favorable media for the development of bacteria and form veritable culture bouillons. 3. Select eggs as being less putrefactive in the intestine.

Fatty foods: 1. Avoid the fats of meat for they increase putrefaction. 2. Give fresh butter in preference to meat fat, for its action is much less harmful.

Farinaceous foods: 1. Introduce into the diet as large a propor- 
tion of farinaceous substances as possible. But to obtain a result it is necessary to saturate the intestine with carbohydrates. The word "saturate" is used because it is not sufficient simply to add a few farinaceous substances to the diet. The patient must be filled with farinaceous aliments. 2. In autointoxication from acute enteritis, or in the acute recurrences, an exclusive farinaceous diet must be maintained for several days, for it plays the same part in acute enteritis that a liquid diet does in acute catarrh.

Chalmers Watson requires that meat shall be excluded from the diet in cases of autointoxication, as do most present day writers on dietetics. If this counsel were followed by all suffering from autointoxication in any degree, it would lead to the disuse of meat, for autointoxication is an almost universal disease in civilized communities. It is for this reason, perhaps, that its full significance as a cause of disease is so often overlooked.

Wegele places first among the list of causes of autointoxication the use of meat; that is, a diet which encourages putrefaction in the intestine, both by the introduction of material which readily undergoes putrefaction and with it $B$. Welchii and other germs which give rise to putrefaction.

Tissier of the Pasteur Institute requires his patients to adhere closely to a strict non-flesh dietary. Writing the author in 1911, Professor Tissier said:

You know that all our efforts have been in the direction of preventing intestinal putrefaction, the predisposing cause of intestinal infection, and sometimes the determining cause of digestive and nervous disorders. To prevent intestinal putrefaction, we introduce as much sugar as possible into the intestine, and certain microbes ferment these sugars and thus produce acids. The acid produces arrest of the bacteria of putrefaction. But sugar is rapidly absorbed by the mucous membrane of the stomach and intestines. It does not reach the large intestine. The sugar that goes the farthest is that of the date, which can be recovered in the cecum. Of all the hydro-carbons, starch alone arrives in the large intestine, where it is decomposed and absorbed by the mucous membrane. We may increase the quantity of the sugar produced, and thanks to the ferments which produce acids from sugar, for instance the bifidus and lactic-forming bacilli, putrefaction may be arrested more quickly.

These views have been abundantly verified by clinical experience, and are fully sustained by recent researches, conducted by Drs. Torrey, Rettger and others, as well as by the writer.

In an extended series of bacteriological studies of the stools of dogs under various conditions by Torrey, the results of which 
were published in the Journal of Medical Research, it was shown that lactose and dextrin have a very marked effect in modifying the character of the intestinal flora. When lactose or dextrin were given in sufficient amount, putrefactive bacteria almost entirely disappeared, the lactic acid-forming bacteria, particularly the $B$. acidophilus, taking their place. Similar but less marked results were obtained by the use of cane sugar.

Better results were obtained with boiled milk than with unboiled. With the latter the $B$. coli predominated. Potatoes, bread; and beans, when freely used, tended to lessen the putrefactive bacteria, while meats "markedly encouraged the growth and activity of putrefactive bacteria." The casein of milk produced less tendency to putrefaction than did meat. Vegetable proteins "did not offer the slightest encouragement to the growth of intestinal putrefactive types of bacteria."

\section{According to Rettger,}

Lactose, milk and mixed grains (wheat, oats, etc.) are specific articles of diet which exert an influence on the intestinal bacteria. Lactose, when fed in sufficient quantities ( 2 or 3 grams daily), brings about complete transformation of the flora of white rats within three or four days: milk requires a longer time, and does not bring about a complete change. Milk and lactose together form the most practical and effective diet, at least for man. Grain feeds tend to increase the number of aciduric bacteria, but their influence is comparatively small.

Meat or other high protein diet increases the indol-producing bacteria and other organisms of the so-called "putrefactive" type, like B. coli and B. Welchii.

The marked influence of a high lactose diet upon the intestinal flora of man, which was demonstrated in these experiments, has been confirmed by other investigators (Torrey) working with typhoid patients, who found that eating milk sugar ( 250 to 300 grams, 8 to 10 ounces, daily) brought about a marked change in the intestinal flora where the initial flora had been of a distinctly putrefactive type.

The above observations show beyond room for doubt the importance of supplying to the colon the carbohydrate necessary for promoting the growth of acid-forming bacteria. The only way in which this can be done is by proper regulation of the diet and increasing peristaltic activity. The regimen must supply forms of carbohydrate which will be slowly absorbed so that some of it may have a chance to reach the colon.

With the fruit regimen, 4 to 8 ozs. of lactose, or lacto-dextrin, are given daily, the amount found necessary for producing the 
change of the colon flora. The milk sugar is given dissolved in plain water, lemonade or fruit juice of some sort, in doses of three ounces, three times a day. A specially prepared lacto-dextrin is preferable for this purpose as the ordinary dextrin of the drug stores has a very unpleasant flavor and is difficult to take. Milk sugar in large amount causes nausea in some cases, while lactodextrin may be taken as easily as any breakfast food.

With the fruit regimen, also, the action of the intestine is hastened, and a sufficient amount of starch and fruit sugar reaches the colon to encourage the needed acid fermentation. In the after-diet, brose, or half-cooked cereals, supplies the raw starch needed to feed the sugar-forming and acid-forming bacteria and to secure normal bowel action and arrest of putrefaction in the colon and of chronic intestinal toxemia which results therefrom.

\section{Increased Intestinal Activity.}

The multiplication of bacteria in the intestine is more actively encouraged by stasis or stagnation of the intestinal contents than by any other factor aside from an excessive amount of protein in the dietary. A most important means of lessening the multiplication of bacteria in the intestine is acceleration of the movement of the food residues along the intestinal tract. Delay of the intestinal contents at any point along the twenty-seven feet of small and large intestine is quickly followed by the development of active bacterial changes. While active digestion is taking place, putrefaction and fermentation are restrained. When the several digestive cycles have been completed, fermentation or putrefaction becomes active - the nature of the process being determined by the dominant character of the food residues. If these are chiefly carbohydrates, fermentation results; if protein, putrefaction.

Normally, the stomach becomes empty in about four hours after the beginning of a meal and the small intestine discharges the last remainders of the meal into the large intestine four hours later. In other words, the process of digesting and absorbing a meal normally occupies about eight hours. Three or four hours later, in the case of a perfectly normal person, the undigested residues of foodstuffs, mixed with excretory substances derived from the liver and mucous membrane, may be discharged from the body. Under such circumstances, the opportunity for putrefactive changes is so slight that the bacteria developed are comparatively few, and the amount of poisons produced is accord- 
ingly small. In carnivorous animals, the time required for the transit of foodstuffs through the alimentary canal is much shorter. Results of experimental researches published by the United States Government show that the time elapsing between the taking of food and the discharge of the unused residues is, in the barnyard fowl, only three and one-half hours. In the fruit-eating bat of South America the time is only one hour, and the feces have the odor of bananas, upon which these bats feed. The alimentary tube of the sheep is twenty-six times the length of the animal's body and the time of transit is necessarily considerably longer. Metchnikoff many years ago showed by extended researches that those animals live longest that have the shortest colons. Some animals, such as the eagle, which live on an exclusively flesh diet, live to a great age because of the extreme shortness of their colons, which gives no opportunity for stasis and hence little chance for putrefactive changes.

It must be clearly understood, however, that by increased frequency of bowel movement is meant not simply one bowel movement a day, but at least three full bowel evacuations. In many cases, it is desirable that as many as four evacuations should be secured. Briefly stated, the reasons are these:

In healthy infants, the bowels usually move soon after feeding. The same is true of healthy animals and active, healthy boys and girls who are fed upon a natural diet.

The savage moves his bowels three times a day, as does also the Turk. Said a Bushman to a missionary doctor whom he consulted for relief: "Doctor, I am horribly constipated; my bowels move only once a day."

The chimpanzees and other big apes in zoological gardens move their bowels four to six times a day.

The colon must be thoroughly cleared of its residues daily. The use of bran, psyllium seed, lacto-dextrin and paraffin in sufficient quantities will accomplish this. The amount of bran and psyllium seed used should be increased until the results desired are secured.

To insure complete evacuation of the colon, an enema at bedtime (three or four pints, $110^{\circ}$ to $115^{\circ} \mathrm{F}$.), at least three times a week, is a wise precaution. In cases of extreme toxemia, it is well to repeat the enema daily for several weeks. 


\section{The Displacement of Harmful, Putrefactive Bacteria by Harmless Acid-Forming Organisms.}

According to Burnet, the idea of supplanting the putrefactive bacteria present in intestinal toxemia by beneficent acid-formers originated with Quincke.

By extensive experiments conducted under the supervision of the writer in the bacteriological laboratory of the Battle Creek Sanitarium, it has been demonstrated that when boiled or even slightly cooked starch is present to the extent of two per cent. the growth and activity of putrefactive organisms in the feces are controlled; acids are produced in place of ammonia, and fermentation predominates over putrefaction. The same is true when milk sugar, dextrin or lacto-dextrin, is taken in sufficient amount; that is, three ounces three times a day (Torrey).

The principal battle waged between beneficent and pernicious bacteria in the intestine is, according to Burnet, between $B$. $W$ elchii on the one hand and $B$. acidophilus and B. bifidus on the other.

Those bacteria that produce the largest amount of acids are most active in resisting the growth of putrefactive organisms. The $B$. bifidus and the $B$, acidophilus cease to grow only in solutions containing one per cent of acid, while $B$. Welchii is inhibited by an acid solution of the strength of one-sixth of one per cent.

Some 20 years ago (1907), the writer began an experiment for the purpose of testing the ability of lactic acid-forming organisms to suppress putrefaction. A pound of beefsteak was immersed in a two-quart jar of buttermilk. Although the flesh was slightly tainted when purchased, after a few days in the buttermilk it was found to be entirely free from taint. This experiment is still in progress (1927), the buttermilk being changed every week. The beefsteak remains absolutely free from any putrefactive change. Bacteriological examination shows no putrefactive bacteria present. The flesh has the appearance of fresh meat made a little paler than usual by the long continued maceration to which it has been subjected.

This method of preserving meat was practiced by the pioneer women of our western frontier, who often kept fresh meat for several weeks by immersing it in pans of sour milk. A gentleman who has traveled extensively in the East reports that a 
similar practice is also in vogue among the Arabs, who preserve their fresh meat in camel's milk.

The philosophy of this method is exceedingly simple. The $B$. acidophilus, introduced with the food, finds its way into the colon and there produces lactic acid and so develops a condition unfavorable to the growth of putrefactive bacteria, which do not thrive in an acid medium. Bienstock, Tissier and Martilly showed that in a medium containing one per cent of sugar, a putrefactive flora does not flourish, an aciduric flora taking its place. The $B$ bulgaricus, a buttermilk germ, so closely resembles the $B$. acidophilus of the intestine that it was for a long time mistaken for it and was recommended by Metchnikoff to change the flora, which we now know it will not do for it does not thrive in the intestine.

Two conditions are necessary for the success of this method:

1. The B. acidophilus shall exist in the colon in sufficient numbers to be able to produce enough lactic acid to acidify the colon's contents.

2. It is necessary that the $B$. acidophilus shall find in the colon the food necessary for its sustenance and by the aid of which alone it is able to produce lactic acid, viz., carbohydrate.

Unfortunately, both these conditions are somewhat difficult to secure. The B. acidophilus is likely to be destroyed by the digestive fluids on its way to the colon, while the sugar which is essential for its development is almost certain to be absorbed in the small intestine, so that the acid-forming bacilli speedily starve to death after reaching the colon, for lack of suitable nourishment.

It is necessary that large quantities of very active cultures should be taken and special measures must be adopted to supply to the $B$. acidophilus when in the colon the carbohydrate which it requires for efficient growth.

One way of accomplishing this is by the administration of freshly prepared acidophilus buttermilk in connection with the milk regimen. It may also be accomplished by giving fresh whey cultures of the $B$. acidophilus in massive quantities, a pint two or three times a day.

The $B$. acidophilus is always present in the intestine, but generally in such small numbers that it is overwhelmed by the putrefactive bacteria.

By the administration of three ounces of milk sugar or dextrin three times daily, the $B$. acidophilus will increase in 
numbers, and by the end of a week or ten days may be obtained from the stools in almost pure culture. The Welch's bacillus and other putrefactive and injurious organisms will be found to have disappeared. By adherence to an antitoxic dietary, an occasional return to the milk sugar feeding, or the constant use of a moderate amount (4-6 ounces) of lacto-dextrin daily, the putrefactive flora of the intestine may be held in abeyance and the colon kept free from putrefaction.

Fuld (Deut. Med. Woch) mentions a method which has been tried for changing the intestinal flora by injecting into the colon cultures of the colon bacillus. This method has been extensively exploited in this country, but cannot be regarded as other than irrational and utterly incapable of securing any definitely beneficial results,

The character of the intestinal flora is wholly determined by the nature of the residues left in the colon after digestion and absorption are completed. It is evident, then, that the character of the flora will change with the diet, and that the change of flora can be maintained only so long as the diet and other measures by which the change has been accomplished are maintained. When they are discontinued, the pernicious bacteria, that is, a putrefactive flora, reappears within a few days. The changes in the character of the flora may be easily followed by noting the odor of the stools. A non-putrefactive flora has little or no odor. Sometimes a faint acid odor is perceptible. There should be no trace of putrescence, An ammoniacal or rancid odor signifies putrescence. As the flora becomes changed for the worse, these offensive odors become pronounced. By noting the odor of the stool from day to day the state of the flora may be judged with considerable accuracy.

When the cecum is adherent, the descending colon spastic, the pelvic colon prolapsed and adherent or impacted, the change of flora is more difficult of accomplishment because of the delay of residues whereby opportunity is given for the absorption of the lactose, permitting the development of the putrefactive organisms. In such cases, the colon must be emptied thoroughly by an enema daily, or even twice a day. By a persevering effort and the use of larger quantities of lactose or dextrin ( 4 ounces three times daily) the change will finally be accomplished, although the time required may be sometimes so long as two or three weeks.

After the flora is changed, continue the same measures moder- 
ated to keep the stools frequent and free from putrid odor. An occasional laboratory test should be made when possible.

With lactose feeding, as with the milk regimen, there is not infrequently at first a very marked looseness of the bowels, due, possibly, to the temporary increased development of the Welch's bacillus. A lessening of the dose to one ounce three times a day will usually correct the looseness at once; then the smaller dose may be taken and as the flora is changed the tendency to diarrhea will disappear.

The first effect of large doses of dextrin or lactose is greatly to increase gas production. This inconvenience will disappear in a few days, as soon as the Welch's bacillus and the colon bacillus have been greatly reduced in numbers. The disappearance of putridity of the stools and of gas are an indication that the change of flora has taken place, and with this change the production of colon poisons and the mischiefs which they produced will have ceased.

The temporary inconvenience from gas formation may be relieved at once by means of a hot enema. The temperature should be $105^{\circ}$ to $120^{\circ} \mathrm{F}$, the quantity two quarts. The enema may be repeated two or three times a day for a day or two, if necessary. It is important to keep the colon as nearly empty as possible. The best time for taking the enema is just before retiring at night. The bowels should be trained to move three times a day. An enema at bedtime will be of benefit whenever the colon has failed to empty itself without assistance during the day. A sense of fullness or heaviness or other abdominal discomfort is usually an indication of stasis or retention of residues which can never be beneficial in any way and may be the source of great mischief, since putrescible residues in the colon undergo the same putrefactive changes as similar refuse elsewhere in the presence of warmth and moisture.

\section{Change of the Intestinal Flora Without the Use of Cultures.}

At the present time much interest is being manifested by the laity as well as by the profession in the change of the intestinal flora by means of cultures, numerous brands of which are being put upon the market and extensively sold. By far the great majority of people who have made use of these cultures have found the results very disappointing. It could not be otherwise, for it is as irrational to suppose that a spoonful or two of any 
culture administered two or three times a day could effect a change of the condition of an infected alimentary canal as to undertake to produce a grass covered front lawn with a teaspoonful of seed, or to disinfect a whole back yard with a teaspoonful of chloride of lime. Whatever the agent employed for changing the intestinal flora, it is necessary that the whole alimentary canal should be flooded with it, and this flooding must be continued for many days in succession if any degree of success is to be attained in ousting the pernicious parasitic bacteria which have for years held undisputed possession of the territory.

The parasitic bacteria are found not only upon the surface of the mucous membrane, but in its deeper layers. The bacteria sometimes even pass through the whole thickness of the intestinal wall and find their way into the bladder, kidneys and other organs and are hidden in innumerable folds and pockets of the bowel, in the appendix, the gall bladder, the bile passages and the ducts of the intestinal glands.

After long and thorough study of the subject and observations in many hundreds of cases, the writer has found that cultures are not only not indispensable for changing the intestinal flora, but that the change may be more certainly, definitely and permanently accomplished without cultures than by the use of cultures alone.

The bacillus acidophilus and other protective acid-forming crganisms are implanted by Nature in the intestine not only of human infants but of the young of all mammals within a few days after birth without the administration of cultures. The protective organisms are evidently very widely dispersed for they never fail to make their appearance in the stools of the nursing infant, the suckling calf or the milk-fed chicken. The droppings of milkfed chickens are, in fact, almost pure cultures of the B. acidophilus. Once introduced into the intestinal tract these organisms are never wholly lost although their numbers may be very greatly reduced, and even if they were lost Nature has an abundant supply ready at hand for adults as well as for infants when conditions are made favorable for their growth. It is evident, then, that all that is necessary to secure a change of the intestinal flora is an abundant supply of such nutrients as will encourage the growth in the colon of the protective flora and discourage the growth of the pernicious bacteria.

Combe and his associate, Amman, showed many years ago 
that the flora may be changed by flooding the intestine with carbohydrates. Distaso, Torrey, Rettger and others, have since called attention to the same fact. It is now well known, as pointed out by the British Medical Journal, which advises that "Before plunging into acidophilus culture therapy," by the use of cultures, "it should be remembered that $B$. acidophilus is a normal inhabitant of the human intestine. It is there for good or ill within every one of us, and the miracle of transforming the intestinal flora is actually nothing more than the making of this microbe sufficiently numerous for the bacteriologist to be able to distinguish it without too much effort."

Fresh meat placed in a solution of lacto-dextrin, or even ordinary sugar, may be preserved indefinitely without undergoing putrefactive changes.

Lactose and dextrin are the only carbohydrates that can be relied upon to encourage a luxuriant growth of $B$. acidophilus in the colon. These carbohydrates are absorbed much more slowly than others and are thus able to reach the colon before complete absorption. To make certain that a sufficient amount of lactodextrin reaches the colon to change the flora, that is, to encourage the growth of the B. acidophilus sufficiently to make it dominant and discourage the growth of the putrefactive organisms, it is necessary that large doses should be taken. Lactose alone has in practice been found to be objectionable for several reasons. First, it sometimes gives rise to nausea; second, it becomes after a few days repugnant to most persons; third, it is difficult to dissolve; fourth, and perhaps the most important of all, the use of such large quantities of sugar for a considerable period sometimes causes the appearance of sugar in the urine. This might prove to be highly injurious to a person already having a tendency to diabetes. Dextrin is equally as valuable as lactose for changing the flora, but its use for human beings is impracticable because its taste is so disagreeable it is practically impossible to swallow the quantity required. The objectionable features of both lactose and dextrin have been overcome by combining the two, not by simple mechanical mixture but by adding lactose to a specially prepared dextrin in such proportions as to produce a readily soluble, highly agreeable product which may be used without difficulty for an indefinite period of time. The combination of a colloid with the crystalloid lactose serves to protect the stomach from the wellknown irritating effects of sugars of all sorts in concentrated 
form. This product, known as lacto-dextrin, not only changes the intestinal flora but is a highly concentrated food, each ounce of it having a food value of about 120 calories.

For rapidly changing the intestinal flora it is necessary to administer large doses, sufficient to fill the alimentary tract, or otherwise the total amount taken may be absorbed before reaching the colon where its presence is necessary to encourage the growth of the $B$. acidophilus. This amount varies in different persons. On the average the required dose is about three ounces. This quantity must be taken three times a day. In exceptional cases four or five ounces may be required for a few days. These are cases in which the intestine is badly crippled and a long time is required for the lacto-dextrin to reach the colon so that nearly all is absorbed. In these cases large doses must be taken until the flora is changed, and afterwards doses half as large will generally suffice to maintain the change, that is, to prevent the return of the intestinal putrefaction and consequent autointoxication.

Lacto-dextrin may be used advantageously at any time, with meals as an addition to fruits, or between meals as a drink-it makes a very agreeable beverage. An excellent time for taking lacto-dextrin in ordinary cases is about one hour before each meal. It should be taken with a liberal quantity of water. A full dose requires about 12 to 16 ounces or two large tumblerfuls of water. The product is much more soluble in hot water than in cold, hence hot water is decidedly preferable. A convenient way for taking is to put about one-half the dose, three heaping dessert spoonfuls, in a tumbler, add an equal quantity of hot water, stir quickly with a fork till smooth, then add hot water to fill the tumbler. After swallowing this, prepare and take another similar dose. Repeat this before each meal.

Not infrequently the taking of a large dose of this concentrated food a short time before a meal lessens the appetite. This need not give alarm, for it is to be remembered that lacto-dextrin is a highly concentrated food. A heaping dessert spoonful represents about 100 calories, and the full day's dose represents fully half the amount of food required by the average individual engaged in light employment. Persons who are overfat or of average weight and hence do not desire to add to their weight must necessarily reduce the amount of food eaten while taking lacto-dextrin. Such persons should make the diet consist chiefly of coarse vegetables such as cabbage, cauliflower, green peas, 
string beans, lettuce, celery, brussels sprouts, asparagus and fresh fruits of all sorts with a moderate allowance of bread, potatoes, cereals, milk, buttermilk, etc. Persons who are thin in flesh and desire to add to their weight should take the lacto-dextrin about three hours after meals. When taken at this time lacto-dextrin does not lessen the appetite for other food and so may be taken in addition to the regular full diet and will thus insure a decided and usually a rapid gain in flesh.

\section{To Keep the Flora Changed.}

This is not a case in which the good Methodist doctrine of "Once in grace always in grace" is applicable. Even when the flora has been completely changed, if the measures employed to effect a change are completely withdrawn, the old putrefactive flora will be found back in full force within a few weeks. To keep the flora changed requires a constant battle.

The things which must be done without fail and systematically are the following:

1. The bowels must be thoroughly emptied every day. To insure this, the bowels should be trained to move three times a day or after each meal. In many cases an additional movement occurs before breakfast and this is advantageous. To secure this efficient action of the bowels the use of large quantities of bulky foods is necessary together with proper lubrication. An ounce to an ounce and a half of bran is required in obstinate cases and paraffin oil in liberal quantities is also needed. Fresh fruit, greens and such vegetables as lettuce, celery, cabbage, beets, parsnips, etc., should be freely used. Bulky or laxative foods must be used in liberal quantities, not once a day only, but at every meal. If one of the daily meals is taken away from home at a lunch counter, hotel or restaurant, a quantity of "ballast" may be taken along in the pocket. Laxa is especially excellent for this purpose. This is a combination of bran and agar, which is the most efficient form of roughage with which the writer is acquainted. It is prepared in the form of a biscuit which may be carried in the pocket and is quite palatable and easily eaten either with or without other food. If the ordinary meal is omitted, one or two laxa biscuit may be taken with a little fruit so as to avoid interrupting the normal intestinal rhythm.

2. An antitoxic diet must be closely followed, that is, meats of all sorts, including fowl, fish, etc., must be rigorously excluded 
from the diet. This is necessary not only for rapidly changing the intestinal flora, but after the flora has been changed to prevent reinfection of the intestine. A single chop or serving of fish or chicken may suffice to re-inoculate the intestine with the putrefactive flora and render necessary a new campaign to change the flora by means of large doses of lacto-dextrin. In some cases even eggs must be used very sparingly, the yolk of the egg in preference to the white, being less putrescible and less likely to encourage putrefaction in the colon. When eggs are eaten the greatest care should be taken to insure perfect freshness. In hot weather an egg will not remain fresh for more than two or three days, and ordinary commercial eggs are quite liable to contain bacteria even when perfectly fresh. Bacteriological examinations of eggs have shown that about one egg in seven is infected when laid. It is necessary that the food and surroundings of chickens should be thoroughly clean in order to insure that the eggs are wholly free from bacteria even when fresh. Eggs from milk-fed chickens are of the best sort.

3. Three or four ounces of lacto-dextrin should be used daily. In many cases a single morning dose of two to four heaping dessert spoonfuls is found to be efficient. This dose in many acts as a decided laxative. Changing the flora always increases the efficiency of the colon. If acidophilus buttermilk is used, about two pints should be taken daily to maintain a good intestinal flora. Perhaps the best plan of all is to combine the buttermilk and lacto-dextrin. When this is done only a half dose of each will be required, say two or three tablespoonfuls of lacto-dextrin daily and one or two glasses of buttermilk.

Change of the intestinal flora should not be neglected as a necessary measure in any attempt to combat constipation. This is true for the reason that putrefaction gives rise to ammonia and other alkaline products which paralyze the colon. That all alkalies have this effect is a well established physiologic fact, while it is equally well established that acids excite the colon to normal activity. The addition of the juice of one or two lemons to the water employed as an enema is one of the most efficient of all known means of stimulating the bowel to action when stimulation is required. When the flora has been changed, the normal acid-forming organisms, B. acidophilus and B. bifidus being dominant, acids are constantly formed in the colon which excite it to normal activity and tend to restore it to a normal condition. 


\section{When Is the Intestinal Flora Changed?}

The intestinal flora is changed when the stools are soft, frequent (three times a day), and free from putrid or rancid odor. Examination by a bacteriologist should show a luxuriant growth of $B$, acidophilus and the gram stain should give positive 80 and negative 20 (80-20), which means 80 per cent of the acid-formers and 20 per cent of other bacteria. In a bad flora, the percentage will be the reverse, 20-80. In some cases, a percentage of 5-95 is found. In healthy, nursing infants, the flora shows gram 80-20. The feces of milk-fed chickens give the same. Of course, a change from $20-80$ or worse to $40-60$ or $50-50$, is a decided improvement, but this should not be considered at all satisfactory. Old troubles may still continue, perhaps somewhat modified. But when the change reaches 75-25, marked improvement will be evident, and the more complete the change of flora, the more decided will be the change for the better in the patient's symptoms.

While the change is taking place, it is most desirable to have made, when possible, frequent bacteriological examinations so as to be able to note the progress being made. However, it is entirely possible to make a thorough-going change of the flora without the aid of the bacteriologist by carefully following the suggestions above made. By continuing the use of a laxative and antitoxic diet with daily use of lacto-dextrin, the flora may be kept changed and with such great profit as will amply repay all the expense and trouble involved. In considering the matter of expense, it should be remembered that the special carbohydrates used possess high food value, equaling in calories about twice their weight of ordinary beefsteak.

In attempting to change the intestinal flora it is of the utmost importance to insure quick transit of residues through the colon. The use of carmine as an indicator is most helpful. Ten grains taken at breakfast will color the stool red. This should be wholly removed by an enema at bedtime, repeated if necessary.

\section{Diseases in Which the Intestinal Flora must be Changed.}

Among the maladies in which the defensive mechanisms of the body are crippled may be mentioned especially the following: Achylia, gastric ulcer, cancer of the stomach, duodenal ulcer, inflammation of the gall bladder, gallstones, abscess of the liver, cirrhosis of the liver, pancreatitis, diabetes, exophthalmic goiter, toxic goiter, cystic goiter, myxedema, Addison's disease, Bright's 
disease, pyonephrosis, arteriosclerosis, jaundice, syphilis, multiple neuritis, intestinal toxemia, dementia praecox, manic depressive, toxic neurasthenia, eczema, acne, degenerative disorders of the nervous system, pernicious anemia, splenic anemia, cancer in any part of the body, chronic appendicitis, chronic bronchitis, bronchial asthma, cardiac asthma, migraine, or so-called sick headache, so-called biliousness or torpid liver, acidosis, apoplexy, osteo-arthritis or chronic rheumatism, chorea, colitis, chronic diarrhea, premature old age, sprue, pellagra, fever.

Sir Arbuthnot Lane enumerates the following symptoms and conditions as due to intestinal toxemia, in all of which the antitoxic diet is necessary:

Wrinkles, atrophy of the skin, pigmentation of the skin, browning of the eyelids, also the skin about the eyes, the axillae, the abdo. men, cyanosis, coldness of the hands and feet, sweating of the hands and feet, livid skin, roughness and dryness of the skin, wasting of the muscles, general muscular weakness, curvature of the spine, wasting of the abdominal muscles, prolapse of the viscera, weakness of the hieart, degeneration of the aorta, dilated heart, melancholia, imbecility, headache, symptoms resembling brain tumor, epilepsy, diseased conditions of the mammary gland-a nodulated condition, induration, cystic degeneration and later cancer-goiter, adenoids, enlarged tonsils-the result of lowered vital resistance-pyorrhea, endometritis, uterine cancer, various diseases of the eye and baldness. In certain persons increased growth of the fine hairs covering the general surface of the body. tuberculosis, rheumatoid arthritis.

In all the above conditions a change of flora is most positively indicated. It is not always possible to accomplish this easily, although in the majority of cases a well directed effort will show definite results within three or four days. The best means is the fruit regimen already described in preceding pages. This should be used for three to five days, after which the antitoxic regimen should be definitely and permanently adopted and scrupulously followed. A departure from a strict antitoxic diet may often be followed by relapse within a short time. It must be constantly borne in mind that disorders and symptoms such as above described do not appear until after some essential part of the defensive mechanism of the body has been broken down, so that dietetic digressions which produced no apparent effect when the body defenses were intact, even when indulged to an immoderate degree, become disastrous even when the departure from a strict antitoxic regimen is comparatively slight. It must be remembered, 
also, that when the results of intestinal toxemia have become well developed and have existed for a considerable length of time the damage to the defensive mechanism has become so serious that it is probably to a considerable degree irreparable, and for this reason relief from the existing disorders can only be secured by a thorough change of flora and the most exemplary following of a carefully arranged antitoxic regimen with no digressions.

When the kidneys have been badly damaged, as shown by the presence in the blood of an abnormal amount of non-protein nitrogen, the protein of the diet must be kept down to the very lowest limit and should never exceed one calorie per pound of body weight. In many cases the protein may be with benefit reduced to half this amount for periods of a few days at intervals, and in extreme cases it is desirable to eliminate the protein from the bill of fare entirely for short periods so as to make the work of the kidneys as light as possible.

In chronic cases of intestinal toxemia it is often necessary to repeat the special regimen for changing the flora (fruit regimen) at intervals of a week or two for some time in order to secure a radical change in the intestinal flora, and the fruit regimen with lactose and acidophilus cultures should be returned to whenever the putrid odor of the stools reappears.

\section{THE ANTITOXIC DIET}

(See also page 982)

According to Herodotus, who wrote in the fifth century, B. C., being one of the earliest of all historians, the early Egyptians recognized the importance of maintaining the activity of the colon. He says: "For three successive days in each month they purge, hunting after health with emetics and clysters (enemas), and they think that all the diseases which exist are produced in men by the food on which they live; for the Egyptians are from other causes also the most healthy of all men next after the Libyans."

The fact that the Egyptians recognized, as shown by the above paragraph, the pernicious influence of constipation as a cause of disease indicates the great advancement they had made in the knowledge of hygiene, as shown also by their numerous sanitary regulations, many of which were incorporated into the code of laws formulated by Moses for the Israelites while they were wandering in the wilderness. 
Bouchard taught us that the body is a factory of poisons. Besides those which it normally produces, the colon when infected produces a long list of disgusting putrefaction products which are absorbed and often overwhelm the poison-destroying and poison-eliminating mechanisms of the body to such a degree as to interfere seriously with the ordinary life processes. This condition is termed autointoxication or intestinal toxemia, or alimentary toxemia, and is the predisposing or exciting cause of most chronic and many acute maladies.

The antitoxic dietary has five essential characteristics.

1. It must be laxative so as to prevent stagnation in the colon, thus giving the residues and wastes no opportunity for putrefaction.

2. It must be free from putrefaction products and from the bacteria which produce putrefaction and the resulting poisonous products.

3. It should be poor in substances capable of undergoing putrefaction; that is, it must be low in protein.

4. It should be rich in carbohydrates which encourage the growth of protective bacteria in the intestine.

5. For the greatest efficiency it must include considerable raw'starch or other carbohydrates such as lactose or dextrin which are absorbed with sufficient slowness to permit them to reach the colon and thus furnish food for the protective bacteria which, by encouraging fermentation in the colon, prevent putrefactive changes, thus cutting off the source of the toxins which give rise to intestinal toxemia.

In arranging an antitoxic bill of fare:

1. Meats of all kinds, including fish, flesh, fowl, and shellfish, together with many varieties of cheese, as limburger and roquefort, must be wholly discarded.

2. Eggs must be used sparingly. The yolks of eggs may be eaten more freely than the whites. Raw whites must be wholly discarded. It has been demonstrated that the raw white of egg is not well digested in the human intestine. The yolks of eggs, even when hard boiled, are quickly and very completely digested. Hard boiled whites of eggs often pass through the alimentary canal without digestion. The greatest care must be taken to avoid the use of eggs which are not perfectly fresh. Eggs are likely to be infected even under most favorable conditions. The bacteria 
found in eggs will escape destruction unless they are well cooked. When freely used, eggs, even when free from germs, promote intestinal putrefaction.

3. In some persons milk produces such decidedly toxic effects it must be avoided. The ill effects are shown by coating of the tongue, loss of appetite, headache and often constipation. The symptoms may be due to a special sensitivity against milk. They may also result from toxemia arising from the putrefaction of undigested curds in the colon. It should be noted, however, that milk, as shown by Tissier, Torrey and others, is much less likely to encourage putrefactive changes in the colon than is either meat or eggs. Buttermilk has some merit as an antitoxic diet if properly prepared and used in sufficient quantity. Three or four pints of acidophilus buttermilk taken daily will produce within a week a very marked change in the character of the intestinal flora.

4. Greens and such coarse vegetables as turnips, carrots, parsnips, celery, lettuce, cabbage, etc., should be freely used together with bran and coarse cereals. Fine flour bread and other superfine cereal products should be avoided. Polished rice may be used, but only in connection with the coarser foods mentioned. A liberal supply of roughage should constitute a portion of every meal.

5. Certain vegetables, such as carrots, seem to possess a special antitoxic value, discouraging the growth of bacteria probably by carrying a considerable amount of undigested starch into the colon. Metchnikoff found that the stools of animals fed upon carrots were sometimes nearly sterile: Salads prepared from uncooked materials, edible raw fruits and vegetables of all sorts are valuable as antitoxic foods.

6. Milk sugar, because of its slow absorption, affords an excellent means of getting carbohydrate into the colon where it is needed to suppress putrefactions. Milk sugar has the additional advantage that it encourages the lactic acid fermentation. On this account a few ounces of lactose or milk sugar is always a valuable addition to the antitoxic bill of fare. The best effect is obtained by taking the sugar before eating. It should be mixed with a little water and swallowed quickly.

7. Dextrin seems to possess the same power to encourage the growth of the $B$. acidophilus in the colon shown by milk sugar and is better suited to many cases. 
8. Raw, or imperfectly cooked starch is digested more slowly than well cooked starch, and hence has a better chance to reach the colon and encourage the growth of a non-putrefactive flora. This may be provided by the use of fresh fruit and such fresh vegetables as lettuce, cucumbers, radishes and salads of various sorts, and especially by the use of scalded oatmeal; that is, steelcut oats cooked six to ten minutes like the brose of the Scotch Highlands.

9. Fats, especially animal fats, must be used in moderation as an excess of fats encourages intestinal putrefaction.

10. In order to secure promptly the maximum amount of benefit from the antitoxic diet it is important to initiate the effort with a few days' special regimen to change the intestinal flora. The fruit regimen is best for this purpose. After the flora has been changed, then the antitoxic diet should be followed systematically for an indefinite length of time.

Says Tibbles, an eminent English authority on dietetics: "A course of lacto-vegetarian dietary is exceedingly useful in the state of high arterial tension which precedes arteriosclerosis, in some forms of renal disease, - e. g., Bright's disease, in Grave's disease, gout, gravel, chronic rheumatism, liver trouble, constipation, intestinal fermentations, autointoxication, skin diseasese. g., chronic urticaria and psoriasis-in neurasthenia, neuralgia, sciatica, cardiac and gastric neuroses, etc. A pure vegetarian diet is good for some of the patients; for others a lacto-vegetarian diet is better."

Torrey found that a diet of bread and milk produces a good or acid-dominant flora. .

It was long ago shown by Bachman (Zeitschrif for Klin. Med., 1902) that while intestinal putrefaction is increased by fat and albumin, it is decidedly decreased by a farinaceous diet as well as by milk.

The diet should be made very simple and laxative. Fresh green vegetables and fruits should constitute a large bulk of the diet. Protein should never constitute more than 10 per cent. of the day's ration. Cottage cheese, buttermilk or egg yolk may be used once a day in most cases. Greens should be used daily. When buttermilk disagrees, Malted Nuts may be substituted for milk with great advantage.

Nearly all modern writers on dietetics recognize the importance of suppressing intestinal putrefactions. Taylor well says: 
"In normal bacterial disintegration of foodstuffs in the alimentary tract no known toxic substance is found." This is perfectly true since normal bacterial disintegration which takes place in the alimentary canal is fermentative and not putrefactive. Consequently, when Taylor further says: "Although protein putrefaction yields phenol, skatol, indol and cresol from aminoacids and hexone bases, none of these are toxic," he is certainly in error. For example, Lee, of Columbia University, found that the skatol and indol submitted to him by Herter for physiologic tests were powerful fatigue poisons, and a long series of observations carried on by Metchnikoff and his students in the Pasteur Institute, of Paris, showed that indol given to small animals produced marked changes in the blood vessels in the course of a few months. Bois, of Paris, showed by experiments upon animals that fecal matters mixed with their ordinary diet, even in small quantities, produced cirrhosis of the liver and other cegenerative changes. Taylor himself in directing the treatment of autointoxication, both acute and chronic, gives the following wise advice.

"Absolutely prohibit those forms of nitrogenous foods that favor the development of putrefactive bacteria, particularly animal protein except milk, e. g., meat, fish and eggs," and names among additional foods to be especially avoided, bouillon, meat soups, meat juices and jellies, meat extracts, white of egg or dishes which are made of it, and further directs that "in autointoxication from acute enteritis an exclusive farinaceous diet must be given for several days."

\section{THE LOW PROTEIN DIET}

\section{(See also page 981)}

The value of the low protein regimen is no longer questioned. Since the epoch-making experiments of Chittenden, supplemented by those of Folin of Harvard, Hindhede of Copenhagen and other investigators, the sufficiency of a low protein diet to support life and health can no longer be questioned. Folin, in fact, states that ordinary foodstuffs contain a sufficient amount of protein to supply the needs of the body, so that a person taking a variety of fruits, grains and vegetables in his daily bill of fare need have no apprehension of injury because of the restriction of the amount of protein eaten, although flesh foods and even eggs may be excluded. Combe and many others have shown by many observations that by eliminating from the diet foods rich in pro- 
tein, putrefaction may be controlled. When milk as well as meat and eggs is excluded, nuts must be eaten.

This fact has also been very clearly established by the experience of the writer and his colleagues in the Battle Creek Sanitarium, where for more than forty years a low protein dietary has been employed as a prominent feature in the general therapeutic program employed in the treatment of more than one hundred and thirty thousand patients. The great benefit derived from the reduction of the amount of protein has in most cases been so pronounced that the patient, as well as the physician, has been thoroughly convinced of the value of this regimen.

It is not necessary at this place to enter more fully into the discussion of the low protein ration, this subject having been considered at length elsewhere in this work. This diet is indicated for all persons suffering from intestinal toxemia, which includes nearly all chronic invalids, and is especially indicated in cases of acute or chronic Bright's disease, arteriosclerosis, myocarditis, and mental and nervous disorders. In detail, the low protein diet is much the same as the antitoxic diet. The same foods may be eaten, care being taken to keep the protein intake at a low level, not exceeding one calorie per pound of body weight. Green vegetables, greens and fruits should make up the bulk of the dietary. In most cases eggs as well as meats should be discarded, and milk should be used in moderation, not to exceed a pint a day. Even milk may be eliminated with advantage in many cases, four to six ounces of nuts or Malted Nuts being substituted for a time at least.

\section{PROTEIN-FREE DIET}

\section{(See also page 984)}

A protein-free diet is of special service as a means of changing the intestinal flora, and in certain cases of nephritis in which uric acid and other nitrogenous wastes are retained in the blood and tissue fluids. It affords the most efficient means of eliminating from the body surplus and toxic nitrogenous compounds and combating uremia and acidosis.

The amount of nitrogen excreted daily during a period of starvation is about seven grams, representing thirty times as much muscle tissue, or seven ounces. The loss of muscular tissue does not begin, however, until about the fourth day when the "floating" nitrogen, chiefly waste matters, has been eliminated. During this 
four days the intake of protein may be reduced to nothing without doing any damage to the vital machinery. This fact opens the way for a vital housecleaning which may be repeated at intervals as frequent as may be necessary, provided the period of complete abstinence from protein is not at any time prolonged beyond four or five days.

Meat and meat products, eggs, milk and other dairy products are all excluded from this dietary, together with nuts, legumes and all cereals with the exception of rice.

In the strictest form, the protein-free dietary will consist exclusively of juicy fruits, fruit juices and sugar water (malt sugar or milk sugar). Honey water may be used in moderation. The juices of sweet fruits, such as raisins and especially orange juice and the freshly expressed juice of sweet apples, are most valuable. All fruit juices are suitable, also dates.

The nutritive value of fruit juices varies from about 10 to 20 calories per ounce. Sweet grape juice, as found in the market, has a somewhat higher calorie value, about 24 calories to the ounce. The most highly nutritive fruit juices have a calorie value equal to that of full milk, while the more dilute juices equal skimmed milk and buttermilk in value.

At 20 calories to the ounce, a pint of fruit juice supplies 320 calories, and hence four or five pints daily, supplying 1,300 to 1,600 calories, would suffice for a person of average size while confined to the bed. This amount of liquid can be easily taken in divided doses.

A good plan is to administer half a glassful (4 ounces) of juice every half or three-quarters of an hour. Fruit juice should be substituted for other fluids. In case the full amount of fluid indicated is more than the patient can easily take, its nutritive value may be increased by the addition of malt sugar or milk sugar. Two ounces of malt sugar added to a pint of apple juice will supply approximately 400 calories. Three to three and onehalf pints of the mixture will furnish a fair day's ration.

It is well to supply the patient with as large a variety of fruit juices as possible. A good substitute for fruit juice, which is better liked by some patients, may be prepared by adding to a pint of water four ounces of malt sugar or milk sugar and the juice of one lemon. Purées of ripe fruits may be used instead of fruit juices, or even the ripe fruits themselves. Watermelon, 
canteloupe, peaches, apricots, strawberries, huckleberries, raspberries and other small fruits may be served with malt sugar. Rice flakes, boiled rice, rice jelly and other preparations of rice may be used in most cases and help to relieve the monotony of this regimen when necessary to do so.

Other foods which may be used are the following: agar jellies, green vegetables of all kinds, particularly tomatoes, cucumbers, turnips, cauliflower, cabbage, celery, squash, artichokes, lettuce and greens. When anemia is pronounced, purées of spinach and other greens should be freely used twice a day.

Within four or five days any surplus or "floating" nitrogen which has existed in the tissues will be removed and hence it will not be necessary to continue this dietary for more than five days. The regimen may be repeated at intervals as required.

The protein-free regimen may be used advantageously in the following conditions: in acute nephritis, temporarily in chronic Bright's disease with low renal efficiency, very pronounced cases of autointoxication, in fever accompanied by a very high temperature in which there is reason to apprehend kidney involvement, etc.; in all cases in which the blood nitrogen is high, that is, above 40 ; in acute severe eruptive fevers and in eczema.

One or two ounces of agar or an ounce of sterilized bran and three to six ounces of paraffin oil should be used daily to promote intestinal activity. In addition, an enema should be used twice a day to insure thorough evacuation of the colon. These measures are highly important, and should always be employed when the patient is placed upon a liquid diet or other regimen which does not supply the necessary bulk to secure sufficient bowel action.

\section{FEVER DIET}

\section{(See also page 983)}

Hippocrates fed his fever patients ptisan or barley gruel. Niemeyer was an enthusiastic advocate of fruit soups in fever cases. We are just beginning to learn how to feed fever patients, after having for years first starved and then stuffed them with milk and whiskey. When the writer was walking the wards of Bellevue Hospital more than forty years ago whiskey and milk constituted the universal diet in typhoid, and this practice still continues in certain regions, although whiskey was long ago discredited and milk affords the best possible medium for the cul- 
ture of the typhoid germ and has been shown to be responsible for the tympanites, putrid stools, and even perforations which were common occurrences in typhoid cases under the old regime.

A better understanding of the metabolism of the body and of the significance of fever has led to radical changes in the dietetic management of fevers of all sorts, especially typhoid and other fevers of severe type. It is now known that under the influence of a high temperature vital combustions are incomplete. This is especially true of fats and nitrogenous substances. A natural consequence of this is the production of acidosis, which is responsible for the so-called typhoid state. Starvation only increases the tendency to acidosis, while abundant carbohydrate feeding has the opposite effect. Carbohydrates afford, in fact, the only efficient means of combating acidosis. Another very pronounced advantage in the use of carbohydrates is the fact that by their free use the character of the intestinal flora may be profoundly influenced, putrefactions may be largely suppressed, and thus the absorption of toxins from the colon may be lessened.

It is evident, then, that the dietary for fever patients should consist chiefly of carbohydrates. The imperfect oxidation of fats precludes the free use of this class of foods, but carbohydrates, being well utilized, may be freely employed.

The old adage, "Stuff a cold and starve a fever" has long been discredited, and yet there are still many who do not recognize the fact that there is no essential difference between a cold and a fever. As a matter of fact, a cold is a fever, a mild infection. A generation ago fever patients often became very greatly reduced by the starvation regimens to which they were subjected. A bill of fare consisting chiefly of meat extracts, broths, thin gruels, etc., is a starvation regimen. On such a dietary the patient is not only starved but poisoned by the large quantities of meat extractives taken, which afford no nourishment and greatly tax the already overworked liver and kidneys.

Dr. Austin Flint many years ago called attention to the fact that the chemical analysis of beef tea very closely tallies with that of urine. Meat preparations of all sorts should be suppressed in fevers. Since fever is generally due to toxins developed in the body by infectious organisms it is manifestly unwise to increase the burden by the addition of toxic foods. Consequently, an antitoxic diet is especially indicated in fever, no matter what the particular infection or condition to which it may be due. 
A low protein diet is certainly indicated for the evident reason that a high protein diet encourages putrefactive changes in the colon and resulting toxemia. The amount of protein should certainly not be greater than that required for repair of the tissues, which is probably about one calorie per pound of body weight.

Rubner, Lusk and others have shown, also, that proteins stimulate metabolism and otherwise excite the body cells to abnormal activity. This effect is certainly not needed in fever; hence particular pains should be taken to keep the protein content of the food low. Eggs as well as meat may well be discarded, though occasionally an egg yolk may be permitted. Milk must also be used in moderation. Buttermilk may be used more freely and whey may be employed liberally.

In fever there is increased production of heat; the combustion of the vital processes in the body are accelerated. If fuel is not supplied from outside sources, the body itself will be consumed. This is the cause of the great emaciation occurring in prolonged fevers. Carbohydrates are evidently needed to furnish fuel to supply the loss occasioned by the fever process and to prevent the destruction of the tissues. When food is supplied in proper amount the great emaciation seen in badly managed cases does not occur. Carbohydrates should be supplied in liberal quantities in all febrile cases. The best forms of carbohydrates are gruels sweetened with malt sugar, fruit juices to which malt sugar or milk sugar has been added, purées of fruits and vegetables and fruit soups.

The loss of lime and iron is greatly exaggerated during febrile conditions, and there is a special need for vitamins to aid in repairing the damage done to the tissues.

Great care should be taken to see that these important elements are supplied in abundance, as they are necessary to aid Nature in the healing process. The importance of this cannot be overestimated. An examination of the fever diet and the so-called liquid diet in ordinary use in hospitals and prescribed in textbooks on dietetics will show that both lime and iron are often almost wholly lacking. When milk is used, lime is supplied in sufficient quantity; but iron is almost universally lacking, and the patient becomes anemic not only as the result of the disease from which he is suffering but because of the iron and vitamin starvation to which he is subjected by a deficient dietary.

Purées of spinach and other greens are of special value be- 
cause of the large amount of lime and iron which they contain. By the free use of greens the pronounced anemia and the heart weakness which results from the depreciation of the blood and from the deficiency of food lime is prevented. Not infrequently, fever patients are fed continuously on water gruel or some other monotonous diet which is lacking not only in lime and iron but also in vitamins, so that to the damage done by the fever is added the injury resulting from a dietary lacking in these three great food essentials. Fever patients often present symptoms of incipient scurvy or beri-beri due to this cause.

Bran or agar in various combinations should be freely used in all cases of fever except in cases of marked gastrointestinal disease. This is necessary to combat the constipation to which there is usually a decided tendency in febrile conditions. Brau may be mixed with cereals in liberal proportions. The average fever patient needs an ounce of bran or agar daily.

In fevers and when a very low protein diet is needed, a smaller amount of protein will suffice if the food is given in six portions instead of three. The reason for this is that protein is totally digested and utilized, and the surplus excreted in six to nine hours from the time it is eaten, so if the protein is all taken in one dose, after nine hours the body will have to draw upon its own tissues for such protein as may be required. This has been proven experimentally.

The following foodstuffs have been found especially valuable in the feeding of fever patients:

Gruels, especially oatmeal, barley and rice gruel

Purées of vegetables Purée of potato

Purées of fruits and ices Purée of banana

Fruit juices

Whey Milk sugar

Malt sugar

Agar jellies

Lacto-Dextrin

The amount of food given the fever patient should be equal at least to that required by an individual of the same size and weight when leading a moderately active life. It is especially important to insure a large intake of water in these cases. Water is the best means of washing the tissues free from the fever toxins and securing their elimination through the kidneys. The amount of water taken daily should be three to six quarts, a glassful of 
water being given the patient every half hour when he is awake. The water may be flavored with fruit juice, lemon juice or any other fruit juice, or malt sugar or milk sugar. Water should be administered by enema when sufficient is not taken by mouth.

Tibbles gives the following list of diseases in which a fever diet is indicated:

\begin{tabular}{ll} 
Abscess of various parts & Boils, furunculosis \\
Appendicitis & Bronchitis, acute \\
Bronchial catarrh & Mumps \\
Broncho-pneumonia & Nephritis \\
Burns and scalds, extensive & Oophoritis, acute \\
Carbuncle & Osteo-myelitis \\
Catarrh, acute & Otitis media \\
Cellulitis & Pancreatitis, acute \\
Cerebro-spinal fever & Pemphigus, acute \\
Chicken-pox & Pericarditis \\
Cystitis & Peritonitis, acute \\
Delirium tremens & Perityphilitis \\
Diphtheria & Pharyngitis \\
Empyema & Plague \\
Endocarditis & Pleurisy \\
Epididymitis & Pneumonia \\
Erysipelas & Poliomyelitis \\
Fevers & Psoriasis, acute \\
Gangrene & Puerperal fever \\
Gastritis, acute & Pyaemia \\
Glossitis, acute & Pyelitis \\
Hepatic abscess & Pyonephrosis \\
Herpes zoster & Rheumatism, acute \\
Influenza & Salpingitis \\
Intermittent fever & Scarlet fever \\
Laryngitis, acute & Septicaemia \\
Lymphangitis & Surgical fever \\
Malarial fever & Synovitis, acute \\
Mastoiditis & Tetanus \\
Measles & Thrombosis \\
Meningitis, acute & Tonsilitis \\
Meningitis, tubercular & Typhoid fever \\
Meningitis, cerebro-spinal & Typhus fever \\
& \\
\hline &
\end{tabular}


In all these maladies meats of all sorts should be discarded together with beef tea, bouillon and meat extracts of all kinds. Fruit juices should be freely used. Fruit soups, fruit and vegetable purées, especially potato soup or purée, cereals of all sorts in the form of mushes and gruels and purées of greens are the proper foods. The use of malt sugar and dates in place of cane sugar because of their richness in iron is important in febrile and many other acute conditions.

In mild fever cases, after convalescence begins in typhoid, and in most cases in which the fever accompanies some localized infection or inflammation, the antitoxic diet may be followed. In all cases attention must be given to the stools. Milk sugar or lactodextrin should be used in sufficient quantity to change the flora. The amount required is usually two or three ounces three times a day. To prevent nausea the sugar should be well diluted. Twelve to sixteen ounces of water or fruit juice is not too much. Take slowly in the course of ten or fifteen minutes, especially if there is a tendency to nausea. Lemon or other fruit juice or syphon water may be added to overcome repugnance or prevent nausea.

\section{THE LAXATIVE DIET}

(See also page 986)

This dietary is chiefly addressed to the colon, although its beneficial effects are participated in by the whole body; and not only indirectly through improved elimination, but directly through the rich supplies of vitamins, lime, iron and other essential food minerals which it affords.

Most important of all as a means of increasing intestinal activity is change of the intestinal flora. The products of putrefaction are alkaline, often strongly ammoniacal, and paralyze the colon. The normal acids produced by an aciduric flora are the natural stimulants of the colon. Increased frequency of bowel movement as the result of change of the intestinal flora was noted by the author some years ago (1915) and has been a matter of constant observation since. Kopeloff and other investigators have recently demonstrated the importance and efficiency of suppression of putrefaction in the colon as a means of overcoming constipation in the most obstinate cases.

The most laxative foods are those which contain the largest amount of cellulose. The amount of cellulose found in each one 
of the ordinary foodstuffs is shown in tables given elsewhere in this work. (See Tables XXI, XXII, XXIII.)

The foods in the following list are especially rich in cellulose and have precedence in the order named:

$\begin{array}{lll}\text { Wheat bran } & \text { Red raspberries } & \text { Spinach } \\ \text { Huckleberries } & \text { Celery } & \text { Currants } \\ \text { Oatmeal } & \text { Turnip } & \text { Cauliflower } \\ \text { Green peas } & \text { Pumpkin } & \text { Figs } \\ \text { String beans } & \text { Peas } & \text { Tomatoes } \\ \text { Cabbage } & \text { Beets } & \text { Lentils } \\ \text { Parsnips } & \text { Asparagus } & \text { Barley } \\ \text { Beans } & \text { Blackberries } & \text { Lettuce } \\ \text { Brussels sprouts } & \text { Cranberries } & \text { Onions } \\ \text { Kohl-rabi } & \text { Carrots } & \text { Gooseberries }\end{array}$

Bran.

When it is necessary to combat obstinate constipation, it is usually essential that wheat bran be used in liberal quantities. The laxative virtues of bran have long been known and utilized by stock raisers, but it is only within the last twenty years that the value of this article as a food laxative has begun to receive the recognition which it deserves. When the writer first began the advocacy of bran as a laxative food some forty-five years ago, it was very hard to convince the average man that in taking bran he was not running great risk of doing violence to his stomach and intestines, perhaps creating ulceration, inflammation, hemorrhoids or some other distressing disorder. On purely theoretical grounds and perhaps with a commercial purpose, some one years ago launched the notion that bran irritates the gastric and intestinal mucous membrane. The patent flour manufacturers have industriously cultivated this false notion until it has come to be widely held and deeply rooted in the popular mind. The millers can well afford to spend a considerable amount of money in persuading people to eat fine flour bread, for this will make them constipated so that they will later be obliged to resort to the use of the same miller's bran, for which they will be asked to pay fifteen to twenty-five cents a pound after having paid one or two cents extra per pound for the superfine flour which has made them ill. Thus the miller by his shrewd scheme not only makes an extra profit on his superfine flour, but gets triple price for the precious 
bran which should never have been separated from the other components of the wheat berry.

It should be remembered that bran does not irritate; it titillates. It passes through the mouth without inflicting injury and likewise passes along over the mucous membrane of the whole alimentary tract, stimulating the intestine to action but doing it no damage.

The amount of bran required to produce decided laxative effects is considerable. A quarter ounce at each meal is often needed, and it must be taken at every meal without exception. The quantity should be increased until the desired effect, three bowel movements daily, is produced.

There are many convenient methods of using bran. Here are a few. Bran brose may be prepared by mixing together equal measures of bran, cornmeal and steel-cut oats. Stir into boiling water and cook three to ten minutes, never longer. This is an excellent breakfast dish. Dry bran may be served upon the table and eaten with corn flakes or rice flakes, oatmeal, cream of wheat or other breakfast foods. One or two heaping tablespoonfuls is not too large a dose for the average individual. Some require more. At dinner bran may be added to the soup or mixed with mashed potatoes or purées of some sort.

Bran bread, bran gems and other similar combinations will be found efficient and satisfactory. The important thing is to use a liberal quantity of bran and to take it at every meal without exception. Agar, another form of cellulose, is equally efficient in many cases and occasionally produces more satisfactory results than does bran.

It is to be remembered, also, that bran is rich in lime, iron and vitamins and so has a real food value aside from its laxative properties.

\section{When the Laxative Diet is Indicated.}

The laxative diet is indicated in almost every case of chronic disease, either alone or in combination with some other specific dietary as may be required in any individual case. It is always indicated when the bowels move less than twice a day and when fecal residue is found in the colon after the last daily movement. Normally, the colon empties itself at least three times a day. Many persons, even some physicians, take alarm at this suggestion; but a short experience of the benefits of prompt and thor- 
ough clearance of the body refuse will convince the most skeptical of the necessity for frequent and complete bowel movement.

In every case of obstinate constipation the motility test should be employed. If the delay is two days or more, an X-ray exâmination should be made and the possibility of mechanical hindrance should be considered.

The laxative regimen is indicated especially in colitis, a very common malady which is due to infection, the result of chronic constipation. Even in cases in which there are frequent bowel movements, the laxative regimen is needed so long as the stools are putrid. There is no danger of irritation from the use of bran and laxative foods. The frequent movements are due to the fact that the colon is never properly emptied, and to the irritation caused by putrefaction products. A clean colon is the first essential for recovery. A daily enema or even two enemas daily, if not always essential, will be found helpful in many cases, in addition to a very laxative regimen.

In chronic tuberculosis, so-called neurasthenia, insomnia, skin diseases, arteriosclerosis, Bright's disease, hyperchlorhydria, duodenal ulcer, and the whole long category of digestive, nervous and nutritional disorders, a laxative regimen is of first importance.

\section{TISSUE-BUILDING OR FATTEINING REGIMEIN}

(See also page 985)

Fluctuations in the weight of the body are chiefly due to changes in the amount of adipose tissue. After maturity has been attained the number of body cells does not materially change although the muscle fibers may increase in volume. A muscle does not increase in size, however, as the result of feeding alone. Exercise must be combined with feeding to stimulate muscular development. The tissue-building or fattening diet, then, relates chiefly to increase of adipose tissue. It is, however, important that means should be adopted also for increasing the blood cells and the volume of blood in the body since the increase of adipose tissue results practically in a dilution of the blood which is spread out through an increased area of tissue. This fact is shown by the fall in the percentage of hemoglobin which usually accompanies an increase of weight. This decrease in hemoglobin may be prevented, however, by the adoption of a diet which will increase the hemoglobin along with the increase of weight. 
Thinness of flesh may be due to any one of many causes, such as fever or other wasting diseases, excess of work, loss of sleep, or high tension due to worry, anxiety, suspense, apprehension or other cause. Whenever the energy intake through eating is less than the expenditure through internal or external work, there will necessarily be a loss of weight. An increase of weight can only be induced by reversing the balance. To accomplish this, the causes of excessive energy expenditure must be removed or reduced as much as possible. When a rapid gain of flesh is desired, it is usually best to keep the patient in bed or at least to keep him horizontal and as quiet mentally and physically as possible, by this means reducing tension as well as muscular work.

In arranging a fattening bill of fare, the relation of the several food principles to adipose tissue must be kept in mind. For fattening purposes it is evidently useless to increase the protein intake, at least so long as the patient is at rest, for the reason that protein cannot be stored in the body. It can be used only for repair and growth. If the growth of the muscles is not being stimulated by exercise, there is not only no demand for an extra intake of protein, but there is no way in which the body can be made to store a surplus of it beyond what is absolutely necessary for repair, which amounts to less than one calorie per pound of body weight.

The case is quite different with carbohydrates and fat. These food principles, when taken into the body in larger quantity than is needed for fuel purposes, are retained and build up the adipose or residual tissue. Fats are absorbed and carried direct to the fat depositories of the body. Carbohydrates, after conversion into dextrose, the sugar of the body, are converted into fat if not needed for immediate use as fuel. Each ounce of surplus fat taken in the food becomes an ounce of fat in the body. In the case of carbohydrates, starch and sugar, however, the case is different, each ounce adding to the body weight three or four ounces instead of one. It is evident, then, that of the several food principles, carbohydrates are best adapted to increase body weight. Carbohydrates have the further advantage, also, that they are much more quickly and easily digested and assimilated than are the fats.

It is possible, also, to greatly increase the intake of carbohydrates without producing disturbance of digestion or of any other function. An increase of protein, through the use of meat, 
eggs, oysters, etc., increases the intestinal putrefactions and so tends to create toxemia. Fats, when freely taken, are likely to impair the appetite by delaying the movement of food through the stomach and intestines and also by increasing the putrefactive changes in the colon. A moderate increase of fat and a very considerable increase of carbohydrates is the physiologic formula for securing a rapid increase of weight. The free use of meats offers no advantage whatever and is indeed a positive disadvantage, since protein does not make fat and consumes energy in excessive heat production and by overworking the liver and kidneys in the elimination of surplus waste.

The amount to which the food intake is increased will depend upon the rate of increase in weight required or expected and the appetite and digestive powers of the patient. Not infrequently the emaciated patient will be found to be greatly underfed, the daily intake of food often being not more than two-thirds the amount required for full nutrition, sometimes even less. In such a case the amount of food habitually taken must be doubled. In general, the calorie value of the daily ration must be made 25 to 50 per cent. more than the amount required for bare maintenance. By reference to the table (see Table II), the number of calories required for maintenance may be found, and this should be increased by one-fourth to one-half. If the patient is put to bed and all exercise prohibited, the base ration may be made the foundation for the feeding and should be increased 50 to 100 per cent. The maximum increase should not be made at once, but the amount of food should be increased from day to day, the maximum being reached by the end of the first week and maintained until a satisfactory increase of weight has been secured.

Whenever possible, the patient's metabolism should be tested. By this means, much time may often be saved. If the metabolism rate is much increased, this will at once explain the loss of flesh and will show the necessity for complete rest in bed as the most efficient means of reducing metabolic activity. The systematic use of clinical metabolism tests, first employed at the Battle Creek Sanitarium, is now in use in several of the progressive clinics of this country.

As a preliminary to the fattening regimen, the employment of the fruit regimen for three or four days has the advantage in most cases of greatly promoting appetite and facilitating the gain in flesh when the fattening regimen is established. 
The dietary must include foods rich in lime, iron and vitamins as well as carbohydrates and fats. This is important for the reason that in many cases of emaciation the cause is in large part a lack of the essential growth-promoting vitamins which are abundant in fresh milk and greens but are found only in very small quantity in most other foodstuffs, being almost wholly lacking in meats and in cereals as commonly used. Foods rich in vitamins also serve to increase appetite for food and ability to utilize it.

In connection with the fattening diet it is important to adopt efficient measures for increasing appetite; that is, moderate cold bathing, particularly such measures as the cold mitten friction, cold towel rub, and for persons of average strength the cold wet sheet rub or cold shower bath. Out-of-door sleeping and almost continuous life out of doors are other measures of highest value and should not be forgotten. Abundant sleep should be secured. Ten to twelve hours daily is none too much.

Among the foods of special value for fattening purposes may be mentioned malt sugar, milk sugar, malted nuts and fresh cream. A food par excellence is fresh cream or butter combined with malt syrup, malt honey or meltose.

In many cases it is of very great advantage to give the patient, in addition to the three regular meals, two or three minor meals consisting of malt sugar or milk sugar dissolved in water, either with or without fruit juices of some sort. (See Supplementary Feeding.) Carbonated water may often be used with advantage, as the gas set free in the stomach has the effect of stimulating gastric activity and hastening the emptying of the stomach. In order that the intake should be as large as possible, it is best that it should be simple in character so that the tax upon the digestive organs may be as little as possible. Complicated dishes should be avoided and only a few different dishes served at each meal. When eggs are eaten, the white should be discarded. The yolk of an egg possesses twice the food value of the white and is much more easily digested, while supplying also a rich store of lime, iron and vitamins which are deficient in egg white.

In connection with the rest cure, it is unwise to allow patients to stay in bed too long. A week or two is usually sufficient. At the end of this time systematic exercises should be begun, gradually increasing in vigor from day to day. With the increase of 
exercise the appetite will also increase, and when things are managed well the patient's gain in weight will continue without interruption and may be substantially increased because of the improvement in appetite.

The fattening regimen is indicated especially for persons who have been reduced in weight by acute or chronic disease or by malnutrition resulting from any cause. Persons who are convalescent from an acute illness and consumptives are excellent subjects for the fattening regimen.

The milk regimen is one of the most efficient of all methods of creating a rapid gain in flesh. When it can be made acceptable to the patient, and is not contraindicated, this regimen may be adopted with advantage. There are, however, many cases in which the patient is sensitized to milk or in which for some other reason it cannot be tolerated.

Cane sugar should never be employed as a means of increas. ing the intake of carbohydrates because of its irritating properties. Malt sugar and the sugars of sweet fruits and fruit juices may be used to any extent which may be desirable without injury.

Milk sugar is sometimes useful in doses of three ounces three times a day as a means of changing the intestinal flora. Large doses of milk sugar sometimes give rise to nausea. In such cases lacto-dextrin may be used or acidophilus buttermilk.

Malt sugar may be used in divided doses to the extent of ten or twelve ounces daily. When carbohydrates are used in large quantities for some time it is a wise precaution to apply the Bergel test to the urine, for the purpose of detecting any elimination of imperfectly utilized carbohydrates. When possible, it is also well to make a determination of the blood sugar.

One of the most important indications for the carbohydrate diet is the preparation of patients for severe surgical procedures, instead of fasting the patient. In cases in which it is desirable that the intestinal tract shall be free from residues the result desired may be secured by feeding the patient on carbohydrates exclusively for a few hours before the operation. Malt sugar is best adapted to this purpose. The commercial product consists of approximately equal parts of maltose and dextrin, which are quickly digested and absorbed and produce no disturbance.

The old teaching respecting carbohydrate indigestion has literally no scientific foundation. When such flatulence appears after the free use of carbohydrates it is not to be attributed to 
the carbohydrates themselves, but rather to the presence in the intestine of gas-producing micro-organisms which do not belong there, and which disappear when the dietary is managed in such a way as to produce a transformation of the intestinal flora, restoring the normal and beneficent $B$. acidophilus. This change may usually be effected in a week or ten days by feeding two to three ounces of milk sugar or lacto-dextrin three times a day.

Gas in the intestine is the result of the action upon carbohydrates of Welch's bacillus, an organism which causes gas gangrene and is also the cause of putrefaction in the intestine and elsewhere. The colon bacillus is also to a less extent productive of gas. These organisms produce little gas on a flesh diet; but when they are present in large numbers in the colon, the addition of considerable carbohydrate to the diet will give rise to much gas. The acid-forming organisms do not produce gas; hence, as soon as the flora is completely changed or nearly so the gas disappears. So while it is true that a carbohydrate diet gives rise to gas in many cases, the remedy is to be found not in less carbohydrate but more.

\section{Fattening Diet.}

Carbohydrates are best. One ounce of carbohydrates represents about 100 calories. This amount is found in each of the following foods in the quantities named:

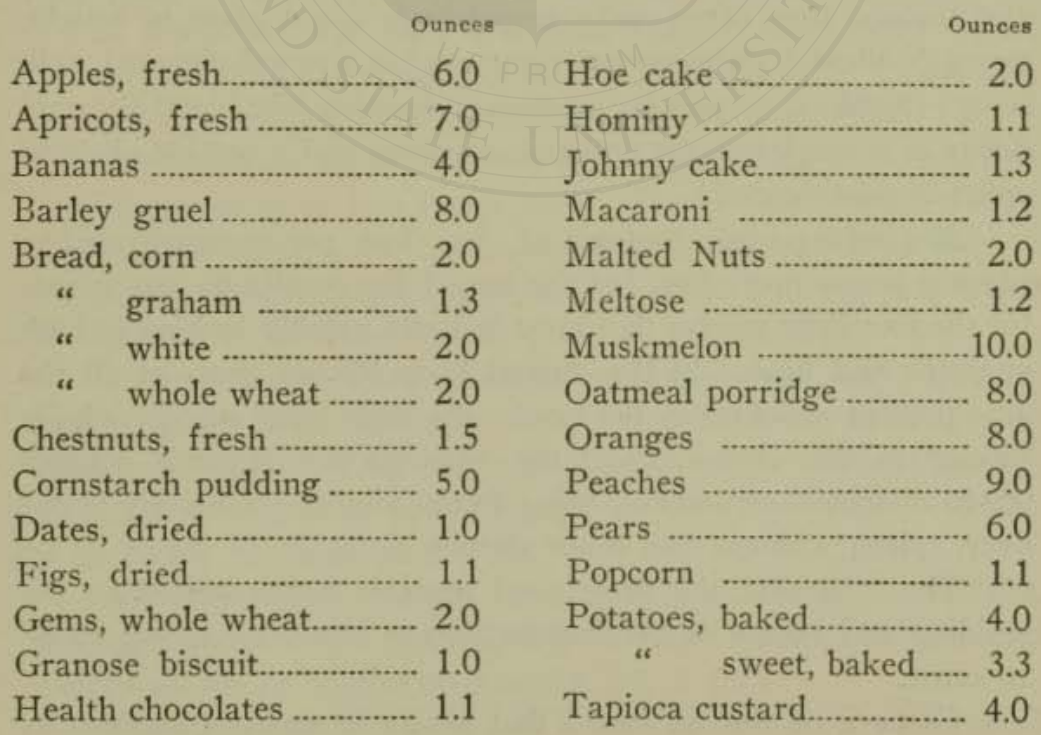




\section{THE RESTORATIVE REGIMEN}

(See also page 988)

The discovery of vitamins has in recent times brought into clear view the cause of a great number of cases of chronic malnutrition which were formerly highly obscure and were often subjected to treatment during long periods without satisfactory results. Extended researches by Bunge of Switzerland, and Sherman of this country, together with Mendel, Osborne, McCollum and others, have also thrown much light upon these cases. It is now known that in a large proportion of instances in which individuals suffer from general feebleness, lack of vigor, endurance, efficiency, and sense of well-being; a state of semi-invalidism or valetudinarianism without evidence of any clearly defined malady; their troubles are due to malnutrition, the result of a deficiency of vitamins, lime, iron, and other salts in the food. There is no doubt that in this country alone there are many tens of thousands of persons suffering from a deficiency of these highly essential food principles in their daily food. Such persons often derive great benefit from short vacations, partly perhaps because of a change from their accustomed inadequate bill of fare to one which supplies some of the lacking elements. On returning home they find themselves quickly relapsing into the old conditions. When such individuals are placed upon a dietary which supplies the needed diet factors, they often make surprisingly rapid gains in weight, strength, mental and physical vigor, and in general physical wellbeing; the blood corpuscles increase in number, the blood becomes richer in hemoglobin, the appetite improves and a notable physical uplift is experienced.

Not infrequently patients of this class are recommended to adopt a heavy meat diet, but the hoped for results do not appear for the excellent reason that meat is itself notably lacking in both vitamins and lime. In the animal body 99 per cent. of all the lime present is found in the bones, the flesh being almost wholly lacking in this element, and the vitamins are likewise concentrated in special structures, being found almost exclusively in the liver, spleen, kidneys and other glands.

The idea that the protein of meat is better suited to the building and repair of the human tissues has been shown to be an error.

Caspari, of Berlin, found that casein is superior to flesh as 
a source of protein. This was observed to be true in both the human subject and the dog.

Among restorative foods will be found those foodstuffs which are particularly rich in the elements that are needed for refurnishing the blood, bones, nerve and tissue fluids with the elements needed for complete nutrition. Greens of all sorts, milk, butter, cottage cheese and other dairy products; all grains, particularly oatmeal and millet; fruits, especially the orange, the tomato and the apple and fruit juices of all sorts; potatoes, artichokes, turnips and other roots and tubers; the onion, properly cooked; these are all highly valuable, also salads prepared from fresh, uncooked materials. (See Raw Diet.) The lentil should not be forgotten because of its richness in lime and iron. Bran should be freely used at every meal because of its rich store of lime, iron and other salts as well as vitamins.

A vegetable purée or consommé prepared from a variety of vegetables may be used with great advantage for both adults and infants whose tissues are poor in mineral constituents in consequence of long feeding on a diet deficient in food minerals. The mixture may consist of string beans, shell beans, especially the Hahto bean, green peas, young beets with the tops, radishes, turnips, young turnip tops, celery, artichokes, spinach, tomatoes, mustard greens, and almost any other green or fresh vegetables. Add cold water enough to cover, bring to a boil and simmer five or ten minutes. Put into a fireless cooker over night. Pass through a colander, taking care to save all the water. Serve as a consommé, or add Japanese isinglass and allow to jelly.

Greens, fresh vegetables and fresh fruits should be taken in liberal quantities at two meals daily. Better results still will be obtained by taking greens at every meal in quantities of four to six ounces. Most surprising results in blood building may often be obtained by the very abundant use of greenstuffs.

Tomato juice, either fresh or canned, potato soup, oranges, lemons, grape fruit and tamarinds are rich in the antiscorbutic vitamin which is often needed in these cases. Wheat germs, sprouted soy beans, or better Savita, an extract of yeast, are also to be highly recommended.

All edible green things, such as lettuce, celery, cucumbers, cabbage, green corn (uncooked), carrots, turnips, salads, and melons should be conspicuous in the bill of fare.

The daily use of a pint and a half or two pints of certified 
milk, a quart of yogurt buttermilk and liberal quantities of cream and butter is conducive to rapid improvement. Cottage cheese is especially valuable for the lime and vitamins which it furnishes.

Elsewhere in this volume will be found lists of foods which are rich respectively in vitamins, lime and iron (see Tables XVII, XVIII, XIX, XIV, XII), which should receive careful study. Foods which contain an abundant supply of lime and iron are certain to contain a sufficient amount of the other mineral salts required by the body.

\section{THE FASTING REGIMEN}

In recent times numerous attempts have been made to exploit the idea of fasting as a panacea for human ills. However, there is no danger that this drastic method will ever become a popular fad. Only heroic souls and fanatics are likely to become enthusiastic devotees of a method which requires the amount of selfcontrol and endurance incident to the withholding of food during periods of one to six weeks.

Voluntary fasting probably originated as a religious rite. It was prescribed as a penance, or was undertaken for the purpose of encouraging a state of spiritual elevation.

That a person may live without eating for a considerable period has been clearly demonstrated by the experience of Cetti and Succi, the Italian fasters, Doctor Tanner, and others. Professor Luciana, who observed the faster, Succi, during a thirty days' fast, found him a demented person (formerly insane) who believed himself to be "the son of God," and who fasted for the purpose of demonstrating the indestructibility of his divine body.

Various professional fasters as well as fasting animals have since that time been studied by physiologists in different parts of the world. The uniform testimony of these expert observers has been that the withholding of food does not exercise the prompt control over intestinal putrefactions that might be expected. Food is not the only source of putrescible material in the colon. The bile, intestinal mucus and other intestinal secretions that are constantly poured into the alimentary canal furnish a sufficient amount of putrescible material to maintain a luxuriant putrefactive flora. When these materials are promptly discharged from the body there is not, however, sufficient time for the development of the putrefactive process, which requires much more time than 
is needed for the development of the fermentative changes to which carbohydrates are subject. In fasting, however, there is almost complete inactivity of the intestine. Food is the natural excitant of peristaltic activity. Whenever food is taken into the stomach, active contraction waves begin at once, traversing the stomach at the rate of three to five a minute, and passing along down the whole alimentary tract. When food is withheld, these movements cease, the biliary and other excretions accumulate and undergo putrefaction; the poisons are absorbed, the tongue becomes coated, the breath foul, the urine loaded with the products of putrefaction and all the evidences of intestinal autointoxication become intensified.

In the case of the faster, Cetti, observed by Lehmann, the first stool after the beginning of the fast occurred on the ninth day. The amount of phenol, a putrefaction product, found in the urine had increased in the meantime to nearly five times the amount present at the beginning of the fast. The condition of the urine was the same as that which is usually observed in cases of obstruction from peritonitis or other causes, the result of the paralyzed condition of the bowel due to complete abstinence from food. Examinations of the urine of Succi, the famous Italian faster, made by Apollo and Solard by Bouchard's method, showed its toxicity to be greatly increased. These facts show most conclusively that fasting is not, as claimed, an efficient means of cleansing the body from poisons.

Starvation suspends the secretion of the digestive juices. "The secretion of saliva diminished in acute starvation even when water was taken ad libitum. Thus Succi, on the seventh day of his fast, only produced as much saliva by the movements of his jaws in three hours as under ordinary circumstances is secreted in five minutes" (Von Noorden).

In fasting, the stools were highly putrid and "similar in appearance to the feces passed when the diet is mainly composed of meat" (Von Noorden).

Says the same author: "The blood atrophies."

Statkewitsch studied the effects of fasting in a large number of animals-cats, dogs, rabbits, pigeons, frogs, lizards and other animals - and found that after prolonged fasting the cells of the heart, liver, muscles, kidneys, pancreas and other glands were the seat of degenerative processes. These processes were most marked in the muscles and the glands. 
Zander found evidences of degeneration of the heart muscles in pigeons after fasting eight to twelve days.

Gaglio found cloudy swelling of the muscles, granular and fatty degeneration of the liver in fasting frogs.

\section{The Fasting Body Feeds Upon Itself.}

So long as life continues, the fasting animal body continues to consume energy although at a reduced rate. The energy output of the body during fasting is three-fifths to three-fourths the ordinary output. In the absence of the usual source of energy food, the body feeds upon itself and is consumed at the rate of about one-eightieth of its weight each day. The stores of fat are first drawn upon, then the muscles, glands and other structures are consumed to keep the fires of the body burning. The heart muscles and some other organs may lose as much as one-half their weight. This process of degeneration may be carried so far that the body cannot recuperate itself. According to the London Lancet, the professional faster, Succi, although only thirty-eight years of age, had the appearance of a very aged person; not only his face but his entire body was covered with deep wrinkles. His muscles were so atrophied and degenerated that he looked like a skeleton and had the appearance of a person suffering from incipient marasmus.

\section{Loss of Weight During Fasting.}

A man of average size when at rest and fasting, burns up daily about 75 grams $(23 / 4 \mathrm{oz}$.) of protein (muscle and gland tissue), 25 grams $(5 / 6 \mathrm{oz}$.) of glycogen (animal starch stored in the liver and muscles), and 200 grams $(62 / 3 \mathrm{oz}$.) of fat (adipose tissue), amounting in all to 10 ounces of tissue consumed. But this does not represent the total loss of weight for the reason that with each ounce of carbohydrate or protein (glycogen or muscle) consumed, three times the same weight of water is lost, so that the actual loss in weight for the muscle and glycogen burned is 100 grams $\times 4=400$ grams or $13 \frac{1}{2}$ ounces. Adding the $62 / 3$ ounces of fat, the total loss in weight is 20 ounces, or double the weight of the actual solid matter consumed by the body processes.

A fasting man burns about 40 to 60 grams of protein and 120 to 140 grams of fat. The amount of protein burned is at the beginning 1 part to $3 \frac{1}{2}$ parts of fat, and toward the end 1 part of protein to $2 \mathrm{t} / 2$ parts of fat.

The following table (Von Noorden) shows the percentage of 
loss sustained by some of the principal organs and tissues of the body during fasting:

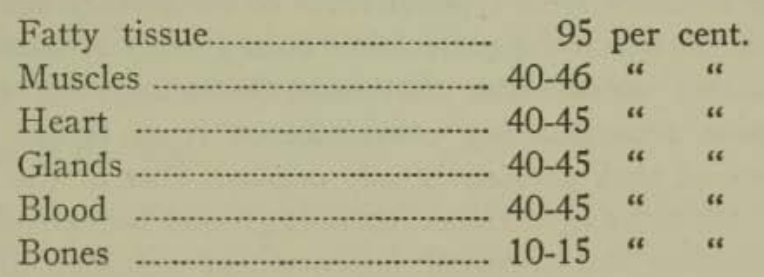

Many persons have passed through the ordeal of a long fast and have survived, and in some instances there has been evidence of a notable improvement in health following a long fast. There are, in fact, certain advantages in fasting which may be briefly stated as follows:

1. By fasting, surplus body fat may be disposed of. During a fast the body feeds upon its reserves.

2. Any accumulation of surplus or "floating" nitrogen or wastes which may be present will rapidly disappear during a fast. It is true that the amount of surplus protein and tissue wastes which may accumulate in the body can never exceed a very small amount, certainly never more than a few pounds; but that these objectionable substances may be present cannot be denied, and by a fast they are gotten rid of.

3. Fasting creates an appetite by producing an imperious demand for food and perhaps at the same time increases the ability of the tissues to assimilate food. This effect of fasting may be an advantage in certain cases, particularly when it is desirable to produce a rapid gain in flesh by subsequent overfeeding.

4. There is some evidence that a prolonged fast may in some cases produce a sort of rejuvenescence of the tissues. It has been shown by experiments upon animals that in certain lower organisms, particularly in certain mites, a remarkable rejuvenescence results from a fast prolonged to such a degree as to reduce the creature to a fraction of its original proportions. These experiments, however, have been made only upon animals of the lowest orders, and there is so far no evidence that a similar change can be effected in human beings. However, the observation has been made that after a prolonged fast, when the body has been built up by proper feeding, there is apparently present 
an unusual degree of vigor and an enhanced sense of well-being. It is to be noted, however, that a similar observation is often made following recovery from typhoid fever or some other acute wasting disease in which the patient has been greatly reduced. It is to be further noted, also, that notwithstanding this apparent rejuvenation accompanying convalescence from fevers the life expectancy of such persons is only one-half that of the average person of the same age. Hence, there is ground for believing that, notwithstanding the apparent improvement resulting from the fast as well as from the fever, a certain constitutional damage is done, the effects of which become apparent later. It is certainly irrational to suppose that great benefit can come from prolonged and painful resistance of a natural instinct with which the body is endowed by Nature for its protection by securing the prompt and regular meeting of its essential requirements. Hunger is a sensation through which Nature serves notice upon the consciousness that the energy resources of the body are running low and need to be re-enforced; in other words, that food is needed. Thirst is a sensation which notifies the intelligence of the need of water. On purely a priori grounds it would seem to be highly unreasonable that man should use his intelligence to thwart this means of automatic defense of the body against injury.

Arguments could be offered in favor of water fasting as well as of food fasting. Indeed, why should not one restrain himself from gratifying the sense of air hunger which prompts constant rhythmic action of the lungs? Air hunger, water hunger, and ordinary hunger are simply Nature's demands for supplies of different kinds of foods which the body needs for its protection and the maintenance of its functions.

When food fasts were practiced by the empiric Priessnitz at Gräfenburg in Germany more than a century ago, a neighbor set up on the opposite side of the mountain a rival establishment at which water fasting was the method employed, and Dr. Schrott, the founder of this absurd method, claimed as wonderful cures as those which have been exploited by the fasting faddists of recent years. Furthermore, notwithstanding the apparent advantages which have been mentioned above as possible results from fasting, it is evident that there must be certain disadvantages; and when these have been considered, it will be found that the disadvantages of prolonged fasting very far outweigh any advantages which may be derived therefrom. And not only this, it will 
also be found that all the advantages derived from complete abstinence from food, the "absolute diet" of the French, may be more easily and more comfortably obtained by a scientifie regulation of the diet and without any of the dangers and disadvantages which attach to the fasting method.

\section{Objections to Fasting.}

Here are some of the serious disadvantages which are properly attributable to fasting, and the force of which cannot be denied.

1. Fasting produces a harmful destruction of the "noble" tissues of the body; that is, while a prolonged fast rids the body of surplus fat and of accumulated wastes, it at the same time involves a notable wasting of the muscles, including the heart, and shrinking of the liver, kidneys and other tissues, a marked reduction of the blood volume and destruction of the blood corpuscles and a great loss of the essential salts, lime, iron and other materials of which the bones, brain and blood are robbed.

2. The body contains, almost wholly in the blood, about forty-five grains of iron. More than a fifth of a grain of iron is lost daily. The life of the blood cells is about six weeks. When the cell dies it gives up its iron, which is eliminated in the bile by the liver and becomes a waste product. Experiments upon animals have shown that the body loses 40 per cent. of its store of iron within three weeks when the daily intake of iron is wholly suspended. In a fast of four or five weeks the body would lose more than half of its blood iron, and must necessarily likewise lose a proportionate amount of its oxygen-carrying capacity. In like manner the body, when fasting, daily loses a certain amount of lime, which in the course of several weeks amounts to a quantity sufficient to rob the bones and the blood of an element essential to their integrity.

3. The body is also continually losing vitamins which are essential for the promotion of the processes of repair and the maintenance of the various vital functions. The daily supply of vitamins is as necessary as the daily supply of air and water. Vitamins cannot be produced by the animal body. They are an exclusive product of plant life. The liver hoards a small store of vitamins sufficient to serve in emergency, but the supply is not sufficient to last indefinitely and, as shown by the experience of sailors who contract scurvy and those who become the victims of 
beri-beri through living upon polished rice, the vitamin store of the liver soon becomes exhausted and the body then falls into serious disorder. Fasting deprives the body of vitamins and thus involves the risk of serious injury for which no adequate compensation is offered. The total loss of all vitamins must certainly involve greater damage than the loss of one only, yet the absence of but one of the three known vitamins for even a short period produces noticeable injury. Certainly the body can be in no way benefited by the deprivation of food iron, food lime, and other food salts, and of the precious vitamins, the activators of the vital processes.

4. A fasting person is starved only in a conventional sense. As a matter of fact, his body feeds as usual, but instead of obtaining his food supply from outside sources, it feeds in part upon reserve fat which has been laid up in store for the purpose, and in part upon the living machinery of the body, muscles, nerves, brain, glands, etc., as already noted. In other words, a fasting person feeds upon himself, upon animal flesh, a strictly carnivorous diet. $\mathrm{He}$ is not only a flesh eater but a cannibal and even worse, he is autophagic. The effect of a strictly flesh diet is always unwholesome. Even the dog suffers physical impairment when fed exclusively upon flesh. So must the flesh-feeding faster.

5. Examination of the blood of a fasting person shows that there are present poisonous acids, particularly beta-oxybutyric acid and diacetic acid, two highly poisonous substances which seriously impair the power of the blood to carry $\mathrm{CO}_{2}$. The cause of these poisons is the imperfect oxidation of the body fats which is always the result of a deficiency of carbohydrates. It has been shown by Naunyn and others that the body requires daily 200 or 300 calories of starch or sugar (2 or 3 ounces) to enable it to burn up the fats of the ordinary diet and when the body is compelled to subsist largely upon its own fats, acidosis quickly develops. It thus appears that long fasting, instead of clearing the body of poisons, produces new and dangerous poisons which grow out of the fact that the fasting body is compelled to feed upon itself. The free use of fruit juices and a moderate use of unsalted mashed potatoes, greens, juicy fruits, with perhaps the addition of a few ounces of malt sugar or milk sugar, will prevent the evil effects of fasting, while at the same time securing all the results desired from underfeeding.

6. It is true, as above admitted, that during a prolonged 
fast any surplus of protein which may be present in the body may be used up and accumulated tissue wastes may be lessened in amount. But the amount of such accumulations is too small to justify the expensive method adopted by the faster for their elimination. For example, Gautier has shown that the total tissue wastes or soluble salts of the tissues amount to only about 10 per cent. of the total solids. In a man weighing 150 pounds, the soft parts and viscera have a weight of about 125 pounds. Threefourths of this is water, giving less than 32 pounds as the total solids of the soft parts. Of this, 10 per cent., or 3.2 pounds, represents the maximum of excretory products and stored protein which fasting is supposed to be able to eliminate. In a prolonged fast, losses of weight of 25 to 30 or even 40 or more pounds frequently occur. It is at once apparent that the faster, in order to free himself of 3 pounds of waste or undesirable material, sacrifices an amount of healthy and useful tissue ten times as great. Why should one throw away 30 pounds of good muscle, brain, nerve, heart, blood and other useful, vital machinery in order to get rid of 3 pounds of waste material which can be easily gotten rid of by simply restricting the diet and increasing the intake of water?

The surplus protein and tissue wastes are derived exclusively from the protein of the food. Fats and carbohydrates leave no poisonous residues behind but are converted into harmless water and carbon dioxide which quickly escapes from the lungs.

It is evident, then, that all that is necessary to secure all that can be accomplished by a long fast in freeing the body from tissue poisons may also be secured by the adoption of a proteinfree dietary which will compel the body to use up its surplus protein and give it an opportunity to eliminate any surplus tissue wastes, while at the same time keeping the body supplied with fuel to furnish animal heat and muscular energy and thus preventing the necessity for the tearing down of its vital machinery and the loss of blood, vitamins and other essential elements.

Fasting is certainly the most wasteful and ineffectual method of body purification that could possibly be conceived. The daily drinking of two or three extra quarts of water, with proper exercise, and a reasonable limitation of the intake of protein and fats will accomplish even more than the fast and without incurring any of its risks.

7. But the eminent absurdity of the fast as a method of 
depuration becomes apparent when some of the conditions present in fasting are considered. Food is the natural laxative. Under the influence of food the stomach and the entire alimentary canal are stimulated to rhythmic activity through which the food is mixed with the digestive juices, spread out over the mouths of the absorbents, and when reduced to a small remnant of indigestible and unusable residues is dumped into the colon and finally dismissed from the body. When food is not taken, these rhythmic movements disappear, and no indigestible and nonabsorbable material is passed along into the colon. Consequently, the colon remains inactive. The faster argues: "I am eating nothing, hence there is nothing for my bowels to remove and no occasion for bowel movements." The argument seems conclusive but it is not. The colon has another function than that of removing food residues. A highly important and essential part of its function is the removal of the body wastes which are excreted by the liver in the bile and also extracted from the blood by the intestine itself ; in other words, the colon is an excretory organ as well as a garbage disposal plant. This excretory function has been quite overlooked by the exploiters of the fasting method. They have thought only of the food residues.

\section{Safe and Scientific Fasting.}

There are several methods of fasting by means of which one may secure all the advantages of the prolonged fast without any of the dangers and inconveniences which long fasting involves. A one-day fast now and then is found by many individuals highly beneficial in recovering lost appetite, relieving headache, dullness, depression and various other unpleasant symptoms. The fasting should be accompanied by copious water drinking. Four to six quarts is none too much. A good plan is to take a glassful of water every hour. A little fruit juice may be added to the water to give it flavor. An ounce of agar or bran should be taken during the day and two to four ounces of paraffin oil. It is also well to take, morning and night, an enema consisting of two or three pints of water at 80 to 90 degrees.

A second method of fasting which will yield excellent results is to live for a week on greens and fruit. Fresh or canned tomatoes should be used liberally with fruit juices of all sorts and melons. Twice a day a liberal serving of greens, four to eight ounces, should be eaten with large servings of lettuce and celery. 
One to two ounces of bran should be taken during the day or an ounce of agar. A couple of ounces of paraffin oil should be taken morning and night.

The chief danger from fasting is the acidosis which develops after the first few days. Usually, symptoms of acidosis, nausea, headache and lassitude make their appearance by the second or third day, due to the fact that the glycogen or carbohydrate of the body has been consumed so that the fats and proteins of the body are not well burned and the poisonous acid products resulting, are beginning to accumulate in the tissues. Recent metabolism studies have shown that by repeated short fasts the body may be trained to utilize its own tissues better, so that acidosis is postponed and is less pronounced. By this method, the patient fasts first for one or two days, then eats sparingly for two or three days, then fasts again for two or three days. After another interval of low feeding a still longer fast of four or five days may be taken without developing unpleasant symptoms. It seems probable that the body not only learns by repeated fasts to make better use of its own tissues, but also improves in its ability to store and utilize carbohydrate in the form of glycogen. This method of fasting may be employed for fat reduction in certain cases, when fasting is resorted to for clearing the body of toxins or to temporarily reduce the work of the liver and kidneys to a minimum. The protein fast by means of a protein-free diet affords the ideal method rather than absolute fasting.

\section{THE BLAND OR NON-LAXATIVE DIET}

The bland diet consists of foods which are lacking in the qualities which stimulate either secretion or motility; that is, foodstuffs which are smooth, emollient, and nearly tasteless. Condiments of all sorts are excluded, with the possible exception of salt, which may be allowed in small amount. Cane sugar and fruit acids must also be avoided. Rice gruel, oatmeal jelly, barley water, and cornstarch or arrow-root gruel, are typical bland foods. Such very bland foods are required only in very exceptional cases, as acute inflammations or burns of the mouth and throat from hot liquids or chemicals, or acute inflammation of the stomach.

In general, milk may be used in combination with the foods named or even by itself. Buttermilk with rice or cornstarch is often preferable to fresh milk. 
Purées of potato and other vegetables, mushes, porridge, cream toast, panada, and most breakfast cereals are suited to cases which do not require the blandest foods. Egg nog (yolk only), coddled eggs and custards are also allowable in many cases, as well as mildly flavored jellies prepared from agar or vegetable gelatine. Malt sugar may be used when cane sugar and other sweets are not tolerated.

There is much less frequent occasion for the use of a bland liquid diet than is generally supposed. The mere fact of frequent bowel movement is by no means an indication for a bland or non-laxative diet. Frequent bowel movement is very often due to the fact that the colon is completely filled with putrefying residues, the frequent but incomplete evacuations being simply the overflow of an overfilled reservoir. In such cases, the feeding of bran and various other food laxatives is indicated rather than a bland dietary. It seems doubtful, indeed, whether the slightest harm ever results from the use of a highly laxative diet except in cases of acute diarrhea or dysentery. Food is a natural and harmless laxative. Bulky food, even roughage, does not stimulate peristalsis by causing irritation, but by titillation. If more bulk than necessary is taken, the increased colon activity may cause some inconvenience but will do no harm, for there is no infection, no inflammation, no production of toxins, and the result will be increased sense of well-being rather than malaise and weakness, the presence of which in ordinary diarrhea is the result of the absorption of the toxins produced by disease-producing bacteria and not by the frequent movements.

The bland liquid diet is most clearly indicated in cases of feeble patients who cannot chew, in patients who are too ill to chew hard or dry food, in certain surgical cases, in cases in which tube feeding is required for duodenal or gastric ulcer, sometimes in cases of insane persons who refuse to eat and must be nourished by tube feeding, and in inflammations of the mouth.

It should be remembered that, in general, a bland diet is highly constipating. In infants and in many adults acid fruit juices and malt sugar solutions have a laxative tendency. Agar jellies are somewhat laxative. Purées of figs, dates, prunes and other fruits should be used when possible, though, in general, acid and saccharine foods must be avoided. Care must be taken to cleanse the colon twice a day by means of a warm enema. When 
colonic irritation exists, a pint of thin warm starch solution should be introduced into the colon after each enema.

All meals should be light, and frequent feedings (four to six daily) should be given if necessary.

A diet of cereal gruels is often of greatest service in cases of intestinal infection with looseness of the bowels, especially in children, not because of its bland or non--laxative character, but because it floods the intestine with carbohydrate, a kind of nutriment which encourages the growth of beneficent acid-forming organisms and discourages the growth of the putrefactive and disease-producing bacteria which are the cause of the diarrhea. The addition of milk sugar to the diet is advantageous, hastening the change of flora and so terminating the bowel disorder.

In cases of stomatitis or after burns of the mouth and throat the rice regimen is most useful. Well-cooked rice gruel or jelly will be tolerated when contact of almost any other food is painful.

\section{CARBOHYDRATE-FREE DIET}

The belief held by many, even by some physicians, that the free use of farinaceous foods is likely to give rise to certain forms of dyspepsia, gout, rheumatism and other disorders, has no scientific foundation. This doctrine, much more widely current during the latter part of the last century than at the present time, was largely due to the teaching of Dr. Salisbury, who held that the free use of starch foods led to the development of yeast in the intestine and in the blood. These migrating yeast cells he held to be the cause of tuberculosis, Bright's disease and various other maladies. He prescribed a starch-free diet, consisting largely of lean meat, for the purpose of starving out the dangerous yeast cells.

On one occasion the writer, having been called to Cleveland to see in consultation a patient who had for some time been under the care of Dr. Salisbury, had an opportunity to see a demonstration of the so-called yeast cells in the blood by an assistant to Dr. Salisbury, his nephew. The demonstration consisted of the exhibition of a mass of disintegrated white blood cells collected together and crushed by a peculiar method of manipulation, but which the demonstrator claimed to be yeast cells. The writer suggested that since yeast is a living organism and will grow if planted, this biologic test should be applied to the so-called speci- 
men of yeast. The proposition was agreed to but never carried out.

Nothing could be more ridiculous than the theory upon which the Salisbury method was based, and it is surprising that this pernicious teaching still survives in current treatises on diet. Starch is the most easily digestible and the least likely to do injury, even when eaten to excess, of any of the several food principles.

An excessive intake of fat causes slow digestion and soon gives rise to a condition known as biliousness, which is caused by an unusual degree of putrefaction in the colon. In children, an excess of fat is likely to give rise to acidosis.

An excess of protein overtaxes both the liver and the kidneys, and promotes intestinal autointoxication from the putrefaction of food residues. An excess of starch, however, leads to no greater damage than an undue accumulation of fat. That a farinaceous diet can in any way be responsible for gout, rheumatism or even diabetes is an implication against this highly inoffensive foodstuff which has no scientific support.

A carbohydrate-free diet is called for in only one class of cases, namely, persons suffering from diabetes, and then only as a temporary expedient to clear the urine from sugar by reducing the blood sugar. The prolonged exclusion of carbohydrates from the dietary is not only unnecessary but is highly dangerous, and is the most certain method of producing acidosis, a condition which is wrongly attributed to the use of carbohydrates. It is impossible to maintain the body in health without a daily intake of at least two or three ounces of carbohydrates.

\section{FAT-FREE DIET}

The researches of Hindhede show conclusively that fats may b. practically eliminated from the diet without danger. He kept a man in good health for twenty-three months on a diet consisting of bread, potatoes and greens, all fat being eliminated except that naturally found in the three foodstuffs named, certainly a very small amount. Dr. Hindhede stated, however, in a letter to the writer, that the subject of his experiment found it necessary to eat large quantities of greens, doubtless to supply the needed fat soluble vitamin, lime and iron, all of which are deficient in bread and potatoes. 
Persons who have a tendency to take on fat readily should avoid the use of butter and cream and should reduce the fatintake to a minimum. A certain amount of fat seems necessary to produce a sense of satisfaction or satiety after meals.

Those whose stomachs produce a deficiency of hydrochloric acid should avoid the free use of fats, since fats tend to discourage the production of gastric acid, as shown by Pavlov.

The free use of fats is also to be avoided by those whose stomachs empty slowly because of lack of nerve tone. Fats should be used sparingly in cases of achylia because of the tendency to intestinal putrefaction due to lack of gastric acid, a tendency which is increased by the free use of fats.

\section{DRY DIET}

(See also page 990)

In this diet, the consumption of fluids is limited to fifteen ounces daily. It is recommended by Von Noorden, Oertel and others in cases of senile heart, fatty heart, beer drinker's heart and all cases in which there is a very pronounced myocarditis or loss of muscular tone with ascites or general dropsy and passive congestion of the liver. In the writer's opinion this severe restriction of fluids is a drastic measure which must be used with great caution and never for more than a few days consecutively. By careful hydriatic treatment the congestion of the liver and kidneys may be in most cases so far overcome as to permit the passage through the tissues of a sufficient amount of water to allow a daily intake of two or three pints. This may be accomplished by fomentations over the liver and kidneys and by the moist abdominal bandage worn continually and changed every two or three hours. If the heart is very weak, a cold compress $\left(60^{\circ} \mathrm{F}\right.$.) should be placed over the heart and chest during the application of the fomentation and each alternate hour during the day. When fluids are too long restricted, there is likely to be an accumulation of waste products in the blood and tissue fluids, the result of which is necessarily harmful.

It is highly important to remember that when the quantity of fluids is greatly restricted, salt must be withdrawn entirely (see Salt-Free Diet).

Deep breathing should be practiced hourly for two to five minutes, to aid the hepatic circulation. 
The diet should consist of thin slices of well toasted graham bread with butter, baked potato, spinach, and other greens, blanched almonds, hazelnuts, oatmeal mush, boiled rice and other foods of high caloric value, with lettuce and celery. The small amount of liquid should be taken in quantities of three ounces at intervals during the day.

\section{THE POTATO REGIMEN}

The potato regimen consists of potatoes served in various forms, baked potatoes, steamed potatoes, potato soup or porridge, and potato purée. A little salt may be taken with the potatoes but not much other seasoning should be added. A little cream or savora gravy is most suitable. In addition to potatoes this regimen may include greens of any sort. Among the best are New Zealand spinach, narrow or curly leaved dock, chard, beet tops and turnip tops. Cantaloups, cucumbers, lettuce, celery and tomatoes are also admissible.

All these garden products are rich in soda and potash, which increase the alkalinity of the blood and tissue fluids and so antagonize acidosis and increase the solubility of the uric acid and other wastes which are likely to occur in excessive quantity in the tissues of sedentary persons and those who have been high livers and in all sufferers from chronic constipation and the resulting intestinal toxemia. Hindhede believes that by following a potato diet for some little time the size of impacted renal calculi or gravel may be diminished and that they may thus be gotten rid of. The writer has several times observed the passage of renal calculi which had lodged in the ureter, after putting the patient on a potato diet accompanied by water drinking, water being given to the extent of three or four quarts daily. This method is certainly well worth trying in cases of this sort and may be adopted in all cases of so-called uric acid diathesis.

If necessary, the potato regimen may be followed for a long time. This has been shown by Hindhede, who fed a young man exclusively on potatoes and fat for several months. In a research conducted at Columbia University by Miss Lenna Cooper, in which the diet consisted exclusively of potatoes and clarified butter, the nitrogen balance was maintained on a protein intake of only one and one-fourth calories of potato protein a day per pound of body weight, from which it is evident both that this 
small amount of protein is sufficient and that the protein of the potato is of very good quality.

Dr. Leipiner recently reported (1919) in the German medical press remarkable results from the feeding of dropsical patients on a potato diet.

\section{FRESH OR RAW DIET}

\section{(See also page 991)}

Much nonsense has been talked and written by food charlatans within the last twenty years about the virtues of the raw diet. The writer has never been able to endorse the extravagant claims made by the promoters of the raw food fad, and there is no doubt that considerable harm has been done by the unscientific methods and unwarranted claims made by these false prophets whose pseudo-dietotherapy has, through the prejudice created, hindered greatly the progress of real diet reform.

It must be remembered, however, that in his original state primitive man lived upon the products of the earth as presented by the bountiful hand of Nature in forest and field. For long ages before the discovery of fire and the art of cookery our hardy and vigorous ancestors subsisted upon the natural products of the earth. Referring to the Golden Age "that fed on fruit, nor durst with bloody meals their mouths pollute:"

While earth not only can your needs supply,

But, lavish of her store, provides for luxury,

A guiltless feast administers with ease,

And without blood is prodigal to please.

There can be no doubt that the natural products of the earth afford all the necessary means for the sustenance of human beings as well as for other animals. Some years ago Dr. Slonaker, of Leland Stanford University, made a feeding experiment with rats from which he drew the conclusion that human beings cannot subsist upon natural foodstuffs of purely vegetable origin; but the researches of McCollum, of Johns Hopkins, confirmed by observations made in the research laboratory of the Battle Creek Sanitarium as well as by the personal experience of hundreds of persons now living, have fully demonstrated that life can be well sustained on a dietary consisting wholly of natural foods unchanged by any artificial process.

Butter, cream, and other dairy products may be easily eaten 
in the raw state. They are, indeed, for the most part habitually used in this way. To this list may be added green corn, certain varieties of which, particularly the Golden Bantam and a dwarf Mexican sweet corn, are more palatable and digestible when raw than after cooking. Wheat when in the milk state may also be eaten raw, and when properly served is not unpalatable. Turnips and carrots, when properly prepared, as well as lettuce. celery, cucumbers, radishes and even turnips, make very palatable as well as wholesome salads.

Milk is one of the most important of all available raw foods. Unfortunately, most of our commercial milk reaches the consumer contaminated with such a load of filth that it is scarcely safe to eat it without pasteurization or sterilization. Buttermilk, sour milk, and cottage cheese are somewhat safer than sweet milk. The best of all is certified milk, or milk produced under certified conditions.

The milk regimen owes the good results which it produces in large measure to the vitamins and food lime which it supplies.

Some of the cereals may also be eaten partially cooked. The Scotch from time immemorial have made scalded oatmeal their staple food. More than two hundred years ago a historian praised the Scotch soldiers for their wonderful endurance and military prowess, which he attributed to the fact that they subsisted upon scalded oatmeal.

Another excellent method of preparing rolled oats is to stir the meal into boiling water and remove from the fire at the ena of one minute. When prepared in this way the usual pasty quality is wholly avoided. The oat grains remain distinct like well boiled rice and may be eaten with relish and satisfaction

The American method of cooking oatmeal until it becomes a pasty mass is doubtless responsible for much chronic indigestion and is particularly promotive of constipation. When cooked in the manner above described, oatmeal becomes an excellent laxative food as well as an efficient means of promoting the growth in the colon of the protective organisms which Nature implants in the intestine at birth and which are lost by errors in diet and neglect to evacuate the bowels with normal frequency and thoroughness.

A special virtue of the uncooked dietary which was never recognized or even suspected by its advocates, and to which the writer first called attention some years ago, is due to the fact that 
uncooked starch is digested less readily than cooked starch and hence is less rapidly absorbed. As a result, when cereals are eaten in an imperfectly cooked state a certain proportion of the raw starch reaches the colon where its presence is greatly needed to encourage the growth of the lactic-acid-forming organisms, and thus prevent the putrefactive processes likely to be active in this part of the intestine.

Recent experiments by Langworthy and Deuel (1920) show that raw potato starch is digested much more slowly than the raw starch of wheat, oats and rice. So large an amount as six ounces of raw wheat starch or eight ounces of raw corn starch was found to be completely digested; whereas, when six ounces of raw potato starch was given with other food nearly one-fourth of it was found undigested in the stools. The interesting fact was noted that the raw potato stools when burned gave off no fecal odor, the odor resembling that of scorched bread.

The uncooked eggs often included in the raw diet destroy the chief advantage of this regimen, since the raw white of egg is known to be indigestible in the human alimentary canal, at least in large part, and hence must greatly encourage putrefaction in the colon.

A diet consisting wholly of fresh, uncooked fruits, vegetables and cereals greatly aids intestinal asepsis. This diet aids the change of flora and is most efficient in mantaining the change. Acidophilus buttermilk may be advantageously used.

While not to be recommended as a steady diet, the fresh or raw regimen may be resorted to temporarily as an aid in combating intestinal toxemia, constipation and obesity, for short periods as a wholesome change. The adoption of such a diet is by no means a hardship to one who does not mind taking the trouble to supply himself with the numerous varieties of delicious fruits, nuts and fresh vegetables which are now available at almost any season of year.

\section{RECTAL FEEDING}

Rectal feeding renders great service in a variety of conditions, particularly in cases of obstruction of the esophagus, inoperable cancer of the cardiac orifice or of the pylorus, in cases of ulcer of the stomach or duodenum, particularly when hemorrhages are present, and in cases in which the patient is unable to swallow 
because of coma, or because of obstruction of the mouth as the result of a burn or other injury. In certain cases of great emaciation with very feeble digestion, but with absence of appetite, rectal feeding renders service.

The use of a long rectal tube is not necessary. It presents no advantages. If the food mixture is warm, $100^{\circ}$ to $102^{\circ} \mathrm{F}$, and if it is introduced slowly, not more rapidly than one or two ounces a minute, the nutritive material will readily find its way from the rectum to the cecum. A long rectal tube offers no advantages for the reason that it cannot be passed into the bowel for more than a few inches and invariably coils up in the rectum. Only by means of the proctoscope can a tube be passed more than 6 or 8 inches into the bowel. Fortunately this high intubation is not necessary.

There has been much discussion concerning the ability of the colon to digest or to absorb nutrient material, and on this account it has been the usual fashion to use for nutritive enemata pancreatized milk or fresh pancreas suitably prepared. The writer is very doubtful whether the addition of pancreas or pancreatic products is likely to be of any material service. In the writer's opinion, nutritive enemas are of little value unless given in sufficient quantity, not only to fill the colon but to pass the ileocecal valve and enter the lower part of the small intestine. It is more than probable that this happens in those cases in which the patient appears to be well sustained or even to gain in flesh during rectal feeding. Incompetency of the ileocecal exists in a large proportion of chronic invalids and in not a few instances is unquestionably a factor in the production of chronic disease.

Rectal feeding is, then, not so very different from ordinary feeding. The small intestine is the principal organ of digestion and absorption. The stomach and the colon are organs which have comparatively little to do with digestive work. The stomach absorbs almost none at all and the colon also absorbs comparatively little. The small intestine is the great absorbing as well as the great digestive organ. It absorbs liquids daily to the extent of six quarts or more, while the colon absorbs only one-twentieth as much. The body is fed when food is introduced into the small intestine either at its upper or lower end.

In general, it may be said that malt sugar is about the only food the absorption of which through the colon can be depended 
upon. This carbohydrate may be absorbed and utilized without undergoing digestion, whereas fats and protein require digestive changes which cannot be effected in the colon. Most of the benefits which can be derived from the nutritive enema may be secured by copious injections of solutions of malt sugar, two ounces to the pint. Six or eight pints should be administered daily. The solution should be at the temperature of the body, and should be introduced slowly, the patient lying down and remaining quiet for half an hour after the introduction of the solution so as to afford opportunity for absorption.

It is an excellent plan to make use of vegetable broths, such as bean broth, vegetable bouillon and solutions of yeast extract (Savita, Marmite). By this means, vitamins and food salts may be supplied.

Nutritive enemas consisting of raw eggs, meat juice and animal broths often do more harm than good. They encourage the growth of putrefactive organisms, which give rise to noxious products, and so increase the burden of toxins with which in a large percentage of cases the patient is already overwhelmed. Malt sugar solutions encourage the growth of acid-forming organisms, which discourage putrefaction and thus help to change the intestinal flora.

In the employment of rectal feeding it is necessary, first of all, that the colon should be properly prepared. To this end the colon should be thoroughly washed out by a cleansing enema half an hour before the feeding takes place. Suitably prepared foods should be administered at intervals of three or four hours during the day. A good plan is to feed at 7:00 A. M., 11:00 A. M., 3:00 P. M., and 7:00 P. M.

Care must be taken to see that a sufficient amount of liquid is taken and retained. The amount required is at least two quarts daily. The amount of urine will be a good measure of the amount being absorbed. The quantity of urine should not fall below one quart.

Patients sometimes suffer from irritation produced by the frequent introduction of the tube into the anal canal. This difficulty may be obviated by lubricating the tube with yellow carbolated vaseline. Care should also be taken to cleanse the anal region thoroughly after each evacuation, using soap and water for the purpose. Absolute cleanliness is the best preventive of fissure and other anal troubles arising from mechanical irritation. 
The addition of lactose or lacto-dextrin to the nutritive enema is a distinct advantage in suppressing putrefaction by encouraging the growth in the bowel of the B. acidophilus and other acidforming organisms.

Boaz holds that rectal feeding is of little value and if at all beneficial is chiefly through mental effect. It supplies practically nothing but water. It should not be depended upon for anything else. Rectal feeding in case of gastric ulcer with hemorrhage is injurious because it interferes with the rest which is necessary to secure the results desired.

\section{INTRAVENOUS FEEDING}

Intravenous feeding is now practiced with great success in certain cases. This method consists in the injection into a vein of a suitable liquid containing sugar (pure glucose).

The services of a surgeon are, of course, required and the most perfect asepsis is necessary.

By intravenous feeding, acidosis may be successfully conbated when not amenable to any other measure. It is a precious resource when feeding by any other method is impossible. Its chief service is rendered in surgical cases.

\section{WATER DRINKING}

Water drinking is an internal bath; it dilutes the fluids of the body in which the cells and fibers are bathed; it purifies the body by diluting the medium in which it lives. By the free use of water the movements of the mass of liquid in which the living elements of the human body perform their work are quickened, and the stream of life runs clear and pure. It has been shown that water is absorbed from the stomach very slowly. Absorption takes place chiefly in the intestine. Absorption is stimulated, moreover, by the presence of carbon dioxide gas. The presence of mineral salts of any kind lessens the rate of absorption.

Examination of the urine shows not only that the quantity is increased by water drinking, but that the urea and other solid constituents are also increased.

Baron Liebig showed long ago (and his observations have been many times confirmed) that water drinking powerfully influences metabolism, increasing both assimilation and disintegration, but especially the former.

When the amount of water supplied to the body is insuf- 
ficient, the condition of the body becomes in some degree comparable to that of a stagnant pool; while an abundant supply of liquid so encourages its activities that it may not inaptly be compared to the flowing mountain stream. Water is not a mere mechanical conveyor of poisons out and of food in ; it is a powerful vital stimulant.

Priessnitz required very many of his patients to drink large quantities of water, many patients taking as high as twenty to forty glasses a day. The deluging practice of Priessnitz is still recommended by the German water-cure empirics and by some hydrotherapists, but cases are certainly very rare is which such quantities of cold water can be swallowed with advantage. As a measure of treatment, water drinking may be generally practiced to the extent of six or eight or even ten glasses a day with advantage, but not infrequently three or four glasses are sufficient, and there are cases in which it is more important to interdict the use of liquids by the stomach than to commend their use. In such cases water may be introduced by the enema in small quantity, to be retained.

Water drinking is especially indicated in all the cachexias and diatheses. In rhenmatism, it is useful as a means of diluting the blood so that it can dissolve and carry out of the body a larger amount of waste matters. As a means for encouraging activity of the skin and kidneys, it is always useful in this disease.

In diabetes, the free use of water is not injurious but advantageous. The blood contains an excess of sugar. All the sugar that is not oxidized must be removed from the body by the kidneys. The specific gravity of the urine in these cases is always high, indicating a similarly high specific gravity of the blood. It is evident, then, that water is needed in cases of this sort for the purpose of maintaining a proper degree of fluidity of the blood and of facilitating the removal of the unused sugar, the presence of which interferes more or less seriously with the various vital functions.

Free water drinking is also important in diabetes to aid in the elimination of acetone and diacetic acid and in preventing acidosis. Free water drinking in cases of acidosis and diabetic coma is highly important. While the free use of water in diabetes will, of course, have the effect of increasing the quantity of urine daily discharged, the amount of sugar, which is a matter of most serious importance in this disease, is not increased. Indeed, the 
amount of sugar has appeared to be somewhat decreased, and sometimes greatly lessened, doubtless as the result of the increased oxidation which takes place within the body under the influence of free water drinking.

In diabetes insipidis, the disposition to drink large quantities of water should be resisted to a moderate extent, water being taken only when the thirst becomes very intense.

In fevers, water drinking is essential as a means of aiding the kidneys and the skin in the elimination of the toxins to which the rise of temperature is due, of aiding the liver in its work of destroying the fever poisons, and promoting the reduction of temperature by causing increased evaporation from the skin.

Water drinking is of the highest importance in the treatment of acute infectious diseases of all sorts. The paralysis which sometimes occurs as a complication of diphtheria and typhoid fever and which regularly makes its appearance in poliomyelitis, as well as the nephritis which may appear in severe acute cases in nearly all of the acute infections, is due to the bacterial toxins characteristic of these disorders. The safety of the patient depends upon the rapid elimination of these poisons. This can best be encouraged by copious water drinking, which not only lessens the intensity of the effect of the poisons by diluting them, but promotes in the most effective way possible their elimination.

For a small child of two to four years, the rule should be an ounce of water every half hour, the total given each twenty-four hours amounting to not less than one quart. For a child of five to eight years, two ounces should be given every half hour. For a child of twelve years and upwards, the amount should be not less than four ounces every half hour, or half a pint per hour. In addition, an enema should be given twice a day. If the bowels move, as they should do, a second enema should be administered immediately afterward, a considerable part of which should be retained. The size of the first enema should be one to three pints, according to the age of the individual, or half a pint for a child of three or four years. The quantity of the second enema should be about half as much.

When the proper amount of water is being given, the fact will be made evident by a copious flow of urine. If the urine remains scanty and high-colored, the fact is almost conclusive 
evidence that the patient is not receiving the proper amount of water.

Fruit juices of various sorts may be added to the water to render it more acceptable to the patient. In most cases much less difficulty will be experienced in giving a small quantity of water frequently than in giving a large quantity at one time. In certain cases the water may be given in teaspoonful or dessertspoonful doses, repeating every few minutes.

In cases of chronic inactivity of the skin, cold water drinking is an exceedingly valuable measure, but it must be employed with discretion as inactivity of the skin generally means an inactive mucous membrane, so that liquids are absorbed with difficulty.

In cases of pyloric obstruction, intestinal obstructions or persistent vomiting, water drinking, while indicated as a means of relieving the general condition, is inadmissible, and water must be introduced by way of the rectum, using either small enemas or the drop method.

Copious water drinking is one of the most effective means of relieving a common cold, by aiding in the elimination of tissue poisons, the accumulation of which gives rise to the difficulty known as a "cold."

One or two glasses of cold water taken half an hour or an hour before breakfast prove in many cases an almost perfect panacea for chronic inactivity of the bowels. At a meeting of the New York Academy of Medicine many years ago, a leading physician of New York City stated that he had successfully treated more than one hundred cases of constipation by this simple means.

Chronic biliousness, which is nothing more nor less than chronic toxemia resulting from the putrefaction of food residues in the colon, requires the free use of water. Eight or ten glasses a day would be none too much in cases of this sort and the colon should be cleared by an enema twice a day. The flora must be changed.

In cases of gall-stones and infectious jaundice, water drinking is certainly indicated. The amount taken should be ten or twelve glasses a day if possible so that the liver shall be thoroughly flushed. The liver is always more or less crippled in these cases by extension of the infection and needs assistance.

In cirrhosis of the liver arising from either indigestion or alcohol, water drinking is essential as a means of aiding the liver 
and the kidneys to perform the work required of them in the removal of the large quantity of colon poisons in addition to the wastes naturally produced within the body by the physiological processes of tissue change.

In the treatment of the opium, alcohol, tobacco and other drug habits, water drinking renders important service by hastening the elimination of the drug and in aiding liver work and general oxidation.

The temperature of the water drunk should ordinarily be about $70^{\circ} \mathrm{F}$. In special cases water at $60^{\circ} \mathrm{F}$. and even $50^{\circ} \mathrm{F}$. may be employed. Very cold water is indicated only in fevers, in constipation, and in small quantities in hypopepsia.

The quantity of water taken must depend on the effect desired. In hypopepsia, one-third or one-half a glassful of cold water may be taken half an hour before eating. For inactivity of the bowels, one or two glasses of cold water should be taken on retiring at night, and as much more on arising in the morning.

Thirst is almost always an indication that water may be taken with advantage, no matter whether such use is in harmony with the established canons of hygiene or not. It must be remembered, however, that when water drinking is indicated it should be taken without reference to the sense of thirst. Not infrequently thirst as well as hunger is absent, although both food and drink may be greatly needed. It is safer to trust to the natural instincts than to pin one's faith to a theory. Almost the only decided contraindication is in connection with meals, when free water drinking prevents proper insalivation of the food.

Whenever water drinking is employed as a therapeutic measure, all the urine should be collected daily and carefully measured. In general, the amount of urine should be not less than half the total amount of water taken, or equal to the amount of water taken as such. When a large amount of water is taken, the proportion passed as urine will be greater. Aside from liquids taken at meals, the food should be reckoned as about three-fourths water. Patients often think they drink more than they do. The actual measurement of the urine is necessary as a check. When the skin is very active as the result of exercise or exposure to heat the amount of urine will of course be diminished, and the intake of water must be increased, to avoid the injury which comes from exposure of the kidney tissues to a concentrated solution of tissue wastes and other poisons. 
Minkowski has shown that, in general, the amount of water taken daily should be such as to maintain a daily output of urine amounting to three or four pints.

An excess of water in cases in which the kidneys do not eliminate water readily may give rise to a marked increase in blood pressure and may encourage dropsy. It is important to remember this in advanced cases of disease of the heart and kidneys. In such cases the intake of water must be restricted.

When the patient cannot be made to swallow a sufficient amount of water, and introduction of water through the colon fails, a duodenal feeding tube may be used with advantage. As much as ten quarts of water have been introduced daily for a week by this method.

The purest water is universally the best. Whatever beneficial effects are obtained from water drinking must be attributed to the water itself, and not to any ingredients which it contains. Mineral waters are simply diluted drugs.

In general water obtained from public supplies should be boiled; this precaution is a wise one under nearly all circumstances.

Very hard water should always be boiled for a long time to eliminate, so far as possible, the lime which it contains.

\section{HOT WATER DRINKING}

Hot water drinking is an excellent means of cleansing the stomach. The stomach, like every other cavity of the body which is exposed to infection from the exterior, needs constant and thorough disinfection.

The mouth is cleansed by the saliva and buccal mucus which not only sweep away the germs which enter the mouth from the air and in the food, but when produced from clean, healthy blood possess the remarkable property of hindering the growth of germs even though not able to actually destroy them.

In addition to this there is an active destruction of germs by the white blood cells which enter the mouth in great numbers with the several salivary secretions and the secretion poured out by the mucous glands of the mouth.

Those wonderful cells, the phagocytes, are always present in the saliva which bathes the gums and the teeth and when examined under the microscope may be seen to be filled with germs which they have captured and destroyed. 
The stomach is disinfected in a similar manner. Healthy gastric juice contains free hydrochloric acid which is a powerful disinfectant. The stomach produces daily from one-third to onehalf ounce of pure hydrochloric acid.

The hydrochloric acid, aided by pepsin, not only digests the food but disinfects it, and after the food leaves the stomach, the gastric acid disinfects the stomach itself. This is highly important as a preparation for the next meal.

Two or three glasses of water should be taken four hours after each meal, the time when the stomach should be emptied of the last remnants of the last meal. The temperature of the water may be hot, $105^{\circ}$ to $110^{\circ} \mathrm{F}$.

The quantity of water taken should be about a pint, and the best time for taking is about an hour before eating.

\section{Lavage of the Blood and Tissues.}

Flushing and washing of the blood and tissues may be accomplished by means of the duodenal feeding tube. The tube may be left in place for several days, and water may be passed in almost continuously at the rate of one and one-half pints an hour, or two or three gallons daily. The result is very free diuresis and often large watery stools. By addition of milk sugar or malt sugar in proportion of one or two ounces to the pint, any desired amount of carbohydrate food may be introduced.

\section{GAVAGE}

Feeding by means of the stomach tube or duodenal feeding tube sometimes proves to be a highly practical and successful measure of treatment. It is especially valuable in cases of gastric or duodenal ulcer, as it affords the stomach and upper duodenum an opportunity for rest. The amount of food given at each feeding should be small, not more than 3 to 6 ounces. The feeding should be repeated every two hours and the food should be introduced very slowly. In many cases the quantity should be reduced to a half ounce or an ounce and the feeding should be repeated every 30 or 40 minutes. The ideal method is almost continuous feeding by means of a syphon, which the patient can be taught to manage for himself.

The following formula has been much used by the writer and his colleagues in duodenal feeding: 


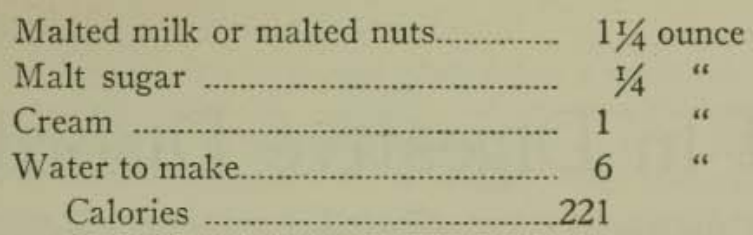

To be given six times a day at two hour intervals $(6 \times 221$ calories $=1326$ calories). After one week, increase amount of each feeding to nine ounces, which will aggregate approximately 2000 calories.

When duodenal feeding is relied upon for more than two or three days, care should be taken to include suitable iron-containing foods in proper quantity. Yolk of egg hard boiled and rubbed smooth and purée of spinach are best for this purpose. Four to six ounces of spinach and half a dozen egg yolks are required daily to supply the iron and vitamins which are especially needed in these cases. Malted nuts may be advantageously used when milk does not agree and especially in cases of anemia, this preparation containing a good percentage of iron, which is deficient in malted milk. 


\section{Diet in Digestive Disorders}

Most diseases of the digestive organs are the result of errors in diet or in eating habits; it is evident, then, that in diet and regimens are to be found the most efficient remedies for disorders of this class. It must be borne in mind, however, that the modes and times of eating and the quantities of food eaten are matters equal in importance with the quality of food. In individual cases, in fact, any one of the factors named may be the dominant influence in causing the particular disturbance present.

Furthermore, it is important to note the fact that other factors besides food profoundly influence the digestive process. The woodchopper, or the athlete, is able to manage without difficulty a dietary which would paralyze the digestive powers of a judge or a college professor. Yet there are sedentary persons who are blessed with such extraordinarily vigorous digestive organs that for many years they are able to disregard every principle of rational dietetics with apparent impunity. But judgment day is certain to come at last. These exceptional persons may escape the miseries of ordinary digestive disturbances, but Bright's disease, cancer, apoplexy, premature senility or some other degenerative disorder will sooner or later appear as the penalty of their unbiologic conduct.

Exercise, cold bathing and hydrotherapy in other forms, outof-door life and biologic or rational living in all particulars are measures which must be faithfully employed in connection with regulation of the diet in all cases of gastric or intestinal disease.

\section{GASTRIC SYMPTOMS}

As Brinton pointed out many years ago, the stomach is devoid of common sensibility. As this sagacious author observed:

"It has a special sensibility of its own; not one whit less marvelous (but surely more so) for possessing a certain independence of the cerebrospinal centre. Closely related to this centre by the feelings of hunger and satiety - nay more, dictating to it (so to speak) those exertions which the proper alternation of these two states imperiously demand from the mass of mankind-it has a sphere of action altogether its own. And the study of digestion has shown us how admirably and silently the stomach fulfills its 
various and complex tasks; and how, incidentally to these, the unfelt particle of food no sooner touches its mucous surface than it excites the flow of a variety of secretions, both far and near, and provokes movements in the muscular substance of its walls and vessels, as well as in the analogous structures of neighboring parts. To these acts, which respectively constitute the sensation and motion of the healthy stomach, its morbid states afford an instructive parallel. And just as the kind of sensibility specific to a healthy muscle-the feeling of its strength, its equilibrium, its measurable force-seems to be traceable, by gradual modifications, through healthy fatigue to the feverish soreness of over-exertion, and through this to the universal muscular pain and prostration of various grave general ailments; so the indistinct sensation of the healthy stomach affords us the best clue to trace a scale of similar kind - from satiety to repletion; from repletion to distension and weight in the epigastrium; and hence to the dull heavy aching of dyspepsia, the gnawing or burning pain of ulcer, and the sharp agony of cancer of the stomach."

\section{The Significance of Symptoms}

The nomal sensations of the stomach are,-appetite, hunger, satiety or satisfaction and repletion.

Appetite is a "conditioned reflex."

Hunger, appetite, satisfaction and repletion are indicators which measure muscular sensibility and the motor efficiency of the stomach, but give no information concerning the secreting function of the stomach.

Abnormal or diseased sensations are pain, nausea, vomiting, flatulence, regurgitation, anorexia or loss of appetite.

The stomach is devoid of all common sensibility (Brinton). The sensibility of the stomach is apparently located, almost wholly, if not entirely, in its muscular structures rather than in the mucous membrane.

Gastric pain and other discomforts such as a sinking sensation, pressure, fullness and what patients sometimes describe as flatulence are all due to exaggerations of the factors which give rise to appetite, hunger and repletion. This remark applies only to visceral pain, which must be distinguished from referred pain (Hurst). A symptom referred to as pressure in the epigastrium is intermediate between the sinking sensation and hunger pain. These sensations all come from the same cause, increased gastric tonus, the order of intensity being a sinking sensation, pressure, hunger pain. The sinking sensation is often a precursor of duodenal ulcer. As Ryle has pointed out, gastric symptoms are, in general, due to either over-activity of the muscular walls of the stomach, 
or to fatigue or exhaustion of the stomach muscles. This is true of such symptoms as hunger pain, pressure, sinking sensation and other unpleasant sensations that are relieved by the taking of food.

\section{Reversed Peristalsis a Possible Cause of Gastric Symptoms}

There is little room to doubt that most gastric symptoms are due to disturbed reflexes. The disturbance may originate either in the stomach or in some remote organ. One of the results of this reflex disturbance which has received little attention is a reversal of peristalsis, due, in many cases perhaps, to overloading of the colon and lower small intestine. Normally, as pointed out by Cannon, the residues of a meal should reach the lower colon ready for evacuation within 12 to 14 hours after the food is eaten. That is, the breakfast residues should be evacuated before retiring, leaving behind only the residues of two meals, dinner and supper. Unfortunately, the average colon that evacuates once a day retains meal residues 53 or 54 hours, and the result is the distension of the colon with a half dozen meal residues or even more when constipation exists. This overloading of the colon must be a most disturbing factor and naturally tends to give rise to inhibitory reflexes and even a reversal of peristaltic movements.

Much confusion and blundering in the dietetic treatment of indigestion has resulted from lack of accurate knowledge of the underlying causes of the most common and troublesome symptoms of so-called indigestion. Little by little modern studies of the alimentary tract by means of the X-ray and the ingenious methods of the physiologic laboratory have thrown great light on this subject.

The researches of Grützner, Bernheim, Alvarez and various others have afforded ground for the belief that many of the symptoms which are included under the general name of indigestion are the result of reverse peristaltic movements. It is now known that the reverse peristaltic movements first observed by Cannon are constant not only in animals but in human beings. These movements are normally confined to the colon. They begin in the middle portion of the transverse colon and pass backward to the cecum, their purpose being to retain the liquid material which enters the cecum from the small intestine in this part of the 
bowel for a sufficient length of time to permit absorption of the larger part of the water content. In constipation, and particularly in cases of colitis with spastic contraction of the descending colon, these reverse peristaltic movements are greatly exaggerated, and when the ileocecal valve is incompetent, these reverse movements push the contents of the cecum back into the small intestine.

Case and others have observed movements beginning near the lower end of the colon when this part of the bowel was distended. Case has reported cases in which the reflux has extended even to the duodenum. Treves, the London surgeon, reported a case in which castor oil and water colored with an aniline dye were vomited within ten minutes after they were introduced by enema into the colon. The surgeon thought such rapid movement of liquids from the rectum to the stomach must be impossible without the existence of a fistula connecting the stomach with the colon. He accordingly operated with expectation of finding a fistula, but to his surprise found the stomach and intestines to all appearance perfectly normal. A number of other similar cases have been reported. Evidently, incompetency of the ileocecal valve must have existed in the case cited. This condition is probably always present in cases of chronic gastro-intestinal disease and infections of the gall bladder and biliary passages which Lord Dawson and other eminent medical authorities hold may result from extension of infections of the colon and appendix. Boldyreff has demonstrated the transmission of substances from the rectum to the duodenum and stomach in dogs and human beings.

There is accumulating evidence, however, that a very slow, almost imperceptible reverse peristalsis is an exceedingly common phenomena and is the real cause of a majority of the symptoms associated with the stomach. These reverse movements may be caused by various morbid conditions, especially by chronic appendicitis, disease of the gall bladder, duodenal ulcer, pregnancy, diseased conditions of the womb and ovaries in women and of the bladder and prostate in men; but the most common of all causes appears to be the accumulation of food residues in the colon. Chronic constipation, or intestinal stasis, is thus being shown to be the cause of most of the disorders of the alimentary canal as well as the origin, through intestinal toxemia, of a vast number of general disorders. Alvarez inclines to the opinion that 
the disturbances caused by fecal accumulations in the colon are chiefly of a mechanical nature, while others hold to the view that the principal factor is the absorption of poisons resulting from the putrefactive changes which are always associated with delay of food residues in the colon. The probable truth is that both of these factors are active. It is certain that relief of the constipation and changing the intestinal flora are the most effective means of combating many of the most annoying symptoms connected with the stomach and intestines as well as the various conditions commonly attributed to intestinal toxemia or autointoxication.

\section{Vomiting.}

Vomiting is undoubtedly the result of a reverse movement of the intestine. During X-ray observations, peristaltic waves have been seen to pass from the duodenum to the stomach, and it is impossible to account for the vomiting of fecal matters and substances introduced experimentally into the rectum except by the occurrence of reverse peristalsis. It was even noted by Hippocrates that purging usually ceases when vomiting begins. Purging has even been treated by the administration of emetics, and it has been found possible to nourish pregnant women suffering from prolonged vomiting by introducing nutritive enemas, which were doubtless carried from the colon into the small intestine. Feeding in this manner will of course be facilitated by incompetency of the ileocecal valve, which exists in a large proportion of patients who have long suffered from constipation. Regurgitation, as Alvarez suggests, is simply mild vomiting. The reverse peristaltic waves are sometimes too slight to produce the violent movements of vomiting, in which case only small quantities of liquid are raised from the stomach to the mouth. Distention of the colon causes it to give rise to reverse waves of sufficient intensity to reach the stomach, although with a force barely sufficient to lift from the stomach to the motth small quantities of the stomach contents. The material lifted to the mouth may be either fresh food in which the process of digestion has not yet been begun or highly acid gastric juice from the lower part of the stomach, or even duodenal fluids, bile and pancreatic juice which, when the pylorus is open, may be carried upward along the lesser curvature of the stomach to the esophagus. 


\section{Nausea.}

This symptom is due to relaxation of the gastric walls. Under the X-rays the lower border of the stomach was seen to drop an inch when the subject was nauseated by a bad odor. Disgusting sights or associations may cause nausea, and fainting is often accompanied by nausea. Fats may cause nausea in persons with gastric hypotonus and poor appetite, especially in hot weather. It is common in chronic gastritis, in gall bladder disease and in anemia. It often results from severe pain. It may be caused by diseases of the eye or of the labyrinth of the ear.

The nausea of pregnancy is a perversion of hunger and may be relieved by food.

Many persons experience a sensation of nausea when the interval between meals is too greatly prolonged. In such cases the cause of nausea may be the sudden relaxation which occurs at the end of the hunger contractions.

Nausea without either vomiting or regurgitation is a common symptom of mild reverse peristalsis. The accumulation of fecal residues in the colon is undoubtedly a very common cause of nausea, although it is entirely possible that the toxins absorbed from putrefying residues in the colon may also give rise to nausea.

\section{Sense of Fullness and Discomfort}

Various minor gastric discomforts described as fullness, heaviness, "sinking" sensation, "goneness," tension, etc., are due to the same cause as pain, that is, increase of gastric tonus, but much less in degree than when pain is present. These symptoms are usually relieved by taking small quantities of food. The bill of fare should be made up of simple, easily digestible foods, carefully excluding exciting and irritating articles, such as condiments, tea, coffee, meats and meat extracts. Over-eating and hasty eating are particularly harmful, and eating when worried, apprehensive, or "nervous." A half hour's rest before meals is advisable. In taking food between meals, fat should be avoided, also milk and confectionery. Ripe, juicy fruits, fruit juice or a little rice or gruel will tax the stomach least and hence will least interfere with the next meal.

Chronic dyspeptics often complain of difficulty in eating because of a "sense of fullness," which appears shortly after a few 
mouthfuls of food have been eaten. Cannon, Carlson and others have shown how that the normal stomach gradually enlarges to accommodate the increasing volume of contents during the intake of food. Any interference with this reflex may cause an increase of tension in the gastric walls and the abdominal muscles which are normally influenced by it. Distention of the colon may well give rise to this condition. In duodenal feeding the too rapid introduction of foods will quickly develop the sense of fullness in the stomach although no food whatever has entered the stomach. It is quite possible that disease of the gall bladder, ulcer of the duodenum or a chronically inflamed appendix may be a cause of these reverse movements; but in the great majority of cases chronic constipation may be held responsible for this condition. By waiting a few moments, especially by diversion of the mind from the condition present, as by pleasant conversation or the reading of the morning paper, the symptom will pass off and eating may be resumed. Worried mothers and mentally depressed business men often complain of this symptom. In these cases the explanation may be easily found in the increased "tension" caused by the mental state.

\section{Lack of Appetite}

Lack of appetite or aversion to food is due to low gastric tonus or to rigidity of the gastric walls. It may be the result of fatigue. The gastric muscles may become tired as well as other muscles. General loss of tone due to loss of sleep, exhaustion, worry, fever, mental depression or intestinal toxemia may cause loss of appetite.

\section{Belching-Aerophagia}

There is naturally air in the upper part of the stomachthe air bubble. Air is swallowed with the food. In disease, gas in the stomach is usually atmospheric air which has been swallowed.

\section{Belching.}

Alvarez has discribed three different kinds of belching. (1) A slight gurgling sensation felt in the esophagus; (2) the eructation of gas from the stomach; and (3) a movement of air downward into the stomach or a voluntary or semi-involuntary swallowing of air.

Instead of seeking relief by the swallowing of air, persons 
suffering from this inconvenience should obtain temporary relief by sipping a glassful of very hot water, perhaps with the addition of a little soda. This will open the upper orifice of the stomach and allow the escape of disturbing liquid or gas and permit the reverse waves to run out as Alvarez suggests, and thus bring relief; but at the same time the real cause of the trouble, which probably exists lower down in the bowel, must be sought out and removed.

\section{Heartburn}

Heartburn, waterbrash, and regurgitation are symptoms of gastric disorder, but are located in the esophagus rather than the stomach (Ryle).

Heartburn is a burning sensation felt behind the lower end of the sternum and is probably caused by a spasm of the cardiac sphincter of the stomach. It is not produced by introducing acids into the stomach and occurs in persons whose stomachs produce no acid. Soda relieves heartburn by producing gas which increases the size of the air bubble and causes the sphincter to relax.

\section{Waterbrash}

Waterbrash is regurgitation of a clear, waterless fluid, consisting of water and mucus, which has accumulated in the esophagus because of spasm of the cardia. It sometimes occurs in duodenal ulcer.

\section{Regurgitation}

This symptom is often confused with heartburn. It frequently occurs in cases of hyperhydrochloria.

Regurgitation of food is due to relaxation of the cardiac orifice. It may be caused by general exhaustion, low tone, overeating, or accumulation of food in the esophagus from spasm or obstruction of the cardiac orifice.

Belching is most likely to occur with gastric hypertonus. The patient experiences a sense of fullness in his stomach high up under the sternum, across the chest or at the angle of the left scapula, which is sometimes described as a bursting sensation, due not to distension of the stomach, but to excessive tension of the gastric walls. He seeks relief by belching, but gulps or swallows air into his stomach instead. The taking of air into the stomach may cause a relaxation of the gastric walls or permit 
some air to escape as the cardiac orifice is opened, and so affords temporary relief.

Belching is frequently present in cases of duodenal ulcer or gall bladder disease. It may also be caused by chronic or acute sore throat. Patients undergoing general anesthesia sometimes gulp air in such quantities that it becomes necessary to evacuate the gas by means of a stomach tube. The writer has more than once met with this difficulty when operating on the stomach.

Belching is most frequent and troublesome in cases of socalled nervous dyspepsia. It sometimes results from insufficient chewing of the food, especially in persons who have lost their teeth. Painting the throat with iodine will induce air-swallowing or aerophagia.

\section{Globus.}

This very umpleasant sensation felt in the esophagus is known to be the result of violent contraction of the muscular tube. It seems to be the result of the meeting of two peristaltic waves, one coming from above and the other trom below. Hysterical patients suffer from globus more than others because they are universally constipated and hence the subjects of pronounced reverse peristalsis. Globus is sometimes a result of an attempt to swallow when a wave or regurgitation is passing upward.

\section{Biliousness.}

There is probably no simple explanation of the condition known as biliousness. Many observations made by the writer lead him to the conclusion that this condition is, in many cases at least, due to an accumulation of toxins in the body fluids. The dingy skin and dull eyes, the general malaise, the mental depression and confusion, lack of energy and endurance are all indications of a toxic state. The great relief which follows the removal of a quantity of bile, either by purging or by means of the duodenal tube, suggest that one source of the toxemia may be a concentrated condition of the bile which, as shown by Lauder Brunton and by Schiff, may by re-absorption become abnormally concentrated, that is, overcharged with toxic substances. This condition naturally is one of the results of intestinal stasis or constipation. The long delay of the excretions in the colon affords an opportunity for excessive re-absorption. Persons who have no gastric acid or too little gastric acid are particularly subject 
to biliousness. This may be in part due to the lack of gastric acid, one of the important uses of which is to precipitate or render insoluble the various toxic substances found in the bile. A lack of the disinfecting properties of the gastric juice may also be a contributing cause since it permits the entrance of putrefactive bacteria into the intestine and the consequent excessive production of putrefactive poisons.

It is entirely possible, also, as suggested by Alvarez, that reverse peristalsis may be a strong factor in the production of biliousness. There is strong indication of this in the fact that nausea, vomiting, and especially vomiting of bile, are common symptoms in severe attacks of biliousness.

Numerous experiments have been made for the purpose of finding substances which may be used to stimulate the formation of bile by the liver. It was long supposed that calomel increases the activity of the liver, but Bennet, of Edinburgh, proved the falsity of this claim more than a hundred years ago. It has been found by actual experiment, in fact, that nearly all of the drugs supposed to increase the activity of the liver actually diminish its activity. According to Meissner,* this effect has been noted from the use of cod liver oil, experiments appearing to prove that this commonly used drug lessens the production of bile by the liver.

This may account for the injurious effects upon rats noted by MeCollum when too large doses of cod liver oil were given, and caution in the use of this remedy may be necessary. It is to be remembered, however, that cod liver oil is used in place of butter by the natives of Iceland in the winter season and apparently without ill effect.

\section{Coated Tonguse and Foul Breath.}

These conditions which accompany biliousness are doubtless in part due to a general lowering of vital resistance with depreciation of the germicidal properties of the blood serum, as a result of which the saliva loses to a certain extent its power to prevent the growth of bacteria in the mouth, which appears to be one of the important functions of this secretion. It appears, however, from the experiments of Grützner and others that the coating of the tongue, which is known to be due to the growth of bacteria, may be the result of reverse peristalsis. Grützner showed that bacteria and lycopodium introduced into the rectum were within a 
few hours carried to the mouth. Examinations of the tongue coatings, made in the bacteriological laboratory of the Battle Creek Sanitarium several years ago and again recently, show that in many cases colon bacilli are found present in large numbers. Since it is known that bile frequently enters the stomach during sleep, and since it is also known that colon bacilli are frequently found in the bile, it will readily be seen that these pernicious organisms may easily find their way by regurgitation to the mouth, and if the saliva has lost its normal power to inhibit the growth of these organisms the natural result will be an unusual development and the densely coated tongue and foul smelling breath which are found in persons suffering from the condition commonly known as biliousness.

\section{Gastric Pain}

Gastric pains are of three sorts: (1) Immediate pain whieh develops soon after eating; (2) Delayed pain which develops later when the stomach is emptying; (3)Pain not associated with eating, due to extragastric causes.

Visceral pain is due to perverted function rather than to structural disease.

Immediate pain is produced by peptic ulcer. Delayed pain is associated with duodenal ulcer.

\section{Abnormal Peristalsis}

The patient experiences griping or colicky pain. This may be the result of indigestion from the taking of unwholesome food. It is most severe when due to obstruction of the pylorus or the upper part of the duodenum. Visible peristalsis is always due to obstruction of the pylorus.

The X-ray evidences of abnormal peristalsis are: (1) increase in the number and depth of the waves; (2) irregularity in the occurrence of the waves; (3) antiperistalsis; (4) absence of peristalsis.

Hyperperistalsis may be the result of a reflex from a diseased gall bladder or a diseased appendix, conditions which are supposed to produce spasm of the pylorus.

Peristalsis disappears after long standing obstruction and in cases of organic changes which cause rigidity of the gastric wall, as in leather bottle stomach. 


\section{Gastric Motility}

The best means of testing gastric motility is by means of the gruel meal. A delay beyond three hours is likely to be caused by obstruction from some cause.

The clinical signs of obstruction are vomiting, visible peristalsis and enlargement of the stomach. Vomiting may be due (1) to irritation of the mucous membrane of the stomach; (2) general fatigue; (3) gastric fatigue from overeating; (4) poisoning; (5) lesions of the central nervous system; (6) mental disturbance; (7) spasm or organic obstruction of the pylorus or duodenum; (8) hour-glass stomach; (9) obstruction lower down in the intestinal tract.

Vomiting is due to closure of the pylorus, pressure of the diaphragm, contraction of the abdominal muscles and sometimes reverse peristalsis.

Rapid emptying of the stomach may be due (1) to duodenal ulcer with hypertonus; (2) to cancer with rigid pylorus; (3) achylia gastrica with pyloric insufficiency; (4) gastro-jejunostomy, partial gastrectomy, Polya operation.

The symptoms of rapid emptying are a sinking sensation or hunger pain developing unusually soon after eating in case of duodenal ulcer.

In the gastrogenous diarrhea which occurs in achylia gastrica and Addison's anemia and in abdominal discomfort after eating following gastro-jejunostomy, the X-ray shows evacuation of the stomach in such cases in two hours or less.

In cases of hypermotility, or too rapid emptying of the stomach, starch disappears after a gruel meal in half an hour to one hour.

When the hypermotility is due to hypertonus, the acid curve is high. If it is due to a patent pylorus the curve is low or there may even be achlorhydria. It is possible, also, that a hyperchlorhydria may exist with a hypomotility, there being nothing in the stomach to dilute the gastric acid. 


\section{DIET IN HYPERCHLORHYDRIA OR HYPERACIDITY}

(See also page 992)

This, one of the most frequent and important of all functional disorders of the stomach, was the despair of therapeutists until the researches of Pavlov made clear the nature of the difficulty and pointed out rational methods of cure. The pain of hyperhydrochloria usually occurs two or three hours after eating, but may occur during the meal or immediately after. In many cases there is constantly present in the stomach a larger or smaller quantity of intensely acid secretion. The gastric crises which occur in locomotor ataxia and certain forms of migraine and in connection with certain other maladies are usually associated with hyperhydrochloria.

Haneborg claims there are three kinds of gastric secretion:

1. The psychic secretion produced by the sight, smell and taste of food. Less pronounced in man than in animals.

2. Secretion produced by physical contact of water and all kinds of foods with the gastric mucous membrane.

3. The chemical secretion produced by certain substances which, when absorbed, produce a gastric secretion, alcohol and xanthin.

The acidity of the gastric juice is regulated by the reflux of bile and pancreatic juice from the duodenum, also by the alkaline saliva and the alkaline secretions of the pyloric region of the stomach. Campbell thinks that the chief importance should be attached to the alkaline secretion, or pyloric part of the stomach.

It is necessary to estimate the chlorids of the gastric juice to determine whether hyperacidity is due to excessive secretion or to failure of neutralization. It is also necessary to determine when free $\mathrm{HCl}$ is absent whether the absence is due to achylia or to excessive neutralization.

Ryle holds that the important factors in causing hyperacidity are:

1. Excessive secretion.

2. Pyloric hypertonus which prevents duodenal reflux and prolongs the stay of the foodstuffs in the stomach, thus giving opportunity for the accumulation of acid.

3. Rapid emptying of the stomach, causing increase of acidity because of rapid diminution of the gastric contents. 
Experiments of Carlson and others show that the pyloric closure may be produced by stimulating the duodenum, not only with acid but with fats, alkalies, or mere mechanical contacts.

Every tonic phase of the stomach is followed by relaxation, so that its tension falls below that of the duodenum, thus giving opportunity for regurgitation. Closing of the pylorus is probably never more than momentary. When apparently closed, a small opening remains sufficient to allow passage of fluids to and fro.

The most common cause of hyperchlorhydria seems to be failure of the pylorus to open sufficiently to permit the usual degree of neutralization by the pancreatic juice (Boldyreff, Ryle).

Ivy has shown that hypersecretion may result from putrefaction in the small intestine. This condition is most pronounced when there is an excessive intake of protein combined with stasis in the colon and incompetency of the ileocecal valve.

Fuld has called attention to the fact that constipation is both a result and a cause of hyperchlorhydria, and Ebstein has shown that hyperchlorhydria is improved by relief from constipation.

Professor Taylor, of Philadelphia, mentions the fact that certain experiments have shown that absorption of poisons from the colon may excite an excessive production of gastric acid.

Roger, who made an elaborate study of the toxins produced in the alimentary canal under various conditions, demonstrated that one of the functions of the stomach is to excrete poisons from the blood. He holds that these poisons may be derived from intestinal putrefaction. One of the functions of the stomach seems to be to eliminate the poisons absorbed from the intestines. Roger found the toxicity of the contents of the stomach of the vegetable-feeding rabbit to be only one-third as great as that of a dog fed on meat.

It appears to the writer that the evidence is very strong that the intestinal toxins excreted by the stomach are a leading factor in the production of hyperhydrochloria, and that the excessive secretion of hydrochloric acid is a part of the defensive effort of the body in combating these toxins. It has been shown that the gastric juice destroys a considerable number of the enterotoxins, and it may be for this purpose primarily that the excess of acid is poured into the stomach.

In the treatment of hyperhydrochloria it is evident, then, that the first and most essential thing to be done is to combat 
intestinal autointoxication. The writer's experience in the practical application of this theory to the treatment of cases of hyperhydrochloria has been most satisfactory. But there are many other causes of hyperacidity which must be taken into consideration.

The causes which may give rise to hyperacidity are numerous. The following may be mentioned as those which are best known:

Meats. Pavlov showed by experiments upon dogs that meats, that is, animal flesh, fish, fowl and shellfish as well as ordinary meats, when taken into the stomach cause the production of highly acid gastric juice. The normal gastric juice produced by dogs is for this reason much more acid than that produced by human beings. It is interesting to note that when a man eats the diet to which a dog's stomach is naturally adapted rather than his own proper bill of fare, his gastric glands are stimulated to meet the emergency by producing a gastric juice like that ordinarily produced by the dog. Protein requires a highly acid gastric juice for its digestion. This is doubtless due to the fact that protein absorbs hydrochloric acid, entering into combination with it as does soda and other alkalies. For this reason persons suffering from hyperacidity are usually made very comfortable by a meal consisting largely of meat. This fact is deceptive and led to the practice of feeding patients suffering from hyperacidity on a meat diet. Prior to the publication of Pavlov's epoch-making book on the digestive glands, scraped beef, raw beef and meat in other forms as an exclusive diet, so-called Salisbury steaks, was the universal remedy for hyperacidity. A meat diet gives temporary comfort in these cases by neutralizing the hydrochloric acid but at the same time stimulates the gastric glands to produce an increased quantity of acid.

Meat Extracts. Pavlov's observations also demonstrated the fact that the extractives of meat stimulate the acid-forming glands of the stomach to a very high degree. Hence bouillon and animal broths of all sorts must be discarded in the treatment of hyperacidity.

Milk. Next to meat, milk, including cheese and other dairy products, stimulates the acid-forming glands of the stomach, this action being due to the large percentage of protein present in milk in the form of casein. 
Cane Sugar. Of all the sugars cane sugar is least well tolerated. Boldyreff has shown that cane sugar does not excite the gastric glands to activity, but clinical experience clearly shows aggravation of distress in cases supposed to be suffering from hyperhydrochloria. The cause must be an increase of hypertonus due to the irritation which it certainly produces as shown by the experiments of Ogata. Malt sugar rarely gives inconvenience even in cases which are highly sensitive to cane sugar.

Dextrin. Pavlov showed that crude dextrin is a powerful excitant of the gastric glands. This fact affords an explanation of the frequent occurrence of heartburn as the result of the free use of oatmeal in the form of porridge and other cereals eaten in the pasty condition resulting from long boiling. New bread is objectionable for the same reason, the central or soft portion of the loaf being often swallowed in doughy lumps which reach the stomach without being acted upon by the saliva.

Raw Starch. It has recently been shown by Marshall that undigested starch in large amount in the stomach absorbs the pepsin of the gastric juice and thus prevents the acid from entering into combination with the protein of the food and so increasing the free hydrochloric acid.

Highly Flavored Foods. These create an excess of highly acid appetite juice by over-stimulation of the gustatory nerves.

Hot Foods. These over-excite the gastric glands, congest the stomach, and when excessively hot may even give rise to injuries of the gastric mucous membrane which may ultimately cause ulcer and, according to Dr. Willjam Mayo, may become a cause of cancer. While over-production of gastric acid under the influence of heat has not been demonstrated by laboratory experiment, there are clinical reasons for believing that excessively hot food and drinks do produce this effect.

Ices. When ices are taken with the food they interrupt the process of digestion and delay the exit of the food from the stomach, thus giving rise to the production of an excess of free acid.

Water. Pavlov showed that when water is taken in more than very small quantities, gastric acid is increased. Hence, free water drinking at meals must be forbidden for persons suffering from hyperacidity.

Fruit Acids. The citric acid, malic acid, and tartaric acid found in fruits are much less stimulating to the gastric glands 
than is hydrochloric acid, but are more or less stimulating and in most cases of hyperacidity must be on this account forbidden until the patient's condition has been improved. It should be noted, however, that in many cases the intolerance of acid fruit is due, not to an excess of acid, but to a hypersensitiveness due to a disordered condition of the gastric nerves rather than to over-stimulation of the gastric glands. The same is doubtless also true as regards the acid of the gastric juice. The distressing symptoms of which patients complain may be due not to an excess of acid but hypersensitiveness resulting from disease.

Tomatoes. These contain citric acid and, with rhubarb, a poisonous plant which contains oxalic acid, are objectionable on the same grounds as acid fruits. As patients recover, tomatoes are better tolerated, especially moderately acid varieties, but rhubarb should be definitely discarded as unwholesome and poisonous, irrespective of its injurious effect upon the stomach.

Butyric Acid. As found in "strong" butter and rancid oils, this is highly irritating to the acid-forming glands of the stomach.

Tannic Acid, found in large amount in tea, is objectionable on the same ground; it also precipitates pepsin leaving the gastric acid free and so irritating.

Vinegar. Fecause of the acetic acid present, vinegar is always unwholesome and is particularly injurious in cases of hyperacidity. It interferes with starch digestion.

Buttermilk, Buttermilk contains lactic acid and so is objectionable in severe cases of hyperacidity, though ordinarily well tolerated.

Alcohol. It has long been known that alcohol excites the activity of the gastric glands. It has been used for this purpose by physiologists in experiments upon the stomach. The observations made by Chittenden and Sir William Roberts show that while alcohol stimulates the stomach to make an increased amount of gastric juice, including gastric acid, it destroys the activity of the juice by precipitating the pepsin. This action of alcohol, by preventing the combination of the free acid with the protein of the food, renders it a direct cause of hyperacidity and necessitates its total prohibition in any form in persons suffering from hyperacidity. So-called light wines and beer are objectionable just in proportion to the amount of alcohol which they contain.

Tobacco. It is well known that smoking often gives rise to the excessive production of acid in the stomach. It has been 
shown experimentally by Gy and others, that digestion is hindered by smoking.

Caffein. This active constituent of tea and coffee directly excites the acid-forming glands of the stomach. The theobromin of cocoa produces the same effect. Tea, coffee, cocoa, and maté or Paraguay tea, are objectionable for the same reason. Kaff, Hag, though said to be free from caffein, is nevertheless objectionable because of the tannic acid and phenolic bodies which it contains, which excite the acid-forming glands besides being otherwise injurious. Most cereal coffees are harmful for the same reason.

Condiments. Under this head are included mustard, pepper, cayenne, ginger, cloves, spices, horse-radish, Worcestershire sauce, and all similar substances, which excite the acid-secreting glands of the stomach and thus produce hyperacidity. It is true that after being used for some time, the gastric secretion is lessened, but this only occurs when the glands have been destroyed by degeneration, the result of chronic irritation and overwork.

Common Salt. When taken in considerable amount, salt induces hyperacidity through interfering with digestion (Debove), by furnishing an excess of the material from which the gastric acid is made. In hyperacidity the use of salt should be restricted at least to very minute quantities.

Carbonated Water. It is objectionable in hyperacidity because the gastric glands are stimulated by carbonic acid.

Errors in the Manner of Eating. These are without doubt in a large measure responsible for hyperacidity in many cases.

Hurricd Eating permits the food to enter the stomach in large masses which, being retained for a long time, may cause the over-accumulation of acid in the stomach.

It has been shown that cooked starch, which enters the stomach without being mixed with a sufficient amount of saliva to change it to dextrin, absorbs the pepsin of the gastric juice and thus leaves in the stomach contents an excess of free or uncombined hydrochloric acid, causing acidity.

Over-Eating, by over-stimulation of the gastric glands, likewise produces an excess of acid.

Too frequent eating produces hyperacidity by giving the stomach no opportunity for rest. When food is taken into the stomach the gastric mucous membrane becomes congested and the glands are filled with blood. After the completion of the diges- 
tion of a meal, the stomach should have an opportunity for rest and complete clearance before the next meal is taken, so that the blood-vessels may be relieved. When a meal is taken before the stomach is empty, the stomach has no opportunity for rest or clearance and hyperacidity is one of the resulting ill effects.

Eating between meals is objectionable for the same reason as too frequent meals. It is rare that more than three full meals a day can be taken without injury. The only exceptions are cases in which the amount of food taken is small and the kind of food such as will leave the stomach in an hour or two. In such cases, four meals or even more may be necessary to supply the body with the proper amount of nutriment. If food is to be taken more than three times a day, it is highly important that close attention should be given to the kind of food taken and the exact spacing of the meals so that they shall not overlap. The common practice of recommending patients to take milk or other food between meals, while at meal times taking ordinary foods which do not usually leave the stomach within less than five or six hours, is strongly to be condemned.

Excessive Chewing must be mentioned as another cause of hyperacidity. Chewing, as shown by Pavlov, produces appetite juice. The stomachs of persons suffering from hyperacidity usually contain constantly an excess of acid. Consequently, in such persons it is not necessary that the stomach should be prepared for a new intake of food by prolonged chewing, as urged by $\mathrm{Mr}$. Fletcher; in fact, long chewing becomes in hyperacidity a means of aggravation of the existing morbid conditions. On this account persons suffering from hyperacidity to an extreme degree should take all their foods in the form of purées or porridges. The food should be swallowed with very little chewing. Thin soups should be avoided because they contain an excessive amount of water which stimulates acid formation (Pavlov).

Cereals and other farinaceous foods which have been well dextrinized, are best suited for use when much chewing is to be avoided. Zwieback softened with hot water or milk and such foods as Granola, Zo, Vita-Bits, Wheat Flakes, Grape Nuts and other dextrinized cereals softened with some hot liquid, are suited to cases of this sort.

Constipation. It is a quite common observation that chronic constipation is an almost universal accompaniment of hyperhydrochloria. An explanation is to be found in the fact that in 
constipation the long retention of the foodstuffs in the colon affords good opportunity for a considerable amount of putrefaction in which there is a digestion of proteins by bacteria and the production of poisonous peptones. Pavlov showed that peptone is one of the most powerful of all stimulants of the gastric glands. This is shown to be true even when the peptone is introduced per rectum through the absorption of the peptone by the mucous membrane of the colon and its excretion into the stomach. The same occurs in cases of colitis in which the filtering power of the mucous membrane is largely lost. Absorption of the poisonous peptones and other products of putrefaction takes place in still larger amounts in cases of incompetency of the ileocecal valve, in which there is a backing up of putrid materials from the colon into the small intestine where absorption takes place with great facility. This condition is very common in hyperhydrochloria.

Mental and nervous influence may give rise to hyperacidity through the gastric nerves. That worry and anxiety cause this condition is well known.

It is also well known that the reflex influence of a diseased gall bladder or duodenal ulcer may give rise to a very high degree of hyperacidity, which disappears when the cause is removed.

Another probable cause of gastric and duodenal ulcer is the reverse peristalsis which recent experiments have shown to be of much more frequent occurrence than was formerly believed. X-ray observation and laboratory experimentation, as well as clinical experience, indicate that the contents of the colon are often transported upward, reaching not only the duodenum and the stomach but even the mouth by a gentle, almost imperceptible, antiperistaltic movement which is especially active in persons who are constipated. It has long been known that duodenal liquids are frequently found in the stomach. It is entirely possible that this action is not purely local, but that it begins in the colon and is simply an extension of the natural, now well recognized, antiperistalsis of the right half of the colon upward along the small intestine. Through this action peptones and other products of digestion which are known to be highly stimulating to the gastric glands, are carried backward, and when brought in contact with the gastric glands stimulate them to abnormal activity. It is entirely possible that this is the cause of the continuous and exaggerated gastric secretion which is found in a considerable number of cases. 


\section{Influences Which Lessen the Secretion of Hydrochloric Acid.}

Pavlov showed that fats of all sorts, and particularly olive oil, have a powerful influence to check the formation of acid by the gastric glands. This is only true, however, when the fats are perfectly fresh, that is, free from butyric acid, shown by a rancid flavor and which excites the gastric glands.

Pavlov also showed that cereals give rise to a gastric secretion which is low in acid but high in digestive power, that is, rich in pepsin. The observations of Pavlov have been confirmed by the experimental work of Carlson, of the University of Chicago, and Boldyreff, Chief of the Pavlov Physiological Institute of the Battle Creek Sanitarium.

By taking the food in the form of purée, so that no chewing is required in passing it through the mouth quickly, the gustatory nerves are only slightly stimulated and less appetite juice will be produced.

The observations of Hemmeter showed in experiments upon animals that the withdrawal of salt from the food may cause the total disappearance of acid from the gastric secretion. It is known that the gastric acid is lessened in cases of fever. It has been determined that in fever there is a marked tendency to the retention of chlorid of sodium in the tissues. It is quite possible that a positive relation may exist between these two facts. It is certain that when it is desirable to lessen the seeretion of hydrochloric acid by the stomach, the intake of chlorid of sodium should be reduced to the lowest limit or at least common salt should be taken only in very small amounts.

In the majority of cases of hyperacidity complete relief may be obtained by the following simple measures, which have been used with success in the treatment of many hundreds of cases:

1. The discarding of meats, condiments and all other things which are known to excite gastric secretion. (See above.)

2. All food should be taken in the form of purée or porridge and should be chewed very little. Dextrinized cereals should be the principal foods.

3. In many cases, milk must be avoided but generally cream may be taken in moderation. In some cases butter is preferable and, in general, butter should be freely used. When milk is used it should be taken freely to avoid the formation of tough curds. In some cases the best remedy in cases in which milk disagrees is more milk. 
4. No salt should be added to the food and the amount used in seasoning the food should be greatly reduced. By gradual diminution its absence will not be missed. The butter used should be without salt. If such butter is not obtainable, the butter should be washed so as to remove the salt present.

5. The bowels should be made to move freely three or four times a day by the use of a laxative diet (see Index), a quarter of an ounce of bran or a third of an ounce of agar at every meal, and half an ounce of paraffin oil in some form taken before and after every meal. An enema should be taken at bedtime two or three times a week. If considerable fecal matter is shown by the enema to be present, the enema should be used every night until the colon has been trained to more efficient action. There is no ground for the fear that bran will aggravate the difficulty by irritating the stomach. It cannot be too often repeated that bran does not irritate; it titillates.

6. Measures should be used to maintain the intestinal flora.

7. The following foods should be absolutely and permanently discarded: Cane sugar, tea and coffee, alcoholic drinks of all sorts, carbonated waters, condiments of all sorts, meats of every description, also meat broths and extracts, much salt and salty foods, vinegar and pickles; and water drinking at meals must be avoided.

8. Olive oil must be used freely. The quantity should not be less than half an ounce at each meal and twice the amount is required in some cases. The amount should be increased to the point of tolerance if the acidity is not relieved without. It should be remembered, however, that gastric pain, which is usually attributed to hyperacidity, may in some cases be due to disease of the gall bladder, in which case the use of olive oil may give only slight relief or even none at all. If the oil "repeats," it should be taken in teaspoonful doses at first, the quantity being gradually increased.

It is also to be borne in mind that pain accompanying hyperacidity is not always due to an excess of acid but is often due to an abnormal sensitiveness of the gastric mucous membrane, and this condition may exist even when there is no excess of acid, or when acid is absent.

In all cases in which relief is not obtained by the measures herein suggested for hyperlydrochloria, tube feeding, as recom- 
mended for duodenal ulcer, should be resorted to. In these obstinate cases, it is quite probable that gastric or duodenal ulcer may exist, either with or without disease of the gall bladder. Careful investigation should be made to determine whether or not disease of the gall bladder is present, by X-ray examination and also by the Graham test and a careful clinical study of the case.

In many, possibly the majority of cases of so-called hyperchlorhydria, there is really no disorder in the stomach itself; the disease in the gall bladder, the appendix, the duodenum (ulcer), or some other organ. The pain, in many cases, is due to the failure of the pylorus to relax and open when food is brought to it by a gastric peristaltic wave.

\section{GASTRIC AND DUODENAL ULCER-PEPTIC ULCER}

(See also page 993)

The cause of gastric and duodenal ulcers is still a subject of discussion. The existence of the so-called peptic ulcer has recently been called in question. To the writer, it seems most reasonable to regard the ulcer as due to infection resulting from loss of general or local resistance, or both. Recent observations showing the probable relation between pyorrhea and a dietary lacking in vitamins, suggest the possibility that gastric and duodenal ulcers may be due to the same cause, and may be associated with focal infections of the mouth.

Ryle of London maintains that "the majority of dyspeptic cases which would formerly have been diagnosed as 'hyperchlorhydria' are now shown to have either a duodenal ulcer or some other organic irritative lesion. The majority of cases showing achlorhydria are found to be suffering from pyloric insufficiency with gastric hypotonus due to general causes; from local diseases such as gastritis or carcinoma involving the gastric mucous membrane; or from general disease such as Addison's anemia."

The pain of peptic ulcer is not due to irritation of acid or the contact of food, but is the result of hypertonus, excessive peristalsis and failure of the pylorus to relax, all of which factors increase intragastric tension and tension of the muscle fibers of the gastric walls (Hurst). These factors may be so intense as to cause spasm of the pylorus.

Acid may give rise to pain by causing excessive muscular tension. Hurst found that 4 ounces of a .5 per cent. solution of 
hydrochloric acid did not increase the pain of gastric ulcer. The pain of gastric ulcer associated with hypochlorhydria is just as great as in cases of hyperchlorhydria. Food gives rise to pain by causing exaggeration of gastric tonus and peristalsis.

Delayed Pain. The delayed pain of duodenal ulcer is not due to the contact of food with the ulcer, as shown by the fact that food begins to pass out of the stomach very soon after it is eaten. The pain is greatest when the stomach is nearly empty because then the tonus of the stomach is increased.

The pain of duodenal ulcer disappears when food, liquid or gas is introduced into the stomach for the reason that the distention of the stomach causes a relaxation of its tonus by the reflex adaptation of the stomach walls to the gastric contents. It is this relaxation or adaptation which relieves hunger and also hunger pain.

Alkalies are useful in relieving hunger pain probably because they give rise to the formation of gas. Bicarbonate of soda is the most effective because it produces the most gas. An effervescent mixture affords greatest relief (Poulton and Turner). The pain of gastric ulcer is also relieved by the taking of milk, water, and even small quantities of food.

Acid alone is not the cause of the pain. The acidity of the fasting juice found in the morning before breakfast is higher than that of gastric juice produced after eating yet no pain is produced by it. Pain may occur in the absence of free acidity. In such cases alkalies do not give relief for the reason that they do not give rise to the formation of gas. Effervescent powders might be given in such a case.

All the symptoms of duodenal ulcer-hunger pain, night pain, relief by food and alkalies - may be produced by gall bladder disease or disease of the appendix. In other words, all the subjective symptoms of gastric and duodenal ulcer may be produced in a perfectly healthy stomach by a disease far removed from the stomach.

Duodenal pain is usually persistent after it begins, rarely if ever rhythmical or intermittent. This fact agrees with the view that the pain is due to hypertonus rather than to increased peristalsis.

All the symptoms of duodenal ulcer may be produced not only by disease of the gall bladder and the appendix but by tobacco smoking, worry, overwork and other extragastric causes. 
The pain of ulcer on the lesser curvature develops within a quarter of an hour to an hour after eating and is relieved by vomiting, washing out the stomach, fasting and alkalies. The $\mathrm{X}$-ray shows spasm of the stomach wall opposite the ulcer. This is not the cause of the pain as the contraction may persist after the pain disappears. The pain is due to the fact that the spasm prevents the adaptation of the tonus or "posture" of the stomach walls to the gastric contents. When food is introduced the intragastric tension is increased because of the lack of adaptation; hence relief is best obtained by vomiting or by washing out the stomach.

The pain is sometimes relieved by removing gas from the stomach and may be reproduced by introducing gas into the stomach. In duodenal ulcer introduction of gas into the stomach does not produce pain. Bicarbonate of soda relieves the pain by producing gas, the belching of which lessens the contents of the stomach and so relieves intragastric pressure and tension of the gastric walls.

The symptoms of gastric ulcer may result from reflex effects of disease of some remote abdominal organ. In such a case the pain is confined to the left half of the stomach. This is the explanation of pain in the stomach due to disease in the appendix or produced by pressure over McBurney's point.

Either duodenal or gastric ulcer may be simulated by disease of the gall bladder or of the appendix.

Persons of the hypersthenic type have symptoms of duodenal ulcer while those of the hyposthenic type are most likely to have symptoms of gastric ulcer.

It has been definitely shown that smoking may cause peptic ulcer. It has long been known that the functions of the stomach and intestine are maintained by carefully balanced action of two important nerves, the sympathetic and the pneumogastric, or vagus nerve. It appears to be the function of the vagus nerve to stimulate the action of the secreting glands. One of the functions of the sympathetic nerve is to control or regulate the action of the vagus nerve. The sympathetic also controls the pylorus and the ileocecal sphincters. The constant and carefully balanced action of these two nerves is necessary for the maintenance of a healthy state of the stomach and intestine.

It has long been known to laboratory workers that tobacco paralyzes the sympathetic nerves. The effect of tobacco in this 
respect is so prompt and specific that nicotine is constantly in use in animal experimentation in the physiological laboratory as a means of paralyzing the sympathetic nerve.

These facts have naturally led specialists, particularly surgeons who have to deal with the stomach, to look upon tobacco as a possible cause of gastric duodenal ulcer, and clinical experience appears to support this conclusion. Moynihan, of Leeds, an English surgeon of international renown, lays great stress upon the importance of prohibiting smoking after operations upon the stomach.

Dr. H. Tyrrell Gray, of the West London Hospital, in a recent article in the British Medical Journal (June 14, 1924), reports the result of a careful study of a large number of cases of duodenal ulcer, which shows that recurrence of the symptoms after treatment by either surgical or non-surgical measures, is four times as frequent in smokers as in non-smokers. He prohibits smoking in all his cases and finds that of patients who abandon the habit, 90 per cent. make good recoveries, while of those who continue to smoke, only about one-half as many, or 47 per cent., were cured.

In view of the fact, that cancer of the stomach is usually preceded by ulcer, it is evident that the use of tobacco must receive consideration in a study of the causes of gastric ulcer.

Consistent with this view is the fact that cancer of the stomach is much more frequent in men than in women. The great increase in the use of tobacco in recent years may account, in part, for the simultaneous increase in cancer, and may especially explain the fact that cancer is increasing among men much more rapidly than in women.

Of all dietetic problems, the feeding of cases of gastric and duodenal ulcer is one of the most important, most interesting and most difficult of solution. It is now generally recogrized that, aside from surgical procedures, dietetic measures are practically the only ones which afford substantial relief in these cases. Nearly all cases may be more or less relieved by proper dietetic treatment and by far the greater majority of cases, at least nine out of ten of the milder class of cases of duodenal ulcer, may be temporarily, if not permanently, relieved.

There is in these cases an inveterate tendency to relapse. This tendency is so marked that a well-known surgeon being asked the question, "When should surgery be resorted to in cases 
of duodenal ulcer," replied, "When they have been cured nine times." It is probable that in a large proportion of cases relapse might be prevented by very careful adherence to a specific dietary. Unfortunately, however, the average ulcer patient does not consider himself cured of his ulcer unless able to return to his ordinary diet, which is invariably the dietary well calculated to develop ulcer and very likely to lead to a relapse. Relapse is in such cases almost inevitable for the reason that the first occurrence of the ulcer is evidence that the resistance of the body to the causes which produce ulcer of the stomach and duodenum has so broken down that the development of ulcer can in the future be prevented only by careful avoidance of all the causes known to be provocative of these conditions, particularly those foodstuffs which unduly tax the energies of the stomach and weaken its defense by prolonging the digestive period with overstimulation of the gastric glands, such as meats.

The first symptoms of the disease, cancer of the stomach, which carried off Napoleon, made their appearance some seven years before, after he had eaten a shoulder of mutton cooked with garlic. He probably had at that time a gastric ulcer, which was a prominent factor in his downfall, and on which later developed the cancer which caused his death.

Every case of gastric and duodenal ulcer should be studied by an X-ray expert to determine, if possible, the location of the ulcer and whether or not it is a so-called penetrating ulcer. This examination is also important for the reason that it often throws light upon the question of the presence of cancer, always a possibility in these cases, especially when the seat of disease is the stomach. When cancer is present an operation, if the case is operable, is the only course which offers any hope for the patient.

An operation is also imperatively indicated when the ulcer involves the pylorus; for the reason that an ulcer in this region is certain sooner or later to cause delay in the emptying of the stomach, because of mechanical obstruction at the outlet. When marked obstruction is actually present, as shown by long retention of the food in the stomach, operation is positively indicated. Very profuse hemorrhage or repeated hemorrhages, whether from the stomach or duodenum, as shown either by the vomiting of blood or by bloody stools, is an urgent indication for operation.

According to Charles H. Mayo, an investigation made in the Mayo Clinic of 438 cases of duodenal ulcer which had been 
operated on, showed that, from the standpoint of the patient, 70 per cent. considered themselves well following gastro-enterostomy, 25 per cent. were improved and 3 per cent. unimproved. The mortality was about 1.6 per cent.

An analysis of 500 cases of duodenal ulcer treated at the Battle Creek Sanitarium, made by Dr. E. L. Eggleston, (Jour. of A. M. A.), showed a cure with no relapse within three years in 70 per cent. of all cases treated. A course of treatment extending over not less than five weeks was found necessary for a cure and it was also found to be essential that the patient should follow permanently a carefully regulated regimen.

If the X-ray examination shows that the ulcer is penetrating in character, that is, if it has formed a deep pocket through more or less destruction of the muscular walls of the stomach, an operation is clearly indicated for the reason that these ulcers are seldom, if ever, permanently cured by other than operative measures and are dangerous because likely to penetrate into the peritoneal cavity.

It is now generally conceded by experienced practitioners that most well-developed gastric ulcers require surgical attention, for radical or permanent relief is not likely to be obtained by any other means. Some gastric specialists, who claim to be able to cure practically all cases of gastric and duodenal ulcer are evidently not thoroughly informed respecting the history of their patients after they pass out from under their care. The writer remembers some years ago having been informed by a famous London gastric specialist that he felt no hesitancy in promising a cure in practically all cases that came under his care. A week or two later during a visit to Moynihan's clinic at Leeds, reference was made to this statement, when the famous surgeon remarked, "Yes, Dr.— cures them and then they come to me for operation. We generally have some of his patients with us."

\section{DIETETIC TREATMEINT IN CASES OF GASTRIC AND DUODENAL ULCER}

(See page 687)

Cases of gastric and duodenal ulcer usually make rapid improvement when the stomach can be permitted to rest from digestive activity. A most efficient plan of treatment introduced in this country by the elder Flint, was starvation. More than 40 years ago, the writer treated cases of this sort with success by a 
fast of one to two weeks. In one case thus treated more than 30 years ago, in which the patient was nearly exsanguinated by loss of blood, the patient remained in good health for more than 10 years and then suffered a relapse which was cured in the same way, after which the patient remained in good health for a number of years before she was lost sight of.

Complete fasting is, however, no longer necessary since the duodenal feeding tube has been devised. By duodenal feeding. the stomach can be given rest while the body is being supplied with needed nourishment. The duodenal feeding tube is so small and flexible that it is easily swallowed, and after the first few hours it gives practically no inconvenience, so that it may be retained in place for several days, even two or three weeks, without giving rise to either serious discomfort or injury of any sort.

Thus tube feeding is beneficial in cases of gastric and duodenal ulcer, not only through giving the stomach rest but because of the reflux of bile which allays irritation by neutralizing the acid gastric contents. The bile also precipitates the pepsin and so stops its digestive action on the ulcer. The tube thus secures results similar to those of the operation of gastroenterostomy and so may sometimes take its place.

Some excellent gastroenterologists question the value of the duodenal feeding tube on the ground that it produces mechanical irritation. It is entirely possible that the brilliant success which has followed the use of the tube would have been equally or even more successful if suitable oral feeding had been employed. The writer feels certain that any case of gastric or duodenal ulcer which does not require surgery can be cured by gastric rest and carefully graduated feeding.

It is now generally conceded by experienced practitioners that most well developed gastric ulcers require surgical attention for radical relief.

It is a common practice to administer through the tube six to nine ounces of food every two hours. A better practice is to give a small amount every half hour. The food should be very slowly introduced. It should be remembered that the duodenum normally receives food very slowly, during a period of about four hours. Three times a minute, the stomach ejects about half a teaspoonful of liquid through the pylorus into the duodenum, or one ounce every five minutes. When large quantities are forced into the duodenum at one time, the result is a reflux into the 

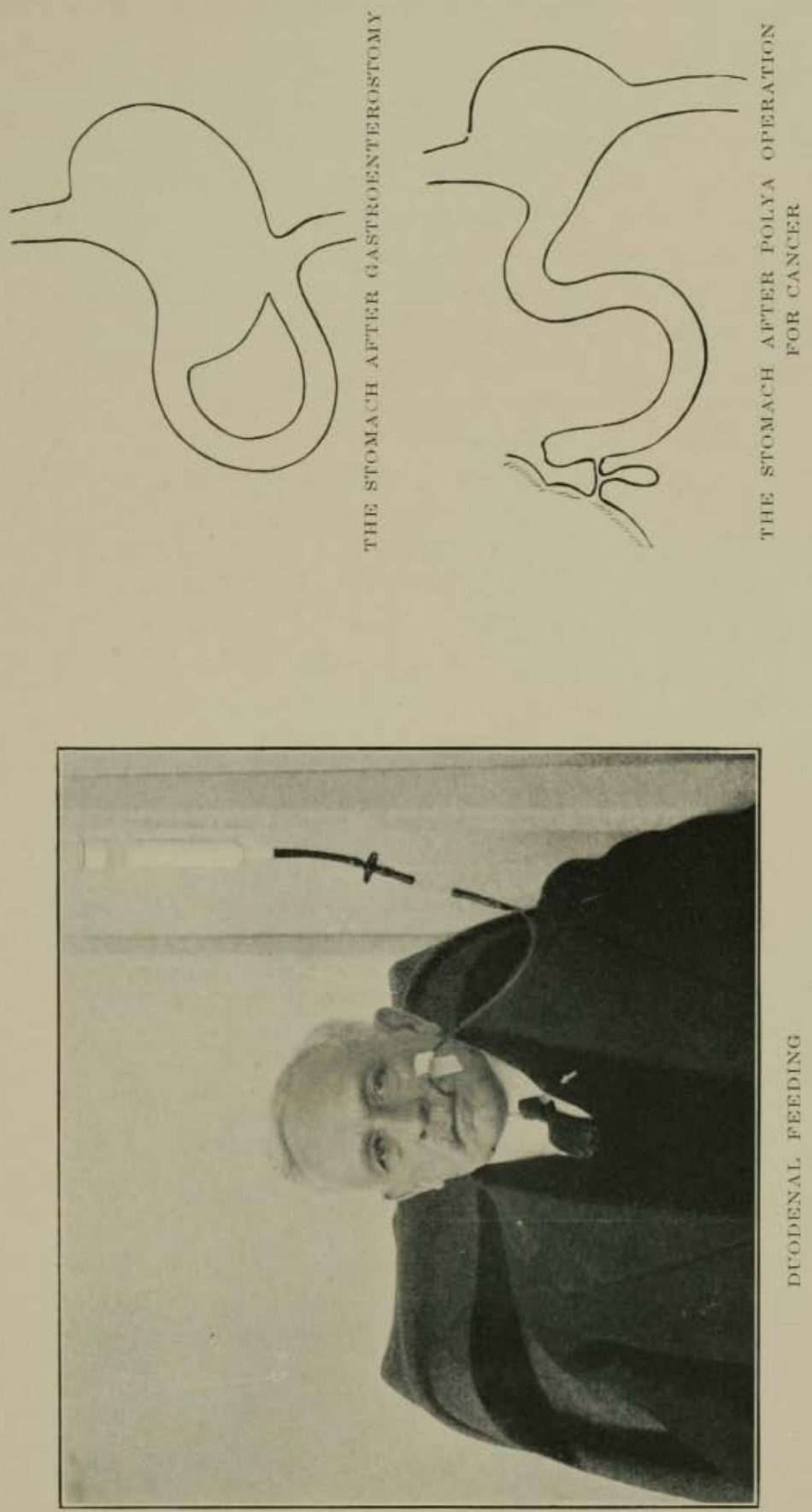


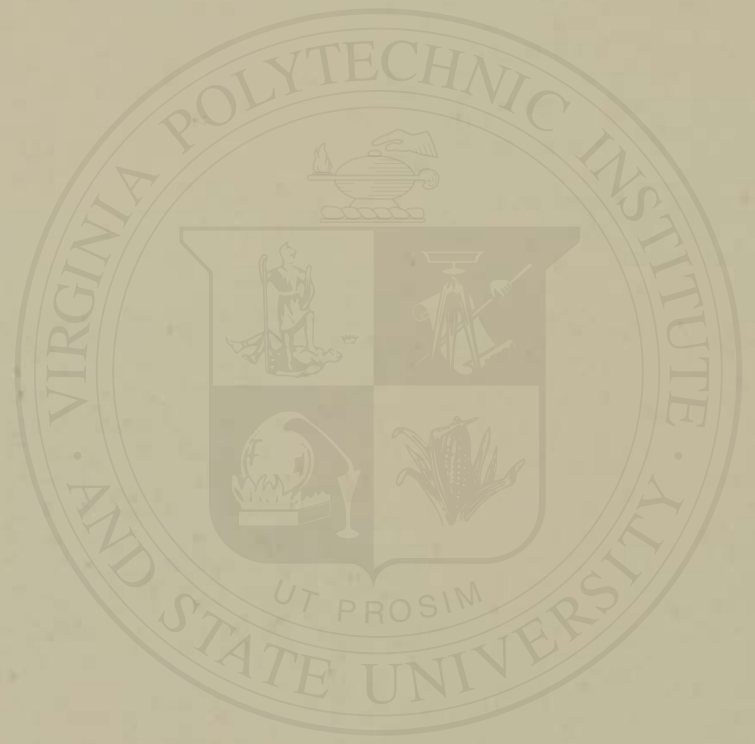


stomach. This, of course, in part defeats the purpose of this special mode of feeding, which is to give the stomach rest, so that the diseased surfaces may not be subjected to the irritating effect of contact with food.

The ideal plan of 'feeding is to introduce the food at the rate of about two ounces every fifteen minutes, omitting the feeding the last quarter of each hour. Continue this for four hours, then allow a rest for one hour. A good schedule is to start the feeding at seven o'clock in the morning; continue until eleven o'clock; rest for one hour; resume at twelve o'clock; continue until four; rest again from four to five; begin feeding again at five o'clock and continue till eight or nine o'clock.

The food having an energy value of 30 calories to the ounce, the hourly intake would be 180 calories, and the amount for each feeding period 720 calories. The total intake for the day will depend upon the number of hours during which the feeding is continued. Eight hours will give 1440 calories; ten hours, 1800 calories; twelve hours, 2160 calories.

The food should never be forced into the duodenum by means of a syringe. Instead, it should be permitted to run in slowly by gravity, as shown in the accompanying cut. The patient soon learns to regulate the inflow in such a way as to produce no discomfort or other inconvenience.

The amount of liquid introduced daily should be two or three quarts.

The patient should not only remain quiet but should remain as much as possible in the open air. The cure is facilitated by a fomentation over the upper abdomen, repeated two or three times a day. In the intervals between the fomentations, the heating compress should be applied. The cold mitten friction or cold towel rub should be employed twice a day, and light massage of the arms, legs, back, and lower abdomen should be given daily. Sun baths and arc light baths are also exceedingly helpful.

Care should be taken to empty the colon at least twice a day by a thorough enema, which should consist of three or four pints of water at $105^{\circ} \mathrm{F}$., followed, after evacuation of the bowels, by two pints at $80^{\circ} \mathrm{F}$. It is well, also, to administer an ounce of paraffin three times daily. The patient may take sips of water through the mouth, but large quantities of water should not be taken for the reason that water in quantity stimulates the production of gastric acid, as shown by Pavlov. Two or three pints 
of water should be introduced into the colon daily to insure an adequate water intake.

As regards gastric ulcer, it must be admitted that there is so strong a tendency to relapse that serious cases usually fall into the hands of the surgeon at last. The danger of fatal hemorrhage or of the later development of cancer is sufficiently great to justify operation in every severe case of ulceration of the stomach or duodenum.

There is little danger of cancer from duodenal ulcer, and the results of treatment are usually better than in cases of gastric ulcer. It must be remembered, however, that every patient who has once suffered from gastric or duodenal ulcer and who has been relieved by treatment or by operation must faithfully adhere to a careful, antitoxic regimen, avoiding meats, tea and coffee, much salt, condiments and other things that excite the gastric glands.

The foods best adapted to duodenal feeding are gruels reenforced with malt sugar or milk sugar and egg yolks or purées of spinach. An emulsion known as malted nuts has proved to be an excellent food in these cases, for which it has been very extensively employed for more than 20 years at the Battle Creek Sanitarium. Milk or buttermilk with yolks of eggs and an addition of cereals make an excellent combination. The egg yolk should be cooked until thickened, then rubbed smooth with a little milk and more milk added until a uniform mixture is obtained. Raw white of egg is indigestible, encourages intestinal putrefaction, and should never be used.

Sugar Formula. In 15 ounces of hot water, dissolve 9 ounces of milk sugar. When completely dissolved, add water sufficient to make 20 ounces. Each ounce of the mixture will represent 50 calories of carbohydrate. The mixture should be boiled to sterilize it and then kept on ice to prevent fermentation. This sugar solution is to be used as an addition to other food mixtures and also to water and fruit juices. Twenty ounces is the amount to be used daily. Milk sugar is not only a food, but aids greatly by encouraging the establishment of a normal intestinal flora.

Lacto-Dextrin. In many cases, dextrin or lacto-dextrin is found superior to milk sugar (lactose). It is equally valuable as an aid to growth of the B. acidophilus in the colon (change of flora) and does not produce nausea, as lactose does in some cases. It should be used in the same quantities. 


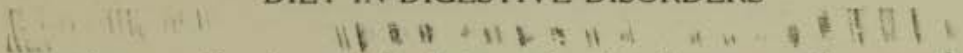

As combinations suited for tube feeding, may be mentioned egg yolks and buttermilk, egg yolks with fruit juice, sugar water, or gruels prepared from rice, oatmeal, millet, soy flour, toasted rice gruel, gruel prepared from brown rice. Most excellent are malted gruel, potato gruel, banana flour gruel, purée of spinach, Lean broth, orange juice, tomato juice, acidophilus buttermilk and whey cultures of $B$. Acidophilus.

It is important to note in duodenal feeding, as in all other special dietaries, that the necessity for a daily adequate supply of vitamins, lime, and iron must not be overlooked. These elements are equally as important as are fats, carbohydrates, and proteins.

Vitamins are found in rich abundance in greens, orange juice, tomato juice, milk and yolk of eggs. In many foodstuffs vitamins are deficient, especially in cereals. Butter or cream is an essential ingredient of duodenal feeding mixtures because of the important fat-soluble vitamin which it supplies. Careful balancing of the diet is as necessary in duodenal feeding as in ordinary feeding. Yeast extract (Savita) is most useful as a source of iron and vitamin. Malted nuts is rich in iron and in this respect is preferable to milk or malted milk.

Each of the following formulas consists of 9 ounces and is sufficient for one hour's feedings.

Malted nuts oz.

Curdled egg yolk. one

Orange or tomato juice.. $2 \mathrm{oz}$.

Water $6 \mathrm{oz}$.

Protein, 40; fat, 120; carbohydrate, 70; total calories, 230.

20 per cent. gluten meal. $1 \mathrm{oz}$.

Cream $3 \mathrm{oz}$.

Tomato juice. $2 \mathrm{oz}$.

Savita (yeast extract) ful

Water $3 \mathrm{oz}$.

Protein, 30 ; fat, 150 ; carbohydrate, 150 ; total calories, 330.

Rice gruel $6 \mathrm{oz}$.

Cream $2 \mathrm{oz}$

Egg yolk $1 / 2$ oz.

Savita 1 teaspoonful 
Protein, 21; fat, 145 ; carbohydrate, 45 ; total calories, 211.

Potato purée
Bean broth
Cream
Savita
Water

Protein, 24; fat, 88; carbohydrate, 112; total calories, 224.

It is not wise to use the same formula continuously. The formula should be changed each day, or two or three of the above formulas may be used in alternation on the same day.

In feeding with the duodenal tube, it is highly important to avoid introducing too large a quantity at once. It is to be remembered that the food is passed into the duodenum from the stomach in small quantities, about a teaspoonful at a time, and at the rate of one teaspoonful a minute. It is evident, then, that the introduction of large quantities of food into the duodenum would be likely to create disturbance. The more nearly continuous the feeding can be made the better. Two ounces every 15 minutes is an ideal plan. In most cases the patient himself can learn to manage the feeding with proper instructions.

\section{The Gluten-Feeding Method.}

It is well known that proteins enter into combination with the hydrochloric acid of the gastric juice. This combined acid is far less irritating than free acid. This fact is the basis of the glutenfeeding method. Forty per cent. cooked gluten meal is used for the purpose in the form of gruel with the addition of cream, butter or olive oil. The butter must be perfectly sweet. The following is a feeding schedule which will be found highly satisfactory.

First Day. Feeding formula: gruel prepared with 40 per cent. gluten meal. Teaspoonful of Savita. One ounce of gluten meal, eight ounces of water. Either no salt or a very little. Two ounces of cream. Water to make eight ounces. Calories, 200. The mixture supplies 25 calories per ounce.

Give two tablespoonfuls (one ounce) every hour from 7 A. M. to 9 P. M., 375 calories.

Second Day. Feeding formula: the same as the first day.

Feed one and a half ounces every hour from 7 A. M. to 9 P. M., 560 calories.

Third Day. Feeding formula: two ounces 40 per cent. 
gluten; three ounces cream; one and a half ounces milk sugar or lacto-dextrin. Water to make one pint. Food value 40 calories per ounce.

Feed one and a half ounces every hour from 7 A. M. to 9 P. M. Total, 900 calories.

Fourth Day. Feeding formula: the same as the third day with the addition of one egg yolk at the $8,10,12 \mathrm{~A}$. M. and 2, 4 and 6 P. M. feedings. Total calories, 1,272.

Fifth Day. Feeding formula: may be exactly the same as the fourth day with the addition of another ounce of milk sugar to the pint. Total calories, 1,422.

Sixth to Ninth Day. Feeding formula: 40 per cent. gluten mush, cream, rice purée, olive oil, curdled egg, feeding every two hours, 7, 9,11 A. M., 1, 3, 5, 7 P. M. Total calories, 2,100.

Tenth to Fifteenth Day. Feeding formula: the diet consists of gluten mush, peas purée, cottage cheese, boiled rice, rice flakes, granola mush, cream, graham toast, potato purée, brose with butter, purées of lentils or spinach and other fresh vegetables, malted nuts, olive oil, butter. Total calories, 2,200 to 2,500 .

After a course of treatment for gastric or duodenal ulcer, the diet for hyperchlorhydria should be adopted permanently.

The old plan of rectal feeding while giving the stomach rest has largely been abandoned. It was found, as a matter of fact, that the introduction of foodstuffs into the lower bowel sets up at once retroperistaltic movements which create a state of unrest of the whole intestinal tract. There is reason to believe, also, that rectal injections may excite gastric secretion. This may be brought about by reflex action through the absorption of putrid substances and perhaps more actively still by the actual transportation of some of the injected material from the colon to the stomach by antiperistaltic action.

\section{Lenhart $z$ 's Method.}

This method, which has been greatly extolled and widely employed, consists in keeping the patient in bed and feeding for several days on a mixture consisting of milk, beaten eggs and sugar. The first day the diet consists solely of two raw eggs and 100 c. c. ( $31 / 2$ ounces) of milk. The second day three eggs are given and the milk increased by $100 \mathrm{c}$. c. Each day till the sixth day the amount of food is increased by one egg and the addition 
of 100 c. c. of milk. Each following day up to the tenth day, the milk is further increased by the addition of 100 c. c., and the patient takes every day in addition four beaten raw eggs and four soft cooked eggs with approximately an ounce of sugar. Beginning with the seventh day, the patient is given a little more than two ounces of scraped beef and three ounces of boiled rice. The boiled rice is continued daily and gradually increased to ten ounces. On the eighth day an ounce of zwieback is given and increased day by day to two ounces by the end of two weeks. After the tenth day an ounce or two of chicken is allowed.

If anybody ever recovered from gastric ulcer by the aid of this treatment it must certainly have been because the previous diet had been so exceedingly unwholesome that even this absurd diet was an improvement.

\section{The Alkaline Treatment.}

The alkaline method consists in the administration of a large dose (one dram) of subnitrate of bismuth before the first feeding and between feedings every morning; before the first meal and half way between feedings, ten grains each of magnesia and bicarbonate of soda, alternating with a powder consisting of 20 to 30 grains of bismuth and 10 grains of bicarbonate of soda. For the first two or three days the food consists of milk and cream, equal parts, one to two ounces every hour from 7:00 A. M. to 7:00 P. M. After two or three days soft boiled eggs and well cooked cereals are added. At the end of ten days the diet will consist of three ounces of milk and cream every hour and one soft boiled egg and three ounces of cereal of some sort, each three times a day. Care is taken to avoid giving more than six ounces at one time. Frequent tests are made of the acidity and care is taken to make sure that no free acid exists in the stomach during the night. This is prevented by giving two or three alkaline powders between 7:00 and 10:00 P. M. By the third week, the patient is taking a sufficient amount of food to secure an actual gain in weight. Irritating foods are avoided for many months. Meats are permitted.

It is objected to this plan of treatment that the administration of alkalies, while temporarily neutralizing the free hydrochloric acid present, tends to increase the amount of acid secreted by the gastric glands. 
The hydrochloric acid of the gastric juice protects the body against the poisons eliminated through the bile by precipitating the bile salts and thus preventing their reabsorption. It is possible that injury may be done by the use of soda in large doses through the prevention of the precipitation of the bile salts. These salts are highly poisonous. When they are absorbed, they are again secreted. They thus become more and more concentrated and may become the cause of headaches and other disturbances. It is now generally conceded that the alkaline treatment is rarely successful and often does harm.

\section{Gastric Acidity Due to Organic Acids.}

The stomach is remarkably insensitive to hydrochloric acid, tolerating a percentage of this acid which no other tissues in the body can endure; but the gastric mucous membrane is often highly sensitive to organic acids. This condition may be the result of a morbid state developed by the free use of these acids in certain foods as, for example, the use of acetic acid in the form of vinegar, pickles, hard cider or sour wines. In some cases the mild organic acids of sour fruits cannot be tolerated. This is particularly true of tartaric acid, the acid of grapes, and the oxalic acid of rhubarb. The milder acids of apples (malic) and oranges (citric) are better borne, though even these are not always well tolerated. Certain stomachs will not even bear the mild acidity due to the lactic acid of sour milk. The stomach is often highly sensitive to butyric acid as found in butter or oils which are not perfectly fresh and in old cheese. Occasionally lactic, acetic and even butyric acid may be formed in the stomach as the result of fermentation. This usually occurs in cases in which there is long delay of the foodstuffs in the stomach either from atony or more frequently from organic obstruction of the pylorus.

In these cases the acids which produce discomfort must be avoided and the patient must be given a diet as free as possible from acidity. Certain fruits, such as very ripe bananas in the form of purée, fresh pears, sweet apples, dates and raisins are usually acceptable; but even these non-acid fruits must not be taken in too concentrated form. Cane sugar must be avoided as it usually has the same effect as acids. Candy of all sorts and sweet desserts must likewise be prohibited. The diet must be as unirritating as possible and must consist largely of purées of 
cereals, potatoes and other vegetables. Psyllium may be used in these cases with good results. Care must be taken to keep the bowels free from putrefying residues. The daily use of the enema is sometimes required for this purpose, but the free use of bran and paraffin will usually secure adequate bowel movements. The colitis which is frequently present in these cases must likewise receive attention.

\section{POST-OPERATIVE FEEDING}

Sir Berkley Moynihan, the leading abdominal surgeon of Great Britain, lays great stress upon the connection of the patient's habits in cases of peptic ulcer upon which he has operated. He says, "Among the most harmful habits for all these patients is smoking. We have found that in many cases of jejunal ulcer a hyperchlorhydria is present, and may be extreme. If a Rehfuss meal is given to a patient accustomed to tobacco at a time when he is not smoking his normal 'curve' may be recorded; if a second meal is given while a pipe is being smoked the increase of gastric acidity is very striking. An 'attack' of dtodenal ulcer often follows an orgy of tobacco; and many attacks are checked by abstinence from it. 'Attacks' ascribed to duodenal ulcer are sometimes due only to nicotine poisoning, and I have not seldom rescued patients from impending operations by noticing their deeply-stained fingers and by prescribing for them a respite from tobacco for a few months and a diminished indulgence in it forever. The close mimicry of 'hunger-pain' in nicotine intoxication appears to have escaped notice.

"At Leeds Infirmary the following instructions are given on a printed form to all patients after operation:

"1. Masticate the food thoroughly. Never hurry over meals.

"2. Do not smoke for three months after the operation and always in strict moderation. The greatest danger lies in excessive smoking.

"3. Avoid vinegar, lemon, condiments and sauces, salads, raw vegetables (celery, lettuce, etc.), pickles, pastry, sour fruit, currants, raisins, figs, lemon-peel, nuts, new bread, tough meat.

"4. Take in small quantities only green vegetables (preferably passed through a sieve); meat cooked a second time.

"5. Take small, light meals of simple foods for the first few months and have a very light meal (e. g., boiled milk, Benger's food or arrowroot with a biscuit) at or near 11 a. m. 
"6. Alcohol, if taken at all, only in moderation; no clarets.

"7. Butter and cream may be taken freely.

" 8 . The teeth and gums and the inside of the cheeks should be brushed with care two or three times daily, and visits to the dentist should be made regularly."

\section{Gastric Atony.}

Atony of the stomach is rarely, if ever, a purely local disease. It is only a part of a general condition of debility and lack of tone. Not only the stomach is relaxed but there is generally a relaxed condition of all the muscles of the body. There is usually a sense of fulness in the stomach and inability to deal with liquids, lack of appetite and frequent regurgitation. Splashing sounds in the stomach due to the presence of both liquid and air are sometimes so loud and frequent as to be a source of embarrassment. A state of atony or debility affects not only the stomach but the whole alimentary canal. There is usually obstinate constipation. There may be colitis with frequent bowel movement, but a spastic contraction of the descending colon is likely to be found present. The relaxation of the abdominal muscles is evidenced by the protruding lower belly. The patient usually sits in a completely relaxed or slumped position, and in the standing posture the forward carriage of the head and loss of the normal contours of the spine are evidence of the relaxed condition of the muscles of the trunk and clearly indicate the lack of energy characteristic of these cases. Examinations of the stomach fluid show either achylia or an excess of gastric acid.

The scraped meat usually prescribed for cases of this sort is highly objectionable. A crippled stomach is much less well prepared to deal with foods of this sort than is the normal stomach, and the result is to promote to a high degree infection of the intestinal tract and the development of the intense toxemia which may be the primary cause, a condition which presents an insurmountable obstacle to recovery as long as it exists.

The food should be thoroughly masticated. The meals should be small, never more than five or six hundred calories. Even smaller meals are better in certain cases. Water should be taken at meals only in very small quantities, the less the better. The same is true of bouillon and other liquids. Even milk and buttermilk are better avoided. It is a good plan for the patient to take 
a couple of glasses of hot water a full hour before each meal. The putrid stools which are usually present in these cases indicate the importance of changing the intestinal flora. This may be best accomplished by the use of lacto-dextrin in liberal doses continued over a considerable period of time. The dose should be from two to four ounces three times a day, sufficient to flood the alimentary canal with the carbohydrates which are best calculated to promote a luxuriant growth of the B. acidophilus. Meats of course must be carefully avoided, particularly fish and other "sea food." Bran and bran preparations should be freely used. It is one of the best forms of roughage for cases of this sort. Mineral oil should also be used in liberal doses. When considerable acid is present, the food should be taken in the form of purées and one or two dessert-spoonfuls of olive oil should be taken at each meal. A moderate amount of fresh butter may be used, but beef and mutton fats should be carefully avoided. A moderate amount of sterilized cream may be used. Nut butters and vegetable fats are preferable to animal fats. Fried foods, rich pastry, tea and coffee and all other unwholesome foods must of course be strictly avoided.

\section{DIET FOR HYPOCHLORHYDRIA AND ACHYLIA}

(See also pages 997, 998)

Hypochlorhydria and achlorhydria are due (1) to excessive neutralization by reflux from the duodenum; “(2) neutralization by alkaline and albuminous secretion from a malignant growth in the stomach; (3) neutralization and inhibition of acid secretion by an excessive output of mucus, as in chronic gastritis."

Mucus by coating the mucosa blocks secretion by preventing contact of the soluble food substances with the mucous membrane which is necessary to set up the reflexes which induce activity of the gastric glands.

Achylia is attributed (1) to atrophy of the gastric glands; (2) destruction of the glands by gastritis; (3) congenital defect.

Hurst thinks gastritis results from achylia more often than the achylia from gastritis. Brinton showed that scarlet fever may produce a severe desquamative gastritis resulting in achylia.

Achylia is most frequently found in Addison's anemia, in which it is constant, and in infective arthritis, appendicitis, and gallstones (Hurst and Bell). It has also been noted in pellagra, sprue and gout. 
A deficiency or total absence of hydrochloric acid is encountered in about half the cases of gastric disorder. The overwork of the acid-forming glands which causes hyperchlorhydria naturally results in the premature wearing out of the glands so that hypochlorhydria is a natural sequence of hyperchlorhydria. In the advanced stages of chronic gastritis and in advanced malignant disease, either of the stomach or of any other part of the body, hydrochloric acid is likely to be absent. Pepsin may be either absent or present. It is important to determine whether or not the pepsin-forming glands have undergone degeneration. This may be ascertained by a simple test. If the digestive activity of the stomach fluid obtained after a test meal is restored by the addition of hydrochloric acid, this means that pepsin is still formed. It is possible that pepsin may be absent from the gastric juice although the glands which secrete pepsin may not be degenerated, lacking only the necessary stimulus to bring them into activity. In such cases the administration of acid protein (Acidone) will stimulate the activity of the glands.

Normally about one-fourth of the hydrochloric acid secreted by the stomach is absorbed or neutralized by mucus. In cases of gastric catarrh, the enormous excess of mucus may neutralize nearly the entire gastric secretion. In such cases, acid protein should be freely used if well tolerated.

The functions of hydrochloric acid in the process of digestion are so numerous and varied that the loss of this element of the gastric juice is necessarily attended by more or less grave consequences. This will readily appear when we recall that the hydrochloric acid of the gastric juice not only aids the digestion of protein but closes the upper or cardiac orifice of the stomach, disinfects the food, activates pepsin, opens the pylorus, closes the pylorus, stimulates the flow of the bile and of the pancreatic juice, activates the pancreatic juice and hence is highly essential to good digestion. One of the worst of the consequences of the lack of hydrochloric acid is infection of the stomach and intestine through the loss of the disinfecting function of the gastric acid. Hence it is that bacteriological examination of the stools reveals the presence of vast numbers of pernicious bacteria, in consequence of which the stools are very foul and in many cases colitis is present. The ileocecal valve is generally incompetent, gastric catarrh and catarrh of the gall bladder are found in a 
very large proportion of cases and the occurrence of gallstones is frequent.

The diet in hypochlorhydria is less restricted than in almost any other condition except that of perfect health. Acid fruits should be used freely. To a slight extent the acids of fruits are a substitute for gastric acid and aid digestion when pepsin is present. The intake of fat should be small. An ounce a day is sufficient to meet all physiologic needs. Large quantities of fat are objectionable because their presence in the stomach, in some way not understood, hinders the secretion of hydrochloric acid when this function is not wholly lost. Fat also delays the passage of food from the stomach. This action appears to be entirely independent of the secretion of acid by the stomach. When fat is taken in large amount it is likely to be retained in the stomach a long time and, in the absence of gastric acid, fermentative and putrefactive changes are likely to take place, with the result that the fat becomes rancid and the patient may suffer from heartburn and great distress with vomiting of rancid gastric contents.

Persons suffering from achylia and with very marked hypochlorhydria should make daily use of acid protein. Acid protein (Acidone) is prepared by adding to pure vegetable protein about 15 per cent. of C. P. hydrochloric acid. This preparation may be used freely and without inconvenience by mixing it with other foodstuffs, such as thick soup, porridge, mashed potato or other vegetable purées. It not only aids the stomach in performing its digestive work, but stimulates the gastric glands to produce their normal secretion. Hydrochloric acid, as ordinarily used, accomplishes little because of the extreme smallness of the dose compared with the amount required to do actual digestive work. As ordinarily administered, the amount of acid given is less than one-twentieth the amount normally produced for the digestion of a single meal. The amount is too small to render appreciable service.

Acids. Acids of all sorts increase the production of gastric acid. Mineral acids and acid protein, that is, protein with which acid has been combined, produce this effect in a very high degree. Pavlov recommended the administration of gastric juice obtained from the stomachs of dogs as a means of stimulating the stomach to produce an increased amount of acid in cases of hypohydrochloria, or deficient formation of acid. This method has never become popular. 
It is an error to suppose that flesh foods are to be recommended when hydrochloric acid is deficient or absent. Since an acid gastric juice is necessary for the disinfection as well as the digestion of meats of all sorts, it is evident that when this condition is present meats should not be eaten, for in the condition in which they are eaten practically all meats are greatly in need of disinfection before being accorded a place upon the bill of fare or at least before or after entering the stomach. Ordinary cooking does not destroy the vast numbers of germs present.

It is known that gallstones are due to infection of the gall bladder. Diminished gastric acid may be either a cause or a consequence of the gall bladder disease. Probably both conditions result from the same cause, intestinal toxemia, a condition which is responsible for a wide range of disorders of the human body.

It is evident, then, that a strict antitoxic dietary, change of the intestinal flora, and frequent and thorough evacuation of the body residues (three times a day) are the dietetic measures likely to prove really efficient.

\section{Pepsin of Little Value.}

For half a century an enormous business has been done in the manufacture of pepsin from pigs' stomachs. Many tons of extract prepared from the lining scraped off the inside of the stomachs of hogs in our great slaughtering establishments are sold every year in the expectation that the deficiency of human pepsin may be made good by digestive ferment borrowed from the pig. Scientific research showed long ago that this enormous consumption of pepsin is of little or no value. As a matter of fact, the stomach rarely fails to make an adequate supply of pepsin. When gastric digestion is deficient, the fault is usually in the failure of the gastric glands to manufacture the necessary amount of gastric acid, so the thing wanted is not pepsin but acid. Notwithstanding the revelations which have been made by the exhaustive experiments of Morro and others, the pepsin business still flourishes. Pepsin is always secreted in abundance when acid is present. It is true, however, that in cases of extreme hyperacidity the pepsin is sometimes destroyed by the gastric acid, but this condition will not be remedied by the taking of pepsin. If the excess of acid is overcome, no further remedy is needed. How this may be done has already been pointed out. 


\section{Bitters do not Stimulate the Stomach.}

Another widespread delusion leads to the use of bitters in cases of this sort. The observations of Pavlov, Carlson and others have clearly demonstrated that bitters do not increase the production of gastric juice. Reichmann and Scheffer have shown. in fact, that the presence of bitters in the stomach has the effect of lessening the secretion of gastric juice. From these experiments, it is evident that the age-long faith in bitters as a means of promoting digestion has no scientific basis. Bitters hinder digestion instead of helping it. Scientific experiment does not even afford evidence that the use of bitters promotes appetite, or at least that any other than a slight psychic effect is produced.

\section{Special Dangers in Achylia.}

In persons suffering from achylia or extreme hypohydrochloria, the disinfecting action of the gastric juice is lost and bacteria and often molds are found present in the stomach and small intestine in great numbers. As a result the alimentary canal becomes thickly populated with putrefactive and other bacteria. A state of pernicious autointoxication is developed. In view of this fact, it is evidently important that foods which are likely to introduce bacteria into the intestine should be avoided. It is equally important to avoid foods which will encourage the growth of bacteria in the intestine, for the reason that the natural defense against these pernicious organisms has been broken down. On this account, meats of all sorts must be strictly prohibited. As shown elsewhere in this work, meats of all kinds, as supplied by the butcher, are swarming with bacteria and are in a state of active decomposition. The only exception to this general statement is found in the case of canned meats, which are necessarily made sterile by exposure to high temperatures in processing. Game, fish, shell-fish and oysters are especially detrimental and likely to give rise to grave and acute symptoms in persons suffering from achylia, when they might be eaten with impunity by persons possessed with gastric juice strong enough to exercise its normal disinfecting function.

Acute intestinal toxemia, or even ptomain poisoning, is not always due to the use of food containing pernicious organisms. Such attacks may as well be the result of taking food which encourages the unusual development of virulent bacteria already present in the intestine. 
Cold storage meats are especially objectionable, as are salted and smoked meats of every description.

For the same reason, certain varieties of cheese, such as limburger, brie and all forms of cheese which are far advanced in the so-called "ripening" process, must be carefully avoided.

Eggs may be eaten in moderation when they are fresh and well cooked. Raw and half-cooked eggs must be avoided..

Strong butter and rancid foods of all descriptions are exceedingly unwholesome, and are certain to increase the intestinal toxins by encouraging intestinal putrefactions.

Ordinary market milk is always unsafe, and is particularly dangerous in these cases, as well as for young infants, because of the presence of putrefactive organisms of various sorts, including the dangerous Welch's bacillus. Pasteurizing does not destroy the spores of these organisms and so does not render unclean milk safe. Only certified or clean milk should be used. Malted nuts may be commended as a safe and very satisfactory substitute for milk in achylia.

Lettuce, celery, all forms of salad vegetables and fresh fruits must be carefully disinfected before being eaten.

Persons suffering from achylia are much more exposed than are others to danger from infection by the embryos of animal parasites which develop in the intestine and with which raw fruits or vegetables are likely to become infected, both in the fields and when exposed for sale in the markets.

Such persons must also be continually on their guard against the use of bad water. Water containing typhoid germs, and even cholera germs, is often taken with impunity by persons whose stomachs contain an abundance of gastric juice, but in cases of achylia this protection is lost and water-borne bacteria pass through the stomach into the intestine and there find conditions favorable for their development. If not known to be perfectly safe, water should always be boiled. In traveling, it is a good plan for persons known to have achylia to carry with them a small supply of water known to be pure, or made safe by boiling. In emergency, chlorid of lime (bleaching powder) may be used to render water safe. Five grains of fresh chlorid of lime added to a gallon of water will destroy in a few minutes any dangerous germs that may be present.

Persons suffering from achylia should avoid large meals, for the reason that in achylia the stomach empties quickly. This is 
due to the fact that the pylorus is not closed by the presence of the gastric acid in the duodenum, as in the normal stomach. On this account, large meals must be avoided, otherwise the small intestine is likely to be overcrowded with food material and its function thereby disturbed. Meals should be made small and frequent. Some persons may take four small meals a day with advantage. Care must be taken to select foods which are quickly digested and to give preference to foods which are capable of being completely digested in the small intestine. Fortunately, this is true of most foods with the exception of raw or underdone meats, which are digested only by the gastric juice. This is a sufficient reason for avoiding meats.

Thorough chewing is necessary not only because the foodstuffs remain but a short time in the stomach, but because the stomach has, to a large extent, lost its power of breaking up the particles of food and reducing them to liquid form, which is a necessary preliminary to intestinal digestion. Such liquid or semi-liquid foods as buttermilk, fruit juices, gruels, broths, purées of fruits and vegetables, especially purées of tomatoes, spinach and other greens are to be most highly recommended in this condition. All fruit juices are valuable, and particularly the juices of acid fruits, because fruit acids are capable of replacing to some degree the normal acidity of the stomach. Fruits rich in vitamins are especially indicated for the reason that achylia is a degenerative state and suggests the probable existence of other degenerative processes which may very likely have been occasioned by a diet deficient in vitamins. Orange juice is for this reason especially valuable.

Whole meal preparations should be used instead of fine wheat flour, macaroni and polished rice. In addition to whole grain meals, bran should be freely used. The laxative diet, elsewhere described (see Index), is especially indicated in cases of achylia. Constipation is invariably present, together with chronic intestinal autointoxication, hence a change of intestinal flora is essential (see Index).

In every case of achylia, the intestinal motility should be determined at the outset of treatment. The stools should be examined and the fruit regimen with the other necessary measures elsewhere mentioned should be employed until the flora is thoroughly changed, as indicated by the character of the stools, the disappearance of intestinal gas and of intestinal putrefaction and clearing of the tongue. 
Two or three glasses of hot water may be taken with advantage half an hour before each meal as a means of cleansing the stomach.

A glass of orange juice or other dilute acid fruit juice half an hour before meals is especially valuable as a means of preparing the stomach for the intake of food.

\section{ACUTE DILATATION OF THE STOIMACH}

Serious gastric dilatation is much less frequent than was formerly supposed. The diagnosis should be based upon a careful $\mathrm{X}$-ray examination by a competent roentgenologist.

Acute dilatation of the stomach may occur after administration of gas, ether or chloroform as an anesthetic, or as the result of grave lesions of the central nervous system, but most commonly follows serious operations upon the intestinal tract. Chronic dilatation rarely exists except in connection with an organic obstruction of the pylorus. Of the greatest importance are cases of acute dilatation of the stomach following abdominal operation. In these cases an X-ray examination should be made for the purpose of determining the exact condition present. Vomiting may be relieved by the use of the duodenal tube, which in many cases may be with advantage left in place for a day or two. Accumulating fluids may be withdrawn and a few ounces of a. 10 per cent. solution of lactose or lacto-dextrin may be introduced. The sugar water has the effect to suppress putrefaction while at the same time affording some nourishment. When the liquids withdrawn from the stomach are no longer brownish in color and offensive in odor small amounts of other foods than lactose may be given, such as fruit juices, rice gruel and purées of dates, bananas, and a little later even spinach. In cases in which the stomach is known to be dilated it is well to prop the patient up in bed or to have him lie partially turned to the right side so that gravity will favor the movement of kiquids from the stomach. Great care must be taken to keep the bowels well emptied as a clogged state of the colon is often the chief cause of trouble through setting up reverse peristaltic movements. 


\section{MERYCISM}

This term is applied to the regurgitation of food from the stomach, somewhat like rumination in animals. The regurgitated liquid may be acid or bitter, or may retain its natural flavor. Regurgitation is the result of loss of tone in the cardiac sphincter, in consequence of which it does not close the entrance to the stomach with sufficient vigor to prevent the contents from being forced upward to the mouth by the contraction of the stomach which takes place during digestion.

In certain persons, regurgitation only occurs on stooping or coughing violently soon after eating. Merycism is most likely to occur in persons who eat hastily, swallowing much air with their food, or on drinking too much liquid.

In some persons the act of swallowing fluids is accompanied by a gurgling sound, which indicates that air is being swallowed with the liquid. In such cases, rumination is likely to occur from distension of the stomach and from the presence of a large quantity of air which, accumulating in the upper part of the stomach, prevents contact of the gastric juice with the cardiac orifice, in consequence of which the sphincter muscle does not contract with sufficient vigor to keep the orifice closed. Moderate eating, thorough chewing of the food and avoidance of liquids at meals are the dietetic measures indicated. The habit of regurgitation of the food when once formed becomes automatic and must be firmly and persistently resisted until overcome.

\section{AEROPHAGIA}

Many persons suppose that they are suffering from formation of gas in the stomach, when, in fact, the air which escapes from their stomach is first swallowed by a half involuntary effort. With the epiglottis closed, air is sucked into the stomach instead of into the lungs. The cause of air-swallowing, according to Hurst, is loss of the opening reflex of the pylorus. The stomach cannot empty itself and discomfort is experienced for the reason that the gastric walls do not adapt themselves to their contents. By swallowing air, the pressure within the stomach is increased considerably and some of the food is forced through the pylorus, thus affording relief. The patient should resist the tendency to draw air into the stomach by taking ten deep breaths while hold- 
ing the mouth widely open. It is well, also, to drink two or three glassfuls of very hot water.

Possible causes of reflex disturbance of the stomach should be sought out and removed. Change of the intestinal flora, relief of constipation and careful diet usually effect a speedy cure. The food should be moderate in quantity and rather dry, and should be thoroughly chewed.

\section{CYCLIC VOMITING}

In this distressing ailment, which occurs most frequently in children, meat and even eggs and dairy products should be carefully withheld. During the attack, no food should be taken into the stomach. The patient should be nourished by means of sugar water introduced by the colon. Malt sugar or syrup of raisins should be used for the purpose; two or three ounces of malt sugar to the pint, or the juice extracted from four ounces of stewed raisins, with the addition of water sufficient to make a pint.

The sugar water may be given even before vomiting ceases if it does not greatly aggravate the vomiting. Some portion of it will be absorbed, and this will help to combat the acidosis to which many cases of vomiting are due. In extreme cases in which the vomiting has been long continued and large quantities of fluids have been lost, a half pint or a pint of normal saline solution may be injected subcutaneously. After the vomiting has stopped for twelve hours the patient may be given cereals. Among the best for this purpose are rice gruel and 20 per cent. gluten with the addition of lactose or malt sugar. Orange juice and other fruit juices are most excellent. Skimmed or buttermilk may be used in small quantities. Fats must be avoided. sometimes for a long time.

\section{HICCOUGH OR SINGULTUS}

Hiccough may be induced by eating too rapidly or swallowing food in too large masses, or it may be caused by drinking carbonated waters or the generation of gas in the stomach.

It may also result from the reflex irritation originating in disease of the gall bladder or in the pelvic viscera.

Hiccough is also often due to an accumulation of irritating liquids in the stomach. Washing out the stomach, drinking co- 
piously of hot water and care to avoid the errors in eating above referred to are measures which sometimes afford relief. When the hiccough occurs only after taking food, the patient may be nourished for a few days by nutritive enemas. Hippocrates recommended tickling the nose with a feather to cause sneezing for hiccough. There is no panacea but the measures above have been found useful.

\section{ACUTE GASTRO-ENTERIC INFECTION}

This common disease of children requires the immediate and complete interdiction of milk and all animal foodstuffs. Thin barley or oatmeal gruel, rice jelly or rice gruel sweetened with malt sugar or milk sugar or sweet fruit juices and vegetable broths should be made the exclusive diet. The patient should be fed small quantities of food, the amount being gradually increased as tolerance is established. After a few days the diet may be made more varied. Purées of potato or other vegetables seasoned with a little butter may be added. Purées of spinach and other greens are especially valuable because of the rich stores of iron, lime and vitamins which they supply.

\section{STOMATITIS}

Rice jelly or a smooth rice gruel should be the exclusive food for a few days. Paraffin oil should be given to counteract the constipation, and the bowels should be made to move twice a day by the enema if necessary. Add a little cream and yeast extract for vitamins.

\section{URTICARIA}

This disease is due in most cases to anaphylaxis. The offending substance may usually be determined by the skin test for sensitivity. Proper materials for this test are now provided and it may be made by any physician who is familiar with the technic. In many cases the skin tests do not show sensitivity. In these cases, the cause is probably bacterial poisons produced in the colon.

When an offending food can be identified, this article must be scrupulously avoided. In all cases, the intestinal flora must be changed and a strict antitoxic dietary adopted. The writer has seen many cases recover as the result of the persevering use of these measures. 
In many cases, the sensitivity to a particular foodstuff may be overcome by gradually accustoming the individual to it, beginning with very minute doses and gradually increasing. For example, in a case in which strawberries caused urticaria the patient was given one strawberry the first day, two the next, and so on, adding one strawberry each day until a full serving could be eaten with no inconvenience. Relief from the intolerable itching may be obtained by touching with very hot, wet cloths $\left(120^{\circ}\right.$ to $\left.130^{\circ} \mathrm{F}\right)$.

\section{A CLASSIFICATION OF DYSPEPSIAS BASED UPON CAUSES}

Most dyspepsias, according to Ryle, are included in the following category: Nervous, toxic, irritated, mechanical, habit. The following classification is based upon that of Ryle:

\section{Dyspepsias Due to Erroneous Habits}

Over-eating
Under-eating
Over-work, mental or muscular
Lack of occupation
Lack of exercise
Posture-relaxed sitting
Eating too fast
Irregular meals
Too frequent meals
Too long without food
Constipation

Nervous Dyspepsias

Worry

Anxiety

Fear-fear of certain foods

Despondency

Hysteria

Nervous exhaustion

Aerophagy

Disappointment

Homesickness

Discontent 


\section{Toxic Dyspepsias}
Alcohol
Tobacco
Coffee or tea
Condiments
Intestinal toxemia
Tuberculosis
Colitis
Teeth
Tonsils
Ileocecal valve incompetent
Gastritis-sensitization
Pernicious vomiting of pregnancy
Viscero ptosis
Sensitivity

Irritative or Reflex Dyspepsias

Gastric ulcer

Duodenal ulcer

Gastro-jejunal or jejunal ulcer

Appendix

Gall bladder

Astigmatism

Mechanical

Pyloric obstruction

Duodenal obstruction

Cancer of stomach

Extensive ulceration

Adhesions

Post-operative complications

Hour-glass stomach

Habit Dyspepsia. The most common symptoms are discom: fort or fullness soon after eating, flatulence, loss of appetite in the morning, sometimes a capricious appetite. The discomfort sometimes occurred later after eating and was relieved by the taking of food. The causes are likely to be too frequent meals, too bulky meals, going too long without meals, neglect of exercise and other adjustments of irregular habits. These symptoms, according to Ryle "may be traced to the fact that the work demanded of the muscle fibre is out of proportion to the requirements of 
the body at the time. In other words, there is fatigue, through overloading or overactivity, of the muscle fibre. By diminishing the work of the stomach or increasing the requirements of the body on the one hand, or by more food and physical relaxation on the other, we rest or refresh the muscle and relieve the dyspepsia."

Nervous Dyspepsia. The most common symptoms are air swallowing, worry, fear and anxiety. Fastidiousness about diet. Fear of various foodstuffs. A tendency to restrict the diet. The nerve supply of the stomach may be influenced in such a way as to either increase or decrease the tonus or sensibility of the gastric muscular walls.

The term nervous dyspepsia is usually applied to obscure cases of gastro-intestinal disorder in which no clearly defined disease can be made out. In the writer's experience, these cases are nearly always highly toxic. Constipation is always present.

The proper procedure is, first of all, to change the patient's flora by the proper regimen (see Index). When this is accomplished, the patient should adopt a laxative and antitoxic diet (see Index), and an outdoor life. If these measures are thoroughly applied and carefully followed, the result will almost certainly be rapid progress toward health.

Toxic Dyspepsia. Alcoholic gastritis the most common. Achlorhydria very common. Likely to be associated with alcoholic cirrhosis of the liver. The gastric acid is absent altogether or develops very slowly, not appearing until after an hour. In gastritis the fasting sample is small in amount and very viscid, consisting almost wholly of mucus, sometimes a peculiar rancid odor, and may show a large number of pus cells. It should be compared with the spittle.

Tobacco Dyspepsia. Langley has shown that nicotine paralyzes the synapses of the sympathetic nervous system. The effect in habitual smokers would be to produce excessive action of the vagus nerve. Ryle reports a case of a man who was an excessive smoker of strong tobacco and who suffered with typical hunger pains which were relieved by food and who had discovered for himself "he could invariably and completely relieve his symptoms by abstaining from smoking." His purpose in seeking medical advice "was to obtain a remedy which would enable him to go on smoking."

The muscular structures of the stomach may be disturbed, 
either irritated or depressed, by poisons of various sorts, as from the use of tea, coffee or condiments or alcohol. Tobacco causes increase of gastric tonus by inhibiting the sympathetic, but other toxins, those produced by infectious disorders as well as those absorbed from the intestine, have a depressing effect.

Irritative Dyspepsias. Brown has shown that pain is excessively severe in the case of ulcers adherent to the pancreas. The pain is not only severe but lasts for periods of an hour or more, or until the stomach has completely emptied itself or has been refilled by eating or emptied by vomiting. The night pain is longest and lasts for many hours because it is not relieved by the taking of food. The pain is produced and aggravated by hunger, by cold, worry, fatigue and smoking. It is relieved by food, rest, warmth, peace of mind, also by alkalies, atropine and belladonna. The appetite is usually good. The fasting juice is usually large in quantity, 80 to 100 c.c. or more. It is clear, limpid, highly acid, with little or no mucus. The initial fall resulting in the swallowing of the gruel is followed by a steady rise to a high point and continuous secretion after the starch has left the stomach. This condition is regarded by Ryle as nearly pathognomonic of duodenal ulcer.

In this class of dyspepsias there is always an increase of gastric tonus, the result of a direct irritation of a gastric or duodenal ulcer or of reflex irritation from a diseased appendix or gall bladder or from irritating nerve disturbances from the central nervous system as in cases of locomotor ataxia or disturbances from the eye, as astigmatism, or from the ear, as in Meniere's disease.

Mechanical Dyspepsias. There may be rigidity of the gastric wall due to malignant or other disease, pyloric obstruction, extensive ulceration, hour-glass contraction or complications arising from gastro-jejunostomy.

\section{ACUTE GASTRIC CATARRH IN CHILDREN}

Jacobson reports that in cases of colitis in children he finds that the stomach shares the catarrhal condition of the bowels. He has treated these cases successfully as follows:

The child is restricted to water only for twelve hours, then for a few days raw oatmeal soup is administered; then half a pint of milk is given during the day, followed the next day by half a pint of milk gruel. The next day a pint of milk, a half 
pint of milk gruel and half a pint of water gruel. The diet is then made more liberal by the addition of toasted bread, sago soup, porridge and mashed potato. Milk sugar may be given with advantage, a teaspoonful three or four times a day.

The bland diet (see Index) and buttermilk (acidophilus) should be employed together with treatment for colitis as in adults (see Index).

\section{DIET IN ACUTE GASTRITIS}

This disease is rather rare. Most cases of so-called gastritis are simply cases of indigestion, not accompanied by actual inflammation of the gastric mucous membrane. When gastritis is actually present, the stomach should be given complete rest for one to three days. This does not mean, however, that nothing should be given the patient to swallow. Water at the temperature of the body should be given frequently in small doses. The addition of milk sugar or malt sugar to the water, in proportion of an ounce to the pint, is advantageous. Disease-producing bacteria will not grow, or at least are not virulent and active in producing toxins, in the presence of sugar. Mild fruit juices, as the juice of sweet oranges and of raspberries well diluted, may also be advantageously used. Sometimes rice water or rice jelly is preferable, especially when great irritation is present. Whey, or still better whey culture of the B. acidophilus, is indicated.

When nausea, vomiting and other distressing symptoms have ceased and water or rice water is well borne, well-boiled rice or rice gruel may be added, with buttermilk (acidophilus). As improvement progresses, the bland diet (see Index), may be added.

Perfectly sweet butter and cream may be taken a little later.

Strong acids, spices, as well as tea and coffee, animal broths and animal fats, must likewise be avoided.

Meat especially must be carefully avoided, as most harmful.

Care must be taken to avoid a relapse, which is very liable to occur. Great precaution must be exercised to avoid overeating and to masticate the food very thoroughly.

The bowels must be kept active by the use at each meal of psyllium seed and, if needed, paraffin oil. The flora must be changed and kept changed by the use of lactose or lacto-dextrin and the avoidance of putrescible foods. An enema at bedtime ( 3 or 4 pints at $110-115^{\circ} \mathrm{F}$.) will assure against colonic stasis which is most harmful. 


\section{DIET IN CHRONIC GASTRITIS}

This disease is usually the result of frequently repeated attacks of acute gastritis occasioned by abuse of the stomach; alcohol, tea and coffee, overeating, the free use of meat, condiments-mustard, cayenne pepper, capsicum, horse-radish, hot sauces, vinegar, spices, pickles and smoking. Gastritis often accompanies cirrhosis of the liver, due to the same causes, and precedes and accompanies cancer of the stomach. It is usually present in pernicious anemia, and often accompanies chronic Bright's disease.

When vomiting is present, the same diet as in acute gastritis is indicated until the vomiting ceases; then farinaceous foods, with buttermilk, potato purée, rice pudding, bread pudding, cereal flakes, toast, lactose to change the flora. Enemas must be given twice a day while the bland diet is used.

When free hydrochloric acid is present in the stomach, butter may be used sparingly, also cream, olive oil, egg yolk and egg yolk nog. As the stomach becomes less irritable, fruit juices and purées of fruits may be added. In all cases, purées of greens, spinach, lamb's quarters, red root, and other greens should be freely used as soon as the stomach will tolerate them. Herschell recommends fresh fruit alone for one meal daily in chronic gastritis, attributing special virtue to the fruit acids, which are doubtless highly beneficial. Cane sugar must be avoided. Psyllium seed is an emollient laxative most useful in these cases.

Things to Avoid: Meats of all sorts, broths, meat extracts, fish, oysters, pickles, vinegar, condiments, new bread, tea and coffee, much liquids at meals, pastry, cane sugar, preserves, confectionery, ice cream, ices.

\section{AMYLACEOUS DYSPEPSIA}

A general characteristic of so-called amylaceous dyspepsia is inconvenience from the presence of gas in either the colon or small intestine or both. When the small intestine is involved, the central portion of the abdomen, in the region of the umbilicus, is the chief seat of discomfort. The presence of much gas in the small intestine is due to two causes; excessive formation of gas in the colon and incompetency of the ileocecal valve, permitting the gas to pass from the colon into the small bowel.

Intestinal gas is chiefly produced by two organisms, the 
colon bacillus and Welch's bacillus. The last named organism, also known as the gas bacillus, is the great gas producer of the intestine. These organisms give rise to both fermentation and putrefaction. From protein they produce highly offensive gases; from starch or sugar they produce enormous quantities of carbonic acid gas.

The normal flora or bacterial vegetation of the human intestine produces harmless acids and little or no gas. In the presence of the "wild" (Herter) putrefactive bacteria, saccharine or farinaceous foodstuffs give rise to much gas; but when the flora is changed, the gas at once disappears. This is a constant observation in the use of the fruit regimen or the milk sugar or dextrin method of changing the flora. At first, the gas is much increased; in a few days, however, the gas disappears although the amount of carbohydrates is not diminished or even is increased. So the cure for so-called carbohydrate or amylaceous or farinaceous dyspepsia is more carbohydrate!

In many cases, a cause of gas formation is the accumulation of food residues in the colon, especially the cecum. In such cases relief is readily obtained by means of a copious warm enema (three pints). The diet should be decidedly laxative, with bran or agar and paraffin. When adhesions of the cecum exist, the enema should be regularly used at night to insure complete emptying of the colon. Adhesions usually exist in cases of chronic appendicitis and in cases in which the appendix has been removed.

Distension of the bowels with gas is also a common symptom in colitis because of the spastic or contracted condition of the lower colon almost always present, causing retention of food residues and consequent fermentation and putrefaction with gas production in the cecum and ascending colon. A change of flora followed by a laxative and antitoxic diet will afford definite relief.

\section{DIET FOR DECAY OF THE TEETH}

One of the first to study the bacteria of the mouth was Miller, an American dentist practising in Berlin. He held that the cause of decay of the teeth was the fermentation of sugar and starch in the mouth. Miller experimented by placing teeth in fermenting mixtures of bread and saliva. He thought that he saw evidence of decay after several months.

Howe, of Harvard University, repeated the experiments, 
using saliva from persons with badly decayed teeth in mixtures of grape sugar, malt sugar, cane sugar, milk sugar, dextrin, and white flour bread. It was only after six months that he noticed etching of some of the teeth.

Many years ago (1895), the writer became convinced that decay of the teeth is not due to the formation of lactic acid by the fermentation of sugar or starch in the mouth as has been generally believed, a doctrine which has been held by dentists for half a century or more and is still believed and taught by the majority of dental practitioners. Observations on numerous patients convinced the writer that the cause of decay of the teeth is a general nutritive disturbance affecting the whole body, particularly a lowered vital resistance from some cause.

One recognized cause was intestinal toxemia; that is, a pollution of the blood through the absorption of the products of putrefaction from the colon. Dr. Percy R. Howe has recently demonstrated another cause. He has shown a lack of vitamins, particularly of the water-soluble B and water-soluble C, or antiscorbutic vitamins, produce a disturbance of the calcium metabolism, that is, interfere with the assimilation of lime so that the teeth are robbed of their lime and are thus softened and made subject to decay as well as other injuries.

It is a notable fact that the teeth of primitive people are almost invariably free from decay. Dr. Ottofy, who examined the teeth of many Igorot children in the Philippine Islands, found more than two-thirds with perfect teeth, and the defects in the remainder were so slight as to be almost undiscoverable.

In one of his interesting papers, Dr. Howe remarks as follows :

It is evident to any practitioner of dentistry that the people of many different nationalities have excellent teeth when they live on their natural food products. The Esquimau is practically free from caries. He does not live on muscle meat alone, but he eats practically the whole carcass of the animals which form his food. He gets his calcium from the bones and some of the tissues. He gets his vitamins from many of the glandular organs, but with a change of diet, his teeth decay. Stefansson has written me that concomitant with the influx of our refined foods, caries has crept into the camps of the Esquimaux and Icelanders. The natives upon the Labrador coast, now subsisting on bread and tea in a great measure, have extensive decay, while formerly they were exempt from it.

Wells has shown that the Scotch Highlanders have excellent teeth, but that as they obtain city foods their teeth decay badly. We might 
mention any number of such cases. We know that many immigrants have excellent teeth upon arrival in this country, but in a short time after living on our foods their teeth decay.

Modern diets are deficient in calcium and in vitamins, both of which are needed for sound teeth, and if we desire to practice preventive dentistry we need to begin here. The other many effects described indicate the need of a similar diet for sound bodily development and for vigor. For the fullest bodily development, every effort should be made to restore the teeth to their usefulness and to retain them.

Soft foods, foods poor in lime and vitamins, and meats of all sorts encourage dental decay and pyorrhea. The fibers of meat caught between the teeth encourage the growth of the sort of germs which cause decay of the teeth.

The use of hard, dry foods which require vigorous use of the jaw muscles and promote the flow of saliva is essential for good development of the teeth. Greens, coarse vegetables, bran and acid fruits which stimulate the flow of saliva are all important means of promoting the health of the teeth. Cane sugar, fine flour bread, very hot foods and drinks and meats are most objectionable from the standpoint of dental hygiene.

Mellanby showed that the fat-soluble vitamin A is necessary for the proper development of the teeth. Marked difference was found between the teeth of animals fed on linseed oil and those fed butter fat. Butter fat contains the fat-soluble $\mathrm{A}$ in abundance, whereas linseed oil contains little or none.

At the suggestion of Dr. Vida A. Latham, the Indians confined in government jails have been required to give special attention to cleansing the teeth in accordance with the rules of oral hygiene. The result has been that the sickness from dysentery, which had formerly been very prevalenit in the jails, has been decreased more than 50 per cent. There is a growing mass of facts which clearly indicate that a foul condition of the mouth may lead to infection not only of structures immediately connected with the mouth but of the stomach and intestines as well. Infection of the mouth and decay of the teeth are encouraged by the use of flesh foods more than by any other means.

The substitution of milk for meat in the dietary, the free use of greenstuffs which abound in food lime, avoidance of confectionery, the use of malt sugar, dates, figs or raisins in place of cane sugar, and especially the use of dry, hard food that will give the teeth and jaws exercise are the most important dietetic pre- 
cepts relating to dental dietetics. Every child should have at least one quart of good clean milk daily.

The intestinal flora must be changed (see Index) and kept in a normal state. The bowels must be kept free by the use of bran, psyllium or agar and mineral oil and a laxative diet.

\section{DIET IN CONSTIPATION}

In recent times the colon has been the subject of much controversy. The question is, shall it be removed or reformed? Anatomists have declared the colon to be a useless appendage a vestigial remnant left over from a prehistoric state. Bacteriologists have charged it with being an incubating chamber of poison-forming germs, a hold of unclean and hateful parasites, a veritable Pandora's box of disease and degeneracy. Surgeons have removed the offending organ, and thus proved that it may be dispensed with, and have claimed wonderful advantages from this abbreviation of the primæ viæ.

Barclay Smith, the great English anatomist, first suggested the uselessness of the colon. Metchnikoff proved that animals that possess the longest colons have the shortest lives, and announced that the colon bacillus is the germ of old age. Sir William Arbuthnot Lane, the eminent London surgeon, cites a long list of grave maladies, ranging from tuberculosis to rheumatism, cured by removal of this offending organ.

In the treatment of every chronic disease and most acute maladies the colon must be reckoned with. That the average colon in civilized communities is in a desperately depraved and dangerous condition can no longer be doubted. The colon must either be removed or reformed. From the beginning of the colon controversy and for many years before, the writer has been a very earnest student of the questions involved and has formed very definite opinions, the validity of which he together with his colleagues of the faculty of the Battle Creek Sanitarium has had opportunity to test in the treatment of many thousands of sufferers from colon and colon-caused maladies. The writer believes that methods have been worked out by means of which the colon may be reformed and made to do its work efficiently, not only in ordinary cases, but in by far the great majority of those cases which are thought by enthusiastic colon surgeons to be suitable subjects for surgical treatment. 
Until very recent years almost nothing has been known of the physiology of the colon. This part of the body has been almost a terra incognita. The physiology of digestion stopped at the ileocecal valve. How the colon dealt with its contents, how the very necessary act of defecation was performed, nobody knew. The discovery of the X-ray enabled Cannon and, later, Hertz to study the colon while in action in animals and man. Elliot, Keith and other anatomists studied the intestine in dogs, and finally Case, by perfecting the X-ray technic of colon examinations, completed the physiologic study of this previously neglected organ. The combined result of the extensive labors of these investigators has been a great flood of light upon one of the most obscure questions in physiology. These new facts, not yet known to the general public, have rendered the greatest service in the development of rational methods of dealing with that most common and most destructive disease of civilized peoples-constipation.

Forty years' experience and observation in dealing with chronic invalids, and careful study of the results of the modern $\mathrm{X}$-ray investigations of the colon, together with observations made at the operating table in many hundreds of cases, have convinced the writer:

1. That constipation with its consequences is the result of the unnatural habits in relation to diet and colon hygiene which prevail among civilized people.

2. That patients are not constipated on general principles, but that there exists in every case of constipation some particular condition which is the immediate cause of the delayed intestinal movement, and which must be removed before definite relief can be obtained, and that in the great majority of cases this cause is some real and tangible difficulty which may be removed by changing the intestinal flora or by other available means.

3. That practically every case of constipation is curable, and in all but exceptional cases without the air of surgery. It must be added, however, that by cure is not meant the working of such a miracle that the colon will perform its function normally without attention to diet or other means which encourage colon activity, but rather that by observing certain rules and the faithful and continuous use of safe and simple means the colon may be made to perform its functions in a regular and efficient manner without the use of irritating laxative drugs.

Constipation, though aggravated by errors in diet, is by no 
means wholly due to this cause, hence it must not be expected that by dietetic means alone all cases of constipation are curable. It is certainly true that food is the natural laxative. The act of taking foods stimulates peristaltic activity and it is more than probable that if from the start an individual adhered closely to the proper dietary eaten in a proper manner and took care to attend promptly to the call of Nature the normal intestinal rhythm would be maintained. These conditions, however, very rarely exist, and in consequence constipation is so nearly universal that the average individual feels himself the proud possessor of a normal colon if his bowels move once in twenty-four hours, whereas the savage declares himself to be "horribly constipated" when his bowels move but once a day. Normal bowel rhythm unquestionably demands at least three evacuations daily.

The alimentary canal is long in man, ten times the length of his body. The colon is also long and sacculated like that of herbivorous animals. This fact predisposes him to constipation, which becomes inevitable under the ordinary conditions of our modern, civilized life. Constipation is universal among civilized people. A large proportion of the people of the United States are habitual users of laxatives of some sort. Cathartic or laxative pills, aperients, liver regulators and mineral waters are manufactured and used in enormous quantities, more extensively than any other drugs. Thousands of men and women who congratulate themselves that they are in good health because their bowels move regularly once a day are nevertheless suffering from chronic intestinal stasis; that is, stagnation of the food residues and body wastes in the colon. The colon of a person whose bowels move once daily is constantly filled with putrefying residues which are retained in the body from two to three days undergoing decomposition and flooding the body with putrefaction products instead of being dismissed in 15 to 20 hours from the time the food was eaten, which is the normal intestinal rhythm.

Modern studies of the physiology of the colon have demonstrated that the bowels should move at least three times a day, or once after each meal. An intake should naturally be followed by an output. Normally, no putrefaction occurs in the colon or in any part of the alimentary canal. When the food residues are dismissed from the body with normal promtpness, there is no time for putrefaction, the stools are yellowish in color and 
are free from the putrid and loathsome odor which is the usual characteristic of the stools of the average individual.

Attention must be called to the fact that a person who has suffered from chronic constipation for many years has a colon so badly crippled that a dietary which would secure normal movements in a person possessed of a normal colon may be found inefficient. Thoroughgoing measures alone will succeed. These will include a very liberal supply of roughage, much more than a normal colon would require, and in addition copious lubrication, which is quite necessary for the reason that in the crippled colon, mucus, the normal lubricant, may be largely lacking owing to the destructive action of colitis upon the mucus-secreting glands. These chronically crippled colons often present a mucous surface so dry that fecal matters adhere to it and the food residues are pushed along only with the greatest difficulty.

A person suffering with chronic constipation should first of all take the carmine capsule test (see Index) for the purpose of determining the motility of his intestine; that is, the time which elapses between the taking of a meal and the discharge of the unusable residues from the body.

The next step is to follow faithfully the laxative regimen. (See Index.)

The third step required is a change of the intestinal flora; for it is quite impossible to secure regular and efficient activity of the colon without first restoring the normal, protective, acidproducing organisms, since the acids developed by these beneficent bacteria are the natural and necessary stimulants of the colon, as pointed out by A. Schmidt, many years ago. (For the method of changing the intestinal flora, see Index.)

Cellulose and the layers of cork cells in bran are the chief laxative elements in foodstuffs. Being indigestible, when present in sufficient quantity, cellulose gives to the alimentary residues the bulk necessary to excite the muscular sense of the intestine and so give rise to the peristaltic contractions by which the unusable residues and body wastes are transported through the colon and discharged from the body. Cellulose is found only in vegetable foodstuffs; hence animal foods of all sorts, meat, eggs and dairy products are constipating. Being wlrolly digestible and absorbable, they leave behind little residue. But the small residues of these animal substances which reach the colon readily undergo putrefaction, and the products of putrefaction being 
alkaline paralyze the colon, which is rendered inactive by alkalies and stimulated to activity by acids.

In addition to increasing the bulk of the food, lubrication is usually required. This may be secured by the use of paraffin in some form. The Russian paraffin oil renders the most valuable service in these cases. Examination of the bowels and rectum generally shows, in cases of chronic constipation, a dryness of the mucous membrane. By the use of paraffin oil, which is not a food, undergoes no change and acts merely as a mechanical agent, the food residues and body wastes are so lubricated that they do not adhere but slip readily along, even when redundant development may have given rise to folds and kinks which are more or less obstructive, or when adhesions exist that offer mechanical obstacles to the progress of the alimentary residues along the colon. Half an ounce to an ounce of washed white Russian paraffin oil should be taken at each meal. Paraffin which melts at the body temperature (Paramels) is much more efficient than liquid paraffin.

Much has been claimed for the special advantages of heavy mineral oils over the lighter oils. Bastedo, who made a careful comparison of the effects of oils of different specific gravity, found no difference between them. Experience has shown, however, that a paraffin which melts at the body temperature, being solid at ordinary temperatures, is much more efficient than mineral oils, and has the further advantage that it does not soil the clothing.

By the application of the two measures above outlined, a low protein diet and some simple means for accelerating movement along the alimentary canal, putrefactive processes in the intestine may be controlled to a wonderful degree and the number of bacteria may be greatly reduced. For best results, either bran or agar and the lubricating paraffin should be mixed in the entire meal instead of being taken before or after the meal or at some other time, as just before retiring at night. It is the residues which need the roughage and the lubrication. These physiological aids to bowel movement do not act by irritation but by promoting physiologic action.

This method of taking bran or agar and paraffin has been found by experience to be highly important. The method must be insisted upon in obstinate cases, for the reason that the food is not thoroughly mixed together in the digestive organs, as 
might be imagined but, unless readily soluble like sugar or a fruit acid, the foodstuffs pass along down the intestine in practically the same order in which they are eaten. If bran and oil are taken before the meal, they proceed before it; or, if taken after, they follow along behind. For best results, it is essential that these helps to colon activity should not only accompany a meal, but each part of it. Otherwise efficient bowel action may not occur. The oil especially will be likely to be discharged by itself, thus giving occasion for unfortunate accidents and often much inconvenience; and the food residues, often including the bran or agar, are likely to remain behind in the cecum, which is frequently crippled badly by overstretching and adhesions, thus becoming a sort of cesspool in which putrefying refuse may be retained for days in succession.

In persons whose colons are badly crippled by disease, through adhesions of the cecum or pelvic colon, and in cases of spastic constipation, the use of paraffin oil in connection with bran or agar is essential in addition to the use of laxative foods. Agar and paraffin oil should not be regarded as medicines. They are not drugs in the ordinary sense. Agar is a form of cellulose similar to that found in many foodstuffs. Paraffin oil is simply a mechanical lubricant. It has no resemblance to ordinary oils and fats except in appearance. It is a mineral product. It undergoes no change in the alimentary canal and is not absorbed. When properly refined and purified it may be used for an indefinite period without liability of injury, and in most persons who have suffered long from obstinate constipation the continued use of bran and paraffin or agar and paraffin is as much a necessity as the regular and continued use of foods. These substances should be regarded as supplementary foods.

Paraffin should be taken with the meal and not before or after. It should be taken in divided doses so as to be distributed through the meal and thoroughly mixed with all the food eaten. It is the food which needs to be lubricated, not the intestine. If the intestine is lubricated, the mucous secretion always forming will quickly remove the lubrication. If the food is lubricated, the lubrication travels with it and facilitates its movement along the intestine. In its ordinary form paraffin cannot be easily taken with food; but in emulsion diluted with water or milk it may be easily taken. Paraffin tablets, a form of paraffin which is solid at ordinary temperatures but melts at the body temper- 
ature, is also easily taken in divided doses with food. In this form paraffin is much more efficient than in the liquid form and does not "leak," a property which removes one of the greatest objections to the use of paraffin.

What has been said of paraffin is equally true of agar, bran and roughage in general. Bran, agar or greens of some sort, must be taken at every meal, and should be taken in divided doses so as to be mixed with the whole meal.

\section{The Use of the Enema.}

In certain cases of constipation the colon is so crippled that it cannot be readily made to assume its normal rhythmical action. In some such cases surgery may be needed to relieve some mechanical obstacle, but in the great majority of these cases by the proper use of the enema in connection with proper diet and the other measures named the colon may be trained to do its work in a fairly normal manner. In these cases the enema must be used regularly and daily. The best time is just before retiring at night, and the hour should be approximately the same daily. About three pints of warm $\left(102^{\circ} \mathrm{F}\right.$.) water should be used, never more than four pints. The enema may be repeated if necessary to completely empty the colon, this being essential. After emptying the colon a cool or tepid enema, $80^{\circ}$ to $70^{\circ} \mathrm{F}$., should be used, quantity one pint. This will "tone" the bowel and prevent any possible injury from the use of this measure. The evening enema will not necessarily interfere with the after breakfast movement the next day and will prevent the overnight absorption of putrefaction products and overdistension of the colon with gas.

When the ordinary enema does not empty the colon, it is useful to add some harmless colon stimulant. A natural stimulant of the colon is provided by Nature in the acids produced in the normal colon by fermentation. These are lost when putrefaction takes the place of fermentation and the ammonia and other putrefaction products produce paralysis of the colon. The effects of these constipating poisons may be overcome by an acid enema. This method occurred to the writer many years ago (1899) and has been used in hundreds of cases with much satisfaction. Either citric or lactic acid may be used. The amount required is the juice of a large lemon or a dram of lactic acid added to the enema.

The introduction into the colon at night of half a pint or more of cottonseed or some other vegetable oil is sometimes 
helpful in securing a morning bowel movement, but is likely to do harm by causing headache and nausea and lack of appetite, doubtless on account of the large quantity of oil and the depressing effects of the oil upon gastric juice formation. When oil is to be used for this purpose, a special paraffin which melts at the temperature of the body should be employed.

Dr. Winfield, of Brooklyn, New York, has published (1912) an account of the successful treatment of psoriasis by irrigation of the colon with lactic acid. Of 40 cases treated, 23 were reported cured, 16 improved.

In cases in which the measures above indicated do not secure prompt relief from constipation, an X-ray examination by the aid of the barium meal should be made. By this means, a minute inspection of every part of the intestine is possible. In many cases, contractions, adhesions, displacements, and other impediments to normal bowel action are found, which may be corrected by the application of special measures. Such an examination should be made in all cases of obstinate constipation.

Food wastes should never be retained more than twenty-five or twenty-six hours at the longest, and the normal period is probably sixteen hours or less. Longer retention results in putrefaction and toxemia.

Constipation is one of the greatest causes of disease, misery, inefficiency and premature senility, and should be most earnestly combated. By a persevering effort and the application of known and well tested measures, every case of constipation may be relieved.

One of the most obstinate forms of constipation and one of the most common is the spastic constipation of chronic colitis. This form of constipation derives its name from the fact that its cause is a spastic or continuous contraction of the colon, usually the descending and pelvic colon. Because this condition accompanies colitis, it is often thought by many that bran and other roughage should be avoided. This is entirely an error. Roughage is more needed in this form of constipation than any other. Bran is of signal benefit in these cases. It is most important that the mucous membrane should be kept clean. The amount of roughage and lubrication should be sufficient to secure two or three free evacuations daily, and a hot enema $\left(115^{\circ}\right.$ to $120^{\circ} \mathrm{F}$, two quarts) should be given at bed-time. Carmine capsules or charcoal should be taken frequently to make certain 
that the colon is thoroughly emptied (page 563). The diet should contain an abundance of bran or agar and other roughage, which is needed to stimulate the dilated right portion of the colon to efficient activity. Paraffin oil is necessary as a lubricant, as long continued catarrhal inflammation causes degeneration of the mucous glands and a loss of the normal lubricating mucus.

\section{Rules for Care of the Colon to Combat Constipation.}

First of all, change the flora (see Index) and then:

1. Never neglect a "call." Visit a toilet at once even at much inconvenience. A lost "call" is not easy to recover.

2. Train the bowels to move three times a day. After breakfast, after dinner or luncheon and before retiring are the times when residues are ready for dismissal. If an extra movement occurs before breakfast, so much the better.

3. Visit the toilet at regular times even if no "call" is present.

4. Take plenty of time at the toilet. Read the morning paper. Crippled colons move slowly.

5. Place a footstool eight inches high in front of the toilet seat. Squatting is the normal position during evacuation.

6. Deep pressure with the fist just above the left groin often aids movement.

7. The meals must be regular to make the bowels regular. Don't miss a meal. Eat something if only an apple or an orange or two with fig bran or psyllium seed.

8. Take plenty of time to eat. Chewing the food stimulates the colon to push the residues along.

9. Eat no white flour bread and avoid condiments, meat and other constipating foods.

10. Eat whole grain breads and cereals. Add an equal measure of bran to oatmeal and other cereals. Eat liberally of greens, fresh vegetables, dates, figs, prunes and all laxative foods.

11. Eat fruit, nothing else, at $11.00 \mathrm{~A}$. M., 5.00 P. M. and bedtime, with fig bran or psyllium seed.

12. Drink a glassful of cold water at bedtime, on rising in the morning and every time the bladder is emptied. 
13. Every night and morning lie on the back and raise the extended legs high as you can ten to forty times. Get a set of good exercise records* and use morning and night. Walk 3 or 4 miles, saw or split wood or work in the garden every day.

14. Take a cold bath on rising.

15. Take bran bread or biscuit and mineral or paraffin oil in some form at each meal, and in such a way as to mix with the whole meal; that is, not only at the beginning and end of the meal but during the meal.

16. Take a hot enema ( 2 quarts, $115^{\circ}$ to $120^{\circ} \mathrm{F}$.) at bedtime occasionally to make sure that the colon empties well, or make the carmine capsule test (page 563).

If necessary, take an enema every night (three or four pints; repeat if necessary; see Index). Keep clean inside. If there is pain in the bowels, much gas or mucus in the stools, make the enema $105^{\circ} \mathrm{F}$. to relax the bowel. After evacuation, take a second enema at $80^{\circ} \mathrm{F}$. to tone up the bowels. This prevents any injury from the enema and helps to train the colon to more efficient action.

17. Beware of Drugs. All laxative medicines, including mineral waters, are highly injurious. They make constipation worse and cause colitis. Agar, bran and mineral or paraffin oil are the only harmless laxatives. These are accessory foods rather than drugs. They may be used indefinitely without injury.

19. When there is no "call," stimulate the rectum with a small enema at $80^{\circ}$ to $70^{\circ} \mathrm{F}$. (half pint to pint). In case the enema does not return well, add the juice of a lemon.

20. When the bowels are once regulated, be careful not to interrupt the rhythm. Make no experiments. Keep right on doing the things which succeed, every day and at every meal.

\section{THE ELIVIINATION OF DISEASE GERIVIS FROII THE ALIVIENTARY TRACT}

The elimination of germs from the alimentary tract is one of the most important things to be accomplished in dealing with most cases of chronic disease. Nature has provided very wonderful means of protecting the body against the invasion of disease-

\footnotetext{
"The "Battle Creek Sanitarium Health Ladder" is a good series. Made and sold by the Columbia Graphaphone Company. May be obtained of the Modern Medicine Publishing Company, Battle Creek, Mich.
} 
producing germs, but the defense is often broken down, making the employment of special measures necessary.

Stagnation or stasis is one of the conditions which favors bacterial development. It is for this reason that the bacteriology of the alimentary tract relates especially to the six cavities in which fluids or food residues are likely to remain for longer or shorter periods-the mouth, the stomach, the duodenum, the gall bladder, the cecum, and the lower colon. The methods of dealing with these several parts to rid them of disease-producing bacteria may be briefly summarized as follows :

The Mouth. Diseased tonsils must be removed by the surgeon or destroyed by the X-ray. Diseased teeth and gums must be dealt with by the dentist and the mouth put in a thoroughly sanitary condition by the dental hygienist, and kept sanitary by the proper use of the tooth-brush, the orange stick and paper polisher (Hygos) and the use of dry food.

The Stomach. Normally, the stomach keeps itself clean and aseptic by its acid secretion, hydrochloric acid, which is produced at a strength of one-half of one per cent., equivalent to 1.25 per cent. of the C. P. acid of the pharmacy. When the acid-forming glands lose their function (achylia), the stomach becomes a dangerous breeding place for bacteria which rapidly spread to the intestine and infect connecting organs.

The stomach may be cleansed to a degree by lavage, which is most useful in cases of pronounced catarrh of the stomach.

Copious draughts of hot water an hour before meals is a crude but more or less efficient mode of cleansing the stomach which often renders great service in preparing the stomach for the next meal in cases of achylia. The free use of orange juice and other acid fruit juices is another highly useful means of combating the molds and bacteria which thrive in these deteriorated stomachs.

The free use of acidophilus buttermilk is another measure of real value.

Another efficient means is the use of acid protein (Acidone), a dram or two at each meal, the best known substitute for the normal gastric acid, containing about 5 per cent. of $\mathrm{HCl}$ in loose combination with protein.

The employment of chemical antiseptics is useless, even worse than useless, because of the extra labor laid upon the liver and kidneys to destroy, distoxicate or eliminate them. 
The liberal use of fresh or uncooked foods and adoption of the two-meal-a-day plan with an interval of seven hours between meals are measures of great service in keeping the stomach and intestine free from bacteria.

The Duodenum. This expanded, upper part of the small intestine receives the food from the stomach sterile, when the stomach produces a normal gastric juice, but often swarming with bacteria when gastric acid is lacking. Here, also, the bile is poured out. The bile may be a source of grave infection when the gall bladder has become an incubating chamber for typhoid or other disease germs, as is often the case after typhoid fever, in cases of infectious jaundice, and probably in many cases of diabetes. The duodenum is the receptacle of poisons constantly poured into it from the blood after absorption from the colon.

Infection of the duodenum may also occur by the backing up of infectious material from the colon, a reflux permitted by an incompetent ileocecal valve. The duodenum is one of the great storm centers of the body. Bacteria and their poisons concentrate here and initiate troubles which extend to other parts.

The Gall Bladder. The liver secretes bile more or less continuously. The gall bladder stores up the bile and pours it out in small portions to neutralize the acid fluids coming in jets through the pylorus at frequent intervals during gastric digestion. Most of the bile is re-absorbed. It thus becomes concentrated and, when infected, the infection inevitably becomes more and more intense. The infection may extend into the pancreas and cause pancreatitis or diabetes. Ulcer may develop and, in rare cases, cancer. Constant exposure of the liver to these concentrated poisons after a time results in sclerosis.

The duodenum and the gall bladder must be dealt with together. By means of the duodenal tube, the duodenum may be washed out and even disinfected. The disinfection may be chemical or biologic. The chemical disinfection is simply an imitation of the normal process. A solution of hydrochloric acid (onefifth of one per cent.) is slowly passed into the duodenum during one hour three times a day. Biologic disinfection is accomplished by passing through the tube a whey culture of $B$. acidophilus and a solution of lactose.

The gall bladder is drained once a day and the bile examined. The draining is repeated daily until the bile is light colored. By this means, the concentrated toxin-laden bile is gotten rid of. 
For most thoroughgoing effects, the duodenal tube will be kept in situ for some days, a week if need be, and two or three gallons of water will be passed through daily. The nourishment should exclude protein and fats almost wholly. Milk sugar, malt sugar and fruit juices should constitute the nourishment. Rice gruel, vegetable broths, savora broth and purées of fruits and vegetables may be added after the first three or four days. Most of the large quantity of water introduced will find exit through the kidneys. Some of it may be discharged as watery stools.

By these thorough-going measures, the duodenum and gall bladder may be cleared of mischief-making bacteria, the bile as well as the flora may be changed and the tissues of the body cleared of tissue wastes and accumulated poisons.

The Cecum. The head of the colon normally receives the food residue, absorbs two-thirds of its water, and then pushes the mass across the transverse colon and so gives it a good start toward the exit. But the cecum often becomes a widely dilated, adherent, inert pouch, a stagnant reservoir of putrefying food residues and a source of almost infinite mischief. This condition is very common in cases which have undergone operations for appendicitis, and especially in cases of so-called chronic appendicitis. The ileocecal valve is always incompetent and hence there is no hindrance to the extension of the infection into the small intestine and the development of all the evil results of autointoxication. Headache and neurasthenia in most pronounced forms are frequently associated with this condition.

The pernicious flora of the cecum may be attacked from both directions. By feeding massive doses of lactose or acidophilus culture or of the two combined, the colon bacillus, Welch's bacillus, B. putrificus and other putrefactive bacteria, may be driven out. By flushing the colon with copious enemas twice daily and the injection of cultures of $B$. acidophilus, the change of flora may be greatly aided.

The Lower Colon and Rectum. These parts are more easily reached and quite readily yield to the combined attack of lactose feeding and colon cleansing followed by freshly prepared cultures of active strains of $B$. acidophilus.

The importance of keeping the lower intestine free from putrefying residues is emphasized by the recent discovery of the facility with which matters are transported upward from the colon by reverse peristalsis. Charcoal introduced into the colon at night 
may often be found in the stomach in the morning. X-ray experts (Case and others) have witnessed the return over night to the duodenum of opaque meals which had reached the transverse colon and have seen material almost instantly transported from the lower colon to the cecum. That reverse peristalsis is a very common cause of general infection of the upper intestine, especially in cases of spastic constipation, cannot be doubted. When the ileocecal valve is incompetent there is no protection against this cause of broadcast infection of the whole intestine from colon to stomach, together with the gall bladder and gall ducts and even the stomach itself. Both ends of the alimentary canal must be safeguarded. The mouth must be closed against infectious material in food or drink; the colon must be kept freely open for the evacuation of putrescible residues.

After the use of all these measures, an anti-toxic laxative diet must be scrupulously followed for an indefinite period, meats of all sorts must be rigorously excluded from the dietary, and bran or agar and mineral oil must be freely used to keep the colon free from residues. By these means the body may be freed and kept free from its most deadly and insidious foes, the bacteria which infect the alimentary canal.

\section{DIET IN CHRONIC COLITIS}

Chronic colitis is an exceedingly common disease which seems to be increasing. It is especially prevalent in cities and not infrequently affects whole families.

The cause of colitis is chronic infection of the mucous membrane of the colon. The disease may involve simply the lower part of the bowel or the entire colon. In time, the disease may extend to such a degree as to involve the entire intestinal tract from the stomach to the rectum. In the majority of cases, the disease is most active in the descending colon and the pelvic colon. In the rectum, the disease is known as proctitis. The cause of the disease is infection of the mucous membrane. The condition of the mucous membrance in colitis has been compared to the state of the colon in eczema. The two diseases exist simultaneously in many cases. The infection is not known to be due to a specific organism but is the result of the contact with the mucous membrane of the various putrefactive organisms, common pus-forming germs which abound in street dust, in putrefy- 
ing meat, and are found especially in the dejecta of meat-eating animals.

In some chronic cases, the disease is aggravated by the presence in the intestine of protozoa-minute animal organisms. This form of colitis is very frequent in the tropics.

Colitis is an infection of the colon. The disease owes its origin chiefly to the use of flesh foods, a fact first pointed out by Combe years ago.

Chronic colitis may follow an attack of typhoid fever or of paratyphoid, or it may be the result of acute infection, as of botulism and of meat poisoning or an attack of dysentery or diarrhea.

First of all, it is necessary for the intestinal flora to be changed (page 592), then kept clean by a laxative diet (see Index), and, if necessary, by the daily use of the enema. The introduction into the colon of large quantities of cultures of protective ferments such as $B$. acidophilus is a measure of value.

It is necessary to lay great emphasis upon the importance of roughage in the treatment of this disease, a fact to which Von Noorden was one of the first to call attention. The colitis is not due to mechanical irritation, but to the chemical irritation of bacterial poisons. It should be noted, also, that bran and cellulose of any sort, in the form in which it reaches the colon, is not capable of causing mechanical irritation. Its condition is comparable to that of wet paper. Bran does not irritate, but titillates, a fact which needs to be often reiterated, for the reason that the laity, as well as many physicians, have become so thoroughly imbued with the idea that roughage is liable to produce harmful irritation-an error which has been productive of an enormous deal of harm and is very hard to uproot.

In cases in which the patient's apprehension respecting the possible harm from the use of bran is so great as to produce a bad psychological effect, agar may be substituted. It must be freely used and nearly always in connection with paraffin. Lubrication is needed for the reason that in many cases the mucous glands have become so diseased that they have ceased to produce the normal lubricating mucus and the mucous membrane is left in a dry condition, which causes the coherence and retention of food residues, even when an excess of mucus is present. The mucus is often of such a character that it encourages obstruction of the bowel rather than clearance. Paraffin, especially a solid form of 
paraffin, which melts at the temperature of the body, is of great service in these cases. Not infrequently, the injection of half a pint of warm paraffin with a melting point of $102^{\circ}$, is found of great service in promoting good bowel action the following morning.

The idea that bland foods should be used in these cases is wholly erroneous. Whole grain preparations, such as oatmeal, graham bread, etc., even bran, greens and other fresh vegetables and stewed fruits, should be freely used.

Lactose, or lacto-dextrin, may be introduced directly into the colon with advantage. A good plan is to wash the bowel out with a hot enema $\left(115^{\circ}\right.$ to $120^{\circ} \mathrm{F}$.), and after the colon is completely emptied, to introduce a half pint or a pint of warm starch water containing half an ounce of cornstarch and an ounce of lactose or lacto-dextrin.

A diet which encourages intestinal putrefaction will promote colitis. Hence, meats of all sorts must be discarded. All flesh foods swarm with the bacteria which give rise to colitis.

Tissier has recently shown that eggs are often infected with the paracoli or typhoid bacillus, which is the cause of white diarrhea in fowls and of colitis in man. Eggs should be avoided because they leave a highly putrefactive residue in the colon. In some cases of chronic colitis, milk must be eliminated from the diet, a measure always necessary in acute colitis. The avoidance of animal proteins of all sorts is advantageous because they are much more readily putrescible than plant proteins.

Kohnstann rightly insists that meat should be excluded from the diet of constipated persons because it will intensify the autointoxication. In colitis the danger of autointoxication is great because the filtering power of the intestine is greatly diminished. This was demonstrated by Hamburger and Monte, who experimented with antitoxin. Their observations showed that, although taken into the system very readily when injected under the skin, antitoxins introduced into the normal rectum are not absorbed to any appreciable amount. This is an excellent demonstration of the filtering power of the normal intestine by which it offers resistance to toxins generated in the alimentary canal. It was found also that when colitis exists, this filtering power is largely lost. The same loss of filtering power has been shown to be 
produced by the administration of calomel, saline laxatives and other cathartic or laxative drugs.

According to Fuld, colitis is rare in Russia, a fact which he attributes to the vegetarian diet of the Russian peasant.

The so-called mucous colitis does not essentially differ as regards either the methods of its production or the treatment required from other forms of chronic colitis. It is not a nervous disease, as has been assumed by many writers, but an infection which is accompanied by nervous symptoms, the natural result of the poisons which are freely absorbed by the irritated, congested mucous membrane. In cases in which parasitic infection exists, the use of suitable anthelmintics is necessary, in addition to dietary regulation.

\section{Acute Colitis-Dysentery.}

This disease is an acute infection of the colon due to invasion of either bacteria or minute animal organisms known as protozoa. This may be a new infection or simply an exaggeration of an old one. The attack may involve the colon only or both colon and small intestine. The usual management of acute colitis is bad. The first measure generally suggested is a laxative for emptying the bowel, which is much like applying a mustard plaster to an inflamed skin surface. It should be remembered that only in cases of mechanical obstruction is there likely to be an accumulation in the small intestine. This part of the intestinal tract is practically always found empty within six to eight hours after the last meal. The small intestine always faithfully performs its part in pushing on into the colon reservoir everything which should be disposed of in this way.

In view of these facts, it is evident that catharsis in colitis is a most irrational procedure. The colon is simply a pouch with an external opening and it can be emptied of its contents almost as easily as a pocket in one's coat. By means of the enema repeated as often as may be required, the offending contents of the colon may be readily removed. The cathartic leaves the whole intestine in a state of increased irritation and damages the bowel in such a way as to lessen its power of resistance to bacteria and bacterial poisons. A simple enema, on the other hand, cleanses the diseased surface, and by the use of warm starch water (onehalf ounce to the pint, boiled for five minutes) for the enema, a decided emollient effect is produced. This may be repeated sev- 
eral times a day, or often as needed. The addition to the enema of half an ounce of lactose to the pint is an exceedingly useful means of combating the development of the bacterial causes of the condition and promoting a luxuriant growth of non-putrefactive, acid-forming organisms.

The diet should be strictly antitoxic. It should first consist of cereals only in the form of gruels, to which lactose or dextrin should be added in the proportion of two ounces to the pint. Three or four pints of gruel may be used in the course of twentyfour hours. This will greatly aid in changing the intestinal flora. It is better to withhold meats of all sorts, as well as eggs, for several days or until the acute symptoms have wholly subsided.

During the first twenty-four hours, the diet may consist of sugar water and oatmeal or barley water, with lacto-dextrin, two ounces to the pint. As the acute symptoms diminish, which usually occurs within twenty-four hours or less, gruels and cereal preparations of greater consistency are added.

Next, vegetable broths and purées of vegetables, sweet and sub-acid fruits may be taken. Banana purée and purée of dates may be taken quite early, together with purée of spinach.

The direction given by some authors to feed albumin water early in the disease and later scraped meat, is quite irrational and wholly out of joint with the clear indications of pathology and bacteriology. Animal proteins of all sorts must be strictly avoided because they encourage the development of pathogenic organisms. Eggs as well as meat (fish, flesh, fowl and shellfish) and even milk must be excluded from the dietary, not only during the acute stage of the disease but during convalescence and for some time afterward. The measures necessary for thorough changing of the intestinal flora (page 592) must be assiduously employed and when possible bacteriological examinations of the intestinal flora should be made at frequent intervals until the pernicious bacteria have disappeared, and an aciduric flora is established. To prevent relapse, the diet recommended for chronic colitis should be faithfully followed.

The recommendation of whisky and claret in this disease, found even in quite recent works on dietetics, is worthy only of most unqualified condemnation as having no sound basis whatever in experimental therapeutics or clinical experience.

When the patient has recovered from an acute attack of 
colitis, care should be taken to follow a strict anti-toxic diet and to keep the flora changed by frequent and liberal use of lactose or lacto-dextrin. Great care must be taken to keep the colon empty. This may require for some weeks a daily enema at bedtime, since acute colitis usually leaves the colon, especially the descending colon, in a highly irritated and spastic state, which may continue for several weeks after acute symptoms have subsided.

Although Bogota (S. A.) has a remarkably healthy climate, the death rate in young children is very high, which Dr. Torres Umana has shown to be due to the use of bad milk; that is, milk contaminated with animal excreta. Such milk always contains the Welch's bacillus in great numbers. In an epidemic of acute colitis in which the stools were examined in 150 cases, the Welch's bacillus was found in a great number of children and was not present in any case in the stools of 50 healthy children. In cases in which the Welch's bacillus was found in the stools of the sick children the same germ was found in the milk which the child had been eating.

In the treatment of these children it was found most essential to suppress animal proteins of all sorts completely, that is, milk as well as eggs, meat, meat juice and meat broths were found to be very harmful. The reason is not far to seek. Welch's bacillus and other putrefactive and disease producing organisms thrive best in a medium containing animal proteins. Tissier, Torrey and other investigators have shown that vegetable proteins do not encourage the growth of these organisms as do proteins of animal origin. The children did best on vegetable broths containing about one ounce of lactose to the pint. Lactose encourages the growth of the protective organisms and discourages the growth of Welch's bacillus and other putrefactive bacteria.

It is probable that the use of impure milk, that is, milk contaminated with stable filth is one of the chief causes of colitis in young children. The use of impure milk is so common it is probable that few children in this country escape infection. The degree of infection may be so slight that it is easily overlooked and becomes chronic. In the writer's experience the total exclusion of animal proteins is not infrequently as necessary for the complete cure of chronic colitis in adults as acute colitis in infants. This is particularly true in cases of chronic colitis in which acute attacks are frequent. 


\section{DIET IN INTESTINAL OBSTRUCTION}

In cases of intestinal obstruction, surgical measures are indicated and should be resorted to at the earliest possible moment. In the meantime, while preparation for more radical measures are being made, several things of importance may be done.

Half a pint of warm water with an ounce of malt or milk sugar should be given by enema hourly. When possible, the duodenal feeding tube should be used. By this means, the putrid and highly toxic duodenal contents may be pumped out and sugar water introduced, greatly to the patient's advantage. Some portion of the sugar will be absorbed, giving valuable re-enforcement to the patient's flagging energies, especially sustaining the heart much more effectually than caffein or any other drug can do. The sugar also has the effect of modifying bacterial changes, preventing the development of putrefactive products and other bacterial toxins. Sugar water is prepared by dissolving in warm water two ounces of malt sugar or milk sugar.

In the writer's hands this plan of feeding a patient suffering with intestinal obstruction has proved invaluable and in several instances has been the means of saving the patient's life.

After an operation for enterostomy has been performed for intestinal obstruction, lactose or malt sugar, two ounces to the pint, should be used for irrigation of the intestine, the effect being both to nourish the patient and to suppress putrefactive changes. An antitoxic diet of liquids and purées is indicated.

The observation by Case that intestinal obstruction may be discovered by examination with the X-ray, which reveals the abnormal distension of the intestine, is highly important as a means of early diagnosis.

\section{DIET FOR ULCERATION OF THE COLON}

The diet in ulceration of the colon is the same as that for colitis (see Index). It is not necessary to suppress bran and other roughage. This element of the food is necessary to promote bowel movement. The thing necessary for recovery in these cases is not a bland diet but cleanliness of the colon. Noth-ing is so irritating to the diseased surfaces as the presence of putrefying residues. The irritation of the colon is caused, not mechanically but chemically, by the products of putrefaction. 
Agar may be used freely, and paraffin oil should be taken with each and every meal. Large enemas of starch water with milk sugar (an ounce to the pint), and, if possible, several ounces of whey cultures of the $B$. acidophilus should be used daily. Lactose or lacto-dextrin one to three ounces three times a day for one or two weeks should be given to change the intestinal flora. This is always necessary.

Ulcer of the colon is usually the result of chronic colitis. In some cases protozoa are present as an active cause of the ulceration. This is particularly true in the case of persons who have lived in the tropics. In such cases suitable antiparasitic remedies should be employed. Ulceration sometimes follows typhoid fever. In the great majority of cases this condition accompanies chronic colitis and is most frequently located in the upper part of the rectum or in the pelvic colon. Bad cases require rest in bed and occasionally surgery is required. Remarkably rapid results have often been obtained by the aid of appendicostomy, an operation which consists in the establishment of a fistulous opening into the head of the colon through the appendix. By irrigating the colon several times a day, thus keeping it free from putrefying residues, ulcerations, even though quite extensive, may often be rapidly healed. The colon should be irrigated with cultures of $B$. acidophilus and sugar water. Whey cultures of $B$, acidophilus are the best for this purpose. Flushing with starch water is also useful. The temperature of irrigations should be $110^{\circ}-115^{\circ} \mathrm{F}$.

\section{Diet in Hirschsprung's Disease.}

In this, fortunately, rather rare disease, the colon is enormously enlarged. The condition generally makes its appearance in infancy and is commonly regarded as congenital, at least in most cases. Removal, or abbreviation of the colon, is recommended by many surgeons, and has been practised in some cases, but in the writer's opinion is rarely required, provided the subject is willing to submit to the necessary restrictions in diet.

The essentials of the dietetic treatment are: 1. The careful exclusion from the diet of meats of all sorts and careful adherence to a strictly antitoxic diet, with no digressions. The dietary must be low in protein, all the surplus being avoided. Even eggs must be used sparingly. It is better to avoid the whites of eggs entirely. It is even necessary to use some care with reference to 
the use of milk. When milk is not well digested, the curds which reach the colon are likely to undergo decomposition and may give rise to an intense toxemia. It is well to remember that vegetable proteins are far less likely to undergo putrefactive changes than animal proteins. On this account, nuts are to be highly recommended as a source of protein in place of meat, milk or eggs. Walnuts, almonds, filberts, and even peanuts, may be made a part of the regular bill of fare with advantage. An emulsion of nuts known as malted nuts is a convenient and very acceptable nut preparation. The intestinal flora should be changed and kept changed by the use of lactose or lacto-dextrin.

The diet should be bulky, consisting very largely of coarse, green vegetables. Bran or agar should be ised at every meal. Paraffin in some form should also be used at every meal. All the measures recommended for the relief of constipation should be systematically and continuously employed. The colon should be emptied daily by a copious enema to which the juice of one or two lemons or one or two teaspoonfuls of lactic acid should be added. If necessary, several enemas may be employed to make sure that the colon is thoroughly emptied.

\section{DIET FOR HEIMORRHOIDS}

The diet to be employed in a case of hemorrhoids is essentially an antitoxic and laxative dietary. Bran shoth be freely the extent of one-half to an ounce daily The bowels should be kept loose; should be moved three times daily. Patients sometimes complain of increased irritation due to frequent bowel movements, particularly when the enema is used. This difficulty can be usually obviated by taking care to cleanse the parts thoroughly after the bowel movement, and to apply to the anal canal a little carbolated vaseline.

Large hemorrhoids may often be made to disappear by the daily application of a powder consisting of equal parts of starch and calomel, after first carefully cleansing the parts.

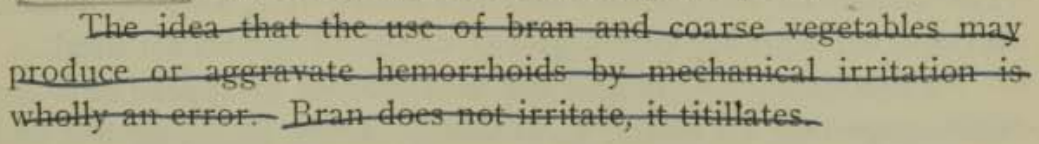




\section{DIET IN APPEINDICITIS}

Appendicitis is the result of an extension of infection from the colon to the appendix. Nothing can be done by diet for an acute attack of appendicitis since all food should be suppressed until after the patient gives marked indications of recovery or has been subjected to the operation indicated. It is highly probable, however, that appendicitis may be prevented by a proper regimen, or at least that it is much less likely to occur when a special regimen is followed, and so-called chronic appendicitis is usually curable by changing the intestinal flora.

Many years ago, Dr. Lucas-Championnière, the surgeongeneral of the French army, discovered that appendicitis is a disease to which meat-eaters are especially liable. During a military campaign in Africa he noted that appendicitis was very common among the French soldiers who were fed canned meats, but almost unknown among the Arabs, who lived upon a diet of wheat cakes, camel's milk and vegetables.

He later observed that appendicitis, except in the case of newly admitted inmates, was practically unknown in the prisons and insane asylums of France, where meat is not used.

Senn, of Chicago, observed that appendicitis was unknown among the vegetarian natives of the east African coast.

Dr. McCord, who has practised for more than thirty years among the natives of Natal, states that appendicitis is the rarest of maladies among these people, by whom meat is rarely eaten.

Crichlow reports that appendicitis is unknown among the natives of the Solomon Islands so long as they adhere to their native diet, which is almost wholly of vegetable origin.

Dr. Snyder, for ten years physician to the Shah of Persia, met only ten cases during that time in Teheran, three of whom were Europeans and one a native student just back from Paris.

Dr. Magnon, physician to the French Embassy at Pekin, met among the Chinese only one case of appendicitis in five years. The staples of the Chinese diet are millet, rice, sweet potatoes, turnips, cabbage, soy beans, greens, cornmeal and wheat meal.

Flesh-eating encourages the development of appendicitis for the reason that all meats, as ordinarily eaten, including fish and fowl, contain in a state of luxuriant growth virulent forms of bacteria which are capable of producing infection of the colon and of the appendix. For this reason, game, cold storage meats 
and meat that has been hung until tender are particularly likely to give rise to this disease.

Persons who have been subject to attacks of appendicitis and have recovered should discard meats of all sorts, especially fish, including oysters, and should take care to keep the colon empty by three regular movements daily.

Efficient measures should be taken for changing the intestinal flora (see Index). The stools are always putrid in persons subject to attacks of appendicitis. Such persons should not rest until they have made such modifications of their diet as to cause a decided change in the stools, including the disappearance of the putrid odor and of the mucus which is nearly always present on account of the coexistence of colitis.

After an operation for appendicitis, the antitoxic diet (see Index) should be permanently adopted and should be faithfully adhered to as a permanent regimen. There is no danger of the recurrence of inflammation of the appendix, since this organ has been removed, but the same sort of infection which produced the appendicitis still exists in the colon and will be perpetuated by the continuance of the diet which induced it in the first place. The danger from infection of the colon is less acute and immediate than that from infection of the appendix, but the autointoxication which it induces may later produce equally calamitous results.

\section{DIET IN DISORDERS OF THE LIVER}

There is probably no other organ of the body whose functions are so often overtaxed either temporarily or permanently as those of the liver. The liver suffers even more than does the stomach from dietetic errors. The stomach may summarily dispose of its offending contents; but the liver acts as a strainer or filter. It must secrete and retain in its cells substances found in the portal blood likely to do the body harm, and must then, little by little, destroy or detoxicate the offending matters. The great multiplicity of its functions and the enormous overwork we require of this faithful body servant, the monstrous abuses we subject it to by our poison habits-the use of tea and coffee, the tobacco habit, the use of alcoholics, the high protein diet of meateaters, and probably worst of all, the great flood of toxins absorbed from the intestines and poured through the liver as the result of constipation and the use of cathartics and other drugs- 
prematurely wear out the liver cells and cripple this most important of all the body defenses against extraneous poisons as well as those generated within the body.

\section{Tests for the Liver Function.}

The great number of vitally important duties performed by the liver render highly important the determination of the state of its function. Although the great desirability of this information has long been appreciated, it has only been within recent years that tests have been devised by means of which the condition of the liver function could be determined with any degree of exactness. Within a few years numerous methods have been brought forward so that at the present time it is possible to apply fairly accurate tests to the liver functions by means of which any considerable degree of insufficiency may be readily discovered. Because of the many different functions of the liver, the tests which may be applied to it are now more numerous than those which are employed in the functional examination of any other. vital organ.

One of the simplest and most practical methods is that known as the hemoclastic crisis of Widal. This test is based upon the fact that at the beginning of the process of digestion some of the products of protein digestion are absorbed before their transformation is entirely completed. Normally these substances, which in this crude form cannot be utilized by the body, and act as poisons, are distributed by the circulation, retained and destroyed or detoxicated by the liver. When the liver is diseased, this protective function is impaired, so that the imperfectly digested proteins are permitted to pass into the general blood stream. The result is a more or less distinct anaphylactic reaction, the first indication of which is an increase in the number of white blood cells. This increase of white cells is a part of the defensive reaction of the body against the foreign proteins. When the liver is the seat of organic disease or its cells functionally crippled, the increase of white blood cells does not occur. Instead the white cell count decreases, and the more, the greater the deficiency.

This decrease may occur as the result of taking milk even in so small amount as one or two ounces in cases in which the liver is very considerably diseased. A larger amount is usually employed, however, so that the smaller degrees of hepatic impairment may be discovered. 
The method of applying Widal's test is as follows: The examination is made in the morning before breakfast, care being taken that the patient has eaten nothing since the night before. The blood is examined and the number of white blood cells determined. The subject is then made to swallow seven ounces of milk and at the end of 20 minutes another specimen of blood is obtained and examined. Each 20 minutes the examination is repeated. When the liver is diseased the blood cells instead of increasing as in health are found to diminish. This change may appear at the end of the first 20 minutes. In the majority of cases the most marked difference is observed at the end of 40 minutes. The decrease in the number of white blood cells may amount to 50 or even 75 per cent. This is a very delicate test for impairment of the function of the liver, and it sometimes gives positive results when no other indication of liver disease is observable. By means of this test, disturbance of the liver function may be clearly demonstrated after the administration of chloroform or treatment by salvarsan or other arsenical preparations. It is highly useful, also, in detecting cases of sensitivity to special proteins and is employed by Widal and his associates in all cases of urticaria and asthma. When in a case of this sort a positive reaction occurs, a further search is made for the purpose of determining the individual protein to which the subject is sensitized.

Temporary inefficiency of the liver exists in most cases of acute infectious disease. It is marked in pneumonia. Whether death or recovery occurs will depend in many cases upon the degree of permanent inefficiency which existed before the development of the acute disease. In general, when marked renal inefficiency exists, inefficiency of the liver is also present.

Hepatic inefficiency may involve all the liver cells and hence all the functions of the liver, or it may concern only a single function. It is possible that a considerable degree of organic disease of the liver may exist without noticeable functional deficiency.

It is thus apparent that tests for hepatic efficiency are highly important and should be employed in all cases in which there is reason to suspect impairment of the functions of this great vital organ. To judge of the conditions of the liver only by the color of the skin or of the stools, is not sufficient. The inquiry must look into other of the less obvious but equally important phases of liver work. As many tests as possible should be employed. 
One of the most valuable tests for liver efficiency yet devised is that of Graham.

The use of tests for hepatic efficiency is especially important in connection with diet regulation, for in no way can the liver functions be so greatly influenced as by the food intake. Certain foods profoundly affect the liver, exciting it to activity when properly employed, and overwhelming it with unnecessary work when unsuitable in quality or quantity. It has even been shown* that certain foods (carbohydrates) may render invaluable service by enabling the liver to repair its damaged cells after they have been injured by poisons. Since the time of Claude Bernard it has been known that the glycogen stored in the liver is an essential aid to the liver in the performance of its poison-destroying function. This fact offers hope to a large class of sufferers from crippled livers, the result of chronic intestinal toxemia. In changing the intestinal flora by the free use of carbohydrates (lactose. dextrin), the toxemia may be cured and the liver damage repaired at the same time.

\section{DIET FOR "BILIOUSINESS"}

"Biliousness," an unscientific term, is generally used to designate a condition of general malaise, loss of appetite, dullness, constipation, and general unfitness. This condition is usually attributed to torpidity or inactivity of the liver and calomel is the usual prescription, especially in the South, one of its chief properties being supposed to be to increase the activity of the liver.

As a matter of fact, calomel does not increase the activity of the liver. This was shown by Bennett, of Edinburg, more than a hundred years ago by experiments upon dogs. Bennett found, in fact, that calomel lessens the amount of bile produced by the liver instead of increasing it. Modern investigators have confirmed these findings; but the use of calomel still continues and the users testify that they find relief by its use, at least temporarily. This is not to be disputed. It is this immediate relief which maintains confidence in this antiquated drug.

The explanation is' to be found in the fact that "biliousness" is not due to deficient secretion of bile or to sluggishness of the liver, but to autointoxication, arising from retention of putrefying food residues and body wastes in the colon. Calomel acts

- Fole et Pancreas. M. J. Castalgne, Paris, 1920. 
as a laxative and a disinfectant. Its use causes the discharge of a quantity of bile which has become concentrated and toxic by re-absorption. Getting rid of these poisons affords relief from the symptoms commonly called "biliousness" and other evidences of toxemia.

Unfortunately, the relief is only temporary; and still more unfortunately, the relief is not the only result. Some of the calomel is converted into corrosive sublimate and is absorbed as such, and poisonous effects are produced. The liver is damaged, and other evil effects result, among which is colitis.

It is thus most desirable to avoid the use of calomel, and to combat the evil conditions for which it is employed in some other way. Fortunately, this may be done and by very simple means. Here are the measures which may be adopted with the certainty of good results,

Omit from the diet meats of all sorts and for a time, also, eggs. Buttermilk may be used freely and all sorts of fresh vegetables, particularly turnips, greens, celery, cabbage and lettuce, with fruits and fruit juices. Two large tablespoonfuls of bran should be taken at each meal.

The flora must be changed by the fruit regimen with three ounces of lactose or lacto-dextrin three times daily for a week or ten days. A cleansing enema should be taken every night to thoroughly empty the colon.

After recovery from a "bilious attack," the antitoxic and laxative dietaries should be definitely and permanently adopted. Care must be taken to move the bowels three times a day, using an enema at bedtime if necessary to secure complete emptying of the colon.

\section{DIET FOR GALL BLADDER DISEASE}

Infection of the gall bladder, either with or without gall stones, as is the case with other cavities of the body is chiefly due to stasis or stagnation of its contents. When the gall bladder is not fully and frequently emptied, it becomes an incubating chamber. The bile is not a real antiseptic and when charged with mucus, as it always is when diseased, it becomes a most excellent medium for the growth of putrefactive bacteria and other disease-producing organisms. On this account, it is highly important in cases of this sort to secure as complete and frequent emptying of the gall bladder as possible, thus lessening the op- 
portunity for the growth of bacteria and the development of virulent strains of bacteria.

Something may be done toward the emptying of the gall bladder by the cool enema $\left(85^{\circ}\right.$ to $75^{\circ} \mathrm{F}$.), which stimulates contraction and thus empties the bile reservoir. Free water drinking ( 5 to 6 pints daily) thins the bile and encourages its passage into the intestine. But the most effective means of exciting contractions in the gall bladder is the taking of food. When food is taken into the stomach and begins to pass out into the duodenum through the pylorus, the contact of the acid mixture of gastric juice and food with the lining membrane of the duodenum, excites powerful contractions in the gall bladder. On this account, it is better for these patients to take small quantities of food at frequent intervals, preferably four times a day. Two major meals may be eaten and two minor fruit meals, the latter two hours before meal-time, so as to secure complete emptying of the stomach before the next meal. The late Professor DujardinBeaumetz called attention to this fact so long as thirty years ago. A point which the professor overlooked is the need of making a careful selection of foodstuffs when four meals are taken. First of all, the food should be easily digestible, and of a sort which leaves the stomach quickly. Fruit fills the indications exactly, and should constitute the sole food taken at the minor meals. Rice gruel or plain potato purée may be used when fruit does not agree.

Persons who suffer from gall stones should avoid foods rich in cholesterol-containing foods. They should particularly avoid much cream or butter and yolk of eggs, which are rich in cholesterol.

Test for liver efficiency should be made in all cases when gall bladder disease is believed to be present. In operating for drainage or removal of the gall bladder the writer has had an opportunity to inspect the liver during life in some hundreds of cases. In practically all cases there has been observed some evidence of disease of the liver itself, after marked cirrhosis has been found present and not infrequently enlargement. In view of these facts it is evidently necessary that in all cases of gall bladder disease great care should be taken to maintain a normal intestinal flora by a strict antitoxic diet. The flora should be changed in every case and the stools should never be permitted to become putrid nor the patient constipated. The bowels should be made to 
move three times a day and care must be taken to see that the colon is well emptied. It is well to empty the colon by means of an enema every night at bedtime whenever there is ground to suspect that there is retention of residues in the colon.

The presence of gallstones or pronounced disease of the gall bladder is always an indication for operation, and in general the gall bladder should be removed because it is a focus of infection not only for the liver, but for the pancreas, and for the whole body. A diseased gall bladder is a far more serious menace than a diseased tooth or tonsil.

\section{CIRRHOSIS AND OTHER DEGENERATIONS OF THE LIVER}

The liver is crippled in all its functions. It has been required to deal with such enormous quantities of toxins that it has been prematurely worn out. Its work must be made as light as possible by changing the intestinal flora with lactose or lecto-dextrin or other of the means pointed out in other chapters, so as to suppress the formation of putrefaction products in the colon, and also by avoiding all excess in the use of fats, since it is a part of the duty of the liver to metabolize fats. Meats of all sorts, condiments, all excess of food and especially of animal protein must be carefully avoided. Copious water-drinking and attention to personal hygiene in all particulars are necessary measures.

M. T. V. Tallqvist (La Semaine Médicale) in extended dietetic experiments made upon persons suffering from catarrhal jaundice, inflammation of the gall bladder, gall stones, and hepatic sclerosis due to cardiac diseases and other forms of disease of the liver due to intestinal autointoxication, observed that in order to produce nitrogenous equilibrium it was necessary to employ much larger proportions of carbohydrates than in the normal state. This is doubtless due to the disturbance of the hepatic functions set up by the toxins absorbed from the alimentary canal. The fruit regimen is of great service in these cases. A diet rich in carbohydrates aids the crippled liver.

\section{DIET IN DISEASE OF THE PANCREAS}

This disease, formerly considered rare, is now known to be of comparatively frequent occurrence. The pancreas is especially exposed to disease because of its connection with the gall duct. The outlet duct of the pancreas and the bile duct generally enter 
the intestine through the same opening, the two ducts usually joining just back of the opening into the intestines. When infection with chronic inflammation of the gall bladder and gall ducts occurs, a common result is a partial obstruction of the outlet, which causes a backing up of bile in the pancreatic duct. The bile, reaching the pancreas, probably activates the pancreatic juice and causes autodigestion of the pancreas, which in turn gives rise to infection and inflammation, either acute or chronic. In many cases this process goes on for years without producing any considerable amount of pain, although a common result is diabetes, which accounts for the observation made by several eminent authorities that meat eating is an active cause of diabetes. Sometimes, through the formation of cysts, the development of calculi or other causes of obstruction, pancreatitis may be accompanied by paroxysms of severe pain.

It is evident that in all cases of pancreatitis a strictly antitoxic dietary should be followed. The diet should be essentially the same as that recommended for gall stones and cases in which operations have been performed upon the gall bladder. It is especially important that meats of all kinds should be discarded and that the colon should be kept free from putrefying residues. The diet should be such as to secure three full movements daily, the enema being used daily, if necessary, to accomplish this.

While surgical drainage of the gall bladder affords the only radical means of relief, regulation of the diet is of the highest importance.

In cases in which sugar has not appeared in the urine and the blood sugar has not increased, as well as in cases of diabetes, the intestinal flora must be changed by the use of lactose or dextrin and the diet must be strictly antitoxic.

Copious water drinking and the use of acid fruit juices are highly beneficial. Condiments of all kinds must be excluded from the bill of fare, together with tea and coffee.

\section{DIET IN DIARRHEA}

Diarrheas are generally due to infection, the increased activity of the bowels being a remedial effort to eliminate poisons of bacterial origin. There are many micro-organisms which are capable of giving rise to diarrhea. Those which are most active are found in impure drinking water, usually being derived from the 
stools of human beings through contamination of the water with sewage, in milk, eggs, meat and stale fruits.

Certain articles of diet, particularly cheese, oysters, contaminated milk, cold storage meats and game, are frequent causes of diarrhea not only in individuals but in whole families, sometimes affecting entire communities. Pork eaters should remember that diarrhea is one of the symptoms of infection with the hog parasite trichina.

Chronic diarrhea is often a symptom of inactivity of the colon. It is not infrequently found in cases in which eight or ten bowel movements occur daily that the colon, especially the right half of it, is filled with fecal matters, never being perfectly emptied; and this retention of irritating, putrefying material is the real cause of the diarrhea. In such cases, it is necessary to increase the efficiency of the colon in order to unload it before any progress can be made toward the control of the disease. The hot enema $\left(110^{\circ}-150^{\circ} \mathrm{F}\right.$.) repeated as many times as necessary, should be employed daily. Carmine should be used as an indicator, so as to insure complete evacuation of the colon.

The use of cathartics, though frequently employed in the treatment of enteritis, is most irrational. The small intestine is always found empty six or eight hours after the last meal, the food residues and wastes being pushed on into the colon from which it may be removed by a simple mechanical means, the enema. Cleansing the colon by means of the enema, which may be repeated several times if necessary, is all that is required. The hot enema (temperature $110^{\circ}$ to $115^{\circ} \mathrm{F}$.) has a remarkably quieting effect upon the colon, relieves pain, relaxes spasm, and in conjunction with hot applications to the abdomen affords the patient very great comfort.

Bran or agar and paraffin oil are indicated in connection with the laxative diet. The intestinal flora must be changed and the antitoxic diet closely followed. Boil drinking water and disinfect fruits, lettuce and celery.

\section{Diet in Acute Diarrhea.}

When the diarrhea is due to acute infection, the condition is usually accompanied by rise of temperature.

First of all, in case of acute diarrhea, the patient should be put to bed and the diet should consist of water only, or water containing two or three ounces of milk sugar to the pint, or of 
thin oatmeal or rice gruel. Eight or nine ounces of milk sugar should be taken daily by an adult. Whey cultures of the $B$. acidophilus, in doses of half a pint by mouth three or four times a day, are most useful. Lacto-dextrin is still more efficient.

Milk, eggs, animal broths, all foodstuffs rich in protein, should be avoided. The colon should be washed out two or three times a day by a hot enema, and a fomentation followed by a moist abdominal bandage should be applied to relieve the intestinal congestion. Repeat the fomentation two or three times a day, not only to relieve pain, but to relax the contracted colon and thus aid in completely emptying it of its irritating contents.

In children, milk should be avoided altogether. Instead, for seasoning, a little almond cream or nut meal may be added to the gruel. Buttermilk, prepared with the B. bulgaricus or B. acidophilus, is an excellent diet for a few days after convalescence begins and helps to complete the change of flora. One to two quarts of buttermilk should be used daily.

Loeper has shown that the loss of calcium through the stools in diarrhea may be so great as to give rise to puerpural hemorrhages, tetany, epilepsy, urticaria, itching of the skin, erythema, neurasthenia and loss of weight.

It is evident that these serious effects of the loss of calcium may easily be aggravated by the long use of a diet consisting only of bland gruels prepared from cornstarch, fine flour and similar preparations which are known to be lacking in lime and calcium as well as vitamins, to the absence of which some of the symptoms noted may be due.

No attempt should be made to correct the diarrhea by means of a constipating diet. The looseness is not due to mechanical causes but to infection. It is necessary that the flora should be changed as promptly as possible. In most cases this is accomplished by the free use of dextrin or milk sugar, which should be given in quantities of two and a half to three and a half ounces, or two ounces of lacto-dextrin, three times a day.

As soon as the diarrhea is checked, foods rich in lime should be administered. Cottage cheese, whey, buttermilk, purées of spinach and other greens are the best foods for rapidly making good a deficiency of lime.

The diet should also include sweet or mildly acid fruits, purées of vegetables, cereal, gruels and porridges. A day or two later, oatmeal porridge, bran brose, graham bread and stewed 
fruits may be added, and within a day or two more the regular antitoxic and laxative diet may be adopted, and should be adhered to as a regular regimen.

If the stools again become very foul or strong smelling, the intestinal flora certainly needs to be changed. It is well to apply the motility test (see Index).

\section{DYSENTERY}

In dysentery, the plan of feeding should be essentially the same as that for diarrhea in the acute form. In addition, a warm starch enema should be administered three or four times a day. The addition of half an ounce of milk sugar to a pint of starch solution is a most excellent means of combating the colonic infection.

In chronic dysentery the diet should not exclude roughage, but care should be taken that the roughage is of the right sort. Agar may be used safely in quantity from one ounce to one and a half ounces daily. Even bran, when well cooked, may also be used in connection with other cereals. Greens should be used freely in form of purée as roughage and for their iron content and vitamins.

Meats of all sorts must be strictly excluded, of course, as they will only aggravate the disease by promoting the growth of the bacteria to which the infection is due. Rice, buttermilk, brose, potato soup, vegetable broths and purées of greens are particularly suitable for cases of this sort.

The intestinal toxemia which often follows diarrhea or dysentery requires a special regimen to change the intestinal flora.

\section{DIET IN TROPICAL DYSENTERY}

The diet in tropical dysentery does not differ from that of ordinary dysentery, and should be employed in connection with proper medication and other measures necessary for the eradication of the infection.

\section{DIET IN PARASITIC DISEASE OF THE INTESTINE}

Most of the parasites which infest the intestine are scavengers and thrive best in a medium rich in protein residues. Constipated children are the ones that suffer from worms. While changing the intestinal flora and increasing the activity of the intestine by natural means cannot be offered as wholly efficient means of 
cure, these measures are of great value in all forms of parasitic disease of the intestine in connection with the use of emetin, thy$\mathrm{mol}$, phenyl salicylate and other appropriate specific remedies. An antitoxic diet, strictly excluding meats of all sorts, and the maintenance of an acidophile flora are efficient means of lessening the liability to relapse.

An intestinal parasite, Balantidium Coli, a unicellular flagellate organism, a native of the tropics, has been observed in this country with increasing frequency during the last thirty years, especially in the Southern States, but more frequently in all parts of the country. This parasite penetrates the intestinal mucous membrane and becomes encysted and resists the emetin and thymol and other remedies which destroy most other intestinal parasites.

Scully and Greene report uniform success in the treatment of these cases with a milk and fruit diet. The patients were given two and a half quarts of milk a day in small doses at frequent intervals. Stewed fruits were given to aid bowel action. After some days one or two soft boiled eggs were added to the diet.

It seems probable that the improvement in these cases may be due to the presence of the lactose of the milk or the lactic acid produced by the acidophile flora, the growth of which is encouraged by a milk regimen. It seems probable that the regular milk regimen, with the use of lactose dextrin to change the flora, would expedite the cure of these cases.

\section{DIET IN INCOMPETENCY OF THE ILEOCECAL VALVE}

Although the ileocecal valve has been known as an anatomical structure since 1579 , or nearly 350 years, its significance has not been fully appreciated until very recent times. The small intestine is the chief digestive and absorbing organ of the body. Within eight hours after the eating of a meal, the digestible and useful portions of the food have been digested and absorbed and the unusable residue has been pushed through the ileocecal valve into the colon. When intact, the valve closes after the food residues have passed through it and prevents their return to the small intestine. This is highly necessary for the reason that in the colon the food residues, if long retained, undergo putrefactive changes through which highly toxic substances are produced.

The structure of the colon is such that these poisons are absorbed only to a small extent and are mostly filtered out, while in the small intestine absorption is very rapid. 
Another and very important reason why the ileocecal valve is essential is a curious function which was first discovered by Cannon in animals and demonstrated by Case in human beings.

While digestion is taking place in the stomach and small intestine and strong peristaltic waves are forcing the food residues into the colon through the ileocecal valve, reverse or antiperistaltic waves, starting at the middle of the colon, travel backwards for the purpose of holding the liquid materials for a brief period in the cecum, where they have been deposited from the small intestine. This action in cases of incompetency of the ileocecal valve is sometimes so strong, especially after the work of digestion in the stomach and intestines has been completed, that several feet of the small intestine may be filled with fecal matters forced back from the colon.

In this way the putrid materials with which the colon is often filled, may find their way into the intestine and the infection traveling upward may reach the gall bladder, the pancreas, even the stomach, giving rise to catarrhal inflammation, gall stones, pancreatitis, probably also duodenal and gastric ulcers and other disorders. According to A. Schmidt, putrefaction should never occur anywhere in the intestine except the colon and in perfectly normal conditions with a proper dietary, which must necessarily exclude meats, putrefaction does not occur even in the colon. Says Schmidt: "The ileocecal valve forms a sharp line of demarcation above which putrefaction never occurs except under pathological conditions. If protein decomposition extends up into the small intestine, far greater quantities of indican appear in the urine than ever occur through the absorption in the colon."

From the above it will readily appear that a rigid antitoxic diet is of the highest importance in cases of this sort. The food should be of such a character that the residues will not encourage putrefaction even if delayed for some time in the colon. If putrefaction in the colon is suppressed, the injuries resulting from a backing up of the colon contents into the small intestine will be largely obviated. In such cases the diet must be not only antitoxic, excluding eggs and flesh meats of all sorts, but also highly laxative so as to encourage bowel action. The bowels should be made to move three times daily, by diet if possible, ordinary foods being supplemented by the free use of bran or agar and paraffin oil. If the bowels do not move freely three times a day. 
the colon should be emptied at night by means of an enema. Quantity, three or four pints. Temperature, $110^{\circ}$ to $118^{\circ} \mathrm{F}$. The intestinal flora must be changed.

\section{ATONIC CONSTIPATION}

Constipation is often attributed to an atonic condition of the colon. In this state the colon is widely dilated, as shown by $\mathrm{X}$-ray examination, and constantly contains large quantities of gas. The walls of the colon seem paralyzed, the gut appearing to have lost its power to expel its contents. This condition is usually regarded as a disorder of the nerves which supply the colon. In the writer's opinion, the condition is due rather to the effect upon the colon of the putrefying materials with which it is filled. In such cases the stools are always highly putrid and have a most repulsive odor because of their long retention and the very advanced stage of putrefaction. In such a condition a large amount of ammonia and other alkaline substances is present in the colon. It is well known that alkaline substances paralyze the colon while acid substances stimulate the muscular structures of the colon and cause contraction. That the colon is not paralyzed even in these cases is shown by the fact that by the introduction of an enema containing citric or lactic acid vigorous bowel movements may be secured. The addition to the enema of the juice of a lemon or a half tablespoonful of lactic acid rarely fails to secure prompt expulsion of the colon contents. This may be expected, in fact, in all cases in which there is no serious mechanical obstacle to bowel movement.

It is of the highest importance, then, in these cases to change the intestinal flora, so that the colon contents will become acid instead of alkaline. This must be done to secure permanent improvement. This may be accomplished by means of the fruit regimen. Much more rapid progress may be made in changing the flora by introducing into the colon, daily, cultures of the $B$. acidophilus or $B$. bulgaricus or a mixture of the two. The introduction of a half pint of an active whey culture of the B. acidophilus into the colon after cleansing it by a warm lemon juice or malt sugar enema (Index) greatly facilitates the change of the intestinal flora. Lacto-dextrin by mouth and rectum is most efficient. Psyllium seed is the best aid to bowel action.

The diet should be made permanently laxative and antitoxic (see Index). It should contain sufficient quantities of bran or 
agar, fresh fruits, fresh vegetables, especially greens, to secure three full bowel movements daily. A hot enema should be used daily, at bedtime, to secure complete clearance of the bowel.

\section{TAPEWORII}

There is no dietetic treatment by which the tapeworm may be expelled from the alimentary canal. Suitable vermifuge treatment is necessary. It is important, however, to impress upon the patient the fact that this disease can be contracted in no other way than by the use of flesh food, either beef, pork or fish.

The fish tapeworm produces more serious injury to health than does either the beef or pork tapeworm, often giving rise to a very pernicious anemia. This parasite is fortunately rare.

In the treatment of tapeworm, it is very important that the patient should eat very sparingly for several days before the treatment is undertaken, and to prevent a recurrence of the disease it is equally important that flesh foods, which are likely to harbor the parasite, should be entirely discarded.

\section{DIET IN ESOPHAGEAL STRICTURE}

In this condition, the diet must be fluid. It is a mistake to use meat broths, bouillon, albumin water, etc. These preparations have little food value and encourage acidosis, which on account of the restricted amount of food which can be taken is likely to supervene.

Malt sugar and lacto-dextrin are of great value. From 1200 to 1600 calories may be given daily. When malt sugar is not available, the sugar of raisins may be utilized. Raisin juice may be obtained by boiling four ounces of raisins in a pint of water for half an hour. This amount contains about 300 calories of sugar.

Rice jelly, or rice purée, egg yolk nog, vegetable broths, potato purée, spinach purée, sweet corn pulp and similar preparations are indicated. Farinaceous foods of all sorts should be well mixed with saliva before being swallowed. These dietetic measures are of course not curative. They are to be used simply to keep the patient alive until radical means of relief can be applied. The bowels must be emptied by the enema daily. 


\section{Diet in Disorders of $\mathrm{Nu}^{-}$ trition}

While food is by no means the only agent by which the nutrition of the body may be influenced, it is certainly the most powerful. By suitable feeding, the weight may be increased or decreased, the iron, lime and other saline constituents of the tissues may be augmented or diminished, cell activity may be excited, the activities of the kidney and other glands may be promoted or lessened and the work of various parts of the digestive organs may be increased, facilitated or lessened.

The significance of body weight as an indication of disease has been greatly emphasized by modern studies in metabolism. The popular idea that rotundity is an evidence of health and vigor has been shown by the statistics of insurance companies, as well as by laboratory studies, to be a grievous error. The insurance companies have shown that the lowest mortality at ages under 30 is to be found among persons whose weight is about 10 pounds above the average, whereas after the age of 30 the lowest mortality is found among persons whose weight is below the average, and at the age of 50 years and after the minimum mortality is found in persons whose weight is as much as 30 or 40 pounds below the average. It is thus apparent that excessive weight, especially in persons advanced in years, is highly undesirable.

Joslin has called attention to the fact that every over-fat person is a potential diabetic. The overfeeding which results in an excessive accumulation of fat overworks the pancreas and ultimately leads to a failure of its function, which is to produce a hormone that enables the body to burn sugar. It is evident, then, that every obese patient should make haste to divest himself of his burden of excessive fat. It has been wisely suggested that every over-fat person should have made on every birthday an examination of the urine for the presence of sugar. For greater safety, a chemical examination of the blood should also be made, for the appearance of sugar in the urine is often preceded for a considerable period by an increase of the blood sugar. The slight- 
est indication of the loss of the ability to utilize carbohydrates should lead to an immediate restriction of the intake of carbohydrates so as to prevent further damage to the pancreas.

\section{DIET IN OBESITY}

Before beginning a course of diet to reduce weight, it should be determined how much the weight is to be reduced. Suppose, for example, a person whose original and normal weight was 120 pounds now weighs 200 pounds; it would be most unwise to reduce such an individual to his original proportions. Ordinarily, in a case of this sort it is wiser to be content with reduction of the weight to 160 or 165 pounds; that is, not more than half or two-thirds of the surplus weight should be removed. In the case of persons who have become exceedingly obese, the weight being increased from two and one-half to three times the original weight, it is not best to undertake to remove more than one-third of the surplus. After this has been accomplished, some further progress may be made by very slow reduction so as to permit the tissues to accommodate themselves to the changed relations which result from the removal of a large amount of accumulated internal fat.

When should the weight be reduced?

In persons over thirty years, a weight 10 to 15 per cent. above the average for a person of the same height is an indication for weight reduction.

Weight reduction is advantageous (1) in all cases of diabetes when the weight is above the average, (2) in cases of high blood pressure even when the overweight is slight, and (3) in cases of disease of the heart and (4) in Bright's disease.

Persons advanced in years even though 10 or 12 per cent. over weight should not undertake a reduction in weight but should carefully avoid taking on more weight.

It is important to notice before beginning a course of diet for fat reduction just where the largest masses of fat are deposited, especially whether the surplus abdominal fat is outside or inside. In many cases, the excessive accumulation is almost wholly within the cavity of the trunk, the fat being deposited in the omentum, the mesentery, about the kidneys, around the heart and in other parts of the chest and abdomen. In other cases, the fat accumulation is almost wholly external, large 
masses being heaped about the abdomen, the hips, sometimes about the shoulders, upper arms and thighs. In women, the breasts are not infrequently the seat of overfat accumulation.

A simple method of detecting the location of the abdominal fat consists of having the patient lie down and raise the extended legs. If the fat accumulation is within the abdomen, the play of the abdominal muscles may be easily seen as they are made tense when the legs are raised; and if the skin and underlying fat is grasped between the hands at the lower abdomen when the legs are raised, it will be noticed that the contracted muscles are readily felt and the mass which may be gathered between the hands when the muscles are lax is snatched away as soon as the muscles are tensed by the raising of the legs. On the other hand, if the fat is external, a large mass remains between the hands when the legs are raised. When the chief accumulation of fat is internal, the patient usually suffers greatly from shortness of breath because of the great deposit of fat about the heart, and fatty degeneration as well as fatty infiltration of the heart muscle may exist. This condition often presents an insuperable obstacle in the way of taking a sufficient amount of exercise to accelerate the fat reduction to any considerable degree.

Having decided how much fat reduction is required, the next question is the determination of the amount of the daily ration; that is how many calories shall the patient eat for breakfast, dinner and supper. First of all, we must know what would be the normal ration for the individual. If he weighs 210 pounds, and we determine to reduce his weight to 170 pounds, then by reference to Table II we may ascertain what is the basal ration for a person weighing 170 pounds. We find this to be about 1,900 calories. This makes no allowance for exercise, which would probably increase the patient's ration about 50 per cent., making it 2,800 calories. Halving this will give us 1,400 calories for the daily intake of the first week.

The amount of food taken should be determined by careful measuring and weighing and should not be guessed at. The average person eats a great deal more than he thinks he does.

The next question is what kind of food shall the patient be allowed to eat? First of all, it is important that the patient should be instructed concerning foods which are highly fattening and which on this account must be avoided. These are, particularly, fats of all sorts, sugar, including candy, rich gravies, pastry and 
potatoes. Breads and breakfast foods must also be greatly restricted. On the other hand, there are certain foods which the patient may eat freely, almost without restriction. These are, especially, fresh vegetables of all kinds, with the exception of potatoes, and juicy fruits, green things, particularly cabbage, lettuce and fresh cucumbers. Lettuce and greens should constitute a large part of each meal, so as to supply an abundance of vitamins and of food iron and lime.

Many over-fed persons hesitate to undertake a course of diet for losing weight because of the dread of hunger. Such persons will be encouraged by learning that when a patient is willing to restrict the kind of food which he eats, there is no necessity whatever for suffering any inconvenience from hunger.

The sense of satiety, or the satisfaction of hunger, is largely the result of distension of the stomach, and hence bulky foods will satisfy the patient and make him feel that he has enough to eat even though the intake of food as reckoned in calories is very small. It is well, also, to allow butter in very small amounts as this greatly aids in producing the sense of satiety. The amount should not exceed a half ounce daily.

Persons who suffer much from hunger may generally find relief by taking a small amount of food at frequent intervals. Von Noorden feeds his obese patients five times a day. Hunger is simply an indication that the stomach is empty and disappears when even a small amount of food is taken. A small apple or some other juicy fruit or a few spoonfuls of gruel will temporarily afford relief of hunger as well as a larger amount of food and may be taken whenever discomfort from hunger is experienced.

Greens and greenstuffs, especially uncooked foods, are particularly valuable and should be made the major part of the diet because of the large amount of vitamins which they contain. In reducing the diet, the intake of vitamins is proportionately reduced, but there is no possible benefit from such a reduction. On the other hand, the diet of the average person is very deficient in vitamins, so in the obese patient more rather than less vitamins should be given and this can only be accomplished by the free use of greens and fresh foods. The reduction of food also tends to produce anemia. This danger is also obviated by the free use of greens, which supply an abundance of food iron.

The amount of protein required is not increased and should 
never be more than one calorie per pound of body weight. An excess of protein is the grievous fault in all the many regimens heretofore recommended for fat reduction. It is true that lean meats do not contribute largely to the production of fat, but it is equally true that the large intake of protein imposes an enormous and onerous burden upon the liver and kidneys while serving no useful purpose to the patient. Surplus protein, as shown by Folin, is simply eliminated as urea and contributes nothing to the welfare of the body. A meat diet greatly increases the liability to acidosis.

The necessary bulk can be easily secured by the free use of greens, fresh fruits, fresh vegetables, and bran or agar. A half ounce of bran should be taken at each meal or a third of an ounce of agar. Paraffin oil should also be taken to secure active movements of the bowels, which are likely to become constipated when the intake of food is cut down. A tablespoonful of paraffin oil should be taken before and after each meal and also, if necessary, at bedtime. Buttermilk, whey, skimmed milk, and cottage cheese may be freely used and are a good source of food lime and vitamins.

The fat reduction is to be secured by reducing the intake of fats and carbohydrates. Fats may, in fact, be wholly eliminated from the dietary for long periods without injury, as shown by the observations of Hindhede referred to elsewhere in this work. (For further suggestions see "The Reducing Diet.")

It is sometimes advantageous to put the patient upon a "mono" diet. For example, he may be allowed one day to eat apples, another day peaches, still another day pears, another day lettuce, celery and salads of various sorts, either alone or combined with fruits. Even nuts may be allowed in moderation. A very acceptable diet may be arranged on a menu consisting of apples, bananas, almonds or Brazil nuts. It is only necessary to take care always to keep the total intake of calories within the limits, that is, not exceeding half or two-thirds the normal ration.

After the first week or ten days the intake of food should be increased 50 per cent., and this reduced dietary, which furnishes about three-fourths of the total energy requirement of the individual, may be continued for several weeks or even months. After a few weeks, the amount of food should be increased a little more, to four-fifths the normal requirement, and later to 
five-sixths. Care must be taken to supply each day all the protein, vitamins, lime and iron the individual can possibly need, and when this is done no possible harm can come from the dietetic restrictions. The injury which many persons suffer when trying to reduce the weight by dieting results from some one of the following causes:

1. Too long fasting, producing injury from acidosis and loss of muscular tissue, weakening of the heart, etc.

2. Excessive intake of protein, overworking the liver and kidneys and increasing intestinal putrefactions.

3. Deficiency of vitamins, leading to general malnutrition and sometimes symptoms of scurvy or polyneuritis.

4. Deficiency of food lime, leading to a condition of lassitude, loss of muscular tone and a state of general asthenia.

5. Deficiency of iron, leading to anemia and general loss of vigor and vitality.

6. Constipation and intestinal toxemia, resulting from reduction in the volume of the food and deficiency of roughage.

\section{Intermittent Fasting.}

Observations made at the Carnegie Nutrition Laboratory of Boston, (Folin and Denis), showed that the calorie value of a pound of human flesh is 1500 , consequently a reduction of the maintenance ration of an individual, that is, the number of calories required to prevent the loss of weight to the extent of 1500 calories, will result in the loss of one pound of weight.

If a person whose maintenance ration is 2500 calories undergoes an absolute fast, the result would be a daily loss of $12 / 3$ pounds. By means of vigorous exercise, in addition to fasting, the energy output may be increased to 3000 or 4000 calories, which would cause a loss of weight of two pounds or more. Fasting is undoubtedly the most expeditious mode of reducing weight, but this method is open to the objection that it produces acidosis, a condition in which there is an accumulation in the blood and tissue fluids of imperfectly burned fats, which give rise to headache, weakness, sometimes nausea and great depression. This objection may be overcome by intermittent instead of continuous fasting.

The patient is asked to make a series of short fasts, following each fast by a day or two of feeding, limiting the intake of food, however, to the bare maintenance ration. 
Folin and Denis observed that when a series of such fasts is undertaken, acidosis becomes less and less severe with each fast and appears later.

A very good plan is the following: Fast one day, drinking freely. An ounce of bran or agar and Psyllium should be taken with Savita broth, weak lemonade, with little or no sugar, or cereal coffee, or some other beverage with a very low nutritive value. Two or three ounces of paraffin oil should also be taken several times a day to insure bowel movements. After one day fasting, take a small amount of bulky food not exceeding the maintenance ration, that is, 1500 or 1600 calories. After a couple of days, renew the fasting for two days. Follow this by three feeding days, making the ration consist chiefly of bulky foods not exceeding 1500 or 1600 calories a day.

After three days of this ration, renew the fasting for a couple of days and so continue fasting one or two days after three days of light diet, until the weight has been reduced to the proportions desired.

\section{Reduction of Weight by Exercise.}

The comparative inefficiency of exercise as a means of reducing weight will be clearly shown by a little computation:

According to the observations of Zuntz, the amount of work done by a man walking 13.4 feet, at the rate of three miles an hour, is the same that would be required in the lifting of his body, vertically, one foot. In walking a mile at this rate, one would do work equivalent to lifting his body 400 feet vertically.

The amount of mechanical work done by a 200 pound man, then, walking a mile at the rate of three miles an hour, would be 80,000 foot pounds $(400 \times 200$ ), equal to 80 calories.

At this rate, a man weighing 200 pounds would have to walk 8 or 9 hours, and to travel 25 to 30 miles in order to reduce his fat half a pound. The actual reduction in weight might be four times this because of the water associated with solids in the tissues, although the water loss often follows a little later.

Automatic exercise, first employed by the author by means of the sinusoidal electrical current some thirty years ago, has proved itself to be efficient as a means of reducing weight. When vigorously applied, this exercise may increase the energy output of the body to eight times the ordinary rate. The actual loss of weight in a half hour by automatic exercise sometimes amounts 
to as much as two pounds, but of course this great loss in weight is chiefly due to excessive perspiration, and does not represent actual fat reduction.

The rational method of dealing with cases of obesity requires the combination of exercise and diet restriction, the last being most efficient.

\section{Obesity Due to Endocrine Disturbance.}

In cases of obesity due to disturbance of the internal secretions, an entirely different course must be pursued. The patient's increase of weight is not due to an excessive intake of food or fuel, but to diminished vital combustions. The fuel-food is not consumed as rapidly as it should be and hence accumulates, producing much the same effect as would result from an excessive intake of food. A metabolism test will probably reveal this condition when present. The metabolism rate is diminished.

The obesity resulting from disturbed metabolism is likely to be regional in character. The principal seats of accumulation are the abdomen, the hips, shoulders or breasts. Obesity from disturbed internal secretions is the most common form of overfatness encountered in boys and girls. It makes its appearance before puberty, and in well pronounced cases there is marked delay in the development of the genital organs.

By proper tests, it may be shown that in these cases there is a diminished metabolism. The treatment consists not in greatly restricted diet and exercise but in the administration of preparations of the thyroid, pituitary or other internal glands.

Still another form of obesity may be mentioned which is sometimes termed anemic obesity. The patient is pale, the tissues are soft and flabby and contain an excess of water. The patient may be said to be "water-logged." Metabolism is deficient. These cases recover rapidly under the influence of tonic baths combined with light baths and massage. The diet should be rich in food iron. This means that the patient should eat a liberal serving of greens of some sort twice a day and should select for his bill of fare foods rich in iron.

In the dietetic management of obesity a few important points to be remembered are these: The protein intake should be not more than normal in amount. Fat may be almost wholly excluded from the dietary and the proteins and carbohydrates considerably reduced. If too great a reduction in the total food intake is made. 
the result will be loss of body protein, which is not desirable. Carbohydrates protect the body proteins more than do fats, hence they must not be too greatly reduced.

Greens should be used in abundance at every meal. A little butter taken at the beginning of the meal helps to cause a comfortable feeling of satiety.

Broths, such as bean broth, vegetable bouillon, and broths prepared from yeast extract (savita), should be freely used to supply vitamins.

\section{The Water Intake.}

A person undergoing treatment for the cure of obesity requires less water than a normal person under the same conditions for the reason that in the state in which they exist in the human body the solids are in biologic combination with a considerable amount of water. This water content of the tissues does not always leave the body as rapilly as do the solids in cases undergoing fat reduction. It is on this account that patients do not always lose in weight as rapidly as they are naturally expected to do, judging from the amount of the food intake and the elimination of solids taking place. When the elimination of water is slow it is only necessary to persevere in the application of the measures employed with the certainty that the effects desired will in due time make their appearance. Such cases are greatly benefited by the use of sweating baths, and also by the disuse of salt.

In the preparation of foods for obese patients, little or no salt should be used. The importance of this will be recognized when it is remembered that for every ounce of salt in the tissues, more than one hundred ounces of water must be retained to hold it in solution; that is, for every ounce of salt which the tissues retain, over six pounds of water will be retained with it. A certain amount of salt is eliminated daily in the urine and the perspiration. If the salt thus lost is not replaced by salt in the food, the result will necessarily be a proportionate loss of weight. The daily salt requirement of the body is not more than one dram (four grams) but the majority of people take with their food three or four times this amount. If the intake be reduced even so much as one dram, the result would be the reduction of the body salt to an equal extent and with it the loss of more than one hundred times as much water and reduction of weight to the extent of one pound. 


\section{Karell's Method.}

Weight Reduction. This method, originated by Dr. Phillip Karell, of St. Petersburg, physician to the emperor of Russia, more than fifty years ago, was the first systematic plan for the reduction of weight. The method consists in restricting the food intake to three or four pints of milk daily. The milk is divided into four portions and taken at intervals of about four hours. This method has been much used in Germany, especially in the treatment of cases of diseases of the heart or kidneys with dropsy and obesity.

\section{Oertel's Method.}

The patient is given daily five to six ounces of protein, two and a half to four ounces of carbohydrates and an ounce and a half of fat, or $1150-1600$ calories. The intake of fluids was restricted to three pints. Tea and coffee and wine were allowed. Patients were required to exercise vigorously, especially in hillclimbing. This method is certainly not to be recommended.

\section{Von Noorden's Method.}

This author divides his patients into three classes, which he distinguishes as "slight, moderate and pronounced." The maintenance ration for the first class of patients is diminished onefifth; for the second, two-fifths; and for the third, three-fifths. The objectionable features are the free allowance of beef broth, wine and an excess of protein, of which the patient is given $480-720$ calories, fully three times the required amount.

\section{Banting's Method.}

This once famous method required the patient to subsist upon a diet consisting largely of meat and wine. Three ounces of toasted bread were allowed, with green vegetables. Fluids were restricted to one quart. As these consisted chiefly of wine, the amount of water supplied to the tissues was not sufficient to insure the proper elimination of the tissue wastes, enormously increased by the great excess of protein. The patient was allowed four meals a day, all but one of which consisted chiefly of meat and wine. A most unscientific method now wholly obsolete. 


\section{The Salisbury Method.}

This method, considerably in vogue forty years ago, is still mentioned by writers of dietetics, although it should have been long ago forgotten, as it had no scientific basis whatever. Dr. Salisbury conceived the idea that most human ailments are due to the development of yeast in the alimentary canal and in the blood. He maintained that by excluding carbohydrates from the diet, the yeast cells might be starved out. He employed this method in the treatment of tuberculosis, Bright's disease and nearly all chronic maladies, including obesity. The diet consisted almost exclusively of chopped or scraped beef, hot water, weak tea and broths. The patient was given one pound of meat three times a day. The weight may be reduced, without doubt, by a diet of this sort, but the remedy will be found worse than the disease and will produce disorders far more serious than obesity.

\section{DIET IN EMACIATION}

A person may be under weight, that is, below the average weight for a person of his height and yet enjoy excellent health; indeed, in persons 50 years of age or over, as shown by the statistics of the Metropolitan Life Insurance Company, the life expectancy of persons who are under weight, even to the extent of 30 or 40 pounds, is greater than that of persons of average weight. In persons under 30 years of age, however, the opposite is true. Youtig persons weighing slightly above the average show the lowest mortality. Rotundity is a mark of high health in youth. In persons below the age of 30 under weight is significant of malnutrition. This is the age when tuberculosis claims the greatest number of its victims and loss of fat is one of the early symptoms of this disease. Persons who are threatened with tuberculosis or in whom a formerly active tubercular process has become latent, should take care to maintain their body weight a little above the normal. Persons suffering from Bright's disease and diabetics should carefully avoid overweight. Neurasthenics generally are improved with the taking on of weight.

Fat, or residual tissue, is like a bank account. The amount depends upon the balance between income and expenditure. When the intake of foods is greater than the amount consumed, the surplus is normally deposited as fat, held in reserve for some future time when the intake may not be equal to the outgo. 
The problem, then, when a gain of weight is desired, is to adjust the relation between the intake of food and the expenditure of energy so that there will be a balance left for deposit as fat. Ordinarily the solution of the problem is not difficult, but there are certain cases in which an abnormal expenditure of energy in a manner not easy to control becomes a serious obstacle. With reference to these cases it is especially important to bear in mind that the largest expenditure of energy, and hence the largest draft upon the food supply, is made by what is known as muscular tension or tonus.

The muscles are constantly in a state of rhythmic automatic activity. When a living muscle is cut the ends instantly separate because of tonus. This tension is maintained by a continuous series of nerve impulses which reach the muscles from the controlling centers at the rate of 16 to 20 impulses per second. Evidence of this continuous muscular activity may be obtained by pressing the tip of the finger into each ear while contracting the muscles of the arms. The pulsating tone heard corresponds to the low notes of a great pipe organ. This muscle tonus is the chief means by which the normal temperature of the body is maintained, heat being the by-product of the muscular action. According to the physiologists from 30 to 50 per cent. of the entire intake of energy in the form of food is consumed by muscle tension. This continuous automatic form of muscular work is exceedingly variable. In a person who is in a highly nervous state, through worry, fear or for any other cause, the tension is high and hence the consumption of energy very much greater. Such persons often make no gain in weight even though eating much more than the normal requirement. They do not gain for the reason that the expenditure is greater than the normal rate.

It is to be remembered, also, that the body, like a growing plant, makes its chief gains during sleep; hence it is essential for a person who desires to gain in weight to eliminate all sources of nervousness and worry so that tension may be reduced to a minimum and that abundant sleep should be secured. For a very rapid gain rest, even to the extent of confinement to bed or to the horizontal position, is essential. The patient should be kept as quiet as possible and should sleep as much as possible. In general it is not necessary that the patient should remain in bed. It is only important that active exercise should be avoided. It is to be remembered, however, that even what might be called moderate 
exercise may increase the expenditure of energy 25 to 50 per cent. So slight an exercise as sitting increases energy expenditure 5 per cent., while standing causes an increase of 10 per cent. Sewing causes an extra loss of 13 per cent., and moderate walking increases the energy expenditure to the extent of 250 per cent.

Generally, the patient may read, chat, and entertain himself, and may be entertained with music and in other ways, but should avoid violent excitement of all sorts.

Care should be taken to see that while the patient lies in bed the trunk is properly supported by a firm cushion placed at the small of the back. When this precaution is neglected patients who lie long in bed often suffer from subluxation, or strain, of the sacro-iliac synchrondrosis, the joint formed by the junction of the sacrum and the lateral bones of the pelvis. Many patients when lying in bed suffer from backache due to lack of back support.

It is also wise for the patient to lie with the body inclined forward toward the right side. This position facilitates emptying of the stomach, especially in cases in which the stomach is dilated or prolapsed. If nausea is present, the patient should lie upon the left side to prevent strain upon the cardiac orifice (Brunton).

Absorption of the digested food material from the intestine may be greatly promoted by application of pressure upon the abdomen. In the majority of cases in which the milk regimen is employed, the patient will be found to have very relaxed abdominal muscles and in consequence the intra-abdominal pressure is greatly diminished if not altogether absent. This greatly hinders the absorption of the digested foodstuffs. By the application of a sand bag or a shot compress to the abdomen the necessary pressure may be secured and absorption greatly facilitated.

Instead of applying weight to the abdomen the patient may obtain essentially the same result by lying upon the face so that the abdomen will be compressed by the weight of the body. By lying over a cushion or a pillow the effect of the prone position may be greatly increased.

Sand bags weighing ten to twenty pounds are of great service in supporting the patient in a position of complete relaxation and rest. More than half the internal work of the body is due to muscle tension. Lying in bed is often far from being the complete rest that one naturally conceives it to be. The evidence of 
this is seen in the fact that the patient gets tired and most uncomfortable and requires frequent change of position.

The chief cause is undue muscle tension, due to unconscious muscular effort in maintaining the body in position. That this is considerable is shown by the instant relief and sense of comfort experienced when sand bags or other suitable "props" are properly applied.

The tendency to slip down in bed if the head and shoulders are raised even a little is unconsciously antagonized by involuntary muscular action involving waste of energy. This may be easily prevented by a twenty-pound sand bag placed against the buttocks. When the patient lies upon the side the addition of a couple of ten-pound bags placed against the small of the back and the shoulders give the patient a feeling of support and security that is most agreeable and restful. In many cases an additional bag placed in front against the lower abdomen is highly advantageous. If pain is present, or if colitis and a spastic condition of the colon exist, a warm or hot bag in front (especially the lower left side) is most useful, relieving pain and relaxing the contracted bowel at the same time that it gives support and compresses the relaxed and distended abdomen.

Surrounded by these supports, the sense of tension that makes patients restless in bed and keeps them turning from side to side or otherwise changing position is soon lost and the patient feels at perfect rest, cradled in a comfortable nest in which he may relax completely and thus save a useless waste of energy that will not only hinder the accumulation of fat, but will also interfere with the storing up of energy in the cells of the brain and spinal cord, one of the essential aims of the "rest cure" in these cases.

Observations by Benedict and others have shown that a considerable restriction in the diet for two or three days is likely to be followed by a more active assimilation of food, and hence facilitates fattening. It is well on this account to employ the fruit regimen for three or four days before beginning the surplus feeding. Lactose or lacto-dextrin should be used in liberal doses for changing the flora and care should be taken to empty the bowels thoroughly as an aid to elimination of toxic products.

In arranging a fattening bill of fare special care should be taken to place the protein of the diet at a low level since it is well known that protein in excess of the actual needs of the body increases nerve and muscle tension and thus wastes energy. 
For a rapid gain in flesh the milk regimen is one of the most efficient means. By the milk regimen is not meant milk diet, but systematic feeding of milk in connection with other foodstuffs necessary to make good the deficiencies as explained elsewhere in this work. The free use of lactose and lacto-dextrin with the use of milk is of great value. Either lactose or malt sugar may be used in doses of one or two ounces well dissolved in two-thirds of a pint of water between meals. By repeating doses of sugar water several times a day the total intake of food may be considerably increased. In general, the increase should be about 25 per cent. above the maintenance ration.

Further directions concerning the technic of feeding to secure a gain in weight will be found under the headings "Fattening Regimen" and "Fattening Diet."

\section{DIABETES}

In this disease, the patient has lost his power to burn the sugar which the blood takes up from the liver and so the kidneys must eliminate it to prevent the mischiefs which would necessarily arise from an accumulation of sugar in the blood. The primary fault is believed to be in nearly all cases in the pancreas, which normally creates and sends into the blood substances which enable the muscles and other tissues to burn the blood sugar, but in diabetes fails to produce the necessary amount of these hormones.

Sugar appears in the blood in excessive proportion before it can be detected in the urine by the ordinary tests.

By modern laboratory tests the sugar content of the blood may be easily determined and watched from day to day. Normally, the amount of sugar is about one part in one thousand of blood, or 0.08 to 0.12 per cent. In diabetics, the sugar of the blood is usually much higher than normal, rising to 0.30 per cent. or even more.

Experiments with insulin show that the sugar content of the blood is nicely regulated to body needs, and when it is much reduced, the results are more serious than when it is raised. A very low blood sugar may give rise to stupor and even convulsions and death. Fatal effects are easily produced in animals, although an injection of glucose, if made soon enough, will cause the menacing symptoms quickly to disappear. 
An excess of blood sugar may result from (a) an excessive intake of sugar ( 6 to 8 ounces); (b) lessened use of sugar by the muscles; (c) failure of the liver to store sugar or glycogen or increased production of sugar by the liver; (d) increased production of sugar or diminished storage by the muscles; (e) lessened fat production at the expense of sugar.

The blood sugar is usually higher in persons of advanced age than in young persons. It is also higher in advanced cases of diabetes. In such cases, it should not be too much reduced.

In occasional cases, sugar is found in the urine even though the blood sugar is not raised. All these facts must be taken into consideration in dealing with diabetics.

In recent years, a new test known as the Bergel test has been developed by means of which the pre-diabetic state may be detected by examination of the urine; that is, a condition in which a moderate excess of sugar is found in the blood and in which carbohydrates are not well utilized. The writer has found this test very useful in detecting cases of potential diabetes. In one instance a father brought to the Battle Creek Sanitarium his daughter-a young girl of 12 years, a very severe case of diabetes. He had lost two children with the disease at about the same age. Her urine showed no sugar, but responded to the Bergel test. Examination of the blood showed it to contain a large excess of sugar.

The failure of the pancreas to produce the sugar-burning hormone is probably due, in most cases, to chronic pancreatitis, one cause of which is associated with infection of the gall bladder and biliary passages.

Conroy, who examined the pancreas of a considerable number of patients who died of diabetes, found quantitative or qualitative changes in every case. Diabetes as a cause of death is becoming increasingly prominent in the mortality statistics. While the death rate from this cause varies in different parts of the country from 6 to 22 per hundred thousand, the average is now 17 . The deaths from this cause in the United States have reached the great total of nearly 19,000 annually in 1922, an increase of 10 per cent. over the preceding year. It is estimated that there are in this country more than one million diabetics, nearly all of whom will be dead within five or six years and their places will be filled by a still larger number with the same dismal prospect.

The new methods of treatment have increased the diabetic's 
life duration more than a year, but the life expectancy of patients under 20 years of age is still less than three years and that of older patients less than six years. There is hope, however, that by the very recent great advances which have been made, not only in feeding methods but through the use of the pancreatic hormone first isolated by Banting, much more may be accomplished in prolonging the lives of these unfortunates than has heretofore been done. It is evident, however, that the most important of all the many problems remaining unsolved with reference to this disease is that of causation and prevention. Some progress has been made in this direction.

It is the writer's belief that diabetes has its origin chiefly in three common, almost universal, dietetic errors:

1. The excessive use of carbohydrates in the form of cane sugar and candy and other sweets.

2. The use of an excessive amount of protein, particularly in the form of meats, by which intestinal putrefaction is encouraged, resulting in infection of the bowel through which the body is flooded with toxins and pernicious organisms which induce degenerative and destructive changes in the pancreas, by which this organ finally becomes so crippled that it is no longer able to perform its part in the metabolism of sugar by the production of the necessary quantity of the hormone insulin, or "iletin."

3. Neglect to provide in the daily bill of fare the necessary amount of bulk in the form of bran and other roughage to secure the prompt and thorough evacuation of the food remnants and body wastes which is necessary to prevent putrefaction of these residues. This results in intestinal toxemia by which the glands which destroy are overworked and damaged, together with other glandular structures, especially the pancreas, the liver, the kidreys and other great glandular organs.

Another cause of degenerative changes in the islands of Langerhans is no doubt the circulation of poisons in the blood, the sources of which may be as various as in the case of similar degenerations in the liver and other glandular structures. Doubtless the most common source of these poisons is putrefactive processes resulting from stasis in the colon and lower small intestine.

In cases in which a hereditary predisposition to diabetes exists, it is possible that careful avoidance of these dietetic errors would prevent the appearance of the disease. 
These several causes co-operate together in a remarkably efficient manner for the destruction of the pancreas by increasing the work required of it, while at the same time lessening its functional capacity. When it is borne in mind that the average American consumes not less than 102 pounds of cane sugar annually, or four and one-third ounces every day, together with one hundred and seventy pounds of meat, or half a pound daily, a quantity of protein which Chittenden has shown to be more than double the amount which the body can utilize-it is no wonder that diabetes is rapidly increasing in this country.

Gall stones are frequently present in cases of diabetes, and in many cases recovery from diabetes has occurred when gall stones have been removed. In other cases, a cure has been effected by draining of the gall bladder.

R. T. Williamson (British Medical Journal, February 2, 1918) lays great emphasis upon three general factors which seem to play an important rôle, at least as predisposing causes, in the production of diabetes mellitus. These are: habitual consumption of sugar and sweets; prolonged and intense mental anxiety, worry, overstrain or sudden shock; a family history of diabetics.

Dr. Magnus-Levy, who has made a study of conditions in Germany since the war, reports that diabetes has practically disappeared, doubtless one of the many beneficent results following from the food restriction made necessary by the war.

The X-ray study of a number of diabetic cases made by Dr. James T. Case showed that ileac stasis, that is, stagnation of the contents of the small intestine, exists in nearly all cases of this disease. This condition is produced in most cases, at least, by incompetency of the ileocecal valve, which is, in turn, due to dilatation of the cecum, a result of obstruction to normal bowel movement usually existing in the descending or pelvic colon. Infection gradually travels up from the lower bowel until it reaches the pancreas. Diabetes is only one of the disorders which may be due to this chronic intestinal toxemia.

\section{The Dietetic Treatment of Diabetes.}

In the dietetic treatment of this disorder, it is important to give consideration to the cause of the disease as well as to its results. The moderate suppression of sugar in the urine is by no means sufficient. The urinary sugar is merely a symptom of the disease. The most important thing of all is to check the pro- 
gressive disease of the pancreas; in other words, to suppress the intestinal infection. This requires first of all relief of the intestinal stasis, which usually begins in the lower part of the bowel and gradually ascends until the small intestine becomes involved.

Chronic colitis, a spastic condition of the colon, stasis in the cecum, and any other conditions found present by careful examination must be removed so far as possible by proper treatment.

The colon must be kept emptied by securing three full evacuations of the bowels daily. Agar-agar, specially prepared bran from which the starch has been removed, and paraffin oil are of essential service in accomplishing this end. If necessary, the warm water enema should be used once or twice daily, to secure thorough evacuation of the colon.

A strictly antitoxic diet must be adopted. This of course requires the elimination of meats of all sorts. If milk is used, only certified milk should be employed and it should be free from contamination with Welch's bacillus. Eggs must be perfectly fresh and when possible should be obtained from milk-fed hens. Mouth asepsis must be carefully maintained. In some cases gall bladder drainage may be practised with great advantage.

The next step is to arrange for the patient a bill of fare which will cause the disappearance of sugar from the urine and reduce the blood sugar. The amount of sugar normally found present in the blood is 9 to 12 parts in 1000 . This may be increased in diabetes to two or three times the normal amount. The determination of urinary sugar is much more easily accomplished than that of the blood and it is a convenient means of watching the daily progress of the case. From time to time the urinary examinations should be supplemented by an examination of the blood.

The treatment of diabetes is chiefly dietetic, although without doubt other accessory measures are important and have been quite too much neglected, particularly exercise and the employment of means to combat intestinal toxemia. Within the last ten years there have been wonderful advances in our knowledge of this malady and revolutionary progress in the methods of treatment. Formerly a diet consisting almost wholly of meats was a routine prescription, and it was thought necessary to increase very considerably the intake of food in order to make good the losses which the diabetic suffers through the escape of sugar in the 
urine. It is now known, however, that not infrequently these overfed patients were actually starving because of their inability to utilize the food eaten. It has been learned, also, that an excess of protein produces even worse effects than an excess of carbohydrates by increasing acidosis as well as the loss of sugar since sugar may be derived from protein as well as from carbohydrates.

One of the first suggestions which led to the development of modern methods was perhaps the observation by the eminent Bouchardat, who noted during the siege of Paris that many diabetics lost their sugar. This led Bouchardat to require his diabetic patients to fast occasionally for the purpose of clearing the urine of sugar. Some years ago Guelpa of Paris adopted fasting as a routine method in diabetes and read several papers on the subject before the faculties of Paris and also presented a paper before the profession of Great Britain and wrote a book on the subject. But the fasting method was made known to the people of this country chiefly through the work of Allen and Joslin. Woodyatt, Newburgh and numerous others in this country as well as in France and England have within recent years made extended experimental and clinical researches which have resulted in the development of new methods which promise to improve very greatly the outlook for this class of sufferers. Hundreds of cases have been treated at the Battle Creek Sanitarium by modifications of the new methods proposed and with results which justify the belief that still farther progress may be made in dealing with this hitherto intractable malady.

In mild cases of diabetes the urine may be made free of sugar by fasting two or three days, and by proper regulation of the diet may be kept free. Cases are rare, in fact, in which by sufficiently careful dietetic management the urine cannot be kept free from sugar.

Complete fasting should be resorted to only when the urine cannot be cleared of sugar by less drastic restrictions in the diet. It needs to be remembered that a fasting patient loses very nearly one per cent. of his tissues daily. To make up this loss may require several days or even several weeks in one whose powers of assimilation are considerably depreciated. A year might be required to recover the loss of weight resulting from a complete fast of five or six days. For each pound of body weight, a person loses about sixty grams of protein, one hundred and twenty-two 
grams of fat and seven grams of carbohydrates, or 1366 calories. Joslyn estimates the value of a pound of human flesh at 1500 calories.

Sakaguchi, who has made an extended research in relation to the effects of food on sugar elimination in diabetes, has shown that tolerance for carbohydrates is least at breakfast and the elimination of sugar highest ifter the first meal of the day. He thinks this is due to the lessened production of glycogen in connection with the breakfast. He finds that the elimination of sugar following breakfast may be reduced by giving a small amount of carbohydrate an hour or two before breakfast.

$\mathrm{He}$ also noted that carhohydrates are best burned if taken four or five hours after the preceding meal. If the interval between meals is prolonged to six hours or more, sugar elimination is increased.

The same investigator observed that the maximum increase of blood sugar occurs two to four hours after the meal it eaten.

The normal sugar tolerance differs considerably for the different sugars, According to the best authorities, a normal individual should be able to utilize completely the several sugars in the quantities named, as follows:

Lactose

120 grams

Cane sugar $150-200$

Levulose

Glucose or malt sugar $200-250$

The above quantities of sugar taken at a single dose should not be followed by the appearance of sugar in the urine. If sugar appears, the patient is probably diabetic.

The discovery by Banting of a method by which the pancreatic hormone, insulin or iletin, may be obtained from the pancreas of slaughtered animals, has provided a new and invaluable remedy for use in the treatment of this disease. By the proper use of insulin, it is indeed possible to dispense with the complete and prolonged fasting first employed by Guelpa and systematized by Allen and Joslin,

By careful administration of insulin the urine may be freed from sugar and the blood sugar may be quickly reduced to normal or nearly normal limits. Insulin does not effect a cure but simply supplies the lacking hormone which is necessary to enable the body to utilize carbohydrate. Insulin should always be em- 
ployed under the supervision of a physician who has been properly instructed in its use. While invaluable as an aid in the treatment of diabetes, its use does not obviate the necessity for careful regulation of the diet; neither does it restore the pancreas to its normal condition. It simply supplies to the body a lacking element which is essential. Its use must be continued indefinitely in cases in which a moderate restriction in diet is not sufficient to keep the blood sugar within safe limits. Neither does the use of insulin remove or combat the original causes of diabetes, whatever they may be. It does, however, afford a means of giving the pancreas rest and so encourages repair. Recently, also, cases have been reported in which by the judicious use of insulin the carbohydrate tolerance has been increased to such a degree that by careful dieting its further use appeared to be, for the time at least, unnecessary.

The readiness with which absolute fasting usually clears the urine of sugar, together with the enthusiastic reports made by the promoters of this method, led to its rapid adoption by physicians throughout the country, but the results were on the whole disappointing. The fasting method has its dangers as well as its advantages and there is at present a decided reaction against the extreme, drastic measures first employed. At the present time, in fact, some of those most successful in the treatment of this disease have eliminated the fast altogether. In cases in which the urine contains a large amount of sugar, however, there is an undoubted advantage in restricting the diet for one to three days to such a degree as to amount to almost an absolute fast. During this period the patient should remain quiet in bed or lying on a couch in the open air. He should drink three or four quarts of water daily. The water may be slightly flavored with some acid fruit, or a dilute broth (yeast extract) may be used instead of water. It is well, also, to have the patient take three or four times a day a half ounce of agar agar or diabetic bran, that is. bran from which all the starch has been removed, not by washing, but by actual digestion.

In arranging the dietary of the diabetic patient, each of the following points must receive consideration:-

1. The amount of protein, which should be sufficient to meet the patient's requirements without excess.

2. The total carbohydrate should be as great as the patient can utilize, that is, should be equal to the patient's sugar tolerance. 
3. The total number of calories should approach as nearly as possible to the patient's actual requirement, and should not be permitted to fall below the patient's basal requirement for any considerable length of time.

4. The supply of vitamins and mineral salts should be sufficient.

5. An adequate amount of roughage should be added to secure normal bowel action.

\section{The Fat Allowance in Diabetes.}

Newburgh and Marsh maintain that fats may be used in much larger quantities than have heretofore been regarded safe provided the protein is kept low (.66 gram per kilogram of body weight, or 1.2 calories per pound). They also dispense with fasting, maintaining that undernutrition is not desirable. They also keep the carbohydrate very low, never allowing more than 35 grams ( $11 / 6$ ounces) daily. They begin the diet with very low protein and very little carbohydrate and a considerable amount of fat and rapidly increase the fat until the requisite number of calories required by the patient is provided, always keeping the carbohydrate and protein within the limits stated. They claim that the results found in the treatment of 200 cases fully justify their conclusion that the danger of acidosis from the free use of fats is much less than has been supposed and that the urine may be kept free from sugar more easily by this method. The results of Newburgh and Marsh seem to agree with those of Labbé and Leclerq with reference to the pernicious influence of a high protein regimen in diabetes.

\section{The Low Protein Ration Established.}

It seems at last to be clearly established that a low protein diet is highly important in a disease which for many years has been supposed to require a very highly nitrogenous regimen. These observations also give color to the view already expressed that excessive use of protein may be one of the fundamental causes of diabetes.

Carbohydrate tolerance is found to be greatly increased by the disuse of meats. Von Noorden found that without meat five times as much starch could be taken without increasing the sugar of the urine as when meat was used with the starch.

After Sydenham and Rollo discovered, something more than a hundred years ago, that the urine of the diabetic patient could 
be made sugar free by a diet consisting exclusively of lean meat and fat, a fat and protein diet consisting almost wholly of flesh foods came into general use and has been practically the routine method until very recent years. A few years ago it was pointed out by Mosse, of France, and others that a flesh diet greatly increases the danger of diabetic coma. Von Noorden announced several years ago that "much meat is not good for diabetics." Straub and other eminent authorities have shown that "meat is especially harmful as compared with vegetable albumin," such as gluten and the protein of peas and beans. Reach believes that "raw (rare cooked) meat has a toxic action which paralyzes the function of the pancreas," and in this way exercises an injurious effect in diabetes.

Practically all authorities on diabetes now agree that vegetable albumin or protein is much to be preferred to meat or animal protein in the treatment of this disease. That vegetable proteins are entirely sufficient to maintain health and strength is now conceded by the ablest physiologists.

The advantages of a non-flesh dietary for diabetics are:

1. Vegetable proteins may be used in larger quantity than animal proteins without increasing the urinary sugar.

2. Vegetable proteins permit a larger carbohydrate intake.

3. Vegetable proteins, being less putrescible than animal proteins, as printed out by Tissier, do not encourage intestinal putrefaction as do animal proteins, and hence are useful in combating the intestinal toxemia which is probably a leading factor in the causation of diabetes.

4. All sorts of fresh, smoked or salted meats, fish, flesh, fowl and "sea foods," are usually in a state of decomposition when eaten, containing millions of undesirable colon germs per gram $(1,000,000$ to $95,000,000$, according to Roderick). Vegetable proteins are free from bacteria. If animal proteins are required, eggs or casein may be used.

5. Avoidance of meats lessens the danger from acidosis and diabetic coma.

\section{The Best Food for Diabetics.}

Nuts. The high fat and protein content and the very small proportion of carbohydrates present in most nuts render them almost ideal food for diabetics. Butternuts, for example, contain 
only 2 per cent. of carbohydrate, estimated on total energy value, and Brazil nuts only 4 per cent. Nuts of almost any sort may be freely eaten by the average diabetic patient. They are not, of course, suited for an exclusive dietary as they are much too concentrated, being almost wholly lacking in the cellulose essential for bulk. The one thing essential for the thorough digestibility of nuts is complete mastication. Each particle must be chewed until of a smooth, creamy consistency.

Legumes. These highly nitrogenous vegetables find a useful place in the dietary of the diabetic when combined with a proper amount of fat. Peas, beans and lentils have a high food content of which nearly one-third is protein.

The soy bean is a remarkable legume, the carbohydrates of which are lactans and not digestible, and so may be disregarded. This bean is nearly free from starch and is very rich in protein of high quality.

Green Vegetables. Green vegetables must always constitute the bulk of the bill of fare of a diabetic patient. Green vegetables furnish a liberal supply of alkaline salts which help to neutralize the acids which form in the tissues in excess in this disease. Green vegetables are also of special service because they supply bulk with practically no starch and very little food value and are rich in food lime and iron and also vitamins.

\section{Foods to Be Wholly Avoided by Diabetics.}

Sugar

Sweet desserts

Sweet fruits

Honey
Tea and coffee

Potatoes (unless prescribed)

Alcoholic drinks

Meats (fish, flesh, fowl)

Pickles, vinegar, condiments

The introduction of insulin has practically rendered thriceboiled vegetables unnecessary so that further details about their use is unnecessary. This is fortunate, as these thoroughly denatured products were a very poor substitute for food, being nearly devoid of flavor and lacking almost wholly the salts and vitamins essential for nutrition.

\section{To Remove Starch from Vegetables.}

Green vegetables contain very little starch and only a small amount of dextrin and sugar. By repeated boiling or soaking 
in warm water nearly all the carbohydrates present may be removed. When thus treated, the vegetables are said to be "extracted." It is very difficult, however, to remove the last trace of carbohydrates, although O'Reilly and McCabe have shown that lettuce, celery, and vegetable marrow may be made completely free from carbohydrates by thrice boiling. For best results it is necessary that the vegetables be boiled in twenty times their volume of water. Canned spinach, canned asparagus, beets, onions, and turnips may be made nearly free from carbohydrate, while thrice-boiled cauliflower, pumpkin, cabbage, carrots, and string beans contain about one-half of one per cent. of carbohydrate. The addition of one-twentieth of one per cent. of bicarbonate of soda greatly favors the removal of the carbohydrate, especially in the case of cauliflower.

Since the introduction of insulin, thrice-cooked vegetables, formerly almost essential in the feeding of diabetics of severe type, have become of comparatively little importance. However, instances may occasionally develop a necessity for the use of carbohydrate-free vegetables.

The following vegetables prepared by thrice-boiling contain about one calorie of carbohydrate per ounce, a quantity so small that it may be ignored except in the gravest cases:

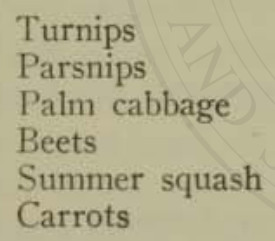

Vegetable oysters French artichokes Rutabagas

Onions Eggplant Cabbage

The use of thrice-cooked vegetables is now practically abandoned since the use of insulin permits the intake of a considerable amount of carbohydrate, more in fact than the small quantities found in green vegetables. 


\section{List of Foods Showing the Quantities of Each in Which Are Found Five Grams of Carbohydrates.*}

\begin{tabular}{|c|c|c|}
\hline FrUITS & FrUITS & Puncen \\
\hline Almonds & Prunes, fresh .......... & 1 \\
\hline Apples, fresh............. 11/4 & “ $\quad$ stewed ...... & $2 / 3$ \\
\hline “ baked ............... $2 / 3$ & Raisins .................... & $1 / 4$ \\
\hline " juice …. 11/4 & Raspberries & $11 / 4$ \\
\hline " sauce ……....... $2 / 3$ & Raspberry juice, black... & 1 \\
\hline Apricots, fresh $\ldots \ldots \ldots \ldots . . .11 / 3$ & " $"$ red $\ldots . . .$. & $2 / 3$ \\
\hline $\begin{array}{lll} & \text { canned ..................... } & 1 / 2 \\
\text { " } & \text { stewed .............. } & 1 / 2\end{array}$ & Raspberries, red, stewed- & \\
\hline Avocado (Alligator pear) 4 & $\begin{array}{l}11 / 2 \text { tablespoonf } \\
\text { Strawberries, fresh }\end{array}$ & $21 / 2$ \\
\hline Banana & " canned. & $\begin{array}{r}27 / 2 \\
1 / 4\end{array}$ \\
\hline Blackberries _............12/3 & Strawberry juice ......... & $3 / 4$ \\
\hline $\begin{array}{l}\text { Blackberry juice- } \\
12 / 3 \text { tablespoonfuls }\end{array}$ & Watermelon .......... & $21 / 2$ \\
\hline Blueberry juice- & VEGETABLES & \\
\hline $11 / 2$ tablespoonfuls & Artichokes & $11 / 4$ \\
\hline Cataloup & Asparagus ............... & 6 \\
\hline Cherries & Beans, baked....... & 1 \\
\hline “ canned .................... & " string ..... & $21 / 2$ \\
\hline Cucumbers ....................... & Beets, buttered.... & 2 \\
\hline Currants ......................... & Beet greens................. & $61 / 2$ \\
\hline Dates ……....... $1 / 4$ & Brussels sprouts...... & 6 \\
\hline Figs, dried. & Cabbage .................... & 3 \\
\hline " fresh ........ & Carrots & $21 / 2$ \\
\hline Grapes & Cauliflower, buttered. & 4 \\
\hline Grapefruit- & Celery …… & 6 \\
\hline Four tablespoonfuls & Dandelion greens.... & 2 \\
\hline Lemon juice- & 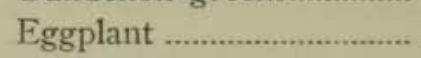 & 1 \\
\hline Four tablespoonfuls & Lentils, baked.... & 1 \\
\hline Orange juice- & Lima beans, green . & 1 \\
\hline Three tablespoonfuls & Onions, fresh .............. & $12 / 3$ \\
\hline Peach juice …… 1 & “ boiled .......... & $21 / 2$ \\
\hline 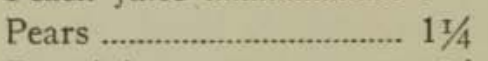 & “ stewed .......... & 2 \\
\hline Pear juice & Parsnips, browned.... & $11 / 2$ \\
\hline Pineapple, fresh & Peas, green & 1 \\
\hline " juice .............. 1 & Potato, baked ...... & - $3 / 4$ \\
\hline Plums, fresh & " boiled ... & 1 \\
\hline
\end{tabular}




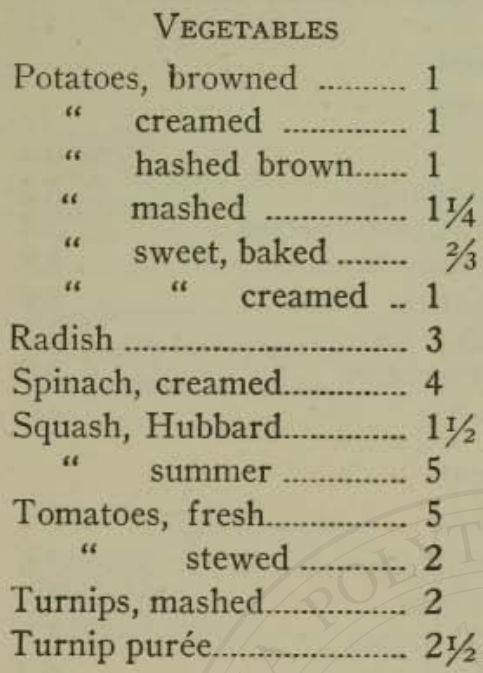

\section{Cereals}

Bran Biscuit.

Bran mush.

Bran, sterilized.

Bread, gluten

Bread, graham

Bread, white.

Bread, whole wheat.

Breakfast toast..

Corn bread

Gluten bread (40\%)

Gluten mush.

Oatmeal, boiled

Pop corn.

Rice, boiled.

Oats, boiled

Shredded wheat.

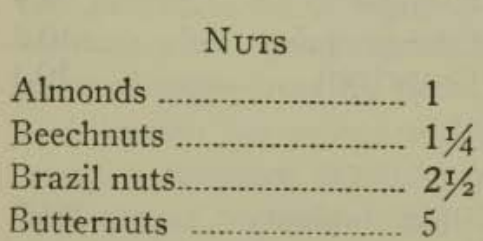

Cocoanuts ……………......... $2 / 3$

Filberts ……................... 11/2

Hazelnuts ............................ 11/2

Hickory nuts .................... 11/2

Nuttolene ............................ 3

“ baked ............... 3

Peanuts .............................. $3 / 4$

Pecans ............................... 11/4

Pignolias ............................ 21/2

Pine nuts (pinon) .............. 1

Protose …………............ 3

Walnuts ….......................... 11/2

Soups

Bean …........................... 11/4

Bean, lima …............... $21 / 2$

Lentil ........................... $21 / 2$

Pea ……............................. 2

Potato, cream of................. 2

" savory, $1 / 2$ cupful

Vegetable

\section{BouILLONS}

Tomato, 4 tablespoonfuls

“ $\quad 1 / 2$ cupful

Vegetable

BRoTHS

Bean, $1 \frac{1}{2}$ tablespoonfuls

Protose, $1 / 2$ cupful

Gruels

Barley, 2 tablespoonfuls

Cornmeal

Farina

Gluten

“ cream of.................. 2

Oatmeal, $3 / 4$ cupful

Rice 


\section{Foods Grouped According to Carbohydrate Content}

\section{Per Cent}

\section{Vegetables}

\begin{tabular}{|c|c|c|}
\hline Chinese Cabbage & $\begin{array}{l}\text { Per cent } \\
1.4\end{array}$ & Asparagus \\
\hline Lettuce & & Brussels sprout \\
\hline Endive ......... & 3.0 & Sauer kraut \\
\hline Cucumbers & 3.1 & String Beans \\
\hline Spinach ....... & 3.2 & Sea kale \\
\hline Beet greens & 3.2 & Tomatoes \\
\hline elery & 3.3 & \\
\hline
\end{tabular}

\section{Per Cent}

\section{Vegetables}

Cabbage sprouts

Cauliflower

4.3 Cabbage 5.6

Chard

4.7

Leeks

Eggplant

Radishes

Pumpkin

5.1

Collards

Kohl-rabi

\subsection{Mushrooms}

Fruits

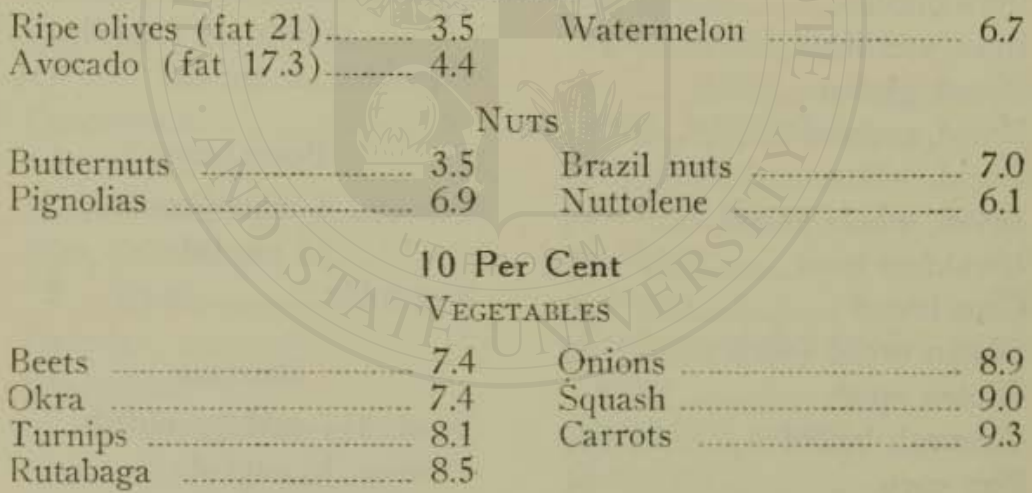

\section{FrUITS}

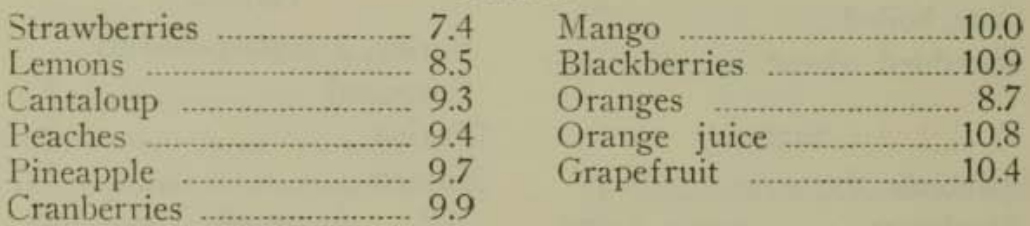

\section{Nurs}

Paradise nuts 10.2 Black walnuts 


\begin{tabular}{|c|c|c|c|}
\hline & $\begin{array}{l}15 \text { Per } \\
\text { VEGETA }\end{array}$ & $\begin{array}{l}\text { Cent } \\
\text { BLES }\end{array}$ & \\
\hline Parsnips & $\begin{array}{l}\text { Per cent } \\
13.5\end{array}$ & Artichokes & $\begin{array}{r}\text { Per cent } \\
16.7\end{array}$ \\
\hline Lima beans (canned) & 14.6 & Green peas & 16.9 \\
\hline Macaroni & 15.8 & & \\
\hline
\end{tabular}

Fruits

\begin{tabular}{|c|c|c|c|}
\hline & & & \\
\hline Raspberries & 12.6 & Pears .. & 14.1 \\
\hline Currants .... & 12.8 & Apples ............. & .14 .2 \\
\hline Blueberries & .12 .8 & Huckleberries & 16.6 \\
\hline Apricots & .13 .4 & Cherries ....... & 16.7 \\
\hline & & & \\
\hline Filberts & 13.0 & Pecans ..... & 15.3 \\
\hline Walnuts (English) & 13.0 & Pistachios & .16 .3 \\
\hline Beechnuts ............... & .13 .2 & Pine nuts & .17 .3 \\
\hline Peanut butter & 14.0 & Almonds & 17.3 \\
\hline & $20 \mathrm{~F}$ & Cent & \\
\hline & VEG & BLES & \\
\hline Baked beans & 19.6 & Potatoes ........ & 20 \\
\hline Green corn & 19.7 & Rice (boiled) & 24.4 \\
\hline & & & \\
\hline Plums & 20.1 & Bananas & \\
\hline & & & \\
\hline $\begin{array}{l}\text { Imonds } \\
\text { lmond butter }\end{array}$ & $\begin{array}{l}17.3 \\
18.0\end{array}$ & $\begin{array}{l}\text { Peanut } \\
\text { Peanut butter }\end{array}$ & \\
\hline
\end{tabular}

\section{Insulin-The Pancreatic Hormone.}

The recent discovery of insulin has begun a new era in the treatment of diabetes, the developments of which are already wonderful although the experimental stage is as yet not finished.

The theory that the pancreas produces an internal secretion which somehow enables the body to burn sugar and fats long regarded as most probable, was definitely proven by Banting of Toronto, who was the first to succeed in obtaining a product from the pancreas, the injection of which lowers the blood sugar and, in dogs whose pancreas has been removed, causes the disappearance of sugar from the urine.

Insulin has proved to be a reliable help in cases of diabetic coma, a condition heretofore justly regarded as most formidable and almost certainly fatal. In a recent case a patient in a state of coma was in one hour relieved and the next morning was feeling as well as usual. 
Properly used, insulin can be relied upon to lower the blood sugar and to lessen the urinary sugar to such an extent as to render unnecessary the drastic dietetic measures.

As would naturally be expected, the use of so powerful a remedy as insulin is not wholly without danger. It is safe only in the hands of skilled experts and must be used in connection with the greatest observation of the blood sugar.

Winter and Smith, of Cambridge, England, have recently discovered the presence in yeast of a substance which seems to be possessed of the same qualities as insulin, and which, in rabbits, reduces the blood sugar with the same degree of certainty as does insulin.

These later discoveries may prove to be of greater value than that of Banting, as it should result in greatly lessening the cost of the remedy which, at the present time, is so great as to be a serious handicap to its use except for the well-to-do. Fortunately this disease is much less common among the poor than among the well-to-do. The hard-working laboring man is little liable to suffer from diabetes or obesity. These are the favorite diseases of over-fed persons who lead inactive lives.

Collip has discovered in various vegetable products a substance which he calls glucokinin, which much resembles insulin and which produces more lasting effects than does insulin.

\section{The Use of Insulin (Iletin)}

The injection of insulin is a simple matter; but the proper dosage of insulin and especially the management of the diet of a patient under treatment by insulin are matters which require scientific knowledge and technical skill.

It is important that every patient for whom insulin is to be used should be clearly informed at the very outset that insulin does not take the place of dieting. Most misleading are the announcements made by sensational newspapers and by some ignorant or unprincipled medical practitioners that by the aid of insulin the diabetic may disregard all restrictions of diet and "eat anything he likes."

Two important results may be justly attributed to insulin. By its use the necessity for complete and prolonged fasting with the great reduction in weight and loss of strength resulting is eliminated. And further, experience has already shown that by the intelligent and continuous use of insulin the restriction of carbohydrates may be very greatly lessened. 


\section{TABLE OF RATIONS FOR DIABETIC PATIENTS}

\section{Balanced for Sugar Tolerance and Acidosis}

\begin{tabular}{|c|c|c|c|c|c|c|c|c|c|c|c|c|c|c|c|c|c|c|c|c|c|}
\hline \multicolumn{2}{|c|}{ Carbo. Tolerance } & \multirow[t]{2}{*}{10.} & \multirow[t]{2}{*}{20} & \multirow[t]{2}{*}{30} & \multirow[t]{2}{*}{40} & \multirow[t]{2}{*}{50} & \multirow{2}{*}{$\begin{array}{r}60 \\
60 \\
8 \\
892\end{array}$} & \multirow{2}{*}{$\begin{array}{r}70 \\
77 \\
16 \\
1077\end{array}$} & \multirow{2}{*}{$\begin{array}{r}80 \\
94 \\
25 \\
1266\end{array}$} & \multirow{2}{*}{\begin{tabular}{r|}
90 \\
110 \\
33 \\
1442
\end{tabular}} & \multirow{2}{*}{$\begin{array}{r}100 \\
127 \\
41 \\
1627\end{array}$} & \multirow{2}{*}{$\begin{array}{r}110 \\
144 \\
50 \\
1816\end{array}$} & \multirow{2}{*}{$\begin{array}{r}120 \\
160 \\
58 \\
1992 \\
\end{array}$} & \multirow{2}{*}{$\begin{array}{r}130 \\
177 \\
66 \\
2177\end{array}$} & \multirow{2}{*}{$\begin{array}{r}140 \\
194 \\
75 \\
2366\end{array}$} & \multirow{2}{*}{$\begin{array}{r}150 \\
210 \\
83 \\
2542 \\
\end{array}$} & \multirow{2}{*}{$\begin{array}{r}160 \\
227 \\
91 \\
2727 \\
\end{array}$} & \multirow{2}{*}{$\begin{array}{r}170 \\
244 \\
100 \\
2916\end{array}$} & \multirow{2}{*}{$\begin{array}{r}180 \\
260 \\
108 \\
3092\end{array}$} & \multirow{2}{*}{\begin{tabular}{r|}
190 \\
277 \\
116 \\
3277
\end{tabular}} & \multirow{2}{*}{$\begin{array}{r}200 \\
293 \\
125 \\
3457\end{array}$} \\
\hline $\begin{array}{c}\text { Protein } \\
80\end{array}$ & $\begin{array}{l}\text { Fats } \\
\text { Carbo. } \\
\text { Cal. }\end{array}$ & & & & & & & & & & & & & & & & & & & & \\
\hline $\begin{array}{c}\text { Protein } \\
75\end{array}$ & $\begin{array}{l}\text { Fats } \\
\text { Carbo. } \\
\text { Cal. }\end{array}$ & & & & & $\begin{array}{r}47 \\
2 \\
731\end{array}$ & $\begin{array}{r}63 \\
10 \\
907\end{array}$ & $\begin{array}{r}80 \\
18 \\
1092\end{array}$ & $\begin{array}{r}97 \\
27 \\
1281\end{array}$ & $\begin{array}{r}113 \\
35 \\
1457\end{array}$ & $\begin{array}{r}130 \\
43 \\
1642\end{array}$ & $\begin{array}{r}147 \\
52 \\
1831\end{array}$ & $\begin{array}{r}163 \\
60 \\
2007\end{array}$ & $\begin{array}{r}180 \\
68 \\
2192\end{array}$ & $\begin{array}{r}197 \\
77 \\
2381\end{array}$ & $\begin{array}{r}213 \\
85 \\
2557\end{array}$ & $\begin{array}{r}230 \\
93 \\
2742\end{array}$ & $\begin{array}{r}247 \\
102 \\
2931\end{array}$ & \begin{tabular}{r|}
263 \\
110 \\
3107
\end{tabular} & $\begin{array}{r}280 \\
118 \\
3292\end{array}$ & $\begin{array}{r}296 \\
127 \\
3472\end{array}$ \\
\hline $\begin{array}{c}\text { Protein } \\
70\end{array}$ & $\begin{array}{l}\text { Fats } \\
\text { Carbo. } \\
\text { Cal. }\end{array}$ & & & & & $\begin{array}{r}49 \\
4 \\
737\end{array}$ & $\begin{array}{r}65 \\
12 \\
913\end{array}$ & $\begin{array}{r}82 \\
20 \\
1098\end{array}$ & $\begin{array}{r}99 \\
29 \\
1287\end{array}$ & $\begin{array}{r}115 \\
37 \\
1463\end{array}$ & $\begin{array}{r}132 \\
45 \\
1648\end{array}$ & $\begin{array}{r}149 \\
54 \\
1837\end{array}$ & $\begin{array}{r}165 \\
62 \\
2013\end{array}$ & $\begin{array}{r}182 \\
70 \\
2198\end{array}$ & $\begin{array}{r}199 \\
79 \\
2387\end{array}$ & $\begin{array}{r}215 \\
87 \\
2563\end{array}$ & $\begin{array}{r}232 \\
95 \\
2748\end{array}$ & $\begin{array}{r}249 \\
104 \\
2937\end{array}$ & $\begin{array}{r}265 \\
112 \\
3113\end{array}$ & $\begin{array}{r}282 \\
120 \\
3298\end{array}$ & $\begin{array}{r}298 \\
129 \\
3478\end{array}$ \\
\hline $\begin{array}{c}\text { Protein } \\
65\end{array}$ & $\begin{array}{l}\text { Fats } \\
\text { Carbo. } \\
\text { Cal. }\end{array}$ & & & & & $\begin{array}{r}52 \\
7 \\
756\end{array}$ & $\begin{array}{r}68 \\
15 \\
932\end{array}$ & $\begin{array}{r}85 \\
23 \\
1117\end{array}$ & $\begin{array}{r}102 \\
32 \\
1306\end{array}$ & $\begin{array}{r}118 \\
40 \\
1482\end{array}$ & $\begin{array}{r}135 \\
48 \\
1667\end{array}$ & $\begin{array}{r}152 \\
57 \\
1852\end{array}$ & $\begin{array}{r}168 \\
65 \\
2028\end{array}$ & $\begin{array}{r}185 \\
73 \\
2213\end{array}$ & $\begin{array}{r}201 \\
82 \\
2393\end{array}$ & $\begin{array}{r}217 \\
90 \\
2569\end{array}$ & $\begin{array}{r}234 \\
98 \\
2754\end{array}$ & $\begin{array}{r}251 \\
107 \\
2943\end{array}$ & $\begin{array}{r}268 \\
115 \\
3132\end{array}$ & $\begin{array}{r}285 \\
123 \\
3317\end{array}$ & $\begin{array}{r}301 \\
132 \\
3497\end{array}$ \\
\hline $\begin{array}{c}\text { Protein } \\
60\end{array}$ & $\begin{array}{l}\text { Fats } \\
\text { Carbo. } \\
\text { Cal. }\end{array}$ & & & & $\begin{array}{r}37 \\
1 \\
577\end{array}$ & $\begin{array}{r}54 \\
10 \\
758\end{array}$ & $\begin{array}{r}70 \\
18 \\
938\end{array}$ & $\begin{array}{r}87 \\
26 \\
1123\end{array}$ & $\begin{array}{r}104 \\
35 \\
1312\end{array}$ & $\begin{array}{r}120 \\
43 \\
1488\end{array}$ & $\begin{array}{r}136 \\
51 \\
1668\end{array}$ & $\begin{array}{r}154 \\
60 \\
1866\end{array}$ & $\begin{array}{r}170 \\
68 \\
2042\end{array}$ & $\begin{array}{r}187 \\
76 \\
2227\end{array}$ & $\begin{array}{r}203 \\
85 \\
2407\end{array}$ & $\begin{array}{r}219 \\
93 \\
2583\end{array}$ & $\begin{array}{r}236 \\
101 \\
2768\end{array}$ & $\begin{array}{r}253 \\
109 \\
2953\end{array}$ & $\begin{array}{r}270 \\
118 \\
3142\end{array}$ & $\begin{array}{r}287 \\
126 \\
3327\end{array}$ & \\
\hline $\begin{array}{c}\text { Protein } \\
55\end{array}$ & $\begin{array}{l}\text { Fats } \\
\text { Carbo. } \\
\text { Cal. }\end{array}$ & & & & $\begin{array}{r}39 \\
4 \\
587\end{array}$ & $\begin{array}{r}56 \\
12 \\
772\end{array}$ & $\begin{array}{r}73 \\
21 \\
961\end{array}$ & $\begin{array}{r}90 \\
29 \\
1146\end{array}$ & $\begin{array}{r}106 \\
37 \\
1322\end{array}$ & $\begin{array}{r}123 \\
45 \\
1507\end{array}$ & $\begin{array}{r}139 \\
53 \\
1683\end{array}$ & $\begin{array}{r}156 \\
62 \\
1872\end{array}$ & $\begin{array}{r}172 \\
70 \\
2048\end{array}$ & $\begin{array}{r}189 \\
79 \\
2237\end{array}$ & $\begin{array}{r}205 \\
88 \\
2417\end{array}$ & $\begin{array}{r}221 \\
96 \\
2593\end{array}$ & $\begin{array}{r}238 \\
104 \\
2778\end{array}$ & $\begin{array}{r}255 \\
112 \\
2963\end{array}$ & $\begin{array}{r}272 \\
221 \\
3152\end{array}$ & & \\
\hline $\begin{array}{c}\text { Protein } \\
50\end{array}$ & $\begin{array}{l}\text { Fats } \\
\text { Carbo. } \\
\text { Cal. }\end{array}$ & & & & $\begin{array}{r}42 \\
7 \\
606\end{array}$ & $\begin{array}{r}59 \\
15 \\
791\end{array}$ & $\begin{array}{r}76 \\
23 \\
976\end{array}$ & $\begin{array}{r}92 \\
31 \\
1152\end{array}$ & $\begin{array}{r}108 \\
40 \\
1332\end{array}$ & $\begin{array}{r}124 \\
48 \\
1508\end{array}$ & $\begin{array}{r}141 \\
56 \\
1689\end{array}$ & $\begin{array}{r}158 \\
64 \\
1878\end{array}$ & $\begin{array}{r}175 \\
73 \\
2067\end{array}$ & $\begin{array}{r}192 \\
81 \\
2252\end{array}$ & $\begin{array}{r}208 \\
90 \\
2432\end{array}$ & $\begin{array}{r}224 \\
98 \\
2608\end{array}$ & $\begin{array}{r}241 \\
107 \\
2797\end{array}$ & $\begin{array}{r}258 \\
115 \\
2982\end{array}$ & & & \\
\hline $\begin{array}{c}\text { Protein } \\
45\end{array}$ & $\begin{array}{l}\text { Fats } \\
\text { Carbo. } \\
\text { Cal. }\end{array}$ & & & $\begin{array}{r}28 \\
1 \\
436\end{array}$ & $\begin{array}{r}44 \\
10 \\
616\end{array}$ & $\begin{array}{r}61 \\
18 \\
801\end{array}$ & $\begin{array}{r}78 \\
26 \\
996\end{array}$ & $\begin{array}{r}94 \\
34 \\
1162\end{array}$ & $\begin{array}{r}111 \\
42 \\
1347\end{array}$ & $\begin{array}{r}127 \\
50 \\
1523\end{array}$ & $\begin{array}{r}144 \\
58 \\
1708\end{array}$ & $\begin{array}{r}160 \\
66 \\
1884\end{array}$ & $\begin{array}{r}177 \\
75 \\
2073\end{array}$ & $\begin{array}{r}193 \\
84 \\
2253\end{array}$ & $\begin{array}{r}210 \\
93 \\
2442\end{array}$ & $\begin{array}{r}226 \\
101 \\
2618\end{array}$ & $\begin{array}{r}243 \\
110 \\
2807\end{array}$ & & & & \\
\hline $\begin{array}{c}\text { Protein } \\
40\end{array}$ & $\begin{array}{l}\text { Fats } \\
\text { Carbo. } \\
\text { Cal. }\end{array}$ & & & $\begin{array}{r}30 \\
4 \\
446\end{array}$ & $\begin{array}{r}47 \\
12 \\
631\end{array}$ & $\begin{array}{r}63 \\
21 \\
811\end{array}$ & $\begin{array}{r}81 \\
28 \\
1001\end{array}$ & $\begin{array}{r}97 \\
36 \\
1177\end{array}$ & $\begin{array}{r}113 \\
45 \\
1357\end{array}$ & $\begin{array}{r}130 \\
53 \\
1542\end{array}$ & $\begin{array}{r}146 \\
61 \\
1718\end{array}$ & $\begin{array}{r}163 \\
69 \\
1903\end{array}$ & $\begin{array}{r}180 \\
78 \\
2092\end{array}$ & $\begin{array}{r}196 \\
86 \\
2268\end{array}$ & $\begin{array}{r}213 \\
96 \\
2461\end{array}$ & $\begin{array}{r}229 \\
104 \\
2637\end{array}$ & & & & & \\
\hline $\begin{array}{c}\text { Protein } \\
35\end{array}$ & $\begin{array}{l}\text { Fats } \\
\text { Carbo. } \\
\text { Cal. }\end{array}$ & & & $\begin{array}{r}33 \\
7 \\
465\end{array}$ & $\begin{array}{r}49 \\
15 \\
641\end{array}$ & $\begin{array}{r}66 \\
23 \\
826\end{array}$ & $\begin{array}{r}83 \\
31 \\
1011\end{array}$ & $\begin{array}{r}99 \\
39 \\
1187\end{array}$ & $\begin{array}{r}116 \\
48 \\
1372\end{array}$ & $\begin{array}{r}133 \\
56 \\
1557\end{array}$ & $\begin{array}{r}149 \\
64 \\
1733\end{array}$ & $\begin{array}{r}166 \\
72 \\
1922\end{array}$ & $\begin{array}{r}183 \\
81 \\
2111\end{array}$ & $\begin{array}{r}199 \\
89 \\
2287\end{array}$ & $\begin{array}{r}215 \\
99 \\
2471\end{array}$ & & & & & & \\
\hline $\begin{array}{c}\text { Protein } \\
30\end{array}$ & $\begin{array}{l}\text { Fats } \\
\text { Carbo. } \\
\text { Cal. }\end{array}$ & & $\begin{array}{r}18 \\
1 \\
286\end{array}$ & $\begin{array}{r}35 \\
9 \\
471\end{array}$ & $\begin{array}{r}52 \\
18 \\
660\end{array}$ & $\begin{array}{r}69 \\
26 \\
845\end{array}$ & $\begin{array}{r}85 \\
34 \\
1021\end{array}$ & $\begin{array}{r}101 \\
42 \\
1197\end{array}$ & $\begin{array}{r}118 \\
51 \\
1386\end{array}$ & $\begin{array}{r}135 \\
59 \\
1571\end{array}$ & $\begin{array}{r}152 \\
67 \\
1756\end{array}$ & $\begin{array}{r}168 \\
75 \\
1932\end{array}$ & $\begin{array}{r}185 \\
84 \\
2121\end{array}$ & $\begin{array}{r}202 \\
92 \\
2306\end{array}$ & & & t & & & & \\
\hline $\begin{array}{c}\text { Protein } \\
25\end{array}$ & $\begin{array}{l}\text { Fats } \\
\text { Carbo. } \\
\text { Cal. }\end{array}$ & & $\begin{array}{r}20 \\
4 \\
296\end{array}$ & $\begin{array}{r}37 \\
12 \\
481\end{array}$ & $\begin{array}{r}54 \\
21 \\
670\end{array}$ & $\begin{array}{r}71 \\
29 \\
855\end{array}$ & $\begin{array}{r}87 \\
37 \\
1031\end{array}$ & $\begin{array}{r}103 \\
45 \\
1207\end{array}$ & $\begin{array}{r}120 \\
53 \\
1392\end{array}$ & $\begin{array}{r}137 \\
61 \\
1577\end{array}$ & $\begin{array}{r}154 \\
70 \\
1766\end{array}$ & $\begin{array}{r}171 \\
78 \\
1951\end{array}$ & $\begin{array}{r}187 \\
86 \\
2127\end{array}$ & & & & , & & & & \\
\hline $\begin{array}{c}\text { Protein } \\
20\end{array}$ & $\begin{array}{l}\text { Fats } \\
\text { Carbo. } \\
\text { Cal. }\end{array}$ & & $\begin{array}{r}23 \\
6 \\
311\end{array}$ & $\begin{array}{r}40 \\
14 \\
496\end{array}$ & $\begin{array}{r}57 \\
23 \\
685\end{array}$ & $\begin{array}{r}73 \\
31 \\
861\end{array}$ & $\begin{array}{r}90 \\
39 \\
1046\end{array}$ & $\begin{array}{r}106 \\
47 \\
1222\end{array}$ & $\begin{array}{r}123 \\
56 \\
1411\end{array}$ & \begin{tabular}{r|}
140 \\
64 \\
1596
\end{tabular} & \begin{tabular}{r|}
156 \\
73 \\
1776
\end{tabular} & $\begin{array}{r}174 \\
81 \\
1970\end{array}$ & & & & & & & & & \\
\hline $\begin{array}{c}\text { Protein } \\
15\end{array}$ & $\begin{array}{l}\text { Fats } \\
\text { Carbo. } \\
\text { Cal. }\end{array}$ & $\begin{array}{r}8 \\
0 \\
132\end{array}$ & $\begin{array}{r}25 \\
8 \\
317\end{array}$ & $\begin{array}{r}42 \\
16 \\
502\end{array}$ & $\begin{array}{r}59 \\
25 \\
691\end{array}$ & $\begin{array}{r}76 \\
33 \\
876\end{array}$ & $\begin{array}{r}92 \\
41 \\
1052\end{array}$ & $\begin{array}{r}108 \\
49 \\
1228\end{array}$ & $\begin{array}{r}125 \\
58 \\
1417\end{array}$ & $\begin{array}{r}142 \\
66 \\
1602\end{array}$ & $\begin{array}{r}159 \\
75 \\
1791\end{array}$ & & & & & & & & & & \\
\hline $\begin{array}{c}\text { Protein } \\
10\end{array}$ & $\begin{array}{l}\text { Fats } \\
\text { Carbo. } \\
\text { Cal. }\end{array}$ & $\begin{array}{r}11 \\
3 \\
151\end{array}$ & $\begin{array}{r}28 \\
11 \\
336\end{array}$ & $\begin{array}{r}45 \\
19 \\
521\end{array}$ & $\begin{array}{r}62 \\
28 \\
710\end{array}$ & $\begin{array}{r}79 \\
36 \\
895\end{array}$ & $\begin{array}{r}95 \\
44 \\
1071\end{array}$ & $\begin{array}{r}111 \\
52 \\
1247\end{array}$ & $\begin{array}{r}128 \\
61 \\
1436\end{array}$ & $\begin{array}{r}145 \\
69 \\
1621\end{array}$ & & & & & & & & & & & \\
\hline
\end{tabular}


It is highly important, moreover, that every patient should be impressed with the fact that, to secure the best possible results, which are almost never all that could be desired, it is necessary that every means by which experience has shown that recovery may be promoted should be applied with persevering thoroughness.

In the practical application of insulin the services of a competent trained physician or dietitian, or both, is indispensable. Insulin is a powerful drug. It reduces the blood sugar rapidly. Curiously, a small deficiency of blood sugar is far more dangerous than a much greater excess. When the blood sugar is lowered to 3 parts in 10,000 of blood, unconsciousness occurs and death may follow. A small overdose causes nervousness or tension which with a larger dose will increase to trembling. Hunger and sweating are other symptoms following an overdose. Fortunately, these symptoms, if given prompt attention, may be relieved within a few minutes by a glassful of orange juice or two or three teaspoonfuls of sugar dissolved in a glassful of hot water. Adrenalin solution (1:1000 in dose of $1 \mathrm{cc}$. or 15 minims) injected hypodermatically is a most valuable antidote and should be given, also, when possible.

Insulin is quantitatively designated as units. One unit is an amount sufficient to reduce the blood sugar of a small fasting rabbit (one kilo) to 4.5 parts in 10,000 or .045 per cent.

In giving insulin, it is safest to start with one unit. This is given a short time before the meal ( 15 minutes to an hour and a half). After the meal the urine is tested and if sugar is found two units are used before the next meal, and before the next, three units, and so on up to three units three times a day (Joslyn). But the increase should not be continued indefinitely. The average, total daily dose, in the hands of such experienced practitioners as Joslyn, is about 10 to 12 units. Very few patients require so much as 40 or 50 units daily and a few get on well with 2 units before each meal. In general, 1 unit will secure the utilization of 2 grams of carbohydrate. Patients differ in this regard, however, so that caution is needed.

\section{A Simple Method of Approximating the Proper Ration for a Diabetic Patient.}

The difficulty in arranging the ration, or bill of fare, for a new case of diabetes is to know where to begin. The ordinary 
"cut and try" method often involves a great waste of time and may work to the serious disadvantage of the patient. This method is clumsy and unscientific and is about as reasonable as would be the action of a tailor if, instead of measuring his customer for a coat, he should make a guess as to the dimension of the garment, make and try it on, and if he found it a misfit, make another, and if he found this a misfit, proceed to make still another, and so on continuing until by gradual approximations a proper fit might in time be attained, then charging up to the patient the total cost of the time and material employed in wasteful experimentation.

All competent internists who have had much to do with the treatment of diabetic patients by modern methods have abandoned the routine, drastic starvation of the Guelpa-Allen method. It seems to be a general practice, however, to begin with the average patient by putting him upon a very low ration and then working up until the limit of carbohydrate tolerance is reached. In addition to loss of time, this method may involve unnecessary loss of flesh and sometimes of strength and vital resistance, which it may be difficult, if not impossible to regain, and so should be limited in use to cases in which the carbohydrate tolerance is so low as to make it necessary.

By the use of mathematical formula (Woodyatt) or computations by the aid of tables (Holmes), a suitable ration may be arrived at when the carbohydrate tolerance is known, this determining factor being ascertained by simply subtracting the urinary sugar from the total carbohydrate of the diet, taking account of the carbohydrate content of the protein and the fat, together with the starch, sugar and dextrin. While accurate and scientific and an immense advantage over the "cut and try" methods in common vogue, this method involves so much figuring and reference to tables, that a very busy internist having to deal with a considerable number of diabetic patients, will be tempted when crowded for time to lapse into the old empirical way of guessing at the patient's requirement instead of making a close approximation to his needs by the application of a more exact and scientific method.

The author has prepared a table by the use of which the laborious calculations referred to may be saved. The table presents several hundred daily rations which have been calculated for different degrees of sugar tolerance, from 200 grams to 10 
grams and for different quantities of protein varying from 80 grams to 10 grams.

The basis of the calculations by which the figures given in the table have been arrived at is to be found in the following wellknown facts which have been established by modern metabolism studies :-

1. One gram of carbohydrate will suffice for the burning of $1 \% 3$ to 3 or 4 grams of fat. The lower figure is very conservative.

2. Protein is ketogenic to the extent of 46 per cent, of its weight, the equivalent of 51 per cent. of fat.

3. Protein when metabolized yields 58 per cent. of its weight of carbohydrate.

4. Fat when metabolized yields 10 per cent. of its weight of carbohydrate (glycerol).

In arranging a diet for a diabetic patient, first consideration must be given to the amount of protein, which should be sufficient to meet the needs of the body without loading it with an excess. Sherman has shown that half a gram of protein per kilogram of body weight ( 1 calorie per pound) is sufficient to meet all physiologic needs. Newburgh finds that 0.66 grams per kilogram of body weight ( 1.2 calories per pound) is ample. Woodyatt employs 1 gram per kilogram of body weight and some practitioners recommend a still higher intake of protein. All are agreed, however, that excess of protein is injurious in diabetes, a fact pointed out by Von Noorden many years ago. Since Folin has shown that all surplus protein is directly eliminated by the kidneys after having been converted into urea by the liver without making any useful contribution to the body needs, the writer considers it wise to keep the protein intake as low as compatible with the satisfaction of physiologic requirements; and hence accepts as a good basis two-thirds of a gram of protein for each kilogram of body weight or approximately one-third of a gram of protein for each pound of body weight. This amount is found ample provided the proteins employed are of high quality.

By the aid of the above data, with the patient's sugar tolerance known, it is easy to calculate at once a diet which will supply such quantities of protein, carbohydrate and fat as the patient should be able to utilize without the appearance of sugar in the urine and without the production of acidosis.

We first ascertain the patient's sugar tolerance. To do this it is only necessary to know exactly what he eats during 24 hours 
and how much, and the amount of sugar in the urine of 24 hours. Let us suppose, for example, that the patient's diet contains the following:

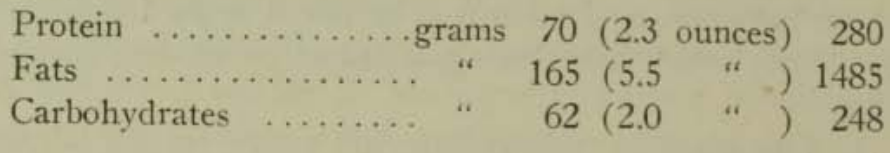

Calories .2013

Examination of the urine shows 70 grams of sugar.

To find the carbohydrate tolerance we first find the total amount of carbohydrate in his diet. This is not fully represented by the amount of starch and sugar eaten. When metabolized in the body, the protein yields 58 per cent. protein and the fats 10 per cent. The total sugar of the diet, then, will be approximately 41 grams from the protein $(70 \times .58=40.6), 16.5$ grams from the fat $(165 \div 10=16.5)$, and 62 carbohydrate, - in all, in round numbers, 120 grams $(41+16.5+62=119.5)$. If from this we subtract the 70 grams of urinary sugar, we have left 50 grams as the sugar tolerance of the patient.

Knowing the sugar tolerance, we can now proceed to tormulate at once a dietary which the patient should be able to utilize without the appearance of sugar in the urine. First, we will fix the protein at such a point as will be just sufficient to meet the patient's actual requirements, or two-thirds of a gram per kilogram of body weight. Supposing, then, that the patient weighs 60 kilos or 132 pounds, we will fix the protein at 40 grams per diem.

Next we will determine the fats. The amount of fat the patient can burn is determined by his carbohydrate tolerance. Assuming that one gram of carbohydrate will insure the complete oxidation of one and two-thirds grams of fat, and the patient's sugar tolerance being 50 grams, he can easily burn one and twothirds as much fat, or 83 grams. However, we cannot add this amount of fat in substance to his bill of fare, since it must be remembered that protein contains a molecular group which in metabolism behaves like fat. This amounts to 51 per cent. of the protein, practically one-half its weight. The protein being 40 grams, we must subtract half of this from 83, leaving 63 grams as the fat of the ration.

Now we may find the carbohydrate constituent of the diet. For this, it is only necessary to subtract from the carbohydrate 
tolerance $(50)$ the carbohydrate of the protein which is 23 $(40 \times .58=23.2)$ and that of the fat which is $6(63 \div 10=6.3)$. This gives 21 grams $(50-29=21)$ as the amount of carbohydrate in substance which may be included in the day's ration. The ration which should free the patient's urine from sugar will thus be,

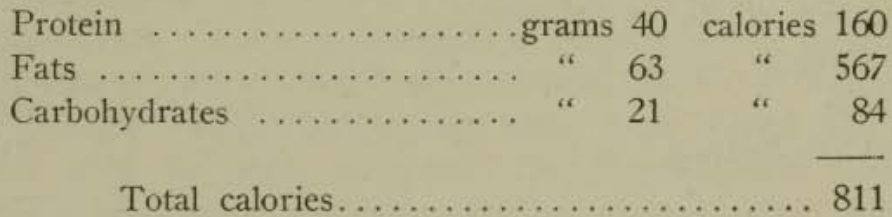

This diet is, of course, not sufficient for a maintenance ration, affording little more than half the energy required by the basal metabolism of a man weighing 60 kilograms. It will free the urine of sugar, however, and will give the pancreas relief from the overwork to which it has been subjected and without the reducing fast required by the Guelpa-Allen method, and may be increased by the addition of fat and carbohydrate by the use of insulin, adding about 2 grams of carbohydrate for each unit of insulin given.

As a labor-saving device, the writer has prepared a table (see Appendix) which gives a series of two hundred rations based upon the protein intake and the carbohydrate tolerance. By the use of this table, a suitable ration may be selected without making the calculations above detailed, thus saving considerable time, a matter of serious moment when many cases are awaiting attention. Another table indicates suitable foods and quantities.

First of all, the patient's sugar tolerance must be found. This is easily done thus: Select from the table a ration which will supply about the number of calories required by the basal metabolism of the patient. For a patient not working and weighing 60 kilos (132 pounds), this will be about 1500 calories (Dubois). Taking the patient's protein requirement as 40 grams (two-thirds of his weight in kilograms), we locate on the chart ration $40-90$ as furnishing the requisite number of calories. This ration supplies

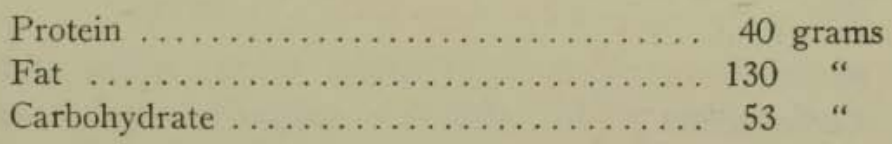

Calories.................... 1542 
It is only necessary to put the patient on this ration for 24 hours and subtract this from the known carbohydrate content of the ration. Supposing the urinary sugar for 24 hours to be 40 grams, subtracting this from 90 we have 50 grams as the patient's sugar tolerance.

Referring again to this table, we find in the column under 50 , ration $40-50$, as follows:

Protein ........................... 40

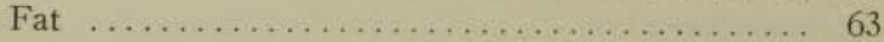

Carbohydrate $\ldots \ldots \ldots \ldots \ldots \ldots \ldots \ldots \ldots, 21$

Calories......................... 811

On this ration the patient will be sugar free. Thus, by the use of the table, the necessity for time-consuming calculations is wholly avoided and the appropriate initial ration for any degree of sugar tolerance may be quickly arrived at.

Having found the ration on which the patient is sugar free, the next thing to be done is to raise the energy value of the diet to the actual body requirement as rapidly as possible. Here is where insulin (iletin) comes in as a time-saving and life-saving remedy of priceless value. By giving the patient 5 units of insulin, his sugar tolerance will be increased 10 or 11 grams. Following the table, we will find the appropriate ration in the 60 column on the 40 protein line, ration $40-60$. This raises the calorie intake to 1001, an increase of 190 calories. The addition of another 5 units of insulin raises the ration to $40-70$, with 1177 calories. Thus within a few days the ration may be brought up to the patient's full basal requirement, say 1542 calories which is provided by ration 40-90 with 20 units of insulin. From this point on the iats need not be increased. The protein remains fixed at 40 grams.

We have now found a solid scientific basis on which to build the patient's daily ration and bills of fare. It is now only necessary to increase the carbohydrate intake by the further daily increase of the dose of insulin. For every 5 units of insulin, 10 or 11 grams of carbohydrate may be added to the ration. With 40 units of insulin, the ration would become,

Protein ........................... 40

Fats ............................... 130

Carbohydrate $\ldots \ldots \ldots \ldots \ldots \ldots \ldots \ldots \ldots \ldots \ldots \ldots$

Calories..........................1702 
After some days or weeks the insulin should be lessened a little for a day and if sugar does not appear, an equivalent amount of carbohydrate may be added to the ration, and this may be repeated until a full ration is supplied that is sufficient for the full requirements of the patient's basal metabolism plus his energy expenditure in work. This will be about one-third more than his basal metabolism.

As there is often a marked gain in sugar tolerance under the use of insulin with a scientifically adjusted dietary, it is wise to omit half the insulin for a day, after some weeks, and determine anew the sugar tolerance. This determination may be used as a basis for a new ration-building process, which will be carried out as already detailed.

In very bad cases, with a sugar tolerance as low as 10 or 20 grams, for example, the protein must necessarily be very low, not more than 20 or 25 grams, and sometimes as low as 10 or 15 grams for a few days at the start. The lower the protein intake, the more fat and carbohydrate may be given.

In these cases the table renders especially valuable service as will be shown by an example. Supposing the carbohydrate tolerance to be found to be 10 . The initial ration selected will be $10-10$,

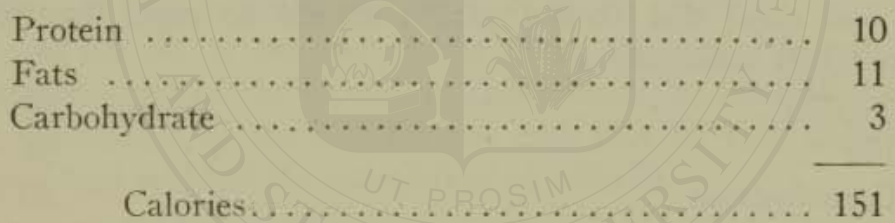

This is a starvation ration and must be increased as rapidly as possible. It is first of all important to increase the protein to the fundamental requirement as quickly as possible. Giving 5 units of insulin will raise the tolerance to 20 which will enable the patient to take ration $30-20$.

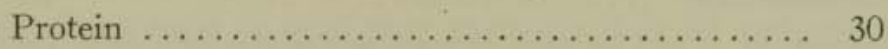

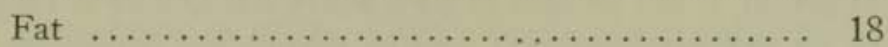

Carbohydrate $\ldots \ldots \ldots \ldots \ldots \ldots \ldots \ldots \ldots \ldots \ldots$

Calories................. 286

This ration affords nearly twice as many calories as the former, and gives 30 grams of protein. Adding another 5 units to the dose of insulin will raise the tolerance to 30 and give the 60 - 
kilo patient 40 grams of protein, which is sufficient, the complete ration (40-30) being

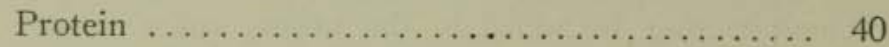

Fats ............................ 30

Carbohydrate $\ldots \ldots \ldots \ldots \ldots \ldots \ldots \ldots \ldots \ldots, 4$

Calories....................... 446

The protein of the ration is now fixed and we may begin at once to build up the ration by daily additions of insulin as before until the basal requirement is reached, then gradually adding carbohydrate until the normal diet is approached as nearly as the patient's carbohydrate tolerance will permit.

If at any time sugar appears in the urine, a new determination of the sugar tolerance should be made.

The rations shown in the chart being in physiological balance under the given conditions of protein intake and carbohydrate tolerance, they may be used as a check upon the daily bills of fare and will quickly show in what direction modification is required.

Suppose, for example, the patient's carbohydrate tolerance is found to be 50 and the protein intake 60 . Reference to the table will show that such a patient should be sugar free with ration $60-50$ consisting of

Protein .......................... grams 60

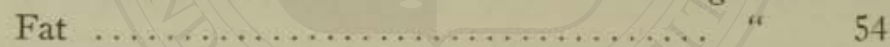

Carbohydrate ................... " 10

Calories......................... 758

If, instead of the above ration the patient is receiving, say,

Protein ................................ 60

Fat $\ldots \ldots \ldots \ldots \ldots \ldots \ldots \ldots \ldots \ldots \ldots \ldots \ldots \ldots \ldots \ldots$ " 70

Carbohydrate $\ldots \ldots \ldots \ldots \ldots \ldots \ldots \ldots$ " 20

by comparison, it will be seen at once where the trouble is (excess of both fat and carbohydrate), and by putting the patient on the diet indicated in the table, he will almost certainly at once become sugar free. Thus by the use of the table, it may be seen at once whether the pancreas is being subjected to a strain likely to injure it or whether, on the other hand, there is a wider margin between the sugar tolerance and the actual intake of carbohydrate than is necessary. 
By a little practice, the table may be used in such a way as to greatly facilitate the fixing of the initial diet and also in the daily adjustment of the diet that changes in the patient's carbohydrate tolerance may necessitate. The table will be found useful, also, in checking up the patient. When his tolerance is known and a ration has been selected for him from the table, the appearance of sugar in the urine will probably mean a surreptitious intake of carbohydrate in some form; or, the failure of the ration selected from the table to make the urine sugar free may indicate that the patient failed to save the whole amount of urine passed.

In the normal diet the proportion of total carbohydrate to fat is about five to one. It is not to be expected that the diabetic will be able to utilize this amount of carbohydrate even with the aid of insulin; but while it is possible for a diabetic to live on a diet in which the proportion of carbohydrate is 1 to 2 of fat, or ten times the usual amount of fat, it is most desirable to increase the amount of total carbohydrate. The amount of carbohydrate in the daily ration (starch, sugar, dextrin) should when possible at least equal the fat in weight. By the proper use of insulin this may be accomplished in most cases and in some cases the proportion of carbohydrate may be made much greater.

As an aid to the use of the table, the writer has had arranged a series of corresponding daily rations, by the use of which the adjustment of the diet for any case may be very quickly accomplished without the calculation of calories.

\section{Diabetes and Renal Disease.}

In cases of diabetes which show evidence of disease of the kidneys - casts, albumin or a high non-protein nitrogen, especially when analysis of the blood shows an excess of uric acid or creatinin, the protein of the diet must be kept as low as possible and meats of all sorts must be rigorously excluded from the bill of fare. Eggs must be used very sparingly if at all and only "sterile" eggs should be eaten. Casein, casein gluten preparations, soygluten biscuit, nuts and to-fu (See Index) are the best sources of protein. There is special danger of acidosis in these cases.

Diabetes in the Aged.

In the treatment of diabetes in aged persons great care must be taken to prevent gangrene, which is very common and often fatal in these cases. By keeping the urine quite free from sugar 
by a judicious dietary with the use of iletin, healing may often be secured in cases in which the disease has not advanced too far.

\section{Pregnancy with Diabetes.}

Pregnancy is less likely to occur in a woman who is diabetic than in a normal woman, but does occur and is a complication which menaces both the mother and the fetus. An experienced obstetrician reports ten cases with death of the fetus in every case. Later experience has shown, however, that by careful dietetic management, much better results may be obtained. The dietetic management of a case of this sort does not differ from that of others. The same general rules should be followed. At confinement, gas should be used rather than ether or chloroform if an anesthetic is required.

In cases in which sugar appears in the urine at the beginning of lactation, the condition appears to be due to a temporary disturbance of metabolism which may be made to disappear in a few weeks by careful dieting.

\section{Diet in Diabetic Coma.}

Perhaps the greatest boon conferred by insulin is its efficiency in combating coma, for which it is so nearly a specific that when it is available, death from diabetic coma should not occur. Coma should be avoided by proper feeding and treatment. If by accident, symptoms of coma develop, prompt action is needed. The patient should go to bed at once, and should drink copiously of hot water and take a glassful of orange juice every hour. In the absence of orange juice, thin oatmeal gruel or better, a dessertspoonful of malt sugar dissolved in a glassful of hot water. Every three hours an enema should be given consisting of three pints of water at a temperature of $105^{\circ} \mathrm{F}$. and containing the juice of two lemons and two ounces of malt sugar.

Ten units of insulin should be given every hour in coma until, if needed, three or four doses have been given, then every other hour; but more than 60 units should seldom be given in twelve hours, although as much as 100 units have been required in some cases (Joslyn). When the patient is in a state of profound coma, carbohydrate may be given intravenously if necessary in the form of glucose, 


\section{Examination of the Urine (see Index).}

Diabetic patients should learn to examine the urine for sugar and dangerous acids. If the tests show sugar or acids, even a trace, the diet must be changed at once.

A twenty-four hour specimen of urine should be obtained and a quantitative examination of sugar made and the carbohydrate tolerance determined. The proper dietary is selected from the chart and the ration increased from day to day by the use of insulin, as previously indicated.

\section{Saccharine.}

The use of saccharine by diabetics should be discouraged. The diabetic should be educated to forget sweets and to be contented without them. A few weeks of discipline will accomplish this. Heitler, of Vienna, claims to have shown that saccharine is a heart poison. Under its influence, the heart's action is lessened in vigor and its continued use may give rise to serious injury.

\section{Recipe for Gluten Bread.}

Make a rather stiff dough by adding to the best quality of spring wheat flour the proper amount of water. Allow to stand for two or three hours, then knead until a strong, elastic, tenacious dough is formed. Now place on a sieve or coarse cloth under a spigot and knead under a small stream of water until no more starch can be washed away; that is, until the water runs away clear. The rubber-like mass left is almost pure gluten. This may be molded and cut into small masses and baked in a quick oven. Surprisingly large rolls may be made from very small pieces of this wet gluten. If 40 per cent. gluten is desired instead of merely pure gluten biscuit, flour may be added to the gluten in right proportion to produce the desired percentage. In general, about one pound and a quarter of spring wheat flour will be required for each pound of wet gluten. Gluten bread is more easily made by the use of 40 per cent. gluten flour.

\section{Gluten Products.}

In the summer of 1889 the writer devised a method for preparing standardized gluten preparations. Prior to that time the only gluten products known were gluten biscuit, prepared by washing the starch out of wheat flour, and the so-called 
"gluten flours" which contained little if any more gluten than good whole wheat flour. The writer had made for use at the Battle Creek Sanitarium gluten flours containing 40 per cent. of gluten and meals containing 20, 40,60,80, and 90 per cent. of gluten. For many years these products were the only glutens produced in this country of known value, as noted by the late Dr. Osler in his writings on diabetes. Many years later the U. S. Government under the Pure Food Law established 35 per cent. as the legal standard for gluten flour and other products to which the term gluten is applied. Most manufacturers have adopted our original standard of 40 per cent., but it would seem to be desirable to make the proportion of gluten 50 per cent. for convenience in prescribing.

Gluten biscuit are especially useful in the feeding of diabetic patients. Gluten contains more lime and iron than any other food product. A pure gluten biscuit weighing one-half ounce contains as much protein as a small beefsteak weighing two ounces, besides more iron and lime.

Eighty per cent. and 40 per cent. biscuit are exceedingly useful, almost indispensable in arranging a bill of fare for the diabetic. As prepared by the Battle Creek Food Company, these gluten products are presented in biscuit of nearly uniform size weighing about 3.5 grams each. The 80 per cent. biscuit contain each 3 grams of protein and .5 grams of carbohydrate. The 40 per cent. biscuit contain 1.5 grams of protein and 2 grams of carbohydrate. These biscuit are much more palatable than gluten bread and, when freshly toasted, are exceedingly crisp and toothsome. Their bulk is so large that they aid greatly in utilizing the large amount of fat which is required in patients whose carbohydrate tolerance is very low.

Two recent improvements have been made in gluten products at the writer's suggestion, in the addition to wheat gluten of a certain proportion of casein and a concentrated vegetable extract (prepared from yeast) rich in vitamin B. The purpose of adding casein to gluten is to supplement its incomplete proteins by means of the trytophan of milk, an amino acid which is deficient in the proteins of all cereals. This is known as "aminized" Gluten. By the addition of vitamin B. to gluten its dietetic properties are notably improved. This "vitamized" and "aminized" gluten more than replaces the proteins of meat in nutrient value. 
Soy bean products are highly valuable not only because of the small amount of carbohydrate which this legume contains but because of the superior quality of its proteins which, like the proteins of milk and nuts, are of the "complete" class.

Acidophilus Buttermilk.-A good culture of B. acidophilus in skim milk or whey is of great value in diabetes as a means of combating the intense intestinal toxemia which is at least one of the potential factors to which this disease must be attributed. The carbohydrate content of acidophilus buttermilk and whey is small, only 4 per cent., so that a half pint will supply less than 10 grams of lactose along with the highly beneficent protective organism, B. acidophilus, of which a first-class whey or buttermilk culture contains about $200,000,000$ to the c. cm. (Kopeloff), or $6,000,000,000$ to the ounce.

\section{Sugar-Free Milk.}

Foodstuffs wholly free from carbohydrates are of less importance since the discovery of insulin; but in cases in which the free use of milk would be desirable except for the considerable amount of lactose which it contains, a formula for preparing a sugar-free milk may be found useful.

First it is necessary to prepare a one per cent. solution of the salts of milk. These are said to consist of the following, combined in the proportion indicated:-

\section{MILK SALT}

Potass. chloride.

Sodium chloride

9.9 grams

Mono potass. phosphate.

11.5 "

Di-potass. phosphate

13.8

Dimagnesium phosphate

10.0 “

Dicalcium phosphate

4.0 “

Tricalcium phosphate

8.0 “

Potassium citrate 9.6 “

Magnesium citrate

5.9 “

Calcium citrate

4.4 "

Calcium oxide 25.5 "

Sodium carbonate. 5.5 “ 40.0 “

148.1

For a quart of sugar-free milk prepare one quart of one per cent. solution of milk salts by dissolving in a quart of hot water 
two and one-half drams of the above mixed salts. Put the solution on ice to cool. Now precipitate the curd of three pints of full milk by adding one dram and a quarter of glacial acetic acid mixed with one quart of water. Drain off the whey on a thick cloth filter. Wash with water until free from acid then dissolve the curd in the solution of milk salts.

\section{DIET IN GOITER}

Plummer, of the Mayo Clinic, who has made a very extensive study of goiter, divides cases of this disease into three classes: first, simple goiter; second, adenomatous goiter; third, exophthalmos goiter.

Medical and surgical treatment of this disease and recognition of its several forms is very necessary, but from a dietetic standpoint the classification is less important. A diseased goiter is a crippled goiter. Its function is disturbed. To secure relief, the causes of the disturbance must be, as far as dietetic management of all forms of goiter. That there is a close relation between diet and the thyroid gland and its function has long been known, and is made clearly evident by many undisputed facts.

It is believed by most surgeons that in adenomatous goiter, that is, goiterous enlargement which presents one or more hard nodular masses, operation is always indicated, at least when hyperthyroidism exists.

In simple goiter, in which there is general enlargement of the thyroid gland, without nodules, and without hyperthyroidism, the enlargement usually disperses quite readily under the use of small doses of iodine or the careful use of dry thyroid, or thyrolysin (?) Such medication, however, must be very carefully managed to avoid producing hyperthyroidism. There is, on this account, reason to doubt the wisdom of recommending the general use of iodized salt. Iodine must be given in cases requiring it, but may do harm in cases in which it is not required, or when its use is too long continued.

The diet should be made antitoxic, including liberal quantities of greens, and particularly the following foods, which analysis has shown to contain iodine in appreciable quantities: Turnips; melons, lettuce, beets, tomatoes, French beans, green peas, radishes. The carrot, parsley and potato also contain con- 
siderable quantities of iodine, while the cucumber, pumpkin, spinach and kidney beans contain only very small quantities.

Agar and Irish moss are very rich in iodine and for this reason should be freely used by persons suffering from the simple form of goiter.

It is quite possible that concentrated extracts prepared from such vegetables as the turnip, radish, French beans and beets, used daily in proper quantities, might be of substantial service in these cases.

Observations of Chalmers Watson suggest the special value of oatmeal in the feeding of persons suffering from simple goiter.

Lack of iodine in drinking water and in foods raised in regions where the soil is deficient in iodine is believed to be the cause of simple goiter, which appears with great frequency in certain regions. This condition prevails in the northern half of the United States, through the greater part of which nearly 50 per cent. of the school children show evidence of thyroid enlargement. The drinking water in this region has been found to contain less than one grain of iodine in 400,000 barrels of water. In the southeastern States, the amount of iodine contained in water is 100 to 500 times as much, and in these regions simple goiter is seldom seen.

According to the best Swiss authorities, a child requires daily about $1 / 650$ of a grain of iodine. In goitrous regions, the water often contains so little iodine that one to two thousand gallons would be required to furnish the amount needed by a child for one day. An adult requires in addition to its daily supply of iron a store in its thyroid gland of about 200 times as much iodine, or one-third of a grain. Fortunately, a considerable amount of iodine, as above indicated, is found in certain foods. Plants store iodine.

The following table shows the amount of iodine found in some common foods produced in goitrous regions, according to J. F. McClendon, of the University of Minnesota: 


\section{IODINE PER TON IN COMMON FOODSTUFFS}

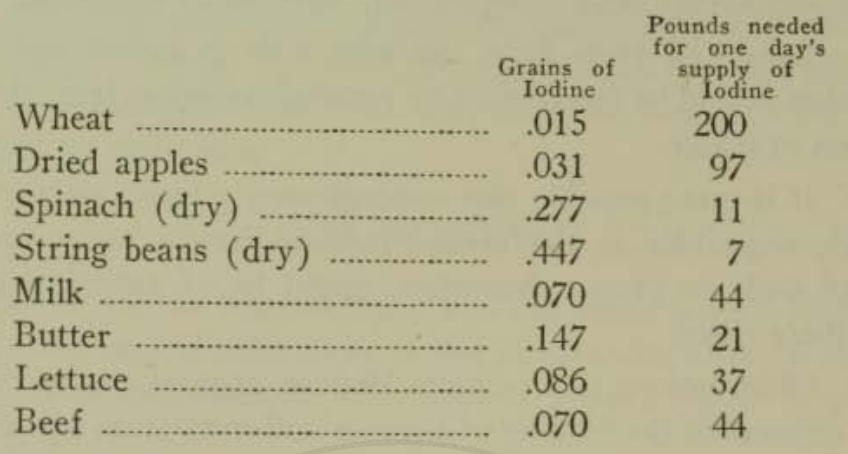

It is evident that many foodstuffs are very greatly lacking in iodine. Food produced in non-goitrous regions contains several times as much iodine as that grown in goitrous States. People living in such goitrous territories as the lake region should use imported rather than home grown foodstuffs.

In the early experiments on the function of the thyroid gland the gland was removed from dogs. It was noticed that these dogs soon became subject to convulsions and other grave disorders and after a few weeks died. It was later learned, however, that if the dogs were fed on bread and milk instead of meat, death did not occur. This led to the theory that a function of the thyroid gland is to destroy the poisons which develop in the body as a result of flesh eating.

That such poisons do develop has been well known since the classical experiments of Bouchard, who found that solutions prepared from the stools of meat-eaters were twice as toxic as those prepared from the stools of non-flesh eaters. Herter, of New York, observed that animals were quickly killed by solutions prepared from the stools of carnivorous animals, but did not succumb to similar solutions prepared from herbivorous animals.

Numerous highly virulent poisons have been isolated from the stools of flesh-eating men and carnivorous animals. The putrid character of the stools of meat-eating animals and men clearly indicates the presence of toxic products.

Extensive laboratory research has shown that the thyroid gland is actively concerned in the destruction of poisons circulating in the blood. The first line of defense against poisons is the intestinal mucous membrane. Next, the liver offers an important barrier against toxic invasion. The third line of defense is the 
thyroid gland and other glands of internal secretion which are associated with it.

In typical cases of hyperthyroidism the thyroid gland is enlarged and its activity enormously increased. A general disturbance of the body results from the abnormal increase of thyrotoxin or thyroidin, which increases greatly the activity of the body cells, producing hypermetabolism, sometimes to the extent of even doubling the normal cell activity of the body. According to Plummer this increase affects especially the protein metabolism, which is also known to be increased in febrile conditions generally.

It may be said, then, that.in exophthalmic goiter the body is suffering from an intense toxemia in which, in addition to poisons produced in the alimentary canal and perhaps elsewhere, the body is also disturbed by thyrotoxin produced by the thyroid gland itself.

It is evident, then, that in the dietetic treatment of exophthalmic goiter, or hyperthyroidism, it is of primary importance to suppress all foodstuffs calculated to increase the production of poisons in the body or which will tend to in any way stimulate the activity of the gland. That meats, meat extracts, bouillon, and broths are active stimulants of the thyroid gland is now generally recognized. Says Plummer, of the Mayo Clinic: "Unquestionably the diet should be one that will reduce metabolism to the lowest point. This indication is best met by a diet low in proteins." Fish, shellfish, meats, tea, coffee, alcohol and tobacco are all well known to be stimulants of the thyroid gland and their use must be positively prohibited. This practice the writer has followed for more than forty years, and by this method he succeeded in curing a considerable number of severe cases of exophthalmic goiter long before relief of this condition by operation was even suggested.

Tsuji, a Japanese investigator, has found that when rats are fed on large quantities of egg yolk or milk their thyroids enlarge. There was also enlargement of other organs such as takes place when animals are fed on thyroid. This observation agrees with the clinical fact that persons suffering from hyperthyroidism, the so-called exophthalmic goiter, are also practically always found to be suffering at the same time from intestinal toxemia or autointoxication, the result of putrefaction of the food residues in the colon. It is doubtless for this reason that meat feeding is found so highly detrimental in cases of thyroid disease. Dr. Crile has 
frequently called attention to the importance of excluding meats from the diets of patients who have undergone operations upon the thyroid. He insists upon the exclusion of meats for at least a month after the operation, and when asked, "Would it be better to exclude meats from the dietary permanently?" at once replied, "Of course, of course." Every physiologist is familiar with the facts upon which this recommendation is based. The surgeons are rapidly becoming informed. It is important that the information should be passed along to the patients as rapidly as possible.

Curschmann, in an article in Riforma Medica, reports that in Italy during the war as meat became scarce exophthalmic goiter steadily decreased in frequency. Twenty-two prominent physicians and surgeons who were questioned with reference to the frequency with which this disease was observed during the war reported, without exception, a notable decrease in the disease. Deneky, of Hamburg, Germany, who observed in the five years preceding the war 217 cases, in the five years from 1915 to 1920 observed but 42 cases.

While there are still a few authors who recommend the use of meat in this disease, the ablest authorities, both English and American, recommend that meat should be wholly discarded and that the diet should consist of milk and farinaceous foods with fresh vegetables and fruits.

Thompson forbids the use of meats of all kinds, including sea food and discourages the use of tea and coffee.

Chalmers Watson claims to have demonstrated that oatmeal also stimulates the thyroid and on this account this cereal is usually excluded from the dietary of patients suffering from Graves' disease. It is difficult to understand how a simple cereal should behave in this manner, and the writer has never observed any injurious effect from the use of oatmeal. Nevertheless, until further light is obtained upon the subject, it will be well to avoid the use of oatmeal in more than small amounts in feeding these patients. Foodstuffs known to be rich in iodine should be used very sparingly or altogether discarded in this disease.

McCarrison finds that a deficiency of vitamins in the food, particularly of the antiscorbutic vitamin, lessens the resistance of the thyroid gland and renders it "susceptible to the harmful action of intestinal bacteria, with resulting atrophic changes."

McCarrison has also called attention to the fact that the feeding of onions to animals suffering from intestinal toxemia 
because of an excess of fat and protein, restrains the growth of putrefactive bacteria in the intestinal tract and retards the absorption of their products and, to a marked degree, delays the development of the thyroid enlargement which usually develops rapidly in pronounced intestinal toxemia. McCarrison was led by this observation to recommend onion juice as a remedy for restraining the over-development of the gland in exophthalmic goiter, or Graves' disease. Evidently, a still better measure is to be found in a change of the intestinal flora, thus eliminating the putrefaction altogether.

The excessive activity of the body cells results in a rapid wasting of the tissues, a loss which must be met by an increase in the food intake. The basal metabolism of a person suffering from hyperthyroidism may, in extreme cases, be double the normal basal metabolism so that in addition to keeping the patient very quiet, an essential factor in treatment, there must be an increase in the total number of calories eaten. The proteins should not be increased, as this will still further increase the abnormal activity. The increase should be confined to the fats and carbohydrates, chiefly the latter.

In all cases operated upon for relief of exophthalmic goiter, the dietary above described should be strictly followed, not simply as a temporary measure but permanently. Otherwise the disorder is almost certain to recur, making another operation necessary.

In cases of exophthalmic goiter with diabetes the patient must be put upon a diabetic diet. In cases of this sort the newly discovered insulin is a very precious resource indeed.

Mental influence is certainly a factor which must be considered in the treatment of exophthalmic goiter. Worry unquestionably disturbs the thyroid. One author reports a series of fifty cases treated successfully with optimism, which he prefers to iodine. In adults suffering from thyroid disease, iodine must be used with great caution and only under judicious medical supervision. The use of iodine by healthy adults is likely to result in serious injury.

\section{HYPOTHYROIDISIM}

It is now generally conceded that the thyroid gland is the seat of iodine metabolism. This gland removes from the blood minute quantities of iodine and therewith produces its special secretion, thyroidin, which is in excess in hyperthyroidism but 
deficient in hypothyroidism, giving rise to certain well-marked morbid conditions. Well marked hypothyroidism in children is known as cretinism and in adults or adolescents as myxedema. In this condition there is a diminished activity of the body cells due to the lack of the stimulating influence of the thyroid secretion. The body cells are overburdened with waste products. This is clearly shown in the skin, which becomes puffy, dry and inactive. The whole body becomes sluggish, including the brain, and in young children cretinism, a condition bordering on idiocy, is developed.

The myxedematous patient usually has a cachectic appearance suggesting Bright's disease. The speech is slow and sluggish, as though the tongue were swollen. The patient moves slowly and clumsily. There appears to be a mental as well as a physical slowness.

These patients generally have a tendency to increase in weight and are often large eaters. Care must be taken to restrict the amount of food to actual needs. A reducing diet for a week or two is advantageous at the outset of treatment.

Mild forms of hypothyroidism occur much more frequently than is generally recognized. This condition almost universally develops in advanced age and is indicated by dryness of the hair and skin and the development of a depression just above the sternum, the result of atrophy of the thyroid.

In this condition, the natural conclusion would be that a radically different dietary would be indicated and perhaps flesh meats might be admissible on account of their iodine content and their stimulating influence upon the thyroid.

But this is not the case. While oatmeal and vegetable foods containing iodine are especially indicated, meats of all kinds are certainly contraindicated for the reason that putrefaction of undigested remnants of flesh foods in the colon gives rise to toxic products with which the crippled thyroid is not prepared to deal, it being remembered that dogs whose thyroid glands have been removed, died when fed meat but lived with a diet of bread and milk. It is a well-known fact that in cases of myxedema a meat diet will "sometimes induce tetanic attacks or convulsions." Flesh foods of all sorts must be wholly and permanently avoided.

Rosenbloom recommends a diet containing a minimum amount of protein. The low protein diet is necessary to lessen the intestinal putrefaction which enormously increases the work 
of the thyroid. Foods rich in iodine are useful because iodine is necessary to enable the thyroid to do its work.

Another point of essential importance in these cases is the feeding of thyroidin, thyroid extract or dried thyroid gland. This feature of the diet, however, should rather be classed as medication and should be carried on under the supervision of a competent physician, since it is quite possible to do harm as well as good. The good effects of thyroid feeding in most cases quickly become apparent. Much harm may result from excessive thyroid feeding, which should be carefully avoided. It is a grave error, however, to depend exclusively upon thyroid feeding.

Regulation of the diet in such a way as to lessen the amount of poisons circulating in the blood, thus diminishing the work required of the thyroid, is of first importance. This with rest in bed and changing the intestinal flora will secure radical improvement even in grave cases of myxedema, although much more rapid progress is made when thyroid feeding is added.

Murray reported in 1920 the death of the first patient treated for myxedema by thyroid extract. The patient began treatment by this remedy in 1891 at the age of 46 years. She continued the treatment until her death in 1919 at the age of 74. Her life was doubtless greatly prolonged by the use of thyroid extract.

The great number of operations now-a-days being done for relief of exophthalmic goiter will, in the writer's opinion, produce an epidemic of surgical hypothyroidism a few years in the future. Operators not infrequently remove so much of the gland that not more than a fifth, or even a tenth of the original gland structure is left. When an operation of this sort is performed and no change is made in the patient's dietetic habits, over-stimulation of the gland will continue and the small fraction of gland left will be overworked to a greater extent even than the whole gland would have been, although the unpleasant effects arising from the excessive production of thyroidin may not appear. Overwork of a gland always results sooner or later in degeneration and diminished functional activity. It is inevitable that these abbreviated thyroids should suffer a very great shortening of the duration of their functional activity.

To say that a man is as old as his thyroid is equally as true as to say that a man is as old as his arteries, for the thyroid function is an important link in the chain of vital activities which are essential to the prolongation of life. 
Deficient functioning of the thyroid, or hypothyroidism, is one of the active factors in inducing the changes characteristic of senility, particularly the changes in the hair and the skin. When the skin becomes thin, dry, parchment-like, inelastic and covered with brown patches, the function of the thyroid gland is impaired. These external evidences of old age degeneration are only the outward sign of similar degenerative changes taking place all through the body, which are in part at least the result of the loss of the thyroid function. To live long and well, one must take good care of his thyroid and must keep it intact. To accomplish this it is necessary that the thyroid should not be overworked by the use of flesh meats and other toxin-containing foodstuffs and by such neglect of the colon as will permit it to become an incubator of noxious germs and a veritable reservoir of virulent poisons.

In many forms of skin diseases, as well as in premature old age, hypothyroidism is found to be the dominant factor. In all these conditions the dietary suggestions made above may be followed with marked advantage.

Another of the consequences of thyroid inefficiency is an irritation of the skin which results from the chapping due to excessive dryness, especially in winter and in old people.

These conditions indicate the necessity for increased activity of the thyroid, which may be encouraged by the use of foods rich in iodine, and if necessary, by thyroid feeding.

In conclusion, attention must be called to the importance of maintaining a sanitary condition of the colon. This requires three bowel movements daily, or an emptying of the colon after each meal. To accomplish this, it is necessary that a much larger amount of roughage than that found in the ordinary bill of fare should be made a regular part of the dietary (see laxative diet, index). Bran to the extent of a quarter ounce at each meal and an abundance of greens are probably the best forms of roughage for regular use. Agar-agar may sometimes be employed advantageously, and the continuous use of mineral oil both before and after each meal is necessary to serve as a lubricant in cases in which constipation has existed so long that the natural lubricating function of the colon has been impaired.

The amount of thyroid needed is nuch less when a strict antitoxic diet is followed and the intestinal flora changed and kept free from putrefactive bacteria. 


\section{DYSPITUITARISIM}

In this disorder there is a remarkable tolerance for carbohydrates. This is not a morbid symptom but simply an instinctive or automatic indication of a special need. The craving for carbohydrates should be curbed only to such a degree as to prevent undue indulgence in confectionery and other sweets. If the patient is asked to avoid the use of candy and sugar, confining the indulgence in sweet things to sweet fruits and fruit juices, malt sugar and malted preparations, no harm will be likely to result from the gratification of the appetite for saccharine and farinaceous foods. Proteins should be kept at a low level. The appetite must be curbed in cases in which there is a marked tendency to accumulation of fat, and the intake of butter and other oleaginous foods may be reduced to a minimum. Potatoes should be largely used instead of cereal foods. This is advantageous, first, because they are more bulky and hence will diminish the tendency to overeating; and second, because potatoes, like other farinaceous roots, are rich in organic bases which are needed to neutralize the acid wastes with which the body is likely to be overcharged. Potatoes are also richer in iodine than are cereals.

Special attention must be given to the colon and to the intestinal flora, which always needs to be changed, so as to lighten so far as possible the burden upon the thyroid, which is also usually crippled to a marked degree in these cases.

The bowels must be kept active, at least three movements a day, by a laxative diet (see Index) and the use of bran, agar, and mineral oil. The flora must be changed.

The same principles of treatment apply as in disease of the thyroid. Evidence of pituitary deficiency requires the administration of the dried gland in connection with proper diet. Thyroid deficiency may exist at the same time, in which case feeding of thyroid may also be found necessary. The regulation of the dose of thyroid or pituitary gland substances requires the attention of a physician skilled in this particular class of ailments.

\section{ADDISON'S DISEASE}

While little in the direction of a cure can be accomplished either by diet or other means in this disease, much can be done to better the patient's condition and prolong life by careful regulation of the diet. The essential things to be done are to change 
the intestinal flora and then to suppress intestinal putrefactions by an antitoxic diet. Meats of all sorts must be excluded. The bowels must be kept active by a laxative diet, supplemented by daily enemas. Carbohydrates may be freely used with advantage. In some cases, benefit has seemed to follow the use of liberal doses of dried adrenal gland.

The patient is always toxic, and hence the flora should be changed by the fruit regimen with lactose or lacto-dextrin.

\section{UREIMIA}

The diet in uremia should be the same as indicated for acidosis (see Index). It should consist exclusively of fruit juices and such carbohydrates as malt sugar and raisin water (see Index). Malt sugar is superior to the juice of raisins in these cases, for the reason that the latter contains some tartaric acid, which is less perfectly oxidized in the body than is citric acid and may tend to increase the acidosis. Orange juice is of great value and may be freely used with advantage in cases of renal acidosis as well as in acidosis due to defective metabolism, the so-called ketonic acidosis. Protein should be wholly excluded from the diet for a few days. Potato in various forms should largely replace cereals on account of the alkaline salts which it supplies, which lessen the tendency to acidosis.

Enemas containing two ounces of malt sugar to the pint may be given in quantities of half a pint to a pint every hour, to be retained. The colon should be emptied by a large enema every four or five hours.

\section{THE ANEMIAS}

In anemia there is a notable lack of iron and of blood cells. The problem is to supply the body with the iron needed for the production of new red blood cells, and to encourage the bloodmaking process. It is now generally recognized that this can be best accomplished only through the means of appropriate foodstuffs and the application of other natural curative measures and not by the use of drugs. Neither is feeding alone sufficient. Out-of-door air, exercise in proper cases, sunlight, cold bathing, and attention to all health-promoting measures, are almost equally important.

The diet, in addition to a sufficient amount of food iron, must also present to the body an abundance of vitamins, lime, 
complete proteins and other essential tissue-building elements. Hence, the restorative regimen is required (see Index), especial care being taken to include foods rich in iron in sufficient quantity to double the ordinary normal intake of iron. The ordinary amount of iron excreted by the body daily is about one-sixth of one grain. Since the total amount eaten is never assimilated, it is necessary that the daily intake or the food iron ration should be about one-fourth of a grain. The amount of each one of the ordinary foodstuffs which contains this quantity of iron is given in the table shown elsewhere in this work (see Table XI).

Anemia is coming to be very widespread in this country because of the neglect of the use of green stuffs, which in every other country are extensively employed.

An explorer for the United States Bureau of Plant Introduction informed the writer that the average Chinaman eats four times as much greens as does the average American. When it is realized that it is from greens that our supply of iron is chiefly derived it becomes apparent that the national anemia must be the consequence of this serious dietetic neglect. Every effort should be made to instruct the public with reference to this grave error, almost universal in this country.

The one-fifth to one-fourth of a grain of iron necessary to make good the daily loss represents about one two-hundredth part of the total iron content of the body.

Each drop of 1 per cent. in the hemoglobin content of the blood means a loss of 0.43 grains of iron. A loss of 20 per cent., as in a case showing a hemoglobin content of 80 per cent., means a deficiency of 8.6 grains, the amount of iron found in 34 pounds of spinach.

By eating half a pound of spinach a day, this amount of iron might be absorbed in sixty-eight days or a little more than two months. It must be remembered, also, that the bill of fare must be so constructed as to supply the patient in addition his full normal daily ration of iron, which may be easily done by the aid of the table above referred to.

In cases in which anemia is due to loss of blood, the iron of the body may be quickly restored by proper feeding. provided there is no recurrence of hemorrhage. The same is true of all secondary forms of anemia. When the cause is removed, proper feeding will quickly restore the patient.

An important point to which attention should be especially 
called is the fact that flesh foods are not particularly valuable for building up the blood. The popular idea that red meat of some sort is indicated in anemia has no sound scientific basis. Red meats often have been prescribed as a remedy for anemia with most unsatisfactory results. Although red meats and blood are rich in hemoglobin, it appears that the iron of hemoglobin is not so readily assimilated as is iron in the form in which it is furnished in plants, particularly green leaves (Sherman).

Grigoriew has shown that rabbits rendered anemic by bleeding improve much more rapidly when given chlorophyll, or organic iron found in green leaves, than when given iron in a metallic state.

Abderhalden and others have shown that the iron found in foods is necessary for supplying the iron requirements of the body. Even the so-called organic compounds were found to be not so readily assimilated as the iron supplied in foods.

Attention should also be called to the fact that there are many vegetable foodstuffs which are far richer in iron than is lean beef, while the fat of meat contains no iron at all. For example the following common foodstuffs (20 in number) are all richer in iron than is beefsteak, namely: almonds, barley, beans, egg yolk, hazel nuts, molasses, malted nuts, malt sugar, peas, protose, 40 per cent. gluten flour, rye, entire wheat, wheat bran, figs, dates, prunes, mustard greens and many other green stuffs. A few vegetable foods contain nearly double the amount of iron contained in beefsteak, among which may be mentioned lima beans, lentils and wheat bran. And it must not be forgotten that the iron found in these vegetable products is more easily assimilated than that found in meats.

Even that humble vegetable, the onion, contains a notable amount of iron which is said to be of particularly fine quality; while the yolk of egg contains as much iron as more than twice its weight of the best beefsteak.

A person suffering from chronic anemia must make radical changes in his bill of fare. Meats must be discarded. Nuts, nut meats, and glutens should take the place of meats. Foods rich in iron should be the staples of each meal. Cane sugar should be replaced by malt sugar, dates, figs and raisins. Malted nuts (vegetable milk) is rich in iron and may well in part replace milk, which is very poor in iron. Lentil soup and greens of all sorts 
must enter largely into the dietary. The restorative regimen is indicated (see Index).

A cup of savita (yeast extract) broth contains as much iron as half a pound of beefsteak besides food lime and vitamins and highly palatable osmazome.

Much more interesting and useful data respecting the iron content of foods may be obtained by a careful study of the Table XI.

\section{Pernicious Anemia.}

In pernicious anemia, leukemia, Addison's disease, and other of the grave forms of anemia, the problem is by no means so simple as in ordinary anemia. In these cases, there is undoubtedly a profound toxemia, the seat of which in many cases at least is the intestinal canal, as shown by the highly putrid character of the stools.

On this account, it is of the utmost importance to give attention to the alimentary canal and to suppress foodstuffs calculated to promote intestinal putrefactions.

Persons suffering with pernicious anemia often present symptoms suggesting scurvy. Changes in the nervous system due to degenerative changes of the spinal cord and degenerations of the gastro-intestinal tract are also common, complete absence of the gastric juice being a very constant symptom. This has led Doctors Koessler, Maurer and Loughlin to the conclusion that:

"1. The blood changes and the changes in the gastro-intestinal tract may be due to vitamin A underfeeding over a long period of years.

"2. The nervous symptoms might be related to an absence or deficient quantity of vitamin B.

"3. The tendency to hemorrhages found in severe anemias may be due to the partial or complete lack of vitamin C."

These authors made an extended series of experiments upon rats with dietaries arranged to test the effects of a deficiency of each of the vitamins, A, B and C. They found that the administration of vitamin $\mathrm{A}$ to an animal in a condition resembling that of pernicious anemia, was followed by a rapid formation of new, red blood cells.

The final conclusions reached by these observers are as follows : 
"1. Blood regeneration cannot take place without the presence of vitamin $\mathrm{A}$.

"2. The addition of vitamin A to the diet of animals, long depleted in their vitamin A reserve, brings about rapid formation of new blood cells."

The failure of the gastric glands to produce hydrochloric acid, a condition present in most cases of pernicious anemia, deprives the intestinal tract of the normal disinfectant action of the gastric juice. In such cases, putrefactive bacteria taken with the food are not destroyed in the stomach but are left to grow and thrive in the intestine, especially in the colon, exposing the body to the pernicious influence of a great variety of putrefaction products. Some of these products destroy the red blood cells when brought into contact with them, a fact which was believed by Herter to explain the disappearance of the red blood cells in pernicious anemia.

Fortunately greens, which are absolutely essential in the feeding of anemic patients, are among the most laxative of foodstuffs. These must be freely used and also bran, which should be eaten to the extent of one ounce daily or at least a large tablespoonful at each meal.

Bran may also be added to potato and other purées and thick soups of all sorts or it may be eaten with bread and milk. It is necessary to insist that it should be taken at every meal.

Fortunately, bran is also rich in iron. An ounce of bran contains 0.034 grains of iron, and the daily dose of one ounce of bran would thus contribute a.sufficient amount of iron to lessen the required intake of spinach by two ounces.

By reference to Table XXIX it will be seen that some other greens, particularly the narrow leaved dock and red root, contain double the amount of iron found in spinach.

The apparent benefit often secured by the use of arsenical preparations, such as cacodylate of soda, has led to the widespread use of these remedies to the neglect of proper feeding. That this practice is a great error, however, has been pointed out by Chaippori (Riforma Medica), who found that in the administration of arsenic in cases of anemia there is an increase of red corpuscles, but no increase of white cells. In one case the red cells were found to be increased one million within an hour after injection. The increase in the red cells was found to be due to 
the throwing into the circulation of a great number of young and immature red cells. These young cells possessed so little resistance that they were quickly destroyed and eliminated, as shown by an increase of urobilin in the urine. Poggi confirmed these observations, which clearly show the futility of depending upon arsenic to cure anemia. The taking of more food iron in the food, relief of autointoxication and the proper use of exercise, phototherapy, and cold frictions with other forms of hydrotherapy, are the most efficient means of combating anemia.

Drugs cannot be relied upon to restore the blood or any other wasted tissue. The use of ox blood and preparations of blood in severe anemia, once so common, has no scientific foundation.

If blood iron were readily available for replenishing the wasting iron losses of the body, carnivorous animals would never suffer from anemia and vegetable-eating animals would be especially subject to blood impoverishment; but the very opposite is the case. Carnivorous animals are more subject to anemia than are herbivorous or grass-eating animals (Sherman).

Pernicious anemia is rare in parts of the world where less cane sugar, muscle meats and dairy products are used, than in those parts of Europe and America, where the disease is common (Minot and Murphy).

The fat should be reduced because it encourages putrefaction in the intestine and because of the possible harm from the effect of lipoids, as shown by Baker and Carrell.

If the iron of blood and flesh were a superior source of iron for replenishing the iron losses of the body, such losses would not occur except as the result of hemorrhage from accident or other cause. This is evident from the fact that the waste iron is excreted through the intestine.

There can be no doubt that the iron of plants is the natural and the best source of iron for blood making. Hence, in anemia, such vegetable foods should be selected as will supply the largest amount of iron. Fortunately, the number of such foods is ample for needed variety. More than a score of different vegetable foods supply more iron than dots average beefsteak, as will be seen by reference to Table XII.

Pure gluten contains fourteen times as much available iron as does average beefsteak; 40 per cent. gluten six times as much; egg yolk and lentils nearly four times; and molasses, wheat bran, yellow dock greens and 20 per cent gluten, an average of three times 
as much. A long list of foods furnish twice as much or more iron than does moderately fat beefsteak, of which may be mentioned soy beans, peas, protose, whole wheat, malt sugar, malted nuts, mustard greens, brose and hazelnuts. The iron content of beefsteak is exceeded by that of all the whole grain cereals, cooked whole wheat, oatmeal, cracked wheat, wheat flakes, shredded wheat, etc., dates, figs, prunes and olives, and by nearly all the nuts and nut products.

Recent investigations show that for rebuilding the red blood cells something more than iron is needed. In hemoglobin the iron is associated with hematoporphyrin. This element must be supplied along with the iron and perhaps also other factors derived from plants and associated with chlorophyll. Iron preparations containing extracts of greens have been found most difficult in promoting blood regeneration in anemia.

Keeping these facts in mind, it is easy to arrange a bill of fare containing an abundance of food iron in most available form. In general, the plan for the bill of fare must be as follows. For cane sugar, which contains no iron, substitute malt sugar and such sweet fruits as dates, figs, Zante currants and raisins, molasses and maple syrup. Discard white bread, polished rice, rice flakes, hominy, corn flour and all bolted cereals, and use only such whole grain products as oatmeal, cracked, shredded, or puffed wheat, wheat flakes, brown rice, graham bread, Boston brown bread, rye bread, bran and bran preparations. Make a liberal use of lentils, dried peas, navy and lima beans, and soy beans. Make greens a staple article of food, serving at least twice a day a liberal portion. Substitute nuts or nut products for meats of all sorts. Use egg yolks in moderation. Malted nuts may be in part substituted for milk because milk is deficient in iron, or altogether in cases in which the patient is sensitized to dairy products.

Fresh fruits, especially citrus fruits, and fresh vegetables, particularly tomatoes and carrots should be freely used to insure an ample supply of vitamins, a deficiency of which is known to be a cause of anemia. It is well to use tomatoes in some form every day.

Says the eminent Von Noorden respecting the feeding of children, and the same principles apply with equal force to the feeding of adults: 
The necessity of a generous supply of vegetables and fruits must be particularly emphasized. They are of the greatest importance for the normal development of the body and of all its functions. As far as children are concerned, we believe we could do better by following the dietary of the most rigid vegetarians than by feeding the children as though they were carnivora, according to the bad custom which is still quite prevalent.

Transfusion with animal blood was once in vogue, but was soon abandoned. Good results follow the transfusion of human blood of the right type. This method is now much more often used than formerly and often with results which seem little short of miraculous. Too often the effect is more or less transient, but the operation is a comparatively simple one and may be repeated many times if necessary.

Walinski has recently shown that by the injection of five to ten units of insulin twice a day in addition to transfusion of blood, very great improvement may be secured. The effect of insulin is to improve appetite and digestion.

Recent observations by Wearn, Warren and Ames (.4rch. Int. Med., April, 1922) show that the life of blood cells when introduced by transfusion is much longer than has been supposed. By ingenious methods they were able to prove that transfused cells survive from 59 to 113 days, an average of 86 days or more than 12 weeks. These observations confirm the increasing confidence in transfusion supported by clinical experience and suggest its use earlier and more frequently. In the case of a patient treated at the Battle Creek Sanitarium the blood was brought from 30 per cent. of the normal to 80 per cent. and at the time of this writing. a year and a half since the patient returned home, the improvement is still maintained. Of course the benefit received could not be entirely attributed to the transfusions. The patient was placed upon a diet rich in iron and thoroughly antitoxic, and the intestinal flora was changed by the method above pointed out. In another case in which the hemoglobin had reached the unusually low point of 14 per cent., the blood was restored by diet and physiotherapy to 90 per cent., and the patient after sixteen years is still in excellent health and active in business as a travelling salesman. No transfusions were employed in this case, but the patient has adhered very closely to an antitoxic (no meat) regimen. 


\section{DIET FOR ACIDOSIS}

The blood and tissue fluids are slightly alkaline in reaction. This slight degree of alkalinity is essential to the maintenance of health. When, through disordered metabolism, the body becomes charged with an excess of acids, so that the alkalinity of the blood and tissues is diminished, the result is a condition known as acidosis. Among the symptoms due to acidosis may be mentioned nervousness, mental depression, mental confusion, drowsiness, convulsions and coma. Air hunger with shortness of breath is one of the early symptoms of acidosis. This condition is most pronounced in advanced cases of diabetes and is encouraged by a meat diet, particularly by the use of an excess of fats. Fasting persons always show a condition of acidosis after fasting for a day or two. Professor Fischer believes that this preponderance of acids, or chronic acidosis, is the exciting cause of Bright's disease. It is believed that acidosis favors the development of disease of the skin. A slight degree of acidosis is manifested in loss of endurance, physical and mental inefficiency and slight shortness of breath.

When the increase of acids in the blood and tissue fluids is due to imperfect oxidation or disturbed metabolism, the condition is termed ketonic acidosis. When the condition is due to deficient elimination of acid wastes by the kidneys, as in uremia, it is known as renal acidosis.

A simple test for acidosis, suggested by Henderson, is holding the breath. One of the first symptoms of acidosis is shortness of breath and a sensation of air hunger. A normal person at rest after taking a full breath can hold the breath for 40 seconds or longer. When acidosis is present, the time for which the breath can be held is reduced to 20 seconds, one-half the normal or even less. When the breath cannot be held more than 20 seconds, the degree of acidosis is sufficient to render general anesthesia a highly dangerous procedure (Stange).

The opposite condition, known as alkalinosis, is believed to be associated with a condition known as tetany.

It is evidently important that attention should be given to the acid and alkaline constituents of the food. When food containing an excess of acids other than malic and citric acid is taken in considerable quantity and for some time the result is likely to be an over-accumulation of acids. This does not occur 
in the case of citric and malic acids for the reason that these acids are combined with soda and potash and in the body the acids are burned, leaving the alkalies behind, so that the effect of taking these acids in the combinations in which they are found in foodstuffs is to increase the alkalinity of the body fluids rather than to diminish it. Tartaric acid is probably burned to some extent in the body, but less readily than malic and citric acid; while acetic, butyric, oxalic and benzoic acids, which are also frequently found in foods, often in considerable quantity, are not burned in the body and hence are not utilized.

When food is burned, the ashes are found to contain sulphuric acid, phosphoric acid and hydrochloric acid in combination with lime, magnesia, soda and potash. In some foods these acids are preponderant. This is particularly true of meats. Milk, fruits and vegetables of all sorts contain a preponderance of the alkalies or bases. A meat diet necessarily tends to acidify the tissue fluids because of the large amount of sulphur, chlorin and phosphorous which meats contain. A diet of cereals has the same tendency although to a less degree.

Carnivorous animals appear to be equipped by Nature for dealing with these acids in a way in which non-flesh eating animals are not prepared to do. From the lean meat of their diet, they are able to produce abundantly the alkali ammonia, thus neutralizing the excess of acids derived from their diet. Human beings and other animals are not prepared to do this except to a limited extent, and hence are likely to suffer from the use of foods containing an excess of acids.

Another source of acidosis is imperfect combustion of fats. Although sugar is the current fuel of the body, more or less fat is burned daily. The chief consumption of fat occurs some hours after a meal has been taken. More fat is consumed in the early morning hours than at any other time because at this time the starch and sugar taken in the previous day's meals have been largely consumed. Exercise before breakfast or when fasting is on this account more efficient in burning up the body fat than when taken soon after eating.

That fats are less easily utilized by the body than are starches and sugars is shown by the observation that while a person may for some days without injury subsist upon a diet consisting exclusively of carbohydrates this is not true in relation to fats. On a diet consisting exclusively of fats, a state of acidosis of the 
tissue fluids very rapidly develops. In this condition $\mathrm{CO}_{2}$ is accumulating in the tissues because the blood is unable to carry it off with sufficient rapidity. As a natural consequence, the amount of carbon dioxid exhaled in the expired breath will be diminished. The state of acidosis is thus most readily shown by an examination of the expired air and a determination of the so-called carbon dioxid tension. The normal tension is about 40 . When the tension falls lower than 34 , the condition of acidosis is present.

Hasselbalch has shown that the carbon dioxid tension of a meat-eater is only 37.8 ; that of a person living on a mixed diet is but little higher, 38.3; while the tension of a non-flesh eater is 43.3. These figures are highly significant. The non-flesh eater's tension is nearly ten points above the danger line, while that of the meat-eater is less than four points; that is, the hearty meateater lives continuously in a condition bordering on acidosis, and the mixed feeder is only a little better off, whereas the fleshabstainer enjoys a wide safety margin.

Here we find the explanation of the greater endurance of the flesh-abstainer as compared with the meat-eater. Hunters have long noted that dogs do not run well when fed meat. When riding on the top of a touring coach through the Highlands of Scotland many years ago the writer, in chatting with the driver, learned that he was a shepherd and kept a number of dogs. When asked the question: "What do you feed your dogs?" the Scotchman replied: "The same as I eat meself, sir-brose, bannocks and potatoes."

"And why do you not give meat to your dogs?"

The instant reply was: "Because they hae nae so guid wind; they canna' rin',"

Vegetable-eating animals are notable for their powers of endurance, whereas the strict carnivora, lions, leopards and other members of the cat tribe, even though possessed of powerful muscles, become quickly exhausted when pursued.

Acidosis develops on a diet of fat because of the incomplete burning or oxidation of the fat.

Naunyn has shown that it is necessary for a person to eat daily at least two or three ounces of carbohydrates (200 to 300 calories), starch or sugar, to prevent acidosis. Carbohydrates in some mysterious way not fully understood seem to enable the 
tissues to utilize fats more perfectly than is possible without them. As Naunyn remarks: "Fats burn in a fire of carbohydrates." The normal diet contains 1,600 to 2,000 calories of carbohydrates or even more, while the proportion of fats is normally not more than 30 per cent. of the total food intake, although in the case of diabetics this proportion must often be considerably increased.

While reducing the protein to a minimum and eliminating fats from the diet, persons suffering from a chronic tendency to acidosis should also avoid meats and should greatly reduce the use of cereals and other foods known to have a tendency to acidify the tissue fluids. In addition, they should select for their bill of fare foodstuffs which are known to be rich in base-forming, or alkaline, elements. See table XVI.

When the stools are foul, which is generally the case, the flora must be changed at once. Protein must be reduced to a low level, not to exceed one calorie per pound of body weight, and fats must be used sparingly.

In acute acidosis, orange juice and malt sugar should be freely used. The juice of a dozen large oranges and half a pound of malt sugar may be given every twenty-four hours, or even more.

The relative excess of acid or of base-forming elements found in some common foodstuffs is shown in table XVI.

Because the tissues are constantly forming acid products which must be removed by the kidneys, lungs and skin, there is always a tendency to acidosis. Collazo and Morelli have recently shown that the blood of man contains on an average about 10 to $20 \mathrm{mg}$. (1/7 to $1 / 3 \mathrm{grs}$ ) per 100 c. c. (3 1/3 ounces) of blood. The blood of swine contains two to five times as much. Evidently the hog is in a state of chronic acidosis.

Lactic acid is always being formed in the body. Everv heart beat, every muscular movement, generates lactic acid. During violent exercise, the acid may be generated at the rate of one dram every second.

Most chronic invalids and especially persons suffering from high blood pressure, are in a state of chronic acidosis or what might be termed subacidosis. It is not uncommon to find the urine of such persons fifty to one hundred times the normal acidity. The elimination of meat (fish, flesh and fowl) and eggs 
from the dietary, and the adoption of a strictly basic dietary is highly essential in such cases and often such a change is folle wed by a notable reduction of the urinary acidity and, of course, a corresponding increase of the alkali reserve of the blood and tissue fluids.

A vegetable diet tends to combat acidosis. The urine of carnivorous animals is highly acid while that of herbivorous animals is alkaline.

\section{Infantile Acidosis.}

The acidosis of infants usually results from overfeeding. The condition is indicated in young infants by convulsions and symptoms of rickets; in other children, by cyclic vomiting, headaches and "biliousness."

In the case of a young child, purées of vegetables, especially potato, tomato juice and orange juice, should be added to the diet and the amount of food should be diminished, particularly fats. Change the intestinal flora (see Index).

In the case of older children, cane sugar should be avoided in all forms, including candy. Malt sugar may be allowed in moderate quantities. Butter and fats should be used sparingly. Meats should be avoided entirely, also animal broths. Potatoes should be largely used in place of bread. Greens should be made a part of the daily diet. A liberal serving of spinach should be eaten at least once a day. In young infants give spinach in purée. Spinach should always be parboiled.

The colon should be kept empty by the free use of bran or agar and paraffin oil. It is well to empty the colon by an enema at $110^{\circ} \mathrm{F}$. at least once a day for several days.

\section{Post-Operative Acidosis.}

Acidosis always occurs in general anesthesia no matter how induced. Dangerous acidosis is most common after chloroform anesthesia, but may occur after ether or nitrous oxide. Prophylaxis is most important in these cases. Instead of being deprived of all food, as is usual, the patient should be given malt sugar freely during the twelve hours just before the operation. Eight to sixteen ounces of sugar, well diluted, should be taken during this time. A little lemon or other fruit juice may be added, to suit the patient's taste. Immediately following the 
operation, malt sugar should be administered per rectum. Half a pint of water containing an ounce of malt sugar should be introduced into the rectum every hour. The introduction should be made slowly, if necessary employing the drop method to insure absorption. As soon as the nausea has disappeared, malt sugar may be administered by mouth, dissolved in water or diluted fruit juice. In severe cases, duodenal feeding (see Index) with sugar water should be resorted to.

Surgical acidosis is now preventable by the intravenous injection of dextrose during the operation. This method is in regular use in the surgical department of the Battle Creek Sanitarium and has given excellent satisfaction.

\section{SPRUE}

This form of intestinal toxemia is apparently simply an advanced stage of tropical diarrhea. The disease generally originates in tropical countries, but after it has once become well developed may continue in spite of removal to a different climate.

The first dietetic indication is to secure a change of the intestinal flora. In addition, it is highly important to include in the dietary an abundant supply of vitamins and iron and lime with the other organic salts associated with them. These indications are best met by a diet consisting of milk and fruit.

At the beginning, milk should be given warm and in small quantities, not more than half a pint, at intervals of two hours. The first day the quantity should be not more than two or three pints. The amount should be gradually increased to seven or eight pints, when the patient will usually begin to show a gain in weight.

Strawberries have been the most highly recommended of any fruit, but in cases under the writer's observation other fruits have appeared to be quite satisfactory. One or two pints of strawberries should be eaten daily, or three to six bananas, or bananas and strawberries may be used together. The fruit should be thoroughly ripe.

When fit for use, the skin of the banana is brown. Bananas should be eaten only in the form of purée, produced by removing the coarse fiber and mashing or putting through a colander. It is a good plan to mix the banana with a portion of milk or cream and beat with an egg beater. 
Lacto-dextrin may be used with advantage both by mouth as well as by enema. Two or three ounces (smaller doses at first) should be administered three or four times a day. It should be dissolved in a glassful of hot water to which a glassful of milk should be added. The use of lacto-dextrin in large doses should be continued until the stools lose their putrid odor. The dose may then be reduced to one or two ounces three times a day and continued indefinitely.

According to Thim, sprue is due to a poison which develops in certain media, particularly in such a medium as is supplied by flesh foods, but appears to be unable to develop in milk or the juices of fruits. He explains in this way the great benefit derived from a diet of milk and strawberries or other fruit.

Yogurt buttermilk, and particularly milk soured with $B_{\text {. }}$ acidophilus, greatly facilitates the process of changing the intestinal flora. Purées of spinach and other greens to the extent of six to eight ounces daily may be used with advantage for the purpose of furnishing iron, which is deficient in milk and is found in only comparative small proportions in most fruits. The superior value of the strawberry in these cases may be in part due to the fact that it is richer than other fruits in iron.

Hot cornstarch enemas should be used twice daily to thoroughly cleanse the colon and hasten the change of flora. An ounce or two of milk sugar or lacto-dextrin may be added to the enema with advantage to still further encourage the development of $B$. acidophilus in the colon. Enemas of whey cultures of $B$. acidophilus or lacto-dextrin are also highly useful.

Brown (Am. Jour. Med. Sci.) reports a number of cases of sprue treated successfully with five to ten grains of pancreatic extract three times a day. The extract is given two hours after meals in connection with 20 grains of bicarbonate of soda. This method of treatment was adopted because of the finding of the complete absence of pancreatic ferments in the duodenal contents. Patients remained well so long as they continued the use of the pancreatic extract. The diet consisted of buttermilk, bread and milk, fruit purées and fruit juices, an excellent antitoxic diet.

Canned fruit may be used in place of fresh fruit if the latter is not obtainable. Apples may be used instead of strawberries. 


\section{Pellagra.}

This disease in some respects resembles sprue. Its nature is not yet fully understood, but the many researches which have been made indicate that it is a general failure of nutrition with lowered vital resistance, associated with intense intestinal toxemia. The dietetic management of this disease should be essentially the same as that indicated for sprue.

Dr. Joseph Goldberger, who has for many years given special attention to the study of the causes of pellagra, in a recent research conducted by himself and Dr. Tanner under the auspices of the U. S. Public Health Service, has apparently demonstrated that pellagra is the result of the long-continued use of a food deficient in protein of high quality. They found, for example, that in one case in which the patient was supplied with an abundance of vitamins, salts and every other necessary food element and food accessory, including milk, pellagra appeared when the milk was reduced in quantity and disappeared when the milk was increased to one and one-half pints, the amount formerly used. Hindhede maintains that this view is erroneous and offers plausible evidence of the correctness of his contention.

It is noteworthy that in Dr. Goldberger's experiments there was no evidence whatever that meat was essential to prevent the development of pellagra. An increase in the amount of milk taken was the only thing found necessary to cause a disappearance of the symptoms characteristic of this disease. Milk protein is of the highest quality. The protein of nuts is of equal value.

The writer has observed recently several cases of pronounced pellagra which have been apparently cured by the use of "606," in connection with a fruit and milk regimen and change of the intestinal flora. At present, this method seems to offer to pellagra patients the best hope for a cure.

\section{HODGKIN'S DISEASE}

The patient is subjected to the influence of toxins continually absorbed from the seat of the disease, and hence the greatest care should be taken to reduce the alimentary toxins to a minimum. This may be accomplished by first changing the intestinal flora, by methods elsewhere described (see Index), and then adhering closely to the antitoxic and laxative diet. 
Anemia and demineralization should be combated by the free use of greens and other foodstuffs rich in iron.

\section{HEMOPHILIA}

The diet should aim to enrich the blood in lime. A milk regimen is especially indicated. Three or four quarts of milk a day, half a pound of spinach, and an ounce and a half of bran may with benefit constitute the regimen for one to three weeks. The most efficient remedy is a transfusion of blood, the effect of which is immediate but, unfortunately, not permanent.

\section{DIET IN SCURVY}

Scurvy has been long recognized, and an efficient mode of treatment has been known, but only recently has the disease come to be well understood. It is now known that natural foods contain a subtle element, the antiscorbutic vitamin (p. 186), the absence of which leads to the terrible consequences sometimes seen in persons deprived of foods containing this vitamin in sufficient amount. This vitamin is, of all the vitamins, the most easily destroyed. Ordinary cooking destroys it, as does also drying and other means of preservation. It has recently been shown that quick drying and drying in the absence of air, prevents the destruction of the antiscorbutic vitamin by preventing oxidation.

Observations by Plimmer (Biochemical Journal) have recently demonstrated that pigs are much subject to scurvy, which is shown by stoppage of growth, lack of appetite, swollen joints and other symptoms observed in scorbutic animals. This disease is the result of using cooked food, which in the winter time is the common food of pigs. Cooking destroys the antiscorbutic vitamin. This disease is sometimes called "pigs gout, rickets, or rheumatism."

Thousands of human beings are unwittingly suffering to a greater or less extent in a similar way as the result of the too exclusive use of cooked foods.

Preserved foods cannot be made an exclusive diet without the development of scurvy. The body stores up vitamins in the liver and other tissues, sometimes more and sometimes less. The length of time one can live on a diet lacking the scurvy-preventing vitamins without contracting the disease will depend upon 
how much of this particular vitam in he happens to have in store. A few weeks at the most will exhaust the reserve, and then the disease makes its appearance and nothing but a new supply will cause the distressing symptoms to disappear.

Umber, of Berlin, recently reported to the Berlin Medical Society several cases of scurvy, some of which had developed in a hospital as a result of feeding on long cooked porridge, the regulation hospital diet. Among the symptoms noted in these patients was the so-called jumping-jack sign, which consists in the jerking upward of the shoulder and arm when the calf of the leg is suddenly pressed upon.

The scurvy was speedily cured by the use of fresh vegetables or fresh fruits, or by a cup of pine needle tea given three times a day.

Fresh foods are the only remedy for scurvy. The fresh juices of oranges and lemons are particularly rich in the antiscorbutic vitamin. The lime has little value, as has been shown by the failure of lime juice to prevent the disease. The juice of tomatoes, even of canned tomatoes, is perhaps as good as orange juice. Several ounces a day of these juices or their equivalent are required to prevent or cure scurvy.

Turnip juice, cabbage, lettuce, all green and uncooked vegetables, are of proven value. The potato is strongly antiscorbutic, even when cooked, as well as the tomato.

Skimmed milk (unboiled), whey, cottage cheese and buttermilk, are good antiscorbutics. Pasteurized milk is better than boiled milk but is inferior to fresh, raw milk. Almost any sort of green stuff will serve in emergency, as alfalfa or clover leaves or even grass. The fact that gardens were little cultivated in the Middle Ages and green vegetables on this account little used doubtless explains in large part at least the great prevalence of scurvy during that period.

The idea that meat is essential as a preventive of scurvy is an error. In the famous siege of Kut-el-amara in Mesopotamia, during the World War, scurvy was very prevalent among the British soldiers, whose rations consisted of white bread or biscuit and tinned meats, but long unknown among the Indian soldiers, who ate no meat but added to their wheat bread a legume known as dhal. The East Indians finally succumbed to scurvy through starvation, but were cured by three ounces daily, per man, of green herbs collected from the plains. The use of 
yeast extract was found of great value in preventing and curing beri-beri (Brit. Med. Jour.).

Recent studies of the antiscorbutic properties of various foodstuffs have shown that cows' milk, even when fresh and neither pasteurized nor sterilized, possesses rather feeble antiscorbutic properties, notably less than mothers' milk. This is probably due to the fact that the calf, being born with teeth, when a few days old begins nibbling at tender green shoots of grass and herbs and thus begins to supply itself with the important antiscorbutic vitamin which is found in all green leaves as well as in the juices of most acid fruits and fresh vegetables.

Another fact of recent discovery is that the antiscorbutic properties of cows' milk vary greatly with the food of the cow. In winter when the cows are fed chiefly upon hay, oil cake and other dry foodstuffs the antiscorbutic properties of the milk are very greatly diminished, and bottle-fed infants using the milk of such cows are likely to develop scurvy even though perfectly fresh, certified milk may be used. Orange juice or tomato juice is just as necessary in such cases as when sterilized milk is employed. When cows are fed on swede turnips the milk is richer in the antiscorbutic vitamin, which is found in abundance in swede turnips although not in the rutabaga. Silage is lacking in the antiscorbutic vitamin, which is destroyed by fermentation. As a means of safety, all infants fed on cow's milk should be given an ounce or two of orange or tomato juice, daily, so long as cow's milk or milk and cereals remain their chief diet.

Scurvy in partially developed forms generally known as malnutrition is one of the most common of diseases. Thousands of puny, weazened and short-lived infants and perhaps millions of ailing and inefficient adults are victims of this nutritional disorder, to correct which is one of the great and worth-while tasks set before the rising generations of dietitians.

Greens and salads are of great use in the prevention of scurvy as well as other deficiency diseases. The following cultivated plants make good greens : radish tops, turnip tops, beet tops, Swiss chard, kale, Chinese cabbage leaves, upland cress, French endive, cabbage sprouts, asparagus, New Zealand spinach, dandelion, native mustard and horse radish leaves, Russian mustard, collards, rape, tender tips of alfalfa, lettuce. In addition to the above cultivated greens the following uncultivated ones are good to eat and some of them are superior to most of the cultivated 
varieties of greens; wild dandelion, wild lettuce, sour dock, lamb's quarter or pig weed, wild mustard, pepper cress, smartweed sprouts, purslane, marsh marigold, tender sprouts and young leaves of milkweed, young thistles.

It was shown by Fürst that the antiscorbutic, or scurvypreventing vitamin, is produced in the germination of plants. Fortunately, this vitamin is not destroyed by heating to a temperature of $158^{\circ} \mathrm{F}$., which is necessary to gelatinize starch. For this reason non-alcoholic malt extracts are useful in the scurvy of infants. Sprouted grain or soy beans may be used as salads.

Rickets occurs with great frequency in all regions in both human beings and animals in regions in which there is a deficiency of sunlight. On this account, it is widely prevalent in the lake region. In these diseases there is a disturbance of the calcium metabolism. This form of metabolic activity is regulated by vitamin $\mathrm{D}$, the lack of which in the food may be the cause of rickets. The worst results are to be seen where there is both a deficiency of vitamin D and the sunlight. Sunlight, acting upon certain elements of the food, particularly cholesterol, produces changes in the food which enables it to prevent the occurrence of rickets, or to effect a cure of rickets, when this exists.

Experience has shown that in the feeding of children suffering from rickets, it is necessary not only to supply food rich in vitamin D, such as butter, cod liver oil and purées of greens, but also to see that the intake of salts, particularly lime and phosphorus, are properly balanced.

Daily exposure to the rays of the sun is also highly important. When this is not possible, treatment by the arc light is highly important. Rickets rarely occurs in children under six months of age. It is most likely to be developed in badly fed infants. It may be easily prevented by keeping the infant as much as possible in the open air and exposure to sunlight daily, first, for a few minutes, and then for a longer period, as the skin becomes accustomed to contact with light. The free use of purées, of greens, and of other fresh vegetables, beginning with the sixth or seventh month, is highly useful as a preventive measure, 


\section{PURPURA}

The diet should be the same as in pernicious anemia, with special attention to antiscorbutic foods.

A pint to a pint and a half of orange juice daily, one or two pounds of fresh tomatoes, the juice of half a dozen turnips, potato soup, sprouted barley or sprouted soy beans with lettuce and other salads are especially helpful food remedies.

\section{SEPTICEIMIA}

The patient should take an abundant supply of water, at least three to four quarts daily. Malt sugar or raisin water should be freely used. A good plan is to add to each pint of water administered two ounces of malt sugar or the juice of four ounces of raisins. A glassful of water should be given at intervals of every half hour during the day. If the patient cannot take the full amount of water by the stomach, it should be given per rectum in such a manner as to secure absorption.

Purées of greens should be used liberally to maintain the blood in a condition to combat the invading germs. Meats of all sorts should be discarded. The fever diet should be carefully followed until convalescence, after which the antitoxic and laxative diet will be indicated.

\section{OSTEOIMALACIA}

This disease requires a reconstructive regimen. Milk, whey, buttermilk, greens of all sorts and other foodstuffs rich in lime are especially indicated. Feeding alone will not effect a cure in this disease but it will help to make good the deficiency resulting from the extraordinary loss of minerals which usually continues in spite of all measures of treatment. The administration of adrenalin seems to have a favorable influence upon the course of the disease and castration has been suggested as a remedy. As the stools are usually putrid, change of the intestinal flora by the use of lactose or lacto-dextrin is important. Care should be taken that the carbohydrate feeding does not lessen the intake of foodstuffs rich in lime.

\section{ANOREXIA}

Ryle has shown that in anorexia there may be either diminished tonus or rigidity of the stomach, as in a leather bottle stomach. Mild anorexia may be due to severe fatigue. Fatigue 
affects the gastric muscle as well as other muscular structures, causing loss of tone. Loss of appetite may occur from worry, which lowers the gastric tone through the sympathetic nervous system. It may be due to lack of sleep or deficient rest. A common cause of loss of appetite is autointoxication, as in neurasthenia. It is also caused by the toxins which cause lowered muscular tone in fevers. Mental disease, chronic alcoholism and cancer, either of the stomach or any other part of the body, are common causes of loss of appetite. Loss of appetite is most conspicuous in the morning. This may be due to the diminished activity of the lungs, liver, kidneys and various poison eliminating and destroying organs during sleep and the absorption of poisons from the colon over night.

The absence of appetite is one of the most constant effects of profound toxemia. When the intestine is loaded with putrefying material, the products of putrefaction are absorbed into the blood in great quantities and produce profound disturbance of all the nutritive functions. The thirst center as well as the hunger center is often depressed to such an extent by the bacterial toxins circulating in the blood that the appetite for water as well as for solid food is almost wholly lacking. The tongue is coated, there is a bad taste in the mouth, and the very thought of food is often nauseating. There is complete perversion of the gustatory sense in certain cases, and lack of appetite even when the tongue is clean; but in these cases, also, stasis in either the large or small intestine is rarely absent.

McCarrison has shown that lack of vitamins may be a cause of loss of appetite and that appetite may be greatly stimulated by the free use of vitamin B.

In the majority of cases, the X-ray will show incompetency of the ileocecal valve, as the result of which there is a reflux of putrid material into the small intestine and absorption of toxins into the general circulation.

The first thing to be done, of course, is to remove the cause by changing the intestinal flora. In the meantime, even though the patient may be weak and emaciated and greatly in need of food, it would be useless and even highly detrimental to urge the taking of rich or hearty foodstuffs. Even milk often aggravates 
the situation rather than relieving it. Foods rich in protein or fats are least suitable, and are often most objectionable to the patient. Fruits and fruit juices (especially orange juice) with liberal amounts of malt sugar or gruels mixed with fruit juices, with lettuce and other fresh, raw vegetables are especially useful. Fruit jellies prepared with vegetable gelatin are usually very acceptable to the patient. These foods should be given in liberal amounts and at intervals of two or three hours. The fruit regimen may be employed for two or three days with great advantage (Index). A large enema (three or four pints) should be administered twice a day, care being taken to empty the colon completely. To accomplish this the juice of one or two lemons should be added to the enema or in place one or two drams of citric or lactic acid. Liberal amounts of bran should be given if possible. It may be added to the vegetable jellies. Paraffin oil in some form also is needed in liberal amounts.

In the majority of cases, the patient will by the second or third day under this dietetic regimen acquire a natural appetite. Not infrequently the appetite becomes so keen that the simplest food is taken with zest. If the patient is much emaciated, it is well to administer in the intervals between meals eight to ten ounces of milk sugar daily. The sugar should be taken dissolved in water or fruit juice. Three ounces of milk sugar or lacto-dextrin three times a day with the fruit regimen is the most effective means of changing the intestinal flora.

When the tongue clears off readily after two to four days of the fruit regimen the patient, if very thin in flesh, should be put upon the milk regimen for two to four weeks (see index).

In the application of the milk regimen and the fruit regimen, the greatest care must be exercised to observe every detail indicated elsewhere (see index).

In cases in which the appetite still remains deficient and fickle after the application of the above measures, the patient must be encouraged to eat liberal quantities of food, even though appetite is lacking. This may best be accomplished by administering the food in very simple, rather concentrated form, in small quantities at frequent intervals. Fats and proteins should be eaten sparingly, the diet consisting at first chiefly of carbohydrates.

Carbohydrates, especially in the form of fruit juices, with or without the addition of malt sugar, are readily taken when foods 
rich in fats and proteins are not relished. It is highly necessary that the protein constituent of the food should be reduced to a minimum. By so doing, the production of toxins in the intestine is lessened and the liver and other excretory organs are relieved of the task of eliminating unnecessary and unusable protein.

Fats should be taken in the form of emulsion as cream or butter or cream mixed with an equal or greater amount of Malt Honey. This combination is very readily accepted by stomachs unable to take fat in any other form and is highly palatable.

By reference to the table of food values, it will be noticed that many fruits have a caloric value considerable in amount; and by the addition of malt sugar, with or without cream, the caloric value can be greatly increased, more than doubled in most cases.

The application of an ice bag over the stomach for half an hour before eating will often induce an appetite. Continuous living out-of-doors day and night, a cold bath morning and night, and a moist abdominal bandage, are other measures which greatly encourage appetite. Care must be taken to keep the colon empty by employing the enema twice a day if necessary, and by the free use of laxative foods and other laxative means, and the intestinal flora must be changed and a good flora maintained.

\section{PICA}

Recent observations indicate that a deficiency of vitamins may be the cause of a form of perverted appetite commonly known as pica, which leads to the eating of chalk, clay, hair, sand, coal. paint, cloth and various other indigestible and unwholesome substances.

These perversions of appetite are indicative of a disturbance of nutrition. The absence of an adequate supply of vegetable salts and vitamins in the diet is a cause which may be easily avoided by the free use of purées of fresh vegetables, spinach and other greens, of whole grain preparations. Free exposure of young children to the sun's rays will aid in the prevention of these normal tendencies. 


\section{Diet in Joint Diseases}

\section{CHRONIC RHEUMATISM}

Within the last few years, since it has become known that infections of the teeth and tonsils are often associated with chronic inflammation of the joints, so-called chronic rheumatism, it has become a fashion to attribute all cases of this disease to mouth infections. This is an error. There are many rheumatics who still suffer from painful joints after having not only their tonsils but all their teeth removed. It is becoming more and more evident that in a considerable proportion of cases the real seat of the disease is in the colon. Infections of the colon such as are always present in colitis, at least in incipient form, whenever the stools are putrid or foul-smelling, may be found to be the chief source of chronic rheumatic affections of various forms.

In the treatment of this disease, the colon should always receive attention, even when the teeth and tonsils are found diseased. The diet should be so regulated that the stools lose their foul odor and the bowels move three or four times daily. It is generally necessary to empty the colon by means of the enema at least once a day, even when using agar or bran, paraffin oil, fresh fruits and vegetables and various other dietetic means by which peristalsis may be encouraged.

It is clear that the indications in rheumatism are for a strictly antitoxic diet and the free use of laxative foods after the intestinal flora has been changed by the use of lactose or lacto-dextrin.

The popular notion that acid fruits should be avoided in rheumatism is wholly an error, for the reason that fruit acids are oxidizable in the body like sugar and behave like carbohydrates, not as mineral acids. In all foods in which they occur they are found in combination with the organic bases, soda and potash. When eaten the acids are burned or oxidized, setting free the bases, so while the acid has a sour flavor and produces acid effects in the mouth and stomach, when absorbed into the blood the acid disappears, leaving behind the soda and potash which serve a useful purpose in rheumatism by neutralizing the acid products of tissue wastes which have a tendency to accumulate in this 
disease. Melons, and especially the muskmelon, are especially valuable in rheumatism because they tend strongly to alkalinize the blood and tissue fluids. The cataloup is in this respect more efficient than any other fruit. Potato soup or purée should appear often on the bill of fare of the rheumatic patient on account of its rich store of bases which alkalinize the tissue fluids, and potatoes and root vegetables should largely take the place of bread and cereals.

Pemberton has shown that a temporary reduction in the food intake produces brilliant results in the treatment of this disease. The food should be reduced to the base ration, or about two-thirds the usual amount of food eaten. In some cases a still further reduction is necessary. Pemberton has also shown that in this disease there is a marked increase of creatinin, which is a clear indication for the elimination of meats of all sorts. It is, of course, to be understood that these dietary restrictions are not to be relied upon as the only therapeutic measure but are to be used in connection with hydrotherapy, especially local applications and sweating baths, sun baths, the arc light, hot applications to the affected joints and other rational measures.

Recently cases of chronic rheumatism have been reported in which the disease had resisted treatment by the most approved methods without benefit and which were either cured or greatly relieved by injections of milk. The milk is sterilized by boiling. The amount used is from a gram to a gram and a half the first dose. The dose is increased from one-fourth to one-third at each successive treatment. The treatment is repeated once in three to seven days. A reaction consisting of chill, fever, headache, nausea and vomiting is present in about half the cases. The more severe the reaction the better the results are said to be.

\section{OSTEOARTHRITIS-RHEUMATOID ARTHRITIS}

Dr. Helen Baldwin, who made in Herter's laboratory in 1904 a study of 21 cases of rheumatoid arthritis, found excessive intestinal putrefaction constantly present in cases in which the disease was active. There were also found in the urine abnormal organic acids which had evidently escaped oxidation in the body.

This disease is without doubt, in many cases, the result of intestinal toxemia and other forms of autointoxication. On this account, the diet should be strictly antitoxic. The bowels should be made to move very freely and in bad cases should be completely 
emptied by enema once a day. Changing the intestinal flora by means of the fruit regimen and by the introduction of the $B$. acidophilus into the colon, as well as taking the culture by mouth are measures which have shown themselves to be of value in dealing with many cases of this disease.

\section{ACUTE POLYARTHRITIS}

This disease is now generally thought to be due to focal infection. The source of infection may be diseased tonsils, the teeth, an infected appendix or gallbladder, a chronic infection of the cervix uteri, gonorrheal infection, or an infection of the colon (colitis). The last named is probably the most common source of infections of this sort.

The modern treatment of this condition consists of rest in bed, the-application of plaster casts to secure complete rest of the joints, fomentations and heating compresses to the joints. After the most acute stage has passed, the application of the arc light, the hot and cold pour to the joints, and the general electric light bath are highly useful.

Most important of all is regulation of the diet, which in general should be like that of other febrile conditions (see Fever). The flora should be changed by the fruit regimen combined with lactose or dextrin feeding, three ounces three times a day for a week or two, until the stools show B. acidophilus dominant. Greens, fresh fruit, and fresh vegetables should be freely used to supply lime, iron and vitamins. Cereals should be used sparingly. Potatoes should largely replace bread. The bowels should be emptied at night by enema, even though there may be one or two natural movements.

\section{Diet in Acute Rhermatism.}

Acute rheumatism has in recent years been proved to be due to bacterial infection. In general, it may be regarded as a streptococcic infection with which the serous membranes are particularly affected. A peculiar characteristic of this disease which must receive special consideration in the arrangement of the dietary is the highly acid state of the excretions due to the production of an extraordinary amount of acids which are eliminated by the kidneys and the skin. During the first stage of this disease in addition to giving water in liberal quantities, fruit juices and thin 
gruels should be very freely used. Potato soup made with very little seasoning, flavored with yeast extract, is very acceptable. Potato and other vegetable purées should be used largely in preference to cereals because of their large content of alkaline salts which are useful in neutralizing the excess of acids present. Purées of fruits and vegetables with a moderate amount of cream may be given in large amounts as the fever diminishes.

Care must be taken to keep the colon free from putrefying residues. An enema should be given twice daily and when the temperature is high more frequent enemas are useful. Irritation of the rectum may be avoided by taking care to lubricate the enema tube with carbolated vaseline. Lactose or lacto-dextrin should be used in quantities of 4 to 8 ounces daily as a concentrated form of carbohydrate which furnishes a liberal supply of calories while at the same time promoting the development of a normal flora.

\section{DIET IN SO-CALLED URIC ACID DISORDERS}

The modern refined methods of chemical examination of the blood has led to the discovery of the fact that uric acid is not infrequently present in the blood in excessive amount. It has also been established that this excess is one of the earliest indications of renal failure. This fact is now recognized as of great diagnostic value. It is equally important as a dietetic indication. When the kidneys are no longer able to keep the blood normally free of uric acid, the fact shows that the process of renal degeneracy has reached a pretty advanced stage. The margin of safety which Nature provides for emergencies and to draw upon in advancing age has been used up. The kidneys are old, in other words, and Father Time is preparing to execute judgment.

In the condition commonly known as gout there seems to be in addition to an increased production or diminished elimination of uric acid, or a combination of these two factors, another element which leads to the deposit of uric acid at certain points. It was observed by Ebstein that the deposits of uric acid were always preceded by a destructive process which prepared the way by changes in the circulation. Other authors regard the deposit of uric acid to be the result of obscure nervous disturbances. Guelpa, of Paris, has recently insisted that the acute attacks to which gouty patients are subject are a remedial effort set up by the 
body for the purpose of eliminating the poison. Haig many years ago put forward the same idea. It must be admitted that the real cause of the characteristic features of gout aside from the accumulation of uric acid are at present only imperfectly understood. There may be a marked retention of uric acid even when the non-protein nitrogen is not in excess.

While it is more than probable that many disorders for which uric acid is not responsible have been erroneously charged to its account, it nevertheless cannot be denied that uric acid is a highly toxic excretory product and that it is certainly associated with gout. Since it is now known that the human liver is not able to convert uric acid into urea or some allied non-toxic substance, it is clearly evident that one who wishes to live long and preserve his liver and kidneys intact to a good old age must eliminate uric acid from his dietary as fully as possible. There is no doubt of the truth of the statement by Tibbles that "The persistent consumption of foods containing a large percentage of purin bodies is apt [certain] to lead to their accumulation in the organism."

\section{Gout.}

Much less importance is in recent years attached to uric acid as a cause of specific disease than in former times. However, it is perfectly well known that the output of uric acid in the urine is diminished in gout, while uric acid is found in the blood in increased amount. The association of uric acid with gout is unquestionable; hence it is clear that foods rich in uric acid should be avoided in this disease. This requires exclusion of meats of all kinds and animal extracts and broths of every description. Foods rich in oxalates or oxalate of lime should likewise be forbidden. Cereals should also be restricted because they leave behind in the tissues a preponderance of acids which lessen the alkalinity of the blood and tissue fluids and so diminish the solubility of uric acid and encourage its accumulation in the tissues. The free use of potatoes and other root vegetables is indicated because of the fact, demonstrated by Hindhede, that the rich store of sodium and potash salts found in these vegetables increases the solubility of uric acid and helps to remove it from the body. With an ordinary mixed diet, including meat, the urine was found to be completely saturated with uric acid and would not take up even a small additional quantity, whereas 
when potatoes were freely used the urine was able to dissolve considerable additional quantities of uric acid.

It is generally recognized that gout is little known among people who live chiefly on vegetable foodstuffs. It is essentially a meat-eater's disease. For this reason gout, while common among city dwellers in England is little known among the Scotch and is also uncommon among the people of southern Europe. Gout is unquestionably one of the consequences of chronic intestinal toxemia. The kidneys become worn out by the demand made upon them for the elimination of toxins, and the result is accumulation of uric acid in the blood and tissues. The toxins produced by the putrefaction of meat residues in the colon excite cell activity, especially in the endocrine glands and so increase the production of uric acid. One of the first things to be done in this disease is the suppression of colonic putrefaction by the exclusion of meats (fish, flesh, fowl) and change of the intestinal flora (Index). It is the writer's belief that the putrefaction of meat residues is a much larger factor in the production of gout than the uric acid found in meats.

To avoid uric acid, it is practically necessary to do nothing more than to exclude meats from the dietary, for the amount of uric acid found in beans and other vegetable foods is too small to be of any significance. For example, beans contain 4 grains of uric acid to the pound. A pound of baked beans containing one grain of uric acid is practically equivalent in food value to a full pound of meat containing 14 grains of uric acid in the case of beefsteak, or 70 grains in the case of sweetbreads.

Meat extracts, bouillon and animal broths of all sorts must be rigorously excluded from the dietary. We are glad to find in a recent work on dietetics* the following lucid statement, which is thoroughly in accord with the known facts of physiologic chemistry :

Soups made with meat stock may be labeled "poison" for gouty people, containing as they do such a high percentage of extractives, almost a solution of purines. In fact these patients might much better eat meat from which the soup is made than the soup itself and a safe rule for them is to forget that such a thing as a clear or meat soup exists.

If meat soups and broths are "poisons" for the gouty, as they are justly called by the authority quoted, they are "near" poisons

-Nutrition and Clinical Dietetics by Carter, Howe and Mason; Lea \& Febiger. 
for everybody, since the human organism cannot destroy uric acid, and the first symptom of renal disease is increase of uric acid in the blood through failure of the kidneys to eliminate it.

If meats, meat extracts, broths and soups prepared from meat stock are excluded from the dietary, little further restriction is required for the reason that the so-called purin bodies are found in vegetable foods in such minute quantities that they are not likely to do harm. Tea and coffee must of course be strictly avoided. The use of salt should be reduced to a minimum. Achard and others have shown in recent years that the food normally contains a sufficient amount of sodium chlorid, so that salt is needed to satisfy only a cultivated taste. It may be dispensed with entirely without harm. By gradually reducing the amount of salt in the food it may finally be omitted entirely and will be scarcely missed.

An excellent soup stock may be prepared from vegetables. Fruit juices, greens and milk with a great variety of green vegetables, of which our markets now have a supply at all seasons of the year, leave no excuse for the use of meat or meat flavors to satisfy a reasonable appetite.

It is highly necessary in cases in which uric acid elimination is deficient to combat intestinal toxemia by a laxative and antitoxic diet and by changing the intestinal flora (Index). The ability of the kidneys to eliminate other poisons as well as uric acid is lowered. These essential organs are seriously crippled, and their task must be made as easy as possible. (See Bright's disease.)

All foods which lessen the alkalinity of the fluids will certainly increase the predisposition to gout. Hence, besides eliminating meats and to a large extent eggs from the diet, care must be taken to give special prominence in the bill of fare to such foods as potatoes, turnips, beets, cabbage, cauliflower, spinach, greens of all kinds, Brussels sprouts and other fresh vegetables.

The influence of diet upon the nervous system must also be considered in this disease, as illustrated by the case of the famous surgeon, John Hunter, of whom it is related that "when not yet fifty years of age he suffered an attack of gout, accompanied with cardiac symptoms. At fifty-five he had an attack of angina and was never quite well afterward. . . The attacks were easily induced by anger, and he used to say that his life was at the mercy of any knave who should chance to worry him." The 
irritating effects of a flesh diet are well known, as shown in the influence of meat feeding upon dogs and other animals.

A fruit diet aids in the elimination of uric acid by increasing the alkalinity of the blood and thus its solubility. This statement does not rest on purely theoretical grounds but may be demonstrated by anyone who will take the trouble to make the simple experiment of placing himself for a few days upon a fruit diet and making careful comparative examinations of the urine.

Some years ago the writer made a study of the effects of fruit upon the elimination of uric acid in a number of medical students who were placed upon a diet consisting almost exclusively of fruit, the only addition being a small amount of dry toast. The urea was, of course, diminished as the nitrogen intake was greatly reduced, but the output of uric acid was very considerably increased, on the average doubled and in some cases more than doubled. A variety of fruits was given, chiefly grapes, bananas, apples, oranges and peaches. The source of the increased output of uric acid was doubtless the quantity of this waste element which had been stored up in the tissues. By the increased alkalinity of the blood, its ability to dissolve the uric acid from the tissues and transport it to the kidneys was increased and thus the output was increased. In a later experiment, in which the fruit diet was continued for more than a week, it was observed that the increased output of uric acid continued only for a few days. After the first three or four days, the acidity of the urine decreased rapidly, and at the end of the week the urine was nearly neutral.

In every case of gout the condition of the colon must receive careful attention. The bowels should be made to move thoroughly three times every day by means of a laxative diet and necessary accessory measures. An enema at bedtime for a few weeks to make certain that the colon is completely evacuated will be found of great service. The flora should be changed by the use of lactose or lacto-dextrin or large doses of whey cultures of $B$. acidophilus. If at any time the stools show a putrid character greater diligence to keep the colon free of putrefying residues should be exercised. It is not only necessary to change the intestinal flora but to keep it changed. 


\section{Diet in Disease of the Heart and Blood Vessels}

In these disorders degenerative changes take place in the heart and blood vessels in various parts of the body. Sometimes there is a progressive rise in the blood pressure as the disease advances. In many, perhaps the majority of cases, the blood pressure is only slightly or not at all raised.

As regards the cause of the disease, Huchard says arteriosclerosis "begins by intoxication, continues by intoxication and ends by intoxication." This intoxication may have various sources, but in the opinion of Huchard and other equally eminent authorities, the most common origin is the intestine through the putrefaction of undigested remnants of food proteins, especially flesh meats, and the absorption of the putrefaction products in quantities greater than the liver is able to destroy or the kidneys to eliminate.

Another remark by Huchard is well worth quoting: "The origin of the disease is alimentary, the lesion vascular, but the danger is renal. As the disease advances, the kidneys become less and less efficient and the final catastrophe is in a large percentage of cases due to renal failure."

Professor Huchard also held that the great frequency with which arteriosclerosis is encountered in modern times is the result of the increase in flesh eating which in recent years has been very marked in all civilized countries.

Boix, of Paris, produced arteriosclerosis by feeding rabbits extracts of feces and cultures of colon bacteria.

Dr. Herbert Fox, director of the laboratory of Comparative Pathology, Philadelphia Zoological Garden, has shown that arteriosclerosis occurs in certain wild animals when subjected to the conditions of captivity.

Among the interesting observations made by Dr. Fox is the fact that the glands of internal secretion, the pituitary, adrenal 
and thyroid glands, are larger in cats, dogs and eagles-flesheating animals-than in lemurs, rodents and rabbits-animals which live on fruits, nuts and tender shoots.

Dr. Fox also observed that arteriosclerosis is found most definitely developed in animals living upon a meat diet and hence subject to autointoxication.

On the whole, arteriosclerosis is found much less frequently in lower animals than in human beings, doubtless for the reason that in general animals live more physiologically than do human beings.

An interesting contrast was found between horses and dogs in the fact that it is doubtful whether arteriosclerosis ever occurs in horses, although they often attain very great age. On the other hand, in the case of dogs arteriosclerosis is comparatively frequent. Dr. Harvey, who examined the body of Old Parr, who was much more than a centenarian (reputed age at death 152 years), found no evidence of arteriosclerosis in his body. Parr's diet consisted of potatoes, greens, coarse bread and buttermilk. He rarely ate meat.

The observations of Dr. Fox have been strikingly confirmed by those of Newburgh, of Ann Arbor, whose experiments on rabbits have shown that rabbits very quickly develop arteriosclerosis when a moderate proportion of meat powder is added to the bread with which they are fed. To the objections brought in reference to the significance of his observations that rabbits are not accustomed to a meat diet and hence more likely to be affected injuriously by it, Dr. Newburgh very properly cited the observations of Fox above referred to.

These observations are highly important for they show that notwithstanding the fact that carnivorous animals are supplied with superior means of defense against the poisons of flesh meats, they still suffer from arteriosclerosis, which seldom occurs in non-flesh eating animals.

Dr. Lorand, one of the best known of European physicians, has recently called attention to the importance of a non-flesh diet in cases of chronic disease of the heart. The Journal of the American Medical Association thus summarizes his views:

"Lorand has often noticed that persons on a predominantly vegetable diet do not get fatigued so readily as on an abundant meat diet. $\mathrm{He}$ has also often noted a remarkable improvement in the muscular weariness of diabetics when they are placed on 
a vegetable diet. There seems to be something in vegetables and fruits that enhances muscular activity. This may be the explanation for the almost unbelievable capacity of the Bulgarian peasant for muscular work without fatigue; his food consists only of vegetables with black bread and a little cheese. Vegetables contain potassium, and the importance of this in the diet has been recently emphasized anew by Urbeanu, who found that fowls and rats kept on an abundant albumin and ample calories diet, but deprived almost entirely of potassium, developed paralysis, hemorrhages, and other symptoms characteristic of deficit diseases, with fatal cachexia. If potassium was fed to them in time, they recuperated perfectly and grew strong again.

"Lorand's conclusion is that organic compounds of potassium are indispensable to keep nerve and muscle substance in normal condition. Human muscles, including the heart muscle, contain large proportions of potassium. Everything that promotes muscular functioning helps to keep the heart muscle in good condition, as well as the other muscles. Muscular fatigue is a prominent symptom in diseases of deranged metabolism, such as myxedema, exophthalmic goiter, and severe diabetes. The muscular fatigue under these conditions shows marked improvement on a vegetable diet. This supplies the needed potassium and phosphoric acid.

"Lorand thinks that the instinctive longing for bread and potatoes is due to their content in salts, etc., more than to their carbohydrate value. No other articles of food are missed so much as these in special diets. With a weak heart, particular care must be paid to these intangible elements in the diet. An unbalanced diet is especially dangerous with a weak heart. The heart action should be constantly supervised on an anti-diabetic diet, or obesity starvation diet; also on an anti-gout diet, and in the elderly with weak hearts.

The first thing to be done in arranging the regimen of a person suffering from arteriosclerosis is to change the intestinal flora. The flora of this class of patients always needs changing. In a very large proportion of cases, Barger and Walpole demonstrated the production of pressure-raising poisons by putrefaction. According to Dixon, of King's College, London, pressure-raising poisons are produced in the intestine, and Bain demonstrated the presence of these poisons in the blood and urine of persons having high blood pressure. Experiments have shown 
that a non-flesh diet reduces the amount of these poisons found in the urine. A biologic diet must be closely adhered to, not merely for a few weeks or months, but so long as the individual lives.

For most complete results in efforts to suppress intestinal putrefaction, eggs as well as meat should be discarded. It is at least equally important that food residues shall not be left long in the colon. The bowels should move after every meal; that is, at least three times a day. Even when the bowels move several times a day, putrescible residues are often left in the colon in cases in which the cecum is dilated or the colon is spastic. This may be true although meat is excluded, because bile and mucus are putrescible, as well as the nitrogenous remains of the various digestive fluids and some undigested proteins from the foods.

The diet to be followed in arteriosclerosis is a strictly antitoxic and laxative one. All foods containing uric acid in appreciable amount must be discarded. This naturally excludes meats of all sorts, fish, flesh, fowl and shellfish, together with meat extracts, broths, bouillons. Reference to the table showing the uric acid content of foodstuffs will show at once the necessity for elimination of the articles named.

Tea and coffee must be discarded for the same reason. A cup of coffee contains more than double the amount of purin (uric acid) found in normal urine. Uric acid is chemically a xanthin compound. Caffein is also a xanthin compound, tri-methylxanthin. Mendel and Wardell have shown that an intake of caffein is followed by an increase of uric acid in the urine.

Foods rich in oxalic acid, such as sorrel and rhubarb, must be discarded.

Eggs must be used sparingly. The yolks are preferable to the whites. Raw eggs should not be eaten.

Potatoes and other farinaceous roots should be largely used in place of cereals, breads and breakfast foods, because of the rich supply of organic bases which they afford and which neutralize the acids arising from tissue wastes always present in excessive quantity in persons subject to arteriosclerosis or high blood pressure. Fruits should be freely used and greens in sufficient amount to furnish an adequate supply of lime and iron.

Bran, agar and paraffin oil should be used freely enough to secure three bowel movements daily and the enema should be 
used at night, in addition, two or three times a week, when a considerable amount of residue is found to be still present after the last bowel movement as ascertained by administering an enema.

In every case of arteriosclerosis the carmine test (see Index), should be applied to determine how long the food residues remain in the colon. If more than 24 hours elapse before the red color disappears from the stools, further stimulation of intestinal activity is necessary and the amount of bran and paraffin oil should be increased. The daily use of the enema of moderate size ( 2 to 4 pints) is in no way harmful, while drug laxatives of any sort are highly injurious when frequently resorted to.

\section{DIET IN HIGH BLOOD PRESSURE}

The diet in high blood pressure should be the same as that suggested for arteriosclerosis (see above). Tea, coffee, tobacco, condiments and flesh foods of all kinds must be discarded. The bowels must be made to move freely (three times a day), by the use of laxative foods, bran, agar and paraffin oil. Vigorous measures for changing the intestinal flora should be adopted and continued or repeated until the stools cease to be putrid. In many cases the colon is so crippled that the fruit regimen for changing the flora (see Index), must be repeated every few weeks in order to maintain a normal flora ; that is, to prevent the recurrence of putrid stools. The adoption of an antitoxic and laxative regimen after changing of the flora in these cases generally results in a pronounced lowering of the blood pressure within a few days, at most within a month. In the case of young persons, or when the high pressure has existed only for a short time in older persons, the pressure can often be brought back nearly to normal. It is not an uncommon experience to see the pressure drop from 200 to 150 or even lower within a few weeks, and by a careful regimen it may be kept down for years and useful life and activity may thus be prolonged. Dr. M. A. Mortensen, of the Battle Creek Sanitarium medical staff, recently reported the case of a patient, a man past middle age, whose blood pressure was reduced from 260 to 160 and still remained at this point after seven years, with the disappearance of other symptoms of ill health. Equally good results have been obtained in many similar cases. Dale showed that para-hydroxylphenyl-ethylamine, a poison found in decaying meat, acts like suprarenal extract, and raises the 
blood pressure at once from 110 to 260 . Hence, the great importance of a low protein and fleshless diet in these cases.

Dr. Arthur Hunter, actuary of the New York Life Insurance Co., has shown that the average blood pressure in China and Japan is appreciably lower than in this country, and attributes the fact to the large consumption of meat in the United States. We quote as follows from Mr. Hunter's most valuable paper:

"While there is little doubt that a reduction in blood pressure of Americans in the United States would result in greater longevity, a question might arise as to the effect on the quality and quantity of their work. Among brain workers and those in sedentary occupation there would be, in my judgment, as great efficiency. Among manual laborers there does not appear to be any satisfactory proof that a low protein diet decreases physical endurance. Taking the population of the United States as a whole, I believe that a better adjusted diet, with less animal food, would result in a lower blood pressure and in greater longevity with an equal ability to carry on their occupations."

Evidently, the pernicious propaganda of the packers has not convinced the life insurance companies that the interests of their business would be promoted by an increased consumption of meat.

Reduction in weight is one of the most important means of reducing blood pressure. In over-fat persons the pressure often falls one point for each pound reduction of weight.

Sansum and Blatherwick have recently reported excellent results in the treatment of cases of high blood pressure by a basic diet, that is, a diet from which meat and eggs are excluded and the use of cereals greatly restricted. Such a diet greatly lessens the work of the kidneys, and by increasing the alkali reserve permits the oxidation of wastes.

It is to be noted, also, that such a diet is highly antitoxic and lessens the absorption of toxins from the intestine, some of which have been shown to be pressure-raising poisons.

\section{DIET IN APOPLEXY}

The diet in apoplexy should be the same as that for arteriosclerosis. Special care must be given to the regulation of the bowels, on which account a large amount of roughage, together with paraffin oil in liberal doses, may be needed because of the 
patient's inactivity. For the same reason, the intake of food should be considerably diminished. If the patient is obese, the diet may be reduced to two-thirds of the standard dietary for the individual until there is an appreciable diminution in weight. The flora must be changed and the antitoxic and laxative diets must be closely adhered to. The stools should never be allowed to become hard or putrid. The bowels must be moved thoroughly three times daily. The enema should be used at night once or twice a week or more often, to insure complete emptying of the colon. It is well, once a month, to resort for a few days to the fruit regimen to keep the flora in good condition.

Overeating and late suppers should be especially avoided. In many cases of apoplexy the history shows that the attack has followed a large meal. Persons with high blood pressure, especially when the cerebral blood vessels are known to be involved to a marked degree, should be carefully cautioned against hearty eating. The diet should be rather meager. The weight should be reduced to the average and if possible a few pounds below. Two meals a day are better than three in these cases for the reason that less food is likely to be taken in two meals than in three. If supper is eaten it should consist exclusively of fruits. Ice cream, pastry, rich desserts and other hearty foods should be strictly excluded from the last meal. Six o'clock dinners are perilous for the apoplectic.

\section{DIET IN ANGINA PECTORIS}

The proper dietary is the same as that for arteriosclerosis and high blood pressure. Great care should be taken to avoid overdistention of the stomach and foods which are known to "disagree." Hot drinks, ices, pickles, and indigestibles of all sorts must be carefully avoided. Flesh meats should be discarded wholly and eggs should be eaten sparingly. The diet indicated for arteriosclerosis (see Index) should be carefully followed.

\section{DIET IN MYOCARDITIS}

This disease is not an inflammation of the heart, as the name would naturally indicate, but a degeneration of the muscle fibers of the heart, which become more or less fibrous in character, thus lessening the efficiency of the heart as a muscular organ. The 
causes of this disease are essentially the same as those of the senile heart, but the disease often develops in persons who are not sufficiently advanced in years to justify a diagnosis of senile heart. The excessive use of tobacco is one of the most common causes of this early deterioration of the heart muscle; alcohol acts in a similar manner. Probably the most common of all causes is to be found in the numerous poisons of alimentary origin with which the blood stream is constantly contaminated in cases of chronic intestinal toxemia. Heavy meat eaters are very prone to suffer from this disease. Men suffer much more frequently than do women, the number of males affected being more than double the number of females. A probable cause for this is to be found in the fact that men are much more addicted to tobacco than are women and are much larger consumers of meat.

In the dietetic management of myocarditis, the first attention must be given to changing the intestinal flora by the means which have already been pointed out (see Index). Great care must be taken to secure prompt discharge of food residues and body wastes. The bowels should be trained to move regularly three times a day, and if there is any ground for suspicion that the colon is not thoroughly emptied, an enema should be taken at bed-time so as to insure complete clearance of the colon. Free use should be made of fruits and root vegetables. Potatoes should be used largely in place of cereals. A potato diet increases the alkalinity of the blood and the tissue fluids and thus aids in the uxidation of body wastes, which have a tendency to accumulate in the blood stream in cases of this sort. Copious water-drinking should be encouraged. Of the highest importance is the complete change of the intestinal flora. The gram stain count should be brought to 120 and maintained at this point. By this means, the intake of colon toxins will be reduced to a minimum. The constipation with putrid stools, commonly encountered in cases of this sort, have undoubtedly a causative relation to the degenerative changes in the heart muscle, and hence great care must be taken to see that the stools are kept free from odor and that the bowels are made to move freely. An enema should be taken at bed-time if necessary. The daily use of lacto-dextrin, paraffin oil and agar or bran are important means which should be combined with a strict antitoxic diet.

In ordinary mild cases of cardiac disease, an antitoxic and laxative dietary is indicated. If the patient is anemic or ema- 
ciated, a reconstructive diet is indicated (see Index). In cases of obesity with myocarditis, or other grave heart lesion, very careful dieting is of greatest importance. Vigorous exercise must be avoided in these cases because of the cardiac weakness; hence, the reduction of weight must be accomplished by restriction of the diet. A low protein regimen, such as is afforded by the antitoxic diet (see Index), with a proper diminution of the amount of food eaten, is usually sufficient. Meats are, of course, contraindicated for the reason that they encourage the degenerative process, which is the essential factor in the case. Fats must also be restricted and need not constitute more than 10 per cent. (calories) of the dietary. The protein should not exceed one calorie per pound of body weight. The total ration may be reduced from one-third to two-fifths the normal amount for an individual of a given height. The Karell method of milk feeding has been highly recommended in cases of this sort and has often been used with success. It is doubtful, however, if it has any specific virtue other than the reduction of the amount of food ingested and the elimination of meats.

In cases of marked heart weakness coupled with dropsy and inability of the kidneys to eliminate the normal amount of water, a saltless diet is essential, together with great restriction of the fluid intake. The amount of fluids taken should not exceed two or three pints for a few days. In some cases the amount may be still further reduced for one or two days (salt-free diet and dry diet, (see Index). But the low fluid intake must not be continued too long. The fluid food should consist of malt sugar in water or fruit juices. A moderate intake of food secures better results than complete fasting for the reason that it promotes the elimination of the sodium chlorid, which is responsible for the accumulation of water in the tissues. Care must be taken to keep the bowels oper. by means of the enema and the free use of bran or agar and paraffin oil.

By the adoption of these measures and close adherence to a non-toxic diet, so great improvement may often be secured that the patient may be safely assured of a very considerable increase of his life expectancy. The writer has seen many cases in which the life expectancy was doubled and even tripled by close adherence to this regimen. 


\section{DIET FOR THE SENILE HEART}

The heart grows old with the rest of the body. The parchment-like, senile skin, marked by brown spots, is an outward sign of the general senile changes which have taken place throughout the body. The heart muscle, cells and other tissues have deteriorated because of the continuous contact of a blood stream containing poisons which the liver has failed to destroy or the kidneys to eliminate.

The most important thing to be done is to lessen the absorption of poisons from the alimentary canal. Tea, coffee, and of course tobacco, must be excluded and the dietary must be made such as will not encourage the putrefaction in the colon and lower small intestine. In other words, meats, including fish and fowl, oysters, as well as red meats, must be wholly excluded from the dietary, together with cheese, with the exception of cream cheese and cottage cheese.

The patient's flora should be changed by the free use of lactose or lacto-dextrin or acidophilus buttermilk and by enemas of lactose or lacto-dextrin. In general, the dietary should be the same as that for arteriosclerosis. 


\section{Diet in Nervous Disorders HEADACHE}

Most headaches are toxic. Many are no doubt the result of the breakdown of the liver defense. As the late Professor Lauder Brunton wisely remarked: "The liver is the porter which stands at the gate of the organism to prevent all deleterious substances which enter the blood vessels from the intestine from reaching the general circulation. These substances are caught up by the liver and destroyed, or excreted unchanged into the intestine, where they may pass away with other feces. But many of these substances may be re-absorbed, and so they form a constant round from bowel to liver and liver to bowel, until at last they amount to so much that the liver cannot deal with them, and they pass into the general circulation. A period of time is required for this accumulation, which varies even in the same person, and occurs in less time with a highly nitrogenous diet. Absorption from the liver is influenced by the emotions, so that after emotion a person may become jaundiced; therefore we may expect anything which is circulating with the bile would be rapidly absorbed. Anxiety, grief or sorrow is apt to bring on a headache, and there are good reasons for attributing the headache to the presence of abnormal constituents circulating in the blood. Why do the toxins fasten on the head and cause a headache instead of causing a pain in the intestines or big toe? It is because there is some factor, perhaps a local lesion, which determines the pain to the head. The most common determining causes are decayed teeth, astigmatism, myopia, hypermetropia, presbyopia, nasal or pharyngeal trouble, disease of the antrum of Highmore, or of the frontal or ethmoidal sinuses, glaucoma, periostitis, or neuritis.

There are now few authorities who question the toxic origin of migrain. Starr, one of the leading neurologists of this country, holds that migrain is due to an unidentified toxin and that hemicrania, a somewhat similar disorder, is due to the same cause. Other investigators attribute the disorder to uric acid and other toxic bodies derived from protein.

Dr. F. Hare some years ago, and more recently Mercier, have 
called attention to the fact that an excess of fat in the diet is often the cause of headache. Headaches due to this cause are usually worse in the morning. Fats may easily become a cause of headache. When present in excess they increase intestinal putrefaction and autointoxication.

With this explanation of the cause of headache, it is evident that the most promising outlook for relief is in the direction of dietetic control.

Most persons subject to headache are found to present coated tongues and foul breath, an indication of saturation of the tissues with putrefaction products derived from the colon.

The first step is to change the flora of the colon (Index). This must be done thoroughly. Often two or three attempts will be needed for complete success. After four to six days on the fruit regimen a more liberal diet may be allowed for three or four days, and then the fruit regimen should be resumed for another period of four to six days. As the tongue clears and the colon becomes more active, the headaches will grow less frequent and finally disappear. The writer has seen this occur in many hundreds of cases.

In occasional cases, a crippled state of the colon will complicate the situation and delay success. The incompetent ileocecal valve, adherent pelvic colon, adherent appendix or cecum and spastic colon, are serious hindrances which must be reckoned with. A diseased gall bladder is another troublesome complication. Sometimes surgery is necessary.

Many persons have experienced relief from headache after vomiting a quantity of very bitter, evidently highly concentrated bile. The relief experienced from purgation by salts or calomel is doubtless due to the same cause-the getting rid of a quantity of concentrated and highly poisonous bile. Bouchard showed that bile is six times as toxic as urine.

Removal of a few ounces of bile by means of the duodenal feeding tube two or three times a week is a measure which may be worth trying. Four to six ounces may be removed at each passage of the tube until a total of a pint and a half or two pints have been removed. Lemon juice (one to three of water) or a solution of citric acid (two per cent.) may be used to excite contraction of the gall bladder instead of magnesia sulphate. Still better is the use of hydrochloric acid, the normal stimulant of the gall bladder and disinfectant of the stomach. 
Many persons subject to headaches notice the beneficial effects of acid fruits, for which they often have a craving, perhaps due to the fact that the gall bladder and liver are made to discharge more freely while peristalsis is hastened, so that le-s bile is absorbed and thus some of the stale bile is gotten rid of.

After the flora is changed, the laxative and antitoxic diets must be consistently followed.

The free use of Lacto-Dextrin, psyllium seed, Fig Bran and Paramels, while discarding meat and eggs, will often affect a rapid cure of headache by keeping the colon free from putrefying residue. In many cases, a hot enema (112-118 degrees) at bedtime, is found to be necessary to insure thorough emptying of the colon. It is desirable that the residue of the breakfast should be evacuated before retiring. Carmine may be advantageously used as an indicator. (See Index.) In some cases, it is necessary to discard milk as well as eggs and meat, at least for a few weeks. In such cases, nut protein should be used to insure maintenance of the nitrogen balance.

Tea and coffee must be avoided. This is imperative. While a cup of tea or coffee is often resorted to as a means of relief from headache, the benefit is only apparent and is delusive. The cause is not removed, and the disorder is definitely aggravated. Not a few persons are so sensitized to coffee that very small amounts produce almost immediate ill effects. An eminent New York physician recently stated to the writer that a teaspoonful of coffee would in his case produce a severe headache in half an hour or less. Many thousands of persons have gotten rid of persistent headaches by discarding coffee and tea. The headache which coffee relieves temporarily is one of the results of the coffee habit and disappears when the drug is definitely discarded.

Copious water drinking ( 3 to 4 pints daily) is highly important to aid in elimination of toxins.

\section{MENTAL DISEASE}

We quote from a competent medical author as follows:

"Intestinal toxemia is a common complication of mental diseases as seen at the Philadelphia Hospital, and occasionally it is the cause or complication of certain cases of epileptiform convulsions."

Modern studies of the causes of mental disease have made 
clear the fact that in not a small proportion of cases mental alienation is the result of intestinal toxins acting upon a peculiarly susceptible nervous system. Constipation and toxemia lead to insomnia and depression and in time the mental equilibrium is broken down. Change of flora and the laxative and antitoxic diets are potent means for loosing the shackles of mental disease; and it is hoped that at no distant day, tens of thousands of mental sufferers who are now immured behind asylum bars may be set free by the application of enlightened physiologic measures which at present the state fails to supply.

In some cases insanity has been traced to the coffee habit. Williams, of Edinburgh, reports a case of mental disease in which a woman suffered from frequent attacks of angoisse. The attacks stopped entirely when she ceased taking three grains of caffein daily, which she had continued for several months. When it is recalled that a single cup of good coffee contains four grains of caffein, according to Dr. Wiley, it will not appear surprising that mental disturbance sometimes results from the long continued use of tea and coffee, or its use in immoderate quantities.

Dementia praecox has been definitely shown to be, in certain cases, at least, the result of chronic intestinal toxemia. Foul stools and constipation are always present. The writer has seen very marked improvement in numerous cases of this disease as the result of a change of flora followed by strict adherence to an antitoxic dietary, with hydrotherapy and other physiologic measures. Better results have been obtained at the Battle Creek Sanitarium in the treatment of many cases of manic-depressive neurosis. The loss of appetite, insomnia, depression, and mental inefficiency are all directly traceable to the disordering influence of intestinal toxins, the suppression of which by changing the intestinal flora and accustoming the patient to an antitoxic and laxative diet of such character as to secure three or four bowel movements daily, has been attended by the happiest results. If these simple measures could be introduced into all our state hospitals for the insane, the result would be a notable and rapid lessening of the insane population of the country and such a setting free of minds in bondage to disease as would remind the world of the days when miracles were wrought and demons cast out by the performance of mystic rites.

In certain forms of paresis, intestinal toxemia plays an active part and hence the fruit regimen and other methods for changing 
the flora and the antitoxic and laxative diet are measures of essential importance. Even in paresis due to syphilitic infection the added burden of intestinal toxins is a decided handicap to the curative efforts of the body forces and an obstacle to therapeutic success in the use of salvarsan and other specific remedies that may be readily and advantageously removed by the use of the proper dietetic measures.

In cases in which food is refused, tube feeding, especially feeding by means of the nasal tube, becomes a most valuable and necessary means of keeping up and improving the patient's nutrition. The liquid foods prescribed for duodenal ulcer are appropriate. Malt sugar and milk sugar are especially useful and should be freely used. Malt sugar is the most readily assimilable of foods and milk sugar or lacto-dextrin is a most valuable means of combating intestinal toxemia, as has been elsewhere shown. Cultures of $B$. acidophilus should be freely used to aid in restoring the normal intestinal flora and suppress putrefactions. Acidophilus buttermilk is most useful.

Water drinking should have especial attention. The perversion of the natural instincts frequently met in the insane leads often to the neglect by the patient to take water in anything like the proper amount. Often the sense of thirst appears to be lost. Water should be given systematically. A glassful every hour is not too much for the average patient suffering from mental alienation. Fruit juices of any sort may be advantageously added.

The methods recommended have been within the last two or three years adopted in several large State institutions, particularly Dayton, Ohio, Trenton, New Jersey, and Kalamazoo, Michigan. The superintendent of one of these institutions reports the number of cures under the new methods to be two and one-half as great as under the old régime.

\section{EPILEPSY}

The most important points in the feeding of epileptics are the elimination of meats, meat extracts and broths, and the disuse of salt.

The late Doctor Combe, of Switzerland, was one of the first to call attention to the injurious effects of salt in epilepsy. He reported very remarkable results from the saltless regimen.

Another French physician, Dr. Mirallié, has more recently 
made a study of this method and claims it to be highly valuable. This author insists that:

Salt should be discarded absolutely, as also tea, coffee and alcohol. Then the doses of bromides can be materially reduced, and such improvement may then be realized that it amounts to an actual cure. He gives a moderate dose of bromide every day, without interruption. When his patients go thus two or three years without a seizure, he reduces the dose of bromide, but emphasizes that absolute abstention from salt is the main thing. In his first series of 181 cases, only 83 followed his instructions. At that time he ordered merely restriction of salt. In 12 cases the results were negative; in 18 there was improvement and 53 had no further seizures. Since 1912, 52 of the later series of 133 cases followed instructions, and 31 have had no further seizures and 18 have been much improved; more or less benefit has been realized in all but 3 cases. Of 10 men in one institution, all were improved and 5 apparently cured, as also 9 of the 12 women, all showing marked improvement. The youngest of the clinically cured was 7 , the oldest 63 years of age. The seizures in one case had been frequent from the age of 7 to 37 , but there has been none since this treatment was instituted. The best results were realized in families in which the mother did the cooking, and she was able thus to keep all salt out of the bread, the butter, etc. When this was left to servants, the salt was not banished so rigorously. Full success was realized only in the cases with absolute abstention from salt, tea, coffee and alcohol. ***** The benefit was prompter and more pronounced in persons who had previously used much salt. The other symptoms of epilepsy were not modified so early as the actual seizures. The diet, except for salt and stimulants, can be varied and liberal, but must be served entirely without salt. The failures were always in wealthy homes.

Epileptics nearly always have an inveterate tendency to overeat. In many cases, they are also very fond of meats and have a craving for salt. All of these faults must be corrected. Meats must be discarded entirely. In cases in which convulsions occur frequently, the patient should be placed upon a strict saltless diet (see Index). Special attention should be given to changing the intestinal flora (see Index) and keeping the bowels freely open by the liberal use of bran, greens and paraffin oil. Washing the colon thoroughly with the enema daily should be practiced as a routine measure in cases in which other methods fail to eliminate the putrid character of the stools. The epileptic should be taught to chew well and to eat sparingly. The food should be simple and rather bland to avoid temptation to overeating. Vegetables like potatoes, turnips, carrots and beets, properly cooked, should largely take the place of breadstuffs. 
It is now generally recognized that a strict antitoxic diet or lacto-vegetarian diet is one of the most efficient means of controlling this disease. The attacks gradually become less and less frequent until they finally disappear. So long as the kidneys continue to act efficiently, there is little danger of an attack. It has been noted that just before an attack the proportion of urea diminishes to half or two-thirds the normal. The reduction of proteins to the lowest practical level is unquestionably one of the most efficient means of controlling this malady.

That toxins in the body fluids play a leading part in this disease has been clearly shown by several observers. Some years ago the writer had under observation the case of a young woman who had frequent epileptic attacks, sometimes remaining in a status epilepticus for two or three days. A study of the toxicity of the urine by Bouchard's method of determining the urotoxic coefficient showed a notable lessening of toxicity during the height of the attack and a remarkable increase as it was passing off. Evidently a highly antitoxic diet and copious water drinking are clearly indicated in this disease, together with the elimination of salt from the dietary and conscientious following of the biologic life. The patient referred to was greatly helped by these measures though not wholly cured.

Great claims have been made for fasting as a cure of epilepsy. All the benefits derived from fasting may be secured by a proteir. fast; that is, by reducing the protein to a minimum or even excluding it from the dietary for a few days. It is indeed much safer and more rational to put the patient on a carbohydrate dietary (see Index) for a week or two than to require an absolute fast.

Good results have followed the operations of appendicostomy and removal of a part of the colon; but there is good reason to believe that the benefit following these operations was the result of a lessening of the toxemia from putrefactive processes in the colon and that the same relief of symptoms may be secured by a thorough change of the intestinal flora (see Index) and persistent adherence to the antitoxic regimen. The writer has seen many epileptics greatly benefited by these dietetic measures and not a few cures. In the epileptic ward of a large insane asylum, the adoption of a fleshless diet diminished the number of "attacks" fully one-half. 


\section{HYSTERIA}

This condition requires no specific dietary other than to avoid the use of nerve-irritating and stimulating foods and condiments and to increase the activity of the colon by a highly laxative diet (see Index). Tea, coffee, mustard, pepper, pepper-sauce, vinegar, pickles, much salt, broths and meat extracts, as well as meats of all kinds must be discarded. For emaciated patients, the milk regimen for two or three weeks often secures good results. The milk regimen should be introduced by three or four days of fruit regimen to get the bowels active, eliminate the fetor of the stools, and to create an appetite. A laxative diet is especially indicated. The amount of roughage, bran, greens, and fresh vegetables should be increased until the bowels are made to move three times daily. The enema should be employed in cases in which diet alone is insufficient. Change of the intestinal flora is of first importance.

\section{NEURASTHENIA}

Neurasthenia is not in itself a disease but is rather a symptom of disease. It may have for its foundation various morbid conditions, but the most common of all is intestinal toxemia. The writer has maintained this view of the nature of this disease for many years. It is also held by many eminent French physicians. M. Lepine, of Paris, holds that neurasthenia is usually due to autointoxication. The source of the toxins, according to Lepine, is intestinal putrefactions. He finds that the symptoms of exhaustion and neurasthenia disappear quickly when the eliminative organs are made active, and especially when the patient is placed upon an antitoxic dietary by the elimination of flesh foods or the so-called lacto-vegetarian regimen. Consequently, the proper dietetic measures are the fruit regimen to clear the tongue and render the stools non-putrid, followed by the milk regimen for two or three weeks if the patient is emaciated. This course should be repeated until the flora is thoroughly changed, the tongue clean, and breath and stools no longer foul.

After a sufficient gain of flesh has been secured, the patient should follow permanently the antitoxic dietary, taking care to include in the bill of fare for every meal a sufficient amount of roughage to secure three bowel movements daily. This method the writer has followed with much success in the treatment of many hundreds of neurasthenics. 
The diet must also include a considerable amount of greenstuffs or uncooked food to supply daily an adequate amount of vitamins, a lack of which is often a cause of the neurasthenic symptoms.

\section{SITOPHOBIA}

Sitophobia is the term applied to a condition in which there is a morbid fear or aversion of food. The patient fancies that some particular food, ordinarily perfectly innocuous, is certain to do him harm, and as a result the food, when eaten, through the patient's perverse mental attitude apparently does disagree, producing various digestive and other disturbances. A person who has been made sick by some particular article, even though it may be something of which he has previously been very fond, is very likely to develop a sitophobia for the article in question.

The gravity of this condition depends upon the extent. of the list of foodstuffs which are tabooed by the disease. If the patient has an aversion to all food, the situation may be very serious. The use of the nasal or duodenal feeding tube may be necessary to prevent starvation. When only one or two articles are involved, wholesome, agreeable equivalents may be substituted. Not infrequently the patient may be cajoled out of his morbid mental state. In other cases suggestion or other careful psychic treatment is required.

These patients are usually neurasthenic. Indeed, sitophobia is really a neurasthenic symptom. There are nearly always present marked symptoms of intestinal toxemia and colitis. The flora must be changed, and the bowels made to move three times a day. In some cases, the patient's aversion is due to sensitivity rather than to a morbid psychology.

\section{NEURALGIA-NEURITIS}

Pains to which the terms neuralgia, neuritis, sciatica, etc., are commonly applied are in general due to toxins. The patient requires an antitoxic and laxative dietary. Frequent bowel movements, at least three times a day, are necessary to keep the body free from the toxins with which it has long been flooded. In many cases there is incompetency of the ileocecal valve, which permits the reflux of putrefying material into the small intestine, where it is rapidly absorbed. Change of the intestinal flora is essential. 
Genuine neuritis, or inflammation of a nerve, is usually the result of traumatism or of a specific infection. The dietary required is the same as that indicated when fever is present; that is, antitoxic and laxative.

\section{VAGATONIA}

This is a condition in which the autonomic nervous system is abnormally irritable, as indicated by a tendency to excessive contraction of the pylorus, spastic contraction of the colon and sometimes of the anal muscle. Diet alone is not sufficient in these cases. Rest, sedative baths, changing the intestinal flora, out-ofdoor life, quiet surroundings, relief from worry, and general reconstructive measures are essential. The diet should be unstimulating. Meats must be discarded. Fruits and green vegetables should be given in great abundance to alkalinize and enrich the blood and farinaceous foods to increase adipose tissue, which is nearly always deficient. The antitoxic and laxative diets are indicated.

This rather vaguely defined condition is supposed to be present in cases of hyperacidity, spasm of the pylorus and gastric hypersecretion, conditions which may be complicated with gastric or duodenal ulcer, or disease of the gall bladder or other lesions. The diet must then be modified to suit the conditions known to be present, as shown by the X-ray or other means. In general, it should be bland and unstimulating and yet should include a suitable amount of roughage to stimulate intestinal activity, for constipation is practically always present in these conditions. The colon is often spastic and there is generally dilation of the cecum and incompetency of the ileocecal valve as a result of the longcontinued over-distension of the large intestine.

Copious drinking of hot water is highly beneficial in most cases of this sort. As soon as the first symptoms of pain appear a tumblerful of hot water should he swallowed. The temperature of the water should not be more than $105^{\circ} \mathrm{F}$. The hot water drinking may be repeated if the pain recurs. In cases in which the pain appears three or four hours after eating, a copious draught of hot water, two to three glasses, will often afford complete relief by diluting the gastric contents and relaxing the pyloric spasm. The good effects of the hot water drinking are encouraged by lying upon the right side for a few minutes after 
the water has been swallowed. A hot bag over the stomach, either with or without the abdominal bandage, is helpful in these cases, as is also a large hot enema ( 3 or 4 pints), in cases in which the pain is accompanied by distention of the colon. In cases in which hot water drinking does not afford relief, bland food may be taken in small quantity. The best food for this purpose is rice jelly or a smooth rice gruel, taken warm, with the addition of a little unsalted butter or sweet cream. A tablespoonful to a cupful of bland food will often cause complete relief from pain by stopping the violent contractions of the stomach which accompany and cause the so-called hunger pain. In cases in which the pain occurs in the night, it is well for the patient to have a little food by his bed to take at once when the pain occurs, as it is much more easily relieved if food or hot water is taken at once when the distress first makes its appearance, instead of waiting until it becomes unbearable.

Since this condition is generally associated with intestinal toxemia and colitis, a change of intestinal flora and treatment for colitis are essential.

\section{DIET IN SEASICKNESS-CARSICKNESS}

The writer once asked an intelligent steward on a big ocean liner what he thought was the cause of seasickness. He replied at once: "It is because they all eats too much. They just goes down into the saloon and stuffs themselves with all sorts of vittles and then when the ship rolls a little they heaves it all up. Why, there's the captain. He's been to sea more than twenty years, and yet he gets seasick every time the water is a little rough. He eats plum puddin' and all sorts of rich things so of course he is sick. I don't eat them fixin's and I am never sick; but I would get sick mighty quick if I ate like the captain does."

While diet may not be the dominating factor in seasickness and carsickness, it nevertheless is important. Constipation, overeating, and especially the eating of rich food, meats and fats, greatly increase the liability to seasickness. If one is about to take a voyage he should take care to have his bowels in thoroughly good condition before embarking; the tongue should be clean and the breath sweet. He should be free from any symptom of intestinal toxemia. Such a person, by the exercise of a little care, is not likely to suffer from seasickness. A highly susceptible person 
may find it necessary at first to remain quiet in his berth during very stormy weather, but he may not suffer from nausea, and by keeping his cabin well ventilated and maintaining a horizontal position he will have no serious discomfort. The diet in the meantime should be spare, consisting of plainly cooked vegetables, cereals and fruits, with an abundance of bran and other roughage. Meats of all kinds and even eggs must be avoided. Buttermilk is preferable to sweet milk. Take fats sparingly.

\section{MYASTHENIA}

The dietary of myasthenia is the same as that suggested for neurasthenia (Index).

The inability of these patients to take much exercise renders a liberal use of bran or agar and paraffin oil essential to promote intestinal activity. The protein intake should be small and fat should be used rather sparingly. A liberal quantity of perfectly sweet butter and yeast extract (Savita) should be used to insure a good supply of vitamins.

Greens in abundant quantity should constitute a part of each day's bill of fare.

Water should be taken to the extent of two to three quarts daily. The intestinal flora should be changed and kept ehanged by the antitoxic and laxative diet (see Index).

In cases with marked emaciation, the milk regimen for two or three weeks is indicated. Care should be taken to make the diet supply an adequate amount of vitamins of all sorts and also food lime and iron.

\section{HERPES ZOSTER}

In severe cases, especially when the disease is accompanied by fever and infection involving the central nervous system, a fever diet should be taken for several days (Index). Agar or bran with paraffin oil should be administered to move the bowels. If the tongue is foul, the intestinal flora should be changed by means of the fruit regimen with milk sugar and cultures of $B$. acidophilus. After the fever has disappeared, the antitoxic and laxative diet should be employed (see Index). 


\section{TIC-DOULOUREUX}

The diet in this disease should be the same as indicated for neuralgia and neuritis.

Many cases are greatly benefited by changing the intestinal flora and the adoption of an antitoxic regimen, but in quite a large proportion of cases, other than dietary measures are essential for the accomplishment of the results desired.

\section{NERVOUSINESS}

A state of high nervous tension and an unusual degree of nervous and mental irritability, a certain mental instability-in short, the nervous condition generally known as nervousness is, to a remarkable degree, amenable to dietetic treatment. In the great majority of these cases, the real cause is not nervousness due to a weak or diseased nervous system, but a toxic state which may be more or less readily relieved by changing the intestinal flora and the adoption of an antitoxic dietary. Of course, fresh air sleeping, out-of-door life, sun-baths, proper exercise, proper clothing, mental hygiene,- everything, in short, that relates to biologic living may render service in promoting recovery in cases of this sort; but, in the writer's experience, the greatest benefit is realized from the employment of measures for cleansing the blood stream from the toxins which disturb the central nervous system. This can only be accomplished by careful regulation of the dietary and by promoting intestinal activity.

Many sufferers from nervousness are constantly being victimized by psychoanalysts, Christian Scientists and the promoters of various pseudo-healing cults. It is a mistake to suppose that these patients are to be cured by psychic measures alone. Couéism, or suggestion in some other form, unquestionably produces iemporary benefit in many cases, but the benefit received is necessarily only temporary for the reason that the remedy does not trike at the root of the disease. The real cause of the condition is physical rather than psychic and can be removed only by the use of such measures as will suppress morbid influences and restore normal conditions. Couéism, suggestion, faith-healing and allied measures may promote recovery through removing some grave obstacle in the way, such as fear or worry, but the cure must be accomplished by the establishment of normal physical conditions, one of the most important of which is a biologic diet. 


\section{Diet in Disorders of the Genito-Urinary Organs}

\section{DIET IN DISEASES OF THE KIDNEYS}

The kidneys eliminate two-thirds of the tissue wastes. Each one of the four million renal cells produces about one-fourth of an ounce of urine in a life-time of sixty years, or four drops in one year. This delicate machinery is easily damaged when compelled to eliminate poisons unusual in character or amount; hence, the injury done by typhoid, scarlet fever and other infectious diseases, and by smoking and other drug habits, the use of tea and coffee and of irritating condiments.

Disease of the kidneys may be regarded as chiefly due to two causes:

1. Injury from acute infections. Tuberculosis, scarlet fever, measles, typhoid fever, smallpox, even chicken-pox and other acute infections may injure the kidney structures to such a degree as to greatly cripple it, so instead of being able to bear the wear and tear of life for 60 or 70 years, they are worn out at 40 or 50 .

In all cases of albuminuria and especially in advanced cases of Bright's disease, the low protein diet must be strictly followed. A large intake of protein, as in eating freely of meat or even eggs, may result in such an over-taxing of the kidneys as to cause an acute nephritis and even death. Such results have often followed a single indiscretion in eating.

When the efficiency of the kidneys is greatly impaired, as shown by the increase of non-protein nitrogen in the blood, the amount of protein eaten should be reduced to a minimum of not more than one calory per pound of body weight daily, and in some cases, the amount should be even less. And in such cases it is advantageous to divide the double amount of protein into five or six small portions, to be taken at intervals during the day instead of taking larger quantities at three meals. Protein is utilized and the surplus eliminated within six to nine 
hours. By reducing the amount ingested at one time, the load upon the kidneys is lightened as much as possible.

2. Overwork. The normal kidney is without doubt able to do the natural work of the body not only for an average lifetime but for 100 or 120 years or more. But if the work required daily and hourly of the kidneys is two or three or ten times as much as is required of these organs under normal conditions, the delicate filtering structures of these wonderful organs become prematurely old and unable longer to perform their function. Of course a breakdown due to overwork will occur sooner in a person whose kidneys have been damaged in early life by an infectious disease than in those who have fortunately escaped such injury.

The cause of overwork of the kidneys is of course the circulation in the blood of an excessive amount of toxins. The normal work required of the kidney is comparatively small, but various causes may increase the burden to an enormous extent.

One of the most common sources of excessive kidney work is a high protein diet. Folin has shown that all of the protein eaten beyond that which the body can use in the repair of its tissues is directly transformed by the liver into urea to be eliminated at once by the kidney; that is, surplus protein never becomes a part of the living structures of the body and does not enter into its useful work. It is simply excreted like so much waste matter. Comparative analyses of the urine of low protein feeders and those who take an ordinary mixed diet show that even moderate meat eaters require of their kidneys three times the amount of work in the elimination of nitrogenous wastes that is demanded of the kidneys of flesh abstainers. While the kidneys are young they are usually able to bear this extra burden so that no evidence of injury appears; but as they become worn with advancing age they become unable to do their work efficiently. Poisonous products begin to accumulate in the blood. Then the blood vessels begin to suffer from contact with a contaminated blood stream and degenerations begin in the heart, liver and various other vital organs.

According to Nencki, MacFayden, and other investigators, one-seventh of the protein eaten undergoes putrefaction instead of being converted into human albumin adapted to the nutrition of the body. 
In view of the above facts it is highly important that an excess of protein should be avoided, since a large intake of protein is certain to be followed by an increase of the amount of toxins produced in the colon. A low protein diet is of the greatest importance in all cases of Bright's disease.

Another prolific source of overwork of the kidneys connected with the high protein diet is the production in the colon through putrefaction of the food residues of large quantities of bacterial poisons. At first these toxins are very largely destroyed and detoxicated by the liver so that their effect upon the kidneys is less pronounced, but later the liver begins to fail in its efficiency and then the injury to the kidneys as well as other structures becomes greater.

Another source of poisons which leads to overwork of the kidneys is to be found in tea and coffee which contain a dozen or more poisons, some of which, like caffein and tannic acid, are found naturally in the coffee berry while others are produced by the roasting process.

Tobacco smoking introduces a long series of highly potent kidney poisons, more than 20 in all, of which nicotine is perhaps chief although exceeded in virulence by several of the poisons associated with it.

Metallic poisons of all sorts are extremely damaging to the kidneys. This is well shown in the nephritis which follows the taking of corrosive sublimate either by accident or intent. The long-continued use of mineral drugs even in small quantities may also result in great damage to the kidneys. There can be no doubt that the use of arsenical preparations in the treatment of syphilis and skin diseases does more or less damage to the kidneys.

The elimination of the excess of protein eaten, of chlorid of sodium, mustard, pepper and other condiments, of the alkaloids of tea and coffee and of other unusable materials taken with our food, together with the poisonous products of putrefactive processes in the colon, constitute by far the greatest part of the work required of the kidneys of modern man. This fact is made to appear in a very strong light by the examination of the urine of a fasting person. Although the ordinary life processes, including muscular activity, may be going on as usual, the amount of solids eliminated in the urine is reduced to a small fraction of that ordinarily found. 
The chief aim of diet should be to lessen the intake of toxins and the production of toxins in the body. The first object may be accomplished by suppressing drug habits of all sorts, including the use of laxative drugs, patent medicines, tonics, and soporifics or sleep-producing drugs as well as irritating condiments, alcohol, tea, coffee, tobacco, meat, meat extracts, broths and vegetables containing considerable quantities of oxalic acid, such as pieplant and sorrel.

Meats of all kinds and meat products should be avoided because meat eating almost inevitably leads to an excessively high protein intake, which necessarily imposes extra and more or less damaging overwork upon the kidneys. Folin has shown that all the protein eaten beyond a small amount required for repair of the tissues, which is never more than two ounces per diem, generally less, is at once converted into urea and eliminated through the kidneys as soon as possible; that is, surplus protein is treated like tissue waste, rendering the body no service, but laying an extra burden on the kidneys.

Squier and Newburgh have also studied the effects of a high protein diet on the human kidney. Two groups of subjects were employed. One group consisted of five healthy young men, who were carefully examined and found to possess healthy kidneys and to be normal in every way. They were then given a pound of beefsteak at each of two successive meals. In each case, examination of the urine showed the presence of blood cells, indicating so intense a degree of congestion produced by the meat that blood had escaped from the blood-vessels into the urinary ducts. Examination of the urine before the meat was given showed not the slightest trace of blood cells or any other evidence of disease.

A second group consisted of persons suffering from high blood pressure. The urine in each of these cases showed some evidence of disease of the kidneys. When given a high protein diet, red blood cells and albumin appeared in the urine, if not before present, and increased if previously present. There was a like increase in all the other symptoms of Bright's disease present.

There can be no doubt that the excessive use of flesh foods is one of the chief causes of the very rapid increase of deaths from Bright's disease in this country and other civilized lands. This has been noted particularly during the last 20 or 30 years, during which time the consumption of meat has greatly increased. Modern methods of examination of the blood have attained 
such a degree of accuracy that the influence of diet can now be very definitely determined. Myers has recently shown that in cases of Bright's disease in which the amount of urea in the blood is greatly increased the urea content may be notably decreased by a low protein diet. The retention of urea in the blood also occurs in pneumonia, in cases of intestinal obstruction, in syphilis and other diseases.

A strict basic diet should be closely followed by every nephritic patient. (See Basic Foods, Index.)

The use of so small an amount as a single small beefsteak which would double the work normally required of the kidneys, might be a matter of serious moment when these organs are crippled by disease. Certainly, the constant aim should not be to tax the kidneys to the limit of tolerance but to diminish the work required of them to the lowest minimum.

Game, cold storage meats and such putrid foodstuffs as limburger cheese are so highly injurious in renal disease that in cases in which the renal efficiency is considerably reduced a single meal of such food may be sufficient to overwhelm the crippled organs completely and produce a fatal uremia. Many a Christmas, New Year's or Thanksgiving feast has been the introduction to a tragedy of this sort.

Meat extracts, animal broths and bouillon are especially harmful. In an interesting paper on the treatment of Bright's disease, (Bulletin General de Therapeutique), Sapelier, an eminent French physician of Nanterre, in prescribing the dietetic regimen suited to patients suffering from this disease absolutely prohibits the use of bouillon and all juices and extracts of meat, remarking of bouillon that it is a "veritable solution of ptomaines."

Smoking is particularly injurious to the kidneys, nicotine acting as an irritant to these organs. Gy, of Paris, has shown by experiments upon animals that the effect of the poisons of tobacco smoke is to cause degeneration of the kidney cells. Doctors who permit their patients to smoke moderately simply say to them: "You may damage yourself a little but do not do yourself as much harm as you might." The late eminent Professor Lauder Brunton maintained that in order to be benefited by renouncing the use of tobacco the smoker must abandon the habit in toto, so that the last trace of nicotine may be eliminated from the body in order to give the recuperative forces an opportunity 
to rally and so far as possible repair the damage done. This agrees with the writer's experience.

Doctors who prohibit smoking to their nephritic patients in so doing do not expect to effect a cure of the disease but only to postpone the patient's funeral. A longer and more certain postponement would certainly have been possible if the smoking had been prohibited earlier, before the appearance of the kidney disease; in other words, prevention by the avoidance of the poison habits is a far more effective means of combating disease of the kidneys and other grave maladies than abstaining after the mischief has been done.

Condiments of all sorts contain essential oils which are chiefly eliminated through the kidneys, and are objectionable because of their irritating properties. Chlorid of sodium should be used very sparingly and in cases in which a dropsical condition exists a salt-free diet should be employed. Tea and coffee, cocoa and chocolate are objectionable for the same reason as meat and meat broths. A single cup of coffee contains on an average three grains of caffein, which is practically identical with uric acid. When coffee is taken, the uric acid of the urine is increased (Mendel). Three cups of coffee daily more than double the amount of work normally required of the kidneys in the elimination of uric acid. The toxic element of cocoa and chocolate is theobromine, an alkaloid very closely related to caffein, which produces when taken into the body essentially the same effects as would be produced by an intake of uric acid.

It is important to remember in the treatment of chronic Bright's disease that every patient suffering from this malady has not only a diseased condition of the kidneys but also of the heart and blood vessels. The affection of the heart is in many cases even more immediately dangerous than the changes in the kidney. In these cases care must be taken to avoid administering too large quantities of fluids, since the weakened heart may be overloaded and dilation may occur. When the heart affection is serious and there is a tendency to dropsical accumulation the amount of fluids should be limited to about three and one-half pints in persons of average size. The food taken may contain a pint and a half of water in addition to that taken as fluid. The amount of urine should be about equal to that of the fluid taken as such. Once a week the tissues should be flushed by doubling 
the intake of fluid. This is important to prevent accumulation of tissue wastes.

The writer is quite in accord with the dictum of Doctor Vaughan, of the University of Michigan, who holds that in Bright's disease the blood stream carries to the kidneys toxins which give rise to degenerative changes. To suppress these toxins, Vaughan forbids all flesh food and commends a diet consisting largely of potatoes, green vegetables, fresh fruits, cream and butter, and cereals in moderation.

It is perfectly true, as Vaughan suggests, that after a few weeks patients lose all desire for meat and eggs. This is quite natural because of the fact that the appetite for a high protein diet is an artificial one. The appetite for meat quickly falls away for the reason that man is not naturally a meat eater. Vaughan affirms that even a hard-working man can easily live on a diet from which both eggs and meat are excluded.

As Vaughan observes: "The chief value of vegetarian food is that it is a low protein diet, containing less extractives and purin bases than the ordinary mixed diet." It is for this reason that it is to be recommended not only for persons who suffer from Bright's disease but for those who do not wish to become the victims of Bright's disease and wish to keep their kidneys intact to a ripe old age. Sooner or later these delicate and essential mechanisms fail through the development of senile changes. Why wait until the catastrophe comes? Wise forethought well suggests that prophylaxis is better than cure, but in the case of Bright's disease the damage is irreparable and cure impossible. Prevention is the proper thing. Hence the advantage of the biologic diet.

Special care should be taken to avoid the eating of raw or insufficiently cooked white of egg. Egg albumen in this form is almost wholly indigestible and greatly promotes putrefactive changes in the colon. If eggs are eaten, it is best to reject the whites for the reason that in cases of nephritis egg albumin seems to be less readily appropriated than most other forms of protein.

The intake of protein by a person suffering from Bright's disease should not be more than one calorie per pound of body weight.

Care should be taken in making up the bill of fare of a nephritic to see that the base or alkaline elements of the food are strongly predominant (see Index). This will not only require the 
elimination of all flesh foods but also decided restriction of cereals, which should never be made the staple dietary in cases of nephritic disease for the reason that their exclusive or dominant use tends to diminish the alkalinity of the blood and tissue fluids. Potatoes and other vegetables should largely take the place of cereals.

The use of mineral waters is to be avoided in nephritis for the same reason that medication of all sorts is interdicted. The ingredients of mineral waters, like other drugs, only add to the burden required of the kidneys and thus hasten the time when their margin of safety will be wholly consumed and they will no longer be able to keep the blood free from toxins and these noxious elements will be permitted to accumulate to such a degree that uremia will supervene and death occur.

In cases of renal sclerosis which accompanies arteriosclerosis or cardiovascular-renal disease it is sometimes necessary to limit the amount of water for periods of one to three days. This measure is especially necessary in cases of general dropsy with dilated heart (see Dry Diet). In nephritis with dropsy, salt should be wholly excluded from the dietary until the dropsy disappears. It may then be used in very great moderation. The "buttermilk cure" and the "skimmed milk cure" for renal dropsy, first suggested by Karell, are chiefly valuable because of the small amount of liquid and very small amount of salt which they supply. This method really seems to present no advantage which cannot be as well secured by a diet consisting chiefly of fruit juices and fruit purées (the Fruit Regimen), which has advantages over the Karell method in the small amount of protein supplied and the laxative effects secured.

In acute nephritis, the diet of the patient for several days may be largely, or even wholly, confined to fruit juices or sugar water prepared by the addition of two ounces of malt sugar to a pint of water, or in place of malt sugar the sugar of raisins may be used. Soak four ounces of raisins in a pint of water, stew for 20 minutes, pour off the juice and increase to one pint. A pint of raisin juice prepared in this way contains 200 calories of sugar which is ready for immediate absorption. Lactose or milk sugar may also be used.

Von Noorden is a very strong advocate of fruit in renal disease, as is well known by all who have visited his clinics. He says in his "Metabolism": 
It is the theory of many physicians that nephritic patients should be given a diet poor in proteins (Senator, F. Hirschfelt, Albu, and others.) It is said that such a diet puts less strain on the diseased organs. No doubt this is true, for acute nephritis and for the acute relapses of chronic nephritis, as I have emphasized elsewhere. For two years I have been of the opinion that in acute and dangerous cases no nitrogen should be given in the food. I have given nothing but sugar water and fruit juice for from three to eight days at a stretch (often 200 to 300 grams of sugar daily). It was my impression that this form of treatment was very useful, and that uremic symptoms were obviated, or if already present, were removed.

in acute nephritis on the one hand, and in acute inflammatory exacerbations of chronic renal disease, a large protein intake undoubtedly exercises an injurious effect on the albuminuria. Even a milk diet is too rich in protein for this form of kidney trouble. When the inflammation is at its worst I give nothing but sugar-water (about 150 to 200 grams daily) and strained rice broth, with cream or butter added. This regimen reduces the work done by the kidneys to the lowest limit. I add milk to the diet only when the patient begins to be convalescent.

The fruit regimen is still more efficient in cases of renal disease than the fruit diet of Von Noorden, because of the increased intestinal activity and change of the intestinal flora, which lessens the absorption of toxins and the work of the crippled kidneys.

In the employment of the fruit regimen it is important to remember that prunes, cranberries and plums must be avoided because they contain a considerable amount of benzoic acid which the body is not able to utilize but must be eliminated through the kidneys. Frothingham and Smillie urge the importance of a low protein diet in chronic nephritis to prevent an increase of nitrogenous wastes in the blood. Goodall observed that in nephritic patients on a low protein diet the nitrogenous wastes, the socalled non-protein nitrogen of the blood, was diminished. $\mathrm{He}$ also observed a simultaneous fall in blood pressure.

\section{Bichlorid Nephritis.}

The almost complete urinary suppression induced by this toxic nephritis quickly leads to an accumulation of nitrogenous wastes in the blood. The indication is to place the patient for a few days upon a strictly carbohydrate diet of rice or potato gruel with sugar water by mouth, by enema, and if necessary, by intravenous injection of glucose. It should be given in such 
quantities as will give the patient 1,200 to 1,500 calories daily. By this means, the patient's strength may be maintained and the heart action sustained, while the mercury is being eliminated. The patient should receive 3 to 4 pints of water or liquid in twentyfour hours.

In cases of poisoning by corrosive sublimate the stomach should be washed out, and after the washing an ounce of lactose should be dissolved in a pint of milk and water, equal parts, and introduced into the stomach.

\section{Water-Drinking.}

Modern clinical experience has shown that the old practice of indiscriminately recommending the sufferer from Bright's disease to drink large quantities of water is not altogether safe, since in numerous cases the liver loses its ability to eliminate water even while still able to deal efficiently with the waste substances brought to it by the blood stream. The water intake of the average person for each twenty-four hours is about four pints, of which from a pint to a pint and a half will be taken as food, which, in general, is about three-fourths water. From onefourth to one-fifth of the fluid intake is eliminated by the lungs and the skin; the balance, or about three-fourths, should appear in the urine. When the kidneys are unable to eliminate this amount of water, it is not only useless but harmful to increase the water intake. In such cases, edema is likely to appear and the water intake must be eliminated.

The exclusion of salt from the dietary and a very low protein diet will greatly lessen the water requirement, as it will greatly reduce the amount of solids which the kidneys have to eliminate. If the amount of salt consumed is reduced by one gram, the intake of water may be reduced one pint and the kidneys will have this much less work to do. Before a patient is encouraged to drink an increased amount of water, care should be taken to make sure that he is able to eliminate the amount of water which he is already taking.

\section{Diet in Cases in Which One Kidney Has Been Removed.}

In cases in which one kidney has been removed, a strict antitoxic, basic and laxative diet (see Index) should be carefully adhered to. On a high protein diet the amount of urea excreted by the kidneys is 33 to 37 grams, or an ounce and a 
quarter. On a heavy meat diet the amount may rise to 100 grams, or three and one-third ounces. On a meat-free diet the urea output falls to 9 grams, less than one-third of an ouncè.

From these facts it is evident that the kidneys of the meat eater are compelled to do from two to five times as much work in the elimination of urea as is required of the kidneys of a low protein feeder. Since Folin has shown that all the urea excreted by the kidneys represents surplus protein, that is, food protein which has never been assimilated and hence has not been utilized for tissue building, it will readily appear that a high protein diet is one of the most efficient methods by which the kidneys may be prematurely worn out and life thus abbreviated. By means of careful dieting, the work of the kidneys may be reduced to onehalf or even one-third that required by the ordinary dietary and thus, when one kidney has been removed, life and useful activity may be prolonged for many years; whereas, without the relief afforded by careful dieting, the single kidney would soon be worn out and the patient's career brought to an untimely end.

\section{ALBUMINURIA}

When albuminuria occurs in connection with a nervous disorder of some sort, such as epilepsy or apoplexy, the patient should be placed at once upon a low protein and salt-free dietary. When albumin appears in acute Bright's disease, all food except fruit juices and malt sugar should be withheld for a short time. In acute nephritis occurring as a complication of scarlet fever and in the acute albuminuria of pregnancy, the same dietary should be adopted for a few days until the intensity of the disease is controlled.

The statistics of life insurance companies show that the life expectancy of persons who have suffered from typhoid fever or other severe infectious maladies is diminished to a marked degree. In a very large number of cases of chronic nephritis a history of some acute infection such as scarlet fever, measles, typhoid or smallpox is obtainable. Evidently the kidney is injured in these cases and so a rational preventive procedure would be to place every such person, even in youth, on a strictly antitoxic diet, excluding meats of all sorts, with tea, coffee and excess of salts, and to take care to change the intestinal flora and to keep it 
changed. A strictly basic diet should be followed. These precautionary measures, if adopted in early life, would doubtless prevent the later development of nephritis in many cases.

\section{OXALURIA}

Besides eliminating from the diet foods rich in oxalic acid, meats of all sorts should be discarded and special care should be taken to avoid the use of hard water. An antitoxic laxative diet should be permanently adopted. Pieplant, tea and coffee must be scrupulously avoided and greens, especially spinach, must be parboiled.

\section{DIET IN ABSCESS OF THE KIDNEY}

When the kidney is the seat of abscess or of infection with rise of temperature, the dietetic indications are the same as in fever. It is especially important to keep the bowels active by a laxative diet and to change the flora so as to lessen the work of the kidneys as much as possible.

\section{GRAVEL AND STONE}

This condition is most often due to faulty metabolism. The 3lkalinity of the body fluids is diminished and there is excessive formation of oxalic acid and other organic acids which, when present in concentrated urine, lead to the deposit of insoluble salts in the kidney or bladder. A diet consisting chiefly of cereals and meats with a small intake of water strongly predisposes to this condition. It only requires the absorption of a small quantity of water from such concentrated urine to cause the deposit of a minute amount of solids in the urinary tract, and when this is done the formation of a calculus has begun. It has been noticed that calculi do not form in domestic animals when they are allowed to graze at pasture in the summer or are fed liberally on potatoes, ensilage, turnips, pumpkins and other foods rich in alkaline salts and containing an abundance of water. Hard water tends to increase the concentration of the urine and so encourages the formation of calculi, which accounts for the frequent occurrence of these concretions in both men and animals in limestone regions.

Many eminent authorities, including Klemperer, insist strongly that all foods containing uric acid or oxalic acid must be 
strictly prohibited. This interdiction includes not only meats of all kinds but tea, coffee and cocoa as well, also sorrel, rhubarb, and even spinach and beans, although beans and spinach may be rendered wholesome by parboiling.

Cereals should be eaten sparingly and potatoes should be used largely instead of bread because of the alkaline salts which they supply. Fruits should be freely used for the same reason. Muskmelons are particularly beneficial.

When magnesia is present in water in considerable quantity, the tendency to formation of calculi is increased because of the readiness with which magnesia is precipitated in the presence of ammonia, which is developed in the bladder in cases of catarrhal inflammation of the mucous membrane, and is likely to occur as the result of infection with the colon bacillus and other germs.

Intestinal asepsis is important in these cases because of the more or less crippled condition of the kidneys. The flora should be changed and the colon kept free from putrefaction by a laxative and antitoxic diet.

\section{PYELITIS}

In this disease there is an infection with an excessive production of mucus and other matters in the pelvis or vestibule of the kidney with the consequent tendency to accumulation of material which is likely to become more or less obstructive. On this account it is important that the volume of urine should be very considerably increased so that the secretions may be diluted and washed away. By increasing the volume of urine the toxic matters which it contains will be diluted and hence less irritating to the hypersensitive structures of the kidney. Irritants of every sort should be eliminated from the diet, as well as everything which will tend to increase the work of the kidneys; hence tea and coffee, condiments, as well as meats of every description, cheese and more than minute quantities of salt, should be avoided. The more completely salt is eliminated from the diet the better. The dietary in general should be the same as that for nephritis. It is well to substitute potatoes largely for cereals because of the large amount of alkaline salts which may thus be introduced into the circulation.

In a large number of cases, the cause of the infection is the colon bacillus, and the reason for the infection is a lowering of the vital resistance of the body and the entrance into the blood 
stream of an excessive number of bacteria absorbed from a colon filled with highly virulent organisms. Cases of this sort require the services of a specially trained expert.

The diet should consist of gruels, purées, fresh vegetables, fruit juices and fresh fruits, with bran and other food laxatives. The patient should take a half glass of water every hour so as to keep a steady stream of liquids passing through the kidneys.

\section{INFLAMIMATION OF THE PROSTATE GLAND}

The indications are the same as for disease of the kidneys and cystitis. Meats and condiments tend to increase the irritation of the prostate. Constipation greatly aggravates this disease and on this account the dietary should be highly laxative, containing large amounts of bran, at least half an ounce to an ounce a day, with greens and other roughage and a sufficient amount of paraffin oil to secure three full and soft bowel movements daily. The enema should be used daily if necessary. Laxative drugs should be avoided.

\section{DIET IN URETHRITIS - GONORRHEA}

Condiments of every description, and especially asparagus, rhubarb and other foodstuffs which contain irritating substances which find their way out of the body through the kidneys must be avoided. Water should be taken very freely, at least a glassful every hour, to render the urine neutral. Beer, ale and other alcoholic liquors and tea and coffee should be avoided, also meat dishes, pickles and other unwholesome foodstuffs of all sorts. The diet should be essentially the same as in cases of calculus.

\section{CYSTITIS}

In cystitis the indications are the same as in urethritis. By copious water drinking the urine may be diluted so that the irritation produced by contact of this excretion with the inflamed mucous membrane of the bladder will be lessened. When urination is frequent and painful, copious water drinking is especially important. A glassful every hour is not too great a quantity. The diet should be laxative and antitoxic (see Index). When the urine is slightly acid, fruits and fruit juices should be freely used, with the exception of prunes, plums, grapes and cranberries. Potatoes should be used instead of cereals. 


\section{Diet in Diseases of Women}

While there is no specific diet indicated for the disorders peculiar to women in general, it is to be noted that women are, on account of their sedentary life, much more subject to constipation than are men. It may be said, also, that many of the distresses from which women suffer should be attributed to the intestinal toxemia, colitis and other colonic infections which are the result of constipation rather than to disease of the sex organs. This is especially true of the headaches, backaches, lack of appetite, lassitude and general lowered vitality which are most often charged to disease of the pelvic organs and various local affections, such as leucorrhoea, cervicitis (erosion), menstrual pains and irregularities and urinary affections, which are in many cases either a direct or indirect result of constipation.

In general it may be said that practically all civilized women need to adopt more biologic habits in diet. Tea and coffee must be given up. The confections and pastries should be replaced by coarser and simpler dishes. Bran should be made a part of every meal. Greens and fresh fruits and vegetables should figure largely in every day's bill of fare. The laxative and antitoxic and blood building dietaries (see Index), may be safely recommended in nearly all cases. The average American woman is anemic. Milk and greens should take the place of beefsteak. Coarse graham or bran bread should replace white bread and soda biscuits. More and better blood acquired by eating and assimilating appropriate food in ample quantity will do more to restore an invalid woman to health than all the drug tonics and widely exploited nostrums to be found in the drug shops.

\section{DIET DURING PREGNANCY}

The expectant mother needs to be well fed for the sake of her unborn child as well as for her own welfare. The intake of food must be increased, and Nature has provided for this by giving to woman a stomach, liver, and other digestive and eliminative organs notably larger than in man. The dietary of the expectant mother should include especially foods rich in lime, iron, 
vitamins and roughage. The body of an infant weighing 6 pounds contains more than an ounce of lime, all of which must be stored up during the months of gestation and most of it within the last three months. This would require an average daily addition of five grains of lime, or one-third the ordinary ration of 15 grains. This extra amount of lime will be furnished by three ounces of spinach and 6 ounces of milk.

Again, the infant's body contains about 10 grains of iron, nearly half of which is stored in its liver for use in blood-making during the nursing period, necessary because of the fact that milk contains only a very small percentage of iron. It is thus evident that the pregnant mother should take care to make her daily menus rich in food iron. The deficiency cannot be made up by swallowing iron pills or tonics or drugs of any sort. The iron must be furnished by suitable foodstuffs. Fortunately, Nature supplies an abundance of iron in greens of many sorts (Table XXIX). Six ounces of spinach added to a varied diet of fruits, whole grain products and fresh vegetables will supply the full amount of iron required. Numerous other foodstuffs are also rich in iron, even richer than is beefsteak, while affording a superior kind of food iron (Table XII).

The expectant mother should take at least a full quart of milk daily. The diet should be substantial. Pastry and tidbits as well as tea, coffee and condiments must be strictly avoided. Flesh foods are not needed, and are likely to do much harm. The same is true of meat extracts and botillons.

The intake of food by the expectant mother near time for confinement should be at least 10 per cent. greater than that of the non-pregnant woman under like conditions. A tendency to overfatness will demand restriction to actual requirements.

Free water drinking is likewise important as an aid to free elimination. The water intake, aside from the water of the food, should be at least three or four pints daily.

Especial attention must be given to the bowels. By three daily bowel movements and care to thoroughly empty the colon every day, intestinal putrefactions may be suppressed and the danger of serious complications greatly lessened. The expectant mother should adhere closely to an antitoxic and laxative diet (see Index), should make free use of bran, Psyllium and paraffin oil and if necessary should resort to the use of the daily enema. 


\section{The Vomiting of Pregnancy.}

The vomiting of pregnancy has been shown (Titus and others), to be due to a carbohydrate deficiency resulting in acidosis. The placenta stores carbohydrates for the fetus. When the mother eats too little carbohydrate, the supply stored in the placenta is soon consumed, the liver store is exhausted, and the result is an improper use of fats, and acidosis, which causes nausea and vomiting. The vomiting occurs especially in the morning because of the long interval since the last meal, so that the carbohydrate stored in the liver is consumed.

When the glycogen of the liver is used up, the liver cells are attacked and undergo degeneration, so that great and permanent damage may be done in severe and protracted cases.

Morning sickness may be prevented by taking care to supply the pregnant woman with an abundance of carbohydrate. Malt sugar and lactose or lacto-dextrin are especially suitable. The diet should be decidedly basic; that is, meat and eggs should be wholly discarded and cereals should be used sparingly. Potatoes should take the place of bread and breakfast food. Such foods as pickles, as well as vinegar, tea and coffee, should be avoided. Fats should be used very sparingly. The bowels must be kept active by the use of fig-bran, paraffin, psyllium seed and other accessories which combat constipation. The enema should be used at night to insure complete evacuation of the colon.

To relieve morning sickness, the patient should be given liberal quantities of carbohydrates, especially malt sugar and lactose, or lacto-dextrin, one ounce every three hours.

Copious water-drinking is required; four to six pints daily, (including all liquids). Water may be given by enema if necessary.

Food may be introduced by the duodenal feeding-tube if it is not retained by the stomach when given in small quantities at short intervals.

In severe cases, 5 per cent glucose solution may be given intravenously or by hypodermoclyster.

After the vomiting has ceased, feeding in small amounts at frequent intervals should be continued.

\section{DIET OF THE LYING-IN WOMAN}

The lying-in period is one of the most critical in a woman's life experience. By proper feeding, serious dangers may be 
warded off. In general, the directions already given for the feeding of the expectant mother should be followed by the lying-in woman. During the first two days, the diet should be light, consisting chiefly of ripe fruits, fruit juices, purées of vegetables, oatmeal porridge with bran, buttermilk and baked or mashed potatoes. Spinach is especially indicated. Meats must be wholly excluded and eggs may be omitted with advantage for a few days. Three ounces of milk sugar three times a day for the first four or five days aid in keeping the intestinal flora in good condition. The aim should be to make the diet sufficiently laxative to insure three bowel movements a day without the use of laxative drugs other than paraffin oil bran or agar and psyllium seed. Copious water drinking, two or three quarts in twenty-four hours, greatly protects the kidneys by encouraging elimination of toxins.

Some authors forbid the use of fruits for lying-in or nursing mothers, particularly very acid fruits, because they are likely to produce colic in the infant. It is doubtful whether this notion is well founded; but when any fruits are found to disagree with the mother or the infant they should naturally be excluded from the diet.

\section{DIET IN ECLAMIPSIA}

The colon should be thoroughly emptied by repeated enemas. Milk sugar should be given in doses of three ounces three times a day for a few days to change the intestinal flora. It is well also to administer enemas containing two or three ounces of milk sugar or malt sugar to the pint of water to suppress intestinal putrefactions. Fasting is a measure of value. It is especially important to suppress proteins, particularly animal proteins. Eggs and meats of all kinds should be carefully suppressed. Fruit juices and purées and sugar water are the only foods permissible. 


\section{Dietetic Manag ment of Fevers}

The recognition of the fact that in fevers a state of general acidosis exists, has led to a radical change in the general method of dietetic management of this class of maladies. The old plan of starving fever patients was most unwise as it encouraged to a high degree the development of acidosis. Equally objectionable was the gross feeding with meats and miscellaneous foodstuffs advocated forty or fifty years ago when Brand was demonstrating the value of the hydriatic method of treating fevers, especially typhoid fever. Incomplete oxidation and acidosis may result either from excess of food or from starvation.

Probably no other measure can do more for the amelioration of the patient's sufferings and his safeguarding against complications than appropriate regulation of the dietary. The normal functions of food are to supply the body with fuel and with material for tissue repair. Certain foodstuffs, particularly proteins, when supplied in more than the amount needed for repairs act as stimulants or excitants and cause an enormous and most unprofitable loss of energy, far exceeding in amount that actually devoted to useful work. Carbohydrates are the most economical of foodstuffs, as they require the least expenditure of energy, and give most in return for the energy expended. Malt sugar and the sugars of fruits and fruit juices are the foods par excellence for fever cases because of the ease with which they are appropriated and because they are least stimulating and also supply alkaline residues which neutralize the disturbing acids always present and leave behind no toxic residues.

Milk sugar and dextrin or lacto-dextrin are the best carbohydrates for combating the intestinal infection and putrefactions, for the reason that they are slowly absorbed and pass quickly through the small intestine, and so are certain to reach the colon and to arrive there more quickly than other sugars. Lactose and dextrin are the best foods to encourage the growth in the colon of the protective organism B. acidophilus, the natural guardian 
of the colon against the dangerous putrefactive organisms always present in the colons of adult human beings.

Lactose, or lacto-dextrin, may be used in liberal quantities; that is, six to ten ounces a day, with marked benefit. Torrey showed that by feeding these carbohydrates the flora may be changed in typhoid fever, greatly to the benefit of the patient. Either lactose or lacto-dextrin may be used with advantage in place of ordinary sugar for sweetening lemonade and other dilute fruit juices.

In the feeding of fever patients, one of the first considerations is an abundance of water to dilute and carry away the toxins through the kidneys. From four to six quarts of fluid are needed daily, by mouth or per rectum or both. This is a matter of the highest importance. A glassful of water should be given every half hour when the patient is awake. The patient often objects, but must be induced to take the amount required. Weak lemonade or diluted fruit juices, of any agreeable sort, are ideal fever beverages. Lacto-dextrin, two ounces to the pint, is a pleasant and perhaps the best of all food beverages for a fever patient.

Another indication is to combat the tendency to emaciation. This is accomplished by rest, control of the temperature, and the liberal feeding of carbohydrates in the form of cereals, fruits and fruit juices and malt sugar.

On account of the tendency to retention of chlorids in the tissues, salt should be as far as possible eliminated from the diet. Loss of lime, iron and vitamins should be made up by the use of fresh vegetables, greens and vegetable broths. The liver and kidneys are compelled to do an enormous amount of overwork in fever, in the destruction and elimination of poisons produced by the fever. On this account the greatest care must be taken to avoid adding burdens to these overworked organs, and to lessen their work as much as possible by supplying an abundance of water and by withholding from the patient anything likely to increase renal work. Condiments of all kinds-mustard, pepper and vinegar-must be strictly prohibited. Alcoholic liquors and tea and coffee are especially damaging because of the poisons which they contain. For this reason, also, meat broths, extracts, beef tea, chicken broth, etc., must be strictly avoided, as well as meats themselves. Because of the special tendency to intestinal putrefaction in cases of fever, all foods which have a tendency 
to putrefy must be prohibited. This is the reason why not only meats of all kinds but eggs must be discarded.

The substitution of malt sugar for cane is important for the reason that this sugar contains much iron, lime and vitamins, not found in cane sugar. Purées and other dishes prepared from dates, figs and raisins should take the place of dishes sweetened with ordinary sugar because of the richness of these fruits in iron (see Table XII). Change the intestinal flora with milk sugar or lacto-dextrin.

Special attention should be given to the colon in all cases of fever. Even when the bowels are loose, the colon should be emptied by an enema twice a day in order to insure against fecal accumulation. Except in enteric fevers, bran, agar, mineral oil and laxative foodstuffs should be used in quantities sufficient to insure efficient action of the colon. Even in typhoid there seems to be no good reason why agar and mineral oil may not be used, and even cooked bran when constipation is present.

The amount of food given in fevers should be sufficient to prevent great wasting. There is perhaps at the present time a tendency to overfeeding. This should be avoided for the reason that the liver, the kidneys and associated organs are so greatly overtaxed that all rational means should be employed to lessen the work required of these poison-destroying and eliminating oragns. No harm will be done if the patient loses a few pounds in weight. The loss will consist only of adipose tissue and will be readily made up when convalescence is established. It is only when the loss of flesh is carried to such a degree that the muscles and other living tissues are attacked that harm is done.

Special care should be taken to avoid an excess of protein as protein more than any other food element increases the work of the liver and kidneys, since any excess of protein must be treated like so much waste matter and is passed directly out of the body without rendering any service. It also encourages intestinal putrefaction, which has a tendency to increase during fever and which enormously increases the burden of the liver and kidneys. The free use of lactose or lacto-dextrin will greatly aid in suppressing intestinal putrefactions.

In the selection of food great care must be taken to see that there is no deficiency of vitamins, which are notably lacking in the ordinary fever diet of gruels, broths, etc. Purées of green vegetables and fruit juices, especially orange juice, fresh or 
stewed tomatoes and yeast extracts (Savita), should be freely used not only occasionally but several times a day. On account of the diminished secretion of hydrochloric acid in the stomach, acid fruits are particularly advantageous and may be freely used without injury.

The amount of food eaten should be fully equal to that of the base ration of the patient; that is, the amount required for a person lying in bed taking no exercise. This would be about 12 calories per pound of body weight. As the temperature falls, the amount of food may be increased. When perspiration occurs feeding should be temporarily discontinued or greatly reduced.

After hemorrhage the feeding should be discontinued for 10 or 12 hours, after which the first food given should be rice gruel.

As little salt as possible should be added to the food of fever patients. This is important for the reason that the kidneys are already overtaxed with the elimination of the enormously increased body wastes together with the specific poisons which are produced by the bacterial cause of the fever.

\section{Foods Which May be Used in Fevers.}

All fruit juices, fresh if possi-

ble. Lemonade, sweetened with

lactose or malt sugar.

Fruit soups

Fruit purées

Fresh, ripe fruits

Banana purée

Soaked prunes

Blanched almonds

Nuttolene

Malted nuts

Almond cream

Malt sugar
Meltose
Rice
Wheat flakes
Gruels of oatmeal, cornmeal,
granola
Lettuce
Vegetable broths
Vegetable purées
Acidophilus buttermilk
Lacto-Dextrin
Buttermilk

\section{Foods to be Avoided in Fevers.}

Foods which must be strictly prohibited in fevers are meats of all sorts-fish, flesh, fowls, oysters, etc.-eggs, mushrooms, pickles, cheese, condiments, much salt. 


\section{Acute Infectious Fevers.}

The dietetic indications in the several infectious fevers are essentially identical. Fever diet is indicated in all. In scarlet fever and diphtheria, bland foods must be employed when the throat is sore. Rice gruel is especially indicated. In cases of post-diphtheritic paralysis in which the patient is unable to swallow, the food must be administered with a small feeding tube. A laxative and reconstructive diet is indicated as soon as the fever patient is able to eat. If for some days during the height of the disease the patient refuses gruels and similar foods, strength may be maintained by the free use of sugar water. The latter is prepared by adding two ounces of malt sugar or milk to a pint of water. The sugar water may be taken by the stomach or by enema, or both.

In scarlet fever the amount of protein in the diet should be very greatly reduced. Eggs must be wholly excluded and milk should be used very sparingly if at all. No harm will be done if the patient's diet consists almost exclusively of fruit juices and lactose or malt sugar for a few days.

Whooping Cough. While the diet in this disease is essentially the same as in other fevers, a special difficulty arises in the fact that the violent coughing often induces vomiting and loss of a large part of a meal which may have just been eaten. Any disturbance of the stomach likewise has a tendency to increase the coughing, so a sort of vicious circle is formed. The food must be appetizing, but bland and lukewarm. If either hot or cold, violent coughing may be induced with vomiting. Gluten gruel, vegetable broths, rice, rice gruel, oatmeal gruel, cream toast, brose and breakfast cereals with bran are foods especially suitable to this condition. When the coughing is violent, the child should be kept in bed for two or three days if necessary until the paroxysms are less intense. The patient should be kept in the open air practically all the time.

Poliomyelitis. The dietetic indications are the same as in typhoid and other fevers.

Other Fevers. The regular fever diet is also indicated in yellow fever, malarial fever, relapsing fever, sleeping sickness, and plague. In malarial fevers care should be taken to avoid giving food just before the time of an expected chill.

In smallpox, as in scarlet fever, the protein should be reduced very low because of the great tendency to nephritis. 
In foot and mouth disease, rice will be especially grateful to the patient as it is bland and absolutely unirritating and can be swallowed with less distress than almost any other food.

Cholera. During the acute stage of the disease, the patient should take nothing but water or water containing a small proportion of sugar, say an ounce of malt sugar or lactose to the pint. Notwithstanding the persistent vomiting the patient should drink copiously. The effect will be to dilute the poisons of the stomach and to wash out infectious material. At the same time large enemas should be administered. Cold rubbing combats shock and promotes the skin circulation. If vomiting and purging continue to an extreme degree, liquids may be introduced by intravenous injection of glucose in Ringer's solution, and it is possible in an extreme case that life may be saved by transfusion of blood, an operation which has now become quite common since the dangers and inconveniences have been practically eliminated by the discovery of a method by which a suitable donor may be selected. The intravenous injection of dextrose is the most efficient method of combating acidosis. Milk sugar with B. acidophilus in larger doses should be tried.

Typhoid Fever. This disease presents typical indications for the fever diet. Alcohol, eggs and animal broths, as well as meat and fish should be prohibited in this disease. Rice, strained oatmeal, granola, toasted flakes of various sorts, zwieback, stewed fruit, fruit juices, and especially purées of greens, potatoes and other vegetables should be given the patient in such quantities as to furnish about 2,000 to 2,500 calories daily. When this is done, the great loss of flesh which often occurs in this disease will not take place, the disease will run a shorter course and convalescence will be more rapid.

Torrey has shown (1915) that by the feeding of 8 to 10 ounces of milk sugar (900-1100 calories) the intestinal flora of typhoid fever patients may be changed from the ordinary, mixed type with putrefactive flora dominant to an acidophile flora with $B$. acidophilus dominant. Such a change is exceedingly advantageous to the typhoid fever patient by the suppression and absorption of putrefaction products which increase the labor of the kidneys, already enormously overtaxed in dealing with the typhoid toxin, the specific product of the typhoid bacillus. Excellent results may be obtained by administering large doses of milk sugar or lacto-dextrin. The latter is preferable to lactose 
for the reason that it is more agreeable to the patient, less liable to cause disturbance of the stomach and presents, besides the carbohydrates, lactose and dextrine, the antiscorbutic vitamin $C$. This method not only changes the intestinal flora but supplies the patient with a considerable amount of nutritive material of exactly the sort needed as has been shown by Coleman.

Milk is not a good routine diet for use in typhoid fever. It often promotes intestinal autointoxication through the retention in the colon of considerable quantities of undigested curds. Whey cultures of $B$. acidophilus are especially valuable in typhoid feeding. Cautley commends whey as a substitute for milk. Milk sugar may be added with advantage, two ounces to the pint. Four to six pints may be taken daily. Malted nuts, lentil soup, the fruit soups of the Germans, and savita are of special value.

Typhus Fever. The dietetic requirements of this disease are the same as those indicated in typhoid. Fruit juices, sugar water, malt or milk sugar, boiled rice and oatmeal gruel are the most appropriate foods.

The great loss of water through vomiting must be made good by the regular introduction of water into the colon at frequent intervals. Lactose or malt sugar should be added to the water in proportion of one ounce of sugar to the pint. This sugar water should be taken by mouth in the intervals between the vomiting spells.

Erysipelas. The dietetic indications are the same as in typhoid and other fevers.

Influenza. The diet requirements are the same as in pneumonia and typhoid. Alcohol should be avoided as also meat extracts and broths.

Mumps. The diet should be the same as in other infectious fevers. Food is best taken in liquid form and by the aid of a large feeding tube, which minimizes the use of the jaws. The necessary intake of bran or roughage and paraffin oil for lubrication should not be neglected.

Pyemia. The diet should be strictly antitoxic. Fruit juices and sugar water should be used in sufficient quantity to give the patient 16 to 18 ounces of carbohydrate daily. A carbohydrate diet greatly aids in promoting resistance.

Tetanus. The diet in lockjaw should be the same as that in other febrile diseases. On account of the difficulty in moving the jaws, liquid diet is especially indicated as in mumps. Milk 
sugar and malt sugar should be freely taken in solution in water or in whey. When swallowing is seriously interfered with, food and drink may be introduced into the stomach by means of a small tube passing through the nose or the duodenal feeding tube.

\section{PNEUIMONIA AND PLEURISY}

In lobar pneumonia, bronchial pneumonia and other forms of inflammation of the lung tissue as well as in pleurisy, the fever diet should be employed (see index).

There seems to be at present a decided tendency to overfeeding in pneumonia. Nothing could be more absurd than the advice of some authors to feed pneumonia patients every two hours night and day, giving in the aggregate an amount of food sufficient to support an able-bodied laboring man. Pneumonia is a disease of short duration and on this account the withholding of food almost entirely for two or three days would do no particular harm. It is most of all important to withhold proteins. Carbohydrates may be used freely in the form of fruit juices, malt sugar or, better, lactose or lacto-dextrin. This will furnish just the sort of material the body needs to support the heart action and to combat putrefactive changes with toxin production in the intestine. Gastric acid is low in amount in pneumonia as well as in other fevers, hence fats should be used very sparingly and preferably in the form of cream.

In addition, it should be noted that in inflammation of the lungs, especially in lobar pneumonia, there is a marked tendency to retention of sodium chlorid. Hence, common salt should be omitted from the food until it re-appears in the urine. Meat, meat broths and extracts as well as eggs should be rigorously excluded in this disease. Copious water drinking to the extent of three or four quarts daily is most important.

After the attack is over, the restorative regimen should be employed to aid in the building of blood and tissues and to restore the tissue salts.

In empyema, hay fever and other chronic febrile conditions of the lungs and air passages, the fever diet should be followed closely during periods when there is a rise of temperature, and in the intervals the reconstructive diet should be employed. Milk, cottage cheese, buttermilk, butter, greens, potatoes, rice, fresh 
fruits and vegetables of all sorts, especially in the form of purées and soups, constitute a suitable diet for cases of this sort.

The bill of fare should be varied from day to day so as to promote appetite, but too many different kinds should not be taken at the same meal. The quantity of food should be 25 to 50 per cent. more than the amount needed for a maintenance ration, so as to secure a gain in flesh until the normal weight has been attained or slightly exceeded.

Malt sugar and malted nuts and other malted preparations are especially useful in promoting flesh-building.

\section{Pleurisy with Effusion.}

The diet should be very low in protein, not more than one calorie per pound of body weight. Salt should be wholly excluded from the diet. Suppression of intestinal putrefactions by the use of lactose or lacto-dextrin will greatly facilitate recovery. The bill of fare should be so arranged as to give the patient an abundant supply of vitamins and food salts.

\section{DIET IN ASTHMA}

There are various forms of asthma, all of which are characterized by obstructed breathing and all are unquestionably influenced more or less by diet. A person who is subject to asthma from any cause whatever is liable to experience an attack, especially after overeating. Eating heartily at night is likely to bring on an attack during the night. This is true of persons in whom the real cause of the attacks is reflex, as from adenoids or other diseased conditions of the nasal cavity and in cases of so-called cardiac asthma, as well as in cases in which the disease is primarily due to errors in diet.

In the writer's experience, fully nine-tenths of all cases of asthma may be attributed to intestinal toxemia, or autointoxication. Constipation, with the coated tongue, foul breath, sallow complexion and highly colored urine, which accompany intense toxemia, is a prominent feature in nearly all cases of chronic asthma. Changing the intestinal flora and normal colon activity will secure prompt and permanent relief in a great majority of cases of chronic asthma of adults. The bowels must move three times a day and the colon must be kept so thoroughly free from 
decomposing residues that the stools will be free from putrid residue or ammoniacal odors.

A laxative, antitoxic diet (see Index) and the continued use of lacto-dextrin will suppress intestinal putrefactions and thus relieve the lungs of the irritation produced in the pulmonary mucous membrane by the effort to remove from the blood through this channel offensive gaseous poisons developed in the breath. The fecal odor of the breath in many asthmatics is very pronounced. The attacks become less frequent and appear only when the stools become putrid through errors in diet or neglect to keep the colon free from putrefying residues.

Meats must, of course, be discarded. Eggs, if eaten at all, must be eaten sparingly and great care must be taken to make sure that they are perfectly fresh. It is difficult and requires as assiduous attention to keep the colon free from pernicious organisms as to keep a garden free from weeds.

A warm enema should be taken at night to make sure that the colon is completely evacuated, until constipation has been fully corrected and the colon kept free from debris by three free bowel movements daily. The enema at night does not interfere with the morning evacuation, but, rather, aids by preventing constant over-distension of the colon, by which its walls are weakened by the production of ammonia and other poisonous products which paralyze the bowel, and by leaving in the colon more or less water, which prevents the excessive drying out of the fecal residues. When the lower part of the colon is filled, the contents often become so dried out and hardened over night as to render the morning movement difficult or impossible. Any possible ill effect of the repeated use of the warm enema may be wholly obviated by introducing into the colon a half-pint of cooler water $\left(80^{\circ} \mathrm{F}\right.$.), at least a portion of which may be retained without inconvenience.

In many cases, asthmatic attacks are in the form of anaphylaxis (see Index). Hay fever is a notable example of this sort. Asthmatic attacks may be induced by the inhalation of various substances to which the body has been sensitized, as dust from horses, cats or other animals, the odor from feathers, etc. It is rrobable that many poisons of this sort are produced in the colon.

In many cases, the asthmatic is found to be sensitized to certain foods.

In all cases of asthma Ewald's test for liver efficiency should 
be employed (see Index). A positive reaction is regarded by Ewald as proof that the patient is sensitized to protein. This fact having been ascertained, such sensitivity tests should be applied.

Fish, milk, strawberries and many other foods, have been shown to be the cause of the attacks in individual cases. The writer is very confident, also, that in not a few instances the poisons produced in the colon act in a similar manner. By the application of special tests which are now available, the particular kind of foods which give rise to the attacks may be discovered. These foods should be avoided and by carefully graduated training, the sensitivity should be overcome. Persons who suffer from Bright's disease are frequently subject to asthma, which is also, in the writer's opinion, often associated with impairment of the liver function. In these cases, especial attention must be given to changing the intestinal flora and avoiding toxic foods of all sorts.

Strong coffee and alcohol are recommended by many writers on dietetics for temporary relief in case of asthmatic attacks. The writer is thoroughly convinced that these drugs are highly detrimental and should be carefully avoided in all cases of asthma. When temporary relief is afforded, which is by no means often the case, it may easily lead to the formation of a drug habit the result of which will be to make matters only worse rather than better. 


\section{Diet in Chronic Infections}

PULMONARY TUBERCULOSIS

It must be frankly conceded that there is no specific dietary adapted to tuberculosis as in diabetes and in certain other maladies. The important thing is to build up the patient's nutrition to the highest level possible.

The general indications are for a diet rich in fats and carbohydrates, vitamins, iron and lime, and sufficiently bulky to secure three or four bowel movements daily. When necessary, agar as well as bran should be added to the dietary and mineral oil should be taken before and after each meal.

Malt sugar in the form of syrup, combined with olive oil or butter or cream, is perhaps the most efficient of all food combinations in promoting the development of fat. Malt sugar is much better tolerated and more readily absorbed and utilized than any other form of sugar, and it is tolerated in much larger quantities than is cane sugar. Fats when combined with malt sugar, are much more readily tolerated and do not cloy the appetite as they are otherwise likely to do. In equal quantities, malt sugar syrup (Meltose) and butter make a very palatable combination and one which can be taken at every meal and in considerable quantities, and which will rapidly secure a substantial gain in weight.

On a dietary such as is here suggested, a gain of a pound a day is frequently secured, and the weekly gain for a short time may be as much as eight or ten pounds.

It is more than probable that a deficiency of vitamins in the diet encourages the development of pulmonary tuberculosis and other diseases which result from low vital resistance.

In many cases the milk regimen (see Index) is advantageous. When the tongue is coated and the breath foul, indicating autointoxication, the fruit regimen (see Index), should be employed for a few days to change the intestinal flora before starting the milk regimen.

Buttermilk prepared with the $B$. acidophilus may be freely used with great advantage. About three pints of buttermilk a 
day is required to influence the intestinal flora to a marked extent. Practically every tuberculous patient may be substantially benefited by a change of the intestinal flora. There is no way in which the curative functions of the body can be more efficiently reinforced than by eliminating the poisons arising from the putrefaction of food residues in the colon. By the methods presented elsewhere in this work this may be easily and quickly accomplished.

It is now well known that milk proteins are equal if not superior to meat proteins and are more easily assimilable. One quart of milk daily will certainly supply to any consunptive all the real value he can possibly receive from the usual daily intake of meat, and in better form. Several quarts may be taken if needed.

The tendency of tuberculous patients to renal disease ( 86 per cent.) is a most weighty reason for lessening intestinal toxins by withholding meats.

Says an authoritative writer in the Journal of the American Medical Association: "The favorable course of many cases of pulmonary tuberculosis is interrupted by attacks of intestinal toxemia. The diagnosis of this complication is absolutely necessary for success in the treatment of tuberculosis."

There has been a marked tendency on the part of the majority of those who have given special attention to the treatment of this class of patients to insist upon overfeeding as the most efficient method of combating the wasting disease.

This is certainly a serious error. No patient can be benefited by an amount of food in excess of his needs, that is, an amount sufficient to maintain his weight at the normal average. An excess of food throws an enormous amount of extra work upon the organism. Not only the liver and kidneys are overburdened, but the lungs also are required to do an extra amount of work in the elimination of the oxidized products of the surplus food. Surplus food increases the tendency to a rise in body temperature. This is particularly true of protein, which produces wasteful metabolism to the amount of 30 per cent.

Certain specialists have demanded a very high protein diet, which in a few instances comprised not less than 1,000 calories of protein per diem, or more than five times as much as the normal requirement according to the standards of Chittenden, Sherman and Folin. Some years ago an institution was started in Ger- 
many which undertook to feed the tuberculosis patient on an exclusively flesh diet, and in this instance the diet was not only wholly made up of meat but of raw meat. It is hardly necessary to add that the enterprise was a complete failure, in fact collapsed within six months of the opening.

For many years the writer has urged the employment of a low protein regimen as best adapted to tuberculosis cases. Some of the dangers of the high protein regimen are pointed out in the following paragraphs.

The marked tendency of the tuberculosis patient to loss of flesh and to the development of anemia has naturally directed special attention to the importance of encouraging the fat-building and blood-making processes of the body. But there are other indications which are equally deserving of attention and which should be permitted to influence the dietetic management of these cases. The materials ingested must be metabolized and eliminated as well as digested and absorbed. Incidentally, while undergoing digestion, they may also undergo fermentations and putrefactions which may so change their nature as to render them not only useless as nutrients but toxic and in the highest degree damaging. Hence the welfare of the liver and other toxin-destroying glands and of the kidneys, poison-eliminating glands, must be considered as well as the need of the organism for an increase of adipose tissue.

A simple increase in the thickness of the panniculus adiposus cannot be of any very great advantage to a man whose body has been invaded by a vast army of tubercle bacilli. Before the infection took place there had been a depreciation in vital resistance, a deterioration of tissue and function which made the invasion possible, and with each advance of the disease this weakening of the ability of the organism to battle against its foes is increased. Recovery can be secured only by improving the integrity of tissue and function to such a degree that the ability of the organism to combat its assailants will be raised higher than before the invasion occurred.

The average subject of tuberculous infection presents a number of special pathological conditions which must be considered in preparing a therapeutic regimen. Among these may be especially enumerated the following:

1. Diminished Alkalinity of the Blood (Lawrason Brown). -This signifies diminished vital resistance, diminished efficiency 
of the blood as a germicide, diminished power to develop immunity, diminished power to repair damaged tissues, diminished power to oxidize wastes. Anything which tends still further to diminish the alkalinity of the blood must be to that degree a hindrance, rather than a help, in the battle of the organism against the tubercle bacillus. One of the notable and constant effects of a high protein dietary is to diminish the alkalinity of the blood.

2. Diminished Hepatic Efficiency-In consumption the liver is nearly always seriously crippled. Ulldom declares that passive congestion of the liver is found in nearly every case of pulmonary tuberculosis. This is the natural result of diminished respiratory activity, since the venous circulation of the liver is largely influenced by the movements of the chest in respiration. To the evidences of chronic passive congestion of the liver are added, according to Ulldom, many other evidences of a lowered vital state in this organ, among which are amyloid and fatty degeneration, tuberculous infection, and fibrosis or cirrhosis. Ulldom expresses the opinion that fibrosis of the liver is not due to the tubercle bacillus, but to other etiological factors. Boix and others have shown what these factors may be. According to Boix the toxins formed as a result of putrefactive processes in the intestine are the chief cause of hepatic fibrosis. He was able to produce cirrhosis of the liver in rabbits by mixing with their food cultures of colon bacilli and also by feeding them extracts of feces.

That a high protein ration, that is, a dietary consisting largely of flesh foods, favors hepatic congestion is a fact long known to physicians. A diet which encourages intestinal putrefaction and an abundant anaerobic flora in the intestine must in the highest degree encourage hepatic congestion and various functional and organic changes in the liver, and thereby break down this important defensive barrier and prepare the way for invasion by the tubercle bacilli. It need not, then, be a matter of surprise that Ulldom found in thirty-seven autopsies in cases of pulmonary tuberculosis a tuberculous condition of the liver in 81 per cent. of the cases. Fatty degeneration of the liver was found in 24 per cent. of the cases, and in only two cases ( 5.4 per cent.) was the liver found approximately normal; that is, the liver was found diseased in 94.6 per cent. of the cases. Arnold reported tuberculosis of the liver as an almost constant finding in pulmonary tuberculosis. Simmons found 78 per cent. 
The passive congestion of the liver which naturally results from the overwork of the right heart and the diminished mobility of the chest, together with the special exposure of the liver to infection through the portal circulation and the extra work required of it in metabolizing the excessive nitrogenous waste which accompanies the febrile stage of pulmonary tuberculosis, certainly suggest the importance of guarding the integrity of this vitally important organ in every possible way, and contraindicate a high protein dietary which imposes exaggerated and unnecessary hepatic work.

3. Degeneration of the Thyroid Gland.-Entirely in harmony with the considerations presented above is the interesting fact to which Roger, Garnier, De Quervain, Sarbuch, and others have recently called attention, viz., the occurrence of general sclerosis of the thyroid gland without tuberculous lesions of the gland itself in cases of tuberculosis affecting other parts of the body.

In a case of tuberculosis of the lungs reported by Carnot and Delion, death occurred from tetany, and post-mortem examination showed caseous degeneration of the parathyroids. This condition of the thyroid and parathyroid is attributed to "the effect of the action of the diffusible toxins produced by the tubercle bacilli." It is well known that other toxins, especially those produced by putrefactive changes in the intestine, are capable of producing diseased conditions of the thyroid, the natural result of the overwork of the glands occasioned by excessive saturation of the blood with these toxic substances.

The enormous burden added to the labor of the defensive organs of the body by a high protein diet, and the great injury which must result when these organs are weakened, is illustrated by what happens when certain toxin-destroying glands are removed from the body or rendered functionally inactive. It is well known, for example, that the removal of the thyroid gland (Breisacher, 1890) of a dog is quickly followed by the death of the animal if it is fed upon a meat diet, while life is prolonged indefinitely and the animal enjoys good health when fed upon a diet of bread and milk. When such a dog is fed upon meat which has been well boiled, it suffers less and lives much longer than when fed upon raw meat, evidently because of the removal of poisonous meat extractives in the boiling. Blum holds that the function of the thyroid gland is to neutralize poisons derived 
from the putrefaction of albumin in the intestine. Kishi holds that the thyroid destroys a poisonous substance, a nucleo-protein which is formed in the intestine after the ingestion of meat.

These facts are alone sufficient to condemn a high protein or flesh diet in a disease in which the thyroid gland is already overworked, and in which it is likely to be seriously crippled. Is it not possible, indeed, that the effect of such a diet in a case of pulmonary tuberculosis with degenerated thyroids may be almost as pernicious as in dogs deprived of their thyroids?

In the ingenious Eck fistula experiment an anastomosis is made between the portal vein and the vena cava, a ligature being applied to the portal vein close to the liver, thus cutting out the liver from the portal circuit. A dog thus prepared and fed upon meat dies in three days; when fed upon a diet of bread and milk, the animal lives in excellent health for an indefinite length of time. The urotoxic coefficient of such a dog was found to be increased to nearly three times the normal after the tying of the portal vein, from which the conclusion may be drawn that with a high protein meat diet the poison-destroying work of the liver and of the body is three times as great as on a low protein or non-flesh dietary.

4. Disease of the Adrenals.-Authorities agree in regard to the great frequency with which the adrenals are involved in connection with tuberculous affections of the lungs. The relation of these glands to oxidation, to general vital resistance, and to the toxin-destroying functions of the body gives great significance to this fact. Behring showed that blood serum (sheep's) possessed sterilizing properties far superior to those even of the stronger solutions of carbolic acid and corrosive sublimate, which were formerly employed in surgical operations. This property of the blood being, very probably, largely due to the adrenals as well as the ability of the blood and tissues to oxidize or destroy toxins. It needs no argument to indicate the importance of promoting intestinal asepsis in all possible ways in pulmonary tuberculosis, and especially by avoiding a dietary which must flood the blood and the tissues with toxic products to an extraordinary degree, thus weakening or destroying its bactericide properties.

5. Concurrent Disease of the Kidneys.-Drs. Flick and Walsh state that "nephritis occurs very frequently in tuberculosis." Among the causes they mention the effects of "the toxin excreted through the kidneys from lesions elsewhere." Grancher 
and Martin produced nephritis in dogs by attempts to establish immunity with increasing doses of tubercle bacilli. "The longer the animal lived and the higher the resistance it attained, the greater were the chances of nephritis." Drs. Flick and Walsh regard nephritis as "one of the complications in tuberculosis most to be dreaded"; especially "because it is a complication which comes through the very process which leads to recovery, namely, immunization." These eminent observers assert that "many patients with tuberculosis die by way of nephritis as the real cause of death," and indicate as the symptoms pointing toward nephritis "a pasty skin, unusual fatigue upon slight exertion, shortness of breath, high specific gravity of urine, hyalin and granular casts, albumin, etc."

The above observations are certainly impressive. They suggest most emphatically that the tuberculous patient should be treated and fed not simply with reference to disease of the lungs, but with reference to the possible complications involving other vital organs which may occur, especially disorders of the kidneys and also of the liver because of its close association with the kidweys in protecting the body against toxins.

The Third Annual Report of the Phipps Institute 1905, contains an exceedingly interesting resume by Joseph Walsh of the autopsy findings, from which it appears that 84 per cent. of the cases showed chronic changes in the kidneys. Tuberculosis of the kidneys was observed in 58 per cent, or about two-thirds of the cases in which the kidneys were affected. In the other one-third, the changes were wholly due to the excessive amount of toxins which the kidneys were compelled to eliminate.

In the face of such facts it would seem to be quite unnecessary to urge the necessity of exercising every possible care to avoid excessively taxing the kidneys, not only by suppressing irritating condiments, the importance of which is recognized, but by avoiding any unnecessary increase of the nitrogenous wastes, of which the kidney is the chief outlet.

It seems proper then to raise the question whether the feeding of large quantities of protein in tuberculosis may not aggravate one of the most grave and incurable of the lesions incident to this malady. Indeed, we may even inquire whether a high protein dietary may not be very largely responsible for the great number of cases of renal disease observed as a complication of 
pulmonary tuberculosis. Certainly it cannot be denied that the kidneys must be considered when arranging the bill of fare of the patient suffering from phthisis pulmonalis.

Every modern author on the dietetic treatment of renal disease insists that the amount of work required of the kidneys must be diminished as much as possible, and that hence the proportion of protein should be reduced, that the extractive matters of meats should be especially avoided, and even that the amount of sodium chlorid should be reduced to the minimum. Hutchison urges that flesh foods should be allowed only in very small quantity in renal disease, and advises that the proteins should be "derived largely from vegetable, sources." Freidenwald and Ruhrah entirely prohibit strong meat broths and beef teas, and reduce the protein to 30 or 40 grams. They also limit the chlorid of sodium and state that "meat should be used cautiously."

This is good advice as far as it goes, but there is certainly no justification for permitting meat to be used even "cautiously." The reasons given for prohibiting meat broths, beef tea, and reducing the protein, are good and sufficient reasons for excluding meats altogether.

Hirschfeld emphasized the inability of the diseased kidney to eliminate nitrogen. The diseased kidney is like an obstructed filter. With a diet of 70 grams of albumin a healthy kidney eliminates 10.1 grams of nitrogen, the diseased kidney 9.3 grams. When the albumin ration is increased to 130 grams, the healthy kidney eliminates 14.5 grams, and the diseased kidney only 11.7 grams. When the ration of 130 grams is continued for a few days, the disproportion increases, the diseased kidney eliminating only 12.7 grams, while the healthy kidney eliminates 18.6 grams. Thus the diseased kidney under a ration furnishing 533 calories of protein - considerably less than the amount often given consumptives-is able to eliminate only two-thirds of the nitrogen intake.

It is evident, then, that the consumptive patient who is fed on a high protein diet is thereby not only rendered more liable to fatal hepatic and renal complications, but through the accumulation of the retained products of protein metabolism all his functions are hindered and all his tissues are exposed to a damaging influence.

Gautier emphasizes the necessity of reducing, in renal disease, "the toxins and other offensive products to the minimum 
by diminishing the consumption of the food principle from which they originate (protein.)" He permits meat in small quantities, 20 grams of protein, but recommends especially bread and cereals, cereal soups, purées of vegetables, potatoes, rice, and all fruits, giving as the reason that "these foods do not produce, or only in very small quantity, urinary toxins." W. Gilman Thompson says: "Whatever views may be entertained in regard to the causation of albuminuria, it is agreed by all that cooked meats, eggs, and highly seasoned foods in general are injurious in all cases." Senator, of Berlin, in cases of serious renal disease prefers to prescribe only vegetables, salads, fruits, or an exclusive milk diet of two liters per diem. It seems a just conclusion from the facts presented that the recommendations made in relation to diet in renal diseases are equally applicable to cases of pulmonary tuberculosis.

If a high protein ration in general is open to suspicion in tuberculosis, a flesh diet is particularly questionable for several reasons :

1. Flesh proteins more readily undergo putrefactive decomposition than do vegetable proteins.

2. Flesh foods as eaten always contain, ready formed, a considerable amount of toxins which have resulted from putrefactive processes which take place in the flesh after killing, while being "ripened" in preparation for the market.

3. Flesh foods always contain multitudes of putrefactive bacteria in active growth, and hence introduce into the intestine an agent in the highest degree calculated to encourage intestinal autointoxication and to aggravate some of the gravest features of the disease.

4. Lusk, Rubner and various other physiologists have shown that protein causes a waste of energy by overstimulation of cell activity. With a high protein ration the body cells are so overstimulated that they expend more energy than the protein supplies. In a tuberculous patient the cells are already suffering from overstimulation due to toxins produced by the tubercle germ. This is the cause of the fever, weakness, emaciation and wasting of the tuberculous patient. It is evident that a diet which increases this destructive tendency cannot be otherwise than harmful; and that a diet rich in flesh proteins produces this effect cannot be questioned in the light of modern physiologic research. 
While the writer is not a specialist in the treatment of pulmonary tuberculosis, he has during more than forty years had cases of this sort constantly under his care and has had considerable opportunity to observe the effects of both a low protein and a high protein dietary. A very considerable number of patients have been seen to make good recoveries from well pronounced tuberculous disease of the lungs on a dietary which furnished not more than 200 calories of protein in the form of eggs, milk and vegetable protein, and often less. On the other hand, there has frequently been a rise of temperature and an aggravation of other symptoms when a digression was made to a diet of flesh foods such as beefsteak, etc. Several times patients have come under our care who had been subjected to forced feeding with disastrous results. The enthusiastic advocacy of forced feeding by Débove, Broca and Dujardin-Beaumetz and the slavish way in which for a time the example of these clinicians was followed, have led in not a few instances to most untoward results. The chief evils of these large dietaries doubtless result from the excess of protein and fats.

Bardswell and Chapman found that "patients make much less satisfactory progress on the very large diets than on diets of considerably smaller nutritive value." The same investigators observed that "any considerable increase in the protein of the diet produced a disproportionate excretion of nitrogen, an increase in the amount of imperfectly oxidized proteins in the urine, a decrease in the percentage of nitrogen absorbed, and an increase in the amount of aromatic sulphates excreted, indicating increased intestinal putrefaction." These authors conclude that the most satisfactory diet for the consumptive "is one which represents only a moderate increase of a diet suitable for him in ordinary health."

Dr. Lawrason Brown notes that the method of forced alimentation rarely yields permanent success, and also warns against the use of foods which are likely "to give rise to toxic action of the liver and kidneys."

Of all foodstuffs flesh meats are the ones which "encourage toxic action of the liver and kidneys," or rather toxic effects due to excessive demands upon the liver and kidneys for the destruction and elimination of poisons. If used too freely, eggs may give rise to similar symptoms, particularly the whites of eggs and especially when eaten raw as in the form of eggnog, as 
elsewhere pointed out. Milk proteins and particularly milk in the form of acidophilus buttermilk present this necessary element in a form in which it is in the highest degree digestible and assimilable. And it should be remembered that Sherman has shown that the addition of a few ounces of milk to a diet otherwise made up of cereals, affords a supply of complete proteins sufficient to wholly meet the needs of the body.

The facts relating to the feeding of tuberculous cases may be summarized as follows:

1. A low protein dietary, 0.80 to 1.00 gram of protein per kilogrami of body weight per diem (one calorie per pound of body weight) is entirely consistent with health, vigor, and a high degree of efficiency and endurance in health.

2. While a patient suffering from pulmonary tuberculosis doubtless requires a small increase in the intake of nitrogen, an excessive increase involves grave dangers to the patient, both $(a)$ by decreasing his general vital resistance and $(b)$ by imposing unnecessary and dangerous burdens upon the liver, kidneys, thyroid, and other organs which are already overburdened and often seriously crippled in this disease.

3. There is no evidence that a larger proportion of consumptives recover under a high protein diet than under a protein ration sufficiently above the Chittenden standard to replace the nitrogen loss due to febrile conditions in certain states of the disease.

4. In consumption the organism is required to deal with various highly virulent poisons which overstimulate and ultimately cripple or destroy the thyroid, adrenals, liver, and other antitoxic organs. A high protein diet produces similar effects in healthy animals and persons, and destroys life in animals whose poison-destroying functions are seriously impaired.

5. A high protein diet is recognized as an important factor in the causation of renal disease and is universally condemned in grave affections of the liver and kidneys. Vegetable proteins are much less objectionable than flesh proteins for the reason that they are entirely free from toxins and very much less readily undergo putrefactive changes in the intestine.

A copy of the above statements was sent to a number of physicians specializing in the treatment of pulmonary tuberculosis, including superintendents of tuberculosis sanitariums. In 
the considerable number of letters received there was a general consensus of opinion against the overfeeding of tuberculous patients, then and still in great vogue, and especially against the very high protein diet. The late Dr. Sternberg, who after retiring from service as surgeon general of the United States Army for some years conducted a sanitorium for tuberculous patients, in a letter to the writer endorsed most heartily the views above expressed and stated that he had demonstrated to his entire satisfaction by his own experience that tuberculous patients are injured by a free use of meat.

Numerous papers have appeared in the medical press calling attention to the dangers of a high protein diet in tuberculosis. Dr. F. J. Walter, in the Journal of the American Medical Association, 1919, calls attention to the danger of a too heavy nitrogenous diet in this disease, showing that a patient by this means may become the subject of nephritis and renal tuberculosis.

The report of observations made by MeCann and Barr, of the Russell Sage Foundation at Bellevue Medical College of New York City, on the metabolism of consumptives has placed the question of the amount of the food intake in these cases on a sound scientific basis. According to these observers, the total amount of food taken by the tuberculous patient need not be more than 20 or 25 per cent. greater than that required by a patient at rest, or 500 calories more than required by the basal metabolism of the individual; and the amount of protein is not more than half that required by the old standard of Voit and only two-thirds of that demanded by the standard of Atwater. In a dietary balanced on this basis there is naturally no room for steaks, chops and similar meats, for in a dietary including a moderate amount of milk, eggs, cereals and such other foods as ordinarily compose a lacto-vegetarian dietary, with the exclusion of meats, there will be as much protein as the body can make use of and more than this must be clearly recognized as a detriment. Malt sugar contains more iron than the same amount of beefsteak and much more than cow's milk. Change of the intestinal flora is of the greatest importance in all cases of pulmonary disease. Patients always give a history of chronic intestinal stasis. The intestinal autointoxication resulting from constipation inevitably lowers vital resistance and prepares the way for the development of tubercular disease. By the use of milk sugar or 
lacto-dextrin the change may be readily made within a few days and should be repeated whenever the stools become foul smelling. Meat feeding will of course at once restore the putrefactive flora, an excellent reason for its disuse. An antitoxic and restorative regimen should be faithfully adhered to. The fattening regimen is needed when the patient is under weight. Special care should be taken to secure a large intake of food lime, at least double the normal requirement or 200 per cent. Milk, greens, cottage cheese, legumes, certain nuts (almonds, filberts), bran, oatmeal, and malt sugar are the best sources of lime (Table XIV). Meats of all sorts are highly deficient in lime. Protose, gluten bread (40 per cent), and gluten biscuit are rich in lime, iron and protein and on this account most valuable foods for a consumptive. Yeast extract (Savita, Marmite), should be used daily to insure a full supply of vitamins.

Hindhede, who has recently made a study of the dietaries of various institutions for the treatment of tuberculosis, correlates his observations with the results obtained in the several institutions studied. He reaches the conclusion that the richer the dietary the poorer the results, and recommends as most suitable for the tuberculous, as well as for healthy persons, the lactovegetarian regimen. He found that the average daily consumption of food at Rollier's, which was the most successful of all the European institutions, was only 2,742 calories, and "the consumption of meat was exceedingly small." The average consumption at eight public sanitoriums was 3,725 calories, and at Saugman's sanitorium the average was 4,738 calories.

It is to be hoped that the application to the treatment of the tuberculous of the new facts relating to human nutrition which have been developed in recent years will soon lead to a reform of the dietaries in public sanitoriums.

\section{THE DIET IN INTESTINAL TUBERCULOSIS}

In general, the diet should be the same as in pulmonary tuberculosis. Carbohydrates (starchy foods and malt sugar) are especially needed to combat emaciation. The intestinal flora must be changed and kept non-putrefactive.

When the patient suffers from diarrhea, milk, milk porridge, rice, millk toast, purées of potato, greens and sweet fruits should constitute the dietary. Use also milk sugar or lacto-dextrin. 


\section{THE DIET IN SYPHILIS}

In syphilis the liver and kidneys suffer because of the toxins with which they have to deal, even when they may not be the seat of specific lesions. It is important to note that the huge doses of drugs which are necessary to destroy the parasite of syphilis are by no means without deleterious effect upon the liver and kidneys. It is, in fact, simply a question of the choice of evils. The powerful arsenical preparations used to destroy the syphilitic parasite inevitably injure the liver and kidneys, not merely temporarily but doubtless more or less permanently, just as does a severe attack of typhoid fever, small-pox or any other grave infectious malady. For example, it has been shown by life insurance statisticians that an attack of typhoid fever diminishes life expectancy 50 per cent. Certainly a person who has had syphilitic infection cannot expect to become a centenarian even though he may have undergone most thorough antisyphilitic treatment, since the treatment itself involves no small amount of damage to vital organs, although far less than would have been produced by the syphilitic parasite and the poisons produced by it if the treatment had not been applied.

Bailey and Mackay have called attention to the toxic effects which frequently appear in patients under antisyphilitic treatment, causing among other tissue damage changes resembling those of yellow atrophy of the liver; while Davis and Whipple have shown the importance of reducing protein and fats to a minimum in cases in which the liver has been damaged by chloroform given for anesthesia or by other drugs. The increase of urea and cholesterol in the blood following the use of salvarsan point to injury to the kidney as well as the liver. The observations made by these investigators, together with facts long known concerning the function of the liver in defending the body against metallic and organic poisons, show clearly the importance of protecting this vital organ by reducing the protein and fat of the diet to a minimum while supplying it by means of a diet rich in carbohydrates with the glycogen necessary to assist it in its work of destroying and detoxicating not only the syphilitic poisons but the toxic substances used to destroy the parasite. An eminent authority, writing upon this subject, remarks: "A diet of cooked skeletal muscle is not favorable for rapid liver repair." "Cooked skeletal muscle" is a fine scientific euphemism for beefsteak, 
roast beef, mutton chops and other forms of meat, all of which are unfriendly to the liver and kidneys because of the increase of intestinal putrefactions to which they give rise.

It has long been known to those familiar with the advantages of a non-flesh dietary that the disuse of flesh foods of all sorts is of very great importance in the treatment of syphilitic patients.

The diet in syphilis should be a combination of the antitoxic, laxative and reconstructive dietaries. It is important to reduce the alimentary toxins to the lowest point possible and by all means available to raise the patient's vital resistance and recuperative powers.

An eminent French physiologist* has recently called attention to the interesting and most useful fact that by a liberal use of carbohydrates the liver may be enabled to repair the damaged cells when the injury is recent and the changes not too far advanced. Lactose, lecto-dextrin and carbohydrate foods of all sorts should enter largely into the dietary in all syphilitic cases.

In cases of syphilitic stomatitis, rice gruel with the addition of a little butter or cream should be the almost exclusive diet for a few days on account of the bland character of this preparation.

In syphilitic cirrhosis the diet which has been recommended for ordinary hepatic cirrhosis should be followed.

Patients preparing to undergo treatment with salvarsan should first have a change of flora and then faithfully follow the antitoxic diet. Numerous observers have noted that the inconveniences and dangers of the treatment may be avoided by the disuse of meats of all sorts. In the treatment of a very large number of cases in which this regimen has been employed at the Battle Creek Sanitarium, most strikingly favorable results have been obtained. Says Dr. W. F. Martin, specialist of the Battle Creek Sanitarium staff, "I consider a low protein antitoxic dietary of essential importance in the treatment of cases of syphilitic disease."

It is highly important that the condition of the liver should be determined by means of Widal's test for hepatic insufficiency (see Index), both before beginning treatment and at frequent intervals during a course of treatment. It is generally agreed that in cases of visceral syphilis, arsenical preparations should

- Foie et Pancreas, Paris, 1920. 
not be employed. It is also equally important that the liver should be permitted to recover from the injurious effects of one dose of salvarsan before another dose is administered.

\section{CHRONIC PARASITIC INFECTIONS}

Actinomycosis. In this disease the indication is for an antitoxic and reconstructive diet. On account of the restricted activities of the patient special care should be taken to keep the bowels active by the free use of bran or agar and paraffin oil.

Trichinosis. In this disease, which is due to the invading of the body by trichina, the pork parasite, the fever diet is indicated during the acute febrile period. If the patient should have the good fortune to recover, he should forever after abjure pork in every form, the ordinary source from which this dreadful infection is derived. Many persons give a history indicating repeated infections by the parasite. Unfortunately this disease is incurable. There is no known method for destroying the parasite after it has entered the tissues. The permanently crippled organism requires scrupulous adherence to an antitoxic dietary.

\section{DIET IN DISEASES OF THE SKIN}

During more than forty years the writer and his colleagues have successfully treated various forms of skin disease, chiefly by regulation of the diet. Nearly forty-five years ago the writer had under his care a prominent educator, a professor in a state college, who suffered from eczema of such a severe type that his entire body was covered. With a strict non-flesh dietary and without other applications to the skin than simple warm baths and soda lotions he was soon wholly free from the disease. Hundreds of similar cases might be cited.

Many years ago Dr. L. D. Bulkley, of New York, began the advocacy of a rice diet in psoriasis and eczema. At first he only required his patients to avoid beef and mutton. Of late years he has made the diet more strict, excluding entirely all flesh foods, even meat soups, poultry, eggs and fish. He has had a number of patients for years on an absolutely vegetarian diet, allowing only butter and restricting the use of milk.

Bulkley thus describes the result of this dietary:

The effect of this cutting off of the supply of animal nitrogenous foods has been very remarkable and striking in many instances (a 
considerable amount of nitrogen is still supplied by certain vegetables, as the legumes and oatmeal). Patients continually notice the change in the color and character of the eruption, it paling and becoming less scaly, and even entirely disappearing in some weeks, with absolutely no local treatment.

Note has been made repeatedly that when there has been a neglect of the dietary element there has been a recurrence of the eruption, which again yielded rapidly when stringent measures were enforced.

On the other hand, there has been a number of patients who have faithfully pursued this plan of treatment, in whom a longexisting psoriasis has remained absent, and who, having become accustomed to the diet, say that they have lost the desire for flesh food and will not touch it again.

Little need be said in regard to the general subject of a vegetarian diet, for abundant experience has shown its value under many conditions of health and disease. The opinion is, I believe, gaining ground both among the medical profession and the laity that far too much meat is eaten by those who can get it. . . In my experience patients have felt remarkably well when this was rightly directed and carried out, and in numerous instances I have found distinct and steady gain in weight in the spare, and loss of weight in the obese, when tested repeatedly on the same scales.

The results of Dr. Bulkley's tests were so successful that he called upon the writer for a dietitian trained in the School of Home Economics of the Battle Creek Sanitarium and established the meatless regimen in the Skin and Cancer Hospital of New York City, where it has been employed now for many years and in the successful treatment of many hundreds of cases.

Ecsema. Eczema and most other forms of skin diseases are greatly influenced by diet, Various skin maladies are commonly associated with chronic intestinal toxemia and are apparently due to the lowered vital resistance resulting from the absorption into the blood of large quantities of putrefaction products developed in the intestine. In some cases the skin eruption may be due to anaphylaxis. These eruptions may occur in persons who are sensitized to some particular foodstuff. The food substances most often incriminated are oysters or other shellfish, pork, mutton, veal, strawberries, buckwheat, eggs, sometimes milk and even tomatoes. In all cases of eczema and other forms of skin eruption a strict antitoxic diet should be adopted. Meats of all sorts s culd be eliminated and a search should be made for the particular substances to which the body may be sensitized by means of tests with the various allergens which are now prepared for 
this purpose. These tests must be conducted by a physician who is acquainted with this line of research.

In beginning a course of treatment, the intestinal flora must be changed; any existing colitis must be cured by diet and treatment of the colon and the antitoxic dietary must be followed.

The dietetic treatment of acne and other chronic skin disorders is essentially the same as that required for eczema. Acne often requires a very considerable restriction of the use of animal fats, and special attention must be given to the colon.

Eczema in Infants. M. Langstein (La Semainé Médicale, September, 1908) confirms the observations of M. Finkelstein in relation to the beneficial results obtained by withdrawing chlorid of sodium from the food in eczema of infants. In four children suffering from eczema the disease disappeared entirely in four weeks on a diet from which four-fifths of the amount of salt ordinarily present had been withdrawn.

Gershun ( ratch) calls attention to the fact that skin diseases in infants are frequently due to poisons in the mother's food. Psoriasis and eczema have been shown to be due to this cause, the disease disappearing when the mother discards meats.

Special attention should be given to the bowels, which should be made to move three times daily by the use of bran, paraffin oil and spinach. The flora should be changed by use of milk sugar or lacto-dextrin and an antitoxic laxative diet. Psyllium is most helpful.

Certain infants are sensitized to cow's milk. In such cases, Malted Nuts or almond milk may be substituted for milk. Butter should be given in potato soup, and a soup of malted barley may be advantageously used. The cure will be facilitated by the use of lanolin cream, of which the following is the formula: lanolin, 2 drams; boroglyceride, 1 dram; cold cream made with white vaselin, 6 drams. Apply freely several times a day.

\section{DIET IN CANCER}

Dr. William Mayo has for some years maintained that the eating of hot food is the cause of the great frequency of cancer of the stomach. It is known that burns give rise to cancer. For example, cancer of the skin of the abdomen is common among the natives of Kashmir as the result of burns due to the kangri, a basket of coals which these mountaineers carry under their cloth- 
ing as a sort of "warming pan," Cancer in this region is almost unlinown among other people.

Cancer of the gullet is rather frequently observed among the men in China, but is rarely met among the women. The men in China eat their rice very hot while the women eat it cold, at the second table.

Dr. Mayo finds a similar explanation of the greater frequency of cancer of the stomach in men in the fact that the wives pour the coffee and serve their husbands first and themselves last, and hence take their coffee at a lower temperature.

Dr. Carl Petersen, the eminent statistician, in a recent article confirms the observation of Professor Maynard that there is a close relation between diabetes and cancer, the coefficient of correlation being according to Petersen 71 per cent.

Careful study of the statistics of these two diseases shows that whenever there is an increase in the mortality from cancer there is a like increase in the number of deaths from diabetes. and the reverse. Williams has shown that the mortality from cancer increases in proportion to the amount of meat eaten. He has accumulated an enormous mass of evidence which seems to demonstrate the close relation between flesh-eating and cancer.

Hoffman, the noted statistician of the Prudential Life Insurance Company, has fully confirmed the observations of Williams in a world-wide study of mortality statistics, the results of which he exhibited at the Panama-Pacific Exposition.

Ross, by observations made in the Lister Institute of Experimental Medicine, showed that putrescin, cadaverin, cholin and perhaps other products of putrefaction of meat, together with caffein and uric acid, increase cell proliferation to an extraordinary degree.

So long ago as 1880 , Beneke called attention to the greater frequency of cancer in meat-eating animals and men.

Dr. Charles H. Mayo finds that cancer is extremely frequent in dogs, being found in 25 per cent of all dogs eight years of age, increasing to 50 per cent in dogs twelve to fourteen years of age.

Recent observations have shown that the suppression of vitamins, particularly of vitamin $\mathrm{C}$, tends to check the growth of cancer. This is the basis of the diet of Centanni.

The writer saw in the laboratory of Ehrlich, who made extensive studies of the effect of diet upon cancer, rats in whom 
well-developed cancerous growths had very largely disappeared under special feeding. Professor Ehrlich remarked that "While the development of cancer could be to a large degree controlled by feeding, it was found necessary to starve the animals. nearly to death to secure a pronounced effect."

Gastric Cancer. A bland reconstructive diet is indicated. Rice is in many cases more acceptable than any other food. Sugar water, prepared by the addition of two ounces of malt sugar or milk sugar to a pint of water, may be used to advantage when more solid food is rejected. The introduction of sugar water by enema is also of great service in sustaining the patient's energies and especially in preparing him for a radical operation when this is deemed advisable.

Sugar feeding not only supplies the patient with the sort of nourishment he most needs, but at the same time serves as a biologic disinfectant and antiseptic for the stomach, preventing the growth of the putrefactive organisms which swarm in the cancerous stomach in these cases, because of the absence of hydrochloric acid.

An antitoxic diet, that is, a diet which disconrages the development of putrefactive poisons in the intestine, is specially to be commended as a means of combating cancer. Such a dietary should be adopted by all persons suffering from cancer, and also by those who are so unfortunate as to have a cancer heredity. During the last forty-five years the writer has had unusual opportunities for observation in relation to the influence of a nonflesh dietary upon the occurrence of cancer. Of many thousands of flesh-abstainers with whom he has been acquainted, he has known during this period of only four cases of cancer in persons who had been for a long time flesh abstainers, and in one case recovery occurred without removal of the cancerous growth. There can be no doubt that among the thousands of persons under observation who escaped the disease, as the writer believes through flesh abstaining, there must have been a considerable number who were especially susceptible to cancer because of heredity and who were able to overcome this special susceptibility by a non-flesh ciietary. This conclusion seems inescapable when it is remembered that ten to fifteen per thousand of all persons past middleage die of cancer.

An antitoxic dietary excludes not only flesh foods but tea. 
coffee, alcohol, tobacco, vinegar, mustard, pepper, peppersauce and other condiments, as well as all other toxic substances.

A liberal use should be made of fresh fruits and vegetables because of the large amount of potash which they contain. Potatoes, carrots, raw cabbage, lettuce and cucumbers are especially to be commended.

\section{Cancer of the Colon.}

This disease, like cancer of the stomach, is more frequent in. men than in women; and, like cancer of the stomach, its frequency is increasing steadily from year to year. Unquestionably, one of the great causes of cancer of the colon is the unusual exposure to injury and contact with putrefactive bacteria and the poisons which they produce. This idea is suggested by the fact that the cecum and the pelvic colon, the two portions of the colon in which foodstuffs remain the longest, are the chief points at which cancer attacks the colon.

Regular and frequent, at least tri-daily, evacuation of the colon is the most important preventive measure which can be brought to bear in cases of this sort, if the colon is kept in a healthy condition by changing the flora at frequent intervals and maintaining a non-putrefactive condition of the seat of cancerous clisease. 


\section{Diet in Surgical and Hos- pital Cases}

The ordinary hospital dietaries are open to severe criticism, especially in the light of modern discoveries in human nutrition. There is almost a total lack of lime, iron or vitamins in the regular hospital "light diet," and the various gruels and other liquid preparations are well calculated to encourage constipation and intestinal toxemia. Milk contains lime, but is lacking in iron. Bouillon and broth are the chief stock in trade of the hospital cook. These time-honored preparations fill such a large place in the current hospital bill of fare that it may seem almost equal to sacrilege to denounce them; but in the light of modern knowledge they must go. They are too pernicious to be allowed a place in a bill of fare from which everything toxic or germbreeding should be excluded. For more than forty years the writer has conducted a surgical service through which many hundreds of cases have passed every year and from which all meats and meat broths and bouillons have been excluded. The advantages of this regimen was early demonstrated. More than twenty-five years ago unusually good results were obtained by the author in several series of abdominal operations, which were attributed to the special system of feeding employed. In a series of 395 abdominal sections within six years (1889-1895) the mortality was 2.3 per cent. The above figures include a series of 233 cases of non-septic ovarian cysts with one death, and 165 successive cases of ovarian tumors or diseased appendages with no deaths. The significance of these figures will be appreciated when it is remembered that at the period named our modern surgical technic had not yet been developed. Rubber gloves were not worn, and the usual mortality rate in abdominal cases was 15 to 20 per cent. or more.

The usual hospital regimen greatly needs reformation. The bill of fare should be enlarged by the addition of many foods rich in vitamins, lime and especially iron. Bran is needed for roughage, with agar and other forms of cellulose. Bran is also a 
good source of food lime and iron. Vegetable broths, rich in vitamins and salts, should take the place of the animal extracts and bouillons, which are nothing less than solutions of uric acid and other poisons, as pointed out long ago by the elder Flint and eminent French physicians. Meats of all sorts may well be dismissed from the hospital larder. All, without exception, are septic, putrescent, swarming with the terrible bacillus of gas gangrene, $B$. Welchii, and other highly virulent mischief makers. Put into the intestine of a feeble patient who has barely survived a grave operation, nothing could be devised in the shape of food more likely to become a cause of disaster.

Lucas-Championnière, of Paris, surgeon-general of the French Army, observed that a high proportion of urea in the urine, a necessary result of meat eating, was unfavorable to recovery after an important operation or injury.

Diet before Operation. After the flora has been changed by a few days of fruit regimen, a non-toxic dietary should be adopted before the operation, as well as after the operation, and until convalescence is fully established. This requires a reduction of the protein to as low a level as safety will permit, certainly not exceeding 10 per cent. of the total calorie value of the ration.

Bouillon and meat extracts of all sorts must be discarded. Meats must necessarily be discarded because of the readiness with which the undigested residues undergo putrefaction in the colon, besides introducing myriads of putrefactive bacteria which are always present in fresh, salted and smoked meats of all kinds.

The famous surgeon, Paget, in his lessons on surgery many years ago asserted that "the high death rate from operations in cities, as compared with rural districts, is due to the fact that city dwellers eat large quantities of meat, while country dwellers in Europe taste meat only on occasions."

More than a dozen years ago Tissier called attention to the fact that vegetable proteins are only about "half as prone to putrefy as animal proteins," and Torrey has recently demonstrated the same fact. Neither bouillon nor meat broths are ever served to the writer's surgical patients. Tea and coffee as well as wines are also scrupulously excluded from the bill of fare.

Storing up Glycogen. It is most desirable that that wonderful poison-destroying organ, the liver, and the poison-eliminating kidneys should be gotten into as efficient a condition as 
possible before the operation, so as to be prepared to deal with the anesthetic, itself an active poison, as well as the great influx of poisons naturally following the operation. For accomplishing this, it is especially important that the patient's diet should be rich in carbohydrates so that the patient's liver, muscles and body cells may be well stocked with glycogen.

It is current practice in abdominal cases to give the patient no food on the day of the operation. This plan has the disadvantage of bringing the patient to the operating table fasting and necessarily with his store of precious glycogen greatly reduced. Glycogen is necessary not only to support the heart in its activity, but to aid the liver in dealing with the great flood of toxins thrown into the blood by the anesthetic and the traumatism of the operation and which are the principal cause of shock, as shown by Cannon. An abundant store of glycogen in the liver and the body cells is the best possible means of protection against shock. In order to enrich the patient's store of glycogen, the writer has for years adopted the practice of giving the patient eight or ten ounces of malt sugar between the last meal the night before the operation and the hour of the operation. Four to six ounces of malt sugar dissolved in water may be given in divided doses the morning of the day of the operation.

The value of this ready-to-use nutriment is very great, not only as a means of replenishing the liver's store of glycogen but to sustain the heart, which consumes a fraction of a calorie of sugar at every beat, and especially to combat the acidosis invariably produced by the anesthetic.

Renal Efficiency. If a high non-protein nitrogen content is shown by the blood test, or the urinary test ration shows renal inefficiency, the operative hazard may be enormously lessened by a few weeks' careful treatment with an atoxic diet, changing the intestinal flora and improving elimination.

Prophylaxis against Acidosis. If the carbon dioxide tension is found to be less than 34 , which is not infrequently the case in meat eaters and in persons whose nutrition has been seriously interfered with by fever, low diet or other cause, the body's store of glycogen must be replenished and the carbon dioxide tension raised to normal by carbohydrate feeding, particularly by the use of malt sugar and milk sugar, of which a half ounce may be given in a glass of water every hour for a day or two before the operation, or even every half hour if the 
patient is able to absorb so much fluid. If necessary, retaining enemas of malt or milk sugar, two ounces to the pint, are given.

\section{Post-Operative Feeding.}

The post-operative feeding of surgical cases is a matter which has received far less attention than its importance demands. In many cases, the patient's nutrition has been seriously interfered with for some time before the operation by the conditions which make the operation necessary. The starvation of patients, either before or after operation, is not only unnecessary but is highly prejudicial to the patient's interests, lessening the chance of recovery and increasing the danger of serious complications. Food is a most important regulator of metabolism. Assimilated food furnishes the material upon which the cell works, both in the performance of its functions and in the repair of its structure. If material is not supplied in the form of food the body must draw upon its own tissues.

Food, likewise, is the only source of energy for the body. The heart alone consumes approximately two ounces of sugat every twenty-four hours.

Furthermore, food furnishes a physiological stimulus to the living cells. The rapid loss of strength and endurance as the result of fasting and the marvelously rapid restoration of energy by appropriate nourishment afford ample evidence of the helpful stimulus which may be secured through proper feeding. This stimulation is doubtless due in large measure to the presence of vitamins which abound in certain foods and in which vegetable broths are rich. These vegetable broths differ from bouillon and animal broths in the fact that, while rich in vitamins, they contain no urea and other waste matters of which animal broths are largely made up while lacking vitamins almost altogether. Yeast extracts (Savita, Marmite) are especially valuable.

The eating habits of most patients lead to an accumulation of protein wastes in the tissues. Since all excess of protein must be eliminated as urea and other nitrogenous wastes, both the liver and kidneys are overtaxed by this excessive protein metabolism, causing a predisposition to acidosis and uremia. In addition to these waste products from excessive protein metabolism, the blood and other body fluids are likely to be saturated with toxins of bacterial origin originating from the putrefactive changes in food residues in the colon. On this account it is most unwise to 
feed a prospective surgical patient meats of various sorts as a means of preparing him for the operation, or to advise such a diet for promoting convalescence after operation. On the other hand, a low protein diet for a few days before the operation as well as immediately following the operation is most advantageous. The blood may be more rapidly built up by a lacto-cereal diet, with the addition of liberal quantities of greens than by heavy meat feeding.

There is usually a sufficient amount of fats stored up in the tissues to supply the needs of the body for a few days, and the fat intake in post-operative cases may be advantageously diminished because of the inhibitory effect of the operation upon the secretion of gastric juice. Gastric juice is important in stomach cases to prevent the putrefaction of stagnant gastric fluids and so maintain a condition favorable to healing. It is well to remember, also, that the body is able to manufacture fat from carbohydrates and that starch and sugar are, under normal conditions, the chief sources of body fat.

Abundant carbohydrate feeding is hence of great interest in relation to surgical cases. Farinaceous foods of all sorts, fruit juices and malt or milk sugar are the most appropriate materials for feeding a patient on the days before operation and two to five days succeeding.

\section{Feeding After Gastro-Enterostomy, Pylorectomy and the Polya Operations.}

The feeding should begin early-as soon as vomiting is controlled-for several reasons: The sooner feeding can be begun the less will be the danger of exhaustion, since the food supply rather than the tissues of the patient will thus be the source of the energy.

Another benefit to be derived from early feeding, which is especially noticeable in operations upon the stomach and gall bladder, is the suppression of "hunger pains" which Carlson has shown to be due to violent contractions of the stomach. Bland food introduced into the stomach is often more effective than morphia or any other anodyne in controlling the pain following operations on the gall bladder or the stomach.

It is well to remember, also, that food is a natural laxative. Taking food into the stomach sets up peristaltic waves which travel over the entire intestinal tract, thus encouraging evacua- 
tion and the elimination of waste matters from the colon. This early peristalsis has the effect of straightening ont kinks in the intestine and breaking up newly-formed adhesions which have not yet become organized. It is even advantageous in many cases to make use of moderate quantities of agar-agar or gruels containing bran. By supplying these colon stimulants, the early restoration of normal bowel action may be greatly encouraged.

Careful early feeding is especially advantageous in cases of gastric surgery, even in gastrojejunostomy, pylorectomy and the polya operations, as well as in other cases.

The objection that foods may be irritating does not hold good for the reason that bland foods of the sort mentioned are by far less irritating than the acrid matters invariably present. Such foods certainly will be as well tolerated by the stomach as the stagnant, germ-infected, often foul-smelling and irritating material usually found in the stomach after operations on this organ. The effect of taking food in such cases is to dilute or wash away the stagnant fluids resulting from oozing, involuntary swallowing of the saliva, etc. Displacing the putrefying gastric contents by a small quantity of bland and sterile rice gruel often produces a most favorable effect, relieving nausea, hunger pains and other distressing symptoms.

The fact that taking fluid into the stomach may give rise to gastric secretion cannot be regarded as an objection to early feeding for the reason that the gastric juice is the normal disinfectant of the stomach, its presence will help to suppress putrefactive changes in the gastric fluids, and will hence promote the healing process rather than prevent it.

When the patient does not vomit, malt sugar water containing two ounces of malt sugar to the pint or fruit juice of some sort containing the same proportion of sugar is given in spoonful doses hourly, and affords the patient exactly the kind of nourishment required. Pathogenic organisms do not grow in sugar water, fruit juice or gruel, while they thrive in blood serum, bouillon and broths. Of course, it is important to avoid overfeeding. A half ounce of rice gruel every two hours while the patient is awake, the first day, increased by half an ounce on the second day, causes no disturbance in the average case. After the first few days, spinach purée may be addẹ to promote blood building and thorough evacuation of the colon.

Bouillon, beef juice, broths and similar preparations are 
carefully avoided, and, in fact, are never admitted to the writer's ward. Vegetable broths, which are quite as appetizing as any of the preparations mentioned and wholly free from unwholesome properties, are freely used. A bouillon prepared from yeast extract (Savita, Marmite) so closely resembles bouillon that it is readily accepted in place of it and is, in fact, more palatable.

Gruel diet is relied upon only for a very short time, a few days at the most. Simple purées of vegetables and fruits are allowed after the first two or three days, and these are accompanied by agar jellies or special bran preparations to encourage bowel action. Purées of spinach or other greens should appear daily on the menu in some form. These lime and iron containing foods are essential to rapid blood building and tissue repair and help to sustain the heart in its arduous work. Green foods are also rich in the vitamins which are as essential for the rapid repair and rebuilding of the tissues as for the normal growth of young animals.

Lacto-Dextrin, psyllium seed, Fig Bran and Paramels render great service in combating constipation.

Every meal should be carefully balanced, not only for proteins, fats and carbohydrates, but for cellulose, food lime and iron and other salts and vitamins.

In cases in which a great portion of the stomach has been removed, great care must be taken in the mastication of the food or else it should be taken in the form of purées. Food must be taken in moderate quantities, and on this account it is necessary in some cases that the time between meals should be shortened, the patient taking four meals a day instead of three. This is especially necessary during the first few weeks following the operation. In several cases in which the writer has found it necessary to remove the stomach, X-ray examinations made by Dr. Case after the operation have shown that Nature has developed a new pylorus which behaves in a remarkably normal manner, holding the food back in the duodenum and the stump of the stomach for three or four hours as in the healthy stomach.

After the polya operation, intense and constant hunger is often experienced for some months. In such cases frequent small meals may be necessary for comfort when the hunger pain is severe. 


\section{Diet in Post-Operative Peritonitis.}

In peritonitis the chief indication is to maintain the strength of the patient by such food as will supply energy without encouraging the development of toxins. The patient's liver and kidneys are already overwhelmed with toxins which are being absorbed from the peritoneal cavity, and there is likely to be pronounced intestinal stasis; hence, foods rich in protein should be withheld, also foods rich in fat, which when present in large amount tends to increase stasis. Carbohydrates encourage peristalsis, combat acidosis and at the same time supply the body with the most readily available source of energy. Malt sugar is exceedingly valuable for use in this class of cases, a fact which seems to have been largely overlooked by writers on dietetics. The writer has made use of this valuable nutrient in the feeding of many thousands of surgical cases within the last 25 years and has found increasing reason for confidence in its value. Sugar water may be used by enema and also by mouth. When vomiting is persistent, a Rehfuss tube should be introduced into the stomach or duodenum and through this the accumulating fluids may be pumped out and sugar water introduced in their place, with great benefit to the patient. By this plan the amount of toxins absorbed into the blood may be greatly reduced and at the same time much needed nutriment may be introduced.

When there is stagnation of fluids in the stomach, as indicated by vomiting, the stomach should be frequently emptied by means of the duodenal tube, which may be left in position and liquids withdrawn every hour or two, as may be required. After the withdrawal of liquid, two ounces of sugar water (one ounce of malt sugar or milk sugar to the pint) should be passed in through the tube. This is especially important in cases in which the fluids have an offensive odor. The sugar water answers a double purpose. It feeds the patient and prevents the putrefaction of stagnant fluids.

Jejunal Feeding. When an intestinal fistula is established for the relief of obstruction, sugar water, preferably milk sugar, malt sugar, or lacto-dextrin, two ounces to the pint, should be introduced instead of plain water. Three or four quarts may be introduced in fractional doses in 24 hours. By this means the patient's energy is sustained and acidosis is prevented. 


\section{Diet After Operation on the Gall Bladder.}

In cases of gall bladder operation, very careful regulation of the diet is essential to insure the results sought by the operation. During the first four or five days the diet should be the same as after operations on the stomach. Later, a strictly atoxic or antitoxic diet should be adhered to without deviation. Meats of all kinds must be carefully discarded. Raw eggs should never be eaten. Meats and raw eggs greatly encourage intestinal putrefactions and continually reinoculate the intestine with the very bacteria which has made necessary the operation.

The diet should be very "loosening," and hence should include a liberal quantity of wheat bran. A quarter of an ounce three times a day is generally none too much, or two-thirds the quantity of agar, with an abundance of fruits, fresh vegetables, greens and other laxative foods, not as frequent items on the bill of fare but as a part of every meal. The regular use of paraffin oil is needed in most cases for the purpose of lubrication, so as to secure three full evacuations daily.

Especial care must be taken to masticate the food thoroughly. Prolonged mastication prevents too rapid swallowing of the food and hence too rapid discharge of food from the stomach into the duodenum, which is important when the gall bladder has been removed, because of the loss of the storage reservoir for bile.

Taking the food at frequent intervals is advantageous by causing the liver to contract and expel the bile from its ducts, thus helping to combat the infection in which the liver is always involved when the gall-bladder is diseased. High carbohydrate feeding is especially indicated for the reason that this is a means by which the liver may be enabled to repair its damaged cells.

\section{Diet in Accidents and Emergency Cases.}

When the accident or emergency is of such a character that the patient must be confined to bed, especial attention to the dietary is of great importance. First of all, the food intake should be diminished in quantity. The food requirement of a person in bed is not more than half or two-thirds that of the same person engaged in ordinary activities. A hard working man requires while he is resting in bed less than half the amount of food usually eaten by a man engaged in hard work. An excessive intake of food under such conditions is certain to aggravate the evils which are naturally the result of inactivity in a person 
who has been accustomed to an active life. Indigestion, so-called biliousness and other inconveniences in many cases very quickly make their appearance. Inactivity produces a tendency to constipation, which will be increased by the lessened volume of food unless care is taken to avoid this by increasing the amount of roughage. On this account, bran should be used in liberal quantities-three or four heaping dessertspoonfuls at each meal, or half as much agar.

Greens should be largely used also. In cases in which there has been a considerable loss of blood, the use of greens, lentils and other foods rich in lime and iron is especially important (see Table XV).

Dates, figs, raisins or malt sugar should take the place of cane sugar and other sweets. Fruits should be very freely used, especially acid fruits and tomatoes, and such coarse vegetables as cabbage, cauliflower, turnips, lettuce and celery.

In cases of bone injury, care should be taken to make the dietary rich in lime. It should be remembered that the cereals are poor in lime, also potatoes and most vegetables. Légumes provide lime in abundance. An ounce of peas, beans or lentils contains several times as much as an ounce of beefsteak. Buttermilk and skimmed milk are also good sources of lime, as are gluten preparations, almonds and hazelnuts.

Gluten preparations such as bread made from 40 per cent. gluten flour or gluten biscuit should be more freely used by surgical patients. Forty per cent. gluten biscuit supply six times as much food iron as does ordinary beefsteak.

\section{DIET IN COIMA}

In coma or unconsciousness from any cause, the two nutrients of which the body stands most in need are water and carbohydrate (dextrose).

Water is needed to dilute and carry away through the kidneys and other emunctories the poisons which are the cause of the condition. If the patient can be made to swallow readily without choking, a glassful ( 8 ounces) of water should be given hourly by the mouth. The colon must be thoroughly washed out by enema twice a day, and water may be given by enema by the drop method or by introducing into the rectum half a pint of warm water every two or three hours to be retained. 
A most efficient method of introducing water is by means of the duodenal tube. When necessary a tube may be introduced through the nose. The tube may be introduced and retained for several days. Water should be introduced at the rate of two ounces every fifteen minutes or eight ounces every hour. Not more than four ounces should be introduced at one time. The amount of water given in twenty-four hours should be not less than five or six quarts. The effect should be to produce a profuse flow of urine. It is important to save and measure the amount of urine as a check upon the water intake. The amount of urine should be not less than three or four quarts.

Carbohydrate must be given in the form of sugar-not cane sugar, but dextrose, levulose or malt sugar. Malt sugar is usually available. It is readily tolerated, is quickly utilized, and is nonirritating, which is not true of cane sugar. Starch is not a suitable form of nourishment in these cases for the reason that it requires digestion and so will be utilized but slowly if at all, while malt sugar is quickly changed to dextrose and absorbed.

Pure dextrose is now available and may be used in place of malt sugar.

Sugar should always be given in very dilute solution. The best plan is to add malt sugar or dextrose to the water in proportion of one ounce to one and a quarter ounces for each pint of water given.

Lacto-dextrin is perhaps the best of all forms of carbohydrate for use in these cases for the reason that it not only supplies carbohydrate but also suppresses putrefaction by inhibiting the growth of pernicious organisms such as B. Welchii, B. proteus and the colon bacillus. In addition, lacto-dextrin affords in abundance the valuable vitamin $C$ which prevents scurvy, which is not present in cane sugar.

An excellent source of carbohydrate for use in coma is fruit juice. The pure juice of any sweet fruit is suitable. Orange juice especially is excellent. Apple juice, grape or loganberry juice, the juice of raspberries, cherries, huckleberries and blackberries are all suitable for carbohydrate feeding. The amount of sugar present in each variety of fruit juice will be found in the tables given elsewhere in this work (Table XXXV). The amount of sugar varies greatly in different varieties of fruit. Orange juice, apple juice and blueberry juice afford about two and a half ounces of sugar to the pint. If any one of these juices were sub- 
stituted for one-half the water given, the proper amount of carbohydrate would be administered. Blackberry and raspberry juice contain about twice as much sugar as does the orange; and grape, strawberry and pear juice about one-half more.

Another excellent source of sugar is the raisin. Soaked and then stewed for an hour, the raisin surrenders to water its fruit sugars which are ready for immediate absorption and assimilation.

In extreme cases intravenous injections of dextrose in sterile normal saline solution must be given by a competent specialist.

It is to be understood, of course, that this water carbohydrate regimen is not to be continued indefinitely. Coma is an emergency and the method of feeding is an emergency diet to be followed for a day or two or possibly a week in an extreme case. As the patient rallies, gruels and purées containing a minimun amount of protein are added to the sugar feeding.

In diabetic coma, orange juice should be given with water as above directed. Other carbohydrates may be given carefully. Chief reliance must be put upon the newly discovered pancreatic extract, Insulin, the pancreatic hormone, by the proper administration of which diabetic coma may be almost certainly controlled.

In case of shock, intravenous feeding with glucose in normal saline solution renders great service. Intravenous feeding may also be employed in cases of great cardiac weakness in preparing a patient for operation. Woodyatt thinks that the tissues may assimilate as much as four-tenths of a gram (six grains) of glucose, per pound of body weight, per hour. Two pints, or even more, of a 5 per cent. solution of glucose, may be employed when necessary and the feeding may be repeated several times in twenty-four hours. Care must be taken that the solution is thoroughly sterile.

The usual formula is one part of chemically pure glucose, or dextrose, to nineteen parts of Ringer's solution.

\section{A Meatless Hospital Dietary.}

That dispensing with meat and meat extracts in the treatment of hospital cases is entirely practicable, as well as advantageous, is clearly shown by the recent action of the management of the great Beth Israel Hospital of New York City. After making a careful study of the results of the elimination of meats by a personal visit to the Battle Creek Sanitarium, the physician-inchief and superintendent of the hospital recommended to the 
board of trustees that, in opening their new three million dollar building which had been several years under construction, meats should be excluded from the bill of fare. The trustees unanimously decided to pursue this course, first having fortified their position by sending out a questionnaire to leading physiologists and other authorities in nutrition in this country, the replies to which were published, together with the proposed meatless regimen in "The Modern Hospital." The replies received to this questionnaire were unanimous in the endorsement of the plan proposed.

Said Dr. Francis G. Benedict, director of the Carnegie Nutrition Laboratory, Boston:

My good friends at the Battle Creek sanitarium would, I am sure, assure you that your project is hygienically and physiologically sound. Looking out for the food accessory substances (vitamins) I should be quite inclined to feel that your plan was a safe one.

Prof. Russel H. Chittenden, Sheffield Scientific School of Yale University, expressed the opinion that

It is quite possible, and indeed a desirable thing, for many reasons, to establish a lacto-vegetarian dietary in the wards of your hospital.

Said Prof. Graham Lusk, Department of Physiology, Cornell University Medical College, New York:

I believe that the lacto-vegetarian diet could be established in any hospital without detriment to the health of the patients.

Said Prof. E. V. McCollum, the Johns Hopkins University School of Hygiene and Public Health, Baltimore:

I have not the slightest hesitation in saying that a vegetarian diet supplemented with fairly liberal amounts of milk, is the most satisfactory type of diet that man can take.

Said Prof. Lafayette B. Mendel, Sheffield Laboratory of Physiological Chemistry, Yale University:

Inasmuch as I myself lived upon a diet devoid of meat, fowl, and fish for nearly a year,for purely experimental purposes, and remained in excellent health and vigor during that period, I have no fears regarding the wholesomeness of such a procedure.

If you have ever visited the Battle Creek Sanitarium, you will have noted that life is possible and good health is maintained without the use of meat. 


\section{Diet in Diseases of the Eye, Ear, Nose and Throat}

Dr. J. A. Stuckey, of Lexington, Kentucky, (Medical Review of Reviews, July, 1909) holds that diseases of the ear, nose, and throat are generally the result of intestinal autointoxication. In several hundred cases of disease of the nose and accessory sinuses, and diseases of the middle and internal ear, the doctor has found in all marked evidences of intestinal autointoxication. Examination of the urine has shown in nearly every case quantities of indican and other poisons formed in the intestine. It was also found that by treating the patient with the proper measures to combat intestinal autointoxication great relief was obtained from the ear, nose and throat disorders. The effect of various drugs such as quinine, for example, upon the ear is well known. It is evident that poisons generated within the body may similarly irritate the nerves and other tissues of the ear, nose and throat, as shown in asthmatic attacks, rheumatic pains, and various painful affections of the eye, ear, nose and throat. Changing the flora and adherence to a strict antitoxic regimen are essential for the most successful treatment of affections of the nose, ear and throat. It is especially important to secure efficient bowel movements three or four times a day. This may be accomplished by the methods elsewhere detailed.

\section{DIET IN DISEASES OF THE EYE}

The supreme importance of diet in the treatment of certain forms of eye disease has not been appreciated until very recent years. Dr. Ernest Clarke, an eminent English oculist, tells us that the eye registers in a very delicate manner the effects of intestinal toxemia. The mode of registration he thus explains:

In quite early youth the crystalline lens is practically a small bag of semi-fluid jelly, and accommodation takes place by its being squeezed by the action of the ciliary muscle in such a manner that its anteroposterior diameter is enlarged. So great is the squeezability (if I may use the term) of the lens in the very young, that an accom- 
modation power of 20 D. can often be recorded. As age advances, a hardening process, or sclerosis, goes on in the lens as in all the other tissues of the body, and so its elasticity becomes less and less, until a point is reached when the near point of accommodation, which represents the fullest accommodative power, has so far receded that the normal eye requires assistance in the shape of a convex lens in order to see near objects distinctly. This hardening of the lens may be delayed by the absence of, and accelerated by the presence of, certain poisons in the system, and intestinal toxemia takes a very high place in the list.

One individual has only an accommodative power of 2.5 D., while another has $8.5 \mathrm{D}$. What is the difference between these two individuals? In the one aged forty with $2.5 \mathrm{D}$. accommodative power the lens has hardened prematurely and become equal to the lens of a man aged fifty-five. That is, he is suffering from premature senility, and in the great majority of cases, in physical appearance, habits and powers, he is aged fifty-five. There are many causes which help towards this premature senility, but the factor common to a very large majority of them is intestinal stasis. On the other hand, those whose accommodative power is higher than normal look much younger than their years, and are in every way younger, and on going into their history it will be found invariably that they have taken the greatest care to avoid the least suspicion of intestinal stasis.

These facts, in connection with the premature senility of the lens, are another proof of the correctness of the old saying that "a man is as old as his arteries"; the premature sclerosing of the vessels often taking place at the same time as the process in the lens.

I think I have said enough to prove that premature hardening of the lens is a very constant sequela of intestinal stasis, and this is a very useful and easily ascertained index.

Here are three cases which have come under the writer's observation and which fully confirm the observations by Prof. Clarke :

A young woman of eighteen years found her sight failing. Examination by an eye specialist showed that the young woman's eye accommodation was so much impaired that she needed glasses such as are usually worn by a person of fifty years. By a change of regimen and improvement of bowel action, as recommended in this work, the abnormality in a few weeks disappeared and her eyes were young again.

A college professor of fifty years, wearing glasses adapted to a presbyopic person of his age, after following for a few months the regimen recommended in these pages found his eyes improved to such a degree that he had the same range of accommodation as a normal person of thirty-five years. 
A physician sixty years of age, who had for some time adopted a highly laxative, antitoxic regimen and who had taken care to change his flora, found his glasses uncomfortable, and on examination by an oculist discovered that these glasses, which had been very carefully fitted some years before, were more than ten years too old for him. A change was made to "younger" glasses, and he is still wearing them at the age of seventy, with entire comfort, although they are adapted to a person of fortyseven years.

In the above cases the rejuvenation of the eyes was accompanied by the disappearance of numerous symptoms of senility, and a great increase in physical and mental vigor and endurance.

Dr. Hugh Blake Williams, of Chicago, professor of ophthalmology in the Illinois Medical School, reports a case of interstitial keratitis which proved to be caused by intestinal autointoxication. The patient had been suffering with attacks of vomiting and diarrhea alternating with constipation prior to the appearance of eye symptoms. Local applications of atropine and dionin gave only temporary relief, but after the patient had been put upon a non-flesh dietary the cornmeal opacity cleared thoroughly and the ocular inflammation completely subsided.

A number of cases of neuroretinitis have been reported which were evidently associated with intestinal toxemia, the patients showing large quantities of indican in the urine. Pains in the eye as well as in other parts of the body attributed to neuritis, rheumatism and gout, are without doubt in many cases due to toxins of intestinal origin.

In view of the above facts, it is evident that the eye is very sensitive to conditions which may result from errors in diet, particularly intestinal toxemia. In both acute and chronic eye affections, thorough attention should be given to elimination of food residues by the aid of a laxative diet and the diet should be strictly antitoxic. The intestinal flora should be changed by an efficient application of the fruit regimen with lactose or lactodextrin and this should be from time to time repeated whenever the stools show any trace of putridity. 


\section{Infant Feeding}

The very best possible food for a very young infant is its own mother's milk, unless the mother is ill. The mortality of bottle-fed infants is several times that of those who are breastfed. Mother's milk differs from cow's milk. The milk of animals differs as does their blood. Mother's milk contains subtle elements designed by nature for the human infant, which are not found in cow's milk and is richer in the things a human infant needs. Mother's milk is so important for the infant that a very little, even so little as a spoonful or two daily, is of value to a bottle-fed infant as a supplement to its artificial diet.

Woman's milk contains only one-third as much casein as does cow's milk, and two-thirds as much total protein; but it contains nearly twice as much milk sugar (seven per cent.) and about the same amount of fat as is found in Holstein milk, so that its total calorie value is slightly greater, 19 for woman's milk and 18 for cow's milk. The iron content of woman's milk is about three times that of cow's milk, an important fact which makes possible a long period of nursing.

The mother should never forget that her own milk, even if deficient in quality and quantity, is better than any kind of artificial food. Only under the pressure of urgent necessity and positive orders of a competent physician should she give up nursing her baby.

If the child does not thrive on its mother's milk the physician should be consulted and the mother's diet should be carefully regulated.

If the mother eats carelessly for a single day or even a single meal, the milk may be affected and the child may suffer.

After the mother leaves her bed there frequently follows a period of depression, due to her physical weakness, and she is likely to feel discouraged and want to give up nursing. She fears that she is not going to be strong enough to furnish the necessary food and to stand the drain upon her own strength. She should, however, not allow herself to yield to this fear. If necessary, artificial feeding may be used to supplement the mother's milk for a short time, if the supply is deficient. In such a case, the 
directions given elsewhere for milk modification should be carefully followed.

The quantity of milk furnished by nursing mothers differs greatly. The amount may be only sufficient to supply the infant with a few ounces two or three times a day, making supplementary feeding necessary, or there may be much more than any child can take.

Certain foods increase the flow of milk. This is especially true of nuts. Malted nuts has a well deserved reputation for greatly increasing the flow of milk and improving its quality (Hoobler). It is extensively used for this purpose in lying-in hospitals.

In their excellent work, "Nutrition and Clinical Dietetics." Carter, Howe and Mason call attention to this remarkable property of nuts. "A diet of nuts and cereals and vegetables has recently been shown to be a satisfactory diet for the production of milk by women,"

The nursing mother should take daily not less than three pints of water in addition to the water taken at meals and in food.

The bowels must be kept well open, that is, moving three times a day by the use of bran and paraffin. Greens and fresh vegetables should be used daily and freely and fruits should be made a part of every meal.

Mother's milk is clean, whereas cow's milk is always more or less contaminated with stable filth. The danger is only partly obviated by pasteurizing, for while the process of pasteurization destroys the germs of tuberculosis, typhoid fever, scarlet fever, diphtheria and septic sore throat, infectious diseases which are likely to be borne by milk, the putrefaction germs derived from the cow's colon which are produced from spores are not destroyed. It is for this reason that sterilized milk undergoes putrefaction, while raw milk sours. This is especially true in the intestine of the chiid, since the sugar of milk which tends to prevent putrefaction is absorbed in the small intestine of the child, leaving undigested curds to undergo putrefactive changes in the colon.

The stools of a child fed on pasteurized or sterilized milk usually become dark colored and putrid, with a very offensive odor, while those of a breast-fed child remain yellowish in color and slightly acid in odor because of the presence of acid fermen- 
tation instead of putrefaction, one of the remarkable means adopted by Nature for the protection of the child against autointoxication. Intestinal toxemia in a child is a much more serious matter than in an adult, for the reason that an adult has acquired a certain resistance to the action of intestinal toxins.

Chapin has called attention to the fact that the character of the food of a young infant has much to do with the development of its alimentary canal. He points out that the hard, tough curds formed by cow's milk are adapted to a digestive apparatus in which a large part of the work of digestion is done in the stomach. In the case of the cow, goat and sheep, the stomach constitutes more than half of the digestive tract. In animals like the horse and donkey, which do not ruminate, the stomach forms only 10 per cent. of the digestive tract, nearly ail the work of digestion being done in the small intestine.

The digestive apparatus in man is much like that of the horse, the stomach forming about 20 per cent. of the whole digestive tract. The milk of the mare and the ass, like human milk, differs from that of ruminating animals in the fact that it does not form large tough curds destined to be retained in the stomach for a long time, but small flocculent masses which pass quickly into the intestine, where the principal work of digestion is done.

It is a great advantage to an infant to have the benefit of breast feeding for the first few weeks of its life, even though artificial feeding must be adopted later, not only for the reason that the character of its food has a remarkable influence in the development of its digestive apparatus, as shown by Chapin, but because of a fact pointed out by Langer, who discovered that so long as the child is breast fed the colon bacilli develop slowly in the colon, and apparently do little or no harm; whereas, later, when cow's milk is fed, the colon bacilli develop rapidly and acquire a high degree of virulence, which shows itself in indigestion, arrested growth and in various other ways. Langer does not assign any reason for the interesting fact which he records, but the writer would suggest that a sufficient reason may be found in the fact that breast milk contains 50 per cent. more lactose than does cow's milk. It is well known that lactose discourages the growth of the colon bacillus and encourages the development of $B$. acidophilus and other beneficent acid-forming organisms. It is also to be noted that in the presence of lactose the colon germs cease to develop toxins, thus losing their power for mischief. 
A further and important reason why a mother should nurse her infant is found in the fact pointed out by Fannileuer and others some years ago, that the milk contains, especially during the first weeks, immune bodies by means of which at least a certain amount of immunity against communicable diseases which the mother has had may be transmitted to the infant. The colostrum is especially rich in immune bodies. An opportunity to employ an immune wet nurse might sometimes be advantageously improved.

The number of feedings daily and the quantity for differen. ages is shown in the following table:

\section{FEEDING SCHEDULE}

$3 r d$ day to

3rd month

6 A. M.

9 A. M.

12 noon

3 P. M.

6 P. M,

9 P. M.

12 midnight ard month to

Sth month

$6 \mathrm{~A} . \mathrm{M}$.

9:30 A. M.

1 P. M.

4:30 P. M.

8 P. M.

12 midnight
Sth month to

12 th month

6 A. M.

$10 \mathrm{~A} . \mathrm{M}$.

2 P. M.

6 P. M.

10 P. M.

During the day the infant may be awakened from sleep to nurse. The habit of regularity is quickly established. It is not necessary to disturb the child at night, provided he is healthy and seems to be thriving. In fact, when he is normal and growing it will be better for the infant and for the mother to miss the midnight feeding. If in the interval he is restless, give a half feeding of warm boiled water from a bottle. This can be done without thoroughly awakening the baby.

Perfect regularity in nursing is especially to be emphasized. The child should be fed at exactly the same hours each day. This is quite as important in breast feeding as in bottle feeding.

The child must not be kept at the breast for more than twenty minutes. Overfeeding is more likely to occur than underfeeding. Sometimes the mother's milk is too rich and the baby must not be allowed to nurse so long. A little water should be then given to supply the amount of fluid needed. 


\section{A Simple Plan for Artificial Feeding of Infants.}

In beginning the use of cow's milk, the quantity given should at first be very small, as in some infants there exists an idiosyncrasy against cow's milk so great that symptoms of poisoning appear when milk is given and a large feeding might even produce fatal results. At first the cow's milk mixture should be given only once a day. If there are no signs of disturbance, a second feeding may be added after a few days.

The amount of food required by a child depends upon its age and size. Children require much more food in proportion to their size than do adults. For example, a child one week old may require one-sixth as much food as an adult weighing twenty times as much; while a child one year old may require one-third as much food as an adult weighing seven times as much.

Milk Mixtures. Pure cow's milk is too highly concentrated a food for an infant which has been breast fed. The stomach of the child must be gradually accustomed to the milk by feeding at first milk mixtures to which considerable water has been added. As the child grows older, less water is added. It is also necessary to add malt sugar or milk sugar as mother's milk contains much more sugar than does cow's milk. Cane sugar should never be used.

Many complicated methods and formulas for artificial infant feeding have been proposed and recommended by various authorities. Numberless special foods have been prepared and offered for sale at high prices. But extended experience has conclusively demonstrated that complex and troublesome methods of food preparation and expensive foods possess no real advantages; the best results may be attained by very simple and inexpensive means.

The first feeding of cow's milk should be very dilute. A good formula is two tablespoonfuls of milk, one level tablespoonful of milk sugar and ten ounces of boiled water. Every two or three days the milk and lactose may be increased a little until the proportions are seven to eight ounces of milk, an ounce and a half of lactose and twelve ounces of water.

The complicated formulas which have been much used in recent times have no advantage over the following simple formulas which have been well tested in the feeding of hundreds of babies, and which may be easily prepared in any home by an intelligent mother : 
First month: One-third milk, two-thirds water, with two ounces malt sugar to the quart. One ounce gives thirteen calories.

Second to sixth months: Half water, half milk, with two ounces of malt sugar to the quart. One ounce gives seventeen calories.

Seventh to ninth months: Two-thirds milk, one-third water, two ounces of malt sugar to the quart. One ounce gives twentyone calories.

Tenth to twelf th months: Use full milk, adding two ounces of malt sugar to the quart. One ounce gives twenty-eight calories. Infant Feeding Table.

\begin{tabular}{|c|c|c|c|c|c|c|}
\hline Age & & Weight & $\begin{array}{l}\text { Quantity in } \\
24 \text { Hrs. } \\
\text { Feeding }\end{array}$ & $\begin{array}{l}\text { No. of } \\
\text { Feedings }\end{array}$ & $\begin{array}{l}\text { Amt, of } \\
\text { Feedings }\end{array}$ & $\begin{array}{c}\text { Calories } \\
\text { Daily }\end{array}$ \\
\hline Birth & 7 & pounds & 20 ounces & 8 & $2 \mathrm{r} / 2$ ounces & 350 \\
\hline $1 \mathrm{mo}$. & 9 & pounds & 24 ounces & 6 & 4 ounces & 400 \\
\hline $2 \mathrm{mo.}$ & $10 \%$ & 6 pounds & 28 ounces & 5 & $51 / 2$ ounces & 475 \\
\hline $3 \mathrm{mo}$. & 12 & pounds & 30 ounces & 5 & 6 ounces & 510 \\
\hline $4 \mathrm{mo}$. & 13 & pounds & 32 ounces & 5 & $61 / 2$ ounces & 545 \\
\hline $5 \mathrm{mo}$. & 14 & pounds & 34 ounces & 5 & 7 ounces & 575 \\
\hline $6 \mathrm{mo}$. & 15 & pounds & 36 ounces & 5 & $71 / 4$ ounces & 612 \\
\hline $7 \mathrm{mo}$. & 16 & pounds & 32 ounces & 5 & $6^{\mathrm{t} / 2}$ ounces & 640 \\
\hline $8 \mathrm{mo.}$ & 17 & pounds & 33 ounces & 5 & $71 / 2$ ounces & 660 \\
\hline $9 \mathrm{mo}$. & 18 & pounds & 34 ounces & 5 & 8 ounces & 680 \\
\hline $10 \mathrm{mo}$. & 19 & pounds & 28 ounces & 5 & $52 / 3$ ounces & 700 \\
\hline $11 \mathrm{mo}$. & 20 & pounds & 29 ounces & 5 & $53 / 4$ ounces & 725 \\
\hline $12 \mathrm{mo}$. & 21 & pounds & 30 ounces & 5 & 6 ounces & 750 \\
\hline
\end{tabular}

Infants under one year should not take more than thirtytwo to thirty-six ounces of food daily. In changing to a stronger diet, that is, from half milk to two-thirds milk or two-thirds milk to full milk, the quantity should be reduced at first. After the child weighs eight pounds, the amount may be increased one ounce of the milk mixture for every six ounces that he gains in weight.

An excellent and simple method of determining the amount of milk to be given when full milk feeding is begun has been proposed by Bartlett. To determine the calorie value of the day's ration, multiply the child's weight in pounds by forty-five. From this subtract 120 calories, the value of the ounce of milk sugar required to supplement the sugar of cow's milk. The remainder 
divided by 20 , the calorie value of an ounce of milk, will give the number of ounces for a day's feedings. If the weight of the child is 15 pounds, the calculation will be made as follows: $15 \times 45=675 ; 675-120=555 ; 555 \div 20=27.7$. This will give $51 / 2$ ounces for each of the five daily feedings. For each ounce increase in weight one small teaspoonful of milk should be added to the total of the daily feedings.

Supplementary Feeding. One of the most important of all foodstuffs which may be usefully added to milk in infant feeding is malt sugar. This is the only sugar which is native to the body, as it is produced in the body by digestion of starch.

The free use of lactose sometimes produces digestive disorders, and an infant will assimilate more maltose. However, the use of lactose or Lacto-Dextrin is desirable for the reason that cow's milk contains less than two-thirds as much milk sugar as does mother's milk. This sugar is highly essential to encourage the growth in the intestine of protective bacteria, thus combating intestinal toxemia and colitis.

Cane sugar is irritating to the stomach and should never be used in infant feeding. Candy should never be given to infants or young children. The use of this unwholesome sugar is the cause of much disease and suffering in both children and adults.

Orange Juice. The juice of sweet oranges is a most important, almost essential aid in the artificial feeding of infants. Orange juice is rich in vitamins. When sterilized milk is used, orange juice is necessary to supply the vitamins which boiling destroys in the milk and which are absolutely essential for the healthy development of the infant. The observations of Holt and others show that every bottle-fed child requires daily at least one ounce of orange juice when cow's milk is used. The orange juice should be given in doses of two or three teaspoonfuls at intervals during the day. When malt sugar and cereals are used, the amount of orange juice must be increased in proportion to the amount of sugar or cereals added to the milk. The more sugar or starch, the more orange juice is needed. In children who show evidences of scurvy, four or five ounces of orange juice must be given daily to supply the needed vitamins. It is now known that in cases in which it was formerly supposed that infants were suffering from an excess of cereals in the food the real fault was the absence of vitamins in the carbohydrate given. Fine wheat flour and prepared infant foods are lacking in vita- 
mins. This discovery has already been the means of saving thousands of infant lives. Every mother should know that orange juice or tomato juice is indispensable in the artificial feeding of infants of all ages. These highly valuable juices should be used in all cases of artificial feeding. All "baby foods" are deficient in vitamins. The same is true of all cereal preparations, and even certified milk is often rather deficient in these essential food principles. It is now known that tomato juice, either fresh or canned, is fully equal to orange juice.

Tomato or orange juice should be given to all infants whether nursing or bottle-fed after the fourth month. All bottle-fed infants should be fed either tomato juice or orange juice daily. Infants four months old may take a teaspoonful of orange juice or tomato juice two or three times a day. If the infant is nursing, the juice should be given half or three-quarters of an hour before feeding; if bottle-fed, give between the feedings.

Purées of Fruits. Purées of ripe fruit such as sweet apples, pears, and bananas, prepared by pressing the fresh pulp through a fine colander, are exceedingly wholesome and useful additions to the dietary of the bottle-fed infant. Modern physiological experiments have shown the necessity for variety in foodstuffs to insure a full supply of vitamins and salts.

\section{Vegetable Broths.}

While meat extracts, bouillon and animal broths of all sorts should never be given under any circumstances to infants, vegetable broths are very useful. The following is a good formula.

\section{Savita Vegetable Broth.}

$\begin{array}{llll}21 / 2 & \text { quarts water } & 1 & \text { cup diced celery } \\ 3 / 4 & \text { cup diced carrots } & 1 & \text { cup diced onion } \\ 1 & \text { cup shredded cabbage } & 1 / 2 \text { cup strained tomato } \\ 1 & \text { cup diced potatoes } & 11 / 2 \text { tablespoonfuls Savita }\end{array}$

3 tablespoonfuls butter

Prepare the carrots first and allow them to cook while preparing the other vegetables. Add these and cook slowly one and one-half hours. Pour through a collander and add to the clear liquor the butter and savita, and boil for five minutes. Should make one quart of broth. 


\section{Vegetable Broth for Babies.}

To one cup of Savita vegetable broth, add one-half cup of water.

Two or three ounces of vegetable broth may be given daily with great advantage. Vegetable bouillon supplies nutritive salts which are needed to promote growth and tissue building.

\section{Malt Sugar.}

Malt sugar in place of cane sugar. Cane sugar is a pure carbohydrate product. It contains nothing but sugar and is intensely sweet. Malt sugar is less sweet-an advantage, because in this respect it more nearly resembles lactose, the natural sugar of milk.

Malt sugar has the great advantage of containing water-soluble B vitamin in considerable amount, as well as iron, lime and other things essential to nutrition. Malt sugar, in other words, is something more than sugar. Purées of dates, prunes and raisins may also be given to infants with great benefit in many cases because of the richness of these fruits in lime and iron.

Malt sugar has also another important advantage which has only recently come to be appreciated; namely, the considerable amount of dextrin which it contains. Pavlov showed the great value of dextrin as a peptogen, that is, a substance which actively promotes the digestive processes. This makes malt sugar of great importance as an aid to the feeble digestive powers of the average infant.

Torrey, Rettger and others have recently shown dextrin to be one of the best of all substances for promoting the growth of the protective germs.

Certain acid-forming germs, the B. bifidus and B. acidophilus make their appearance in the colon of the breast-fed infant within a few hours after birth.

When cow's milk is fed, these essential protective germs tend to disappear, their place being taken by putrefactive germs which produce poisons causing dark, foul-smelling stools, fretfulness, restless sleep, lack of appetite, delayed growth and other symptoms of the chronic poisoning induced by dangerous germs which, unfortunately, are always found in great numbers in commercial cow's milk.

Torrey, experimenting with white rats, found that by feeding dextrin the protective germs could be restored within three 
or four days, the putrefactive and colon germs disappearing almost wholly. This is a discovery of very great importance and helps to explain the benefits derived from the feeding of barley gruel, malt soup, potato soup, and other preparations containing more or less dextrin. In malt sugar, dextrin is found in greater abundance than in any other food suitable for young children, a fact which in part doubtless explains the great benefits following the substitution of malt sugar for cane sugar in the diet of bottle-fed infants, an observation which has led modern specialists in the care of children to recommend malt sugar as greatly superior to either cane sugar or milk sugar in infant feeding.

The malt sugar should be added to the milk or milk and water mixture in the proportion of one ounce to the pint. Malt sugar is now obtainable at most drug stores.

\section{Vitamins.}

Gruels prepared with wheat germs are very rich in vitamins and are advantageously used in supplementing the dietaries of artificially fed children.

Wheat germs may be combined with oatmeal, barley or any other cereal suitable for gruels in proportion of one part of the germs to nine or ten parts of cereal meal. Boil half an hour and strain.

Recent observations show that mother's milk as well as cow's milk is often deficient in vitamins because the food of the mother or of the cow is lacking in these food accessories. The food of cows in winter, when they eat much dry feed, is certain to be deficient in growth-stimulating elements. The winter food of mothers is likely to be deficient for a similar reason, unless pains are taken to supplement the usual dietary with liberal quantities of greens, fresh vegetables, tomatoes, and fresh fruits.

\section{Fats Often a Source of Disturbance.}

An excess of fat in cow's milk is one of the most serious causes of trouble in the artificial feeding of infants. Many infants are able to digest fats only in small amounts and when the food contains an excess, the undigested portion undergoes decomposition in the intestines, forming irritating and poisonous substances. An excess of fat is one of the most common causes of indigestion and bowel troubles. This difficulty is overcome by diluting the milk with skimmed milk or with water, or by removing a part of the cream. 
The fat of cow's milk consists of globules much larger than those of human milk, which is one of the reasons given for the inferior digestibility of cow's milk. The milk fat of Holstein and Guernsey cattle occurs in smaller globules than that of other breeds of dairy animals.

The common practice of taking the baby to the table and permitting him to "nibble" all sorts of foods is most pernicious. The infant's stomach is able to digest only simple foods.

\section{Dangerous Foods.}

Beef tea, meat juice, and meat broths are in the highest degree unwholesome for infants. These preparations have practically no food value. They represent the excretory products in the meat and encourage putrefaction in the intestines.

Eggs are also a dangerous food for young infants. Many infants are "sensitized" to-eggs so that a single feeding may produce highly poisonous, even fatal effects.

\section{Anemic Infants.}

Many infants become anemic soon after the sixth month because of the small amount of iron in cow's milk. This may even occur in the case of breast-fed infants, for mother's milk contains little iron. The liver of the child born at full term contains iron enough to last it for eight or nine months, but it is reasonable to suppose that a premature infant born from three to eight weeks before full term must be launched into the world with a deficient store of iron in its liver. Such infants will need a supplementary supply of food iron earlier. Test the blood of the infant for hemoglobin whenever there is any ground for suspicions that its supply of iron may be insufficient. Malted nuts may be used in alternate feedings with much advantage.

One teaspoonful of purée of steamed spinach, or steamed carrots may be given daily after the sixth month. Three ounces of malt sugar daily will insure the infant an adequate supply of food iron. Cane sugar and milk sugar supply no food iron.

Cereals for Infants. Oatmeal is a specially wholesome food for all young children who have attained the age of six months, and may often be used earlier with advantage. According to Chalmers Watson, oatmeal and milk constitute a better food than bread and milk because of the stimulating action of oatmeal on the thyroid gland, which tends to accelerate growth. 
It is better that the oatmeal should not be too long boiled. Just sufficient boiling to render it liquid enough to feed conveniently is quite sufficient. Long boiling impairs the precious vitamins. An eminent specialist in the diseases of children records that he found bottle-fed babies did well on a diet consisting of milk with the addition of fifty grams daily of raw starch, which ignorant mothers had administered through failing to follow directions. All the cereal starches are digestible in small amounts in the raw state and aid bowel action and combat intestinal toxemia.

Vita Wheat contans the wheat germ and when well boiled and strained, is a most excellent cereal for infants. Cream of wheat and cornmeal may be used if a rounded teaspoonful of wheat germ meal is added for each ounce of the cereal. The wheat germ meal is a necessary addition when degerminated cereals are used. In place of wheat germ meal, Savita or Marmite, an extract of yeast, may be employed.

"Zo" is an excellent cereal for infant feeding because of its richness in vitamins and iron.

Dry toast is another form of cereal food which may be given to infants in small amounts after the teeth make their appearance. Graham or whole wheat bread should be cut in thin slices and placed in a moderately hot oven until brown through the whole thickness of the slice. This toast is much superior to grdinary commercial zwieback.

\section{Vegetable Soup.}

Potato soup prepared without meat or other seasoning than a little butter or cream, may be given once a day at nine or ten months. Soup or purées prepared from potatoes and spinach mixed has the advantage that it supplies iron, which is deficient in potato.

Butter Soup for Infants. Czerny and Kleinschmidt suggest for the feeding of very young infants, especially premature infants, butter soup combined with milk. Butter soup consists of $71 / 4$ level teaspoonfuls of melted butter, $51 / 4$ level teaspoonfuls of sugar (preferably malt sugar), 143/4 level teaspoonfuls of wheat flour and $17 \frac{1}{2}$ ounces of water. The food value of soup prepared in this way is about 28 calories to the ounce. For an infant weighing 7 pounds, 14 ounces of this soup should be given daily mixed with 7 ounces of whole milk. The food should be 
given at 7 feedings. The food value of the mixture will be about 24 calories to the ounce. This diet is particularly rich in vitamin $\mathrm{A}$, which is needed by the young infant to promote growth.

Buttermilk for Infants. Marfan, an eminent specialist in diseases of children, gives the following directions for the prepration of buttermilk for infants suffering from diarrhea, claiming it to be an almost infallible remedy:

A little sour milk is added to the milk to be prepared. This is set aside for 24 hours and allowed to sour. At the end of this time it is skimmed, then churned for 40 minutes and passed through a fine sieve. An ounce of malt sugar and a tablespoonful of rice gruel are then added with a little salt and the whole is very slowly heated to boiling while constantly stirred with a cream whip. This preparation should be made the sole food of the child until the diarrhea disappears. Buttermilk prepared with $B$. acidophilus is doubtless the best of all buttermilks for the reason that the $B$. acidophilus is the normal protective organism of the intestine. Acidophilus buttermilk should be taken without cooking, as heat will quickly destroy the bacilli which it is desired to plant in the colon.

Water for Infants. Infants often suffer from lack of water. The crying of young children is often due to thirst rather than hunger. A child under one year of age should receive for each pound of body weight two ounces of liquid, either in the form of water or liquid food. Children over a year old require not less than one quart of water daily.

Lime Water. The extensive use of lime water as an ingredient of modified milk used for bottle-fed children renders important a brief reference to this widely used mineral solution. Lime water differs little from hard water except in the fact that it contains more lime. Tibbles says that the value of lime water is disputable. Bunge declares that inorganic lime is not assimilated. As a matter of fact, the amount of lime in lime water is less than that found in cow's milk. A pint of cow's milk contains 26 grains of lime $(\mathrm{CaO})$ while a pint of lime water contains only 20 grains or less than four-fifths as much. In milk and other foodstuffs the lime is in organic combination with the casein and so is prepared to be utilized by the body, whereas in lime water it is in its native mineral form and needs to be acted upon by the cells of the living plant before it is suitable for 
human food. It is believed by Tibbles and Bunge that to insure utilization, lime "must be organically united to the proteins of the food."

The addition of lime water or soda to milk is an objectionable practice for the reason that these alkalies injure and may destroy the vitamins upon which the development, health and even the life of the infant depend.

Cane Sugar Poisoning. According to Fischl, cane sugar, in excess, may in young infants give rise to fever, with rapid breathing, stupor, tendency to collapse, and the occurrence of sugar in the urine. This is especially likely to occur from the use of milk preparations containing much sugar, such as the Swiss condensed milk and similar preparations.

Cane sugar should be excluded from the diet of infants.

Malt Soup and Scurvy. Experience has shown that malt soup or malt soup extract, when fed to young children, is likely to lead to the development of scurvy. When malt soup or any other artificial food is used in infant feeding, orange juice or tomato juice should be added in proper quantity.

The Deleterious Effects of Meat Diet for Children. Professor Sherman of Columbia University, an able physiologist, several years ago called the attention of the profession to the fact that meat is lacking in lime salts. This fact alone renders it an unsuitable food for children.

Dr. Winters maintains that one of the many evil consequences of the early use of meat by children "is the loss of relish it creates for the physiological foods of children-milk, cereals and vegetables." Says Dr. Winters, "A child that is allowed a generous meat diet is certain to refuse cereals and vegetables. Meat, by its stimulating effect, produces a habit as surely as does alcohol, tea, or coffee, and a distaste for less satisfying foods. The foods which the meat-eating child dislikes contain in large proportions certain mineral constituents which are essential to bodily nutrition and health, and without which the processes of growth and development are stunted."

Dr. Winters attributes to the use of meat many of the disorders to which children are subject, particularly incontinence of urine, rheumatism, chorea, rheumatic inflammation of the tonsils and torticollis, night terrors, urticaria, angio-neurotic edema, anemia, convulsions and petit mal.

According to this author, "there is more so-called nervous- 
ness, anemia, rheumatism, valvular disease of the heart, and chorea at the present time in children from an excess of meat and its preparations in the diet than from all other causes combined." No doubt these observations are well founded. A meat diet is as unnatural for a young infant as for a young gorilla.

\section{The Infant's Stools.}

In the feeding of children of all ages, careful observation of the character of the flora is of the utmost importance. This may be done in a crude but practical way by simple examination of the stools. If the stools are dark in color and of putrid ammoniacal odor, putrefactive germs or flora may be known to be dominant. The flora needs to be changed by regulation of the diet in a manner elsewhere recommended for this purpose. Porter, Morris and Meyer (Am. Jour. Dis. of Chil., 1919), who have made a special study of the intestinal bacteria in children, tell us :

In children who suffer from certain types of alimentary intoxication with malnutrition, the intestinal flora departs in a uniform manner from the normal, and this departure is always characterized by the establishment of bacterial types predominantly putrefactive. The return of these children to normal health is coincident with a regression of the intestinal flora toward predominantly fermentative types and a later swing to balance between the two types. Such changes in the intestinal flora can be brought about in the intestine of the human infant by withdrawing of animal protein and persistently feeding large amounts of lactose (from 2 to 4 ounces daily), and other carbohydrates (the period which may be necessary to produce this variation being from 10 to 40 days). . . The progressive cessation of the symptoms of intoxication and a return of toxemic patients to nutritional health coincides with the recognizable dominance of a fermentative flora (B. acidophilus). Lactose and dextrins are the carbohydrates most effective in encouraging the rapid establishment of a fermentative flora in the intestines of infants and children.

\section{Nursing.}

Observation of the habits of primitive people and lower animals indicates that the nursing period naturally continues until some time after the eruption of the first teeth is complete. Garner noted that apes, our nearest kin in the animal world, nurse their infants from five to seven months. Their first teeth are complete by the third month. Young camels nurse for a year although they begin to eat with their mothers a few weeks after they are born. 
Among primitive people nursing continues until the first teeth are complete, or for three years, although considerable other food is eaten. Pictures of young children standing at the breast of savage mothers are common. Referring to this fact, Dr. Vander Bogert well says: "But we must remember that these are primitive people in their natural state and that because of natural conditions they are able to produce a better milk supply and for a longer time." This simple statement contains much food for thought. In how many other ways is the tame or civilized human being inferior to his wild or savage brothers and sisters because of unnatural conditions of life!

It is a matter of general observation that the modern civilized woman is unable to produce a good quality of milk for a longer time than one year. Babies that are breast-fed for a longer time do not develop well and in later life develop physical defects and digestive disorders which are attributed to too long breast-feeding.

\section{Weaning.}

Artificial feeding is by no means a full substitute for breastfeeding, hence a child should never be weaned before the age of nine months except for unavoidable reasons. No food can be so well adapted to the young infant's needs as its mother's milk. Mother's milk contains three times as much iron as does cow's milk, which explains the fact that artificially-fed infants are usually pale, anemic and far more subject to illnesses of various sorts than are breast-fed children because of their low resistance. Breast milk contains, in addition to the food principles necessary for nourishing the infant, subtle substances derived from the mother's blood known as immune bodies, by means of which at least some degree of the immunity acquired by the mother against disease is transmitted to the infant.

It is well to prepare the infant for weaning by beginning bottle feeding early and increasing the amount gradually as the infant advances in age. Bottle feeding may be substituted for breast feeding once a day, after three months, with great advantage. At this age, infants accept the bottle much more readily than later. When bottle feeding is begun as early as three months, the proportion of milk employed should be about six ounces of milk to fourteen of water. When bottle feeding is begun at the age of six or seven months, a larger proportion of 
milk may be employed, say eight ounces of milk to twelve of water. For feeding program, see Table XXX.

Malted nuts or lacto-dextrin may be added to the mixture in proportion of two ounces to the quart. This will supply practically the same amount of carbohydrate ( 7 per cent) found in breast milk. Lacto-dextrin is superior to either lactose or malt sugar for the reason that it supplies vitamin, which is never in excess in cow's milk and is always deficient in milk mixtures.

\section{Mixed Feeding.}

Infants do far better when breast milk is very gradually withdrawn. Even though the mixed feeding may cover a very considerable period, a small amount of breast milk is a great advantage to the infant, not only because it is richer in iron but because of other elements which it supplies which are not found in cow's milk.

When the infant has reached the age of five or six months, two or even three bottles may be given daily in place of breast feeding and the child may be given small quantities of purées of vegetables and fruits of various sorts so as to accustom it to the flavors of these foodstuffs. Well boiled oatmeal gruel may also be given in moderate amounts, slightly sweetened with lactose or malt sugar.

The proper rule seems to be to begin feeding wholesome simple food in small quantities as soon as the first teeth appear, especially purées of greens and of fresh vegetables, fruit juices and gruels. From week to week the quantity and the variety of foods may be increased. Orange juice and tomato juice may be given almost from the outset, and encourage growth, appetite and bowei action.

Children should be taught from a very early age to make use of sweet fruits instead of cane sugar. Dates, figs and raisins, first in the form of purées, then in their natural state, should be made a regular part of the child's bill of fare.

\section{Vomiting of Infants.}

Vomiting in bottle-fed infants may be due to swallowing air while feeding. To prevent, hold the bottle so as to keep the neck wholly filled with milk.

If large curds are present, the milk mixture is probably too strong. More water should be added. 
If the vomit has a rancid odor, the stomach must be washed out. Less fat should be given and more malt or milk sugar.

If vomiting is very persistent, there may be obstruction of the pylorus requiring operation. In such cases, operation should not be delayed. An X-ray examination should be made.

Simple regurgitation of the food after eating, without the presence of tough curds, has no significance other than that the child may have eaten a little too much.

Hot applications over the stomach before and after feeding will often control troublesome vomiting.

\section{Diarrhea in Infants and Cholera Infantum.}

In cases of intestinal irritation with diarrhea or cholera infantum, the child should be fed on a thin barley or oatmeal gruel until the bowel looseness is checked, and then malt soup should be employed.

\section{Pyloric Obstruction.}

Congenital obstruction of the pylorus is by no means a rare condition in infants. If the obstruction is complete, as shown by the vomiting of everything eaten and rapid emaciation, an operation should be performed at once. If the obstruction is not complete, as shown by the fact that only a portion of the food is vomited, and even a slight gain in weight, an attempt should be made to build the patient up by breast-feeding and by the feeding of lactose or malt sugar by enema. One ounce of sugar should be dissolved in a pint of water, and 3 or 4 ounces of this sugar solution, at body temperature, should be introduced by enema three or four times a day. Breast milk may be given, together with an equal quantity of sugar water. Time should not be lost in ineffectual efforts to build the child up. The case should be placed in the hands of a competent surgeon for operation.

An hour after the operation, half an ounce of sugar solution or equal parts of sugar solution and breast milk may be given. During the first twenty-four hours the sugar water should be given every hour and a half. After the second day an equal quantity of mother's milk may be mixed with the sugar water. If mother's milk is not obtainable, cow's milk may be givenat first, one part to nine of sugar water; the next day, one to eight; third day, one to seven, gradually increasing the milk until the proportion is one to three of sugar water. 
After the first day, the amount of food given at each feeding is gradually increased from half an ounce to one ounce at the end of the fifth or sixth day. Orange juice may be given as to any other infant. Vegetable broths are needed to supply food salts.

\section{Feeding During Fever.}

During an attack of fever from any cause, less fat and protein are required, but the amount of sugar should be increased. On this account, the feeding mixture should be diluted by the addition of an equal quantity of water. The added water should contain an ounce of sugar to the pint. Cane sugar should not be used because of its irritating effects. Lactose, malt sugar or better, lacto-dextrin are suited to the infant's needs. Orange juice should be given in proportion to the age of the child. An infant too feeble to take its food in the usual way, or unconscious, may be fed by means of a soft catheter passing into the stomach. Water as well as food should be given. In addition to a quart of food, a pint or a pint and a half of water will be required to aid elimination.

\section{Hypersensitiveness of Infants to Certain Foodstuffs-Anaphylaxis.}

For many years it has been known that children, as well as adults, may be made very ill by small quantities of foods and other substances which are ordinarily harmless. So long ago as 1831 Elliotson noted the connection between the pollen of plants and hay fever. Hutchinson, in 1884, reported the observation that certain patients suffered from nausea, vomiting, and abdominal pain after eating eggs. Within recent years a large number of similar cases have been reported. It is now known that both children and adults are frequently sensitized to cow's milk, shellfish, meat and other substances. The following case, reported by Dr. Blackfan, of Baltimore, will be found interesting as an example of the effects of food upon a sensitized person:

An infant that had been exclusively breast-fed was given, when six weeks old, a small amount of cow's milk. This was immediately vomited. One month later one-half teaspoonful of milk caused a severe general reaction. The baby vomited and had diarrhea. He became cyanosed and so prostrated that the physician considered him dangerously ill. Bread prepared with cow's milk brought on a similar reaction, as did the administration of three drops of one in 
32 dilution of condensed milk. Cow's milk in any form and in infinitesimal amounts always caused romiting, loose stools, cyanosis, irregular respirations and prostration. He was able to take goat's milk without any symptons. In order to determine that the symptons were due to cow's milk the protein of cow's milk was applied to the skin. This was followed by an area of edema and erythema after four hours. When introduced in this manner cow's milk caused the same general symptons as when taken by mouth. Protein from beef, egg, horse serum, barley, human milk and goat's milk caused no reaction when applied to the skin. The baby was nursed at the breast and given supplemental feedings of goat's milk until ten months old, after which minute amounts of cow's milk were given by mouth. Gradually the amount was increased until the hypersensitiveness to cow's milk was overcome. The paternal aunt had an idiosyncrasy to shellfish and the paternal grandfather and great-grandfather had asthma.

An infant aged two years was breast-fed for two months, after which he was given a formula of cow's milk. Cereals, broths and vegetables were added subsequently. Eczema appeared when he was given a soft-boiled egg. This was immediately vomited and he was sick for several hours. Six months later egg was given and he again vomited. The protein of egg, cow's milk, barley, horse serum and beef gave a marked reaction when applied to the skin. By omitting eggs, meats and milk from the diet the eczema improved, and by giving them in small amounts and gradually increasing them he was finally able to take these foods without a return of the eczema. The mother had eczema when she was an infant.

A girl of ten suffered from fever, vomiting, diarrhea, great prostration, edema and intense general itching whenever she ate meat. This child showed no intolerance for milk.

Methods now exist by which a person may be tested for hypersensitiveness by simple means which may be employed by any physician familiar with the technic. No child should be given cow's milk without first being tested for hypersensitiveness; in other words, in changing from breast milk to cow's milk, the cow's milk should be given first in very small quantities. The amount at first should be not more than a teaspoonful and should be well diluted with water, and after it is given several hours should elapse before another dose is administered, to give opportunity for the development of any possible unfavorable reaction.

By means of allergens, prepared from various foodstuffs, sensitivity tests may be made for more than a hundred different articles which have been incriminated clinically of causing anaphylaxis. The test is usually made after the method of vaccination. A slight abrasion of the skin is made and the substance to 
be tested is rubbed upon it. The appearance of a macula within a few minutes shows sensitivity.

Hypersensitiveness may be inherited. In some instances every member of a family of children are found to be sensitized to the same foodstuff. Sensitivity may appear in very young infants or develop a few months later. Very marked sensitivity may disappear in a manner wholly unaccountable.

The foods to which infants and young children are most often sensitized are cow's milk, oatmeal, potato, eggs, peas, beef juice, chicken, fish and strawberries.

A new and apparently valuable method for discovering cases of this sort has been recently brought forward by Widal. This sagacious observer has discovered that the application of his simple milk test for hepatic insufficiency is one of the best means of discovering sensitivity. The test is a simple one, as elsewhere described (see Index). It owes its value to the fact that when sensitivity exists a slight anaphylactic effect is observed, the evidence of this renction being a more or less considerable fall in the white blood count. According to Widal, when this reaction is found, the patient is sensitized to something, and the particular form of protein to which he is sensitized may be worked out by the application of the appropriate tests.

\section{Infant Foods.}

Most infant foods consist of starch which has been fully or in part digested by means of malt or exposure to a dextrinizing temperature.

According to a report of the Connecticut Agricultural Experiment Station, most infant foods are prepared by very simple methods from ordinary foodstufts.

Horlick's Malted Milk consists of a malt-digested mixture of wheat flour and milk.

Fessenden's Food is made from arrowroot, wheat, rye, and barley malt. starch.

Ridges' is simply "baked flour," consisting largely of raw

Mellin's Food consists of wheat flour which has been digested with barley malt.

Allenbury's Milk Food consists of a mixture of pasteurized milk and malted wheat. 


\section{Vegetable Milk.}

The Chinese have for ages made from the soy bean a preparation which so closely resembles the dairy product that it may not inappropriately be called vegetable milk. A much better preparation is made from walnuts, almonds, and even peanuts. These nut milks so closely resemble ordinary milk that they may to a large extent take its place in the diet. Milk prepared from walnuts and almonds is used in China, the Philippines and throughout the Orient, as a food for nurslings for whom a wet nurse can not be provided. This custom prevails extensively in those Asiatic countries in which cow's milk is but little used.

A nut milk commonly known as "Malted Nuts" has been made in this country for more than thirty years and has come into extensive use.

Nut creams prepared from almonds and other nuts are also valuable. These plant protein milks render great service especially in the treatment of infants and invalids who are sensitized to cow's milk. They are also valuable resources in the treatment of colitis in infants and in cases in which change of the flora is found difficult, so that the suppression of all animal proteins becomes necessary.

\section{Humanized Milk.}

A preparation which often renders great service in the feeding of babies with whom cow's milk disagrees because of the formation of curds, consists of malted nuts or nut cream combined with ordinary milk, one ounce of malted nuts dissolved in six ounces of hot water. To this is added a tablespoonful of milk.

The effect of adding nuts is to cause the milk when brought in contact with the gastric juice in the child's stomach, to form small, soft, flocculent curds similar to those formed by human milk. A large part of the casein is replaced by the more easily digestible plant proteins.

The composition of this preparation so closely resembles that of human milk that it may be said to be "humanized." 


\section{Diet of Young Children}

\section{One to Five Years of Age}

The physiological needs of a child are practically the same as those of adults with this exception, however, which must be constantly kept in mind, that the child is a developing organism, that its body is not yet formed, but is growing rapidly and hence requires not only special attention to the kinds and quality of food eaten but also special consideration as to quantity. A child requires a much larger intake of food in proportion to its weight than does an adult. Between the ages of two months and five years, a child requires, in fact, practically double as much food in proportion to its weight as does an adult; that is, a boy of seven weighing fifty pounds requires as much food as an adult weighing one hundred pounds; and after the ages of ten or twelve, active children require practically as much food as do adults who are not engaged in hard, muscular work. The stomach of a child of ten years is scarcely half the size of the average adult, and having practically the same amount of work to do as has the adult stomach, it may easily be overtaxed, either by overeating or by the use of foods difficult of digestion.

In general, it may be said as regards the kind of food suitable for children of three years of age and afterwards, that the child may be permitted to eat anything that is really suitable for its parents. The presence of teeth indicates the suitability of foods requiring mastication, and if only such foods are eaten as are perfectly adapted to meeting the biologic or physiologic requirements of the human body, there is no reason why a child may not eat anything which his parents eat, although, as will be noted later, there are certain important particulars in which the dietetic requirements of the child are essentially different from those of the adult, to which careful attention must be given in the preparation of bills of fare for growing children, especially as regards the need of greater quantities of lime and iron.

While it is true that a biologic diet is safe and wholesome for children that have acquired teeth, it is important that attention should be called to the fact that many articles which usually 
enter into the daily bill of fare of adults, but which are better ${ }^{*}$ excluded because of their unwholesomeness, are particularly unwholesome for children, and hence must be rigorously excluded from their dietaries. Among articles of this sort may be mentioned particularly condiments of all sorts. Mustard, pepper, peppersauce, cayenne, horseradish, cloves, even nutmeg, and vinegar and pickles of every sort, while unwholesome for adults, are pernicious and even positively dangerous for young children. None of the substances named have any food value whatever. They supply no body need. They are instead poisons which injure the stomach and work various mischiefs in the body.

A properly trained child has no relish for any of the articles mentioned above. They are unnatural substances, an appetite for which is cultivated and must be regarded as a perverted taste. Hot condiments of all sorts over-excite the stomach, wear out the glands which secrete the gastric juice and do other mischiefs to which attention has been called elsewhere in this work (see Condiments). Even salt should be used sparingly.

The use of tea and coffee is another practice which works great injury to the growing child. The caffein of tea and coffee, as every one knows, tends to wakefulness. The child needs a great amount of sleep. Ten or twelve hours out of every twentyfour, or even more, in the case of very young children, are required to afford the body opportunity for tissue building bones, muscles, nerves and other tissues, a process which proceeds rapidly only during sleep. Plants grow much faster at night than in the daytime and the same is true of children. The use of tea or coffee tends to lessen the hours of sleep through its effect upon the nervous system and thus lessens the rate of growth and dwarfs the child.

Coca Cola has precisely the same effect as coffee owing to the caffein which it contains. Many parents being unaware of this fact, permit children to use Coca Cola ad libitum, a practice which cannot be too strongly condemned. The Coca Cola habit is easily formed and will naturally lead later in life to other drug habits.

The idea that meat is highly essential for children has been in recent years widely propagated by various unreliable syndicate writers and, unfortunately, has sometimes been countenanced by physicians who have not given special study to dietetics to qualify them to give expert advice upon the subject. The 
studies of Professor Sherman, of Columbia University, McCollum, of Johns Hopkins, and of numerous other authorities who have conducted nutrition researches in laboratories especially equipped for the purpose, have shown most conclusively that meat is not essential for the feeding of children. In fact, the very opposite is the truth. Every food essential which can be secured by the use of meat is supplied by milk and in a form far better adapted for the use of the child than in meat. Meat, on the other hand, is highly lacking in certain elements which are absolutely essential for children. This is particularly true of the salts essential for building up not only the bones but other of the bodily tissues. But the greatest objection to the use of meat for children is to be found in the fact that it is practically always swarming with bacteria of the most pernicious sort. Fresh meat, fish, chicken, fresh oysters, meats in all forms, in fact, are fairly swarming with putrefactive bacteria.

In the process of slaughtering animals, their tissues become thoroughly infected with the manure germs which are found upon their bodies. These bacteria rapidly penetrate the tissues and within a few hours after death the carcass of the animal is everywhere swarming with these filthy intestinal bacteria, which are not destroyed by cooking. When flesh is eaten, these parasites infect the intestine, particularly the colon, and cause diarrhea, appendicitis and various other infections.

Feeding meat to young children is not only absolutely unnecessary but is one of the most certain methods of laying the foundation for life-long misery from constipation, autointoxication and various disorders which grow out of these conditions.

Every mother should know that in order to supply a child with the things essential for its nutrition, foods must be taken as nearly as possible in their natural state. Unfortunately many foods which come to our tables are denatured by the manufacturers of breakfast foods and other staples. A child fed upon bread and milk, a dish which is perhaps more generally used than any other in the feeding of children, is certain to be poorly developed, to suffer from poverty of blood, constipation and possibly more serious maladies. A bread and milk diet, as well as a bread and meat diet, is a most incomplete bill of fare. Food to be suited to the needs of children must be particularly rich in vitamins, lime and iron. It is unnecessary to repeat here the facts in relation to vitamins, lime and iron which have been fully presented else- 
where in this work (see index). We wish only to emphasize the fact that children require at least double as much of these food essentials as do adults because of the fact that they are developing organisms and require not simply a sufficient amount of new material to replace that which has been worn out and lost from the body, so-called "repair material," but a large amount of building material out of which to construct the framework of the body, the bones and various soft parts attached thereto.

There are few foods which are richly supplied with all three of the food essentials mentioned, lime, iron and vitamins. It is only by a proper combination of foodstuffs that the necessary supply of these elements can be insured to the child. Many common foods are greatly deficient in all three of these essentials. This is especially true of many breakfast foods, which are woefully lacking in lime, iron and vitamins. Of the various cereals, oatmeal is one of the best. It contains a considerable amount of lime and some iron, though deficient in vitamins. By the addition of milk, oatmeal becomes very nearly a perfect food, for milk is rich in lime, although deficient in iron. On a diet of white bread and milk, or of rice, rice flakes, or corn flakes, the diet would be deficient because of the almost entire absence of iron. A breakfast food ought to supply, with the addition of milk, all of the elements required for complete nutrition and in such abundance as to make good the deficiency of other foodstuffs. Such a breakfast food has within the last year or two been put upon the market and is sold under the name of " $\mathrm{ZO}$."

A very excellent breakfast food for children may be prepared by the addition of sterilized, cooked bran to oatmeal. The two may be combined in equal parts by measure. Cooking should not be continued longer than ten or fifteen minutes. Longer cooking lessens the food value.

Ordinary cornmeal is deficient in lime and iron, but may be improved by the addition of wheat bran. The coarser the bran the better. Bran not only adds lime and iron, but also supplies the necessary intestinal stimulant. It should be remembered that bran is not irritating, but is simply titillating; that is, it stimulates the intestine by its presence, but not by irritation. It may be used by the youngest children without injury and in liberal quantities. A law prohibiting the manufacture and sale of fine flour bread and similar denatured products would be of great service as a health-promoting measure. 
Fresh vegetables are of great service as a source of vitamins and salts, and should be used freely in the feeding of children. The Irish potato, in particular, is a food of great value for children, tending to prevent acidosis, which is likely to develop from a too exclusive diet of cereals and milk. When meats are given to children, acidosis is still more likely to develop, with constipation and autointoxication. The potato is of almost equal value with bread as a nutrient, though of course it must be used in larger quantity. Baked potatoes are easily digestible and, combined with milk or buttermilk, they supply all the elements required for good nutrition, with the exception of food iron and roughage. These should be supplied through the free use of bran and greens.

For very young children, vegetables should be used in the form of purées of carrots, turnips, spinach and other fresh vegetables, and greens.

Dates, raisins and figs should be largely used instead of cane sugar and cakes. Candies might well be excluded altogether. Children are as well satisfied with raisins or dates, which are in every way preferable to candy, not only because they are less intensely sweet but because they contain vitamins, lime and iron, all of which are lacking in candies. Raisins contain more iron than do ordinary meats, and dates are richer in iron than the average beefsteak.

Cane sugar could be banished from our tables with advantage. The practice of eating sugar freely on cereals is highly objectionable. For all table purposes it is better to use molasses, maple sirup, maple sugar or sorghum than the fine crystalline product usually used, for the reason that these sweet products contain the lime and iron which are excluded in the process of crystallization and purification to which fine sugars are subjected. In recent years, malt sugars have been produced which may be substituted with great benefit for cane sugar and corn sirups which are now in general use. Malt sugar is not only rich in lime and iron, but also vitamin $B$ and is a natural sugar which may be taken by children freely without injury. The slightly laxative effects of malt sugar are also of service in many cases.

The quantities of food required by children at different ages is shown in the accompanying tables (pp. 954, 955). The following menus will be found useful in arranging bills of fare for children at different ages. 


\section{BILLS OF FARE FOR CHILDREN}

For Children of Two Years

Breakfast

\begin{tabular}{|c|c|c|c|c|}
\hline & Oz. & lorles & & Calorles \\
\hline Banana purée ........ & $\begin{array}{l}2 \\
3\end{array}$ & $\begin{array}{l}56 \\
36\end{array}$ & Butter …….... & 28 \\
\hline Malt sugar ...... & $1 / 4$ & 25 & Total & 336 \\
\hline Milk & 8 & 157 & Orange Juice & 45 \\
\hline Bran Bread Toast & $. .1 / 2$ & 34 & & \\
\hline
\end{tabular}

Dinner

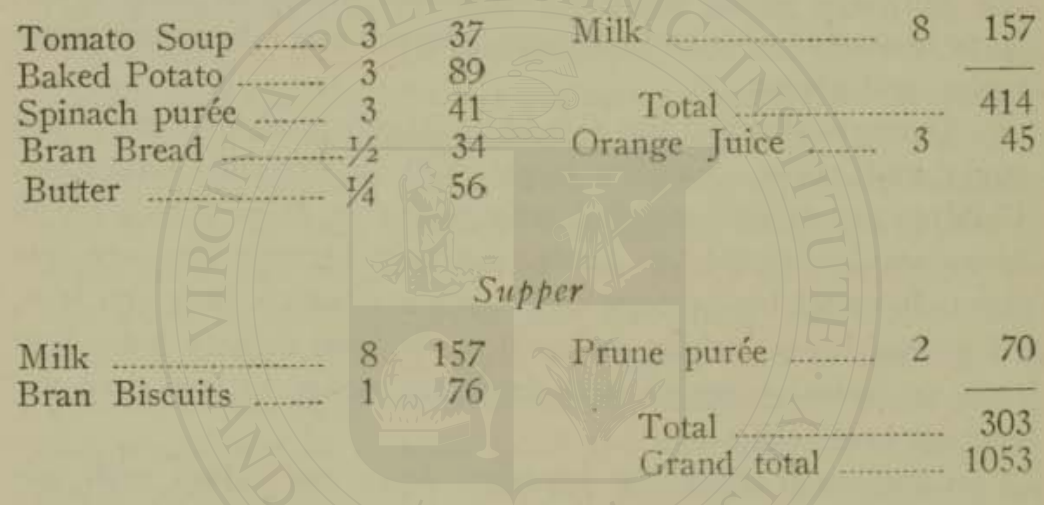

For Children of Six Years

Breakfast

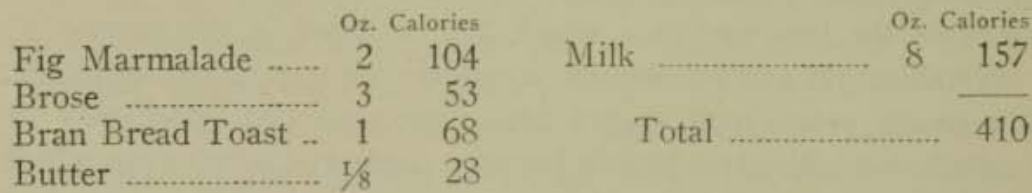

Dinner

Lentil Soup ……..... $3 \quad 37$

Scalloped Potato .... 334

Spinach ……......... 344

Stewed Tomatoes .. $4 \quad 124$

Bran Bread …........ 168

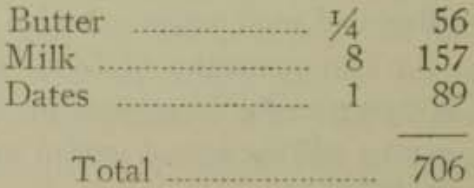




\section{Supper}

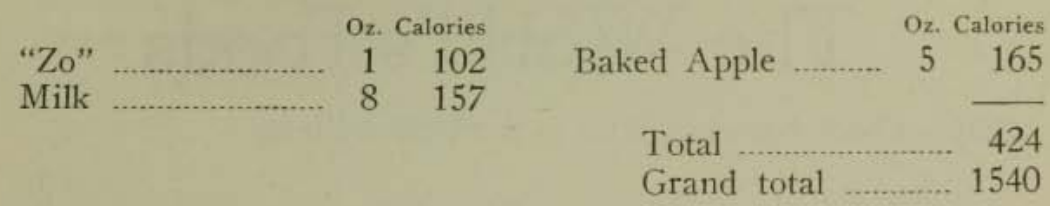

\section{For Children of Ten Years}

\section{Breakfast}

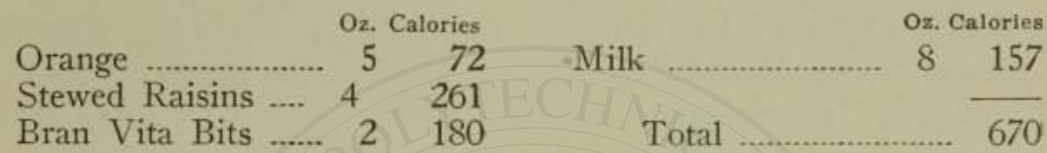

\section{Dinner}

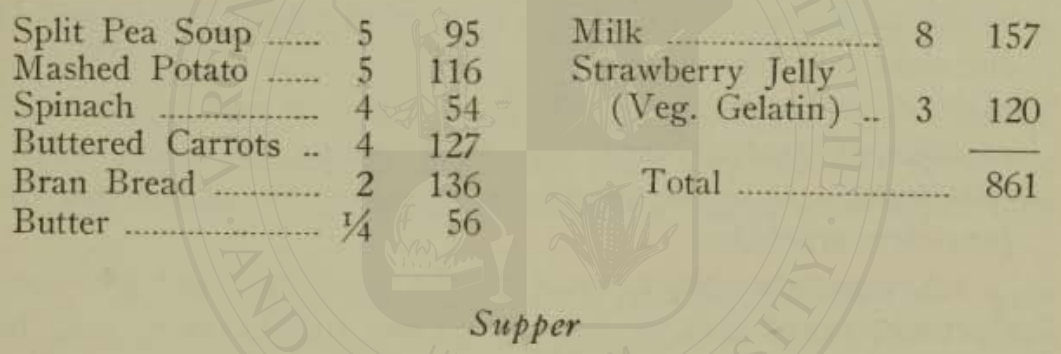

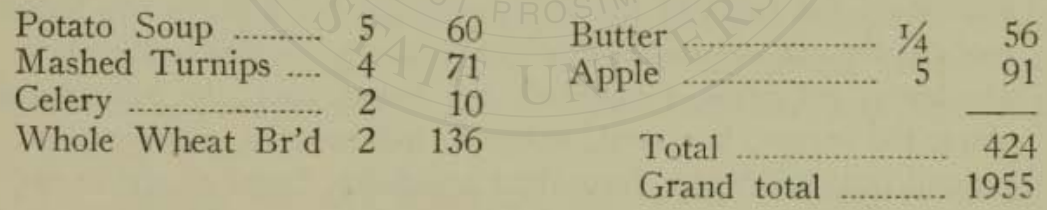




\section{The World's Foods}

Edible Foods Growing Wild in the United States.

The North American Indians, when this country was discovered by Columbus, were by no means such large consumers of flesh as many have supposed. They subsisted very largely upon the natural products of the earth. In various parts of New England and other localities they had extensive gardens. A British officer in describing the results of an expedition against the Indians soon after the arrival of the Pilgrims at Plymouth, announced among other evidences of his sweeping victory that he had destroyed extensive corn fields and several fine orchards which the Indians had planted.

The Indian and other aboriginal Americans gave to the world, as the result of centuries of careful cultivation, several of our most valuable food plants, among which are all the different varieties of Indian corn, including sweet corn and pop corn, 15 varieties of garden beans, the white potato, the sweet potato, watermelons, squashes, pumpkins, tomatoes, the peanut and the Jerusalem artichoke.

Among other plants eaten by the aborigines, and to a considerable extent still in use by various Indian tribes, may be mentioned the roots of the wild parsnip, lily bulbs, camas bulbs, roots of the cat-tail, of fern, several grasses, various water plants, of clover, eel grass, cinquefoil, the kouse root, the arrowhead plant, the carum, bur reed, valerian, balsam root, prairie potato, ground nut, milkweed, Jerusalem artichoke, "man of the earth," brake, tule, wild sago; also wild rice, wild oats, wild wheat, wild rye, tomatoes, peanuts; besides acorns, walnuts, chestnuts, beechnuts, hickories, pecans, and a great variety of other forest products. These foods were not only eaten in their season but were preserved by drying. Fruits were pulped and dried in the sun or in a basket over fire. Roots were stored in the ground.

The Indians understood various methods of cookery, some of which yielded products quite equal to the very latest refinements of the culinary art. Indeed, their method of steaming food for a day and a night by means of heated stones and boiling 
in water-tight baskets by dropping hot stones into the water enabled them to produce from the crude products of the forest dishes capable of tempting the palate of a modern gourmand. The Indians have known for ages how to produce highly palatable and wholesome vegetable dishes, not a few of which have been adopted by the civilized people who have supplanted them. Hominy, hoecake, and succotash, roasting ears, popcorn, and cornmeal mush are examples. They used various sorts of greens and knew how to prepare delicious and nourishing creams and soups from walnuts, hickory nuts, beechnuts, and almost an infinite number of mushes or porridges from pinole, a common name used for a meal prepared by parching and grinding the seeds of the scores of different wild plants with which they were acquainted. Sugar was prepared from the sap of the box elder, the willow and the agave, as well as the maple tree. It was also prepared by boiling chips of the hickory and from watermelons. They made large use of honey and gathered sweet products from the sugar pine and Douglas fir. In Mexico the fleshy leaves of the agave were roasted and eaten together with the fruit.

Some Indian tribes seem to have appreciated the necessity for an ample supply of food lime, since they carefully saved the bones of fishes and ground them into a fine powder which they added to their food.

The aboriginal Americans were excellent botanists and were thoroughly acquainted with the products of the forest. They readily distinguished between poisonous and non-poisonous plants, and cultivated in their gardens many of the useful food plants which they redeemed from the forest, together with medicinal herbs.

Neither tea and coffee nor any other harmful or intoxicating drink was indulged by the aborigines of this country, although they made many agreeable beverages from the pulp of fruits of various sorts. These primitive people, like the wild animals which shared their forests with them, refrained from drinking at meals, as do all primitive people.

The Eskimos are by no means exclusive meat feeders. They eat all the vegetable foods they can get. Certain tribes gather masu roots for food, which they eat either raw or cooked, sometimes fermented. They also make a sort of salad of a species of sorrel mixed with seal oil. The cloudberry, barberry and crowberry are eaten by most Eskimo tribes, together with reindeer 
moss and other plants found in the stomachs of caribou and other species of reindeer.

The writer is indebted to Dr. Goddard, of the department of anthropology of the American Museum of Natural History, for much information concerning the diet of the western Indians. The California Indians make large use of acorns. They first grind the acorns, then extract the tannin by leaching with warm water. They spread the ground acorns out on a bed of sand which is hollowed out like a shallow bowl. They pour warm water on this and let it leach out. A squaw sits by, weaving and pouring on the warm water occasionally. The process is completed in about half an hour. The water has just the right temperature to dissolve out the tannin quickly but without cooking the meal, which would make it pasty. When the leaching is done, the squaw very carefully removes the acorn pulp, avoiding the sand. The layer next to the sand is removed by pressing the hand upon it. Both sand and acorn pulp adhere to the hand. She dashes a little water gently against the hand, which washes away the sand without washing off the acorn pulp.

The mush made from the acorn is very wholesome and tasty. The Indians thrive on it and prefer it to "white man's food." It constitutes the principal part of the sustenance of some tribes of Indians in northern Mexico. They eat it without salt. They use no salt with their food but occasionally eat small quantities of kelp, a sort of seaweed which they get from the seashore, to which they make occasional visits for the purpose.

The Indians do not relish beef. It was at first difficult to persuade them to eat it.

\section{WILD FOODS}

The following foods grow wild in the United States, and have long been used by the Indians and also to some extent by hunters, explorers and early settlers*:

" Useful Wild Plants of the United States and Canada," by Charles Francis Suunders, Robert M. MeBride, New York. 


\section{Stems or Leaves}

$\begin{array}{ll}\begin{array}{l}\text { Bisnaga } \\ \text { Bledo }\end{array} & \begin{array}{c}\text { Milkweed (sprouts, buds } \\ \text { and pods) }\end{array} \\ \text { Bracken } & \text { Miner's lettuce } \\ \text { Bulrush } & \text { Nettle } \\ \text { Cabbage palmetto } & \text { Pokeweed } \\ \text { Cactus } & \text { Purslane } \\ \text { Chickory } & \text { Red maids } \\ \text { Clover } & \text { Red root } \\ \text { Dandelion } & \text { Scurvy grass } \\ \text { Desert trumpet } & \text { Sotol } \\ \text { Dock } & \text { Spanish bayonet } \\ \text { Lambs' quarters } & \text { Watercress } \\ \text { Mescal } & \text { Wild } \\ & \text { Wintercress }\end{array}$

\section{Roots and Tubers}

Arrowhead

Biscuit-root

Bitter-root

Camas

Cat-tail

Chufa

Conte

Coontie

Florida arrowhead

Groundnut

Harvest bread-root

Indian potatoes

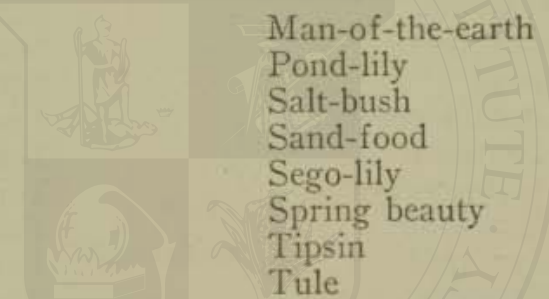

Man-of-the-earth

Pond-lily

Salt-bush

Sand-food

Sego-lily

Spring beauty

Tule

Jack-in-the-pulpit

Jerusalem artichoke

Virginia tuckaho

Water chinquapin

Lotus

Wild anise

Wild onion

Wild potato

Yamp

\section{Seeds}

$\begin{array}{ll}\text { Amaranth } & \text { Salt-bush } \\ \text { Brickeye } & \text { Songwae } \\ \text { Chia } & \text { Sunflower } \\ \text { Goosefoot } & \text { Tarweed } \\ \text { Indian millet } & \text { Wild fiax } \\ \text { Islay } & \text { Wild oats } \\ \text { Jojoba } & \text { Wild wheat } \\ \text { Juniper } & \text { Wild sage }\end{array}$


Nuts

Acorn

Beechnut

Chestnut

Chinquapin

Groundnut

Hickcry

Hog peanut

Walnut

Piñon

\section{Fruits}

\begin{tabular}{ll} 
Barberry & Oregon grape \\
Blackberry & Papaw \\
Black haw & Persimmon \\
Buckthorn & Pumpkin \\
Buffaloberry & Raspberry \\
Cactus & Salal \\
Cranberry & Sahauro \\
Currant & Screw-bean \\
Elderberry & Serviceberry \\
Fan-palm & Squash \\
Gooseberry & Strawberry \\
Grape & Summer haw \\
Ground cherry & Teaberry \\
Hawthorn & Tomate del campo \\
Huckleberry & Tomatillo \\
Manzanita & Tomato \\
May apple & Tuna \\
May-pop & Watermelon \\
Mesquit & Yucca \\
Mulberry & \\
\hline
\end{tabular}

Plants Cultivated by the Natives of the

New World Before 1492.*

Agave

Alligator pear

Arrowroot

Bean, kidney

Bean, lima

Cashew nut

Cherimoya

Corn

Gourd

Guava

Jerusalem artichoke

Maize
Manioc

Potato

Papaw

Peanut

Pineapple

Prickly pear or Indian fig

Pumpkin

Quinoa

Squash

Star apple

Sweet potato

Tomato

-The Ameriean Indian, by Wissler. 


\section{EDIBLE PLANTS}

As regards the number of different plants in current use as foods by human beings, information was sought from the office of Dr. David Fairchild, chief of the Plant Introduction Bureau of the U. S. Department of Agriculture. The request brought in return the following lists of cereals, vegetables, nuts and fruits :

\section{Cereals}

All plants yielding farinaceous seeds:

Amaranthus sp. -4 kinds at least

Bamboo

Barley

Brush grass

Buckwheat

Carob

Corn

Durra

Emmercorn

Job's tears

Kafir corn

Lotus

Manna grits

Millet (several kinds)

Oats

Quinoa

Ragi

Rice

Rye

Sorghum -6 kinds at least

Spelt

Teff

Teosinte

Tocusso

Wheat

\section{Vegetables}

F1-flowers, R-roots. Unless part eaten is evident from name, stem or leaves are eaten if no abbreviation follows:

$\begin{array}{ll}\text { Abutilon esculentus-FI. } & \begin{array}{l}\text { Beet } \\ \text { Adzuki bean }\end{array} \\ \text { Angelica } & \text { Brusels sprouts } \\ \text { Anise } & \text { Cabbage } \\ \text { Arracach } & \text { Cabbage palm } \\ \text { Arrowhead-R } & \text { Caper-buds eaten } \\ \text { Arrowroot } & \text { Cardoon } \\ \text { Artichoke } & \text { Carosella } \\ \text { Asparagus } & \text { Carrot } \\ \text { Balm } & \text { Cassava-R } \\ \text { Bamboo } & \text { Cat-tail-R } \\ \text { Basil } & \text { Cauliflower } \\ \text { Bean, Bonavist } & \text { Celeriac } \\ \text { Bean, common } & \text { Celery } \\ \text { Bean, lima } & \text { Chard } \\ \text { Bean, mung } & \text { Chayote } \\ \text { Bean, soy } & \text { Chervil } \\ \text { Bean, string } & \text { Chick pea }\end{array}$


Chicory

Chinese artichoke

Chinese cabbage -2 kinds

Chives

Clary

Cornsalad

Cress- 3 or more species

Crosnes

Cucumber

Dandelion

Dasheen-R

Egg plant

Endives

Fennel

Fenugreek

Ferns -5 kinds - R

Garlic

Goa bean

Goosefoot

Guar bean

Horehound

Horseradish

Ice plant

Illipi-Fl.

Indian pea

Jerusalem artichoke- $\mathrm{R}$

Kafir potato

Kale

Kohlrabi

Leek

Lentil

Lettuce

Licorice-R

Lotus-R

Mauritius palm-stem

Mayna-R

Mitsuba-stems

Mustard

New Zealand spinach

Oca-R

Okra

Onion

Parsley

Parsnip

Pea, field

Pea, garder

$\mathrm{Pea}$, pigeon
Pepper

Phog-F1.

Pokeberry

Pomme blanche

Potato

Potato bean

Pumpkin

Puya

Queensland arrowroot-R

Radish

Raisin tree-stems

Rape

Rhubarb

Rice bean

Risga-R

Rocambole-R

Rocket salad

Roselle-Fl.

Sago palm-pith

Salep-R

Salsify

Scorzonera

Scullion

Seakale

Shallot-R

Skirret-R

Snake gourd

Sorrels -5 kinds

Spinach

Squash

Sugar cane

Sweet potato

Sword bean

Tahiti arrowroot

Takka-R

Tarragon

Tomato

Turnip

Tania-R

Udo-stems

Ulluco-R

Umara-R

Wax gourd

Winter purslane

Yam

Yam bean-R

Yautia-R 


\section{Nuts}

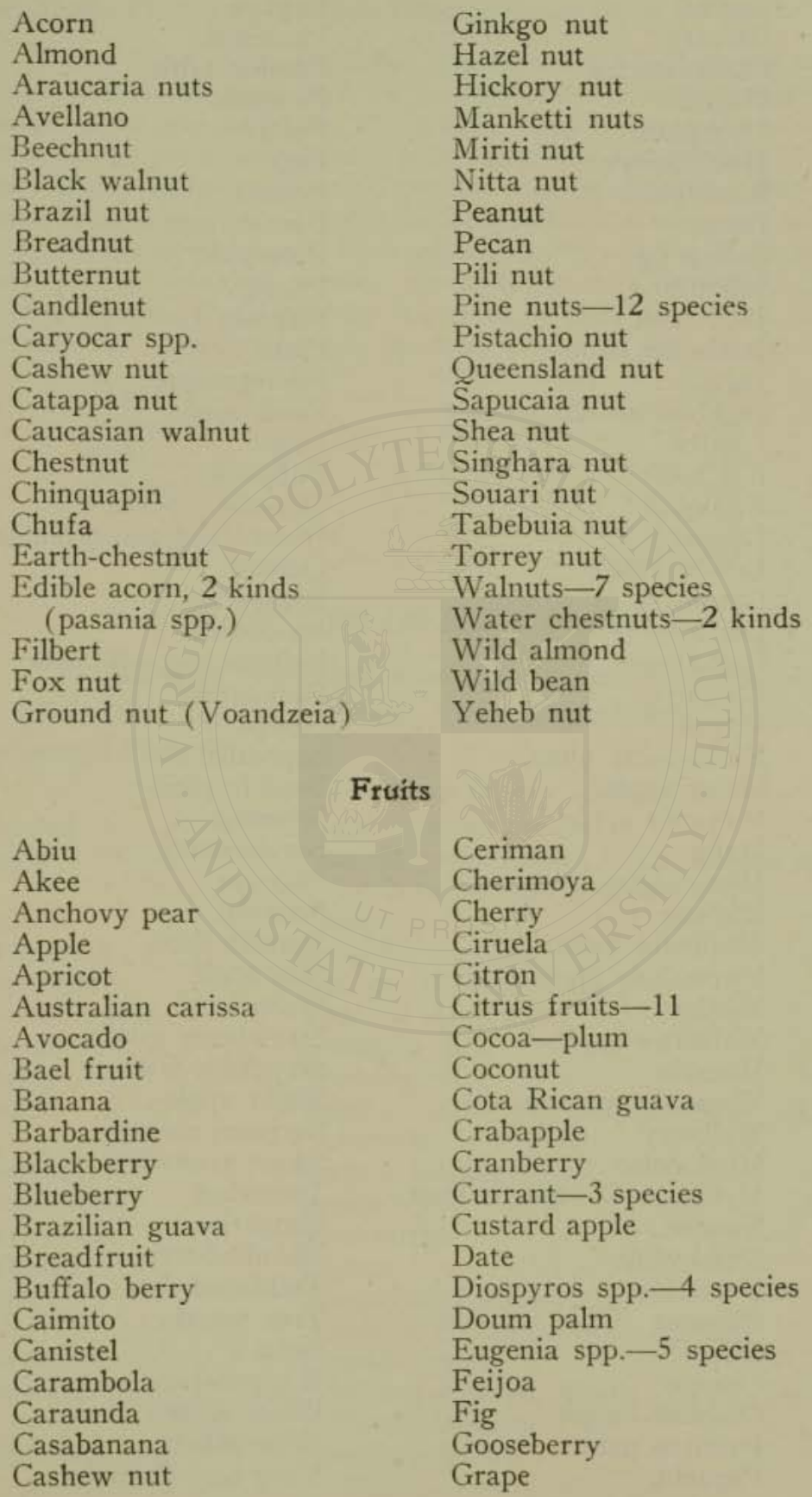


Grape fruit

Guava

- Guisaro

Gumixameira

Hagam

Hog plum

Huckleberry

Icaco plunı

Ilama

Indian fig

Jaboticaba

Jack fruit

Jambolan plum

Jujube

Juneberries-25 species eaten

Kei apple

Kumquat

Lamsat

Lemon vine

Lemon

Leichee

Loganberry

Longan

Loquat

Madagascar plum

Malay apple

Mammey apple

Mang

Mangosteen

Marang

Maracujá melao

Marmalade plum

Medlar

Molucca-apple

Mombin

Mountain soursop

Mulberry

Muskmelon

Mytaceae -7 kinds at least

Nanche

Natal plum

Nectarine

Oleaster

Olive

Orange

Otaheite-apple

Palmyra palm

Papachi
Papaw

Papaya

Pasionaria

Passion fruit

Pawpaw

Peach

Pear

Pepino

Persimmon

Pineapple

Pitanga

Plantain

Plum

Pomegranate

Pond apple

Prune

Pulassan

Quandong-peach

Queensland pomegranate

Quince

Rambutan

Raspberry

Rollinia spp. -6 kinds

Rose apple

Saguarro

Sapodilla

Seaside plum

Sebesten

Serviceberry

Soncoya

Soursop

Spanish lime

Star apple

Strawberry

Strawberry guava

Strychnos fruit

Sugar apple

Surinam cherry

Sweet granadilla

Tamarind

Tangerine

Thimbleberry

Trebizond date

Tree tomato

Tuta

Watermelon

White sapote

Whortleberry

Yang tao-2 kinds 
In relation to the above list of food plants, Mr. P. G. Russell of the Plant Introduction Bureau, wrote us as follows:

I have listed about 200 fruits; this I believe would be not more than a third of all fruits used for food by native peoples throughout the world, or perhaps 600 in all. I have listed 142 vegetables; if we count all the plants occasionally used for greens and salads, and the many plants whose roots are occasionally eaten, I suspect that the number would be at least 500 . I have listed 70 kinds of nuts; this might easily, in the same manner, be increased to 200 , and the 35 kinds of cereals, if we take into account the native grasses, amaranths, and chenopodiaceous plants used by the tribes of India and Africa, could be made 100 . Thus we have as the total number of food plants:

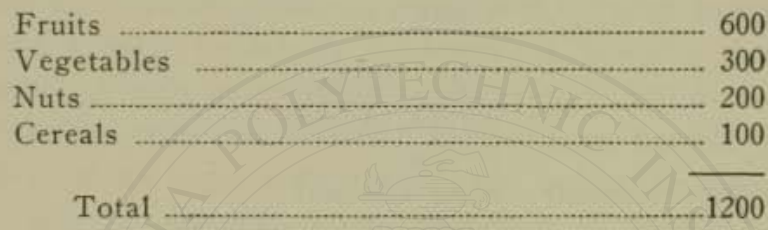

\section{THE ORIGINS OF FOOD PLANTS}

The following condensed information concerning the origin of food plants is chiefly derived from de Candolle's "Origin of Cultivated Plants":

Almond

Apple

Apricot

Arrowroot

Asparagus

Avocado pear

Banana

Barley

Bean

Beetroot

Bread fruit

Buckwheat

Cabbage

Carrot

Celery

Cherry

Chestnut

Chick pea

\section{Region Where Indigenous}

Mediterranean region

Anatolia; was known to the ancient lake dwellers of Switzerland

\section{China}

Tropical America

Europe, Western Asia

Tropical America

Southern Asia

Western Asia

Region south of Caspian Sea. Also America.

Canary Islands

Sunda Isles

Manchuria

Europe

Temperate Europe, Asia

Southern Europe, North Africa

Caspian region

Southern Europe, North Africa, Japan, North America

Caspian region 
Cucumber

Currant

Date palm

Field pea

Fig

Gooseberry

Guava

Hickory nut

Indian corn, maize

Lemon

Lentil

Lettuce

Litchi nut

Mango

Melon

Millet

Mulberry

New Zealand spinach

Oats

Okra

Olive

Onion

Orange

Papaw

Pawpaw

Peach

Peanut

Pear

Persimmon

Pineapple

Pistachio nut

Plum

Pomegranate

Potato

Quince

Quinoa

Raspberry

Rice

Rye

Sorghum

Spinach

Strawberry

Sugar cane

Sweet potato

Tomato

Turnip

Walnut. Persian

Walnut, black

Wheat
Northwest India

Europe, North India, United States

Western Asia, Africa

Italy

Syria

Southern Europe, North Africa, Himalayas

Tropical America

North America

South America

India

Western Asia, Greece, Italỷ

Southern Europe, North Africa

China

India

India, Guinea

Egypt, Arabia

Persia

New Zealand

North of Caspian Sea

Tropical Africa

Syria

Persia, Palestine

China

America

Tropical America

China

Brazil

Southern Europe, Asia

Eastern United States

Tropical America

Syria

Persia

Anatolia

Peru

Persia

New Granada, Peru

Europe, Asia

India, China

Temperate Europe

Tropical America

Persia

Europe, Asia, North America

China, Japan, Java

Tropical America

Peru

Europe, Siberia

Temperate Europe, Asia

North America

Region of Euphrates 


\section{Making the Bill of Fare}

The making of the bill of fare is one of the greatest of all household responsibilities and should not be left to chance, individual whim or the gross ignorance of the average cook. It is a task for the woman who as wife and mother directs the household functions. It is never to be forgotten that we are made of what we eat. What we eat today will be walking about and talking tomorrow. Nature performs wonderful miracles in the transmutation of humble foodstuffs into sentient, forceful, intelligent human beings, a truly marvelous transfiguration ; but even Nature must be supplied with raw material. And Nature herself does not undertake to make brass rods out of steel ingots, or muscles out of fat or starch. Quality is as important in foodstuffs as in fabrics. All is not gold that glitters. All is not food that fills the mouth or thrills the palate.

The construction of a bill of fare is a simple problem for an ape or a savage. The anthropoid knows instinctively what will satisfy his body needs. The savage fills his stomach with what he finds, which, fortunately, is usually fairly well suited to his physiologic requirements, thanks to the fact that he is so close to Nature that his primitive tastes are still dominant and are a fairly safe guide to his fundamental needs, the means of gratifying which have been determined by the accumulated experiences of many generations.

But the civilized man has so completely lost his instinctive nutrition guides that he must take careful thought about his eating, being guided by the results of the thousands of laboratory experiments which have been made in state experiment stations and in nutrition laboratories.

Fortunately, the whole matter of diet has within recent years been so simplified by extended clinical experience that it is possible, by taking a little care and without the aid of elaborate calculations, to arrange a bill of fare for one's self or for a family that may be followed with full assurance of satisfaction and safety. It is necessary to give attention to eight items; viz., the amount of the food, the protein, the fats, carbohydrates, vitamins, 
scilts (lime and iron) cellulose, and variety in the foodstuffs composing the menus.

1. Amount of Food. The amount of food must be adapted to individual needs. A slight excess is better than a deficiency. In general, an average of 2500 calories each will be ample for an ordinary family, including two or three small children. This is enough for an office man. Hard working men require more. If one chews his food thoroughly, the appetite will be a safe guide as to quantity. The body weight should be watched.

The following is an excellent formula for calculating the daily ration: For each pound of normal body weight allow one and one-third calories of protein. Multiply the protein by four for fat, and multiply the fat by two for the carbohydrates. Thus, supposing the normal weight of a man to be 150 pounds (height, $5 \mathrm{ft} .7 \mathrm{in}$.) his ration would be found as follows:

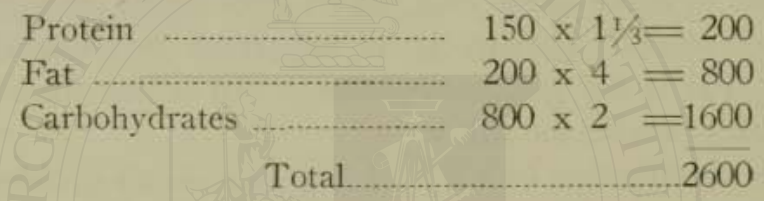

2. The Protein. If care is taken to provide for each child a quart of full milk daily and for each adult a pint of milk, two or three ounces of cottage cheese, or four to six ounces of nuts, no further consideration need be given the question of protein, provided the diet comprises the usual variety of grains, fruits and vegetables. No meat, fish, fowl or shellfish are needed, and if eaten will simply burden the body with an excess of protein which must be eliminated as urea, throwing this extra burden upon the kidneys. Eggs may be eaten sparingly in place of milk, but are inferior as a source of protein. Excess of protein is one of the most serious of all dietetic faults. Beans, lentils, cottage cheese and milk are not needed at meals at which eggs are eaten. Eggs must be strictly fresh to be safe and wholesome, and should be produced by fowls that are cared for in a cleanly manner and given no meat and only clean food such as bran, buttermilk, grain and green food. Eggs laid by hens affected with "white diarrhea" are infected with the bacteria which cause colitis (Tissier).

One calorie per pound of body weight per diem is all the protein needed to meet physiologic requirements (Sherman).

3. Fats. Little thought need be given to fats except to 
avoid taking too much. One-half, or at most two-thirds of an ounce of butter at each meal is quite sufficient in addition to the fat usually taken as seasoning in food and in milk or cream. Fat is the least essential of the food elements. When taken in excess, digestion is delayed and colon putrefaction is increased. Two or three calories per pound of body weight per diem will amply meet all body needs. More than this may slowly but surely lead to obesity. When olive oil is freely used with salads or otherwise, the amount of butter eaten should be diminished, especially if there is a tendency to take on flesh.

4. Carbohydrates. Most of the carbohydrates should be taken in the form of whole grain preparations and potatoes (Irish or sweet). Cane sugar should be used sparingly on account of its irritating and somewhat toxic character, and because it is wholly lacking in lime, iron and vitamins. These farinaceous foodstuffs should constitute the staples of the bill of fare. the foods which are relied upon to sustain the body, the fuel to keep up steam in the vital engine. It is a mistake to depend too much upon breads and breakfast foods, because they lack vitamins and salts. Discard white bread for graham or bran bread. Use oatmeal or cornmeal with a liberal amount of bran added (an equal measure is not too much). The potato should be much more largely used than at present. When well cooked, it is the most quickly digested of all farinaceous foods.

The use of honey and of dates in place of cane sugar, to some extent, at least. is advisable. Malt sugar may well take the place of cane sugar for table use. The sugars made from corn by chemical processes do not deserve a place in a biologic bill of fare.

5. Food Salts, Lime and Iron. Care must be taken to provide an abundance of these essential blood and bone-building elements, for they are woefully deficient in most bills of fare. Lime is abundant in milk, greens, wheat bran, beans, lentils, peas and some nuts (see Table XIII). Greens, bran, whole grains, malt sugar and gluten preparations are rich in iron, richer even than average meats. Egg yolks are rich in both lime and iron. Milk is very deficient in iron. Malted nuts contain two and a half times as much iron as does milk. Some of these iron-and lime-rich foods should appear on every bill of fare (see Table XV). They are greatly neglected by the makers of menus. Children need more than adults; and nursing or expectant mothers need more than other adults. 
6. Vitamins. To insure an adequate supply of the trio of vitamins, fresh, uncooked foods of some sort should be made a part of every meal. Unpasteurized milk supplies all the vitamins, as do also tomatoes (fresh or canned), raw cabbage, carrots and lettuce. On this account, one or more of these foods should appear daily on the bill of fare. (See Tables XVII, XVIII, XIX, $\mathrm{XX)}$.

7. Cellulose. Roughage must be provided for every meal. Fresh fruit, greens, fresh vegetables, and especially bran or agar in liberal quantities should be used, not daily only, but at every meal. Each meal must have its due quantum of indigestible material to furnish a residue which will stimulate the colon to pass it on to the exit, carrying with it the bile, mucus and other secretions which so readily undergo putrefaction in the colon when allowed to stagnate. It is well to add bran to the oatmeal, cornmeal, or other cereal. Bran may be combined with vegetable purées.

8. Balancing the Bill of Fare. A balanced bill of fare must contain not only the proper proportion of complete proteins, carbohydrates, and fats but also the essential amounts of food lime, iron and other food salts with vitamins and cellulose, or roughage. The most important thing, of course, is to make sure of a sufficiency of each one of the six essential food constituents; namely, proteins, fats, carbohydrates, food salts, vitamins, and cellulose. A little excess of any one of these is really a matter of no particular consequence, and indeed no great harm could result from a considerable temporary excess of any of the several food constituents with the exception of protein and fats. As has elsewhere been shown, an excess of protein lays a great and unnecessary burden upon the liver and kidneys, which must transform and eliminate all the excess as so much waste matter, none of it being utilizable except in an incomplete way.

An excess of protein imposes another great burden upon the body by flooding the blood and tissue fluids with highly virulent toxins which raise blood pressure, irritate and intoxicate the brain and nerves, set up degenerative processes in the liver and other glands, cause changes in the blood vessels which result in arteriosclerosis, angina pectoris, apoplexy and other grave mischiefs and enormously increase the work of the kidneys, through which they are eliminated, wearing them out prematurely, thus prepar- 
ing the way for Bright's disease and general senility and premature decay.

It is thus important to watch the protein content with vigilance. Fortunately, the danger of an excessive intake of protein almost wholly disappears when meats of all sorts (fish, flesh and fowl) are eliminated from the bill of fare. A pint of milk, or even a smaller amount, to a bill of fare made up of fruits, grains, and vegetables will fully meet the body requirements for protein.

An excess of fat may do harm by delaying the movements of food along the alimentary canal, particularly prolonging the time of gastric digestion. An excess of fats also encourages putrefaction in the colon and hence should be avoided. A deficiency can be easily compensated for by carbohydrates, so the only real danger is from excess. If the total intake of fats does not exceed two and one-half to three ounces there is little possibility of excess.

As regards the food salts and vitamins, there is no evidence whatever that any harm can possibly result from an excess; in fact, it is wise to make constant use of a superfluity by the free use of foods rich in salts so as to make sure of an abundant absorption of these essential elements.

If meats are excluded from the bill of fare, as they certainly should be in the light of well established physiologic facts, the dangers from an excess of alimentary acids will be largely removed. However, it is to be remembered that eggs, especially egg yolks are even more acid than meats, and that cereals are also acid and must not be used too freely.

As regards cellulose or roughage, there is a constant danger of a deficiency but no danger of an excess, provided always that the bulk is not so great as to overtax the capacity of the stomach. It is to be remembered always that roughage is an essential part of every meat and under ordinary circumstances never to be omitted.

9. Variety. The staples which furnish the bulk of the food value should be varied from day to day, and to these should be added as large a variety as can be reasonably secured, so as to make certain of securing a full complement of the various subtle elements needed for complete nutrition. The variety should not be great at a single meal, but the bill of fare should be varied from day to day. 
10. Appetizers. Mustard, pepper, vinegar and the long list of unwholesome condiments are of course excluded from a biologic dietary: but their place may be more than filled by wholesome physiologic gustatory and peptic stimulants, such as lemon juice, verjuice (juice of green sour grapes), celery, lettuce and other fresh green stuffs such as cabbage, chard, endive, etc. SalSavita, a new table relish, not only creates appetite but aids gastric digestion and is an excellent source of vitamin $B$ and food iron.

Breakfast. This is too often a makeshift meal, affording little support for the day's labors. The fault does not lie altogether with the cook. The seven o'clock dinner has spoiled the American breakfast. The late diner rises with little or no appetite, because his exhausted stomach is unprepared for a digestive task. A cup of coffee with a bit of toast and bacon or griddle cakes with corn sirup are the miserable substitutes for a real breakfast which are swallowed hurriedly by many thousands of business men. Oatmeal with bran and cream, an egg or a glass of milk, fresh fruit, lettuce or celery, with bran bread and butter, will constitute a breakfast much more in harmony with physiologic requirements. For a vigorous man, a total of 800 calories will be needed to fortify the body for a good day's work.

Dinner. The heartiest meal should be taken at midday or at least not later than four or five p. $m$. The total number of calories at the midday meal may be 1000 to 1200 .

Supper. Brain workers do much better to omit supper. Better sleep is secured and better digestion. If nourishment is needed at night, it is by all means best to confine the menu to fresh or stewed fruit with malt sugar, dates or raisins. A few nuts may be added if very well chewed and bran with rice, gruel or oatmeal porridge may be taken or some dextrinized cereal. The evening meal should be light; 500 to 700 calories should be sufficient when two previous hearty meals have been eaten.

The accompanying diet lists and bills of fare may serve as models by the aid of which suitable menus may be readily arranged for almost any patient or class of patients. By the addition of carbohydrates in the form of malt sugar, cereals or potatoes, the energy value or number of calories may be increased to meet the needs of practically any case requiring increase of flesh or a large output of energy in muscular work. 


\section{BILL OF FARE FOR LOW PROTEIN DIET}

\begin{tabular}{|c|c|c|c|c|c|c|}
\hline \multirow[b]{2}{*}{ Breakfast. } & \multirow[b]{2}{*}{ Pro. } & \multicolumn{2}{|c|}{ Catories } & \multirow[b]{2}{*}{ Total } & \multicolumn{2}{|c|}{$\begin{array}{l}\text { Per cent of } \\
\text { day's ration }\end{array}$} \\
\hline & & Fat & Carbo. & & Lime & Iron \\
\hline$\ldots \ldots \ldots, \ldots, 5$ & 5 & 3 & 66 & 74 & 7 & 2 \\
\hline Blueberries $\ldots \ldots \ldots \ldots \ldots . . . . .215$ & 2 & 3 & 42 & 47 & 1 & 4 \\
\hline Cream of wheat ........... 4 & 6 & 1 & 42 & 49 & 7 & 8 \\
\hline Cream $\quad \ldots \ldots \ldots \ldots \ldots . . . . . . .21 / 4$ & 7 & 111 & 12 & 130 & 7 & 1 \\
\hline Malt sugar $\ldots \ldots \ldots \ldots \ldots \ldots \ldots 1 \ddot{y}_{4}$ & 0 & 8 & 210 & 218 & 3 & 18 \\
\hline Graham toast ............. 1 & 10 & 6 & 52 & 68 & 1 & 5 \\
\hline Butter ................... $1 / 2$ & 0 & 54 & 0 & 54 & & \\
\hline Yogurt buttermilk .........6 & 24 & 5 & 36 & 65 & 28 & 3 \\
\hline Bran $\ldots \ldots \ldots \ldots \ldots \ldots \ldots \ldots$ & 5 & 3 & 13 & 21 & 1 & 4 \\
\hline $\operatorname{Total} \ldots \ldots \ldots \ldots \ldots$ & 59 & 194 & 473 & 726 & 55 & 45 \\
\hline \multicolumn{7}{|l|}{ Dinner } \\
\hline Celery soup ............... 3 & 1 & 8 & 7 & 16 & & \\
\hline Baked potato $\ldots \ldots \ldots \ldots \ldots .3$ & 9 & 1 & 78 & 88 & 1 & 3 \\
\hline Spinach $\ldots \ldots \ldots \ldots \ldots \ldots$ & 7 & 51 & 9 & 67 & 7 & 23 \\
\hline New beets ............... z & 3 & 14 & 21 & 38 & 2 & 2 \\
\hline Waldorf salad $\ldots \ldots \ldots \ldots \ldots, 21 / 4$ & 8 & 55 & 29 & 92 & & \\
\hline Bran bread ............... 1 & 6 & 32 & 62 & 100 & 1 & 5 \\
\hline Butter ................. 1/2 & 0 & 108 & 0 & 108 & & \\
\hline Golden nectar ............ $3 \frac{1}{1 / 2}$ & 1 & 1 & 64 & 66 & 1 & 1 \\
\hline Cantaloup ............. $6 \frac{1}{2}$ & 4 & 0 & 69 & 73 & 5 & 4 \\
\hline Pecans............ .15 & 6 & 94 & 9 & 109 & 1 & 2 \\
\hline Yogurt buttermilis ... . . . 6 & 24 & 5 & 36 & 65 & 28 & 3 \\
\hline 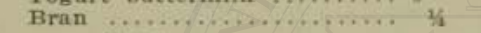 & 5 & 3 & 13 & 21 & 1 & 4 \\
\hline Total $\ldots \ldots \ldots \ldots \ldots \ldots$ & 74 & 372 & 397 & 843. & 47 & 52 \\
\hline \multicolumn{7}{|l|}{ Supper } \\
\hline Potato sorp .... & 5 & 16 & 38 & 59 & & \\
\hline Stuffed tomato $\ldots . . . \ldots \ldots$ is & 10 & â & 55 & 103 & 1 & 5 \\
\hline Lettuce ................ $1 y_{2}$ & 1 & 1 & 6 & 8 & 3 & 1 \\
\hline Bran bread toast $\ldots . . \ldots \ldots .1$ & 6 & 32 & 52 & 100 & 1 & 5 \\
\hline Butter .................. & 0 & 54 & 0 & 54 & & \\
\hline Yogurt buttermilk ......... 6 & 24 & 5 & 36 & 65 & 28 & 3 \\
\hline Apple $\ldots \ldots \ldots \ldots \ldots \ldots \ldots \ldots 1 / 2$ & 2 & 7 & 88 & 97 & 2 & 3 \\
\hline Bran ................ is & 5 & 3 & 13 & 21 & 1 & 4 \\
\hline Total $\ldots \ldots \ldots \ldots \ldots \ldots, \ldots$ & 53 & 156 & 288 & 507 & 35 & 21 \\
\hline Grand total $\ldots . . . .$. & 186 & 722 & 1158 & 2076 & 187 & 118 \\
\hline
\end{tabular}

\section{LOW PROTEIN DIET LIST}

Fruits: All fruits and fruit juices. Figs, prunes and dates. Chestnuts and litchi nuts. (Avoid other nuts and olives.)

Vegetables: All green vegetables, especially fresh vegetables, greens and salads.

Cereals: Rice, barley and rice flakes.

Miscellaneous: Butter, olive oil, honey, malt sugar, malt honey, malted nuts, buttermilk (one-half pint daily).

Accessories: Bran, agar, laxa and paraffin preparations. Lactodextrin.

When a very low-protein diet is required, as in certain cases of nephritis, the diet must be confined to malt sugar and fruit juices. 


\section{BILL OF FARE FOR ANTITOXIC DIET}

\begin{tabular}{|c|c|c|c|c|c|c|}
\hline \multirow[b]{2}{*}{ Breakfast } & \multicolumn{4}{|c|}{ Calories } & \multicolumn{2}{|c|}{$\begin{array}{l}\text { Per cent of } \\
\text { day's ration }\end{array}$} \\
\hline & Pro. & Fat & Carbo. & Total & Lime & Iron \\
\hline Cantaloup $\ldots \ldots \ldots \ldots \ldots \ldots \ldots 61 / 2$ & 4 & 0 & 69 & 73 & 5 & \\
\hline Stewed raisins $\ldots \ldots \ldots \ldots \ldots 3^{12}$ & 6 & 17 & 173 & 196 & 5 & \\
\hline Brose $\ldots \ldots \ldots \ldots \ldots \ldots \ldots .41 / 4$ & 10 & 8 & 36 & 54 & 7 & 20 \\
\hline Cream $\quad \ldots \ldots \ldots \ldots \ldots \ldots \ldots \ldots .214$ & 7 & 111 & 12 & 130 & 7 & \\
\hline Graham gems ............. & 27 & 46 & 139 & 212 & 6 & \\
\hline $\begin{array}{l}\text { Malt honey } \ldots \ldots \ldots \ldots \ldots \ldots 21 / 4 \\
\text { Cereal coffee }\end{array}$ & 0 & 0 & 194 & 194 & 2 & \\
\hline Bran $\ldots \ldots \ldots \ldots \ldots \ldots \ldots \ldots, 1 / 4$ & 5 & 3 & 13 & 31 & 1 & \\
\hline Dinner Total $\ldots \ldots \ldots \ldots \ldots \ldots$ & 59 & 175 & 636 & 880 & 33 & 56 \\
\hline Vegetable soup with noodles. $4 \% / 4$ & 3 & 19 & 22 & 44 & & \\
\hline Baked sweet potato ......6 6 & 12 & 10 & 188 & 210 & 4 & \\
\hline Buttered asparagus ......... 3 & 6 & 94 & 11 & 111 & 3 & \\
\hline Swiss chard-lemon ....... 3 & 7 & 51 & 9 & 67 & 19 & 15 \\
\hline Cabbage salad ........... 2 & 4 & 37 & 17 & 58 & 6 & \\
\hline Bran bread ................ 2 & 12 & 64 & 124 & 200 & 2 & 10 \\
\hline Butter $\ldots \ldots \ldots \ldots \ldots \ldots \ldots{ }_{1 / 2}$ & 0 & 108 & 0 & 108 & & \\
\hline Yogurt buttermilk $\ldots \ldots \ldots \ldots 6^{2}$ & 24 & 5 & 36 & 65 & 28 & \\
\hline Watermelon ............ 8 & 3 & 4 & 76 & 83 & 4 & \\
\hline Bran .................. $1 / 4$ & 5 & 3 & 13 & 21 & 1 & \\
\hline Total $\ldots \ldots \ldots \ldots \ldots \ldots$ & 76 & 395 & 496 & 967 & 67 & 52 \\
\hline Supper & & & & & & \\
\hline Okra soup $\ldots \ldots \ldots \ldots \ldots \ldots \ldots, 4 \%$ & 2 & 1 & 9 & 12 & & \\
\hline Baked cornlet $\ldots \ldots \ldots \ldots \ldots .3$ & 13 & 91 & 90 & 194 & 1 & \\
\hline Shredded carrot-lemon .... 2 & 2 & 2 & 22 & 27 & 4 & \\
\hline Whole wheat bread ......... 2 & 22 & 4 & 112 & 138 & 2 & \\
\hline 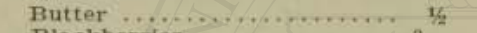 & 0 & 108 & 0 & 108 & & \\
\hline Blackberries $\ldots \ldots \ldots \ldots . . .3$ & 5 & 8 & 38 & 51 & 2 & \\
\hline Bran $\ldots \ldots \ldots \ldots \ldots \ldots \ldots$ & 5 & 3 & 13 & 21 & 1 & \\
\hline Total $\ldots \ldots \ldots \ldots \ldots$ & 50 & 217 & 284 & 551 & 10 & 19 \\
\hline Grand total ........... & 185 & 787 & 1416 & 2398 & 110 & 127 \\
\hline
\end{tabular}

ANTITOXIC DIET LIST

Soups: Vegetable soups without cream and bouillons.

Relishes: Tomatoes, celery, lettuce, endive, cucumber, radishes, shredded cabbage, shredded carrots and turnips. Ripe olives, bromose, meltose, meltose with butter. Vegetable and fruit salads without eggs.

Vegetables: All vegetables, especially fresh vegetables, raw and cooked.

Cereals: Brose (scalded oatmeal), cereal flakes, vita-wheat, shredded wheat, granola, macaroni, corn meal, rice, bran.

Breads: Graham at least 24 hours old. Zwieback, dry toast, bran gems, gluten bread.

Beverages: Fruit juices, cereal coffee, malted nuts, malted milk, buttermilk (yogurt, acidophilus).

Fruits: All fresh and cooked fruits. Tomatoes are particularly beneficial.

Desserts: Fruit tapioca, fruit gelatines, fruit ices, fruit purées, fig marmalade, prune marmalade, dates, figs, raisins.

Miscellaneous: Butter, cream, buttermilk, cottage cheese, to fu, nuts, malted nuts, malted milk, protose.

Accessories: Bran, agar, laxa, paralax, paramels and other paraffin preparations, lacto-dextrin. 
BILL OF FARE FOR FEVER

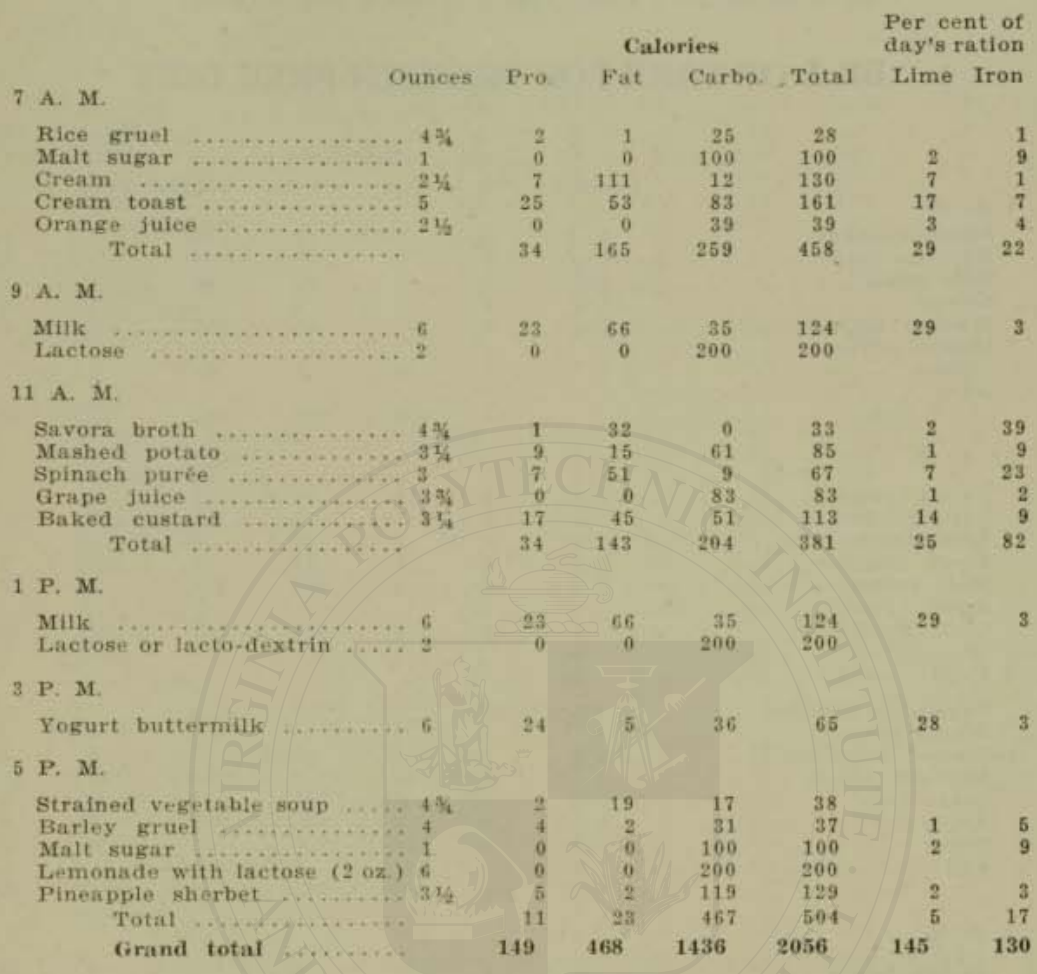

\section{FEVER DIET LIST}

Fruits: Purées and juices of all sorts, especially orange juice, fig and prune marmalades (meltose).

Cereals: Gruel and porridge of all cereals, cereal flakes, granola, graham toast, all dextrinized cereals, granose biscuit, rice biscuit, shredded wheat.

Vegetables: Purées of all vegetables, potato soup, vegetable soup, cream of corn soup. purées of spinach and other greens, vegetable broths.

Miscellaneous: Buttermilk (acidophilus), whey, malt sugar, mait honey, malted nuts, malted milk, savora broth, gluten gruel $(40 \%)$, cream gluten gruel, lactose, lacto-dextrin.

Accessories: Bran, (except in typhoid), agar, laxa, paraffin (paramels, paralax, petrolatum, etc.) 


\section{BILL OF FARE FOR PROTEIN-FREE DIET}

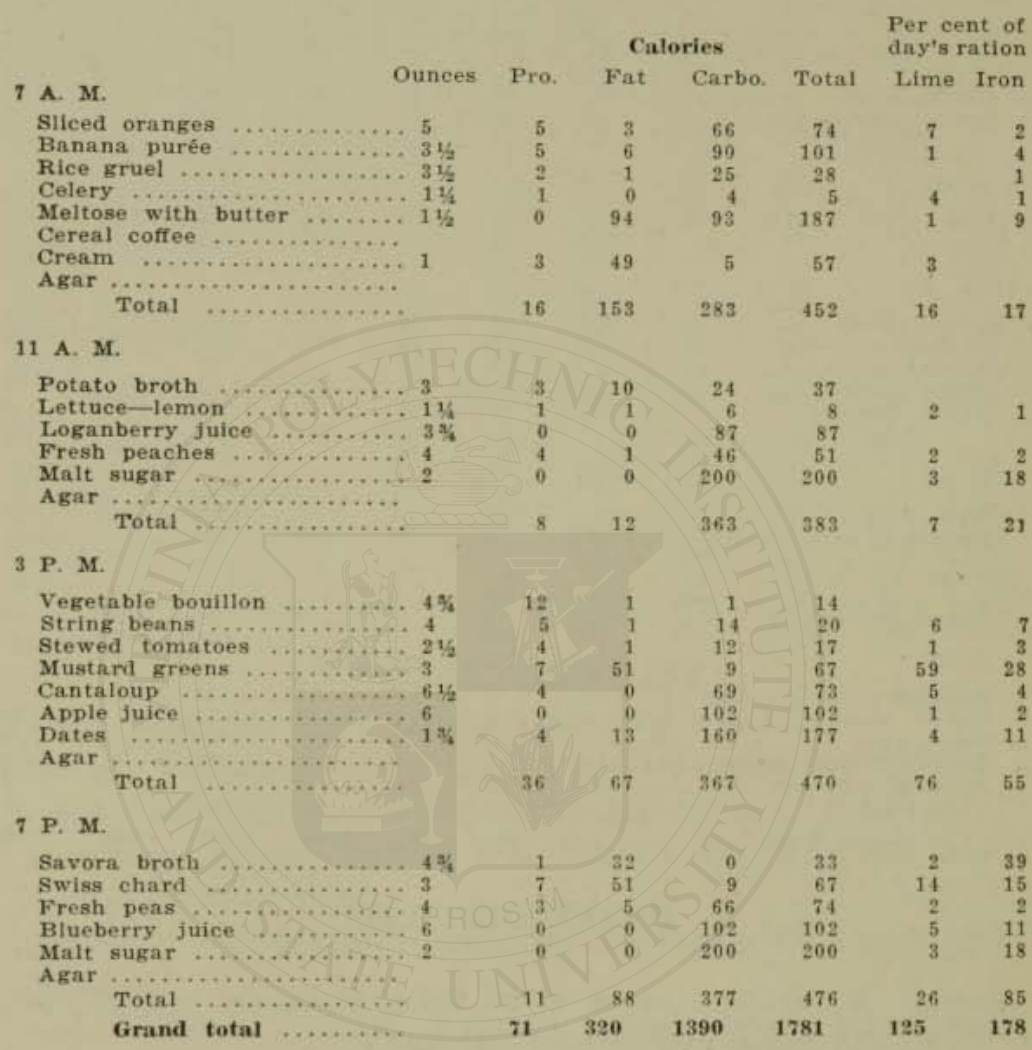

\section{PROTEIN-FREE DIET LIST}

All fruit juices, peaches, pears, apples, bananas, honey, malt sugar (meltose), malt honey, raisin juice, corn starch, rice gruel, oatmeal water, vegetable broths, lactose, lacto-dextrin, whey. Greens of all sorts, celery, lettuce, tomatoes, ripe olives, litchi nuts. 


\section{BILL OF FARE FOR FAT'TENING DIET}

\begin{tabular}{|c|c|c|c|c|c|c|}
\hline \multirow[b]{2}{*}{ Breakfast } & \multirow[b]{2}{*}{ Pro. } & \multicolumn{2}{|c|}{ Calories } & \multirow[b]{2}{*}{ Total } & \multicolumn{2}{|c|}{$\begin{array}{l}\text { Per cent of } \\
\text { day's ration }\end{array}$} \\
\hline & & Fat & Carbo. & & Lime & Iron \\
\hline Grapefruit $\ldots \ldots \ldots \ldots \ldots \ldots \ldots 3{ }^{*}$ & 3 & 2 & 44 & 49 & 3 & 2 \\
\hline Steamed rice with raisins $\ldots 41 / 2$ & 15 & 7 & 171 & 193 & 3 & 6 \\
\hline Malt sugar .............. 2 & 0 & 0 & 200 & 200 & 3 & 18 \\
\hline Cream $\ldots \ldots \ldots \ldots \ldots \ldots .21 / 4$ & 7 & 111 & 12 & 130 & 7 & 1 \\
\hline Baked potato ............. & 9 & 1 & 78 & 88 & 1 & 8 \\
\hline Celery $\ldots \ldots \ldots \ldots \ldots \ldots, 1^{1 / 4}$ & 1 & 0 & 4 & 5 & 4 & 1 \\
\hline Bran gems toasted ........ 2 & 16 & 67 & 55 & 138 & 2 & 10 \\
\hline $\begin{array}{l}\text { Butter } \ldots \ldots \ldots \ldots \ldots \ldots \ldots \ldots \ldots \\
\text { Cereal coffee } \ldots \ldots \ldots \ldots \ldots \ldots\end{array}$ & 0 & 108 & 0 & 108 & & \\
\hline Bran ......................... $1 / 4$ & 5 & z & 13 & 21 & 1 & 4 \\
\hline Total ............... & 56 & 299 & 577 & 932 & 24 & 50 \\
\hline \multicolumn{7}{|l|}{ Dinner } \\
\hline Cream of pea soup ........ 4 /4/4 & 12 & 33 & 25 & 70 & 6 & \\
\hline Mashed potato ........... $31 /$ & 9 & 15 & 61 & 85 & 1 & 8 \\
\hline Mustard greens ........... 3 & 7 & 51 & 9 & 67 & 89 & 28 \\
\hline Creamed carrots ........... 34 & 9 & 41 & 36 & 86 & 4 & 2 \\
\hline Yogurt buttermilik $\ldots \ldots \ldots 6$ & 24 & 5 & 36 & 65 & 28 & 3 \\
\hline Bran bread ................ 2 & 12 & 64 & 124 & 200 & 2 & 10 \\
\hline Butter $\ldots \ldots \ldots \ldots \ldots \ldots \ldots . . . . . .1 / 2$ & 0 & 108 & 0 & 108 & & \\
\hline Meltose ................. 2y & 0 & & 193 & 193 & 2 & 7 \\
\hline Baked apple ............... $4^{1 / 3}$ & 1 & 3 & 82 & 86 & 1 & 2 \\
\hline Pecans $\ldots \ldots \ldots \ldots \ldots \ldots . . . . .1 / 2$ & 6 & 94 & 9 & 109 & 1 & 2 \\
\hline Bran ................... & 5 & 3 & 13 & 21 & 1 & 4 \\
\hline Total $\ldots \ldots \ldots \ldots \ldots \ldots$ & 85 & 417 & 588 & 1090 & 135 & 66 \\
\hline \multicolumn{7}{|l|}{ Supper } \\
\hline Savory potato soup $\ldots \ldots \ldots .4 \pi$ & 2 & 27 & 20 & 49 & 1 & 35 \\
\hline Cereni flakes ............ & 11 & 1 & 92 & 104 & 0 & 0 \\
\hline Cream $\ldots \ldots \ldots \ldots \ldots \ldots . . \ldots \ldots$ & 7 & 111 & 12 & 130 & 7 & 1 \\
\hline Whole wheat bread ........ 2 & 22 & 4 & 112 & 138 & 2 & 6 \\
\hline Butter ............... $1 / 2$ & 0 & 108 & 0 & 108 & & \\
\hline Fresh strawberries ........ 4 & 15 & 6 & 34 & 55 & 5 & 4 \\
\hline Malt sugar ............. 1 & 0 & 4 & 120 & 124 & 2 & 9 \\
\hline 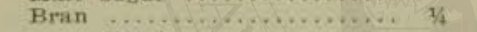 & 5 & 3 & 13 & 21 & 1 & 4 \\
\hline 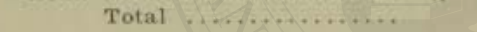 & 62 & 264 & 403 & 729 & 18 & 59 \\
\hline Grand total $\ldots \ldots \ldots \ldots$ & 203 & 980 & 1568 & 2751 & 177 & 175 \\
\hline
\end{tabular}

\section{FAT'TENING DIET LIST}

Fruits: All fruits, but especially dates, figs, avocadoes, ripe olives.

Nuts: All nuts, especially almonds, walnuts, pecans and peanuts, peanut butter, almond cream, malted nuts.

Milk: Certified milk, cream, butter, buttermilk, cottage cheese, cream cheese, clotted cream, malted milk.

Cereals: All cereals, especially rice, granola, zo, vita-bits and other dextrinized cereals, breads, zwieback, mushes, etc.

Vegetables: All vegetables. Potatoes, sweet potatoes, greens.

Miscellaneous: Malt sugar (meltose), lactose, lacto-dextrin. Accessories: Laxa, agar, paraffin preparations (paralax, paramels, petrolatum). 


\section{BILL OF FARE FOR LAXATIVE DIET}

\begin{tabular}{|c|c|c|c|c|c|c|}
\hline \multirow[b]{2}{*}{ Breakfast } & \multicolumn{4}{|c|}{ Calories } & \multicolumn{2}{|c|}{$\begin{array}{l}\text { Per cent of } \\
\text { day's ration }\end{array}$} \\
\hline & Pro. & Fat & Carbo. & Total & Lime & Iron \\
\hline Orange juice , & 0 & 0 & 76 & 76 & 6 & 2 \\
\hline Stewed prunes $\ldots . . . . \ldots \ldots \ldots . . .33$ & 3 & 1 & 99 & 103 & 2 & 6 \\
\hline Brose $\quad \ldots \ldots \ldots \ldots \ldots \ldots \ldots \ldots, 41 / 6$ & 10 & 8 & 36 & 54 & 7 & 25 \\
\hline Cream $\quad . . \ldots \ldots \ldots \ldots \ldots \ldots . . .21$ & 7 & 111 & 12 & 130 & & 1 \\
\hline Bran gems ............... & 16 & 67 & 55 & 138 & 1 & 8 \\
\hline Butter ................... 1/2 & 0 & 108 & 0 & 108 & & \\
\hline $\begin{array}{l}\text { Cereal coffee } \ldots \ldots \ldots \ldots \ldots \ldots \\
\text { Lettuce }\end{array}$ & & & & & & \\
\hline $\begin{array}{l}\text { Total } \\
\text { Totace }\end{array}$ & 37 & $\begin{array}{r}1 \\
296\end{array}$ & $284^{6}$ & $\begin{array}{r}8 \\
617\end{array}$ & $25^{2}$ & $\begin{array}{r}1 \\
43\end{array}$ \\
\hline \multicolumn{7}{|l|}{ Dinner } \\
\hline Tomato soup ...... & 6 & 22 & 29 & 57 & & 1 \\
\hline Baked potato ................ & 9 & 1 & 78 & 88 & 1 & 8 \\
\hline 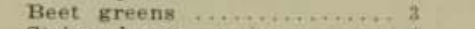 & 7 & 51 & 9 & 67 & 8 & 23 \\
\hline String beans $\ldots \ldots \ldots \ldots \ldots \ldots 4$ & 5 & 1 & 14 & 20 & 6 & 7 \\
\hline sliced cucumbers ............ z & 2 & 1 & 7 & 10 & 1 & \\
\hline Cottage cheese.....$\ldots \ldots \ldots$ i & 20 & 12 & 5 & 37 & 28 & 2 \\
\hline Bran bread $\ldots \ldots \ldots \ldots \ldots \ldots q$ & 12 & 64 & 124 & 200 & 2 & 10 \\
\hline Butter ................ & 0 & 108 & 0 & 108 & & \\
\hline Apple and date salad $\ldots . . . .21 / 2$ & 4 & 8 & 94 & 106 & 2 & 5 \\
\hline Lemon jelly, whipped cream $31_{2}$ & 1 & 23 & 115 & 139 & 1 & 1 \\
\hline Bran ................... $1 / 4$ & 5 & 3 & 13 & 21 & 1 & 4 \\
\hline Total ... & 71 & 294 & 488 & 853 & 50 & 61 \\
\hline \multicolumn{7}{|l|}{ Supper } \\
\hline Vegetable consomme $\ldots \ldots \ldots .4 \pi / 2$ & 12 & 1 & 2 & 15 & & \\
\hline Asparagus on toakt $\ldots \ldots \ldots \ldots .3$ & 14 & 85 & 67 & 166 & 2 & 7 \\
\hline Shredded carrots $\ldots \ldots \ldots \ldots z$ & 3 & 9 & 22 & 27 & 5 & 2 \\
\hline Celery ............ & 1 & 0 & 4 & 5 & 4 & 1 \\
\hline Graham bread & 20 & 12 & 104 & 136 & 2 & 10 \\
\hline Butter .......... & 0 & 108 & 0 & 108 & & \\
\hline Pecans .. & 6 & 94 & 9 & 109 & 1 & 2 \\
\hline Grapes .................... 5 & 6 & 16 & 83 & 105 & 4 & 3 \\
\hline Yogurt buttermilk ..........6 & 24 & 5 & 36 & 65 & 28 & 3 \\
\hline Bran ................... $1 / 4$ & 5 & 3 & 13 & 21 & 1 & 4 \\
\hline Total $\ldots \ldots \ldots, \ldots \ldots \ldots$ & 91 & 326 & 340 & 757 & 47 & 32 \\
\hline Grand total $\ldots \ldots \ldots$ & 199 & 916 & 1112 & 2227 & 122 & 136 \\
\hline
\end{tabular}

Intermediate of fruit only may be eaten at 11.00 A. M., 5.00 P. M., and just before retiring.

\section{LAXATIVE DIET LIST}

All foods rich in cellulose (See Tables XXI to XXIII).

Fruits: All fruits, especially fresh figs, dried figs, purple figs (soaked), figs purée, prunes, prune purée, meltose marmalades, tomatoes, berries and nuts.

Cereals: Oatmeal, brose (cooked only 6 to 10 minutes), cracked wheat, whole wheat, cooked bran, bran mush, graham bread, bran bread, bran muffins, shredded wheat, wheat flakes.

Vegetables: Carrots, turnips, rutabagas, parsnips, beets, spinach, dandelion and other greens, string beans, green peas, egg-plant, celery, lettuce, cuycumber, radishes, salads, cabbage. Miscellaneous: Malt sugar (meltose), malt honey, malted nuts, malted milk, buttermilk (acidophilus, yogurt), marmalades. Accessories: Agar, laxa, paraffin preparations (paramels, paralax, petrolatum, etc), lacto-dextrin, psyllium seed. 


\section{BILL OF FARE FOR FRUIT REGIMEN}

\begin{tabular}{|c|c|c|c|c|c|c|}
\hline \multirow[b]{2}{*}{ Breakfast } & \multirow[b]{2}{*}{ Pro. } & \multicolumn{2}{|c|}{ Calories } & \multirow[b]{2}{*}{ Total } & \multicolumn{2}{|c|}{$\begin{array}{l}\text { Per cent of } \\
\text { day'a ration }\end{array}$} \\
\hline & & Fat & Carbo. & & Lime & Iron \\
\hline Tomato broth $\ldots \ldots \ldots \ldots \ldots \ldots+\frac{\pi}{4}$ & 4 & 1 & 11 & 16 & & 1 \\
\hline 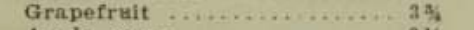 & 3 & 2 & 44 & 49 & 3 & 2 \\
\hline Apple sauce $\ldots \ldots \ldots \ldots \ldots .31 / 2$ & 1 & 3 & 73 & 76 & 2 & 3 \\
\hline Steamed figs ............... 2 & 9 & 1 & 148 & 158 & 4 & 4 \\
\hline Lettuce $\ldots . . \ldots \ldots \ldots \ldots \ldots \ldots .11 / 4$ & 1 & 1 & 6 & 8 & 2 & 1 \\
\hline Lactose $\quad \ldots \ldots \ldots \ldots \ldots \ldots \ldots, 2$ & 0 & 0 & 200 & 200 & 0 & 9 \\
\hline Bran $\ldots \ldots \ldots \ldots \ldots \ldots \ldots \ldots$ & 11 & 6 & 26 & 43 & 2 & 8 \\
\hline Total $\ldots \ldots \ldots \ldots \ldots \ldots$ & 29 & 14 & 507 & 550 & 13 & 19 \\
\hline \multicolumn{7}{|l|}{ Dinner } \\
\hline Vegetable boullion .......... 4 a & 4 & 1 & 11 & 16 & 0 & 1 \\
\hline Turnip tops $\ldots \ldots \ldots \ldots \ldots .3$ & 7 & 51 & 9 & 67 & 41 & 20 \\
\hline Cauliflower $\quad \ldots \ldots \ldots \ldots \ldots . . .3$ & 2 & 15 & 5 & 22 & 12 & 3 \\
\hline Celery $\quad \ldots \ldots \ldots \ldots \ldots \ldots \ldots \ldots 11 / 4$ & 1 & 0 & 4 & 5 & 4 & 1 \\
\hline Strawberry jelly ,......... 3 & 0 & 0 & 120 & 120 & 1 & 1 \\
\hline Fruit nectar $\ldots \ldots \ldots \ldots \ldots .5$ & 2 & 2 & 96 & 100 & 1 & 1 \\
\hline Dates ................... $1 \mathrm{~g} / \mathrm{h}$ & 4 & 13 & 160 & 177 & 4 & 11 \\
\hline Bran $\ldots \ldots \ldots \ldots \ldots \ldots \ldots$ b. & 11 & 6 & 26 & 43 & 2 & 8 \\
\hline Lactose ................. 2 & 0 & 0 & 200 & 200 & 0 & 0 \\
\hline Total $, \ldots \ldots \ldots, \ldots \ldots$ & 31 & 88 & 631 & 750 & 65 & 46 \\
\hline \multicolumn{7}{|l|}{ Supper } \\
\hline Savora broth $\ldots \ldots \ldots \ldots \ldots, 4 y_{6}$ & 1 & 32 & 0 & 33 & 1 & 39 \\
\hline New beets . . . . . . . . . . . . . 21/4 & 6 & 1 & 19 & 26 & 2 & 2 \\
\hline String beans ............. 4 & 5 & 1 & 14 & 20 & 6 & 7 \\
\hline Sliced tomato ............ 4 & 4 & 4 & 18 & 26 & 1 & 3 \\
\hline Pear sauce $\ldots \ldots \ldots \ldots \ldots \ldots .4$ & 3 & 4 & 93 & 100 & 2 & 2 \\
\hline Apple $\ldots \ldots \ldots \ldots \ldots \ldots \ldots . . .516$ & 2 & 7 & 88 & 97 & 2 & 3 \\
\hline Bran $\ldots \ldots \ldots \ldots \ldots \ldots \ldots \ldots$ & 11 & 6 & 26 & 43 & 2 & 8 \\
\hline Loganberry juice, lactose ... $5 \%$ & 0 & 0 & 287 & 287 & 3 & 4 \\
\hline Total $\ldots \ldots \ldots \ldots \ldots \ldots$ & 32 & 55 & 545 & 632 & 19 & 68 \\
\hline Grand total & 92 & 157 & 1683 & 1938 & 97 & 133 \\
\hline
\end{tabular}

\section{DIET LIST FOR FRUIT REGIMEN}

Fruits: Fresh apples, oranges, grapefruit, dates, figs, raisins, all berries, plums, peaches, cantaloups, watermelon, honeydew melon, osage melon, grapes, apricots, pears, pineapple, cherries, bananas, fruit sauces, fruit juices-orange, lemon, apple, grape, raspberry, blackberry, loganberry, etc.

Vegetables: (1) Uncooked-Tomatoes, celery, lettuce, endive, cabbage, cabbage (shredded), radishes.

(2) Cooked-Spinach, mustard, turnip tops, dandelion greens, red root, Swiss chard, dock, tomatoes, asparagus, string beans, potato gruel, vegetable broths, Savita broth.

Miscellaneous: Malt sugar, malt honey, fig marmalade, prune marmalade.

Accessories: Bran, laxa, agar, paraffin preparations (paralax, paramels, etc.).

Bran: 2 large tablespoonfuls each meal.

Cereal coffee (unroasted) without cream.

Lacto-dextrin to change the intestinal flora. 


\section{BILL OF FARE FOR RESTORATIVE OR BLOOD- MAKING DIET}

\begin{tabular}{|c|c|c|c|c|c|c|}
\hline \multirow[b]{2}{*}{ Breakfast } & \multicolumn{4}{|c|}{ Calories } & \multicolumn{2}{|c|}{$\begin{array}{l}\text { Per cent of } \\
\text { day's ration }\end{array}$} \\
\hline & Pro. & Fat & Carbo. & Total & Lime & Iron \\
\hline Orange juice $\ldots \ldots \ldots \ldots \ldots \ldots 5$ & 0 & 0 & 76 & 76 & 6 & 2 \\
\hline Oatmeal with dates $(10 z.) \ldots 5$ & 14 & 17 & 142 & 173 & 5 & 12 \\
\hline Cream ................. $2^{1 / 4}$ & 7 & 111 & 12 & 130 & 7 & 1 \\
\hline Bran bread toast ......... 2 & 12 & 64 & 124 & 200 & 2 & 10 \\
\hline $\begin{array}{l}\text { Butter } \ldots \ldots \ldots \ldots \ldots \ldots \ldots \ldots \ldots \\
\text { Cereal coffee } \ldots \ldots \ldots \ldots \ldots \ldots \ldots\end{array}$ & 0 & 108 & 0 & 108 & & \\
\hline Bran $\ldots \ldots \ldots \ldots \ldots \ldots \ldots . . .$. & 5 & 3 & 13 & 21 & 1 & 4 \\
\hline Total $\ldots \ldots \ldots \ldots \ldots \ldots$ & 38 & 303 & 367 & 708 & 21 & 29 \\
\hline \multicolumn{7}{|l|}{ Dinner } \\
\hline Savora rice soup .......... $4 \pi$ & 4 & 16 & 33 & 53 & 2 & 39 \\
\hline Scalloped potatoes ........ 41/4 & 16 & 57 & 74 & 147 & 2 & 12 \\
\hline Mustard greens-2 egg yolks 4 & 25 & 131 & 9 & 165 & 64 & 44 \\
\hline Sliced tomatoes ............ 4 & 4 & 4 & 18 & 26 & 1 & 3 \\
\hline Yogurt buttermallk ........ 6 & 24 & 5 & 36 & 65 & 28 & 3 \\
\hline Bran bread ............ 2 & 12 & 64 & 124 & 200 & 2 & 10 \\
\hline Butter ................ 1/4 & 0 & 108 & 0 & 108 & & \\
\hline Fresh strawberries $\ldots . \ldots \ldots, 4$ & 5 & 6 & 34 & 45 & 7 & 7 \\
\hline Malt sugar $\ldots . \ldots \ldots, \ldots \ldots, \ldots 1$ & 0 & 0 & 100 & 100 & 2 & 9 \\
\hline Bran $\ldots \ldots \ldots \ldots \ldots \ldots \ldots \ldots$ & 5 & 3 & 13 & 21 & 1 & 4 \\
\hline 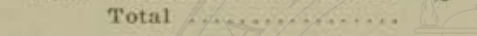 & 95 & 394 & 441 & 930 & 109 & 131 \\
\hline \multicolumn{7}{|l|}{ Supper } \\
\hline Lentil soup ............... $4^{1 / 4}$ & 35 & 19 & 86 & 140 & 3 & 14 \\
\hline Baked potato ............ 3 & 9 & 1 & 78 & 88 & 1 & 8 \\
\hline Lettuce sandwich ......... 2 & 12 & 123 & 63 & 198 & 2 & 10 \\
\hline Ripe ollives $\ldots \ldots \ldots \ldots \ldots 1^{1 / 4}$ & 2 & 92 & 7 & 101 & 7 & 9 \\
\hline Raspberries $\ldots \ldots \ldots \ldots \ldots .4$ & $\overline{5}$ & 0 & 59 & 64 & 8 & 4 \\
\hline Malt honey ............ $21 / 2$ & 0 & 0 & 194 & 194 & 2 & 7 \\
\hline \multirow{3}{*}{ Grand total $\ldots \ldots \ldots, \ldots$} & 5 & 3 & 13 & 21 & 1 & 4 \\
\hline & 68 & 238 & 500 & 806 & 24 & 56 \\
\hline & 201 & 935 & 1308 & 2444 & 154 & 216 \\
\hline
\end{tabular}

\section{RESTORATIVE OR BLOOD-MAKING DIET LIST}

This dietary includes most foods rich in lime and iron salts (See Tables XII, XIV, XV), especially whole grain preparations, graham bread, cracked wheat, oatmeal, browned rice, wheat flakes, shredded wheat, zo, gluten biscuit, gluten mush, gluten bread ( 40 per cent), beans, lentils, peas, soy beans, to $\mathrm{fu}$, spinach, turnip tops, lettuce, salads, milk, cream, whey, buttermilk, cottage cheese, malt sugar (meltose), malt honey, malted nuts, savita, vegetable broths, tomatoes, tomato juice, orange juice, lemon juice, egg yolks, nuts of all sorts, dates, figs, raisins.

It is not necessary that the diet should be strictly confined to the above foods, but they should constitute the staples of the dietary, so as to make sure of an abundant supply of vitamins, iron, lime and other food salts. When the hemoglobin is low, the iron and lime content of the food should be doubled until the deficiency is made good. That is, the percentage of the day's ration of these elements should be 200 . 


\section{BILL OF FARE FOR REDUCING DIET}

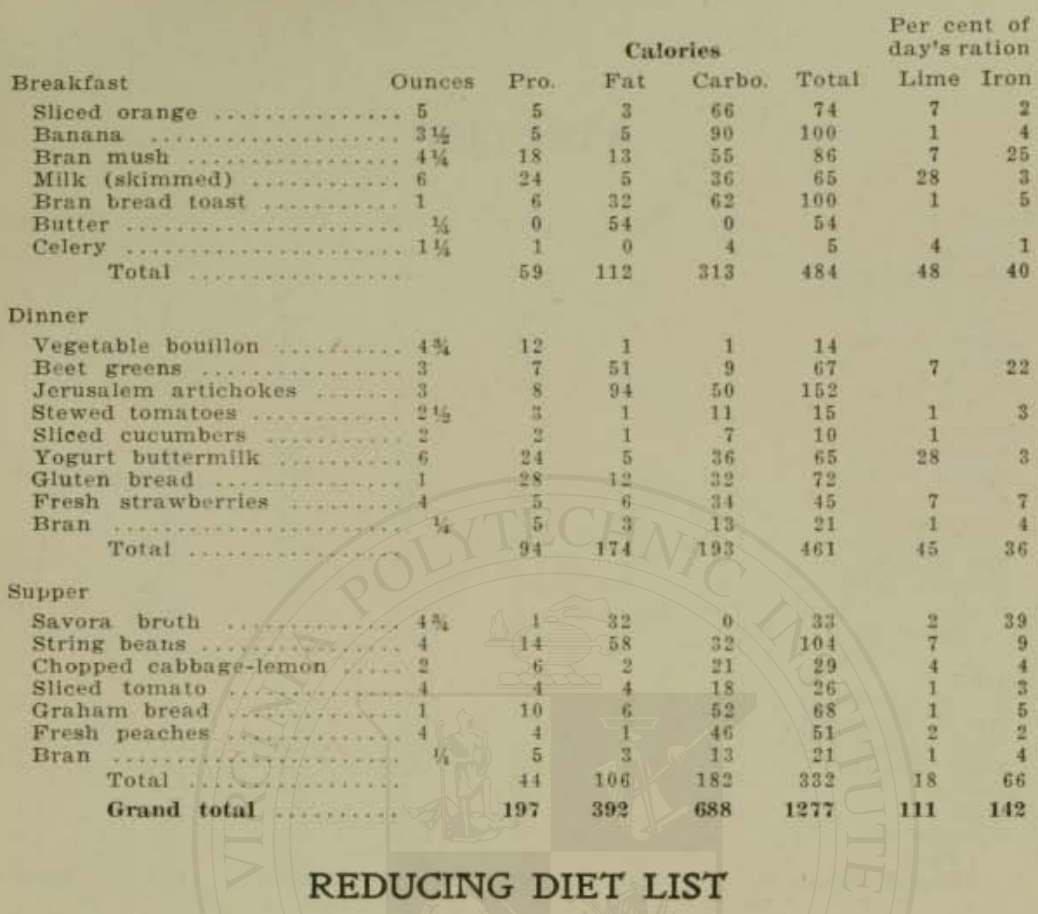

Fruits: All fresh, juicy fruits, especially acid and sub-acid fruits, including tomatoes both cooked and raw. Avoid figs, dates, raisins and prunes. Also avoid nuts.

Cereals: Bran mush (bran three parts by measure, rolled oats one part. Cook six minutes). Bran bread toasted, one slice at each meal, or rye bread toasted. Gluten bread ( 40 per cent), gluten gems, and gluten biscuit instead of ordinary bread. Avoid breakfast foods, flakes and dextrinized cereals.

Vegetables: Most vegetables, especially the artichoke, the Jerusalem artichoke, greens of all sorts, turnips, cabbage, celery, lettuce, cucumber, radish, salsify, Brussels sprouts, and avoid the potato, sweet potato and the dasheen.

Miscellaneous: Whey, cottage cheese, skimmed milk, buttermilk (yogurt, acidophilus), butter (one-half ounce daily), cereal coffee.

Accessories: Laxa, agar, paralax, paramels and other paraffin preparations, 


\section{BASIC DIET}

\begin{tabular}{|c|c|c|c|c|c|c|c|}
\hline Breakfast & Oz. & Pra. & Fat & Carbo. & Total & Lime & Iron \\
\hline Orange $\ldots \ldots \ldots$ & 5 & 5 & 3 & 66 & 74 & 7 & 2 \\
\hline Steamed Figs .... & 4 & 10 & 2 & 168 & 180 & 12 & 11 \\
\hline Fig Bran Flakes. & 1 & 19 & 9 & 62 & 90 & 14 & .2 \\
\hline Cream $\ldots . . . .$. & 3 & 8 & 142 & 15 & 165 & 7 & .9 \\
\hline Basie Biseuft ... & 1 & 3 & 55 & 10 & 68 & 3.3 & 11.0 \\
\hline Butter $\ldots \ldots \ldots \ldots$ & $1 / 4$ & 0 & 54 & 0 & 54 & & \\
\hline Total,$\ldots \ldots$ & & 45 & 265 & 321 & 631 & 29.7 & 25.1 \\
\hline \multicolumn{8}{|l|}{ Dinner } \\
\hline Tomato Soup ... & 5 & 6 & 24 & 30 & 60 & 0 & 1.0 \\
\hline Baked Potato .... & 6 & 18 & 1 & 156 & 175 & 2 & 10.0 \\
\hline Spinach $\ldots \ldots \ldots \ldots$ & 4 & 10 & 42 & 12 & 64 & 11 & 27 \\
\hline Parsnips $\ldots . . .$. & 4 & 6 & 15 & 60 & 81 & 9 & 5 \\
\hline Lima Beans ..... & 4 & 32 & 15 & 100 & 147 & 4.4 & 15,2 \\
\hline Soy Biscuit ..... & 1 & 8 & 3 & 3 & 14 & 6.3 & 19.0 \\
\hline Butter $\ldots \ldots \ldots$ & 16 & 0 & 108 & 0 & 108 & 1.0 & 1.0 \\
\hline Melon ......... & 8 & 5 & 0 & 74 & 78 & 5.6 & 4.4 \\
\hline Total $\ldots \ldots \ldots$ & & 85 & 208 & 435 & 727 & 39.3 & 82.6 \\
\hline \multicolumn{8}{|l|}{ Supper } \\
\hline String Berns .... & 4 & 4 & 15 & 11 & 30 & 3.6 & 2.2 \\
\hline Baked Dasheens.. & 4 & 14 & 2 & 135 & 151 & & \\
\hline Apple Date Salad & 3 & 5 & 10 & 109 & 124 & 2.0 & 5,0 \\
\hline Basic Biscuit .... & 1 & 3 & 55 & 10 & 68 & 3.3 & 11.0 \\
\hline Butter $\quad \ldots \ldots \ldots$ & $0^{1 / 4}$ & 0 & 54 & 0 & 54 & & \\
\hline Milk $\ldots \ldots \ldots \ldots$ & 8 & 30 & 82 & 46 & 158 & 38.4 & 3.7 \\
\hline Strawberry Jelly. & & $\theta$ & 0 & 120 & 120 & 1.0 & 1.0 \\
\hline Total $\ldots \ldots$ & & 56 & 218 & 431 & 705 & 48.3 & 22.9 \\
\hline Grand Total. & & 206 & 691 & 1187 & 2063 & 117.3 & 130.6 \\
\hline
\end{tabular}

Basic biscuit (garbanzo, soy bean, chestnut, taro, almond), potato, sweet potato, dasheen, all green vegetables, all root vegetables, fruits of all sorts except prunes, plums and cranberries, peas, beans, string beans, soy beans, chestnuts, milk, cream, butter and nuts. 


\section{BILL OF FARE FOR FRESH OR RAW DIET}

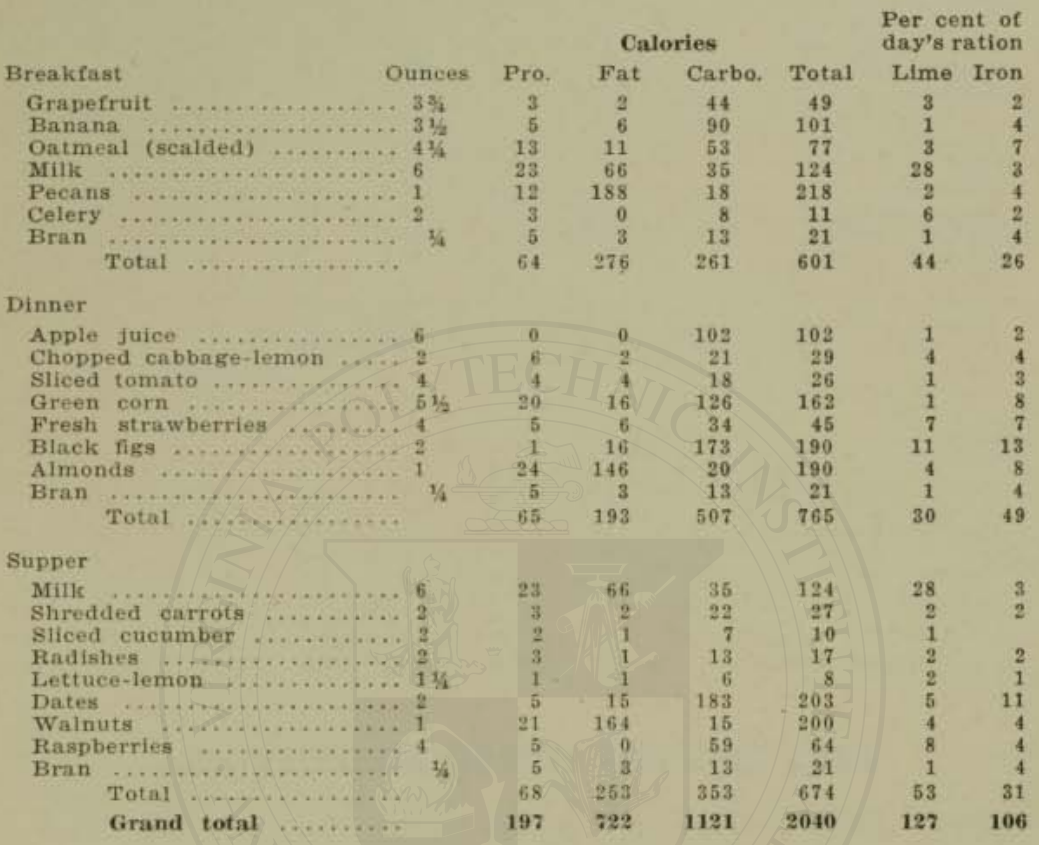

\section{FRESH OR RAW DIET LIST}

Fruits of all sorts and fruit juices.

Lettuce, celery, radish, cucumber, cabbage (uncooked), carrot, turnip, tomato.

Certified milk, whey, buttermilk (yogurt, acidophilus), eggnog (yolks).

Brose (oatmeal scalded), bran brose (oatmeal and bran equal parts, cooked six minutes), green corn (uncooked).

Nuts, especially almonds, hazelnuts or filberts, walnuts and pecans. Malt sugar may be used with acid fruits. 


\section{BILL OF FARE FOR HYPERCHLORHYDRIA}

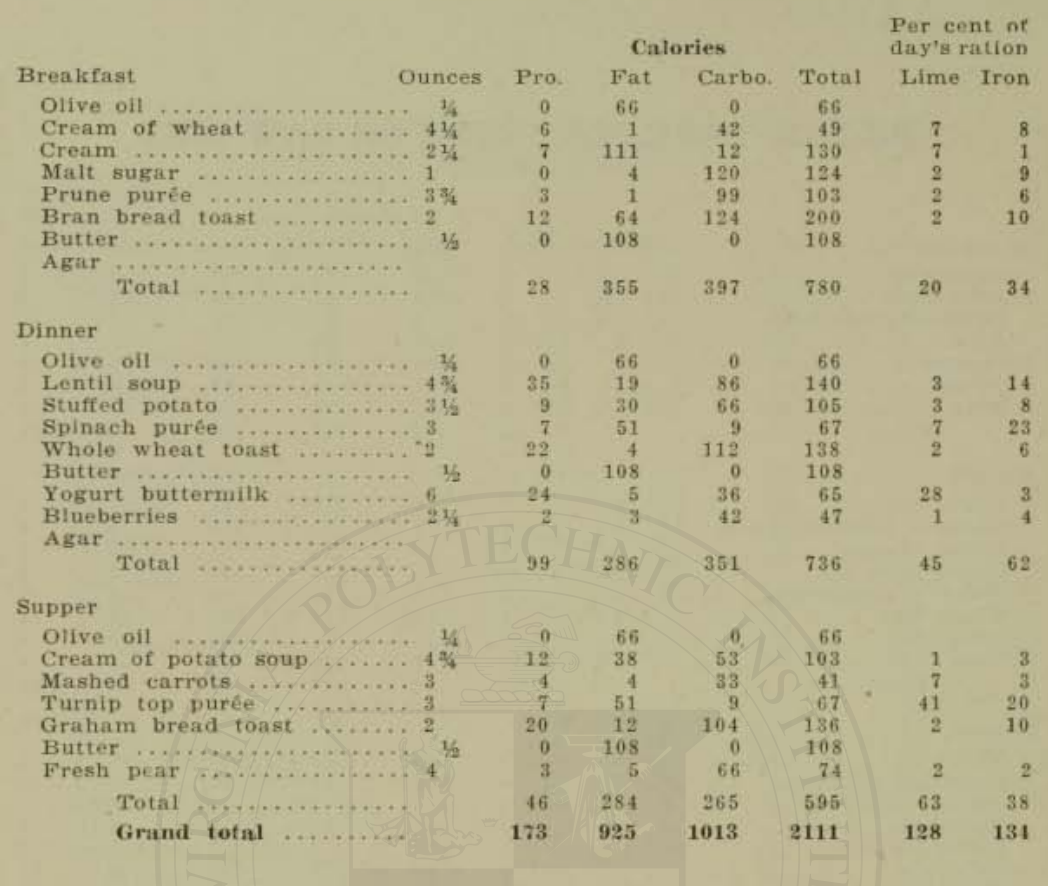

\section{DIET LIST FOR HYPERCHLORHYDRIA}

Soups: Plain and cream vegetable soups.

Relishes: Tender celery, lettuce with olive oil, ripe olives.

Entrees: Macaroni and spaghetti (buttered, creamed or with egg sauce), soft cooked eggs (poached or boiled), protose, nuttolene.

Vegetables: Purées of corn, peas, greens, beets, carrots, beans, oyster plant, squash, asparagus, soy beans, potatoes.

Cereals: All flakes, cream of wheat, shredded wheat, oats, cornmeal, hominy, gruels of all kinds, especially gluten, bran.

Breads: Dextrinized breads such as rice biscuit, granose biscuit, browned rice, zwieback, granola, thin crisp toast.

Fruits: Sweet apples, dates, fresh pears, blueberries, very ripe bananas, white cherries, cantaloup, malaga or tokay grapes.

Beverages: Buttermilk with cream, milk and cream, malted milk, malted nuts, unsweetened eggnogs (made with yolks only), unsweetened cocoa and minute brew. 
Desserts: Custards (slightly sweetened), ices and sherbets, jellies made with bland fruit juices, ice cream, frozen cream and fluffs made with sub-acid fruits.

Miscellaneous: Pecans, pinenuts, cream, butter, malt honey, with butter, olive oil, bromose, malted nuts, cottage cheese with cream, yogurt cheese.

This dietary should consist of foods which require little mastication and all irritating and stimulating foods should be eliminated.

Accessories: Bran, laxa, colax, agar, paraffin oil, paralax, paramels.

\section{BILL OF FARE FOR ULCER DIET}

\begin{tabular}{|c|c|c|c|c|c|c|}
\hline \multirow[b]{2}{*}{ Breakfast } & $\bullet$ & \multicolumn{2}{|c|}{ Calories } & \multirow[b]{2}{*}{ Total } & \multicolumn{2}{|c|}{$\begin{array}{l}\text { Per cent of } \\
\text { day's ration }\end{array}$} \\
\hline & Pro. & Fat & Carbo. & & Lime & Iron \\
\hline Rice gruel ,..... & 2 & 1 & 25 & 28 & & 1 \\
\hline Soft posched egg yolk ...... 1 & 18 & 80 & 0 & 98 & 11 & 20 \\
\hline Oven baked toust (moistened) 1 & 10 & 6 & 52 & 68 & 1 & 5 \\
\hline 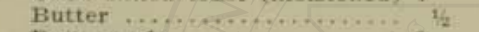 & 0 & 108 & 0 & 108 & & \\
\hline Date purée $\ldots . . \ldots \ldots \ldots . . . .2$ & 5 & 15 & 183 & 203 & 5 & 11 \\
\hline Total $\ldots$. & 35 & 210 & 260 & 505 & 17 & 37 \\
\hline \multicolumn{7}{|l|}{10 A. M. } \\
\hline Milk $1 / 3$ eream & 21 & 148 & 34 & 198 & 26 & 3 \\
\hline \multicolumn{7}{|l|}{ Dinner } \\
\hline Cream of lentil soup .......4 4 y & 44 & 80 & 97 & 221 & 8 & 14 \\
\hline Spinach souffle ...... & 25 & 78 & 146 & 249 & 10 & 10 \\
\hline Carrot puree $\ldots \ldots \ldots \ldots \ldots$ & 5 & 19 & 31 & 55 & 7 & 3 \\
\hline Oven-baked toast (moistened) 1 & 10 & 6 & 52 & 68 & 1 & 5 \\
\hline Butter $\ldots \ldots \ldots \ldots \ldots \ldots \ldots \ldots$ & 0 & 108 & 0 & 108 & & \\
\hline Malted milk ............. 115 & 20 & 14 & 132 & 166 & 2 & 2 \\
\hline Total. & 104 & 305 & 458 & 867 & 28 & 34 \\
\hline \multicolumn{7}{|l|}{$\begin{array}{l}4 \text { P. M. } \\
\text { Milk } 1 / 3 \text { cream } \ldots \ldots \ldots .\end{array}$} \\
\hline Milk $1 / 3$ cream $\ldots \ldots \ldots ., 6$ & 21 & $1+3$ & 24 & 198 & 26 & 3 \\
\hline \multicolumn{7}{|l|}{ Supper } \\
\hline Potato soup $\ldots \ldots \ldots \ldots \ldots \ldots \ldots+436$ & 5 & 16 & 38 & 59 & & \\
\hline 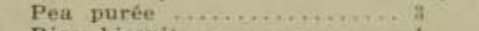 & 19 & 24 & 52 & 95 & 4 & 11 \\
\hline Rice biscuit ............. 1 & 9 & 1 & 97 & 107 & & \\
\hline Butter $\ldots \ldots \ldots \ldots \ldots \ldots \ldots \ldots .1 / 2$ & 0 & 108 & 0 & 108 & & \\
\hline Banana puree ............ $31 / 2$ & 5 & 5 & 87 & 97 & 1 & 4 \\
\hline Malt sugar $\ldots \ldots \ldots \ldots \ldots \ldots \ldots . . .2$ & 0 & 0 & 200 & 200 & 3 & 18 \\
\hline Total ,...................... & 38 & 154 & 474 & 666 & 8 & 33 \\
\hline Grand total ............ & 219 & 955 & 1260 & 2434 & 105 & 110 \\
\hline
\end{tabular}

\section{ULCER DIET LIST}

Soups: Strained cream soups of vegetables, such as peas, beans, asparagus, potato, corn, rice and oatmeal.

Vegetables: Purées of vegetables such as spinach, peas, asparagus, beets, carrots, corn, celery, and egg plant. Mashed or baked potato. 
Cereals: Rice flakes, wheat flakes, cream of wheat, granola, soft buttered toast, cream gluten mush, rice and gruels of all kinds.

Breads: Toasted bread, granose and rice biscuit.

Beverages: Malted nuts, malted milk, cream and milk, acidophilus buttermilk, with cream, unsweetened eggnog (made with yolks only).

Fruits: Banana purée, date purée, baked sweet apple, baked banana, avocado.

Desserts: Frozen cream, unsweetened custards, and jellies made from bland fruit juices.

Miscellaneous: Olive oil, butter, cream, soft cooked eggs, cottage cheese with cream, yogurt cheese.

Accessories: Agar, bran, paraffin oil.

\section{BILL OF FARE FOR MODIFIED ULCER DIET}

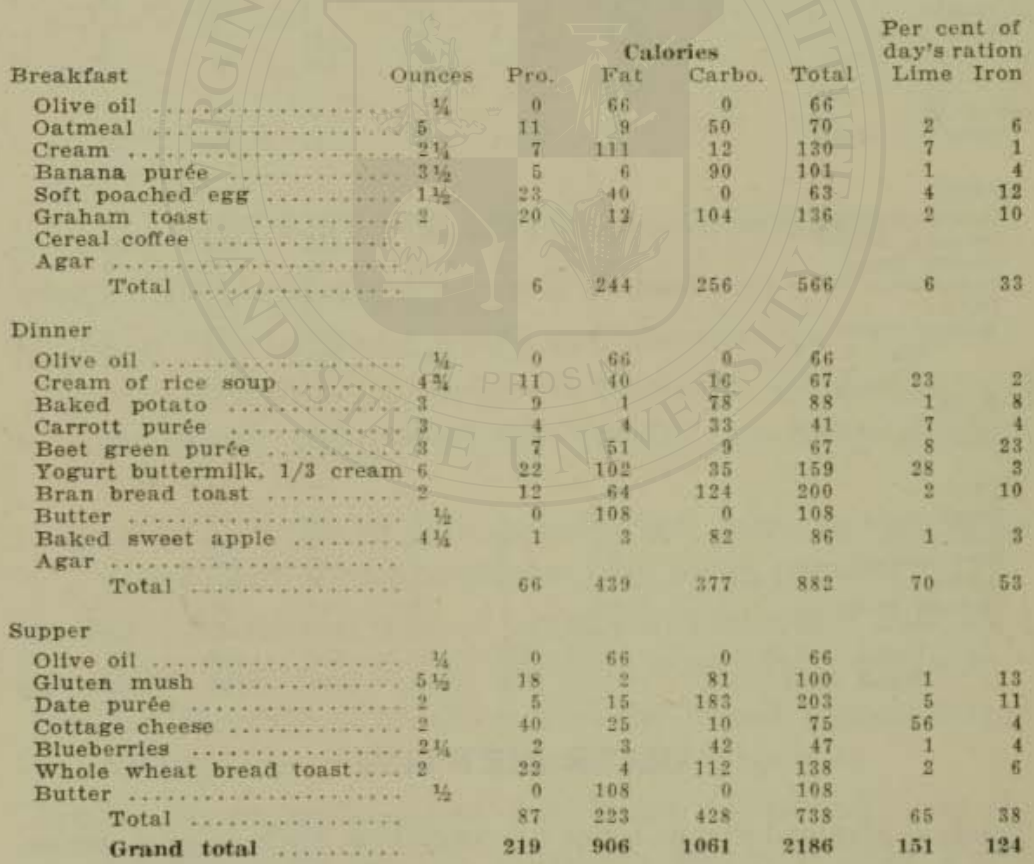




\section{BILL OF FARE FOR HYPOCHLORHYDRIA}

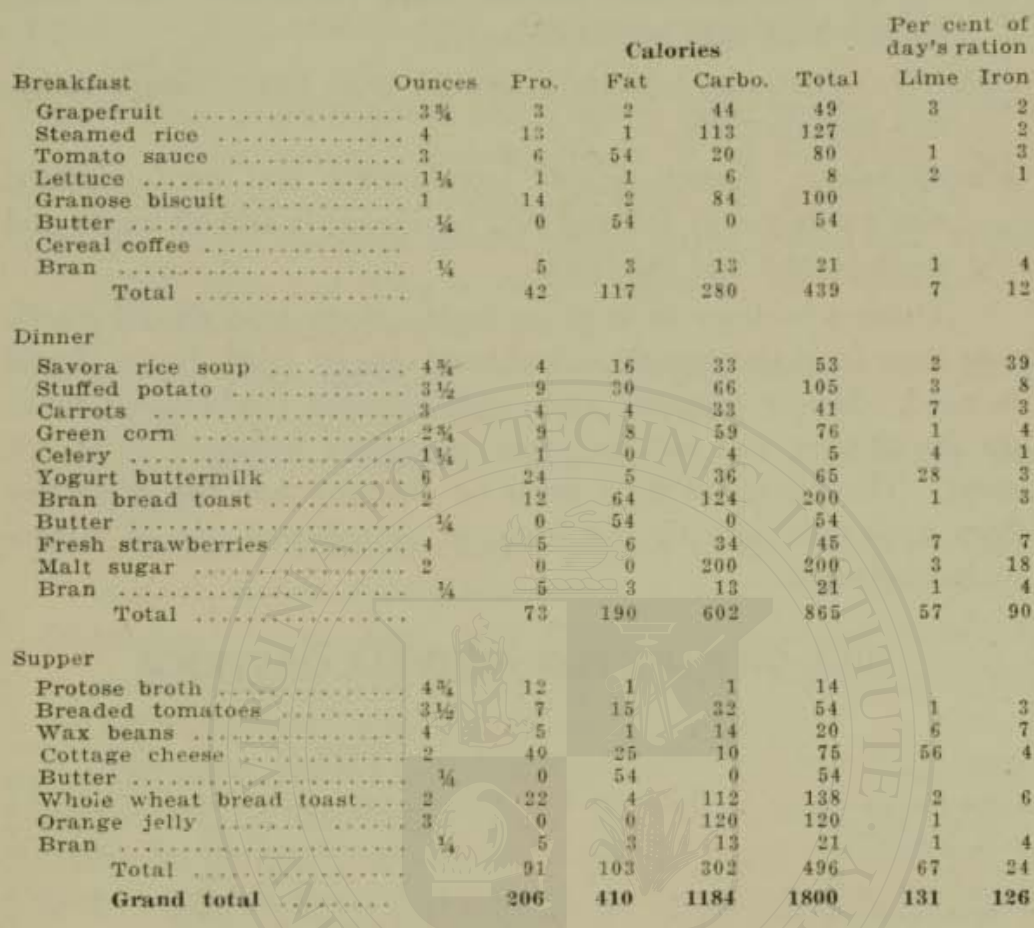

\section{DIET LIST FOR HYPOCHLORHYDRIA}

Soups: Clear vegetable broths and cream vegetable soups.

Relishes: Ripe olives, celery, lettuce, endive and watercress.

Entrees: Macaroni, spaghetti and soft cooked eggs.

Vegetables: Baked, mashed or stuffed potatoes, asparagus tips, tender string or wax beans, spinach finely chopped, new peas, beets purée, tomatoes, corn, carrots and purée of navy and lima beans.

Cereals: Flakes, zo, granola, cream of wheat, shredded wheat, rolled oats, oatmeal with bran, hominy grits, rice and cornmeal mush.

Rreads: Dry toast (whole wheat, bran, graham), zwieback, graham wafers, soda, butter or water crackers. 
Fruits: All fresh and cooked fruits. Acid fruits are especially indicated.

Beverages: Malted nuts, malted milk, acidophilus buttermilk, minute brew and health cocoa.

Desserts: Custards, tapioca, gelatines, fruit fluffs, simple cakes, ices and sherbets.

Miscellaneous: Avoid pickles, condiments and confectionery, also an excess of fat and rich heavy foods and all meats and meat broths and extracts.

Those who have little or no hydrochloric acid should masticate their food thoroughly. An abundance of fresh fruits should be used. Simply prepared and easily digested foods should make up the dietary and not too great a variety indulged in at one meal. The intestinal flora must be changed and the protective flora kept dominant by the frequent or constant use of lactose or lacto-dextrin.

\section{BILL OF FARE FOR ACHYLIA GASTRICA}

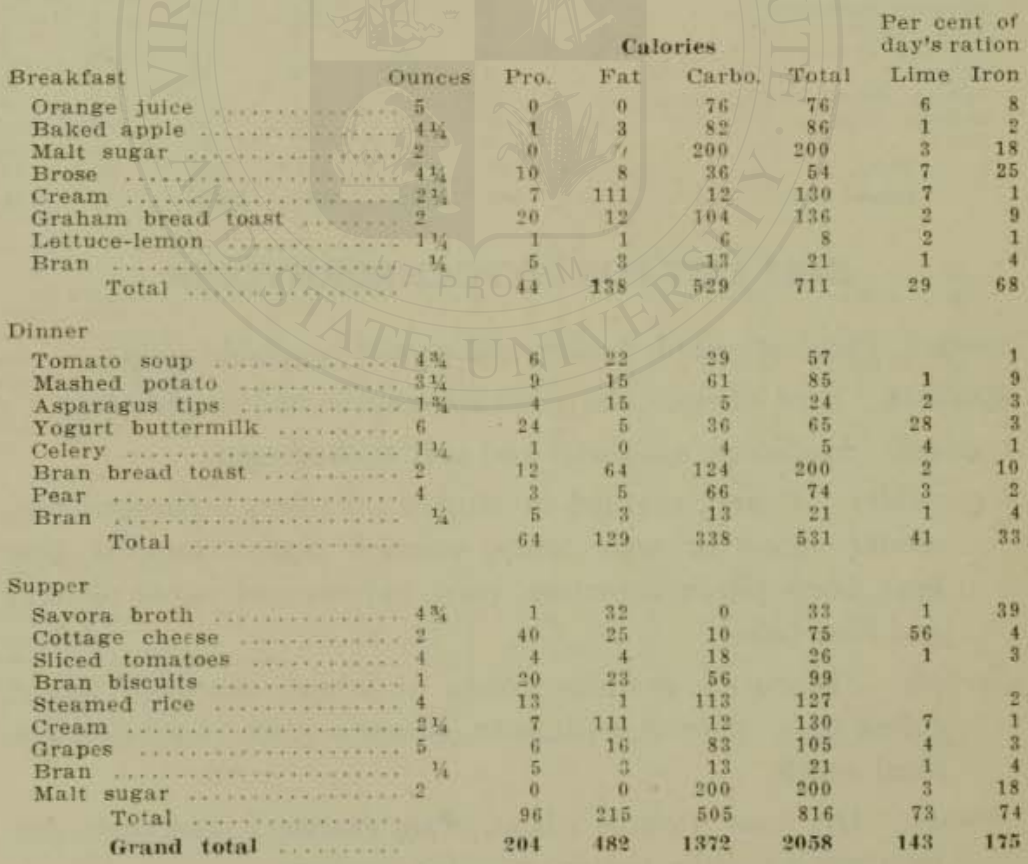




\section{DIET LIST FOR ACHYLIA}

Soups: Plain vegetable soups and purées.

Relishes: Celery, lettuce, endive and watercress.

Entrées: Soft cooked eggs, macaroni, spaghetti, protose and nuttolene.

Vegetables: Irish potatoes, peas, navy, lima, kidney, soy, wax and green beans, tomatoes, greens of all kinds, egg plant, carrots, squash, corn, beets, artichokes and asparagus.

Cereals: Rice, shredded wheat, brose, farina, rolled oats, cereal flakes, bran and other cereal preparations, with breads.

Fruits: All fresh and cooked fruits.

Beverages: Fruit juices, malted milk, malted nuts, minute brew, cocoa, buttermilk and yogurt buttermilk.

Desserts: Gelatine custards, ices, sherbets, plain cakes and tapioca.

Miscellaneous: Cottage cheese, yogurt cheese, meltose (malt honey), honey, butter and cream in restricted quantities.

Accessories: Bran, agar, and paraffin preparations should be used regularly with each meal, also acid protein (acidone) when free acid is wholly absent or nearly so.

The food must be thoroughly masticated and the dietary kept laxative by the use of bulky foods such as fresh fruits, vegetables and greens. Acid fruits should be freely used, also fruit juices. The intestinal flora should be changed frequently by the use of lactose or lacto-dextrin.

\section{Table XLIII}

\section{DIGESTIBILITY OF FOODSTUFFS AND TIME RE- QUIRED FOR THEIR DIGESTION}

Both the digestibility of foodstuffs and the time required for their digestion depend much upon the condition and the manner in which they are eaten and the quantity of material taken at one time. The amount of material absorbed from the digested food depends largely upon the amount of cellulose or roughage present. Indigestible material hastens the foodstuffs along the alimentary tract and thus lessens the amount absorbed. The amount of food material lost in this manner, however, is of little consequence compared with the importance of the prompt dismissal of unusable food residues, for the accomplishment of which a considerable amount of roughage is highly essential. Even in the case 
of foods which contain considerable cellulose, such as legumes, nuts and some fruits, digestion and absorption are satisfactorily performed provided the foodstuffs are thoroughly chewed or well comminuted before eaten.

The following table shows the degree of digestibility of various foodstuffs as determined by various authorities (Tibbles):

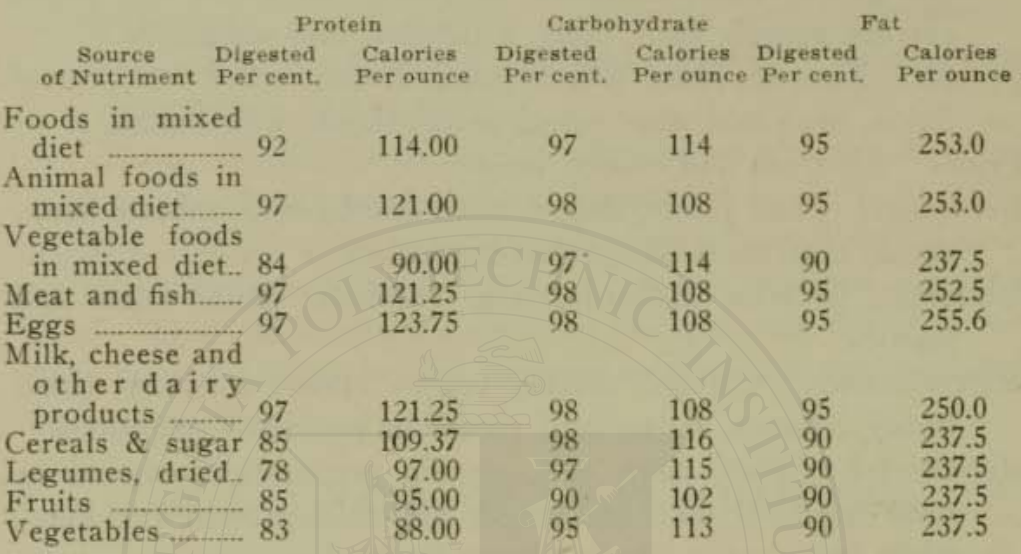

\section{Digestibility of Fruits and Nuts.}

The following table (Tibbles) shows the digestibility of fruits and nuts in various combinations:

\begin{tabular}{|c|c|c|c|}
\hline & $\begin{array}{r}\text { Foo } \\
\text { Protein }\end{array}$ & Fat & $\begin{array}{l}\text { Per cent. } \\
\text { Carbohydrate }\end{array}$ \\
\hline Olives, grapes, tomatoes. & 44.43 & 67.76 & 95.84 \\
\hline Brazil nuts, gra & 84.17 & 91.20 & 95.06 \\
\hline razil nuts, wheat flakes. & 85.44 & 84.32 & 97.45 \\
\hline Valnuts, grapes, wheat flakes & 79.76 & & 97.18 \\
\hline $\begin{array}{l}\text { Peanuts, persimmons, wheat flakes and } \\
\text { milk }\end{array}$ & 86.80 & 88.70 & 91.22 \\
\hline Valnuts, pears, milk and wheat flakes... & 84.10 & 91.41 & 96.98 \\
\hline coanut and pears & 75.12 & 91.27 & 97.44 \\
\hline Jalnuts, figs, apples.... & 70.79 & 88.22 & 97.85 \\
\hline anuts, dates, apples. & 78.14 & 83.29 & 97.16 \\
\hline cans, dates, apples............. & 76.16 & 91.53 & 97.78 \\
\hline alnuts, raisins, apples.... & 80.70 & 87.10 & 98.28 \\
\hline
\end{tabular}

\section{Dr. Beaumont's Digestion Table.}

The following table presents the principal facts shown by Beaumont in his experiments upon St. Martin in relation to the time required for the gastric digestion of various foodstuffs named: 


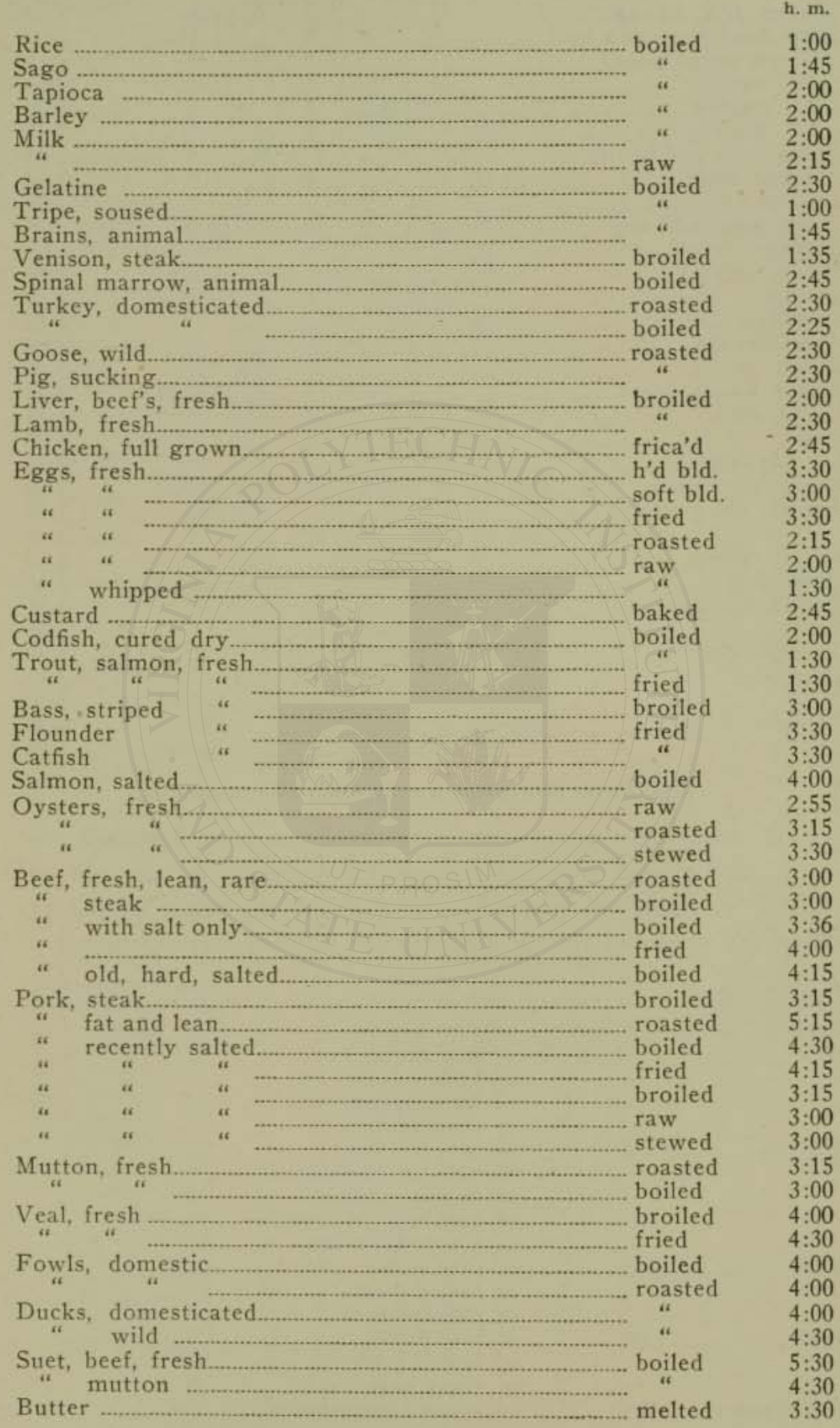


Cheese, old, strong.

raw

Soup, beef, veg. and br'd. boiled

marrow bones.

“ bean

“ barley

" mutton

Green corn and beans.

Chicken soup.

Oyster soup.

Hash, meat and veg ......................................................... 0 :

Sausage, fresh.

Heart, animal

Tendon

Cartilage

Aponeurosis

Beans, pod.

"

Bread, wheat, fresh

Cake corn. boiled

$5: 30$

$4: 15$

3:00

$2: 30$

$3: 30$

$3: 15$

3:00

$2: 30$

Dumpling, apple.

3:00

Apples, sour, hard

2:50

2:00

Parsnips

mellow

$1: 30$

2:30

Carrot, orange

Beets

Turnips, flat

Potatoes, Irish

Cabbage, head.

boiled

3:15

$3: 45$

$3: 30$

$3: 30$

$2: 3 \mathrm{C}$

$2: 30$

baked

raw

boiled

$4: 30$

\section{The Time Required for Gastric Digestion.}

Within recent years numerous observers have renewed the study of the time required for gastric digestion by the aid of modern methods of research. The following table (Tibbles) shows the results of the most recent and reliable investigations :

\begin{tabular}{|c|c|c|c|c|c|}
\hline Kind of Food & & uantit & $y$ of Food & Food let & $t$ the sto \\
\hline Beef ……................ & $31 / 2$ & ances & of raw beef & & hours \\
\hline & & 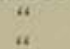 & of half-boiled beef & $21 / 2$ & " \\
\hline & & .* & of horled beer & 3 & " \\
\hline & & “ & of roasted beef & 4 & " \\
\hline Mutton & $31 / 2$ & " & of raw mutton & & “ \\
\hline & & " & of half-boiled mutton & $21 / 2$ & 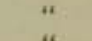 \\
\hline & & " & of boiled mutton & 3 & " \\
\hline & & $\ddot{~}$ & of half-roasted mutton & $\begin{array}{l}3 \\
4\end{array}$ & " \\
\hline Veal & $3 \pi / 2$ & “ & of raw veal & $21 / 2$ & " \\
\hline & & “ & of boiled veal & $33 / 4$ & " \\
\hline & & " & of roasted veal & & " \\
\hline Pork & $31 / 2$ & " & of raw pork & 3 & “ \\
\hline & & “ & $\begin{array}{l}\text { of Dolled pork } \\
\text { of roast pork }\end{array}$ & & “ \\
\hline Sweetbread ..... & 91 & " & of cooked sweetbread & $23 / 4$ & \\
\hline
\end{tabular}

Time in which the Food left the Stomach 


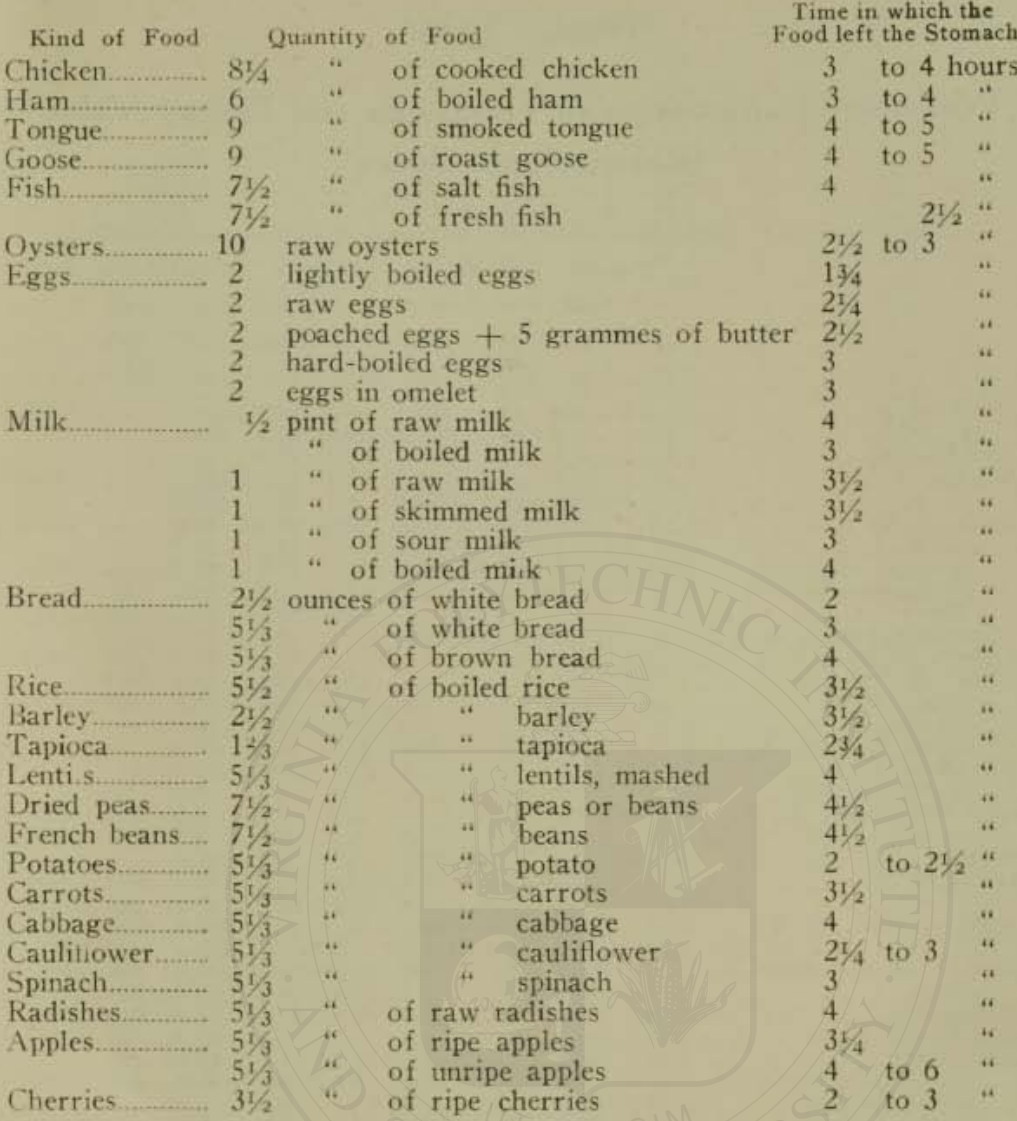

In the use of the above table, it should be remembered that the times given relate wholly to gastric digestion. The time required for the total digestion and absorption of the average meal and the transmission of the indigestible residues into the colon, is eight hours. The digestibility of foodstuffs and the time required for digestion are now known to be of much less importance than formerly supposed. Some of the most unwholesome foods are digested the most quickly. That the bill of fare shall be sufficient in amount and properly balanced and of such character as to maintain a normal non-putrefactive flora in the intestine are the matters of primary consequence. 


\section{TABLE XLIV.}

\section{Percentage Composition of Foodstuffs and Energy Value (Calories) per Ounce*}

(The letters E. P. stand for edible portion and A. P. for as purchased.)

\begin{tabular}{|c|c|c|c|c|c|c|c|c|c|}
\hline & \multicolumn{2}{|c|}{ Protein } & \multicolumn{2}{|c|}{ Fat } & \multicolumn{2}{|c|}{ Carbo. } & \multirow{2}{*}{$\begin{array}{c}\text { Total } \\
\text { Cal. }\end{array}$} & \multirow[t]{2}{*}{ Lime } & \multirow[t]{2}{*}{ Iron } \\
\hline & & Cal. & & Cal. & & Cal. & & & \\
\hline & Per & Per & Per & Per & Per & Per & Per & Per & Per \\
\hline & cent & oz. & cent & oz. & cent & oz. & oz. & eent & cent \\
\hline Acorns, E, P. . . . . . . . . . & 8.1 & 9.2 & 37.4 & 95.4 & 48.0 & 54.4 & 159.0 & & \\
\hline A. P. $\quad \ldots \ldots \ldots \ldots \ldots \ldots$ & 5.2 & 5.9 & 24.1 & 61.5 & 30.9 & 35.0 & 102.4 & & \\
\hline 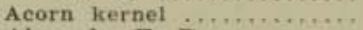 & 4.2 & 4.8 & 5.4 & 13.8 & 54.6 & 61.9 & 80.5 & & \\
\hline Imonds, E. P. $\quad \ldots \ldots \ldots \ldots$ & 21.0 & 23.8 & 54.9 & 139.9 & 17.3 & 19.6 & 183.3 & .335 & .0039 \\
\hline A. $\mathbf{P}, \ldots \ldots \ldots \ldots \ldots \ldots$ & 11.5 & 13.0 & 30.2 & 77.0 & 9.5 & 10.8 & 100.8 & & \\
\hline pples, E. P. ............. & .4 & .5 & .5 & 1.3 & 14.2 & 16.1 & 17.9 & .010 & .0003 \\
\hline A. P. $\quad \ldots \ldots \ldots \ldots \ldots \ldots$ & -3 & 3 & +3 & .8 & 10.8 & 12.2 & 3.3 & & \\
\hline dried, A. P. . . . . . . . & 1.6 & 1.8 & 2.2 & 5.6 & 66.1 & 5.0 & 82.4 & .045 & .0015 \\
\hline crab, canned, A I..... & .3 & .3 & 2,4 & 6.1 & & 7 & 8.1 & & \\
\hline juice $\quad \ldots \ldots \ldots$. & $\cdots$ & $\cdots$ & $\cdots$ & +1 & & & & .011 & .0002 \\
\hline sauce, canned, A. P.... & -2 & .2 & 8 & 9.0 & 37 & & & & \\
\hline pricots, E. P. , . . . . . . . & 1,1 & 1.2 & 2.2 & $\therefore \ldots$ & 13,4 & 5.2 & 16.4 & .02 & .0003 \\
\hline A. P.,$\ldots \ldots$ & 1. 0 & 1.1 & & & 12 & 3 & & & \\
\hline aried, A. P. & 4. 7 & 5.3 & 1.0 & 9.6 & 62.5 & & 78.8 & .092 & .0014 \\
\hline canned, A. P. $\ldots \ldots \ldots$ & .9 & 1.0 & & & 17. & & 20.6 & $\cdots$ & $\ldots$ \\
\hline sauce, canned, A, P... & 1.9 & 2.2 & 1.3 & 3.3 & & & & $\therefore$ & \\
\hline rtichokes, A, P. ........ & 2.6 & 2.9 & 2 & .5 & 16.7 & 18.9 & 29.3 & . & \\
\hline 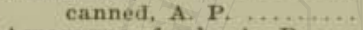 & .8 & 9 & & & 5. & 5. & 6.6 & & \\
\hline sparagus, fresh, A. P. ... & 1,8 & 2.0 & .2 & .5 & 3. & 3. & 6.2 & .03 & .0010 \\
\hline canned, A. P. ......... & 1.. & 1.7 & 1 & .3 & 2. & 3. & 5.2 & .040 & .0010 \\
\hline vocado,$\ldots \ldots \ldots \ldots \ldots \ldots+\ldots$ & 2.2 & 2.5 & 17.3 & 44.1 & 4. & 5. & 51.6 & & \\
\hline Banana, E. P. ...... & 1,3 & 1.5 & .6 & & 22. & & & & \\
\hline A. P. $\quad \ldots \ldots \ldots \ldots \ldots$ & .8 & 9 & 4 & 1.0 & 14.3 & 16.9 & & & \\
\hline Bacon, smoked, E. P....... & 10,5 & 11.9 & 64.8 & 165.2 & $\ldots$ & $\ldots$ & & & \\
\hline A, P. $\quad \ldots \ldots \ldots \ldots \ldots$ & 9.5 & & 59.4 & & & & & & \\
\hline Barley meal and flour. ..... & 10.5 & 1.9 & 2.8 & 5.6 & 72.8 & 82.6 & & & \\
\hline entire $\ldots \ldots \ldots \ldots \ldots \ldots$ & 9.0 & 0.9 & & & 66. & & & & \\
\hline pearled ........ & 8.5 & 9.6 & 1. & 2.8 & 77.8 & 8.2 & & & \\
\hline Bass, black, whole ........ & 20.6 & 23.4 & 1.7 & 4.3 & & & & .031 & .0008 \\
\hline 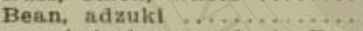 & 21,0 & 23.8 & 7 & 1.8 & 56.7 & 1.3 & & & \\
\hline baked, canned, A. P... & 6.9 & 7.8 & 2,5 & 6.4 & 19. & & & .06 & 021 \\
\hline butter. green & (4.7. & 5.3 & -3 & 8 & 14.6 & 6.6 & 28 & & \\
\hline 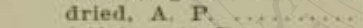 & 22.5 & 25.5 & 3.8 & 4.6 & 59.6 & 7.6 & 97.7 & .22 & .0070 \\
\hline frijoles, A. P. . . & 21.9 & 24.8 & 1.8 & 3.3 & 65.1 & 73.8 & 101.9 & $\ldots$ & $\ldots$ \\
\hline $\begin{array}{l}\text { little green, } \\
\text { canned, A. P. ...... } \\
\text { haricots, verts. }\end{array}$ & 1,2 & 1.4 & 1 & 3 & 3.4 & 3.9 & 5.6 & & \\
\hline canned, A. P. .......... & 1.1 & 1.2 & 1 & .3 & 2,5 & & & & \\
\hline $\begin{array}{l}\text { jack } \\
\text { red kidney, dried. }\end{array}$ & 23.8 & 27.0 & 2.6 & 6.6 & 50.4 & 57.2 & 90.8 & & \\
\hline A. $\mathbf{P}, \quad \ldots \ldots \ldots \ldots$ & 7.0 & 7.9 & .2 & .5 & 18.5 & & & & .0073 \\
\hline lima, dried .. & 18.1 & 90.5 & 1.5 & 3.8 & 65.9 & & & & 70 \\
\hline Hma, fresh, A. P. & 3.2 & 3. & 3 & .8 & 9.9 & & & & 020 \\
\hline limn, fresh, E. P. .... & 7.1 & 8. & .7 & 1.8 & 32.0 & & 34 & .07 & .0040 \\
\hline lima, canned, A. P... & 4.0 & 4. & .3 & .8 & 14.6 & 6 & 91 & & $\ldots$ \\
\hline mesquite, dry, A. P. . & 12,2 & 13.8 & 2.5 & 6.4 & 77.1 & & 10 & & \\
\hline and pod, mesquite $\ldots$ & 13.0 & & 3.1 & 7.9 & 46.7 & 0 & 75 & & $\ldots$ \\
\hline mung $\ldots \ldots \ldots \ldots \ldots$ & 23.8 & 27.0 & 2.0 & 5.1 & 54.8 & 62.1 & 94.2 & & $\ldots$ \\
\hline soup, homemade, A. P. & 3.2 & 3.6 & 1.4 & 3.6 & 9.4 & 10. & 17.9 & & $\ldots$ \\
\hline $\operatorname{soy} \ldots \ldots \ldots \ldots \ldots \ldots$ & 39.5 & 44.8 & 18.5 & 47.8 & 17.7 & 20.1 & 112.1 & & \\
\hline string, cooked, E. P... & .8 & .9 & 1.1 & 9.8 & 1.9 & 9.8 & 5.9 & & \\
\hline string, fresh, E. P.... & 2.3 & 9.6 & .3 & .8 & 7. & 8.4 & & .06 & .0011 \\
\hline string, fresh, A. P.... & 2.1 & 2 . & -3 & .8 & 6. & 7. & 11. & .06 & .0011 \\
\hline string, canned, A. P... & 1.1 & 1. & 1 & .3 & 3.8 & 4.3 & 5.8 & .035 & .0008 \\
\hline wax, ennned, A, P. ... & 1.0 & 1.1 & 1 & 3 & 3,1 & 3.5 & 4.9 & $\ldots$ & $\ldots$ \\
\hline Beechnut, E. P, ......... & 21.9 & 24.8 & 57.4 & 146.4 & 13.2 & 15.3 & 186.5 & $\cdots$ & $\cdots$ \\
\hline A. P. $\ldots \ldots$ & 13.0 & 14.7 & 34.0 & 86.7 & 7.8 & 8.8 & 110.2 & - & . \\
\hline $\begin{array}{l}\text { ef, briske } \\
\text { fat, E }\end{array}$ & 5.8 & 17.9 & 28.5 & 79.8 & 4 & & 90.7 & .012 & .0024 \\
\hline
\end{tabular}

- This table is complled from Bulletin No. 28 of the U. $\mathbf{S}$. Agricultural Department. Sherman's Chemistry of Foods and other authoritative sources. 


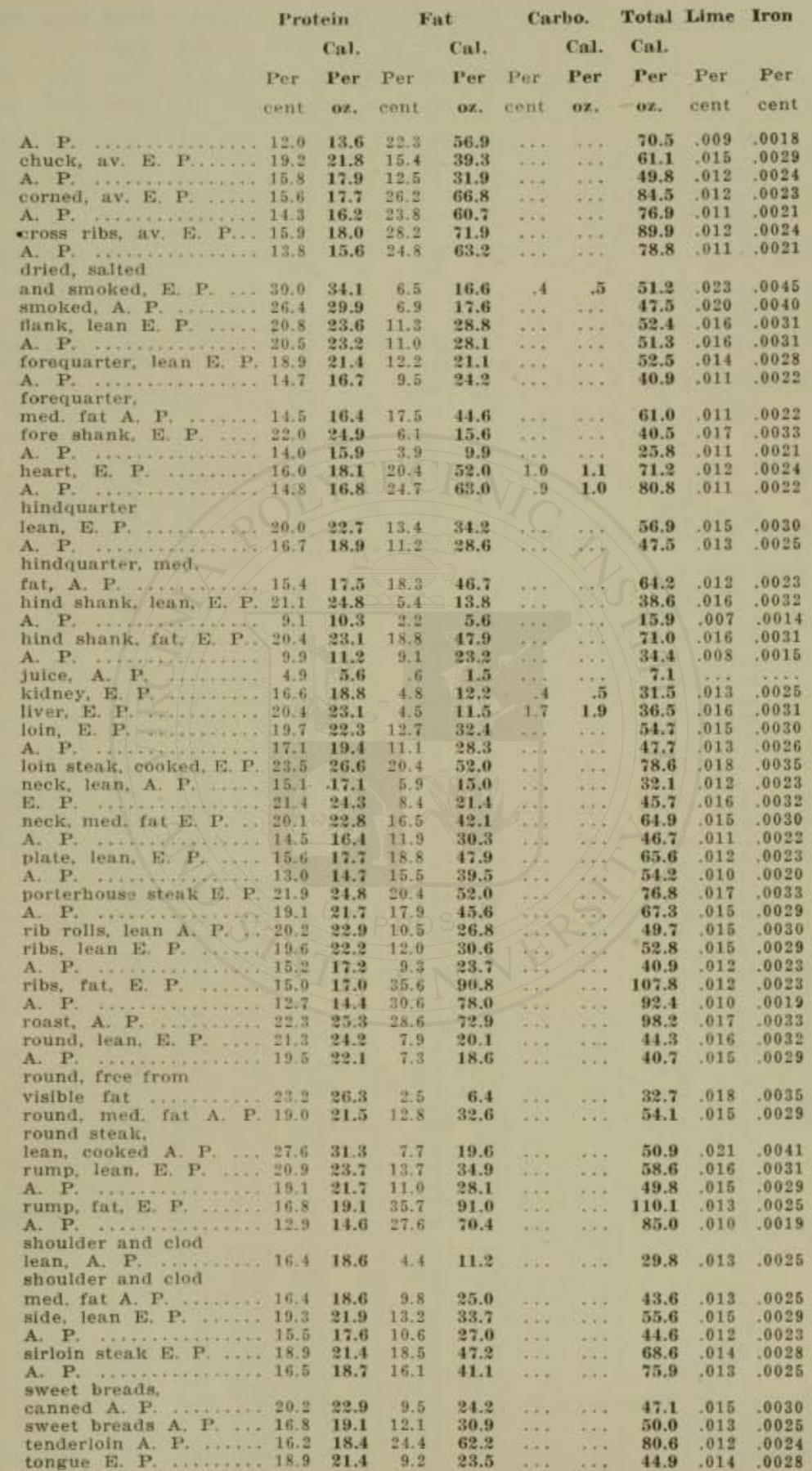




\begin{tabular}{|c|c|c|c|c|c|c|c|c|c|}
\hline & \multicolumn{2}{|c|}{ Protein } & \multicolumn{2}{|c|}{ Fat } & \multicolumn{2}{|c|}{ Carbo. } & \multirow{2}{*}{$\begin{array}{c}\text { Total } \\
\text { Cal. }\end{array}$} & Lime & \multirow[t]{2}{*}{ Iron } \\
\hline & & Cal. & & Cul. & & Cat. & & & \\
\hline & Per & Fer & Per & Per & Per & Fer & Per & Per & Per \\
\hline & & oz. & cent & & & & & & cent \\
\hline A. $\mathbf{P}, \ldots \ldots \ldots \ldots$ & 14.1 & 16.0 & 6.7 & 17.1 & & & 33.1 & .011 & .0021 \\
\hline leets, cooked E. P. ....... & 2.3 & 2,6 & .1 & .3 & 7.4 & 8.4 & 11.3 & & \\
\hline fresh E. P. & 1.6 & 1.8 & 1 &. .3 & 9.7 & 11.0 & 13.1 & .041 & .0006 \\
\hline Blackberries A. P. & 1.3 & 1.5 & 1.0 & 2.6 & 16.9 & 12.4 & 16.5 & .024 & .0006 \\
\hline canned A, P. & .8 & .9 & 21 & 5.4 & 56.4 & 64.0 & 70.3 & $\cdots$ & $\cdots \cdot$ \\
\hline juice (eanned) & & & & & 26.6 & 30.3 & 30.3 & & \\
\hline Blackfish E. P. ... & 18.7 & 21.2 & 1.3 & 3.8 & $\ldots$ & $\cdots$ & 24.5 & .028 & .0007 \\
\hline A. P. $\ldots \ldots$ & 7.4 & 8.4 & .7 & 1.8 & & & 10.2 & .011 & .0003 \\
\hline Blueberries, fresh & .6 & .7 & .6 & 1.5 & 16.6 & 18.8 & 21.0 & .028 & .0009 \\
\hline canned A, P. & .6 & .7 & .6 & 1.5 & 12.8 & 14.5 & 16.7 & $\cdots$ & $\ldots$ \\
\hline 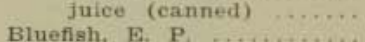 & & & & & 16.6 & 18.9 & & & \\
\hline $\begin{array}{c}\text { Bluefish, } \text { E. P. } \\
\text { A, P. }\end{array}$ & $\begin{array}{l}19.4 \\
10.0\end{array}$ & $\begin{array}{l}22.0 \\
11.3\end{array}$ & $\begin{array}{r}1.2 \\
.6\end{array}$ & $\begin{array}{l}3.1 \\
1.5\end{array}$ & $\ldots$ & $\cdots$ & $\begin{array}{l}25.1 \\
12.8\end{array}$ & $\begin{array}{l}.029 \\
.015\end{array}$ & $\begin{array}{l}.0008 \\
.0004\end{array}$ \\
\hline Boulllon, canned, A. P..... & 2.2 & 2.5 & 1 & .3 & .2 & .2 & 3.0 & .050 & .0012 \\
\hline $\begin{array}{l}\text { cooked, A. P. } \\
\text { soup, eream of, canned. }\end{array}$ & 2.1 & 2.4 & 3.3 & 8.4 & 2.2 & 2.5 & 13.3 & .050 & .0012 \\
\hline A. $\mathbf{P}, \quad \ldots \ldots \ldots \ldots \ldots$ & 2.5 & 2.8 & $-3,2$ & 8.2 & 5.5 & 6.2 & 17.2 & .040 & .0010 \\
\hline Branola .......... & 12.0 & 13.6 & 2.1 & 5.4 & 68.0 & 77.7 & 96.7 & .097 & .0054 \\
\hline Bran, corn & 9.7 & 11.0 & 5.7 & 14.5 & 62.4 & 70.8 & 96.3 & & \\
\hline rys & 12.2 & 13.8 & 2.8 & 2.1 & 56.6 & 64.2 & 85.1 & .250 & .0140 \\
\hline wheat $\ldots$ & 12.5 & 14.2 & 3.0 & 3.7 & 41.6 & 17.2 & 69.1 & .168 & .0181 \\
\hline Brazil nuts, E. P. & 17.0 & 19.3 & 66.3 & 170.3 & 7.0 & 7.9 & 197.5 & & \\
\hline A. P. $\ldots \ldots$ & 8.6 & 9.8 & 33.7 & 85.9 & 3.5 & 4.0 & 99.7 & $\ldots$ & \\
\hline read, biscui & & & & & & & & & \\
\hline made, A. & 8.7 & 9.9 & 2.6 & 6.6 & 55.3 & 62.7 & 79.2 & .130 & .0012 \\
\hline biscuit, soda, A. P. . . & 3.3 & $10 . \overline{5}$ & 13.7 & 34.9 & 52.6 & 59.6 & 94.5 & .150 & .0014 \\
\hline biseuit, white, A. P. .. & 8.0 & 9.1 & 1.4 & 3.6 & 54.3 & 61.6 & & .130 & .0012 \\
\hline bran,$\ldots \ldots \ldots \ldots . . . .$. & 8.8 & 10.0 & 2.4 & 6.0 & 54.6 & 62.0 & 78.0 & & \\
\hline brown, $\mathrm{A}-\mathrm{P} \quad \ldots \ldots \ldots$ & 5.4 & 6.1 & 1.8 & 4.6 & 47,1 & 53.4 & & .180 & .0030 \\
\hline corn (johnnyeake) A.P. & 7.9 & 9.0 & 4.7 & 12.0 & 46.3 & 52.5 & 73.5 & .013 & .0009 \\
\hline & 26.0 & 29.0 & 2. 0 & 5,0 & 29.0 & 32.0 & 68.0 & & \\
\hline tham. A. P & 8.9 & 10.1 & 1.8 & 4.6 & 52.1 & 59.1 & 73.8 & .070 & .0025 \\
\hline milk, A. P. , & 9.6 & 10.9 & 1.4 & 3.6 & 51.1 & 57.9 & 72.4 & & \\
\hline rolls, French, A. P. .. & 8.5 & 9.6 & 2.5 & 6.4 & 55.7 & 63.2 & 79.2 & .024 & .0010 \\
\hline rolls. plain, A. P. ... & 9.7 & 11.0 & 4.2 & 10.7 & 59.9 & 67.9 & 89.6 & .028 & .0010 \\
\hline rolls, vienna, A. P. . . . & 8.5 & 9.6 & 2.8 & 5.6 & 56.5 & 64.1 & 79 & .036 & .0010 \\
\hline rolls, water, A. P. ... & 9.0 & 10.2 & 3.0 & 7.7 & 54.2 & 61.5 & 79.4 & .036 & .0010 \\
\hline rye, A. P. & 9.0 & 10.2 & -6 & 1.5 & 53.2 & 60.3 & & .034 & .0016 \\
\hline rye (blacis) A. P. & 9.5 & 10.9 & -6 & 1.5 & 48.9 & 55,5 & 67.9 & .036 & .0018 \\
\hline rye (whole) A. P. & 11.9 & 13.5 & .6 & 1.5 & 35,9 & 0.7 & 7 & .040 & .0022 \\
\hline usted & 11.5 & 13.0 & 1.6 & 4.1 & 61.2 & 69.4 & & .036 & .0011 \\
\hline wh & 9.7 & 11.0 & .9 & 2.3 & 49.7 & 6.4 & .7 & .070 & .0016 \\
\hline Lemade & 9.1 & 10.3 & 1.6 & 4.0 & 53.3 & 60 & & & \\
\hline ne: & 9.4 & 10.7 & 1. 2 & 3.1 & 54.1 & 61.3 & & .036 & .0 \\
\hline zwieback & 9.8 & 11.1 & 9.9 & 95.2 & 73.5 & 83.3 & 119.6 & .040 & .0011 \\
\hline romose, nut .. & 18,3 & 20.8 & 27.5 & 70.1 & 52.1 & 42.1 & 133.0 & .038 & .0021 \\
\hline & & & & & & & & & \\
\hline & 1.5 & 1.7 & \pm & 3 & 3.4 & 3.9 & 5.9 & .038 & .0011 \\
\hline Buckwheat .... & 10.8 & 12.2 & 2.5 & 6.4 & 62.2 & 70.5 & 89.1 & .036 & \\
\hline flour ..... & 6.4 & & 1. 2 & 3.1 & 77.9 & 88.3 & 98.7 & .055 & .0012 \\
\hline Bung, hoteross & 7.9 & 9.0 & 4.8 & 19.2 & 49.7 & 56.4 & 77.6 & .024 & .0007 \\
\hline er, A. P, .. & 1.0 & 1.1 & 85.0 & 216.8 & & & 217 & .021 & .0002 \\
\hline Buttermilk, A. P. & 3.0 & 3.4 & .5 & 1.3 & 4.8 & 5.4 & 10.1 & .147 & .0003 \\
\hline Butternuts, E. P. & 27.9 & 31.6 & 61.2 & 156.1 & 3.5 & 4.0 & 191.7 & $\ldots$ & .... \\
\hline A. P. $\quad \ldots \ldots$ & 3.8 & 4.3 & 8.3 & 21.2 & 5 & .6 & 26.1 & 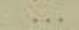 & \\
\hline Cabbage, E. P. ... & 1.6 & 1.8 & .3 & .8 & 5., 6 & 6.4 & 9.0 & .063 & .0011 \\
\hline & 1,2 & 1.4 & & & 1. & 1.6 & 3.0 & $\ldots$ & .... \\
\hline A. P. . & 4.1 & 4.6 & .6 & 1.5 & 6.2 & 7.0 & 13.1 & & \\
\hline sprouts, E, P. . . . . . & 4.7 & 5.3 & 1.1 & 2.8 & 4.3 & 4.9 & 18.3 & .148 & .0018 \\
\hline & & & & & & & & & \\
\hline A. 1 . & 6.2 & & 8.1 & 20.7 & & & & & \\
\hline$P$ & 7.1 & 8.1 & 7.5 & & 63 & 71.7 & & $\ldots$ & ... \\
\hline cake, A. P. . & 5.9 & 6.7 & 9.0 & 23.0 & 68.5 & 77.7 & 107.4 & $\ldots$ & .... \\
\hline drop. A, P. ......... & 7.6 & 8.6 & 14.7 & 37.5 & 60.3 & 68.4 & 114.5 & & \\
\hline frosted. A. P. & 5.9 & 6.7 & 9.0 & 23.0 & 64.8 & 73.5 & 103.2 & $\ldots$ & $\ldots$. \\
\hline fruit, A. P. ........ & 5.9 & 6.7 & 10.9 & 27.8 & 64.1 & 79.7 & 107.2 & $\cdots$ & ..... \\
\hline ging rbread, A P. P... & 5.8 & 6.6 & 9.0 & 23.0 & 63.5 & 72,0 & 101.6 & ... & $\cdots$ \\
\hline A P $\quad \ldots \ldots$ & 6.3 & 7.1 & 10.7 & 27.3 & 65.9 & 74.7 & 109.1 & $\cdots$ & $\ldots$ \\
\hline cookies, molasses, A. P. & 7.2 & 8.2 & 8,7 & 29.2 & 75.7 & 85.8 & 116.2 & $\cdots$ & ... \\
\hline gingersnaps, A. P. ... & 6.5 & 7.4 & 8.6 & 21.9 & 76.0 & 86.2 & 115.5 & ... & $\ldots$. \\
\hline s foot jelly, A. P. ........ & 4.3 & 4.9 & $\ldots$ & $\ldots$ & 17.4 & 19.7 & 24.6 & & \\
\hline & .6 & .7 & & .... & 9.3 & 10.5 & 11.2 & .024 & .0003 \\
\hline
\end{tabular}




\begin{tabular}{|c|c|c|c|c|c|c|c|c|c|}
\hline & \multicolumn{2}{|c|}{ Protein } & \multicolumn{2}{|c|}{ Fat } & \multicolumn{2}{|c|}{ Carbo. } & \multirow{2}{*}{$\begin{array}{c}\text { Total } \\
\text { Cal. }\end{array}$} & \multirow[t]{2}{*}{ Lime } & \multirow[t]{2}{*}{ Iron } \\
\hline & & Cal. & & Cal. & & Cal. & & & \\
\hline & Per & Per & Per & Per & Per & Per & Per & Per & Per \\
\hline & cent & oz. & cent & ox. & cent & oz. & oz. & cent & cent \\
\hline Capon, cooked. E. P. ....... & 27.0 & 30.6 & 11.5 & 29.3 & & & 59.9 & .020 & .0040 \\
\hline Carrots, fresh, E, P, ...... & 1.1 & 1.2 & & 1.0 & 9.3 & 10.5 & 12.7 & .078 & .0006 \\
\hline Cashew nut, E. P. .......... & 9.7 & 11.0 & 47.1 & 120.1 & 5.9 & 6.7 & 137.8 & $\cdots$ & $\ldots$ \\
\hline Cassava, dri d, A, P. ..... & 3.8 & 3.2 & .5 & 1.3 & 84.1 & 95.4 & 99.9 & $\cdots$ & $\cdots$ \\
\hline sweet. A. P. ........... & 1.1 & 1.2 & .2 & .5 & 30.2 & 34.2 & 35.9 & $\because$ & $\ldots$ \\
\hline 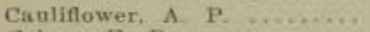 & 1.8 & 9.0 & -5 & 1.3 & 4.7 & 5.3 & 8.6 & .172 & .0006 \\
\hline $\begin{array}{l}\text { Celery, E. P. } \\
\text { soun. carned. }\end{array}$ & 1,1 & 1.2 & .1 & .3 & 3,3 & 3.7 & 5.2 & .109 & .0005 \\
\hline croam of, A P. $\ldots \ldots$ & 2.1 & 2.4 & 2.8 & 7.1 & 5.0 & 5.7 & 15.2 & $\cdots$ & $\cdots \cdots$ \\
\hline Cerealine, A. P. ........... & 9.6 & 10.9 & 1.1 & 2.8 & 78.3 & 88.8 & 102.5 & .015 & .0011 \\
\hline $\begin{array}{l}\text { Chard, Swiss ................... } \\
\text { Cheese, American }\end{array}$ & 3,2 & 3.6 & .6 & 1.5 & 5.0 & 5.7 & 10.8 & .210 & .0025 \\
\hline pale, A. P .......... & 28.8 & 32.7 & 35.9 & 91.5 & 3 & .3 & 121.5 & 1.303 & .0013 \\
\hline American Red, A. P... & 29.6 & 33.6 & 38.3 & 97.7 & & & 131.3 & & $\ldots$ \\
\hline cottage, A P. ........ & 20.9 & 93.7 & 1.0 & 2.6 & 4.3 & 4.9 & 31.2 & .300 & $\ldots \ldots$ \\
\hline full cream, A. P. ..... & 25,9 & 99,4 & 33,7 & 85.9 & 2.4 & 2.7 & 118.0 & $\cdots$ & $\cdots$ \\
\hline cheddar, A. P, . . . . & 27,7 & 31.4 & 36.8 & 93.8 & 4.1 & 4.6 & 129.8 & $\ldots$ & $\ldots \ldots$ \\
\hline Fromage de Brie, A. P. & 15.9 & 18.0 & 21.0 & 53.6 & 1.4 & 1.6 & 73.2 & $\ldots$ & $\cdots$ \\
\hline limburger, A, P. ..... & 23.0 & 26.1 & 29.4 & 75.0 & 4 & .5 & 101.6 & $\ldots$ & $\cdots$ \\
\hline Neuchatel, A. P. ...... & 18.7 & 91.2 & 27.4 & 69.9 & 1.5 & 1.7 & 99.8 & $\therefore$ & $\ldots$ \\
\hline pineapple, A. P $\ldots \ldots$ & 29.9 & $3: 9$ & 38.9 & 99.2 & 2.6 & 2.9 & 136.0 & $\cdots$ & $\cdots$ \\
\hline $\begin{array}{l}\text { roquefort, A. P } \\
\text { partly skimmed milik: }\end{array}$ & 22.6 & 95.6 & 29.5 & 75.9 & 1.8 & 3.0 & 102.8 & $\ldots$ & $\ldots$ \\
\hline A) P. $\quad \ldots \ldots \ldots$ & 25.4 & 98,8 & 29.5 & 75.9 & 3.6 & 4.1 & 108.1 & $\cdots$ & $\cdots$ \\
\hline sirimmed mille, A. P... & 31.5 & 35.7 & 16.4 & 41.8 & 2.2 & 2.5 & 80.8 & $\ldots$ & $\ldots \ldots$ \\
\hline Swiss, A. P. $\quad \ldots \ldots \ldots \ldots$ & 27.6 & 31.3 & 34.3 & 89.0 & 1.3 & 1.5 & 191.8 & $\cdots$ & $\cdots$ \\
\hline Cherries, E, P. A......... & 1.0 & 1.1 & .8 & 9.0 & 16.7 & 18.9 & 99.0 & 027 & .0004 \\
\hline A. P. . & .9 & 1.0 & 8 & 9.0 & 15.9 & 8.0 & 21.0 & $\ldots$ & $\ldots$ \\
\hline canned, $A, P, \quad \ldots \ldots$. & 1.1 & 1.2 & 1 & .3 & 21.1 & 23.9 & 95.4 & $\ldots$ & $\cdots$ \\
\hline jelly, A, P & 1.1 & 1.2 & ... & & 77.2 & 87.5 & 88.7 & $\cdots$ & \\
\hline juice, A. P. .... & 8 & 3 & -16 & & 4.6 & 5.9 & 5.5 & $\therefore$ & .0003 \\
\hline Chestnuts, fresh, F: $\mathrm{P}$. & 6.2 & 7.0 & 5.4 & 13.8 & 42,1 & 47.7 & 68.5 & .048 & .0007 \\
\hline A. P. $\ldots \ldots \ldots \ldots$ & 5.2 & 5.9 & 4.5 & 1.5 & 95.4 & 40.1 & 57.5 & $\ldots$ & $\ldots$ \\
\hline 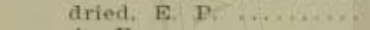 & 10.7 & 19.1 & 7.0 & 17 & 74.2 & 84.1 & 114.1 & $\ldots$ & $\cdots$ \\
\hline A. $\mathbf{P}, \quad \ldots \ldots \ldots \ldots \ldots$ & 8.1 & 9.2 & 5.3 & 13.5 & 56.4 & 61.0 & 86.7 & $\cdots$ & $\ldots$ \\
\hline Chicken, broflers, E. P. & 21,5 & 94.4 & 2.5 & 6.4 & & & 30.8 & 016 & .0032 \\
\hline fricasseed, E P $\ldots . .$. & 17.6 & 20.0 & 11.5 & 99.3 & 2.4 & 9.7 & 52.0 & .013 & .0026 \\
\hline gumbo, canned, A.P... & 3.8 & 4.3 & .9 & 23 & 4.7 & 5,3 & 11.9 & & $\cdots$ \\
\hline sandwich, canned, $\lambda$. P. & 20.8 & 23.6 & 30.0 & 76.5 & & & 100.1 & .016 & .0031 \\
\hline soup canned. A. $P, \ldots$. & 8.6 & 4.1 & 1 & 3 & 1.5 & 1.7 & 6.1 & $+\cdots$ & $\cdots$ \\
\hline soup, homemaite, E. P. & 10.5 & 11.9 & .8 & 9.0 & $3 / 4$ & 9.7 & 16.6 & $\ldots$ & $\ldots$ \\
\hline Chinese cabbage ............... & 1.2 & 1.1 & & & 1.4 & 1.6 & 3.0 & $\ldots$ & $\ldots$ \\
\hline Chinquapin, E. P. ........ & 10.9 & 19.4 & 7 & 1.8 & 73.8 & 83.7 & 97.9 & $\ldots 2$ & \\
\hline Chocolate,$\ldots \ldots \ldots \ldots . . .$. & 12.9 & 14.6 & 48.7 & $191 ?$ & 30,3 & 34.4 & 173.2 & .129 & -0.0 .927 \\
\hline $\begin{array}{l}\text { Clams, long, fresh, E. P. } \\
\text { Clam chowd } r \text {. }\end{array}$ & 8.6 & 9.8 & 1.0 & 9.6 & 2.0 & 2.3 & 14.7 & .174 & $+\cdots$ \\
\hline homemade, A. P. . & 18 & 90 & .8 & 9.0 & 6.7 & 7.6 & 11.6 & & \\
\hline round, canned, A. P... & 10,5 & 11.9 & .8 & 2.0 & 3,0 & 3.4 & 17.3 & .148 & $\cdots$ \\
\hline 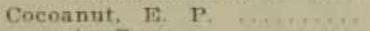 & 5.7 & 6.5 & 50.6 & 199.0 & 27.9 & 31.6 & 167.1 &, 034 & , n. \\
\hline A. $P . \ldots \ldots \ldots \ldots$ & 3.9 & 8.3 & 25.9 & & 14,3 & & & & $\ldots$ \\
\hline preparea, A. P. ... & 6.8 & 7.1 & 57.4 & 146.4 & 31.5 & 35.7 & 189.2 & .083 & $\cdots$ \\
\hline Cod, dressed, A, P. ... & 11.1 & 12.6 & .2 & $\sqrt{5}$ & $\ldots$ & $\ldots$ & 13.1 & .015 & .0004 \\
\hline salt, $\mathbf{E}, \mathbf{P}, \ldots \ldots \ldots$ & 25,4 & 98.8 & .3 & .8 & & ... & 29.6 & .038 & .0010 \\
\hline salt (boneless) E. P. ... & 27.3 & 31.0 & .3 & .8 & $\cdots$ & $\cdots$ & 31.8 & 041 & .0011 \\
\hline whole, E. P, ......... & 16.5 & 18.7 & .4 & 1.0 & $\cdots$ & $\ldots$ & 19.7 & .015 & .0004 \\
\hline Collards, E, P., .......... & 4.5 & 5.1 & .6 & 1.5 & 6.3 & 7.1 & 13.7 & $\cdots$ & ..... \\
\hline Consomme, canned, A. P... & 2.5 & 9.8 & & $\ldots$ & 4 & .5 & 3.3 & $\cdots$ & $\cdots$ \\
\hline Corn, green, sweet, E. P.... & 3.1 & 3.5 & 1.1 & 9.8 & 19.7 & 92.3 & 98.6 & .008 & .0008 \\
\hline $\begin{array}{l}\text { green, canned, A. P. . . } \\
\text { soup, cream of, }\end{array}$ & 2.8 & 3.2 & 1.2 & 3.1 & 19.0 & 91.5 & 27.8 & $\cdots$ & $\ldots$. \\
\hline canned, A, P..... & 2.3 & 9.8 & 1.9 & 1.8 & 7.8 & 8.8 & & & \\
\hline bran $\ldots \ldots \ldots \ldots \ldots$ & 9.7 & 11.0 & 5.7 & 14.5 & 62.4 & 70.8 & 96.3 & & \\
\hline mature $\ldots \ldots \ldots \ldots$ & 10.1 & 11.5 & 5.0 & 12.8 & 70,9 & 80.4 & 104.7 & .030 & .0029 \\
\hline flour $\ldots \ldots \ldots+\ldots$ & 7.1 & 8.1 & 1.3 & 3.3 & 78.4 & 88.9 & 0.3 & & \\
\hline meal, granular ... & 9.2 & 10.4 & 1.9 & 4.8 & 75.4 & 85.5 & 100.7 & .025 & .0009 \\
\hline unbolted $\quad \ldots \ldots \ldots \ldots+\ldots$ & 8.4 & 9.5 & 4.7 & 18,0 & 74.0 & $8: 3.9$ & 105.4 & ..e & $\ldots$ \\
\hline oil $\quad \ldots \ldots \ldots \ldots \ldots \ldots \ldots$ & & & 100.0 & 955.0 & & & 255.0 & $\ldots$ & $\ldots$ \\
\hline parehed ........... & 11.5 & 13.0 & 8.4 & 21.4 & 72.3 & 89.0 & 164.0 & & \\
\hline soft $\ldots \ldots \ldots+\ldots \ldots$ & 7.4 & 8.4 & 3.8 & 9.7 & 56.0 & 63.5 & 81.6 & & \\
\hline sweet, mature ....... & 11.5 & 13.0 & 7.9 & 20.1 & 67.2 & 76.2 & 109.3 & & .0008 \\
\hline syrup $\ldots \ldots \ldots \ldots \ldots \ldots$ & $\ldots$ & She & & & 83.5 & 94.7 & 94.7 & & \\
\hline
\end{tabular}




\begin{tabular}{|c|c|c|c|c|c|c|c|c|}
\hline Pre & ein & & & & bo. & Total & Lime & Iron \\
\hline & Cal. & & $\mathrm{Ca}$ & & Cal & Cal. & & \\
\hline er & $\begin{array}{c}\text { Per } \\
\text { oz. }\end{array}$ & $\begin{array}{l}\text { Per } \\
\text { cent }\end{array}$ & $\begin{array}{c}\mathrm{Pe} \\
\alpha z\end{array}$ & $\begin{array}{l}\text { Per } \\
\text { cent }\end{array}$ & $\begin{array}{c}\text { Per } \\
\text { oz. }\end{array}$ & $\begin{array}{c}\text { Per } \\
\text { oz. }\end{array}$ & $\begin{array}{l}\text { Per } \\
\text { cent }\end{array}$ & $\begin{array}{l}\text { Per } \\
\text { cent }\end{array}$ \\
\hline
\end{tabular}

Cornstarch

Cettolene

Cottonse ........................

$\cdots 100.0$

255.0

2100.0

90.0

s.

cent

$\begin{array}{lllll}\text { Cow peas, Ary } & \text { A. . . . . . . . . } & 44.1 & \mathbf{5 0 . 0} & 9.1 \\ 21.4 & \mathbf{9 4 . 3} & 1.4\end{array}$

Crabs, hardshelt

Crackers, Boston, A. P. ... 11.0 butter, A. P. ....... 9.6

$\begin{array}{rrr}\text { cream, A. P. } & \ldots \ldots \ldots & 9.7 \\ \text { egg, A. P. } & \end{array}$

12.6

flatbread, A. P. $\ldots \ldots, 14.9$
graham, A. P. $\quad \ldots \ldots .010 .8$

oatmeal, A. P . . . . 11.8
oyster, A, P

oyster, A. P. $\ldots \ldots \ldots \ldots 1.3$
saltines, A. P. $\ldots \ldots \ldots .10 .6$

soda, A P

water, A. P

10.6
9.8

Cranberries, A. P

Cream

Cucumbers, E, P

Currants A $P$, fresh 1. Zante, dried, A. P. ... 2.

Dandelion greens .......... 2.

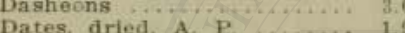
dried, E. P . . . . . .

Doughnuts

Eels, E. P.

Eggs, uncooked, A P .... uncooked, E. P. ..... 13 bolled. E. P.

boiled whites, E. P Is boiled yolks, E. P. ... 15.

$18.8 \quad 2.0$

$17.9 \quad 1.5$

$12.5 \quad 8.5$

10.910 .1

$\begin{array}{ll}11.0 & 12.1 \\ 14.3 & 14.0\end{array}$

$16.9 \quad .5$

$11.3 \quad 9.4$

$13.4 \quad 11.1$

12.810 .5

$12.0 \quad 12.7$

11.1 9.1

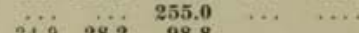

$\begin{array}{llllll}23.2 & 25.0 & 28.4 & 101.6 & -371 & \ldots\end{array}$

$\begin{array}{llllll}3.6 & 60.8 & 69.1 & 97.0 & .150 & \ldots\end{array}$

$\begin{array}{llllll}5.1 & 1.2 & 1.4 & 25.3 & \ldots & \ldots \\ 3.8 & .7 & .8 & 9.5 & & \end{array}$

$\begin{array}{lrrrrr}21.7 & 71.1 & 80.6 & \begin{array}{lll}114.8 \\ 2.8\end{array} & \cdots & \cdots\end{array}$

$\begin{array}{llllll}25.8 & 71.6 & 81.2 & 117.9 & \cdots & \cdots\end{array}$

$\begin{array}{llllll}30.9 & 69.7 & 79.0 & 120.9 & \ldots & \ldots\end{array}$

$\begin{array}{llllll}35.7 & 66.6 & 75.5 & 125.5 & \ldots & \ldots\end{array}$

$\begin{array}{rrrrrr}1.3 & 73.6 & \mathbf{8 3 . 5} & \mathbf{1 0 1 . 7} & .027 & .0015 \\ 0.0 & 73.8 & \mathbf{8 3 . 7} & \mathbf{1 1 9 . 0} & 055 & .0057\end{array}$

$\begin{array}{lllllll}28.3 & 69.0 & 78.2 & 119.9 & 090 & 0027\end{array}$

$\begin{array}{lllllll}26.8 & 70.5 & 79.9 & 119.5 & .032 & .0017\end{array}$

$\begin{array}{lllllll}32.4 & 68.5 & 77.7 & \mathbf{1 2 9 . 1} & .030 & .0016\end{array}$

$\begin{array}{llllll}23.2 & 73.1 & 83.9 & 117.2 & 028 & 0015\end{array}$

$\begin{array}{llllll}2.2 .4 & 71.9 & 81.5 & 116.0 & .030 & 0016\end{array}$

$\begin{array}{llllll}1.5 & 9.9 & 11.2 & 13.2 & .025 & .0006\end{array}$

$\begin{array}{rrrrrrrr}2.8 & 18.5 & 47.2 & 4.5 & 5.1 & 55.1 & .120 & .0002 \\ .9 & & & 5.3 & 3.5 & 4.9 & 022 & 0002\end{array}$

$\begin{array}{lllllll}1.7 & 12.8 & 14.5 & 16.2 & .036 & 0005\end{array}$

$\begin{array}{llllllll}8.7 & 1.7 & 4.3 & 74.2 & \mathbf{8 4 . 1} & \mathbf{9 1 . 1} & \mathbf{1 1 5} & .0025\end{array}$

$\begin{array}{lllllllll}9.7 & 1.0 & 2.6 & 10.6 & 12.0 & 17.3 & 147 & .0027\end{array}$

$\begin{array}{lllllllll}3.4 & 2 & .5 & 29.8 & 33.8 & 37.7 & \ldots & \ldots\end{array}$

$\begin{array}{llllllll}\mathbf{2 . 4} & \mathbf{2 . 8} & \mathbf{7 . 1} & \mathbf{7 8 . 4} & \mathbf{8 8 . 9} & \mathbf{9 8 . 4} & .100 & .0036\end{array}$

$\begin{array}{llllllll}7.6 & 21.0 & \mathbf{5 3 . 6} & 53.1 & \mathbf{6 0 . 2} & \mathbf{1 2 1 . 4} & \ldots 13 & \ldots \ldots\end{array}$

\begin{tabular}{lllllllll}
1.1 & 9.1 & 93.2 & $\cdots$ & $\ldots$ & 44.3 & .013 & 0003 \\
\hline & 9.3 & 23.7 & & & 37.2 & 094 & 0030
\end{tabular}

$\begin{array}{rrrrrrrrr}13.5 & 9.3 & 23.7 & \ldots & \ldots & 37.2 & .094 & .0030 \\ 15.2 & 10.5 & \mathbf{9 6 . 8} & \ldots & \ldots & \mathbf{4 2 . 0} & .094 & .0030\end{array}$

\begin{tabular}{ll|lllllll}
15.0 & 12.0 & 30.6 & $\ldots$ & $\ldots$ & $\mathbf{4 5 . 6}$ & .093 & .0030
\end{tabular}

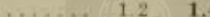

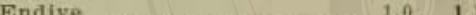

Farina

Fig, fresh, E P $\mathrm{P} \ldots \ldots \ldots \ldots$

dried, A. P.

12.5 Filbert, E. P. . . . . . . . .
A. P.

Flaxseed …............ $32.6 \quad 25.6 \quad 33.7$ E. P. 14.216.

Flour, gluten, pure ....... 81.292 .1 gluten, $40 \%$........ 42.5 48. buckwheat .......... $6.4 \quad 7.8$

rye

wheat, entire graham $13.8 \quad 15.6$

wheat, straight, grade $10.8 \quad \mathbf{1 2 . 2} \quad 1.1$ wheat, average,

high and medium ... $11.4 \quad 12.9 \quad 1.0$

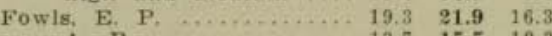
A. P. . . . . .

Frankforts, sausage, A. P. $19.6 \quad 28.218 .6$

Frog's legs, E. P. ......... 15.5 17.6 .

Garlie ................. $6.8 \quad 7.7$

Gelatin .............. 91.4103 .6

Gluten bread ........... $20.0 \quad 99.0 \quad 2.6$

Goose, young, E. P. ...... $16.3 \quad \mathbf{1 8 . 5} \quad 36.9$

Granola ................... $13.7 \quad 15.6 \quad 1.0$

Grapes, E. P. ........... $1.3 \quad 1.5 \quad 1.6$

A. P. $\quad \ldots \ldots \ldots \ldots \ldots \ldots+1.0 \quad 1.1 \quad 1.2$

butter, A. P. ........ 1.2 1.

ground, A. P. ...... $2.8,3$.

juice

- $\cdots$

$\begin{array}{lllll}\text { Grapefruit } & \ldots \ldots \ldots \ldots \ldots \ldots \ldots & .6 & .7 & .1 \\ \text { Greens, keet, cooked } & . . . \ldots & 2.2 & 2.5 & 3.4\end{array}$ danclelion, A. P. ..... $2.4 \quad 9.7 \quad 1.0$

$8+$.

30.6

$14.4 \quad 021 \quad 0001$

$\begin{array}{llllll}.8 & 5.1 & 5.8 & \mathbf{8 . 0} & .015 & .000\end{array}$

$\begin{array}{lllll}.3 & 2.0 & 3.4 & 4.8 & .146\end{array}$

$\begin{array}{lllllll}3.6 & 76.3 & 86.5 & 102.6 & .029 & .0008\end{array}$

$\begin{array}{rrrrr}18.8 \quad 21.3 \quad 23.0 & .074 & .000 \\ 8 \quad 74.2 & 81 . & 89.8 & 227 & 003\end{array}$

$\begin{array}{llllll}8 & 40.9 & 46.4 & 48.6 & 080 & .0009\end{array}$

$\begin{array}{lllllll}166.5 & 13.0 & 14.7 & 198.9 & \ldots & .0040\end{array}$

$\begin{array}{lllllll}79.8 & 6.2 & 7.0 & 95.3 & \ldots & \ldots\end{array}$

$\begin{array}{rrrrrr}85.9 & 23.9 & 96.3 & 137.8 & -238 & \ldots 2\end{array}$

$\begin{array}{llllll}1.5 & \ldots & \ldots & \mathbf{1 7 . 6} & .021 & .0006\end{array}$

$\begin{array}{llllll}1.5 & 16.6 & 18.8 & 112.4 & 234 & .0340\end{array}$

$\begin{array}{llllll}9.6 & 42.5 & 48.2 & 99.0 & .094 & .0136\end{array}$

$\begin{array}{lllllll}3.1 & 77.9 & \mathbf{8 8 . 3} & \mathbf{9 8 . 7} & .015 & .0012\end{array}$

$\begin{array}{llllll}2.3 & 78.7 & 89.2 & 99.2 & .025 & .0013\end{array}$

$\begin{array}{rrrrrr}1.8 & 71.9 & 81.5 & 101.9 & .050 & .0025\end{array}$

$\begin{array}{rrrrrr}9.8 & 74.8 & 84.8 & 99.8 & .028 & .0015\end{array}$

$\begin{array}{llllll}2.3 & 75.1 & \mathbf{8 5 . 2} & \mathbf{1 0 0 . 4} & .025 & .0015\end{array}$

$\begin{array}{lllllll}41.6 & \ldots & \ldots & 63.5 & .015 & .0029\end{array}$

$\begin{array}{lllllll}31.4 & \ldots & \ldots & 46.9 & .011 & .0021\end{array}$

$\begin{array}{lllllll}47.4 & 1.1 & 1.2 & 70.8 & .015 & .0029\end{array}$

$\begin{array}{lllllll}.5 & \ldots & \ldots & 18.1 & .027 & \ldots\end{array}$

$\begin{array}{rrrrrr}3 & 27.9 & 31.6 & 39.6 & \ldots & \ldots \\ 3 & & & 103.9 & \ldots & \end{array}$

$\begin{array}{lllllll}5.0 & 29.0 & 32.0 & 68.0 & \cdots & 0 & \end{array}$

$\begin{array}{rrrrrr}92.3 & \cdots & \ldots & 110.8 & .012 & .0024 \\ 3.6 & 73.8 & 88.8 & 109.0 & 054 & .0060\end{array}$

$\begin{array}{lllllll}4.1 & 19.2 & 91.8 & 97.4 & 024 & 0003\end{array}$

$\begin{array}{lllllll}3.1 & 14.4 & 16.3 & 90.5 & \ldots & \ldots\end{array}$

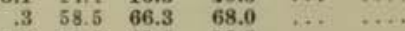

$\begin{array}{lllllll}1.5 & 60.5 & 68.6 & 73.3 & \text { 20. } & \ldots 0\end{array}$

$\begin{array}{llllll}.3 & 12.2 & 13.8 & 14.8 & 029 & 0003\end{array}$

$\begin{array}{rrrrrr}8.7 & 3.2 & 3.6 & 14.8 & \ldots . & \ldots\end{array}$

$\begin{array}{rrrrrr}2.6 & 10.6 & 12.0 & 17.3 & .147 & .0027\end{array}$ $\begin{array}{llllllllll}\text { turnip salad. A. P. } & 4.2 & 4.8 & 6 & 1.5 & 6.3 & \mathbf{7 . 1} & \mathbf{1 3 . 4} & 500 & \ldots\end{array}$ 


\begin{tabular}{|c|c|c|c|c|c|c|c|c|}
\hline Pro & tein & & & & bo. & Total & Lime & Iron \\
\hline & Cal. & & Cal. & & Cal. & Cal. & & \\
\hline $\begin{array}{l}\text { Per } \\
\text { cent }\end{array}$ & Per & $\begin{array}{l}\text { Per } \\
\text { cent }\end{array}$ & Per & Per & Per & Per & Per & \\
\hline
\end{tabular}

Haddock, E. P

$\begin{array}{lll}17.2 & 19.5\end{array}$

A. $P$.

\begin{tabular}{rr}
19.5 & \\
9.5 & \\
17.4 & 4. \\
21.1 & 5.2 \\
28.4 & 14. \\
28.1 & 14. \\
17.4 & 28.9 \\
15.3 & 25. \\
22.5 & 20.8 \\
19.8 & 18. \\
9.1 & 28. \\
\hline
\end{tabular}
steak, F, P 18.6

Ham, fresh lean, E. P. .... 25.0 A. P. $\ldots \ldots \ldots \ldots \ldots \ldots 24.8$ fresh med., E. P. ..... 15.3 A. P. $\ldots \ldots \ldots \ldots \ldots$. 13.5 A. P. . . . . . . . 17.5

Hazeinut $\ldots \ldots \ldots \ldots \ldots .8 .0$

Herring, E. P. $\quad \ldots \ldots \ldots \ldots \ldots$
$\mathbf{A} . \mathbf{P}$. smoked, E. P ...... 36.9

A. P

Hickory nuts, E. P. . . . 15.4

Hominy ny

5.8
8.3 pearled

Honey, A P

Huckleberries, fresh, $p$ canned .......... 6

Julienne, canned, A. P .... 2.7

Kaffir corn, A. P.

Kaoliang grain, A P $\quad 10.5$

Kohlrabi, E, P. A

Koumis

.....

Lamb breast, $\mathrm{E}$. $\mathrm{P}$.

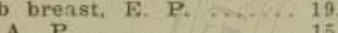

chops brolled $P$ P foreguarter, E. P. .... 18.3 A. P. … 14 hindquarter, $\mathrm{E}$. P. . 19.6 A. P. . . . . . . . . . 16.5 leg roast $\ldots \ldots \ldots \ldots \ldots 19.7$
side, $\mathrm{E} . \mathrm{P} . \ldots \ldots \ldots \ldots 17.6$ A. P. $\ldots \ldots \ldots \ldots \ldots 1.14$ loin, E, P ......

Lard, refined

Leeks, E. P

Lemons, E. A. P.

$$
\text { juice . }
$$

Lentils, A. P

Lettuce, $\underset{\mathbf{P}}{\mathbf{E}}$. P

Litchi nut, E. P

$$
\text { A. } \mathbf{P} \text {. }
$$

Linseed meal

Liver beef, $\mathrm{E}$. $\mathrm{P}$ veal, E. $P$

Lobster, fresh, $P$. whole, A. P.

Loganberry juice, eom

Macaroni cooked

19.

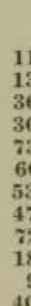

$\begin{array}{rrr}.8 & \ldots & \ldots \\ 11.5 & \ldots & \ldots \\ 13.3 & \ldots & \ldots \\ 36.7 & \ldots & \ldots \\ 36.2 & \ldots & \ldots \\ 73.7 & \ldots & \ldots \\ 66.0 & \ldots & \ldots \\ 53.0 & \ldots & \ldots \\ 47.2 & \ldots & \\ 72.7 & 11.5 & 13.0\end{array}$

$\begin{array}{lll}20.3 & .026 \quad .0007\end{array}$

$\begin{array}{lll}10.0 & .013 & .0003\end{array}$

$\begin{array}{lll}8.6 & .023 & .0006\end{array}$

$\begin{array}{lll}34.4 & 013 & 0003\end{array}$

$\begin{array}{lll}65.1 & 019 & .0038\end{array}$

$\begin{array}{llll}64.3 & .019 & .0037\end{array}$

$\begin{array}{llll}91.1 & .016 & .0023\end{array}$

$81.3 \quad 010 \quad .0020$

$\begin{array}{llll}75.5 & .015 & .0030\end{array}$

$12.7 \quad 3.9$

$41.8 \quad 15.8$

18.

$\begin{array}{llll}67.0 & .013 & .0026\end{array}$

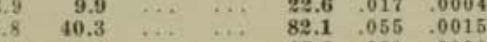

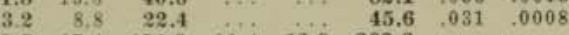

$\begin{array}{rrrrrrrr}6.6 & 67.4 & 171.9 & 11.4 & 12.9 & 202.3 & \ldots & \ldots\end{array}$

$\begin{array}{llllllll}9.4 & .6 & \mathbf{1 . 5} & 79.0 & \mathbf{8 9 . 6} & \mathbf{1 0 0 . 5} & .015 & 0009\end{array}$

$8.8 \quad 3.3$

.5

3.7

3.1
7.5

11.9

$\begin{array}{lll}.5 & 17.8\end{array}$

.6

$\begin{array}{llll}8.4 & 76.1 & 86.3\end{array}$

$\begin{array}{rrrrr}81.2 & 98.1 & 98.6 & .006 & .0007\end{array}$

$\begin{array}{llllll}1.5 & 16.6 & 18.8 & 21.0 & .028 & .0009\end{array}$

$\begin{array}{llllllll}.6 & 1.5 & 12.8 & 14.5 & 16.7 & \ldots & \ldots\end{array}$

$\begin{array}{llllllll}3.8 & 9.7 & 70.6 & \mathbf{8 0 . 1} & \mathbf{9 7 . 3} & \cdots & \cdots & \cdots\end{array}$

$\begin{array}{rrrrrr}11.0 & 71.9 & 81.5 & 104.4 & 0 & 0\end{array}$

$\begin{array}{rrrrrrrr}3.2 & 2.1 & 5.4 & 5.4 & \mathbf{6 . 1} & \mathbf{1 4 . 7} & 0.5 & 0029\end{array}$

$\begin{array}{llllllll}17.5 & 19.1 & 48.7 & \ldots & \ldots & 66.2 & .012 & .0023\end{array}$

$\begin{array}{lllllllll}24.6 & 39.9 & 76.2 & \ldots & \ldots & 100.8 & .017 & .0033\end{array}$

$\begin{array}{llllllllll}20.8 & 25.8 & 65.8 & \ldots & \ldots & \mathbf{8 6 . 6} & .014 & .0027\end{array}$

\begin{tabular}{l|l|l|l|l|l|l|l|l}
16.9 & 21.0 & 53.6 & $\ldots$ & $\ldots$ & 70.5 & .011 & .0022 \\
\hline
\end{tabular}

\begin{tabular}{llllllllll}
22.2 & 19.1 & 48.7 & $\ldots$ & $\ldots$ & 70.9 & .015 & .0029 \\
\hline
\end{tabular}

$\begin{array}{lllllllll}18.7 & 16.1 & 41.1 & \ldots & \ldots & 59.8 & .013 & .0025 \\ 22.3 & 12.7 & 92.4 & \ldots & \ldots & 54.7 & .015 & .0030\end{array}$

$\begin{array}{lllllllll}20.0 & 23.1 & 58.9 & \ldots & \ldots & 78.9 & .013 & .0026\end{array}$

$63.7-011 \quad 0021$

$\begin{array}{lll}93.4 & .014 & .0028\end{array}$

72.2

\begin{tabular}{lr|rrrrrr} 
& 100.0 & $\mathbf{2 5 5 . 0}$ & $\ldots$ & $\ldots$ & $\mathbf{2 5 5 . 0}$ & 0.3 & $\ldots$ \\
1.4 & .5 & $\mathbf{1 . 3}$ & 5.8 & $\mathbf{6 . 6}$ & $\mathbf{9 . 3}$ & .081 & $\ldots 00$ \\
1.1 & .7 & $\mathbf{1 . 8}$ & 8.5 & $\mathbf{9 . 6}$ & $\mathbf{1 2 . 5}$ & .050 & .0006
\end{tabular}

$\begin{array}{rrrrrr}1.8 & 8.5 & \mathbf{9 . 6} & \mathbf{1 2 . 5} & .050 & .0006 \\ 1.3 & 5.9 & \mathbf{6 . 7} & \mathbf{8 . 8} & \ldots & \ldots . .\end{array}$

$\begin{array}{rrrrrrrr}.8 & .5 & 1.3 & 5.9 & 6.7 & 8.8 & \ldots 3 & \ldots\end{array}$

\begin{tabular}{rrrrrrrrr}
25.7 & 29.1 & 1.0 & $\mathbf{9 . 6}$ & 59.2 & $\mathbf{6 7 . 1}$ & $\mathbf{9 8 . 8}$ & .150 & .0086 \\
\hline & 1.1 & & 8 & 9.9 & 3.3 & $\mathbf{5 . 5}$ & .060 & .0007
\end{tabular}

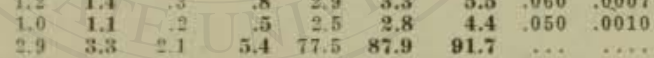

Mackerel, A. P. ......... $10.2 \quad \mathbf{1 1 . 6} 4.6$ E. $P$. $\ldots \ldots \ldots \ldots \ldots 18.7 \quad 21.2 \quad 7.1$ $\begin{array}{lllllllll}\text { Balt, E } & \text { P } & \ldots & 21.1 & 23.9 & 22.6\end{array}$

$\begin{array}{llllllr} & \text { Balt, A. P. } & \ldots \ldots \ldots \ldots \ldots & 16.3 & \mathbf{1 8 . 5} & 17.4 \\ \text { Malt } \ldots \ldots \ldots \ldots \ldots \ldots \ldots & 18.0 & \mathbf{2 0 . 4} & 3.7\end{array}$

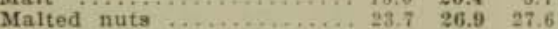

Mango, $F$.

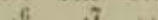

Maple syrup

Marmalade (orange peei)

Meat stew, homemade, A.P.

Milk

condensed, sweetened

cond. uns'veetened

skimmed

\begin{tabular}{rrr}
\multicolumn{6}{c}{} & \multicolumn{1}{c}{.7} & .1 \\
4.6 & 5.2 & 4.3 \\
3.3 & 3.7 & 4.0 \\
8.8 & $\mathbf{1 0 . 0}$ & 8.3 \\
9.6 & $\mathbf{1 0 . 9}$ & 9.3 \\
3.4 & 3.9 & .3
\end{tabular}

$\begin{array}{lll}.3 & 45.2 & 51.3\end{array}$

$\begin{array}{llll}7.4 & 36.3 & 41.2\end{array}$

53.5

$91.4 \quad 578$

$36.5 \quad .016 \quad .0031$

$\begin{array}{llllll}13.5 & 1.7 & 1.9 & 36.5 & .015 & .00315\end{array}$

$\begin{array}{rrrrrr}4.6 & 4 & .5 & 93.7 & \cdots & \cdots \\ 1.8 & .2 & .2 & 8.7 & \cdots & \cdots\end{array}$

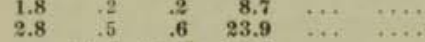

$\begin{array}{llll}30.9 & 35.0 & 35.9\end{array}$

$\begin{array}{llllll}2.3 & 74.1 & \mathbf{8 4 . 0} & \mathbf{1 0 1 . 5} & .031 & .0012\end{array}$

$\begin{array}{llll}3.8 & 15.8 & 17.9 & 25.1\end{array}$

$\begin{array}{llll}38.8 & 65.2 & 73.9 & 120.1\end{array}$

$\begin{array}{llllll}10.7 & \cdots & \cdots & 22.3 & .015 & .0004\end{array}$

$\begin{array}{lllllll}18.1 & \ldots & \ldots & 39.3 & .028 & .0007\end{array}$

$\begin{array}{llllll}57.6 & \ldots & \ldots & \mathbf{8 1 . 5} & 032 & 0008\end{array}$

$\begin{array}{lllllll}44.4 & \ldots & \ldots & 62.9 & .025 & .0007\end{array}$

$\begin{array}{lllllll}\mathbf{9 . 4} & 60.6 & \mathbf{6 8 . 7} & \mathbf{9 8 . 5} & .060 & .0040\end{array}$

$\begin{array}{llllll}71.2 & 43.9 & 49.9 & 148.0 & \ldots & \ldots\end{array}$

$\begin{array}{llllll}\ldots & 10.0 & \mathbf{1 1 . 3} & \mathbf{1 2 . 0} & .030 & \ldots \\ \ldots & 71.7 & \mathbf{8 1 . 3} & \mathbf{8 1 . 3} & .150 & .0030\end{array}$

$\begin{array}{rrrrrr}.3 & 84.5 & 95.8 & 96.8 & \ldots & \ldots \\ 11.0 & 5.5 & 6.2 & 99.4 & & \ldots\end{array}$

$\begin{array}{llllll}10.2 & 5.0 & 5.7 & 19.6 & .168 & .0002\end{array}$

$\begin{array}{lllllll}21.2 & 54.1 & 61.3 & 92.5 & .420 & .0006\end{array}$

$\begin{array}{lllllll}23.7 & 11.2 & 12.7 & 47.3 & .504 & .0007\end{array}$

$\begin{array}{llllll}.8 & 5.1 & 5.8 & 10.5 & .171 & 0002\end{array}$ 


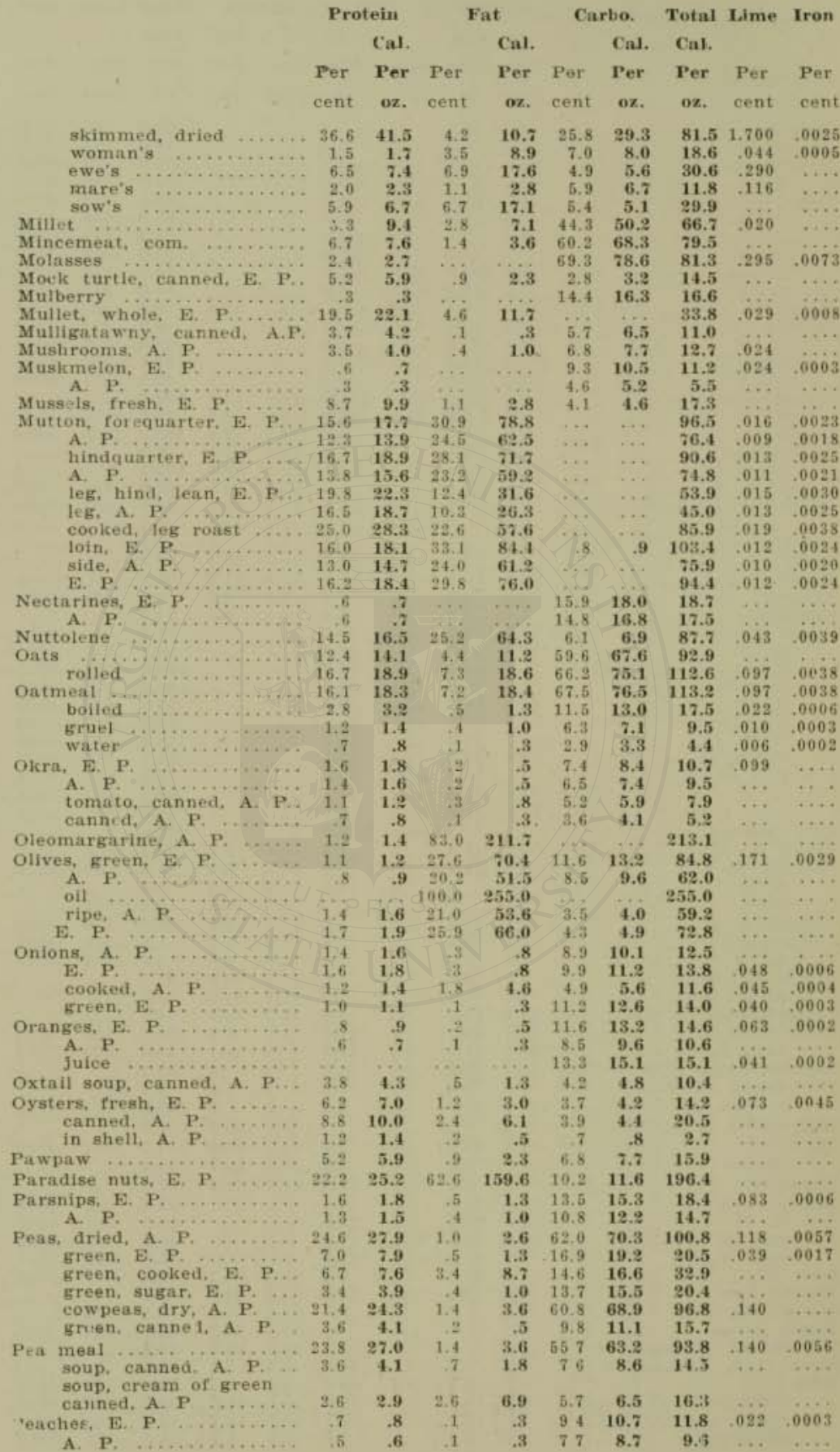




\begin{tabular}{rrrrrrrr} 
Protein & \multicolumn{2}{c}{ Fat } & \multicolumn{2}{c}{ Carbo. } & \multicolumn{2}{r}{ Total Lime } & Iron \\
Cal. & & Cal. & Cal. & Cal. & & \\
Per Per & Per & Per & Per & Per & Per & Per & Per \\
cent oz. & cent & oz. & cent oz. & oz. & cent & cent
\end{tabular}

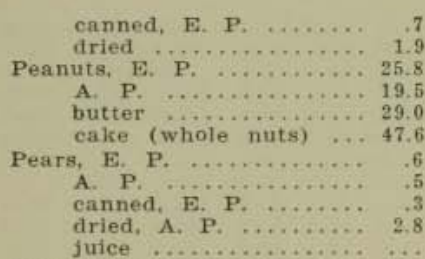

Pecans, unpolished E. P

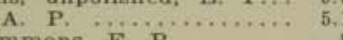

Persimmons, E. P.........

Pickerel, pike (whole) E. P. 18.7

Pie, apple, A. P. ........ 3.1 custard, A. P. ..... 4.2 mince, A. P. . . . . 5.8

Pineapple, E. P.
canned, E. P. canned, E. P nuts (Pignolias) E.P. 33.4

Pine nuts (Pignolias) E.P.. 14.6 A. P. $\because \ldots \ldots \ldots \ldots$ 8.7

Pistachios, E. P. ....... 22.3

Plums, E. P. . . . . . . . 1.

Pomegranates, E. P ...... 1.5

Pompano (whole) E. P. ... 18.8

Pop corn ................ 10.7

Pork chops, med, E. P. . . 16.6 A. P.
chuck ribs and shoulder, E. P. ...... 17.3 A. $P, \ldots \ldots \ldots, \ldots, 14.1$ fat, salt, A. P. .... 1.9 loin chops, fat, E. P. 14.5 side, E. P. ......... 9.1 A $P$ P 8.6 tenderloin, A. P. . . . 18.9 salt, lean, ends, E. P. 8.4 sausage, bologna, E. P. 18.7 steak, cooked, A. P... 19.9 29.6.

Potatoes, raw, E. P. ...... 2.2 A. P. ….......

evapornted, A. P. .... 8.8 cooked, chips, A. P, $\ldots 6.8$ cooked, boiled, A, P. - 2,5 sweet, canned, A, P. 1.9 sweet cooked, A. P.... 3.0 chips, A P. ........ 6.8 flakes, dried . . . . 7 . flour $. \ldots \ldots \ldots \ldots \ldots, 2$.

Prunes, E. P Aried $\mathrm{E}$ P Aried, E. P.
dried, A. P. $\ldots \ldots \ldots \ldots$
1.8

Pudaing. Indian Meal, A. P. 5, 5 rice custard, A. P. . . 4.0

Pumpkins, E. P. A. P..... 1.0 canned, A. P. ....... 8

Quail, canned, A. P. ...... 21.8

Quinoa flour ............. 19.

Radishes, E. P. $\quad \ldots \ldots \ldots \ldots \ldots$
Raisins, A. P. . . . . . . . . 2.

Raisins, E, P, $\ldots \ldots \ldots \ldots \ldots$. 2.6

Raspterries, black, E, I: .. 1.7

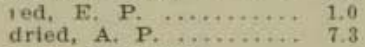
juice, sweetened ...... 5

Rhub:ırb, E. P. . . .

\begin{tabular}{|c|c|c|c|c|c|c|c|}
\hline .8 & .1 & .3 & 10.8 & 12.2 & 13.3 & & \\
\hline 2.2 & .3 & 8 & 25.4 & 28.9 & 31.9 & .048 & .0012 \\
\hline 29.3 & 38.6 & 98.4 & 24.4 & 27.7 & 155.4 & .100 & .0020 \\
\hline 2.1 & 29.1 & 74.2 & 18.5 & 21.0 & 117.3 & & \\
\hline 33.0 & 45.9 & 117.0 & 16.7 & 19.0 & 169.0 & .100 & .0020 \\
\hline 54.0 & 8.0 & 20.4 & 23.7 & 26.9 & 101.3 & & \\
\hline .7 & .5 & 1.3 & 14.1 & 16.0 & 18.0 & .021 & .0003 \\
\hline .6 & .4 & 1.0 & 12.7 & 14.4 & 16.0 & $\cdots$ & $\ldots$ \\
\hline .3 & .3 & 8 & 18.0 & 20.4 & 21.5 & $\cdots$ & \\
\hline 3.2 & 2.2 & 5.6 & 72.9 & 82.7 & 91.5 & 013 & \\
\hline 10.9 & 70.5 & 179.8 & $\begin{array}{l}22.6 \\
15.3\end{array}$ & $\begin{array}{l}25.6 \\
17.4\end{array}$ & $\begin{array}{r}25.6 \\
208.1\end{array}$ & .105 & .0026 \\
\hline 5.8 & 37.9 & 96.6 & 8.2 & 9.3 & 111.7 & & \\
\hline & .7 & 1.8 & 31.5 & 35.7 & 38.4 & .031 & \\
\hline 21.2 & .5 & 1.3 & & & 22.5 & .028 & .0007 \\
\hline .6 & -3 & .8 & 2.7 & 3.1 & 4.5 & $\cdots$ & $\cdots$ \\
\hline 3.5 & 9.8 & 25.0 & 42.8 & 48.5 & 77.0 & $\ldots$ & \\
\hline 4.8 & 6.3 & 16.1 & 26.1 & 29.6 & 50.5 & & $\ldots$ \\
\hline 6.6 & 12.3 & 31.5 & 38.1 & 43.2 & 81.3 & .040 & \\
\hline .5 & 3 & 8 & 9.7 & 11.0 & 12.3 & .025 & .0005 \\
\hline .5 & .7 & L.8 & 36.4 & 41.3 & 43.6 & $\ldots$ & .... \\
\hline 38.4 & 49.4 & 126.0 & 6.9 & 7.8 & 172.2 & $\ldots$ & \\
\hline 16.6 & 61.9 & 157.9 & 17.3 & 19.6 & 194.1 & $\ldots$ & \\
\hline 9.9 & 36.8 & 93.8 & 10.2 & 11.6 & 115.3 & $\ldots$ & \\
\hline 25.3 & 54.0 & 137.7 & 16.3 & 18.5 & 181.5 & & \\
\hline 1.1 & $\ldots$ & $\ldots$. & 20.1 & 22.8 & 23.9 & .028 & .0005 \\
\hline 1.0 & & & 19.1 & 21.7 & 29.7 & & \\
\hline 1.7 & 1.6 & 4.1 & 19.5 & 22.1 & 27.9 & .015 & .0004 \\
\hline 21.3 & 7.5 & 19.1 & & & 40.4 & .028 & .0008 \\
\hline 12.1 & 5.0 & 12.8 & 78.7 & 89.2 & 114.1 & & \\
\hline 18.8 & 30.1 & 76.8 & $\ldots$ & $\ldots$ & 95.6 & 013 & .0025 \\
\hline 15.2 & 24.2 & 61.7 & $\cdots$ & $\ldots$ & 76.9 & .010 & .0020 \\
\hline 19.6 & 31.1 & 79.3 & $\ldots$ & $\cdots$ & 98.9 & .013 & .0026 \\
\hline 16.0 & 25.5 & 65.0 & $\ldots$ & $\ldots$ & 81.0 & .011 & .0021 \\
\hline 2.2 & 86.2 & 219.8 & $\ldots$ & $\ldots$ & 222.0 & .002 & .0003 \\
\hline 164 & 44.4 & 113.2 & $\ldots$ & ... & 129.6 & .011 & .0022 \\
\hline 10.3 & 55.3 & $1+1.0$ & ... & $\ldots$ & 151.3 & .007 & .0014 \\
\hline 9.1 & 49.0 & 125.0 & ... & $\ldots$ & 134.1 & 006 & .0012 \\
\hline 21.4 & 13.0 & 33.2 & & $\ldots$ & 54.6 & .014 & .0028 \\
\hline 9.5 & 67.1 & 171.1 & $\ldots$ & $\ldots$ & 180.6 & .007 & .0013 \\
\hline 21.2 & 17.6 & 44.9 & $\ldots$ & & 66.1 & .014 & .0028 \\
\hline 22.6 & 45.4 & 115.8 & $\ldots$ & & 138.4 & .015 & .0030 \\
\hline 2.5 & $P+1$ & S .3 & 18.4 & 20.9 & 23.7 & .020 & .0013 \\
\hline 2.0 & 1 & .8 & 14.7 & 16.7 & 19.0 & & \\
\hline 9.6 & .4 & 1.0 & 80.9 & 91.7 & 102.3 & .064 & .0052 \\
\hline 7.7 & 39.8 & 101.5 & 46.7 & 53.0 & 162.2 & $\ldots$ & \\
\hline 2.8 & 1 & .3 & 20,9 & 23.7 & 26.8 & & \\
\hline 2.0 & .7 & 1.8 & 27.4 & 31.1 & 34.9 & .027 & .0005 \\
\hline 2.2 & -4 & 1.0 & 41.4 & 46.9 & 50.1 & $\cdots$ & \\
\hline 3.4 & 2.1 & 5.4 & 42.1 & 47.7 & 56.5 & . & $\ldots$. \\
\hline 7.7 & 39.8 & 101.5 & 46.7 & อั3.0 & 162.2 & . & \\
\hline 8.1 & -3 & .8 & 73.6 & 83.5 & 92.4 & & \\
\hline 3.1 & .8 & 2.0 & 81.3 & 92.2 & 97.3 & & \\
\hline 25.6 & 9.6 & 24.5 & 8.9 & 10.1 & 60.2 & .058 & .0057 \\
\hline 1.0 & $\ldots$ & $\ldots$. & 18.9 & 21.4 & 22.4 & $\ldots$ & \\
\hline .8 & $\ldots$ & .... & 17.4 & 19.7 & 20. & & \\
\hline 2.4 & $\cdots$ & & 73.3 & 83.1 & 85.5 & .076 & .0030 \\
\hline 2.0 & & & 62.2 & 70.5 & 72.5 & $\ldots$ & .... \\
\hline 6.2 & 4.8 & 12.2 & 27.5 & 31.2 & 49.6 & & \\
\hline 4.5 & 4.6 & 11.7 & 31.4 & 35.6 & 51.8 & & \\
\hline 1.1 & .1 & .3 & 5.2 & 5.9 & 7.3 & .032 & .0008 \\
\hline 9 & .2 & .5 & 6.7 & 7.6 & 9.0 & & \\
\hline 24.7 & 8.0 & 20.4 & 1.7 & 1.9 & 47.0 & .017 & .0033 \\
\hline 21.5 & 5.0 & 12.8 & 60.0 & 68.0 & 102.3 & & \\
\hline 1.5 & .1 & $\therefore$ & 5.8 & 6.6 & 8.4 & .029 & .0006 \\
\hline 2.6 & 3.0 & 7.7 & 68.5 & 77.7 & 88.0 & & \\
\hline 3.9 & 3.3 & 8.4 & 76.1 & 86.5 & 97.6 & .090 & .0021 \\
\hline 1.9 & 1,0 & 2.6 & 12.6 & 14.3 & 18.8 & .969 & .0006 \\
\hline 1.1 & & & 12.6 & 14.3 & 15.4 & .069 & .0006 \\
\hline 8.3 & 1.8 & 4.6 & 80.2 & 90.9 & 103.8 & & \\
\hline .6 & & & 49.9 & 56.6 & 57.2 & .030 & \\
\hline .7 & .7 & 1.8 & 3.6 & 4.1 & 6.6 & .062 & .0010 \\
\hline
\end{tabular}




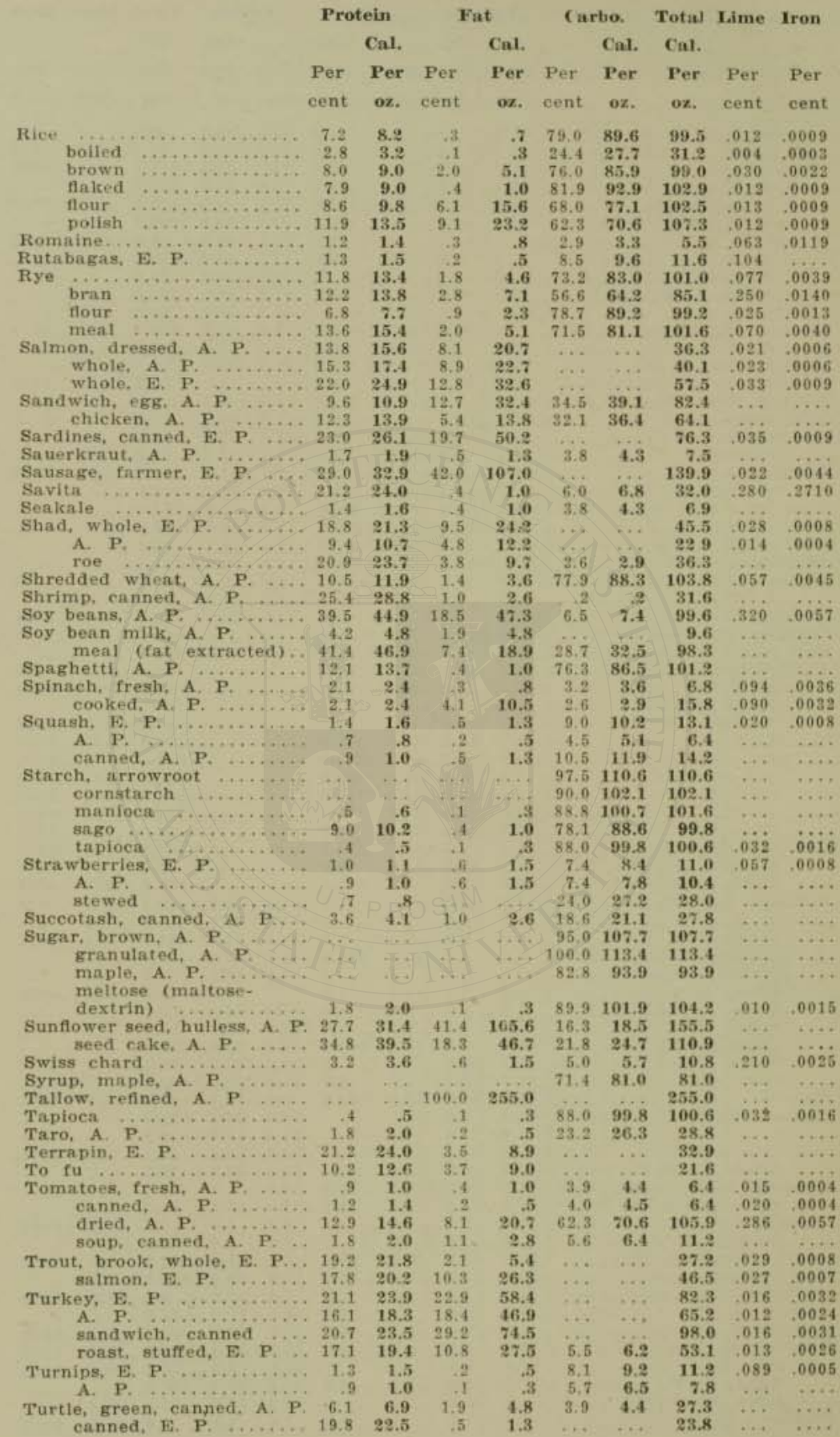




\section{Protein \\ Fat \\ Carbo. Total Lime Iron}

Cal. Cal. Cal. Cal.

Per Per Per Per Per

Per Per Per Per

cent oz, cent

oz. cent

oz. oz. cent cent

Vegetable soup. canned ... 2.9

Veal, breast, E. P. ....... 20.3 A. P. ........... 15.3

chuck, lean, E. P. ... 20,6

med. fat, E, $\mathrm{P}, \ldots \ldots, 19.7$

cutlets, E. P. …..... 20.3

A. P. …....... 20.1

forequarter, E. P. ... 20.0

A. P. $\ldots \ldots \ldots \ldots \ldots \ldots \ldots, 15.1$

hindquarter, E. P. . . 20.7

A. P. . . . . . . . 16.2

ade, E. P....... 20,2

loin med. fat, E. P... 19.9

Vermicelli .............. 10.9

a.s

$\begin{array}{lllll}\cdots & .5 & .6 & 3.9 & .035\end{array}$

$\begin{array}{llllllllll}28.1 & \ldots & \ldots & 51.1 & .015 & .0030\end{array}$

$\begin{array}{llllll}21.9 & \ldots & \ldots & 51.3 & .015 & .003\end{array}$

$\begin{array}{llllll}4.8 & \ldots & \ldots & 28.2 & .016 & .0031\end{array}$

$\begin{array}{lllllll}16.6 & \ldots & \ldots & 38.9 & .015 & .0030\end{array}$

$\begin{array}{lllllll}19.6 & \cdots & \cdots & \mathbf{4 2 . 6} & .015 & .0030\end{array}$

$\begin{array}{llllll}19.1 & \cdots & \ldots & 41.9 & .015 & .0030\end{array}$

$\begin{array}{lllllll}20.4 & \cdots & \cdots & \mathbf{4 3 . 1} & .015 & .0030\end{array}$

$\begin{array}{llll}15.3 & 32.4 & 012 & 0023\end{array}$

$\begin{array}{llllll}15.3 & \cdots & \cdots & 44.7 & .016 & .0031\end{array}$

$\begin{array}{llllll}\mathbf{2 1 . 2} & \cdots & \cdots & \mathbf{4 4 . 7} & .016 & .0031 \\ \mathbf{1 6 . 8} & \cdots & \cdots & \mathbf{3 5 . 2} & .016 & .0023\end{array}$

$\begin{array}{lllllll}\mathbf{2 0 . 7} & \cdots & \cdots & \mathbf{4 3 . 6} & .015 & .0030\end{array}$

$\begin{array}{llllll}16.1 & \ldots & \ldots & 33.8 & 016 & 0023\end{array}$

$\begin{array}{lllllll}\mathbf{2 7 . 5} & \ldots & \ldots & \ldots & \mathbf{5 0 . 1} & .015 & .0030\end{array}$

Walnuts, Callornia, E. P $, 18,4$ A. P.
Calif. Black. E. P. . . . 27.6 A. P $\ldots \ldots \ldots \ldots \ldots, 7.2$

Callif, soft shell, E. P. 16.6
A. P.

$\begin{array}{lllrlll}5.1 & 72.0 & 81.6 & 99.1 & \ldots & \ldots \ldots\end{array}$

$90.9 \quad 64.4$

$\begin{array}{rrrrrr}\mathbf{1 6 4 . 2} & 13.0 & \mathbf{1 4 . 7} & \mathbf{1 9 9 . 3} & .125 & .0021 \\ \mathbf{4 4 . 1} & 3.5 & \mathbf{4 . 0} & \mathbf{5 3 . 7} & \ldots & \ldots \ldots\end{array}$

$\begin{array}{llllllll}31.3 & 56.3 & 143.6 & 11.7 & 13.3 & 188.2 & \ldots & \ldots\end{array}$

A. P. ............. $6: 9$

$8.2-14.6$

$\begin{array}{llllll}37.2 & 3.0 & 3.4 & 48.8 & \ldots & \ldots\end{array}$

Water chestnut ........... 1.5

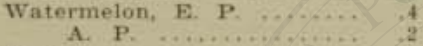

$\begin{array}{ll}7.8 & 26.6\end{array}$

$18.3 \quad 198$

$\begin{array}{lllllll}67.8 & 6.8 & 7.7 & 83.3 & \ldots & \ldots\end{array}$

$\begin{array}{rrrrrr}67.8 & 6.8 & 7.7 & 83.3 & \ldots & \ldots \\ .4 & 15.6 & 17.7 & 19.8 & 120 & \ldots\end{array}$

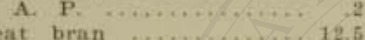

Wheat bran $\ldots$ ind crushed 12,5 tlaked $\ldots \ldots \ldots \ldots \ldots \ldots$
germs $\ldots \ldots \ldots \ldots \ldots \ldots$
13.4 germs
shredded $\ldots \ldots \ldots \ldots . .10 .5$

winter .............. 11.7

spring $\ldots . . . . . . .2 .5$

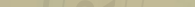

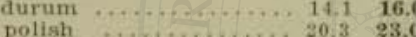

middilings, standard

phort

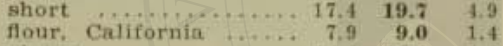

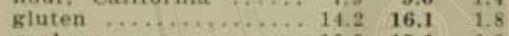

graham …......... $13.3 \quad 15.1 \quad 2.2$

baker's grade .......... 13.3 15.1 1.5

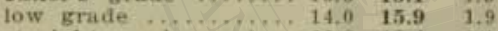

straight grade, spring. $10.8 \quad 12.4 \quad 1.1$

$\begin{array}{llll}.5 & 6.7 & 7.6\end{array}$

$\begin{array}{rrrrrr}.3 & 2.7 & 3.1 & 3.6 & 7.6 & \\ 7.7 & 41.6 & 47.2 & 69.1 & .168 & 0078\end{array}$

$\begin{array}{llllll}4.3 & 75.5 & 85.6 & 109.5 & 061 & 0059\end{array}$

$\begin{array}{llllll}\mathbf{3 . 6} & 74.3 & \mathbf{8 4 . 3} & \mathbf{1 0 3 . 1} & \ldots & .0066\end{array}$

$\begin{array}{llllll}\mathbf{5 . 1} & 76.0 & \mathbf{8 6 . 2} & \mathbf{1 0 3 . 2} & .099 & .0141\end{array}$

$\begin{array}{llllll}3.6 & 77.9 & 88.3 & 103.8 & .061 & .0063\end{array}$

$\begin{array}{lllllll}\mathbf{5 . 1} & 71.6 & 81.2 & \mathbf{9 9 . 6} & 061 & 0053\end{array}$

$\begin{array}{lllllll}5.6 & 70.5 & 79.9 & 99.7 & \ldots & .0050\end{array}$

$\begin{array}{llllll}6.4 & 68.6 & 77.8 & 100.2 & \ldots & .0050\end{array}$

$\begin{array}{llllll}4.3 & 64.2 & 72.8 & 100.1 & \ldots & \ldots\end{array}$

Whey winter trate 12.8

$13.9 \quad 1.1$

$\begin{array}{llllll}12.5 & 56.8 & 64.4 & 96.6 & \ldots\end{array}$

$\begin{array}{llllll}3.6 & 76.4 & \mathbf{8 6 . 6} & \mathbf{9 9 . 2} & .025 & .0015\end{array}$

$\begin{array}{lllllll}4.6 & 71.1 & 80.6 & 101.3 & .116 & .0057\end{array}$

$\begin{array}{llllll}5.6 & 71.4 & 81.0 & 101.7 & .061 & .0051\end{array}$

$\begin{array}{lllllll}3.8 & 72.7 & 82.4 & 101.3 & .040 & .0025\end{array}$

$\begin{array}{lllllll}\mathbf{4} .8 & 71.2 & \mathbf{8 0 . 7} & \mathbf{1 0 1 . 4} & .040 & .0012\end{array}$

$\begin{array}{lllllll}2.8 & 74.8 & 85.7 & \mathbf{3 0 0 . 9} & .018 & .0011\end{array}$

$\begin{array}{llllllllllll}\text { efish, whole, E. P. } \ldots \ldots & 22.9 & \mathbf{2 6 . 0} & 6.5 & \mathbf{1 6 . 6} & \ldots & \ldots & 42.6 & .034 & .0009\end{array}$

A. P. . . . . .

$\begin{array}{llllllllllll}\text { Whortleberries, A. P. } & \ldots . & .7 & .8 & 3.0 & 7.7 & 13.5 & 15.3 & 23.8 & .043 & \ldots\end{array}$

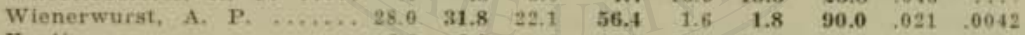

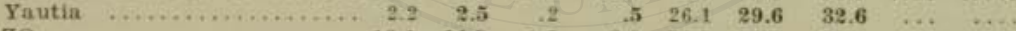

$\begin{array}{llllllllllll}\text { Zo } \ldots \ldots \ldots \ldots \ldots \ldots \ldots \ldots & 13.1 & \mathbf{1 4 . 9} & \text { i. } & \mathbf{3 . 8} & 70.8 & \mathbf{8 0 . 4} & \mathbf{9 9 . 1} & .090 & .0021\end{array}$ 


\section{INDEX}

Aberhalden $\ldots \ldots \ldots \ldots \ldots$ 426, 816

Abscess of kidney, diet in ... 880

Accidents, diet in .......... 927

Acetic acid ........... 232, 474

Achard ............... 477, 844

Achylia .............. 702, 997

diet for .......... 996, 997

Acids ................ 189, 704

Acid, acetic ............... 232

benzoic $\ldots \ldots \ldots \ldots \ldots \ldots \ldots .237$

butyric $\ldots \ldots \ldots \ldots \ldots .238,680$

citric ................. 231

foods $\ldots \ldots \ldots \ldots \ldots 189,192,230$

fruit $\ldots \ldots \ldots \ldots \ldots 679,333,334$

in gastric ulcer .......... 699

hydrochloric of gastric juice. 53

lessening....$\ldots \ldots \ldots \ldots .684$

lactic .................... 240

malic .................. 231

meat .................... 394

mineral ................ 241

organic .............33, 330

oxalic ................... 232

phytic ................. 231

tannic .............237, 680

tartaric ................... 230

unwholesome ............ 232

uric ................. 239

Acidity, gastric ........ 560, 699

Acidone .................. 704

Acidophilus Buttermilk ...... 803

Acidosis ................ 408, 421

diet for .............. 822

infantile .............. 826

post-operative ........... 826

tests for ........... 572, 822

Acorns .................... 379

Actinomycosis .......... 516, 913

Adametz, bacteria in cheese 446,505

Adami, bacteria of intestine.... 547

Addison's disease ............. 813

Adrenals, disease of ........ 903

Adsuki bean ................ 321

Adult body, comp. of ........ 28

Adult, food requirement .. 119, 120

Adulteration of foods ......... 517

Aerophagia ........... 710, 670

Agar ...............225, 300

Age, change takes place ....... 115

food required ............. 116

Aged, diabetes in ........... 799

Air hunger ................ 96

Akeley .................. 252

Albu, on flesh diet ......... 420
Albuminuria $\ldots . \ldots \ldots \ldots \ldots . .679$

Alcapton .............. 552, $68 \mathrm{C}$

Alcohol ............... 485, 681

Alimentary Canal, nerves of ...75

Alimentary tract, elimination of disease of ........... 731

Alkaline balance ........... 189

Alkaline treatment of ulcer ... 698

Alkalinity of blood .......... 900

Alkalinosis .............. 822

Allen and Joslin ........... 780

Alligator pear .............. 360

Almonds ................. 374

Almond milk .............. 375

Alvarez, gradient theory ...... 549

Alwens, salt in ascites ....... 488

Ameba coli ................ 519

Ames, life of blood cells ....821

Amino acids ........... 31, 143

Amylaceous dyspepsia ........ 718

Amylase...$\ldots \ldots \ldots \ldots \ldots .62$

Anaphylaxis ............... 33

in infants ....... 422, 449, 953

Anemia ....... 162, 422, 449, 814

pernicious ............. 817

Anemic infants .............. 945

Angina abdominis .......... 505

Angina pectoris ........... 852

Animal foods ................ 387

Animal heat, source of .... 102

Anorexia ................ 834

Antineuritic vitamin ......... 200

Antiscorbutic vitamin ........ 197

Antitoxic diet $\ldots \ldots \ldots \ldots 6613,982$

Apoplexy ............. 421, 534

Apoplexy, diet in ........... 851

Appendicitis ............. 422, 558

diet in ....................... 744

Apperly .................. 51

Apple ...................... 341

acid in ................ 341

juice ................... 342

Appetite ................. 98

juice ................... 46

lack of ..................6 670

Appetizers ................... 980

Arctic animals, bacteria

free .................. 133

Aretoeus, milk cure ......... 579

Arsenic in fruit ............ 342

Arteriosclerosis ...... 409, 421, 533

Arthritis rheumatoid ......... 839

Artichoke .................299

Jerusalem .................. 313 
Ascites, tuberculosis ......... 489

Asparagus ................. 297

Asthma .................. 422

diet in ................ 895

Atonic constipation ......... 758

Atony, gastric ............. 701

Autointoxication ............ 545

diseases caused by .........6 611

symptoms of ..............6 611

Avitaminosis ............... 207

Avocado .................... 360

Bachman, farinaceous diet . . . . 616

Bacillus acidophilus ...........6 602

bifidus ..................6 602

Bulgaricus ................602 602

putrificus ................ 405

Welchii ................602

Bacteria in animal droppings .. 408 of cheese ............ 446, 514 in children ............... 949 destroyed in intestines ...... 553 in eggs ................ 433 of fruit $\ldots \ldots \ldots \ldots \ldots . .335,516$ in intestine .............. 546 in meat ................ 405 on milk bottles ...........453 of mouth ................ 545 of stomach ............... 545

Bäeltz, diet of Japanese ...... 416

Bailey ................... 401

Baker and Carrell ............ 819

Baking powders .............. 262

Bananas .................. 355

Banana figs ............... 357

flour ................... 357

Bandisch .................. 167

Bannocks .............. 273

Banting .......... 769, 780, 789

Barberry .................. 350

Bardswell and Chapman ......907

Barium meal ................ 561

Barley .................... 275

Barr and McCann, tuberculosis. 909

Basal metabolism ........... 100 table ................... 103

Bases of foods ............. 189

Bastedo, on oils ............ 726

Bayliss, on egg white ....... 427

Beans ..................... 320

adsuki $\ldots \ldots \ldots \ldots \ldots \ldots \ldots, 321$

mung $\ldots \ldots \ldots \ldots \ldots \ldots \ldots . \ldots . \ldots 321$

shelled .................. 322

string $\ldots \ldots \ldots \ldots \ldots \ldots \ldots \ldots \ldots \ldots \ldots \ldots \ldots+321$

string, botulism from ....... 522

soy ......................... 322

Beaumont's digestion table ....998 experiments .... 45, 84, 88, 436

Beaver, on raw rice ......... 251

Beechnuts ............... 379
Beef ................... 395

not antiscorbutic ......... 418

Beets ..................... 310

Belching ................. 670

Benedict .................... 488

on emaciation .............. 773

on meat diet ............ 931

Beneke, cancer and meat eating 916

Benzoate of soda ............ 520

Benzoic acid ............... 237

Bergeim .................... 158

Beriberi .........200, 421, 449

Bernard, observations ........4 489 effects of egg on urine ...... 435

Berries .............347, 350

Bichlorid nephritis ......... 877

Bienstock, on B. coli ........ 594 sugar in toxemia .......... 586

Bifidus bacillus ............ 602

Bigelow, flesh abstainer ....... 415

Bile .............63, 81,560

Biliousness ............... 672

diet for ................. 748

from eggs ............. 429

Bill of fare, balancing of ....978 for children .............. 962 making of . ............ 975

Bitters .......... 56, 95, 706

Blackberries .............. 347

Blackfan, anaphylaxis in infants 953

Black spot in meat ......... 510

Bland diet ............... 645

Blatherwick, on acids in fruit.. 344

and Sansum $\ldots \ldots \ldots \ldots \ldots$...... 851

Blood ....................... 567

alkalinity of $\ldots \ldots \ldots \ldots \ldots .900$

chemical examination ....... 568

lavage of $\ldots . . \ldots \ldots \ldots \ldots, 662$ pressure ...........4 421, 483

pressure, high, diet in ....... 850

useless in anemia .......... 392

waste constituent ........... 569

Blood-making diet list ....... 988

Blood vessels, diet in disease of 846

Blueberries ................ 349

Body weight ................ 572

Bugota ................... 740

Boix, arteriosclerosis ....... 846

butyric acid .............. 238

pepper ............... 472

Boldyreff $\ldots \ldots \ldots \ldots .50,88,684$

Bolicoh beans ............. 321

Borax versus salt .......... 489

Borow ................... 479

Bouchard .................. 81

poisons in the body ......... 613

recognition of $\ldots \ldots \ldots \ldots \ldots 134$

toxicity of stools $\ldots \ldots .552,806$

toxins in the colon .......... 548

Bouchardat on diabetes ...... 779 
Botulism ............ 517, 522

Botulinus in olives .......... 345

Boys, growth of ........... 112

Boys, weight of $\ldots \ldots \ldots \ldots \ldots$. 111

Brain of adult .............. 28

Brain of infant ............ 28

Bran ................ 218, 626

how to use ............... 264

not irritating $\ldots \ldots \ldots \ldots \ldots 223$

Brand, diet in fevers ........ 887

Brandl ....................... 150

Brazil nuts ......... 3.8.0. 370\%

Breads, deficient in lime ....... 257 making .................. 261 gluten, recipe for ......... 801

iron in ................ 254

Breakfast ................. 980

test ................. 559

Breath, foul ...............673

Brenzcatechin ............. 552

Bright's disease ......421, 628, 875 caused by salt . . . . . . . . 485

Brillat-Savarin ......... 84, 499

Broca, forced feeding ........ 907

Broccoli ,................... 297

Brose .................... 273

Brown ................... 828

forced feeding . .......... 907

Brugsch .................. 63

Brunton, on bile ............ 856 observations of bile .......6.672 tobacco in kidney disease .... 873

Brussels sprouts ............ 296

Bryant, William Cullen, diet of 415

Buckner and Martin, lime .... 188

Buckwheat ................. 277

Bulimia ................. 94

Bulkley, diet in psoriasis ...... 913

Bullard, tea poisoning ......... 493

Bunge ............... 189, 217

lime water in diet ........ 947

effects of meat diet ........ 418

salt theory ............. 480

Burbank, Luther, origin of corn 267

Burmann, exp. with coffee ..... 495

Burton on eating ............ 538

Butter ...................... 445

color .................... 446

soup for infants ......... 946

coconut .................. 379

peanut $\ldots \ldots \ldots \ldots \ldots \ldots \ldots . \ldots . \ldots 381$

Buttermilk .......... 449, 688 acidophilus ............... 803

Butternuts ................. 377

Butyric acid ........... 238, 680

Cabbage,$\ldots \ldots \ldots \ldots \ldots \ldots . \ldots 294$

Chinese .................. 297

palm ..................... 297

Caffein, cause of goiter ...... 503 poisoning $\quad \ldots \ldots \ldots \ldots \ldots \ldots .491$

and uric acid ............. 849

in Kola drinks ........... 502

in various products ....491, 681

Cajori, on nuts ............ 368

Calcium of body ........... 27

Calorie .................. 99

required $\ldots \ldots \ldots \ldots \ldots \ldots . . .103$

in foodstuffs .............. 1002

Campbell ............... 51, 676

Cancer ...........4 422, 535, 543

colon ................. 918

diet in .................. 915

gastric .............. 917

Candy bill, Nation's ......... 158

Cane sugar ............... 679 poisoning $\ldots \ldots \ldots \ldots \ldots .948$

Cannon ....... 65, 77, 89, 95, 219

on colon ................ 723

observations of ........... 218

on ileocecal valve ......... 757

reversed peristalsis ........ 666

on stomach .............. 670

Car sickness ............... 866

Carbohydrates ....... 33, 64, 145

content of foods ........... 786

Carbohydrate free diet .......647

Carbonated water $\ldots \ldots \ldots \ldots .681$

Carbolic acid in cheese ....... 447

Carbon of the body ........... 27

Carbon monoxide ........... 505

Cardiac disease ............. 483

orifice of the stomach $\ldots \ldots, 62$

Carlson ........45, 65, 68, 89, 677 on bitters ............... 706

obs, on stomach .......... 670

Carrell and Baker ........... 819

Carrots .................. 311

Cartilage, composition of .... 28

Cassava ................... 291

Case ..............65, 72,229

on barium meal ........... 562

on colon $\ldots \ldots \ldots \ldots \ldots \ldots .723$

obs, of ileocecal valve ...... 757

reversed peristalsis .........6.666

X-ray study of diabetes .... 777

Cashew nut ................ 380

Casein superior to meat ...... 634

Caspari, milk exp. .......... 438

Catarrh, bronchial .......... 422

gastric $\ldots \ldots \ldots \ldots \ldots \ldots \ldots .422$

gastric in children .......... 716

Catalase ................. 418

Cauliflower .................. 296

Cecum .................. 734

Celeriac ................... 313

Celery ...................... 298

Cellulose .............217, 220

in cereals (table) ......... 220

foods rich in ............6 626 
in fruits ................ 221

in vegetables ............. 220

Cereals ............... 253, 969

breakfast ............... 278

cellulose .................. 221

flakes .................... 279

for infants ............. 945

iron in ................. 254

lime in ................. 255

protein of ......128, 137, 247.

Cetti, effects of fasting ...... 637

Chaipori, treatment of anemia.. 818

Chapin, diet and development .. 937

Chapman and Bardswell, diet in tuberculosis ........... 907

Charrin, toxins in colon ....... 548

Chayote .................... 316

Cheese ..................... 446

bacteria ........... 446, 514

lime content of .......... 440

poisoning ............... 515

Chemical balance ........... 189

juice .................... 48

secretion test ............ 561

test of blood ............. 568

Cherries .................... 343

Cherrie, Dr. George K. ...... 267

Chestnuts .............. 378

Chestnut, water ............ 293

Chewing ............ 78, 682

Cheyne-Stokes ........ 97, 487

Chick and Delf, scurvy ........ 198

Chicken ................... 396

Chickory, white .......... 2999

Children, bacteria of ........ 949

bills of fare for .......... 962

diet of $\ldots \ldots \ldots \ldots \ldots .948,956$

food requirement .. 115, 118, 119

gastric catarrh ........... 716

water requirement of ..... 526

Chinquapin ................ 378

Chittenden ..............118, 488

effects of alcohol .........680 680

meat diet ............... 931

low protein diet ...........6.617

protein standard ............ 126

protein in diet ............. 535

Cholera ................... 892

infantum ................ 952

Cholin $\ldots \ldots \ldots \ldots \ldots \ldots \ldots \ldots, 507$

Cholesterol ................ 425

Chronic infections, diet in .... 898

Circulation and heart ........ 573

Cirrhosis of liver $421,483,659,751$

Citric acid ................ 231

Citrus fruit $\ldots \ldots \ldots \ldots \ldots \ldots 350$

Clairmont .................. 42

Clark, Sir Andrew ........... 85

Clams, poison in ........... 513

Clay, laxative effects .......229
Cloud berries .............. 350

Cob nuts .................. 378

Cobez de Baca ............ 179

Coccidium oviforme ........ 519

Cocoa ...................... 495-not there?

Coca cola ............... 958

Coca drinks .............. 501

oxalic acid in .............. 233

tannic acid in ............. 237

Coconut ................. 379

Cocks, meat diet in ........ 402

Coffee .............. 490, 491

raises blood pressure ....... 493

decaffeinated ............. 500

a drug .................. 490

heart ................. 494

cripples the liver .......... 495

insomnia ................ 499

poisons . . . . . . . . . . . 495

substitutes .............. 503

and tea, effects of ........ 496

tannic acid in ........... 237

increases uric acid ......... 874

Cold, common .......... 71, 659

Collozo ................. 825

Colitis ...................... 420

acute $\ldots \ldots \ldots \ldots \ldots \ldots \ldots . . . \ldots 738$

chronic ............... 735

diet in ............... 735

Collip, glucokinin ......... 790

Colon, care of .............. 730

cancer of ................ 918

diet in ulceration of ........ 741

movements of $\ldots \ldots \ldots \ldots \ldots 72$

putrefactions in ........... 551

diet for ulceration of ..... 741

Coma .................. 928

diabetic diet in .......... 800

diet $\ldots . . \ldots \ldots \ldots \ldots . . . . .928$

Combe, rules of diet ....... 597

salt in epilepsy ............ 860

low protein diet .......... 617

toxins in the colon ........ 548

Composition of foodstuffs ....1002

Condiments ............ 471, 681 use of ................ 540 as preservatives ......... 477

Constipation ................. 682 atonic $\ldots \ldots \ldots \ldots \ldots \ldots \ldots .758$

diet in $\ldots \ldots \ldots \ldots \ldots \ldots, 722$

rules for relief of .......... 730

Conroy, examination of pancreas ................ 775

Convulsions .............. 487

Cook, toxicity of foods ....... 520

Cookery ............... 245, 249

Cooper ....................... 203

potato diet $\ldots \ldots \ldots \ldots \ldots 287,650$

Corn ..................... 267

flakes ................... 271 
oil $\ldots \ldots \ldots \ldots \ldots \ldots \ldots \ldots . .279$

popped $\ldots \ldots \ldots \ldots \ldots \ldots \ldots .270$ products, composition of ... 277 syrup ................... 279 sweet, green ............. 280 meal ...................... 268

Corn meal mush and milk .... 269

Cornstarch ................. 269

Cows, tuberculosis .......... 512

Crab, Roger .............. 244

Crabs ................. 397

Cranberries ................... 349

benzoic acid in ............. 237

Crato on eating $\ldots \ldots \ldots \ldots \ldots .538$

Cream ...................... 445

Creatin .................... 398

Cresol .................... 551

Crichlow .................. 744

Crile, diet in goiter ......... 807

Cucumber ................. 315

Currants ................. 347

Curschmann .............. 808

Cyclic vomiting ........... 711

Cystitis ............. 422, 882

Darwin .................. 88

Dasheen .................... 290

Dates ............... 155, 358

Davis and McCollum ........ 199

Day's ration ............... 100

Débove ............... 49, 907

Delf and Chick, scurvy ...... 198

Delf and Eddy ............. 200

Dementia praecox .......... 859

Deneky on goiter . . . ......... 807

Denis ....................... 766

Deuel ..................... 149

Development, influence of food 117

Dewberries ................ 350

Dextrin ........33, 151, 615, 679 effects on the stomach .....694

Dextrose ................. 152

Diabetes ....... 487, 535, 564, 774 in the aged .............. 799

diet in $\ldots \ldots \ldots \ldots \ldots \ldots \ldots \ldots, 777$

fat allowance in ........... 782

with pregnancy ........... 800

protein ration ............ 782

and renal disease ......... 799

Diabetic coma .............. 800

ration ..................... 791

Diarrhea ................... 485

diet in .................. 753

acute, diet in ............ 754

in infants ................ 952

Diet, antitoxic ........613, 982 abcess of the kidney ........ 880

Diet, accidents ............. 927

achylia gastrica ............ 996

acidosis $\ldots \ldots \ldots \ldots \ldots \ldots . . .622$ angina pectoris $\ldots \ldots \ldots \ldots .853$

asthma ................. 895

appendicitis ............. 744

basic .................. 990

bland or non-laxative ...... 645

carbohydrate free ..........647

carsickness .............. 866

children .................. 957

constipation $\ldots \ldots \ldots \ldots \ldots .722$

deficiency ............... 536

and development ........... 117

diabetes .................... 779

in digestive disorders .......664 66

dry ...................6 649

dysentery ................ 755

errors .................. 533

esophageal stricture ....... 759

eclampsia ................ 886

emaciation .............. 770

fattening $\ldots \ldots \ldots \ldots \ldots \ldots, 628$

fat-free .................. 648

fever ..............620, 983

raw food ........ 243, 651, 991

fruit ................... 337

hemorrhoids ............. 743

of Grecians ............... 414

genito-urinary diseases ...... 869

goiter ............... 804

gall bladder disease ........ 749

in heart and blood vessel

diseases ........ \&46, 850, 855

hyperacidity ..........676, 992

hypochlorhydria .......... 995

for infants .............. 939

mixed for infants ......... 951

schedule for infants ........ 938

supplementary for infants ...9941

intestinal obstruction ......... 741

ileocecal valve ............ 756

Japanese ............... 416

joint diseases ............ 838

kidney diseases .............. 879

laxative, when indicated ....6 627

laxative .........625, 745,986

liver disorders ........... 745

meat, pernicious effects .... 409

and metabolism ............. 117

misinformation about ........ 542

monotonous ............... 540

mother's .................. 936

nervous disorders ......... 856

obesity ...................... 761

pancreas ................... 751

parasitic disease .......... 755

post-operative .............. 700

protein .................. 595

low protein ..........6.617, 981

protein-free ...........617, 984

raw ................6. 651, 991

rheumatism ................. 840

salt-free ................ 486 
in skin disease ........... 913

scurvy ................ 830

teeth decay ............... 719

ulcer ............... 993, 996

of colon ............ 741

United States army ........ 118

urethritis ................ 882

uric acid disorders .........841

diseases of women ....... 883

lying-in women ........... 885

Dietary, meatless ........... 930

Dietaries and regimens ...... 574

Dietetics, medical ........... 531

Dinner ................... 980

Digestibility of foodstuffs ..... 997

Digestion aided by liver ..... 79

alcohol hinders ............. 488

mouth ................ 39

small intestines ........... 60

gastric, tests of .......... 561

table ..................... 998

time required .............1000

Digestive fluids ........... 39

processes ................ 39

Diseases requiring antitoxic diet 611

Disease germs, elimination of . 731 requiring change of flora ....6 611 of women, diet in ....... 883

Dixon, poisons in intestine ... 848

Drinking at meals ......... 539

Drinking water ......... 489,661

Dropsy .......4 421, 482, 487, 649

Drug, alcohol ............. 489 effects of, on peristalsis ... 75

Drummond ..............201

Dry diet .................. 649

Dubois .................... 104

Dujardin-Beaumetz ......... 907 putrefaction in fish ....... 396

Duodenal feeding ........... 662 formulas for ............. 695

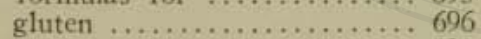

lacto-dextrin ............... 694

sugar formula ............ 694

duodenal ulcer ......4 423, 691, 993

diet, Lenhartz's ............ 697

von Leube's ................ 698

Duodenum ............... 733

Dupratz ................... 267

Dwight, apple cider for headache $\ldots \ldots \ldots \ldots \ldots \ldots \ldots .338$

Dysentery ........ 645, 738, 755 tropical ................ 755

Dyspepsia, amalacious .... 713, 718 nervous $\ldots \ldots \ldots \ldots \ldots \ldots \ldots . \ldots \ldots$

Dyspituitaryism .......... 813

Ear disease ............... 932

Eating, hasty .......... 538, 681 physiology of ............ 121
Ebstein .................. 841

Eclampsia, diet in ......... 886

Eck, fistula ............... 903

Eczema ........... 422, 620, 914

in infants .............. 915

Edelmann, bacteria in meat .... 510

Edema ................... 483

Egers .................. 168

Eggs ................... 423

bacteria in .............4 433

bad .................. 432

biliousness from .......... 439

candling of ............. 433

composition of ........... 424

cooking of .............. 428

digestion of $\ldots \ldots \ldots \ldots \ldots \ldots 428$

when contraindicated ....... 435

influence of food on ....... 428

iron of ................ 429

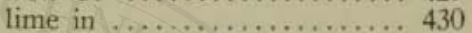

poisoning ............... 434

preserved ............... 431

production, lime .......... 181

protein of ............. 390

urticaria from ............ 434

substitutes ..............4 431

whites $\ldots \ldots \ldots \ldots \ldots \ldots \ldots .426$

yolks ................. 425

Eggplant ................ 415

Eggleston on duodenal ulcer ..691

Ehrlich experiment .......... 194

Eichhorn, bacteria in meat ... 510

Einhorn tube .............. 558

Eiskamp Park ............. 553

Elderberries .............. 350

Emaciation, diet in ........ 770

Emergency cases, diet in ...... 927

Emerson and Manny ......... 110

Emmet and Luros ............ 201

Endive-white chicory ........ 299

Endocrine disturbance ....... 766

Endurance, Fisher's exp. . . . . 417

Energy output of body ....... 99

and food requirement ...... 108

of foodstuffs .............. 1002

Enema ............... 72, 533

use of $\ldots \ldots \ldots \ldots \ldots \ldots \ldots .728$

English walnuts .......... 376

Epilepsy . . . . . . . . . 422, 860

Ergotism ................ 516

Erysipelas ........... 482, 893

Escherich, bacteria of

intestine ............. 547

germs in milk ........... 514

toxins in colon ......... 548

Eskimos, non-use of salt .... 484

Esophageal stricture ........ 759

Evelyn, John, on salads ...... 309

Ewald's test breakfast ....... 559

Ewald's test dinner ........ 561 
Exercise in obesity ........ 766

Eye, diet in diseases of ..... 932

Fasting, intermittent ........ 765

objections to ............ 641

regimen ............... 636

scientific ................ 646

loss of weight in .......6 638

Fatigue poisons ............ 616

Fats ......... 35, 64, 141, 615 effect on gastric juice ...... 48 cause of headache ......... 491 in infant diet ............ 944 allowance in diabetes ....... 782 vegetable ............... 143

Fat free diet ............ 648

Fat soluble vitamin ........ 203

Fattening diet .....628, 633, 985

Feeding, duodenal ......... 662 after gastro-enterostomy ...993 intravenous .............656 post-operative ........... 922 rectal ................. 653 tube, supplementary ........ 590

Ferments in plants ............ 252 made by liver .............. 80

Fever ................ 420, 572 acute infections .......... 891 feeding in .............. 890 foods to be avoided ......... 891 dietetic management of .... 887 diet .............6. 620,983 eruptive ................ 620 in infants, diet in ........ 953 malarial ................. 891 relapsing ................ 891 scarlet .................. 488 yellow ................ 891 water drinking in .......... 657

Figs ..................... 357

Filberts .................. 378

Finkelstein ............. 915

Fischer exp. with protein .... 552 acidosis and Bright's disease.. 822

Fischl, cane sugar in diet ..... 948

Fish .................... 396

Fisher, Irving . . . . . . 44,417

Fleckseder ............... 43

Flesh diet cause of acidosis ... 408 effects on animals ......... 402

Flesh foods, characteristics of .. 388

Flick and Walsh ........... 903

Flies, infection from ........ 517

Flint, beef tea and urine .. 487.621 treatment for ulcer .........6691

Flora, changing of ........ 592 change of without cultures ..6 605 intestinal ...........6.608, 610

Flour, banana .............. 357 peanut ............... 382
Fluke, blood .............. 519

Fluke, liver .............. 519

Folin, protein in diet $.535,766,870$

Food acids ............ 189, 230 and basis ................ 189

Foods, adulterated ........... 519 animal .................. 388 calorie value of $\ldots \ldots \ldots \ldots \ldots 243$ carbohydrate ............. 786 rich in celluiose ......... 626 cold ................... 541 combinations, bad ........ 539 cookery ................. 245 definition of $\ldots \ldots \ldots \ldots \ldots .25$ edible, wild .................. 964 diabetic ................ 783 energy of $\ldots \ldots \ldots \ldots \ldots \ldots . . . .69$ highly flavored ...........6679 hot ................... 541 indigestible $\ldots \ldots \ldots \ldots \ldots \ldots .541$ infant ................ 469 influence upon gastric

secretion ............ 56

insipid .............. 540 transportation by intestine .. 65 rich in iron ............ 175 poor in iron ........... 89 iron, where found $\ldots \ldots \ldots 167$ lime, content of .......... 180 rich in lime (Table) ....... 188 passage of ............... 69 poor in lime .............. 189 deficient in lime .......... 183 rich in lime and iron (Table) 188 stored by liver ............. 79 influence upon milk ....... 439 parasitic infections of ..... 518 plants, origins of ........ 973 poisoned ............... 507 poisoning, cheese ........ 515 principles $\ldots \ldots \ldots \ldots \ldots \ldots \ldots .30$ potato ............... 650 raw .................... 250 requirement $\ldots \ldots \ldots \ldots \ldots 108,118$ of men and women .... 119, 120 salts .............. 36, 160 highly seasoned .......... 539 sodium chloride in ........ 486 source of ............... 29 passage of from stomach .. 64 time for digestion ......66, 997 toxicity of $\ldots \ldots \ldots \ldots \ldots \ldots .520$ toxins of $\ldots \ldots \ldots \ldots \ldots \ldots . .508$ transportation of $\ldots \ldots \ldots \ldots 65$ uncooked ...............250 uric acid in ............. 240 values of $\ldots \ldots \ldots \ldots \ldots \ldots . . .998$ vitamin content ........... 213 water in (Table) .......... 248 wholesome ..............243 
work equivalent of ....... 99 the world's .............. 964

Foodstuffs, digestibility ....... 997 composition of .............. 1002

Foot and mouth disease ......891

Fox, arteriosclerosis in animals. 846 disease in animals ........ 410

Fowls .................. 396

Fractional test meal ......... 559

Freidenwald and Ruhrah ..... 905

Frothingham and Smillie .... 877

Fruits ............ 971, 968

Fruit acids ..............6.6. 679

arsenic in ..................341

canned .................. 363

cellulose in ............. 221

citrus .................. 350

composition of (Table) .... 333

diet $\ldots \ldots \ldots \ldots \ldots \ldots \ldots \ldots . . . \ldots 37$

dried $\ldots \ldots \ldots \ldots \ldots \ldots \ldots . . . .362$

digestibility of ........... 998

how they differ ......... 574

disinfection of ........... 335

germicidal properties ...... 338

iron content of .... ...... 339

juices ................. 363

juices, composition of ...... 366

pectin in ................ 331

lime content of ........... 339

purées for infants ........ 942

regimen .............. 574

when indicated .......... 578

ripening process in ........ 340

seeds ................ 336

skins, germs of .......... 516

sugar in (table) .......... 331

vegetable $\ldots \ldots \ldots \ldots \ldots \ldots \ldots .316$

Fuld, changing flora ......... 603

on constipation .........6.677

Fullness, sense of ........ 679

Funk, vitamin ...... 37, 200, 217

Fürst, vitamin in plants ...... 833

Gaglio, fasting $\ldots \ldots \ldots \ldots \ldots 638$

Galen, milk cure .......... 579

Gall bladder disease.. 421, 683, 733 diet for ................. 749 drainage...$\ldots \ldots \ldots \ldots \ldots .750$ examination of ............ 564 operation, diet after ........ 927

Gallstones ............ 421, 659 and diabetes $\ldots \ldots \ldots \ldots \ldots \ldots, 777$

Garbanzos ................. 329

Garlic ................... 293

Gas, intestinal .............. 718 in stomach ..............6.672

Gaspard, putrefying substances, 401

Gastric acid ................ 49 influences which lessen .... 684 how increased $. . \ldots \ldots \ldots \ldots, 53$
Gastric acidity . ......... 49, 699

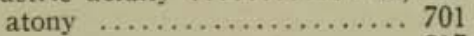
cancer ................. 917 in children ............. 716 digestion $\ldots \ldots \ldots \ldots \ldots \ldots \ldots .44$ time of digestion ..........1000 dilatation ................ 79 disorders ................. 543 glands, test of ........... 561 juice $. . \ldots \ldots \ldots \ldots \ldots .53,56$ effects of salt on ......... 479 due to organic acids ....... 699 pain .................6.674 spasm ............... 554 symptoms .............. 664 toxicity ............. 554 ulcer ...... 71, 423, 686, 691

Gastritis ........... 543, 993 acute $\ldots \ldots \ldots \ldots \ldots \ldots \ldots . \ldots \ldots$ chronic, diet in ......... 718

Gastro-enteric infection, acute. 712

Gastro-enterostomy, diet in.... 923

Gautama Buddha ............ 139

Gautier, analysis of beef tea ... 399 effects of coffee ........... 497 toxins in renal disease ..... 905 salt in diet ............... 480

Gavage .................. 662

Genito-urinary disorders ...... 869

Germs, elimination of ...... 731 of fruit skins .......... 516

Germicidal properties of fruits. 338

Gershun, skin diseases in infants 914

Gies ..................... 42

Girls, growth of $\ldots \ldots \ldots \ldots \ldots 113$ weight of $\ldots \ldots \ldots \ldots \ldots \ldots 111$

Givens .................. 315

Globus ................ 672

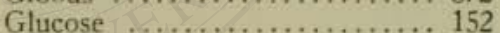

Glutelin ................... 267

Gluten feeding for ulcer ...... 696

Gluten products .......... 801

Glycogen ............... 34, 920

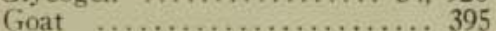

Gofio ................... 271

Goiter, simple, diet in ....... 804 caffein, cause of .......... 502

Goldberger and Tanner ....... 829

Gonorrhea, diet in ......... 882

Goodall, observations of ..... 877

Goodhart and Campbell ...... 51

Goodrich and Hindman ...... 397

Gooseberries .............. 347

Gout .............. 421, 842

Graham,(Sylvester),........ 265 -

Graham, Robert ............ 518

Grain, average crop ......... 419

Grapes ................ 346

Gray ..................689 689

Gravel ................. 880 
Greens ............... 302, 615 lime in ............ 306, 308 as roughage ............. 304 Green regimen ............ 577

Grierson ................. 147 digestibility of starches ....283

Grigoriew, diet in anemia ... . 816

Grindley, starches ........... 247 vegetables (Table) ......... 249

Grits ...................... 269

Ground nuts ............ 379

Growth .................. 109 of boys ................ 112 of girls ................ 113

Grützner .................. 672

Guava ................. 359

Guelpa, fasting in diabetes ...780

Guerithault, M. ............. 160

Gy ................. 873

Haberland, food value of wood. 223

Hall ......................... 167 uric acid .............. 239

Hamburger and Monte ...... 737

Hamburger steak ........... 509

Hamilton, exp, with coffee .... 496

Hanburg ................ 676

Hansen, manna ............. 300

Hare ..................... 856

Hart, cellulose experiment .... 222

Hart and Steenbock .......... 178

Hartridge ................. 505

Hasselbalch ............... 824

Harvey ................... 846

Hawk ................. 50

Hazelnuts ................ 378

Chinese ................. 385

Headache ............. 500, 856

Heat, animal .............. 102 effects of .............. 71

Heart of adult ............. 29

Heartburn ..............6.671

Heart and circulation, tests of. 573 disease ................ 482 diet in disease of ......... 846 disease caused by coffee .... 495 of infant ................. 29 senile, diet for .......... 854 tobacco ................ 504

Height of boys and girls.. 112, 114 and weights of men and women .............. 120

Heitler ................... 156

Hematogen .............. 429

Hemmeter, obs, on stomach ... 684

Hemophelia .............. 830

Hemorrhoids ................. 534 diet for ............... 743

Henderson, test for acidosis... 822

Henry, Prof. .............. 468
Hepatic abscess ............ 421 Hepatic efficiency diminished.. 901 Herbst, food lime .......... 180 Herpes zoster ................ 867 Herter ........................ 549 Herter and Kendall .......... 594 Hertz ................. 65, 68 on colon ............... 723

Hess, carrots .............. 311 on scurvy .................. 198

Heynemann, germs in milk ... 516 Hiccough $\ldots \ldots \ldots \ldots \ldots \ldots \ldots .711$ Hickory nuts ............. 376

Hindhede ............... 35, 148 on bran ................. 222 on fat-free diet ...........648 on greens ............... 305 on potato diet ........ 286, 649 on diet in tuberculosis ...... 910

Hinman and Goodrich ....... 397

Hirschfeld ............... 125 diet in diseased kidneys ..... 905

Hirschsprung's disease, diet in. 742

Hippocrates, cellulose ....... 214 on diet in fever ...........620 milk cure .............. 579 water drinking ..........6.656

Hodgkin's disease .......... 829

Hoffman ................ 916 milk cure ................ 579

Holcomb, diet of Chinaman ... 415 Holmes, digestibility of soy bean 324 Holst, report of ........... 196 Holzknecht ................ 74

Honey ................... 158

Hoobler, malted nuts ........ 383 orange juice .............. 195

Hookworm ................ 519

Hormones in liver ......... 80 in pancreas ............... 789

Horseradish ............... 312

Horton, diet of Grecians ...... 414

Huchard on arteriosclerosis ... 846

Housework, energy expended .. 108

Howe, dental disorders ....... 198 experiments with saliva .... 719

Huckleberries .............. 349

Humbolt, banana yield ...... 356

Hunger, absence of ......... 92 air $\ldots \ldots \ldots \ldots \ldots \ldots \ldots \ldots .96$ increase of .............. 93 modifications of .......... 94 mental and nervous influence. 92 salt ..................... 480 in infants ............... 91 pains $\ldots \ldots \ldots \ldots \ldots \ldots \ldots \ldots .94$ during sleep ............... 92 starvation ............... 91

Hunt, Dr. Reid ........... 140

Hunter, Arthur ........... 851 
Hunter, John ........... 50, 161 apples ................... 333 on diet $\ldots \ldots \ldots \ldots \ldots \ldots . .844$

Hurst $\ldots \ldots \ldots \ldots \ldots \ldots \ldots \ldots \ldots \ldots \ldots$. 74

Huxley, diet restriction ....... 533

Hydrochinon .............. 552

Hydrochloric acid ....... 678, 684 uses of $\ldots \ldots \ldots \ldots \ldots \ldots \ldots, 53$ acid of stomach ............. 49

Hydrogen of body .......... 27

Hyperacidity ............... 533 diet in ............6.676, 992 caused by meat .......... 423

Hyperchlorhydria ...........6.676 diet in ............6.676, 992 caused by meat .......... 423

Hypochlorhydria, diet for.. 702, 995

Hyperthyroidism ............ $4 \overrightarrow{20}$ diet in ............... 809

Hypothyroidism ........ 421, 809

Hysteria ................. 863

Ice cream ................ 513

Ices $\ldots \ldots \ldots \ldots \ldots \ldots \ldots .679$

Iceland moss ................ 299

Ileocecal valve .............. 756

Iletin ............. 585, 790

Incompetency of the ileocecal valve $\ldots \ldots \ldots \ldots \ldots \ldots \ldots . \ldots 756$

Indigestion, tea and coffee .... 500

Indol in cheese ............ 447 with different diets ........ 134 fatigue poisons ........... 616

Inebriety ................ 401

Infantile acidosis .......... 826

Infants, anaphylaxis in ...... 953 anemic ................ 945 artificial diet of ........... 939 body, composition of ...... 28 buttermilk for ........... 947 dangerous foods for ....... 945 diarrhea in ................ 952 diet schedule for ........... 938 eczema in .............. 915 feeding $\ldots \ldots \ldots \ldots \ldots \ldots \ldots . . .635$ feeding oranges .......... 352 feeding program .......... 940 foods ................... 469 food, composition of ....... 470 food requirement ............ 118 hunger in $\ldots \ldots \ldots \ldots \ldots \ldots . .61$ nursing of ............... 949 protein requirement ........ 127 pyloric obstruction in ....... 952 ration ................ 940 vomiting of $\ldots \ldots \ldots \ldots \ldots .951$

Infantile acidosis ........... 826

Influenza ................... 893

Inosite .................. 154
Insanity $\quad \ldots \ldots \ldots \ldots \ldots \ldots \ldots, 422$

Insomnia ............ 422, 535

caused by coffee ........... 499

Insulin, use of .............. 789

Intestinal absorption and excre-

tion $\ldots \ldots \ldots \ldots \ldots \ldots \ldots, 64$

activity $\ldots \ldots \ldots \ldots \ldots \ldots, 600$

antiseptics .............. 553

bacteria in arctic region .... 133

flora, method of changing.. 592 juice...$\ldots \ldots \ldots \ldots \ldots \ldots .61$ obstruction ............. 741 parasitic disease, diet in ....755 putrefaction ............. 133 rhythm ................ 77 toxemia .................. 545 tuberculosis, diet in ....... 910

Intestine $\ldots \ldots \ldots \ldots \ldots \ldots .64$ excretion by ............ 64 effects of heat and cold .... 71 movements of ............. 71 small, digestion in ......... 60

Intravenous feeding ......... 656

Invertase $\ldots \ldots \ldots \ldots \ldots \ldots .63$

Iodine in body ............ 27 in foods ................ 806

Irish moss . . . . . . . . . . . 300

Iron in body .............. 27

in cereals and bread .......254

daily requirement .......... 164

in eggs .............. 429

in food, where found ...... 167

foods, rich in ............ 175

foods, poor in .......... 189

in foodstuffs $\ldots \ldots \ldots \ldots 169,171$

in fruits .............. 339

in greens ................ 308

in legumes $\ldots \ldots \ldots \ldots \ldots \ldots, 318$

and lime, foods rich in...... 188

in meats ........... 389, 391

in milk ............... 443

in nuts ................. 372

pregnancy .............. 883

ration $\ldots \ldots \ldots \ldots \ldots \ldots \ldots .616$

how to determine ......... 174

tables ........... 173, 175, 189

Ishiguro, beriberi .......... 200

vegetables . .............. 283

Ivy $\ldots \ldots \ldots \ldots \ldots \ldots \ldots, 677$

Jacobson, colitis in children ... 716

Janeway, uric acid ........... 239

Jansen, experiment ........... 380

Jaundice ................. 421

Javal and Widal ............478

Jejunal feeding .............. 926

Jerusalem artichoke .......... 313

Johns, nuts ................ 368

Joffe $\ldots \ldots \ldots \ldots \ldots \ldots \ldots . . \ldots 67$ 
Joint diseases $\ldots \ldots \ldots \ldots \ldots \ldots 838$ Jolles ..................... 251

Joly, Major P. ............ 139

Jordon $\ldots \ldots \ldots \ldots \ldots \ldots \ldots$ 229, 246

Joslin $\ldots \ldots \ldots \ldots \ldots \ldots, 760,780$

Juneberries $\ldots \ldots \ldots \ldots \ldots \ldots .350$

Kaa lee, Paraguay .......... 157

Kaffee Hag ............. 500

Kaiser, germs in milk ....... 514

Kakowsaki ................ 301

Kale .................... 297

Kaoliang ................. 277

Karrel, milk diet ....... 580, 769

Katalases ................ 251

Kellner ...................... 160

Kennan .............. 228, 477

Keratitis, diet in .......... 934

Kidney diseases.. 421, 482, 869, 903 diet after removal ......... 878

Kitasato, exp. with fruit acids. 338

Kishi, function of thyroid ....993

Klemperer ................ 125 diet in kidney disease ..... 880

Knapp, on rice eaters ...... 273

Kohl-rabi ............... 312

Kohnstann, on meat exclusion.. 937

Kola ..................... 491

König, composition of meat ... 398

Kopoloff ................ 625

Kronecker, effects of coffee .. 498

Kumagawa ............... 124

Labbe and Leclerq, fats in diabetes .................... 782

Lactans ................... 145

Lactic acid ............... 239

Lacto-Dextrin ............ 695

Lane, diseases due to toxemia. . 611

Langer, colon bacillus ....... 550

Langworthy and Hunt, meat ... 392

Langworthy and Deuel ....... 653

Lanoline cream .............. 915

Lasegue, on the tongue $\ldots \ldots \ldots .555$

Latham, importance of clean teeth $\ldots \ldots \ldots \ldots \ldots \ldots \ldots, 721$

Lathyrism ............... 516

Lavage of tissues .......... 662

Laxative diet .........6.625, 986

Lee, on skatol and indol ...... 616

Leek ................... 293

Legendre, poisons in meat .... 400

Legumes .................... 317 for diabetics ............. 784

digestibility of $\ldots \ldots \ldots \ldots \ldots 317$

iron in $\ldots \ldots \ldots \ldots \ldots \ldots \ldots . . . . .318$

lime in .................... 319

Lehmann, on caffein ......... 491

Leipiner, potato diet ........651
Lemoine, experiment with eggs. 434

Lemons ................. 353

Lenhartz's duodenal feeding ... 697

Lentils ................... 329

Lepine, on neurasthenia ..... 863

Leszynski, coffee injures children ................... 496

Lettuce .................. 298

Leucin ................. 130

Levin ..............65, 546

on bacteria ............. 133

Levulose ................ 154

Liebig, diet of bear ......... 416 water drinking ............ 656

Lily bulbs ................ 293

Lime in body ............. 27 in cereals ............... 255

in cheese $\ldots \ldots \ldots \ldots \ldots \ldots .440$

in foodstuffs ............. 180

table .................... 184

daily loss of $\ldots \ldots \ldots \ldots \ldots . . .176$

foods deficient in . . . . . . 183

deficient in breads ........ 257

and egg production ......... 180

in eggs $\ldots \ldots \ldots \ldots \ldots \ldots .430$

foods poor in ............. 189

foods rich in ............. 188

content of fruits .......... 339

in greens ................. 305

and iron ................. 188

in legumes .............. 319

deficient in meats ............ 393

in milk ............... 440

in nuts $\ldots \ldots \ldots \ldots \ldots \ldots . \ldots \ldots 2$

ration .................. 175

starvation .............. 176

in tuberculosis ........... 910

in vegetables ............. 285

juices, composition of ..... 367

Limes ................... 355

Lime water for infants . ...... 947

Linnaeus, cherry cure ....... 572

on strawberries ............ 333

Linossier, effects of salt ...... 475

toxalbumin in eggs ........ 428

Lipase $\ldots \ldots \ldots \ldots \ldots \ldots \ldots . .62$

Lister .................. 88

Litchi nut ............... 385

Littlejohn, tuberculosis ....... 404

Liver ................. 79, 81

of adult ................ 29

effects of coffee ........... 497

congestion of ............ 655

cirrhosis of .............751

disorders, diet in ......... 745

degenerations of $\ldots \ldots \ldots \ldots .751$

fluke $\ldots \ldots \ldots \ldots \ldots \ldots . . . . .519$

of infant $\ldots \ldots \ldots \ldots \ldots \ldots, 29$ 
spots ................ 421

functions, test for ......... 746

Lockjaw .................. 893

Lobsters ................ 397

Loganberries ................ 349

Lorand ................ 847

Loufer, report on salt ....... 479

Lucus-Championniére ........ 744

Lungs of adult .............. 29 of infant ................ 29

Lusk ..................... 139 protein experiment ........621 protein in tuberculosis ...... 906 on meat diet ............. 931 milk replaces meat ......... 414

Luxus protein ............. 130

Lyon, diet of hog .......... 411

Lysin ................. 32

McCallum ................. 548

McKay and Bailey ...........9911

MacFayden ........... 552, 870

Magendie, on saliva ......... 558

Magnesium in body ......... 27

Magnon, appendicitis and diet..744

Magnus Levy, on diabetes .... 777 milk diet .............. 582

Malaria .................... 423

Malarial fever ............. 891

Malic acid ............... 231 in apples ................. 341

Malted nuts ............... 382

Maltose ................ 153

Malt sugar $\ldots \ldots \ldots \ldots \ldots \ldots$ 152, 591 for infants .............. 943

Malt soup in scurvy ........ 948

Mango ...................... 359

Manna .................... 154

Mannite ................. 154

Manny and Emerson ......... 110

Manyalla ............... 228

Martelly, sugar in toxemia .... 586

Mastication ................. 82

Martin, nephritis ............ 904

Martin, W. F. ............ 912

Maté ...............491, 501

Maurel and Quatalet .......... 16

Maxwell ................. 44

Mayer, on salt ........... 487

Mayo, on duodenal ulcer ...... 690 cause of cancer ........... 541 food in cancer ............. 915

McCann and Barr ......... 909

McCarrison .............. 202 on deficiency diseases ........ 195 diet in goiter .............. 809 vitamins in diet $\ldots \ldots \ldots \ldots .835$

McClendon ............... 805

McCollum .............. 37, 148 on beans $\ldots \ldots \ldots \ldots \ldots \ldots .320$

diet for children ........... 958

flesh foods .......... 391, 931

importance of milk ........ 438

value of milk ............. 389

McCollum and Davis ........ 201

McCord, appendicitis and meat. 744

Meakins ................. 98

Meal, barium ................. 584

Meals, drinking at ......... 540

Meats ................ 390, 678

acidosis from ........... 408

bacteria ............ 398, 407

black spot in .............. 510

canned, bacteria in ........ 412

harmful for children ....... 948

pernicious effects of .......4409

effects on animals ......... 408

not essential ............. 413

extract, toxicity of .....398, 679

condemned by Roger ....... 398

fatal to $\operatorname{dog}$............ 400

extractives ............... 398

increases metabolism ....... 117

moldy ................ 510

in tuberculosis ............. 898

source of poisons ......... 135

contraindications .......... 420

high $\ldots \ldots \ldots \ldots \ldots \ldots \ldots .400$

and indol $\ldots \ldots \ldots \ldots \ldots . . . .134$

iron of $\ldots \ldots \ldots \ldots \ldots \ldots .391$

lime, deficient in ............ 393

and nut $\ldots \ldots \ldots \ldots \ldots \ldots .383$

parasites of $\ldots \ldots \ldots \ldots \ldots, 411$

poisons of ................... 398

putrefaction in ........... 402

purins in ................. 399

Meat fed cocks ............. 402

Meatless hospital dietary .... 930

Meals, irregular ........... 537

Medical dietetics ........... 531

Melic, infected vegetables ..... 516

Mellanby ................ 721

vitamin A $\ldots \ldots \ldots \ldots 210,721$

Melon sapote ............. 359

Men, food requirement of. 115,119

Mendel ............. 118, 148, 266 on coffee $\ldots \ldots \ldots \ldots \ldots 487,849$

on meat diet ............. 931

Mendel and Osborne ........ 222

Mental disease ............. 858

Mental work, metabolism in .. 105

Merycism ............... 710

Metabolism ............... 100

basal .................. 101

diet $\ldots \ldots \ldots \ldots \ldots \ldots \ldots . . \ldots 117$

increased by meat ......... 117

in plants ............... 101

during sleep $\ldots \ldots \ldots \ldots \ldots, 101$ 
tests

and work $\ldots \ldots \ldots \ldots \ldots \ldots . \ldots \ldots$

Metchnikoff ............ 233, 549

on the colon ............ 722

Metzer-Lyon method ......... 564

Michele, poisons on milk ..... 513

Migrain .................. 422

Milk ............... 436, 678

almond ................. 375

of animals, composition of... 469

bitter .................. 453

bottles, bacteria on ....... 453

buddized ................ 457

canned .................. 449

certified ................ 457

clean ................... 451

composition of .......... 436

condensed .................. 449

and corn meal mush ........ 268

cost of .............. 468

dried ................. 444

hickory milk ............ 376

feeding $. . \ldots \ldots \ldots \ldots \ldots \ldots . \ldots 53$

how to eat .............. 466

iron content of ............443

lime content of ............. 440

medical uses of ..........4 465

mixtures ............... 939

modified ................. 451

pasteurizing $\ldots \ldots \ldots \ldots \ldots \ldots 455$

peanut ................ 382

poisoning .............. 464

products, composition ......4 436

protein ................4 438

regimen ...... 579, 585, 590, 632

regimen, technic of ....... 581

salts .............. 440, 803

sensitization to .......... 463

skimmed ................. 448

slimy and colored .......... 453

souring of ..........4 437, 452

soy bean ................ 325

sterilized ............... 455

effect on stomach ......... 678

sugar ....... 154, 591, 597, 615

sugar free ............. 803

sugar in typhoid fever ...... 892

from tuberculous cows ..... 512

infection due to ..........443

rich in vitamins .......... 439

Miller, experiments .. 47, 118, 719

effects of tea and coffee .... 497

microbes in mouth ....... 545

Millet, vitamins in ......... 277

Mineral acids ........... 241

mineral salts ........... 36

waters .................. 527

in nephritis ............ 876

Minkowski, daily water intake. 661

Mohler, bacteria in meat .... 510
Molds, poisons of ......... 515

Morelli ................. 825

Morganroth, on oleomargarine. 453

Moro ............... 439, 705

Morse, W. J. ................ 322

Mortensen, heart test ..... 571, 850

Moss, Iceland . . . . . . . . . . . 299

Irish $\ldots \ldots \ldots \ldots \ldots \ldots \ldots \ldots 300$

reindeer ................... 299

Mossé, meat in diabetes ...... 783

Motility test .............. 563

Mouth, care of ......... 589, 732

digestion $\ldots \ldots \ldots \ldots \ldots \ldots \ldots .39$

examination of .......... 555

Moynihan ................ 700

Mulberries ............... 350

Muller, indol .............. 552

effects of meat extract .... 400

Mumps ................ 893

Mung bean ................ 321

Murlin and Miller ........... 118

Murphy ................ 819

Murray, treatment of myxedema ............ 811

Muscarin ................ 507

Muscles, composition of ...... 28

Muscle tension .............. 104

Muscular work, metabolism in. 106

Mushrooms ............... 301

Muskmelon ............... 316

Mushroom intoxication ...... 508

Mutton .................. 395

Myasthenia, diet in ........ 867

Myelitis, diet in .......... 868

Myer's diet in urea ......... 872

Myer and Rose, bananas ......256

Myocarditis ........... 421, 852

Myasthenia .............. 867

Mytilotoxin ............... 507

Myxedema ................ 572

Nansen, effects of tea and coffee 492

Naunyn, bodily requirement ... 825 on acidosis .............. 825

Nausea ................ 91, 669

Nencki, on protein ....... 551, 870

Nephritis ............. 237, 877 bichloride $\ldots \ldots \ldots \ldots \ldots \ldots .877$

from common salt ........ 485

mineral waters in ......... 875

mushrooms, harmful in .... 301

Nerves of alimentary canal ... 75

Nervous disorders, diet in ....856

Nerve sensibility ............4 489

Nervousness .............. 868

Neuralgia ........... 535, 864

Neturasthenia ....... 422, 628, 863 from tea and coffee ........ 498

Neuridin ................. 507

Neurin ................. 507

Neuritis ............... 864 
Newburgh $\ldots \ldots \ldots \ldots \ldots \ldots \ldots .8 . \ldots 3$ arteriosclerosis in animals .. 847

Newburgh and Marsh, fats in diabetes ................. 782 protein for diabetics ........ 791 meat experiment ............. 409

Newburgh and Squier ........ 872

Nicate and Schulz ...t.

Nicotine ................... 69 injurious to kidneys ....... 873

Niemeyer, diet in fever ......6.620

Nitrogen of body ............ 27

Non-laxative diet ........... 645

Nose, disease of ............. 931

Nursing infants .............. 949

Nutrition, disorders of ......7760

Nutritive functions, test of ... 555

Nuts ................ 368, 971

composition of . . . . . . . . 369

creams ..................... 386

for diabetics ............ 787

digestibility of ........... 998

iron in .................. 372

lime in ............... 372

malted .................... 382

meats ........................ 384

meals ................... 386

milk (Table) .............. 374

protein ................ 136

vitamins in ............ 374

Nuttal and Thierfelder ........ 546

Nuttolene ...................... 384

Oats

272

Obesity ........ 767

diet in $\ldots \ldots \ldots \ldots \ldots \ldots . .761$

exercise ................ 760

Occupations, energy consumed in 108

Oertel, on dry diet ............. 649

method ................. 760

Ogata ................... 151

Oils, effect upon gastric secretion .............. 59

Oils, kernel ................ 143 vegetable ................... 142

Okada, white of egg .........4 426

Okra ....................... 299

Old age, changes in .......... 115 food requirement ........... 116

Oleomargarine, bacteria in .... 454

Olives ..................... 345 botulism from ............. 522

Onions ..................... 292

Operations, diet before ....... 919

Oranges .................. 350

Orange juice, for infants ..... 941

Organs in infants and adults... 29

O'Reilly and McCabe ........ 785

Origin of foods ............ 973

Osborne .................... 139 and Harris ................. 368

and Mendel ........... 89, 326

Osler .................... 802

Osteoarthritis ............. 839

Ottofy .................... 720

Osteomalacia .............. 834

Overeating ............ 533, 681

Oxalic acid .................. 232

in food (Table) ............ 237

Oxaluria ................... 880

Oxygen of body ............. 27

inhalation in pneumonia ..... 98

Oyster juice, bacteria in ...... 397

typhoid from ............. 513

vegetable ................ 312

Paget, meat eating ............ 919

Pain, delayed ................ 687

Palm cabbage ............... 297

Pancreas, diet in disease of ... 751

Pancreatic hormone ......... 585 juice ...................6. 61

Papaw ................... 359

Paradise nuts ............. 379

Paraffin .................. 58, 727

Paralysis .................. 421

Parasites in food ........... 518 of meat .................4 411

Parasitic infection ......... 738, 913 disease of intestine .........755

Paré, Ambrose, parasites in meat 411

Parsnips .................. 310

Pasteurizing milk .......... 455

Pavey, diet of Scotchman ..... 417

Pavlov .............4 46, 676, 693 on bitters ..............7.706 effects of chewing ......... 682 experiments with meats .... 678 observations on stomach .... 684

Pawpaw ..................... 359

Peaches .................... 343

Peanuts ................... 381

butter ..................... 382

flour ................. 383

milk ................... 383

oil ....................... 382

Pears .................... 343

Peas .................... 328

Pecans ................. 376

Pectin in fruits ............. 331

Pellagra ....... 215, 421, 516, 829

Pelvic colon ................. 60

Pemberton ................. 839

Pennington, bacteria in eggs... 433

Pentosans ,.................. 145

Pepper .................. 233

Pepsin ............... 51, 705

Peptic ulcer ............. 686-986

Peristaltic waves ............ 71

Peristalsis, abnormal ....... 674 
Peritonitis, diet in .......... 926

Pernicious anemia ........... 817

Persimmons ................ 443

Petersen, Carl ............... 916

Petroschky, germs in milk .... 514

Pezard, on flesh fed cocks.... 402

Phosphorus of body ......... 27

Phthisis ................ 579

Physiology of eating $. . . \ldots \ldots, 121$

Physique and diet .......... 116

Phytic acid ................ 231

Pica .................... 837

Pickerill ................. 42

Pickles .................. 474

Pidau ................... 431

Pietro, study of pellagra .....516

Pin worm ................ 519

Pineapple .................... 360

Pine nuts .................. 377

Plague ................. 891

Plants, edible .............. 969

Plant foods, poisons in ....... 515

Plato .................... 139

Pleurisy .................. 894

Plimmer ................... 207

scurvy in pigs ............ 830

Plums ................... 343

benzoic acid in 237

Plummer ..................... 804

Plutarch .................... 139

Pneumonia .............. 483, 894 salt in ................. 488

Poehl ...................... 593

Poggi ..................... 819

Poisons in clams . ........... 513

in coffee ................ 495

in colon .................. 548

detoxicated by liver ...... 75

of foods ................ 507

of meats ............ 398, 511

metallic ................ 80

in milk ................... 511

of molds ................. 515

organic ................ 80

in plant foods ............. 515

from putrefaction ......... 552

excreted by stomach . ....... 555

Poisoning, cane sugar ....... 948

Poliomyelitis ............... 891

Pollinosis ................ 33

Polya operations, diet after ...9923

Polyarthritis, acute ......... 840

Pomegranate ............... 360

Pop corn ................. 270

Pork ...................... 395

tapeworm .............. 519

Post-operative acidosis ....... 826

feeding in ulcer ........... 700

feeding $. . \ldots \ldots \ldots \ldots \ldots . . .922$

Potassium of body ......... 27
Potato cookery ........... 289

poisoning ................ 288

regimen ................. 650

sweet ..................... 290

white potato ............. 286

Potter, experiment with coffee. 496

Pregnancy, diet .............. 883

diabetes ................ 800

vomiting ................. 885

Prescott .................. 481

Preserved foods and scurvy .. 830

Priessnitz, use of water ....... 640

Prostate gland, inflammation of 882

Proteins ............... 30, 124 complete ............. 31, 136

of cereals ........... 128, 254

diet low ........ 595, 617, 981

diet in kidney work ........ 129

effect of .................. 131

quick elimination of ....... 129

in fever .................621

in intestinal putrefaction .... 133

and kidney work ......... 132

luxus .................. 130

of milk .................. 438

cause of nephritis ..........621

ration $\ldots \ldots \ldots \ldots \ldots \ldots 118,124$

ration in diabetes ........... 782

ration, high .............. 118

ration, low ................ 139

ration of infants . ......... 127

requirement . ............. 138

standard . . . . ............ 126

use in body ................ 129

vegetable $\ldots \ldots \ldots \ldots \ldots \ldots, 31$

Protein free diet . . ......617, 984

Protose ...................... 384

Prunes .................43 343

benzoic acid in ............. 237

Psoriasis ............ 422, 914

Psychic secretion, test for ..... 561

Ptomaines ............. 507, 549

Pulmonary tuberculosis ....... 898

Pumpkin ...................... 316

Purées, for infants ........... 942

Purins ................ 27, 399

Purpura .................. 834

Putrefaction in colon ........ 552

Putrescin ................... 507

Pyelitis ................... 881

Pyemia .................. 893

Pylorectomy, diet ........... 923

Pyloric obstruction in infants.. 592

Pyorrhea ................. 194

Pylorus, action of ............. 63

Pythagoras ................. 139

Quatelet and Maurel ........ 116

Quercin .................... 379

Quince $\ldots \ldots \ldots \ldots \ldots \ldots \ldots \ldots, 343$ 
Quinoa

277

Rabbits, arteriosclerosis in .... 409

Radish $\ldots \ldots \ldots \ldots \ldots \ldots . \ldots . \ldots 312$

Raspberries ............... 350

Ration, daily ........110, 119, 976

Ration for diabetics ........ 971

Raw diet ............... 651

Rectum, care of ........... 734

Rectal feeding ............. 653

Reducing diet ............. 989

Regimens and dietaries ...... 574

Regimen, fasting ........... 636

fattening $\ldots \ldots \ldots \ldots \ldots 628,985$

fruit ............... 574, 987

green .................. 577

milk ................... 581

milk, technic of ............ 583

potato ................650

restorative ..........634, 988

Regurgitation ............6.671

Rehfuss ............. 50, 158

on tea and coffee .......... 497

Reichmann and Scheffer ..... 706

Reid ..................... 153

Reindeer moss .............. 299

Relapsing fever .......... 891

Renal disease and diabetes .... 799 efficiency ................. 919

Repletion ................ 91

Restorative regimen ..... 634, 988

Rettger, lactose and milk ..... 559 bacteria in eggs ........... 433 changing flora ...........6 604

Rheumatism ............... 421 acute, diet in . . . . . . . 840 chronic ................ 838

Rheumatoid arthritis ........ 839

Rhubarb, oxalic acid in ..... 233

Rice ................. 273 eaters, report on ........... 274 polished $\ldots \ldots \ldots \ldots \ldots \ldots . . .274$ wild $\ldots \ldots \ldots \ldots \ldots \ldots \ldots . \ldots . \ldots . \ldots$

Richards, germs on fruit ...... 516

Rickett, salt experiment ...... 478 vitamins in .............. 194

Roberts, Sir William ....... 41, 232 effects of alcohol ........... 680 effects of tea .............. 500 diet in, calculus .............. 881

Robertson, Atkinson ........ 150

Rochelle salts, toxicity of .... 520

Roderick, bacteria in meat .... 407 bacteria in milk ........... 456

Roger $\ldots \ldots \ldots \ldots 48,52,158,677$ experiment ............. 554 toxicity of meat extract .... 398 tuberculous milk .......... 512

Roith ................... 72

Rother .................... 63
Roots ................... 283

and tubers $\ldots \ldots \ldots \ldots \ldots .967$

Root starches ............. 283

vegetables ................ 310

Rose, Dr. Mary S. .......... 311

Rosebloom, low protein diet ...8 810

Rosenau, milk in tuberculosis.. 453

Ross, observations of meat diet. 916 potash .................. 264

Roux, experiment ......... 546

Rubner .................. 139 nutrient value of corn ....... 267 protein in diet ............ 535 protein experiment ........621 on protein ration .......... 127 protein in tuberculosis ...... 906 Ruhrah and Friedenwald ...... 905 Rumford, Count ......... 85, 288 Rusby, caffein as a poison ..... 493 Rush, Benjamin ............ 474 Rye $\ldots . . . \ldots \ldots \ldots \ldots . . .276$ Ryle ......... 50, 89, 686, 834

Saccharine ............. 157, 801

Sago ................... 291

Sakaguchi, on diabetes ....... 780

Salads .................. 309

Salant and Wise, citric acid ...2231

Salisbury .................. 654 method in obesity ......... 770

Saliva ............. 40, 558 effects of acids on ......... 42 deficient...$\ldots \ldots \ldots \ldots \ldots .43$ diminished in starvation .... 591 diseased ................ 43 protective action of ...... 42

Salivary digestion..$\ldots \ldots \ldots .41$

Salkowski, indol .............. 552

Salsify ................... 312

Salt in animal tissues ........ 474 Bright's disease ........... 485 common ............ 474, 681 experiment ............... 481 food .................. 36, 160 in foods (Table) .......... 482 daily loss of $\ldots \ldots \ldots \ldots \ldots, 36$ hunger .................... 476 of milk ............ 440, 803 poisonous effects ........... 479 suicide by ................ 479

Salt-free diet .............. 482

Saltless diet in epilepsy ....... 860

Sanderson, flesh abstainer ..... 423

Sarcin in meat ............... 399

Satiety ................... 90 caused by fats ............ 36

Sauerkraut ................ 295 composition of ........... 296

Savarin, use of coffee ........ 499

Sansum and Blatherwick ...... 851 
Sapelier, diet in Bright's disease 873

Sayre, experiment with coffee.. 495

Scarlet fever .................. 482 salt in ................ 488

Schibkow, acetic acid a poison. 471

Schiff, observations on bile ... 677

Schlossman, proteins ........ 439

Schmidt, on colon .... 549, 725, 753

Schultz and Nicate,

intestinal antiseptics ......... 553

Scientific fasting ........... 644

Scully and Greene, on parasites 756

Scurvy in babies ............. 455

diet in ............... 830

and malt soup ............. 948

meat unnecessary in ....... 832

sprouted grain in .......... 833

Sea foods ................ 397

Seasickness, diet in .......... 866

Secretion, psychic ............ 561

Seeds .................. 967

Senator, diet in kidney disease. 906

Seneca ................... 139

Senn, observations of vegetarians $\ldots \ldots \ldots \ldots \ldots \ldots \ldots \ldots, 744$

Sensitization .............. 953

Septicemia ................ 834

Service berries .............. 350

Sesame seed meal ............281

Shackleton ................ 34

Sherman. 83, 137, 168, 189, 203, 819

on cereal protein ......... 129

diet for children ............ 956

experiments ................ 438

food iron ................. 393

lime starvation $\quad \ldots \ldots \ldots \ldots . . . .177$

iron in meat ... . . . . .......... 389

value of milk ............. 389

protein in diet ............ 535

protein ration of .......... 126

vitamin $\mathrm{A}$................ 201

affect of cooking on vitamins 200

Shoemaker, effects of caffein. . 497

Short, experiments with rats... 194

Sick headache ............... 500

Silicon in body ............ 27

Simmons .................. 893

Sinclair, sour milk cure ........ 580

diet ................... 272

Singultus $\ldots \ldots \ldots \ldots \ldots \ldots \ldots .711$

Sitophobia $\ldots \ldots \ldots \ldots \ldots \ldots \ldots . .864$

Sittlar, on diet .............. 594

Siven ................... 125

Skatol .................... 544

fatigue poison ............ 616

Skin, diet in disease of ...... 913

discases ................. 628

disorders ................. 422

Sleep, hunger in ........... 92

Slonaker, exp. on rats ....... 651
Small intestine, digestion in ... 60

Smallpox ................. 483

Smith, Barclay, on colon ....... 722

Smith, Edward, caffein as a drug 493

Smith, Russel ............... 382

Smoking, injurious to kidneys.. 873

Snyder, appendicitis and diet... 744

cabbage ................. 248

Socrates .................. 139

Soda fountain drinks ......... 503

Sodium, benzoate of ......... 520

Sodium chloride ............ 474

in body ................. 27

in foods (Table) .......... 482

Soil, clean ............... 521

Soper, poisons in clam ........ 520

Soup for scurvy .............. 948 vegetable for infants ....... 946

Soy bean ................. 322

composition of $\ldots \ldots \ldots \ldots \ldots 324$

milk .............. 325, 326

sauce $. . \ldots \ldots \ldots \ldots \ldots \ldots \ldots . . . . .327$

sprouts, composition of ..... 328

Spallanzani .............. 83

Spinach ................ 308

Sprue ................ 421, 427

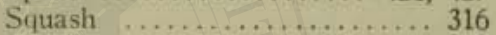

Squier and Newburgh ....... 872

Stange ................. 822

Star ................... 856

Starch ................ 33, 145

digestibility of ...........283

raw, digestibility of ...... 679

Starches, root ............. 283

effect of temperature ....... 247

to remove from vegetables.. 784

Starvation ............... 91

Statkewitsch, fasting ........ 637

Starvation, hunger in ....... 91

Steenbock, vitamins ........ 200

Steenbock and Gross experiment 310

Stefansson, on salt ........ 480

Steinitz, egg white ......... 426

Sternberg, meat in tuberculosis 909

Stiles, parasites in meat ......4 411

Stockard .................. 489

Stomach, acute dilatation of ..709

care of ................ 732

effects of heat and cold .... 71

disinfection ..............6.6. 662

gas in ................. 672

movements of ............ 68

passage of food .......... 69

normally sterile ........... 545

Stomatitis ............... 712

Stone and gravel in kidney .... 880

Stools, examination ........ 566

infant's ................ 949

Strawberries ............... 347

Strength tests ............. 527 
String beans ............... 321

Stuckey .................. 932

Succi, effects of fasting ...... 637

salt experiment ............ 477

Succotash ................ 322

Suet $\ldots \ldots \ldots \ldots \ldots \ldots \ldots . \ldots . \ldots . \ldots 394$

Sugars ........ 145, 149, 155, 632

consumption of .......... 159

tolerance in diabetes ....... 794

formula in ulcer ............ 694

in fruits ................ 331

malt ................. 591

milk ............154, 591, 597

effect on stomach .........679

substitutes for ............. 156

odd .................... 159

Sugar-free milk ........... 803

Suicide by salt ............4 483

Sulphur of body ............ 27

Sun, the source of food ...... 29

Supper ................. 980

Supplementary feeding ........ 590

Surgical cases, diet in . . .....9919

Swallowing ............... 66

Sydenham, milk cure ........ 580

and Rollo ............... 782

Syphilis .............. 423, 488 diet in ................ 911

Tadpoles, effects of feeding ... 118

Tallquist, dietetic experiments.. 751

Tannic acid ............ 237,680 in tea and coffee ..........497

Tapeworm ............... 759

pork .................... 519

Tapioca ................... 292

Tarahumara Indians ........ 271

Taro ........................ 293

Tartaric acid ............... 230

Taste ................... 43

Taylor ....... 41, 53, 55, 262, 677 on acid protein ........... 561 experiment with colon poisons 677 baking powders ............ 263 on protein ration .......... 128 vegetarian diet ........... 413

Tea ...................... 490

and coffee, effects of .......4496 and coffee neurasthenia ..... 498 a drug ................... 490 oxalic acid in ............ 233

Tannic acid in ............. 237

Teeth .................... 557 diet for caries of ........... 719

Tenia echinococcus .......... 519

Tension, muscular ........... 104

Teosinte .................. 267

Tests for acidosis ............ 572 breakfast ................ 559 for chemical secretion ...... 561 of gastric digestion ....... 561

of heart and circulation ..... 573

for liver function .......... 746

fractional meal ............. 559

meal, Klemperer ........... \$61

test of metabolism ......... 571

motility ................ 563

of nutritive functions ...... 555

for psychic secretions ....... 561

of stools ................. 566

of strength ............... 572

of urine ................ 570

of water ............... 527

Tetanin .................... 507

Tetanus _........................ 893

Tetany ..................... 899

Thirst .................. 95

Thompson, effects of coilee.... 497

diet in goiter .............. 808

Threadworm ............... 519

Throat, disease of ......... 531

Thyroid gland, degeneration of. 902

Tibbles, effect of caffein ...... 495 cellulose .............. 222

fever diet ...............6.623

fine flour . ............. 260

lacto-vegetarian dietary .... 616

lime water useless in diet ...9947

nephritis ..............236

salt in diet ................480

effects of tea ............ 498

effects of uric acid ........241

Tic-douloureux ............ 868

Tissier, non-flesh dietary ..... 598

sugar in toxemia .......... 586

toxins in colon ........... 552

Tissues, lavage of ............ 662

Tlascalans, deprived of salt ...481

Tobacco ............... 504, 680 effects of ............... 504

heart poison...$\ldots \ldots \ldots \ldots .504$

To $\mathrm{fu} \ldots \ldots \ldots \ldots \ldots \ldots \ldots, 326$

Tomatoes ............... 316, 681

vitamins in juice ......... 315

Tongue, coated ..... 555, 577, 672

Torindo, use of salt ......... 485

Torrey, bread and milk diet ... 616 exp. with lactose and dextrin .......... 598, 602 milk sugar in fever ........ 892

Toxemia, intestinal ..... 545, 577

Toxicity, gastric ........... 554 of certain foodstuffs ........ 520

Toxins, food .............. 508

Transfusion ................ 823

Treves .................... 187

reverse peristal jis .........666 666

Trichina spiralis ............. 519

Trichinosis ................. 913

True, food lime ........... 180 
Tsuji, on rats $\ldots . \ldots . \ldots \ldots \ldots \ldots, 807$

Tube feeding ............... 692 feeding formula ........... 695

Tubers .................. 283 and roots .............. 967

Tubercular ascites .......... 489

Tuberculosis ............ 422, 628 in cattle ................ 404 intestinal, diet in ........... 910 milk ................. 512 pulmonary ............. 899 pulmonary, diet in .......... 908

Turner, spinach ............... 302

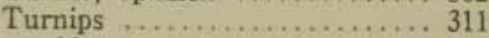
cabbage ................. 312

Typhoid fever ....... 482, 488, 892 caused by oysters ......... 513

Typhotoxin ................ 507

Typhus fever ............... 893

Tyrosin ............... 130

Tyrotoxicon $\ldots \ldots \ldots \ldots \ldots \ldots . . .507$

Ulcer diet duodenal and gastric ..... 423, 628, 686, 691 peptic ...............686 of the stomach ........... 543

Ulceration of colon, diet for. . 741

Ulldom, liver congestion ...... 901

Umana .................. 740

Umber, diet in scurvy ........ 831

Underfeeding ............. 536

Uremia .................. 814

Urethritis, diet in ......... 882

Uric acid ................... 239

disorder, diet in ........... 841

in foods (Table) .......... 243

toxic effects of ............ 243

in tea and coffee .......... 495

Urine ................. 569

collection of ............ 570

in diabetes ................. 801

effects of protein ........... 131

tests of ............... 570

Urticaria ............ 433, 712 caused by eggs $\ldots \ldots \ldots \ldots \ldots 433$

Vagatonia .................. 865

Van Ermengen, fruit acids .... 338

Van Slyke, protein elimination. 129 nuts ................... 368

Van Swieten ............... 580

Vander Bogert ............. 950

Vaughan, cheese poisoning .... 515 toxins in Bright's disease ...875

Vegetables ............. 282, 969 broths for infants ......... 942 cellulose in ..............220 disinfection of ............... 335 dried $\ldots \ldots \ldots \ldots \ldots \ldots \ldots . \ldots \ldots$

fats ................. 143

fruits $\ldots \ldots \ldots \ldots \ldots \ldots . . \ldots \ldots$

greens ................. 294

greens, for diabetics ....... 784

infected ............. 335, 516

and indol ................ 135

iron in ................ 285

lime in ................ 286

marrow ................. 316

oysters $\ldots \ldots \ldots \ldots \ldots \ldots \ldots . \ldots \ldots \ldots$

root ..................... 310

soup for infants .......... 946

starch-free .............. 784

water in .................. 249

Venison ........................ 397

Vinegar ............. 232, 680

Vines, ferments in plant juices. 252

Vinson, on dates ............... 358

Viscera, objectionable ........ 400

Vitamins ............... 37, 194

Vitamin A ............. 203, 208

Vitamin B ........... 200, 209

Vitamin C ........... 197, 209

Vitamins D and E ....... 206, 207

Vitamins, how destroyed ..... 216

and dental decay .......... 720

of foods ............ 210, 214

infants ................... 215

lack of ............... 197

of milk ................... 439

in millet ................. 277

of nuts . . . . . . . . . . . 374

sources of (Table) ......... 215

in tomato juice ........... 315

of yeast extract ..........203

Vogeler ................ 69

Voit, diet standard of ......... 118 experiment on pigeons ...... 178

Vomiting .............6.68, 668 cyclic ................ 711 of infants ............... 951

Von Noorden ........... 162, 175

on cellulose ..............224

on diet for children ....... 820

on dry diet ..............6 649

on fasting $\ldots \ldots \ldots \ldots \ldots \ldots 639$

on fruit in renal disease .... 876

on meat in diabetes ......... 783

method .................. 769

on mineral waters ......... 528

on obesity .............. 763

on uric acid in meats ...... 396

Wahl ................... 262

Wahlinski ............... 821

Walking, energy used in ...... 106

Walnuts .............. 371, 377

Walter, diet in tuberculosis ... 909 
Warren, on blood cells

Water .......................679

brash ...................671 67

carbonated ...............681

chestnut ................293

in body ................ 28

distilled ................. 528

drinking ............ 517, 656

fasting $\ldots \ldots \ldots \ldots \ldots \ldots, 640$

purification by chlorine ..... 529

for infants .............. 947

intake ................ 768

meal ......................561

mineral ............... 528

drinking in nephritis ....... 878

tests for purity ............ 527

qualitative examination ...... 527

secreted by stomach ....... 60

Watermelon ............... 315

Watson, Chalmers, diet in goiter 805 diet in autointoxication .... 598

Weaning .................. 950

Wearn, on blood cells ........821

Weber, food in old age ....... 415

Wedekind, caffein in goiter .... 503

Wegele, meat in autointoxication 598

Weight $\ldots \ldots \ldots \ldots \ldots \ldots \ldots . \ldots \ldots 72$ boys and girls ............ 111 and height $\ldots \ldots \ldots \ldots \ldots \ldots . \ldots . \ldots 114$ loss in fasting ........... 638 reduction by exercise ........ 766

Weinzirl and Newton, bacteria in meat ............... 510

Welch's bacillus ........... 602

Wells and Zilva, vitamins ..... 198

Weseberg, poisons in meat .... 512

West, George ............... 225

Wheat .................. 257

composition of ........... 260

bran ................... 263

Whey .................... 450

in fever ............... 893
Whipworm $\ldots . . \ldots \ldots \ldots \ldots . . .519$

Whooping cough ............ 891

Whortleberries .............. 350

Widal's test .................. 747

Widal and Javal ............. 478

Wiley, benzoic acid .......... 238

caffein in coffee ........... 859

caffein in cola drinks ....... 503

Williams, on caffein ......... 859

on cancer ................ 916

Williamson, on diabetes ...... 777

Winfield, cure of psoriasis .... 729

Winter and Smith, yeast extract 790

Winters, meat for children ....9948

Women, diet in diseases of....883

food requirement of ....115, 119

Woodhead ............... 487

Woodyat, protein for diabetics.. 792

Work, energy expended ....... 106

equivalent of food .......... 99

food requirement ........... 108

metabolism ................ 106

vital .................... 100

mental ................... 105

Worms ...................... 519

Wright .................. 549

Yautia ................... 292

Yeast and yeast extracts ..... 301

in diabetes ............... 790

products ................ 294

vitamins $\ldots \ldots \ldots \ldots \ldots \ldots .203$

Yellow fever ................ 891

Yogurt cheese ................ 443

Youth, food requirement ...... 119

Zander, fasting ...........6 638

Zein $\ldots . . . \ldots \ldots \ldots . . . . .267$

Zilva and Wells, vitamins ..... 198

Zine in body .............. 27

Zuntz .................. 766 


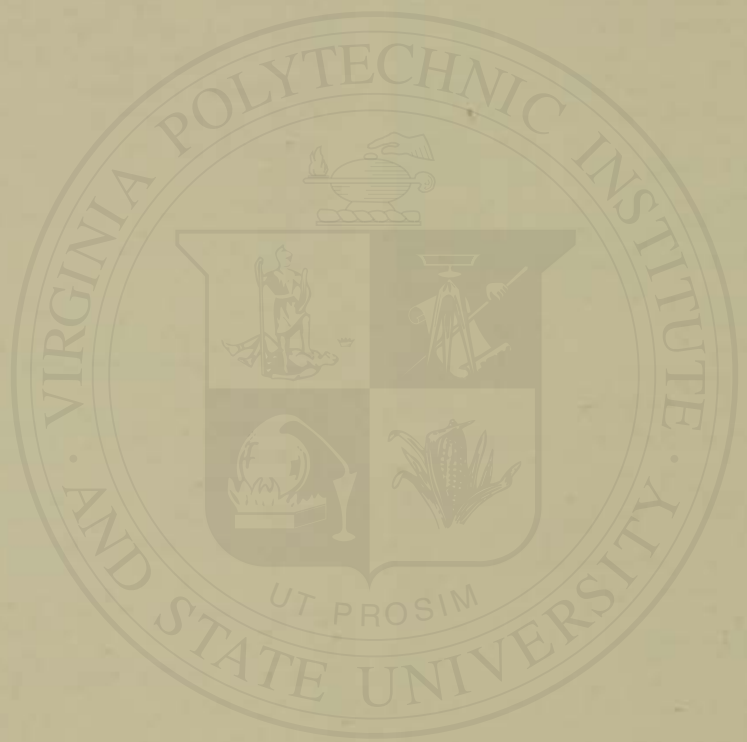




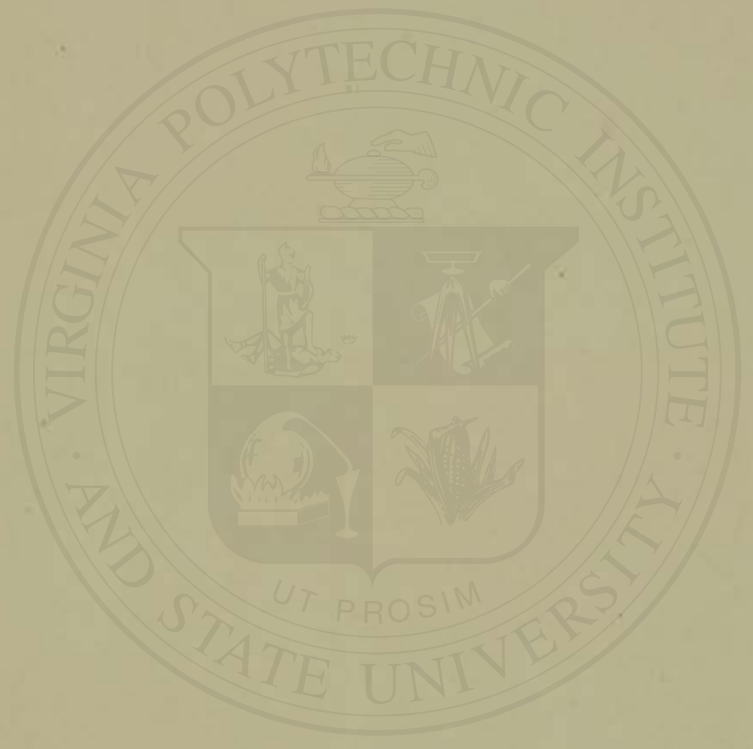




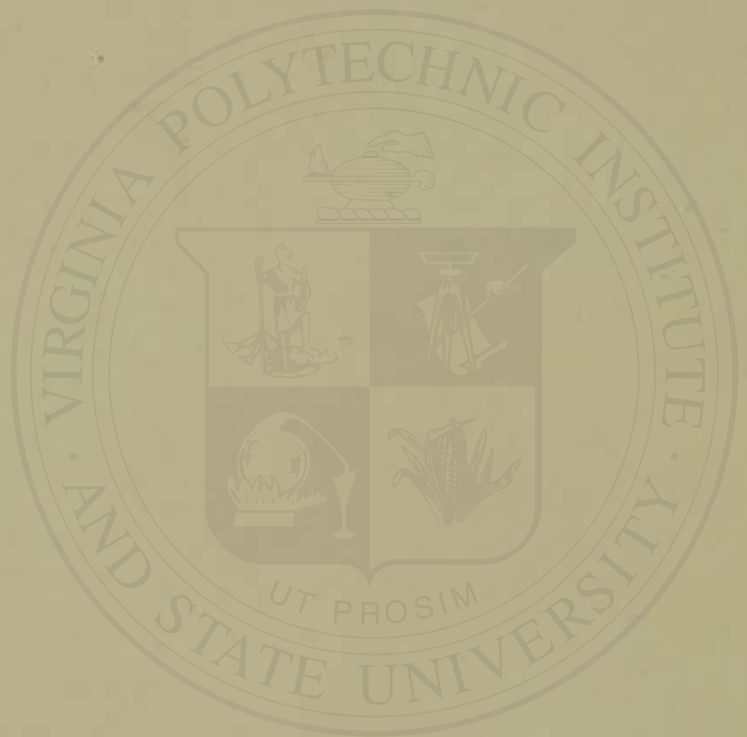




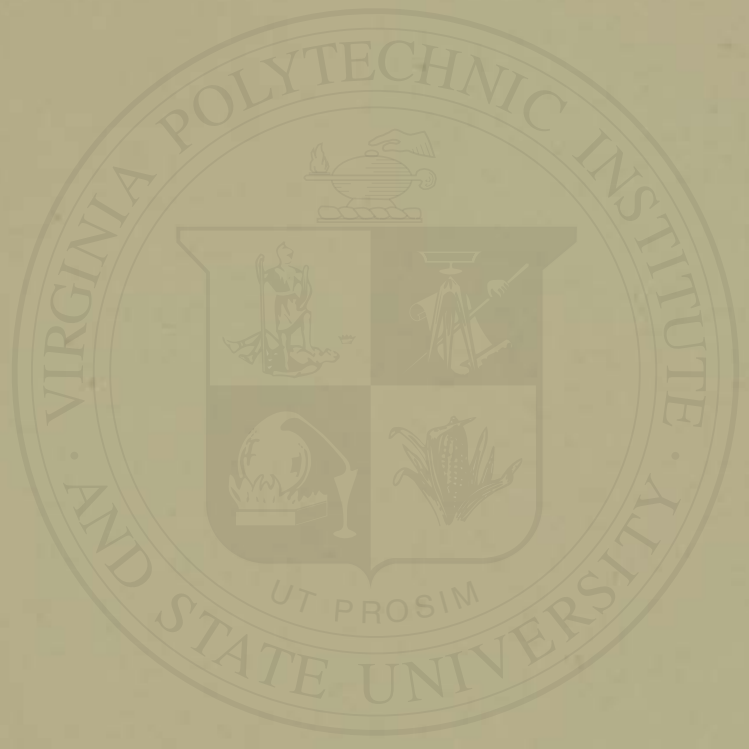


